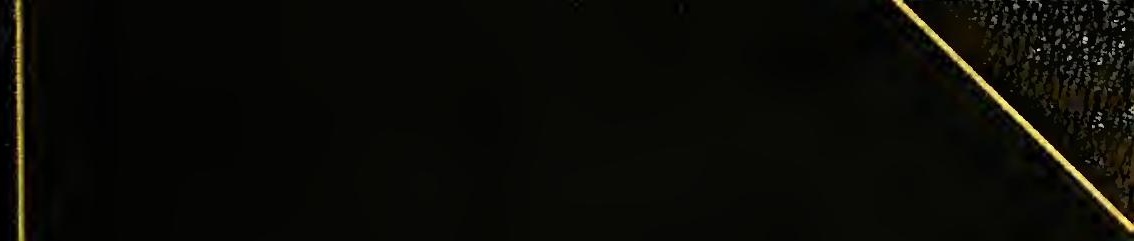




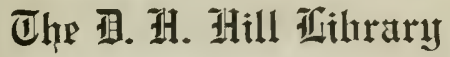

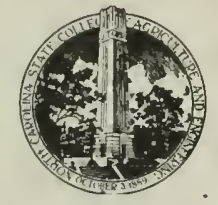

North Carnlina State Collpge

S501

F62

v.1

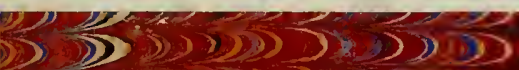

$3 \times 2$

3) 520

2DDD $D 20150$

D)2) 20

D) 201

D) 2.

1) 10312

D)

5) $) 23$

(2)$$
\text { (2) } \rightarrow \text { t } 5
$$

1) 202010

(2)

D) 5

)

D)

2)

()

2

) $)$

D.

S) 5

$52) 2$

D) 2. 7

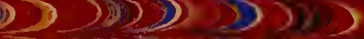

3 2D)

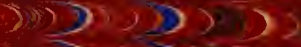

D) 5

1) 2233

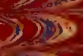

3202

()) $2 \times(2)$

525.9

(3) 20

D) 3

D)D

1.) 23

D 230

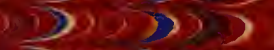

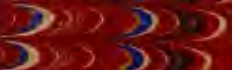

(D) $D$ D D

3

1.)

D)

D2

5

3

3

3

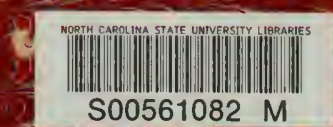

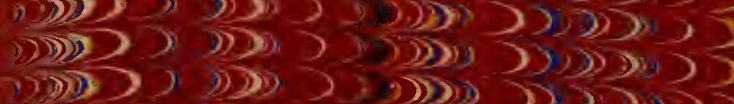

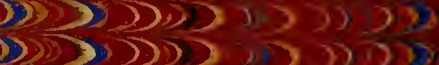

D)DDD.?

(1) $3, \frac{1}{D}$

3.) $3 \times 3$

D) 38

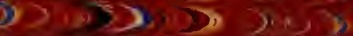

D) $D$ D

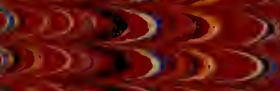

(1)

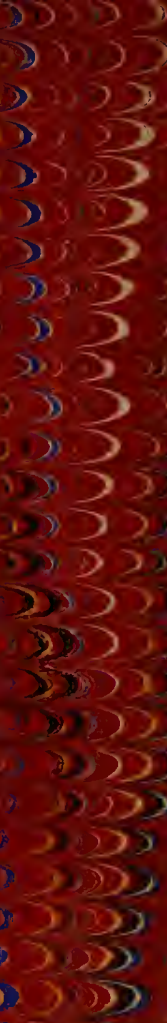

$2,23,513$

(2) 23,5

(1) 020,5

(3) - (2) 3

D. $)(25) D$

32020

2.5)

(2) 3) ) 200.12 3) 2) 2059 D) 000

D) $D$

3020

D)

298

D) $7 \times 3$

(2) 321

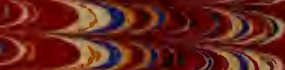

D) 53.35010

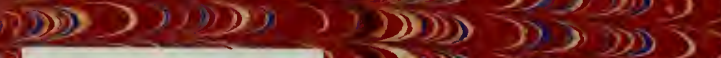

202030303

D) 2312 (D) 130015

D) 1202

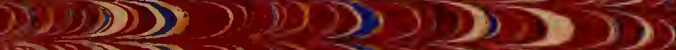

DD) 0215

(2)D 20

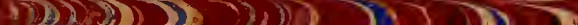

3i) 235 


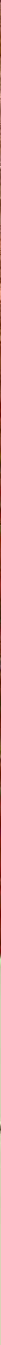


* 


Digitized by the Internet Archive in 2010 with funding from

NCSU Libraries 


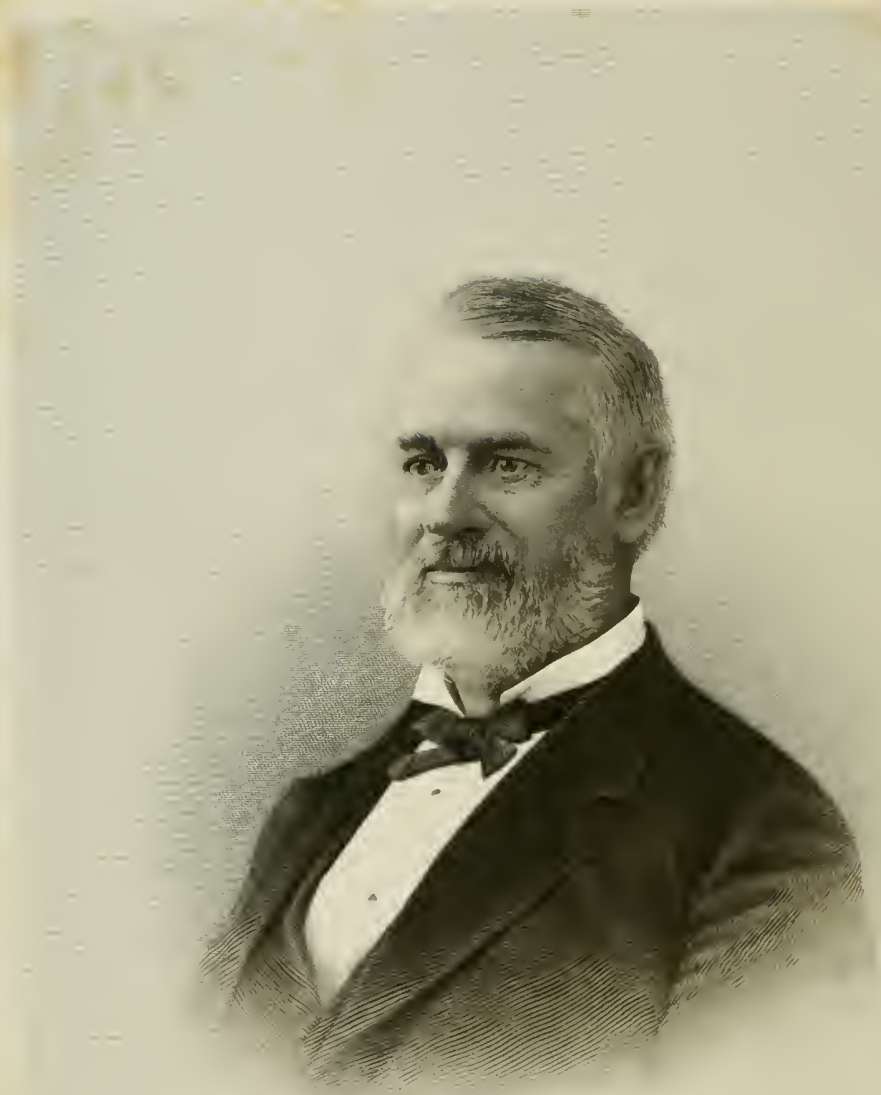

Stlarces hitenin) 


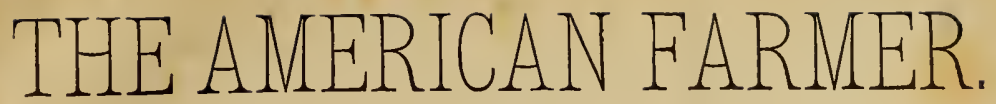

A

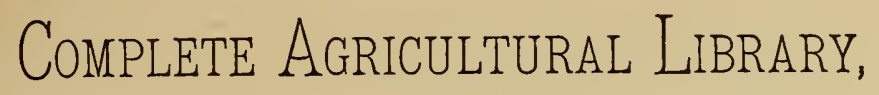

WITH

\section{USEFUL FACTS FOR THE HOUSEHOLD,}

DEVTOTED TO

FARIING IN ALL ITS DEPARTMENTS AND DETAILS, INCLUDING THE DAIRY, ORCHARD; VINEYARD, GARDEN, DOMESTIC ANIMALS, THEIR BREEDING,

MANAGEMENT, AND DISEASES; BEES AND THEIR MANAGEMENT,

FISH CULTURE, SILK CULTURE, ARCHITECTURAL DESIGNS

FOR HOUSES AND OTHER FARM BUILDINGS, IM-

PROVED SANITARY CONDITION OF COUNTRY

HOMES, Eтc., Eтc.

EDITED BY

CHARLES L. FLINT,

Secretary of the State Board of Agriculture of Massachusetts for twenty-eight consecutive years, and Author of "Grasses and Forage Plants," "Milch Cows and Dairy Farming," "Manual of Agriculture,"

Editor of "Hsrris on Iosects," Etc., Etc.

\section{9⿺辶一巛工}

\section{COMPLETE IN TWO VOLUMES. VOL. I.}

\footnotetext{
"Two men I booor and no third. First the toil-worn craftsman, that with earth-made implement lahoriously conquers the earth and makes her man's."-TномAB CARLTLE.

"The city is always recruited from the country. The men in cities who are the centres of energy, the driving-wheels of trade, politics, or practical arte, and the women of beauty and genins are the children or grandchildren of farmers."-RALPH WALDO EMEBSON.
}

HARTFORD, CONN.:

RALPE H. PARK \& CO. 1882 
COPYRIGHTED, 1892,

BY RALPH H. PARK \& CO.

ALL RIGHTS RESERVED.

THE CASE, LOCEWOOD, \& BRANNARD CO.,
PRTTERS AND BINDER. 
To

\section{HoN. MARSHALL P. WILDER,}

PRESIDENT OF THE AMERICAN PONOLOGICAL SOCIETY.

THE ENLIGHTENED AND LIBERAL FRIEND AND PATRON

or

AGRICULTURAL AND HORTICULTURAL PROGRESS IN AMERICA,

This : Notume is :

BY

THE AUTHOR. 



\section{PREF A OE.}

THe prosperity of a country depends chiefly upon the intelligent cultivation of its soil. No one can doubt that. The intelligent cultivation of the soil depends upon information. This is equally plain. Practical and theoretical knowledge is power in agriculture, as in everything else. It lies at the foundation of individual and national wealth. Those who know the most will, in the long run, do the best work at the best time and in the best way. They will work out the best results, both for themselves and for the community in which they live.

The progress made within the last twenty years in the sciences which are intimately connected with the cultivation of the soil is something wonderful. It has opened new fields of thought, of investigation, and of experiment. During that time the science of clemistry has made rapid advances, and its methods of investigation have been greatly improved. It has brought its contributions to agriculture from a great variety of sources, each of which brings something peculiar to itself. It has explained the composition of soils and manures. It has added greatly to our stock of knowledge as to the methods of feeding animals to obtain special results, giving us accurate information of the composition of feeding substances, and the effect of different processes of feeding upon the development of the animal economy.

At the same time botany has solved the mysteries of plant-growth, and, with the aid of vegetable physiology, has thrown a flood of light upon the elements of plant-food, and the means by which the production of crops can be greatly promoted. All the sciences, indeed, have contributed to the development of agriculture, some of them directly, others by leading the minds of men to a higher standard of intellectual activity.

The agricultural literature of the country has grown up almost entirely within the last quarter of a century, and has taken a rank worthy of the importance of the industry to the development of which it is devoted. Thirty years ago there were few works on farming in this country that were at all creditable either in style or the information they contained. Most of the books on the subject were English, or reprints of English publications. They were not well adapted to meet the wants of American farmers. They often contained much that was interesting, but generally lacked that full and practical information based on actual experiment that was well calculated to meet the wants of daily life upon the farm. They abounded in theories rather than facts.

The present work is designed to embody the most practical information upon agriculture, the results of scientific investigation in the laboratory, and of experiment in the field. It is comprehensive in plan, treating of a great variety of subjects so fully as to.make it unnecessary to purchase many books on special topics-a farmer's library, in fact, condensed within the covers of a single volume. It aims to present the most intelligent practice of the best cultivators in all sections of the country, and to meet the wants of every practical farmer who desires to rise above the drudgery of his calling. It does not deal to any extent in theories. The effort has been to present facts. They have been gleaned from many fields, from conversations with intelligent farmers, from the talks at farmers' clubs, from the agricultural press, and from actual experience on the farm. 
Other books of less comprehensive character have appeared from time to time, and they are all more or less valuable and instructive, but it is some years since they appeared, while changes have taken place in the condition of many parts of the country, owing to the rapid growth of the means of transportation, and the consequent modifications in the systems of farming. The farmer, like the merchant, is under the necessity of keeping up with the times, in order not to be left behind in the close competition which he has to meet. Principles may not change, but practice must be flexible enough to adapt itself to the present condition of things and the wants of the market in which the infinite variety of the products of the farm are to be sold.

This volume is committed to the press in the confident hope and belief that it will prove to be of permanent value and helpfulness to every farmer into whose hands it may fall.

CHARLES L. FLINT.

Boston, Mass., January, 1882. 


\section{The Publishers' Announcement.}

In presenting "The AMERican FARMER" to the general public, it is proper to state that it has been the object and aim of the publishers to make it both comprehensive and exhaustive; in brief, an authoritative standard work on general and scientific agriculture, in all its departments and details, and the best work in this class of literature ever published in this or any other country. To this end we have spared neither time nor expense, and flatter ourselves that the "well done" of the American Public awaits us.

The editor of this work, Hon. Chas. L. Flint, of Boston, Mass., needs no introduction to the Agricultural World, nor does he need the encomiums of the publishers ; for his twentyseven annual reports on the "Agriculture of Massachusetts," issued during twenty-eight consecutive years of service as Secretary of the Massachusetts Board of Agriculture, his "Grasses and Forage Plants," "Milch Cows and Dairy Farming," "Manual of Agriculture," "Harris on Insects," and his numerous essays, lectures, and contributions to the press, all stamp him as a practical man, and able authority on all that pertains to advanced agriculture.

Now when we add, that the youth and early manhood of Mr. Flint were passed upon the home farm at Middleton, Mass., this work may be justly regarded as the choice fruitage of a life devoted to the interests of Agriculture-a union of practice and theory; a secure guaranty, as to the entire trustworthiness, of the facts for farmers contained in this volume.

This, his latest production,- - possibly his valedictory to the Agricultural World,-will be welcomed in many a farmer's home, not only for its fullness and completeness in every department of which it treats, but also for the plain and practical discussion of all subjects; the reader understanding clearly the abundant facts, principles, and suggestions found herein, touching the cultivation of the soil, which, when rightly understood and utilized by the intelligent, practical farmers of our country, becomes at once, not only the foundation of our highest national prosperity and wealth, but the very keystone of our body politic. .

The publishers congratulate themselves that they have been so fortunate as to secure the services of so high an authority on practical and scientific agriculture for the eaitor of this work; nor should they neglect to congratulate the American Public that they are to be the recipients of Mr. Flint's valuable work, and able to avail themselves of the many advantages that such a volume as this affords.

As might be anticipated, this work is very appropriately dedicated to the Hon. Marshall P. Wilder, of Boston, the life-long friend of the farmer and the personal friend of Mr. Flint, being officially associated with the latter in the Agriculture of Massachusetts and the country for over thirty years. Col. Wilder, now in his eighty-fourth year, (of whom Mr. Flint gives a brief memoir in connection with our Fruit Department,) was born at Rindge, New Hampshire, in 1798, and has taken an active interest in agricultural pursuits all his life, having been one of the foremost in organizing the "Massachusetts State Board of Agriculture," and also the "State Agricultural College ;" besides being the President of the "Massachusetts Horticultural Society" from 1840 to 1848, and of the "United States Agricultural Society" from 1852 to 1858 , and the President of the "American Pomological Society" since its formation in 1848. In this connection it is proper to remark, that it was through the personal solicita- 
tion of the publishers, that Col. Wilder permitted us to place his portrait in our work; and we are confident that his many friends in the United States and Canada will thank us for the favor. We present also, as a fitting frontispiece to this work, an excellent steel engraving of its editor, whose genial face, as represented, is a speaking picture of the man, of whom we may say truthfully: "All his works do praise him."

Without disparaging any of the few agricultural works now before the public, we may say generally of books of this sort, as Daniel Webster once said of the legal profession: "There's always room at the top"; at this point, only expressing the hope that happily a critical public will accord to our book that honorable place in agricultural literature, which we believe it merits.

The methods of tillage and fertilization of the soil, the cultivation of that portion of the vegetable kingdom here presented, whether of products in the East, West, North, or South, will be found not only entirely reliable, but the latest word on the subject treated, and sug. gestive of still better conditions of improved agriculture in the future.

Broad and liberal, as well as bumane views are here inculcated with reference to the breeding and management of all domestic animals; the hundreds of engravings in steel and wood being an attractive as well as instructive feature of this exhibit.

The systematic arrangement of this work into Parts and Departments, with Sub-headings, greatly adds to its utility by making it convenient for ready reference, and invaluable, if not indispensable, to all farmers, and those interested in general agriculture, as well as to fanciers of fine stock of whatever variety or breed.

In conclusion, it is our agreeable duty to acknowledge our indebtedness to the late United States Commissioner of Agriculture, Hon. Wm. G. Le Duc, and his successor, Hon. George B. Loring, Hon. Marshall P. Wilder, Hon. Spencer F. Baird, Director of the United States Fish Commission, and Seth Green, Esq., from all of whom we have received valuable assistance; to the Commissioners of Agriculture generally in the several States who have favored us with copies of their reports and other important documents; to Prof. C. V. Riley, Entomological Department of Agriculture, Washington, D. C., for valuable printed documents, to Professors Brewer, Johnson, and other leading agricultural writers. Our acknowledgments are also due to the agncultural press generally, to the Librarian of the Massachusetts Agncultural Society, and to the officers of the Boston Public Library, who have greatly aided us in the prosecution of this work; to Hon. J. H. Pope, Minister of Agriculture in the Canadas, who has rendered great assistance by reports and other documents; and finally, to that agrculturist of world-wide reputation who established, and for nearly fifty years has conducted an "Experiment Station" on his estate at his own expense, and solely for the advancement of knowledge in agriculture, the Hon. J. B. Lawes, LL.D., of Rothamsted, St. Albans, England; we would also tender our sincere thanks for the many valuable documents he has furnished us, giving the rich results of his experiments and investigations.

Very respectfully,

RALPH H. PARK \& CO

HARTFord, Ct., Jan. 1882. 


\section{LIST OF FULL PAGE PLATES.}

ENGRAVING OF THE EDitor,

PAGE.

Colored Lithograpil of Trotting Stallion, Gov, Spragde, . . . . . . 2

BARN-TARD GRAss,

Bermuda Grass, Wire Grasi, .

Broom Grass, or SEDGE,

BRoOM SEDGE,

Champion Road-Grader at Work, .

Clevelayd Bay STALlion, "BAY SPLeNDOR,

Chydesdale Stallion, "The o' Day,"

Cock's Foot Griss,. .187

Cotton Gin (Power), . . . . . . . . . . . . . . . . . 398

Crab Grass,

Crow Foot Grass,

Design for a Countri House.

Dog's Tall Grass,

Doctelgeared Plantation Cane Mill,

Draco Prince,

Drop-Seed, Nimble WILI,

Echo Fari Buildings and Jersey Group,

Eclipse Wind Engine,

ENelish Draft Stallion, "Lincoli," .

Egyptian Grass,

Erecting Barb Wire Fence,

Feather Grass,

Field Pitchixg Apparatus,

Fowl Meadow Grass,

Gava Grass,

Gathering TeA,

Grain Dropper,

Grasseoppers iN a Wheat Field, . . . . . . . . . . . . . 261

Half-Blood Percheron Norman Stallion, "Napoleon III," . . . . . . 706

H IYPUETOYIAY STALLION, "SATELLITE,"

HARVESTER AND TWTNE-BINDER, . . . . . 269

Hay and Stgar-Cane SCalies,

Hay Elevator aNd Cirrier,

HaY RaKes,

IIORSE Power,

Hitratlic RaM in Operation,

Improved Fary EygiNe witu Iroy Truck TVIIETEL,

INDIAN Grass,

InoN Mower,

Irox Turbine Wind Engine,

JaCk, "Magnem Bondm," .

JAPAN Clover, 
Jersey Bull, "Litchfield," Johnson Griss, .

Kinm's MANuRE-SPREADER,

Kextucky Buue Grass,

LEAF OF TEA,

Means Grass,

Mextcan Clover,

Model Barn, Nontueast View,

" " NoRThinest View,

" " SOctheast View,

Matrtarn Red-Top, Northern Red-Top,

"Mountain-Side Farm," Front View of Barn,

$$
\text { " " " Rear }
$$

Orloff Stallion, "Sobol,"

Percheron-Norman Stallion, "Eldorado," .

"Rovelos,"

Pigeon Grask, Buille Grass,

Power Cotton Gin,

RED-TOP,

Rescue Grass,

Rocky Mountain Locdst, .

RYSDYK'S HAMRLETONIAN, .

Scene on the Grandin Farm, Dakota.-Twenty Gano Plows in Operation,

Self-Binding Harvester,

Sequoda Gigaftea-Giant Red Wood,

Sirut Grass,

Steav SEgar Works,

TARGART'S ABDALLAH,

Tali Panic Grass,

TALL RED-TOP,

TEA Leaver,

Texas Mrllet,

Tine Silos of II. Augtste Goffart, at Burtin, France,

Traction or Self-Propelling Fatir Exgine,

Trotting Stallion, "Alexander,"

66

“

Vantlia Grass,

Water Grass,

Wim Fescue,

Woop Grass,

Yard Grass,

Youne Tel Plant and Root,
"Gov. Sprague,"

"ThorNdALE," . 


\section{GENERAL INDEX OF ILLUSTRATIONS.}

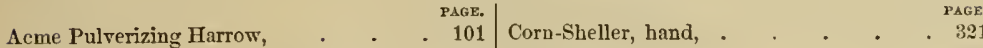

A Country Villa, . . . . . 623 Corn-Sheller, power, . . . . $\quad$. 321

Adamant Cabbage Plow, or Corn Subsoiler, 84 Corn-Sheller for Husked and Unhusked Corn, 322

Adamant Plow, . . . . . . . 94 Cotton-Gin, liand, . . . . $\quad$. $\quad 399$

Advance Chilled Plow, . . . . 97 Cotton-Gin, hand, with Feeder and Con-

Alpha Potato, . . . . . . . 343 denser attached, . . . $\quad .400$

American Hay-Tedder, . . . $\quad 210$ Cotton-Gin, power, . . . . $\quad$. 398

An Ornamental Cottage, . . . $\quad$. 624 Cotton-Seed Huller, . . . . $\quad$. 402

Cotton-Planter, . $\quad \cdot \quad \cdot \quad \cdot \quad 383$

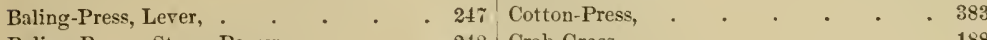

Baling-Press-Steam Power, . . . 248 Crab Grass, . . . . . . 188

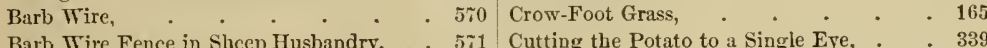

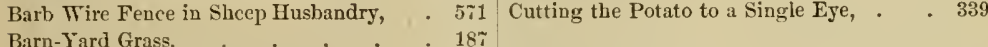

Bastian's Early Red Beet, . . . . . $\quad 366$ Dairy Barns, . . . . . . . . 651

Bastian's Half Long Blood Beet, . $\quad .366$ Deer Spring Cultivator, . . . $\quad$. 316

Beauty of Hebron Potato, . . . . 331 Defiance Wheat, . . . . 266

Bermuda Grass, Wire Grass, . . . 160 Design for a Country House, . . 611

Black Hawk, . . . . . . 728 Dilapidated Barn, Fig. 3, . . . $\quad$. 652

Black Hawk, Jr., . . . . . . 696 Dog Power, . . . . . . 513

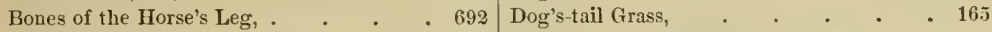

Boston Horse-Hoe, . . . . . 361 Double-Geared Plantation Cane-\$Lill, . . 416

Broadcast Plastel" and Guano Distributor, . 290 Double-Row Stalk-Cutter, . . . 318

Broadcast Seed-Sowers, . . . 289, 290 Draco Prince, . . . . . . 781

Broom Grass, or Sedge, . . . . 180 Drop-Seed-Nimble Will. . . . 155

Broom Sedge, . . . . . 170

Bullard's Hay-Tedder, . . . . 241

Cane-Mill, . . . . . . . 414

Carrot and Cotton-Weeder, . . . 369

Cassaday Sulky Plow, . . . . 88

Champlain Wheat, . . . . . 266

Champion Road-Grader at Work, . . 557

Check-Rowing Corn-Planter, . . . 312

Chinese Sugar-Cane, . . . . . 421

Cleveland Bay Stallion, "Bay Splendor," . 714

Clod-Crusher and Leveler, . . . . 101

Clover-Huller and Cleaner, . . . . 204

Clydesdale Stallion, "Time O' Day," . 686

Cock's-Foot Grass, . . . . . 187

Colossal Asparagus, . . . . . . . 366

Colt's Foot at Two Fears, . . . . . 786

Colt's Foot, Showing Quarters, . . . . 786

Compton's Surprise Potato, . . . 342

Corn-Cultivator,

Early Amber Sugar-Cane, . . . . 421

Early Half-Long Carrot, . . . . $3 \% 0$

Early Half-Long Scarlet Carrot,. . . 370

Early Half-Short Scarlet Carrot, . . 370

Early Scarlet-Horn Carrot, . . . 370

Echo Farm Buildings, and Jersey Group, . 586

Echo Farm Butter-Stamp, . . . . 594

Echo Farm Butter-Worker. . . . 594

Echo Farm Churn, . . . . . 593

Echo Farm Cream-Strainer, . . . 593

Echo Farm Group of Yearling Jersey Bulls, 589

Echo Farm Group of Fearling Jersey IFeifers, 588

Echo Farm, Interior of Cow Stable, . . 591

Echo Farm Jersey Bull, "Litchfield," 592

Echo Farm Triple Strainer Pail, . . 593

Eclipse Wind-Engine, . . . . 538

Eclipse Wind-Engine in motion, . . 539

Eclipse Wind-Engine at rest, . . . 510

English Cottage, . . . . . 625 


\section{GENERAL INDEX OF ILLLSTIRATIONS.}

English Cottage, chamber plan of, Encrlish Cottage, ground plan of, English Draft Stallion, "Lincoln," Ensilage Corn-Planter,

Ensilage Cutter,

Ensilage Cutter,

Egrptian Blood Turnip Beet,

Errytian Grass,

Erecting Barb Wire Fence,

Erecting Barb Wire Fence, Use of Stretcher,

Excelsior Oat,

Farm Barns,

Farm IIill,

Farm Mill,

Farmer's Farorite, Grain-Drill,

Feather Grass,

Field Pitching Apparatus,

Field Roller,

Filled Silo Being Emptied by Fertical

Slicing. .

Towl IIendow Grass,

Tused-Foot, Steel Point,

Gama Grass.

Gatbering Tea,

Gilpin Sulky Plow,

Gleaner IIas and Grain Rake,

Grain-Dropper,

Grapple Har-Fork,

Grasshoppers in a Wheat-Field, .

Group of Yearling Jersey Bulls, .

Group of Yearling Jersey Heifers,

IIalf-Blood Pereheron-Norman Stallion,

" Napoleon III.,"

Hambletonian Stallion, “4

Harrester and Twine-Binder,

Harpoon IIay-Fork,

Har und Sugar-Cane Scales,

IIay-Carrier

Hay-Elevator and Carrier, .

IIilling Cultirator,

Hoosier Grain-Drill,

Horse's Foot.

Horse-Hoe,

Horse Power,

Hangarian Millet,

Hydraulic Ram in operation,

Imperial Sugar Beet,

Improved Farm Engine, witl iron truck wheels,

Iniproved Long Blond Beet,

Improved Peach Blow Potato,
PAGE.

625 Improved Lima Bean,

625 Indian Gras

710 Iron Beam Plow

501 Iron MIower,

503 Iron Turbine Wind Engine,

504

366 Jack, "Magnum Bonum," .

183 Japan Clorer,

568 Johnson Grass,

Justin MLrgan,

Kiemp's Manure Spreader, .

6.50 Kentucky Blue Grass,

139

322

323 La Dows Pulverizing Нагтот, . . . 100

2:t Lampas Iron, . . . . . . . 796

$1 \% 3$ " " . . . . 796

246 Large Globe Artichoke, . . . . 366

103 Large Orange Carrot, . . . . . 370

Large White Belgian Carrot, . . . 370

505 Leaf of Tea, . . . . . . . . . . . . . . .

140 Lever Baling Press, . . . . . 247

93 Liberian Sugar Cane. . . . . . 421

166 Lneation of the Internal Organs of the Horse, 817

458 Long Red Jangel Trurzel, . . . . . 370 89

213 Meadow Fescue, . . . . . 151

276 Meador Fox-tail, . . . . . 146

245 Maple Sugar Making,-rien of sugar house, 431

261 Means Grass, . . . . . . 183

589 Mexican Clorer, . . . . . . 196

588 Iodel Barn,_cow stable. . . . . 649

" " feeding floor and yards, plan

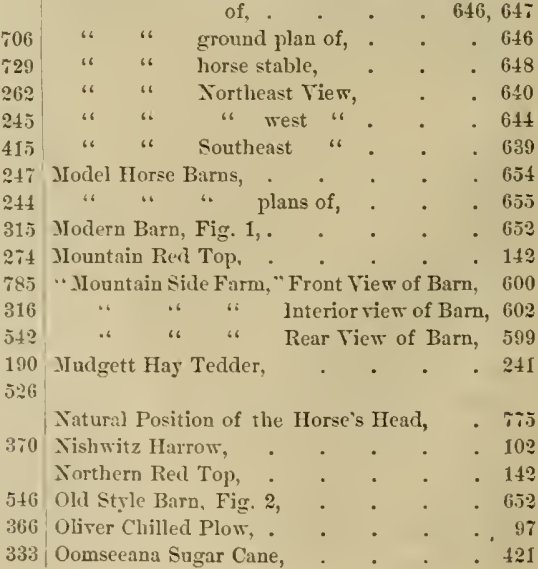


GENERAL INDEX OF ILLUSTRATIONS.

Orloff Stallion, "Sobol," . . . . PAGE.

Osborne Sulky Plow, .

Pasterns,

Percheron-Norman Stallion, "Eldorado," .

Percheron-Norman Stallion "Romulus,"

Pigeon Grass-Bristle Grass, . . 159

Plan of Unitcd Silos, . . . . . . 497

Points of the Horse, . . . . . 683

Potato Coverer, .

Potato Diggers, .

Potato Planter, .

Power Cotton Gin,

Red Top,

Rescue Grass,

Rocky Mountain Locust,

Root Cutters,

Rural Cottage,

" " chamber floor, plan of,

" " ground plan of,

Rye Thresher,

Rysdyk's Hambletonian,

Scene in the Black Tea District,

Scene on the Grandin Farm, Dakota, Twenty

Gang Plows in Operation,

Schrader's Grass,

Self-Binding Harvester, . . .

Sequoia gigantea,-Giant Red Wood,

Share's Coulter Harrow,

Sheep's Fescue, .

Short Stem Cauliflower,

Silver Skin Potato,

Skeleton of The Horse,

Small Gherkin or Burr Cucumber,

Smut Grass,

Snow Flake Potato,

Southern Bent, .

Southern Seed Corn, .

Spring Cultivator for Cotton,

Stallion "Frank Allen,"

Stalk Cutter for Cotton or Corn Stalks in the field,

347

608

178

278

521

101

803

500 397
Stalk Cutters,

Steam Sugar Works,

Stone and Root Puller,

Sub-Soil Eagle Plow,

Sub-Soil Plow, Grub Hook,

Sugar Evaporator,

Stationary Sugar Evaporator,

Swiss Chard Beet,

442 Taggart's Abdallah,

151 Vanilla Grass, . . . . . . 179

370 Vesey Homestead Cottage, . . . . . 623

334 Victor Clover Hachine, . . . . 205

370 Wagon Jack, . . . . . . 667

176 Water Grass, . . . . . . 174

333 Wild Fescue, . . . . . . 177

154 Wood Grass, . . . . . . 184

395 Yard Grass, . . . . . . 165

751 Yellow Globe Mangel Wurzel, . . . 366

PAGE.
17,318

- 410

92

97

419

366

\% 64

169

$$
\begin{aligned}
& 74 \\
& 84 \\
& 85 \\
& 66 \\
& 57
\end{aligned}
$$
(n)

(





\section{CONTENTS.}

\section{PART I. GENERAL AGRICULTURE.}

\section{Farming as an Occupation.}

Agriculture tae basis of civilization and wealth of a country-Its aim and scope compared with other avocations.-Dignity of farm labor.-Its relation to pliysical, mental, and moral culture-Its influence upon National industry and prosperity-Original calling of mankind-Independence of the farmer-Advantages of a farmer being educated to his business-Old and new methods of farm. ing compared-Elements of success in farming-Farming can be made profitable.

Soils.

The origin of soils-The constitution and texture of soils-Their classification-Clay soils and their management-Gravelly soils-Loamy soils-Marly and calcareous soils-Silt and alluvial soilsMuck-Peat soils and their cultivation-Sub-soils-Friableness of the soil-Light colored claysMarls and sands-Color of soils-The Dew Point-Analysis of various soils-Analysis determines the constituents of a soil, but not its mechanical condition-Analysis of plants more satisfactory than that of soils-Both are aids when combined with experience and observation.

\section{Fertilizers.}

Elements of plant food-Elements necessary to supply soils-Organic and inorganic elements-Plant. life and plant food-General propositions concerving plant food-Exhaustion of soil by farm crops -Haterials removed from the soil by various products-Deficiency of potash in common fertilizers and in soils-Common manures and commercial fertilizers-Barnyard manure and its value-Poultry manure-Night soil and poudrette-Sewage as a fertilizer-The sewage of Paris-Sail in a Paris sewer-Composts-Their value-How to construct a compost pile-The value as fertilizers of flesh -Blood-Hair-Horns-Hoofs-Fish-Fish Guano, \&c-Cotton seed as a fertilizer-Castnr and Linseed Pomace-Animal dust--Peruvian Guano-Rectified Peruvian Guano-American GuanoLime-Ground Limestone and shells-Gas Lime-Wood ashes-Coal ashes-Soot-Charcoal dustPeat and peat ashes-Sea-weed and sea-weed ashes-Bones-Mlarl-Gypsum-Salt as a fertilizerPotash-Sources of supply of potash-The Stassfurt potash mines-Composition of German Potash salts-How to use potash salts as a fertilizer-Leaves of trees as fertilizers-Manuring with green crops-Clover for vegetable manure-Various crops for green manure-Sod-Special fertilizers for various crops-Stockbridge Formula for Wheat, Indian Corn, Fodder Corn,-Rye,-Oats, -Buckwheat,-Potatoes,-Ficld Beans,-Swede Turnips or Rutabagas,-Onions,-Hay.-Application of manures-Quantity to be used-The fallow system.

\section{Tillage.}

The advantages of tillage-It aids in supplying plant food-Objections to tillage answered-Judgment and skill necessary to successful tillage-Different soils require different methods-Tillage should always be adapted to the wants of the crops cultivated and the character of the soil-The best implements essential-The plow the principal reliance of the farmer in the labor of tillage-The plow should be suited to the kind of work required,- a light plow should not be used for heary work, nor a sub-soil plow for light work-Sulky and Gang plows, Chilled plows, Ec-Lands that should be plowed in the fall-Lands that should be plowed in the spring-Best method of fall plowingRidging-Manner of plowing a field-Deep and shallow plowing-Depth of plowing must depend upon different conditions, such as the character of the soil and the crops to he cultivated-Subsoil plowing-The principal material for plant food is to be found in the surface or agricultural soilWhen desirable to break up the subsoil-A knowledge of the character of the subsoil essential to 
successful tillage-Benefits occasionally derived from mixing the subsoil with the surface or agricultural soil-An instance given-Clay soils, heary loams, etc., best pulverized by plowing when dry or nearly so-Lse of the harrow-Its use for any purpose most effectual when the soil is dryBest implements required-Various harrows and clod crushers described and illustrated-The use of the Roller-Benefits derixcd from pressing the soil upon the seed-Exterminating noxious weeds.

\section{What Crops to Raise.}

A judicious choice of crops essential to success-Crops that can find a ready market to be cultiratedPrinciple always to be regarded-Choice modified by the character of the soil, etc. - Relative cost and value of different crops.

\section{Rotation of Crops.}

Benefits of a judicious rotation of crops-Theories relatire to the csuse of failure or depreciation of the same kind of crops produced continuously from the same soil-Nature follows a course of rotation -This strikingly illustrated in the succession of different forest trees- "Clover sick" lands- $\mathbf{A}$ scheme of rotation to be so formed as to cultivate the same or similar crops on the same ground at intervals as remote as practicable-The English practice called the "Four-field or Norfolk System "-Rotation in Agriculture in Italy-Various methods of rotation adopted in this countryRotation of crops in the Southern States-Rotation for the rorn lands of the South-Grass-Pasturage- Whatever the rotation, good crops cannot be produced in any section without proper man. agement, and a sufficient supply of manure to the soil.

\section{Irrigation.}

Water one of the sources of supply of plant food-The benefits derived from irrigation- Tarious methods of irrigating lands- - se of the windmill in irrigation- Then irrigation is injurious-An excess detrimental- When irrigation should begin in the spring-Should always be adapted to the charac. ter of the soil and the kind of crops cultivated.

\section{Drainage.}

Various methods-Benefits to be derived from drainage-Difference in temperature of drained and un. drained soils-Drainage tends to render soils warm and more friable-Enables the farmer to plant earlier in the spring-Tends to prerent injury to crops from excess of moisture-To prevent drought-To prevent surface washing and winter killing of grain-Tends to an earlier harrestRenders a location more healthful-Drainage system at the South-Reclaiming the Everglades of Florida by draining Lake Okeechobee-The drainage system two hundred years ago-Partial drainage-Arerage amount of rain-fall- What lands require drainage-Location of drains-Necessity of drains being properly constructed and the work well done-Tile draining the best method-Careful planning of location of drains necessary to secure the best results-Hom to dig the trenchesDepth of drains-How to lay the main drains-How to lay the tributaries-Protection against sand and other obstructions entering at the joints-Filling the trenches-Stone drains-Plank drainsTurf-Brush, etc.-The mole plow-Open ditches-Furrow draining or ridge cultivation-Tile draining the most effectual, permanent, generally satisfactory, and eventually the most economical.

\section{AGRICULTURAL PRODUCTS.}

\section{Grasses and Forage Plants.}

Great importance of the grass product-Tarieties-Description of more than one hundred varieties of grasses-Their cultivation-Forage plants-Varieties-Cultivation-Indian Millet-Common Millet -German or Golden Millet-Pearl Millet-Indian Corn as a forage plant-Japan Clover-Mexican Clover-Red Clover-Time of cutting and method of curing clover-Conditions favorable to fermentation in clorer hay-Cultivating clorer seed-Harvesting clorer seed-Clorer as a fertilizer"Clover sickncss" of lands-Alsike clover and its cultivation-White clover-Crimson cloverAlfalfa or Lucerne-Talue as a forage plant-Cultivation-Sainfoin-Cultivation-Tetch-Spurry -The Cow or field pea-Lupine-Prickly Comfrey-List of grasses and forage plants, with their common and botanical names, their time of blossoming, whether wild or cultivated, and the kind of soil adapted to their growth-Glossary of terms used in describing grasses-Grouping of grassesNutritive value of grasses-Analyses of different varieties-Tables for the mirture of seeds for the following lands-Mowing lands, Permanent pastures, Hay and pssture combined-Rotation and improrement of soil-Jeadow lands-Summer pastures- Tinter pastures-Permanent pastures 
according to English methods-Permanent pastures as practiced in Scotland-Lawn grasses-Fine lawns frequently mown-Hay and pasture in orchards and shady places-Nowing on light landsReclaimed peaty lands-Marshy grounds liable to be occasionally overflown with fresh waterRocky lands-Time to sow grass seed-Selection of seed-Time of cutting and manner of euring hay-Improved implements in hay making-Mowing machines-Hay tedders-Horse rakes-Horse hay forks-Hay carriers-Hay caps-Storage of bay-Stacking of hay-Hay stacking apparatusBaling hay-Aftermath or Roweu-Cultivating grass seed-Fall grazing of mowing lands-The practice injurious to the crop of the following year if done after eutting the aftermath-Manuring mowing lands-Rolling mowing lands and pastures beneficial-Renovating grass lands.

\section{Cereals.}

Importance of the cereal product-The great advance in the production of cereals in the United States during the last ten years-Comparative advancement in the production of the different cerealsWheat-Description of different varieties-Preparation of soil for wheat-Fertilizers-Selection of seed-Time of sowing spring wheat-Time of sowing winter wheat-Quantity sown to the acreSowing broadcast and drill sowing compared-Depth of covering-After-cultivation-Improved implements in the cultivation of whent-Harvesting-Threshing-Diseases and insects of wheatRust-Chinch Bug-Hessian Fly-Rye-Varieties-Soil-Fertilizers-Sowing-Harvesting-Threshing-Diseases of Rye-Oats-Varieties-Soil-Fertilizers-Cultivation-Harvesting-ThreshingDiseases-Barley - Varieties-Soil-Fertilizers - Seeding-Harvesting-Threshing -BuckwheatSoil-Sowing-Harvesting-Corn-Its importance among cereals-Analysis-Varieties described-Selection of seed-Preparation of sced for planting-Soil for corn and its preparation-Time for planting in the different sections of the country-Methods of planting-The mixing of different varieties to be obviated-Hill and drill planting compared-After-cultivation-Harvesting-Fodder -Stalks in the ficld-Husking-Shelling-Shelling unhusked corn-Grinding corn-Grinding unhusked corn-Cob meal-Diseases and enemies of corn-Rice-Its value as an article of food- $\mathrm{Va}$ rietics-Analysis of rice-Lands adapted to its cultivation-Cultivation-Harvesting-Threshing and milling.

\section{Roots and Esculent Tubers.}

The common potato-Native of this continent-Improvement through cultivation-Introduction into Great Britain-Subsequent cultivation-Description of varieties-Soil and its preparation-Fertilizers-Selecting and cutting potatoes for planting-Cutting to a single eye-Number of eyes to the hill-Planting-Hill and drill planting compared-Potato planters-Benefits of early planting-The manner of growth of different varieties illustrated-Hilling and level culture compared-Forcing potatoes-Cultivation-How to raise potatoes from cuttings-Two crops of potatoes a yearHyhridizing potatoes-How to raise seedlings-Harvesting-Potato diggers-Storing-DiseasesPotato rot-Potato Bug or Colorado Bectle-Use of Paris Green, etc. - Scabhy Potatoes-How to tell a good potsio-Sweet potatoes-Native of Southern Asia-Not exclusively.a Southern product, and may be raised with fair success in many of the Northern States-Its nutritive value-Varieties - soil-Fertilizers-Obtaining plants-Transplanting-Cultivation-Harvesting-Storing-The Yam -Varieties-Chinese yam-Cultivation-Turnips-Varieties-Table of nutritive value of rootsSoil-Fertilizers-Preparation of soil-Sowing-Cultivation-Cultivation of spring turnips-Rutabaga or Swedish turnips-Harvesting-Storing-Raising turnip seed-Beets-Varieties-Preparation of soil-Sowing-Cultivation-Mangel Wurtzels-Cultivation-Harvesting-Storage-Root Cutters-Carrots-Varieties-Cultivation-Carrot weeders-Harvesting and storage-ParsnipsVarieties-Preparation of soil-Cultivation-Artichokes-Analysis of artichokes and potatoes compared-Their value as food for swine and other live stock-Varieties-Planting and cultivationYield-Chufas-Cultivation-Ridge culture for root crops-Root pits-Ventilators for root pits essential-How constructed-Onions-Varieties-Soil and its preparation-Cultivation from seedCultivation of Sets-Cultivation of top onions-Potato onions-Shallots-Rareripes-Onion weeders -Cultivation of onion seed-Harvesting and storing onions.

\section{Miscellaneous Products.}

Cotton-The great importance of the product-History-First exportations-Cotton growing sectionBeauty of the cotton blossom-Varieties-Upland cotton-Sea Island cotton-Cotton product of British India and Egypt-Unproductive cotton fields of the South-How the evil may be remedied - Invention of the cotton-gin - Varieties - Soil - Preparation of soil - Fertilizers - PlantingCultivation-Securing the crop-Ginning-Baling-Use of seed-Seed hulling-Facts elicited respecting the cotton product at the Atlanta Exposition in 1881 - Enemies of the cotton 
plant-Sugar-Sources of supply-Importation-Introduction of Sugar cane-Section suited to the production of the sugar-cane-Varieties of sugar-cane-Preparation of the soil-Fertilizers-Cuttings -Setting the cuttings-Cultivation-Harvesting-Manufacturing sugar-Machinery requiredSorgho and Imphees-Soil-Planting-Cultivation-Harvesting-Manufacturing syrup-Sugar Beet - Varieties - Preparation of soil - Sowing - Cultivation - Harvesting - Storing - Manufacturing sugar-Sugar maplc-Obtaining sap-Sugar making-Tobacco-Varieties-Soil-Preparing seed beds-Sowing-Weeding-Preparation of soil-Fertilizers-Transplanting-Cultivation-EnemiesWorming-Topping-Suckering-Cutting-Curing-Stripping-Packing-Coffee-Climate suited to the cultivation of coffee-Soil-Cultivation-Harvesting-Preparing for market-Tea-Southern localities adaptcd to its culture-Varieties-Nethods of cultivation-Gathering-Curing-Peanuts There successfully cultivated-Soil-Fertilizers-Planting-Cultivation-Harresting-Indigo-Cultivation-Flax-Varieties-Preparation of soil-Sowing-Cultivation-Harvesting-Preparation for market-Cultivating seed-Hemp-Soil-Sowing-Jlethod of cultivation-Cutting-DryingCultivating seed-Jute-Cultivation-Harvesting-Preparing for market-Peas-Varieties-SoilCultivation-Beans-Tarieties-Preparation of soil-Planting-Cultivation-Castor Beans-Cultivation-Broom corn-Preparation of soil-Planting-Cultivating-Harvesting-Curing-HopsSoil-Cultivation-Stacking-Harvesting-Drying-Mustard-Varieties-Cultivation-Harvesting.

\section{Ensilage.}

History of the discovery of the ensilage system-Various experiments-Objects to be secured-Minner of building silos-Earth silos-The most profitable crops to ensilage-Cultivation-Cutting-How to fill a silo-Removing fodder from silos-How to feed ensilage-Economic value of ensilage compared with other fodder-Improved farming methods-Ensilage adapted to the Southern StatesAnalysis and composition of corn (or maize) when cut in its green state-Advantages of the ensilage sfstem-Opinions and experiments from variuus authentic sources respecting the system.

\section{Good Seed.}

Necessity of care in the production of good seed-Choice of plants-Improvement by cultivation-How effected-Seed plants to be grown apart from others-Special cultivation should be gircn-Protec. tions afforded against adverse influences-Importance of removing inferior plants-Gathering anu storing seed-Seed from the strongest plants selected-Injury from moisture-Qualities of good seed-Vitality-Vigor-Productiveness-The use of old or poor seed attended with loss to the farmer.

\section{Timber Culture.}

The great importance and value of timber-The destruction of American forests to be deplored-Influence of forests upon rain fall-Climate-Soil and vegetable growth-Healthful influence of forests -Forests should be restored-Tree planting a uecessity-Varieties-Large trees of CaliforniaTime aud manner of transplanting forest trees.

\section{Water Supply of the Farm.}

Importance of a sufficient supply of pure water for the farm-Sources of supply-Springs-PondsBrooks-Rivers-Wells-Contamination of water from various sources-Sickness and death often caused by use of impure water-Surface drainage-Injurious effects of metallic pipes-Artesian Wells-Hydraulic rams-The windmill as a motive power for pumping water-Use of syphon in water supply.

\section{Motive Power for Farms.}

Various powers employed for farm use-Dog and sheep power-Horse power-Wind power-Steam power-Farm engines-Their importance and utility on large farms-Steam power employed ot the farm of Mr. Dalrymple in Dakota-Steam power for tillage.

\section{Farm Teams.}

Good teams essential for farm use-Best animals for the purpose-Horses-Mules-Oxen-Relative cost and value-Number of animals should be in proportion to the work to be performed-Firm animals should have good care and kind treatment.

\section{Roads and Road Making.}

Importance of having good roads-Improper manner in which the early settlers laid out rosds-Eleva 
tions in surveying for roads to be avoided-How roads should be laid out-Should be substantially constructed-A solid and unyielding foundation necessary-Form of the road-bed-Removing obstructions and repairing roads-System of drainage for roads-The constructiou of catch waters or bars-Value of the stone crusher and roller in road making.

\section{Farm Roads.}

The great convenience of roads connecting different portions of the farm-How constructed-The road grader-Farm roads an economy in the saving of time, labor, and accidents.

\section{Farm Fences.}

Necessity of fences on the farm-A surplus found in many sections-Expense and labor in constructing and repairs-Different kinds of fence-Rail fence-Post and rail-Board fence-Stone wallHedge-Wire fence-Arguments for and against the wire fence-Importance of fences being well made and kept in good repair.

\section{Wastes and Wants of the Farm.}

Common waste of fertilizers-Exhaustion of soil from constant cropping with an insufficient supply of manure-Neglect to perform work at the proper time-Labor not always economized-Carelessness or indifference-Slovenly management-Doing work overnice-Use of poor tools-Lack of care of farm implements-Insufficient help on the farm-Poor teams-Inconvenience of farm buildings and arrangement of farms-Farmers should read the best agricultural books and papers and keep up with the times in advanced methods of farming-Less drudgery in farm life and more knowledge and skill in conducting the business-Better farm implements essential-Labor-saving implements to be used wherever practicable-Larger crops should generally be produced from the same amount of land-English agriculture an example-A more perfect system of management in farming-Advantage of keeping an accurate account of receipts and expenditures-Farmers too often put the receipts of the farm into the bank or bonds instead of using them for the improvement of lands or farm buildings-More capital required for successful farming.

\section{PART II. FARMS AND FARM BUILDINGS. Choice of Farms.}

A wise choice essential-Location-Quality of soil-Size-The adoption of improved methods often better than change of locality-A change should not be thoughtlessly made-Desertion of the old homestead too frequently a mistake-Advantage of large farms-Advantage of small farms-Proprietors of small farms should coöperate-The farm should be no larger than can be properly managed-All the capital should not be invested in land.

\section{Model Farms.}

Advantages of a thorough system in conducting a farm-The best methods always the most profitable -Examples of model farms-" Echo Farm "-Its management-Improvements, etc. - "Deerfoot Farm "- "Mountain Side Farm "- "Lorillard Stock Farm "— "Hillhurst"-Long View Farm," etc.

\section{Large Farms of the Country.}

Large farms a necessity in some sections of the country - Western farms-Southern-Eastern-Products -Noted large farms of the country- "The Dalrymple Farm," including Cass, Cheney, The Alton, and Grandin farms-"Quinn farm"- "Glen Farm, etc."

\section{Farm Buildings.}

What buildings are necessary on the farm-All farm buildings should be made convenient, and in as good taste as practicable-Expense-Repairs-Painting buildings-The farm house should be pleasantly located, near the road-Should be convenient, tasty, roomy, light and well-ventilatedThe farmer should make his home attractive to his children-Influence upon character-The exhibition of good taste not necessarily an extravagance-Size should be adapted to the needs of the family-Height of buildings-Plans for houses-Interior arrangement-Expense-WarmthWindows-Doors-Floors-Stairs-Roofs-Chimneys-Mantels and closets-Piazzas-Eave troughs -Blinds-Lightning rods-Ventilation-Cellars-The barn-Granary-Hog house-Poultry house -Wagon house or shed-Wood house-Store bouse-Water closets and vaults-Tool house and repair shop-Ice bouse. 


\section{PART III. DOMESTIC ANIMALS.}

\section{The Horse.}

History - Value to mankind-Qualities desirable in a horse-The farm horse-The carriage and saddle horse-Points by which a good horse may be known-The eye-The ear-The head-The neckThe chest-The bones-The shoulder-The pastern-The foot-The back-The hind quarter-The rump-The thigh bone-The bock-Tarieties-The Clydesdale-Percheron-Norman-English draft -Boulounaise draft-Conestoga-Cleveland Bay-Hambletonian-Messenger-Morgan-OrloffThe trotting horse of America-Noted trotting horses, their description, record, etc. - Progenitors of trotting families of horses-Breeding-Disqualifications for breeding-Influence of the sireInfluence of the dam-Breeding for beauty, style, speed, docility, strength, endurance- What horses to breed-Only thoroughbred or high-grade stallions to be used-Care of breeding maresCare of joung colts-Castration-Breaking or training colts-The old-time method of managing colts-The present system of colt management-How to break or teach a colt-Uniform kindness to be the rule in colt training-Valuable colts often ruined by the ignorance and brutality of the socalled professional "colt breaker"-The training to begin early-Halter breaking-Driving the colt-Teaching a colt to back-The bitting process-Value of good training-Age for workingGeneral management of horses-Use of check-rein-Blinders-The stable-Impaired ejesight from improper amount of light in stables-Horse stables-Proper ventilation of stables-Warmth-Food - Water-Horse shoeing-Importance of having horses properly shod-Different systems of shoeing-Shoe tips-Barefoot-Diseases of horses-Their treatment-Good care will usually prevent disease-Treatment of old horses-Kind treatment at all times conducive to the best results.

677

\section{The Ass.}

Native of Asis-Usefulness-Great endurance-Varieties and characteristics-Breeding in the United States-The progenitor of the mule.

The Mule.

Economy of mule labor-Hardiness and other valuable qualities-MIule and horse labor comparedToo often overworked and otherwise abused-Benefits of kind treatment-Rearing, breaking, and general management-Quality and kind of food-Shoeing-Diseases and their treatment. 



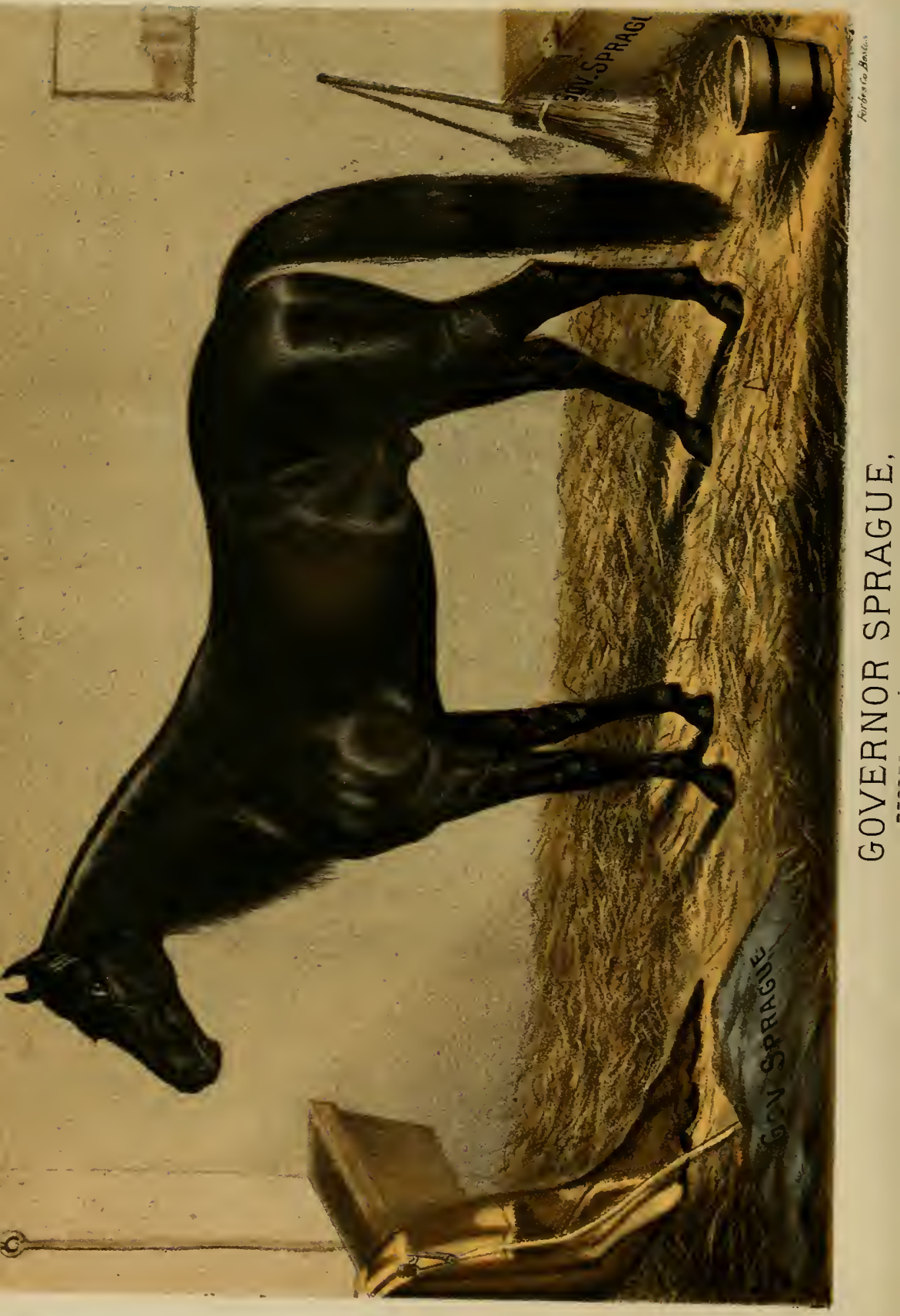




\title{
THE AMERICAN FARMER.
}

\author{
PART I. \\ GENERAL AGRICULTURE.
}

FARMING AS AN OCCUPATION.

A

GRICULTURE is the foundation of civilization; the basis of all other industrial pursuits ; its advancement having a more powerful influence on the prosperity and social elevation of a people than any other of the world's industries. Were it not for this time-honored avocation, mechanics, manufactures, and commerce, would have nothing to supply the material upon which to depend, and mankind be devoid of the resources of physical life. In its aim and scope, the amount and variety of its productions, it stands supreme among man's material interests, underlying and upholding all others.

Webster never uttered a more forcible and important truism than when he said, "All national wealth depends upon an enlightened agriculture;" a truism illustrated in the history of every nation on the globe; prosperity and wealth ever being found proportionate to a nation's advancement in agricultural knowledge and praetice. This will always be true of any country or limited section of a country; and while the fact is recognized with respect to all nations in all ages of the world's history, it is equally true that the decrease in a nation's wealth and prosperity has ever been found to be proportionate to the decrease in the productiveness of the soil, and the neglect of agricultural interests; the depreciation of agriculture being an index of the demoralization and decay of a nation. Agriculture furnishes the sources of all physical and intellectual energy; in fact, the products of all other trades and professions seem insignificant when compared either in quantity, variety, or value with those of the farmer. No one can compute the deplorable results that would follow the suspension of agricultural labor for even a single year; in all probability, famine with its attendant horrors would extend throughout the civilized world, which would soon become, by this means, in a great measure depopulated. This could not result by the suspension of any one of the other branches of industry for one year or several years; in fact, any one of them might be suspended for a succession of years without exerting a very marked influence upon the progress of civilization, all of which proves the supremacy of agriculture. It supplies the indispensable wants of the human race, feeding and clothing mankind ; the materials for this purpose being received by the farmer directly from the inexhaustible stores of earth and air placed there by the bountiful Giver of all good. This brings us to another important consideration in connection with the avocation of the farmer, and that is, its true honor and dignity, and tendency towards the advancement of religion and morality, as well as the opportunities afforded for physical and mental development. It was the original calling of man, appointed by the Creator, who planted a garden in Eden and made it man's duty to "dress and keep it." and when by disobedience he was driven from Eden, it was still his mission to till the ground. As this was the first employment assigned man by God, we have here not only a proof of its vast importance, but every reason to believe it also especially adapted to man's requirements in every respect, all of which has been fully con. 
firmed by the subsequent history of the human race. There is no employment that lrings man into such close communion with nature - a communion so intimate that it must almost of necessity reach to nature's God; hence, we find, as a general rule, that there are fewer skeptics aniong farmers, in proportion to the number engaged in the occupation, than among other branches of industry. Farmers, as a class, are moral and law-abiding citizens, and the embodiment of those principles that constitute the very foundation and framework of good society. In making this assertion, we would not wish to extol the avocation of the farmer beyond its real importance, or place other industries beneath their proper standard; there are exceptions to all rules; honor and worth, as well as the lack of these essentials of true cliaracter, are to be found in all occupations under the sun; but we believe the verdict of the public generally would concur in the opinion previously expressed, viz.: that the avocation of the farmer has a tendency towards inculcating a reverence for the Deity, with its attendant ennobling infuences.

It is true that character makes the man, and not his occupation or surroundings, and that a truly honest man will prove himself such in whatever capacity he may be placed : also, that a man lacking these qualities will not be changed by merely engaging in the most honorable and ennobling occupations ; still it cannot be denied, tnat the associations and influences con. nected with the employment of the hands and brain, do have a powerful influence in forming the character of an individual. Farming, as an avocation, presents one of the most exten. sive fields of varied research and close investigation of any of the various professions and trades represented in the world, and yet how few comparatively that are engaged in it, who realize the opportunity for mental, as well as physical development, their employment affords. As a class, however, farmers are becoming better educated, and thus better fitted for successful achievement in their business than formerly, while the Agricultural Colleges, Associations, and journals of the present time are rapidly increasing these facilities. There is no good reason why the farmer should not be specially educated to his business, and farming rank among the learned professions of the day. It seems to us that scientific farming requires a knowledge of a greater variety of things than almost any other kind of business. As far as the nature and treatment of soils is concerned, some knowledge of mineralogy is very essential ; a knowledge of chemistry, botany, and entomology are all, to a certain extent, equally valuable as far as they apply to the successful growing of crops and their preservation from the ravages of insects. The farmer must also be a manufacturer as far as the making of butter, cheese, cider, curing of hay or preserving of ensilage and crops generally are concerned, while a thorough understanding of the habits, necessities, diseases, etc., of domestic animals is indizpensable to success in the work. Surely the farmer, in order to be well fitted for every department of his avocation, must not only possess a natural taste and aptitude for his respective calling, but should receive special instructions in these several departments : in other words, he should be educated for his business, thereby increasing his usefulness and success in life.

One of the wants of the agricultural interests of the country is a greater facility for the education which is especially directed to the practical business of farming. Our agricultural colleges are supplying this need to a certain extent, but we should have more of them; besides, our public schools practically ignore in their system of instruction everything pertaining to this important branch of national industry. A great improvement upon the present system would be instituted by inculcating a more general knowledge of those sciences which are more intimately connected with it, such as chemistry, mineralogy, geology, botany, and their relations to agriculture, the laws that modify and regulate the conditions of the great mysteries of plant-life and plant growth, together with the best methods of fertilizing, grafting, budding, and the history and practice of everything connected with this pursuit, which affords employment to so large a proportion of our population, and which forms the basis of our national subsistence, wealth, and power. The large number of schools in various portions of Europe, in which practical instruction is given in farming in all its various branches and departments, have obviously done much in bringing the agriculture of those 
sections of the Eastern Continent to the high standard it has attained. In these schools, boys from twelve to fourteen years of age are instructed in the practical business of farming, and also in mechanics, as far as the use and repairing of all kinds of agricultural implements are concerned,-theories being taught only in connection with their practice, which is the only true method of instruction. Science has opened the door to successful progress in agriculture in the past, a progress that will approach nearer and nearer to perfection in the art as time advances. Sir Humphrey Davy may be said to have inaugurated, in a great measure, this improvement in the early part of the present century, although it was not until 1840 that Liebig announced propositions that opened a new field of thought and investigation, and awakened the attention of intelligent farmers to the importance of applying the results of chemical investigations, which in a great measure essentially modified the agricultural practice of all the civilized portions of the world. He opened the way to the whole system of concentrated fertilizers in the following simple words :-

"To manure an acre of land with forty pounds of bone-dust is sufficient to supply three crops of wheat, clover, potatoes, turnips, etc., with phosphates; but the form in which they are restored to the soil does not appear to be a matter of indifference; for the more finely the bones are reduced to powder, and the more intimately they are mixed with the soil, the more easily they are assimilated."

Since that period the progress in improvement, although gradual, has been marked, and the use of commercial fertilizers has become in many sections a necessity ; not only this, but they are now regarded by our fest farmers everywhere as an important and indispensable adjunct to farm-yard manures. What science-which is justly regarded in all civilized countries as the handmaid of successful agriculture-will accomplish in the future, remains to be seen; but if, in the words of King Brobdignag to Lemuel Gulliver, she will cause "two blades of grass to grow where only one grew before," great good will be accomplished.

We are essentially a nation of farmers ; more than one-half of our population relying upon agriculture for support, and no country is more dependent for prosperity on its resources in this respect than our own ; yet, while much has already been done, and great advancement made in this branch of industry during the past half century, there remains much to be accomplished before we, as a nation, shall approximate to that high standard and degree of success that it is our privilege to attain.

We beheve no one would think of disputing the fact that a given area of land, with a similar soil, season, and equally favoring conditions otherwise, would, with an equal amount of time and labor, produce more wheat, corn, grass, cotton, rice, tobacco, or any other product now, than it would twenty-five or fifty years ago. And this is due to the advanced methods of farming, including the use of improved agricultural implements, etc., which practice causes the soil to produce more with the same or less expense of time and labor, and hence, farming is more profitable now than at that period, not only in the amount produced from the soil, which will bring more in return, but in the expense of production in time and labor. It is also true that more can be accomplished with the same amount of labor and expenditure in any of the other departments of business, than could have been performed twenty-five or fifty years ago, owing to new and scientific discoveries, increased skill and mechanical art; therefore, the farmer of to-day need not make his business the drudgery that it was formerly; or, if he works as hard, he can have better returns for his labor in the form of more of the necessaries and luxuries of life. Consequently, farmers, as a class, are not necessitated to labor as hard, or practice as much self-denial in the rigid economies of life, in order to attain success, as in the olden time, and this is all due to advanced science and its application to agriculture.

When we compare the implements for tilling and cultivating the soil that were used by our forefathers, with those of the present time, we are able to realize more fully the benefits bestowed upon agriculture by men of science, inventors, and manufacturers. The improve- 
ments in agricultural machines alone is something wonderful. Formerly the principal farm implements consisted of a one and two-horse plow, a harrow, a few hoes, rakes, and. scythes of the crudest kind, a sickle, a reaping cradle, and a flail for threshing the grain. The only power employed in the use of these rude implements was that of the horse, mule, and $o x$, and the hand of the farmer; the latter being the main reliance, except in propelling the plow and harrow, and even here the holding and guiding of the plow often called for the most severe physical exertion of all the farm labor. Compare these machines with those of to-day, the plows of every conceivable pattern adapted to the various kinds and conditions of soil ; sulky plows that will turn the furrow to any desired depth with but slight manipulation from the driver, who rides, except to hold the reins and guide the horses; the harrows and clod-crushers of various, derices and plans of operation, that pulverize the soil to a degree of perfection that was once never dreamed of ; the drills and planters that deposit the seed and cover it with a uniformity that is truly astonishing; and not only this, but in the vast amount of labor accomplished in a given time, the driver not being obliged to even walk over the ground; the rollers, the cultivators, and horse-hoes, the mowers, tedders, horserakes, forks, carriers, and stackers that facilitate the former laborious and slow process of hay-making, not only enabling the farmer to secure his crop of grass when it is in the best condition for cutting, and not oblige him to leave a portion until it is over-ripe, but also rendering him less dependent upon the weather in securing this crop, as it can be harvested, cured, and put under shelter in mucl less time than formerly.

Thus we have ensilage cutters that dispose of the forage crop in a green state, with considerable less labor and time than by the hay-making method; the reapers and self-binders, that reap and bind the grain as if by magic hands, one machine doing the work of many men in a givem time; the threshers and cleaners, that prepare the grain for market; the stalkcutters, corn-harvesters, huskers, and shellers. In the use of these implements, we have not only the powers appropriated to the purpose in the olden time, but steam as a motive power in the form of farm engines, that can be utilized in threshing, plowing, husking, grinding, and various other kinds of farm work, one such machine often being made to perform the work of over twenty horses at once, the agricuitural implements alone of some well conducted farms now often representing more money than the entire value of a good farm fifty years ago. Hence, it will be seen that the application of science to agriculture has vastly increased the productions of the country, and enabled the farmer to rise above the mere drudgery that formeriy characterized his calling.

But these are not the only advantages emanating from scientific agriculture; the innumerable experiments, investigations, and labors originating from this source, have resulted in giving to the world improved varieties of all kinds of agricultural products, including grain, fruits, vegetables, flowers, etc.; also farm animals of such improved utility and beauty as to be scarcely recognized as akin to their predecessors.

Fifty or more years ago the farmers of the country were generally the hardest laboring class that could be found, their lives being a constant round of severe toil, which left no time for the employment of mind or hands with other business. But little attention was paid to the itaprovement of farm stock-a horse, cow, sheep, or pig being recognized merely as sucl without regard to desirable points of preference in individual animals. Farmers, as a $\mathrm{gcn}$. eral rule, depended almost wholly upon the products of their farms for a living, raising nearly all the supplies for their own tables, and also largely for their clothing, which was manu. factured from the raw material in their own homes.

Whent was more commonly cultivated than now, but not in sufficient quantities to supply bread for the household, flour being rarely bought by the barrel. Rye flour and corn meal were mostly used for making bread, that made from wheat flout being considered quite a luxury, and in many sections was dignified with the name of "cake," and used only on extra occasions, such as when company was entertained. An instance illustrative of this fact 
is said to have occurred in those early days in the eastern part of New England. A hightoned gentleman, known as the "Squire," called at a farm house one day on some business, and when he had finished his errand, and had remounted his horse at the door, the good housewife, wishing to impress the 'Squire with the dignity and thrift of her family, said to him, "'Squire, won't you stop and have some cake and butter,"-thinking it now too late for him to accept her invitation. To her chagrin, the doughty 'Squire replied, "Thank you, marm, I don't care if I do," and promptly dismounted and entered the house.

The poor woman could only explain that to her surprise she found the flour bread all out, and offered him the best she had, some Indian "bannock."

There was little market in those times for farm produce, except in the larger towns, and these were often reached only after long journeys. To these towns, such things as the farmers had to spare were taken and exchanged for "store goods," said "store" usually being some corner grocery, where more rum than any other commodity was sold, it being purchased and used by the most respectable people, and its sale considered as legitimate and laudable a business as any other.

Each farmer owned his flock of sheep, from the wool of which the family were principally clothed. It was carded, spun, and woven at home, and made into garments for the entire family, that from which the "best clothes" of the masculine portion were made being called "fulled cloth," which was woven at home from the finest wool of the flock, and afterward taken to the fulling mill, where it was subjected to a process of fulling or thickening, and afterwards dyed, pressed, and otherwise finished for use. The material for the dresses of the feminine portion of the bousehold, - which were designated "gowns," -was a home-made flannel, which was taken to the mills and pressed so as to present a glossy surface. Each farmer had also a small patch of flax for home use. At the proper season the flax was pulled and spread in rows on the ground, "rotted," and afterward "broken" and "swingled,"-as the process was termed; it was then prepared for combing, carding, and spinning on the small machine which was called the "little wheel," to distinguish it from the larger implement for spinning wool. It was woven into cloth for table covers, toweling, sheeting, shirting, etc., the coarser portion called "tow," which was combed out of the waste material of the flax at the first combing, was spun into a coarse yarn and made into cloth for the summer suits of men and boys, these being usually as harsh and uncomfortable to the wearer as they were coarse and uncouth in appearance. Ready-made clothing being unknown in those times, the hard-worked tailor, with his "goose" for pressing seams, the gossiping tailoress, who carried all the neighborhood news from house to house, and the old-fashioned shoemaker, working in each house in turn till all the family were supplied with a pair of shoes, were important adjuncts of society, as they went their accustomed rounds, plying their individual trades, but they are personages entirely unknown to the present generation.

Great, indeed. has been the change since that period, and were one of these old farmers of the past to awake from his sleep of fifty years, and make his appearance among the gen. eration of to-day, he would scarcely be able to recognize this earth as the same he once inhabited and tilled. Fifty years from the present will possibly mark an era characterized by an equal extent of progress, although it may be as difficult for us to realize it, as it would have been for the generation of half a century ago to predict the present standard of advancement. The future prospects of American agriculture are indeed promising, and its progress may be estimated in a great measure by the thirst for knowledge, energy, and enterprise that everywhere characterizes the American people. In striking contrast with the agricultural advancement of our own nation during the last half century, may be instanced that of the Chinese, where the same rude agricultural implements are employed in tillage to-day that were used by their ancestors thousands of years ago. It is not difficult to understand why the agriculture of an intelligent. free people must ever essentially differ from that which is developed by an order of society less free, and characterized by ignorance and superstition. 
The permanence and independence of the farmer's avocation are strong arguments in its favor in the choice of a business, while there are few callings in which a small amount of capital can be as safely invested and at the same time prove as lucrative. It is also a business that is always sure to give plenty of employment to those engaged in it, with a certainty of a fair remuneration. It is very true that there are many farmers that make but a poor success of it,-if success it could be called without a misuse of the term-but it is equally true that there are failures in every kind of business, and that there is no calling known but what may be represented by incompetent men that are engaged in it, such men as would not make a success in anything they miglit undertake, the fault not being in the business, as such, but in the persons representing it. In any occupation or department in life, more depends upon the man than the business, as regards the ultimate success. In order to be successful in any position, there are certain requisites that cannot be ignored, and the observance of these is quite as essential in farming as in any other employment or profession.

Some persons seem to have a kind of general capacity or aptitude for almost any avocation, and will make a success of anything they may undertake, but these are the exceptions. As a general rule, men have a special talent for certain kinds of business, and in following those to which they are by nature fitted, they will be more successful and happy in their pursuit than in any other. However, a taste for certain avocations can be cultivated where it docs not naturally exist, but at the same time there must be certain natural aptitudes or capacities inherent in the individual in order to make it a success in such cases.

The really successful farmer is he who chooses his vocation for the love of it, and who feels that in it he is in his own native element, as it were; he enters it with a just conception of its nature and demands, and in its pursuit he is not disappointed. The man who has no love for the business, but, on the contrary, detests it, and only pursues it from necessity, ought not to expect to succeed, for he lacks the first great essential in the elements of success. The successful farmer will not only need a love and taste for the business, with a proper appreciation of its nature and duties, but a healthy and vigorous body, and a wellbalanced, cultivated mind. He should get beyond the narrow-mindedness that characterizes so large a class of farmers, and keep abreast of the times, profiting by the improvements, and progress of the age in which he lives, and be willing to believe there may at present be systems of practice in agriculture superior to that of his forefathers, and that the years of labor and investigation since that period have not been spent in vain. What would be the success of the merchant, if he shonld refuse to conform to the demands and customs of the present, and persist in selling only the old-fashioned goods of twenty-five or fifty years ago? He might, it is true, sell a few articles as ancient relics, but his business as a merchant would be very far from proving a success. It is just as essential for the farmer to meet the demands of the times, as for the merchant or any other business man, in order to insure success ; and to accomplish it he must study the wants of the market, and raise those crops that are most in demand, and consequently will bring the highest price; and not only this, but he should inform himself as to the best methods of improving his lands, and cultivating such crops so as to procure the best results. A man of one idea may possibly succeed in some pursuits, but not in farming. The farmer of the highest success must be a man of broad views, cultivated mind, and well-developed resources. The time is passed for the opinion of the olden time to be popularly entertained, when the merest ignoramus was thought to be competent to do well enough on the farm, and that the boys of the family that were intending to be farmers did not require as good an education as those who were intending to follow some other calling or profession. The farmer of the present and future must be well informed to answer the demands of his position; he must be competent to meet the various questions constantly presenting themselves to him from every side in the duties of lis vocation : he must be capable of understanding, as far as possiblo, the nature of the elements with which 
he has to deal, and the laws that control and regulate them. He must be able to appreciate the agricultural literature of the times, and make himself familiar with it, and not only thus to receive the products of other minds, but he must be competent to think and investigate for himself.

Successful farming demands the practice of systematic business principles. No man can succeed well in any employment whose practice lacks method and thoroughness. A slack and slovenly manner of doing work of any kind can never be very remunerative, and in no business is this fact more clearly verified than in farming. No farmer can manage his affairs on strictly business principles who does not keep an account of his receipts and expenditures, and take an occasional inventory to see how he stands financially. A merchant might run the risk of being bankrupt if he did not do this, but how few farmers ever have a systematic financial account kept from year to year.

Farming can be made profitable when the same thought, energy, business tact, and principles are associated with it that are required for other successful enterprises, and under such favoring conditions the profits will compare favorably with those of other employments. It is true that few among the many farmers can be called wealthy, but this is equally true of other vocations ; it is only one among many, in any business the world over, that rises to eminence above his fellows and distinguishes himself by extraordinary achievements of any kind, and those who acquire wealth and power are few compared with the masses in the enjoyment of but a competence. But the farmer can trust in the assurance that if he gives a proper amount of attention to the business, and ntilizes the aids within his reach, he can make it pay, and the compensation will be a fair one in comparison with that of other callings. That farming pays best which will produce the largest amount from a given area, with an equal expenditure of time and labor; in other words, it is the surplus production of each acre over the cost of raising the crop, or the raising of maximum crops at a minimum cost, which constitutes the real profit of farming.

Aside from the other advantages to be derived from agricultural pursuits, there is much of pleasure connected therewith, in an æsthetic point of view, or what might be termed the poetry of farming, and which those with an eye for the beautiful in nature cannot fail to appreciate. There are few persons so constituted as not to enjoy and appreciate, to a certain extent, works of art in the form of beautiful pictures or fine statuary, and those possessing the means will often pay large sums of money for these master-pieces of the best artists, and consider them indispensable in furnishing their homes; but what painting ever equaled the changing scenes of nature of which the farmer is ever a participant or observer; the glory of a beantiful sunrise or sunset, the ever-changing clouds in storm or sunshine; the vast forests and fields of waving grain; the quiet loveliness of the valley among the hills; the grandeur of the distant mountains, or the broad expanse of the green prairies that seem like the ocean with its rolling waves suddenly made stationary by the word of some mighty power. To one with a love for domestic animals, there is much enjoyment in the care of these mute creatures that so willingly recognize man as their lord, and so often look the affection and gratitude they cannot speak.

The farmer needs the painting of no Landseer, Bonheur, or other artist, however distinguished, to cause him to appreciate a fine horse, cow, or other animal, for these are but copies from life, and he has the originals ever about him; hence, if a man with a love for the beautiful has not the means to gratify his taste in art, he can do so to a great extent by becoming a farmer and possessing the subjects of which art furnishes but the copy.

But this is only the poetic side of farm-life, though by no means to be overlooked, and the business of the farmer requires the best energies of hands and brain, much thought and hard Iabor ; and to him life is earnest and real, and he who makes the wisest and best use of these energies will, other conditions being equal, receive the largest remuneration for his labors, in the profits that are the result of intelligent farming. 


\section{SOILS.}

YOILS may be defined as those portions of the upper stratum of the earth which contain a mixture of mineral, vegetable, and animal substances in proportions suited to the growth and nourishment of vegetation; thus containing the food of plants, which assimilate it in some mysterious way, and combining with it the requisite elements supplied by the air, build up their structures, and in their turn furnish sustenance for man and the lower animals.

Since all soils are formed from the disintegration and decomposition of the rocks which constitute the solid crust of the earth, together with a mixture of vegetable and animal remains, they take their character from or are combined mainly of the elements of the rocks from which they take their origin; hence, the great diversity of soils.

This relation is, of course, modified by circumstances, such as the prevalence of one or other of the series, or the greater or less inclination of the strata by which the debris from different sections are more or less mixed, and also by the action of the water in washing certain portions of the surface free from some constituents of the soil, and carrying the debris in a greater or less quantity to others. Hence, the great difference often found between the fertility of the soil on a higher ground from which certain of its elements have been washed, and that of the valley to which they have been conveyed.

The names commonly applied to the different soils, have respect to their qualities; thus they are from their composition designated clay soils, loam, sand, gravel, clalk, peat, etc.; or from their texture. in respect to which, those in which clay predominates are called hear' $y$, stiff, or impervious, and the others, light, friable, or porous. From the tendency of the clay soils to retain moisture, they are often called wet and cold, and the other soils, for possessing the opposite properties, dry and warm. According to their degree of fertility, they are also often spoken of as rich or poor.

The 0rigin of Soils.-It is now a generally accepted theory, and one which does not antagonize with the teachings of the Scriptures, that the greater portion of the soil which covers the earth surface, and which is cultivated by man, was once a solid rock forming the crust of the earth, and that long ages before man's creation and occupancy of the earth, this rock was covered by sheets of ice many thousand feet in thickness in the form of glaciers, that extended over nearly the entire continent; and that by the gradual melting and moving of this vast mass, the rocky surface of the earth was ground, scoured, and pulverized, forming grooves and scratches in the rocks that can even now easily be traced to the extent that the course of the moring mass can be determined, which was from the north in a southerly direction; and also grinding, as if by an immense machine, the loose materials resting upon the surface of the earth to a powder or paste, which forms the basis of the agricultural soil; that by climatic changes, the vicissitudes of heat and cold, terrific storms and various chemical and mechanical changes thus produced, the earth became fitted for the production of a lower order of vegetation. These having matured, died, and decayed, were followed by those of another order, the higher classes of plants appearing as the soil became enriched by the decay of previous vegetable organisms. During these ages it is supposed that the earth was constantly undergoing changes of climate and vegetation, which latter became in time so dense and luxuriant as to store up vast quantities of carbon taken from the air, and in this manner were formed the immense beds of coal which are now found in various parts of the world; thus preparing the earth for man's future necessities long before his creation. It will be found that soils vary much in color; this is caused mainly from the nature of the different rocks from which they are formed. This manner of soil formation,--the mixing up of the ground-up fragments of many kinds of rocks, - have given great variety to soils both in formation and fertility, and in many sections has prevented uniformity to the extent that often adjoining farms, or different portions of the same farm, are very unlike in soil. There is, of course, a great differ. 
ence between the upper and subsoil, the upper being mixed more or less with vegetable matter, besides being modified by atmospheric agencies. The broken and pulverized material lying above the solid rocks is known to geologists by the general name of drifl. With respect to the origin of the agricultural soil, Prof. Agassiz says:-

"There is hardly anywhere in the world an extensive tract of cultivated soil which has not been brought to the place where it exists from considerable distances. It is no doubt the case that the rocks are decomposing in places constantly; and the amount of this decomposition is very great, varying according to climate, and is the result of influences which are also different under different climatic conditions. In our colder northern regions the decomposition is owing chiefly to the filtration of water into rocks, to the frosts following that infiltration, to the splitting of the surface of the rock into fragments in consequence of the expansion of frozen water, and thus the disintegration of the rocks themselves. In more southern climates, where warm tropical rains are incessantly pouring upon the hard rock, it is chiefly that agency and the decaying of the rock by the heat of the tropical sun which pro. duces a similar result. And yet this process, extensive as it is, is not the chief cause, hardly an extensive cause, of the production of agricultural soil. There is another cause which ought to be taken into account-the wearing of the rock by the action of running water. Here again we have an accumulation of an immense amount of loose materials which are the result of the wearing action of running water. And yet even that is only a small portion of the amount of loose materials which are scattered over the earth and form part of the agricultural soil. The main mass of the agricultural soil is derived from an entirely different source, and is produced by an entirely different cause. There has been at work a grinding machine more powerful than the action of the sun, of water, of frost or of wearing currents. It is the agency of ice; and to that agency we owe not only the grinding of the rocks to powder and all the comminuted material which forms the chief portion of the loose coatings above the rocks which serve as the basis for our agricultural operations, but we owe also to that natural machinery the mixture of rocks derived from different regions, which have formed the compound coating over the whole surface of the earth, without which agriculture would be limited to those regions the rocky foundation of which is such as to afford a suitable soil. The agency of ice has been such as to bring together from remote countries the loose materials from the limestone rocks, the slaty rocks, the marl beds, the granite rocks, and the wearing of those materials into paste has transformed them into that coating which really constitutes the bulk of our agricultural soil. Those materials have been remodeled since by atmospheric agencies; they have been rained upon since the time they were deposited, and of course the action of water has carried far off to other places what had been first worked upon by ice. But this is not very extensive and does not constitute a part of the primary formation of agricultural soil.

"It would lead very far to enter into an extended discussion of the manner in which ice can have produced these results. It would probably excite a smile if I were to begin by saying that the whole extent of the United States has at one time been covered with a sheet of ice many thousand feet in thickness; and yet geology can show that it was so. It would probably excite doubt if it were stated that the whole sheet, moving from the north in a southerly direction, has ground the loose materials resting upon the surface of the earth to that paste which constitutes the agricultural basis; and yet it is so. It has been by a succession of observations, starting from small beginnings. that this result has been reached and is now recognized as a fact among geologists"

The Constitution and Texture of Soils.-Since soils are derived manly from the rocks, they will contain all the elements which the rocks originally did from which they were formed; usually, however, in different proportions, and in different chemical combinations, and to this mineral matter is added various materials derived from the air (aside from the 
vegetable or animal substances which they may contain in a greater or less proportion), hence their mechanical texture, as well as their mineral composition, depends upon their origin.

According to texture, soils are commonly termed rocky, stony, gravelly, sandy, silt, marl, and calcareous, loam, clay, 'etc. Though rocks and stones cannot properly be termed a portion of the soil, yet when mingled with the finer soil or found to any great extent upon the surface, these terms are commonly applied to distinguish such soils from others of different characteristics.

Although rocks and stones have long been, and are at present, the great pest to the New England farmer, yet they are not entirely useless, as they frequently benefit crops by the warnith, protection from winds, and the moisture they afford, as well as by the gradual decomposition of those which contain lime, potash, and other fertilizing elements which con. tribute to the support of the soil, these elements being furnished by the decomposition of the rocks through the action of the atmosphere, the crumbling of them by the agency of frost, or by the dissolving of their mineral properties by water-pure water itself being a strong solvent, - while water containing carbonic acid, such as rain-water (which always contains it), will dissolve limestone, chalk, and even the hardest marble in time, though the latter, of course, much more slowly than the former.

Some soils are peculiarly fine in texture, being almost entirely free from stones, gravel, or sand, such as that of certain of the TVestern prairies, while others possess but little mineral element, being composed mostly of vegetable matter, such as peat swamps, and salt marshes.

Prof. S. W. Johnson, Director of the Connecticut Agricultural Experiment Station, who is one of the best authorities on this subject, says, that the fertility of the soil depends, chemically, first, upon the presence in it of all the ash elements and of nitrates in proper quantity, and, second, on their occurrence there in such states of combination as to give a constant and regulated supply; that numerous experiments have demonstrated that a soil destitute of either phosphoric acid, sulphuric acid, potash, lime, magnesia, or oxide of iron, is absolutely barren on account of such deficiency ; and that it is equally certain that a soil which contains the usual amount of potash, but only in the form of foldspar, or of phosphoric acid, but only as apatite, or of magnesia, but only as serpentine, is infertile, because these substances do not yield their elements to the solvent agencies of the soil or plant rapidly enough to serve as plant food.

Also of silica, which was formerly supposed to be a necessary element of the soil in order to sustain a healthy and prolific vegetation, that recent investigations appear to show, that though it is present in many plants, it is an accidental ingredient and in no manner essential to their growth or perfection; and alumina, though an abundant element of soils, is always absent from agricultural plants; that soda likewise appears to be unessential to most of the vegetative processes, for although it is perluaps never entirely absent from cultivated plants, it often occurs in them in extremely minute quantity, so that the soda which is indispensable to the blood and milk of animals must be obtainod, in part at least, directly from mineral sources.

Hellriegel's experiments show that 55 pounds of potash, 55 of phosphoric acid, 17 of magnesia, 17 of soda, 23 of lime, 11 of sulphuric acid, 8 of chlorine, and 54 of nitrogen (in the form of nitrates), are sufficient in soluble condition, in $1,000,000$ pounds of soil, in order to establish there a fertility equal to the production of 33 bushels of barley grain and 2,000 pounds of straw per acre. Good soil, however, may contain a larger proportion of available plant-food than this. The weight of an average loamy soil is estimated to be about $4,000,000$ pounds per acre for each foot of depth.

It is estimated by good authority, that a crop of grain of thirty-three bushels removes from the soil but 140 pounds of ash elements; that is,--forty pounds in the seed and one hundred pounds in the straw. 
We see by this how small a proportion of the ash element is taken from the soil by a single crop of the above mentioned quantity.

There is a wise provision in nature which prevents some soils from being worn out or permanently exhausted, as they otherwise might be, could all their fertilizing elements be extracted by man at will, which is in the slow and gradual solution of their stored-up treasures of mineral wealth, and the chemical changes wrought in time through the agency of air and water.

It is a fact well-known to any farmer that when soils have become poor or infertile from too extensive cropping without sufficient fertilizers of the proper kind to restore in a measure the elements extracted by the production of the crops, often a rest of a few years, without aid from any kind of applied fertilizers, will in a measure restore the fertility. This is caused by the decay of the insoluble part of the soil, which thus assumes a new chemical combination and becomes soluble, these being chemical changes brought about through the agencies of the atmosphere and water.

Prof. Wm. H. Brewer, also of the Connecticut Agricultural Experiment Station, says in relation to this subject:

"If the original rock which furnishes the sand and gravel of a soil, contains all the mineral elements of fertility, such a soil may be impoverished by too hard cropping, but it cannot be exhausted, or, as you would say, worn out. The annual weathering, by making soluble what was before insoluble, is a process of contiuually restoring fertility to a greater or less degree. We are told of certain soils in Greece which have been cropped for thousands of years, and are still reasonably fertile, because of such changes.

A soil having this character in a less marked degree may be temporarily impoverished; we say then, that it is in a low condition, or "run down," and yet it recuperates rapidly with rest, and with proper tillage or pasturage.

On the other hand, there are some soils that have great fertility for a time, and when they run down remain very poor indeed. What fertilizing material the soil had that was mostly in an available condition for plant-food, is used up by a few crops, and then barrenness follows. I have heard of some remarkable cases of this in the Western States, where the soils seemed wonderfully fertile at first, produced a few large crops, then utterly failed, and are now abandoned. These first few large crops took up the plant-food which had been preparing for centuries. It was like a long investment suddenly becoming available in ready cash, which is as suddenly spent, poverty following in its track.

Some years ago I began a microscopic examination of different sands in connection with certain geological studies. Sands from the sea-shore in many parts of the world, from the shores of lakes fresh and salt, the wash of rivers, from placer mines, from mountains and valleys, the drifting sands of the Pacific slope, and from the deserts of the great basin, and finally the sands washed out from varions soils, arable and otherwise. It is curious to see how some of those from soils have been modified by the influences here spoken of-often there is a partial cementing again, by which each larger particle is coated with smaller grains adhering to it; in others the splits and cracks by frost, and more interesting still, the way some show that they have been partly dissolved. Only this week I was examining some from a sandbank deposited in Central New York, near Cayuga lake, so long ago that a ravine four hundred feet deep has been worn in the rock since that sand was deposited. When deposited it was evidently all worn and rounded by the water which left it where we now find it. Now each quartz grain is all roughened by little pits sunk into it by being partly dissolved in the long ages it has lain there. In another, from the old silt left near Niagara Falls before the gorge was made in its present shape, the solution of some ingredients has partially re-cemented others.

The matter of oxidation is a curious one. Wooden posts last very differently in differ. 
ent soils. $\Lambda_{\text {s a }}$ rule, they decay quickest in open sandy soils, and last longest in heavy clays or wet peat. It is just so with all organic matters. It is well known that in some grave. yards human bodies decay in four or five years, in others only in fifteen or even twenty years. Now, this has an important bearing on the use of manures. In some soils the organic part of the manure disappears quickly, particularly in sandy soils, while in others, particularly loams and clays, they remain much longer, modifying both the chemical composition and physical ebaracter.

Again, soluble mineral salts are washed out unequally. As a general rule, those salts which are of most use to plants are held more tenaciously, while those that are injurious wash out easier.

There is another way in which the composition and constitution is affected by the air, that is, through the climate. Alkaline soils and salty soils of various kinds are only found in dry climates. All the saline and alkaline soils of the interior and western parts of our continent, and indeed of all countries (except shores) would be soon purified if the rains were abundant enough for numerous springs and streams. The countries are salt and alkaline because these salts have been developed by the disintegration of the rocks, and the soils have not been sufficiently washed out. Even our Conneeticut coast salt marshes soon lose their saline character and become fresh if the sea water is excluded."

Thus we see that many soils contain in themselves the elements of fertility, which are gradually supplied through the agency of water and air, while others soon become exhausted without the use of manures or other fertilizers to restore the elements that have been extracted by the crops produced.

Classification of Soils. It is a well known fact that soils frequently, by a gradual process, change from one character to nnother, so that a minute classification is almost impossible. We prefer for general reference the arrangement adopted by the noted British authority, Prof. Johnston, which is based upon their chemical constituents.

"1st. Pure clay (pipe-clay), consisting of about 60 of silica and 40 of alumina and oxide of iron, for the most part chemically combined. It allows no silicious sand to subside when diffused through water, and rarely forms any extent of soil.

" 2 d. Strongest clay soil (tile clay, unctuons clay), consists of pure clay mixed with 5 to 15 per cent. of a silicious sand, which can be separated from it by boiling and decantation.

"3d. Clay loam differs from a elay soil, in allowing from 15 to 30 per cent. of fine sand to be separated from it by washing, as above described. By this admixture of sand, its parts are mechanically separated, and hence its free.and more friable nature.

"4th. A loamy soil deposits from 30 to 60 per cent. of sand by mechanical washing.

" 5 th. A sandy loam leaves from 60 to 90 per cent. of sand, and

"6th. A sandy soil contains no more than 10 per cent. of pure clay.

"The mode of examining with a view of naming soils, as above, is very simple. It is only necessary to spread a weighed quantity of the soil in a thin layer upon writing paper, and to dry it for an hour or two in an oven or upon a hot plate, the heat of which is not sufficient to discolor the paper-the loss of weight gives the water it contained. While this is drying, a second weighed portion may be boiled or otherwise thoroughly incorporated with water, and the whole then poured into a vessel, in which the heavy sandy parts are allowed to subside until the fine clay is beginning to settle also. This point must be carefully watched, the liquid then poured off, the sand collected, dried as before upon paper, and again weighed. This weight is the quantity of sand in the known weight of moist soil, which by the previous experiment has been found to contain a certain quantity of water.

"Thus, suppose two portions, each 200 grs., are weighed, and the one in the oven loses $50 \mathrm{grs}$. of water, and the other leaves $60 \mathrm{grs}$. of sand,--then the $200 \mathrm{grs}$. of moist are equal to 150 of $d r y$, and this 150 of dry soil contains 60 of sand, or 40 in 100 ( 40 per cent.). It would, therefore, be properly called a loam, or loamy soil. 
"But the above classification has reference only to the clay and sand, while we know that lime is an important constituent of soils, of which they are seldom destitute. We have therefore,

"7th. Mfarly soils, in which the proportion of lime is more than 5 per cent., but does not exceed 20 per cent. of the whole weight of the dry soil. The marl is a sandy, loamy, or clay marl, according as the proportion of clay it contains would place it under the one or the other denomination, supposing it to be entirely free from lime, or not to contain more than 5 per cent., and

"8th. Culcareous soils, in which the lime, exceeding 20 per cent., becomes the distin. guishing constituent. These are also calcareous clays, calcareous loams, or calcareous sand, according to the proportion of clay and sand which are present in them. The determination of the lime, also, when it exceeds 5 per cent., is attended with no difficulty.

"To 100 grs. of the dry soil diffused through half a pint of cold water, add half a wine glass full of muriatic acid (the spirit of salt of the shops), stir it occasionally during the day, and let it stand over night to settle. Pour off the clear liquor in the morning, and fill up the vessel with water to wash away the excess of acid. When the water is again clear, pour it off, dry the soil and weigh it; the loss will amount generally to about one per cent. more than the quantity of lime present. The result will be sufficiently near, however, for the purposes of classification. If the loss exceed 5 grs. from 100 of the dry soil, it may be classed among the marls; if more than $20 \mathrm{grs}$, among the calcareous soils.

"Lastly, vegetable matter is sometimes the characteristic of a soil, which gives rise to a further division of,

"9th. Vegetable molds, which are of various kinds, from the garden mold, which contains from 5 to 10 per cent., to the peaty soil, in which the organic matter may amount to 60 or 70 per cent. These soils also are clayey, loamy, or sandy, according to the predominant character of the earthy mixtures.

"The method of determining the amount of vegetable matter for the purposes of classification, is to dry the soil well in an oven, and weigh it; then to heat it to dull redness over a lamp or bright fire till the combustible matter is burned away. The loss on again weighing is the quantity of organic matter."

The preceding are commonly recognized as such general divisions as possess properties sufficiently common to require a similar process in testing. The earthy, or what are termed inorganic parts of soils, are found almost exclusively in combination with earths, salts, or minerals, and constitute from less than one-half of one per cent. to over 10 per cent. of all plants; in addition to these inorganic parts, fertile soils must also contain carbon, oxygen, nitrogen, and hydrogen, which are called organic parts of soils, because of their greater proportion in vegetables and animals, of which they constitute in substance from about 90 to over 99 per cent.

Clay Soils and their Management.-From their affinity for water, and a tendency to hold it in such quantity as to often impede a rapid or luxuriant growth of vegetation, clay soils are commonly denominated cold and wet.

Clay owes its origin mainly to the decomposition of other minerals, and consists largely of alumina, which is the most abundant of all the earths, combined with silica and water, its plasticity being dne to the alumina it contains, which being so fine in texture and compact, prevents the escape of water resting upon the surface of such soils, or contained within it. In its pure state, alumina is an earth resembling soft, white powder, withont taste; in its crystaline form, it occurs as sapphire and ruby, which are among the hardest and most valuable of the precions stones.

Prof. Brewer states that when on bottom lands, or in valleys, clays are usually the sediment deposited from muddy water in some earlier age of the earth's history, but when on hillsides, they are often, in part at least, due to the decay of slate rocks. 
The feldspar which by its decomposition largely yields the alutnina of clay soils, coatains also potash and soda; hence, a mixture of calcareous matter with other earths prores beneficial to crops. Clay soils have also a remarkable power for absorbing ammonia and cther substances, which constitute the food of plants.

Under-draining is the best means of removing the water from clay soils, and thus render them more available for cultivation, though open drains are effectual to a certain extent.

Sand, being porous, is very valuable to mix with the too compact clay, thus producing a soil neither too porous or compact; for large fields, this may often prove too expensive for practical purposes. Coarse vegetable manures, such as straw, corn-stalks, fine chips, etc., which have a tendency to reduce the compactness, are recommended by some writers on this subject. Barn-yard manure is also excellent. Lime and gypsum add much to the fertility of such soils.

English writers on agriculture mention the use of burnt clay in many parts of Europe, a practice long in vogue there for the improvement of clay soils, but in this country it would be attended with too great expense to be practicable. Their method is to roll and harrow the field in dry weather till the majority of the clods are about the size of a large walnut; when perfectly dry, coliect them into rows about six yards apart with iron-teeth rakes; about four yards apart in the rows apply faggots and burn the clay slowly, to prevent hardening. When the heaps of earth are cold, they are spread over the surface and plowed in. This method, it is stated, has astonishingly increased the productiveness of some of the poor clay soils in certain localities of England, but as we have previously stated, we doubt the expediency of its adoption in this country, where labor is attended with so much greater expense.

It is desirable to plow clay soils in the autumn, that the action of frost, rain, and heat may aid in pulverizing the earth, after which, if barnyard manure be applied (which we think one of the most desirable manures for clay soils). it acts both chemically and mechanically to reduce it to a mellow condition and perfect its texture, being in a condition to freely admit air and moisture, and consequently warmth and increased fertility, while it is neither too porous or compact, and readily discharges surplus water, yet retains sufficient for moisture; by the fineness and closeness of its particles, the soil settles sufficiently compact around the roots of the plants to increase their absorption of nutritive properties, which produce a rapid and healthy growth. If plowed in the spring or summer, it should not be either too wet or dry; if too wet, it turns over heary and soon becomes hard and lumpy by drying in the hot sun, and subsequent plowing or harrowing will not effectually counteract the evil for a long time, thus often injuring the texture of the soil for many years. The action of frost will afterwards correct this difficulty in a measure, but not wholly. If plowed too dry, the soil is so compact that it renders the task a difficult one; besides, it remains in clods. After the rain has sufficiently moistened such clods, the harrow may be applied effectually to reduce them with considerable success; but it is better to plow such soil when neither too wet or dry, but of medium consistency. Plowing under green crops is very beneficial to clay soils; but one of the most important points in their successful management is to keep the texture in a desirable condition by the judicious use of implements in working the land over, and preventing its becoming lumpy and hard, thus at the same time improving its texture and increasing its fertility in a proportionate degree.

Thorough mixture should never be neglected in any soil, as the change of particles gives the opportunity for the extended union of the fertilizing elements, while new combinations are thus formed, aided by the atmosphere and the increased moisture resulting from the stirring of the soil.

The gradual deepening of clay soils, that is, deepening it some with each season's plowing, is thought by most farmers to be a good rule, though a rery old one. 
Clay soils are strong and durable, and retain more than any other the fertilizing elements that may be added in the form of manures, etc., and when properly managed, are among the most valuable and productive. They are admirably adapted to the production of most of the grains, especially wheat, also clovers of various kinds, and beans. They are also peculiarly suited to grasses, hence are often denominated grass lands, owing to their peculiar fitness for mowing lands and pastures.

Sandy Soils and their Management.-Sandy soils are in almost all respects the reverse of clays-being warm, light, and dry. Under judicious cultivation, for most kinds of crops, no soils yield more readily or bountifully, while they possess the advantage of being ready for working earlier in the spring than clay soils, and are less liable to be affected by frosts. Being leachy, they have but a slight hold on manures, as water soon washes out the fertilizing elements, consequently it is best not to apply them until near the time of planting, such as late in the winter or early in the spring, instead of the fall, as on some other soils.

The texture and quality of sandy soils are much improved by a mixture of clay and humus, or mold, while muck and vegetable matter, liberally applied, are also often very beneficial. With a good supply of humus in the soil, little fear need be entertained of drought.

Allen says: "As clay soils are much benefited by a mixture of sand, so likewise are sandy soils greatly improved by the addition of clay, yet in a much higher degree; for though it would never pay, as a general rule, to add sand to clay, yet the addition of a few loads of the stiffest clay to a light sand, would in almost every instance much more than compensate for the trouble and the expense. For this purpose, the clay should be thinly spread in autumn upon sward land previously plowed, and the winter's frost will effectually separate the particles. It should then be harrowed thoroughly and deeply in the spring, and subsequently plowed if necessary. Such a dressing on a light, crawling land, is more than equivalent to an equal quantity of the best manure, and will be permanent in its effects. Clay and sand are necessary to each other, as they both contain qualities which are essential to a good soil; and that will always be found the best, which has the proper proportion of each.

Sandy soils are improved by the frequent use of a heavy roller: it cannot be used too often. They require to be made more compact, and any treatment that secures this object will be advantageous.

Lime, by its chemical action on the constituents of soils, while it separates clay, renders sand more adhesive; and when cheaply obtained, it is always a profitable dressing for sandy soils, to the full amount they may require. Gypsum, in considerable quantities, has an effect similar to lime, both on clay and sand; and when added in smaller portions, produces a striking increase in the crops of sandy soils. Clay marls, containing either carbonate, sulphate, or phosphate of lime, are of great value to sandy soils. Equally beneficial are ashes, leached or unleached, peat, or vegetable manures of any kind. Some calcareous sands, containing a large proportion of lime, like those of Egypt and extensive regions in the Barbary States, will produce luxuriantly, if supplied with a slight addition of manure and an abundance of water. Sandy soils can never be profitably cultivated till they have acquired sufficient compactness and fertility to sustain a good growth of grass or clover; and when once brought to this condition, they are among the most valuable.

They are at all times easily plowed and worked; they require no draining; and though light and dry, are quick and kindly soils, giving an immediate and full return for the labor and manure bestowed upon them. When in a condition to produce grass, sheep are admirably adapted to preserve and augment their fertility, and by their incessant migrations over it, their sharp hoofs pack the surface closely, producing the same effect as the roller."

For corn or any strong-growing crop in sandy soils, it is well to give the land a large supply of barn-yard manure-applying it after the land is plowed, which should be a few 
weeks before planting, that the soil may be saturated with the fertilizing properties at the time of plantiug. This practice favors quick starting in growth, the roots being fed with the manure that is constantly being diffused through the soil. Sandy soils being warmer and more dry than others, favoring, as we have previously stated, early working, which not only gives an early start to the crops, but furnishes them with the winter moisture therein contained, and enables them to secure a good hold upon the soil before the dry season sets in, and thus many of the bad effects of drouth may be in a measure avoided. Where the season is unusually wet, much of the fertilizing element of the manure will be washed out of the soil and wasted, but where dry weather is more prevalent than wet, the above practice will give the best of results.

Leachy soils generally must have frequent and light applications of manure, as they will not retain, like clay, the wealth of soil till the following year; they will only hold it long enough for the growing vegetation during the season, the remainder passing through the light soil, and is lost. Where there is a considerable amount of clay mingled with the soil, the effect of retaining will be varied proportionately. Clover is exceedingly beneficial in increasing the productiveness of poor, sandy soils that are capable of producing but very light crops. We give the following method from the pen of a farmer whose land at the time of his purchasing it was of such poor, sandy quality as to be considered almost worthless for agricultural purposes.

"Clover is my main dependence for keeping up and increasing the productiveness of my lands. The entire tract ( 300 acres) is now all under cultivation, and the annual yield to the acre of wheat, rye, corn, and clover hay will average with the best land, in Wisconsin or Iowa, which has been the same number of years under cultivation. My favorite plan is to have one field each of clover, corn, and wheat every year. This makes a three-year rotation. The first crop of clover is cut for hay in June. The second crop is allowed to go to seed, and plowed in late in the fall ; in the following spring it is planted with corn; the next spring sown with wheat, and the land will be found to be abundantly seeded to clover. The clover is dressed with one hundred pounds of land plaster to the acre. The manure made on the farm is spread where most needed.

It is sometimes desirable to let the land remain in clover for a longer period. My experience is that clover, being a biennial plant, will winter-kill the third winter after sowing, but leaves behind a great mass of roots that wonderfully enrich the soil. Land laid down with clover is better than money in bank, drawing more interest than any bank can pay and compounding the interest oftener.

Bonssingault took a portion of pure sand, burned it until all traces of organic matter were expelled, then took up some growing clover plants, washed them clean, removed the external moisture with blotting paper, weighed them, and set them in the sand. He then watered them with distilled water, placed them in the air, and in two months' time found that they had tripled their amount of organic matter-thus proving that air, pure water, and sand have all the elements necessary to sustain the growth of clover. In rich soil the results would doubtless have been increased, but we see in this little experiment the elements of all successful agriculture. Had he buried the perfected plant in his sand, and planted again, and continued the process, it is evident that there would be no end to the amount of fertility which might be accumulated, and this would not be in an arithmetical, but in a geometrical ratio, for the presence of manure in the soil would make it easier to accumulate still more from the atmosphere.

Early in the month of September I plowed a field of forty-three acres of well-worn land, from which a crop of rye had been harvested the preceding July, and sowed it again with rye. In November, cattle and horses were permitted to run on it, and continued to do so until March-no snow covering the ground. Early in May the rye averaged nearly three 
feet high, and was beginning to head out, when the plows were set in motion, and the green, juicy rye was turned under and the field planted with corn. The yield was forty-four bushels of No. I shelled corn to the acre. There was less labor and expense attending the plowing and sowing of that rye than there would have been to have hauled sufficient manure from my own barnyard, even if I had had the manure to have spread on the land to produce the same quantity of corn that plowing in the rye did. Again, the corn did not require one-half the labor to keep it clean that it would have done had crdinary barnyard manure, full of all kinds of seeds, been spread on the land and plowed in before planting. Rye is the best of all the small grains to clean land from weeds. The late fall and winter feed which the rye furnished to the stock was of more value than the seed sown.

Some farmers make a practice of sowing rye among their corn in July, just before cultivating the corn the last time. The rye does not make much growth until the corn is ripe and the deadened leaves let the sun on it. After the corn is picked, the cattle are turned in, and thrive on the stalks and green rye. In May, the rye and what is left of the stalks are plowed in, and is said by those who have practiced it to increase the yield of corn ten to fifteen bushels to the acre. There is abundance of evidence that heavy clay lands that have been worn out hy successive cropping, can be restored to at least their original fertility by the same process that I have found so beneficial on sandy land."

Gravelly Soils.-Gravelly soils, being coarser in texture than what are commonly called sandy soils, are peculiarly leachy and permit the escape of manures, both by evaporation and rapid drainage; they are therefore not as valuable as sandy soils. Some of the gravelly soils are composed of essentially the same elements as the coarse granites, viz., sand, clay, and potash, with a small percentage of iron, lime, and magnesia. Carbonic acid is found in the air, water, and soils; when in a state of solution in water it is the principal agent in decomposing the granite rocks and setting free the potash contained in them; when in large quantities in the soil, or, as chemists say, in excess, it forms bicarbonate of potash, soda, magnesia, and lime, all being soluble in water. By different processes new compounds are thus formed which constitute the food of plants, and thus the coarser soils are made available for agricultural purposes. Gravelly soils that are in their nature calcareous, or composed of decomposed limestone, are more valuable for tillage than the former, since they have a tendency to retain more of the elements of plant-food, and are consequently richer and more compact. The poorer gravelly soils are used principally for pasturage, not yielding sufficient returns in crops generally to repay the expense and labor of tillage.

Loamy Soils.-A loamy soil is an intermediate in character between sandy and clayey soils, being lighter and warmer than a clay, and stronger and more retentive than a sandy soil. Most loamy soils contain, in different proportions, silicious sand, clay, carbonate of lime, with more or less oxide of iron, magnesia, and various salts, etc. These soils, when of desirable combination, are generally regarded as among the best for the various agricultural purposes. When they contain a large per cent. of clay they require, like clay soils, careful drainage, this is especially desirable for an orchard, otherwise many of the tender varieties would be liable to be winter-killed. In cultivating such soils they should be treated like either clayey or sandy soils in proportion as they approximate nearest to the one or the other of these soils in characteristics.

Marly and Calcareous Soils.--Marly soils contain a mixture of clay and sand with a considerable proportion of carbonate of lime, the latter being a very valuable ingredient as a fertilizer. They are designated as calcareous, clayey, or sandy, according to the preponderance of one or the other main ingredient. Pure marl, in order to be of much value as a manure, must be rich in carbonate of lime, and sufficiently soft to be excavated by ordinary digging utensils. It is generally formed of the shells of mollusks and other marine animals 
of a former age ground or crushed by various natural causes, and cemented by clay or rendered compact by the pressure of the overlying stratum of earth, or from other causes. South Carolina and New Jersey have immense beds of underlying marl, which are especially valuable as a fertilizing element, and to which much of the fertility of their soil is doubtless due. Marl containing a large per cent. of gypsum, and in which crystals of gypsum are easily descernible to the naked eye, is found in great quantity and readily accessible on the shores of Lake Jessup, Florida. The large amount of gypsum it contains renders it of great value to the agricultural interests of that section. Like loamy soils, marly soils may contain also a greater or less proportion of sand or clay, and should be treated according as they approach nearest the characteristics of either a sandy or clayey soil. These soils are very tenacious of manures, and are what may be termed durable or lasting soils.

Silt and AlluviaI Soils.- Silt being synonymous with sediment, denotes those soils which are formed by water transporting fine sediment or mud into lakes and estuaries, and depositing it in their beds. Alluvial soils are such as have been formed by the deposit of rivers or streams, hence a silt soil, according to Prof. Brewer, may be a river.deposit and consequently alluvial, or it may be a salt inarsh by the sea, or such a marsh reclaimed, and in no way pertaining to a river deposit, in which case it cannot strictly be called alluvial, and properly is not. These soils vary greatly in their characteristics from a mixed clay to pure sand, but generally combine the elements of such soils as are denoted loamy or sandy loams. When of this character, they are exceedingly fertile, and if favored with an annual overflow from a stream above them, thus depositing upon their surface the fertilizing elements of other soils, they yield remarkably large crops constantly without other fertilizing agents. They are suited to the various agricultural purposes, and are easily tilled. Such soils yield large crops of the best of grasses, and when particularly exposed to injury by freshets, this crop is less liable to be damaged by such exposure than any other; therefore it will be safer for farmers having such particularly exposed lands, to appropriate them to the permanent production of grass. The rich bottom lands in the valley of the Mississippi furnish a good illustration of alluvial formations, as also the valley of the Nile.

The drainage of Lake Okeechobee and the Everglades in Southern Florida, thus reclaiming 12,000,000 acres of the best sugar land in the world, will prove a grand scientific agricultural achievement, and one that will result in great benefit, not to Florida alone, but to the United States; since Florida, according to an accurate estimate, is by this means capacitated to produce more sugar than the United States consumes.

Muck.-The principal constituent of muck is of vegetable origin, being formed from decayed vegetation in swampy lands, hence as a soil it is too wet to admit of cultivation, as such, but is principally used as an absorbent for manures, and in composts, in which case it must be well dried before it is suited to absorb the liquid portions of manure. When thus saturated it makes an excellent fertilizer. Muck varies much in value, according to its origin. When originating from a mass of decayed forest leaves and other plants, it must of necessity contain considerable fertilizing matter; if from mosses and coarse sedges, it will contain far less of the valuable elements; and when composed largely of sand, nearly worthless; or it may be impregnated with protoxide of iron, which is a deadly poison to vegetation, lence, its value depends entirely upon its origin. Its chief value when used alone consists in the quan. tity of vegetable matter it contains, and which is well adapted to soils of an opposite nature to muck, or dry soils that require vegetable manures. When thus used it should be well drained to dispose of the surplus water it contains.

The Origin and Age of Muck Deposits.- Wherever stagnant water has existed for a long time a certain class of coarse marsh plants, grasses, and mosses, are usually found, such conditions favoring their rapid and luxuriant growth. These plants year by year mature 
and decay in the impure water that is the source of their origin, and at the same time is likewise their grave. As years go by and centuries succeed each other with their changes of heat and cold, these basins become filled up and other forms of vegetation present themselves; sometimes trees and shrubs cover the spot, together with coarse grasses and mosses, the bed remaining spongy and soft, the annual fall of leaves and the decay of plants constantly contributing to the filling up of the stagnant ponds. In some cases large trees bave fallen and become covered with the accumulated muck and peat, and have thus been preserved from destruction for ages, forming some of the most durable timber known for ship-building and other purposes. Cypress Swamp, located in the southern portion of Delaware, presents an interesting example of this kind. Here for several miles around can be found buried in the muck and peat at various depths, reninants of a fallen forest, with forests of another generation-mostly white cedar-growing above.

The method of obtaining these buried trees is described as follows, by a recent writer:

"The huge trees which lie under the swamp to unknown depths are of the white cedar variety, an evergreen, known scientifically as the Cupressus Thyoides. They grew years ago in the fresh water, which is necessary for their sustenance, and when in time, either by a subsidence of the land or a rise of the seas, the salt water reached them, they died in great numbers. But many of them, ere they died, fell over as living trees, and were covered slowly by the deposits of muck and peat which fill the swamp. The trees that fell over by the roots are known as windfalls to distinguish them from the breakdowns. The trees which were broken off are the ones most sought for commercial uses, and they are found and worked as follows: The log-digger enters the swamp with a sharpened iron rod. He probes in the soft soil until he strikes a tree, probably two or three feet below the surface. In a few minutes he finds the length of the trunk, how much still remains firm wood, and at what place the first knots, which will stop the straight split necessary for shingles, begin. Still using his rod like the divining-rod of a magician, he manages to secure a chip, and by the smell knows whether the tree is a windfall or breakdown. Then he inserts in the mud a saw, like that used by ice-cutters, and saws through the roots and muck until the log is reached.

The top and roots are thus sawn off, a ditch dug over the tree, the trunk loosened, and soon the great stick, sometimes five or six feet thick, rises and floats on the water, which quickly fills the ditch almost to the surface. The log is next sawn into lengths two feet long, which are split by hand and worked into shingles, as well as into the staves used for pails and tubs. The wood has a coarse grain, and splits straight as an arrow. The shingles made from it last from sixty to seventy years, are eagerly sought for by builders in Southern New Jersey, and command in the market a much higher price than the ordinary shingles made of pine or chestnut. In color the wood of the white cedar is a delicate pink, and it has a strong flavor, resembling that of the red cedar used in making lead pencils. The trees, once fairly buried under the swamp, never become waterlogged, as is shown by their floating in the ditches as soon as they are pried up, and, what is more singular, as soon as they rise they turn invariably underside uppermost. These two facts are mysteries which science has thus far left so. The men who dig the logs up and split them earn their money. The work is hard, exacting, requiring lusty manual labor, skill, and experience.

Owing to the fact that the swamps are soft and treacherous, no machinery can be used, and long stretches of mud and water must be covered with boughs and bark before the shingles can reach the village and civilization. The number of the trees which lie below the surface of the ten square miles of swamp is almost countless. In many places the probe will be sunk many times before it fails to strike a log. As the workmen only dig for those near the surface, and none but the best trees are selected, it is certain that only a small fraction of the logs have been exhumed since 1812, when the industry first sprang up. The sunken forests lie in all shapes. Sometımes the trees are found parallel, as though a wind blowing 
from one quarter bad felled them, but usually they lie pointing in every direction, and when, as occasionally happens, the wet soil sinks or dries, the mighty trunks are seen piled upon each other as in a Maine log-jam. What are seen, too, are but the uppermost strata of piles upon piles unseen below."

The time required to form a muck-bed cannot be estimated, the stage of decay of the materials composing it not always indicating the lapse of time. Hundreds of centuries must have been required for the formation of the deposits in the swamp previously referred to, and human computation fails in arriving at any definite conclusion.

Muck differs very materially in texture and color, according to its origin and the degree of change reached by the material of which it is composed. It is generally regarded by chemists and agriculturists as most valuable for agricultural purposes when dried and used as an absorbent of liquid manures, its application alone to soils of any kind not being considered generally of sufficient value to warrant a recommendation of its use for this purpose.

Peaty Soils and their Cultivation. Peaty soils are frequently called vegetable soils, because they consist mostly of vegetable matter, with but little of the mineral element. They are usually found in low swampy localities, but sometimes on declivities where the water is obstructed in its course by basin-shaped cavities. They are formed mostly by the growth and decay of aquatic vegetation. In many swamps the peat is found to be of great depth, sometimes reaching to that of from thirty to fifty feet. In such cases the locality is supposed to have formerly been a lake which was gradually filled up with peat. When peaty swamps are overflowed from time to time by a river that deposits sand or elay, some of the most productive soils will frequently thus be formed. The rich lands of Holland, which support such a dense population, consist of a soil of this character. The islands near the junction of the Sacramento and San Joaquin rivers in California are well-known examples un this country.

Prof. Brewer says: "Peaty soils may shrink on drying even more than clays. This is duc to two causes, - first, the actual shrinkage of the dried material, and second, its oxidation or decay. When such lands are dried and brought into cultivation, the vegetable matter decays. When the Holland peat-bogs have been drained, they have sometimes sunk several feet in the course of years, by such decay, and the same is true of the drained marshes in New Jersey. We have a similar case in New Haven, where the salt water was shut off from a salt marsh over a century ago, and it has since been used for pasture; the embankment was broken last year, and the tides now cover it like a lake; the land has sunk away from a few inches near the edges to more than two feet in the more peaty portions."

When drained and exposed to the air sufficient to become dry, peat in its natural state is often used for fuel, as is the case in Ireland. It is valuable as an absorbent of liquid manures; also as a fertilizer when applied alone to a dry, gravelly or sandy soil.

In England, charred peat has been used as a manure, both when applied alone and when combined with night-soil, liquid manure, sewage, water, etc., which it dries and deodorizes. So great at one time were the expectations of an enormous demand for it, besides the bene. fits to result to Ireland by thus disposing of her bogs, that a royal charter was granted to a company for its manufacture. It is not at present, however, used in that form as extensively as that which is merely dried, the latter being a better absorber and retainer of ammonia than that which is charred. Peaty soils, being naturally wet, should be well drained of sur. plus water and moisture, in order to be successfully cultivated.

It is a good practice to cut up the hummocks or bogs after draining, throwing them into heaps, and after they are sufficiently dried, to burn them and scatter the ashes over the land thus drained. Wood asbes and gypsum are valuable fertilizers for peaty soils.

Subsoils. The character of the stratum or bed of earth lying immediately beneath the surface soil, called subsoil, affects very materially the efficiency of soils in their produc. 
tion. If it is of a clayey nature, that prevents the escape of water, and thus permits an accumulation on the surface, many crops will be seriously injured by heavy rains. Soils having such a subsoil will be greatly benefited by under-draining, and this should be done if possible. Some recommend breaking up and loosening this hard strata with a subsoil plow. This should be cautiously done, however, for unless the subsoil is of a nature to benefit the surface soil by mixing with it (which is rarely the case), or additional manure be applied, the latter will be injured by the mixing; for, while deep plowing is very desirable in some soils, furnishing additional moisture to the roots of crops, and admitting the air, besides increasing the depth of the soil, and obviating the effect of drouth in the hot season, still, with other soils, it is a positive injury, and the few benefits derived are more than counterbalanced by the evil effects upon the soil of bringing up coarse gravel or other undesirable materials to the surface. If the subsoil can be broken or loosened without mixing with the surface soil, much benefit may be thus derived. Since soils differ so greatly in character, only gen. eral rules can be given in this respect the farmer being able to judge of the nature of his own soil, must be guided accordingly.

When the subsoil is porous and leachy, readily permitting the escape of not only water, but the soluble portions of manure and other fertilizing elements, the above-mentioned pro. cess of subsoil plowing would be very injurious, since the sand or gravel would be brought to the surface and mixed with the fertile surface soil. Most writers recommend in such cases the deepening of the surface soil by the addition of vegetable manures, in order to afford greater depth for the roots and thus increase the moisture. This is a laborious and expensive process, bowever, and such lands make usually the best return for what is expended. by being kept as permanent wood or pasture lands.

If the subsoil and surface soil be of such a nature as to be improved by mixing, such as the one being of a sandy or gravelly nature, the other of a clayey or marly character, the mixing of the two soils by deep plowing will be rery advantageous, since the very ingredients wanting to perfect or improve the surface soil will thus be furnished.

Allen, in his American Farm Book, says: "The effect of long-continued cultivation, besides exhausting what is essential to the earthy part of plants, is to break down the coarser particles of the soil, by the mechanical action of the plow, harrow, etc., and in a much more rapid degree, by the chemical combinations which cultivation and manuring produce. A few years suffice to exhibit striking examples in the formation and decomposition of rocks and stones. Stalactites and various specimens of limestone, indurated clays, sandstone, and breccias, or pudding stones, are formed in favorable circumstances, almost under our eye; while some limestones, shales, sandstones, etc., break down in large masses annually, from the combined effect of moisture, heat and frost. The same changes, on a smaller scale, are constantly going forward in the soil, and much more rapidly while under cultivation. The general tendency of these surface changes is towards pulverization. The particles forming the soil, from the impalpable mite of dust to the large pebbles, and even stones and rocks, are continually broken up by the combined action of the vital roots and the constituents of soils, by which new elements of vegetable food are developed and become available, and in a form so minute as to be imbibed by the spongioles of the roots, and, by the absorbent vessels, they are afterwards distributed in their appropriate places in the plant. Where this action has been going on for a long period, a manifestly beneficial effect has immediately followed from bringing up and mixing with the superficial earth portions of the subsoil which have never before been subject to cultivation.

"A subsoil which is permeable to water is sometimes imperceptibly beneficial to vegetation, not only by allowing the latent moisture to ascend and yield a necessary supply to the plants, but a moisture frequently charged with lime and various saline matters, which the capillary attraction brings from remote depths below the surface. It is probably from this 
cause that some soils produce crops far beyond the yield which might be reasonably looked for from the fertilizing materials actually contained in them. This operation is rapidly going forward during the heat of summer. The water thus charged with saline matters ascends and evaporates at and below the surface, leaving them diffused throughout the soil. After long-continued dry weather, a thin white coating of these salts is frequently discernible on the ground.

"Where rain seldom or never falls, this result is noticeable in numerous and sometimes extensive beds of quiescent (not shifting) sand. Deposits ofttimes occur several inches in thickness. Such are the extensive beds of impure muriate of soda and other salts in the arid deserts of Arizona; in the great Salt Lake Basin; in the southern parts of Oregon; the ritrates found in India, Egypt, Peru, and various other parts of the world.

"Besides the qualitiesof soils already noticed, there are several physical conditions which affect their value. They should be of sufficient depth, friable, or easily pulverized; they should possess the right color, and be susceptible of the proper admission and escape of heat, air, and moisture.

"Friableness of the Soil is a quality equally removed from the adhesion of strong clay or the openness of loose sand. Good loams and fertile alluvial soils always possess this property. When stirred by the plow, the spade, or the hoe, the earth should fall and crumble readily, although wet. Such a condition secures a ready admission to the roots, which thus easily pervade the soil, and draw from it in every direction their necessary support. Under draining, and the addition of coarse manures to clay, fermented manures and ashes to sand, and lime and gypsum to both, will materially enhance their friableness.

"Color of Soils.-Color is an essential feature in soils, and, like friableness, it has an important relation to their capacity for heat and moisture. Dark-colored earths, and black in the highest degree, absorb heat more rapidly than any other when exposed to a temperature above their own, and it escapes with equal readiness when their relative temperature is reversed. A rough, pulverized surface, which is seen in the minute inequalities of a friable soil, produces the same result. During the heat of the day, especially when the sun's rays fall upon the earth, the dark friable soil imbibes the heat freely, and transmits it to the remotest roots, thus securing warmth to the plant, which is one of the necessary conditions of its growth. When the temperature of the air falls, on the approach of evening, a reversed action in the soil takes place, by which the heat as rapidly escapes. This immediately brings the surface to 'the dew point,' and secures a copious deposit of moisture, which a friable soil speedily conveys to every part of the roots."

For reasons above given, namely, that dark soils absorb the heat more readily than lighter ones, a dark soil, other conditions being equal, will be more productive than a light one. In cold climates, melons are often ripened by covering the soil an inch deep with char. coal dust, while it is a well-known fact that in Belgium grape-growers spread fragments of black slate over their light soils, and thus greatly improve them. Muck and peat both improve the soil to which they are applied, in this respect, in addition to their fertilizing elements.

The Dew Point.-The temperature at which dew begins to form is called the "dew point," and this is a degree of temperature when the air begins to part with its vapors. It is generally attained when the surface of an object is below the temperature of the air surrounding it, as is often illustrated by the moisture on an earthern, glass, or metallic vessel filled with cold water on a hot summer day, or when set in a warm room in cold weather. The temperature at which the vapors of the atmosphere condense to form dew is usually several degrees below that of the atmosphere in clear weather, varying from four to ten degrees; but in damp or rainy weather the dew point will often be found to correspond 
nearly with the temperature of the atmosphere, proving that any cause vihich contributes to bring the temperature of the air to this point, or the degree at which vapor condenses to form dew, will also condense the moisture of the atmosphere into mist, clouds, rain, snow, etc. The formation of dew and its influence upon vegetation and soil is an interesting subject, as well as an important one in agriculture.

\section{ANALYSIS OF SOILS.}

$\mathrm{W}$ HILE it is true that an analysis of a soil or of any of its products gives us its component elements with sufficient accuracy for us to theoretically estimate its capacity for production or value in nutritive element, we frequently find such estimates at fault in practical experience. However, this should not cause us to discard analyses of soils or their products, for much benefit has been derived thereby in many ways, and valuable knowledge thus obtained which could not have been derived from any other source; for while chemistry can do much to unlock the hidden secrets of Nature and show us the means by which she accomplishes certain results, it cannot do everything, and this latter fact should not cause us to undervalue the light and knowledge it does afford us.

The productiveness of a soil depends much upon its mechanical condition, for although a soil may contain all the elements suited to a luxuriant and healthy plant-growth, if these elements are not in a condition for a plant to assimilate, and thus derive from them its nourishment or plant-food, it is no more benefited by the presence of these nutritive elements in the soil than if they were entirely wanting. For instance, if a soil is hard, or much of the essential substance for the crop production is largely in a coarse, crude state, and not in a condition to furnish the right proportion of the elements in a solvent form, which is the accepted standard of the amount of crop-production in any given soil, we can readily see that the delicate roots of the plants cannot penetrate it or appropriate its various combinations to its use ; and although there may be large quantities of nutrition locked up there, it is practically useless as far as the crops are concerned. Chemistry can separate the different elements, by analysis, and tell us what proportion of each the soil may contain, and what essentials are wanting; it can instruct the farmer how to test his soils, so that he can easily determine the kind of fertilizers they require to render them productive; it can tell him the composition of the different plants he cultivates, and thus show him what kind of nutriment and in what proportion they require to abstract from the soil to produce their own growth; hence, he will be able to determine what kind of fertilizers to apply. But chemistry cannot tell him whether these elements are in a condition for the plant to derive from them present nourislıment; in other words, it cannot tell him the mechanical condition of the soil; hence, a chemical analysis may often show very favorable results from certain soils, while the practical experience of the farmer in its cultivation may result very unfavorably. On the other hand, a chemical analysis may prove certain soils very deficient in some of the elements which plants require for their food, and yet its texture and mechanical condition generally be so favorable that the roots can easily penetrate it and take up almost entirely the nutrition in store there, and thus favorable results reached in the crop obtained.

It has also been found that a mixture of clay and sand soils produces much better and larger crops than either the clay or sandy soil alone. Analysis would prove the clay the most abundant in the nutritive elements, and hence, reasoning from that fact, we would conclude that the mixture of the poorer sandy soil would deteriorate the clay soil instead of increasing its productive qualities. This proves that while the chemist can greatly benefit the farmer by his skill in separating and testing the component elements of the soll, 
such analysis does not determine accurately its degree of fertility, since practical experience proves that successful agriculture depends quite as much, if not more, upon its mechanical condition, as its composition. Notwithstanding these facts, chemistry, as we have previously stated, has been of great value to the farmer, and the high standard attained in agriculture at the present time is due largely to its instructions.

Notwithstanding the inability of chemical analysis to furnish us all the knowledge desired, sufficient information can be obtained through its teachings for all practical purposes. Chemistry teaches that plant nutrition depends upon two great classes of matter-organic and inorganic; and that no plant can accomplish a perfect growth without being supplied with both of these, in such proportions as it needs; and that if a plant has access to all the requisite mineral elements in abundance, these mineral elements of nutrition are valueless in the production of the plant, unless it can at the same time obtain in some way all it requires of the inorganic elements of nutrition; also, that plants derive a large portion of their constituents from the atmosphere, either directly or indirectly, and the remainder from the soil, and that the elements supplied by the one are as essential to plant growth as those of the other. If there were even one of the requisite mineral elements wanting, though there were an abundant supply of all the others, there would not be a perfect growth of the plant; neither could an excess of one element make up the deficiency in the quantity of the others. For instance, potash and phosphoric acid are both essential to plant growth, and this growth cannot be produced without the requisite quantity of each; now, if there should be a deficiency in the quantity of potash in the soil, no surplus quantity of phosphoric acid, however great, could counterbalance that deficiency in potash. We therefore see, that if any one of the requisite elements of the soil becomes exhausted, or so diminished in quantity that it cannot afford a necessary supply for the plant growth, the crop will be a small one until this neces. sary element is restored to the soil. Only a few of the elements of the soil are at all liable to become exhansted. As a general rule, the principal mineral constituents that may become so are phosphoric acid and potash; while nitrogen is the only organic element the farmer will probably ever be required to supply.

Prof. Stockbridge, of the Massachusetts Agricultural College, says that "carbon, of which plants in their dry state contain about fifty-five per cent., the farmer nerer need supply, because in the soil and in the air there is an abundant supply for all the plants that erer have grown or that ever will grow on the earth, and nature made the plant with the capacity to gather it, so that without any aid from man the plant can gather all its carbon. There is no need then that you feed a plant with carbon; it will take care of itself. So with oxygen, and so with hydrogen. In the water and in the air,-with which the plant is filled, -and by chemical action, the union of certain substances in the plant, carbon, oxygen, and hydrogen, the plant can always supply itself with. No farmer need ever feed his plants with these substances to promote their growth. If, however, we come to nitrogen, it has been abundantly proved by scientists that there is here a deficiency. The plant takes large quantities of nitrogen, especially in its seed; it may gather some nitrogen naturally from the air, perhaps in the form of carbonate of ammonia, and from the soil in the form of ammonia taken in soil water. It can gather some, and yet the quantity of nitrogen arailable to the plant is insufficient to supply its wants. Now, it is the farmer's clear duty, so far as these four elements are concerned, to regard only the supply of nitrogen. Give your plant nitrogen, and you may rely upon it as a natural law, that they themselres can take care of the supply of the other organic elements.

Turning now to the inorganic elements, the same law holds good. Take soda, for instance; all our plants require soda, but so far as the farmer is concerned, be will find that any of the soils that are made of micaceous rocks contain enough magnesia and soda to supply all the plants probably that will ever be produced on that soil; he will probably find enough 
sulphuric acid and silica. If, however, we come to the substance that we call potash, we shall find, as a rule, that plants take a large quantity of it,-a large proportional quantity,and that potash is very deficient in the soil. The constant cropping has exhausted the soil of potash, and the farmer must supply it. All scientists agree on this matter, that so far as the farmer is concerned in supplying the wants of the plant, the only substances that he need supply will be potash, phosphoric acid, and nitrogen. My owu experiments seem to show that with one or two plants, he should give them a little of something else, which are mag. nesia, and a little lime for the tobacco and cotton plant."

We see, therefore, that if the above principle is correct, it will be a very easy matter for the farmer to test his soil, and find in what it is deficient. It may be deficient in one or all of these elements, but by careful experiment and noting the effect upon different plots of ground, of each of the above mentioned fertilizers, apphed separately and in combination, he can soon determine by the results, which of these elements are requisite to the improvement of his lands.

These elements can be obtained in commercial fertilizers, or they can be prepared by the farmer himself, who can obtain a good supply of potash in the form of wood ashes; of nitrogen, in the form of fish-guano, and phosphoric acid from a quantity of bones. By careful experiment, any intelligent farmer can thus learn the elements of his soil, and supply the elements of plant-food requisite.

Since soils differ so greatly that those taken from no two localities, or even from different portions of the same field, will be found, on analysis, to contain the same proportion of ingredients, still, a fair estimate of the predominant constituents of different kinds of soil can thus be obtained.

The following are very fertile and arable soils, of which Professor Johnston, an eminent English authority, says:-

"The first had been cropped for 160 years successively, without either manure or naked fallow. The second was a virgin soil, celebrated for its fertility. The third had been unma. nured for twelve years, during the last nine of which it had been cropped with beans, barley, potatoes, winter barley and red clover, clover, winter barley, wheat, oats, naked fallow."

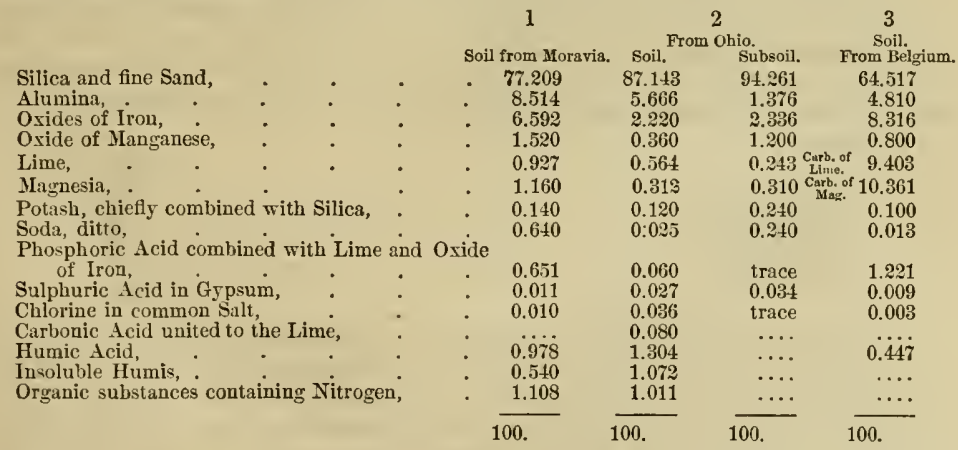

The following analyses were made by Prof. Collier, chemist for the department of agricalture, at Washington, D. C.:-

No. 1.-Green sand marl, from Meridian, Miss.

No. 2.-Clay from Maryland.

No. 3.-Clay from Potomac, Terra Cotta Company. 
No. 4.-Kaolin (porcelain clay), from Maryland.

No. 5.-Kaolin from Virginia.

\begin{tabular}{|c|c|c|c|c|c|}
\hline Constituents. & No. 1. & No. 2. & No. 3. & No. 4. & No. 5. \\
\hline 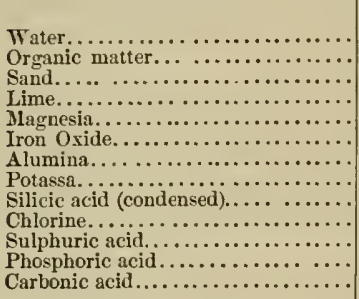 & $\begin{array}{c}\text { Per Cent. } \\
16.80 \\
2.18 \\
36.55 \\
6.00 \\
2.28 \\
15.94 \\
\ldots .69 \\
2.69 \\
\ldots . .0 \\
.14 \\
\text { Trace.. } \\
\text { Trace.. } \\
16.96\end{array}$ & $\begin{array}{c}\text { Per Cent. } \\
3.98 \\
\ldots \ldots \\
58.32 \\
\ldots . .5 \\
.55 \\
1.96 \\
10.60 \\
.30 \\
29.96 \\
\ldots . \\
\ldots \ldots \\
\ldots .\end{array}$ & $\begin{array}{c}\text { Per Cent. } \\
9.16 \\
\ldots \ldots \\
\ldots . .09 \\
38.09 \\
2.33 \\
3.48 \\
7.63 \\
.80 \\
23.31 \\
\ldots \ldots \\
\ldots . \\
\ldots . .59\end{array}$ & $\begin{array}{c}\text { Per Cent. } \\
17.42 \\
\ldots . \\
\ldots . \\
.86 \\
.29 \\
.81 \\
32.34 \\
2.49 \\
45.69 \\
\ldots . \\
\ldots . \\
\ldots . \\
\ldots\end{array}$ & $\begin{array}{c}\text { Per Cent. } \\
10.65 \\
\ldots . \\
\ldots .18 \\
\text { Trace. } \\
\text { Trace. } \\
36.66 \\
\ldots .49 \\
\ldots \ldots \\
\ldots \ldots \\
\ldots .\end{array}$ \\
\hline & 99.54 & 100.45 & 94.39 & 99.81 & 98.98 \\
\hline
\end{tabular}

Gypsum Marl.-A sample of marl said to exist in very great quantity and readily accessible on the shores of Lake Jessup, Florida, gave upon analysis the following:-

Water and organic matter, Sand and clay, Gypsum $\left(\mathrm{CaSO}_{4}, 2 \mathrm{H}_{2} \mathrm{O}\right)$, Limestone $\left(\mathrm{CaCO}_{3}\right)$, Iron and aluminium oxides, . Phosphoric acid,
Per Cent.

6.36

68.94

13.79

3.77

7.14

Traces.

100.00

The crystals of gypsum are easily discernible to the eye, and the large percentage of this fertilizer makes this deposit of very great value to the farming interests of that section.

Unproductive Soil.-The following analysis of soil (which was from Oak Hill, Volusia Co., Florida) shows that its barrenness is very likely due to a lack of constituents suitable for plant nourishment, and not to the presence of any injurious substance.

It contains-

$$
\begin{aligned}
& \text { Per Cent. }
\end{aligned}
$$

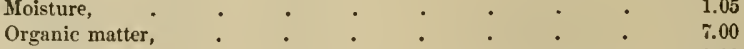

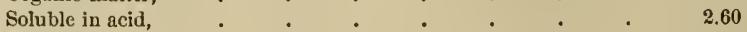

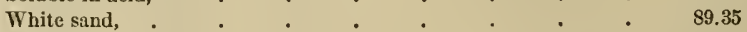

$$
\begin{aligned}
& 100.00
\end{aligned}
$$

One of the most fertile soils of Sweden is said by Bergmann to contain 30 per cent. of carbonate of lime. A very productive soil in France was found by the analysis of Chaptal to contain nearly 25 per cent. of carbonate of lime, beside 7 per cent. of organic matter. Tillet analyzed a soil that contained 37.5 per cent. of carbonate of lime, which was exceed. ingly fertile. Some of the best Mississippi Valley soils are said to yield, upon analysis, from 20 to 25 per cent. of magnesian lime, and from 2 to 3 per cent. of phosphate of lime, while many other soils throughout the United States have been known to yield an equal proportion of carbonate of lime, when analyzed.

As we have seen, chemistry will show the constitnents of the soil, and what elements are lacking that are essential to the growth of certain crops, but will not indicate whether those 
elements contained within it are in a condition to be utilized by the plants. Although this knowledge imparted by chemistry is imperfect, it is nevertheless of value to the farmer, for it tells him what elements are necessary to apply to his lands in order to make them productive. It goes farther than this; it shows the composition of the various plants that he cultivates, and what proportion of the various fertilizing elements each variety of product abstracts from the soil ; therefore the amount of fertilizing material removed from the soil by the different crops can be calculated with considerable accuracy, and by applying a sufficient supply of these elements to his soil he may be sure that it contains the necessary elements of fertility in a form to be utilized by the plants. It will be seen, therefore, that both the analysis of soils and plants are of great use in agriculture, and that when both are combined with experience and observation, the farmer may derive great benefit from the teachings of chemistry. This is a subject of great interest and importance, and will be more fully treated in connection with fertilizers.

\section{FERTILIZERS.}

$\mathrm{P}$ LANTS require food on which to subsist, it being just as essential to their sustenance as with animals. They also require a variety of nutrition for their proper nourishment and growth, which can no more be secured without it, than a perfect animal growth when subsisting constantly on one element. It is also equally true that different plants require different proportionate elements in their food, just as different species of animals require different kinds of nutriment, and they will dwarf and starve unless that nourishment is furnished them. When farmers learn to feed their crops with the same care and consideration that they feed their animals, they will be more successful in their business, and find that farming pays well for the labor bestowed.

Although some soils contain all the requisite elements of plant-food in the proper proportions for certain kinds of crops, and will produce them for a long time without any apparent impoverishing, others contain certain necessary substances of nutrition in smaller quantities, which must be furnished in fertilizers of some kind in order to produce a healthy and luxuriant growth. These elements that are needed in the soil to supply the suitable plantfood may be contained in the proper proportion in vegetable manures plowed into the soil in a green state, stable manure, lime, wood ashes, guano, or any of the other substances used for fertilizing purposes. It depends upon the farmer to ascertain what is needed, and supply the requisite demand; certain it is, that all cultivated soils, however rich, will in time become impoverished with constant cropping, unless a portion of the elements extracted from the soil by the growth of these crops be returned in the form of fertilizers of some kind ; it may be by a deposit of rich mud or sediment drained to impoverishment from other soils by inundation, as is the case with the valley of the Nile and banks of the Ganges, or it may be by other agencies; it makes no difference how the supply comes, the principle remains the same, which is, that soils producing constantly must be supplied with nutriment from some source. The Nile and Ganges rivers have continued by their overflow to furnish for more than 3,000 years all the fertilizing elements requisite to produce crops constantly during that period. When soils remain in their natural state, or are used only for pastures, but little change is perceptible in their character. Changes, however, are constantly going on both in their texture and capacity for production, although so gradual as to be perceptible only after a long period. Soils constantly cropped are the ones to become exhausted soonest. The decay of vegetable matter upon the surface of lands, the fertilizing properties washed down by the rains and melting snows from the steep declivities of hills and mountains, the 


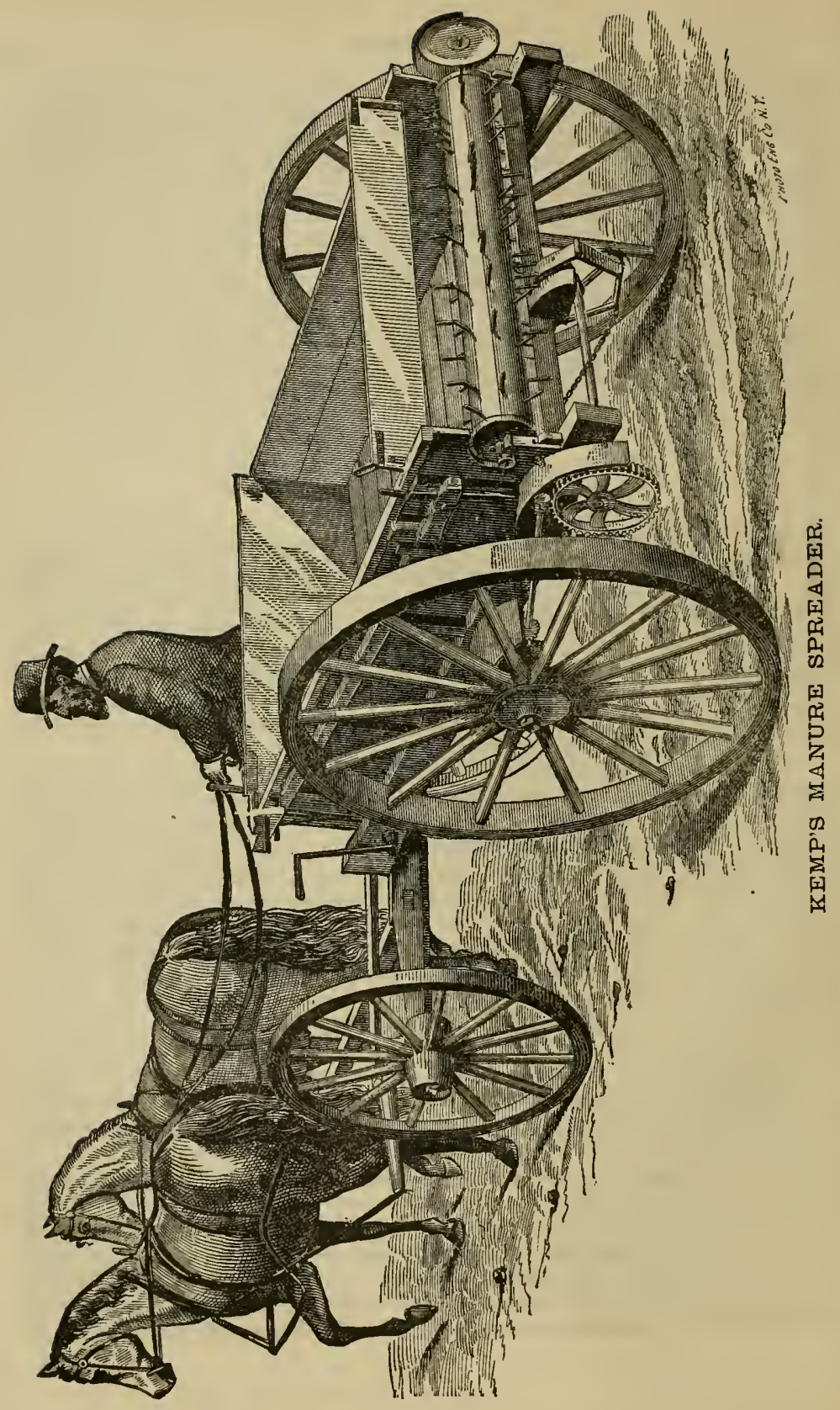


mineral elements contained in the soil and rocks, that are rendered soluble through the agencies of the atmosphere, water, heat, and cold-all these, though silent and gradual in their working, affect the productiveness of soils in a remarkable degree. The washing of the fertilizing properties from the mountains and hills is one great reason why the valleys contain usually the most productive soils.

The food of plants is derived from two sources, viz., the soil and atmosphere. The elements obtained from these two sources, though greatly varying in proportionate quantities, are equally essential, as far as the plant-growth is concerned, and no excess in the amount furnished from one source can make up or counterbalance the deficiency in the quantity furnished irom the other, both being equally necessary.

Elements of Plant-Food. The elements of plant-food necessary to produce plantgrowth are called organic and inorganic elements, and, in a general sense, embrace every material which, if added to the soil, will tend to increase its fertility. Organic elements embrace animal and vegetable substances which have a certain proportion of nitrogen; inorganic elements comprise those that are purely mineral or earthy, and which generally contain no nitrogen. The organic elements, to be in the proper proportion, should be about ninety-five per cent., the inorganic the remaining five per cent. By burning a plant, the organic elements will be converted into gases, while the inorganic elements remain in the form of ashes, called in analysis the ash of plants ; thus the organic element, or the ninety. five pounds out of every one hundred pounds of the dried plant that is burned, and which is derived mainly from the air, disappears, while the inorganic, or the five pounds out of every one hundred pounds that are left behind in the form of ashes, are obtained from the soil. Different plants differ somewhat in the proportion of the organic and inorganic elements contained in them. The tobacco plant, for instance, having a larger proportion of ash than many others, but, on the average, every plant that grows will have about ninety-five per cent. of organic matter and five per cent. of inorganic matter, the organic matter being furnished by the air, the inorganic by the soil. This, in the main, is the unchangeable law of nature.

Since the atmospheric elements are found present in the soil, in a greater or less degree, according to its texture and degree of porosity, it has been estimated that about one-half of the material furnished to plants is derived from the earth. It was formerly believed that plants depended for their nutrition upon the organic matter (or what is called humus) in the soil. This humus theory was set aside by chemistry, which proved that certain soils were extremely fertile with but two or three per cent. of humus in their composition, while certain other soils were sterile with from twenty to forty per cent. of humus contained in them. Afterward it was advocated by even the noted Liebig, that plants for nutrition required only the application of the mineral elements. This theory was met by the nitrogen theory, between which two (the mineral and nitrogen theories) a long contest ensued, until it was discovered that in some respects both theories were right, and in others that both were wrong. Then followed the theory, that in order to ascertain the wants of plants and provide them with proper nutriment, we had only to analyze the soil and determine its wants, also the structure and composition of plants, and by supplying the soil with the elements necessary to certain plants, and in which it was deficient, a perfect supply of nutriment would thus be furnished the plant. Although this theory seemed to approximate nearer the truth than either of the preceding ones, still it had its objections, and its advocates were disappointed in not meeting with that success in its practical application that had been anticipated; for it was soon found that the acids of the chemist, in analysis, would take from the sample of soil certain elements which the plant never could find and appropriate from the soil, owing to its being in such a state that it rendered it impossible to extract it from the soil. For instance, the analysis of the soil might prove an abundant supply of phosphoric acid contained within it, but it might not be in such a solvent state as is necessary for the tender roots to derive 
nourishment from it ; hence, the plant would just as surely starve for the want of that phos. phoric acid as though the soil contained none. In other words, the analysis determined the constituents of the soil, but not its mechanical condition, upon which so much depends in successful agriculture. Besides these objections, different samples of soil taken from different portions of the same field might vary greatly in their composition; hence, an analysis of soil from one portion of a field might not apply to other portions.

Elements Necessary to Supply Soils. With respect to the obstacles met with in chemical analysis, and the elements abstracted from the soil by different crops, Prof. WW. O. Atwater says:

"It was once thought that the chemical analysis of a soil would easily reveal its deficiencies in plant food. But later experience has shown that this is, at best, a costly and very defective source of information. Difierent samples of soil taken from different places in the same field and at different times may vary widely in composition, and, what is a still greater diffculty, the cliemical tests which prove the presence of a given ingredient in the soil, do not tell whether it is already in such a form that the plant can use it, nor whether or how soon it may become so. And there are many other factors of vital importance to the development of tho plant which soil analysis does not reveal.

To find what materials crops remove from the soil is an easier matter. Thousands of analyses of the more important vegetable products have been made, nearly all in Europe, however, and we have tables prepared from them giving the average composition of our field crops. From these any farmer may calculate how much plant-food a given crop takes on the average from his soil. These figures might possibly fit his case no closer than would the cal. culation of his own weight from that of the average European, but they will suftice well enough for practical purposes.

Formulas for fertilizers for special crops are commonly based upon calculations of this sort. For instance, a crop of fifty bushels of corn, with the stalks, would take from the soil on the average,

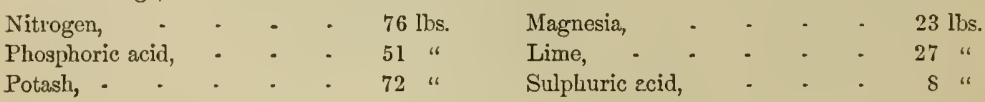

Now we may assume that nitrogen, phosphoric acid, and potash are the only ones the soil will fail to furnish in plenty, or that if lime and sulphuric acid are needed in addition, there will be enough in the superphosphates and other materials to be used to supply them. We have then to provide $76 \mathrm{lbs}$. of nitrogen, $51 \mathrm{lbs}$. of phosphoric acid, and $72 \mathrm{lbs}$. of patash, in forms readily available for plant-food."

The organic elements that enter into the composition of plants are four in numberoxygen, hydrogen, carbon, and nitrogen. All of these, with the exception of nitrogen, are supplied by nature in sufficient quantities; though this is furnished to a certain extent, still it is often found in such small quantities as to require an additional supply applied in order to render its fertility available in the production of good crops.

This mysterious element, nitrogen, in order to be beneficial to plants, must undergo a chemical change. By uniting with oxygen, it forms nitric acid; by uniting with hydrogen, it forms ammonia, which is the form in which nearly all the nitrogen used by plants is taken; hence nitrogen and ammoma are often used, in connection with agriculture, as synonymous terms. Both nitric acid and ammonia are powerful fertilizers. It is estimated by good authority that the nitrogen in organic matter is worth only about three-fifths as much as pure ammonia. Nitrogen in the form of ammonia, also in the form of nitric acid, is washed down to the soil by water. The nitrates are carried in the water to the roots of piants; the leaves also absorb nitrogen from the air, and in this manner the plants obtain their nitrogen. 
The inorganic elements, which form only about five per cent. of the constituents of plants, are eight or ten in number, but most of these are, as a general rule, supplied in abundance by the soil.

Prof. Atwater also asserts that we may take it as pretty well settled "that the only ingredients of plant-food which we need supply to our soils are Potash, Lime, Magnesia, Phosphoric Acid, Sulphuric Acid, and Nitrogen. Iron and chlorine are necessary to the perfect growth of plants, but only in minute quantities. Silica and soda are needed, if at all, in similarly small proportions. Every ordinary soil supplies these last four substances in abundance.

Of the above list the magnesia is rarely deficient in even "worn-out" soils. Sulphuric acid and lime are more often lacking, and hence, doubtless, one reason of the good effect so often observed from the application of hme and plaster. The remaining substances, Phosphoric Acid, Nitrogen, and Putash, are the most apt to be deficient. In some soils one, in others several or all of these may be wanting. When we say the ingredients are "wanting," we do not mean that the soil does not contain them, but that it does not supply the crops as much as they need. It is not so much because our "worn-out" soils have not enough plant food in store, that crops starve upon them, but rather, because the food is locked up in such combinations that the roots cannot get at and use them. Such is the general result of the best testimony that experience and experiment have placed at our disposal."

We find that the elements usually deficient in almost all soils, as above stated, are Phosphoric Acid, Potash, and Nitrogen. Oxygen, hydrogen, and carbon, those elements supphed by the atmosphere, will be abundant and always available, providing the plants to be cultivated are grown in such a manner that the air can have free access to them. When the roots and stalks are so compact that the air and sunlight cannot penetrate the dense mass and the air circulate freely among them, there will never be a perfect or healthy growth of any crop, though some crops require more favoring in this respect than others. Sufficient space must be given the stalks and roots, and with a good supply of air and sunlight, other conditions being equally favorable, a good crop must of necessity follow. Nitrogen, as we have previously stated, although furnished in part by the atmosphere, is not thus furnished in sufficient quantity for a perfect plant growth, and must needs be supplied by man in some form.

Water is also an essential agent in furnishing a source of food supply to plants, since the elements derived from the soil can be appropriated to the use of the plant only in a liquid, or soluble form, and water is necessary to produce this condition of these elements. Some plants require more water than others, but all require it in a greater or less quantity. It is stated from good authority that the cabbage plant is composed of about nine-tenths water, and the potato-tubers nearly three-fourtlis, which shows how large a proportion of water enters into the composition of some plants.

General Propositions Concerning Plant Food. The following important propositions concerning plant food, agriculturally considered, are given by Dr. E. L. Sturtevant of Massachusetts, an authority that will be a sure guaranty of their reliability and true value to the farmer.

1st. The soil contains plant-food.

2d. The plant removes this plant-food from the soil.

3d. Analyses of plants will show the character and quantity of the plant-food removed from the land.

4th. A supply of the elements removed by the plants from the soil in such a way that they can be appropriated by the plants, will produce the plant-growth, under proper conditions.

5th. Plants remove varying quantities of plant-food from the soil, according to their species, their manner of growth, and the proportions and quantities of plant-food present in the soil. 
6th. The constituents of plants furnished from the atmosplere being beyond our control, in studying plant-growth and food supply, we can limit ourselves to the earth alone, and study the air supply only as modified by our control over the elements supplied to our land.

7 th. The soil contains the elements of plant-growth in varying proportions and conditions; some elements far in excess of the needs of our plant, other elements in quantities suffcient only to support a scant growth.

8th. The plant-food in the land must, to be of service to the crop, be in a soluble state, or in a state capable of being taken up by the rootlets of our plants.

9th. When the elements of plant-food, in the form of chemicals or dung, are added to our soil, there is a chemical action taking place between them and the soil constituents, in the presence of moisture.

10th. The soil has the power of decomposing most of the chemicals which are applied, and has a retaining power on their compounds, varying with the character of the element.

11th. As a rule, phosphoric acid is firmly held by the soil, and is not subject to loss through drainage, and has a limited diffusibility; potash is strongly retained, but has a greater diffusibility; nitrogen, in its various forms, is less strongly retained, and has a ready diffusibility.

12th. In proportion to the presence of, and diffusion of these fertlle elements in our soil, other conditions being favorable, will be the amount of our crop.

13th. Waste of fertility in our land arises from drainage, and the changing of soluble plant-food into insoluble forms.

14th. Plants require different proportions of plant-food, and rifferent combinations, at different periods of their growth.

15th. The presence of certain elements in excess has an influence on the growth and composition of the plant.

16th. The seed has an influence, in itself, in determining the crop, an influence apart from the presence of a full supply of food, or the climatzc features of the season.

Phosphoric Acid.-With respect to the three elements requisite to be supplied to lands in increasing their productiveness, viz., phosphoric acid, potash, and nitrogen, we quote the following, also from Dr. Sturtevant, as one of the best authorities in the conntry on this subject:-

"Phosplzoric acid is one of the principal compounds of bone, in the form of a phosphate of lime, and is furnished to commerce either in bone, phosphate guanos, phosphate rock, or

- in the wastes of commerce. It occurs in three forms: an insoluble condition, in which it is unassimilable by the plant; a soluble form, wherein it is readily absorbed, and an intermediate form, the reduced or reverted form, of the soluble, wherein its adaptability as plantfood is questioned, but which we esteem of some value, on account of the condition in which it occurs, and the conditions under which it is usually presented to the plant rootlets. It is commercially prepared from bone or phosphate rock, by the addition of sulphuric acid, which, uniting with a portion of the lime with which the phosphorus is combined, leaves a soluble form. The reaction may be expressed as below:

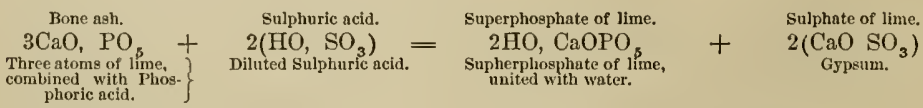

This product, the superphosphate, is the valuable portion of the fertilizers placed on the market under this name, and it may compose from $\frac{1}{2}$ per cent. to 23 per cent. of their mass by weight of soluble phosphoric acid. We thus see the importance of the sale of fer. tilizers by guaranteed analysis under government control. Ordinarily, a good superphos. phate will contain about 10 per cent. of soluble phosphoric acid, and a few per cent. of nitro. 
gen, in addition to insoluble and reverted phosphoric acid. We can estimate its value on the basis of 12 cents a pound for the soluble phosphoric acid, from 4 to 6 cents a pound for the reverted, and 1 or 2 cents for the insoluble. Yet these figures are subject to changes.

When this fertilizer is applied to our land, and the rains fall, it is washed into the soil, but only to a certain distance. The soil bas a retaining power on this element, aud holds it firmly against washing. So far as the phosphoric acid extends through the soil, it is diffused: that is, it is evenly distributed, and the soil holds it in saturation, but in a quantity for a given area dependent on the nature of the soil. What follows from this? If we apply a given quantity to the surface of our land, a quantity sufficient if applied to our plant to raise a maximum crop, so far as this constituent is concerned, the rains wash it down, and it is retained, we will suppose, by the first inch of our soil. Now, as it is retained by the soil in this position, despite the rain-falls, it is evident that in this supposed case, only the root fibers which enter this upper inch of earth can feed on it. If a double quantity be applied, then it will extend down two inches, and the same facts about the plant feeding bolds for this two inches, and so on. It is evident that it can only be by applying phosphoric acid in large quantities, or repeatedly during a series of seasons, that we can so saturate our land with this element, under these assumed conditions, as to furnish the whole root-area with plant-food.

These considerations would lead us into the practice of looking for the full effect of this fertile element from repeated applications, rather than from a single trial. They would also lead us to apply this element within reach of the principal plant fibers in all cases. They show us that it is safe to apply a superphosphate at any period of the year, so far as loss by drainage is concerned. In applying on sod for corn, we like to apply this element of our fertilizer on the surface, so that it may be washed in by the rains before plowing. Then when the sod is inverted, shallow, as is our custom, the fertile strata of the earth is in the right position for the roots.

We also have an action of the acids of our combined fertilizers in rendering more soluble the phosphoric acid which has reverted in the soil, but space will not admit of further considerations.

Potash.-Potash, as supplied in commerce, occurs in three forms, - the carbonate, sulphate, and muriate. The first form occurs in ashes, and we will suppose a bushel of ashes to weigh $48 \mathrm{lbs}$. to the bushel, as has been determined as the average for house ashes as they usually occur. Now ashes contain about 6 per cent, of potash, and about 2 per cent. of phosphoric acid, in a reduced or insoluble form. Hence a bushel of ashes would be held as of the value of 2.8 pounds of potash and .9 pounds of phosphoric acid. If these ashes are worth 30 cents a bushel, and we consider the phosphoric acid worth 6 cents a pound, the pot. ash in our ashes stands us at about $8 \frac{8}{4}$ cents a pound. If leached ashes are used, at 10 cents a bushel, the potash supply is costing about 20 cents a pound. Carbonate of potash is, how. ever, a good form in which to use potash, as the carbonic acid has an action of itself in the soil, in aiding the solution of plant-food.

Of late years, however, the principal agricultural supply of potash is looked for in Kainite or German potash salts. These are the product of the salt mines of Germany, and are put on the market in the form of sulphate of potash and muriate of potash of varying strength. The most economical for the farmer are the high grades. The sulphate of potash which contains 78-80 per cent. of the pure salt, furnishes about 43 per cent. of actual potash, and when the commercial salt is quoted at 4 cents a pound, the actual potash therefrom is costing about $9 \frac{1}{3}$ cents a pound. Muriate of potash, the other potash salt of these mines, when of 80 to 85 per cent. muriate, contains about 50 pounds of actual potash, and furnishes, when the commercial article is selling at 3 cents a pound, the potash at about 6 cents a pound. We thus find that the cheapest supply of potash is in the high grade muriate. To furnish the potash at the price it is procured for in the muriate, we should buy the sulphate of the same grade at about $2 \frac{1}{2}$ cents a pound, and wood ashes at about 17 cents a bushel. 
These salts of potash are supposed to act differently on crops, but we do not consider the matter at all proved at present. Indeed, at present prices, we prefer to apply the muri. ate in nearly every case, or, better still, two-thirds muriate and one-third sulphate.

When we apply our potash compounds to the land, they become dissolved by the rains, and wash into the soil. They are then decomposed into their constituent parts, separating into sulphuric and muriatic or carbonic acid, and potash, and while the acids are not retained at all closely by the soil, but follow the water of drainage until they meet new compounds, and are subject to different actions, the potash is quite strongly retained. It is, hence, easier to saturate our soils with potash than with sulphuric acid, and if our soil is deficient in this element, we can hope to more speedily cause it to be diffused through the soil, and thus become more accessible to the crops.

In applying potash, then, we desire to keep it near the surface at first, as we are quite certain it will pass downward into the root pasturage, and we are never quite sure against a small waste."

Nitrogen.-This element is furnished in various forms by the atmosphere, as well as by the decay of organic materials in the soil. As gencrally supplied, it is in the form of ammouia or a nitrate, such as witrate of soda. It is found quite abundantly in guano, which is one of the important sources from whence this valuable fertilizer is obtained. It is also obtained from dried blood and various waste materials. Its effect on plants is generally speedy, being readily diffused through the soil, and in this way promptly supplied to the roots of plants, hence, for this reason is subject to waste, and as it is the most expensive element of plantfood the farmer has to supply his crops, it is well while proriding a sufficient amount to meet the wants of the crop, to avoid applying an excess.

This element can often be procured from local waste materials, as in the refuse of woolen mills, fish from which the oil has been extracted, powder factories, etc. Leather contains it, but as the decomposition is rery slow, a long time is generally required to render its fertilizing qualities perceptible. Old boots, shoes, or harnesses are often buried under fruit trees, and by their gradual decay prore of considerable value as a fertilizing agent.

The bodies of dead animals added to the compost heap will also furnish in their decomposition a valuable amount of nitrogen. Some soils respond more promptly to its effects than others, but all lands are more or less benefited by its application; especially so are those that have long been under cultivation.

The amount of nitrogen left in the soil by a crop of clover, either where it has been cut for hay, or ripened for seed, is often astonishingly great, and furnishes a source of rich food for cereals. Prof. Voelcker found by careful investigation, that the amount of nitrogen thus deposited by a crop of clover was from two and a half to three tons per acre. He also found that on soils where clover had been grown, not only were rast quantities of nitrogen stored up by this product, but the soil was left in a better condition in the spring than could have been secured by the application of any fertilizer. These inrestigations of Prof. Toelcker were made at different depths of soil; first taking an upper lajer of six inches, then the next six inches below the former, and then the next six inches below that, making eighteen inches of soil in depth carefully analyzed.

\section{Exhaustion of Soils by Crops-The Composition of Manures-Commercial} Fertilizers, etc.-We give the following tables from authentic European sources, showing the composition of many of our common farm products; also, by way of comparison, a table showing the composition of a variety of fertilizers commonly used. These figures, it must be remembered, represent only general averages; in special cases, there might be more or less variation from these percentages, which are sufficiently definite for all practical purposes. As the first table given represents the arerage composition of the different products, it also represents the amount of fertilizing ingredients remored from the soils in producing these 
products; hence the farmer may be able to calculate the extent of exhaustion of his soil caused by certain crops. On the other hand, by consulting the table representing the compo. sition of different manures, he can find what fertilizers will best supply the requisite elements for special crops, or what will best restore the elements extracted by the growing of certain products.

\section{TABLE}

For Calculating the Exhaustion of Soils by Crops and Enriching by Manures. Average Quantities of Water, Organic Matter, Ash, Nitrogen in Organic Matter and Potash, Lime, Phosphoric Acid, and other Ingredients in Ash, of Fresh (Green) and Airodry Materials.

\section{Plants.}

veOETABLE MATERials.

\section{GRAIN AND STRAW.}

Wheat, grain.

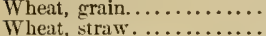

Rye, grain................

Rye, straw...............

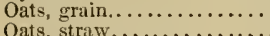

Indian Corn, grain......... do. stalks and leaves.....

Buckwheat, grain.........

Buckwheat, straw........

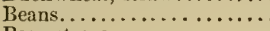

Bean straw............

Pea straw...............

\section{HA Y.}

Average Meadow Hay.....

Timothy Hay..............

Red Clover Hay ...........

Swedish Cloter IIay........

Lucerne (Alfalfa)......... GREEN CROPS.

Young Grass.............

Timothy Grass.............

Fodder Rye...............

Fodder Corn..............

Red Clover in blossom......

ROOTS (tubers) AND TOPS.

Potatoes, tubers. . . . . . . . 750 do. rines nearly ripe.

Turnips, roots............ 920

Turnips, tops................ 898

Sugar Beets, roots.......... 815

Surar Beets, tops............ 897

Carrots, roots.............. 850

Carrots, tops............. 822

Hops, entire plant......... 140

Hops, the cones. ............ $1: 0$

Tobacco, leaves............. 180

\begin{tabular}{|c|c|c|c|c|c|c|c|c|c|}
\hline \multirow{2}{*}{ 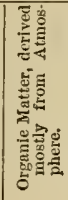 } & \multirow{2}{*}{ 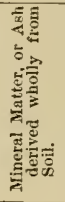 } & \multirow{2}{*}{ 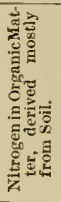 } & \multicolumn{7}{|c|}{ INGREDIENTS OF ASH. } \\
\hline & & & 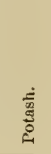 & 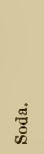 & 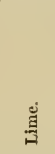 & 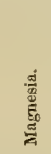 & 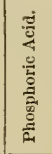 & 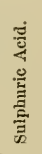 & 节 \\
\hline lbs. & lbs. & lbs. & lbs. & lbs. & lbs. & lbs. & lbs. & lbs. & lbs \\
\hline 840.1 & 16.9 & 20.8 & 5.3 & 0.4 & 0.6 & 2.0 & 7.9 & 0.1 & \\
\hline 812.9 & 46.1 & 4.8 & 6.3 & 0.6 & 2.7 & 1.1 & 2.2 & 1.1 & \\
\hline 839.1 & 17.9 & 17.6 & 5.6 & 0.3 & 0.5 & 2.1 & 8.4 & 0.2 & \\
\hline 816.5 & 40.5 & 4.0 & 7.8 & 0.9 & 3.5 & 1.1 & 2.1 & 1.1 & 22 \\
\hline 830.0 & 27.0 & 19.2 & 4.4 & 0.6 & 1.0 & 1.9 & 6.2 & 0.4 & 12 \\
\hline 816.6 & 40.4 & 5.6 & 8.9 & 1.2 & 3.6 & 1.6 & 1.9 & 1.3 & 19 \\
\hline 843.0 & 13.0 & 16.0 & 3.7 & 0.2 & 0.3 & 2.0 & 5.8 & 0.2 & \\
\hline 808.1 & 41.9 & 4.8 & 9.6 & 6.1 & 4.0 & 2.6 & 5.3 & 1.2 & 11. \\
\hline 848.2 & 11.8 & 14.4 & 2.7 & 0.7 & 0.5 & 1.5 & 5.7 & 0.2 & 0 \\
\hline 788.3 & 51.7 & 13.0 & 24.2 & 1.1 & 9. & 1.9 & 6.1 & 2.7 & . \\
\hline 824.3 & 30.7 & 40.8 & 13.1 & 0.4 & 1.5 & 2.2 & 11.9 & 0.8 & 0 \\
\hline 796.1 & 43.9 & 16.3 & 18.5 & 1.1 & 9.8 & 3.3 & 3.2 & 1.6 & \\
\hline 833.5 & 23.5 & 35.8 & 9.8 & 0.2 & 1.2 & 1.9 & 8.6 & 0.8 & 0.2 \\
\hline 796.0 & 44.0 & 10.4 & 10.1 & 1.8 & 16.2 & 3.5 & 3.5 & 2.7 & 3. \\
\hline 805.5 & 51.5 & 15.5 & 13.2 & 2.3 & 8.6 & 3.3 & 4.1 & 2.4 & 13.8 \\
\hline 794.9 & 62.1 & 15.5 & 20.4 & 1.5 & 4.5 & 1.9 & 7.2 & 1.8 & 22 \\
\hline 783.1 & 56.9 & 19.7 & 18.3 & 1.2 & 20.0 & 6.1 & 5.6 & 1.7 & 1. \\
\hline 800.3 & 39.7 & 24.0 & 11.0 & 1.2 & 13.5 & 5.0 & 4.0 & 1.6 & 1. \\
\hline 777.9 & 62.1 & 23.0 & 15.3 & 1.3 & 26.2 & 3.3 & 5.5 & 3.7 & 3. \\
\hline 179.3 & 20.7 & 5.6 & 11.6 & 0.4 & 2. & 0.6 & 2.2 & 0.8 & 2. \\
\hline 2 & 21.6 & 5.4 & 7.4 & 0.5 & 1.6 & 0.7 & 2.5 & 0.6 & 7. \\
\hline 2 & 16.3 & 5.3 & 6.3 & 0.1 & 1.2 & 0.5 & 2.4 & 0.2 & 5. \\
\hline 16 & 1 & 1.9 & 4.3 & 0.5 & 1.6 & 1.4 & 1.3 & 04 & \\
\hline 206.3 & 13.7 & 5.1 & 4.4 & 0.3 & 4.8 & 1.5 & 1.4 & 0.4 & 0.3 \\
\hline & & & 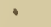 & & & & & & \\
\hline 240.6 & 9.4 & 3.4 & 5. & 0.2 & 0.2 & 0.4 & 1.6 & 0.6 & 0. \\
\hline 210.3 & 19.7 & 4.9 & 4.3 & 0.4 & 6.4 & 3.3 & 1.6 & 1.3 & 0. \\
\hline & & 1.8 & 3.3 & 0.7 & 0.8 & 0.3 & 0.9 & 0.8 & 0 . \\
\hline & 11 & 3.0 & 2.8 & 1. & 3.9 & 0. & 0.9 & 1.1 & 0 . \\
\hline 177 & 1 & 16 & 3.9 & 0.7 & 0.4 & 0.5 & 0.8 & 0.3 & 0. \\
\hline & 18.1 & 3.0 & 6.5 & 2.7 & 2.7 & 2.7 & 1.3 & 0.9 & 0. \\
\hline & 7.8 & 2.2 & 2.8 & 1.7 & 0.9 & 0.4 & 1.0 & 0.5 & 0. \\
\hline 15 & 26.0 & 5.1 & 2.9 & 5.2 & 8.5 & 0.9 & 1.2 & 2.0 & 2.9 \\
\hline & & $?$ & 29. & 2.8 & 18 & 6.4 & 7.5 & 3.7 & 16.4 \\
\hline & 66.8 & ? & 23.0 & 1.4 & 11.1 & 3.7 & 11.2 & 2.4 & 11.1 \\
\hline 769.0 & 151.0 & 34.8 & 30.3 & 5.1 & 62.8 & 17.7 & 4.8 & 5.8 & 13.5 \\
\hline
\end{tabular}


JANCRES.

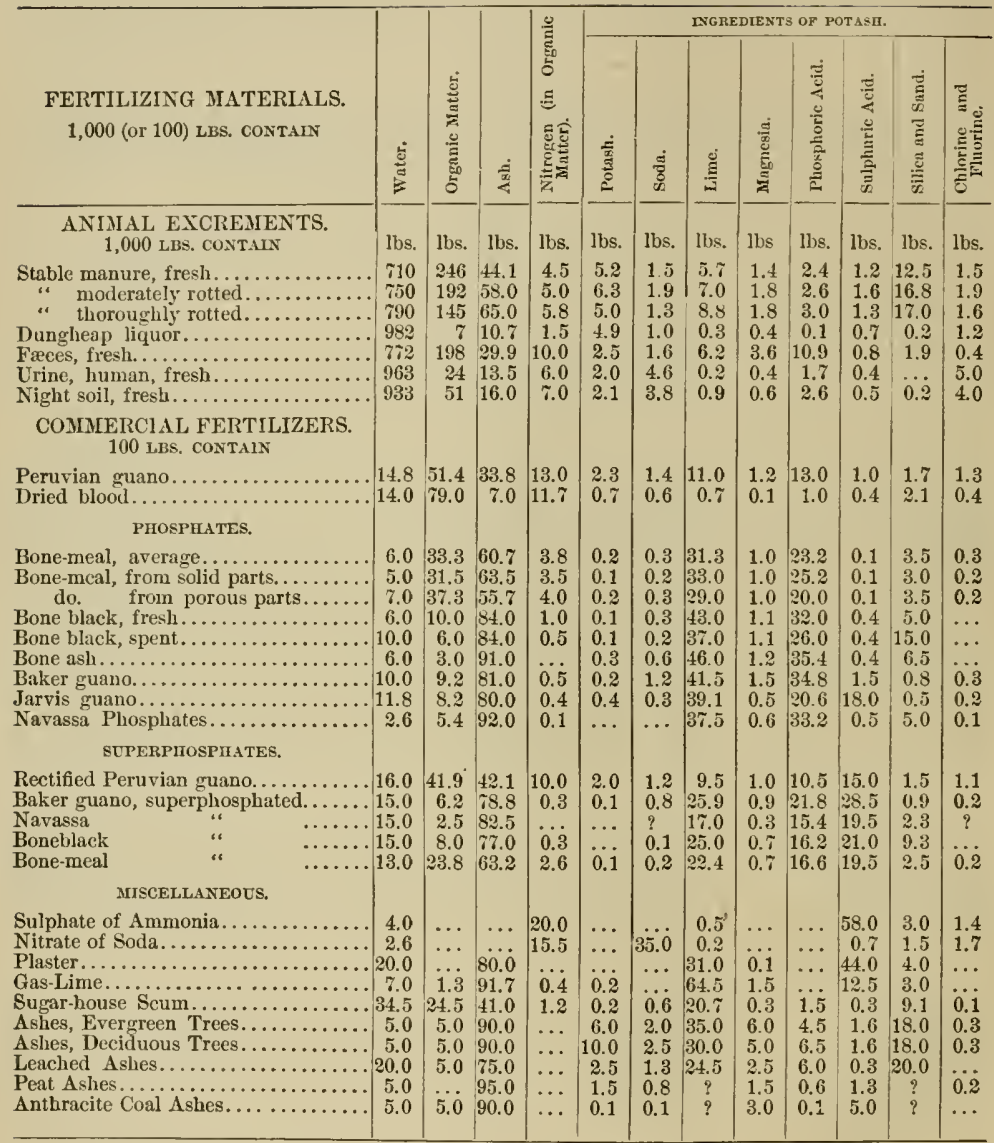

We see by the first of these tables, that in the wheat grain we have, in 1,000 pounds, on the average, about $21 \mathrm{lbs}$. of nitrogen; $5 \frac{1}{2} \mathrm{lbs}$. of potash and $7.9 \mathrm{lbs}$. of phosphoric acid. In the same amount of wheat straw there would be 4.8 pounds of nitrogen; $6.3 \mathrm{lbs}$. of potash and $2.2 \mathrm{lbs}$. of phosphoric acid. In 1,000 lbs. of hay, made from the common grasses, we would have $15.5 \mathrm{lbs}$. of nitrogen; $13.2 \mathrm{lbs}$, of potash, and $4.1 \mathrm{lbs}$. of phosphoric acid. In $1,000 \mathrm{lbs}$. of ordinary clover hay there would be $21.3 \mathrm{lbs}$. of nitrogen; $18 \mathrm{lbs}$. of potash and 5.6 lbs. of phosphoric acid. 
We quote below, a table arranged by Prof. Atwater, based upon the previous table, showing how much of each of the more important ingredients is removed from the soil by various crops of ordinary size.

TABLE.

Materials removed from the Soii by Various Crops.

\begin{tabular}{|c|c|c|c|c|c|c|}
\hline & 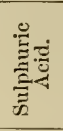 & 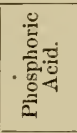 & $\stackrel{g}{\Xi}$ & 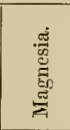 & 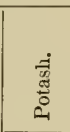 & 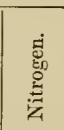 \\
\hline RYE. & Ibs. & lbs. & lbs. & Ibs. & lbs. & lbs. \\
\hline $\begin{array}{l}\text { Grain, } 25 \text { bushels }=1,400 \text { lbs., } \ldots \ldots \ldots \ldots \ldots \\
\text { Straw, } 3,500 \text { lbs., } \ldots \ldots \ldots \ldots \ldots \ldots \ldots\end{array}$ & $\begin{array}{l}0.3 \\
3.8\end{array}$ & $\begin{array}{r}11.8 \\
7.3\end{array}$ & $\begin{array}{r}0.7 \\
12.2\end{array}$ & $\begin{array}{l}2.9 \\
3.9\end{array}$ & $\begin{array}{r}7.8 \\
27.3\end{array}$ & $\begin{array}{l}24.6 \\
14.0\end{array}$ \\
\hline Total, $\ldots \ldots \ldots \ldots \ldots \ldots \ldots \ldots, \ldots \ldots$ & 4.1 & 19.1 & 12.9 & 6.8 & 35.1 & 38.6 \\
\hline Grain, 30 bushels $=960 \mathrm{lbs}, \ldots \ldots \ldots \ldots$ & 04 & 6.0 & 1.0 & 1.8 & 4.2 & 18.4 \\
\hline 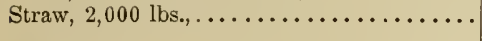 & 2.6 & 3.8 & 7.2 & 3.2 & 17.8 . & 11.2 \\
\hline Total, $\ldots \ldots \cdots \cdots \cdots \cdots \cdots \cdots \cdots \cdots \cdots \cdots \cdots$ & $3.0^{\circ}$ & 9.8 & 8.2 & 5.0 & 22.0 & 29.6 \\
\hline Grain, 20 bushels $=1,200$ lbs., $\ldots \ldots \ldots \ldots$ & 0.1 & 9.5 & 0.7 & 2.4 & 6.4 & 25.0 \\
\hline 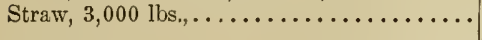 & 3.3 & 6.6 & 8.1 & 3.3 & 18.9 & 14.4 \\
\hline $\begin{array}{c}\text { Total }, \ldots \ldots \ldots \ldots \\
\text { cons. }\end{array}$ & 3.4 & 16.1 & 8.8 & 5.7 & 25.3 & 39.4 \\
\hline Grain, 50 bushels $=2,800$ lbs., ....... & 0.6 & 16.5 & 0.8 & 5.6 & 10.4 & 44.8 \\
\hline Stalks, 6,500 lbs., ............. & 7.8 & 34.5 & 26.0 & 16.9 & 62.4 & 31.2 \\
\hline Total, $\ldots \ldots \ldots \ldots \ldots \ldots \ldots \ldots, \ldots$ & $8 . \dot{4}$ & 51.0 & 26.8 & 22.5 & 72.8 & 76.0 \\
\hline $\begin{array}{c}\text { Mixed grasses, } 1 \frac{1}{2} \text { tons }=3,090 \text { lbs., } \ldots \ldots \ldots . \\
\text { potatoes. }\end{array}$ & 7.2 & 12.3 & $25 \cdot 8$ & 9.9 & 39.6 & 46.5 \\
\hline $\begin{array}{c}\text { Tubers, } 150 \text { bushels }=9,000 \text { lbs.,.......... } \\
\text { товассо. }\end{array}$ & 5.4 & 1.44 & 1.8 & 3.6 & 51.3 & 30.6 \\
\hline $\begin{array}{l}\text { Leaves, } 1,800 \text { lbs., }(1,260 \text { lbs. dry, }) \ldots \ldots \ldots \ldots \\
\text { Stalks, } 1,100 \text { lbs. dry, } \ldots \ldots \ldots \ldots \ldots \ldots \ldots\end{array}$ & $\begin{array}{r}14 \\
3\end{array}$ & 15 & $\begin{array}{l}73 \\
15\end{array}$ & $\begin{array}{r}17 \\
2\end{array}$ & $\begin{array}{l}71 \\
47\end{array}$ & $\begin{array}{l}49 \\
33\end{array}$ \\
\hline Total, . & 17 & 22.5 & 88 & 19 & 118 & 82 \\
\hline
\end{tabular}

These elements that are thus removed from the soil must be supplied in some way, or exhaustion of soil follows. If stable manure could be supplied in sufficient abundance to such lands, not only would all the materials removed from the soil by the crops be returned and the fertility of the land kept up, but it would be gradually increased. But as this cannot be done, owing to an insufficiency of the article, the farmer must depend upon other materials, such as guanos, superphosphates, etc., to make up the deficiency. Prof. Atwater says in this connection, with respect to the

Deficiency of Potash in Common Fertilizers and in Soils._- Most of our artificial manures are only special fertilizers. Guanos, phosphates, fish and bone manures and the like, contain more or less nitrogen, phosphoric acid, and lime; the superphosphates contain sulphuric acid also. With the exception of some to which German potash salts have 
been added, very few of them contain any appreciable quantities of magnesia or potash. They supply part but not all the materials which soils inay lack.

Many farmers find that guano, fish, and other special fertilizers whose action is quick and stimulating, seems to leave their soil in a more exhausted condition than before they were applied. And the complaint is not uncommon that such fertilizers do not bring the same return as formerly.

May we not at least question whether the immediate effect of these special fertilizers has not been, in many cases, to aid the plant to use the more available stores of food in the soil, until these latter have become so far exhausted as no longer to respond to the stimulating action of these special manures?

If the abore supposition be correct, it is clear that what such exhausted soils need, is something to supply, not only the nitrogen and phosphoric acid of the guano, or fish, or bone, or superphosphates, or other special fertilizers, but also the potash and other materials that these latter do not furnish.

Ashes are, for many soils, a standard fertilizer. Places where a tree or a brush-heap has been burned, often show the effects of the manuring for years. It is a trite saying, that 'The land nerer forgets ashes.' Ashes supply directly all the soil ingredients of plant-food, except nitrogen. Their indirect action is also very important in rendering nitrogen and the other materials already in the soil, available to crops. Instead of wearing out soils, they strengthen them. May not this difference be due, in part at least, to the fact that they supply the other ingredients of plant-food that the guano and fish lack?

Stable manure furnishes all the ingredients of plant-food. It is a complete fertilizer. Farmers do not complain that it helps to exhaust their land.

There are soils which, by applications of nitrogen and phosphoric acid, in the form of guano, bone, or nitrogenous superphosphates, may be made to bear good crops year after year. They supply, of themselves, the other materials needed. They have abundant stores of magnesia and potash, and so on, and by weathering, tillage, and the action of the fertilizing materials added, these are worked over from unavailable forms into those which the plant can use.

But such is not always the case. Among the worn-out soils of New England, there are a great many which lack more than nitrogen, phosphoric acid, sulphuric acid, and lime.

This is very strikingly illustrated in the elaborate experiments of Prof. F. H. Storer, at the Bussey Institution, at Jamaica Plain, Mass. These were made upon what Prof. Storer calls 'a very good representative of the light, leachy soils that overhie gravelly drift in New England.' In these experiments, which continued through a series of years, different crops were raised with different manures, the same crop being grown with the same manure on the same plot, year after year. Those raised with fertilizers containing nitrogen and phosphoric acid only, showed very hittle good effect from the manuring, while the potash compounds brought the most satisfactory returns. As Prof. Storer says, the 'crying want' of this land was for potash."

Barnyard Manure.-Farm manures, as a general rule, may be regarded as containing all the essential elements of plant-food, hence, are complete fertilizers. In this respect they differ from commercial fertilizers, any one of which contains but a portion of the requisite elements appropriated by plants in their growth. Animal excrement was formerly almost the only reliance of the farmer for replenishing his lands, he depending mainly for the supply upon what was furnished by the barn-yard, the pig-sty, and the sheep-fold; and even these manures were usually lessened in value by being left until wanted for use, where their most valuable fertilizing elements would be leached out by the rains and evaporated by the sun.

The improvements in the system of agriculture during the last half or quarter of a century, while they bring new aids to the farmer by the use of concentrated fertilizers of 
various kinds, have furnished nothing that has caused this old time-honored fertilizer to be less valued, or less useful; but since the old source of supply is not at present sufficient for the demands of the soil in crops produced, these furnish important and valuable substitutes, or rather are supplementary aids to that which our forefathers depended upon principally.

Though there has been a great improvement within a few years with regard to preventing the waste of stable manure among farmers generally, still there is a great loss in this respect on many farms, which a little pains-taking and care would prevent. The soil needs all the manure that can be made upon the farm, both liquid and solid, which, if saved and applied will result in larger crops, and consequently larger profits; hence, a waste of anything that can increase the productiveness of the farm, is a loss in money to the farmer. Among the fifty important theses published by Liebig as axioms of his theory, he says:

"In the productions of the field, throngh the harvests, a great quantity of the elements of the soil which have become elements of the plants is taken away and removed from the soil; before the sowing, the soil is richer than after the harvest, -the composition of the soil is changed after the harvest.

The lost fertility is again restored by means of manures,-stable manures,-excrement of man and animals.

Stable manure consists of putrefied materials from plants and animals, which contain a certain quantity of soil elements. The excrements of animals and men show the ashes of the food which has been eonsumed in the bodies of animals and men, and derived from plants that have been harvested from the fields. The urine contains the soil elements of nourishment soluble in water, the frees those that are not soluble therein. The manure contains the soil elements of the harvested products of the field."

Hon. Alexander Hyde of Mass., says: "We have yet to learn that there is any effectual substitute for large loads of barn-yard manure and compost. There are two great objections to all these concentrated forms of patent fertilizers, even supposing them to be pure and good of their kind. The first is that they gencrally contain only two or three elements of plant-food, and the second is that they utterly ignore the fact that one great object of putting manure upon the land is the mechanical effect-the putting of the soil in such a condition that it can absorb nutriment from that great reservoir of fertility, the air, and also allow the plant free foraging ground for appropriating to itself all the food that mother earth contains in her bosom."

It is estimated that the urine of the horse is of more value as a fertilizer than the solid manure. Though that of the cow is less valuable than the solid, still it is quite a valuable fertilizer, the average weight of that produced by an ordinary cow being about two thousand pounds in one year, and the estimated worth about three dollars. In some portions of Europe a much higher estimate is placed upon its value as a fertilizer. In Flanders, for instance, its estimated value, according to good authority, is about ten dollars per year. According to the analysis of Sprengel, the average urine of the cow contains 92.6 per cent. of water; that of the horse, 94 ; the sheep, 96; the hog, 92.6; and that of the human species 93.3 . The remainder is composed of various salts, and rich vegetable food, very valuable as fertilizers; but the human is much richer in these elements than any other. Like the solid manures, its quantity and value varies greatly, and depends zpon the quality and quantity of food and liquid taken into the stomach, etc. By reference to the table of analysis of various manures previously given, the composition of these products of the farm, so often wasted, will be ascertained. Many farmers spend large sums of money yearly in the purchase of guanos and other commercial fertilizers, which, with a little forethought in the economy of manures produced upon the farm, would not only render that expenditure needless, but prove the farm product more valuable often for agricultural purposes than the former. Since manures lie at the foundation of all successful husbandry, it becomes a question of importance to the farmer as to how this necessary element can best be utilized and increased; in othor words, how shall the 
manure, both liquid and solid, and the compost piles from all sources on the farm, be saved, increased, and utilized? In answering this question, we would say that the first consideration of importance was the proper construction of the barns or sheds, in order that all the manure made could be preserved, instead of allowing all the liquid portion to be wasted, and throwing the solid manure out of the stable windows to lie in piles, exposed to the leaching process of the storms, permitting the rain and eaves droppings to wash out the most valuable inorganic part of it, and the sun to evaporate a large proportion of the nitrogenous elements, as is still too often the practice with the farmers of our country. Barn cellars, when properly constructed, are very convenient receptacles for both solids and liquids, and also fur'uish protection from exposure to the sun and storms. But whenever used, a sufficient amount of suitable absorbents should be applied to take up all the liquid portions, so that they can be applied to the land as readily and with as little inconvenience to the farmer as the solid manure. We do not, however, recommend the use of farm cellars for the storage of manure, unless more than the ordinary facilities are employed, in order to carry off the gases that must of necessity arise from the decomposing mass in the cellar to the floor above, where the animals are kept. These must be carried off by a perfect system of ventilation. No animal can be healthy and continue to breathe such atmosphere; and animals thus stabled will be compelled to breathe it unless great care is taken in making provision for thorough ventilation; for, although many absorbents employed will prove to a great extent deodorizers, such as dried muck, loam; etc., they will not generally be used in sufficient quantities to become wholly so.

Neither do we approve of the practice, followed by some farmers, of keeping pigs in a barn cellar which is used for the storage of manure. The foul, confined air in such a place, almost destitute of sunlight, cannot be otherwise than injurious to the health of the swine. No animal can be healthy, and hence fit to become the food of man, that does not, while living, have a sufficient supply of pure air and sunlight. Otberwise the blood becomes poisoned, the whole animal system diseased, and the meat thus produced will be anything but a wholesome sanitary diet. In order, also, to prevent, as far as possible, the foul air of the manure thus stored below the barn from arising and vitiating the air where the cattle or horses are kept, it should be mixed with a sufficient supply of absorbents in order to prevent the escape of ammonia and other substances. The barn cellar should also be well ventilated, while all barns where animals are stabled should be supplied with a sufficient amount of pure air by means of proper ventilation; that is, the ventilators should be so arranged as to permit the escape of the foul air of the barn and armit the fresh air from without, without producing a draft upon the animals, which latter would be the means of serious trouble among the stock, - a draft of air being one of the worst exposures for either man or beast to severe colds, with possibly pneumonia or lung fever, etc., that could be found. We have known valuable animals to have died from such exposure through the thoughtlessness or ignorance of their owners.

A very important fact for the farmer to have in mind is, that the better the animal is fed, the better will not only be the meat, the milk, butter and cheese, but the better will be the fertilizing properties of the manure furnished by such animals. Excessive and rapid fermentation should be avoided, as much of the ammonia is often thus wasted. Horse manure is espccially hable to ferment or "burn," as the fermenting process is termed, and is often rendered almost worthless by the loss thus sustained. Many farmers prevent this by mixing it with cow manure, which is colder and less liable to fermentation, thus easily obviating the difficulty. If, however, this is not convenient, owing to the locality of the horse and cow stables, or for any other reason, it is desired to keep them separate, decomposition can be greatly retarded by keeping the manure trodden compactly and saturated with water to keep out the air as far as possible; thus keeping the temperature low, it can be preserved a long time before fermentation commences. 
Where no cellars are provided for the manure taken from the stables, it should be protected from the rain and sun by covered sheds, which will repay the farmer for furnishing, in the amount of rich fertilizing material saved, the quantity thus saved soon repaying expense of the sheds. Where cellars for its storage are used, many farmers prefer muck or peat as an absorbent of the liquid portions; but in such cases it is necessary that the material be perfectly dry, and in order to secure this condition, it should be dug from the muck or peat bed and thrown into heaps, where it is left several months exposed to the air and sun. The drying and decomposing process can be greatly facilitated by shovelling over the piles and admitting the air occasionally. Some advise mixing lime, (three or four bushels to a cord of muck, ) first slacking it with water; wood ashes are also often used for this purpose, about fifteen bushels to the same quantity. When dry and ready for use it should be stored in some convenient shed. Only a small quantity will be required to absorb the liquid accumulations of the stable each day. Dried leaves, straw, and clay are also used for this purpose. Some prefer saw dust for the bedding of stock, and as an absorbent of the liquids of the stable, which is found very valuable for this purpose; but it should be quite dry, or it will be entirely useless. It readily absorbs the liquids, and is easily mixed with the solid manure and soil, when applied to lands. Dry loam has been found to be a valuable absorbent also, when used in the stables for this purpose. Mr. Albert Day of Brooklyn thus describes his method of preserving stable manure:-

"In the preservation and preparation of manure the principle of the old adage holds good-'A penny saved is a penny earned.' Hence, my first care is to guard against the loss of valuable ingredients of the manure caused by the action of the weather, and by the escape of the ammonia set free in the process of fermentation.

"To accomplish this end and to make the largest quantity of the most valuable manure, - my milch cows are kept in the stable nights from about the first of September until about the first of June, and in the winter months eighteen to twenty hours per day. The stables are littered with refuse hay or straw, and the manure is thrown into a cellar beneath, where I usually have some three-cart loads of loam for every animal housed. The manure is spread over a portion of the cellar at intervals and covered with the loam, at the same time throwing on plaster. I have preferred this course to that of putting muck or loam in the stable to absorb the liquid portion, as one involving less expense, and securing equal benefits. The bulk of the other manures upon the farm are from the hog-pen, horse-stable, and barn-yard.

"The compost in the cellar is worked over two or three weeks before use, when it is drawn to the field and ploughed in, and the compost from the barn-yard and hog-pen worked in on the furrows with a Shares harrow. I plow in much less now than in former years, for the reason that from experiments in both methods I am much better satisfied with the results of surface applications. From this statement it will be seen that the manure is applied fresh rather than fermented.

"A slight covering of earth is sufficient to prevent the escape of ammonia and other gases, even from the rapidly fermenting compost heap; and spread in or upon the field, where the process of fermentation is comparatively slow, very little is lost, in the opinion of many good, practical farmers, even by top-dressing."

Tanks for holding the liquid manure from the stables, have long been in use in many portions of Europe and this country, it being conveyed to them in drains from the stables. These tanks or cisterns, which are made of cement or concrete, are so constructed as to be closely covered to prevent the escape of ammonia, which is produced by the fermenting process. This fermentation rapidly progresses in warm weather, but the loss can be retarded by putting in gypsum or charcoal, which absorb the ammonia. A few days after fermentation commences, the liquid is pumped into casks and carried on to the lands. When designed for watering plants, it must be diluted with water to prevent injury. This process is attended with considerable labor, although it may prove a good one where a large number of animals 
are kept, but for the general farmer we think the use of absorbents, as above stated, far preferable. Horse manure is one of the valuable farm manures, and the easiest to ferment. When packed in heaps this process will sometimes commence in twenty-four hours. Even in the severe weather of winter, fermentation will progresss rapidly, if a large quantity is accu. mulated. When not arrested, it becomes in a short time of about as little value as ashes, and loses about nine-tenths of its original weight. The fermentation or "burning," as it is sometimes called, can be arrested or prevented, as we have previously stated, by mixing with other manures, or rendering the mass conpact, and saturating with water to prevent the circulation of air.

Sheep manure, and that furnished by the pig sty, are also very rich fertilizers, the former boing considered by many to be more raluable in producing good crops than even cow manure. It is rendered most available by being composted, as it frequently injures the seed of plants if placed in the hill or near it before the fermentation is completed. By supplying the pig yard with plenty of muck, the material from that source is greatly improved, and proves a valuable fertilizer.

The illustration represents a manure spreader manufactured by the Kemp and Burpee Manufacturing Co., Syracuse, New York, a most valuable acquisition to the agricultural implements of the present time, being, in fact, a manure spreader, pulverizer, and cart com. bined. It not only is a great labor-saving machine, but does the work better than could be done by hand, spreading the manure more uniformly and at the same time pulrerizing it, which increases its value, since the finer the manure is pulverized when applied to the soil the more readily it is assimilated. It will spread all kinds of manure, whether coarse or fine, wet or dry, heavy or light, and is regulated to spread different quantites to the acre, so that the farmer may know just what amount per acre he is using. It can also be attached to the fore wheels of auy ordinary farm wagon.

Poultry IIaure.-This is a valuable fertilizer, and any farmer who has been accustomed to neglect his flock of hens, caring little or nothing for their products or the care they received, would be surprised at the quantity and quality of the guano they manufacture when properly fed, and which might be utilized by being saved from their roosting place. The droppings of the poultry should be secured from under their roosts every few days and mixed with earth, or with a compost, as they are very soluble. It is a good plan to scatter a little sand over the floor of the hen house every day or two, which has a tendency to keep the place clean. When applied to the soil, it should be harrowed in lightly, since the excrement of fowls contains both the foces and urine combined; it is peculiarly rich in the fertilizing elements, and is considered, in this respect, next in value to night-soil. Poultry should al ways be provided with warm quarters for roosting, and we hope no farmer is so far behind the times in this respect or so inhumane as to adhere to the old and barbarous custom of forcing his hens to roost in trees, and take care of themselres on the scanty pittance they are able to glean from the barn-yard.

Night-Soil and Poudrette.-One of the most powerful fertilizers known is human excrement. In China, Japan, and many portions of Europe, it has long been one of the chief fertilizing substances used in agriculture, where the continued fertility of the soil and abundant crops fully attest its value. In our own country, this source of supply has for various reasons been in a great measure ignored and neglected, though it is sometimes found in the market among the various commercial fertilizers, mixed with charcoal, dust, charred peat, or other substances under the name of poudrette or tafeu. By being thus mixed and dried, it is disinfected of its effuvia, and becomes a convenient article for transportation. With regard to this substance, Prof. C. A. Goessmann, of the Mass. Agricultural College, and also State A gricultural Chemist, says:-

"There are sometimes four different kinds of poudretle offered for sale:-

"I. Blood or Meat Poudrettes, which are manufactured from the solid portion of the human 
excretions, with the addition of blood and refuse meat from the slaughter-house, or the carcasses of dead animals. They are usually sold in a dry and pulverized state.

II. Simple Poudrettes, which consist of the dried, pulverized, solid human excretions.

III. Humid Poudrettes. These consist usually of the entire contents of the vaults, which, after being deodorizen, are left in large tanks for evaporation by mere exposure, or receive additions of gypsum, etc., as absorbers of moisture.

IV. Compost Poudrettes. The following course is frequently pursued in their manufacture. the sweepings of the streets, ashes, refuse lime from gas-houses, and various other suitable refuse materials of factories, etc., are screened to remove stones and other worthless materials. The screened mass is subsequently filled in alternate layers with deodorized nightsoil, in large tanks containing water-tight floors. These tanks are frequently large enough to store five hundred tons at one time. After the material has been left for from four to five months for a thorough disintegration, it is cut through from the surface to the floor, and thereby thoroughly mixed. The fertilizers Nos. III and No. IV are, for economical reasons, best adapted for consumption in the vicinity of the manufacturing establishment, whilst Nos. I and No. II, on account of their higher value, may enter with good success more distant markets. There is scarcely any other class of commercial fertilizers which is apt to suffer as readily a depreciation in value from careless mangement of its raw material and its mode of manufacture, as the poudrettes. For this reason, they ought to be sold by analysis; at least with reference to the amount of ammonia, phosphoric acid and potassa. A detailed statement of these substances gives a somewhat more definite idea regarding the nature of the excretions which served in their manufacture. It needs no particular argument to show the great value of the human excretions in the agricultural industry, as long as those of our domesticated animals are considered most efficient for the manuring of our farm-lands.

"The food of man, as a general rule, is much richer in the most valuable elements for plant-growth than that of our farm live-stock; the same relations are true, for obvious reasons, with regard to the excretions of both. To establish that claim among our farmers requires the manufacture of standard articles of definite chemical and physical properties. It is a fact worthy of notice, that in the most densely-populated countries, the superior efficiency of the human excretions for manurial purposes has been most decidedly recognized. Belgium, like China and Japan, is largely indebted for its high state of cultivation to the extensive use of night-soil as a fertilizer. Prejudice against the more general use of the latter for the reproduction of our garden and farm crops contributes largely to the indifference which still prevails among many agriculturists regarding the magnitude of the pecuniary interests involved in the question of securing the human excretion in the most advantageous form for agricultural purposes. The same indifferent management which characterizes quite frequently the treatment of the barn manure causes izsually a most serious depreciation of the contents of the vaults. The wasteful practice adopted in our large cities with regard to the disposi. tion of the human excretion is not unfrequently the outgrowth of considerations which have largely lost their importance in consequence of the accumulated experience elsewhere. An intelligent solution of the sewage question in our large cities touches the pecuniary interest of every farmer. However intricate the various considerations which deserve careful attention may render the problem, the sewage question cannot be considered satisfactorily settled without a due recognition of the agricultural interests of the country."

A noted agriculturist has said that the waste from the kitchen and the contents of the water-closet, if properly composted, would be of greater value than the same amount of many of the commercial fertilizers that are bought at a high figure by our farmers.

Says Liebig: "If we admit that the liquid and solid excrements of man amount on an average to 547 pounds in a year, which contain $16.4 \mathrm{I}$ pounds of nitrogen, this is much more than is necessary to add to an acre of land in order to obtain, with the assistance of the nitrogen derived from the atmosphere, the richest possible crop every year." 
Prof. Hilgard, of the Unirersity of Mississippi, states that all the products of our fields, excepting a portion of the feed crops, ultimately go to serve as food or raiment to man. Hence man's excrement, rags, paper and bone, must and do contain the ingredients withdrawn from our soils; and were we faithfully to return all these things in the proper form and in the right place, we should need no guano islands to eke out the deficiency in the return made in the offal crops and manure of cattle. Prof. J. F. W. Johnston says of it: "Night-soil is the most valuable of all the solid animal manures. When dry, few other solid manures can be compared with it, weight for weight. Dried night-soil is equal to thirty times its bulk of horse manure."

The cesspool and priry are necessary concomitants of every family, but it is the manner in which they are regulated that renders them a source of pestilence and ill odors, or that can transform these unseemly parts of the farm-house into comehness and a source of profit, in converting, by the natural process, these noxious odors into elements of fertility for farm use; thus producing utility and beauty, which may be in the form of farm crops, garden reg. etables, fruits, or flowers, from what would otherwise be a source of noxious effluvia to poison the air, and breed disease. How shall, this be accomplished? In the first place, we would say, let such places be surrounded with some kind of shrubbery or vines, concealing the deformities of art (which are in such cases usually conspicuous) with the beauties of nature. As a disinfectant, there is nothing better than dry earth; a hitle used each day to cover the contents of the vault and absorb the liquids will prevent all offensive odors, and keep the contents in a condition to be easily handled when taken out to apply to the soil. It is necessary that the earth should be perfectly dry, in order to be an effectual absorbent. Earth. closets have long been in use in England and France, and to a limited extent in this country, and have been found to be completely deodorized, when used as above described.

It has been found by experiment, that such soil, after being exposed to the sun and air, and thoroughly dried, can be used repeatedly in the same manner, even six or seren times, without being offensive, which proves the powerful deodorizing effect of earth.

By adopting the earth-closet principle, any farmer can easily secure, free of expense, one of the best fertilizers in quality that can be found. Road-dust, sifted coal-ashes, pulverized charcoal and dry muck, or any kind of dry soil, may be used for this purpose. When either is used in sufficient quantity each day, it can afterwards be as easily handled and removed as any kind of soil; it is also improved in quality by being mixed with earth or some foreign sibstance. By this means also is it not only deodorized, but the evil effect of drainage into wells can be obviated, thus preventing the contamination of the water, which is so often, in the country, the fruitful cause of typhoid fevers, and other diseases.

Sewage as a Fertilizer.-The sewage question, both in a sanitary and agricultural point of view, -in other words, the question as to how the sewage of our cities can best be utilized to fertilize the soil, and thus prove the double benefit of a sanitary and agricultural means, has long engaged the attention of scientific men, and, although no definite solution of the problem or practical plan has yet been adopted, we feel sure that the time is not far distant when this question, which so materially affects the sanitary interests of the country, will be satisfactorily settled in the interests of agriculture, as it has been in various European cities.

Of course, the sanitary view of the subject is first in importance, and demands the high. est consideration, irrespective of agricultural benefits to accrue therefrom. It cannot be otherwise in the nature of things, than that the water supphed by rivers and lakes to our cities, is contaminated with poison, disease, and filth from the sewers that empty into them. When we remember that the city supply furnishes the drinking-water to the inhabitants of those cities, we do not wonder at the various diseases, and the extended death lists that swell the records; we only wonder that they are so few. Gen. N. N. Halsted of Newark, N. J., states as follows respecting this subject: 
"It has been ascertained by Boussingault, that a man in a healthy state passes three pounds of urine daily; and Liebig states, that, in the same state, he roids five ounces and a half of dung. These two quantities give a total annual quantity of 1,220 pounds of liquid and solid manure voided by every person, on th 3 average. Now, taking two million as the population of London, the quantities of those manures voided by the inhabitants of the metropolis amount annually to $1,089,285$ tons. Chemistry has ascertained that the component parts of the excrements of man are as valuable to vegetation as those of guano; and, as the different sorts of guano sell from $£ 6$ to $£ 10$ per ton, we are warranted in estimating the value of night-soil and urine at $£ 8$ per ton, which would give the entire value of this manure in London alone every year at $£ 8,714,280$, or $\$ \$ 3,571,400$ in gold. This may seem like exaggeration; but, put it at half the amount, and the subject is serious enough to address itself to every thinking mind. This waste is not the worst of it. We claim that there is a sanitary point of view, higher in importance than all considerations of moneyed value: we mean so far as this waste of sewage into the rivers affects the supply of drinking.water for our cities. We take two prominent instances within the State of New Jersey; the two largest cities both taking their supply of water from the same source,-the Passaic River. This river, furnishing an illimitable supply, takes the drainage of the large manufacturing town of Paterson, and the smaller towns along the river, to the points where the supply is taken up: in addition to these, the sewage of the city of Newark, with a hundred and twenty-five thousand population, discharged into the Passaic River, is carried up by every flood-tide beyond the city, and to the very conduits where the water is taken for the supply of the two cities, Newark and Jersey City. The operations of the United States Government in removing the reef, and the obstructions to navigation in the river above the city of Newark, have greatly facilitated this flow; and there can now be no question but that the supply of each city is contaminated by this sewage. The joint commission of Newark and Jersey City employed an eminent chemist to analyze the waters of the Passaic as furnished the two cities. We quote so much of his report treating of sewage pollution of rivers as applies forcibly to our subject, and the sanitary point now involved: 'That class of scientists who study microscopic fungi, mycologists (in common with many distinguished scientific physicians), are now settling down to the belief that most epidemic and epizoötic diseases are accompanied (as causes, not merely as effects) by certain fungoid growths: in other words, that these diseases are produced by vegetable parasites. When these fungi take root in live animal tissues, they develop into abnormal and monstrous forms, which have not been recog. nized until lately; but it is now known that the spores discharged (by millions of millions it may be) with the excreta, when cultivated outside the body, come back again to their normal forms, and the fungi are recognizable. Thus in common dysentery and cholera-morbus, the spores, when replanted, produce a common fungoid parasite of wheat; while, during the fearful Asiatic cholera, the spores produced a parasite of the East India rice-plant. These facts (if they must be admitted as such, which seems inevitable) are suggestive with regard to sewage contamination of rivers. One case of cholera brought to Paterson, or any of the towns lying on the upper Passaic, might fill the whole river with the living seeds of the pestilence. A like propagation would take place from Newark throughout Jersey City and Hoboken, and even throughout Newark itself. I am aware that these are appalling consid. erations, and may be rejected by some as contingencies too remote and dreadful to be possible. But human experience, alas I will not counterbalance any such puerile view as this. We must stare these horrors sternly in the face, bring all our science to bear, and study prevention, rather than wait till called upon to endure the svii when it shall have passed beyond our cure.'

It is not only river water that is contaminated by the sewage of the cities, but the wells are also poisoned from which many of the inhabitants derive their sole supply of cool 
drinking-water, in their innocence supposing they are drinking the purest and best. We give a single instance in illustration. A well in Market street, in the city of Newark, standing in front of the office of one of the daily journals of that city, had been so lauded for its cool and sparkling water, that people were wont to stop and refresh themselves with a drink from its pump: nay, instances were frequent of passers-by in the throng of Broad street crossing over and going down to this pump, as if to a favorite soda-fount. It might have been a rival, for aught we know, to some such popular establishment in its vicinity. This well, with two others very much used, was designated by the chief engineer of the water board, to Professor Wurtz, as among those of the city most resorted to. Samples were taken; and, on analysis, the one we have selected was found to contain, in the words of the professor, 'about five grains to the gallon of an ingredient which cannot be traced to any other source than the infiltration of urine of man or beast, or both.' He further recommended the closing of this well to the public use, and it was done.

These words require no comment: they speak for themselves."

We see from the above that for both sanitary and economic reasons, the night-soil should not go into the sewage of the city. The following will show how the French have utilized the sewage of Paris for agricultural purposes.

The Sewage of Paris.-The benefits of appropriating sewage water to irrigating the land is fully shown in the following account given by Prof. Geo. H. Cook, of New Jersey, in his report on Agriculture and Agricultural Teaching in Europe :-

"The 24 th of June was given to an excursion to Gennevilliers, where the sewage water of Paris is used to irrigate and enrich market-garden land. The question of sewage, its removal and purification for sanitary benefits, and its uses for agricultural purposes, have been discussed in Paris much as in other cities. Friends of sanitary improvement, however, have insisted that the first step should be taken and the first expenses incurred in the interests of public health, and, in spite of determined opposition, have carried their point. A very succossful beginning has been made.

In most of the houses of Paris there are two sets of drain-pipes. One of these connects the washing-sinks, bath tubs, etc., with the city sewers; the other connects the water-closets with cesspools in the house-yards, and these last are pumped out, and the contents are carried off to be used on the land. It is only the sewage from the former kind that have to be attended to, and for this purpose the whole contents of the public sewers are collected in very large conduits from both sides of the Seine, and are conducted down to Asnieres, on the river, and just at the outskirts of the city. Here, by means of powerful pumps, about one-third of all the sewage water is raised high enough to flow by gravity orer a large tract of flat land in the bend of the river just below this place. The rest of the sewage is allowed to enter the river here. The amount of sewage carried in the sewers yearly is about one sundred and thirty-three million cubic yards, and-as a cubic yard is not far from two hum. dred gallons-the daily flow of sewage is about seventy-three million gallons. One-third of this, or twenty-four millions, is daily pumped up twenty or thirty feet to distribute it over the peninsula on which Gennevilliers stands, comprising about one thousand acres.

The sewage is carried to various parts of this plot in closed pipes, and is then let out into open ditches, which are a little above the general surface of the ground. From these it is let out into little irrigating ditches, which are drawn parallel to each other, and about three feet apart, so that the whole ground looks as if laid out in garden beds. The beds are planted, and water in the little ditches soaks through and moistens and feeds the roots of the growing plants without defiling or in any way injuring their leaves or stems, and a most luxunant growth is produced. Every year the little ditches are changed, and where those of one year are, is the middle of the bed the next year, and the sediment of the ditches is scraped out to mix with the soil as manure. In winter the water can be distributed over the 
whole surface, but in summer, when the vegetation is active, it needs skill and experience to put on the water or withhold it at proper times. The gardeners, however, have learned to do it successfully-and the land which nine years ago was only an open gravelly sand, so poor that but moderate crops of rye could be grown on it, is now the most productive garden-soil about Paris. When the experiment was begun no one would take the water, and the city bought about twenty-five acres of ground on which to make the first trial. That land is now rich, and rents yearly for $\$ 40$ an acre, and the adjoining land-owners, who have concluded to try the effect of the water, have increased their rents fourfold-the average rent being now about $\$ 36$ an acre. No one who has taken the water has yet discontinued its use.

The first effect sought-that of purifying the water-is produced completely. As it comes from the sewers it is dark-colored, turbid, and of a vile odor. After being filtered through the cultivated ground it collects in the underdrains and runs out clear, cool, without smell or taste, and to chemical tests shows no organic matter. In fact, the little brook we saw looked like the purest and most tempting of spring waters, and those who drank it said it was so.

The crops grown on this soil are cabbages, beets, carrots, beans, artichokes, cauliflowers, garlic, onions, leeks, celery, salsify, pumpkins, potatoes, mint, absinthe, angelica, and nursery and fruit trees.

A committee of investigation who examined this subject in all its relations, reported the following as crops per acre raised on these grounds :-

Artichokes-From 14,000 to 30,000 heads.

Cauliflowers-12,000 to 20,000 heads, weighing from 30,000 to 35,000 pounds.

Garlic-32,000 pounds.

Carrots-52,000 to 72,000 and even 115,000 pounds.

Celery— 87,000 pounds.

Cabbage-120,000 pounds.

Onions-From 52,000 to 72,000 pounds.

Leeks-52,000 pounds.

Potatoes-From 25,000 to 35,000 pounds.

Pumpkins-100,000 to 120,000 .

Salsify-8,000 to 10,000 bunches, weighing 22,000 pounds.

A remarkably fine dairy is kept there, and the cattle are fed on lucerne grown with sewage, and we were treated to strawberries grown on ground irrigated with sewage water, which were as large and unexceptionable in flavor as Durand's "Great American." The vegetables are among the finest-looking in market, and are unquestioned in flavor and good quality. The commission reported that 'the quality of the products, which had been condemned by some persons, was now acknowledged good ; they retain the flavor which properly belongs to them, and do not contract any bad taste.'

The benefits of this use of sewage water, both for its sanitary and agricultural effects, are fully demonstrated, and proves the soundness of the views of those who devised and carried it out. So far, the expense has been borne by the city. No charge has been made for the supply-ditches and drains, or for the water. At the present time the question is being raised, whether the land-owners, whose rents have been increased, and the gardeners, whose crops have been so much enlarged, should not pay a part of the expense. It is, however, generally conceded that the main part of the expense must be paid for the public good, and for the benefit which it brings to health, and that it cannot be expected that sewage water, diluted as it is, can ever be used on agricultural land so as to pay the whole cost of distributing it. The present effort is very encouraging to sanitarians, and has only been reached after a great deal of talk and unsuccessful work for the same end. On the ground where the sewage is now distributed, we saw large reservoirs into which the sewage water was 
formerly drawn, in order to precipitate the fertilizing matters by some chemical process, but it failed. The water was made clear, but the main impurities were not removed, and the works stand there as a monument of the work required to accomplish this end, so important for all cities, where filth will accumulate and must be removed or destroyed."

France has set a good example to other countries in thus disposing of the sewage of her largest city, and though some improvements can doubtless be made in this system, it is admirable in its results thus far practiced. As apropos to the subject, a description of a sail in the Paris sewers, as given by a Boston journalist, may not be devoid of interest to our readers :-

"A Sail in a Paris Sewer.-A visit to the sewers of Paris is indeed a novel one. There are two visiting days per week for the sewerb, and, by writing to the prefect, the person who desires to make them a visit can obtain a pass. This pass is about three inches square, and is as finely embellished and printed as an invitation to a millionaire's reception. The entrance to the sewers is at the Place du Chatelet, and is simply a slab removed from the top of the main sewer. When my turn came to enter into the yawning blackness below, I shuddered as one would who never expected to see the light of day again. Descending the narrow, winding stairs, I found myself in a large vaulted space. This $\pi$ as the main sewer, black as the Styx, and probably many times nastier. Directly over the nastiness, the rails resting on either side of the sewer, were a number of open cars, holding fifteen or twenty each, whose construction would almost warrant the term elegant. Bottoms and sides were of light wicker-work, and at either end shoen two globular lamps. As fast as a car was filled with passengers, two men on either side of the sewer passed a stout bar through fised iron rings in the car-frame, and, bracing themselves against the projecting ends, trolled off and trundled us away into the darkness. At short intervals the noise of marmuring rills and babbling brooks came to our ears. These, as we were rushed by them, we saw to be the tributaries of the main stream. The sewer over which we rode was named from the street under which it ran-the Rue de Rivoli-and the murmuring rills, brooks and rivulets, were called from the streets under which they passed. Some bounded into the main river in joyous little cascades, and the meeting of others with the parent river was condueted in a more quiet, orderly, and solemn fashion. The average smell was simply that of a sewer, somewhat carbolized, perhaps, but still a sewer. We stopped. Here we were to leave the car. Here began the remainder of the passage by water-at least, it is called water, and for navigation the fluid answers the purpose; but it isn't water. We left the car. The barges were loading up. Each would hold fifteen or twenty people. Our barge started. It rolled unctuously in the dark.colored fluid. As the passengers moved about in getting to their seats, it rolled as a ressel might roll in a lake of molasses. It was an unpleasant sensation. The motive power was furnished by a few blouse-covered Frenchmen attached to long ropes, who, in the capacity of mules, hauled us along. Silently we moved on. Not a ripple was heard under the prow. The sewer had no capacity for rippling. Little was seen, save the glimmer of the lamp on the barge ahead of us, and a long, straight, silent stream. The barge, loaded deeply, seemed but a few inches above this Farisian Styx. As we slightly rocked, there came awful suggestions to our minds of the possibility of shipping a sea. On the current we saw straws, corks, and lemon peelings. A young man, actively and unpleasantly curious, thrust his cane in the stream, with a viow of getting soundings. On bringing the cane on board, all in its vicinity mored away. Some folks never can let well-enough alone. We turned into the Rue Royale. The stream grew wider, the current more rapid. Most of the ladies now held handkerchiefs to their faces. They seemod a deeply-affected company. The smell was that of carbonated sewage. It was discernible by the tongue as $\pi$ rell as the nose. The combined ride and sail occupied one hour and a quarter. I suppose we traversed two miles of sewer; anyhow, I was only too glad when the voyage ended and I once more inhaled the pure air of heaven." 
Composts.-We sometimes hear the question asked by farmers, "What is the use of composting barnyard manure? Why not take it directly from the stables to the field and thus save the labor of composting it?" We know of no better answer than this: Since manure contains the elements of plant-food, and must be decomposed and become soluble or gaseous before it can properly feed the little rootlets that are reaching out in the soil for their nourishment, thus to support and promote plant growth,-the sooner this decomposition is accomplished, the earlier will the plant be supported, and the better its growth established, instead of waiting for this process to take place in the soil, after being plowed under, which sometimes requires considerable time, unless well pulverized when applied to the land; besides, even then the manure is so inaccessible to the amount of air required to produce fermentation, that the process of decomposition is much retarded; but when composted before being applied, it is readily assimilated. Another good reason for composting might be the use that can be made of the fermenting manure to produce a similar fermentation in other substances mixed with it, thus utilizing for fertilizing purposes, what might otherwise be entirely useless.

The word "compost" signifies "placed together," hence it means a compound. Alexander Hyde; of Massachusetts, thus defines it in a lecture on this subject:

"Compost is 'hash,' and as we can make hash of fish, flesh, or fowl, using for this purpose cod, halibut, salmon, or any other kind of fish; beef, pork, mutton, or any other kind of meat; brahmas, dorkings, shanghais, or any other kind of fowl, using nice cuts, or the refuse pieces, as economy or skill may dictate; so we may compost out of an almost unlimited number of substances. There are almost as many modes of compounding food for plants as for man, and every house-wife knows that these are endless. Speaking generally, it may be said that everything that has once been organized into plant or animal life, may be made to go the round of life again.

More specifically, as we generally use potatoes as the base of hash, by which to extend the animal food, so we generally use muck, or some substance abounding in vegetable matter, such as sods or leaf mould, as the base of the compost heap, so as to extend the more concentrated animal fertilizers and render them more easily assimilated by plants."

By the use of dry earth, muck, and other substances as absorbents, in stables, pig-yards, and privy-vaults, as has been indicated in the previous pages, these manures are composted and greatly improved in condition, for application to lands, besides the saving by this means of much of the liquid that might otherwise be lost, and preventing the escape of ammonia and other valuable gases. But as it is often desirable to compost by a different process, we will consider the usual and most approved method of doing it.

Many farmers compost their manures under the stable-floors in a barn-cellar for that purpose, where the various manures of the farm are mixed with muck and other absorbents until well fermented; others preserve the liquid manure in tanks under the stable for that purpose, and produce a valuable fertilizer in the form of ammoniated superphosphate, by mixing with it ground phosphate and sulphate of lime. Perhaps the more common method of composting is by making a compost heap.

How to Construct a Compost Pile.-An elevated and dry spot is generally selected as the site of the compost heap, in order that it may not receive the wash from a higher source and thus much of the fertilizing substance be washed away and lost.

Boards should be placed at the bottom to prevent the liquid portion from soaking into the ground, as would be the case were the soil of a sandy or gravelly nature. A layer of muck or loam, from a foot to a foot and a half thick, should be placed so as to form a base for the heap; over this a layer of stable manure of equal thickness should be spread, which forms the fermenting principle of the heap. This may be covered by a layer of leaves, or refuse of any kind, such as straw, cornstalks, decaying vegetables, etc. If in the vicinity of 
a woolen or paper mill, or tannery, the waste substances furnished by these are often utilized in the compost beap with good effect. Some farmers put in old woolen rags, old boots and shoes, bones, brine, soap suds and all the garbage from the kitchen, and old refuse of every kind and description that can be collected together, and which will, in time, become subject to the chemical changes of the compost heap, and produce an excellent fertilizer.

In the cotton growing states, cotton-seed is often utilized as an important ingredient of the compost pile, after having been fermented with wood-ashes to soften and reduce the hulls. Another layer of muck should follow this, then stable manure and waste material, thus alternating until the pile is made of sufficient size. The manure from the sheep fold, pig-sty and poultry-house, together with night-soil, all furnish rich material for this purpose, and are thought by most farmors to be more beneficial to various crops when composted in this manner than when applied separately. The compost heap should be composed of about one load of manure to two loads of muck, in proportionate quantity of each, and from twentyfive to thirty pounds of plaster added to each load. It should be built five or six feet high (if not under cover), to prevent the rains from leaching it. The top of the heap should be covered with muck. Some surmount this with boards on straw, as they finish a hay-stack, which is a very good practice. When built under a cover, leaching by the rain is prevented, but it involves the additional labor of keeping the mass wet. The three essentials necessary to produce rapid decomposition in the pile are air, moisture, and a temperature above $65^{\circ}$, and these conditions, except in quite cold weather, are generally found in a compost heap. Superphosphate of lime, about two hundred pounds to each cord of material, is considered by many a very valuable addition to the compost pile, also finely ground bone, which is a good substitute for the latter when that material is difficult to obtain.

Freshly slacked lime hastens decomposition, and thus gets the mass in condition to be used much sooner than it otherwise would be, but its use is objected to by many as having a tendency to set free much of the nitrogen, and thus involving too much loss to be profitably used. Salt in sufficient quantity to give five or six bushels to the acre is also often a valuable acquisition to the pile. Some mix ashes, both leached and unleached, with the compost, while others prefer to apply the ashes to lands separately.

Alexander Hyde says further respecting composts: "Muck, mixed up with fermenting manure, becomes quickly infected with a tendency to decomposition, the manure acting much like yeast in the housewife's bread-bowl. A little leaven of manure is thus made to leaven the whole lump. When shoddy was not so much in vogue as it is at present, we were accus. tomed to use the refuse of a neighboring woolen-mill for composting with muck, and as the wool was generally oily, ready to absorb oxygen from the air rapidly, and thus undergo a spontaneous combustion, we have seen the pile in an almost boiling condition. A yellow oily substance exuded from it, and a stick thrust into the pile, soon became feverishly heated. In contact with such fermentation, muck is rapidly cooked, and made into good plant-food.

Common barn-yard manure does not ferment so furiously as oily wool-waste, but the effect is similar. A board placed in it rots twice as fast as when in common earth. Even old boots, which, from the tannin in them, resist decay with great pertinacity under ordinary circumstances, placed in the compost heap, become as powerless as Samson with his head shaven, and are as easily torn as so much pasteboard. Horse-manure is more inclined to fermentation than that from cows, and is therefore best adapted to produce this decomposing influence in the compost heap. We have known bones in a pile of horse-manure become so soft that they could easily be crushed.

We are not prepared to assent to the assertion so often made, that no additional virtue is acquired by manure in the process of composting and fermentation. It might as well be asserted that flour acquired no additional nutriment by composting it with a little yeast and water. In the process of fermentation great chemical changes are going on; old compounds are destroyed and new ones formed. 
The power of absorption is not the only recommendation of muck for the base of compost. Pure muck is pure vegetable matter, has been once organized into vegetable life, and in its decay must furnish food for future vegetation. Placed in contact with animal manures, or any dead animal substance, the decomposition of the muck is also accelerated, and tho mutual action is highly favorable for forming a good plant-food.

The quality of muck varies in different localities. We have dug that which had the odor of burning gnnpowder as it first came from the pit. Such muck is a good manure in itself. Other deposits possess less intrinsic value. The goodness depends in a measure upon the character of the vegetation which, by its partial decay, has formed the muck bed. Rushes, ferns, and mosses form a muck of less value than that made from leaves of decidnous trees, and soft, sappy wood, partially decayed, furnish less fertilizing material than hard woods.

But muck is not the only base of the compost heap, and whether we should use it at all will depend somewhat on the nature of the soil to which the compost is to be applied. If this is sandy or gravelly, or a loam in which sand predominates, there is no better compost than that with muck or leaf-mold as the base. On clay land, also well-drained, muck operates favorably in making it loose and friable; but if it is inclined to be wet and cold, muck increases this tendency, as it is a great absorbent and retainer of moisture. We have used a muck compost on a clay loam, pretty well drained too, till we perceived that the vegetation started a hittle later in the Spring, when we changed the base of the compost, using alinvial soil instead of the muck. The change was decidedly beneficial. The soil was apparently pretty much destitnte of vegetable matter, but was rich in silicates and other salts.

The caterer of plant-food finds that plants, like animals, are fond of a variety. Barnyard manure is the staple fertilizer, and, like bread in the animal economy, makes the best steady diet. But this manure is better when made up or composted of the excrements of different animals. Let a farmer feed a piece of land with the droppings of sheep alone, or cows alone, for a series of years, and he will find that the effect is not so great after a time, and that a little horse-manure put for a change upon the same land will work wonders. Night-soil is one of the most efficient fertilizers, and has been much used by market-gardeners near our large cities, yielding in its first applications immense crops, but after a time it loses its magical influence, and a market-gardener once said to us that he was so well convinced of the necessity of a change in plant-food, even where night-soil was used, which he considered the best for the growth of vegetables, that once in the course of six or eight years he would prefer a dressing of muck or rotted sods, or leaf-mold, or even good loam, to any pure nitrogenous manure. A compost made of nitrogenous manure, salt, lime, ashes, bone-dust, refuse vegetables, and refnse of almost every nature, will make a fertilizer of which plants will not soon tire.

Different methods are employed for forming composts, according to the judgment and opinions of different individuals; some preferring a hill-side slope for locating the compost heap to an elevated, level situation.

Dr. Thomas Pollard, late Commissioner of Agriculture in Virginia, gives the following method in his recent report:

"A good plan for a compost heap is to select a gentle slope, and from the place selected for the compost, dig a ditch, say a foot wide and half a foot deep, as long as may be necessary, and sink a keg or barrel at the mouth to catch the drainings. On each side of the compost pile slope the ground with a hoe down to the ditch, so that all the drainings will flow into the ditch; cover the whole bottom where the compost is to be formed with plank and cover the ditch with plank, so that the drainage will get into it and the compost be prevented from clogging it up. To build up the pile straight it will be well to make a temporary barzicade of plank across the ditch about three feet up from the keg. Commence building the compost 
pile against this plank fence. Build up the pile in sections of about six feet to a convenient height, sloping the sides so as to shed rain. Finish each section to the top, sprinkle the ontside with dissolved bone, or if ground tone is used, then with gypsum, and cover with boards or with straw like a stack, unless the compost is made under shelter, as some prefer, to prevent loss of the liquid manure by heavy rains. But this will involve the labor of keeping the pile wet by pouring water over it. When one section is finished proceed to form the next section above and so on. The advantage of finishing off in sections is, that decomposition starts sooner, and by the time the last section is done, the first will be sooner ready.

The constitnents and proportions for the composts must be determined with reference to the crops to be grown, and the soil.

It is particularly important that putrescent manures should be composted, for the quantity can be largely increased with very little or no loss in quality, particularly if phosphoric acid and potash be added. Even without these it would be profitable to compost, for by adding rich dirt, scrapings of fence-comers and ditch-banks or woods' mold, and adding all the hen-manure, hog-manure, urine, soapsuds, etc., from the house, a pile can be made of equal value and much larger than if no compost is attempted, because many things will be saved and added which would be thrown away and neglected. And by adding these things, ammonia, which would escape, will be absorbed and retained, and the fermentation will reach and embrace all the materials in the pile, and 'a little leaven leaveneth the whole lump.' But ground bone or ground phosphate, or these materials in dissolved form, should be added, and potash, if the soils and crops require it. Do not add ashes or lime to the compost unless gypsum is applied at the same time to prevent the ashes driving off the ammonia. It is probable that it is betier not to add lime at all. In making composts, the farmer must study the wants of his different crops and of his soil. If the compost has to be formed in a short time, it will be better to use dissolved bone or phosphate; but if there is sufficient time, then ground bone or phosphate should be used, as the ammonia and other things in the pile will have time to make the phosphoric acid soluble. Observe, we frequently use the term phosphoric acid for the bone or phosphate, in which form it is available to crops. If the dissolved article is used, gypsum will not be necessary. If the ground article is used, then gypsum will be necessary to prevent escape of the arnmonia. Professor Pendleton, using stable-manure as the basis, directs that about the first of January this be removed to an open space, the manure to be chopped and the compost formed by a layer of stable-manure six inches thick, with a good sprinkling of ground phosphate (South Carolina) over it; then a layer of cotton seed (previously saturated with water); then another layer of superphosphate half an inch thick; then a layer of stable-manure, and so on, until the heap is completed, which should be conical in form. (We think the pile had better be flat to catch water enough to promote fermentation and prevent firing:) Over the heap then apply several inches of dry clay soil to prevent the escape of ammonia. But if superphosphate is used this will not be necessary. Professor Pendleton in one place says ground phosphate, and in another superphosphate. This compost is to be put in corn or cotton beds in March and April. As we cannot get cotton-seed, which are rich in nitrogen, then we must substitnte sulphate ammonia or nitrate soda, if we think we have not enough nitrogen for the crop. If we have a clover or pea-fallow, or there is much vegetable matter in the soil, we can do without these. In the cotton states very little grass is raised, and the land is usually clear of vegetable matter. Cotton-seed comes in there very happily, and is very valuable in the production of a cotton crop. In our compost heaps for a tobacco crop we can profitably put in the compost all the tobacco stalks and stems we can get; they should, as far as practicable, be put in before Christmas, to give them time to decompose. So much putrescent manure will not be required in compost with these other materials as when used alone. We may do with ten or fifteen one-horse cart-loads per acre, or less than this if some material, as sulphate ammonia or 
nitrate soda, is added to supply ammonia. In the cotton States they always add cottonseed to the heap, which furnishes as much ammonia, pound for pound, as good, dry stable. manure. Judge Turner, of Hancock county, Georgia, reported to the commissioner of agriculture of that State the following experiment: dissolved bone, applied at the rate of two hundred pounds per acre. yielded only six hundred and seventy-two pounds of cotton per acre, while fifty pounds dissolved bone, seventy-five pounds cotton-seed and seventy-five pounds of stable manure to the acre, yielded one thousand and eight pounds cotton to the acre. This seems a remarkable result for so small a quantity of stable-manure and cottonseed.

When the compost is hauled out, every layer in the heap should be chopped down and well mixed and pulverized. For wheat, it should of course be applied broadcast; for corn, tobacco, potatoes, etc., in the trenches, unless there should be a plenty to apply broadcast."

An agricultural writer from Georgia recommends that a compost, which he has found extremely valuable, be formed according to the following method: "If 360 pounds of cottonseed be well wet and then mixed with four bushels of hard-wood ashes, and permitted to ferment, all the time kept moist, and turned several times, they will form a compost that will saponify the oil in the cotton-seed, break down the hulls, and reduce them to a pulpy mass of most wonderful fertilizing properties. Then mix this mass with one ton of well-rotted stable-manure; let the whole stand three weeks; turn it twice; keep all the time under shelter, and it will be fit for use. I consider this quantity equal to 400 pounds of grano, and think it will bring as large crops as can be gotten from that quantity of guano applied to an acre in the drill.

The practice of composting cotton seeds directly with phosphates and manure is objectionable, judging from my experience. The following are the reasons: The cotton-seeds are made dry and hard, the hulls are not broken up; the benefits from the seeds are not obtained on these accounts. I have noticed the seeds entire two years after using the compost, show. ing they had not given up their valuable properties to plants. The phosphate forms an insoluble fatty acid with the oil in the seed, which coating defies rot and prevents decomposition. To avoid all these inconveniences, I prefer composting the cotton-seed with an alkali, so as to form a soap, as this will break up the hulls, and permit the valuable fertilizing properties of the seed to be readily surrendered to the plant for food. Ashes contain the cheapest alkali for this purpose; 100 pounds of ashes will saponify 300 pounds of cotton-seed, and reduce them to pulp. Hence the above compost is recommended, which has proved emi. nently valuable in my experience."

The fermentation process will commence in a few days after the compost heap is formed, if the weather be moderately warm, which will increase to a certain degree before subsiding. By keeping the mass compact, fermentation can be retarded; water applied will also produce the same effect. The only danger at this stage in the process is, that if the fermentation proceeds too rapidly, some of the most valuable elements, such as ammonia, will escape in the exuding gases and be lost. This, however, can be detected by the odor; and if there be a strong odor similar to ammonia, or what is commonly called "hartshorn," in the vapor that escapes, this proves that these elements are being lost, and the evil will have to be remedied by putting on a covering of wet plaster with a covering of dry earth above it, which is considered the best method of checking it, since wet plaster arrests decomposition as well as retains any ammonia that may have been generated, - or it may be checked by turning on water only.

If the compost pile be made rather late in the autumn, such as the latter part of October in the colder climate, and in December in the warmer, too rapid decomposition might, in a great measure, be avoided by the temperature of the air. It is always a benefit to the com. post thus formed to be shoveled over and mixed before using. The time required to properly 
compost fertilizing materials (which is not done until the materials are all broken up and separated), varies according to the kind and quality of the substances used, the manner in which they are treated, the degree of heat, cold, and moisture to which they are exposed, etc. It is sometimes accomplished in three or four weeks, but often not under six months or a year; some writers say two years, but we think this, as a general rule, is quite too long, though there may be exceptional cases where the materials used would require this length of time. When made, as above described, in the fall, the composted manure will usually be ready for use in the spring.

General Grant states that when in China he was shown a piece of land which had been under cultivation every year for 5,000 years without deterioration of the fertility of the soil. This result is effected by returning to the soil everything taken from it. Fish constitutes a large proportion of the food of the people, and offal not consumed for human food is carefully applied to the soil, and fish is a great fertilizer. Even the roots of the wheat-- which is grown to a very limited extent-are taken and rotted in a compost heap and returned to the soil. All the leaves and garbage are utilized in the same manner. Thus the Chinese have shown us that by returning to the soil the elements extracted from it, deterioration in fertility may be entirely prevented.

Flesh, Blood, Hair, Horns, Hoofs, etc. These substances, when decomposed, afford, in a condensed form, some of the most valuable fertilizing elements, such as nitrogen, phosphate of lime, etc. They are not, however, always procurable by the farmer, but when a loss occurs among the animals of the farm, or if the refuse of a slaughter-house can be obtained and buried in a peat-bed, or rich garden mold, until a perfect decomposition has taken place; or, if put into the compost pile, the fertilizing material thus obtained will well repay the trouble and labor attending it. When buried, a little lime added to the pile before covering will greatly quicken the process of decomposition. It is estimated that a dead cow or horse thus buried and mixed with eight or ten times their weight of soil, will yield from ten to fifteen loads of the richest manure. When the body is cut in pieces and mixed with dry muck or loam, decomposition is hastened and a more uniform mixture produced; but this never should be done if the animal died of a virulent disease. In such a case, the dead animal should be buried deep in the earth and far from any farm building, and never be used as a fertilizer. The compost, in any case, should be made at a distance from the house. Allen says that butchers' offal will give twenty times its weight of more valuable manure than any from the cattle-yard.

Dried blood, when unadulterated, forms a valuable commercial fertilizer. Meat scraps of any kind, fish-offal, shoddy, gelatine, and glue-waste are all valuable sources of nitrogen. Much, however, depends upon their mechanical condition, with respect to their value, such as their fineness, freedom from moisture, etc. Horns, hoofs, clippings from hides, etc., are also rich in organic substances required by plants, when mingled with the soil, but must be very finely pulverized to be available as fertilizers, as their naturally indestructible character prevents them from decaying easily. The horns and hoofs are prepared by first steaming, which renders them soft and pliable like rubber; they are then quickly dried, which makes them very brittle, and are easily ground into a fine powder, which yields, on analysis, over I5 per cent. of zitrogen.

Dried Blood. This fertilizer is one of the most valuable animal substances on account of the nitrogen it furnishes, and its rapid formation of ammonia. The western cities supply largely the markets of the country with this commodity, which has within a few years become to be highly valued in the agricultural world. It is prepared for the market for fertilizing purposes in the following manner: from two to four tons of fresh blood are put into wooden or iron tanks of suitable size, containing double bottoms several inches apart. The upper bottom is perforated with small holes and covered over with a coarse sacking to 
serve as a strainer or filter. These two bottoms of the tank have each a larger opening, which is closed by a long stick fitting into them, and reaching to the surface of the tank which is used to discharge the liquid that has passed through the strainer, after the treat. ment of the blood has been accomplished. A steam-pipe passes down to the filter, which is controlled by a valve. The steam is then turned on and remains so for an hour or more, which heats the blood and changes it into a coagulated mass that collects upon the filter or strainer after the hquid at the bottom has been discharged. I $t$ is afterward dried by steam and pulverized for the market. This substance will contain, when pure, from 10 to 16 per cent. of nitrogen. The amount of nitrogen lost by discharging the liquid is very slight, amounting to only about one-half of one per cent.

A nimal Dust. - This fertilizer is prepared from the blood, meat, scraps, and a part of the bones obtained from large slaughter-houses, some of which, in the vicinity of Boston, are large enough to dispose of three hundred head of cattle and two thousand sheep per day, and the daily production of the fertilizer made from the refuse is equal to about six tons. It is made by the meat scraps and smaller bones being passed through the rendering process to secure the fat; the refuse matter is then mixed with blood and dried by steam heat. When properly dried and ground to a powder, it furnishes a good fertilizer, which is easily decomposed and assimilated by the plants, and is said to be a good substitute for Peruvian guano. Of course, this material varies in its composition and value, and is, like most other commodities in the market, susceptible of adulteration, but the following table from an authentic source, giving the analysis of this article manufactured by a reliable firm, will show about what its average fertilizing properties should be :-

\section{ANIMIAL DUST.}

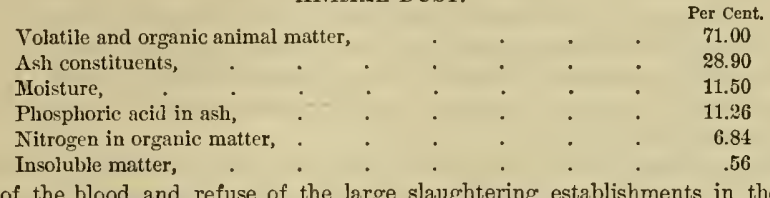

Most of the blood and refuse of the large slaughtering establishments in the Eastern States are used for making this manure.

Cotton Seed, Castor, and Linseed Pomace.-The pomace left after expressing the oil from cotton-seed, linseed, castor beans, etc., is rich in nitrogen, and can be made very profitable for fertilizing the soil, when of a suitable texture, since the finer the texture, the more easily is it decomposed to become available plant-food. Prof. White, of Georgia, in his treatise on the "Complete Analysis of the Cotton-plant," states that the ashes of the hulls of the cotton-seed contain fourteen per cent. of potassium oxide, and seven per cent. of phosphoric acid. Cotton-seed furnishes a valuable ingredient to the compost pile, but when taken whole will become most readily reduced and the hulls brokeu up by first being fermented by having the seeds well wet and mixing with hard-wood ashes, in the proportion of about 360 pounds of seed to four bushels of ashes. This should be kept well wet until thoroughly fermented, as described under the heading of Composts. It is conceded by the best agriculturists, as a general rule, that the waste material of any farm-crop pays best when used upon the same field which served for its production, and that this is especially true in case of the industrial products where a frequent reproduction of the same crop becomes a leading feature of the farm management. In this way, the elements extracted from the soil in the production of certain crops are in a measure restored, and exhaustion of soil prevented.

Fish, Fish Guano, Fish Ponıace, etc.-Fish have long furnished manure for agricultural purposes to this and other countries, and will probably continue to be one of the chief sources of supply for the future, since it is rich in phosphoric acid and nitrogen, two 
valuable elements of plant-food. The aborigines of our country were acquainted with its value as a fertilizer, and were accustomed to place one or two fish in a hill when planting their maize, burying them with the seed, that their decomposition might enrich the soil, by the time the seed had well started in growth. This practice, as well as that of plowing them into the soil, of course, involves much waste, as the slight covering of earth would not be suflicieut to retain the gases produced by the decomposition. Various species of small fish are used for this purpose, the menhaden boing used the most extensively in this country. The best and only proper mode of using them for a fertilizer is to first compost them with dry earth or muck. This should be done by making a compost heap of alternate layers of fish and earth, one above the other'to the desired height, and covering the pile at the top with three or four feet of soil. The pile should have about a foot of earth at the bottom, upon which a layer of fish should be spread from four to five inches dcep; upon this a layer of earth about a foot deep, followed by another layer of fish, and so on till the pile is complete, the top to be covered with a foot of soil. As the fish decomposes, the soil acts as an absorbent and deodorizer, which are so effectual that no annoyance will be occasioned by the escape of any effuvia offensive to the most fastidious olfactories. In a few weeks, if the weather be warm, the pile can be shoveled over and thoroughly mixed with the earth. It may be applied at any time to the soil, but should not be left exposed to the storms, as the rains will leach out its valuable properties; when not intended for immediate use, it should, therefore, be kept under a shed or cover of some kind.

Upon the Atlantic coast, the menhaden fish are used principally for this purpose, being very abundant, and furnish a large portion of our fish guanos. The fish are usually first subjected to a process that extracts a great portion of the oil, and the pomace is then dried and ground, ready for use. The flesh of fish, bke that of all domestic animals, cuntains about fifteen per cent. of nitrogen, and a fish guano that contains the largest amount of nitrogen is, of course, the most valuable. When an undue amount of heat is applied in extracting the oil from the fish, the guano is injured for agricultural purposes in a proportionate degree. Prof. Gocssmann says:

"Nobody familiar with the nature of a good fish guano considers it less efficient for agricultural purposes than any other animal refuse matter of a corresponding percentage of phosphoric acid and nitrogen. In fact, all true guauos, the Peruvian not excepted, owe their most valuable constituents, in a controlling degree, directly or indirectly to the fish. A welldried and finely-ground fish guano is one of our best substitutes for Peruvian guano, and ranks equally high with the best quality of animal dust from our butcher-refuse establishments. It deserves the liberal patronage of farmers wherever a rich nitrogenous phosphate is called for."

In England, an immense amount of fish guano is made from little herrings (clupea sprattus), that are taken in vast quantities off the southern coast of that country.

When the ground material is used, it should be mixed with about twice its bulk of dry earth and usually scattcred broadcast, and oither harrowed in at once or covered by plowing a shallow furrow. When usod in the hill, this mixture should be covered slightly with soil before dropping the seed. The quantity required depends upon the condition of the soil, and the kind of crops to be produced.

Pernvian Gnano.-It is a well-known fact that the deposits of guano upon the Chincha Islands, after furnishing to the world from twelve to fifteen millions of tons of this substance, are nearly exhausted, and that other islands now furnish almost entirely the supply to meet the demand. The Guanape Islands, located about three hundred miles north from the Cnincha Islands, on the coast of Peru, now produce large quantities of it, but will not be 
able to furnish as much as the former, and the quality is not quite equal to that produced by the Chincha, as will be seen from the following analysis of the two varieties compared :-

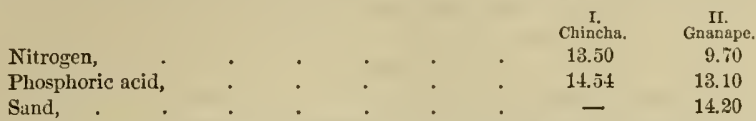

Considerable quantities are also furnished by. the Lobos, Macabi, and Ballistas islands.

The Chincha Islands are peculiarly adapted to furnishing a fine quality of this material, as they are located only about twelve miles from the coast of Peru, in a region where rain never falls, and the air is so dry that the juices of meat will evaporate so rapidly that it can be preserved by drying without salt. Therefore, the accumulations of the excrement deposited there for ages by the sea-birds have never been leached of their valuable fertilizing properties by the rains, and possess the elements suited to plant-growth in an eminent degree. These islands are almost entirely dovoid of vogetation, are very rocky, and covered to an incredible depth with these deposits of the excrement of the sea-fowl, which gather there and feed upon the fish that are so numerous in the waters surrounding their shores. It is said to look in the distance, on approaching the islands, like light-colored hills covered with snow, so great is the amount accumulated. It is, as will be seen by consulting the table giving the analysis of manures on a previous page, very rich in nitrogen and phosphoric acid, and well adapted to most crops and soils. No definite rule can be given with respect to what soils will be most suited to its use, and hence the most benefited by its application, since soils differ so greatly, that this can best be determined by experiment.

If used too long, however, upon any soil without a change to some other manure for a time, the soil will be liable to become exhausted of those elements that it does not largely furnish. This is true of all fertilizers that do not furnish a fair proportion of all the elements of plant-food. It is wonderfully stimulating in its effects when applied to old, exhausted soils. Peruvian guano should not he applied in its pure state to seed of any kind, as it will be liable to injure it. Some farmers recommend mixing it with three or four times its weight in good soil before using. For such crops as grass, wheat, oats, etc., it should be sowed broadcast in the early spring. For corn, potatoes, cotton, beans, peas. etc., the mixture should be placed in each hill, covered slightly with earth, and the seed dropped upon it, thus giving the opportunity of mingling its elements with the soil before the tender rootlets penetrate it. It is best in all cases to apply it in damp weather; in fact, its value is greatly affected by the moisture or dryness of the season whenever used, an unusually dry or wet season being injurious, as the former prevents decomposition, while the latter, in many soils, especially sandy soils, leaches out its most valuable properties, and places them beyond the reach of the plant-roots. The average quantity for use is from two hundred to three hundred pounds per acre. When applied to flowering plants and garden vegetables, it is well to dilute it in water and sprinkle about the roots.

Rectified Peruvian Guallo.-For several years after the introduction of Peruvian guano into the market, it continued to be of uniform quality; after a time, however, it was observed that various admixtures of stones from the underlying rocks, and sand from beach washings, showed that some portions of the supply were becoming exhausted. It also sometimes happened that entire cargoes, or a portion of them, became damaged by sea-water in the transportation, or by being packed in a very damp place. It was decided by parties interested in the guano trade, to endeavor to restore the value thus lost by treating the damaged material with sulphuric acid, by mixing from twenty to twenty-two pounds of the acid with every one hundred pounds of guano when dried, which experiment was found to be very successful, the rectified guano meeting with unusual favor, so much so that when the damaged article was disposed of, the good guanos were subjected to the same process. 
American Guamo.- This name has been applied to the product furnished by the low coral islands in the far western Pacific, and which is inferior to the guanos of the islands on the coast of Peru, owing to its being deposited in a region exposed, to a certain extent, to rains, also moisture from the low character of the surface of the islands, and from being mingled more or less with the soil. These islands are ten in number, the principal being Baker, Howland, Jarvis, Enderbury, and Starbuck. The guanos they furnish differ much from the Peruvian, being free from odor, and resembling brown dust in appearance, while they are mearly destitute of nitrogen, but rich in phosphates. The African and Peruvian guanos are light in color, and have a pungent odor. It is estimated that Baker Island alone contained 160,000 tons before the supply was diminished by transportation, since the inauguration of the guano enterprise. Enderbury also contained about the same amount, while that of the other islands varied in quantity from 20,000 to 50,000 tons. Some of the islands have already been abandoned, their supply being exhausted. As a general rule, a larger quantity of this will be required for most soils than the Peruvian, as it is less rich in fertilizing properties than the latter.

Bat Guano is found in caves in many of the Southern States from Virginia to Texas. It is stated that, before the war, this substance was experimented with for the extraction of nitre, which it was thought to contain in abundance, many of the experiments proving quite successful in obtaining a large per cent. of this compound, but not sufficiently so to warrant continuing the working of it for this purpose. It is, however, thought to be a valuable fertilizing material, most of the samples that have hitherto been analyzed having been found to contain a fair per cent. of organic nitrogen, while many of them contain ammonia and nitrates. In Highland Co., Virginia, also in Tuscumbia, Alabama, these deposits are said to be quite extensive, the latter containing a cave from eighty to ninety feet deep, and alout three miles wide, where it is found in great quantities. The guano furnished from this source has been tried on corn, tobacco, and some other products, with good results. The Commissioner of Agriculture says:

"The continued examination of bat-guano has afforded results confirming those obtained by the former analysis, and proving the wealth of native fertilizing material existing in the South. Indeed, calculations based upon the reported extent of the deposits and the proportion of valuable constituents they are found by analyses to contain, show the aggregate value of this material that may be considered in sight to amount to about $\$ 20,000,000$. Surely Southern cultivators need no urging to induce them to take advantage of these stores of fertility for their poor or exhausted soils."

Dr. Goessmann, of Massachusetts, says the value of bat-guano, as a fertilizer, compares in value favorably with those of the fish products largely manufactured in New England, and even with Peruvian guano. Not having used this substance, or seen the result of its application, we are not able to speak from experience or observation on this subject, but, judging by the estimation placed upon it by those having a knowledge of its use, as well as by the result of chemical analyses, we do not doubt that this product may be utilized to the great advantage of agriculture in many of the Southern States.

Wood Ashes.-Wood ashes are particularly valuable as a fertilizer, containing a large proportion of potash, which is in a condition to act quickly upon the soil in furnishing a stimulating element of plant-food, and are consequently the best of saline manures; they however vary in quality according to the quality of that from which they are produced, ashes from hard wood being more valuable than those from the soft varieties. But any farmer who knows the value of ashes, would not permit to be wasted those from any source; all would be carefully saved and applied to his lands; and when they can bo procured on reasonable terms, it would well repay purchasing them for this purpose. 
Potash is a more lasting manure than some fertilizers, and does not wash so readily from the soil; hence, ashes can be relied upon as a manure of considerable permanence, and have long been, like barn-yard manure, one of the chief dependences of the farmer for feeding his crops. The ashes of seaweed, and plants that grow near the sea, contain large quantities of soda, while bone ashes consist mostly of phosphate of lime.

Ashes are valuable for grass and various other crops, such as grains, potatoes, turnips, clover, lucern, peas, beans, etc. When mixed with bone-dust they are said to be more valuable for all root crops-peas, beans, and clover. They may often supplement the use of stable-manure, but cannot take the place of it, since stable-manure contains all the elements of plant-food, and ashes do not. The quantity to be applied must depend upon the soil and the kind of crop to be cultivater. Soils containing a large amount of potash will require less than those not having a sufficient supply. As a general rule, light soils should have a smaller quantity, and rich, heavy clays a larger supply, but as the constituents of different soils differ so greatly, the reverse will often prove true, and many light and dry soils be benefited to a greater extent by their application than the heavy and damp. Experiment will be the surest guide to the farmer.

As to quantity, we have known some soils to be best benefited by about 50 bushels per acre; others, to be equally benefited by from 10 to 15 bushels, the quantity to be used entirely depending upon the nature of the soil. When used a long time without the application of an equal amount of vegetable or barnyard manure, they will exhaust the soil, manures requiring a rotation, as well as crops.

But they can be applied to grass lands for a longer period without injury to the soil, than to tillaged lands. They should be sown broadcast, and well harrowed in to plowed lands.

Ashes should be kept quite dry until used. They are valuable in reducing bones for fertilizing purposes, and in composts.

Leached Ashes are those from which the potash has been in a great measure extracted in the form of ley, by water, and are composed of carbonate of lime, phosphate of lime, oxide of iron, etc. They will furnish nearly as much phosphoric acid as unleached ashes, but only a small amount of potash; hence, while they are of some value for agricultural purposes, they are not as valuable as the unleached, where potash is needed in the soil. They also furnish a less amount of lime than the unleached.

Coal Ashes have been known to benefit certain heavy soils by rendering them lighter and more porous, but do not in themselves possess much value for fertilizing purposes, as will be seen by reference to the table giving the analyses of various manures on a former page. Their chief value is as a mulch when spread wide and thick enough to smother weeds and grass. They are sometimes useful for spreading around the roots of young fruit-trees, for reasons above given. When used as a fertilizer, and contain many cinders from not having been thoughly burned, they would be injurious to light soils, though they might improve the mechanical condition of such as are heavy and damp.

Soot has long been valued as a top-dressing for cereal crops in the early stage of their growth, and for grasses. It contains ammonia, charcoal, and other desirable fertilizing ingredients. It may be applied at the rate of from 15 to 50 bushels per acre. On light soils, the addition of from 8 to 10 bushels of salt per acre is said to increase its good effect. Like ashes, its best effeet is not appreciable in a very dry season, moist weather being most desirable for the application of either. It is often used mixed with absorbents like earth, marl, or muck, which aid in preventing the escape of ammonia. 1t may be sown broadcast or in trenches, and harrowed in. The mixture of salt and soot is said to be the most powerful of all manures for carrots. 
Charcoal Dust, when applied as a top-dressing to lands, serves to condense anmonia, nitric acid, and the gases of the atmosphere. It has an extraordinary capacity for absorbing gases, and is said by reliable authority to absorb ninety times its bulk of ammoniacal gas. It renders soils warmer by absorbing the sun's rays, and also serves to check rust in wheat, and mildew in other crops.

Peat is used for agricultural purposes-principally for making composts, and as an absorbent of other manures. Dried peat is valuable for absorbing the liquid manures of sta. bles. As it contains from 75 to 90 per cent. of water in its natural state, it should be dug and exposed to the atmosphere several months before being used for this purpose.

It varies in quality according as it is mixed more or less with the soil, some being much more rich in ammonia than others.

Peat Ashes were at one time highly recommended for their value as a manure, both when applied alone, and as an absorbent of night-soil, sewage-water, the liquid manure of stables, etc.

It should first be dried thoroughly. It has been proved by Dr. Anderson of Scotland that peat, when well dried, is a better absorbent and retainer of ammonia than after it has been charred.

In reclaiming peat soils farmers frequently burn the peat and spread the ashes over the beds from which the peat was taken.

Sea-Weed.-To those farmers living within convenient distance of the sea-shore, a valuable manure can be obtained from the drifted sea-weed. It is often thrown upon the sandy coast by the waves in large quantities, and can easily be raked up and carried away after the tide has receded.

It is frequently used as a bedding for cattle, and makes a valuable addition to the compost heap. In England it is frequently applied as a top.dressing to grass and clover. It may be plowed into the soil in the spring with a shallow furrow, three or four inches, and is valu. able for most crops, especially grain, which it is said to produce of a very fine quality. It is also said to be highly valuable in the cultivation of potatoes. Large quantities are recom. mended in using.

Ashes of Sea-Weed.-As sea-weed is quite bulky, and is sometimes inconvenient to remove to a distance, it is often burned where gathered, and the ashes saved for agricultural purposes. These ashes contain soda, potash, and other fertilizing substances. The ashes of sea-weed are known under the names of kelp, barilla, and varec. In Sicily, Spain, and some other countries, the ashes of marine plants, called barilla, were used for making soap. Kelp and varec are terms applied to the ashes of sea-weed made from the algæ and fuci species, which grow upon rocks in great abundance on a large portion of the coast of Great Britain and some other countries. The sea-weeds are dried and burned in large ovens, twenty-four tons of the sea-weed being required to produce one ton of kelp. This is at present the principal material from which iodine is obtained. The name for sea-weed ashes issued in France is varec.

From the above it will be readily seen that sea-weed ashes have alkaline properties that may be valuable as a fertilizer of the soil.

Marl is soil containing clay, carbonate of lime, sand, and sometimes sulphate and phos. phate of lime, differing in its composition in different localities, some containing more or less of these ingredients than others. It is often applied to lands to increase their fertility, the lime it contains being the most essential element. When it is found in the proximity of, or lying under sandy or peaty soils, its application in large quantities is attended with the best effects. 
It is adapted to all soils that have not already a sufficient supply of lime. It is especially adapted to grass and clover. The clay mari produces the best effect on light, sandy soils, and the sandy marl on clay and heavy soils. It is better to be well exposed to the sun and air before applying it to any kind of soil. The green sand marl, of which there are extensive beds in New Jersey, contains a large amount of potash, besides lime, magnesia, sulphuric acid, phosphoric acid, silica, and various other substances, and is a valuable fertilizer. It has long been a staple manure in that State, where it occurs in three distinct deposits, - the upper, middle, and lower marl beds, which stretch across the State from the High. lands of Navesink, near Sandy Hook, to the Delaware River, below Wilmington, and in many localities is easily excavated. It seems especially adapted to the use of vegetation as plant. food, its application having a speedy effect. The result of its use is seen in a remarkable degree in the improvement of the light, sandy soils of Eastern New Jersey, where large tracts of sandy and almost worthless lands have been transformed into a garden. It must be applied in large quantities, often several tons to the acre, in order to obtain the best results, and then furnishes potash and other fertilizing properties with a considerable degree of permanency.

Lime.-Lime is manufactured from himestone, marble, and shells, by burning or subjecting them to intense heat, which expels the carbonic acid. After this acid has been driven off by calcination, it has great affinity for water and carbonic acid, and readily combines with both, on exposure to either water, earth, or the atmosphere; in the latter case it is said to be "air-slacked."

With respect to the manner and extent of benefiting soils by the application of lime, there exists among the farmers of the present day a difference of opinion, some claiming it to be of no appreciable value whatever; others, that it acts, not only as a fertilizer, but by its chemical action upon some of the constituent elements of the soil, renders them more available as plant-food; in other words; it unlocks the stores of rich plant-food, which by the mechanical condition of the soil are locked up beyond the reach of the plant-roots, and hence are useless to them.

Lime enters into the composition of nearly all crops, and when not found in sufficient quantities in the soil, will prove a valuable fertilizer; but most soils contain in themselves a fair proportion of lime for the production of most crops, yet, as has been previously stated, the quantity sufficient for the present requirements of the plant, may not be available; hence experiment alone, as is the case with most fertilizers, will best prove to the farmer whether his lands will be benefited by its use or not. Its application hastens the decay of vegetable substances contained in the soil, and converts them into available plant-food, and by its chemical action upon the coarser particles of the soil, causes them to become broken up and, in a measure, pulverized, thus setting free the mineral properties therein contained, and which are so essential to vegetation. When not contained in any soil in sufficient quantity, it can be applied separately, or combined with sulphuric acid. in the form of gypsum or plas. ter; and since these two ingredients-lime and sulphuric acid-wash out of the soil more readily and freely than most of the other constituents, it must be frequently applied to make up the constant loss of these elements, and keep the soil supplied with the requisite amount.

A farmer of large experience says, he knows of a farm that has not had a bushel of lime applied to it in twenty years, and it is now infested with sorrel; previous to this time, lime was used upon it, and it was very productive; he draws the natural conclusion, that lime would prove a remedy for the evil. We think he argues correctly, and that all such lands as will produce sorrel and other weeds better than the common farming products, will be greatly improved by the application of lime, which corrects the acidity of the soil, and ren. ders it suited to the production of more valuable crops. It also is very beneficial in destroy. ing many noxious insects and worms that injure the roots of plants. Allen says of it:- 
"Lime, next to ashes, either as a carbonate or sulphate, has been instrumental in the improvement of our soils beyond any other saline manures. Like ashes, too, its application is beneficial to every soil, not already sufficiently charged with it. It makes heavy land lighter, and light land heavier; it gives adbesiveness to creeping sands or leachy gravel, and comparative openness and porosity to tenacious clays; and it has a permanently beneficial effect, where generally used, in disinfecting the atmosphere of any noxious vapors existing in it. It does not condense and retain the organic matters brought into contact with it by the air and rains, but it has the better effect of converting the insoluble matters in the soil into available food for plants. It has proved in many instances the wand of Midas, changing everything it touched into gold. It is the key to the strong box of the farmer, securely locking up his treasure till demanded for his own use, and yielding it profusely to his demands whenever required. In its influence in drying the land, and accelerating the growth of plants, the use of hime is equivalent to an increase of temperature; and the farmer sometimes experiences, in effect, the same benefit from it, as if lis land were removed a degree or two to the south. The influence of lime in resuscitating soils after they have been exhausted has been frequent and striking; and it may be stated as an incontrovertible truth, that wherever procurable at low prices, lime is one of the most economical and efficient agents in securing fertility within the farmer's reach.

It has been falsely said to be an exhauster of soils; that it enriches the fathers and imporerishes the sons. So far as it gives the occupant of the land the control over its latent fertility, this is true; but if he squanders the rich products when within his reach, it will be his own fault. Lime gives him the power of exhausting his principal; if he uses aught beyond the interest, his prodigality is chargeable to his own folly, not to the liberality of his agent."

Lime made from shells is the most valuable. Our cultivated crops contain on the aver. age about as much ime as potash.

Lime should be slacked before being applied, except when used to decompose the organic substances in the soil; it can then be applied without slacking, but in any case, while it is nec. essary that it should be thoroughly mixed with the earth, it should be kept near the surface, as it is liable to sink into the soil. In whatever way it is applied, it is well to remember that the carbonic acid which has been expelled from it by the heat is quickly regained from the atmosphere, and it should, in consequence of this, be as little exposed to the air as possible before being applied to the land. It should be put upon the land as soon as practicable after slacking. Some farmers pile it in heaps upon the land, and by mixing it with water apply it at once, and harrow it in. Another method is to place it in large piles and cover it thickly with earth and leave it to gradually slack. In this case there is danger from loss by drench. ing rains, unless the pile be well-protected to prevent washing. Some favor a compost of earth, lime, and salt, in the proportion of one part of salt to three or four parts of lime. It is better to apply lime in small quantities, and do so frequently, than to make a large application of it at longer intervals, from ten to fifteen bushels being considered by good judges a fair allowance for ordinary soils, though we have known 100 or more bushels per acre to be used. Bywatching the effect of a small application, the farmer will be able to judge whether his lands require it in larger quantities, or whether its use on bis particular soil be at all beneficial, since there is scarcely anything that is so dependent upon repeated experi. ment, and so independent of all definite rules, as the fertilizing department of agriculture, owing to the great diversity of soils, the difference in their mechanical condition, and various other causes. white.

Lime is particularly valuable in the cultivation of tobacco, causing the ashes to be very 
Ground Limestoue and Shells are sometimes applied as a fertilizer, and are similar in effect to old air-slacked lime, or shell-marl, being a carbonate of lime in their principal composition.

Its action is more speedy when ground fine than when coarsely ground. It is useful on such soils as are benefited by the application of lime. Ground shells are also sometimes used as a fertilizer. They contain carbonate and phosphate of lime, and often some animal matter. It is better to burn them first, as they are then converted into nearly pure lime; besides, this is the easier and cheaper method of preparing shells for nse.

Gas Lime.-Lime from gas.works is said, by competent writers, to be for agricultural purposes about equal in value to common lime.

It is the refuse lime that has been used in the manufacturing of gas, and consists mainly of carbonate of lime, some sulphur, tar, and sulphate of lime, and must be applied more cautiously or in more moderate quantities than common lime. It is safer to apply it after a few weeks' exposure to the air, as it would be liable to poison vegetation if used fresh from the gas works. It is very important that it be well pulverized, that it may be evenly spread upon the land It may be applied to grass or plowed lands for wheat or other crops, and should in the latter ease be well harrowed in. Some farmers pile it in small heaps in the fall or winter, and spread it in the spring. When designed for plowed crops, it wonld be well to spread it upon the soil late in the fall or winter, that it may be exposed to the air before being harrowed in. It is also valuable for the compost heap.

Its acrid qualities are said to render it valuable as an insect-repeller, and, when strewn sparingly over young turnip-plants, to repel the turnip-fly. Some writers recommend its use at the rate of 25 bushels per acre, but we think it would be well to experiment cautiously with a smaller quantity at first.

Bones contain a large proportion of phosphate of lime and other substances which ren. der them valuable for fertilizing purposes. There is, in fact, no ingredient of the bone that is not useful to vegetation, and it is well adapted to almost all soils and nearly all agricul tural products, especially so to the various kinds of grains, to potatoes, turnips, clovers, and most garden vegetables. Since bones decompose so slowly, one of their chief factors of value in agricultnre is the fineness to which they are reduced before applying, since the finer they are ground the more readily will the plants be able to appropriate them as plant-food.

Bone is especially valuable for fruit-trees. Many farmers use bones to place under the roots of grape-vines and fruit-trees when setting them, putting a peck or more of old bones in the bottom of the place dug for inserting the roots. This will give something for the rootlets to feed upon for years, as they become slowly decomposed. and will be of great value in promoting the future growth and vigor of the trees or grape-vines. As bone-dust is rather slow in decomposing, it is recommended by many writers on agriculture to apply it in autumn or early winter, thus giving the rains and melting snows an opportunity to act upon it, and wash it among the roots of plants. It may be sown broadcast at the rate of from five hundred ponnds to a half ton or more per acre, according to the nature and needs of the soil. In England, the enormous quantity of a ton and a half of bone manure per acre is often applied to pastures of old clay soil in keeping them productive; and English writers on the subject state that there is nothing equal to it, either in the permanency of its effects, or in the production of a sweet, luxurious herbage, of which the cattle are fond.

Pulrerizing and Reducing Bones.-There are varions methods of pulverizing or reducing bones for agricultural purposes. Grinding is the best process when a mill is accessible for that purpose, and the finer they are ground the better. When grinding is impossible, for lack of a suitable mill, many farmers reduce them by the nse of ashes and liquid manure, by first breaking the bones, as well as may be, with a sledge-hammer, and then 
mixing them in a large barrel or hogshead, in alternate layers, with wood ashes, the layers to be each thrce or four inches deep. When the bones are thus disposed of, wet the whole with urine from the cattle or horse stable (which can easily be saved by placing a tank under the stable-floor) - there should be a sufficient quantity of the liquid manure to wet the whole mass thoroughly. After fermenting a few weeks, the bones will be sufficiently soft to be easily crushed with the shovel, and they can then be mixed with dry earth, road-dust, muck, or any other dry absorbent, and are ready for use.

Some use water only, first making a hole in the bottom of the barrel and covering it with straw for a filter, placing a tank underneath to receive the lye. As the lye accumulates in the tank, it is again poured into the barrel. This is to be continued daily, with perhaps occasionally a little addition of water to restore the quantity lost by evaporation. A month or two of warm weather, it is said, will render the bones soft and ready to be mixed for use. The addition of a little caustic potash of commerce will hasten the operation and give increased value to the manure. The use of liquid manure is preferable to water for this purpose.

A recent writer gives the following method of reducing bones with lye:

"Where unleached ashes are easily obtained, bones can be reduced to a very fine state by boiling them in strong lye. This eats them up with great rapidity. We have reduced a half ton of them in a day with two four-barrel kettles. The fine bone must be frequently taken from the bottom of the kettle with a shovel made of the proper shape, to keep it from burning on the bottom. When the bone is reduced to a fine state in this way, it is not all soluble, as in the case of being reduced with acid, but it is so fine that it becomes soluble in a short time, by the action of the air and soil. When the wood-ashes can be obtained, this is an excellent way to reduce bones, as it may be considered very cheap, since the potash or lye is all saved in the manure, and is worth all it costs. The leached ashes are also valuable as a manure, and the only expense that can properly be counted in reducing the bones by this method is the labor. Although the fine bone reduced with lye is not so soluble as that dissolved in sulphuric acid, yet it is more rapidly dissolved by the action of air and soil than fine ground bone. Dry earth is excellent to mix with this lye-reduced bone, if it is to be applied in the hill to potatoes or corn; but if it is to be applied through a drill with grain, or corn planted with drill, it must be dried with some dry, fine sifted material, such as coal-ashes, or dried and sifted clay, lime, or land-plaster.

We have found this the best application for potatoes. We might expect this result, as the ash of the potato is 59 per cent. potash and 20 per cent. phosphoric acid, and these two most important elements are the principal ones in this manure. We have also found it successful upon wheat, oats, rye and grass."

It is a custom with the German farmers to decompose even the ground bone, before applying it to the soil; for this purpose they mix it with sawdust or dried peat, and pour over it liquid manure or water, letting it stand in piles about a week to ferment, then turn it over and mix it with earth and put it upon the field, where it is either plowed under quite shallow, or harrowed under rather deeply. It is often composted with manure to advantage, the bone-dust and manure acting and re-acting upon each other in the process of fermentation, producing a valuable fertilizer.

Bones can be pulverized by burning, but this drives off the organic matter, which contains nitrogen, and hence greatly diminishes their value; it leaves, however, the phosphate of lime, which is a very rich fertilizer.

A very good manure can be made by the use of sulphuric acid in reducing bones, but it must be used with the greatest caution, as it will make the hands sore, and spoil clothing, if they come in contact with it.

Old bones tliat have been lying about the farm for a long time exposed to all kinds of 
weather, are harder to reduce than fresh bones containing gelatine. As a general thing, it will be found more satisfactory to purchase ground bone from the manufacturers, who have every facility for putting them in a condition favorable and convenient for use. The only objection is, that this material is often adulterated by mixing something of inferior value with it, and the fraud is difficult of detection except by chemical analysis, which will neither be convenient nor economical for the farmer to have done. The best safeguard will be to deal only with manufacturers who have a good reputation for honesty in producing a pure article.

Mineral phosphates, as well as bones, furnish a supply of phosphoric acid to the agricultural world. They are found in South Carolina in great abundance, and are sometimes called phosphate marls. They are supposed by geologists to be copro'ites, or the excrementitious remains of fossil animals, that seem to have congregated in great numbers in this region. They are reduced with sulphuric acid, and are considered among the best of fertilizers.

Gypsum, Plaster, or Sulphate of Lime.-The pure gypsum, when ground and subjected to the proper degree of heat, constitutes what is called "Plaster of Paris," which is so extensively used for stucco, hard-finish, and for various purposes in ornamental art.

The most celebrated gypsum-beds are those of Nontmartre, near Paris. In this country gypsum is found in Nova Scotia, New York, Virginia, Tennessee, Michigan, and various other localities. It is valuable as a fertilizer, containing lime, combined with sulphuric acid, and a few other substances.

The pure gypsum is usually white, but the ground land-plaster is white or light gray, and sometimes of a dark gray, like that quarried in central New York. Its agricultural value depends upon its amount of sulphate of lime principally, and cannot be judged of accurately by its color or appearance after being ground.

The use of gypsum is attended with great benefit to certain soils and crops, being best adapted to sandy, loamy, or clay soils, thongh requiring a larger application to such soils as contain a considerable amount of vegetable matter. Its use is especially beneficial to the production of the red and white clovers, grass, lucern, sainfoin, peas, beans, etc. It has been found, by the experience of most farmers, that in the use of plaster, as with wood-ashes and some other fortilizers, that the season has much to do with the benefits derived from its application; that gypsum requires a large amount of water, and when the season is very dry, the benefits resulting, even upon the same field, are greatly inferior to those of a moist season; also, another noticeable fact in its use is, that it almost always shows better results upon the north and northeastern slopes of hills, than upon the southern. This can be accounted for only in the fact that the northern slopes of hills are longer in the shade than the southern, and usually have a heavier deposit of dew; hence the soil there would contain more moisture than the southern slopes, proving the necessity of a sufficient supply of moisture in attaining the best results.

Plaster is a great absorbent, and its efficiency is supposed to be in a measure attributable to its power of absorbing and retaining ammonia, and furnishing it to plants to which it is applied, as they have need. It is a very good absorbent to apply to the manure-heap, compost-pile, and privy vaults, not only acting as a disinfectant, but, by thus mixing it with ammonical manures, a sulphate of ammonia is obtained which is less volatile than carbonate of ammonia, the natural product of these substances.

When applied to the soil, the sulphate of ammonia more readily parts with its fertilizing properties than the carbonate form, which effect is of course modified by the amount of moisture in the soil.

Liebig says on this subject: "Soluble sulphate of ammonia and carbonate of lime are formed, and this salt of ammonia, possessing no volatility, is consequently retained in the soil. All the gypsum gradually disappears, but its action upon the carbonate of ammonia continues as long as a trace of it exists." 
Gypsum seems to produce very different effects, as we have previously stated, on different soils, and in different seasons. Sometimes its results are astonishing in its prompt effect in increasing the yield of crops, as in the case of Dr. Franklin (mentioned in the chapter on clover), whose name, traced by its application to a clover-field, could be distinctly seen from a long distance, by the darker green and more luxuriant growth than the other portion of the field. In other cases its application seems to have no effect whatever. It is often a powerful stimulant to old worn-out lands, as it seems to set free the potash and phosphoric acid in the soil; but it must be used with judicious care, for, if applied too long continuously upon any soil, such lands will become exhausted and cease to produce crops worth cultivating; they will become what is sometimes termed "plaster sick," which is simply a starvation of the soil for want of a greater variety of food, as it furnishes only a part of the elements of plant-food.

Plaster, from its own constituents and by absorption of ammonia from the air, can furnish only two of the many inorganic elements which enter into the composition of plants. If its use conld be followed by clover, as a green manure every three or four years, thus restoring in a measure the elements extracted by its stimulating effects, such soils would be greatly benefited, and its use in this manner could be continued for a long time with no injurious effects.

The necessity of moisture in obtaining favorable results in its use will be apparent when we remember that it requires over 400 parts of water to dissolve one part of gypsum.

For corn, potatoes, and crops cultivated in a similar manner, it is usually put in the hill at the time of planting the seed, though some sprinkle it upon the plants after the crops have been hoed the first time, and when they are wet with dew or rain. For many crops, such as grass, clover, grains, etc., it is sown broadcast, which can be done with almost any broadcast seed-sower.

Experiment alone can determine definitely to what soils its use is best adapted, and the quantity to be applied. Some English writers mention two or three hundred pounds per acre as a proper amount, but American writers generally do not recommend over one hundred or one hundred and fifty pounds per acre. When deposited with the seed by the grain-drill, it should be mixed with three or four times its quantity of soil, otherwise the germ of the seed might be injured.

Salt (Chloride of Sodium).-The use of common salt as a fertilizer of the soil is of remote date. In China and Hindostan, the rice-fields have been kept in fertility for ages by applying sea-water. Its use has also been long known in various portions of Europe. It supplies plants with chlorine and soda, but its principal value as a fertilizer is in its chemical action upon other elements of plant-food in the soil, which it renders more available. It also is a valuable agent in exterminating grubs, worms, and other vermin from the soil, being very beneficial in the culture of garden vegetables, for this reason. It has a tendency to keep the land cool and moist, and thus neutralizes drouth, and is thought by many farmers to glaze and stiffen the straw of grains, and prevent crinkling and rust. Salt mixed with wood-ashes, in the proportion of one part of salt to four of ashes, and applied at the rate of a handful to the center of each hill of corn immediately after planting, is said by good authority to supersede the use of scare-crows and coal-tar, as no worm or crow will touch it; besides, the fertilizing properties of the compound are highly beneficial. When sown on wheat, it is said to destroy the chinch-bug, so destructive to that crop. To lands near the sea-coast and that occasionally receive a supply of salt from the spray that is carried to them by the ocean storms, it usually produces no apparent effect, as they are already supplied with a sufficient quantity of this element. It is a fertilizer that must be used with care, for if too large $\varepsilon$ quantity be applied, it is liable to injure vegetation. When applied in contact with seed, as is sometimes done with grain-drills, only a small quantity should be used, as it may destroy 
the germ. It will destroy the eyes of potatoes, when put in contact with them in the hills at planting. A western farmer and writer states that he has never seen any damage done the wheat-crop by the chinch-bug where there had been two hundred pounds of salt to the acre, sown broadcast, and that the best time for sowing it is when the wheat is about four inches high. The refuse salt from packing-houses is often used for this purpose. Prof. Whitney states that there is no manurial substance of less reliability than salt, for the reason that its effects depend not only upon the kind of crops raised, but upon the character of the land itself, and in no less degree on the other fertilizers with which it may be used. On sandy soils, devoted to the growth of the mangold-wurtzel, the results of its use are often marvelous; under these conditions its application is very common and profitable in England. It rarely benefits stiff clay soils, as it renders them too wet. It is thought by most farmers to be adapted to light soils, such as a light sand or loam; also to those soils that are rich in organic matter, and which contain considerable humus. It is not, however, of advantage to apply it to very loose, sandy soils, or what are termed gravelly soils.

According to Dr Voelcker, the good effect of applying salt is in its power of liberating the ammonia from soils which have been highly manured with decomposed stable manure, and that it is most beneficial when applied to light land after a good dressing of this manure, either alone or with Peruvian guano. English writers generally favor its use in combination with some other fertilizer, such as lime, soot, nitrate of soda, etc. The following are the results of Dr. Voelcker's experiments :-

On a sandy soil, containing only a moderate quantity of soda and potash, and with a deficiency of lime, from a given area, without the use of salt, there was obtained 12 tons, $2 \mathrm{cwt}$., and 76 pounds of mangolds. When salt had been applied at the rate of two hundred pounds per acre, 18 tons, $19 \mathrm{cwt}$., and 93 pounds were produced, showing an increase per acre, from the use of salt, of 58 tons, $14 \mathrm{cwt}$., and 20 pounds. Three hundred pounds of salt per acre were applied, and the increase was slightly less than the preceding. On increasing the amount of salt to eight hundred pounds per acre, the yield was $21 \mathrm{tons}, 18 \mathrm{cwt}$., and 84 pounds, or an increase of over nine tons as compared with the area that was unmanured with salt. From one hundred to two hundred pounds of salt per acre are often beneficial to pasture-lands ; cattle and sheep greatly relish such grass. When applied to coarse grasses, it has a tendency to greatly improve the quality of the herbage.

The quantity of salt to be applied must be determined, in the main, by experiment, as well as the kind of soil to which it is adapted. It would be well for the farmer to apply it to a portion of his field, and carefully note the result. Some writers speak of using at tho rat : of two bushels per acre with good effect; but it seems to us that this is an unusually large quantity; four hundred pounds is about the quantity generally recommended by Engli ' 1 authorities, while many agriculturists of our own country recommend two hundred or three hundred pounds per acre as a sufficient quantity. The quantity required will, of course, depend upon the nature of the soil, the same as with all other fertilizers.

Potash-Sources of Supply-The Stassfurt Potasl Mines, ete.-Since potash is such an essential element in plant-production, and consequently an element greatly needed in the soil, where it is rarely found in sufficient quantities, it is a question of no small import to the farmer as to how this necessary substance can be obtained. Wood-ashes are valuable in this respect, but cannot always be procured in sufficient quantities, or at is rate to render their use an economical fertilizer. Very fortunately for the agricultural interests of the world, an immense deposit of the salts produced from the evaporation of sea-water, and containing a number of compounds, including potassium, has been discovered in northern Germany, which is supposed to be the remains of an ancient sea. An all-wise and kind Providence has thus provided for the wants of man in this respect, as in all others, and has left them there for him to discover and apply to use through the patient labor of his hand 
and brain. These famous German potash mines are located near the Hartz Mountains, partly in Prussia and partly in the small Duchy of Anhalt. These include the mines of Stassfurt proper, the contiguous mines at Leopoldshall, and those more recently opened about ten miles distant at Westeregeln. According to the best authority, the deposits of this region consist chiefly of the various compounds of sodium, potassium, magnesium, and lime, with sulphuric acid, and chlorine. The larger proportion, however, consists of chloride of sodium, or rock-salt, which seems to be stored there in an inexhaustible supply. Lying on the top of the rock-salt deposit are various mineral salts, such as kieserite, polyhalite, kainite, carnallite, etc. The total area of this salt-basin is supposed to be about six hundred square miles; the upper surface of the deposit at Stassfurt being from 350 to 830 feet from the surface. It is stated that in the year 1856 the miners penetrated to the depth of 1,856 feet, finding pure rock-salt in a seemingly inexhanstible supply as they bored down, and that they then said, "We have made the depth equal to the date; the salt layer seems to be as immeasurable as the centuries. Let up stop." Prof. Atwater says of these mines:-

"It is a comparatively few years since the deposits were discovered, but the products hare come into very general use in Germany and other parts of Europe, and are being imported quite largely into this country. When rightly used on soils deficient in potash, they are very profitable fertilizers. A vast amount of experimenting has been done with them, more in Germany than anywhere else. The results indicate that the usefulness of these salts as fertilizers depends not only upon the character of the salts themselves, of which there are various grades, but also on the kind of soil, the mode of application, and the kind of crop.

Composition of German Potash Salts.-The potash salts, as taken from the mines, contain only small proportions of potassium compounds, the bulk consisting of materials which have comparatively little agricultural value, and are sometimes positively injurious. In the early history of the Stassfurt potash industry, many experiments were made with these crude salts, but, with the exception of the 'Kainit,' their use has been attended perhaps oftener by failure than by success. It has been found necessary to subject them to chemical treatment, by which the potash compounds are more or less completely purified. This is done at factories near the mines, where immense quantities of the potash salts are manufactured for technical and agricultural uses. As prepared for market, the potash fertilizers contain potas. sium in the form of either chloride of potassium or sulphate of potash, and, along with these, chloride of sodium, chloride of magnesium, sulphate of soda, and sulphate of magnesia, and small quantities of other materials.

The basis of potash compounds is the element Potassium. This, combined with oxygen, forms potassium oxide, or potassa, or, in familiar language, potash. The same term, potash, is applied to two other potassium compounds. One, which may be considered as a compound made up of potassa and water, is known in the chemical laboratory as potassium hydrate, and, in common language, as caustic potash. The other is a compound of potassa with carbonic acid, and is commonly called carbonate of potash. The ordinary potashes and pearl. ashes, prepared from the lye of wood-ashes, are more or less impure carbonate of potash. Potassium, combined with chlorine, forms potassium chloride, commonly called chloride of potassium, or 'muriate of potash.' Potassa united with sulphuric acid forms potassium sul. phate, or sulphate of potash. By adding to ordinary potash lye from ashes, a proper amount of sulphuric acid, and boiling the liquid down, we might, with proper care, obtain a solid substance, which would be a sulphate of potash. If we were to use hydro chloric (muriatic) acid instead of sulphuric, we should obtain a chloride of potassium, or muriate of potash. Other elements, as well as potassium, combine with sulphuric acid and chlorine to form sulphates and chlorides. Sodium chloride is common salt. Sulphate of soda (sodium sul phate) is known as Glauber's salt, and sulphate of magnesia as Epsom salts. The German potash salts consist of potassium chloride and sulphate, mingled with more or less of the just. 
named compounds. It is customary to reckon the potassium of these salts as 'actual potash.' In the sulphates this term expresses the amount of potassium oxide, potassa, or potash present. In the muriates it represents the amount of potash which the potassium would make if it were combined with oxygen instead of chlorine-100 lbs. of pure sulphate of potash contains about 54 lbs. of 'actual potash.' $100 \mathrm{lbs}$. of sulphate of potash are therefore said to be equivalent to $54 \mathrm{lbs}$. of actual potash, and vice versa, $54 \mathrm{lbs}$. of potash in the sulphates are reckoned as equivalent to $100 \mathrm{lbs}$. of sulphate of potash. In the 'muriates,' $100 \mathrm{lbs}$. of chloride of potassium are reckoned equivalent to about $63 \mathrm{lbs}$ of potash, and vice versa.

How to use Potash Salts as Fertilizers.--"The results of experiments in differ. ent places are so varying that it is impossible to lay down precise rules to apply to all cases. The potash salts sometimes bring a remarkable increase of crop, and sometimes do no good at all. Whether a given soil is deficient in potash or not, can be best told by actual experiment. In general, potash is most likely to be lacking in light, sandy, and calcareous soils, and in those which consist largely of decayed vegetable matter, like peat and muck beds and moors. It is more apt to be plenty in loamy and in clayey soils that come from what the geologists call igneous rocks, which, like the trap rocks of our region, and the granites, syenites, and other rocks that contain felspar and mica, are rich in potash. But in soils of either of these classes the available supply may be reduced by cropping. Where guanos, phosphates, bone, fish-manures, and other fertilizers rich in nitrogen, phosphoric acid and lime, lut furnishing little or no potash and magnesia, do not bring as large returns as formerly. a trial of potash salts is to be recommended.

'the potash salts have proven especially beneficial for fodder crops, like clover, grass, and rye; for leguminous crops, such as beans and peas, and vetches; and for potatoes, roots, tobacco, and fruits. The immediate effects upon grain crops are ustally not so beneficial. When applied shortly before putting in the seed, the result is often unsatisfactory, especially as regards the quality of the grain. For tobacco they have proved highly beneficial, and there is reason to presume they might do the same for corn. They have been found remarkably useful in some cases for fruits, especially grapes, and their use for our failing orchards and other fruit-trees is wortliy, at least, of trial.

In fact, the question whether it will pay to use potash salts in any given case or not, is one that cannot be decided in advance. Chemists can not give prescriptions for your failing soils as doctors do for your diseased bodies. You must settle such questions yourselves by actual trial. I should therefore by no means advise any one to invest in large quantities of these salts before he had found whether they were going to benefit him enough to make it profitable or not.

As I have said, the high grades will, generally speaking, be best for our use. The chlorides are the cheapest, because they are produced at less cost from the crude salts; but for such crops as potatoes and tobacco, the sulphates are safer. The general effect of the chlorides is to increase the quantity rather than to improve the quality of these crops. They are apt to make the potatoes soggy, and to injure the burning quality of tobacco. So if you want your potatoes mealy, use the sulphates; and if you grow tobacco, and would have it good, use the sulphates. The muriates are apt to injure the burning quality of the leaf, giving it a tendency to char and hold fire but a short time, which is not the case with the sulphates.

For fodder crops, like clover, grass, and corn, and for grain, and, indeed, for any of the crops we raise in this part of the country, except the ones just mentioned, the chlorides . may be used to advantage. I have lately noticed some statements, by the way, that the use of the chlorides for sugar-beets is rather on the increase in Germany, the effect on the percentages of sugar and of objectionable materials in the juice not being so bad as had formerly been supposed.

The method of applying potash salts is a matter of great importance. Cases are very 
common-I have known several myself-where crops were injured or destroyed by improper application. The great point is to have the material uniformly diffused through the soil, so as to be within as ready reach of as many of the roots as possible, and not to be concentrated in single places in such quantity as to injure the plants. The best way to secure this distribution is to apply it some time before the seed is put in. For a crop to be sown in the spring, it is well to put the potash fertilizers on in the fall, so that the water from rains and melting snows may have opportunity to carry the potash down into the soil, and thus secure a deep and uniform distribution before it is wanted for the growing crops. This is a particularly good plan with the chlorides, and with the low grade salts, if the latter are used. The chlo. rine is gradually leached down into the lower strata of the soil, and away beyond where it will do harm. There is no reason to fear loss of potash or magnesia in this way, since they are retained by the absorptive power of the soil, and do not get beyond reach of the roots. The only ingredients thus exposed to loss are the soda and chlorine, which have very little value. There may, perhaps, be danger that the chlorine, in leaching out, will take lime with it from the soil, but the loss would probably be of little practical moment. If, however, you cannot apply your potash salts in fall or early spring, the next best plan is to mix them with three or four times their bulk of earth, spread the mixture uniformly, and either plow under or harrow in. Another most excellent way is to mix the salts ${ }^{\circ}$ with stable manure, by spreading them on the heap from time to time as it accumulates. For clover or grass lands, where the potash salts are to be used as a top-dressing, a very good plan is to compost and apply as long as possible before the growth of the crop begins.

It is especially advisable to apply the potash compounds-not alone, but mixed with phosphates and nitrogenous fertilizers. In this way the best practical results have been obtained. Peruvian guano, ammoniated superphosphates, bone and fish, furnish nitrogen, phosphoric acid and lime, and, if superphosphated, sulphuric acid also. The potash salts supply potash with more or less sulphuric acid and magnesia. Such mixtures therefore would form "complete fertilizers."

In Germany, where the potash salts have come into very general use, quantities corresponding to from 200 to 400 , or at most 500 pounds of the higher grades, and from 300 to 600 of the lower grades, per acre, are recommended."

The general conclusion respecting the use of the German potash salts as fertilizers are given by Prof. Atwater as follows:

" 1 . Potassium, the basis of potash compounds, is indispensable to the growth of all our cultivated plants. It has at least one specific office in the nutrition of the plant, that of aiding in the formation of carbo-hydrates (starch). Without a plentiful supply of potash in available forms, full crops are impossible.

2. The German potash salts afford at present the cheapest and most available supply of potash for fertilizers. They supply also more or less of magnesia and sulphuric acid, which are essential ingredients of plant-food, and sometimes deficient in our soils, and of sodium and chlorine compounds, which latter may be beneficial or harmful, according to the circumstances of their use.

3. The higher grades will be, in general, most profitable for use in this country, because they furnish the most potassium with the least admixture of inferior materials, on which costs of freight and handling must be paid. The chlorides (muriates), with $80-84$ per cent. of chloride of potassium, and the sulphates, with 70 to 80 per cent. of sulphate of potash, or the sulphate of potash and magnesia, with 54-57 per cent. of sulphate of potash, are to be especially recommended.

4. For potatoes, sugar-beets, or tobacco, the sulphates are preferable; for other crops, the chlorides, which are cheaper, are equally good.

5. In order to secure uniform diffusion through the soil, the potash salts should be 
applied as long as possible before the crop is sown. It is well to mix with earth, or to compost, before applying, especially if used shortly before sowing the seed. And, in general, potash salts are well adapted for composting with muck, earth, stable-manure, phosphates, fish, and the like.

6. The best results are generally obtained by using potash salts not alone, but with other fertilizers, as superphosphates, guanos, and fish. Mixtures of these latter with potash salt form "complete fertilizers." The proper use of potash salts is as adjuncts to other fertilizers.

7. From $200 \mathrm{lbs}$. to 400 or $500 \mathrm{lbs}$. per acre of the higher, and 300 to $600 \mathrm{lbs}$. of the lower grades, are appropriate quantities.

8. The question of the need of potash in a given soil can be best decided by actual trial. It will be generally advisable to test the question by experiments on a small scale before making large purchases."

Magnesia is contained in many soils, and in limestones, which are often called mag. nesian limestones, when this element is found in them in large quantities.

It is also found in wood-ashes, oyster-shell lime, New Jersey green marl, and various other fertilizing materials.

The waters of the ocean contain magnesia, to the presence of which is attributed the peculiar bitterish taste it possesses. It is estimated that every cubic foot of sea-water con. tains two and a half ounces of magnesia. It is supposed that the celebrated Strassfurt deposit referred to in previous pages, which is one of the principal sources of commercial magnesia and its salts, is the residue of some ancient sea.

The trap rocks of the Hudson River Palisades liave been found on analysis to sometimes contain ten per cent. of this substance. The ashes of grains of some wheat have been found to contain 11.75 per cent. of magnesia; hence, a soil deficient in this element would not produce wheat, however rich it might otherwise be. Since lime is abundant in wheat straw, a good calcareous soil, when lacking magnesia, might produce straw without any wheat, or, if the heads were formed, they would be very imperfect in wheat formation.

Leares of Trees can be made available in furnishing fertilizing substances in the form of potash, phosphoric acid, and lime, in limited quantities. Used in the compost heap, as bedding for stock, or as a covering for plants to protect them in winter, they are valua. ble. When mixed with the compost heap, or with the manure from the stables, they will soon become decomposed and add to the fertilizing properties of the mass, and will well repay the farmer for gathering, providing he is not required to go a long distance to obtain them. The best time for gathering them is when damp with dew or rain, as they can then be put in a more compact form, and be more easily handled. If there are boys upon the farm, a late autumn day could not be spent by them in labor much more profitably or pleasantly than in gathering and storing leaves for the various uses to which they can be applied. Stored under shelter, in some barn or shed, they should be spread and raked over occasionally in order to dry them, if designed for the bedding of stock. Besides being valuable, the gathering of leaves that would otherwise be blown about the farm buildings, giving the premises an untidy appearance, would be of advantage to any farm.

Tobacco Stems.-The refuse of the cigar manufactories, consisting of the stems and midribs of tobacco leaves, have been utilized to a considerable extent as fertilizers, especially in the culture of tobacco.

Analysis proves that they are a good general fertilizer, supplying all the elements of plant-food to a certain extent, and are especially rich in potash and lime. They are liable to be rather variable in composition, especially in respect to moisture. They are generally plowed into the soil a few weeks before planting. On clayey soils, they are frequently ap. plied in autumn, but this practice would not be advisable on a sandy or porous soil, as the 
fertilizing elements would be likely to be leached out by the rains and melting snows, and lost before the time of planting.

In plowing them under, care should be taken not to bury them too deep in the soil, where their fertilizing elements will be beyond the reach of the roots of the young plants.

Manuring with Green Crops is, in the opinion of many farmers, one of the most economical and, to certain lands, one of the surest and most speedy means of improving the mechanical condition or texture and fertilizing properties of the soil that are known in agriculture.

Besides fertilizing the soil by the use of green manures (as most plants have all the elements of fertility in a good proportion), the soil is rendered more mellow and thus better fitted for the production of other crops. The land is also cleared of troublesome weeds by plowing before the seed ripens. Soils are also improved by changing the combination of certain elements in them, which may be done by the simple process of plowing in the crop. If plants derived all their nourishment or fertilizing properties from the soil alone, simply plow. ing in again what the soil had produced would not prove of so much advantage as far as adding fertility; but the plant obtains its constituent elements not from the soil alone, but from the soil and atmosphere combined; many plants also draw a portion of their food from the subsoil, and these elements combine to form compounds in the plant, some of which are found in the leaves, others in the roots, sap, and stems, etc. Nitrogen is contained in some of these compounds, which is a promoter of decomposition. By the decomposition of these, other elements become involved, and new combinations are formed, which we call decay, but which is, in fact, only a change in the elements forming the plant. By this means, the average farmer may derive great benefit to his lands with but slight expense, and we think it a great error in farmers generally that they do not resort to the use of vegetable manures more frequently in obtaining a supply of plant-food for their crops.

The time when the crop is plowed under, and the depth to which it is covered by the furrow, are both very important considerations in this system. With regard to the former, there is a difference of opinion among farmers, some regarding the best time to be when the crop-clover or buckwheat, for instance-is in full bloom; others prefer the period just before blossoming. In no case should it be deferred until the blossoms fall and the seed begins to form. The harder the fiber of the stems, the longer the time required to make them available for plant-food. When green vegetation is allowed to decay in the open air, much of the fertilizing material is evaporated by the atmosphere, and hence lost to the soil. In the other extreme, if the crop is buried too deeply in the soil, it decays more slowly, and also much of the fertility designed for plant-food will be kuried too far from the reach of the roots of many plants; hence it is very essential that the vegetable manure be covered by the soil just enough to prevent the fertilizing elements from passing off into the air, and suffciently for the soil to absorb all these properties. It should be turned down only enough to be simply well covered; in this way the strength of its fertilizing properties lies near the surface, and the heat and light rains will also aid in the decomposition, and if plowed under in the proper stage of its growth, this will be very rapid.

Vegetable manures are especially adapted to such soils as have a large proportion of lime, but when lime does not exist in a fair proportion in the soil, it will be well to apply it. This corrects any sourness in the soil, and aids decomposition. Gypsum and ashes are recom. mended by some as good substitutes when lime or marl are not easily procured.

Some writers recommend vegetable manures for all soils except low, peaty lands. They are beneficial often to a surprising extent, to the two extremes of clay and sandy soils, giving a peculiar mellowness to the one, and a retentive character to the other, improving their texture and color by the necessary humus supplied; also improving their capacity for moisture, especially to the sandy soil. 
The effect of their use will vary according to the peculiar character of the soil upon which they are used, the kind of crop prodnced, and the manner in which the systern is conducted.

For the exhausted lands of New England, upon which certain crops have been continuonsly grown for years, and have thus been rendered but partially productive, and for many of the worn-out soils of the Southern States, which have become exhansted by the constant cultivation of cotton, sugar-cane, and tobacco, etc., this system is highly recommended by many of the best agricultural writers of the country.

Before sowing any crop for green manure, it will be well to apply to the soil well-decomposed and pulverized barnyard manure, bone-dust, guano, or some other good fertilizer, in order to give the plants a good vigorous start, since the more luxuriant and heavy the growth of the crop to plow under, the more fertilizing material will be obtained from it, and consequently the greater benefit to the soil.

Some exhausted soils require considerable time to recuperate in this way, but it does not involve much expense. Often three or four crops of green manure are turned under before certain worn soils will be rich enough to produce a fair product.

For green manuring, various crops are used, but some are more valuable than others. First and foremost in value we place red clover. The crops most commonly used for this purpose are red clover, buckwheat, rye, oats, corn, millet, many of the grasses, the cow-pea, etc. Rape, vetches, mustard, spurry lupine, and the leaves of turnips, after removing the bulbs, are frequently used for this purpose in Europe.

Clover for Vegetable Manure has been styled by one of the best authorities in agriculture (Joseph Harris), "the grand renovating crop of America."

Hon. George Geddes, so well known throughont the United States as a practical, scientific, and eminently trustworthy farmer, says of it:

"The agriculture ef Onondaga county is based on the clover plant. It is used for pas. tnre, for hay, and for manure. Strike this plant out of existence and a revolution would fol. low that would make it necessary for us to learn every thing anew in regard to cultivating our lands. What their value would be without clover, we will not attempt to conjecture. We have this most valuable treasure, and apprectate it.

If our soils require improving, we tnrn the clover crop under, and repeat the operation until there is sufficient fertility to allow us to carry the clover off. The oftener we can fill the soil with roots, and then plow them under, and thus allow them to rot, the sooner do we expect to get our land in condition to bear a crop of grain."

A very considerable part of the cultivated land of this county (Mr. Geddes might have appropriately said "a very considerable part of the cultivated land in Central and Western New York)" has never had any other manuring than this clover and gypsum, and its fertility is not diminishing. Fields that are distant from barnyard manure are rarely treated to any thing but gypsum and clover. These fields are not cropped with grain as often as those that have the benefit of barnyard manure, but they are manured with much less expense.

The cost of clover seed, at $\$ 6$ per bushel, is, . . . . . . . . . . $\$ 150$

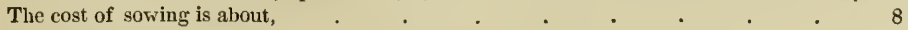

The cost of three bushels of gypsum at the mills is, . . . . . . $\quad 24$

The cost of drawing the same, . . . . . . . . . 12

The cost of sowing at three different times, $\quad$. $\quad$. $\quad$. $\quad$. $\quad$. $\quad$. 38

Total cost of manuring one acre, . . . . . . . . . $\overline{\$ 232}$

A field baving the first year given a crop of hay and another of seed, the second year an acre will nearly or quite pasture a cow from the twentieth of May until the middle of August. If then plowed in the most perfect manner, it will be in the best possible condition 
for winter wheat; the land may be used the second year for pasture the whole season if not wanted for wheat, and put intu curn or any other crop the nexu.'

He also has stated that he had a field which for seventy-four years had been manured with nothing except clover, grown upon it and plowed in, and that this field had produced wheat, corn, oats, barley, and grass. The clover thus used had for fifty years been regularly treated with gypsum, and that the land is constantly increasing in fertility. Suflicient tests have been given clover as a fertilizer, when plowed under, to prove that it can be used continuously on many soils for a long period with good results, many farmers considering it a good substitute for barnyard manure. If it can be thus utilized, it proves a great convenience for use on such lands as are at a distance from the farm buildings, where the carting of yard manure would be attended with considerable labor and expense. As clover will produce two or three crops during the season, many farmers cut the first for hay, and plow under the second for manure, while others secure both crops for hay, and plow under merely the stubble and roots, which are accounted by many as equivalent to a good dressing of stable manure; but, of course, the greater the quantity of this fertilizing material that is left in the soil the greater will be the fertilizing properties of the soil resulting from the system of green manuring, and the sooner-other conditions being equal-will the entire crop used for this purpose restore and enrich the lands upon which it is used. Some recommend sowing buckwheat with the clover seed. As we have never practiced this method, we cannot give the result of it from experience. Clover should be plowed under when perfectly green, having attained a good growth; and most farmers consider the best time for doing this to be when in full blossom; it should then remain undisturbed until the proper time for sowing grain or planting other crops.

Various Crops for Green Manure,-Next in value to clover, buckwheat is thought by many to merit a place, and to be nearly equal to clover in fertilizing properties. It can be easily sown and raised, and requires but a short time for growth; hence, where a crop of wheat, rye, or barley has been removed it is especially adapted to the purpose. It also shades the ground well, keeping it cool, and thus lessens the evaporation of its fertilizing gases and renders it mellow, while it keeps down the weeds. It thus furnishes a very good substitute for clover.

Winter rye is also valuable for green manure. It should be sown early in the fall, and a further growth given it in the spring before turning it under.

Corn also sown broadcast is highly valued by many for this purpose.

The wonderfully valuable properties of the cow-pea, as a renovator of poor soils, are well known in the South, where it is considered the best of vegetables for this purpose. It grows luxuriantly and rapidly, producing two crops in a season, and will thrive on very poor land, but the erop will be greatly improved by the application of about a hundred pounds of plaster per acre.

It should be turned under when most of the pods are about half-grown, and none are near being ripened.

Dr. Pollard, late Commissioner of Agriculture in Virginia, gives the following directions relative to improving partially-exhausted lands in the Southern States, by the use of vegetable mauures:

"By husbanding all animal manures, and sowing peas and clover, we can obtain a large amount of nitrogen for the soil. It may be said that a considerable portion of our lands are too poor to produce clover or even a crop of peas. Then let two hundred pounds of ground South Carolina phosphate, and three hundred pounds of kainit (Dr. Ravenel's 'ash element') be applied to the land, and peas be seeded; turn these under and sow clover with wheat, or oats if thought best, and we shall be apt to get a stand of clover, particularly if the land be limed after the peas are turned under. But the clover will do better by itself than with 
wheat or rye, if a stand can be obtained. The wheat or oats, to be sure, will be some protection in the spring from frost, but grass and weeds will soon spring up to protect the clover. If this plan should be tried, as a means of furnishing nitrogen and improving poor lands, it might be well to sow the clover in the fall as soon as the peas are turned under, if it is deter. mined to grow the clover alone. If this is done, the land should be rolled after seeding clover, as the peas will leave it in a spongy state. But if the farmer cannot get the 'ash element,' let him sow oats or rye early in the fall, or oats early in the spring, turn them under in June, then sow peas, and then clover, if a good growth of peas has been secured, applying lime, however, if possible, after the peas are fallowed in."

Hungarian grass possesses rich vegetable substances, has a luxuriant growth, and turns under very easily, as its fine fibrous roots hold fast to the ground, while the upper portion is turned into the furrow, never clogging the plow. It is a cheap crop and grows quickly, being ready for plowing under usually in about sixty days, requiring only about a dollar's worth of seed per acre. Its growth will be facilitated by finely pulverizing the soil before sowing; and after covering it lightly with a harrow to use the roller, which promotes a quick germination of the seed.

After the crop has been plowed under, the decomposition of the buried vegetable matter will be hastened by harrowing the ground and rolling it. It is said to leave the land in a better condition for wheat than oats used for this purpose.

Sod of any kind of grass is valuable for fertilizing purposes, and the more grass there is on it, when turned down, the better. It is a noticeable fact among farmers in cultivating corn, that the best crops are generally produced when the seed is planted upon the inverted grass turf; the roots and stubble of the grass furnishing an immense quantity of valuable plant-food for the growing crop. Besides the vegetable substances we have mentioned for fertilizing purposes, various others are often used; in fact, every decomposable thing adds to the productiveness of soils, when applied in the proper quantity and manner.

Even weeds of rank growth plowed under can be utilized in furnishing enriching elements to the soil, when suitably decomposed; they should, however, be plowed before any of them have ripened their seed, as they would otherwise become self-sown and produce another crop of weeds. It is as true in agriculture, as everywhere else, that every creation has a purpose, and "nothing is made in vain."

Commercial Fertilizers.-The question often asked by the farmer is, Do commercial fertilizers pay?-in other words, Is the amount expended in artificial fertilizers realized in benefit to the crops? In answering this question, many considerations must of necessity be taken into account.

They may be very beneficial and well repay the expenditure involved in increasing the quantity and often the quality of the crops, or they may result in a serious loss to the farmer, with an injury to the soil; much depending upon the kind and quantity used, and the manner of using. In the use of all fertilizers, the needs of the soil and requirements of the crop to be produced must be taken into account; it must also be properly applied.

If a farmer expends large sums of money for phosphoric acid and applies it to the soil, when nitrogen is what that soil needs in producing the desired crop, disappointment will be the result; or, if he supplies to a soil nitrogen when phosphoric acid is the element needed, the result will be no more satisfactory than the former; or, as is often the case, if too small a quantity of the proper kind be applied to produce any change in the productive capacity of the land, or if an unwarrantable amount of certain kinds be used and in a short time it exhausts certain elements of the soil, with other uudesirable results--in either of these cases the fault would not be with the fertilizer itself, but with the lack of skill in the one who selected and applied it. Soils widely differ in their capacity for supplying crops with plantffood, and correspondingly in their demands for aid from fertilizers; some soils 
will, consequently, give the best results with one kind of fertilizer, and others with another kind, while others still will not respond to any application whatever, until the condi. tions are changed, which can only be effected by suitable drainage, irrigation, the use of lime, or proper tillage.

In order to attain the highest results in agriculture, as with everything else, we must make use of the right materials in the right place; and if artificial fertilizers are thus used they cannot bring results otherwise than satisfactory, providing the season and other circum. stances be favorable. The world-renowned experiments at Rothamsted, England, by Mr. Lawes, embracing a period of over forty years, besides those from other noted scientific agriculturists covering a more limited space of time, all prove conclusively that artificial fertilizers, when rightly used, will prove the most potent aids in agriculture, and that the only means of determining what a soil needs is to study it by careful observation and experiment.

In making this statement favorable to artificial fertilizers, we do not wish to be understood as recommending them as substituting entirely the various farm manures that the farmer has recourse to in improving his lands and crops; we do not recommend them as supplanting, but supplementing the farm manure.

We think it would be a very poor policy indeed for any farmer to allow the farm manures to go to waste, and expend money for commercial fertilizers. We believe in using all the available wastes of the farm possible, for manure-the compost heap, cess-pool, privy-vault, ashes, old bones, and everything that can add to that best of all the fertilizers, stable manure - to aid in furnishing plant-food to crops. It would be very poor economy for a farmer to neglect tc gather his own crops and buy of his neighbor, and it would be equally poor economy for him to neglect to make use of his farm manure, and bny commercial manures to take their place. The poor farm management in the one case would be equai to that of the other.

We simply advise that the farmer use the artificial fertilizers to supplement or help out, as it were, the farm manure, as most farms require more manure than the farm furnishes for keeping them up in their best condition in soil, and giving the best results in crops. It is thought by many of our leading agriculturists, that a liberal supply of barn-yard manure increases the effect of the commercial fertilizers, and vice versa, when the two are used together.

Mr. Wood of Mt. Kisco, N. Y., recently stated at an agricultural meeting that, as a result of his experience in commercial fertilizers, he would warn farmers against their use in small quantities, or without the application of sufficient plant-food to support the growth of the plant after the fertilizers have started it vigorously; that, by the use of phosphates, he can raise one hundred bushels or more per acre of southern white Dent corn, and it will be sufficiently ripe to be cut by the first of September. His lands are made very rich otherwise by the use of barn-yard manures, and the phosphates force the growth of his crops so rapidly that the effect is to give him the results of a climate like that of southern New Jersey.

Prof. Atwater, in giving the result of farm experiments with fertilizers, in connection with the Connecticut Experiment Station, also says:

"17. The common impression among farmers that the best use of artificial fertilizers is to supplement farm manures is, doubtless, in ordinary circumstances, correct."

With respect to the requirements of different crops, a very accurate estimate can be obtained from a table giving their analyses by a competent chemist. Much can also be learned by the experience of the most successful farmers in the country, given in the best agricultural works and newspapers, which will render valuable assistance to the farmer in all the departments of agriculture; but since soils vary so widely in their constituents, the best and surest test in determining the needs of certain soils is found only by careful study and experiment, which each farmer can accomplish for himself in a small way very easily. Haring 
once determined those requirements for his particular lands, important information has thus been obtained and much been accomplished toward successful results.

Prof. Johnson, of Yale College, the author of several agricultural works, and chemist of the Connecticut Experiment Station, suggests that in making such experiments, it is better to have a long and narrow plot of ten or fifteen square rods area to experiment upon, because the inequalities of the soil are less liable to disturb the results. The land being prepared for a crop, a number of measured plots or strips are laid off, and different fertilizing materials are applied to them in appropriate quantities. On one, for example, use gypsum; on another, fresh slacked lime; on another, a superphosphate made from bone-ash or bone-black, or ground bones; on another, pulverized blood and meat-scraps, rich in nitrogen, but nearly free from phosphates; on another, sulphate ammonia; on a sixth, muriate of potash; seventh, a nitrogenous phosphate or a fish guano; eighth, stable manure, etc. Two or three plots with no manure should intervene to make a basis of comparison. The experiments should extend over a series of three or four years, the same plots being each year treated with the same kinds and qualities of fertilizers, but cultivated with different crops. Different fertilizing materials, or the combination of certain substances to form special fertilizers for special crops, could thus be tested. Mr. Lawes, of Rothamsted, states that in order to obtain large crops he would use artificial fertilizers. Prof. Ville, the noted French agricultural chemist, concurs in the same opinion from his numerous experiments. For speedy results, when skillfully used, and for restoring partially-exhausted lands, rendered so by over-cropping, commercial fertilizers, when properly used, are among the most valuable agents of the farmer. By combining stable manure and commercial fertilizers, we think the result in all respects will prove perfectly satisfactory.

Since commercial fertilizers are so frequently adulterated with worthless substances, it is safe to purchase only those manufactured by some well-known firm of reputed integrity, whose products have been well-tested in the markets, or after a sample has been subjected to chemical analysis by a competent chemist. The principal deficiencies of our soils, as we have previously stated, are potash, phosphoric acid, and nitrogen, with often a lack of sulphuric acid, lime, and rarely magnesia. In England, according to Mr. Lawes, nature has furnished, generally, a sufficient supply of potash, and consequently the soils there do not require it artificially to the extent that those of the United States do, the principal demand there being nitrogen; consequently the tests of English soils will not always apply definitely to our own in the production of certain crops; yet, notwithstanding, these tests of foreign soils serve to illustrate the potency of chemical fertilizers as agents in successful agriculture, when applied to any soil deficient in the elements that such fertilizers can supply.

Special Fertilizers.-According to authentic sources, Prof. Johnston, of Scotland, was the first to prescribe special fertilizers in detail for special crops, his general principle being that the manure applied to the soil must contain all those inorganic or mineral substances which the crop we wish to grow carries off the soil, and in the relative proportions in which they are respectively found in the ash of the plant (the ashes remaining after burning the entire plant), the exception being to omit from the application those elements already abundant in the soil. He also favored the addition of organic matter to the soil, in the form of animal or vegetable substances, in order to restore the elements of this nature that the cultivated crops had extracted in their growth.

Prof. Ville, of France, has based a theory of fertilizing upon the following propositions: "1st. Give the earth more phosphates, more potash and lime, than the harvests have taken from it. 2d. Give it fifty per cent. of the nitrogen they contain." He has also given various. recipes for complete fertilizers for special crops, based upon the above principles. Prof. Stockbridge, of the Massachusetts Agricultural College, has arrived at the opinion, after various and repeated experiments at the agricultural college farm, that the only substances 
generally needful to be supplied artificially, in order to produce good crops, are nitrogen, potash, and phosphoric acid, and that to compound these substances in the proportion in which they are contained in crops, according to an average of many of the most reliable analyses, will make a special fertilizer for each crop, which, if applied, will produce the best of results without apparent exhaustion to the soll. The nitrogen, as given in Prof. Stockbridge's formulas for special manures (known as the Stockbridge fertilizers), is supplied in the form of sulphate of ammonia, or its equivalent, potash as muriate or sulphate of potash, and phosphoric acid as super-phosphate of lime. We give a few of the Stockbridge formulas for some of the principal crops, which show how small a quantity of each ingredient is necessary when supplied in the proper proportion. With regard to them, Prof. Stockbridge says:-

"The form in which I have obtained nitrogen, potash, and phosphoric acid to compound for the nutrition of plants, in these experiments, has been in that of a neutral salt for the nitrogen and potash, and a superphosphate for the phosphoric acid. For root-crops axd beans, I have used the potash in the form of sulphate; for grain and forage crops, in the muriate form. No specific rule can be given as to the quantity of the compounds to be used in preparing any of my formulas, because the percentage of nitrogen, potash, and soluble phosphoric acid they contain is quite variable; but having learned the percentages of the com. pounds, the required quantity is easily ascertained.

\section{WHEAT.-FOR ONE ACRE.}

Nitrogen, . . . $41 \mathrm{lbs}$, in the form of Sulphate of ammonia, 205 lbs.

Potash, . . . 24 lbs., " " Muriate of potash, . $48 \mathrm{lbs}$.

Soluble phosphoric acid, 20 lbs., " " "Superphosphate, . 160 lbs.

The crude materials for the above will comprise, as will be seen by the table, over 400 lbs., and are said to increase the crop 25 bushels over the natural yield of the soil.

INDIAN CORN.-For ONE ACre.

Nitrogen, . . . 64 lbs., in the form of Sulphate of ammonia, 320 lbs. Potash, . . . 77 lbs., " " Muriate of potash, : 154 lbs.

Soluble phosphoric acid, 31 lbs., " "Superphosphate, : 248 lbs. The above is designed to increase the natural product of the soil 50 bushels.

\section{FODDER CORN,-FOR ONE ACRE.}

Nitrogen, . . . 20 lbs., in the form of Sulphate of ammonia, 100 lbs.

Potash, . . . 66 lbs., " " Muriate of potash, . 132 lbs.

Soluble phosphoric acid, 16 lbs., " " Superphosphate, . 128 lbs.

Increase over natural yield, two tons.

RYE-FOR ONE ACRE.

Nitrogen, . . . 25 lbs., in the form of Sulphate of ammonia, 125 lbs.

Potash, . . . 24 lbs., " " Muriate of potash, . 48 lbs.

Soluble phosphoric acid, 16 lbs., " " Superphosphate, . 128 lbs.

Increase over natural yield, 20 bushels.

\section{OATS.-FOR ONE ACRE.}

Nitrogen, . . . $23 \mathrm{lbs}$, in the form of Sulphate of ammonia, $115 \mathrm{lbs}$.

Potash, . . . $20 \mathrm{lbs}$, " " Muriate of potash, . 40 lbs.

Soluble phosphoric acid, 12 lbs., " "Superphosphate, . 90 lbs.

Increase over natural yield, 25 bushels.

\section{BUCK WREAT.-For ONE ACre.}

Nitrogen, . . . $37 \mathrm{lbs}$, in the form of Sulphate of ammonia, $185 \mathrm{lbs}$.

Potash, . . . $50 \mathrm{lbs}$, " " Muriate of potash, . 100 lbs.

Soluble phosphoric acid, 15 lbs., " "Superphosphate, . 105 lbs.

Increase over natural yield, 25 bushels. 
POTATOES.-FOR ONE ACRE.

Nitrogen, . . . 21 lbs., in the form of Sulphate of ammonia, $105 \mathrm{lbs}$.

Potash, . . . $34 \mathrm{lbs} .$, " " Muriate of potash, . $225 \mathrm{lbs}$.

Soluble phosphoric acid, 11 lbs., " " Superphosphate, . 85 lbs.

Increase over natural yield, 100 bushels.

\section{FIELD BEANS.-FOR ONE ACRE.}

Nitrogen, . . . 53 lbs., in the form of Sulphate of ammonia, $265 \mathrm{lbs}$.

Potash, . . . $33 \mathrm{lbs}$, " " Muriate of potash, . $198 \mathrm{lbs}$.

Soluble phosphoric acid, $20 \mathrm{lbs}$., " “ "Superphosphate, . 160 lbs.

Increase over natural yield, 20 bushels.

SWEDE TURNIPS, OR RUTABAGAS.-For ONE Acre.

Nitrogen, . . . $11 \mathrm{lbs}$, in the form of Sulphate of ammonia, $55 \mathrm{lbs}$.

Potash, . . . $18 \mathrm{lbs}$., " " Muriate of potash, . $118 \mathrm{lbs}$.

Suluble phosphoric acid, 8 lbs., " “ Superphosphate, . 63 lbs.

Increase over natural yield, 100 bushels.

\section{ONIONS.-FOR ONE ACRE.}

Nitrogen, . . . $11 \mathrm{lbs}$, in the form of Sulphate of ammonia, 55 lbs.

Potash, . . . . 9 lbs., " " Muriate of potash, . 54 lbs.

Soluble phosphoric acid, 4 lbs., " " Superphosphate, . 32 lbs.

Increase over natural yield, 100 bushels.

HAY.-For ONe Acre.

Nitrogen, . . . $36 \mathrm{lbs}$, in the form of Sulphate of ammonia, $180 \mathrm{lbs}$.

Potash, . . . $31 \mathrm{lbs.,} \mathrm{"} \mathrm{"} \mathrm{Muriate} \mathrm{of} \mathrm{potash,} \mathrm{.} 70 \mathrm{lbs}$.

Soluble phosphoric acid, $12 \mathrm{lbs}$., " " "Superphosphate, . $95 \mathrm{lbs}$.

Increase over natural yield, one ton.

The above is founded on an average of the formulas for red clover, white clover, English hay, and timothy.

Having never used the above special fertilizers, we cannot speak of their merits from experience, but from what we have been able to learn concerning them, they seem better adapted to any other soil than heavy clays, the latter giving often unsatisfactory results. If they will accomplish what is claimed for them on other soils, in will certainly be of assistance to the farmer to learn the results of careful experiments by others, in determining the quantity and kind of fertilizers suited to different crops, and spare him the trouble of experimenting for himself; however, in the use of commercial fertilizers of any kind, we favor the application of a liberal supply of barn-yard manure, occasionally, as that is a complete fertilizer, and, hence, contains all the elements of plant-food, and will greatiy aid in keeping the land in a high state of fertility, and, at the same time, causing it to yield abundant crops. Special fertilizers will, without doubt, also aid in restoring lands exhausted by excessive cropping. As we have previously stated, we regard barn-yard manure, of the best quality, the most desirable general fertilizer that can be used by the farmer, and commereial fertilizers as supplementary to that; but where the former cannot be obtained in sufficient quantities, as is the case with most of our farms-the farm-supply not being adequate to the demand, where the lands are fertilized to the extent that high cultivation and successful farming requires-commercial fertilizers are valuable substitutes. Fertilizers of all kinds should be made very fine, that they may be easily taken up and appropriated by the plants, and their fertility, in a great measure, depends upon the fineness to which they are reduced before being applied.

In addition to the above formulas for fertilizers, the Mapes Co. of New York have, for sugar-cane or sorgo, one containing from two to three per cent. of ammonia, from ten to 
twelve per cent. phosphoric acid, and from six to eight per cent. of potash. This is said to prove valuable for either Southern sugar-cane or sorgo, and to greatly increase the quantity of saccharine matter produced by the stalks.

They also have a formula for cotton, containing from three to four per cent. of ammonia and from ten to fourteen per cent. of available phosphoric acid.

Dr. Nichols also has a formula for imitating barn-yard manure in its constituents and results, but which is said to act rather more slowly than that substance, yet is more lasting in its effects. It is as follows:-One cord of seasoned muck, sixty-five pounds of crude nitrate of soda, two bushels of wood-ashes, one peck of common salt, ten pounds of fine bone-meal, two quarts of plaster, and ten pounds of Epsom salts. These are all to be carefully composted before applying to the soil.

The manufacturers of nearly all special fertilizers make use of the same materials, but in different proportions, some giviug potash a larger proportion than others, while some rely more upon nitrogen and phosphoric acid than potash.

Application of Manures.-Formerly, it was the custom to plow in farm manure, even on sod lands, it often being plowed under so deep as to be beyond the reach of most of the plant roots during the first season after its application, while so large a portion of the fertilizing elements were washed still lower by the rains, that they failed to benefit subsequent crops; hence, much of the value of the material applied was wasted and lost to the crops it was intended to benefit.

Light covering and surface application were afterwards practiced with more satisfactory results, and at the present time we find none among the more intelligent farmers, who would think of covering manure heavily with soil.

The reasons for receiving the most benefit with manures near the surface are obvious. In order to obtain a vigorous start, plants need the stimulus supphed by this plant-food in the early stages of life; and in order to be benefited by it, it must lie near them, where their short and tender rootlets can reach it. If it is beyond their reach, they cannot be nourished by it. By having it near the surface, they can grasp it readily, otherwise they will need to wait until they can grow to the depth in which their food is hidden, before they can be benefited by it, and this may be a slow and starving process. By having the plant-nourishment at the start, a much larger crop will not only be secured, but it will mature earlier, and be more perfect in its development.

There is a tendency in manures generally to sink deeper in the soil; this fact argues in favor of surface application. The principal objection brought against the latter is, that when exposed to the atmosphere much of the ammonia contained in the manure is evaporated and lost. This may be true to a certain extent, but we think the loss occasioned in this manner not as great as is generally supposed.

When stable manure is applied to the surface of lands, the moisture dries very quickly, and as soon as dried, all evaporation and consequent loss of ammonia ceases; it is then left for the rains and dews to gradually dissolve and take to the soil, where the rootlets can feed upon it. The greatest loss to manure from exposure to sun and air, is during the process of fermentation; that occasioned by such exposure after this process being very slight, while the benefits resulting from surface application are very apparent, and many times counterbalance the loss occasioned by it.

When green manure is applied to grass-lands, many of our most successful farmers sow a moderate supply of plaster upon it, as soon as spread, in order to hasten decomposition and aid in preventing the escape of the ammonia.

Seeds are generally mixed with unfermented manure, and often noxious weeds, that may prove very troublesome to eradicate, are sown by its application; they, however, will not take root as readily on grass lands, where the turf is compact, as on a pulverized soil; hence 
for all crops on tilled lands, we would recommend that the manure be partially, if not wholly, fermented, or, as is commonly termed, rotted, before being applied. This may be done by composting it with soil, as we have previously described, which process will have a tendency to destroy the germs of any seed it may contain; or it may be occasionally turned, giving the seeds an opportunity to sprout, and die in the process, but the former is the surest method of destroying them. In no case do we approve of applying strong manure directly to the seed of crops. The safer way for hoed crops, such as potatces, for instance, is to have a slight layer of soil cover the manure before the seed is dropped; and as a general rule for all soils, the best mothod of applying it, is to spread it on the surface after the last plowing, and harrow it in with the crop, if practicable.

When manures are covered by heary clay or loam soils, they remain, owing to the nature of the soil, nearly dormant, and as no decomposition takes place, they are of little use to the growing crop. On lighter soils their action is more speedy, but in any case they are more efficacious when near the surface, or upon it, as we have previously stated.

For grass lands, we advise that the application of farm manure be always in the late autumn or early winter, that it may have the benefit of the melting snows and early spring rains in dissolving it, and the finer it is pulverized when applied, the better.

Dr. Nichols says on this subject: "I have had considerable experience in this matter of top-dressing grass lands, and feel competent, therefore, to advise on the subject. In the use of stable dung, we say, apply it rather late in autumn, but not too late, that is, do not wait until the ground freezes, but do it about a month before the usual time of the advent of severe frosts. If applied too early, as in August or September, in the Northern parts of the country, it dries hard in the sun, and is not so readily acted upon by frosts, and besides it sinks deep into the growing grass, and is, therefore, not so well distributed as it should be. The active principles have time enough to undergo chemical change from October to the next April or May. Concentrated fertilizers should not, under any ordinary conditions, be applied in the autumn. The reason is, that by the rapidity of the change the active elements of food are brought almost immediately to the open mouths of plants, and thus by absorption they are stimulated to growth when growth is not desirable."

Application of Commercial Fertilizers.-Respecting the application of commercial fertilizers, we know of nothing better than the following, from the Director of the Connecticut Experiment Station.

"The fertilizers may be applied broadcast, or if more convenient, they may be put in the bill or drill, provided, they are well diffused through the soil. To accomplish this, they had better be diluted with several times their bulk of earth before using. The important points are, that they be:

"1st. Apphied evenly over the plots where they belong and not allowed to get outside.

"2d. Well distributed through the soil.

"Experiments with concentrated fertilizers are often spoiled, just as crops are injured or lost through wrong application. Farmers are apt to think that the manure must be put close to the seed or the plant will not get the benefit of it. This is wrong. It is not the just germinated plantlet that needs the manure, but the plant, from the time it is well started until its growth is done. We want, not only to give the crop a good start, but to help it out on the home stretch as well. The roots and their branching rootlets run out in all directions in search of food, and the fertilizers ought to be where as many of the rootlets as possible can get at them. If we distribute the fertilizers as well as we can, the water in the soil, aided by the chemical and physical forces that nature keeps in operation, will do the rest. In illustration of this. remember how well barn-manure acts when applied as a top-dressing long before the seed is put in.

"But if we concentrate the fertilizers in one place, fewer roots will get them, and these 
may be injured by coming in contact with them or with their concentrated solutions in the soil. The roots will find their way to the manure and develop more where it lies, it is true; still, we should not oblige them to huddle together in one place, but should rather encourage them to spread around where, with the increased capacity the fertilizer gives them, they can get the more from the soil. Roots join with other natural agents in rendering inert stores of plant-food available.

"Above all, do not let the fertilizers come too close to the seed. A coarse, dilute material like yard manure may do the plants no harm, but such concentrated fertilizers as potash salts, dried blood, nitrate of soda, or high grade superphosphates, may kill them."

"The testimony of the experiments is on the whole against applying in the hill or drill. The best results in the majority of cases came where the fertilizers were sown broadcast. Several of the very best were where the materials were scattered over a strip a couple of feet or so wide along the rows. Many of the worst results were where the fertilizers were put in the hill or drill. The nitrate of soda and potash salts thus applied often injured the crops, especially in dry weather."

Prof. Pendleton of South Carolina, on the contrary, says that in his experiments, super. phosphates never produce remarkable results sown broadcast, and he bas found that two hundred pounds of phosphoric acid will produce more effect in the drill than five hundred pounds sown broadcast. The explanation he gives for this effect is. that the superphosphates find plenty of bases, such as clay, iron, lime, etc., in the whole soil to reduce the solubility before the rootlets of the plants find it; while in the drill, sufficient remains in direct contact with the roots to give the plant a vigorous start. He also found by exporiment, that equal parts of lime and superphosphate produced one hundred and thirty-seven pounds of cotton less per acre than the same quantity of superphosphate alone.

When used in the drill, we have, in our experience and observation, found the best results only to be attained when the fertilizers were mixed with four or five, or more, times their bulk of earth and thus diluted. This is very important, as the strong chemical substances contained in them will be liable to injure the germs of the seeds or the rootlets of the young plant. When applied as a top-dressing to growing crops, artificial manure should be applied only in wet weather.

The Fallow System was formerly much practiced in England, and also in this coun. try to a certain extent, but at present is partially, if not almost entirely, discarded by the leading agriculturists of both countries. It is a very ancient custom in agriculture, and is a means of enriching lands by allowing them to rest for one or more seasons, after partial exhaustion occasioned by continuous production. No tillage is required except one or more plowings, and thus exposing the soil to the action of the elements. Such lands are sometimes termed "naked fallows." This system is founded upon the principle that the soil has stored up within it vast quantities of plant-food, which is not in a condition to be available for the present use of vegetation; that the crops which the soil has previously produced have exhausted most of the available soluble food, such as potash, phosphoric acid, etc., and unless these elements that have been extracted from the soil, in the production of previous crops, can be returned to it in some form of fertilizers, suited to the immediate support of plants. time must be given for dissolving (by the disintegrating process so constantly going on in all soils) such elements as are now locked up in the soil, and which will become available before the period of anotlier sowing. The advocates of this practice claim that it not only greatly improves the condition of the soil, but is also the surest means of destroying noxious weeds; while the objections urged against it are not only a loss of the use of the land by the period of idleness, but a great loss of much of the fertilizing element of the soil by evapora. tion and drainage, exposed as it is during the summer to the hot rays of the sun, drying winds, and leaching rains. 
The unlocking of the mineral elements of the soil, or, in other words, changing its mechanical condition, as well as the destruction of troublesome weeds, can, it seems to us, be accomplished in a more economical way, and without wasting any of the soil elements; and that is by the "green fallow," which has generally taken the place of the old-time fallow system. Some green crop, such as clover or buckwheat, is often sown after the plowing, and allowed to grow until at the proper stage of growth for plowing under; in this way the advantages of vegetable manuring are secured, with but slight expense of time or labor, and the soil will acquire more nutriment and benefit by the vegetable manure than if allowed to lie idle, while all troublesome weeds that are plowed under before ripening their seeds will not be self-sown. Where the land lies idle, some recommend that the plowings be about six weeks apart. We consider that land is better prepared for grain, or any other crop, by the use of vegetable manures as above described, than by the fallow system.

Fall Fallowing. - The old-time practice of summer fallowing, or working the soil for a year without a crop, for the purpose of producing a larger crop the second season, is, as previously stated, regarded as nearly obsolete by the best agriculturists of both this country and Europe. Whatever may be the opinion concerning the summer fallow, there can be no doubt whatever of the value of fall fallowing. The benefits derived from the latter practice are very justly set forth by the following extract from one of our leading agricultural journals:-

"The constant turning and working of the ground during the fall months cost nothing but time and labor, at a season when these cannot be otherwise employed, and so, in reality, cost nothing. But the benefits to the soil are very considerable. Especially is this the case with heavy clay soils, and less, in a descending ratio, tbrough the gradations from heavy olay down to light loams-at least, it is so considered by many; and it is reasonable to suppose that if the atmospheric effects upon the particles of a clay soil serve, to some extent, to dis. solve the mineral particles, they may easily do the same service for a sandy soil, and help to set loose some of the potash contained in the granitic or feldspathic particles of such a soil. The mechanical effects of the fall-working are certainly more useful upon clay than a light loam; but there are other purposes to serve than merely to disintegrate the soil, and mellow and loosen it. There are weeds to destroy, and the forwarding of the spring work by the preparation of the ground for early sowing. These services are useful for a light soil as a heavy one, and as it is reasonable to look for some advantage from the working in the way of gain in fertility on light as well as heavy soils, it is advisable that owners of either kind should avail themselves of whatever benefits the practice affords. Fall fallowing consists in plowing and working the soil with the cultivator or the harrow. This may be done at such intervals as may be convenient, or which will help to start some weeds into growth, when these may be destroyed by the harrow or cultivator. Heavy soils should be left in rongh ridges at the last plowing, with as deep furrows between them as possible, in order to expose the largest surface to the effects of frost and thaw. Light soils may be left in a less rough condition, but the last plowing should be so done as to throw the furrows on edge, and not flat, leaving the field somewhat ridged. A very bitle work in the spring will put the ground into excellent order for the early crops, and for spring wheat, especially, this better condition of the soil will be of the greatest benefit. When thus treated in the fall, the soil is remarkably mellow, and is dry enough to work much earlier than the compact stubble-land which remains as it was left after the harvest. As to the time for doing this work, the sooner it is begun and the oftener it is repeated, the better. It is not too late to finish when the ground is frozen, or there is an inch of snow on the ground." 


\section{TILLAGE.}

$\mathrm{T}$ ILLAGE has been defined as the art or practice of preparing land for seed, and keeping it in a state favorable for the growth of crops. The object of tillage is defined by another to be that of extirpating from the soil the growths that we do not want, and putting it in the best possible condition for the growths that we do want. The means employed and the nature of conducting the proper tillage of the soil, together with the benefits to be derived thereby, are among the most important considerations in agriculture, since tillage has relation not only to the mechanical condition of the soil, but to its moisture, warmth, aeration, fertilization, and the many benefits derived from the mixing of different elements of the soil, and setting free those mineral constituents of plant-food that are locked up in all soils; hence, to a certain extent, judicious tillage is equivalent to the application of fertilizers to the land, and many writers have placed so much importance upon it as to assert that if lands were allowed to lie fallow every other season, producing only once in two years, manure would nerer be required to produce good crops, and no deterioration of the soil would result. On the other side of the question, there are those who take the opposite extreme view of the subject, and claim that tillage has a tendency to make land sterile by the exposure of its elements to the air, thus permitting the fertilizing properties contained in the soil to escape. Either extreme has its objections, and the medium position, sometimes denorninated "the golden mean," is, in regard to this, as in most subjects, the most desirable course to pursue. By following the fallow method, the farmer would require twice the area of land that he now uses in order to produce the same amount in crops, while that portion of his land which produced tbe prerious season was lying idle; besides, we very much doubt whether, eren with this rest of alternate seasons, most soils would not suffer a gradual deterioration when entirely unsupplied with manure. The other theory above mentioned sets at naught all the experience of the past with its important teachings, and gives but a partial view of the subject. There are many good reasons for believing that too much plowing, as well as too deep plowing, is injurious to many lands, and has a tendency to make them sterile; the former, by continuous exposure of the fertilizing matter which the soil contains to the air, involving a waste of these elements. and also increasing the porous condition of such soils, while too deep plowing throws up often a gravelly or sandy subsoil, and buries the rich humus of the surface benesth, where it is beyond the reach of the roots of the plants, and consequently is lost to them; but, as a general rule, we believe the loss of plant-food occasioned by this exposure to the air and sun to be very slight, and that the fertilizing properties of the soils are greatly increased by cultivation. As almost all good is liable to be perverted to a wrong or injudicious use, so the good results of tillage may, in some cases, be perverted by the farmer plowing his fields too much, but we believe such cases are rare, and are greatly outnumbered by those where the soil is not plowed enough to produce the successful results that might be obtained by a thorough and judicious tillage adapted to the character and needs of the soil and crops to be produced. Because an orerdose of the proper kind of medicine might prore injurious, and perhaps fatal in some cases, is no reason why all medicine should be discarded and denounced as positively injurious; but such a course would be quite as reasonable as the assertions of those who claim tillage to be injurious to lands because it is sometimes practiced to excess. Success in agriculture requires intelligent thought and action as well as erery other department of business, and the results of ignorance and carelessness are fully as disastrous here as everywhere else. All soils contain the mineral elements of plant-food in abundance, while the crops produced require but a small amount: hence, but little of the mineral substance of the soil is extracted by the growing crops, yet, small as that amount may be, and abundant as this element may be in the soil, it is almost wholly locked up, as it were, beyond the reach of plants, or rather, is not in the condition to be assimilated by them, but requires the agency of the air, sunshine, dew, and rain, and the rarious chemical changes that are constantly being produced, 
to set free or unlock these elements suited to plant-growth. But these agencies are slow in their work of preparing these elements, and do not generally supply them with sufficient rapidity for the use of plants; hence, it is necessary that the farmer should aid the soil by applying the elements needed in the form of manure of some kind, and also in hastening these chemical changes by tillage, both of which are essential to the highest success in most soils, thus adding to the materials suited to plant-food, and also putting in an available form those elements already contained within it. Both are essential aids, and the best results in agriculture are only reached when they are combined. Prof. Johnson explains some of the reasons for tillage as follows :-

"In soils which have long lain undisturbed by the plow, and carry an inferior growth of vegetation, grass or weeds - which have become 'bound out,' in farmers' language-chemical influences doubtless have oftentimes set in with the effect of gradually filling up the pores, and solidly cementing together the particles of earth by the very processes which, operating through long periods of time, have converted into actual rocks what once were soils, either gravels, sands, or clays. The red rock, so abundant in the central parts of Connecticut, at New Haven is plainly seen to be a petrified gravel; at the Portland quarries it is a consolidated sand. The writing and roofing slates we are all familiar with are petrified clays, while the limestones, so abundant in New York and further west, were once the dust and mud of shores and sea-bottoms, full of shells and lime. Geologists assure us that there is evidence to show conclusively that all the rocks of New England, even her granites, were once sediments and soils. In exclusion of air and of surface-water oft replenished with air, soils petrify or turn to rocks, while in free contact with air, and water charged with air, rocks decay or disintegrate, and crumble into soil. Hard-pan, in many or most cases, is clay or clayey gravel cemented in this manner, and many soils, even sands and loose-textured gravels originally, have been made comparatively impervious by some cementing material. That which acts as cement is sometimes carbonate of lime, or carbonate of iron dissolved in water, and deposited by the evaporation of the solvent. In other cases it is humate of iron, the same as bog iron ore, the same also essentially as the moor bed pan, which often forms a thin but impervious bottom to peat beds and muck swamps, even when situated on coarse gravelly sands. In other cases silica and various silicates are the petrifying agents, as in the geysers of Iceland and the Yellowstone. They form in the soil itself by the chemical interaction of the substances there.

"A full consideration of all the facts makes it quite evident that in most soils there are tendencies constantly exerted with more or less vigor, towards mechanical compacting not enly, but towards chemical induration or petrifaction, and one of the important offices of tillage is to counteract these tendencies. Tillage accomplishes this office, not by crushing of solid grains, and not to any great extent by any direct mechanical reduction of lumps of coherent particles, but rather by lifting up masses of soil, turning them and letting them fall so that the close contact produced by rest and moving water is broken, and the grains of sand and the minute aggregations of loam are brought into new positions with regard to each other, and to greater distances from each other. The soil ought to be moist when plowed. Moist sands may be thrown up to a ridgey surface, and the mass be full of considerable cavities, whereas dry sand, if plowed, falls into nearly its original level and compactness. A mass of clay soil in a certain stage of moisture is broken by the plow into a multitude of small lumps, which if let dry a little more will yield to the impact of the harrow teeth and fall to smaller lumps, and in part to powder. The subsequent drying of the earth thrown up by a plow has much to do with its loosening. Some heavy clays of the plastic kind require their spring tillage to be done, as it were, "on the wing." If a trifle too wet, the plow makes paste of them where its pressure is felt, and the upturned soil falls to putty-like clods, which harden on drying, so that the field after plowing is as unfit for a seed-bed as before it was touched. If let stand too long before plowing, such clays bake at the surface to a crust that turns up cloddy, and is difficult to reduce. 
"What really loosens clay soils by detaching the particles from each other is the act of drying. The ten to twenty per cent. of impalpably fine real clay in such soils, distended by physical hydration, is the glue which sticks their coarser parts together. As the water dries

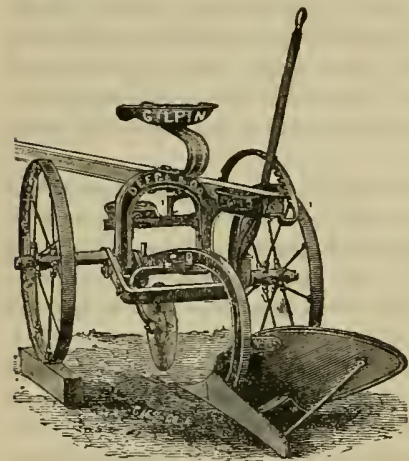

GILPIN SULKY PLOW.

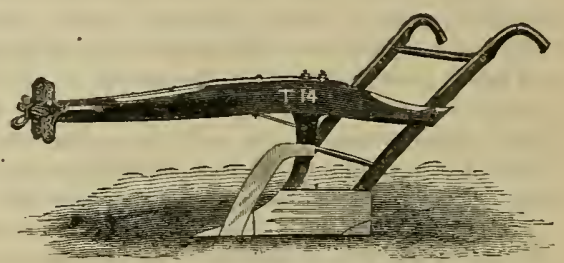

TIMBER LAND PLOW

out, this cement shrinks around the sand-grains, and must either bring these together into cioser contact, or itself crack and break. It does both in fact. A brick just moulded shrinks on drying, and its sides move together. The sides of a field of clay cannot approach each

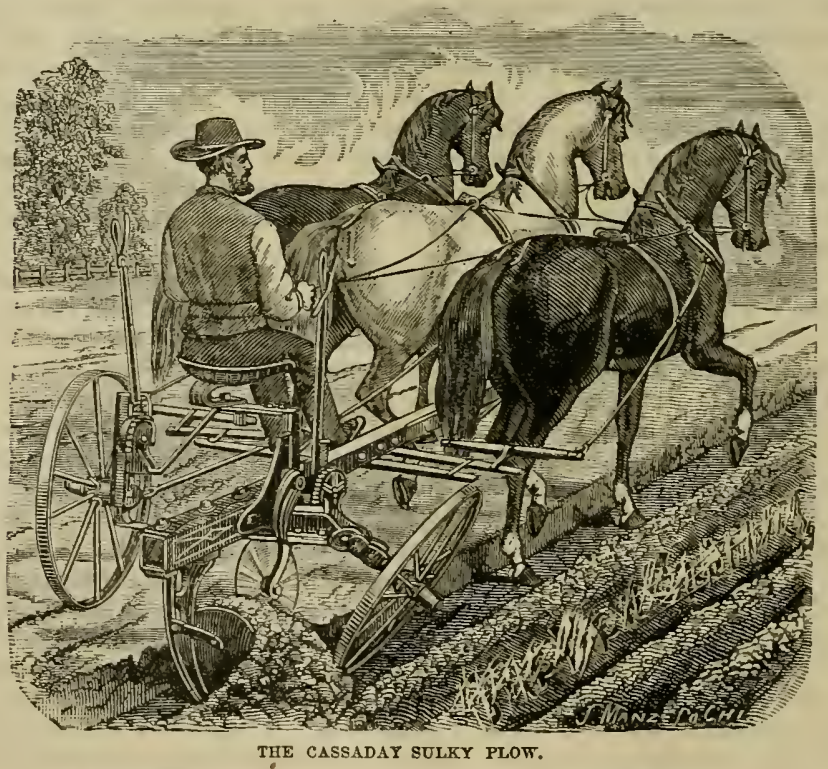

other, and therefore when it dries, innumerable cracks form over all its surface, and penetrate domnwards as the drying deepens. When the soil is so dry as not to be plastic, so that water cannot be squeezed out of a ball of it when worked in the hand, and so that when thrown up 
by the spade it does not stick to the tool, then plowing throws apart the granular masses along the lines of the dry.cracks, and the soil is adequately loosened.

Some clay-lands are very difficult to till at all in spring time. In drying, they form not many small, but a few wide cracks, and when they are dry enough below to crumble as the plow passes, the surface is already baked to clods, so that after plowing, the clod-crusher-a series of toothed wheels revolving independently, but close together, on a common axle-is needed to actually pick and cut to pieces these tough lumps.

Other clay lands that are very close-textured and heavy when wet, slack or fall to pieces as they dry, and on such soils early spring tillage may be practiced, and in short seasons may be necessary to dry out the land. This class of clays, and many heavy loams remain for a long period wet at the surface during the spring rains, as they retain water with great tenac. ity, and although they dry out finally, and come into good condition after a time, yet time may be made and seed got in earlier by putting the plow to work and actually raising the earth and shaking it in the air (as we would lift and agitate a wet cloth), for the sake of drying out the excess of moisture, which, if not removed, would cause planted - seed to rot. This dry. ing of the soil is an essential preliminary to warming it up to a point favorable for the germination of seeds and growth of young plants.

The best time to plow heavy clay lands for loosening their texture is the autumn, soon after the crops have been removed, and before the naked

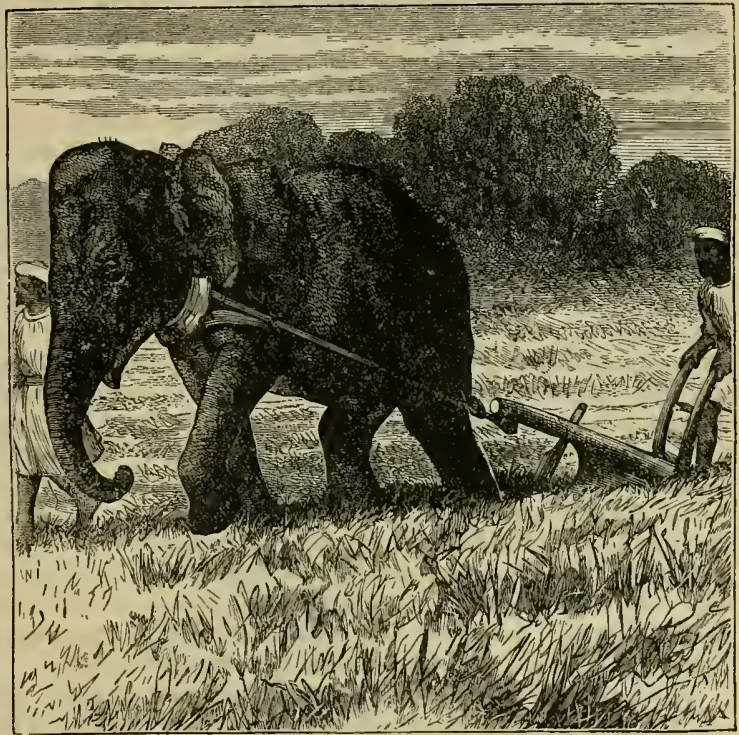

TILLAOE IN INDIA. surface hardens under the late rains. Fall plowing may be done, if needful, when the clay is wet, for if plowed dry it will become wet, and wetting is an essential part of the loosening process. Alternate freezings and thawings of wet clay will loosen it as no other agency can. Water in freezing expands ${ }_{12}^{1}$ th its bulk, and the energy of its expansion is enormous. This energy is exerted throughout the entire mass of the frozen earth, and in its finest pores. But severe frost is needful, for, under sufficient pressure, or in strong capillary tubes, as in the fine pores of brick and sandstone, water either does not freeze because it cannot expand, or it requires a temperature much reduced below $32^{\circ}$ Fahrenheit for its congelation.

The act of freezing not only effects a mechanical separation of adjacent particles of sand and silt between which the water expands into ice, but it has a special influence on the real clay. When water, turbid from suspended clay that would remain without settling for weeks 
together, is frozen, the clay particles, before so attenuated as to be invisible under the microscope, are precipitated in distinct flocky masses, which, under the microscope, transmit much light and have a granular or cellular appearance; and the water may be poured off quite clear and free from milkiness. This result of freezing clay-water appears to be quite analogous to that of freezing a thin starch paste, the starch being separated in glutinous clumps, and very like the action of rennet or vinegar on milk, whereby the casein is coagulated or curdled. Freezing thus coagulates clay, causes the swollen, gelatinous, transparent, clayey substance, so retentive of water and so attenuated with water, to discharge the liquid and condense to a visibly flocky or granular matter.

It is plain that tillage may modify the storage of water in the soil. If a compact clay or loam be loosened in its texture, subsequent rains will more readily pass through the loosened tilth and will penetrate and remain in reserve in the soil immediately underlying. This will be advantageous in two ways. It will, first, give the sowed crop an aërated and therefore healthy root-bed, and second, it will tend to conserve the rain against time of drought.

On coarse-textured soils, deep and frequent tillage may too much promote the descent of rain, and therefore be detrimental to vegetation.

Let us now suppose the surface of the several soils we instanced, after being fully charged with rain, to be equally exposed to the prolonged influence of sunshine and drying winds, whereby the water at the surface and to a certain depth evaporates. At the very sur. face the soils all become quite dry. At some distance down they kept moist, becanse, as the water escapes above, it is raised by capillary action. The larger the pores the deeper the soil dries out, because the larger the pores the less does water rise in them. It will thus happen that so long as there is water in the deeper layers of soil, so long the clay will remain moist at almost the surface, while the quantity of moisture will decrease from soil to soil, in propor. tion to the coarseness of the pores, until in the gravel it may be dry almost throughout.

That stratum of the soil which is subjected to loosening tillage is always dried thereby. A wet soil sometimes may be advantageously plowed for the purpose of drying it. A dry soil may be made too dry by deep and repeated tillage. The reasons are-1st. By loosen. ing, the soil is made to expose a vastly greater evaporating surface to the atmosphere than the compact soil presents. 2d. The capillary connection of the loosened earth with the underlying soil is impaired, and its power of taking up and distributing bottom-water is diminished. 3d. The rain that falls upon it flows more freely and completely into the subsoil. Thus its rate of losing water is increased, and the sources of supply are rendered less rapidly effective. It is evident that the amount (depth and frequency) of tillage which would benefit crops on a clay soil might damage those on a sandy one.

To conserve the water of a loose-textured soil its main tillage should be shallow, so that the bulk of the earth remains compact enough to hold the rain and to transmit bottom-water steadily from the subsoil upwards to the roots of crops. The surface only, which has been puddled by the latter and early rains, need be disturbed in spring-time to prepare a seed-bed and to cover in manure. That surface, as often as it settles to compactness or forms a crust, should be loosened up again with the horse-hoe, so as to maintain orer the water-conducting body of compact earth a relatively non-conducting layer of loose soil, to cut off the escape of moisture into the asmosphere.

Summer tillage, to conserve moisture, should be shallow, and should not extend to the roots, unless, indeed, with the object of compelling them to develop lower down and nearer to the water supply. Generally, however, the depth of the well-manured stratum is but small, and as little as possible of it should be withdrawn by frequent stirring and consequent drying from serving as forage-ground for the roots of crops.

It is, however, easy to see that the effects of surface tillage will differ according to the 
nature of the soil. If, instead of sand or soil made up of solid, coarse particles, it is a loam which spring preparation has reduced, not to dust, but to lumps like sand-grains in size, but themselves porous and easily broken to dust or made finer by the passage of the cultivator, then the loosening effect of tillage on the mass may be more or less compensated by its pulverizing effect on the lumps.

Again, unless there be uninterrupted capillary communication between bottom-water and the surface soil, surface working will not prevent the inevitable drying out of the tilth; nay, it may in the later stages of that drying-out hasten the result.

A light, loamy land, underlaid at a little depth by coarse sand, and deeper still by a loose gravel, will subject the vegetation on it to severe extremes of water supply, for when heavy rains fall, the fine interstices of the surface will remain gorged, because the coarse material below cannot suck the water down, and when, in drought, the surface-water is exhausted, there is no capillary connection between the loam and bottom-water. Subsoils are not, however, necessarily deficient in capillarity because they contain some gravel. A proper kind and amount of porosity insures the ready distribution of water, whatever be the name of the material.

The use of the roller after harrowing in seed, and of striking with the flat of the hoe upon the hill after planting, is obviously to facilitate the access of moisture to the seed. The capillary connection between the surface-soil and the moist earth below is largely interrupted by putting in the seed, for that is, in itself, a loosening process. The soil must be compacted in order to restore that connection and insure to the seed and young plant a steady and sufficient supply of water."

We therefore see that proper tillage-which means tillage adapted to the character and conditions of the soil-not only enables the land to retain a greater amount of moisture in time of drouth, but permits the air to penetrate and aerate the soil, thus increasing the vigor and growth of plants, and in various ways greatly augmenting its productive capacities. The soil, having its origin in the solid rock, and thus being abundantly supplied. with mineral elements, can never become exhausted of these, as long as any soil remains to be cultivated; but the necessity of tillage and other aids will probably always remain, in order to put these elements in the best condition to be taken up by the plants and used as plant-food.

\section{PLOWING.}

$\mathrm{I}$ $T$ is a fact worthy of note that the agricultural implements used by a nation or people are a sure index of that nation's civilization and commercial prosperity; and that where the best are in general use, there will be found the best crops, the most wealthy, enterprising, and intelligent people. The principal reliance of the farmer in performing the labor of tillage, is the plow: it may be followed by a variety of other implements, such as the harrow, roller, etc., to complete the process, but the primary and indispensable utensil is this time-honored implement; hence, plowing, being the most important of the mechanical opera. tions of tillage, should always be adapted to the character of the soil and the crops to be 


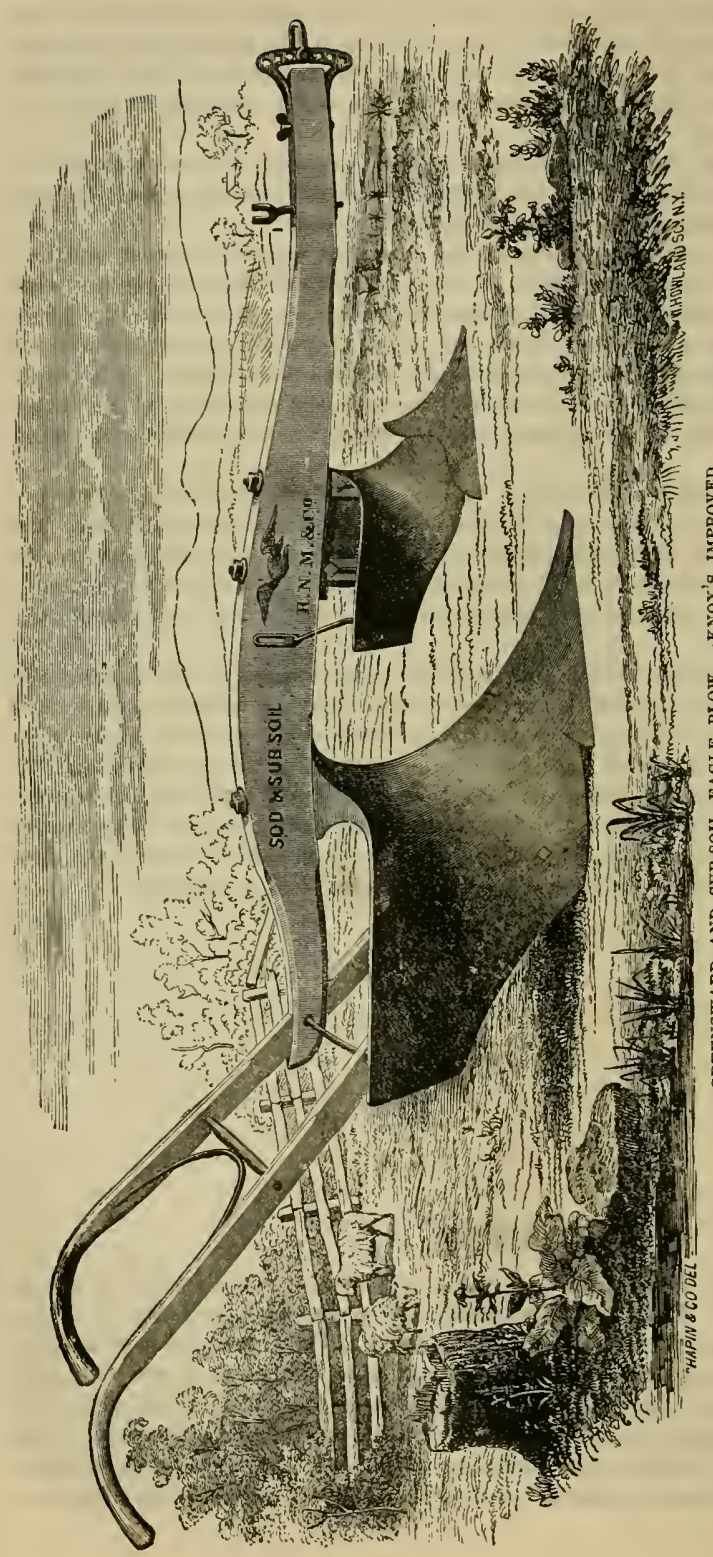

produced. Its object may be simply to invert the sod, to slightly mel. low the surface of the soil, or to penetrate into the depths of the subsoil and break it up without bringing it to the surface, or for various other purposes, therefore it is very im. portant that the plow should be adapted to the kind of labor to be performed, and the depth and time of plowing should vary according to the nature of the soil and the crop to be cultivated. Light or shallow plowing cannot be performed with a plow designed for heavy work, suited to that of the sub-soil plow, neither can deep plow. ing be well accomplished with an implement designed for light surfacework. Great improvements have been made in these implements within the past few years, and one is led, in seeing the complete success of the work performed by some of them, to almost believe that perfection has at least been reached in this department of farm utensils; but as progress and improvement are ever inseparable from intelligence and culture, probably improvements will continue to be made, although it may be diff. cult to tell how it could well be accomplished.

Iron plows are gen- 
erally preferred for stony lands, but the steel plow is better adapted to soils that are of a sticky character, like certain clays, or soft, mucky soils, that adhere to the share in turning a furrow, and with care, will prove the most durable for general use, even on stony fields. The "chilled " plows, which are produced by pouring a fine quality of iron over an iron chill or mould, giving them a hard temper and rendering them very durable, are also very good for all uses.
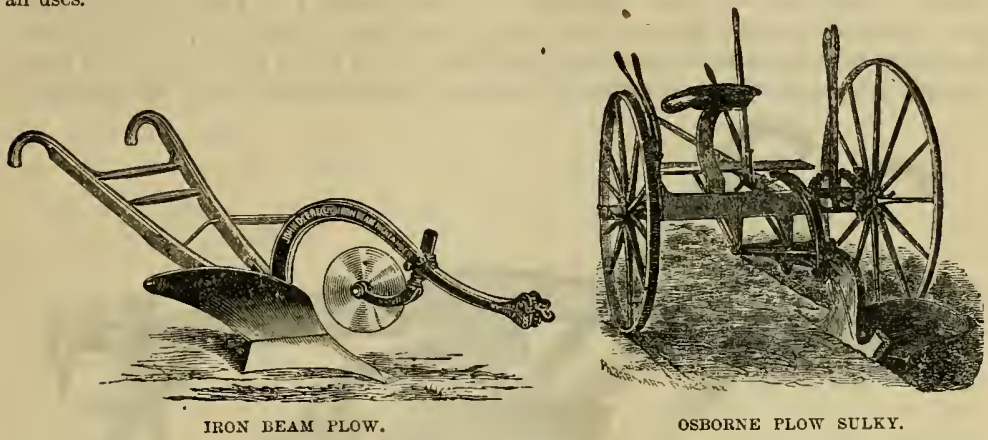

The sulky and gang plows, so extensively used on large farms in the Western States, and to a certain extent in New England, are admirably adapted to perform the most perfect work with facility and rapidity. The ease with which the operator performs his task with one of these plows, as well as the amount of work accomplished per day, forms a peculiarly-striking contrast with the excessive labor and rude implements with which our forefathers tilled the soil, and are an index of our nation's progress in the art of agriculture since that time.

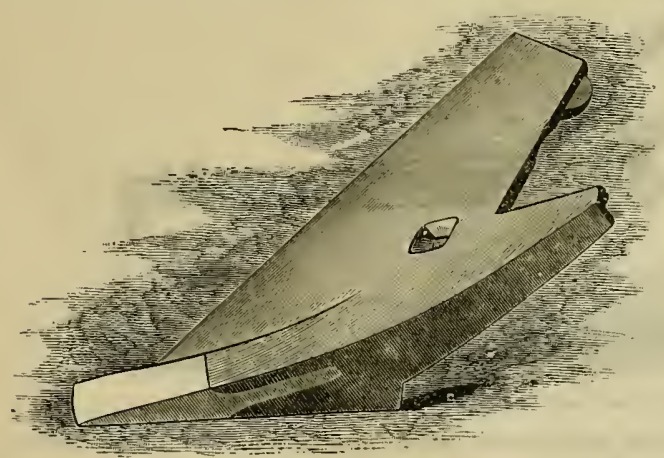

FLSED FOOT, STEEL POINT.

Every farmer should have farm-implements adapted to the different uses of his avocation, and among none of such implements is this adaptation more essential than in the choice of plows, since the yield of crops will depend largely upon the manner and thoroughness with which the plowing is performed. If the plow is a poor one, not adapted to the kind of work to be accomplished, the plowing will be very poorly done. It should be of the proper form and good material. the best being the cheapest in the end, since it will perform better work, and last longer than a cheaper implement that will soon wear out or break. Besides, a plow that is perfectly adapted to the purpose desired will not only perform better work, but that work can be accomplished with less labor and expense than with a poor plow or one ill adapted to the kind of work for which it is used. It is therefore necessary that the farmer have good plows, and those of different styles for the various uses in tillage. Some farmers, 
even on small farms, claim that at least three or four good plows of different styles are neces. sary. while on larger farms a greater number would be found necessary.

The plow which is to invert a sod in the best manner must differ from that designed for a loose soil, and which leaves it in the most desirable condition for seed or tender plants; while the plow designed to turn a deep furrow must differ widely in style from one that turns a shallow furrow. Those generally considered necessary for a small farm are a sod. plow, stubble-plow, and a light, one-horse plow for marking out lands for planting, etc., and for various other purposes that can be accomplished only by such a plow. Additions might be made to these, as the requirements of the soil and crops demand. Good implements will
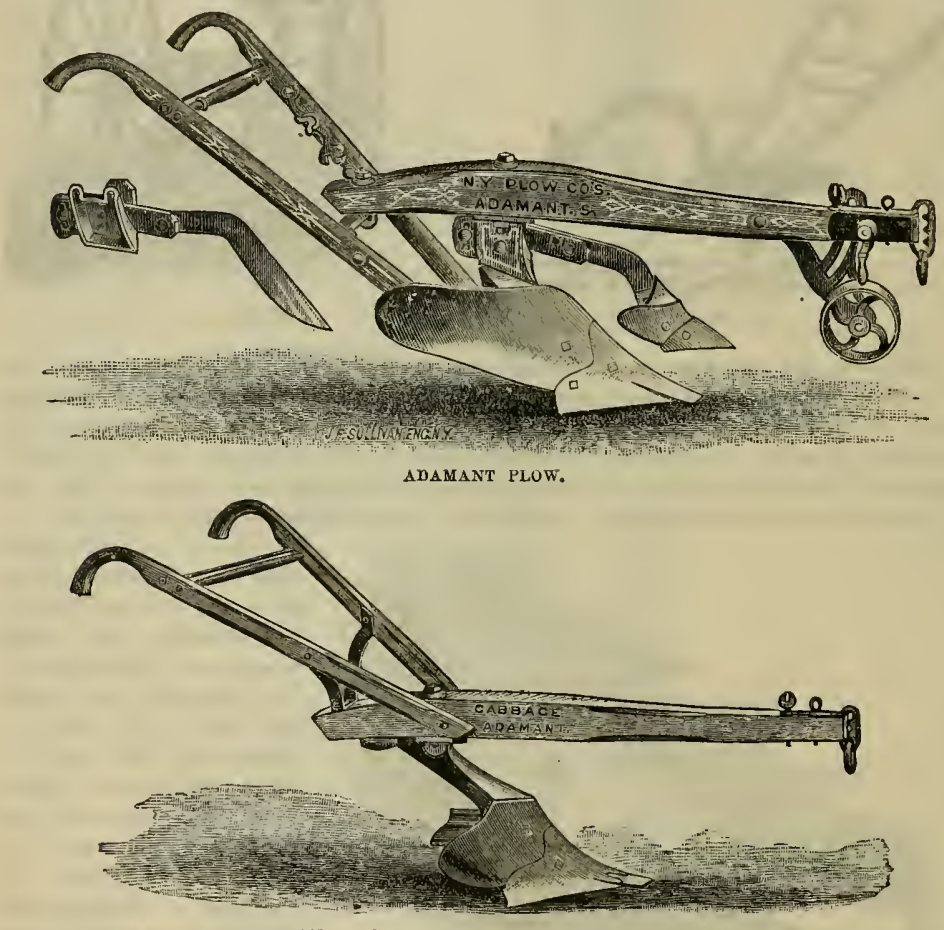

ADAMANT CABBAGE PLOW, OR CORN SUB-SOILER.

a] ways prove a paying investment to any farmer, while cheap, inferior ones will be dear in the end, in the failure of crops for lack of proper tillage of the fields, hindrance and expense from constant breakage and repairs, etc., all of which will prove a great detriment to successful farming.

When to Plow.-Some lands will be more benefited by fall-plowing, others by plow. ing in the spring, and as near to the planting season as possible. Lands that should be plowed in the fall are stiff clays, clay loams, and heavy loams, and any kind of soil that is inclined to become more compact by the rains and become what is termed, in farmers' phrase, "puddled in," Fall plowing will be greatly beneficial to such soils, on account of the action 
of the frost, which pulverizes and reduces them to a finer condition than could be effected in any other manner; besides, such soils become thoroughly aerated, and through the agency of the sun, snow, rain, and air, these wonderful forces of nature, such chemical changes are wrought by the blending and mingling of the different elements contained in the soil, that these elements can readily be taken up by the plants as their proper nourishment. The frost lifts the surface of the soil, rendering it more open, porous, and flexible for the next season's work, as well as increasing its fertility.

Lands that should be plowed only in the spring, and not until wanted for planting, are light, sandy soils, such as dry quickly in the wind. If such lands are plowed in the fall, and thus exposed to the rains, atmospheric influences, and the sun, their fertilizing properties are very liable to be washed out or exhaled, and such soils derive but little fertility from the atmosphere; in fact, not enough to compensate for the loss sustained by the exposure.

How to Plow. - In plowing in the fall, it is always well to so turn the furrows that they will lap over; one upon the other, forming what is called the "lap-furrow," which will admit of a free circulation of air by forming an air-chamber under each furrow the entire length of the field. By this means a better drainage of the land will be secured, the soil aerated, and a greater benefit derived through the agency of the frost, since not only the furrow-slice will be frozen, but the soil beneath it to quite a depth, thus breaking it up, thoroughly pulverizing it, and rendering it more porous and friable. Lands plowed in this manner are in good condition when the frost leaves the soil, and are ready for use much earlier than when plowed in the spring. Some farmers recommend ridging the entire field by turning two furrows together, thus exposing a larger portion of the surface-soil to the action of the frost than by the previous method. Lands tilled in this manner may be crossplowed and cross-harrowed in the spring: or, if this is not done, the furrows should be divided by running a plow through the center of each, and afterwards cross-harrowed.

A. W. Cheever, of Boston, a practical farmer, and editor of the New England Farmer, gives the following sensible advice on the manner of plowing a field:-

"If the field to be plowed is of regular shape, with four right-angle corners, and with no fences to obstruct the team in turning, it may be plowed and left nearly as mellow as by spading, and without leaving the track of a single hoof upon the entire field. If enclosed by fences, the team should start on one side and travel to within a convenient turning-distance from the fence, then reverse and plow back, leaving a strip of uniform width at either end. The plowing should go on back and forth, till a strip of the same width of the ends is left at the side of the field, when the team, instead of coming back at the end of each furrow, should so move as to plow on the three sides with once reversing the plow. This avoids useless turning of the plow at the corners, and prevents excessive tramping of the furrows. Where there are no fences in the way at the ends, if the first furrow turned is carted across the field and neatly laid in the bottom of the last furrow, the field will be as level and as smooth throughout as before plowing. In plowing sod-land for immediate use, whether for planting or for sowing, it is very important that every foot of soil is completely inverted and the furrow-slices laid regularly in their appropriate places, and the furrows should be of uniform depth and width throughout their entire length. If the furrow-slice is too wide for the preceding furrow, its outer edge will be lapped over the previous furrow, and be liable to be turned back during the after cultivation. If the plow dodges its work and takes more land than it can turn, ugly depressions are left, which cannot be properly filled by any amount of subsequent harrowing. It is, therefore, of the greatest importance that the plow should run steadily, and with a uniform width and depth of furrow. Many farmers use too short a chain between plow and team. With a long chain a misstep of the animals is less felt, and the plow less liable to leave its place than with a short hitch, and by using a wheel to govern the depth of furrow, the plow will run just as easily with a long, as with a short hitch, pro. 
vided the amount of work done is the same in both cases. A long yoke or whifiletree, besides being easier for the team, especially among rocks, is more necessary when using a swifel than a landside plow, as a full width furrow is more readily obtained. Farmers hare sometimes condemed swivel plows for no other reason than because they used too short yokes and chains. Farmers also make a great mistake when they neglect to use a good sharp cutter when running plows in sod-land. The difference between plowing with or without a good cutter is about the same as the difference between cutting and tearing cloth or paper. With a cutter running from two to three inches deep, and well forward of the rising furrow-slice, the latter is brought up and turned over with a smooth, straight edge, which folds down into the previous furrow, leaving no ragged or unsightly edges to be hauled back by the harrow. The cutter is also useful in clearing away stubble or rubbish, when plowing corn or grain fields, and prevents the choking and obstructing of the work of the mould-board."

Depth of Plowing. - With regard to the depth of plowing, there has been much discussion, and various opinions expressed; many eminent authorities in scientific agriculture adrocating deep plowing, and asserting that we cannot plow too deeply; others-equally good authorities-have given strong arguments in faror of shallow plowing. We believe, however, that the majority of the best agriculturists at present concur in the opinion that the depth of plowing must depend upon certain conditions, which may be as various as the character of the soils cultivated and the crops grown-some soils and crops requiring much deeper plowing than others; hence, each farmer must be "a law unto himself" in such matters, and by a careful study of the nature of his soil, and by experiment, learn the depth of soil-stirring best adapted to his own particular farm. As a general rule, however, the plowing should be as deep as the soil, or rather, the plowing should go to the subsoil, and should sometimes break up the subsoil, but should rarely bring it to the surface. A deep, mellow soil is, of course, to be preferred to a shallow one that orerlies a hard subsoil.

Mr. Cheever recommends eight inches of well-pulverized, rich, mellow top-soil, as a pretty good standard, as a general rule, to aim for on most farms, and quite as much as most farmers have sufficient manure for, and says that in order to reach the eighth inch with a common plow, he has found great benefit from the use of the steel subsoil plow, without mould-board, - a plow that simply loosens the subsoil without bringing it to the surface.

To turn a flat furrow eight inches in depth, the width should be from fourteen to sixteen inches. The principal material for plant-food is to be found in the surface or agricultural soil; hence, it is always safe to plow as far down as that soil extends, whether it be four, fire, six, or even twelve inches in depth. When the surface soil is very thin and it is desirable to deepen it, it will be well to plow an inch or so deeper each year into the subsoil, mingling it with the surface-soil gradually in this way, together with the application of manure, until the soil attains the required depth, As a general rule, it will not be safe to deepen it much more than this degree each year, as the surface-soil will be liable to become deteriorated by the mix. ture of a great amount of the raw subsoil, since time will be required for it to become suited to plant growth. Many fields have been rendered sterile by too rapid deepening of the soil in this manner, and required years of culture to recover from the effects. The reason for this is very evident: suppose we have a surface-soil of five inches, rich and mellow, overlying a subsoil of coarse gravel or hard clay; now, if we attempt to plow to the depth of eight or nine inches, and throw up this coarse gravel, or compact, lifeless clay that forms the subsoil, we bury the principal portion of the soil that can produce vegetable growth deep in the earth, away from the reach of the seed, or young and tender plants, and have upon the surface a coarse soil unsuited to plant-growth, and in which the plants would starve and die for lack of proper food; and it would probably require several years' time for the action of the air, rains, dews, frost, and the various agencies of nature that aid in the changes and decomposition 
constantly going on in the earth, to bring that soil up to the degree of fertility that it had attained before the plowing.

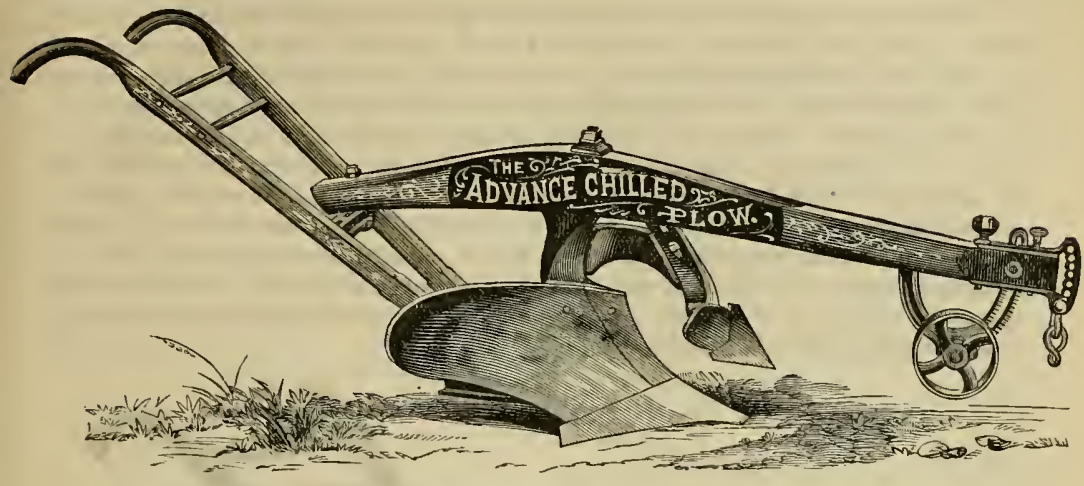

ADVANCE CHILLED PLOW,

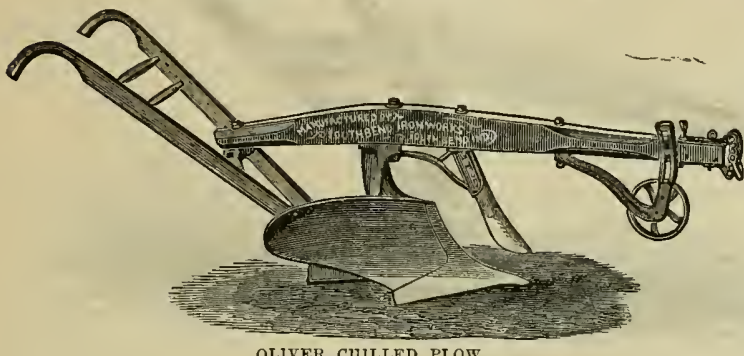

It is sometimes desirable to break up the subsoil, which can easily be accomplished with a good subsoil plow without bringing it to the surface; but the instances are very rare where benefits will be derived by mingling it to any great extent with the surface-soil, and it should always be done with great caution. When the surface-soil is very sandy, with a subsoil of stiff clay, or the reverse, such soils may be improved hv mixing the clay and sand as thoroughly as practicable, and subjecting them to the various processes of proper tillage. Soils of great value are sometimes produced in this way from such as have previously been considered of inferior quality.

Sometimes the subsoil is a hard clay, almost impervious to water; hence, in rainy seasons, the surface-soil is saturated with a surplus of water that can- $\mathrm{THE}$ : not make its escape downward through

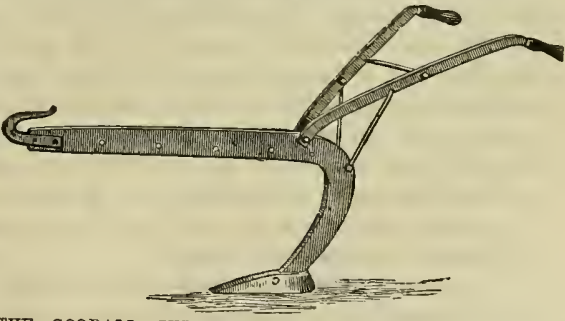
the impenetrable layer beneath, and the land must depend almost wholly upon evaporation for becoming dry enough to promote the growth of crops; in such cases, a breaking up of the hard subsoil will materially improve the condition, as it furnishes room for deep-rooted crops, and a means of drainage by opening a way of escape through it for the surplus water in the soil, and also admits of the moisture from beneath finding access to the roots of plants 
in time of dronght, while the roots also can penetrate deeper in search of the requisite amount of water.

There may be other instances where the breaking up of the subsoil would be a positive injury to crops, such as certain loams or any kind of leachy soil, with a coarse, sandy subsoil that would, if undisturbed, form to a certain extent by its compactness, a barrier to the rapid escape of water and fertilizing properties that would otherwise wash through it and be wasted. It is easy to see that the breaking up of such a subsoil would be very hazardous to crops, and the fertilizing elements contained in the surface-soil capable of being washed out by heavy rains. On old fields that may have a shallow surface-soil, and have been plowed to a certain depth for a succession of years, and never reaching beyond that depth, or, on certain soils formed from the fine sediment of a river, a hard subsoil is often found which seems to resemble hard-pan in compactness, and which is evidently rendered so in a great measure by the plow always going to a certain depth. Such soils are materially benefited by breaking through this hard crust with a subsoil plow.

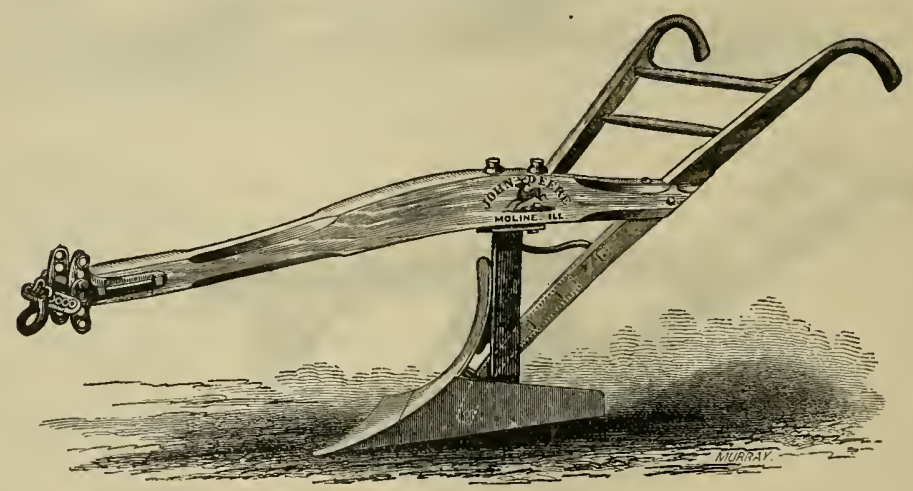

STUBBLE PLOW.

One of the first and most important things for every owner of a farm to ascertainaside from the nature of the surface-soil-is the character and value of the subsoil underlying his land. In many instances it will prove of little value, and should either be left undisturbed, or loosened simply for the purpose of giving greater capacity for supplying moisture, according to the conditions and nature of the soil, as we have previously stated. In other cases, the subsoil has been known to materially increase the value of the agricultural soil when mixed with it, but as we have before stated, it should be done very cautiously and gradually, accompanied with a mixture of manure each year.

A recent authentic writer gives the following striking instance of benefits derived from a mixture of the surface and subsoils:

"An open ditch two feet deep was cut through a portion of a field, and the excavated subsoil scattered to the distance of a rod on each side. The field was afterwards sown with wheat, the season for which was so unfavorable that the field averaged only five bushels per acre, except where the subsoil from the ditch had been scattered. Here the product was estimated at no less than twenty bushels per acre. It would have paid well to dig up enough of the subsoil to scatter over the whole field. A farmer in one of the wheat-growing regions informed us that he increased his wheat ten bushels per acre by running the plow two inches deeper than it had ever gone before, throwing up and intermixing a small portion of the subsoil." 
Several years ago, John Johnston of Geneva made in substance the following statement: "I had some 800 oak and black walnut stumps pulled, bringing up earth from four to six feet deep, and ten feet square or more on the surface. The next year I sowed the field to wheat. Where the stumps stood, the wheat was not so good as elsewhere, but it is now, after some years, in wheat again, and I never saw such wheat as where the stumps were pulled. You could see where every stump had been; the wheat is all of ten inches taller, stands far stockier on the ground, and looks as if a load of barnyard manure had been laid down and not half spread."

We are inclined to believe such instances as above quoted, rare, and as being the exception rather than the rule, although subsoils of value are common in some localities.

As we have previously stated, no definite rule can be given with respect to the depth of plowing. We have specified general rules, and stated various conditions and circumstances, with probable results, but soils differ so widely in character that different methods will be required for different fields, and each farmer, by careful experiment and observation, will be able to best determine for himself the methods most suited to his own soils, always bearing in mind the general principles relative to the character of different soils, and the relation which the surface or agricultural soil bears to the subsoil.

Clay soils, heavy loams, or heavy soils of any kind are best pulverized and made friable by plowing when dry or nearly so. If plowed when wet, they will turn over in smooth, compact furrows, which will dry in the sun into hard lumps that will require often the entire season to pulverize, and usually will remain so until subjected to the action of the frost, which has a tendency to separate their particles and reduce them to a pulverized condition.

Gravelly soils may be plowed when either wet or dry. The accompanying illustrations in this department represent several of the standard plows at present in use.

The Gilpin Sulky, Timber Land, Stubble and Iron Beam Plows represent cuts made from photographs of implements manufactured by Deere \& Co., Moline, Ill.

In the Gilpin Sulky Plow one lever varies the depth of plowing, levels the plow at all depths, and raises it out of the ground when desired. Sulky plows are admirably adapted to large farms and level lands free from stones, and are much used in the Western States. The Deere Gang Plow, -an illustration of which will be seen in connection with another department, on large farms of the country, where twenty of them are employed in a single field,-is similar to the Gilpin Sulky, except it plows two furrows at once instead of one, and is more heavy and cumbersome than the latter, being used only on very large farms. Its use requires three or four horses or mules. It is very strong and not easily broken by striking large stones or other obstructions. The Cassaday Sulky Plow, and Oliver Chilled, by the Whittemore Bros. of Boston, Mass., are also fine implements. The former is especially adapted to lands where there are stones or other obstructions, and for this reason is proving especially valuable to the farmers of New England. Two horses are sufficient for its use.

The Osborne Plow Sulky, manufactured by Gregg \& Co., Trumansburg, N. Y., has a system of levers by which the driver is enabled to regulate the depth and width of the furrows at will.

The Green-sward and Subsoil Eagle Plow, by the well-known firm of Ames Plow Co., Boston, Mass., may be made to turn furrow slices from nine to fourteen inches deep, and from sixteen to twenty inches wide when desired.

The Advance Chilled, and the Goodall Subsoil Plow, also used as a grub hook, stone and root-puller, and manufactured by Joseph Buck \& Sons, Boston, Mass., are too well.known to require comment.

The Adamant plows, by the New York Plow Co., of New York City, are exceedingly durable, being made of metal of uniform hardness. They are so constructed as to be fitted with "slip points" of steel,-of which we give an illustration-by means of which the plows can be kept constantly sharp, while the cost of repairs is thus greatly reduced. 


\section{HARROWING.}

$\mathrm{T}$

$\mathrm{HE}$ harrow is indispensable to any farm, however small, being useful in various ways, principally for pulverizing the soil, and covering the seed when only a slight covering is necessary; it is also often valuable for exterminating weeds. Its use for any purpose is most effectual when the soil is dry and capable of being crumbled or pulverized into a fine mellow condition; therefore, a field should never be harrowed when the soil is wet. But a few years since, the straight-toothed harrow, in the form of a triangle or letter $\mathrm{A}$, was the only har. row known among farmers; although a useful implement in many respects, it was a very un. satisfactory one for fitting green sward for planting or sowing, as well as for some other pur. poses for which the improved harrows in use at the present time are so admirably adapted. A thorough pulverization of the soil will well repay the labor bestowed in rendering it so, since a soil that is reduced to a fine and mellow tillage, other conditions being equal, requires less manure than one that is not thoroughly reduced to this condition, besides, it is au importaut

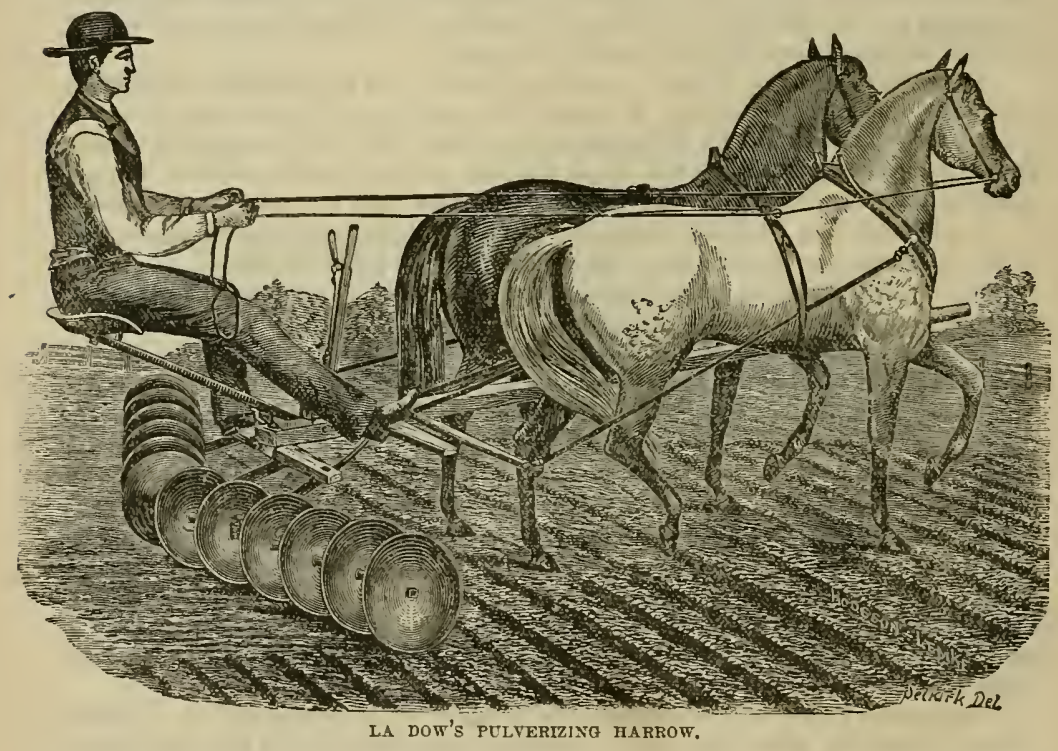

principle in agriculture, that the more thoroughly the soil is mixed and worked over, the better are the crops. Those agricultural machines that will best accomplish this complete pulverization of the soil, are of course the best implements. For reducing heavy soils, such as sodded or stiff clay lands, the efficiency of the harrow is increased by its moving quickly through the soil, and striking the larger lumps with sufficient force to break them in pieces; for this reason an active team is better then a slow-moving one for this purpose, and horses or mules are often preferred to oxen.

The above illustration represents La Dow's jointed pulverizing harrow, manufactured by the Wheeler \& Melick Co., Albany, N. Y. This is a very efficient implement for pulverizing and preparing lands for seed, as the discs are of steel and very sharp, and break or cut through all the solid portions of the soil, leaving it fine, even, and mellow. It cuts uniformly deep the entire length of each gang, and performs very thorough work by once going over 
the ground. Each disc is supplied with a patent scraper bar attachment, which obviates all difficulty in sticky soils by keeping them clean from any earth that may adhere to them; this scraper being controlled by the driver simply moving a lever toward him. The number of discs or wheels in the different sizes of this harrow range from twelve to twenty-four, and the width of the cut from six feet to fifteen feet.

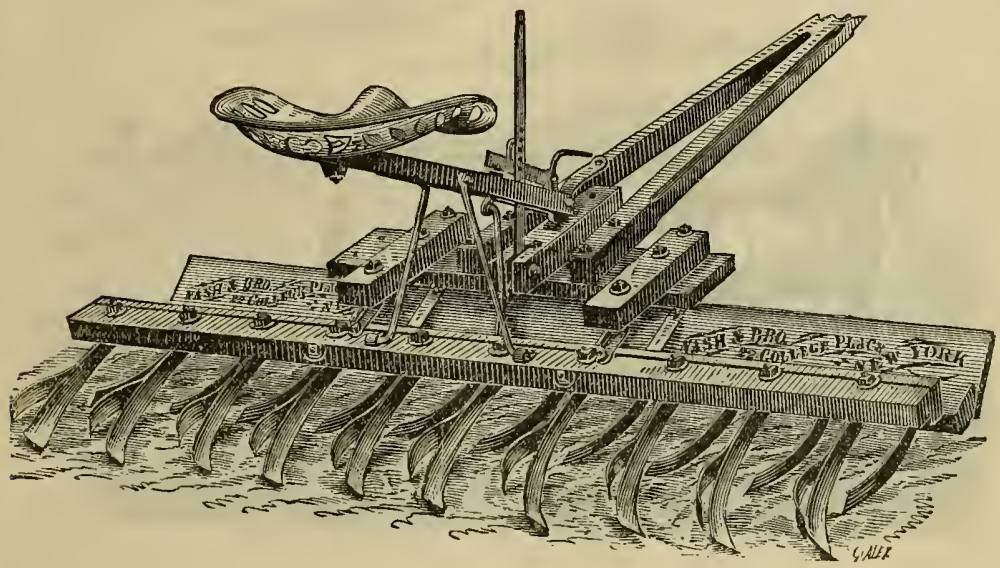

ACME PULVERTZING HARROW, CLOD-CRUSHER, AND LEVELER.

The Acme harrow, manufactured by Nash \& Brother, New York city, is a combined harrow, clod-crusher, and leveler, which very effectually breaks down the hard portions of the soil, and levels the surface, while the curved steel coulters cut and pulverize it to the

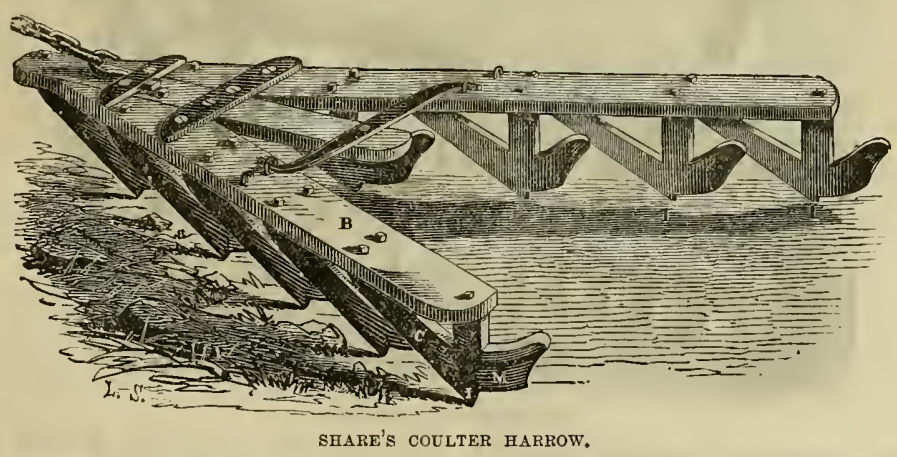

depth of four or five inches, leaving it mellow and fine. It can be made to effectually pulver. ize a field of corn stubble, or run light enough to cover grass seed. It covers seed to a uniform depth, and will also thoroughly mix commercial fertilizers with the soil. The weight of the driver while riding adds to its efficiency. 
Share's Coulter Harrow, by the firm of Belcher \& Taylor, Chicopee Falls, Mass., is an implement, the merits of which are well-known.

The Thomas and Nishwitz harrows, manufactured by Joseph Breck \& Sons, Boston, Mass., have also been extensively used. The essential principle of the former consists in placing the teeth at a double angle, both backward and sidewise in the bar, so that the

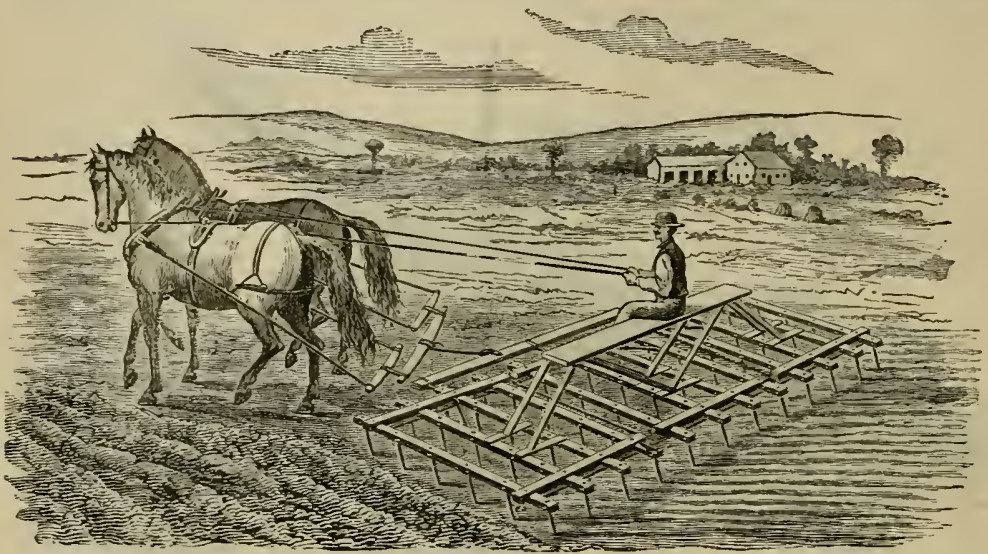

THE THOMAS HARROW.

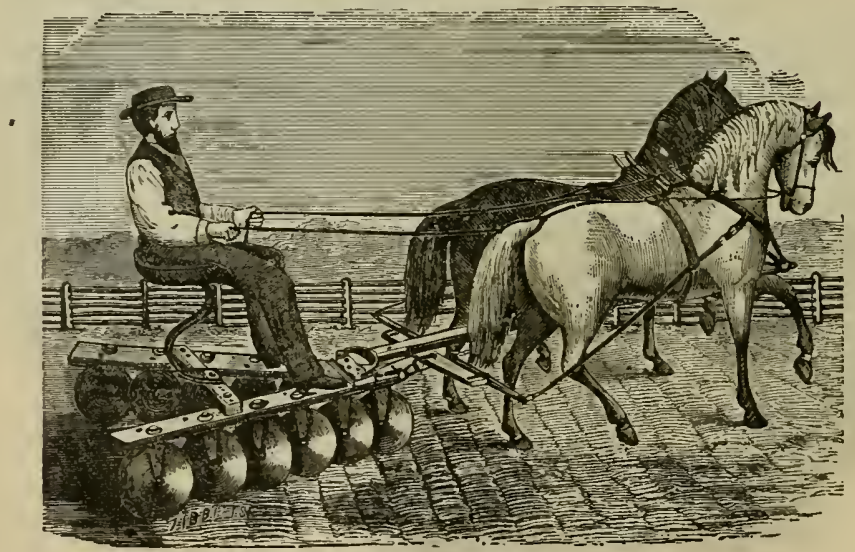

THE NISHWITZ HARROW.

position of each shall be directly backward in the line of draft, or direction in which the harrow is moving. If the bar in which the teeth are placed moved directly lengthwise, all the teeth in a bar would follow each other; and, to avoid this, the bar is made to advance somewhat diagonally or sidewise, so that each tooth will cut a slice for itself, and no two teeth follow each other. The distance between the teeth is a little less than one inch as they 
work in the ground, or about one-third the distance usual in most harrows, thus securing the perfect and minute pulverization of the entire surface. Owing to this position of the teeth, they never clog, keep themselves scoured clean and bright, and bury any rubbish, such as straw, roots, manure, etc., completely beneath the soil.

The latter harrow consists of a series of revolving concave discs, and is somewhat similar in its construction to La Dow's harrow.

\section{USE OF THE ROLLER.}

$\mathrm{T}$ $\mathrm{HE}$ field-roller is a valuałle machine for every farmer to possess, although it is more rarely seen on farms thau almost any other farm implement.

It is very useful for crushing the clods and lumps that are left by the harrow, and for pressing down stones and rendering a field smooth for a mowing machine, also in pressing the soil down upon the seed, which produces a more sure and quick germination by the increased moisture thus secured. It also consolidates soils that are too loose in texture. All lands, whether seeded down in the spring or fall, should be rolled every spring. All grass lands even are greatly benefited by its use, since the action of the frost has a tendency to throw up the ground; the tender roots are thus exposed and will soon dry up if not replaced. By pressing them back again to their proper places with the roller, an increased crop of grass will result which will abundantly repay for the labor. Its use is said to have often doublerl a crop on certain sandy soils that are not sufficiently compact to hold the roots of plants firmly, and retain sufficient moisture for their support.

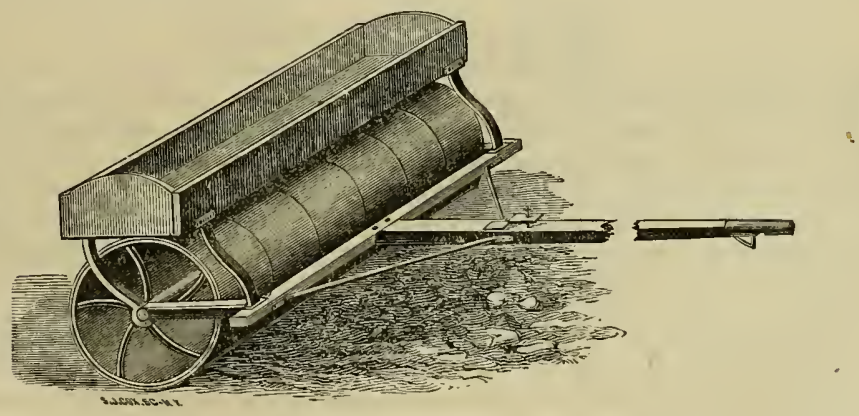

THE FIELD ROLLER.

As a general rule, the tillage operations of the roller, as well as those of the harrow, can be performed to best advantage when the soil is not wet.

Rollers are made in various ways, the material of which they are constructed being of both wood and iron; but the iron roller is the most approved kind, as it does better work and is more durable than wood. It is made in sections of cast-iron. The box attachment is very servicable for holding stones, weeds, roots, etc., picked up in the field, and for supplying weight to the roller, according to the work required.

The above illustration represents one of the best iron rollers in use.

For clay lands that have become very compact and hard, a heavy iron roller which has teeth attached to the external surface is sometimes used in breaking up the soil, but such an 
implement might very properly be superseded by the use of some of the most approved kinds of harrows for stirring and pulverizing the soil, which might be followed by the ordinary roller for crushing the smaller clods that would escape the harrow.

How to Construct a Farm Roller. On small farms where often the moderate means of the farmer would not warrant the purchase of a good iron roller, a substitute for light work is sometimes found in a log drawn over the ground without rolling.

Some farmers use a square piece of timber for this purpose, either of which will do fairly well where the lumps are not very hard, as in some clay soils. In using the log for this purpose, a pair of shafts will be needed for one horse, and a pole like that of a wagon for two horses. Of course, the round stick of timber, or log, will approach nearest the roller, and will be the more desirable substitute, as it is less liable to clog and become obstructed in the work.

A very cheap and serviceable roller can be easily made from a round piece of timber of suitable size and length, (which should be eight or ten feet; if too long it would not be conve. nient to manage in turning round,) and inserting irons of sufficient size and strength in the center of each end, as axles on which the $\log$ shall turn. The box attachment can also be added, and a roller, simulated after the common iron roller in finishing, be made to answer the requirements of a small farm. Of course, it will be necessary that the log of which it is made should be as nearly straight and as near the form of a perfect cylinder as possible. Almost any farmer can find such a log in his woodland, and but little ingenuity and labor would be required in the construction of such an implement, which would be found rery serviceable on those farms where the iron roller is not used.

\section{WHAT CROPS TO RAISE.}

$\mathrm{F}$ ARIING is constantly becoming more of a mercantile business than formerly. In the earlier days, it was the custom for farmers to raise everything for home use, even the clothing worn by the family, and scarcely a farmer was known who did not produce his yearly crop of flax and wool for this purpose. The old maxim was, that farmers should sell and not buy. At present this idea is in a great measure ignored, and although it is customary for farmers to raise what is used on the farm, as far as practicable, there is a tendency towards the opinion that division of labor, or specialties, are as applicable to farming, as to other kinds of business.

That the shoemaker should endeavor to make clothes, hats, and bonnets for his family, mould be thought absurd; and that the tailor should attempt to make the shoes, furniture, etc., for his household would be considered, to say the least, very poor economy, when by working at his trade he could earn many times as much as those articles would cost, and the purchased goods would be in every way superior to any that he could manufacture. That the farmer should endeavor to cultivate everything consumed, or in demand upon his farm, without regard to the expense attending it, the nature of his soil, the relative market value compared with other crops, the demands of the market, and other considerations, would also be very poor policy, since the real profits, or the attainment of the largest possible excess of receipts over expenditures, is the aim of the farmer as well as those engaged in other depart. ments of business.

A Judicious Choice of Crops Essential. There are few things that have so important a bearing upon the success or failure of the farmer's business, as the choice of crops to be produced. Of course his success also depends upon many other considerations in connec. tion with this one great essential, such as the manner of cultivation, judicious management, 
and other things too numerous to mention, which, if ignored, would fail of giving success, even with the most desirable choice of crops that might be made; but where all other condi. tions are favorable, a judicious choice is one of the first essentials to success, and a failure or error here will inevitably lead to disastrous results, - such results as no amount of labor and care, or skill in culture, can to any great extent ameliorate.

Choice of Crops Modified by Demand. It will be generally conceded that the choice of crops is to the farmer what the selection of goods is to the merchant. The selection of goods is to the merchant, a consideration of the first importance in his success, and his study must be to learn the wants of his customers and meet their demands, both in kind and quality of goods with which to continually replenish his stock. If he fails to do this, he soon loses custom, for no one would think of buying things he may happen to have on hand, simply to accommodate him, when they were not what was wanted; and if he did not have the goods desired, his customers would go elsewhere for them, and other merchants would gain the trade that he had lost. One of the great principles of mercantile business everywhere recognized is to meet the demands of the trade, and if this be ignored, fallure of success must inevitably follow. This same principle is equally applicable to the agricultural pursuit, since the farmer as well as the merchant depends upon the sales he makes for the profits in his business, the only difference being that the merchant purchases his goods for sale, and the farmer prodnces his from the soil by skill and labor in cultivation.

If the merchant requires skill and judgment in determining the wants of his customers, and in selecting his goods with a view to meet those wants, so does the farmer require an equal amount of skill and judgment to meet the demand for the class of products he is to supply. And if in the mercantile business, there is constantly a demand for something new, the old going ont of fashion to give place for the new, - so in farming, is there no permanency in the demand for certain products. What is most in demand now, may not be required by the markets a few years hence.

New varieties of fruits and other products are constantly being introduced, some of which find a more ready market than those formerly cultivated, and it is the farmer's business to learu what kinds of products and the varieties of these that are most in demand, and will consequently bring the highest price, and cultivate these. In many instances the varieties most popular with the purchasers may seem no better to the farmer than the old; but since it is but right that the consumer who pays his money for the products should be the one to decide which he will buy, the farmer will find it for his interest to cultivate the kind desired. In other words, the farmer, as well as the merchant, must keep up with the times and the demands of the age in which he lives in order to be successful. The farmer then, as a general rule, must keep himself informed with respect to the state of the markets at which he is to dispose of his crops, and raise such as are in demand there.

Principle Slould be Regarded. There are, however, exceptions to the above rule governing the choice of crops. We cannot recommen it only as far as it does not iuvolve a violation of moral principle. Popular demand should always be subservient to that, and no truly honest man would sacrifice principle for the profits that may be the result. We have a profound respect and admiration for an old New England farmer, who in a time of general scarcity of apples, being offered a high price for those from lis large, well-bearing orchards, for the purpose of manufacturing cider-brandy, replied, "No! though I want the money, I'll let 'em rot upon the ground before I'll sell 'em for such a purpose!"

If all farmers were equally true to principle with respect to the disposal of their products, there would be less perversion of the good and useful, and what the Creator designed for man's sustenance, into evil, and that element that destroys annually morally, mentally, and physically, so many of the human race. He who administers to an evil habit or depraved 
taste, aids in debasing his fellow-man, and is himself debased by the act. To all farmers we would say, -in the cultivation and disposal of your crops, be true to the principle of right and honor; let that be the standard of choice always, and the popular demand secondary to this.

Choice of Crops Modified by the Character of the Soil.-Another very important consideration, in determining the kind of crops to be cultivated, is the character of the soil. No farmer can be very successful in his business who does not understand the nature of his soil and the crops to which it is best adapted. The soil of some farms is better suited to grass and the rearing of stock; others for grain, roots, and other cultivated crops; others still for fruit culture principally. Some farms are better adapted to certain particular kinds of stock-those for special purposes; others still for specialties in farming, while many are suited to a mixed husbandry, or the cultivation of many of the farm products.

In making a choice of crops, therefore, the farmer will find it to his advantage to understand the character of the soil he is to cultivate, as well as the demands of the market, and produce those for which his lands are best adapted. If he attempts to cultivate products that are best suited to a dry, warm soil, in a cold clay or wet lands, or the reverse, he will generally meet with failure, unless by underdraining the wet lands or otherwise changing the condition of the respective soils, they become suited to the growth of the plants for which they are not naturally adapted. Again, some soils may possess more of certain kinds of elements of plantgrowth than of others; for instance,one soil may be more deficient in phosphoric acid than the other desired elements of plant-food; another may have an abundance of phosphoric acid, but be lacking in potash, etc., the same principle applying to a deficiency of any of the elements of plant-food, the stores of which may be capable of a partial or complete exhaustion in a soil. Fortunately for the farmer, science has come to his aid and pointed out to him the way to restore the elements that may be deficient in his lands, through over-cropping without sufficient fertility restored to the soil, or for other reasons, and when once he has determined the great want of his lands, he may, by applying it in the form of commercial fertilizers or farm manure, so change its nature and condition as to adapt it to the successful cultivation of crops that it would not otherwise produce.

He may use special fertilizers for special crops, or he may use barn-yard manure, if it can be obtained in sufficient quantities, since it is a complete fertilizer for all crops, and the commercial fertilizers are not. Special fertilizers that are complete can, however, be made for certain crops by a proper combination in kind and quality of commercial manures, which have given remarkable results even in soils not especially adapted to their production. Barnyard manure is more slow in its results thon commercial fertilizers, since it requires a longer time for it to become assimilated to plant-growth, being coarse in texture, while commercial fertilizers, being reduced to a condition to be readily taken up by the plants, act more quickly in stimulating the growth of crops. For this reason, where barn-yard manure is applied alone, the yield is always modified more largely by the adaptation of the land for the particular crop under cultivation, than where commercial fertilizers are used, and much of the fertility in the soil is left over to the following year, while the fertilizing properties of commercial manures are usually mostly extracted during the first season. With skillful management with special fertilizers-those adapted to certain products-many crops may be grown successfully on lands that otherwise were not well adapted to their production; still, as a general rule, the best permanent results are attained on lands naturally suited to the crop to be cultivated. It is well, however, for the farmer to bear in mind that one of the most important things to be considered is the necessity of thoroughly understanding; as far as can be, the nature of the soil he is to cultivate. The value of this knowledge cannot well be overestimated. The soil is the element with which the farmer has to deal, and unless he possesses a good knowledge of it, and knows the crops to which it is best adapted, he cannot expect to 
succeed. If he mects with any degree of success while working in ignorance, it is by mere chance that he reaches the result-a kind of "guess work" without knowledge of the facts - that lead to it. What could be expected of a mechanic who attempted, in the pursuit of his business, to use tools constructed in a complicated manner, when entirely ignorant of their use and construction? Equally presumptuous would it be for the farmer to expect to cultivate intelligently and with successful results the most complicated of elements combined-the agricultural soil-when entirely ignorant of its composition, or of what it was best adapted to produce.

Relative Cost in Production, etc.-Another important point to be considered in the choice of crops is the relative cost in production, and the value of different crops when harvested. There may sometimes be crops in popular demand in the market, for the production of which the soil of the farmer may be admirably adapted, but the expense of which production may so far exceed that of other crops less in demand, that the latter may be found to be the most profitable. When it costs a farmer fifty per cent. more to produce a bushel of potatoes than it does corn, for instance, or any other crop, and the former brings but twenty-five per cent. more profit than the latter, he will find it for his advantage to cultivate the latter, since by so doing he realizes a larger per cent. of profit; that is, his receipts are larger in excess of his expenditures than on the more expensive crop. It is not profitable to raise large and expensive crops that do not bring a large proportionate profit in return. The fact should be kept in mind that that farming is most profitable that brings the largest returns for what is expended. Farmers should keep an accurate account with respect to the expense of each crop (including cost of fertilizers, expense of cultivation, harvesting, marketing, etc.), and determining relatively what the cost of production and receipts of their sale are, they will thus learn which pays best and which are the most unprofitable. By so doing, a decision as to which are the most desirable to raise can easily be reached.

Where it will be found that the crops for home consumption on the farm can be grown cheaper than they can be purchased, it will, of course, be best to cultivate enough of such, at least, as are necessary for that purpose; but where certain products for home production can be bought for less money than the farmer could grow them himself, it will not pay to cultivate such, when money and labor could more profitably be expended on other products. By giving due thought and attention to the subject of a choice in crops, and taking measures to so inform himself as to obtain all the light possible on the subject, the farmer will be liable to make a more judicious choice than otherwise; and having once taken such steps in the right direction-although he may make some mistakes, and often obtain benefit from such experience-yet lie will, in the main, with other favoring conditions, meet with success. The farmer who profits by the experience of the past, and wisely appropriates to his use the advantages within his reach that the present affords, must win success, however close the competition with which he meets, or difficult the obstacles he has to overcome.

\section{ROTATION OF CROPS.}

$\mathrm{I}$ $\mathrm{T}$ has become an established fact in agriculture that the continued growth for successive years of the same kind or family of plants on the same soil is one of the surest and most speedy means of impairing, and, in many instances, rendering that particular soil unfit for bearing further crops of that kind. More especially is this true if the crop matures and ripens its seed, like the various kinds of grain, etc. It has also been ascertained by long practice, that if a proper system of rotation be adopted, exhaustion of soil will be greatly deferred, and that by the use of fertilizers, which will return an equivalent 
for the elements extracted from the soil by the production of crops, the exhaustion can be largely prevented. Iet, eren in such cases, it is found that for all soils a change by way of rotation is the most satisfactory in the result upon both the soil and crops produced, most writers on the subject claiming that the application or withholding of manures only serves to retard or accelerate this process of exhaustion.

As a general rule, the poorer the soil the greater the necessity of diversifying the crops, consequently the greater the necessity of a rotation, and those crops that are wholly removed from the soil in root, branch, and seed, will exhaust lands sooner than those that permit of a portion remaining on the lands to fertilize it, such as stubble, etc. Eren on tlie rich lands of the $W$ est, it is found that special crops, such as wheat, cannot be cultivated year after year on the same soil without deterioration. When lands became unproductive among the earlier cultivators of the soil, it was conciuded that it was because such lands needed rest; hence, the fallow system was introduced, which was a common practice among the Romans. Their usual course was to permit the land to rest after each crop; a crop and a year's fallow generally succeeding each other, although, where manure was applied, two and sometimes sereral crops were taken between the fallowing periods. Among the ancient Egyptians the fallow system was almost unknown, since their agriculture was confined to the banks and lands adjacent to rivers having an annual overflow, which inundation caused a rich deposit of mud to be left upon the surface yearly, thus furnishing a rich top-dressing sufficient to keep the soil in constant fertility. The practice of changing the crops with more or less regularity, although found to be attended with beneficial results, has never been quite satisfactorily explained.

Theories Relative to the Necessity of Rotation of Crops.-Various theories have from time to time been advanced relative to the cause of the failure or depreciation of the same kind of crops produced continuously from the same soil. One of these theories formerly was, that plants, in growing, exuded or threw off from their roots waste substances, which rendered the soil unfit, to a certain degree, for the production of the same variety of plants, until time had neutralized the deleterious properties thus imparted, but that those properties given off were not injurious to other kinds of plants. This theory is now generally discarded. Another theory, and the one now generally adopted, is that different kinds of plants exhaust certain elements from the soil in different degrees, and that this explains why a change in the production of crops is beneficial. This may be a true reason to a certain extent, but it does not fully answer the question, since it is found that different crops, requiring about the same elements of plant-food in similar quantities, do not affect the soil in the same manner, or, in other words, that the slight difference in the proportionate elements of plant-food of different crops does not account for the great difference between the alternation of these crops, and the successive following of the same, as is the case with wheat and corn, as instanced by the following from Prof. Blount, of the State Agricultural College, Colorado:-

"In the vegetable, as well as the animal kingdom, it is the infallible law of nature that constant cropping and continual feeding of one thing to the exclusion of all others, tend to reduce the strength, vigor, growth, and product. Now corn takes from the soil only about $6 \frac{1}{2}$ pounds of its whole substance when dried, and wheat 7 ponnds. All the rest of the matter comes from the atmosphere in the shape of carbon, oxygen, hydrogen, nitrogen, etc. If a succession of croppings be made, it necessarily takes from the soil these elements to a greater or less degree. Both wheat and corn, chemists tell us, take up the same essential ele. ments, but they fail to make the process or operation clear enough to show why one crop following itself lessens, and following another does not lessen the yield. Corn has its own natural habit of extracting food from the soil; so has wheat. The operation of both cannot be alike, or the exhaustion, lessening of the yield, and the same condition of the soil would 
follow rotation. It is very evident that wheat leaves the soil in a better condition for corn than it does for wheat again, and corn leaves it better for wheat. Clover, peas, and sweet potatoes are among the best crops for preparing or leaving the ground for any crop. Wheat does much better after Irish potatoes and tobacco in some States, than after any other grain or vegetable.

Several instances have come under my observation where sweet potatoes were raised for 10 to 20 years in succession, without any apparent exhaustion, but with great fertility to the soil. In looking into the chemistry of this subject, we find in the analyses that wheat contains about 55 per cent. of starch, and corn 70 per cent. The gluten in the former runs from 10 to 19 per cent., while in the latter it is about 12 per cent. Now if the soil contains a certain average supply of these and other inorganic substances upon which the plant feeds, and the same wheat or corn crop is grown in the same soil year after year, the crops will carry off some of these substances in a greater proportion than others, so that they will become relatively less every season. The result is, the soil will become so impoverished of these substances that no crop of the same kind can be raised, although it may contain a large store of other inorganic substances. When these crops are grown one after the other, one draws especially upon one class of elements and the other upon another, thus keeping up the equilibrium of fertility necessary to support either plant.

In connection with these operations there must be two kinds of exhaustion-general and special-the former consisting, of gradual extraction of all the essential elements upon which each crop feeds, and the latter consisting of a want of one or more of these elements."

There evidently are other reasons for the difference, in connection with the many theories advanced, which are not yet understood, and which are not necessary for the farmer to understand, since he has to deal with the fact itself, and its relations to agriculture. He can afford to leave the explanation of this phenomenon to the agricultural chemists, and give his attention to the benefits that may be derived from a knowledge of the facts. We find that

Nature Generally Follows a Course of Rotation in her various departments of vegetable production. Although mowing lands part with their annual crop of hay, and by a partial rest, or a pasturage of their aftermath or "rowen," will remain fresh and seem to retain the orngnal plants that produced the crop, yet on a close examination it will be ascertained that the varieties of the grasses and other plants will change gradually from year to year, some kinds predominating at one time and others at another, in a continuous round of rotation.

But this fact of change is more strikingly illustrated in the succession of forest trees, which cannot fail to attract the attention of even the most casual observer. We distinctly recall, among our childhood memories, a grand old forest of oak, walnut, and chestnut. Many of these monarchs of the forest succumbed to the fate of the woodman's axe. Soon appeared in their places a thick growth of white pine, which increased with remarkable rapidity. These pines were subsequently cut down, and maple, chestnut, and oak came up with scarcely a pine tree among them. And thus it is always found that when a forest is cut down, and the roots destroyed, another growth of trees succeeds of an entirely different nature, and these are followed by still another variety, completing in time a rotation all probably pro. duced from seeds that may have lain dormant in the soil for centuries, waiting for favoring conditions to germinate.

It is often found that when a soil becomes exhausted to a certain extent by one particular crop, and ceases to give a sufficient return of it, it will still yield a large crop of some other kind that is adapted to it, and which calls for just the elements of plant growth that this soil may contain; this is not only a fact concerning field crops, but the same is true of gardening, and the culture of trees, etc. There would be found few gardeners of experience, if any, who would think of cultivating cabbages, turnips, or peas, on the same piece of land 
without an interval of at least two or three years; and although in some special cases, they may be grown successfully on the same soil for several consecutive seasons, this would be an exception to the general rule; the general and most successful method being to have an interval of two or three years between the crops and this interval to be occupied by plants entirely different from them.

Dr. Daubeny has experimented by cultivating crops on the same and different plots in successive years, and given the arerage taken from five years as follows:

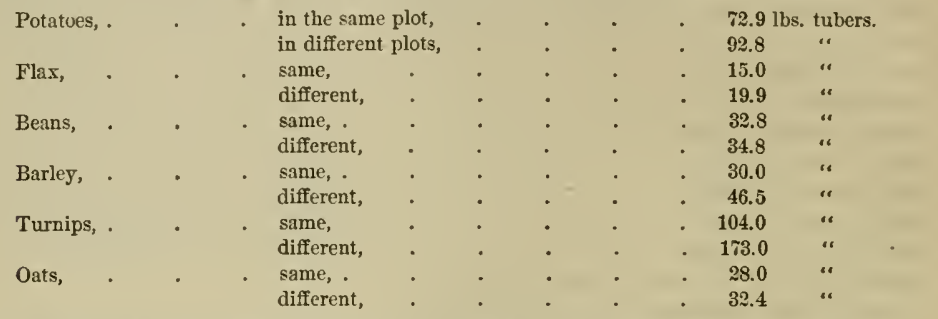

This shows an advantage in change, which varies from one to seventy-five per cent.

A Massachusetts nurseryman, eminently successful in his business, says: "I have witnessed the most extraordinary effects follow the carrying out of the principle of rotation of crops. To illustrate the point, I will say, that in the preparation of the ground for appletrees, for instance, we are obliged to prepare it very nicely, in order to be successful; as nicely as for a vineyard, or for garden vegetables. We plow deep, manure thoroughly, and then, in setting out the small plants, the seedling apples, we calculate that the ground is in a fit condition to carry those trees almost to their maturity. Now you will see that the grow. ing of a crop of apple-trees successfully, which requires three or four, and sometimes five years, exhausts the soil of all its ingredients which the apple-tree calls for, or perhaps any deciduous tree calls for. A nurseryman who understands his business knows that it would be folly for him, after he has taken off that first crop, to attempt to put a second crop upon that land, even if he manured equally as well as he did the first time, because his crop has exhausted the soil of certain things which are peculiarly necessary; and which can only be found in newer soil. My practice has been this: after my apple-trees have been removed, I find the land admirably adapted to the growth of evergreen trees, such as spruce, arbor vitæ, hemlock, etc. Without remanuring that land, without any repreparation, almost, except it be plowing, I can set out evergreens, and get an admirable crop, because the elements which the evergreens call for still exist in that soil; because the elements which the erergreens call for are different from those which the apple-trees call for. I grow them three jears, and then they pass away. What is the condition of the soil then? It is exhausted for the erer. green, it is exhausted for the deciduous tree, and you might say that the soil was entirely exhausted. But such is not always the case. I may plow that land thoroughly and lay it down to grass, without even putting any manure upon it, and raise a splendid crop of grass.

We believe it is because the grass calls for different elements in the soil from either of the varieties of trees specified, and the farmer will find the same principle true respecting his crops." For this reason orchards planted upon old orchard sites seldom do well. The super. intendent of gardens and grounds for the department at Washington states that the same results have been found in the culture of the grape. For a number of years past, it has been customary for the department to propagate several thousands of plants, embracing many varieties of native grapes. These are mostly grown from single eye cuttings in sand beds under glass, and placed singly in pots when rooted. About the end of May they are turned 
out of the pots, and planted out in the open field rather closely, in rows which are about three feet apart. When they have finished growth for the season they, are lifted and removed from the field; the ground receives a coating of rotted manure, which is either plowed in or worked with a spade, leaving the surface rough to be acted upon by the frost. In the following spring the surface is again worked over, and the soil placed in good order for planting. At the proper period young grapes are again planted as before. These are removed at the end of the season, and the ground receives similar treatment to that of the previous year. Notwithstanding this treatment, the third crop is very indifferent, and if a fourth successive crop is planted, it will prove to be an entire failure.

Experience shows, that by selecting a field which has never been occupied with grapes, the young plants will make an average growth of about four feet in length the first year; the average growth of the second year will reach about two feet; the growth of the third year will be exceedingly weak, the best plants reaching to about eighteen inches in length, many weak kinds not reaching the length of one foot.

This result of diminishing yearly growths has not been sensibly affected by the application of different manures, and the question naturally arises, that if a deterioration of growth becomes so marked in so short a time, and with such attention to the soil, what may be expected when acres are closely planted with grapes, as in the case of vineyards, where the entire soil speedily becomes filled with roots? It need not be a matter for surprise if vineyards become unproductive after producing several satisfactory crops.

It is a fact well-known to florists, that even the smaller plants require a change, and such small growths as petunias and verbenas, if continued for a few years on the same ground, will not give satisfaction, however richly fertilized with different manurial applications, and that when it is desirable to grow these plants year after year in the same spot, it is necessary to remove six or eight inches of the soil, and replace it with fresh earth from other sources.

Clover, which is the great renovater of the soil, may be cultivated upon the same land until it becomes "clover sick," and ceases to produce it.

In his experiments at Rothamstead, Mr. Lawes found that with occasional variations due to the character of the seasons, the average annual produce of a certain field for twenty successive years without manure was sixteen bushels of wheat per acre, and sixteen hundred weight of straw. This soil was a strong clay loam resting at a depth of five or six feet upon chalk, and probably produced a larger yield under the circumstances, than most soils. In the case of turnips, when treated in the same manner, he found that after a few years they ceased to grow larger than radishes, and he could not afterward by the application of any kind or quantity of manure obtain a crop equal to the first. The result was very different with the wheat experiment above referred to, for by the application of four hundred weight of Peruvian guano, the crop was at once doubled.

Strawberry plants require a constant change of ground, in order to do well, and are constantly seeking to occupy new territory by throwing out their long "rumners;" and it is said, by those familiar with the cultivation of mushrooms, that they never rise in two succes. sive seasons from the same spot.

Nature has in such, and various ways, sufficiently indicated the law of rotation as the law of successful growth, and we doubt whether any agriculturists can improve upon it. Exceptions to this law are found, but they are exceedingly rare, change being the great demand for all varieties of plant-growth.

By a judicious system of rotation, the soil not only has the different elements of plant. food extracted in various proportions suited to the growth of different crops, thus preventing the exhaustion of a few elements suited to one variety, but permits certain crops, that might be injured by a direct application of manure, to have the benefit of that which has become thoroughly decomposed and blended with the soil, and hence, suited to its successful cultura 
As some crops require more cultivation than others-such as corn and potatoes, for instance, more than wheat or oats-which cultivation necessitates the stirring up of the soil at intervals, and permits the air to permeate it more effectually, thus hastening the changes in its mechan. ical condition and bringing its elements into a state to supply plant-food, and also clears the soil of troublesome weeds, a rotation with such crops is exceedingly beneficial. Besides, the destruction of the weeds also cuts off, in a measure, the food supply of noxions insects, and further reduces the numbers of these pests by disturbing them in their haunts, and in autumn exposes them to the frosts, all of which have a tendency to destroy them.

Still another benefit in rotation may be found in the fact that while some crops are almost wholly removed from the land upon which they have been cultivated, others, like oats or wheat, leave a portion of their product in the form of stubble; or, like clover, leave a waalth of fertility in their long roots, all of which supply more or less organic material to the soil, which, when suitably decomposed, greatly aids in the production of the future crop.

Schemes of Rotation. The choice of crops for rotation will differ with different soils, climates, and conditions; consequently, in deciding upon a scheme for any particular farm, varions considerations must be regarded, such as the nature and capacity of the soil for production, the demand of the markets which may be accessible for the disposal of the crop, and the quantity and kind of manure to be applied. Each farmer will have to decide these for himself. $\overline{\mathrm{I}} \mathrm{t}$ is well to have a scheme made out of from four to six of the best crops, and the lands given to the culture of these in successive order. In all cases the best results will be attained when the soil is abundantly fed with fertilizers of some kind for each crop.

The general rule for a farmer to base his scheme of rotation upon, is to cultivate as large a variety of crops as his soil, circumstances, and the demand of the market will render profitable, and to have the scheme so arranged that the same, or a similar species of plants, shall occupy the same ground at intervals as remote as practicable. In the English practice, called the "four-field or Norfolk system," which is considered, for that country, one of the best for friable soils of fair quality, in which half the lands are in grain and half in cattle crops annually, a great variety of changes may be introduced, which will bring the interval between the same kind of plants on the same soil, one of eight years, instead of four, for one or two of the more important crops.

This is described by Thornton in the following, which we insert simply as an illustration of how it may be accomplished: "Instead of a rigid one-fourth of the land being each year under turnips, barley, clover, and wheat or oats respectively, balf only of the barley division is frequently in practice now sown with clover-seed, and the other half cropped on the following year with beans, peas, potatoes, or vetches. On the same set of fields, coming round again to the same point, the treatment is reversed by the beans, etc., and clover being made to change places. An interval of eight years is thus substitnted for one of four, so far as these two crops are concerned."

Hon. C. C. Andrews gives the following information relative to rotation in agriculture in Italy, based upon personal observations:

"The system of culture and of rotation of crops is not everywhere the same in the valley of the Po. The most fertile land is never left in fallow. Above Turin the rotation is usually Indian corn one year, then wheat two years, followed with clover one year. Around Milan the rotation is, first, white-bearded wheat, sown in November, with clover sown the following Fehruary. The wheat is harvested in July; the next month some clover is cut, and then cattle are allowed to feed in the field. The second year four crops of clover are cut, the land having been periodically overflowed. In the succeeding winter the ground is manured, and clover is cut the third year. The fourth year the ground is plowed once, harrowed four times, sown with hemp in March, and rolled. The hemp is cut in June, during which month the ground is again plowed once, harrowed once, and planted with Indian corn, which is 
harvested in October. The ground is then plowed again, harrowed once, sown in November with wheat, which is harrowed in. The soil about Milan is easy to work, only one pair of oxen being required to draw the plow, while toward Mantua, on account of the stiffness of the clay, five pairs are required.

In the rich rice district of Verceil a three-years' course is the more common, but the best cultivators, including Mr. Malinverni, follow a six-years' rotation, as follows: First year, wheat (which was sown the preceding October, after the harvest of rice, and after deep culture and manuring at the rate of one cwt. of guano per acre.) Clover having been sown in the wheat in the spring, a fair crop of this is cut in August, after the wheat crop. Second year, the clover is hberally manured, irrigated, and cut three times. Third year, Indian corn manured with guano. Fourth, fifth, and sixth years, rice. The rice-fields are inundated five months. There is a depth of 4 inches of water till the grain is up, then the quantity of water is lessened. Some sorts of rice grow to a height of 4 feet, others less. It costs 20,000 francs a year to supply a rice-farm of 600 acres with water. The rice-crop is gathered in September, the harvest sometimes running into October.

In the country above Turin it is a practice to plow twice after a crop of wheat-once at the time of manuring and again at the time of sowing-and but once after a crop of Indian c orn. In preparing the soil for hemp, it is usual there in the autumn to cover the ground with heaps of stubble and brush, in such number as 300 heaps to an acre, and to burn them slowly. This is called half manuring. In spring some manure is added, the ground plowed, and the hemp sown in April. Among the smaller farmers on hilly land, the rotation is: wheat two years, manured each year with stable-manure; the third year, rye, with clover to a part of it. Timothy is not used in the Po Valley."

In this country, it is generally deemed desirable to have grass for one of the principal crops in the rotation system. It is thought by many farmers that wheat succeeds better after peas and corn than after any of the other crops. The following rotation, practiced by $\mathrm{Ml}$. Waring, is considered by those that have followed it a desirable one for some sections: Grass is followed by corn; the next year the land is occupied by either potatoes, carrots, or sugar beets; then follows green forage crops (generally oats or corn), and when the land is cleared of these, winter rye is sown in the fall. The next year the rye is sometimes cut while green, for fodder, and other forage crops are grown upon the land which are ready to be cut earliest. In the fall of the same year wheat is sown, and the land seeded with timothy and clover. When the grain is harvested the following season, the grass remains and soon shows a vigorous growth.

Peas or clover plowed under is thought to be one of the best preparations for wheat; and old pastures, plowed under in the fall, are generally considered precursors of fine crops of corn, while corn-fields are, in turn, followed by good pastures.

Grass followed by corn, then a green fodder crop, and this followed by wheat, and afterward grass again, is recommended by some agriculturists as a good rotation for the Northern and Middle States. Of course, as a general rule, each crop should have its hiberal supply of manure, in some form.

The following systems have been adopted by many in this country and found admirably adapted to certain soils: Grass is followed by corn, the land being heavily manured, and ashes and lime added, if suited to the soil. Gypsum is applied to the corn-plants after the first hoeing, which is as soon as it is well out of the ground; the second year, some kind of root crop occupies the ground; th9 third year, either wheat, barley, rye, or oats, with grass or clover; the fourth year, grass or clover, as the case may be. A rest is often given such lands by pasturing for a year or two.

Another:-Grass followed by corn, or a root crop of some kind; second year, oats or barley; third year, peas or beans, removed early, and sown in the autumn with wheat; fourth year, wheat, which can be followed by grass or clover, or both. 
Grass may also be followed by peas or corn, then wheat; some root crop, followed by barley, with grass and clover seed, which will produce a good crop of grass the following year. In this way, changes can be made in rotation almost ad infinitum, and, as we hare previously stated, a scheme for such can best be made when the character of the soil, and the crops desired are known, since soils differ so greatly that a rotation for one section, or farm even, may not be suited to another, and only the general principles of rotation can be given as applicable to all. We have aimed to lay down general rules simply, the details of which each farmer can best arrange to suit his individual requirements and circumstances.

It has been found by long practice that better results are reached when two crops of grain are not cultivated in succession on the same field, though corn may be an exception to this rule; also, that certain products are mutual fertilizers, being so mysteriously related that the growth of one is the best preparation of the soil for the other.

The following rotation for wheat, corn, oats, peas, and clover, is recommended by the editor of the Country Gentleman:

"Two modes of rotation are adapted for these crops, the most common being to invert sod, and plant corn on it, with good manuring the previous autumn or winter on the surface; follow with oats, barley, or peas; and the same autumn sow winter wheat with a moderate seeding of timothy, and the next spring, clover-seed. The field may remain in grass any number of years, according to the number of your fields. This rotation is modified where the brown cut-worm is prevalent, by first sowing wheat on the well-inverted and pulverized sod, and follow this with corn and the other crops already mentioned. The decaying sod and the manure which is applied give a good crop of corn."

Of course, a rotation suited to one portion of the country would not be adapted to another portion where the products were very dissimilar, such as the Northern and Southern States.

With respect to rotation of crops in the Southern States, Mr. C. W. Howard, of Georgia, says:

"A great defect of Sonthern pianters is, that they do not keep, in the way of fertility, what they get. That is to say, when they make a piece of ground rich, they afterward con. tinue to work it in exhausting crops until all the richness is gone. The true policy is not merely to keep the ground rich, but to make it richer. To illustrate: If a piece of rich land is put in cotton, it may be followed with corn, small grain, with clover being sowed among the corn in A ugust. If the clover is allowed to occupy the ground for two years and to go to seed, even under a longer rotation than the above, it will not be necessary to sow it again. As soon as the ground is at rest, it will be covered with young clover. Three years ago a piece of ground was put in turnips, manured in the ground with farm-yard manure. The turnips were eaten on the ground by the sheep. The next year it was put in corn; the next in cotton worked very clean, and the following year in oats.

After the oats were cut a fine stand of red clover appeared. This seed was never sown, but must have been in the manure applied three years since to the turnips. This is not a solitary case. Many similar instances have occurred within the observation of the writer. It is such plants as clover and peas that not only hold, but increase the fertility of the soil.

In order to illustrate the writer's views of diversified husbandry, the following rotation of crops is submitted as one suited to the agricultural condition of the Sonth.

Tre will suppose a farm of 500 acres of open land under fence. Let 250 acres be devoted to arable purposes, and the rest to grazing. The rotation might be as follows: 1. Cotton and corn in the same field in suitable proportions. 2. Oats sown in August on the cotton and corn land. 3. Rye, or rye and wheat, sown in September, the land having been twice plowed in order to kill the germinant oats. 4 and 5 . Clover, if the land is in sufficient heart to pro. duce it; if not, the fourth year rest ungrazed, and the fifth year sheep and cattle penned upon it every night during the year, using a portable fence. An ordinary farm of 500 acres will 
support five hundred sheep, besides the crop in the above rotation. The oats and rye will feed them during the winter nearly or entirely, without injury to the grain. Five hands would be sufficient to work such a farm and take care of the live-stock."

During the first year with this practice, the same authority thinks the following results might be expected from an ordinary farm.

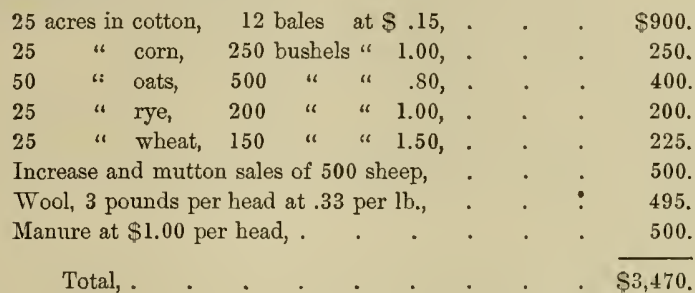

When taken separately each of these estimates in production is small; they however result in the aggregate to more than $\$ 600$ per hand employed. Yet this is about three times what was estimated to be the average product per hand in the cotton states, that of Georgia having been estimated to be $\$ 209$; South Carolina, $\$ 202$; Virginia, $\$ 211$, and North Caroliua $\$ 214$; these being the lowest averages.

In the case above supposed, the amount given is the result of the first year of the rotation.

"The next year the cotton and corn would be more thau double by penning 500 sheep at night on 50 acres. It is the writer's experience that ten sheep regularly penned will manure well, one acre in a year. Five hundred would, therefore, manure well fifty acres. The appear. ance of the ground would not indieate this high manuring; but it should be remembered that the liquid manure, which is equal in valne to the solid, is not visible. If, in addition, a stock of cattle is kept and penned on the same fifty acres, their fertility would be increased in proportion. It should never be forgotten that accumulating, saving and applying manure, is as important a business of the farm as making corn or cotton. At the end of the fiftl year of this rotation, the change in the farm would be equal almost to a transformation, the crops being doubled or trebled, without (which is a most important point) any material increase of labol or other expense.

"This improvement of the soil, accompanied at first by moderate profits, and with a great diminution of vexatious and unreliable labor, should be the great end of the Southern planter. It involves a double profit from increased production and increased salable value of the soil."

Prof. Pendleton, one of the leading agricultural authorities of the Southern section, recommends for the warm lands of the South, a rotation of cotton for two years, followed by corn on the most productive portions, and wheat or oats on the rolling lands, with a fourth year of rest, the land to lie fallow. According to his opinion, cotton will give better results for two successive years, providing the soil is not very deficient in vegetable material, than if an intervening crop is made to occupy it. For lands badly exhausted by constant cropping without sufficient manure to return an equivalent for the elements exhausted, this fallow system may prove quite beneficial; but as a general rule it is thought by most writers on this subject, that the plowing under of green crops, such as clover, peas, etc., combined with the application of an abundant supply of manure will prove more beneficial to most soils than fallowing.

Wherever practicable, pasturage may form a part of the rotation with profit. Especially so on farms where the live stock interest is one of the principal features in the management. By using a portion of the farm for keeping cattle and sheep a few years, and afterwards till- 
ing it for crops, while a field lately tilled is in turn taken for pasturage, the various tilicd fields may often be utilized with profit in completing the rotation.

Sheep especially, are very beneficial in improving the fertility of the soil, as is seen in English husbandry. They can be as successfully reared at the South as in other sections of the country, since it has been found that they are as healthy in the cotton-growing states as those of the North, while the time of grazing is much longer at the South than at the North, thus giving a better opportunity to utilize pasturage in rearing them.

Besides, cotton seed will furnish a cheap and nutritious feed in winter, and aside from the wool product, sheep on cotton plantations are worth their keeping simply for the weeds and briars they will destroy, and the fertilizer they furnish to the soil. We believe that the proper management of sheep on cotton plantations, will prove one of the most potent aids in restoring fertility to pastially exhausted lands, as well as maintaining the fertility of such as have been properly tilled.

Dr. Lawes, of Rothamsted, England, found, in his experiments on unmanured rotation, extending over a period of more than thirty years, that upon an unmanured soil, where the crops were entirely removed, such cereal products as wheat and barley continued to give a considerable yield after what are commonly described as restorative crops have almost ceased to exist on the land. He refers to them as follows:-

"The experiment may be described as a struggle for existence-not one, however, where the fittest survives, but rather where each crop is allowed to get what food it can from an ordinary unmanured soil, while other plants are prevented from interfering with its operations. This experiment has been going on for thirty-four years, and adds one more to the many proofs we possess of the fact that the graminaceous plants which yield the cereal crops have a far greater power of obtaining food from an unmanured soil than any other description of plants. At the commencement of the experiment the land was in high condition, and the first crops of both turnips and clover were large; since then the former have arrived at a point where they resemble weeds rather than turnips; while the red clover, when repeated at the end of the second period of four years, died off altogether; it was then considered advisable to grow in its place beans, which, except on one occasion, have proved a very small crop, amounting to not more than about eight bushels per acre. The last crop of barleythe ninth of the rotation-yielded between twenty-six and twenty-nine bushels per acre, and the wheat crops have also continued fairly good. We have, therefore, evidence that upon an ordinary soil of fair quality, the cereal crops can obtain a supply of food, where the so-called restorative crops have failed to do so."

Thatever the system of rotation may be, or whatever the nature of the soil, it is well for the farmer to bear in mind that good crops cannot be produced without proper management, and that that management has for one of its main features, a sufficient supply of manure to furnish the elements of plant food to the soil that is expected to produce so abundantly. If we are to have satisfactory results in the rearing of live stock, we expect to feed these animals with a sufficient supply of proper food; but many farmers seem to think that lands can con. stantly produce large crops with only a meagre supply of plant-food furnished for this pur. pose. This is a great mistake, as any farmer whose practice involves this principle will learn sooner or later, for as a general rule. lands will produce and remain in a state of fertility and exempt from exhaustion, only in proportion to the proper amount of fertilizing material applied to furnish the elements of growth to the crops they produce; and if they are constantly forced by successire cropping without this aid, exhaustion must follow as a natural result. 


\section{IRRIGATION.}

$\mathrm{W}$

ATER is not only one of the sources of supply of plant food, since all the materials that promote vegetable life must be in a soluble form before being available, but it also furnishes the elements essential to plant life and growth. Most countries, as a general rule, receive this necessary element in sufficient quantities to favor vegetable growth in the supply of rain and dew; but in many sections of different climates irrigation, or the artificial application of water to growing crops, is one of the essentials in successful agriculture. In this country, crops often suffer more from drought than from all other causes combined, and this evil seems to be extending, especially in the west and southwestern portions, where, as the forests disappear through the agency of the axe and fire, the rains are less frequent. The yearly amount of rainfall may not be, and probably is not, much diminished, since this change, but the intervals between the showers are more prolonged, and to the extent that the earth frequently becomes parched and the crops suffer accordingly. Besides, the rains coming between such long intervals generally descend in torrents, and although a portion of the water will be absorbed by the soil, a large portion flows into streams and is lost as far as agricultural uses are concerned. Forests have long been regarded as the regulators of rainfall, and if they could be in a measure restored by tree-planting, in a few years the evil would be largely remedied, and the rain-storms more frequent and less violent. Irrigation is found to be one of the most primitive of agricultural arts, the remains of reservoirs and canals for this purpose still existing in those regions where civilization had its origin, as among the most ancient human relics. Egypt and the Barbary States, Syria, and other parts of Asia, Italy, Spain, and other portions of Europe practiced irrigation extensively hundreds of years ago, and to this means may be traced the high rank in agriculture that was early attained in these regions. Lombardy and Piedmont average from thirty-seven to thirty-eight inches of rainfall during the year, most of it falling between March and October, the irrigating months, and yet it is estimated that $1,000,000$ acres in Lombardy are irrigated by works costing $\$ 200,000,000$, which fact explains in a measure why that section should be one of the most productive of all Italy, and the very garden of that region. In Italy the canals for irrigating purposes are owned principally by the government; in France there are no government canals, they being generally built by the land-owners, yet are periodically inspected by government engineers. In our own hemisphere there are many portions of Spanish America where irrigation has always been necessary to successful agriculture; beside, there are vast tracts, both on the east and west of the Rocky Mountains, which are usually considered an arid desert, and estimated to be equal to nearly one-fifth of the productive area of the United States, portions of which have been irrigated and found to be productive in an astonishing degree, especially for the common cereals, and we believe the time is not far distant when that entire section will, by artificial watering-whether it be by artesian wells or otherwise-be made productive, and its value to the country proportionately increased. Utah furnishes a most remarkable illustration of the wonderful effects of irrigation, which, since the year 1847 , has transformed the Salt Lake Valley, then a barren wilderness, into one of the most productive regions in the world, and this has all been accomplished by means of irrigation alone. Whatever objections we may have toward the Mormons in their religion and practices, we must give them the credit of having one of the most perfect systems of agriculture by irrigation known. There are there at present from four hundred to five hundred irrigating canals, many of them of considerable size and length, and these have not only completely changed this formerly barren desert into a land of luxuriant vegetation and beauty, but have been the means of producing other important and beneficial results, such as cooling the temperature of the atmosphere, and lessening the evaporation of the water of the lake, etc. Prof. Paul A. Chadbourne gives the following respecting this change:- 
"We find now the valley through this entire territory, instead of being a dry, barren plain, over which the wind used to pass so rapidly, a country covered with vegetation, with grass, corn, grain of various kinds, and with groves of trees, so that the wind, in the first place, moving up through the valley, is checked by the trees, and when near the surface of the earth, probably does not move up the valley with half the rapidity that it formerly did. On the surface, instead of passing over hot, barren sand, it passes over fresh herbage, so that we have all through that valley a layer of air that moves comparatively slow, and at the same time is charged with moisture, and when it strikes the great Salt Lake, instead of being a rapidly moving current of air, hot and thirsty, it is moving comparatively slow, is nearly saturated-with water, and has no longer the ability to take up the waters of the lake as it formerly did. Therefore the waters accumulate, and so over the whole surface (and this is true of all these places) evaporation is prevented by the introduction of trees to prevent the rapid movement of the currents of air, and also by covering the whole surface of the earth with this vegetation."

The moist atmosphere of the British Islands and the German provinces, and the generally plentiful summer rains of the Atlantic States, have thus far obviated the necessity of resorting to any extent to artificial watering in these regions, except in market-gardening, but it would be well for many farmers if, during certain dry seasons especially, they possessed the means of irrigating some of their crops, and thus obviate the great loss often sustained by drought. This can often be accomplished with slight expense where the lands are suited to it and the means of irrigation convenient.

All waters are beneficial for irrigating purposes, except those that contain an excess of certain mineral substances that are injurious to vegetation, such as peat-swamp drainage, water from saline and mineral springs, and from ore-beds of various kinds, these often con. taining a solution of iron. Waters that have washed other soils often contain a vast amount of fertilizing stibstances, which they will deposit in the gradual process of irrigation; consequently after heavy raius and floods, the brooks and rivers are usually heavily charged with fertilizing materials. Dr. Dana estimated the quantity of humus and other fertilizing substances which were borne to the ocean, past Lowell, on the Merrimac river, during a year of unusual freshets, to be about 840,000 tons, or enough to have sufficiently enriched 100,000 acres of land. We have already referred to the fertilizing properties of the serwage of cities in previous pages, and a repetition is consequently unnecessary here; suffice it to say, that when such water can be utilized for irrigating purposes, the results on vegetation are often almost incredible. In any case, water should never be permitted to remain stagnant on the surface of lands, both for sanitary reasons and on account of its injurious effects upon the land and vegetation; saturating the soil with an excess of water being equally injurious to a drought. These injurious results are often seen in charging the sub-soil with water, and rendering it cold and "sour," as it is often termed, to the roots of plants which pierce it; it also facilitates the decomposition of both organic and inorganic soluble matter contained in soils; and carries off such matter from it, leaching it of its fertilizing properties to a certain extent. This is sometimes disputed, but it is proven by the fact that constituents of vegetable growth are found in water drained from cultivated fields, and also that grounds impregnated with salts to such a degree as to be incapable of vegetable production, have been made fertile by washing with fresh water. We quote the following on the advantages of irrigation from a treatise on that subject by Hon. George P. Marsh:-

"In elevated and mountainous districts, water is usually abundant, and its sources so numerous that almost any land-holder may secure one or more of them for his own sole use, without clashing with the rights and interests of his neighbor. Hence the division of the soil into comparatively small estates is promotod; for though, in new countries like ours, moun. tain-lands are thinly inhabited and held in large tracts, yet well-watered hill-pastures gradually rise in value, and these at last become the homes of a comparatively dense population, each 
of whom is the lord-not of square miles, indeed, but-of acres of productive soil.

In such territories, irrigation does not injuriously affect the health of the population. Malarious influences are exerted not by flowing, or even by freely-percolating water. It is only when the fluid stagnates on the surface, or in the soil, that it becomes pernicious. In the hills, the inclination promotes the swift flow of water over the ground, or along the canals, and its descent by infiltration is also too rapid to admit it to become a cause of vegetable putrescence. In Central and Southern Europe, almost all the surface of the mountains which has not been swept away by torrents is irrigated through the summer; but fevers and other malarious diseases do not occur in those regions, and they are regarded by many European physicians as especially salubrious, even for persons affected with pulmonary complaints.

The beneficial effects of irrigation in mountainous countries are not confined solely to the watering of the crops. It checks the too rapid flow of the waters of precipitation, and thus exerts an important geographical, if not climatic, influence. A large proportion of the water permitted to spread over the surface, and meander along the canals in the ppland meadows and pastures, is absorbed by the earth and slowly filtered down, refreshing the roots of the plants it encounters in its passage, until at a somewhat lower level it bursts out in the form of springs. It is a familiar observation in all the older American States, that the hills are growing constantly drier and the herbage less abundant, and that the springs which formerly supplied this stock are disappearing. The principal cause of this disastrous change is undoubtedly the destruction of the forests which once clothed the crest of every mountain, and which, it is earnestly to be hoped, will soon be at least partially restored. The replanting of the woods is a slow process, and the continued drying-up of the soil is every day rendering it more and more difficult. In the meantime, the introduction of a general system of irrigation at the highest levels where water can still be found, aided by the excavation of simple reservoirs on the hill.tops, and at other higher points for retaining the water of rains and melting snows unitil it can be applied to the surface by canals, or absorbed by the earth, would do very much to retard the unfavorable chango which is now taking place in the water-supply of our mountain farms, and would, at the same time, greatly augment the product of our grassgrounds, and often of our plow-lands.

It has been observed in Europe, that draining the soil, either by surface or by under. ground conduit, tends to increase the suddenness and violence of inundations by promoting a too rapid discharge of the waters into river-channels. Irrigation in the mountains, or even on the plains, has the contrary effect by retaining much of the water until it can be returned to the atmosphere in the form of vapor. Draining, then, deranges the harmony of nature by interfering with her methods of maintaining a regular interchange and circulation of humidity between the atmosphere, the earth, and the sea. Irrigation is in effect a partial return to the economy of our great material parent, by regulating that circulation in a manner analogous to her primitive processes.

Where springs are numerous, as they usually are in hilly countries, only small and cheap canals, easily accommodated to the accidents of surface, are needed for the diversion of water from its natural channels, to flow over the surface of the ground or to moisten the roots of the grass by infiltration from the artificial water-courses. But the moderate extent and capacity of the necessary canals is not the only advantage of an inclined and undulating surface in the supply of water for the crops. Hilly and winding slopes admit of a simple and efficient mode of irrigation, or rather of a substitnte for the practice which is not a vailable on level soils. The method in question has been practiced with success in many parts of the United States, where it is known by the name of circling, and it is very highly recommended by all European writers.

It consists in horizontally terracing the slopes, or even simply furrowing them with the side-hill plow, and leaving the surface permanently in this condition. The rains and 
melting snow are arrested by the hittle platforms and ditches thus produced, and gradually sink into the ground instead of running off the surface, and thus supply sufficient moisture for vegetation. It has been found that even in the parched provinces of Southern France, soils thus treated produce a vastly increased amount of herbage, or of other small crops, and this system, as has been observed in regard to ordinary methods of irrigation, has a collateral advantage of serious importance in countries subject to inundation. The water of precipitation, which soaks into the ground, instead of rushing swiftly into the tributaries of great rivers, and suddenly swelling them into raging floods, is retained long in the soil, and finally carried off by slow subterranean conduction, or restored to the atmosphere through absorption and exhalations by vegetables, or by direct evaporation from the surface, and thus equilibrium is restored. In a large part of our territory, then, and especially in that best suited to the important branch of dairy husbandry, irrigation would not only be unattended with many of the evils which are in some degree inseparable from it on soils of a champaign-configuration, but might be introduced at a very moderate cost, and probably with very beneficial results, to our agricultural and other social interests."

Methods of Irrigation.-Various means are employed for irrigating lands, depending upon the surface to be irrigated and the water-supply, the usual source being from springs or streams from an elevation. Sometimes reservoirs are made where the water from rains or inundations is collected and utilized. There are many localities where an adequate supply of water can be obtained without resorting to streams or reservoirs. Prof. Marsh states that in Italy, Spain, and many other countries, much of the land is irrigated with water drawn from common wells by cheap machinery worked by horse-power. Artesian and tubular wells are also largely employed for the same purpose, and copious springs are often reached by driving short tunnels into hill-sides.

In Persia, tunnels for this purpose, of incredible length and very simple construction, are frequently excavated. Chardin describes these tunnels in the fourth volume of the Paris edition of his travels, 1811 , and states that they are carried to a distance of twenty or thirty miles, and sometimes much more. This would be almost beyond belief, were not Chardin's accounts confirmed by the recent testimony of Colonel Chesney. On sloping grounds in Lombardy, which receive a subterranean supply by infiltration from mountain lakes and streams, water is cheaply obtained by what are called fontanili. These are small reservoirs, excavated to a moderate depth, and connected by open ditches extending up the slope to small springs, whose water is collected in barrels sunk in the ground to receire it, and if the supply is insufficient, the water from several small sources, aided, perhaps, by an artesian well of little depth, is united, and the whole conducted to a common receptacle.

Another excellent method, practiced with much success in France, is that of conducting the rain and snow water from hollow slopes of grass-ground of a considerable surface into cisterns, or into filtering-receptacles, consisting merely of a relatively small extent of sand or porous earth laid over a pavement or bed of clay some four or five feet below the surface. In this way, a large proportion of the precipitation received by the slope is retained, and perennial springs are formed at a less expense than is very frequently incurred in conducting water from only a moderate distance. Contributions of this sort deserve encouragement, because they render the farmer independent, both of his neighbor and of the public; and even if the first cost of the works is somewhat greater than that of a canal from some source not his own, he will generally be, on the whole, a gainer by procuring a supply absolutely at his control.

In the irrigation of the Salt Lake valley by the Mormons, the mountain streams were utilized; cznals being dug all along towards the base of the mountains, which prevented the water from going into the lake until it had first permeated the soil. These canals are kept full of water, and have small canals connecting them with the lands to be irrigated below, 
and which convey the water to them. Each man's land must have a supply in order that it be perfectly irrigated; consequently, by a system of coüperation, one man bas the use of the canal so many hours on a certain day, and another man the length of time allotted him when his turn comes, and so on. Prof. Chadbourne says, respecting their system of irrigation:-

"Every man knows the time when he can turn the water on his land, and when it must be turned off; and no matter whether it is midnight, or cock-crowing, or any other time, when that moment comes, he must be ready to turn the water on his land. And not only that, but before that time comes, the ditches must be cleared out, and everything arranged, so that, when the water is turned on, it will go where it is needed. There is no time to lose. The whole system makes men wary and watchful, makes them look out beforehand. A man knows, for instance, that to-night, at twelve o'clock, he may turn the water on to his garden for three hours, and that when it has run for three hours, his neighbor can turn it on to his garden, and if he oversleeps, his garden must go dry; there is no help for him. Or if his ditches are not prepared, so that the water can run along readily, his crops must suffer. You see, the man must have everything in readiness, the ditches all arranged properly, and when the time comes, he takes the water from the large canal, and it passes along through the smaller canals in his grounds the length of time that is allowed him, and then the next man takes it. It is so arranged that each man shall have enough for the particular crop that he raises. Nothing but the most perfect coöperation, under a rigid system, could possibly control that thing, among so many people, and with oo many interests."

In California, artesian wells are largely osed for irrigating purposes during the dry season. Hon. Marshall P、 Wilder, in a visit to the valley of Santa Clara, in that State, saw on the premises of one gentleman, three artesian wells, varying from 320 to 340 feet, which gave a constant flow of water. The strawberry grounds there, when irrigated, produce fruit during the entire year; the irrigation being accomplished by carrying the water along the head. lands in wooden flumes about eighteen inches square, stoppers being inserted opposite the spaces between the rows of strawberry plants, so that the water can be turned on or shut off at pleasure. These grounds were supplied with a mile and a half of such flumes.

Dr. A. L. Kennedy gives the following statement relative to irrigation in California: "The lessons on the art of irrigation, taught by the experience of Califormia, have a value far beyond her boundaries, and should be carefully studied by the farmers in not a few of her sister States. Having the lofty Sierra Nevada just inside her eastern line, and the ridge of hills called the Coast Range, parallel with and near to the Pacific, most of her territory is constituted of a vast interior valley. The northern half of this great hydrographic basin is drained by the Sacramento; the southern by the San Joaquin, its lakes and branches. These rivers mingle their waters in the bays tributary to that of San Francisco, and reach the ocean through the Golden Gate. During the summer, drought prevails throughout the great valley, and in its southern third, south of the Merced River, which receives the waters of the Yosemite, crops, with rare exceptions, cannot be raised without irrigation. Passing south. erly over the low rim of the San Joaquin valley, another irrigated region is reached, watered by the Los Angeles, the Santa Ana, and the San Gabriel Rivers. These rise in the spurs of the Sierra Nevada, - here called Sierra Madre and San Bernardino,- - and run westerly to the sea, cutting through the Coast Range. Each of them may therefore be said to drain two val. leys-an upper and a lower one; the former lying between the Sierra Madre and the Coast Range. the latter between the Coast Range and the Pacific.

The number of acres under irrigation in these upper and lower valleys at the close of 1879 was 82,485 ; in the San Joaquin, 188,000 ; in that of the Sacramento, and in the foothills east of it and the San Joaquin, 22,400. As the streams in all these districts descend from hills not very remote, the fall is sufficient to admit of the water being diverted from them for irrigating purposes without resorting to the mechanical appliances for raising water 
employed in Egypt, and in Southern Europe. In the San Joaquin valley, wing-dams extending obliqucly from one shore towards the opposite, at an acute angle up stream, generally afford sufficient head. These are numerous in Kern River, at the head of the valley, one of the most thoroughly irrigated sections, which, being crossed by the Southern Pacific Railroad, is readily accessible. Stone being scarce here, and the river not navigable, the headworks at the entrances of the canals are built entirely of wood, on pile foundations well driven down, and sheathed on both sides with two-inch plank. The wings, which are similarly piled and sheathed, extend fifteen or twenty fcet into the bank on either side of the canal, and at right angles with it. Gates are not used, but posts are set four feet apart, either vertically or inclining backwards at an angle of forty-five degrees. The four feet bays are divided and fitted to receive the weir-boards, which are six to eight inches wide, and slide in groores. Over the tops of those boards the water flows, and the height is regulated according to that of the river and the volume desired in the canal. Where necessary, a side sluice is constructed, by opening which, sand accumulated in front of the head-works may be scoured or flushed out. The natural slope of the ground being too great to admit of the diverted water following it, at intervals, say, of a mile on the canal, weirs or "drops" are introduced. These are similar in construction to the head-works, and by means of sliding weir-boards the water in the canal is kept at the proper height to supply the distributing ditches. Of course the utilization of the water power afforded by these weirs is not neglected.

One of the most important irrigation works, near the Southern Pacific Railroad bridge over Kern River, is the Kern Island Canal, eighteen miles long. It takes its water from the left bank of the stream, where the head-works have a clear width of forty-eight feet and a depth of four. There are two main branches, one of which is ten miles long, width at bottom 20 feet, depth 3 feet, slopes 3 to 1. The other branch, two miles long, supplies the vil. lage of Bakersfield and vicinity, and at the village is a fall of nearly twenty feet perpendicu. lar, the power of which runs a large flour-mill. The canal and its branches supply thirty-one lateral ditches, owned and controlled by individual land-owners, and having a total length of eighty miles. Besides the Kern Island, four other canals, the Old South Fork, the Farmers', the Castro, and the Stine, divert water from the river in this vicinity. Their total length is 110 miles, with ditches extending an additional 140 miles. These five canals are capable of supplying 895 cubic feet of water per second, and the area irrigated in 1879 was 22,750 acres. In 1873 , six years previously, but 5,000 acres were under irrigation.

Water is applied to all crops, but not at the same time. Some farmers prefer to irrigate in November before sowing their wheat, in order to give it an early start, while others, more hopeful, wait for winter rains, and, if disappointed, irrigate during the last of January.

Fields of Lucerne are watered about the middle of that month; and in Narch or April wheat receives one, or, at most, two additional waterings. In May the application to Lucerne is again made; Indian corn receives a share, and the process is repeated, if found necessary, during June and July. After that month, irrigation is not practiced except for Lucerne, late potatoes, and garden vegetables. Twenty pounds of Lucerne seed are sown to the acre, and the average yield during the first year is, from three cuttings, four tons, second year six tons; in subsequent ycars, before putting again under the plow, ten to twelve tons. An acre is capable of supporting five head of horses or cattle, or twenty head of sheep, during the nine or ten months of the grazing scason. The average yield of wheat is $27 \frac{1}{2}$ bushels, and of barley 32 bushels to the acre, but in exceptional instances as high as 50 of the former and 90 of the latter have been raised. The quantity of water required, which depeuds not only upon the kind of crop, but also upon the nature of the soil and subsoil, is supplied to consumers at fixed rates by the Kern Island Irrigating Canal Company above referred to, and by other com. panies in the valley. Sometimes the irrigators own the canals themselves, as shareholders in a coöperative company, the water supply being divided into as many parts as there are shares. 
The gates through which the water passes from the canal into the ditches are so constructed as to allow each shareholder his due proportion. Again, large landed proprietors have con. structed canals, erected buildings, planted orchards, and leased the farms to tenants, the latter having rent and water free, the owner receiving one-fourth of the crops. The leases run for five years, and if the land requires cleaning the tenant has all he can make the first year. At the expiration of the lease he has the privilege of purchasing in installments at an agreed price.

Methods of applying water to crops vary. A simple one is, to surround the fields with ditches, which are kept full of water; another is to run numerous furrows, called carriers, with the plow, these by percolation to distribute the water to crops growing between them on narrow "lands." The flooding of the fields is sometimes effected by ditches, which follow the higher ground. From these the waters flow in a thin sheet over the surface, but the uniform application of the water is difficult. If there be inequalities in the surface it collects in the low spots. If the soil or subsoil be unequally retentive, some portions will be too dry, others surcharged. The favorite plan is to divide the land by flat embankments or levees, into a series of temporary ponds, the size of which is larger as the surface is more level. In laying out the ground, contour lines are run, each level being taken eight to twelve inches below the next preceding, and as far apart horizontally as the slope of the land will allow. Along the contour lines are thrown up flat embankments eighteen inches high, and from fifteen to twenty feet broad at the base. Over these, farm machines can be driven in any direction, crops grow on them, and the long lines of gentle swells, winding, it may be, and more or less parallel, are no deformity. Each space between these embankments is called a check: the levees are therefore check-levees, and the system the check-levee system. Trunks with gates are laid from check to check through the levees, and the water from canal or lateral is first let into the highest check until it covers it, reaching nearly to the top of its lower levee, and but slightly higher than the base of the upper. The water is allowed to stand until sufficiently absorbed, when what remains is let into the check next below; this, through its own connection with a lateral, is filled, and the process is repeated throughout the series. James D. Schuyler, assistant State engineer, to whose able report we are mainly indebted for our facts, gives the cost per acre of check-levees on one-foot contours with about twenty feet base, as follows: Earthworks, $\$ 1.64$; water or drainage gates, $\$ 0.51$; total, $\$ 2.15$; average cost of lateral canals, including necessary regulators and side-gates to supply the lands, $\$ 4$ per acre-making the total cost of preparing ground $\$ 6.15$ per acre.

In the counties of San Bernardino and Los Angeles, the orchards and vineyards are irri. gated by ridging the land into small compartments by means of the plow. The water is first admitted into the highest compartment and retained until the ground is sufficiently soaked. The ridge between it and the compartment below is then broken down and the water admitted, and so on though the series. The supply of water is obtained in the upper valleys of San Gabriel, Santa Ana, and Los Angeles directly from streams, although the country is well adapted to the construction of storage reservoirs, but in the lower valleys, near the sea, artesian wells are the source of supply. These three valleys constitute what is called the Artesian Well Belt, a strip of land about 40 miles long, and from 2 to 12 wide, lying between the Coast Range and the Pacific. There are about 600 wells in this belt, the general depth being from 150 to 200 feet, and the yield 0.2 to 0.3 cubic feet per second. Some of the wells irrigate from 100 to 200 acres each, but a well which will irrigate 40 acres is considered a very good one. As land without water is worth little or nothing, and as the annual yield of an irrigated vineyard is considered worth $\$ 250$ per acre when the grapes are grown for wine, and much more when they are grown for raisins, the value of a good well assumes large proportions. Great as are the profits from irrigated vineyards, they are exceeded by those of irrigated orange orchards, each tree of which, when in full bearing, should field from 
1,000 to 1,500 oranges annually, worth from $\$ 15$ to $\$ 50$ per thousand, according to size and quantity."

Windmills are sometimes used for pumping water from common wells for the artificial watering of lands. Hydraulic force, in the form of the common hydraulic ram, can also be easily utilized for the same purpose. A recent writer in one of the leading agricultural jour. nals, gives the following simple method of irrigating meadows by means of a hillside spring:

"A typical instance may be given: A hillside field is flooded with water, which either flows from springs above it, or bursts out from springs upon it. The water lies there; the land has been poached by cattle, turned on to feed down the coarse grass of early growth, when other pastures were still bare, and the surface has grown up into bogs, tussocks, and bunches of reeds, interspersed with muddy pools. Below this lies a meadow which produces only a light crop of hay or scanty pasture for the cows when they are tired of trampling mud up above. Such a piece of land it was once my lot to own, and my business to renovate. The process was as follows: As I could not control the land above the wet part of my field, I dug a drain 3 feet deep along my fence and laid 3 -inch tile in it. This gathered a large quantity of waver from the land above, and partly dried the land below. But here there were several strong springs, which melted the snow in the winter, and which were always surrounded with luxuriant green grass. From the upper drain, one-inch tiles were laid, cutting through these spriugs and the wettest spots. This effectually drained the wet ground. The flow from these tiles discharged into an open sodded ditch or furrow six inches deep, and about eighteen inches wide. The water from this ditch was directed, in small zigzag channels, over the dry ground below; and here and there it was forced to overflow by placing stones in the waterway to arrest part of the water. In this way the water could be distributed wherever it was needed, and it furnished a steady supply until late in the summer. The effect the first year was to give two heavy crops of hay from each piece of ground, neither of which had given anything of any value whatever before.

Since that time, I have improved many similar pieces of ground at a very small expense, as compared with the beneficial results; and I have seen a much larger number that could be improved and made valuable, if the owners could be induced to do the work for themselves. The full reclamation of the tussocky ground gives the most trouble. This is best done by cutting off the bunches of roots with a heavy grub-hoe, and then running over the surface when dry with a disc-harrow, by which the sod is cut up and fresh earth thrown over it, and the surface smoothed. Some fresh grass-seed, and a dressing of ten bushels of lime or two bushels of plaster per acre, is a great help to the growth of the better kinds of grass. I do not advise such ground to be plowed; generally it breaks up very rough, and gives much trouble to reduce to a fine surface again. After a few years the herbage is totally changed, and timothy, red-top, and fescue grass come thickly into both the drained and the irrigated parts of the ground."

Secretary Gold, in one of his Agricultural Reports of Connecticut, gives, as an example of the value of irrigation, the farm of Albert G. Ayres of Preston, New London County, Connecticut. It consists of about two hundred and fifty acres of land, of average quality, when compared with the other farms of that vicinity, and has been the homestead of the Ayres family for at least three generations. "The beginning of the system of irrigation that prevails upon the farm dates back to the time of his grandfather, a hundred years ago or more. Any good farmer in passing by would notice the exceptional greenness and fer. tility of the southern slope of this farm. This is owing entirely to the free use of water for a hundred years or more upon about forty acres of the farm. The system of irrigation is of the simplest and most inexpensive kind, just such as any enterprising farmer could make for himself without the aid of stone-mason or engineer. Near the north end of the farm a small trout-brook comes in, never big enough for a mill-stream, and in summer often dwindling 
away to a mers rill. It is fed by springs, and in these springs the trout survive, through the heats of summer. This brook is dammed, near the spot where it enters the farm, with a sligh bank of earth and stone. No effort has been made to accumulate water in a reservoir against a dry time, though it could be done at small expense. Only a part of the natural flow of the water has been turned out of its channel.

The irrigating ditches, of which there are several taken from the main stream, are small and narrow, and have a very slight fall. They could be made very rapidly with a plow and ox-shovel. The forty acres put under water, slope gently to the south and east. The water is taken out of these irrigating ditches in shight rills, and passed over the meadow. Any surplus water falls into the ditch below, or is returned to the brook. The distance for which the water is diverted from its natural channel is less than a quarter of mile. The water is kept flowing summer and winter, and the winter flowage carries quite as much fertilizing matter as that of summer, and perhaps more. The refuse vegetable matter gathered in the swamp above, floating leaves, wash of roads and cultivated fields and brook channel, is carried down to these meadows.

The water is often discolored in heavy rains, and even that which seems to be pure carries more or less sediment with it. The hiquid manure may be very thin, but the fact is well established that wherever water runs over well-drained soil, grass springs up in greatest luxuriance. The purest spring water makes grass wherever it flows. Summer and winter, while Mr. Ayres sleeps, this brook is making money for him, as it did for his ancestors. The only expense to him is the slight labor of keeping the ditches clear, and of regulating the flow of the water. The soil is a gravelly loam, and slopes so much that there is no chance for stagnant water. In the opinion of Mr. Ayres the crop of hay is nearly doubled by the i.rigation alone."

The advantages of this system of irrigation are summed up as follows:-

"Besides the large increase of the grass crop, all these forty acres of meadow can be kept perpetually in grass, which is probeb!y the most profitable crop upon Connecticut farms. There need be no more plowing, no more tillage crops. The turf may thicken from generation to generation, and produce that best of all forage, a thick, fine hay made from a mixture of grasses growing upon an old sod. Then top-dressing, when applied to increase the year's crop, can be used to the best advantage. The manure is carried down immediately to the roots of grasses, by the large supply of water upon the surface. There is no loss from evap. oration. The soil is kept to the production of nearly two tons of hay to the acre, without top-dressing.

Upon this the calculation is based of the value of irrigation upon this farm. We suppose the natural production of the land to be not over a ton to the acre. A ton to the acre then would be a fair estimate of the hay made by the irrigation. Hay sells in the neighboring city markets at from $\$ 15$ to $\$ 20$ a ton, according to season and quality. If we call the hay $\$ 10$ a ton standing, it would give $\$ 400$, as the annual dividend declared by the brook. The investment is about as secure as government bonds, which pay four per cent. nearly; the income is about the same as $\$ 10,000$ in United States stocks. Not every farm has the facilities of this for irrigation, but some could irrigate on a much larger scale. Almost every farm that has a brook running through it, or upon its borders, could have some portion of its acres subject to irrigation."

The growth of large forests may also be promoted by irrigation. Damont states in Des Traraux Publics, that in order to hasten the growth of wood on the flanks of a mountain, II. Eugene Chevandier divided the slope into zones" forty or fifty feet wide, by horizontal ditches closed at both ends, and thereby obtained from firs of different ages, shoots double the dimensions of those which grew on a dry soil of the same character, where the water was allowed to run off without obstruction. 
Irrigation is often accomplished by flooding or inundating the lands for a few days, when they are so situated that this is practicable. A good illustration of this means is seen in the Grand Pie Marsh in Nova Scotia, which contain the most fertile lands in the province, and is often called the garden of Nova Scotia. These marsh lands are enclosed by dikes, and whenever it is found necessary to enrich the soil, that may become exhausted by constant cropping, the sluice gates are opened and the tide is allowed to overflow the meadows, which leaves a rich deposit of alluvial matter to the depth of one or more inches on the surface, by which means they are kept in constant fertility.

Any field of a moderate descent can be irrigated by open ditches with but slight expense, where the water is available on an elevation. In Europe, the quantity of water supplied to crops ordinarily cultivated on tilled lands, is from twenty to forty inches during the season; though the rice-fields of Lombardy require a considerably larger amount than this. Experience alone can best determine the proper quantity for certain soils and crops. Much, also, depends upon the season and the amount of rain.fall, etc.

When to Irrigate. - Evening, as a general rule, is the best time for irrigating all crops. Constant watering under a hot sun is not only unnatural to all vegetation, but will often prove very injurious to many plants. All plants require more water in the early part of their growth than when nearly mature. Nany crops, such as grasses, and some of the grains, for instance, would be injured by an excess of irrigation when approaching the lipening process, and it should be at this time carefully aroided. Light, porous soils, according to best authority, are most benefited by artificial watering; sandy and grarelly soils re. quiring it most. Clays, being naturally moist and heavy, are less benefited by it, though are said to be improved by this means when made porous by under-draining. Irrigation often greatly increases the quantity of grass for cattle in pastures that would otherwise, from the condition of the soil, be almost worthless. After a crop of grass has been cut, irrigation will greatly improve the quantity and quality of the aftermath, besides preventing the drying up of the grass-roots by evaporation in the hot sun. The process of irrigating should begin on grass lands very early in the spring, in order to give the herbage a good start. The quantity of water thus supplied to any crop should be adapted to the kind of soil and the crop produced, which can only be determined by experiment, since soils vary so greatly that only general rules can be given in this respect. 


\section{DRAINAGE.}

$\mathrm{D}$

RAINAGE, or the removal of the surplus water from the soil by artificial means, may be accomplished by the use of open ditches, covered trenches, plank or stone drains, tiles, etc., the best and most popular method being tile draining.

Perhaps no part of farm husbandry pays a larger per cent. of profit on the money invested than proper and judicious drainage, where the conditions are such that lands will be benefited by this system, since it not only removes stagnant water from lands, but relieves them of their excess of moisture, rendering them productive, and more healthful to both vegetable and animal life. It also deepens the soil, makes it more friable, and by drawing the water from the surface, not only adapts it to earlier cultivation in the spring, thereby insuring an earlier and more abundant crop, and also prepares it to be worked advantageously in the autumn, but the surface rain-water, being warmer than the soil, such drainage increases the temperature of the earth as it sinks downward.

Mr. Parks, the English Agricultural Engineer, found by making simultaneous obser. vations on a drained portion of a field, that from a mean of thirty-five observations, during the spring and early summer, the drained soil, at seven inches depth, was ten degrees warmer than the undrained, at the same depth. The highest temperature of the undrained soil was forty-seven degrees, while that of the drained went up to sixty-six degrees at seven inches, and forty-eight degrees at thirty-one inches, after a thunder storm. A wet soil can never be properly pulverized, hence, cannot be well tilled; it is also always cold; and as drainage renders the land warmer, the roots of plants which will never go below the water-line, always strike deeper in under-drained soils, which explains the fact, often surprisingly mani. fest, why lands well under-drained should endure the evil effects of a drouth better than those not drained at all. This mystery is solved when we consider that roots in such soils strike so much deeper than in lands not drained, that they are constantly in a moist soil, however dry the surface may be; besides such lands are more porous and permeable to the dews and showers. Under-draining also prevents surface washing, by allowing the water to percolate through the soil, and thus whatever fertilizing material it contains is retained. It renders winter killing of crops less liable, and preserves a more uniform degree of moisture in the soil, since it not only causes its saturation with water to be impossible, but keeps it in such a pulverized and porous condition, that in times of drouth it absorbs moisture from the air and never becomes baked and hard, thus the evil effects of extremes of wet and dry seasons are avoidable. Much land otherwise almost worthless for cultivation has become by this means very valuable. Lands well under-drained can also be used with safety in hauling loads and are less liable to injury from the treading of cattle, \&c.

Thornton, an eminent English authority, sums up the benefits of drainage as follows: "An earlier seed-time and harvest, better crops, a healthier live stock and an improved style of husbandry, are the usual and well-known sequence of judiciously-conducted drainage operations. In short, the most experienced and skillful agriculturists now declare with one consent, that good drainage is an indispensable preliminary to good cultivation."

In England and Scotland the improvement of lands by drainage has long been practiced, and it is by no means a modern improvement, though it has comparatively but recently been reduced to a system based upon scientific principles. The Romans were careful to keep their arable lands dry by means of open trenches, and there are some indications of their having used covered drains for the same purpose. English literature shows that it is at least more than two centuries ago that Captain Walter Blithe wrote the following:

"Superfluous and venomous water which lyeth in the earth and much occasioneth bogginesse, mirinesse, rushes, flags, and other filth, is indeed the chief cause of barrenesse in any land of this nature. . . . Drayning is an excellent and chiefest means for their reducement; and for the depth of such draynes, I cannot possibly bound, because I have not time and oppor- 
tunity to take in all circumstances. . . . And for thy draynung trench, it must be made so deepe that it goe to the bottome of the cold, spewing moyst water, that feeds the flagg and the rush; for the widenesse of it, use thine owne liberty, but be sure to make it so wide as thou mayest goe to the bottom of it, which must be so low as any moysture lyeth which moysture usually lyeth under the over and second swarth of the earth, in some gravel or sand, or else, where some greater stones are mixt with clay, under which thou must goe halfe one spades graft deepe at least; yea, suppose this corruption that feeds and nourisheth the rush or flagg should lie a yard or foure foot deepe, to the bottome of it thou must goe, if thou wilt drayne it to purpose. . . . And for the dreyning trench be sure thou endeavour to carry it as neare upon a straight line as possible.

"To the bottome where the spewing spring lyeth thou must goe, and one spades depth or graft beneath, how deep so ever it be, if thou wilt drayne thy land to purpose. I am forced to use repetitions of some things, beeause of the quitableness of the things to which they are applyed; as also because of the slownesse of people's apprehensions of them, as appears by the non-practice of them, the which whenever you see drayning and trenching you shall rarely find few or none of them wrought to the bottome. . . . .

"Go to the bottome of the bogg, and there make a trench in the sound ground, or else in some old ditch, so low as thou verily conceivest thy selfe assuredly under the level of the spring or spewing water, and then carry up thy trench into thy bogg straight through the middle of it, one foot under that spring; . . . . . . but for these common and many trenches, oft times crooked too, that men usually make in their boggy grounds, some one foot, some two, never having respect to the cause or matter that maketh the bogg to take that way, I say away with them as a great piece of folly, lost labour and spoyle.

After thou hast brought a trench to the bottome of the bogg, then cut a good substantial trench about thy bogg; and when thou hast so done make one work or two just over-thwart it, upwards and downwards all under the matter of the bogg. Then thou must take good green faggots, willow, alder, elme or thorne, and lay in the bottome of thy works, and then take thy turfe thou tookest up in the top of thy trench, and plant upon them with the green sward downwards; or take great pebbles, stones, or flint stones, and so fill up the bottome of thy trench about fifteen inches high, and take thy turfe and plant it as aforesaid, being cut very fit for the trench, as it may join close as it is layed downe, and then having covered it all over with earth, and made it even as thy other ground, waite and expect a wonderful effect through the blessing of God." The instructions of Blithe do not seem to have been extensively heeded by his contemporaries, though they probably were to a certain extent. Since that time, various systems have been practiced in Great Britain and other portions of Europe, the moist climate of the former rendering drainage more essential to successful agriculture than in some other countries. In our own country, the general adoption of a complete system of drainage is comparatively of recent date, but enough has been accomplished in this direction to prove its great value to the agricultural interests of every section, and the time is not far distant when it will doubtless be practiced on a larger and more extensive scale, and many of the vast tracts, now utterly worthless, will be converted into some of the most fertile and valuable of its agricultural lands, besides rendering such localities more healthful.

What Lands Require Draining.-All lands containing an excess of water are greatly benefited by draining, and these are usually heavy, tenacious clays, those soils having a hard clayey subsoil that will not admit of the water passing through them, and peaty or swampy lands. A large proportion of the lands in cultivation are sufficiently drained by nature. No land with a subsoil so porous as never to retain a surplus of water requires an extensive drainage. Sometimes a farm that requires no thorough draining may be benefited by a partial one; for instance, many farms contain small swamps or swales, so located in a field as to greatly hinder cultivation, and retard the growth of crops; these have probably been formed by the water that has passed through the porous soil, meeting with an obstruction 
in the form of an impervious stratum. This stratum may be horizontal, or nearly so, and the water may follow it out to the surface of a lower level, and thus the swale is formed, a permanent nuisance in an otherwise valuable tract of land. In such cases a partial drainage of the lands will entirely remedy this evil. In the United States, the average amount of rain-fall is about three inches per month, and frequently an inch of rain, - which is equal to a little more than three hundred and sixty hogsheads of water, - falls upon an acre of land iu a single shower. The question arises, What becomes of all this water? It must eitlier be disposed of by sinking into the soil, by evaporation, or it remains upon the surface. Moist land conduces to good cultivation, but not wet lands; therefore, if the soil or subsoil is of such a nature that this water does not percolate through it readily, and leave it in good condition, drainage is the only remedy for the evil. When the land is very level, admitting of no surface draining, and the soil retentive and clayey, drainage will be required for any improvement in the conditions.

Swamps and peat-beds frequently occur in a hilly country. Sometimes their origin is in numerous springs from adjoining hills; when this is the case, often a ditch dug around the entire outer edge of it, where it meets the ascending land, will cut off the water supply, and generally remedy the evil; but open ditches are very objectionable for many reasons, and tiles are preferred. It is stated that Mr. John Johnston, of Geneva, New York, who was the first farmer to use tiles for draining in this country, once drained a quagmire so that it produced eighty bushels of corn per acre, and in that case the cost of draining was paid by the increase of one crop. The draining of lakes and marshes requires great capital, and engineering skill, and is sure to become a very important branch of agricultural improvement in our Southern and Western States.

The reclaiming of the Everglades of Florida by draining Lake Okeechobee sufficiently to prevent its overflow, is an instance of what may be accomplished by this means. This may be done by a canal ten miles long, connecting Lake Okeechobee with the headwaters of the Caloo. sahatchie River, which flows into the Gulf of Mexico, the mouth of which is twenty-three and a half feet lower than the lake. It is the overflow of the lake which causes the Everglades to be submerged in water, the lake having no natural outlet, while the Everglades are surrounded by.a limestone ridge which acts as a dam to the water, and after the overflow of the lake, it can only be reduced by evaporation, which, being so slow a process, keeps the ground almost constantly under water. Should the above drainage not prove sufficient to prevent the over-flow and render the lands reclaimed suited to agricultural purposes, another canal to the eastward, cut to the St. Lucie River, twelve miles long, which will carry the water into the Atlantic Ocean, will remedy the evil, the mouth of the St. Lucie River being twentyfive feet lower than the basin of Lake Okeechobee. In Holland, steam-pumps, wind-mills, and tide-gates are used extensively in drainage, the great Haarlemer-meer being not only drained, but is kept so, by steam-power. Prof. Pendleton states that much of the billy land in some of the best farming portions of the Southern States has become impoverished by unskillful culture and by continual washings of soil and fertilizing materials which have been swept into the valleys, that are now so saturated with water as to be unfit for proper tillage, and that in this way many valuable lands have been lost to cultivation, and rendered unhealthy by engendering malarial fevers, etc.

By a judicious system of drainage, these lands may become among the most productive, as well as profitable, of any in that section of the country. Prof. Cleveland, of Chicago, says: - "The necessity of drainage is not to be determined by the quantity of water which falls or flows upon the surface, nor upon the power of the sun to carry it off by evaporation, but upon the character of the subsoil. If that is of sand or gravel, the water will pass off below by natural drainage, but if the subsoil is clay or other impervious substances, the water is checked in its downward course, and remains stagnant or bursts out upon the 
surface in the form of springs. Most of the prairie lands are of the latter character, and wherever such subsoil exists, the land will not only be improved by tile drainage, but it may be said that such drainage is absolutely essential to its proper development and culture. To question the necessity of the process is little less alsurd than it would be to ask whether it would be improved by plowing, instead of making hills for corn in the prairie-grass with a hoe or spade. All lands require drainage which at any season contain too much water, that is, which hold at any time of the year more moisture than is good for the crops, and causes them to suffer, either by freezing ont in winter, or having their roots in water during the growing season."

Drainage has been appropriately defined as "the art of rendering land not only so free from moisture that no superfluous water shall remain on it, but that no water shall remain long enough to injure or retard the healthy growth of such plants as are required for the sustenance of man or beast." There are some plants, such as rice and cranberries, which require a wet soil, but most of those which are useful to man are drowned by being overflowed for a short time, and are injured by any stagnant water about their roots.

We would recommend that in all cases of drainage, it he well done, as the most careful and permanent work of this kind will be the cheapest in the end, and the best-paying in return for the capital invested, while work of this kind carelessly and indiferently performed will prove very expensive eventually, as it will probably fail to accomplish the end sought, and will have to be done over in a short time to be of any practical benefit. The best method is to establish a plan for complete system of drainage for the farm, and when this is once done, the work can be accomplished from time to time as opportunity affords, with but little or no interruption to the regular rontine of farm labor. It can often be accomplished in those seasons when there is little else to do upon the farm, bence at less expense than other. wise. There is no part of farm labor where previous calculation and careful, systematic work pays better than in under-draining. Many farmers have but little to occupy them during the late antumn and winter months, and could devote the time to such work then with no interruption to the other farm work, and with less expense.

Tile Draining. - This is the best method of draining known, being the most effectual and satisfactory in all respects. No person should undertake the task of draining with the expectation of success without first fully informing himself with respect to all the important details, as it is a work which requires much scientific skill and careful planning in order to reach the best results. And, as we have previously recommended, we would advise that the plan be a complete one for the farm, which plan can be gradually carried out from time to time as the farmer feels able to accomplish, either in expense of money or labor.

Determining the 0utlet.-One of the most important steps in drainage is to decide upon a place, or the places of outlet. This must, of course, be enongh lower than any portion of the lands that are to be drained by it, to admit of sufficient fall for the passage of watcr from the heads of the most distant tributaries; yet it must at the same time be high enough above the level of whatever it empties into to prevent the danger of its being overflowed. and the drainage-ivater dammed back in the pipes. In all cases where the inequalities of the surface are not sufficient to render it obvions that every field on the farm that it is desirable to drain can be drained into the mains withont difficulty, the most important point to determine is the lowest level at which the outlet can be located so as to secure a free and constant outflow. For instance, the level of every other point must be high enough above the outlet to secure a grade in the tiles of at least $2 \frac{1}{2}$ to 3 inches per 100 feet, thongh from 6 inches to 12 is better, if practicable. Some writers claim that they have known drainage to prove a complete success where the grade is from one to one and a half inches per 100 feet, but we should fear to risk the cost and labor of constructing drains on that principle. The grade 
should be sufficient to carry off any silt, sand, or other obstructions that may get into the pipes. On very level farms-the prairies, for instance-it may often be difficult to secure the necessary grade, without bringing the upper portions of the drains too near the surface. It is better, therefore, to have the levels all taken before the work is begun (which can be done at slight expense and labor), than to incur the cost of correcting, afterwards, mistakes that may be made at the commencement. We therefore advise that the services of a competent civil engineer be secured, and that these levels be taken on cross lines about fifty feet apart, and marked on a map of the farm, or land to be drained; and when this is done, there is a map for guidance marked in fifty-feet squares, where the relative height of each square is marked at the intersection of these lines, so that, having the lines for drains previously marked, when the farmer digs his drains for laying the tiles, he knows precisely the depth he must go at each point to give a uniform fall, and thus the saving of labor and the avoiding of mistakes will well repay the slight expense required to secure this knowledge. By this means the farmer can have a map to refer to, showing the exact location and depth of every main and side-drain on the premises; and he can go on from year to year doing as much or as little an amount of labor as he chooses, but always working with the assurance that he is on the correct principle and knows what he is about, and that he is working out one definite plan. This same plan will afterwards enable him to find, without any difficulty, any point he may wish to obtain access to for repairs, etc.

In England there is an accredited profession of farm-engineering, regulated and encouraged by the English agricultural laws. It would be a great aid to the agricultural interests of this country if the farmers of every state could have the benefit of just such competent assistance, based upon accurate mathematical knowledge and experience. The members of this profession in England are required to pass a severe and critical examination, proving themselves to be thoroughly competent, both theoretically and practically, for the position, before admission.

Locating the Main Drains and Tributaries.-Having determined where the outlet will be, which, as has been previously stated, is a very important question to decide, the next step will be to locate the main drains; and this can be properly accomplished only by taking into consideration the surface of the land. If the surface is uneven or undulating the main receiving-drains should be carried along the lowest part of the land, in the hollows, while the branches or parallel drains, running in the line of the greatest ascent of the ground, flow into them, often at nearly right angles. Where the land is nearly level, and a fall is obtained only by increasing the depth of the drains at the lower ends, the tiles may run in any direction convenient. The conformation of the land may often be such that a single field may require several distinct sets of drains, lying at different angles, suited to the various slopes of the surface; but in all cases where slopes occur, the pipes should be laid directly up and down the slope, so that the water once entering at the joints of the tiles will not run out again at other joints, but will follow the course of the pipes to the end. In an irregular field, where the slopes are in different directions, of course it will be impossible to have the drains run directly up and down the slope, and at the same time parallel to each other, which would be the most economical.

In varying from parallel directions, there is danger of getting the pipes too far apart, and making the drainage incomplete, or of crowding them so near together as to be quite expensive; hence, there must be the exercise of careful judgment in laying out the system of drains, in order to arrange the slope correctly, and yet accomplish the desired end in drainage without incurring unnecessary expense.

When part of the field is flat, and other portions have a considerable slope, the best authorities advise placing a receiving-drain near the bottom of the slopes, and giving the level ground an independent set of drains. The surface of the ground, convenience of 
outlet, and character of the soil, all have to be taken into account, consequently the plan of no two fields will scarcely ever be the same. The roots of trees and shrubs sometimes obstruct drains, therefore it is well in laying out their course to avoid them as far as practicable. When a drain must of necessity pass near trees, the use of coal-tar is sometimes found beneficial in excluding the roots. Mixing coal-tar and sawdust to the consistency of mortar, and placing a layer of this in the bottom of the trench at such places, and then laying the drain-pipe upon it, and covering it with this mixture to the thickness of an inch, before covering with earth, all trouble from roots will be obviated. Some writers recommend that soft and porous tiles (though not as durable) be used at such plates as are liable to trouble from roots, with the joints laid in cement, the hard tiles being ordinarily used for drains, as they are more durable. All tiles having small holes, called "pin-holes," should be carefully aroided, and all aquatic trees near the line of the drain should be killed before the drain is laid. Mr. W. I. Chamberlain, of Summit County, Ohio, gives the following statement relative to the wandering nature of roots, and their tendency to obstruct drains:-

"The roots of aquatic or water-loving trees, like the willow and some kinds of elm, seem to have almost no limit to their growth, either horizontally or vertically; and they seem to go in search of moisture or richness, as if by instinct, and to know just where to find it. I have traced the roots of a smallish elm some twenty-five feet horizontally. and six feet vertically, to their feeding.place in a grave in an old cemetery; and I have, in plowing, traced the roots of a large elm one hundred feet horizontally by measurement. These roots will enter even a 'pin-hole' in tiles, if they can find running water. Mr. H. B. Camp, of Cuyahoga Falls, Ohio, told me a few days since, that he once helped take up an obstructed drain, whose joints were laid in cement, I think he said. At all erents, a willow root had entered at a pin-hole not larger than a small darning-needle, and spread into a fibrous mass and packed the tile full of roots for several feet-the only connection with the upper world and their lnngs (the tree-leaves) being this small, thread-like root, that entered at the pin-hole. It is better to cut such trees down when they are near a damp drain, and see that they are dead. Prof. Townshend, of the Columbus (Ohio) Agricultural College, exhibited in one of lais lectures recently a dark, stiff, fibrous, sponge-like, solid cylinder, some three feet long and three inches in diameter. When the class had done guessing, he gave its history. It was the willow-root core of his cellar-drain. Knowing the nature of these roots, he cut the willow down before he laid the drain, and burned the stump all he could; but in spite of that, its roots stopped his cellar-drain at a depth of five or six feet !"

When a main drain has parallel drains flowing into it on both sides, these openings into the main drain should not be exactly opposite each other. The evil effects of such a plan are at once perceptible. Some prefer to have two main drains parallel to each other, each to receive the subordinate drains from its side only. If these main drains are located as far apart as the other tiles in the field, but little additional expense will be incurred by having two instead of one, as these receiving-drains, besides taking the water from the others, also perform the same office as the other drains for the lands through which they pass. One of the best English authorities on this subject says:-

"Much of the success of draining depends on the skillful planning of these main àrains, and in making them large enough to discharge the greatest flow of water to which they are exposed. Very long main drains are to be avoided."

"In the whole process of draining," says an experienced engineer, "there is nothing more important than permanent and substantial work at the outlet." It is a place where obstructions are most liable to occur, being most exposed to the effects of frost, while reptiles, cattle and mischievous boys often do great damage, unless it is sufficiently protected. The earth everywhere else is a protection to the tiles. It is, therefore, well to have as few outlets as possible, and these should be secured by a heary frame-work of timber or stone, and an 
iron grating, or heavy wire netting, fine enough to prevent the entrance of reptiles or other animals. One outlet to an area of from ten to fifteen acres is usually considered a good arrangement.

A uniform grade is very desirable, for if any portion of the main be lower than the outlet, there will, of course, be a permanent stoppage, as the water will stand there until there is a deposit of mud, and it will not be forced out by the action of the water. All curves and angles should be avoided, if possible, as they are the places where obstructions will be liable to occur.

Depth of Drains. - The best English authorities advise that the drains should not be less than four feet in depth, and that even a greater depth may be desirable; it depends mainly upon the subsoil; but they are often made very serviceable at a less depth. Sometimes a seam of gravel or other porous material, charged with water, may be found underlying a con. siderable portion of the ground at a greater depth than four feet, and this may render it necessary to dig the drain sufficiently deep to reach that seam. When such, or similar cases occur, it is desirable to have the drains quite deep, as they will perform more efficient service in draining the whole area under which this strata extends, than shallow ones placed nearer together. It has been proved by experiment that drains three feet deep, at forty feet apart, are not so effective as those at five feet deep and fifty feet apart. The depth necessary to lay the tiles will depend much upon the character of the soil and subsoil. As a general rule, it will cost as much to dig the fourth foot as the other three, and if the conditions are such that the benefits arising from the greater depth will more than counterbalance the increased expense, it will be advisable to increase to the fourth or even fifth foot in depth. The farmer must take into consideration the various conditions of soil, and be his own judge in this respect. Many writers advocate three feet, and some even two and a half feet, as sufficient for all practical purposes in most soils; but we prefer, as a general rule, that the depth be four feet. Prof. Shattuck expresses the following opinion in the Drainage Journal :-

"They must be deep enough to be out of the way of frost; they must give sufficient room for the roots of plants above the water-table, because of the evil effects of stagnant water and the intrusion of roots; deep enough to prevent evaporation of the water arising in the soil from the force of attraction, or at least to reduce it to a small amount. Experiments show that capillary attraction operates with considerable power at eighteen inches. They have also shown that water coming from a thirty-inch drain is two or three degrees colder than that taken from a depth of four feet, and that this is a little cooler than that from a greater depth. We must draw the conclusion that evaporation has considerable effect at thirty inches. Four feet, then, seems to be the standard minimum depth; it cannot always be had, but better go deeper than not so deep, when possible.

The above statement is mainly in reference to the requirements of the crop to be grown. I admit that depth and distance between drains should bear a relation to each other, that the character of the soil and subsoil is an important factor in the question. I give the above as a general guide. There is a feeling with many that thirty inches, or three feet in depth, is sufficient. I know that drains at those depths will do good service, but I believe one at four feet will, as a rule, do better. Theory indicates this and practice confirms it.

Of two drains at different depths, the deeper one acts first, and with far more effect; but the greater expense is objected to, though without sufficient reason in many cases, it seems to me. The tiles cost the same in either case, and, from my standpoint, the additional labor of the deeper one is of little account to the general farmer. I venture to say that the majority of Western farmers are unemployed onc-half of the time during three or more months of the year, and at that season when the work of drainage could go on with the least interruption to farm culture.

The labor of the farmer is an important part of his capital, and with proper management 
it will not grow less by use. The improvement of the farm by drainage should be a part of the regular work of each season; when thus carried out, it is not the burden that many make it, in order that the work of years may be put into one. In case of so-called partial drainage, the depth of four feet is even more strongly recommended, as such is usually made with large-sized tiles, and it should be, with the view of making in the future the drain's main, or of a system, at least, of getting the greatest benefit from them."

Scarcely any person whose experience and judgment are of value in successful agricultare, would think of laying tiles at a less depth than three feet, while, comparatively speaking, those soils of clay or loam having a clay subsoil will receive so much additional benefit from a greater depth than this, that we might almost say, no man can afford to place his tiles at a less depth than four feet. As draining is generally an expensive operation, it should be done with care, and with a view to permanency. If this is done, the work will remain as long as the tiles will last, which, if they are of the proper kind, will be a very long time. Waring, as well as many others of our best writers on the system of drainage, states that tiles are much cheaper than stone drains, and by far more durable, being less liable to fill up with sand, mud, or other obstructions. In constructing the drain, it is important that the bottom of the trench be cut out just wide enough to admit the pipe, and no more, as tiles fitted in that way are less liable to become displaced than if it were cut too large and again filled up with loose earth. Various kinds of draining-tools have been invented for this purpose, such as trench-plows, long, tapering spades for digging out the middle and bottom of the trenches, recurved scoops for removing the earth, and pipe-layers, by means of which a workman can hook up a pipe and collar and put them in place in the trench while standing on the margin of the drain. When the bottom of the trench is clay or hard gravel, with a desirable grade, there is no trouble in placing the tile; but when the bottom is sandy, peaty, or soft, it will be found advautageous to put strips of rough boards at the bottom to place the tiles on, and prevent them from settling beyond the common depth and becoming displaced.

Size and Kind of Tile for Drainage, etc. - In the selection of tiles for the mains as well as the tributaries, it is important that they be of sufficient size to take the water as fast as the soil can furnish it in heavy rains. The mains had better be too large than too small, as economy here will prove poor economy in the end. It is often surprising to see how soon, in a shower, the water will percolate through the soil, be collected by the laterals, and reach the mains, and how perfectly the system of drainage works, when everything is constructed properly. Considerable expense may be saved by having the upper portion of the mains smaller, and the size increased as the surface from which it receives the water is increased. It is estimated that a three-inch main will answer a good purpose until it has received the water from the laterals of two acres, then add a four inch to receive the water until four acres are drained, a five inch up to six acres, and so on.

The rule to find how many acres a given main will drain, by squaring its diameter, thus, -a 3-inch main will drain 9 acres; a 4 -inch, 16 acres, a 5 -inch 25 acres, etc., may do very well for some purposes, like a limited range of sizes of tiles, and variations in grade ; but to make allowance for variable and heavy rain storms, this would give too many acres for the size of main. Mr. Chamberlain, of Ohio, gives, we think, a better and safer rule to follow. in what we subjoin :-

For sizes from 3 to 6 inches, and grades less than three feet to the 100, square the diam. eter and divide by 4 . Thus

A 3-inch main will drain $2 \frac{1}{4}$ acres.

4 do. do. 4 do.

For heavier grades it may do to divide by 3 . Thus
A 3-inch main will drain 3 acres.

A 5-inch main will drain $6 \frac{1}{4}$ acres.

4 do. do. $5 \frac{1}{3}$ do.

A 5 -inch main will drain $8 \frac{1}{3}$ acres.
6 do.
do. 12 do. 
But it must be borne in mind that the steeper the grade the greater the danger of sur. face wash, which often causes great loss of manure, and even of soil itself.

As to the proper size of tiles to use, it is diffieult to give a rule that will apply to all cases, so many considerations having to be taken into account ; but, as we have previously stated, it is safer to have the mains and laterals larger than necessary, rather than too small. For this reason we should feel more inclined to follow Mr. Chamberlain's rule than the one previously given, and commonly applied, but which will not meet the requirements of all cases. A two-inch pipe tile is largely used for laterals. By using such small tiles they ean be placed nearer together than more remote larger ones, at no greater expense, hence better drainage can be secured at the same expense, by their use.

Among the various kinds of tile manufactured and in use, the round tile seems to be most in favor. In many seetions tiles round inside and octagonal externally are quite popular, while others still prefer the sole tile and other varieties. Cylindrical pipes with collars are considered the best by English authorities ; the collars being simply short pieces of pipe just wide enough in diameter to admit freely the smaller pipes that form the drain ; hence, in use, one of these collars is so placed as to receive and hold the end of each tube where they join, keeping them in position to form a continuous canal for the free passage of water, and guarding against the entrance of mud, sand, vermin, or other obstmetions. These joints, which are frequent, also admit the water furnished by the soil. It may seem almost impossible that lands could be drained by the water entering only at these joints in the tiles thus laid, but it is proven to be a faet; water will find its way to such places in the drains and make them the permanent places for reaching eventually the mains, and it is surprising to see how soon after a rain commences these mains will begin to discharge their contents received from the laterals, and often to their fullest capacity.

Hard-burnt tiles should be seleeted for the purpose; those having any defect whatever should be discarded. They should be carefully examined before being laid, and whenever there is found one with a lump of unslacked lime, or lime pebbles, or any small hole, it should not be used. To determine whether there is unslacked lime in the tile, immerse it in water and allow it to remain until thoroughly soaked through; if it contains lime it will break. The breakage of such a tile when placed and covered by the soil might make an obstruction that would eause days of labor and expense to find and repair. Waring advises that each tile be examined by a stroke from a hammer, and every one be rejected that does not give a clear metallie ring. Mr. Chamberlain, previously referred to, recommends the glazed tiles as most desirable, and deseribes them as follows:-

"They are made of fire clay or second quality potter's clay, like that used for sewer pipes, burnt hard and glazed. They are about as dark colored and smooth as a 'little brown jug,' and seem likely to be as durable. They are the only kind I shall use hereafter.

I think tiles made of ordinary brick clay are liable to soften and crumble and decay, certainly if exposed to frost. After the first job of draining I did, I had a few of these red brick tiles left. They lay on the ground through one of our changeable winters, and the com. bined action of moisture and frost erumbled most of them to pieces. And unless burnt very hard indeed, I should fear the same result in time, even when laid below frost, for we have noticed that in a mass of brick, even buried in the ground, all the soft ones will crumble in time, even without the action of frost. But with the hard, glazed tiles I now use, with their clear metallic ring, and their glazed surface inside and out, impervious to water, crumbling and dissolution seem impossible. I have tested them very thoroughly in moisture and frost two winters, in small heaps above ground; and I do not see but that they are likely to last as long as a broken bit of a glazed jug, or some of the broken tiles or bricks of ancient eities, unearthed after centuries of oblivion.

Now this seems to me an important matter. Drainage, if properly executed, is a perma- 
nent improvement. It is so regarded by the English laws authorizing government loans in aid of drainage, and permitting drainage to be charged for gradual payment to estates which could not otherwise be burdened with debt under the laws of the entail. If every tile is perfect and the work done thoroughly and well, and the outlets properly constructed and kept clear, I see no reason why drains should not remain perfect for generations and centuries."

It is a common practice to place tiles from 25 to 40 feet apart, according to the conditions of the soil to be drained. If very wet, and the amount of water to be discharged be great, it might be necessary to place them nearer than 25 feet. It is not a good plan to have the trenches dug long before the tiles are laid, as they are apt to cave in, especially in cold, frosty weather. If the work is done in winter, it may be facilitated by removing the sod in the fall and plowing frequently the bottom of the ditches to keep them from freezing; as fast as the trenches are dug to the proper grade, the tile should be laid and covered, remembering that it is very important that the grade be such as to give the drains a contiuuous fall to prevent the accumulation of obstructions in the pipe, or the water standing. Where collars are not used, the pipes are often secured against displacement by two small flat stones placed like an inverted letter $V$ (in this form $\Lambda$ ) against the joints.

Where there is a good fall, sand or mud will not be as liable to obstruct the drain, as it is more readily washed out by the swifter current of water, but where the land is nearly level, with but slight fall, obstructions will more readily accumulate, hence in such cases it is often necessary that great care be taken to pack the joints well with clay to prevent the sand entering and forming an obstruction. This is done by putting directly upon the joints of the tiles the finest, most compact and clayey soil that can be found, and which does not contain any perceptible vegetable matter whatever, such as leaves, grass, straw, or humus, nothing but tlie hard, clayey soil. This should be put in to the depth of seven or eight inches and then carefully beaten down around the tiles to hold them perfectly in place. Mr. Waring says, that he believes this one operation has more to do with the permanence of a welllaid tile drain than any other one part of the work. As fast as the tiles are laid and joints properly secured in place, the ditch can be filled up with a plow or scraper, or in any other way, as is found most convenient and economical.

Stoue Drains come next to tile in value for drainage purposes, but are more expensive in the end than tiles, even where there is a supply of stone on the farm to be disposed of. The labor of making a stone drain is much greater than laying tiles, and the process a slow one, while they are very apt to fill up with sand and mud or other obstructions, and do not discharge the water as readily as tile drains. With great care they can be made serviceable for years, and it may sometimes prove a good way to dispose of the surplus stones on the farm, where tiles are not easily procurable, but as a general rule, we would advise that tiles be used by all means and the work be well done, with a view to permanency. Mr. Waring says that providing the stones were delivered free of cost on the bank of a ditch, the stone drain would be more expensive than tile, and that where plenty of stones are on the ground for making the drain, it will be easier and cheaper to cart them off and put them by the side of the road, or dig a wide deep ditch and throw them into it to dispose of them and use tile, rather than to utilize the stones for drainage purposes, he having tried both methods. Dr. Loring, Com. missioner of Agriculture, states that he has both tile and stone drains on his farm ; that the tile drains were laid in 1857 and the stone drains in 1861, and that there is not a stone drain open to-day, while there is not a tile drain that is obstructed, all being in good order. Farmers who have had practical experience with both stone and tiles almost invariably prefer tiles. The labor of getting together and selecting suitable stones for the purpose involves much time and expense, while with the greatest care they cannot be made as close and free from obstruc. tions or as serviceable as tiles, and the current is never strong enough in them to remove obstruction as it does in tile drains. The labor and expense of digging the trench for stone is 
also much greater than the former; hence tiles are not only better for the purpose, but cheaper. Where the soil is very wet, a complete system of drainage could not well be established by the use of stone drains ; but where a partial drainage only is required, and the farmer may be so circumstanced that he cannot well procure the tiles, stone may be utilized for the purpose. There are various methods of making stone drains. Formerly drains were often made by digging a trench about three or four feet deep and then filling it to within twelve or fifteen inches of the surface with stones, covering the whole with flat, stones to keep out the earth, before filling in the soil, thus furnishing a means for the water to escape by working its way between the stones; but this method is rarely employed at present. Another method is to put small stones in the bottom of the trench to the depth of four or five inches; line one side of the trench with flat stones, and by placing long, flat stones against the opposite side on the pebbles or small stones forming the bottom of the drain, and leaning the top of them against the top of the stones forming the lining, an opening or throat will be formed through which the water will find a passage ; the top will, of course, be covered with flat stones, followed by coarse gravel before covering with soil, -in order to prevent the earth falling or washing in between the chinks of the stones and obstructing the passage. Some care will be necessary in making any stone drain, to fill up all the little openings with smaller stones, as far as possible, as the earth will be very liable to wash in and obstruct the water if this is not done. The best form of stone drain is to make the sides of the ditch slanting and place the stones at the sides for a lining so as to form an acute angle at the bottom, in the shape of a letter $\mathrm{V}$, and cover the top with flat stones, the opening thus formed to be triangular. Special attention should be given to filling up the trench above in such a manner that the sand cannot find its way into it. It is also important that the water have as much fall as practicable, which will facilitate the drainage. As we have previously stated, such drains are much more expensive, owing to the labor and expense of constructing, and also less effective than tile, but are the best substitute for tile drains that are known. In all kinds of drains, a free outlet should be furnished for the water to escape. Open ditches closed at the end with standing water in them are not a very effectual means of draining lands; the water should always have some means of escape by a proper outlet.

Plank Drains.-Planks or heavy boards are sometimes used for making drains, and if properly laid will last many years, especially where they are kept constantly wet; but we would not recommend them for this purpose; since the labor and cost of draining is so great, more durable material should be used.

Turf Drains. - The same could be said of turf drains with respect to their durability. They are constructed by making the sides of the trench very slanting, and fitting in a piece of turf for a covering of sufficient size to go within a few inches of the bottom of the drain, with. out settling into it. This forms a drain that is not at all durable, being very liable to fill up by the falling in of the turf covering, or caving in at the sides. Brush and rails used in the construction of drains have so long been in disuse that they have become among the obsolete things and scarcely require comment here. They were first made use of in the progressive steps towards improvement in drainage, from the open ditches, which are such a nuisance on the farm, - and have long since given place, among our best farmers, to the more complete and perfect system of tile drainage.

The Hole PIow was for a time deemed a great convenience in drainage, but experience proved that it would not work except in a subsoil quite uniform and entirely free from stones, and that it would only do good work in the strong clay soils, while even then the trenches soon filled up with earth, so that no permanent results could be accomplished by it. It can only be used as a temporary means of drainage, and to do service until a more complete system can be adopted. 
Open Ditches for draining are very objectionable, since they are so liable to cave in at the sides and fill up with the mud that is washed into them, and by the treading of cattle over them, that they prove only a temporary means of drainage. They also require considerable land, as teams cannot be driven near their sides, in plowing or harrowing, while with the use of tiles no land whatever is rendered useless.

In crossing them with teams, bridges are required, causing cousiderable trouble and expense in constructing, or subjecting the farmer to great inconvenience without them. Besides these objections, open ditches with standing water are unhealthy, and are often the cause of disease in those sections where used. The digging of such trenches is also attended with considerable labor and expense. The soil removed from them is, however, often valuable for mixing with manure, and when dried, for bedding for stables and hog pens, since it contains more or less of the fertilizing properties, and forms a good compost with both the liquid and solid substances from these sources.

Furrow Draining, which consists of cultivating the land in ridges or furrows, by plowing in such a manner as to leave open furrows for drainage purposes between the ridges of earth, and planting on the top of each ridge, was formerly practiced in England and other portions of Europe to a considerable extent, but has generally been superseded for the most part by under-draining.

It requires considerable labor to prepare the land in this manner, and also a large area of surface to make it practicable, besides not being a very efficient method, is consequently not to be recommended when other and better means can be employed.

Hon. C. C. Andrews, in referring to the peculiar rigid appearance of the wheat fields in some portions of Italy, says that this formation comes from plowing with a view to drain. ing. The furrows are turned so that with the subsequent harrowing, and use of a wooden smoother, the ground is left in oval ridges or beds a foot high, and from 2 to 3 feet wide. The ditch between is scarcely wide enough to place the foot. What is striking about the ridges, is their remarkable regularity and precision. Occasionally, however, and apparently on the better-cultivated farms, the drains are in some cases 10 feet, in others 30 feet, apartthe plowing in such case being done as in the country north of the Alps.

These ridges are usually formed by turning two furrows together from opposite direc. tions, which gives the field a very uniform appearance. As we have previously stated, the most effectual method of draining lands is by the use of tiles, and will be found, all things considered, the most permanent, economical, and satisfactory means that can be employed for this purpose.

\section{GRASSES AND FORAGE PLANTS.}

G RASSES, or plants constituting the order Graminex, are distributed over the entire earth and constitute one of the largest orders of the vegetable kingdom. This order includes all the cereals of temperate climates, together with the grains of the warmer zones, and the bamboos of India and America-many of which have columnar stems reaching to the height of forest trees-yet are all formed on a common type, and therefore belong to the same botanical order as the tiniest spear of grass beneath our feet.

The seeds of many of the varieties and the nutrition of the herbage, form the chief por. tion of the sustenance of mankind, and the more valuable of the domestic animals. Among these are wheat, rye, maize or Indian corn, barley, oats, rice, sugar-cane, sorghum, broomcorn, millet, etc., while the bamboo is indispensable to the natives of India in the construction of their dwellings, the making of mats, cordage, boats, salls, masts, rafts, and even musical 


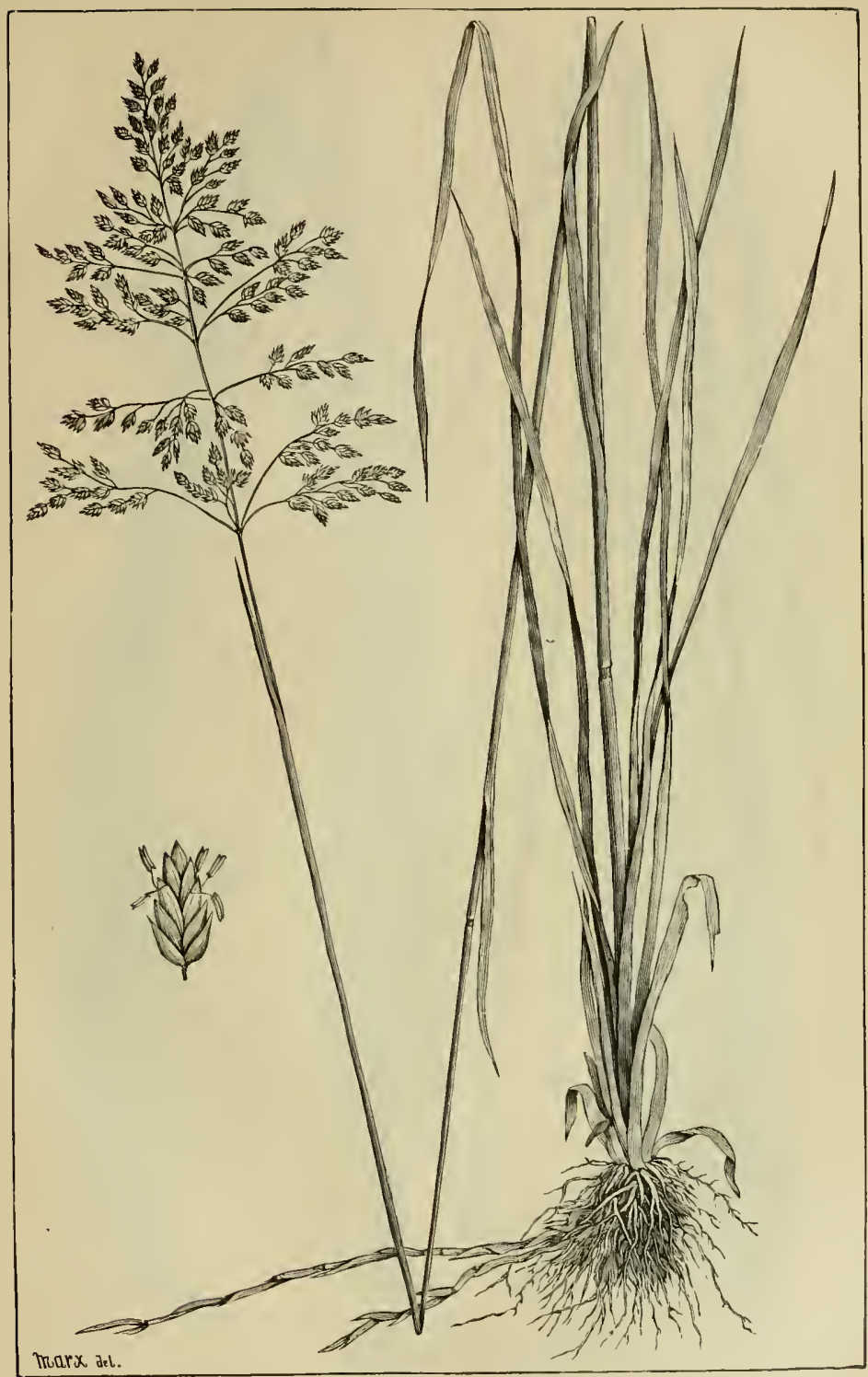

KENTUCKY BLUE GRASS.

(Poa pratensis.) 


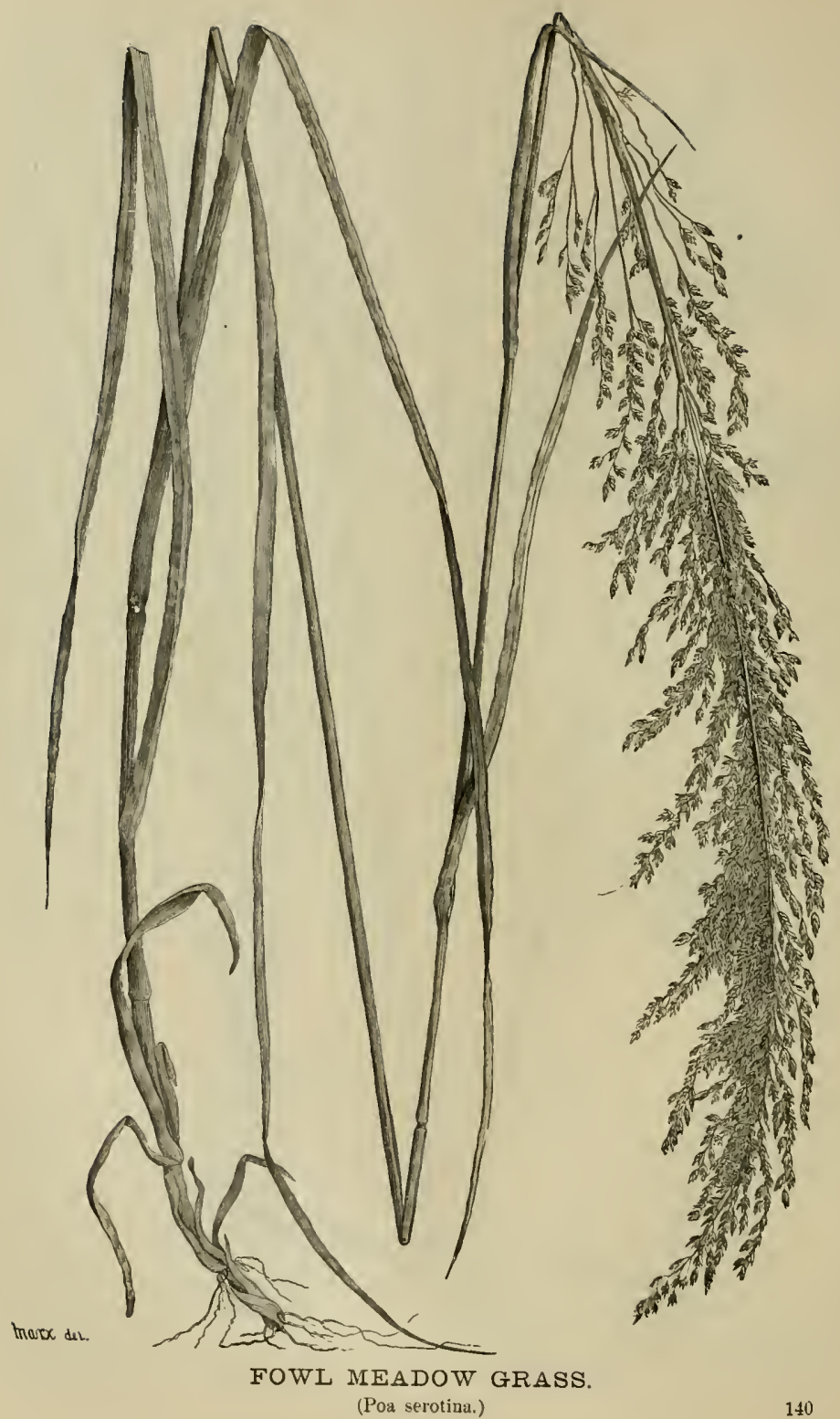




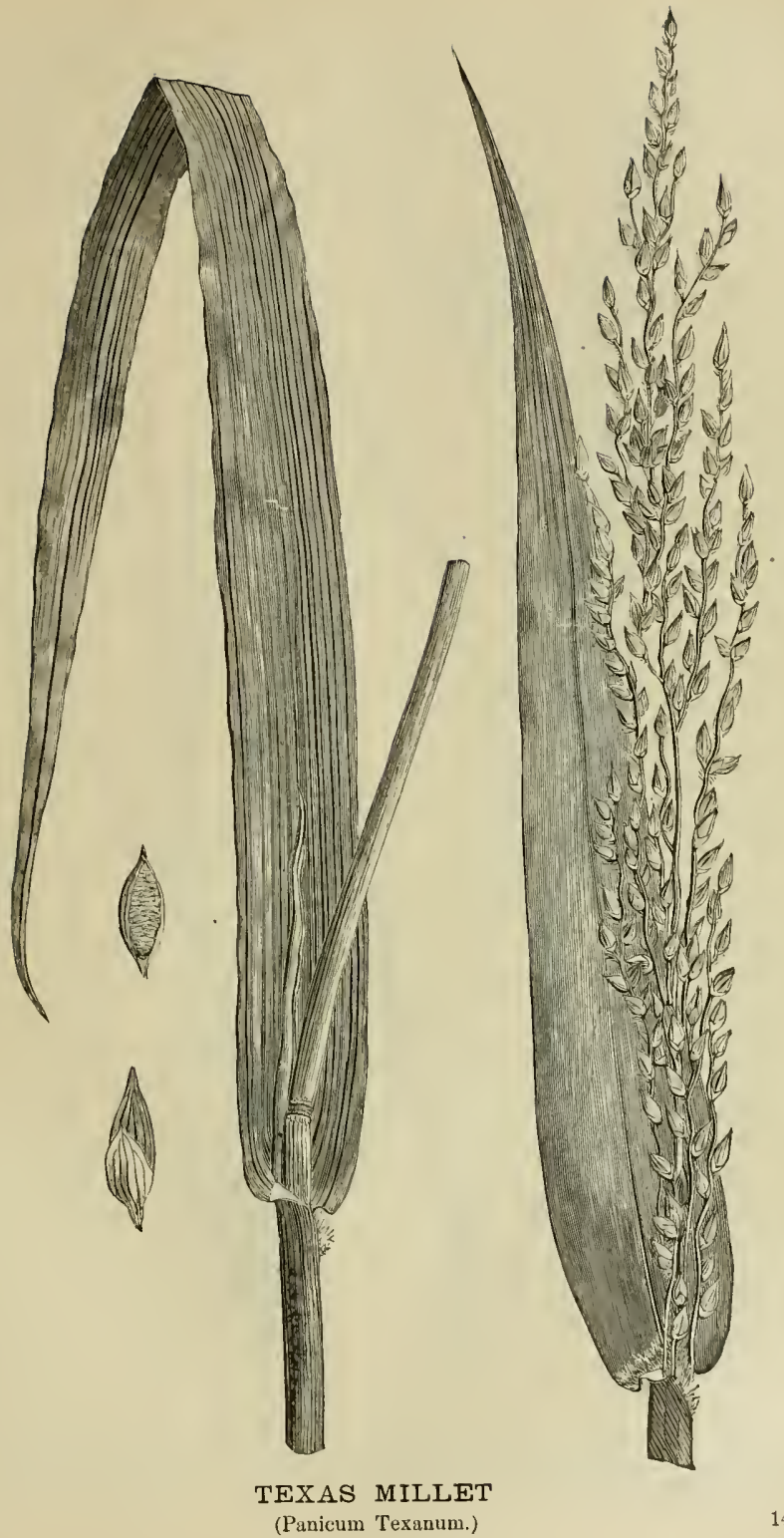




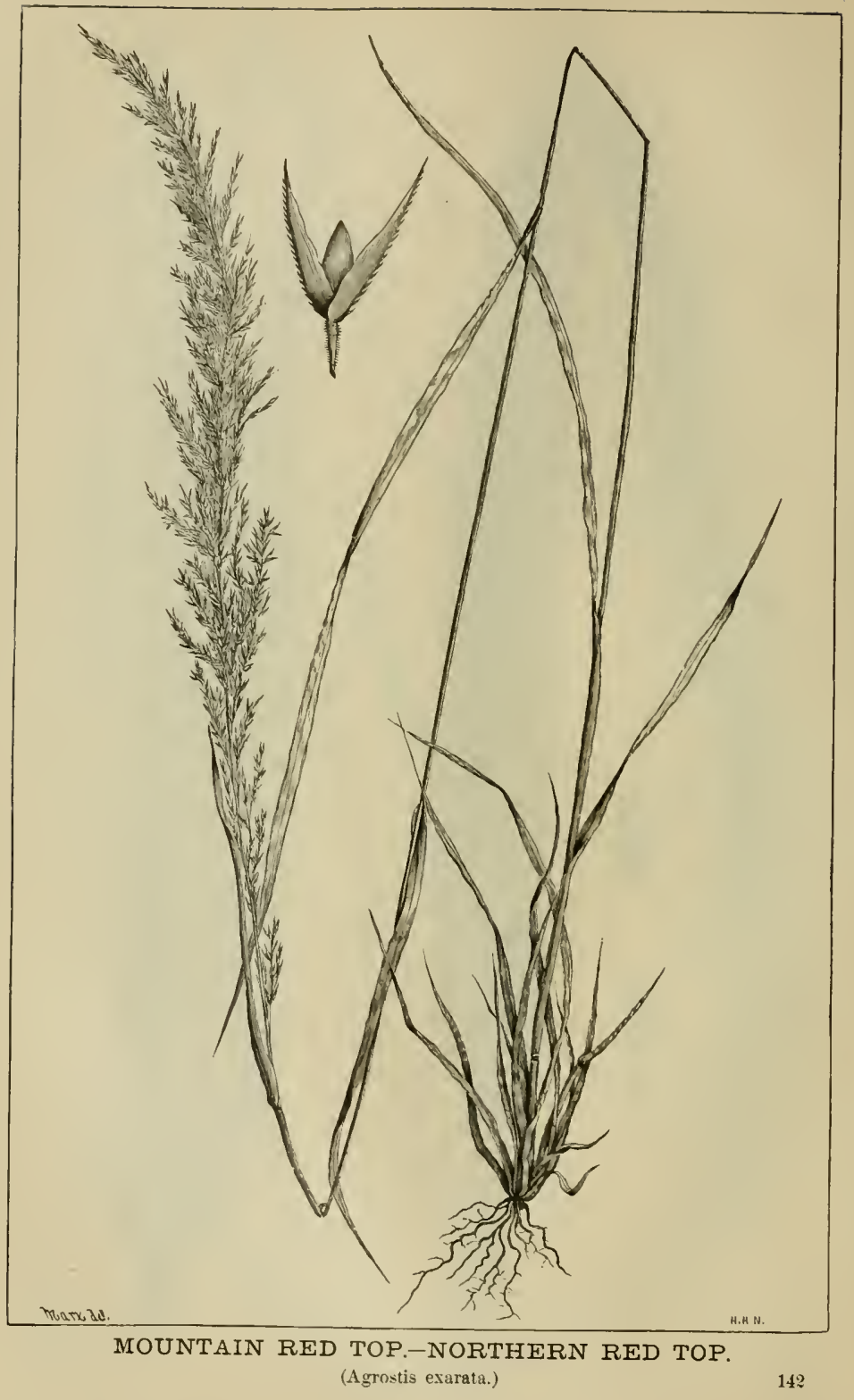


instruments and weapons. A large number of grasses, however, seem to have little or no agricultural value, and, to all appearance, are but little better than weeds on a farm, causing the farmer considerable labor and annoyance in keeping his lands clear of them. There are also probably many valuable species of native grasses growing widd in different sections of our country, now regarded as little better than worthless weeds, which when fully tested may be found to be of great agricultural value. The Department of Agriculture has within a few years past made special efforts in ascertaining the real value of some of these wild grasses, the results of which investigations have been, in a measure, suecessful; but thus far these experiments have been carried on in this country to only a limited extent. The English are far in advance of us in this respect, the most careful experiments there being made with reference to the value of the various grasses for different soils, situations, and climate, as well as in relation to their value as to the season, etc.

There are over two hundred varieties of grasses cultivated in England for the use of domestic animals, while in our own country the number of cultivated grasses is very much less, although there is probably no country on the entire globe that possesses so many varieties of native grasses. A few years in advance of the present will doubtless show great improvement in this department of our agriculture, and the proper tests afforded by science and experience, will acquaint us with the true value of many varieties, now almost unknown.

What render grasses so peculiarly nutritious, and hence valuable to agriculture, are the large proportions of sugar, starch, fatty matter, albumen, and fibrine contained, not only in the seeds, but also in the stems, leaves, and joints of grass before the seeds are fully matured.

The importance of grasses, in all systems of agriculture, can scarely be over-estimated. In fact, the proportion of meadow and pasture lands in any region is regarded as a good crite. - rion of the agricultural wealth of that region.

Wherever the importance of the grass crop has been overlooked in the desire to realize immediate results from special crops, the consequence has generally been an impoverished soil, and an impoverished people; while the farmer with an abundance of pasturage and meadow lands, has in his own hands and under his own control, the very elements of wealth, which, if judiciously employed, cannot fail of good in the result.

It is not to any one species of grass, or a few species only, that we should depend upon for the sustenance of our stock, but the many species intermingled, each doing its part in the great economy of nature,-some starting and maturing early, others late; some preferring low, wet localities, others only dry soils; some seeking the shaded situations for growth, others the most exposed localities, such as the broad open prairies of the West, or the savannas of the South. Some will grow only in the water, others only along the margin of lakes and rivers; some only in fresh water, others only in salt water. Thus we have grasses suited to every section and condition, every soil and climate, from one portion of the continent to the other, and no creation in the vegetable world, or any other department of nature's vast laboratory, will be found in vain or useless, however man may regard it.

The great error in New England farming has formerly been the practice of stinting or robbing the grass lands to feed the hoed crons and the arable lands. Although there is at present a great improvement in this respect over the old-time method, still the practice is not wholly discarded, and more attention should be given to the cultivation of the grass crop than is common in many sections.

A considerable portion of the department, embracing the description, cultivation, and relative value of the different grasses, is taken from "Grasses and Forage Plants," of which the editor of this volume is the author.

As the general appearance of plants is often greatly modified by climate, soil, and modes of cultivation, it is important to fix upon certain characteristics which are permanent and 
unaltered by circumstances, by means of which the particular genus and species may be identified with ease and certainty. It is evident that these characteristics could not be simply in the leaves, or the stems, or the size of the plant, because there will be a great differenee between plants growing in a poor, thin, sandy soil, and others of the same species on a deep, rich loam. Botanists have, therefore, been compelled to resort to other peculiarities to distinguish between different species; and the terms used to express these, like the terms used in other departments of natural history, are technical; and hence, in detailing the natural history of the grasses, the use of technical language to a greater or less extent, eannot be avoided.

The flowers of the grasses are arranged on the stem in spikes, as where they are set on a common stalk without small staiks or branches for each separate flower, as in herds-grass (Phleum pratense), or in panicles, or loose subdivided clusters, as in orchard grass (Dactylis glomerata). A panicle is said to be loose or spreading; as in redtop (Agrostis vulgaris), when the small branches on which the flowers are set, are open, or extended out freely in different directions; it is said to be dense, or crowded or compressed, when the branches are so short as to give it more or less of the spike form.

In different parts of the country a great variety of grasses are known by different common names, - the same name being frequently applied to grasses entirely different from each other; or, a single plant may be known by several different names, which occasions no little confusion where the common or local name is wholly depended upon to distinguish one variety from the other; for instance, the grass that is commonly called "cat's tail" in England, is known as timothy in some sections of this country, and herds-grass in others; and that which is called red-top in one locality, is known as herds-grass in another, and by the various terms of Burdin's grass, red bent grass, summer dew grass, small red-top, fine red-top, and fowl meadow grass in others. What is commonly called blue grass in one locality, is known as green grass in another, etc.; so that it is evident that were the common name to be depended upon alone, in distinguishing one variety from another, considerable difficnlty would be involved in arriving at a correct understanding as to which species is meant. And when we take into further eonsideration the fact of the close resemblance of many of the different species of grasses, espeeially to a person inexperienced in distinguishing one from another, the subject becomes a more puzzling one still, and it could hardly be expected that a correct understanding could be arrived at, when depending entirely upon the common or local name. We therefore see the necessity of the use of the Latin name of the species, and when this is once known, there is no further difficulty in this respect, since the same Latin or scientific name is never applied to but one of a species.

The description of the obnoxious or worthless grasses here given, will also be of use to the farmer in enabling him to recognize them when found, and hence, to take measures for their extermination.

Timothy, or Herds-Grass (Phleum pratense). Generic characters: Panicle spiked, spikelets compressed, palea shorter than the awned glumes, the lower one truncate, usually awnless; styles distinct, filaments hairy, spike dense, rough, or harsh. So called from an ancient Greek term signifying cat's tail, the name by which it is still most frequently known in Great Britain.

Specific characteristics: Spikes cylindrical or elongated; glumes hairy on the back, tipped with a bristle less than half their length; leaves long, flat, rough, with long sheaths; root fibrous, often bulbous--perennial. Grows best on moist, peaty soils. This grass is uni. versally known and highly valued. It is, in fact, generally considered in this country as the best grass for hay that can be produced. It is commonly grown throughout the entire North, and flourishes well on the drained lowlands of the South. It is said to have received its name more than a century and a half ago from one Herd, of Piseataqua, who found it grow. ing in a swamp there. 
The name of timothy, by which it is more generally known over this country and abroad, was obtained from Timothy Hanson, who cultivated it extensively, and, according to some accounts, introduced it into England, from whence it is supposed to have been originally brought to this country. It forms a large proportion of what is called English hay.

For land that is adapted to it, there is, all things considered, no better grass than timothy, and it is probably unsurpassed for hay by any kind now cultivated. If allowed to stand too long, however, it will, as almost every farmer knows, become hard and woody, and its nutritive value greatly lessened, yet if cut when in bloom, it is relished by all kinds of stock,--especially so by horses,-while it possesses a large percentage of nutritive matter in comparison with other agricultural grasses. It was formerly often sown with clover, but the best practical farmers have discontinued this custom on account of the different times of blossoming of the two crops; since timothy being invariably later than clover, the former must be cut too green, before blossoming, when the loss is great by shrinkage, and when the nutritive matter is considerably less than at a little later period, or the clover must stand too long, when there is an equally serious loss of nutritious matter in that. It grows very readily and yields very large crops on favorable soils. We have known instances where its yield was four tons to the acre of the best quality of hay, the timothy constituting the bulk of the grass. It is cultivated with ease, and yields a large quantity of seed to the acre, varying from ten to thirty bushels on rich soils. This grass has long roots which extend deep into the soil, and when once well established, it will withstand drouth better than many other varieties. When a good growth is once well started on a mellow surface-soil overlaying a moist subsoil, this crop is quite safe against any ordinary drouth. It only requires a good start in the spring, so as to occupy and protect the ground. May is the best month for the grass crop, and if it be unusually dry, or cold winds prevail then, the product will be liable to be affected accordingly; but such grasses as have a good soil, and deeply penetrating roots, will endure without permanent injury, the effects of unfavorable weather better than those varieties with roots lying near the surface, as they can draw moisture from the subsoil, and also passes the basis for a good crop the following year.

In one respect, perhaps, it must be admitted that this grass is inferior to meadow foxtail, and that is, in the quantity of its aftermath; for while that of the latter is very great, the aftergrowth of timotlyy is but slight, and if allowed to stand too long and then mown in a dry time, it starts so slowly as to leave the ground exposed to the

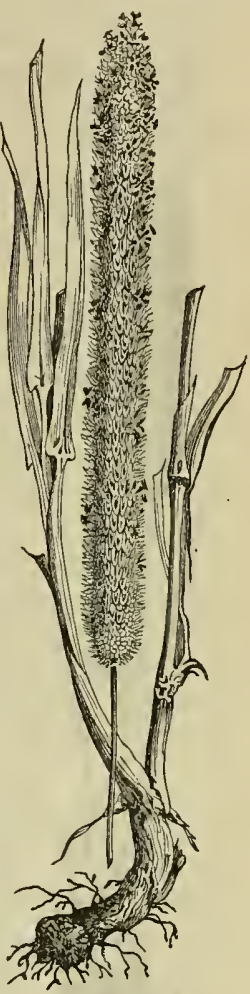

TIMOTHY. scorching rays of the sun, unless there happens to be a rapid growth of clover to protect it. It is frequently attacked by an insect, apparently just before the time of blossoming, which causes the stalk to die.

When pastured, it yields abundantly during the season, starting very early in the spring, and is greatly relished by stock of all kinds. It may be sown on wheat or rye in August, or later, or in the spring. The quantity of seed required per acre depends largely on the soil and its condition. From ten to fourteen quarts is sufficient, where the soil is fine and mellow, while from eighteen to twenty might be required in heavy clay, when sown alone. It is stated by good authority, that a crop of pure timotliy produced three tons to the acre, on the farm of Mr. George Geddes, near Syracuse, N. Y., and also that Mr. John Fisher, of Carroll Co., Md., 
cut from one acre five tons and one thousand six hundred and twenty-two pounds of dry hay. The proper time for mowing Timothy, is when the first dry appearance is seen abore the first joint; if mowed earlier than this, the plant is injured; if left to a later period, the starch and sugar are converted into indigestible woody fibre, and the nitrogenous compounds, on which its value chiefly depends, are transferred from the leaves and culm to the seed, which mostly drop out before they reach the manger. Timothy is not well adapted to hot sands, grave's, chalks, nor hard, sterile clays; but thrives on peaty, damp soils, and especially on most calcareous loams, where it exhibits its fullest perfection.

Headow Foxtail. (Alopecurus Pretensis.) - Generic characteristics: Spikelets, one flowered, glumes compressed and keeled, united at the base, lower palea awned on the back, upper palea wanting, stamens three, styles generally united, stigmas long, panicle compressed

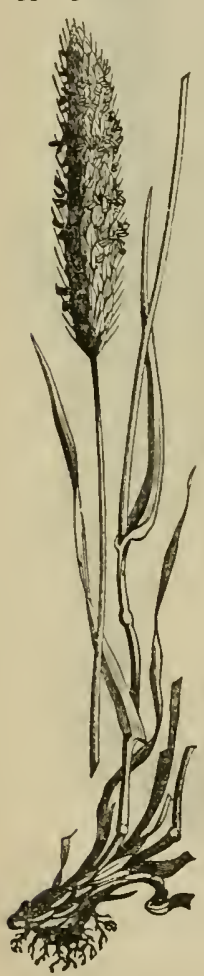
into a cylindrical spike like the tail of a fox, from which it derives its name. Native of Great Britain.

The specific characters are, an erect, smooth stem, two or three feet high, with swelling sheaths, spikes cylindrical, obtuse, equaling the sharp cone-like glumes, awn twisted and twice the length of the blossom. The spike not so long as that of Timothy. Flowers in May, in fields and pastures. Perennial-introduced.

The meadow foxtail closely resembles Herds-grass, but may be distinguished from it as having one palea only. The spike or head of meadow fortail is soft, while that of Timothy or Herds-grass is rough. It flowers earlier than Timothy, and thrives on all soils except the dryest sands and grarels.

It is valuable grass for pastures, on account of its early and rapid growth, and of its being greatly relished by stock of all kinds. The stems and leaves are too few and light to make it so desirable as a field crop. It thrives best on a rich, moist, strong soil, and the quantity of its nutritive matter when raised on such soils is considerably greater than on sandy soils. As a pasture grass, its luxuriant aftermath, being in value nearly one fourtl greater than its first spring growth, recommends it still more highly. In this respect it is superior to Timothy, the aftermath of which is generally but slight. For lands designed to be laid down to permanent pasture it will make a prominent part of the seed. Where it occurs in fields it loses largely its nutritive value if cut in the blossom. It is regarded in England as one of the most valuable of the native pasture grasses, forming there a very considerable portion of the sward, vegetating with great luxuriance, and starting up vigorously when eaten off by stock, producing seed in abundance, and enduring any amount of forcing and irrigation. It does not acquire its full perfection and hold of the soil until three or four years after being sown. The aftermath exceeds the flowering crop in quantity as well as in nutritive matter. The grass loses seventy per cent. of its weight in drying, and the hay contains abont sixtyseven hundredths per cent. of nitrogen.

The seed of meadow foxtail is covered with the husks of the flower, MEADOW Fortail. soft and woolly, while the larger valve is furnished with an awn. There are five pounds of seed in a bushel, and 76,000 seeds in an ounce. An insect attacks the seed while it is forming, and it is a'so subject to blight, and hence the seed is somewhat difficult to procure and is held at a high price. We have many grasses superior to it for cultiva. tion, but for permanent pastures it is superior to Timothy, which is not a suitable pasture grass.

It is found abundantly in our best pastures, is one of the earliest to start in the spring, and the first to mature its seeds. 
Orchard Grass, Rough Cocks Foot, (Dactylis glomerata.)-Spikelets several flow. ered, crowded in clusters, one-sided, panicle dense at the top, branching, glumes two, herbaceous, keeled, long-pointed. Stamens three, seed oblong, acute, free. Named from dactylus a finger.

Orchard grass flowers in dense tufts. Its stem is erect, about three feet high. Leaves linear, flat, dark green, rough on both surfaces, which, with the fancied resemblance of its loose tufts to the foot of a barnyard fowl, have given it the common name in England of rough cocksfoot. Root perennial. Flowers in June and July. Not uncommon in fields and pastures.

This is one of the most valuable and widely known or all the pasture grasses. It is com. mon to every country in Europe, to the north of Africa, and to Asia as well as America. Its culture was introduced into England from Virginia, where it had been cultivated some years previously, in 1764. It forms one of the most common grasses of English natural pastures, on rich, deep, moist soils. It became, soon after its introduction into England, an object of special agricultural interest among cattle feeders, having been found to be exceedingly pala. table to stock of all kinds. Its rapidity of growth, the luxuriance of its aftermath, and its power of enduring the cropping of cattle, commend it highly to the farmer's care, especially as a pasture grass. As it blossoms earlier than timothy, and about the time of red clover, it makes an admirable mixture with that plant, to cut in the blossom and cure for hay. As a pasture grass, it should be fed close, both to prevent its forming thick tufts and to prevent its running to seed, when it loses a large proportion of its nutritive matter, and becomes hard and wiry. All kinds of stock eat it greedily when green.

It is a grass well adapted to sow with clover and other seeds for permanent pastures or hay, but is not generally sown alone except for seed. It exhausts soil less than timothy or rye grass, and will endure considerable drouth. It has been known to grow more than five feet in height, and has produced five and a half tons per acre. It contains nearly as much of the fat and flesh-forming material as timothy, but much less of heat-forming matter. It flourishes well in shady places, and receives its name from its being, for this reason, adapted to orchards. Its disposition to grow in tufts or tussocks may be prevented by good cultivation of the land, and thick sowing. Harrowing and rolling in the spring will also remedy this evil.

It flourishes well in almost all soils and climates, but best in sandy loam. It is known in England as cocksfoot. In this country it is most common in New England, New York, Pennsylvania, and Ohio. It is good for both orchards and pastures, especially for the latter, as it produces good milk, beef, mutton, or wool, and every kind of stock eat it freely and thrive well upon it. After being mowed, or closely grazed, it throws up a new growth more readily than most of the other grasses, and it is not inclined to run out in situations where it once gets a good root and the soil is adapted to it, although as easily subdued as timothy by plowing. It is, however, better than the latter to mix with clover, and it ripens more nearly at the same time. If sown too thin it is apt to be rather coarse; hence, to secure the best results, it should be thickly sown, - from two to three bushels per acre being a liberal quantity, - if sown alone. It is, however, not usually sown alone, except for seed, but with a variety of other grasses blossoming about the same time, when designed for hay, and with both early and late grasses for pasturage. Being an early grass, it requires early cutting, or much of its nutritive value will be lost; the time for cutting being when it comes into blossom.

Green Headow Grass, June Grass, Common Spear Grass, Kentucky Blue Grass, etc., (Poa pratensis.)-The characteristics of the genus Poa, are, ovate spikelets, compressed, flowers two to teu in an open panicle, glumes shorter than the flowers, lower palea compressed, keeled, pointless, five-nerved, stamens two or three, seed oblong, free, stems tufted, leaves smooth, flat, and soft. 
Specific characters: Lower florets connected at the base by a web of long, silky filaments, holding the calyx; outer palea, five-ribbed, marginal ribs hairy, upper sheath longer than its leaf; height from ten to fifteeu inches, root perennial, creeping, stem erect, smooth, and round, leaves linear, flat, acute, roughish on the edges and inner surface; panicle diffuse, spreading, erect. The plant is of a light green color, the spikelets frequently variegated with brownish purple. Introduced. Flowers in June.

This is an early grass, very common on the soils of New England in pastures and fields, constituting a considerable portion of the turf. It varies much in size and appearance according to the soil on which it grows. In Kentucky it is universally known as Blue grass, and elsewhere frequently called Kentucky blue grass, and still more frequently, June grass. It must be regarded as one of the best pasture grasses known. It is common all over the northern part of the country, growing indigenously in all limestone countries lying between the thirty-fourth and forty-fifth parallels, and coming to its highest perfection upon the rich, marly blue limestone soils of some of the central counties of Kentucky. It is said to have been found growing there when the region was first discovered, constituting a natural pasturage that attracted vast numbers of grazing wild animals, countless herds of buffalo, elk, deer, antelopes, etc.

This grass is not so well adapted to a short rotation, and is, therefore, less suited to our mowing-lots, from the fact that it requires three or four years to become well set so as to form a close sward. This habit of growth is less objectionable as a permanent pasture. When a soil is once well sodded with this grass, it will endure the vicissitudes of the seasonsheat and cold, sunshine and shade, droughts and floods-with wonderful persistency. It is the source of wealth in sections adapted to it; and there are pastures of it fifty years of age, still luxuriant and profitable. It throws up flower-stalks but once in the season; but it starts quickly after grazing or cutting, and forms a thick green growth.

On this account, it is highly recommended for lawns. It thrives equally well on ligh or low lands, but is not adapted to a thin, poor soil. In some sections not adapted to its growth, it is regarded as a troublesome weed. Its flower-stalk is short and matures early. It is not to be judged in value by this, but by its long leaves, abundantly produced throughout the season, which form a rich, sweet herbage; in fact, one of the best for milch cows, the dairy product from such milk being of the best quality. On rich soil, the leaves will, frequently attain the length of two feet when allowed to grow.

It grows well in rather a dry soil, but will grow on a variety of soils, from the dryest knolls to a wet meadow. It does not withstand severe droughts as well as some other grasses. It endures the frosts of winter better, perhaps, than most other grasses; and in Kentucky, where it attains the highest perfection as a pasture grass, it sometimes continues luxuriant through their mild winters. As it requires at least two or three years to become well set, and does not arrive at its perfection as a pasture grass till the sward is older than that, it is not suited to alternate husbandry, or where the land is to remain in grass only two or three years and then be plowed up. In Kentucky, the best blue grass is found in partially shaded pastures.

Meadow Spear Grass, Nerved Manna Grass, (Poa nervata,) is the fowl meadow of some farmers, while the grass most commonly called fowl meadow, (Poa serotina,) goes with them under the name of "bastard fowl meadow." It has a broad, open panicle, six inches in length, with slender branches; spikelets small, ovate, oblong, green; leaves in two rows like a fan, a little rough; stem a little compressed, one to three feet high. It is a native American grass, flowering late in June. It is a hardy grass, grows best on wet or moist grounds, and is said also to succeed on light upland soils. It is somewhat coarse, and not particularly relished by cattle, though readily eaten in winter. It would be a valuable ingredient in a mixture for moist pastures. It is not very common. Native of North America. 
Blue Grass, or Wire Grass, (Poa compressa.) - Stems ascending, flattened, the uppermost joint near the middle, leaves short, bluish green, panicle dense and contracted, expanding more at flowering; short branches often in pairs, covered with four to nine flowered, flat spikelets; flowers rather obtuse, linear, hairy below on the keel; ligule short and blunt; height about a foot. It is very common on dry, sandy, thin soils and banks, so hardy as to grow on the thin, hard soils covering the surface of rocks, along trodden walks, or gravelly knolls. It shoots its leaves early, but the amount of its foliage is not large, otherwise it would be one of our most valuable grasses, since it possesses a large per cent. of nutritive matter. Flowers in July. Most grazing animals eat it greedily, and it is especially relished by sheep. Its bluish green stems retain their color after the seed is ripe. It shrinks less in drying than most other grasses, and consequently makes a hay very heavy in proportion to its bulk. It is an exceedingly valuable pasture grass on dry, rocky knolls and should form a portion of a mixture for such soils. This should not be confounded with Kentucky blue grass alluded to above.

Annual Spear Grass, (Poa annua,) is, perhaps, the most common of all our grasses. Its stems are spreading, flattened, panicle often one-sided, spikelets crowded, three to seven flowered, lower palea more or less hairy on the nerves below; leaves of a light green, swordshaped, flat, often crumpled at the margins, smooth on both surfaces, rough at the edges. F'lorets not webbed, and this distinguishes it from the June grass, (Poa pratensis,) and its varicties. The outer or lower palea of this grass has no hairs on the lateral ribs as the Poa pratensis has. This modest and beautiful grass flowers throughout the whole summer, and forms a very large part of the sward of New England pastures, producing an early and sweet feed, exceedingly relished by cattle. It does not resist the drought very well, but becomes parched up in our pastures.

The Rough Stalked Meadow Grass, (Poa trivialis, though not so common as the June grass, (Poa pratensis,) is still often met with, and is found to have webbed florets; outer palea five-ribbed, marginal ribs not hairy, ligule long and pointed, sterns two to three feet high. Distinguished from June grass by having rough sheaths, while in the latter the sheaths are smooth, the ligule obtuse, and the marginal ribs of outer palea furnished with bairs. The rough-stalked meadow grass has a fibrous root, that of the June grass is creeping. It flourishes in moist meadows, where it flowers in July. Introduced.

This is a valuable grass to cultivate in moist, sheltered soils, possessing very considerable nutritive qualties, coming to perfection at a desirable time, and being exceedingly relished by cattle, horses, and sheep. For such soils it should form a portion of a mixture of seeds, producing, in mixture with other grasses which serve to shelter it, a large yield of hay, far above the average of grass usually grown on a similar soil. It should be cut when in seed and not in the flower. Seven pounds of seed to the acre will produce a goud sward. The grass loses about seventy per cent. of its weight in drying. Its hay contains about one and sixty hundredths per cent. of azote, and the nutritive qualities of the aftermath exceed very considerably those of the crop cut in the flower or in the seed.

Wood Meadow Grass, (Poa nemoralis.) - This grows from eighteen inches to two feet high, has a perennial, ereeping root, an erect stem, slender, smooth, the upper sheath no longer than its leaf, with a very short ligule, the base of the floret having a silky web suspending the calyx, leaves light green. It is common in moist, shady places, and appears as a tall, rank grass, with a long, finely arched panicle. It flowers in June and ripens its seed in July.

It is certainly to be classed among the best of shaded pasture grasses, furnishing a fine, succulent, and very nutritive herbage, which stock of all kinds are very fond of. Hay contains one and sixty-four one-hundredths per cent. of azote. The grass loses about fifty-five per cent. of its weight in drying. 
The Creeping Sea Meadow Grass, or Sea Spear Grass, (Poa maritima,) referred by Gray to glyceria, is a beautiful grass which appears in and around salt marshes, growing from six to twelve inches high, and having a perennial, creeping root. Stem erect, round, smooth, leaves mostly folded and compressed, roughish on the inner surface, spikelets linear, with from six to ten florets not webbed, the outer palea or lower floret terminating in an acute point. Flowers in July. Grows naturally near the sea.

The Water Spear Grass, or Reed Meadow Grass, (Poa aquatica) grows in wet soils, is a tall, reedy grass, four or five feet high with a panicle nearly a foot long, diffuse, with smooth, flexuous branches. From its large size and broad leaves it can hardly be mistaken for any of the other species of Poa. Its root is perennial, creeping, stern erect, stout, smooth, joints seven, smooth, spikelets numerous, florets not webbed. Flowers in August. This grass is referred by Gray and others, to glyceria. It is very common in wet meadows and will be easily recognized. More nutritive when in flower than when the seed is ripe. It contains a comparatively large per cent. of sugar. Makes a valuable fodder, and cattle are very fond of it.

Several other species belonging to this genus are frequently met with, as the Branching Spear Grass, on dry, sandy soils, a very elegant species, with a large panicle of sea-green spikelets; the Hair Spear Grass, also an elegant grass growing on similar soils, with a hairy branching panicle over a foot long, leaves linear, nerved. But the most important of all is the Fowl Meadow, or False Redtop.

Fowl Headow, or False Redtop, (Poa serotina.) - The specific characteristics of this species are two to four, sometimes five, flowered spikelets, oval, spear-shaped, ligules elongated, flowers acutish, green, often tinged with purple, roots slightly creeping; wet meadows and banks of streams, very common. Flowers in July and August. In long. continued moist weather the lower joints send up flowering stems. The panicle is erect and spreading when in flower, but more contracted and drooping when ripe. It is perennial. Native of Germany.

It early commended itself to the attention of farmers, for Jared Eliot, writing in 1749 , says of it: "There are two sorts of grass which are natives of the country, which I would recommend, - these are Herds.grass, (known in Pennsylvania by the name of Timothy. grass,) the other is Fowl Meadow, sometimes called Duck-grass, and sometimes Swamp-uire grass. It is said that Herds-grass was first found in a swamp in Piscataqua, by one Herd, who propagated the same; that Fowl Meadow-grass was brought into a poor piece of meadow in Dedham, by ducks and other wild water-fowl, and therefore called by such an odd name. It is supposed to be brought into the meadows at Hartford by the annual floods, and called there Swamp-wire grass. Of these two sorts of natural grass, the fowl-grass is much the best; it grows tall and thick, makes a more soft and pliable hay than Herds-grass, and consequently will be more fit for pressing, in order to ship off with our horses; besides it is a good grass, not in abundance inferior to English grass. It yields a good burden, three loads to the acre. It must be sowed in low, moist land. This grass has another good quality, which renders it very valuable in a country where help is so much wanting; it will not spoil or suffer, although it stand beyond the common times for mowing. Clover will be lost, in a great measure, if it be not cut in the proper season. Spear-grass, commonly called English grass, if it stands too long, will be little better than rye straw; if this outstand the time, it is best to let it stand till there comes up a second growth, and then it will do tolcrably well; but this fowl-grass may be mowed any time from July to October. *** This I wondered at, but viewing some of it attentively, I think I have found the reason of it. When it is grown about three foot high it then falls down, but doth not rot like other grass when lodged; in a little time after it is thus fallen down, at every joint it puts forth a new branch; now to 
maintain this young brood of suckers there must be a plentiful course of sap conveyed up through the main stem or straw; by this means the grass is kept green and fit for mowing all this long period."

This grass grows abundantly in almost every part of New England, especially where it has been introduced and cultivated in suitable ground, such as the borders of rivers and intervals occasionally overflowed. It will not endure to be long covered with water, especially in warm weather. It is well to let a piece go to seed, save the seed and scatter it over low lands.

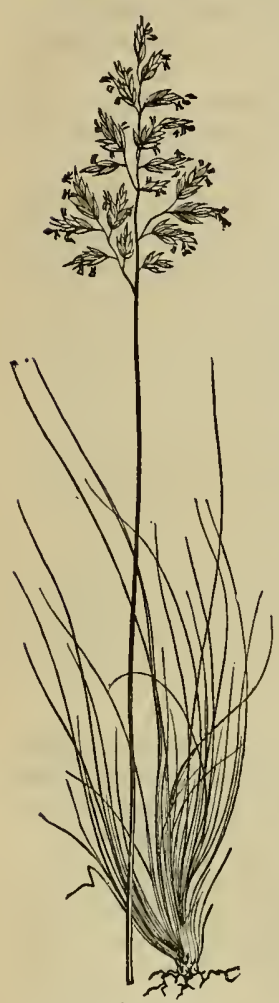

SHEEP'S FESCCE.
It makes an excellent grass for oxen, cows, and sheep, but is thought to be rather fine for horses. It never grows so coarse or hard but that the stalk is sweet and tender, and eaten without waste. It is very easily made into hay, and is more nutritive, according to Sinclair, than either foxtail, orchard grass, or tall meadow oat grass. Owing to its constantly sending forth flowering stems, the grass of the lattermath contains more nutritive matter than the first crop at the time of flowering, hence the names fertilis and serotina, fertile and late flowering meadow grass. It thrives best when mixed with other grasses, and deserves a place in all mixtures for rich, moist pastures.

This species is most common in the Northern States, particularly north of Pennsylvania. It is a good grass for moist meadows. In Wisconsin and some of the other Western States, where many natural meadows of this grass occur, it is highly esteemed.

The Creeping Meadow Grass, (Eragrostis reptans,) is frequently found on the sandy banks of rivers, and is a beautiful and delicate grass. Flowering in July and August. Its leaves are short, nearly awl-shaped, spikelets smooth, long and lance-shaped, flowers acute, sheaths loose, striate and a little

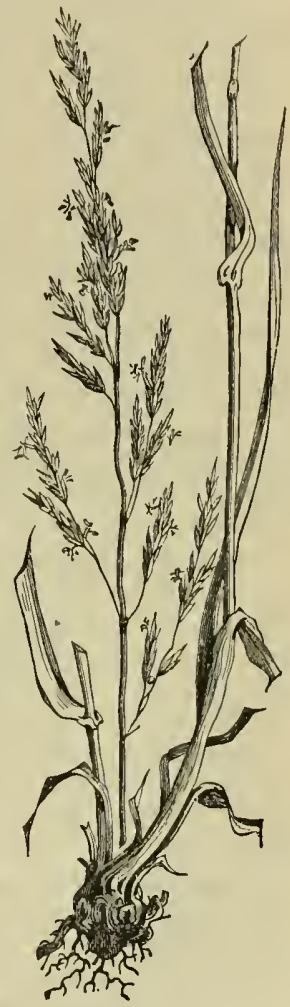

MEADOW FESCUE. hairy on the margin, panicles from one to two inches long. Not a cultivated grass.

Sheep's Fescue, (Festuca ovina, is known by its narrow panicle, short, tufted, bristle-shaped leaves, of a grayish color, somewhat tinged with red, its two to six flowercd spikelets, awn often nearly wanting. It grows from six to ten inches high in dense perennial rooted tufts. It forms an excellent pasturage for sheep. It flowers in June and July, in dry pastures.

Headow Fescue, (Festuca pratensis,) is one of the most common of the fescue grasses. It is said to be the Randall grass of Virginia. Its panicle is nearly erect, branched, 
close, somewhat inclined to one side; spikelets linear, with from five to ten cylindrical flow. ers; leaves linear, of a glossy green, pointed, striated, rough on the edges; stems round, smooth, from two to three feet high, roots creeping, perennial. Its radical or root leaves are broader than those of the stem, while in most other species of fescue the radical leaf is generally narrower than those of the stem. Flowers in June and July, in moist pastures and near farm houses.

This is an excellent pasture grass, forming a very considerable portion of the turf of old pastures and fields, and is more extensively propagated and diffused by the fact that it ripens its seed before most other grasses are cut, and sheds them to spring up and cover the ground. Its long and tender leaves are much relished by cattle.

It is generally sown in mixture with other grasses, as orchard grass, rye grass, or common spear grass. It is of much greater value at the time of flowering than when the seed is ripe. It is said to lose a little over fifty per cent. of its weight in drying for hay.

Tall Fescue Grass, (Festuca elatior, is also found pretty commonly in moist meadows and around farm houses. Its panicle is contracted, erect, or somewhat drooping, with short branches, spreading in all directions; spikelets crowded, with five to ten flowers, rather remote, oblong, lanceolate; leaves flatish, linear, acute; stems two to four feet high, root perennial, fibrous, somewhat creeping and forming large tufts. Flowers in June and July.

It is a nutritive and productive grass, growing naturally in shady woods and moist, stiff soils. Cattle are very fond of it. Said by some to be identical with the meadow fescue.

Hard Fescue Grass, (Festuca duriuscula,) is also found to some extent, though not so commonly as the meadow fescue. It is by some regarded as a variety of the sheep's fescue, taller, and with a panicle more open, leaves flat, and spikelets four to eight flowered. It grows from one to two feet high. Flowers in June, in pastures and waste grounds.

Red Fescue, (Festuca rubra,) by some regarded as only a variety of the preceding, is one of the largest of the varieties of fescue. Its leaves are broadish, flat, root extensively creeping, and throwing out lateral shoots. Found in dry pastures near the sea shore, in sandy soils. It is a grass of better quality than some of the other varieties, but never cultivated here as an agricultural product. The color of its leaves is somewhat more grayish than the preceding and often tinged with red.

Slender Spiked Fescue, (Festuca lotiacea,) is a species nearly allied to the tall fescue and possesses much the same qualities. It grows naturally in moist, rich meadows, forming a good permanent pasture grass, but as it is met with only very rarely among American grasses, and is of no value for cultivation, it scarcely deserves a more extended notice.

Rice Grass, Cut Grass, False Rice, (Leersia oryzoides,) grows very common in wet swampy places. Stems from two to three feet high, panicle erect, spreading with rough, slender branches, leaves narrow or long, sheaths exceedingly rough and sharp to the hand, drawn from the end backward. Florets oval and white, spikelets flat. Flowers in August. Native of the Levant. Name from Leers, a German botanist.

It is a beautiful grass, said to be useful at the South, where it is cultivated to some extent, and may be cut several times in a season. It is said there to make a valuable hay. At the North it is regarded as a weed, and thorough draining will destroy it.

Redtop, Finetop, Burden's Grass, Dew Grass, Herds-Grass of Pennsylvania and Southern States (Agrostis vulgaris). Plants of this genus have one flowered spikelets in a loose open panicle; glumes nearly equal, the lower longer than the palex, which are thin and naked: stamens three-perennial.

The specific characters are, stems erect, slender, round, smooth and polished; roots creeping, panicle oblong, leaves linear, ligule very short, lower palea mostly awnless and three 
nerved. Flowers in July. Pastures and moist meadows very common-introduced. The term Agrostis was the ancient Greek word for field, and was applied to all varieties of grass that grew there.

This valuable grass, so common in all our cultivated fields, has been an inhabitant of our soils for more than a century. It was called simply English grass by Eliot, Deane, and other early writers, and by the English, Fine Bent. Indeed, the whole genus agrostis is commonly known in England as "Bent Grass." This grass is often sown with timothy and clover, in which case the clover, of course, soon disappears, being biennial, when timothy follows, after which redtop usually takes its place, and with some wild grasses forms a close sward. In Pennsylvania and States further south, it is universally known as Herds-grass-a name applied in New England and New York to Phleum pratense alone. It is of somewhat slow growth, but of good or medium quality. It is suited to moist soils, though common to all. This grass is probably rather overrated. It makes profitable crop for spending, though not so large a crop is obtained as from Herds-grass. It is a good permanent grass, and consequently well-suited to Northern pastures, as it endures that climate as well as any other grass. It should be fed close in pastures, for if allowed to grow up to seed the cattle refuse it; and this fact seems to show that it is not so much relished by stock as some of the other pasture grasses. The fact that cattie eat any grass greedily in the spring, is no proof of its excellence or nutritious qualities, since, then, all grasses are tender and full of juice, and many varieties of both grasses and shrubs are readily eaten, which at a more advanced stage of growth are refused.

This grass goes by various names, and is greatly modified by soil and cultivation. On a moist, rich soil it grows larger than on a poor, thin soil, and not only larger but has a darker purplish color, with a stem varying from eighteen inches to two feet or two and a half feet high; while on thin, poor, gravelly soils, it seldom grows over twelve inches, and often not over five or six inches high, while it has a lighter color. In the latter situations it goes by the name of Finetop, and is universally seen in dry pastures. In some sections of Massachusetts, as in Bristol County, it goes by the name of Burden's or Bor. den's grass, or Rhode Island Bent, and is highly esteemed.

Finetop may be regarded as a variety of redtop, produced by the character of the soil.

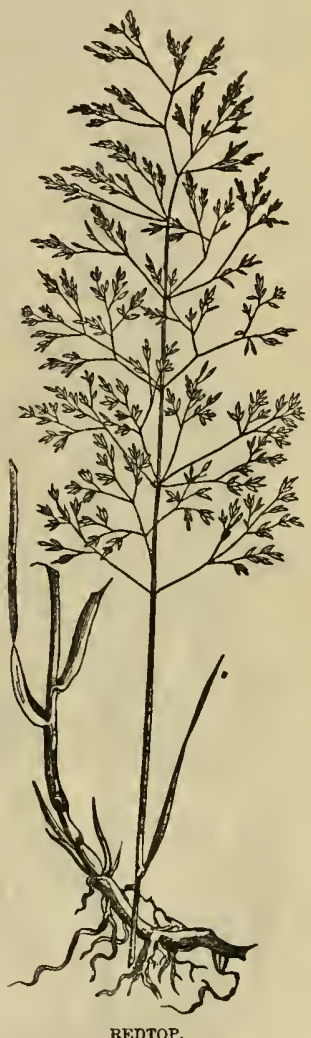

Dew Grass, White Top, White Bent, English Bent, Bonnet Grass, (Agrostis alba.) Generic characters same as those of redtop. Specific characters: Stem erect, round, smooth, polisked, having four or five leaves with somewhat rough sheaths, striated, upper sheath longer than its leaf, crowned with a long, acute, ragged ligule; joints smooth, branches numerous, recumbent, rooting at the lower joints where they come in contact with the ground; panicle somewhat narrower than in redtop, lightish green, or with a slight tinge of purple; lower or inner palea one-half the length of the upper, and shorter than the glumes; five nerved, awnless-perennial. Native of Europe. 
Whitetop may be known from redtop by the sheaths being rough to the tonch from above downwards, and the ligule being long and acute, and the keel of the large glume of the calyx toothed nearly to the base. In Agrostis vulgaris the sheaths are smooth, ligule short and obtuse, and the keel of the large glume toothed only on the upper part.

It may be known from Brown Bent, (Agrostis canina,) by having an inner palea in its floret, while in brown bent the inner palea is wanting. This grass is very common on the Connecticut River meadows where it appears to be indigenous, and is there called the English bent. Fiorin, (Agrostis stolonifera,) is only a variety of the white top, or Agrostis alba, which

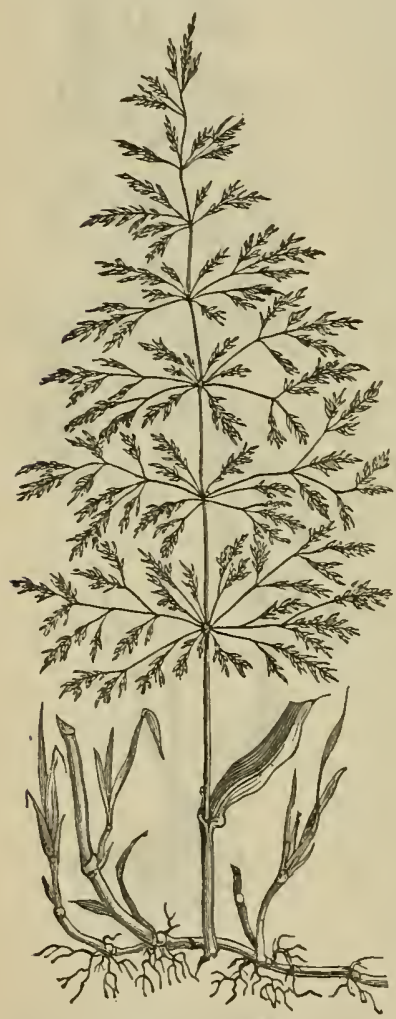

SOCTHERN BENT. gained great notoriety some years ago in Ireland and England, volumes having been written in its praise, while it received the execrations of those who found it troublesome to eradicate on account of its creeping and stoloniferous roots. It belongs peculiarly to moist places which are occasionally overflowed. This grass has often been used in the manufacture of bounets, and is called Dew grass in some sections.

Hair Grass, or Fly Away Grass, Tickle Grass, (Agrostis scabra,) is another species belonging to this genus, with a panicle very loose and spreading, purplish. Flowers in June and July. Mainly remarkable for the long hairy branches of its extremely loose panicle. Common in old fields and drained swamps. It is of no particular agricultural value. Tery common at the West, in Ohio, Illinois, Michigan, and about Lake Superior. The large, loose panicles are exceedingly delicate and brittle when the plant is ripe and $d r y$, and easily break away from the stalk when they are blown about by the wind, scatterng their seeds far and wide; and hence it is frequently called "Fly Away Grass." This illustrates one of the admirable contrivances of nature for the distribution of the seeds of grasses and other plants; sometimes by means of birds, sometimes by a sort of wing attached to the light seed, and sometimes by the force of the wind alone, as in this case, when plants start up where no seed had been sown by the hand of man, and often to our astonishment.

Alpine Brown Bent, Ipright Flowered Bent, and many other species of Agrostis might be mentioned. Of all the species of this genus, the redtop and white top are the most common as agricultural grasses among us.

The Fiorin, (Agrostis alba, var. Stolonifera lati. folia,) or Broad-leared Creeping Bent, has been more highly commended in Europe than either of these. In experiments this last has been found to be inferior in nutritive value to orchard grass and meadow fescue, (Festuca pratensis, ) and superior to meadow foxtail (Alopecurus pratensis).

The Southern Bent, (Agrostis dispar, ) has been highly extolled in France. It is a native of the United States; was at one time highly commended in England, but was rery soon discarded. It furnishes a hay of rather coarse quality, yields a large produce on 


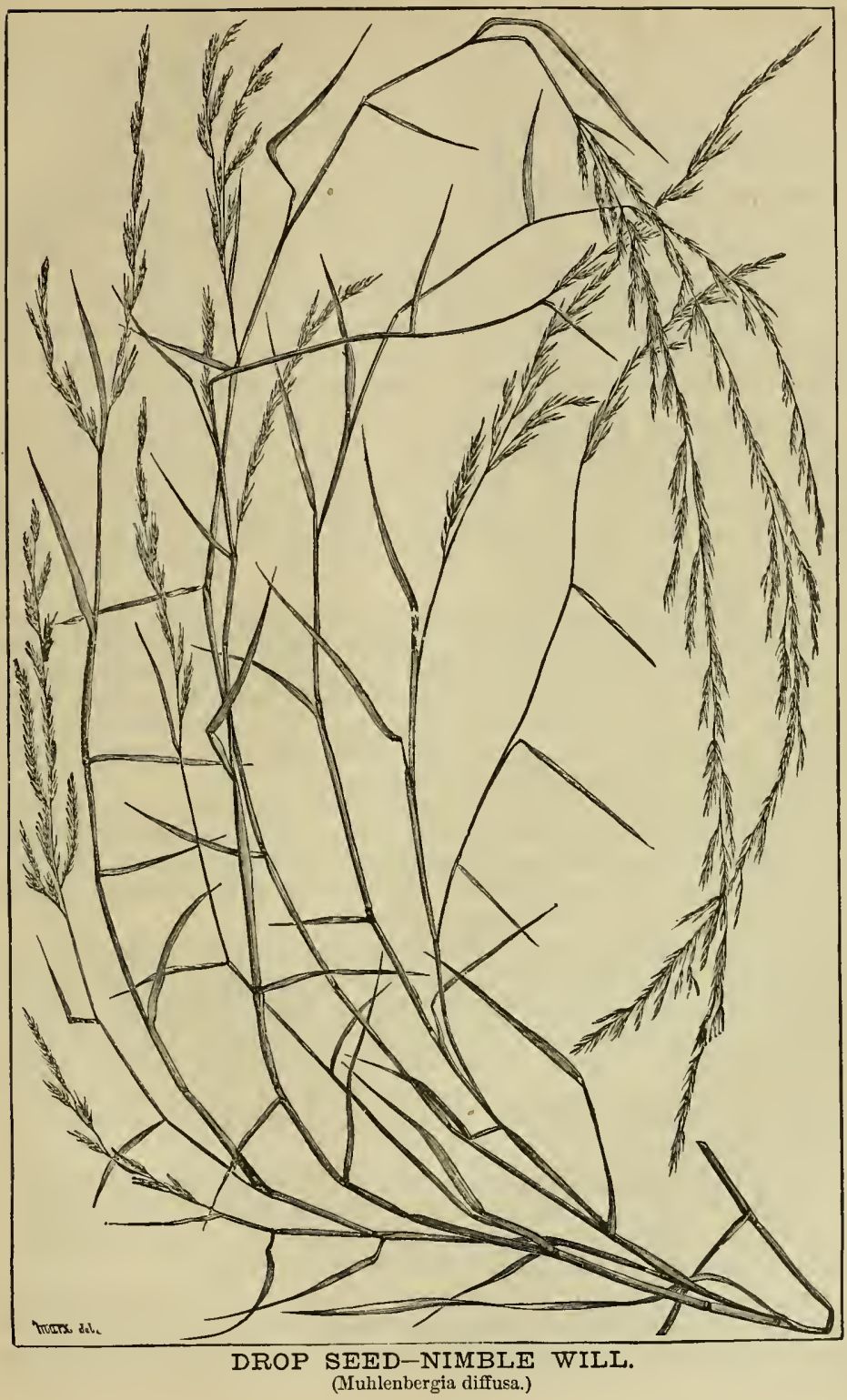


good, deep sands and calcareous soils. It tillers very much, and, when once rooted, is very vigorous and lasting, and consequently makes a good pasture grass. It is very similar in appearance to some of the broad-leaved varieties of Agrosits vulgaris, and is said to yield a larger produce than that commonly known as redtop. It has stronger and more numerous creeping roots, broader leaves, and more upright leafy stems. It is most frequently met with in the southern states and in the south of France.

Monntain or Northern Redtop. (Agrostis exarata.) This variety is a native of the Rocky Mountain region, and is also found in many of the Western States, particnlarly in the low. grounds of Wisconsin. It is more slender in its growth than the common cultivated redtop, (Agrostis vulgaris,) and varies much with different soils and climates. It is said to present so many variations in form and size on the Pacific slope that it has received several specific names, being unistaken for different varieties of grass. The analysis of this plant protes that it possesses considerable value, and we doubt not that it would prove a desirable agricultural product if cultivated on moist meadows and bottom-lands.

Brown Bent, or Dog's Bent Grass, (Agrostis canina,) another variety of agrostis, has for its specific characters a floret of one palea, sheaths smooth, ligule long, and grows from one to two feet high, awnless. The root is perennial and creeping. The stem is erect, slender, leaves flat and linear. The palea shorter than the glume and furnished with a long awn on the back, bent; spikelets at first greenish, afterwards brown or slightly purple. Meadows and pastures, and wet, peaty places. Introduced. Flowers in June and July. It is of no special agricultural value.

Drop Seed Grass, (1fuhlenbergia diffusa,) is a grass which derives its generic name from Dr. Henry Muhlenberg, a distinguished American botanist, pupil of the great Linnæus. It is commonly known in Kentucky and Tennessee by the name of "Nimble-will," and there forms a pasture grass of some value. Its stems are diffusely branched, from ten to eighteen inches high; panicles slender, contracted, glumes minute, awn nearly twice as long as the palea. It is sometimes found on dry hills and in woods. Flowers in August and September. Perennial. Cattle eat it very readily. Not very common.

There is another species of this grass, the Muhlenbergia glomerata, from one to two feet high, much more common than the preceding, with stems upright, somewhat branched; panicle oblong, linear, contracted into an interrupted glomerate spike, with long peduncles or flower stalks and awned glumes. Perennial. Flowers in August and September. Common in swamps and low grounds. Of no agricultural value.

The Mexican Muhlenbergia, (Muhlenbergia Mexicana,) another species of this genus, has been mistaken by some for our fowl-meadow. It has an erect stem, two to three feet high, much branched; panicles lateral and contracted, branches densely spiked and clustered, green or purplish; glumes pointed, awnless, and unequal. It is perennial. Flowers in August. Frequently regarded as a troublesome weed in low grounds; somewhat common at the West, and frequent here in low grounds, the borders of fields, and even in gardens, where its spreading roots are difficult to eradicate. Cattle eat it very readily; and, as it blossoms late in the season, it is of some value.

The Sylran Muhlenbergia. (Mfuhlenbergia sylvatica,) is also rather common in low, rocky woods. Its stem is ascending, branched, spreading diffusely; panicles contracted, densely flowered; glumes nearly equal, bristle pointed, lower palea one-awned, twice or three times the length of the spikelets. Flowers in A ugust and September.

The Awnless Mnhlenbergia. (Muhlenbergia sobolifera,) is sometimes found in open, rocky woods, from New England to Michigan, and farther south. It grows from one to two feet high, with a simple contracted panicle, very slender; glumes long, pointed, nearly equal, 
root perennial, creeping, woody, leaves pale green, sheaths open, ligule wanting. Flowers in August and September.

- Still another species, sometimes called hair grass, (Muhlenbergia capillaris,) is sometimes, though not often, found on sandy soils.

Willdenow's Muhlenbergia, (Muhlenbergia Willdenovii,) is also not uncommon in rocky woods, growing about three feet high, with a slender, simple stem, contracted panicle, loosely flowered, glumes sharp-pointed, half as long as lower palea, which has an awn from three to four times the length of the spikelet.

None of the grasses of this American genus are of great value as agricultural grasses, except as they add considerably to the mass of living verdure which clothes our low lands.

Blue Joint Grass, (Calamagrostis canadensis.) The general characteristics are, oneflowered spikelets, open panicle, contracted or spiked; glumes keeled, about equal to the paleæ, around which, at the base, is a thick tuft of white bristly hairs; lower palea generally with a slender awn on the back.

Specific description: Stems three to five feet high, grayish, leaves flat, panicle often purplish, the glumes acute, lanceolate, lower palea not longer than the very fine hairs bearing an extremely delicate awn below the middle, nearly equal to the hairs. Flowers in July. The blue joint grass is very common on low grounds. It is generally considered a valuable grass. It is eaten greedily by stock in the winter, and is thought by some to be as nutritious as timothy.

The Glaucons Small Reed, (Calamagrostis coarctata, ) is also somewhat common in our wet meadows, open swamps, and along low river banks. Its stems are from three to five feet high, seed hairy, crowned with a bearded tuft ; lower palea shorter than the taper-pointed tips of the lanceolate glumes, almost twice the length of the hairs, with a rigid, short awn above the middle.

Beach Grass, Sea-Sand Reed, Mat Grass, (Ammophila arundinacea,) grows to a height of two or three feet, with a rigid culm, from stout roots running often to the distance of twenty or thirty feet ; leavos wide, rather short, of a sea-green color ; panicle contracted into a close dense spike, from six to twelve inches long, nearly white. It is found in the sands of the sea-shore, where its thick, strong, creeping, perennial roots, with many tubers the size of a pea, prevent the drifting of the sand from the action of the winds and waves, thus forming a barrier against the encroachments of the sea.

This grass is very generally diffused on sea-coasts over the world, and is found inland on the shores of Lake Superior. It has also been cultivated by way of experiment, and with success, on the sands at Lowell, and still farther up on the banks of the Merrimack River. Though not cultivated for agricultural purposes, it is of great value in protecting sandy beaches. It is preserved in England and Scotland by act of Parliament. Flowers in August.

The town of Provincetown, once called Cape Cod, and its harbor, still called the harbor of Cape Cod,-one of the best and most important in the United States,-sufficient in depth for ships of the largest size, and in extent, to anchor three thousand vessels at once, - owe their preservation to this grass. To an inhabitant of an inland country it is difficult to conceive the extent and the violence with which the sands at the extremity of Cape Cod are thrown up from the depths of the sea, and left on the beach in thousands of tons by every driving storm. These sand-hills, when dried by the sun, are hurled by the winds into the harbor and upon the town. A correspondent at Provincetown says: "Beach grass is said to have been cultivated here as early as 1812. Before that time, when the sand drifted down upon dwelling-houses, -as it did whenever the beach was broken,- to save them from burial, ihe only resort was to wheeling it off with barrows. Thus tons were removed every year 
from places that are now perfectly secure from the drifting of sand. Indeed, were it not for the window-glass in some of the oldest houses in these localities, you would be ready to deny this statement; but the sand has been blown with such force and so long against this glass as to make it perfectly ground. I know of some mindows through which you cannot see an object, except to remind you of that passage where men were seen 'as trees walking."

Congress appropriated, between the years 1826 and 1839, about twenty-eight thousand dollars, which were expended in setting out beach grass back of the rillage, for the protection of the harbor. From the seed of this grass it is estimated that nearly as much ground has become planted with it as was covered by the general government. In $185 \frac{4}{4}$, fire thousand dollars were expended most wisely by the general government in adding to the work so nobly begun; and the experience of former years was of great value to the efficiency of this latter effort.

"It may be proper to state," says the same writer, "that this tomn does much in the way of 'beach-grassing' by its 'beach-grass committee,' whose duty it is to enter any man's enclosure, summer or winter, and set out grass, if the sand is uncovered and morable. Br this means we are now rid of sand-storms, which were once the terror of the place, being something like snow-storms, for drifts which were to be remored. Our streets are now hardened with clay which has been imported, and instead of its being buried, as it would once hare been in a few days, I notice that the surreyors hare to resort to sprinkling it with sand in wet weather, so effectually has the culture of beach grass answered its end.

"The mode of culture is rery simple. The grass is pulled up by hand and placed in a bole about a foot deep, and the sand pressed domn about it. These holes are dug about one foot and a half apart. The spring is the usual time of planting, though many do this work in the fall or winter. The roots of the grass, from which it soon corers the ground, are very. long. I hare noticed them ten feet, and I suppose upon high hills they extend down into. wet sand."

Beach grass seems to require the assistance of some disturbing causes to enable it to attain its full perfection. The driving winds in some localities, are sufficient, while in other places, where it does not thrire so well, it is probable that an iron tooth harrow would greatly improre and aid its growth. It has been extensively cultirated or propagated from the seed on many parts of Cape Cod, on Nantucket, and in fact to a considerable extent all along our coast. It comes in of itself along Nantasket Beach from seed borne by the tides, probably from the Cape.

The Bristly Foxtail, (Setaria verticillata,) is a grass sometimes, though rarely, found about farm-houses. It has crlindrical spikes two or three inches long, pale green, somewhat interrupted with whorled, short clusters, bristles single or in pairs, roughened or barbed downwards, short. Not cultirated.

Bottle Grass, sometimes called Foxtail (Osctaria glauca). This is an annual with a stem from one to three feet high, leares broad, hairy at the base, sheaths smooth, ligule bearded, spike two to three inches long, dense, cylindrical, bristles six to eleren in a cluster, rough upwards, perfect flower wrinkled. The spike is of a tawny or dull orange yellow when old. Flowers in July. It is common in cultirated grounds and barnyards. Introduced.

The Green Foxtail, sometimes also called Bottle Grass, (Setaria viridis, has a spike cylindrical, more or less compound, green, bristles few in a cluster, longer than the spikelets, flower perfect, striate lengthrise and dotted. It is common in cultivated grounds.

The Bengal Grass, sometimes called Millet, (Setaria italica,) also belongs to this genus. It has a compound spike, thick, nodding, six to nine inches long, yellowish or purplish, bristles two or three in a cluster. Introduced from Europe. 


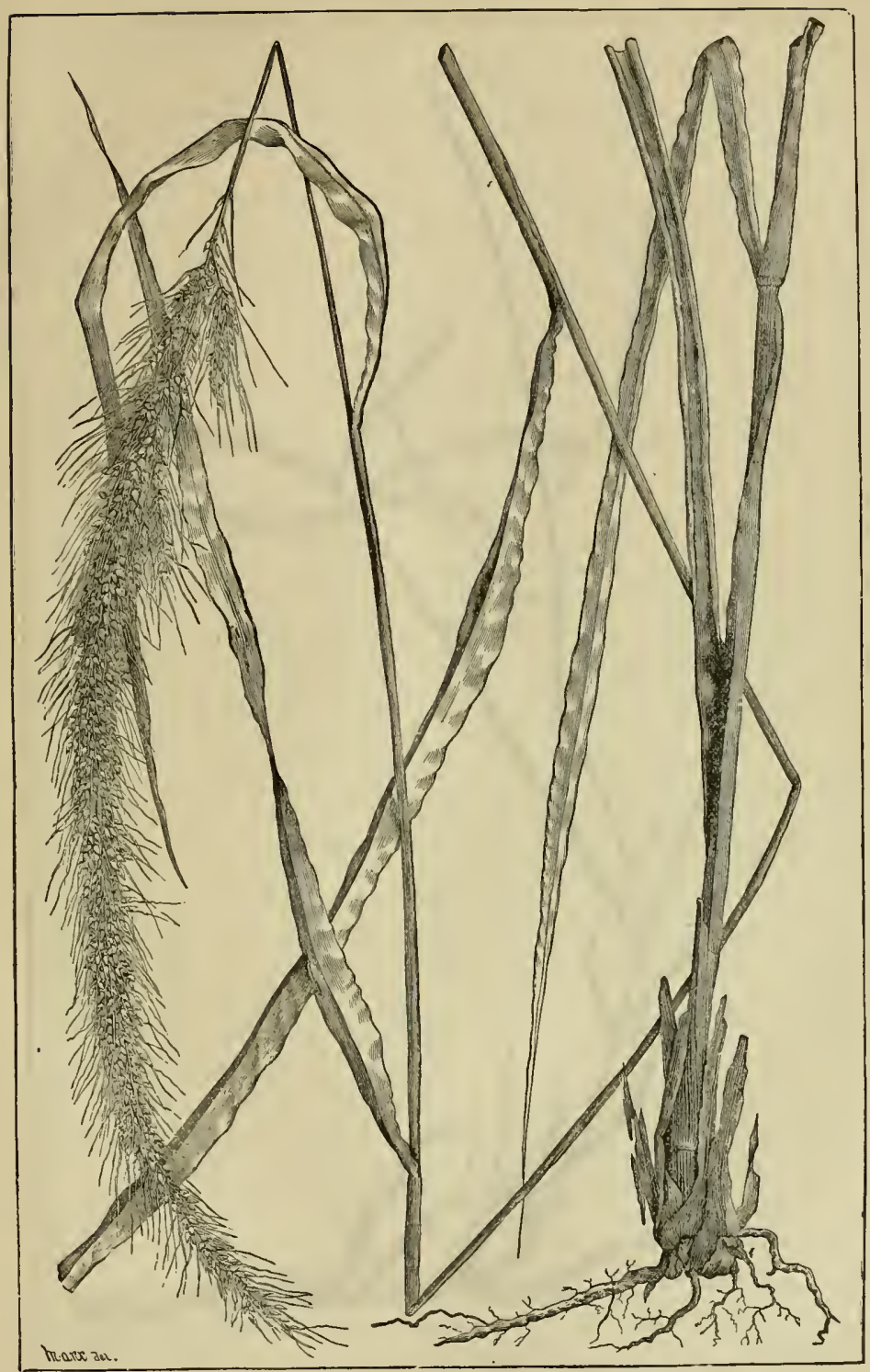

PIGEON GRASS,-BRISTLE GRASS.

(Setaria Setosa.) 
160

THE AMERICAN FARMER.

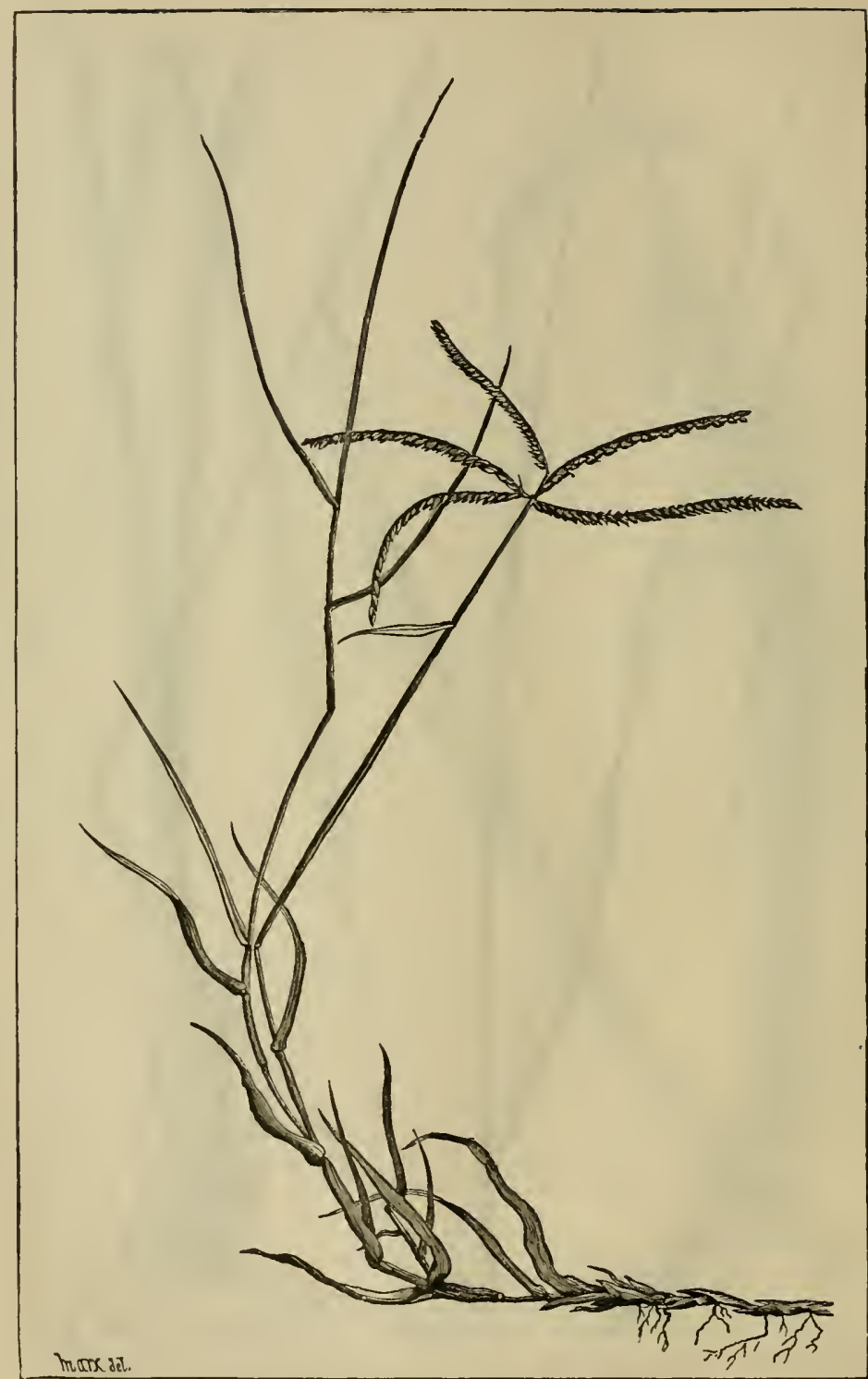

BERMUDA GRASS,-WIRE GRASS.

(Cynodon Dactylon.) 
Pigeon Grass, Bristle Grass (Setaria setosa). There are two species of Setaria which are common in cultivated fields in the Northern States, usually springing up after the cutting of grain, and often yielding a fair crop for mowing. In some of the Southern States, and particularly in Texas, there is another species that is of longer and stronger growth. Its habit is much like that of the Italian millet (Setaria italica), and its value is also much the same as that species.

Quaking Grass, (Briza media,) is sometimes met with in pastures, especially in some portions of New England. Panicle erect, with very slender spreading branches, and large, purplish, tremulous spikelets from five to uine flowered, inner glume finely fringed, entire at the end. It is a very beautiful, light, slender grass, about a foot high, perennial. Flowering in June and July. There is an annual, the Large Quaking Grass, (Briza maxima,) with large many-flowered spikes, cultivated in gardens for ornament.

Perennial Rye Grass common Darnel (Lolium perenne). Generic charactersspikelets many-flowered, solitary on each joint of the continuous rachis, placed edgewise. Specific characters-stem erect, smooth, fifteen inches to two feet high, root perennial, fibrous, joints four or five, smooth, often purplisl, leaves dark green, lanceolate, acute, flat, smooth on the outer surface and roughish on the inner, glume much shorter than the spikelet, flowers six to nine, awnless. Flowers in June.

This grass has had the reputation in Great Britain, for many years, of being one of the most important and valuable of the cultivated grasses. It is probably much better adapted to a wet and uncertain climate than to one subject almost annually to droughts, which often contnue many weeks, parching up every green thing. There is, perhaps, no grass, the characteristics of which vary so much from the influences of soil, climate and culture, as perennial rye grass. Certain it is that this grass has been cultivated in England since 1674, and in the south of France from time immemorial. It is admitted to be inferior in nutritive value to orchard grass, (Bactylis glomerata,) when green.

Whenever it is cut for hay, it is necessary to take it in the blossom, or very soon after, since otherwise it becomes hard and wiry, and is not relished by stock of any kind ; and it changes very rapidly after blossoming, from a state in which it contains the greatest amount of water, sugar, etc., and the least amount of woody fiber,--into the state in which it possesses the least amount of water, sugar, etc., and the greatest amount of woody fiber and other insoluble solid matter. A specimen analyzed about the 20 th of June, and found to contain $81 \frac{1}{4}$ per cent. of water and $18 \frac{3}{4}$ per cent. of solid matter, was found only three weeks later to contain only 69 per cent. water, and 31 per cent. solid matter. It is undoubtedly a valuable grass, and worthy of attention; but it is not to be compared, for the purposes of New England agriculture, to Timothy or to orchard grass. It produces abundance of seed, soon arrives at maturity, is relished by stock, likes a variety of soils, all of which it exhausts ; lasts six or seven years and then dies out.

Italian Rye Grass (Lolium italicum) differs from perennial rye grass in the florets having long, slender awns, and from bearded darnel, (Lolium temulentum.) in the glumes being shorter than the spikelets. It turfs less than the perennial rye grass, its stems are higher, its leaves are larger and of a lighter green, it gives an early, quick and successive growth till late in the fall.

It has the credit abroad of being equally suited to all the climates of Europe, giving more abundant crops, of a better quality, and better relished by animals than the perennial rye grass. It is one of the greatest gluttons of all the grasses, either cultivated or wild, and will endure any amount of forcing by irrigation or otherwise, while it is said to stand a drought remarkably well. The soils best adapted to it seem to be moist, fertile and tenacious, or of a medium consistency ; and on such soils it is said to be one of the best grasses known to cut green for soiling, affording repeated luxuriant and nutritive crops. 
It is sometimes sown in antumn, but those who have had the fullest experience in its cultivation give a preference to spring sowing. As a proof of the fondness of sheep for this grass, it has been observed that when mixed with red clover, and sheep have been turned into the field after it is mowed, they will eat down the Italian rye grass in preference to the clover.

Its comparative value is well known from the thorough and reliable analyses of Prof. Way. By these, it appears that 100 parts of timothy grass, as taken from the field, contain 57.21 per cent. of water, 4.86 per cent. of albuminous or flesh-forming principles, 1.50 per cent. of fatty matters, 22.85 per cent. of heat-producing principles, such as starch, gum, sugar, etc., 11.32 per cent. of woody fiber, and 2.26 of mineral matter or ash, while 100 parts of Italian rye grass taken from the same kind of soil, and in the same condition, green, contained 75.61 per cent. of water, 2.45 of albuminous or flesh-forming principles, .80 of fatty matters, 14.11 of heat-prodncing principles, starch, gum, and sugar, 4.82 of woody fiber, and 2.21 of mineral matter or ash. Of these, the flesh-forming principles, fatty matters, and heat-producing principles, are, of course, by far the most important; and in all these our favorite timothy very far excels the Italian rye grass, showing a nutritive value nearly double. Nor has the Italian rye grass any advantage over timothy or herds-grass in the dried state, though the difference is by no means so marked, the former, dried at $212^{\circ}$ Fahrenheit, containing 10.10 per cent. of flesh-forming principles, the latter 11.36; the former containing 3.27 per cent. of fatty matter, the latter 3.55 ; the former containing 57.82 per cent. of heatforming principles, the latter 53.35.

There are $\$ 32,000$ seeds in a pound of Italian rye grass, and from thirteen to eighteen pounds in a bushel.

Couch Grass, Qnitch Grass, Twitch Grass, Dog Grass, Chandler Grass, ete. (Triticum repens). The chief generic marks of this grass are, three or several flowered spikelets, compressed, with the flat side towards the rachis; glumes nearly equal and opposite, nerved, lower palea like the glumes convex on the back, awned from the tip, upper flattened, stamens three; mostly annuals, but others are perennials, to which the couch grass belongs. The specific characters of couch grass are, roots creeping extensively, stem erect, round, smooth, from one to two or two and a half feet high, striated, having five or six flat leaves with smooth, striated sheaths; the joints are smooth, the two uppermost very remote, leaves dark green, acute, upper one broader than the lower ones, roughish, sometimes hairy on the inner surface, smooth on the lower half. Inforescence in spikes. Flowers in June and July. Introduced from Europe.

This plant is generally regarded by farmers as a troublesome weed, and efforts are made to get rid of it. Its long, creeping roots, branching in every direction, take complete possession of the soil and impoverish it. When green, however, it is very much relished by cattle, and if cut in the blossom it makes a nutritious hay. I have seen acres of it on the Connecti. cut River meadows, where it had taken possession and grew luxuriantly, and is called wheat grass, from its resemblance to wheat. It goes in different sections by a great variety of names, as quake grass, quack grass, squitch grass. It is important to destroy it if possible.

The Downy Oat Grass (Trisetum pubescens) is a very hardy perennial grass, naturalized on chalky soils, and on such soils its leaves are covered with a coating of downy hairs, which it loses when cultivated on better lands. It is regarded as a good permanent pasture grass on account of its hardiness and its being but a slight impoverisher of the soil, and yielding a larger per cent. of bitter extractive than other grasses grown on poor, light soils. It is therefore recommended abroad as a prominent ingredient of mixtures for pastures. It flowers early in July. Formerly classed as Avena pubescens. 
Meadow 0at Grass (Avena pratensis) is a perennial grass, native of the pastures of Great Britain, growing to the height of about eighteen inches. It furnishes a bay of medium quality. Flourishes best on dry soils. Flowers in July.

The Yellow Oat Grass (Avena flavescens, now generally classified as Trisetum flavescens) can scarcely, perhaps, be regarded as naturalized bere. It is a perennial plant of slow growth and medium quality, furnishing a hay containing about 1.79 per cent, of azote or nitrogen; suitable for dry meadows and pastures. It is sometimes regarded as a weed, growing about eighteen inches high. It fails if cultivated alone, but succeeds with other grasses, and is said to be the most useful for fodder, of the oat grasses. It grows best with the crested dog's tail and sweet-scented vernal. It contains a larger proportion of bitter extractive than most other grasses, and for that reason is recommended by some English writers as a valuable pasture grass. It flowers in July.

Tall Meadow Oat Grass, or Tall 0at Grass (Arrenatherum avenaceum) is the avena elatior of Linnæus. Specific characters: Spikelets open panicled, two-flowered, lower flower staminate, bearing a long bent awn below the middle of the back; leaves llat, acute, roughish on both sides, most on the inner; panicle leaning slightly on one side, glumes very unequal; stems from two to three feet high, root perennial, fibrous, sometimes bulbous. It is readily distinguished from other grasses by its having two florets, the lower one having a long awn rising from a little above the base of the outer palea. Introduced. Flowers in June and July.

This is the Ray grass of France. It produces an abundant supply of foliage, and is val. uable either for hay or for pasture, and has been especially recommended for soiling purposes, on account of its early and luxuriant growth. It is often found on the borders of fields and hedges, woods, and pastures, and sometimes very plenty in mowing-lands. After being mown, it shoots up a very thick aftermath, and on this account, partly, is regarded as nearly equal for excellence to the common foxtail (Alopecurus pratensis).

It grows spontaneously on deep, sandy soils, when once naturalized. It has been cultivated to some extent in New England, and is esteemed by those who know it, mainly for its early, rapid, and late growth, making it very well calculated as a permanent pasture grass. It will succeed on tenacious clover soils.

Bermuda Grass, Wire Grass (Cynodon dactylon).-A low, creeping perennial grass, with abundant short leaves at the base, sparingly sending up slender, nearly leafless flowerstalks, with 3 to 5 slender, diverging spikes at the summit. The flowers are arranged in a close row along one side of these spikes. The spikelets are one-flowered, with a short pedicelled rudiment of a second flower. The glumes are pointed, but without awns; the lower palet boat-shaped.

This grass is a native of Europe, and is abundantly naturalized in many other countries. It is said to be a common pasture grass in the West Indies. In the Southern States it has long been the chief reliance for pasture, and has been extravagantly praised by some, and cursed by others, who find it difficult to eradicate it when once established. Mr. C. Mohr speaks of it thus:-

"It thrives in the arid, barren drift-sands of the sea-shore, covering them by its long, creeping stems, whose deeply-penetrating roots impart firmness to a soil, which olse would remain devoid of vegetation. It is esteemed one of the most valuable of our grasses, either in the pasture or cured as hay."

Col. T. C. Howard, of Georgia, says: "The desideratum to the South is a grass that is perennial, nutritious, and adapted to the climate. While we have grasses and forage plants that do well when nursed, we have few that live and thrive here as in their native habitat. The Bermuda and crab grasses are at home in the South. They not only live, but live in 
spite of neglect, and when petted and encouraged they make such grateful returns as astonish the benefactor."

It seems that it rarely ripens any seed, and the usual method of reproducing it is to chop up the roots with a cutting-knife, sow them broadcast, and plow under shallow. Col. Lane states:-

"Upon our ordinary uplands I have found no difficulty in destroying it by close cultiva. tion in cotton for two years. It requires a few extra plowings to get the sod thoroughly broken to pieces."

Professor Killebrew, of Tennessee, states that_- "In Louisiana, Texas, and the South generally, it is, and has been, the chief reliance for pasture for a long time, and the immense herds of cattle on the Southern prairies subsist principally on this food. It revels on sandy soils, and has been grown extensively on the sandy hills of Virginia and North and South Carolina. It is used extensively on the southern rivers to hold the levees and the embankments of the roads. It will throw its runners over a rock six feet across, and soon hide it from view, or it will run down the sides of the deepest gully and stop its washing. Hogs thrive upon its succulent roots, and horses and cattle upon its foliage. It has the capacity to withstand any amount of heat and drought, and months that are so dry as to check the growth of blue grass will only make the Bermuda greener and more thrifty."

Mr. Wall, of Mississippi, says of it: "It is a most valuable grass, and is destined to be the salvation of the hill land in Mississippi. W ith us it has no seed, but can be easily propagated by dropping the cuttings in a furrow, two or three feet apart. It does not endure shade, and can be destroyed by sowing the land with oats, followed by peas. It throws out runners down the sides of the deepest gully, and stops its washing. Hogs thrive upon it and its succulent roots, and horses and cattle upon its foliage."

Crow-foot, Yard-grass, Dog's-tail (Eleusine indica).-This is an annual grass belonging to tropical countries, but now naturalized in most temperate climates. In the Southern States it is found in every door-yard, and in all waste places. The culms or flowering stems rise from 6 to 18 inches high, and are usually coarse and thick; the summit is crowned with from 2 to 6 spikes, resembling those of common crab grass, Panicum sanguinale, but much thicker and heavier. It makes a thick bed of rather coarse but rather long and wide leares, and takes such a firm hold on the soil that it is difficult to pull up a clump by hand. The spikelets are crowded on one side of the flattened spikes, 2 to 6 flowered, and with pointless glumes.

It is said to form a good and lasting pasturage for stock at the South, although it does not appear to be very highly recommended for cultivation in that section.

Gama Grass (Tripsacum dactyloides). A tall perennial grass, with solid clums, broad, flat leaves, and with flower-spikes from 4 to $S$ inches long, produced from the side joints or from the top, either singly or two or three together. The upper portion of these spikes is staminate or male, and the lower portion pistillate and producing the seeds. It grows from three to six feet high, with large broad leaves resembling those of Indian corn. The upper or male portion of the flower-spikes drops off after flowering, and the fertile portion easily breaks up into short joints. These joints are thick and polished, and the flowers and seeds are deeply imbedded in them.

Mr. Howard, in his "Manual of Grasses," says: "This is a native of the South, from the mountains to the coast. The seed stem runs up to the height of five to seven feet. The seeds break off from the stem as if in a joint, a single seed at a time. The leaves resemble those of corn. When cut before the seed stems shoot up they make a coarse but nutritious hay. It may be cut three or four times during the season. The quantity of forage which can be made from it is enormous. Both cattle and horses are fond of the hay. The roots are 


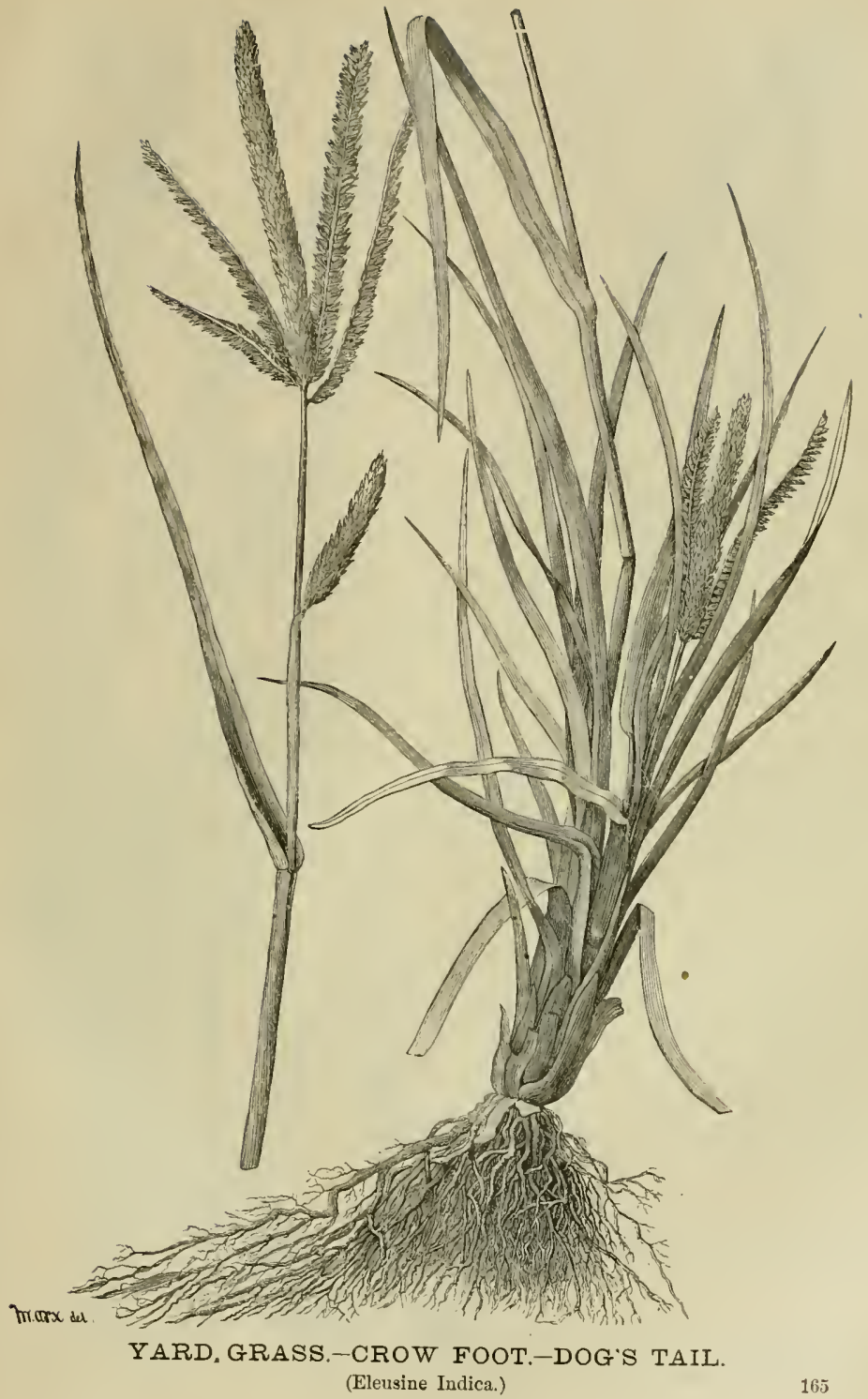




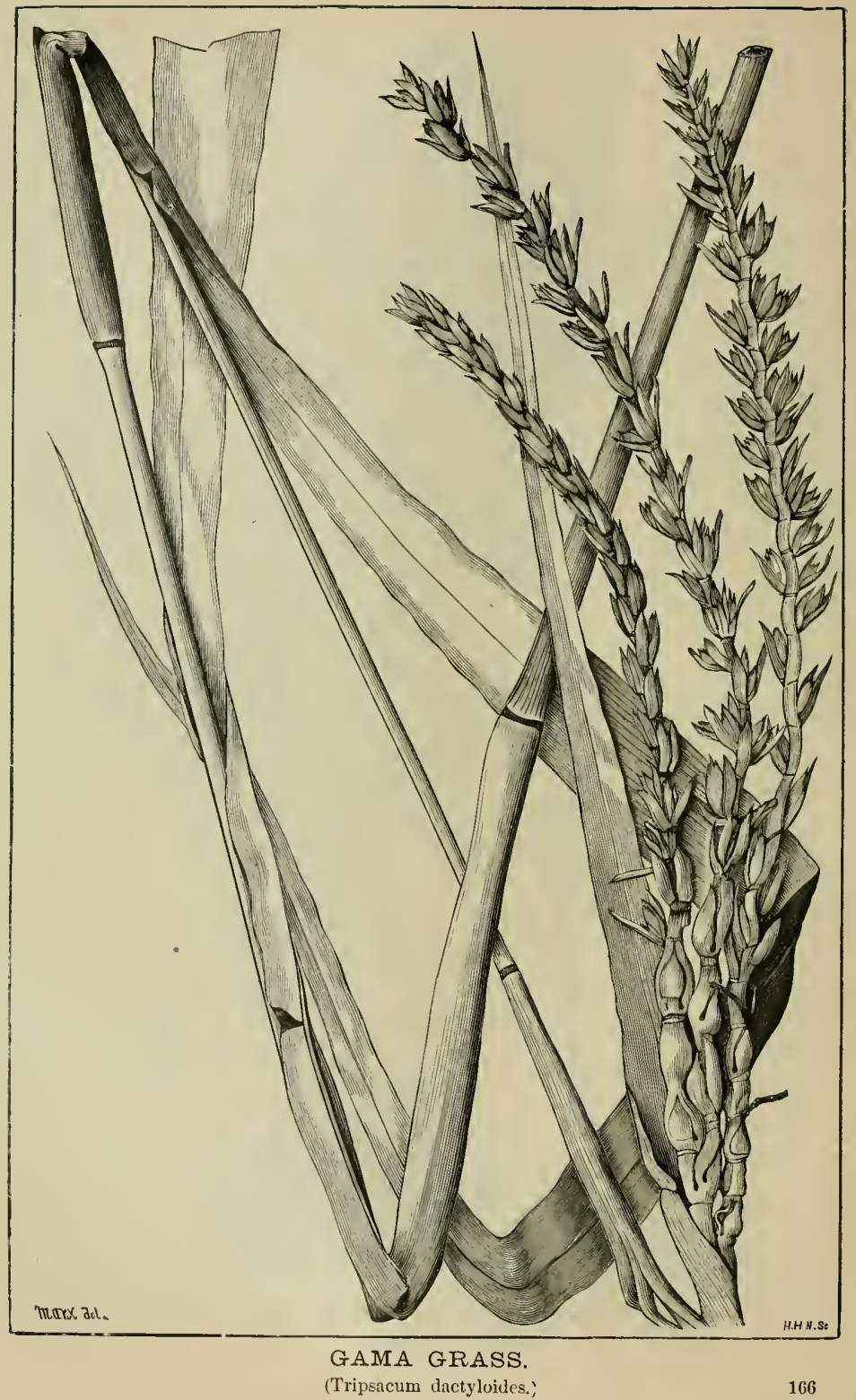


almost as large and strong as cane roots. It would require a team of four to six oxen to plow it up. It can, however, be easily killed by close grazing, and the mass of dead roots would certainly greatly enrich the land. As the seeds of this grass vegetate with uncertainty, it is usually propagated by setting out slips of the roots about two feet apart each way. On rich land the tussocks will soon meet. In the absence of the finer hay grasses this grass will be found an abundant and excellent substitute. The hay made from it is very like corn fodder, is quite equal to it in value, and may be saved at a tithe of the expense."

Rattlesnake Grass, (Glyceria canadensis.) The generic characteristics of glyceria are, many-flowered spikes; mostly flattish; glumes two-pointed, nearly equal, awnless, the lower one obtuse, seven-nerved; roots creeping. Perennial. Wet places and standing water. The name of the genus is from a Greek word signifying sweet.

Rattlesnake grass has an oblong, pyramidal, spreading panicle, with beautifully-drooping spikelets, six or eight flowered, and long, roughish leaves, which together make it an object of interest and search for bouquets and vases. It is very common in wet, boggy places, growing from two to three feet high, but possesses little or no agricultural value. Flowers in July.

The Obtuse Spear Grass, (Glyceria obtusa,) has a dense, narrowly-oblong panicle; spikelets six or seven flowered, erect, swelling; lower palea obtuse, leaves smooth, as long as the stem. This is an aquatic grass, found occasionally on the borders of ponds. Flowers in August. Of no agricultural value.

Long Panicled Manna Grass, (Glyceria elongata,) is a very distinct species; stems one to three feet high, panicle branching, narrowly-elongated, recurving, the branches appressed, spikelets pale, erect, three to four flowered, lower palea obtuse, rather longer than the upper; stamens two, stigmas compound, leaves very long and rough. Flourishes in wet woods and swamps. Flowers in June and July. Perennial. Of no special agricultural value.

Mountain Rice, (Oryzopsis melanocarpa,) is a grass common in rocky woods; the large white-grained mountain rice, (Oryzopsis asperifolia,) common on steep and rocky hill-sides and dry woods, and the Smallest Oryzopsis, (Oryzopsis canadensis, ) are sometimes found. These grasses are easily distinguished from each other. The first has an awn thrice the length of the blackish palea; the second, an awn two or three times the length of the whitish palea; the third, an awn short, deciduous or wanting. The first grows from two to three feet high, the second from ten to eighteen inches.

Feather Grass, or Black 0at Grass, (Stipa avenacea,) is sometimes met with in dry, sandy woods, and is collected for vases and ornaments, but is of no agricultural value. It rises from one to two feet; its panicle is open, leaves almost bristle form, palea blackish, nearly as long as the almost equal glumes, awn bent above, twisted below. Flowers in July.

Porerty Grass, or Three Awned Grass, (Aristida dichotoma,) and Slender Three Awned Grass, (Aristida gracilis,) are found in old, sandy fields, dry, sterile hill-sides and pine barrens, but are of no value for cultivation. One or two other species of three-awned plants also occur on similar soils, as the Aristida purpurascens and the Aristida tuberculosa. None of these species are of importance in agriculture.

Fresh water Cord Grass, (Spartina cynosuroides.) This is found on the banks of streams and lakes, rising to the height of from two to four feet, with slender clum, narrow leaves two to four feet long, tapering to a point, and spikes of a straw color. Flowers in August.

The Salt Reed Grass, (Spartina polystachya,) has a stout culm from four to nine feet high, broad leaves, roughish underneath and on the margins; spikes 20 to 50 in number, forming a dense, oblong, purplish cluster. It is found on the salt marshes 
Rush Salt Grass, (Spartina juncea,) grows from one to two feet high, stems slender, leaves narrow, rush-like, and very smooth. It is common on salt marshes and sandy seabeaches, and flowers in August.

Salt Marsh Grass, (Spartina stricta, var. glabra,) grows from two to four feet high, has from five to twelve spikes from two to three inches long; spikelets crowded and lapping over each other. It is common on the coast.

Sand Grass, (Tricuspis purpurea, is also found on dry, sandy soils, along the coast; flowering in August and September. It is acid to the taste, grows from six inches to a foot high, and has numerous bearded joints.

Tall Redtop, (Tricuspis sesleroides.) This grass grows from 3 to 5 feet high. It is very smooth; the leaves are long and flat, the lower sheaths hairy or smoothish. The panicle is large and loose, at first erect, but finally spreading widely; the branches in pairs or single, naked below, flowering toward the extremities and becoming drooping. The spikelets are 5 to 6 flowered, 3 to 4 lines long, purple, on short pedicels. The glumes are shorter than the flowers, unequal, and pointed; the lower palet is hairy toward the base, having 3 strong nerves which are extended into short cusps or teeth at the summit. It is a large and showy grass when fully matured, the panicles being large, spreading, and of a handsome purplish color. Instead of being called Tall Red-top it would be much more properly called Purple-top.

It grows in sandy fields and on dry, sterile banks, from New York to South Carolina, westward to the prairies, and southwestward to Texas, where several other species also occur.

It is not considered very valuable, but is however cut for hay and eaten by cattle and other stock in those localities where it grows without cultivation, as in the mountain meadows of Pennsylvania. It is said to be very harsh and wiry, and probably would not be eaten by stock if they could have access to hay of a better quality.

Headow Soft Grass, Velvet Grass, (Holcus lanatus,) has its spikelets crowded in a somewhat open panicle, and an awn with the lower part perfectly smooth. The generic characters are, two-flowered spikelets jointed with the pedicels, glumes boat-shaped, membranaceous, inclosing and exceeding the flowers; lower flower perfect, its lower palea awnless and pointless, upper flower staminate only, bearing a stout bent awn below the apex. Stamens three; grain free, slightly grooved.

This species grows from one to two feet high, stem erect, round, root perennial, fibrous, leaves four or five, with soft, downy sheaths, upper sheath much longer than its leaf, inflated, ligule obtuse, joints usually four, generally covered with soft, downy hairs, the points of which are turned downwards; leaves pale green, flat, broad, acute, soft on both sides, covered with delicate slender hairs. Inflorescence compound panicled, of a greenish, reddish, or pinkish tinge; hairy glumes, oblong, tipped with a minute bristle. Florets of two paleæ. Flowers in June. Introduced.

This beautiful grass grows in moist fields and peaty soils, but I have found it on dry, sandy soils on Nantucket, and specimens have been sent me from Boxford and other places where it grew on upland fields, and was cultivated with other grasses. It is productive and easy of cultivation. It is of but little value either for pasture or bay, cattle not being fond of it. When once introduced it will readily spread from its light seeds which are easily dis. persed by the wind. It does not merit cultivation except on poor, peaty lands, where better grasses will not succeed. This grass loses about .63 of its weight in drying, and the hay contains about 1.92 per cent. of nitrogen.

Sweet Scented Vernal Grass, (Anthoxanthum odoratum.) Specific characteristics: Spikelets spreading, three-flowered, lateral flowers neutral, with one palea, hairy on the out. side and awned on the back; glumes thin, acute, keeled, the upper twice as long as the lower; 


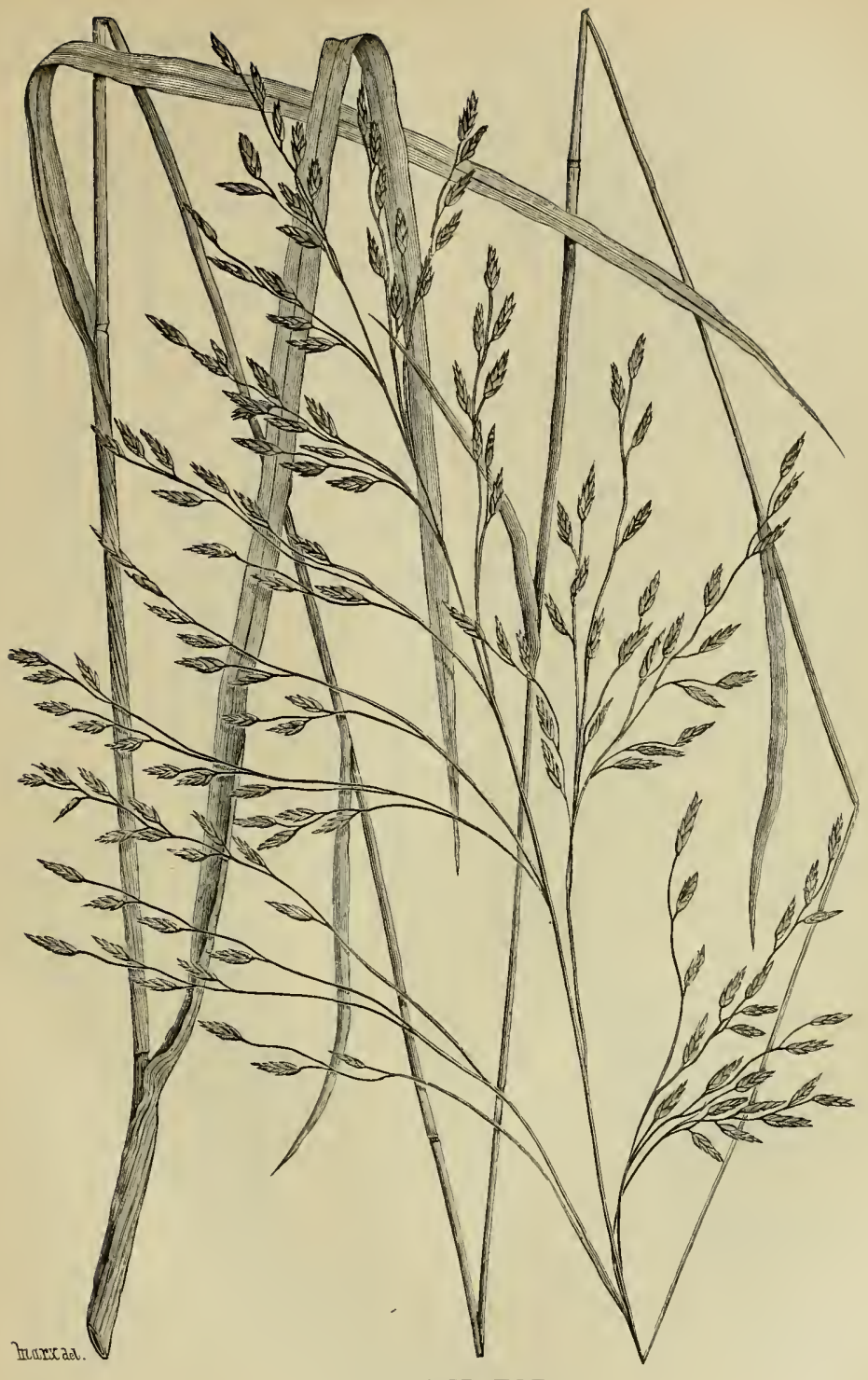

TALL RED TOP.

(Tricuspis sesleroides.) 


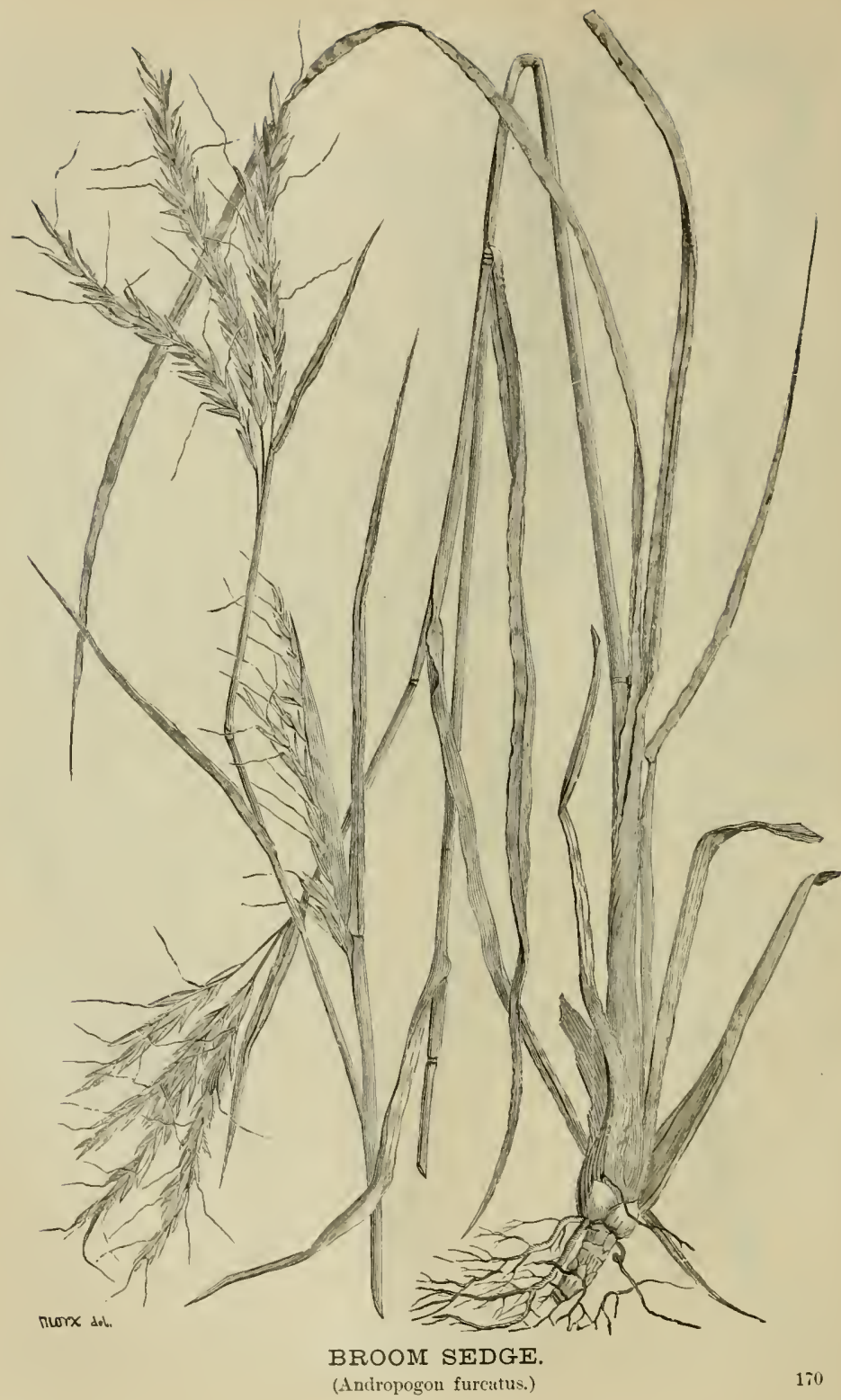


seed ovate, adhering to the palea which incloses it; root perennial. Flowers in May and June. Stems from one and a half to two feet high. Introduced from Europe.

This is one of the earliest spring grasses, as well as one of the latest in the autumn. It is almost the only grass that is fragrant. It possesses a property peculiar to this species, or possessed by only a few others, known as benzoic acid. It is said to be this which not only gives it its own aromatic odor, but imparts it to other grasses with which it is cured. The green leaves when bruised give out this perfume to the fingers, and the plant may thus be known. It possesses but little value of itself, its nutritive properties being slight; nor is it much relished by stock of any kind, but as a pasture grass on almost all soils, and with a large mixture of other grasses, it is very valuable for its early growth, and this gives it the character of a permanent pasture grass.

It is not uncommon in New England pastures and road-sides, growing as if it were indigenous. I have found fine specimens of it on dry soils at Nantucket and elsewhere.

The aftermath or fall growth of this beautiful grass is said to be richer in nutritive qualities than the growth of the spring. Though it is pretty generally diffused over the country, it is only on certain soils that it takes complete possession of the surface and forms the predominant grass in a permanent turf, as it is said to do in some sections around Philadelphia. The flavor of the spring butter sold in that city is ascribed by some to the prevalence of this grass. There seems to be nothing inconsistent in this supposition, since it is well known that disagreeable flavors are often imparted to the milk and the butter by the substances taken as food by the cow, as turnips, for instance, or cabbages, or cauliflowers; and if an objectionable flavor may be imparted by one substance, it is reasonable to suppose an opposite flavor may be given by another.

Dr. Emerson, of Philadelphia, found by experiments in flavoring other grasses with a slight addition of benzoic acid in the form of an essence, previous to feeding them out to milch cows, that the flavor of the best Philadelphia spring butter was thus imparted to the butter made from them.

A curious and beautiful peculiarity is exhibited in the seeds of this grass, by which they are prevented from germinating in wet weather after approaching maturity, and thus becoming abortive. The husks of the blossom adhering to the seed when ripe, and the jointed awn by its spiral contortions, when affected by the alternate moisture and dryness of the atmosphere, acts like levers to separate and lift it out from the calyx even before the grass is bent or lodged and while the spike is still erect. If the hand is moistened and the seeds placed in it, they will appear to move like insects, from the uncoiling of the spiral twist of the awns attached to them.

There are 923,200 seeds in a pound, and eight pounds in a bushel. It cannot be said to belong to the grasses useful for general cultivation.

Reed Canary Grass. (Phalaris arundinacea.) Generic characteristics: Spikelets crowded in a dense or spiked panicle, perfect flower, flattish, with two neutral rudiments of flowers, one on each side at its base, awnless, two shining paleae, closely inclosing the smooth flattened grain; stamens three. Specific description: Panicle very slightly branched, clustered, somewhat spreading when old, glumes wingless, rudimentary florets hairy, stem round, smooth, erect, from two to seven feet high, leaves five or six in number, broad, lightish green, acute, harsh, flat, ribbed, central rib the most prominent, roughish on both surfaces, edges minutely toothed, smooth, striated sheaths. Flowers in July. Grows on wet grounds by the sides of rivers and standing pools, hest suited to somewhat tenacious soils.

A beautiful variety of this species is the RIBBON or STRIPED GRASS of the gardens, familiar to every one. It will bear cutting two or three times in a season, but if not cut early the foliage is coarse. Cattle are not fond of it at any stage of its growth, but if cut early and well cured, they will eat it in the winter if they can get nothing better. 
This grass is common in low, rich soils where the water is either standing or sluggish, and is not unfrequently produced by transplanting the roots of the striped grass into suitable soils. In one instance within my knowledge, it came in and produced an exceedingly heavy erop, simply from roots of ribbon grass which had been dug up from a garden and thrown into the brook to get them out of the way. Several other instances of a similar nature have also come to my notice. One farmer has propagated it extensively in his wet meadows by forcing the ripe seed panicles into the mud with his feet. As the stripe of the ribbon grass is only accidental, dependent on location and soil, it constitutes only a variety of the reed canary grass, and loses the stripe when transferred to a wet and muddy soil.

The panicles of this grass, if allowed to stand after the time of flowering, become filled with ergot, or long, black spurs, issuing from between the glumes, and occupying the place of grain. This, if there were no other reason, would be sufficient to determine that it should be cut at or before the time of flowering. I have never seen rye worse affected than my specimens of this grass are. The effects of this mysterious disease are well known. The noxious power it exerts on the system of animals which receive even a small portion of it, is oftentimes dreadful, producing most horrible gangrenes, rotting of the extremities, internal tortures and agonizing death; it has been known to slough and kill not a few human beings who have accidentally or inadvertently eaten grain or flour infected with it.

The variety called striped grass, (colorata,) is exceedingly hardy, and may be propagated to any extent by dividing and transplanting the roots. In moist soil it spreads rapidly and forms a thick mass of fodder which might bo repeatedly cut without injury, though it is of little value for feeding stock.

The Common Canary Grass, (Phalaris canariensis,) is cultivated in gardens, and to some extent in fields and waste places, for the sake of the seed for the canary bird. It has a spiked, oval panicle, glumes wing-keeled; rudimentary flowers smooth and half the length of the perfect one. Flowers in July and August.

Millet Grass, (Millium effusum,) is found growing commonly in moist, shady woods, mountain meadows, and on the borders of streams. Panicle widely diffuse, compound, glumes ovate, very obtuse, leaves broad and fiat, thin, root perennial, flower oblong. Flow. ers in June. Introduced. Of no value for cultivation, the foliage possessing but slight nutritive qualities. The seeds are sought by birds. It will thrive transplanted to open places.

Feather Grass, (Leptochloa mucronata,) is an annual grass growing in many of the Southern States. It reaches the height of from two to three feet, the flowers being arranged on numerous slender spikes in a long-panicled raceme. They are very minute, from three to four in a spikelet, the uppermost one usually imperfect; the glumes pointed. When full grown it is a beautiful grass, the panicles on thrifty specimens sometimes becoming two feet long, the slender branches arranged along the main stem in a feathery-like manner, from whence it takes its name. It grows in fields and pastures, and as it is less affected with drought than some other varieties, affords a small amount of grazing during the hot months, when the regular pasture grasses are parched and dry. It is not considered of much agricultural value.

Water Grass. (Paspalum laeve.) There are a large number of species of this genus which are to all appearance so nearly alike as to be scarcely distinguishable. They resemble in their flower structure the Panicum, from which they chiefly differ in the want of a lower glume. The flowers are spiked or racemed in two or more rows on one side of a flattened stalk, while the spikelets are one-flowered, or with the rudiment of a second. The flowers are ovate or roundish.

Mr. Phares, of Mississippi, says of this: "It is not called water grass because it grows in and about water, for it does not, but probably because it is very succulent. It is trouble- 


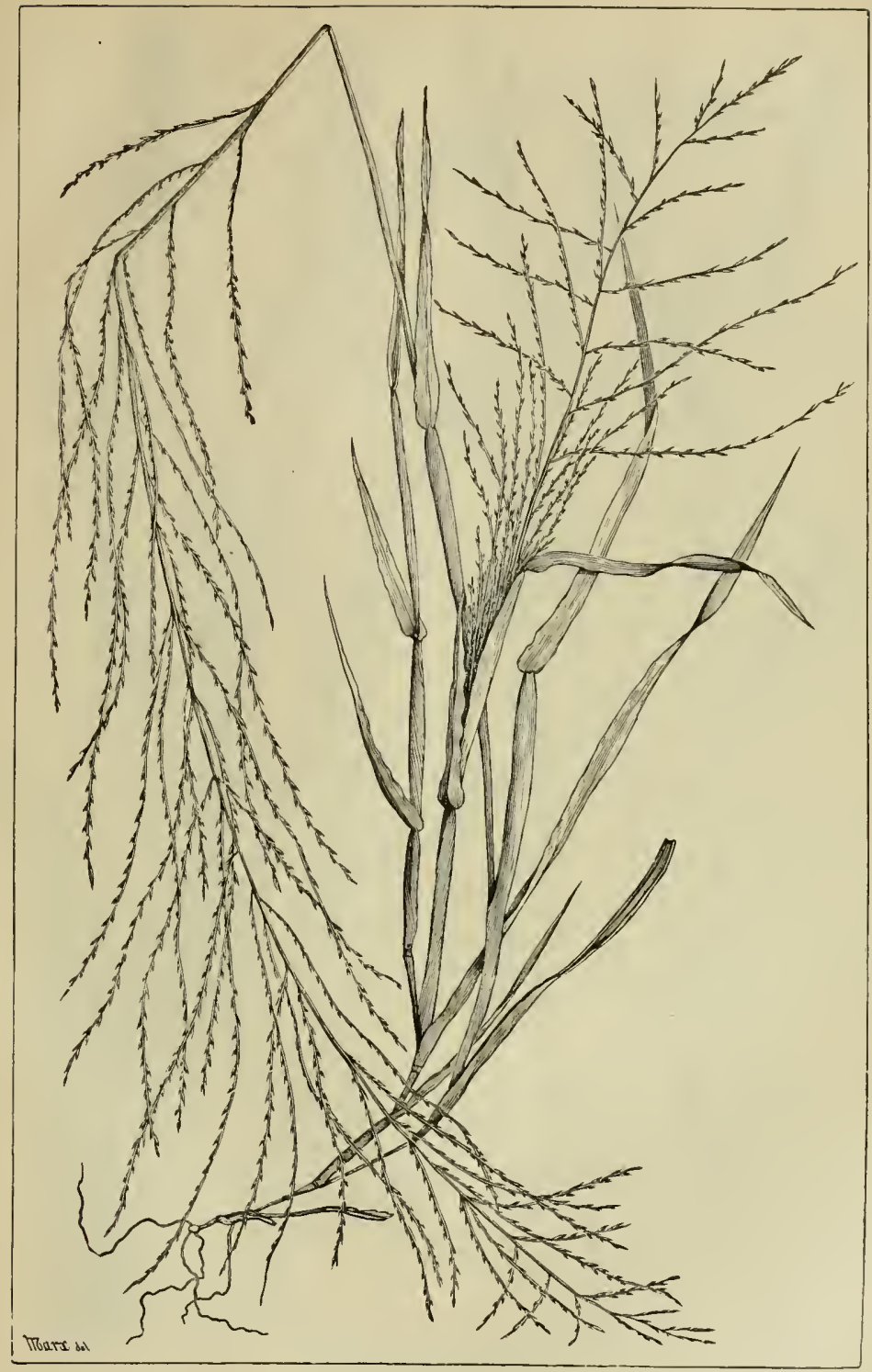

FEATHER GRASS.

(Leptochloa mucronata.) 


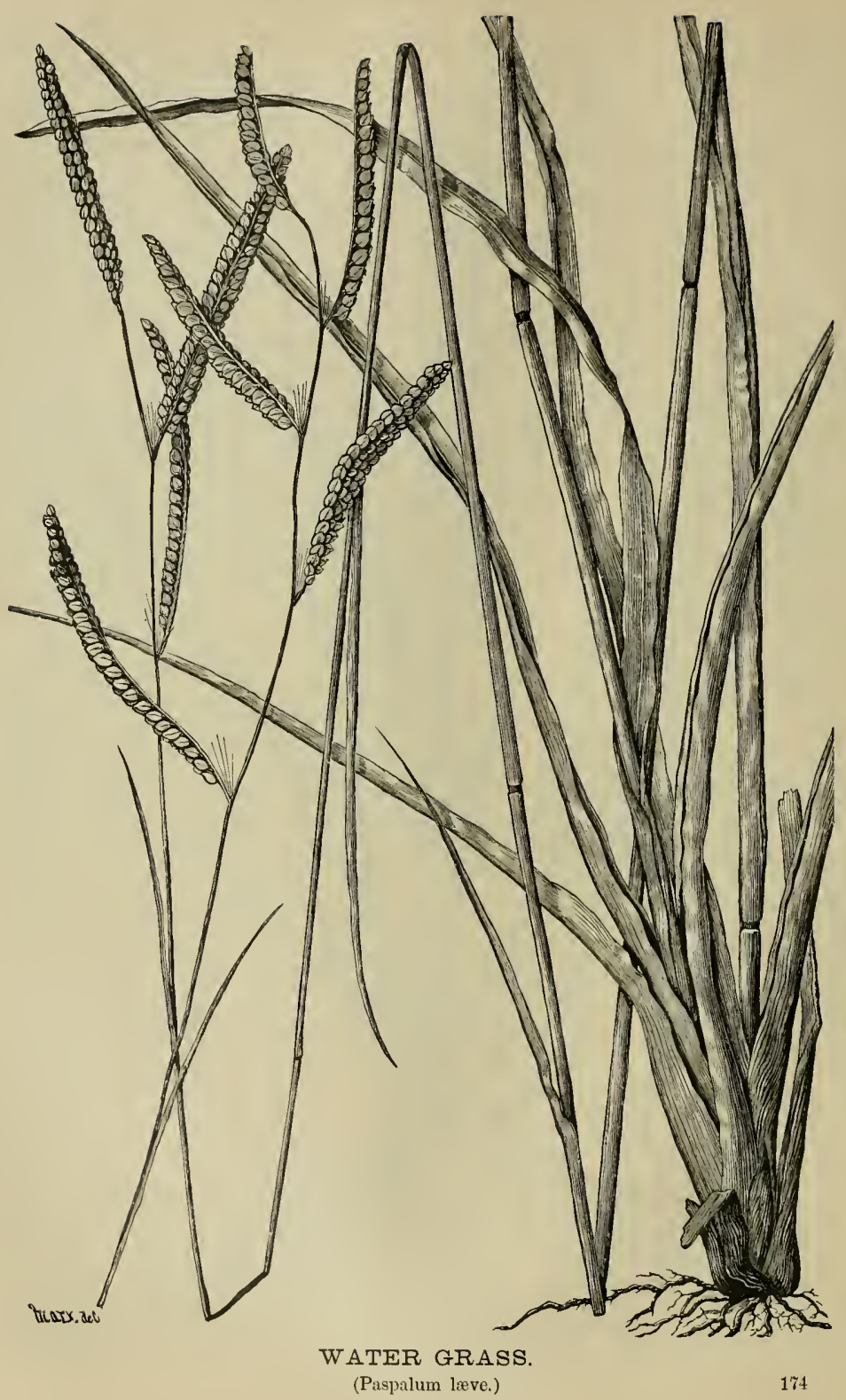


some in crops; live stock are very fond of it in all stages of growth and as dry hay. A neighbor has a meadow of it from which he mows every summer about two tons of hay per acre. It is very easy to set land with it."

Smut Glass. (Sparibolus indicus.) This grass is a native of India, but is now spread over many countries, and is found more or less abundantly in all the Southern States. It is called "Smut grass," on account of its heads becoming affected with a blackish smut after flowering. It is said to grow luxuriantly on uncultivated lands, and is eaten by cattle and norses with evident relish, and seems a valuable plant. Dr. Gattinger, of Tennessee, says of it:-

"All parts of the plant are equally pliant and succulent. It sprouts again after being pastured down, with numerous new culms, and its growing season lasts from May till frost. The culms stand about two feet high, and, as far as I have observed, I found it always grow. ing in patches. It grows in low and small tufts, and sticks firmly to the soil. I would very earnestly recommend to try it under cultivation." The flowers are borne in a long, narrow, spike-like panicle. The spikelets are single flowered, with unequal glumes.

Wild Fescue. (Uniola latifolia.) - This is a beautiful grass with very large spikelets; in form they are somewhat like those of the Bromus unioloides, and, like them, are very flat and compressed. Mr. Charles Mohr, of Alabama, says of it: -

"A fine vernal grass, with a rich foliage, blooming early in May, two to three feet high; frequent in damp, sandy loam, forming large tufts. This perennial grass is certainly valuable, affording an abundant range early in the season; if cultivated it would yield large crops ready for cutting from the Ist of May. It is called, by some, wild fescue or oat grass. It is not found near the coast, consequently I had no chance to observe its growth during the latter part of the summer and in the winter season, and therefore am not able to judge of its value as a pasture grass." It grows as far north as Pennsylvania, but it is less vigorous in growth as it advances northward.

Smootli Brome Grass, or Upright Chess, (Bromus racemasus,) has a panicle erect, simple, rather narrow, contracted when in fruit. Flowers closer than in the preceding, lower palea exceeding the upper, bearing an awn of its own length. Stem erect, round, more slender than in chess, sheaths slightly hairy. In other respects it is very much like Willard's bromus, but may always be distinguished from it as well as from Bromus arvensis, in the summit of the large glume being half-way between its base and the summit of the third floret, on the same side; whereas in Willard's bromus the summit of the large glume is half-way between its base and summit of the second floret. This character is constant, and offers the surest mark of distinction. It is common in grain fields. Flowers in June. It is worthless for cultivation.

Soft Chess, or Soft Brome Grass, (Bromus mollis,) is sometimes found. I pro. cured beautiful specimens of it at Nantucket, where it was growing in the turf with other grasses on a sandy soil near the shore. Its panicle is erect, closely contracted in fruit, spike. lets conical, ovate, stems erect, more or less hairy, with the hairs pointing downwards from twelve to eighteen inches high, joints four or five, slightly hairy, leaves flat, striated, hairy on both sides, rough at the edges and points; summit of the large glume midway between its base and the apex of the third floret, by which it is always distinguished from Willard's bromus. Flowers in June. Birds are fond of the seed, which are large and ripen early. Of no value for cultivation.

The Wild Chess, (Bronzus kalmii,) is another species, found often in dry, open wood-lands. It has a small, simple panicle, with the spikelets drooping on hairy peduncles, seven to twelve flowered and silky; awn only one-third the length of the lance-shaped flower, 


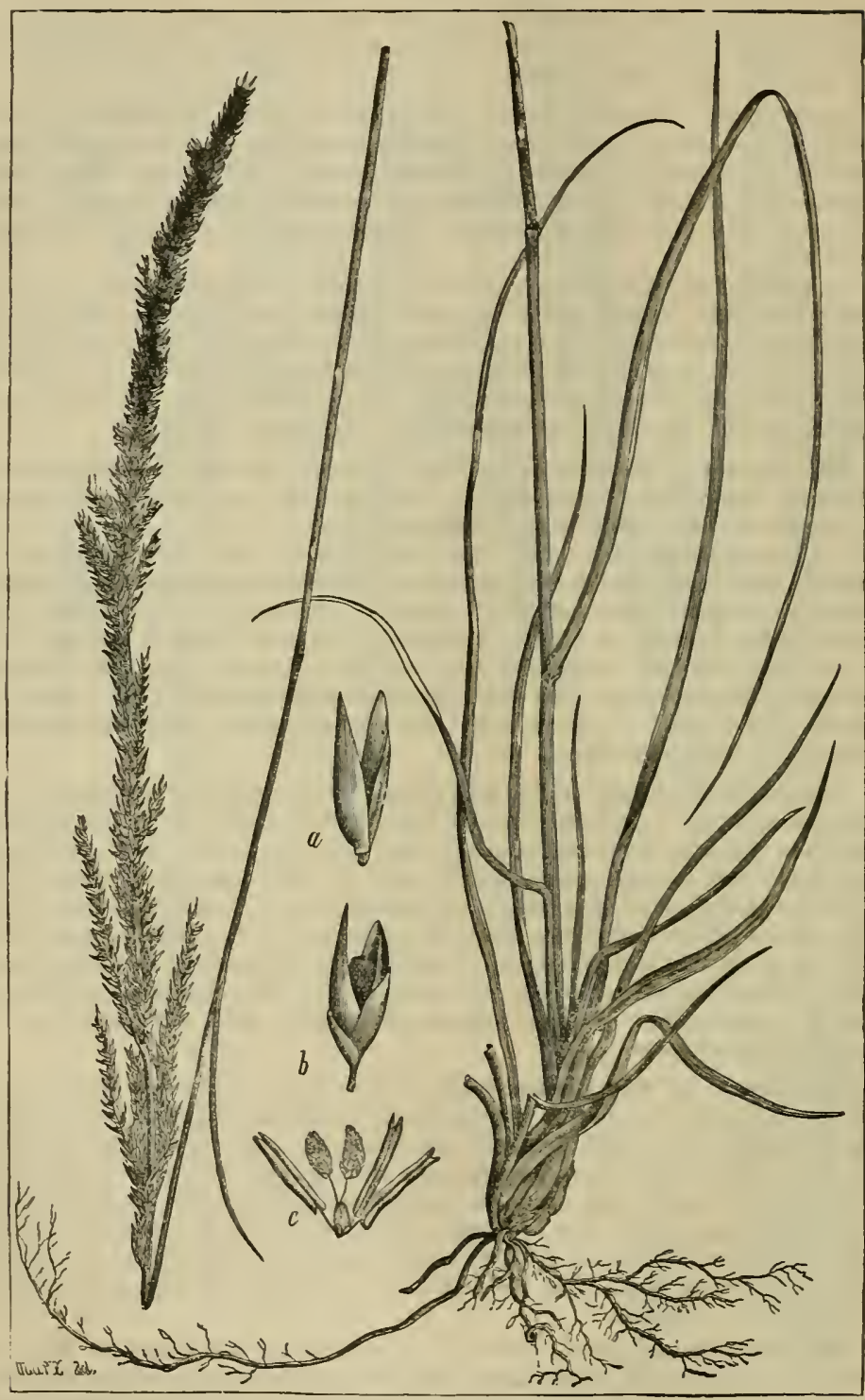

SMUT GRASS.

(Sporobolus Inticus.) 


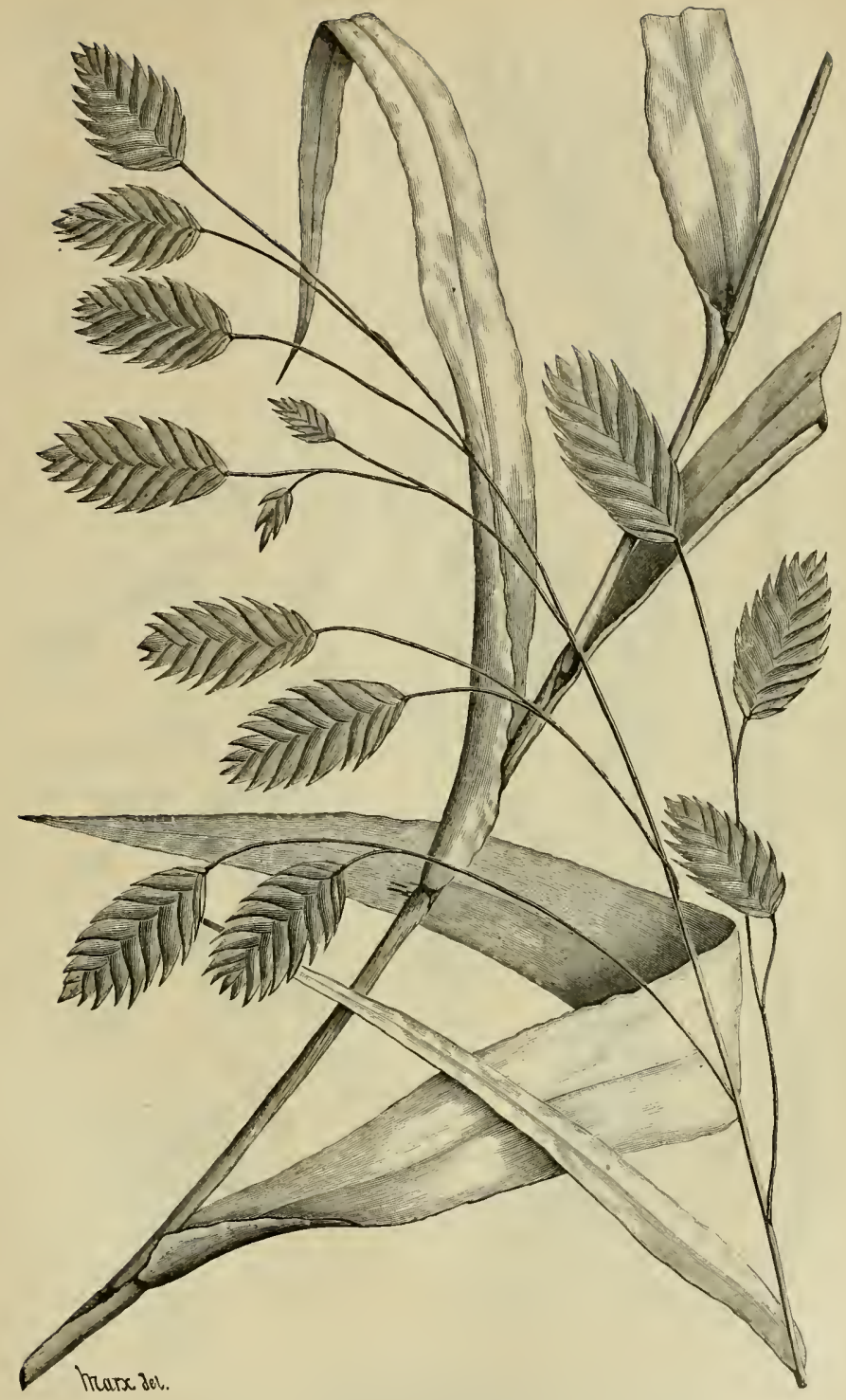

WILD FESCUE.

(Uniola latifolia.) 


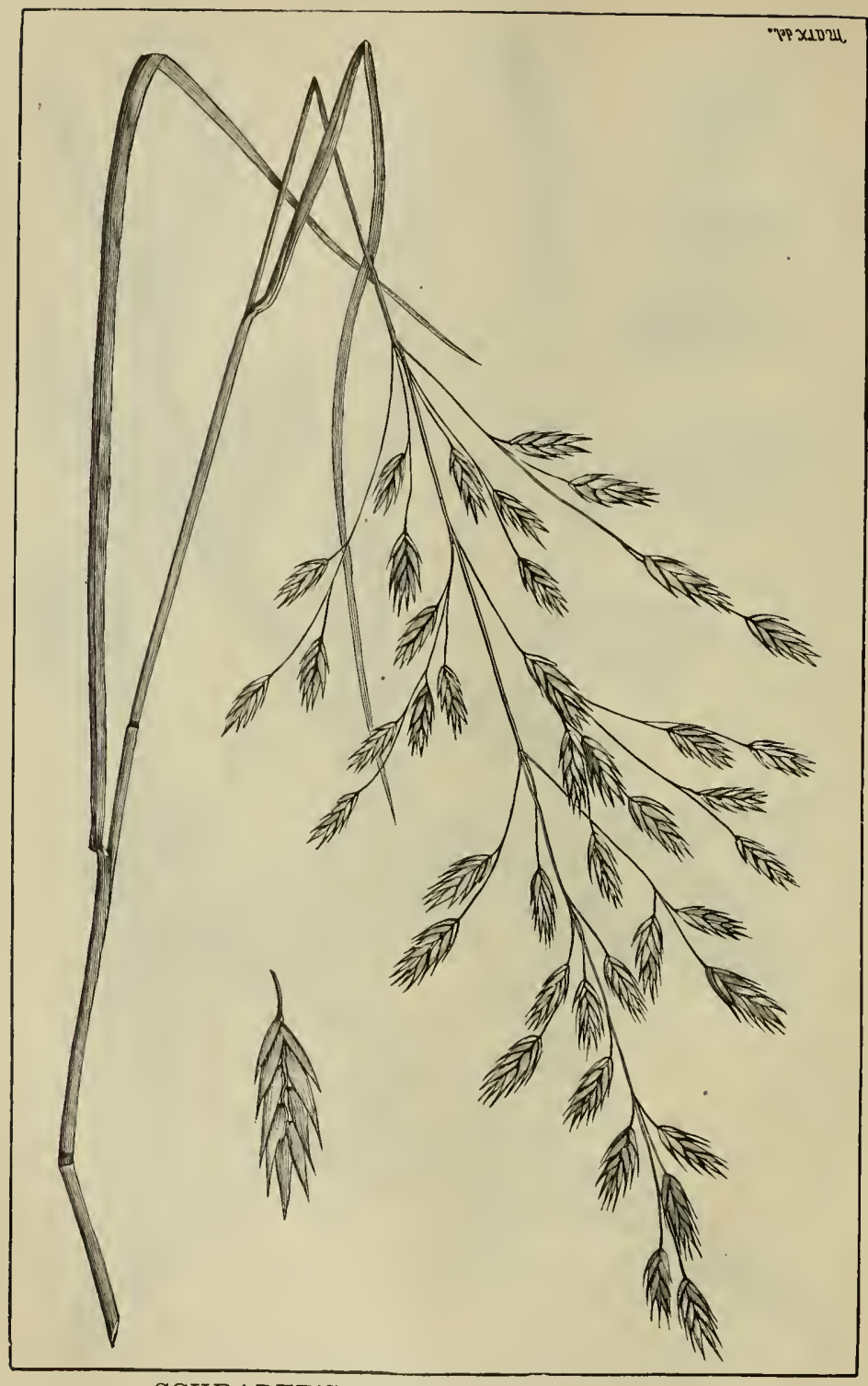

SCHRADER'S GRASS.-RESCUE GRASS.

(Bromus unioloides.) 


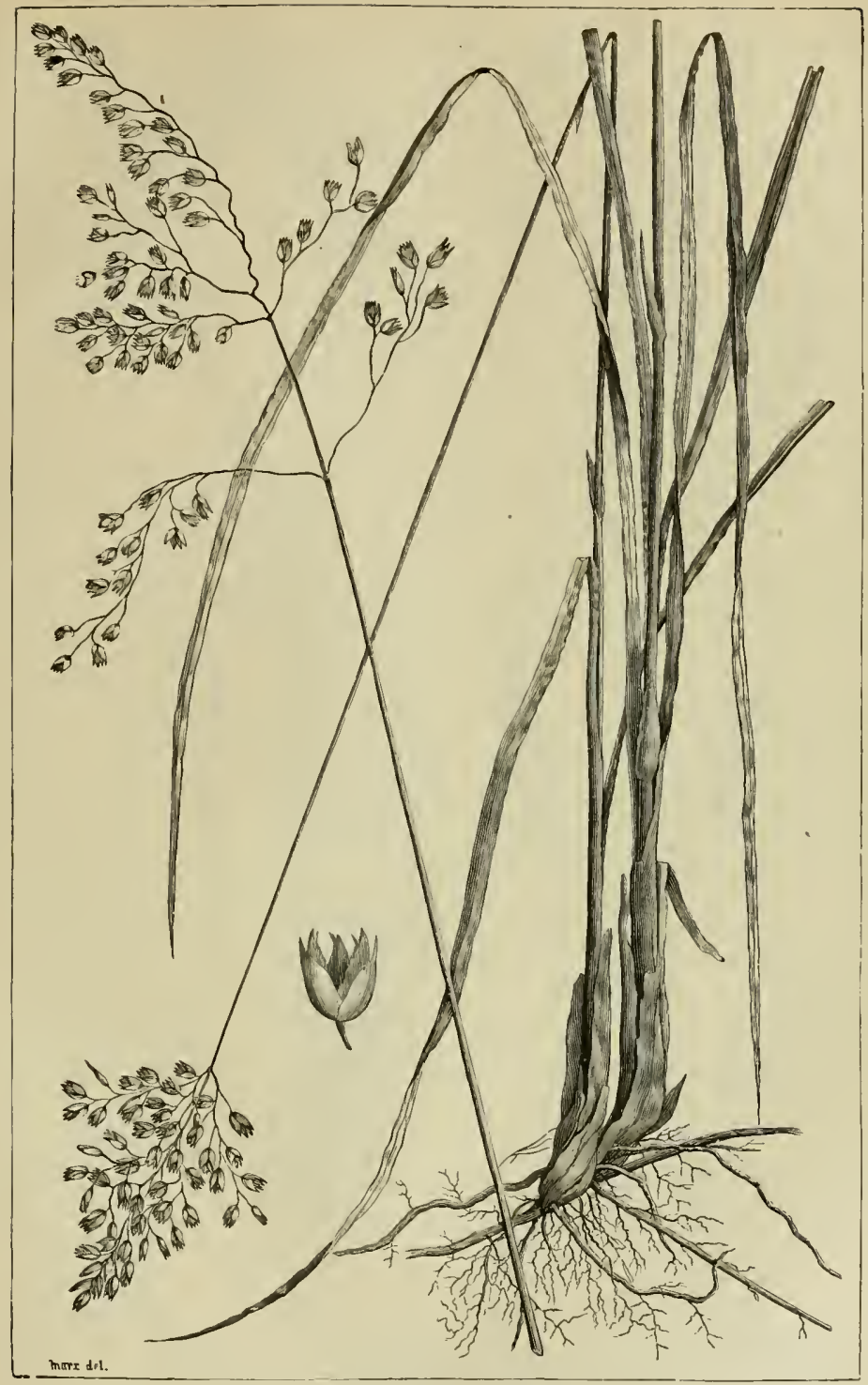

VANILLA GRASS

(Hierochloa borealis.) 


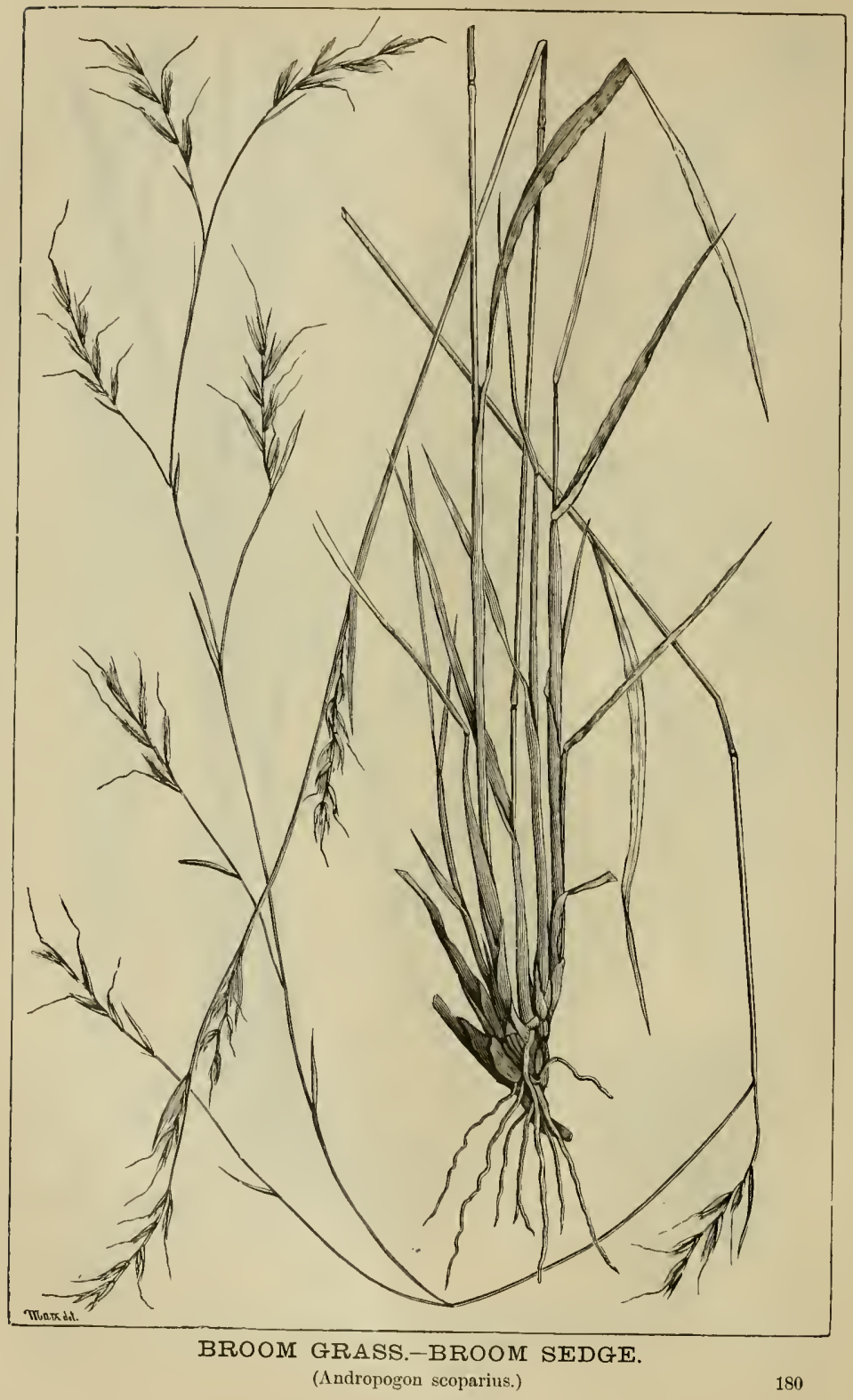


stem slender, eighteen inches to three feet high, leaves and sheaths hairy. Flowers in June and July. Of no value for cultivation.

Schrader's Grass, Rescue Grass (Bromus unioloides). The description of this grass is taken from a recent report of the Department of Agriculture:

"This is one of the so-called winter grasses; that is, it makes a large share of its growth during the winter months. It belongs to the Chess or Cheat family. In its early growth it spreads and produces a large amount of leaves. Early in the spring it sends up its flower stalks, which grow about 3 feet high, with a rather large, open, spreading panicle, the ends of the branchlets bearing the large flattened spikelets, which, when mature, hang gracefully upon their stems, giving them quite an ornamental appearance. These spikelets are from an inch to an inch and a half in length, and composed of two acute lanceolate glumes at the base, and from 7 to 10 flowers, arranged in two rows alternate on each side of the axis. The flowers are lanceolate, or ovate lanceolate, the lower palet extending into a fine point or short awn.

During several years past this grass has been sent to this department, chiefly from Louisiana and Texas, and has been much commended. Many years since the same grass was dis. tributed and experimented with under the name of Australian oats, or Bromus schraderi. It is not adapted to use in a country with severe winters, and hence did not give satisfaction in all places. Mr. C. Mohr, of Mobile, says of it :

"Only of late years found spreading in different parts of this state; makes its appearance in February, grows in tufts, its numerous leafy stems growing from 2 to 3 feet high ; it ripens the seed in May ; affords in the earlier months of spring a much-relished nutritious food, as well as a good hay."

Mr. Williams writes from San Antonio, Texas, describing the introduction and spreading of a patch of this grass. He says: "Inasmuch as Western Texas is the great stock-producing section of the Southwest, and considering the fact that pasturage is scanty, particularly in February, stunting the growth of young cattle, this seems wonderfully adapted to supply just what is greatly wanted, both for milch cows, calves, colts, and ewes just dropping lambs; and besides, this grass grows well on the thinnest soil and crowds out weeds, maturing in March and early April, while not interfering with the native mesquite. I therefore regard this grass as a wonderful and most important discovery."

This grass is said to have been introduced into Georgia by General Iverson, of Columbus, and by him called Rescue grass. The favorable opinion which it at first received does not seem to have been well sustained in that state.

Fringed Brome Grass, (Bromus citiatus,) is often found in woods, and on rocky hills and river banks. It has a compound panicle, very loose, nodding, spikelets seven to twelve flowered, flowers tipped with an awn half to three-fourths their length, stem three to four feet high, with large leaves. Flowers in July and August. Of no value for cultivation.

The Meadow Brome Grass, (Bromus pratensis,) is a perennial weed in the corn-fields of England, and is only recommended in any part of Europe for dry, arid soils, where nothing better will grow.

The brome grasses generally have not been found worthy of much attention as cultivated agricultural grasses, and the cleaner the farmer keeps his fields of them, as a general rule, the better.

Vanilla or Seneca (Grass (Hierochloa borealis). This is a grass growing in the northern latitudes, in moist meadows near the coast, also in low, marshy ground in some parts of Illinois and other Western states, bordering on the great lakes, and in the mountains of Colorado and further north. It derives its generic name Hierochloa, from two Greek words, which signify sacred grass, so called because it was customary to strew it before the doors of the churches 
on festive and saints' days in the north of Europe. It is said to be sold in Sweden to be hung up over beds, where it is supposed to possess some mysterious properties of inducing sleep.

The culms grow from one to two feet high, with short lanceolate leaves and an open pyramidal panicle from two to five inches long. The spikelets are three-flowered; the flowers all with two palets. The spikelets are chestnut-colored and rather ornamental. It is very fragrant and flowers in May. It has not much practical value for cultivation on account of its powerful creeping roots, and slight spring foliage.

Broom Grass, Broom Sedge (Andropogon scoparius). This is a perennial grass, with tough, wiry stems, growing from two to three feet high, with narrow flower-spikes coming out from the side joints and also at the top. The spikelets are in pairs on each joint of the slender axis; one of the flowers is sterile and fringed with soft spreading hairs, the other perfect and with a small twisted awn.

It grows mostly in dry, sterile soil, and is considered a great pest if it takes possession of meadows; but it is also considerably valued as a pasture grass before it shoots up its culm, or stem, after which stock will touch it no more. It is said to be very common at the South, especially in Georgia, Alabama, and other of the Gulf States, where it covers old fields and fence rows, and grows extensively in the dry sandy soil of the pine woods. Though much despised by many as an unsightly, troublesome weed, it is nevertheless valuable, while green and tender, as sustenance for stock, of which it affords, in its season, a considerable share. It blossoms in August.

Andropogon Furcatus, Andropogon Virginicus, Andropogon Macrourus (sometimes called Indian Grass), ( $A$. nutans,) and other species of this genus, which have similar characters with the preceding, form an important quantity of the native grasses of the great prairie region of the West. They are useful as a resource for stock having an extensive range over uncultivated fields, but their places should be filled with more valuable grasses, as early as practicable.

Heans, Johnson, or Egyptian Grass (Sorghum halpense). This is a perennial grass, with strong, vigorous roots and rather broad leaves. The flowers and seeds closely resemble those of broom-corn, but the panicle is finer and more spreading. Its stems attain the height of five or six feet, with a very broad spreading panicle. It is much cultivated in the Southern States, where it is valued both for forage and hay. It is difficult to exterminate when once the ground is occupied by it, and this seems to be the only objection to it. It has been found that plowing the field and leaving the roots exposed to the frost will eradicate it. It is quite nutritious, will grow on ordinary land and yield abundantly. The following history of its introduction into the Southern States is given by Mr. Wall, of Mississippi, in his recent official report as Commissioner of Agriculture :

"A few years before the war, Captain Means, of South Carolina, who commanded a trading vessel to the Mediterranean Sea brought from Egypt a lot of seeds, from which he obtained a spoonful of seeds of an unknown kind. He handed them to a friend with the request that they be sown in his garden. They came up and proved to be the grass named above; but little attention was paid to it until it nearly took his garden. He had the plants dug up by the roots and thrown into a neighboring gully, where they soon began to grow, stopping the wash and spreading all around. It was now seen for the first time that it was greedily eaten by stock. This was suggestive in a country where all the hay had to be imported, and so the seeds were gathered and sown. Thus the Means grass derived its name."

In 1860 Captain Johnson of Marion Station, Alabama, paid a visit to some relatives in South Carolina and heard of this grass, that had in the meantime acquired a great reputation, and on his return, carried home with him a bushel of seeds and sowed them on his plantation. Soon after this be went into the Confederate service and was killed, leaving two little girls. 


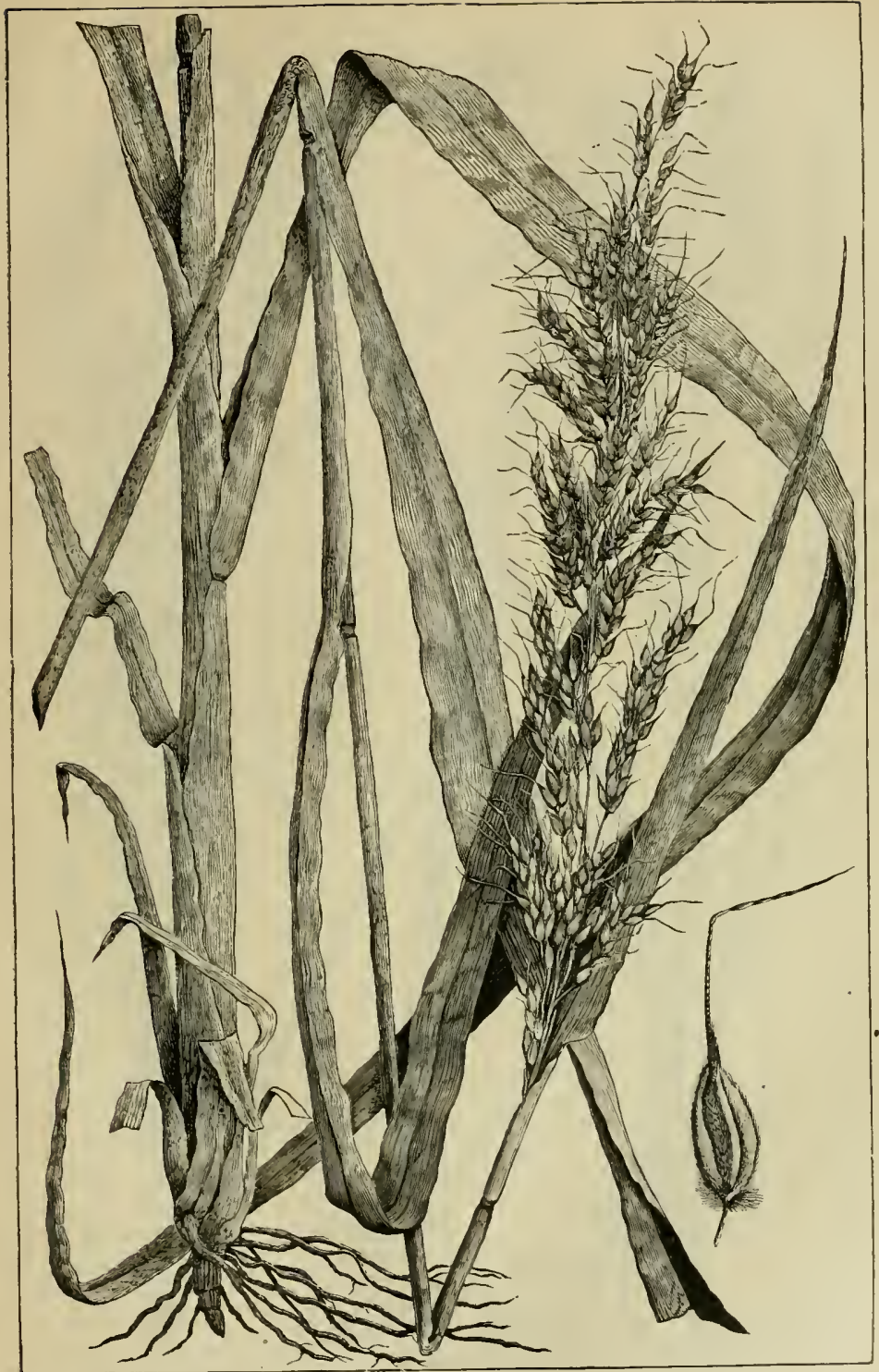

MEANS. JOHNSON, OR EGYPTIAN GRASS.

(Sorghum halapense.) 


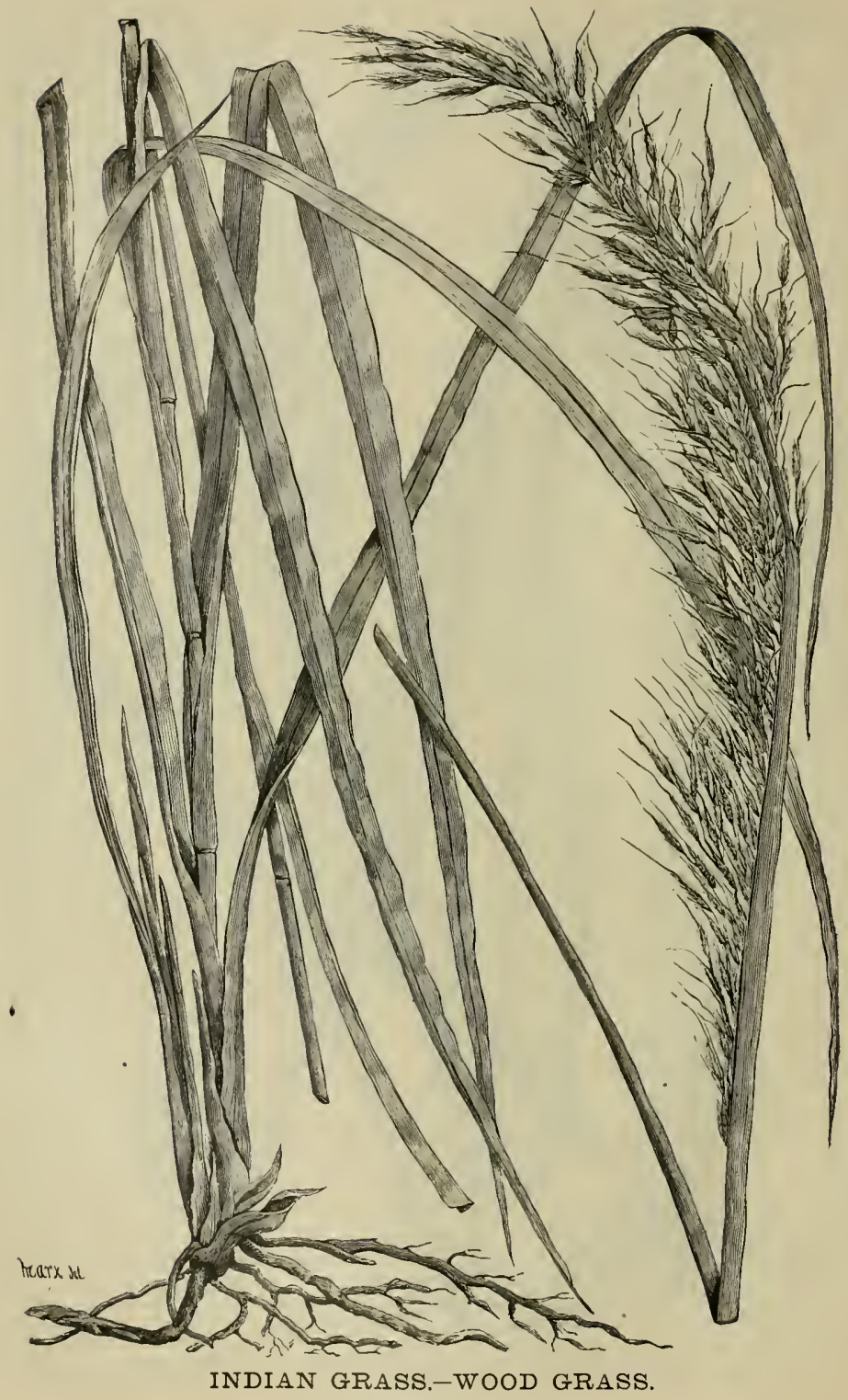

(Sorghum nutans.) 
These girls were sent to school at Tuscaloosa, but having no guardian and no means, the President of the college had a gentlemen appointed guardian who went to Marion Station to see if his wards had anything. In the meantime the plantation was left alone, no one caring for it, and it was unrented. He found it a large place and almost covered with the "Means grass," the winds and stock having set it everywhere. Being a shrewd man, he saw its capability and at once advertised it as a stock farm, and soon rented it to Messrs. R. C. Gardner and J. C. Copeland, of Nashville, Tennessee. They saw their cpportunity at once, and securing a number of baling presses, set to work cutting and baling hay for the Southern market.

The hay proved popular and sold wherever tried, stock delighted in it, leaving all other kinds to eat it. Applications naturally poured in for some of the grass, and so they sold immense quantities of the seed, and also of the roots, getting large prices for both. So great was its popularity that at the end of their five years' lease a company of Northern men outbid them, and have resorted to steam to assist in baling hay for the market.

Egyptian sugar-cane (is its proper name) is a native of the Nile, where it grows fifteen to twenty feet high. So great is its luxuriance there, that it has filled all the upper Nile so that a boat can not be driven through it. Great numbers of cattle and wild animals resort to it, and, in fact, it is the chief food of ruminants in that country.

When young, it is very tender and sweet, the sap being full of sugary juice. It is a peren. nial plant and so vigorous that when once planted, it is a difficult matter to eradicate it, so care must be taken to plant it where it can be kept in bounds. The roots are creeping and throw out shoots from every joint. It is a fine fertilizer. and sown on a piece of poor washed land, will in a few years restore it to fertility. But there is really not much difference where it is sown, for a farmer once getting a good stand will not want to destroy it. It will bear cutting three or four times a year, and in fact, this has to be done, for when it matures seed, the stem and leaves are too coarse and woody for use.

The ground must be prepared as for other grasses, and in September, the earlier the better; it should be sown at the rate of onc bushel of seed per acre. It can be propagated also by the roots, by laying off the rows each way and dropping a joint of the root two feet apart and covering with a harrow.

It gives the earliest pastures we have, preceding blue grass, or clover, a month. Hogs are fond of the roots, and any amount of rooting will not injure it. In fact, it is a stick tight.

For soiling purposes it is not equaled by any grass in our knowledge, as it can becut every two or three weeks. There is a vast amount of land in Mississippi now devoted to gullies, that do not now pay their taxes, which would richly remunerate the owner, if set in this grass. It is not necessary that the land should be broken up to start it; a few sprigs set out here and there in the richest spots, will soon secure a stand.

Many farmers object to it on account of its great tenacity of life, matting the soil in every direction with its cane-like roots, and the rapidity with which it will spread over a field, and the difficulty of eradicating it; but these very objections should be its recommendation to the owners of worn-out fields. To destroy it and keep it down, it is only necessary to pasture it closely one year, and then in the fall, turn the roots up with a big plow to the freezes of winter, renewing the plowing once or twice during the winter, and then cultivating the land in a hocd crop the next spring. There certainly would be no risk in sowing it upon those worn-out hillsides, so many of which form unsightly scars upon the face of nature in Mississippi." The illustration which is given is a very correct representation of this grass.

Indian Grass, Wood Grass (Sorghum nutans). This is a perennial grass, having a wide range over all the country east of the Rocky Mountains. The stalks are smooth, hol. low, and straight, and grown from three to five feet high, having at the top a narrow panicle of handsome straw-colored or brownish flowers, drooping when mature. It flowers in August. 
This grass, though not usually considered of much agricultural value, forms an important part of the native grass of the Western prairies, and, if cut early, makes a good and nutri. tious hay. In the Southern States there are two additional species, or varieties, which are not materially different. These varieties are very common in Texas.

Indian Millet, (Sorghum vulgare,) is a cultivated species, which has many varieties, one of which is the broom-corn. It is sometimes used as a forage plant, and when raised on good soil, and under favorable circumstances, is said to yield abundantly. It is called guinea-corn in the West Indies, dourah in Arabia, and Nagara in the north of China. When raised on good soil, it is said to yield a larger quantity of seed to the acre than any other cereal grass known, its nutritive quality being nearly equal to that of wheat.

Texas Millet(Panicum Texanum).-The branches of the panicle of this variety are rough, spikelets oblong, somewhat pointed, lower glume half or two-thirds the length of the upper; sterile flower with two palets, the lower five to seven, curved, much like the upper glume, and the upper palet thin and transparent. It is an annual grass growing from two to four feet high, sparingly branched, at first erect and becoming widely branched; margin of leaves rough; leaf blades from six to eight inches long and a half to an inch wide, the upper leaves reaching nearly to the base of the panicle, which is from six to eight inches long. The leaves are broad, and rather thin. It is a grass of vigorous, rapid growth, and is capable of yielding a large amount of food. Mr. Pryor Lea of Texas, who has cultivated it for several years, says of it:-

"I consider it far superior to any grass that I ever saw for hay. It is a much more certain crop than millet, and cultivated with less labor, and all kinds of stock prefer it. I expect to report a good second crop on the same ground this year. In this region this grass, in the condition of well-cultivated hay, is regarded as more nutritious than any other grass. It grows only in cultivated land; it prospers best in the warmest fourth of the year; its luxu. rious growth subdues other grasses and some weeds, with the result of leaving the ground in an ameliorated condition." (See illustration, Page 140).

Barnyard Grass, Cock's-Foot Grass (Panicum crusgalli).-This is an annual grass, with thick, stout culms branching from the base two to four feet high. It varies greatly, sometimes being awnless or nearly so, and sometimes with long awns, especially in some of the coarser varieties. It is very common in all parts of the country in waste places. It is, however, cultivated in many sections, especially in some of the Southern States. A writer in Northern Mississippi states that the hay from this grass is very highly esteemed by many farmers, and large fields of it are often mowed. It should, however, be cut early, while it is most juicy and nutritious, which will be when the flowers first make their appearance. In the latter stages of growth, or about the time of ripening its seed. The long and stiff awns of its spikes tend to make it undesirable for stock food.

Crab Grass (Panicum sanguinale).-This is a native of Europe, but has become naturalized in all parts of the country. It springs up quickly in both cultivated and waste grounds, and sends out roots from the lower joints, which take firm hold of the soil and spread rapidly in all directions. In the Northern States it is very troublesome in corn-fields, it being difficult to keep them clear of it. In the Southern States it is used for hay crops, giving a large yield of hay of excellent quality if cut in proper time, which is before the ripening of its seeds. It also furnishes very desirable pasturage during August and September, when the spring grasses are old and dry. Prof. Killebrew of Tennessee says of it:-

"It is a fine pasture grass, although it has but few base leaves and forms no sward, yet it sends out numerous stems, branching freely at the base. It serves a most useful purpose in stock husbandry, and the Northern farmers would congratulate themselves very much if they had it to turn their cattle on while the clover fields and meadows are parched up with 


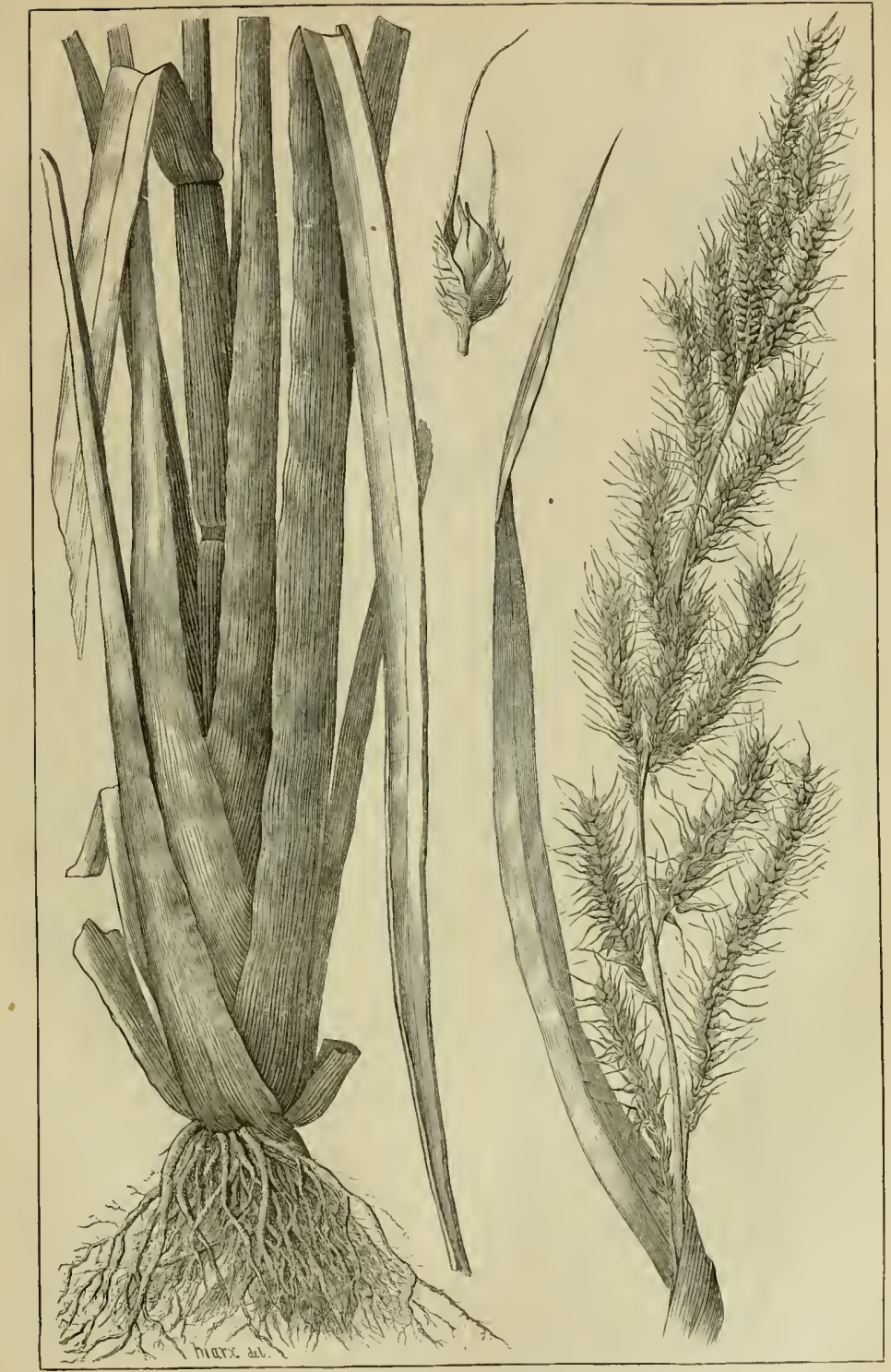

BARNYARD GRASS.-COCK'S FOOT GRASS. (Panicum crusgalli.) 


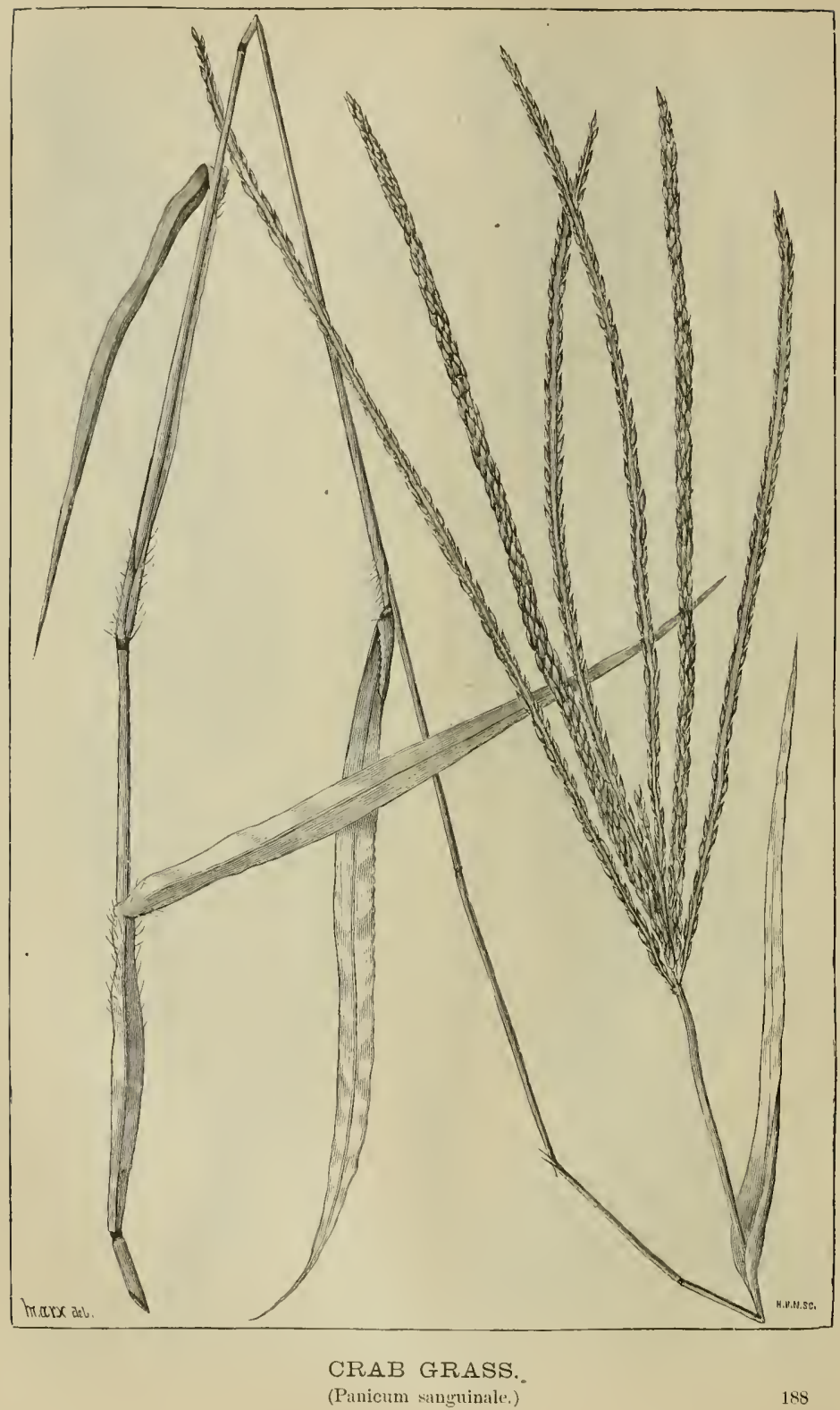


summer heat. It fills all our corn fields, and many persons pull it out, which is a tedious process. It makes a sweet liay, and horses are exceeding fond of it, leaving the best hay to eat it."

Tall Panic Grass, SwitchGrass (Panicum virgatum).-A tall perennial grass growing mostly in clumps in wet or damp soil, especially near the sea-coast. It grows from three to five feet high, and furnishes very good feed for stock if cut when juicy and tender. If cut whon ripe, it is very harsh and unpalatable. It forms a considerable portion of the native grasses of the prairies, especially in those localities where the soil is unusually moist.

Slender Crab Grass (Panicum filiforme).-A native species of crab grass. It is an annual, growing with erect slender culms, which terminate with from three to five slender spikes of flowers; leaves from one to two inches long. The stems are thin and wiry, the leaves small and scanty, furnishing but little herbage, and for this reason it has little or no agricultural value. It grows mostly in dry sandy soil.

Obtuse-Flowered Panic Grass (Panicum obtusom).-This is similar to the Panicum Texanum, or Texas millet, although it does not grow as high as the latter, and is less vigorous. It is found in many of the Southern States, but has never been used very extensively for agricultural purposes.

Guinea Grass (Panicum jumentorum).-This grass is a native of Africa, and is extensively cultivated in tropic sections. Mr. C. Mohr of Mobile says of it: "lt is planted with us in the beginning of April; admits the first cutting during the last week of May; it makes very large bunches, and is to be cut before extending to the height of about eighteen inches. In that stage it is very sweet, tender, and as easily cured as hay. In moderately fertilized land and favorable seasons, it can be cut every five or six weeks, yielding, by its throwing out numerous stolons, increased crops until killed down by frost. The roots are easily protected during the winter by a good covering with ground, like the rattoons of sugar-cane, and allowing of a manifold division, afford the best means of propagation. These root-cuttings are set out in March or the beginning of April." This grass must not be confounded with the Sorghum halapense or Johnson grass, which is often improperly called Guinea grass, from its similar habit and appearance."

Agrostis-like Panic Grass (Panicum agrostoides).-This grass has its stems flattened, upright, two feet high, leaves long, sheaths smooth, spikelets on the spreading branches crowded and one-sided, ovate, oblong, acute, purplish. It is common on wet meadows and borders of rivers. Flowers in July and August.

Prolific Panic Grass (Panicum proliferum) grows on brackish marshes and meadows, and is common along the coast. It sometimes appears on dry places. Cattle are fond of it. It differs from the preceding in having culms thickened, succulent, branched and bent, ascending from a procumbent base, and spikelets appressed, lance-oval, of a pale green color.

Hair Stalked Panic Grass (Panicum capillare) grows in sandy soils and cultivated fields everywhere. Its culm is upright, often branched at the base, and forming a tuft, sheaths flattened, very hairy, panicle pyramidal, hairy, compound and very loose, spikelets scattered on long pedicels, oblong, pointed. Flowers in August and September.

Broad-Leaved Panic Grass (Panicum latifolium).-This is a grass with a perennial, fibrous root, and stem from one to two feet high, and leaves broad, long, taper-pointed, smooth or slightly downy, branches of panicle spreading, spikelets long, obovate, downy. Flowers in June and July. It is common in moist thickets and woods. Of no value for cultivation. 
Hungarian Grass or Hungarian Hillet, (Panicum Germanicum), is an annual grass cultivated as a forage plant, and is a variety of Setoria Germanica, the common millet. It was introduced into France in 1815 , where it has been considerably cultivated. It germinates readily, has a quick luxuriant growth, and is remarkable in withstanding drought, remaining green, even when other vegetation is parched and dry, while if its growth is arrested, in a measure, by dry weather, the least rain will restore its vigor. It is much relished by horses and cattle, as well as all other kinds of stock; when fed in too large quantities, it will act as a diuretic, and hence, in such cases, injurious; but if fed in moderate quantity is very nutritious and harmless. It makes most excellent hay, of good weight, flourishing on light and dry soils, though it attains its greatest luxuriance in soils of medium consistency, well manured, and may be sown broadcast and cultivated precisely like other varieties of millet. When blown down by storms and violent winds, it readily recovers its upright growth. A farmer recently mentions the fact of his field having been blown down three times in succession by storms, and each time it recovered its upright position very readily. It

has been known to attain the height of three and a half feet on rich soil. This grass does well on the rich lands of the West, though it will exhaust soil quicker than some other products. It is sown about the time of planting corn, at from two to three pecks per acre, and should be cured the same as Timothy grass. The editor of the Country Gentleman expresses the following opinion relative to this grass

"Hungarian grass will grow on any soil of sufficient richness and condition to raise good corn and potatoes, the richer the better, provided the manure has been applied to previous crops. Sow when the ground is warm and dry, or immediately after corn planting. If portions of the field are sown with a few days' interval between, it will not be ready to cut all at once, which may prove a convenience in a large field. The ground before sowing should be thoroughly pulverized by harrowing, and made smooth and level, so that the small seed may not be buried too deep. Three pecks broadcast are enough for an acre. Cahoon's sower is well adapted to doing the work evenly. Cover the seed with a fine or smoothing harrow, and then roll the surface. In two months the heads will appear, at which time, if intended for hay, it should be cut with a mowing machine. Two or three days may be required for thorough curing, including turning or shaking up before raking, and opening the cocks. Good land, well managed, will give two or three tons of good hay per acre. It is best as a fodder for cows. The crop is too dense in growth to sow clover or grass seed with it.

Common Millet. (Panicum Milliaceum.) - In growth and manner of bearing seeds, the common millet resembles broom-corn. Its foliage is broad and abundant; heads open; branching panicles. It grows to the height of from two and a half to three and a half feet, according to the nature of the soil, and makes excellent forage for cattle, though not equal to the German, Golden, or the Pearl millet. Allen states that from eighty to a hundred bushels of seed per acre have been raised, with straw equivalent to one and a half or two tons of hay, but an average crop may be estimated at about one-third this quantity. Birds are usually troublesome in destroying the seed when ripened, as they are very fond of it, consequently it is more profitable to cut it for the seed before all the heads bave matured. It 
is cured the same as hay, and yields from two and a half to four tons per acre. All kinds of grazing stock are fond of it. The seeds are glossy, oval, and somewhat flattened. When cut in the seed it is a very rich food, consequently should be mixed with other hay in feeding.

Cultivation.-A rich, dry soil, well pulverized, is the most desirable for the best results, although it will grow on thin soils. It can be sown broadcast or in drills. From the first of May to the first of July is the usual time for sowing at the North, the best time being generally conceded to be in June, when the soil is warm. At the South, it can of course be sown much earlier. From a bushel to a bushel and a half of seed per acre is considered a fair quantity, if sown broadcast; if sown in drills, from eight to ten quarts per acre will suffice. It will ripen in from sixty to seventy-five days after sowing. It should be cut, if for fodder, while the seed is in the milky state, which will be in about six weeks after sowing. It can be cut with a mowing machine, and cured the same as hay.

For green food, it can be cut during its various stages of growth, and will produce a quick after-growth when cut at any stage, and is not liable to be easily affected by dry weather.

It is especially valuable to supplement the hay crop, as a farmer can wait until he can determine the yield of his hay or ensilage crop before sowing, consequently can calculate the amount of millet required to be cultivated to insure his usual amount of feed for his stock for the year, while nearly all other crops require an earlier planting or sowing.

Golden Millet, called in some sections German millet, is considered by many as greatly superior to common millet, both as a forage plant and for hay. It grows to the height of from three to five feet; the heads are closely condensed; the spikes very numerous. The seeds grow in rough, bristly sheaths, and are round and golden yellow. It requires a rich, fertilized soil, which should be well pulverized and mellow. It may be sown broadcast or in drills, and is cultivated the same as the common millet, previously described. It requires a warm soil to thrive well, and should, therefore, not be sown at the North until the middle or last of June. When used for hay, it is cut and cured the same as common millet. It is greedily eaten by all kinds of stock, that thrive well upon it.

Pearl Millet (Penicillaria spicata). This, as a forage plant, has been cultivated for several years with success in many of the Southern States, where it is known as "African Cane," "Egyptian Millet," "Japan Millet," "Cat-tail," "East Indian Millet," and in some localities as "Horse Millet."

But little has been known of it at the North until recently. It closely resembles Setaria in appearance, and the genus is allied to Pennisetum.

Mr. P. Henderson of New Jersey thus gives his experience in experimenting with this plant: "A piece of good strong loamy ground was prepared, as if for a beet or turnip crop, by manuring with stable manure at the rate of ten tons to the acre, plowing ten inches deep, and thoroughly harrowing. The millet was then sown in drills, eighteen inches apart, at the rate of eight quarts to the acre. We sowed on the 15th of May, about the date we plant corn; in twelve days the plants were up so that a cultivator could be run between the rows, after which no further cuiture was necessary, for the growth became so rapid and luxuriant as to crowd down every weed that attempted to get a foothold. The first cutting was made July lst, forty-six days after sowing; it was then seven feet high, covering the whole ground, and the crop, cut three inches above the ground, weighed, green, at the rate of thirty tons per acre; this, when dried, gave six and a half tons per acre as hay. After cutting, a second growth started, and was cut August 15th-forty-five days from the time of first cutting. Its height was nine feet; it weighed this time at the rate of fifty-five tons to the acre, green, and eight tons dried. The third crop started as rapidly as the second, but the cool September nights lessened its tropical luxuriance, so that this crop, which was cut on October 1st, only 


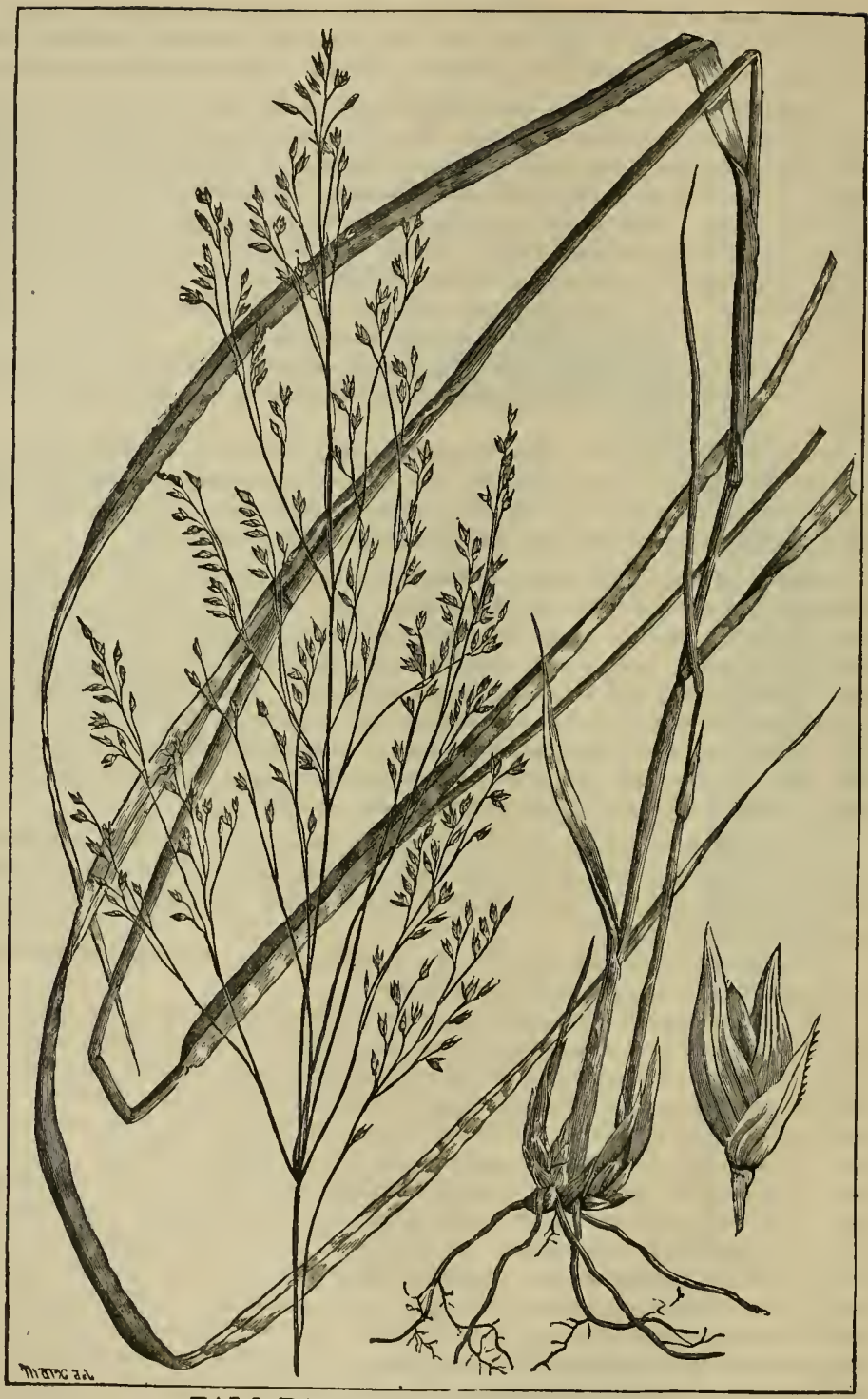

TALL PANIC OR SWITCH-GRASS.

(Panicum virgatum.) 
weighed ten tons green, and one and a half tons dried. The growth was simply enormous, thus: First crop, in forty-five days, gave thirty tons green, or six and a half tons dry; second crop, in forty-five days, gave fifty-five tons green, or eight tons dry; third crop in forty-five days gave ten tons green, or one and a half tons dry, - the aggregate weight being ninety. five tons of green fodder in $\mathbf{1 3 5}$ days from date of sowing, and sixteen tons when dried to hay. This exceeds the clover meadows of Mid-Lothian, which, irrigated by the sewage from the city of Edinburgh, and cut every four weeks, gave an aggregate of seventy-five tons of green clover per acre.

"There is little doubt that pearl millet is equally as nutritious as corn fodder, which it resembles even more than it does any of the other millets. We found that all our horses and cattle ate it greedily, whether green or dry. If sowing in drills is not practicable, it may be sown broadcast, using double the quantity of seed-say sixteen quarts per acre. The ground should be smoothed by the harrow, and again hightly harrowed after sowing; if rolled after harrowing, all the better. I know of no farm crop that will better repay high manuring, but so great is its luxuriance that it will produce a better crop without manure than any other plant I know of. In those parts of the Southern States where hay cannot be raised this is a substitute of the easiest culture, and, being of tropical origin, it will luxuriate in their long, hot summers. Even though our Northern seasons may be too short to mature the seeds, our experiments in New Jersey this summer show what abundant crops may be expected if the similar conditions are secured. Pearl millet as a fodder plant presents a new feature in our agriculture, and I feel sure that within ten years we shall wonder how we ever got along without it. Besides our own testimony given above, we have received the most satisfactory letters from experienced men in different parts of the country to whom we sent seeds of pearl millet for trial, and all are unanimous as to its enormous productiveness and great value. From all we have seen and can learn, we are fully convinced that pearl millet is to be one of the great fodder plants of the future."

Farmers should bear in mind that it is a tender annual, and must not be planted until the weather is settled warm. Wherever and whenever corn flourishes, it will yield bountiful crops of fodder, which cows, horses, and other farm stock are exceedingly fond of. It is equal in nutritive value to corn fodder, while its yield is greater. The fodder is in the best condition for cutting and curing when the stalks are five or six feet high; but, if used for soiling, it may be cut earlier or later, at the convenience of the cultivator; the stumps, sprouting and throwing up a new growth, continue to grow until killed by frosts. The seed should be sown at the time of sowing corn (and not earlier), in drills four feet apart. Sown in this way, two pounds of the seed will plant an acre.

From all we have been able to learn concerning pearl millet, we judge it to be a desirable acquisition to our forage plants, and that with suitable soil and proper cultivation it will abundantly repay the farmer for the care bestowed upon it, in those sections where the climate is well adapted to its luxuriant and rapid growth, and even as far north as some por. tions of New England will prove a profitable crop for cultivation.

Favorable reports of experiments in its cultivation have reached us from portions of the country farther north than New England even, and from the vicinity of Montreal, Canada; but we doubt whether in the latter latitude it will prove a generally profitable crop to the farmer, owing to the shortness of the season, and the cooler temperature.

Indian Corn or Maize as a Forage PIant (Zea mais.) Though one of the most impor. tant and abundant of the cereals, this plant belongs to the order Gramineae, or grasses; and while we shall speak of it elsewhere as a cereal, we refer to it in this connection merely as a forage plant, which, when cultivated as such, is extremely valuable, being very nutritious, and an almost indispensable crop, not only in summer when the drought may greatly reduce the supply from the pastures, but also to be cut and cured for winter use. Cattle, and in 
fact all kinds of stock, are very fond of it, while at the same time they thrive well on such fodder.

The varieties recommended for this purpose are those with the largest and most succulent leaves. Some farmers prefer some of the varieties of sweet corn for sowing in this way. The early varieties of dent corn, as well as Stowell's evergreen sweet corn, are excellent for this purpose, sowing from one to two bushels per acre, according to strength of the soil, etc.

There is a diversity of opinions among farmers relative to the comparative value of corn fodder thickly and thinly sown, some preferring as much as three bushels per acre, others less than a bushel. The thickly-seeded fodder does not grow as tall as the thin, and to first appearance the latter, to a superficial observer, would be pronounced the heavier product of the two. The more numerous stalks of the thick seeding, however, more than make up the deficiency in height, and when the product of an equal area of each is weighed, the thick seeding is always found to be the heavier of the two. On the other hand, the thick sowing bears no ears, and the stalks are more difficult to cure than those of the thin sowing, which bear small ears. The advocates of thin sowing claim that the quality thus produced more than compensates for the lack in weight when compared with the thick seeding. Howerer, it is always admissible that the fine hay from thickly-seeded mowing lands is superior to the coarse hay from poorly-seeded lands.

We should recommend rather thick seeding, the quantity to be regulated by the character of the soil, and to be from one to two bushels per acre, as previously given; poor soils requiring more seed than rich.

We believe it is conceded by the majority of farmers, that it is preferable to cut the corn for fodder before the ears get to any considerable size, and as a general practice, it is most valuable as a forage plant, when sown so thickly that the ears are not liable to form at all.

As a general rule, the earlier the corn is sown, the better; depending, of course, somewhat upon the season. In latitude as far south as Maryland, its growth is more rapid and luxuriant in May aud June than in July and August. Sowing in drills, from twelve to fifteen inches apart, will admit of after-culture. The crop should be worked twice with a cultivator to remove weeds and loosen the soil, and when in tassel, should be cut with a mowingmachine. It requires from forty-five to sixty days to ripen it into tassel, therefore can safely be sown up to the 15th of July. If the land is in good condition, it will yield twenty tons to the acre; and by the use of superphosphates, the crop can be doubled; but this is a matter subject to the will of the farmer. It should be well cured before stacking or storing in barns, as the thickly-sown stalks contain more juice, proportionately, than those cultivated in hills, and require considerable time for drying, so as to be perfectly preserved in large masses; otherwise it will mould and ferment, and, of course, become useless as animal food. By the ensilage process, so commonly practiced in France and other European countries, and which is now being practiced to a considerable extent in this country, the curing process of cornfodder would be entirely obviated, and thus an entire change be instituted in this department of agriculture, which experiments thus far have shown would be of immense advantage to the agricultural interests of the country.

Japan Clover, (Lespedeza striata.) This is a low, perennial plant, not rising much above the ground, but spreading widely on the surface. It belongs to the leguminous family of plants, which includes the common clover, bean, pea, etc. It has small trifolate leaves growing numerously upon the stems. The flowers are small, and are produced in the axils between the leaf and stem; the fruit a kind of flat pod. It seems to be peculiarly adapted to the Southern States, and proves a very valuable plant there for grazing. Sheep are very fond of it. It will grow on soils that are almost unfit for anything else, furnishing good pasture, and also supplies a good green crop for turning under and improving the land. Mr. Wall says, it should be sown in January or February, in the proportion of one bushel of seed 


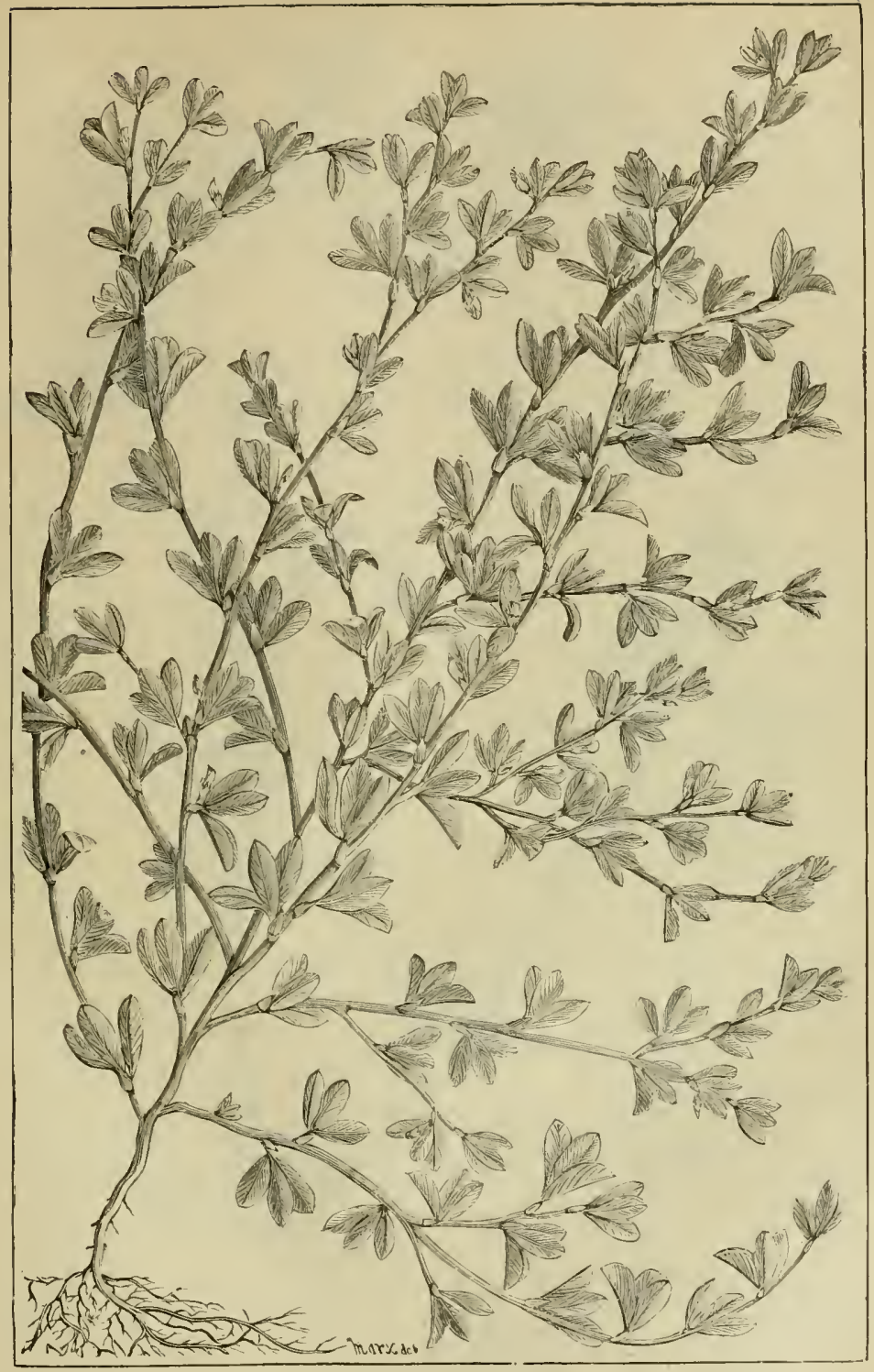

JAPAN CLOVER.

(Lespedeza striata.) 


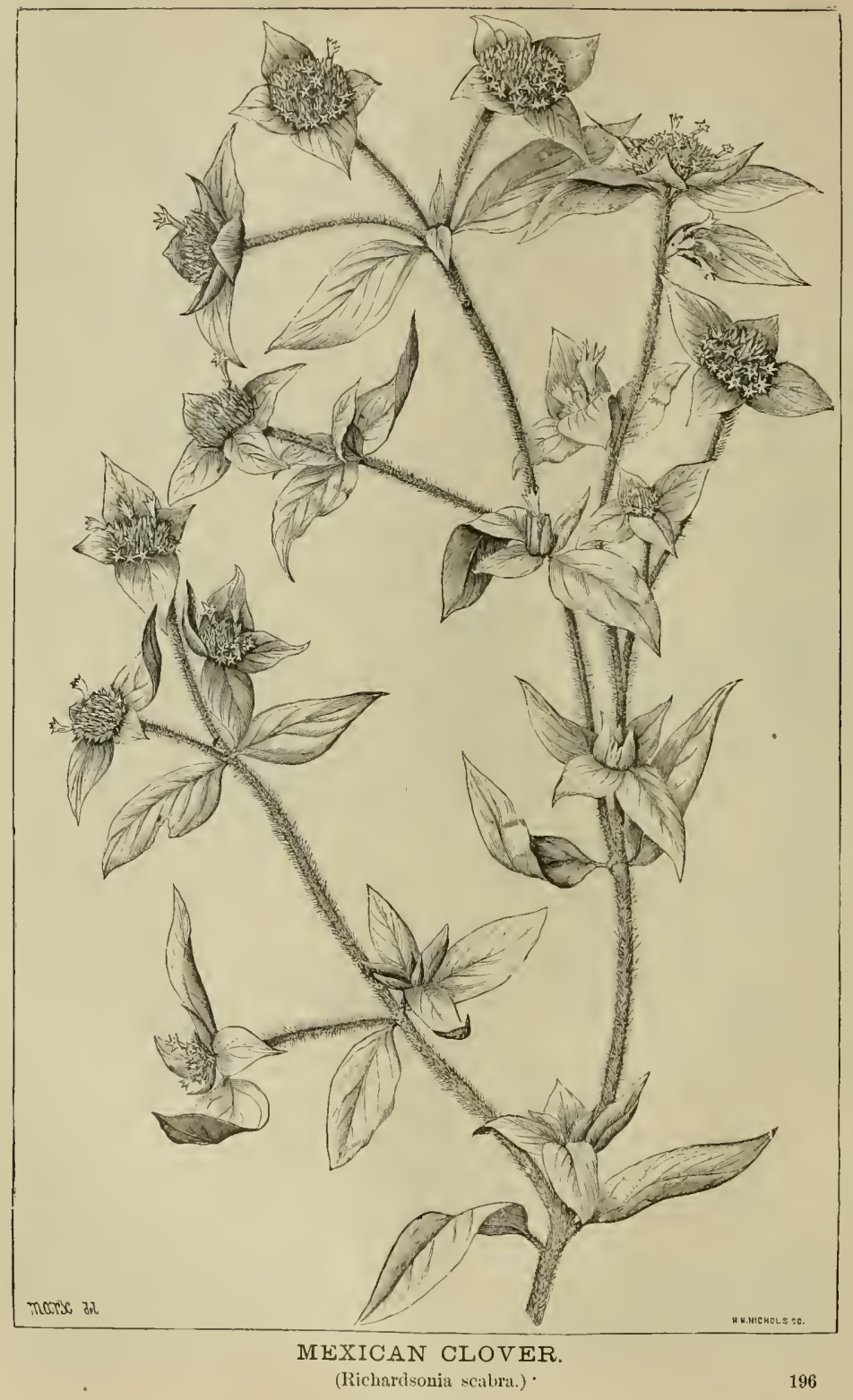


to ten acres, to secure a good start the first year. It is said to be an excellent renovator of old fields, and restores fertility in an incredibly short time.

Prof. Killebrew says concerning it: "About the year 1849 it was noticed in the vicinity of Charleston, S. C., the seeds having probably been brought from China or Japan in tea boxes. A short time afterwards it was discovered at a distance of forty miles from Charleston, and still later near Macon, Ga. It seems especially adapted to the Southern States, not flourishing above $36^{\circ}$, growing with great luxuriance on the poorest soils, and retaining vitahty in its roots in the severest droughts. It is said to be a fine plant for grazing, and, being perennial, needs no resowing and but little attention. On soils unfit for anything else it furnishes good pasturage, and supplies a heavy green crop for turning under and improving the land."

Mr. Samuel McRamsey, of Warren County, Tennessee, says: "This clover made its appearance in that locality in 1870. It is fast covering the whole country; it supplies much grazing from the first of August until frost. It is short, but very hard. Sheep are very fond of it, and cattle will eat it."

It is said to supply grazing from the first of July until frost, and to root out any other plant growing among it. It is found in the South from the Atlantic slope to the banks of the Mississippi. (See illustration.)

Mexican Clover, (Richardsonia scabra.) This is a native of Mexico and South America, and has only within a few years been naturalized in some parts of the South. It is an annual plant of the order Rubiacea, which includes coffee, cinchona, and ipecacuanha plants. It grows rapidly, under favorable circumstances, has succulent, spreading stems, which have small flowers in heads or clusters. The flowers are white and about half an inch long. The leaves are oblong, or elliptical, and from one to two inches long. In some parts of the South it is called Florida clover, in others, water parsley, Spanish clover, and bell fountain. It contains considerable water, and therefore is as difficult to cure as hay. It was formerly regarded as a troublesome weed by farmers, but is now valued for the purpose of green soiling.

Mr. Coleman, of Florida, says of it: "It is called in this locality Spanish clover. The tradition is that when the Spaniards evacuated Pensacola, this plant was discovered there by the cavalry horses feeding upon it eagerly. Five years ago, hearing of it, I procured some of the seed, and have been planting or cultivating it in my orange-grove from that time to the present, as a forage plant and vegetable fertilizer. I find it ample and sufficient. It graws on thin pine land, from four to six feet, branches and spreads in every direction, form. ing a thick matting and shade to the earth, and affords all the mulching my trees require. One hand can mow as much in a day as a horse will eat in a year; two days' sun will cure it ready for housing or stacking, and it makes a sweet, pleasant-flavored hay; horses and cattle both relish it. The bloom is white, always open in the morning and closed in the evening. Bees and all kinds of butterflies suck the bloom."

Dr. Rohmer, of Spring Hill College, Mobile, gives the following account of its medicinal value: "I was the first discoverer of the plant in Blakely, Baldwin County, Alabama, in 1858. When the war broke out I was appointed by the Confederate government botanist for the department of the gulf, for the purpose of having such medicinal plants collected as in my judgment might be rendered useful in the treatment of disease, and subsequently I received the appointment of superintendent of a laboratory established at Mobile for the manufacture of medicinal preparations for the use of the army. The idea suggested itself to me that the Richardsonia might be made to supersede the commercial ipecac, instead of using the Euphorbia corollata, as had been suggested. In the fall of 1863 I had the roots collected, dried, and powdered, and then delivered to the medical purveyor. From the testimony of surgeons, the Richardsonia answered every purpose when given in increased doses."

It is quite nutritious, and cattle, horses, and sheep are very fond of it. It is also valuable 
as a fertilizer when plowed under while green, and a great benefit to the farmers of the lower pine regions. The illustration which we give is a very correct representation of the plant, and the best we have seen.

Red Clover, (Trifolium pratense.) - Although not properly included in the family of grasses, red clover is one of the most valuable, economical, and nutritious of forage plants. It belongs to the family Leguminosae, which includes the larger portion of forage plants, commonly called artificial grasses, in distinction from the Gramineae, the true, and usually called natural grasses. The common red clover is the most valuable of all the varieties of clover, and is a native of some portions of Europe, growing in meadows and pastures. It was introduced into England about the year 1633, and has since that time been extensively cultivated in that country, where it forms the staple crop for soiling. We read of the natural grasses having been cultivated in this country some time previous to the year 1750 , especially the timothy, although clover was not cultivated to any extent until the early part of the eighteenth century, when, as is the common fate of all new departures under the sun, it met with great prejudice at first, which time alone could overcome.

The great value of the different clovers, as forage, was well known to the ancients, and were extensively cultivated by the early Romans. Although the nutritive quality of clover is relatively less than that of some of the grasses, yet in the average amount to the acre it is fully equal to that of any other forage crops, produced at the same expense, and, when properly cured, is a most valuable food for milch cows, calves, and sheep. It matures early, is raised cheaply, and is generally, in this country, a sure crop under proper treatment, producing two crops during the year, and is liable to few casualties or injury by destructive insects.

Although the common red clover, (Trifolium pratense,) is a biennial plant, and consequently two years' growth is all that should be expected of one seeding, yet Mr. J. S. Gould states, in his extended and valuable treatise on Forage Crops, that there is a variety of it, (Trifolium pratense perenne, ) that is truly perennial, though he never saw a whole field of this variety; that it is, however, frequently met with in permanent pastures, on soils naturally adapted to the growth of clover. It may be distinguished, in general, at a glance, by its deeper bluish-green color, the greater narrowness of the leaves, its more straggling growth, and the greater number, greater length, and greater stiffness of its hairs. The root of this variety differs considerably from the biennial kind; being somewhat creeping and very fibrous; whereas the perennial has an almost fusiform root. (Fusiform, spindle-shaped, as in the radish, having comparatively few fibers.) Yet, after all, these characters are sometimes interchangeable. He has seen undoubted perennial clover with a light green color, and very sparsely furnished with hairs, and has occasionally found equally undoubted biennial clover that was of a very dark color and quite abnormally hairy. In doubtful cases, the root is the best test.

The color of the flower varies very much in the species and in the variety; in general, the perennial has much the deepest red, but both kinds are met with, in which the color of the flower is of a pale pink, and rarely of a pure white. The heads of the perennial are generally less crowded with flowers, the flower stalks more slender, and with a greater tendency to curve. When young, the flower head presents a much more woolly appearance than the biennial.

In the present condition of our seed markets, he thinks that the seeds of this variety could not be obtained with any certainty; but when farmers become well acquainted with the distinction between them, an imperative demand for reliable seeds will arise, which will then be met by seedsmen. Mr. Sinclair says that this variety is found in the greatest abundance in Lincolnshire, and recommends that the seeds should be first selected from thence, and then be cultivated until the demand is fully supplied. It flourishes better on clayey or peaty soils than the common red clover, or Trifolium pratense proper. 
There are two varieties of red clover, known among farmers as "the large" and "small clover." The large is considered less valuable for hay or pastures, and is little cultivated, as it yields but a single crop of hay in a season; but where wanted for manure only it is often preferred to the smaller; on account of its heavy growth.

Clover has been used as human food for generations by some tribes of Indians. The Digger Indians of California eat it raw, and also cook it by placing a thick layer of green clover between stones that had been previously heated. When young onions or chives and grasshoppers are mingled with the clover, the dish is considered a great luxury! The A paches make what to them is a very savory and palatable dish, by mixing together clover, pigweed, and dandelions in a vessel, which is afterward filled with water. Heated stones are then put into the vessel, and taken out as soon as their surplus heat is imparted to the water; this process being repeated until the mass is sufficiently cooked.

Cultivation of Clover.- - Red clover will grow on almost all soils, except those that are too wet. It will, however, not prove a very profitable crop on very light, sandy soils. When it is desirable to enrich such soils by green manuring, it is well to sow some other crop for the purpose. Clover is a lime plant, and the soils best adapted to its production are tenacious or stiff loams.

The analysis of Prof. Way found 35.39 per cent. of lime in the inorganic constituents of red clover, that of Boussingault 32.80 per cent., while the term "clover soils" is now almost universally employed to denote a tenacious loam containing more or less lime in its composition.

A great advantage in its cultivation, aside from its superior nutritive properties, consists in its rapid and luxuriant growth, but a comparatively short time being required from the time of sowing the seed before it yields an abundant crop, that is greatly relished by stock of all kinds.

Mr. Gould says:- "The best soils for red clover are the best wheat soils; where the one will grow luxuriantly, there will the other grow luxuriantly also. There are loams and friable clays with some admixtures of calcareous matters to which it is well adapted, though it will grow in tolerably stiff clays, if they are not too wet. In thin, sandy soils it is much more bable to freeze out than in stiffer ones, and there seems also to be less of its natural specific food."

The growth of clover is known to differ very widely upon soils of the same geologic formation, and to be equally luxuriant upon soils of very different geologic characters. The richest clover region of Ohio is in a belt running east and west near the latitude 41 . $^{\circ}$

The best portion of this belt is in Monroe County, upon the Hamilton shales, but it grows in equal perfection further west, on the cliff and carniferous limestone of Marion and Hancock Counties.

One of the finest clover regions of New York is on the Onondaga limestone, which is precisely the same as the Helderberg limestone, being the same deposit; yet the clover growing on the same rock around the Helderberg mountains is much less luxuriant. Some of the finest clover that can be grown is found on the Moscow shales in the town of Romulus.

Clover grows better when it is sown in connection with some other cultivated plant which will give it shade during the early period of its growth; hence, it is generally sown on winter wheat or rye in the spring. The best way of doing this is to sow it on a freshly fallen snow in the latter part of March, which soon melts, and thus leaves the seed to sink into the ground as deep as is desirable without any other care or attention. If it is not convenient to sow it on winter grain, it is put in with oats or barley in the spring, the latter being decidedly preferable.

In the latter case care must be taken not to bury the seeds too deeply, as they will be assuredly lost, if such is the case. Mr. Stirling, of Glenbervie, in Scotland, experimented 
very carefully upon this point with the following results, which are embraced in a report to the Highland Society:-

"The seeds weighed sixty-four pounds to the bushel. There were 16,000 seeds in an ounce (the number of seeds in a pound may of course be found by multiplying this number by sixteen, and if the number of seeds in a bushel is required it may be found by multiplying the latter product by sixty-four). From 0 to $\frac{1}{2}$ inch in depth the greatest number of seeds sprouted. When covered from an inch and a quarter to one inch and a half only half of the seeds came up; and when they were covered two inches deep none of them came through the surface. When the seeds cannot be sown on a snow on winter wheat or rye, as above recommended, it will be advisable to stir up the wheat with a light harrow before sowing it, as it germinates so much more freely in fresh-stirred soil. All that will then be necessary for this, or in case of sowing with spring crops, is to go orer the seeds with a light roller.

"In nothing is there a greater difference of opinion and of practice among farmers than with regard to the amount of seed required for an acre. Some sow only four pounds, and some go as high as sixteen pounds. I have seen crops of clorer sufficiently large to satisfy any farmer grown from four pounds of seed; but in this case the circumstances were all favorable; the seeds were all full of vitality; the soil was exactly adapted to the growth of clover; the temperature, at the time of sowing, and the succession of showers after it, were exactly adapted to promote its growth, and it $w$ as not infested with injurious insects.

"But such a combination of favorable circumstances is very rare, and to provide for con. tingencies it will always be safe, even on good soils, to sow eight pounds to the acre, and in proportion to the poverty of the soil we may go up to sixteen pounds. In all cases the greatest amount of seed must be sown on the poorest soils."

The best time to sow clover seed in New Eugland, is in the spring. It is frequently sown upon the late snows in March or April, by the gradual melting of which it finds its way to the soil, where the moisture soon causes it to germinate and take root.

Clover is sometimes sown broadcast in August or September, but the best results are usually attained, other couditions being favorable, when sown in the early spring. It may be sown with grains, cultivated grasses, or by itself. The quantity of seed required per acre varies according to the kind of soil. Clay soils require more than loams. The usual amount for well-prepared loams is from eight to ten pounds per acre; for heavy clays, from fourteen to sixteen pounds. Then sown with grasses, the proportionate quantity of clorer-seed is from a third to one-half of the amount sown. The larger the amount of seed, as a general rule, with any of the grasses or forage plants, the greater the number of stalks; consequently the finer the quality of the product.

The sowing should be liberal, but not so large as to cause the crowding of plants, which will prevent as heary and strong growth as would otherwise be secured. The corering, as has been previously stated, should be very slight. When sown early in the spring on well. pulverized soil, it will germinate freely without the use of the harrow. Rolling the field will, howerer, press the seed into the soil, and cause it to germinate quicker than without this pro. cess. Gypsum, sown broadcast at the rate of from one to three or four bushels per acre, is very beneficial to the crop, when the soil on which it is grown is suited to its use. This should be done in the spring, just as the leaves are developed.

When grown too frequently on the same soil, land will sometimes become incapable of producing a good crop, and will become what is commonly termed by farmers, "clover-sick." In such cases other crops should follow for four or five years, for the benefit of a change.

It is stated by good authority that little interest had been taken in the cultivation of clover in this country until the wonderful effects of gypsum in stimulating its growth was discovered.

Dr. Benjamin Franklin seems to have been among the first to recognize in this country 
this wonderful power in gypsum, which had been first discovered as a fertilizing agent by a Protestant pastor by the name of Nayer, in the principal city of Hohenlohe, and was the first to call the attention of farmers here to its fertilizing power. Franklin simply traced the letters of his name in powdered gypsum upon a patch of clover, and in a short time his name could be easily read by all the passers-by. It stood up three times as high as the surrounding plants, and was equally distinguished by the dark-green stems and leaves, and the vivid red blos. soms, from the unplastered portion, which was much smaller and less luxuriant, the stems and leaves of a yellowish green, while the blossoms were of a pale pink hue, some of them degenerating to an almost white color. Great multitudes flocked to see the miraculous handwriting on the field, and sometimes the roads around it resembled a county fair from the number of vehicles and horses that were collected. The doctor soon afterwards published an account of the experiment, written in his most attractive manner, that was read by almost every one, and thus it was diffused over the whole country. Every one was talking about it, and a few tried the experiment, and from that time the culture of clover has been extending even to the present day.

Time of Cutting, and Method of Curing Clover.-Of course the time of cutting clovers, as well as grass, differs with different latitudes, and can only be determined by the condition of the crop. The opinion formerly prevailed that this crop should be cut after hav. ing fully blossomed and assumed a brownish hue, or as soon as the earliest heads showed signs of ripening; but it has been ascertained, by many and repeated experiments, that the prope: time for cutting it is just as it is coming into full bloom, as it then has the maximum amount of nutritive matter in a condition best fitted for assimilation.

The amount of loss sustained by cutting clover at improper times was determined by Dr. Voelcker in his admirable experiments made at the agricultural college at Cirencester.

The plot upon which the experiments were tried was a good calcareous clay soil, and was subdivided into plots, each of which was sixteen and a half feet square. Plot No. 1 was cut six times, at intervals of a fortuight. The total amount yielded per acre was five tons, eight cwt. and sixty-four pounds of green clover, and one ton, four cwt. and seventy-six pounds of hay dried, and contained 388.8 pounds of nitrogenous, and 1,646.4 of non-nitrogenous matters. Plot No. 2 was cut a fortnight later, and so each successive plot was cut a fortnight after the preceding one. The clover began to blossom on the twentieth of May. Plot No. 7 was cut June sixteenth, and again on the second of July. These two cuttings yielded eleven tons, seventeen cwt. and sixteen pounds of fresh grass, and three tons, thirteen cwt. and ten pounds of dry hay, 780 pounds of nitrogenous, and 5,680 pounds of non-nitrogenous matters. This was the maximum yield; each successive plot had given a richer yield up to this point. After this, the amount steadily receded, until the last cutting, which was on the twenty-eighth of July, when the fresh produce was four tons, ten cwt.; the dry hay, two tons, four cwt. and ninety pounds. The loss between the cutting of June sixteenth and that of July twenty-eighth was, of dry hay, one ton nine cwt. and ten pounds; of nitrogenous matters, of 480.8 pounds, and of non-nitrogenous matters, $1,315.2$ pounds. The steady decrease of nitrogen is shown in the following table:-

\begin{tabular}{|c|c|c|c|c|c|c|c|c|c|c|}
\hline & & & & & & & & & & $\begin{array}{l}\text { Per cent of } \\
\text { Nitrogen. }\end{array}$ \\
\hline June 16 , & . & . & • & - & . & . & • & . & . & 1.81 \\
\hline June 23, & . & . & . & . & . & . & . & . & . & 1.49 \\
\hline June 30 , & . & . & . & . & . & . & . & . & . & 1.32 \\
\hline July 7 , & . & . & . & . & . & . & . & . & . & 1.27 \\
\hline July 18 , & . & . & . & . & . & . & . & . & . & 1.06 \\
\hline July 28, & . & . & . & . & . & . & . & . & 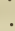 & .97 \\
\hline
\end{tabular}

The above figures speak for themselves, and require no comment. When farmers gen. erally understand better what the real losses in nutriment are which are occasioned by cut- 
ting grass or any of the forage crops at improper seasons, they will be less carelsss and indifferent with respect to it.

In actual practice, the evil effects of rain upon clover, and of too early or too late cutting, are more apparent than would appear from the results of a chemical analysis. When properly cured, animals eat the hay with avidity, increase in flesh, are healthy, and give milk in abundance, but when fed on bay cut too late, or imperfectly cured, they cease to make fat and diminish in their milk. It is conceded by the best authority that in order to secure the perfect curing of clover hay, two things must necessarily be kept in view: first, the clover must be cut when it contains the greatest amount of nutriment, and second, it must have the water that is contained in its stalks and leaves evaporated as rapidly as possible. The first will be accomplished by cutting it when in full bloom; the second, by not cutting it until every vestige of dew and rain has been dried off from it. After it has been wilted by two or three hours sunshine, it should be tedded with great care, or the leaves will be almost entirely whipped off, and the stalks bruised, which would be a great injury to the bay; those tedders being the best that do not strike with great violence, but lift it from the ground and toss it into the air. It must be raked and cocked so as to shed rain before the dew begins to fall, and if there is a prospect of rain, it should be capped. The next day the cocks must be opened as soon as the sun becomes warm, and be deposited in the barn before night.

Well made clover hay furnishes one of the most nutritious articles of food for stock, while that which is badly cured is about as poor diet for animals as could well be found.

When clover is simply dried in the sun without bruising the tissues or beating off the waxy covering that envelopes it, it is as nutritious as it is when green, since in such a process it loses nothing in curing, but simply water.

When clover is cut, it contains a considerable amount of sugar, gum, mucilage, albuminous and some other soluble compounds, all of which are liable to be washed away by rains or dews when exposed to their action.

All feeders of stock have invariably found, that while domestic animals will fatten on well-made, bright clover hay, they remain stationary or go backwards on clover hay that has been injured by exposure to dews and rains.

Dr. Voelcker ascertained the composition of clover hay that produced such unsatisfactory results in feeding, by analysis, and found that it contained:-

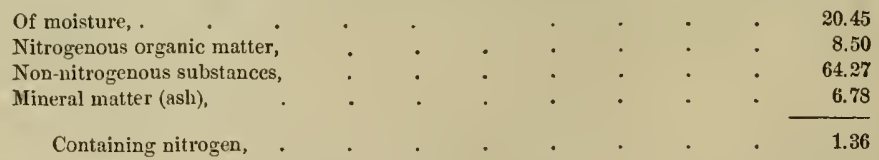

The composition of well-made clover hay, as ascertained by Professor Way, is as follows:-

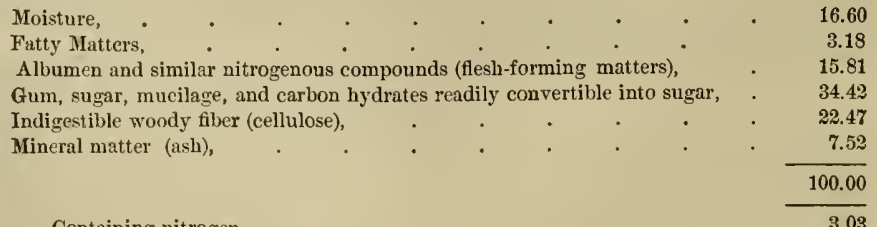

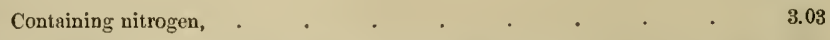

On comparing the above figures, it will be seen that exposure to rain caused, in this experiment, a loss of 7.31 per cent. of nitrogenous substance, or, we may say, that nearly one-half of the nitrogenous matters are wholly lost. Clover hay that is badly cured in the 
field will also lose more weight in the stack than well-cured hay, as well as more of its nutritive qualities, the loss being occasioned by the conversion of the sugar and other nutritive - compounds into those which are innutritious.

Conditions Farorable to Fermentation in Clover Hay.-Air is always essential at the beginning of the process of fermentation, but after it has been once commenced, this process may be continued even after the air has been subsequently excluded. To illustrate this principle, the reason why grapes will not ferment until the juice has been expressed, or the skin broken, is because the air is excluded by the close, tight covering or skin which envelops the pulp.

Fermentation, which is that decomposition or decay which acts to all appearance sponta. neously, upon animal and vegetable substances, generating leat and throwing off gas in the process, is caused by a microscopic fungi; hence ferments always contain the germs of fungi which are more and more fully developed during the process. Moisture and heat will cause any organic body, not living, to ferment. From sixty-five to eighty degrees Fahrenheit is a temperature most favorable to rapid fermentation. When reduced much below this, it is slow in its operation.

Fermentation is also largely affected by the amount of water present. In the process of fermentation, the elements of sugar and other carbonaceous compounds are re-arranged; the carbon uniting with the hydrogen to form alcohol, and with oxygen to form carbonic acid. It is a fact well known to chemists, that if one part of sugar is dissolved in three or four parts of water, with the addition of yeast, fermentation will not occur even in a warm room ; but if an equal weight of water be added to the solution, fermentation will soon commence; and that if the water is increased to the extent that the proportionate quantities are as one of sugar to sixteen or twenty of water, it will either ferment very slowly or turn acid. This proves that too large or too small a proportion of water is unfavorable to fermentation. Applying this principle to the curing of hay, and we can readily understand the reason why grass or clover freshly cut does not ferment as readily as that which is partially cured. When grass is freshly cut, its vitahity has not wholly left it, consequently fermentation is deferred. It also contains too large a proportion of water compared with that of the sugar and gum of its composition, and the albuminoids have not at this period begun to change into ferments.

But after the evaporation of the water contained in the leaves and stalks of the clover or grass has continued for a time, the relations of the different combinations become such as to favor fermentation, and if at this stage the drying process is arrested by any means, such as cloudy or rainy weather, the hay will undergo a process of fermentation in the field which destroys its nutritive properties, and leaves little else than the indigestihle woody fibre.

If the former condition (that of drying) be continued without arrest. long enough to exhaust the amount of water contained in the clover, beyond the point where fermentation can take place, the hay remains exempt from fermentation and is nutritious.

The bruising of hay during the curing process has a tendency to produce fermentation, therefore the less it is stirred or tedded beyond the point of sufficient exposure to air and sunshine, the better.

How Fermentation May be Prevented in the Mow.-Fermentation and mold are often prevented in the mow by mixing, as evenly as possible, from ten to twenty quarts of salt per ton with the hay when storing it, which can be done by alternate layers of hay and salt. Although salt has a tendency to make hay moist, it is a preserving agent, and also renders it more palatable to stock. Another method employed by farmers with success, is that of mixing alternate layers of dry grass, hay or straw with the clover, which absorbs the mo1sture of the clover and thus prevents fermentation, while the juices of the clover, permeating the straw or hay thus placed in layers between, improve the flavor of the latter for fodder. 
Hay that is packed compactly in the mow, excluding the arr as far as possible, is less liable to ferment than where this precaution is not observed.

Cultivating Clover Seed.-Some farmers pasture the clover-field till June, and then leave it to mature a full crop of seed; others mow it early, and leave the second crop to mature the seed; but when this is done, the first cutting should be done before blossoming and seed-forming, as the plant will become too much exhausted to produce a second crop of seed, the seed-forming substance having been used up, in a measure, in the first crop. It is of more importance to place more value upon the seed to be raised, than the hay first secured, as this will prove most profitable in the end. The early mowing has the benefit of removing the weeds, while the second growth of clover will be so rapid that the weeds will be smothered and the clover is then saved comparatively free from other seeds.

An application of plaster to the clover-field in the spring will secure a better crop of seed when matured, while its application to the field freshly mowed, after its first crop, will make the second growth very luxuriant and rank in hay, to the detriment of the seed-crop.

Harvesting Clover Seed.-The best time for cutting clover for seed is when about three-fourths of the heads are brown; at this time there is less liability to loss from shelling while handling; besides, clover heads will ripen considerably after cutting, and those beads not

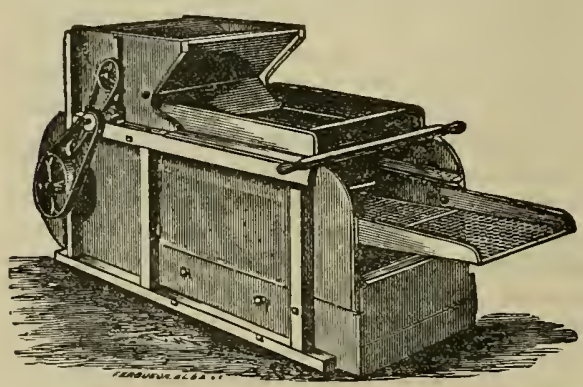

CLOVER HULLER AND CLEANER.

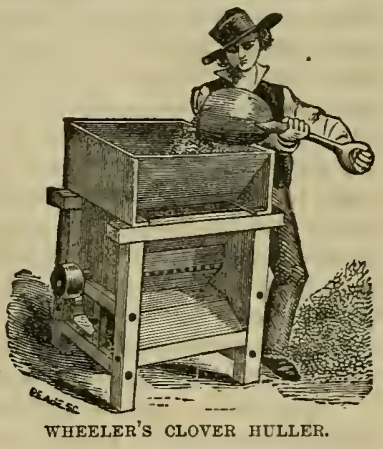

sufficiently advanced, at this period of ripening process, would be of but little value for seed production if allowed to stand till dead-ripe. The hay is also of greater value for fodder if cut at this period than later. It can be mowed with a scythe or mowing-machine, but what is better still is a reaper, arranged as for reaping grain, with a board at the back to retain a large amount of the clover, which can be pitched off in heaps.

Some recommend cutting quite high, as it saves time in curing and labor in handling, and leaves the dryer and coarser portions of the stalk upon the field. It should be well-cured and handled with care to prevent loss of heads; still, it is less liable to injury from lack of curing than either hay or grain. When thoroughly dried, the seed can be threshed out with a threshing-machine, or light flails, which can best be done in extreme cold weather, when there is no dampness in the seed or air. The calyx of the clovers is very firmly attached to the seed, often rendering the separation difficult. A clover-huller, of which there are several kinds, is a good machine for this purpose, in rubbing the seeds out from the chaff, by passing through it several times, until the separation is complete. The first two cuts of clover machines represent implements manufactured by the Wheeler \& Melick Company, Albany, N. Y. The first is somewhat similar to a grain thresher and cleaner, except that it is under instead of overshot, and fed from a hopper over the cylinder, instead of in front of the cylin- 
der. Over the cylinder, in the hopper, is a revolving shaft, with arms, to prevent clogging and to stir up the seed and chaff. The cleaned seed is discharged in a large drawer underneath the machine to be put into bags or barrels. The unthreshed seed-bolls fall at the back end of the machine, and are to be put into the hopper to pass through the machine again. It can be run with a two-horse railway, or three or four lorses to a sweep-power, and is said to clean from ten to twenty bushels of clover-seed per day.

The second represents a compact and simple machine which can be used with one horse. A fan-mill will be required with this machine, as it does not separate the seed from the chaff.

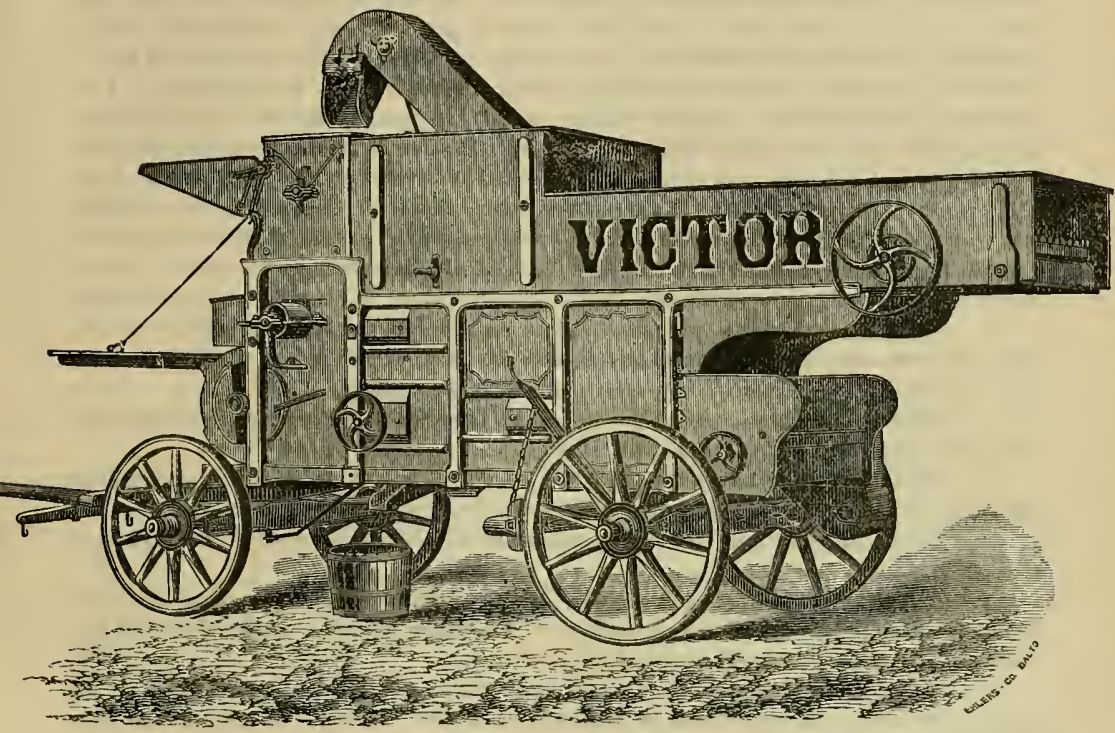

VIOTOR OLOVER MACHINE.

The Victor Clover Machine, manufactured by the Hagerstown Agricultural Implement Manufacturing Company, of Hagerstown, Maryland, has an under-shot six-beater open cylinder, which has important adrantages over the closed or drum cylinder, and over the over-shot cy!inder. This cylinder, being open, the dust is taken through into the separator, where it is confined and carried out at the rear end of the machinery.

The seed is rubbed out by the diminishing of the space between the front edges of the rubbers and the corner of their backs as they pass each other with their fluted sides by the revolution of the cylinder. The process is one, as is readily seen, that will rub out the seed very effectually. It is said by those who have used them to thresh very rapidly, and to do the work very satisfactorily, and can be run with either horse or steam power.

Clover as a Fertilizer.-Clover is not only extremely valuable as a forage plant, but also as a fertilizer of any soil on which it grows. It is often stated that the introduction of clover into England produced an entire revolution in her agriculture; we know its import. ance to the agricultural interests of our own country are beyond estimation, and we wonder how our ancestors could have gotten on in farming without it. 
Clover acts as a fertilizer in various ways. Its long roots not only penetrate the soil, loosening it and admitting the air, thus improving its mechanical condition, but also serve to fix in the soil those elements important to enrich it. When these roots decay, they contribute their substance to further increase the fertilizing properties of the land. The heavy foliage of clover also serves to choke out the weeds that would be liable to spring up on newly seeded land. It also heavily shades the surface, which tends to increase the fertility of the soil by this means. It has been found that while clover takes out of a soil probably as much of some of the elements of fertility as many of the farn crops, even more than wheat or other cereals, it leaves in it a much larger portion of nitrogen and nitrogenous elements than any other crop. And it is a fact worthy of note, that a crop of grain will grow better after a crop of clover than it will after any other crop. The amount of nitrogen left in the soil by a crop of clover was found (as we have previously stated), by the careful investigations of Prof. Voelcker, - who is one of the best antliorities on agricultural chemisty the world has ever known, - to be equal to two and a half to three tons per acre. He also found, that on soils where clover had been grown, not only is all that nitrogen collected and stored up in the soil by the clover, but it is left when spring returns in a much better condition to produce a grain crop than any other fertilizer that could be applied. T'hese investigations were made at different depths of the soil, taking six inches at a time until the depth of eighteon inclies was reached. From the thorough investigations thus made, Prof. Voelcker arrived at the following conclusions:

1. That a good crop of clover removes from the soil more potash, more phosphoric acid, more lime, and other mineral matters which enter into the composition of the ashes of our cultivated crops, than any other crop usually grown in the country.

2. There is fully three times as much nitrogen in a crop of clover as in the average produce of the grain and straw of wheat per acre.

3. Notwithstanding the large amount of nitrogenous matter, and of the ash constituents of plants in the produce of an acre, clover is an excellent preparatory crop for wheat.

4. During the growth of clover, a large amount of nitrogenons matter accumulates in the soil.

5. This accumulation, which is greatest in the surface-soil, is due to decaying leaves dropped during the growth of clover, and to an abundance of roots, containing, when dry, from one and three-quarters to two per cent. of nitrogen.

6. The clover-roots are stronger and more numerous, and more leaves fall on the ground, when clover is grown for seed, than when it is mown for hay. In consequence, more nitrogen is left after clover-seed than after hay, which accounts for wheat yielding a better crop after clover-seed than after hay.

7. The development of roots being checked when the produce in a green condition is fed off by sheep, in all probability leaves still less nitrogenous matter in the soil than when clover is allowed to get riper, and is mown for hay. Notwithstanding the return of the produce in the sheep-excrements, wheat is generally stronger, and yields better, after clover mown for hay, than when the clover is fed off green by sheep.

8. The nitrogenous matters in the clover-remains, on their gradual decay, are finally transformed into nitrates, thus affording a continuous source of food, on which cereal crops specially delight to grow.

It is thus seen that clover is one of the best fertilizers known for preceding almost any crop. Clover is also a most valuable agent in restoring the fertility of exhausted lands by being plowed under when green. The leaves gather food from the atmosphere, and this food it stores up in its roots and stems, which by their decomposition afford a peculiar nitrogenous element to crops. The texture of many soils is also greatly improved by the use of clover as a green manure. This subject of manuring with green crops will be found more fully treated in connection witl Fertilizers, and hence does not require a further consideraation here. 
" Clover Sickness." - When soils have for a series of years borne luxuriant crops of clover, it sometimes happens that they seem suddenly to be unable to continue its production longer, which is evinced by the plant being found stunted in growth and scattered here and there in small patches, surrounded by the bare soil. Sometimes the plant has a yellowish tinge and is covered with brown spots, which are found to be a minute cryptogamic parasite. When these indications occur, the land is said to be "clover sick."

The cause of this disease is not fully understood, various opinions being entertained re. specting it. It is the general opinion, however, that one of the chief causes of the evil is the exhaustion from the soil, by the continual growth of the clover crop, of some of the elements which are essential to its growth.

Dr. Grouven, after a careful study of this subject in Germany, arrived at the conclusion that the chief cause of the clover disease is attributable to a change in the chemical composition of the plant, which change is caused by an altered condition of the soil. The difference between the composition of the ash of liealthy and diseased clover is seen by the following analysis, which shows in the latter a remarkable deficiency of potash and phosphoric acid:

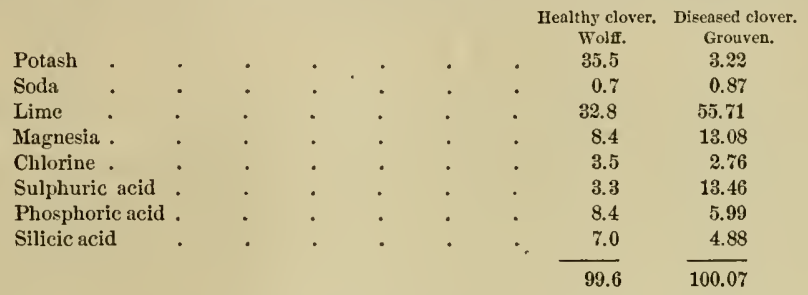

Prof. Buckman refers, in connection with this subject, to the fact that our clover is a derivative plant that has been forced in growth until it is now many times larger than the wild plant from which it sprung, and that this growth demands the continuance of those elements from the soil which were originally the cause of its enlarged growth; hence the lack of a sufficient supply of those elements nay be one of the causes of the disease. Insects injurious to clover are constantly increasing, and this may be one of the causes of the evil. It has been found that one of the best remedies in this disease yet discovered is paring and burning the surface of the soil, which destroy the eggs and larvae of insects injurions to it, and also all the undecomposed vegetable matter that it may contain. A rotation with other crops, for a sufficient length of time to change the properties of some of the constituents of the soil, will also prove highly beneficial.

Alsike Clover, (Trifolium hybridum.)-This plant is a native of Sweden, and is often known by the name of "Swedish clover." It is said to have been brought from Sweden to Scotland by a professional land-drainer of Edinburgh, Mr. George Stephens, and sown in the Meadowbank nursery on the 17th of April. It blossomed on the 25th of August, and the seeds ripened in the early part of the following October, when the plant was twenty-two inches high. In the year 1835, the plants were in full flower on the 18 th of June and ripened their seed in the early part of August, when the plant was about two feet high. From thence it spread gradually into England and was introduced into the United States about the year 1855 , where in some portions it became quite popular. It is a perennial, and endures for a longer or shorter time, according to the character of the soil; on good soils it will do well for four or five years. It is more like the white clover than the red, in its growth and blossom, and is considered a valuable addition to the forage crops. The color of the flower is white with pink markings when fresh blossomed, but which fade out to white before dropping. The general appearance of the stem is more smooth than that of the red clover. The 
lower part of the leaf is sharply serrate, the serrations diminishing in running upwards, and the upper portion is almost entire. The leaf is of uniform light color, having no pale spots like the red clover, and grows a darker green with age. It will do well in many soils where the red clover will not grow luxuriantly, and makes a good substitute for it.

A stiff, clayey soil is best adapted to its growth, particularly a marly clay that is quite moist, and it is thought that it thrives best in such soils as the red-top grass most prefers. It will also do moderately well on cultiveted fens and marshes, where the red clover would scarcely grow at all. It is more slender than red clover, as well as finer in its growth, also more nutritious and sweet. Cattle and other stock are exceedingly fond of it. It is more hardy than any of the other varieties of clover, and is rarely winter-killed. On such soils as heave in frosty weather, so that red clover would be entirely killed, the Alsike will do very well. It yields only one crop in a season, and of less weight per acre than red clover, although growing about the same height, but the hay is of better quality, hence what it falls short in weight, when compared with red clover, it seems to make up in nutrition. When sown on a dry or exposed knoll, and cattle are allowed to graze it too closely, it will be liable to be in. jured and doubtless will not make its appearance the next year; but when sown on soils fairly suited to its growth, it will bring good returns for at least four or five years.

As it throws out many stalks from one root, it should be sown thin in order that the roots and branches may have sufficient room for growth, and the roots to strike deep into the sub. soil. It bears cropping well, and is admirably adapted to pasturage.

It continues longer in condition to be cut than any other forage plant, and throws a large number of heads from one stalk, coming into bloom successively for four or five weeks. Even when the heads are nearly ripe, the stalks and leares are still in condition to make very good hay. The seed crop will make very excellent fodder when the seed has been threshed out. The seed is about half the size of that of the red clover, consequently from five to seven pounds of seed to the acre is sufficient in sowing. The seed is somewhat oval in form, and of a dark color, nearly violet when ripe; when of a yellow green they are not ripe, and will -not germinate. It is one of the best plants known for bees, as the blossoms are peculiarly sweet. Mr. Gould says of it:-

"I believe it to be the best honey-plant in the world. Much attention has been given to the habits of the honey-bee in regard to its choice of flowers, and the quantity and quality of the honey stored in different portions of the season. We often see a new plant recom. mended for cultivation specially for the bees. I think now that bee-keepers generally agree that the surplus honey is all, or very nearly all, stored during the bloom of the white clover. From all other flowers, including buckwheat, but little more is gathered than is consumed daily. I have for several years had on my place a plat of Alsike clover, besides one of the white. I have often called the attention of people to the working bees on the plats, and it was always easy to see that the Alsike had the greater attraction. Equal areas usually show twice the number of bees on the Alsike. The blooming season is longer on the Alsike; the heads are also more numerous. A field of Alsike clover is the best patronized bee-pasture that I have ever seen, exceeding that of an orchard in full bloom. I am sure that in a neighborhood of Alsike clover-fields, bee-keepers would not be searching for other plants for their use.

The Alsike resembles the white clover in its crceping habits, hence it is better to sow it with some stiff grass, such as orchard grass, when intended to cut it for hay, as it will then grow upright, and is ready to be cut at the same time with the orchard grass.

In comparing Alsike with red clover, we must not forget that the former grows very little after mowing, and that it never yields a second crop.

Alsike clover-seed is more easily threshed than red clover. When threshed together, the Alsike always comes out first from the pods, and, therefore, when it can be avoided, it is better not to thresh them together, as the more threshing it receives the more is the straw 
injured for fodder. The heads of the Alsike are, moreover, much more likely to drop off than those of red clover; hence, when it is gathered for seed, still greater care should be taken to avoid the loss of the seed. When ripe, it should always be mowed either early in the morning or late in the evening, when wet with dew. A canvas lining should be used in the carts, so that the seed-pods may not be lost.

The heads are sometimes threshed in the ordinary machines, but the seeds are so small that very many of them are blown away and lost. It is, therefore, better to thresh them with a flail, and farmers who raise their own seed almost invariably do so."

The following is Dr. Voelcker's analysis of Alsike clover:-

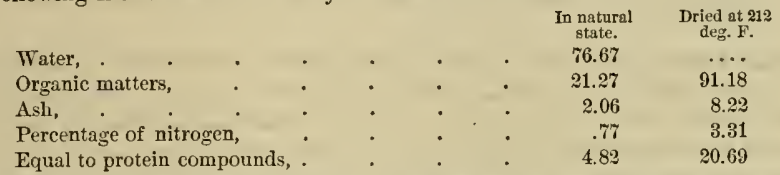

As compared with red clover, it has 3.31 pounds more of solid matter to the hundred pounds in the green state, 1.95 pounds more of protein substances, and 0.31 pounds more of nitrogen.

White Clover, (Trifolium repens,) which is well known in some localities as Dutch clover, honeysuckie, trefoil, and white creeping clover, is said to be also the shamrock of the modern Irish. It is indigenous to both Europe and America, and is widely diffused over both countries. It is so natural to our soil that it is rarely sown, except on lawns, to which it is admirably adapted. Its principal value is as a pasture grass, as its dwarf character ren. ders it less adapted to mowing than the clovers of higher growth. Sometimes it is seen fully two feet high, though this is very rare, its usual growth not being over ten inches; while its creeping branches will often lie so flat upon the ground as to be scarcely recognized without particular attention. It is very hardy, nutritious, and self-propagating, springing up almost everywhere, in all kinds of soils and climates. It grows very rapidly after being grazed by the cattle, and overcomes weeds to the extent that it usually gains the mastery; while it is highly valued by both butter and cheese makers for imparting a peculiarly rich and sweet flavor. Some dairymen go so far in their assertions as to say that good butter cannot be made where this plant is not present in the pastures.

It is said that where it is sown, the length of time that it will remain in the soil will vary from three to thirty years, depending mainly upon the nature of the soil. Close feeding by sheep, as they gnaw the stems down to the root, is said to exterminate it sooner than anything else. In loose soils, they often tear the roots out of the ground in feeding. Bees are very fond of it in procuring honey, of which it makes the whitest and best quality.

When sown forseed, one and a half or two pounds per acre will be sufficient when the soil is adapted to it. In this case the land should be very rich, otherwise it will not grow sufficiently high to secure the heads well in mowing. It is said that in Germany the women and children are employed to cut off the heads with scissors in securing seed.

Crimson Clorer, (Trifolium incarnalum,) is a native of Italy, and has long been cultivated in France and other portions of Southern Eưrope with success. It was cultivated in Great Britain for many years by horticulturists as an ornamental flower, often as a border annual, until it began to be cultivated in fields, its long head of bright scarlet flowers making it very attractive for this purpose. Its efflorescence being centrifugal, it begins blossoming at the base of the head, and requires about a week to reach the top, and remains in blossom longer than any other kind of clover. It blossoms about two months aiter sowing. The seeds are of a salmon color. It began to be cultivated as a forage plant about the year 1821 . Though cultivated with success in Southern Europe, it has never received much attention in 
this country. Several years ago some attempts were made to introduce it here, but rere not aitended with such success as to commend it to agriculturists generally. In England it is culti. vated by sowing either broadcast or in drills. If broadcast, usually eighteen or twenty pounds are required per acre; if in drills, from eight to twelve inches apart, twelve or fifteen pounds per acre is sufficient. It is usually sown on improved stubble-land as soon as a grain crop is removed, and harrowed just enough to slightly cover the seed with fresh earth. It grows rapidly in the spring, and is much valued for food for young lambs, and later in the season for horses and cattle. It can be cut for hay on the first of June, thus leaving the land clear for wheat. It is not equal to the common red clover, either in quantity and quality of hay, or as a fertilizer.

Zigzag Clover, (Trifolium medium.)-This plant is often called by farmers, in some localities, "cow grass." The peculiar bending of the stem from the left hand to the right has given it the name of zigzag clover. It much resembles the common red clover, and would easily be mistaken for it, though the head is less globular and more oblong, and the blossoms of a deeper purple in color and less compact in the head. The leaves are of a darker green, and without the light spot seen in the common red clover. The roots are creeping, and it is always found growing in patches; often on very dry banks and on the top of old stone-walls, and it always excludes all other plants when once it has taken good root. It will flourish in long droughts, and is especially adapted to dry, sandy soils. It is sometimes found on cold, clayey soils, where it grows much smaller and darker in color. The stems are also more woody. It is a pest to the farmer, being almost worthless, as horses, cattle, and sheep will not eat it unless compelled to from hunger. Farmers are therefore warned against it, and advised to destroy it on the farm, as far as practicable.

Buffalo Clover, (Trifolium replexum.) - This is a species of clover growing wild on the prairies and meadows of the Western and Southern States. It has large, handsome heads of a rose-red color, and grows luxuriantly. It is thought by many to be worthy the attention of agriculturists, as a cultivated forage-plant.

Hare's Foot Clover, (Trifolium arvense.)-This variety of clover is often called rabbit-foot, stone clover, pussy clover, etc.; it is found on gravelly banks and old fields, and is of little value, in an agricultural point of view. The heads are cylindrical in form, long, very soft and downy, and seem like a mass of whitish silky hairs. It grows from five to twelve inches high, and flowers in July and August.

Hop, or Yellow Clorer, (Trifolium procumbens.)-This is a small species of clover growing usually on dry, sandy, and rocky soil. It has flowers, which at first are a bright yellow, then fade to a light buff color, and finally take a dark brown when dry. It is called "hop clover" from the fact that its heads, when in blossom, resemble in form small bunches of hops. The seeds ripen toward the latter part of July, wlien the blossoms look dry and withered, presenting a striking and unpleasant contrast to plants in full verdure. Cattle and sheep will eat it, though it is not considered of much value as a forage-plant. As it is an annual, farmers can easily eradicate it, if desired, by close mowing before the seed is allowed to ripen.

Bokhara Clover, (Melilotus luencantha,) is valuable for soiling or mowing, and affords excellent food for bees throughout its season.

Alfalfa, Lucerne, (Medicago sativa.)-There are many varieties of this species of Medicago, but this is the only one that has been cultivated in this country to any extent. Though generally known by the name of Lucerne, the more modern name, Alfalfa, comes from South America, where, according to best authority, it grows wild in the utmost luxuriance on the pampas of Buenos Ayres; it is, however, slightly modified in the United States 
by the change in soil and climate. We find, by consulting the most authentic sources, that this particular plant has been known and cultivated from time immemorial, and that it was brought from Media to Greece in the time of Darius, about five hundred years before Christ; its cultivation afterwards extending to the Romans and through them to the south of France, where it has ever since continued to be cultivated as a forage-plant. It is a plant allied to the clover family. Loudon describes it as "a deep-rooting perennial plant, sending up numerous small and clover-like shoots, with blue or violet spikes of flowers." He speaks of it also as a native of the south of Europe, of unknown antiquity in Spain, France, and Italy, but extensively grown also in Asia, and the province of Lima, South America. Columella also speaks of it as the choicest of all fodder-plants, because it continues to yield for many years without being renewed, and affords from four to six crops during the year. Pliny writes of it with the utmost enthusiasm; he says: "So superior are its qualities that a single sowing will last for more than thirty years. It resembles trefoil in appearance, but the stalk and leaves are articulated. The longer it grows in the stalk the narrower is the leaf." His directions for sowing and cultivating it are precisely the same as are laid down and practiced at the present day, in every essential respect.

Botanists describe it as having an erect, smooth, branching stem; the leaflets in threes; obovate, oblong, and toothed. The roots are long, and have been traced to the depth of thirteen feet by the side of a sand-pit. They grow large, like those of the carrot, having rootlets shooting off from the main root. A plant sending up numerous tall and slender, clover-hike shoots, with spikes of blue or violet-colored flowers; flowering in July. It is extensively cultivated in Chili, and was introduced from thence into California; large supplies of seed are also still obtained from that source. It is now being considerably cultivated in California, as well as other Western and Southern States.

Value of Lucerme or Alfalfa as a Forage-Plant.-The true value of alfalfa has been already pretty well tested in this country, although it has not received much attention until within a few years past. It was, however, introduced here about the year 1800 . It is said by those who have experimented and tested its real worth, that it is one of the best forage-plants known. Its age is certainly in its favor, for if a plant can outlive the centuries in popularity and fame, it certainly must possess considerable merit!

Mr. Loudon says of its use in Europe:- " The principal and most advantageous practice is that of soiling horses, neat-cattle, and hogs; but as a dry fodder it is also capable of affording much assistance; and as an early food for ewes and lambs, may be of great value in particular cases. All agree in extolling it as food for cows, whether in a green or dried state. It is said to be much superior to clover, both in increasing the milk and butter, and in improving its flavor. In its use in a green state care is necessary not to give the animal too much at a time, especially when it is moist, as they may be hoven or blown with it, in the same way as with clover and other green food of luxuriant growth."

Mr. Wycoff says: - "For milch cows it is superior to any other hay. It excites the secretions." He thinks that to make good hay for this purpose, it should not be cut before it has been in bloom ten days. He states that when grazed by cattle and sheep in the spring, while it is growing rapidly and is so abundant that they can gorge themselves quickly, it sometimes gives them the "hoven or colic," with fatal consequences. When grazed closely, no such evil occurs. He represents that it is benefited by being grazed moderately, as soon as the growth is sufficient and dry enough, and that cows are the best with which to graze it the first year. Mr. C. Reed, former President of the California State Board of Agriculture, says, that if cut when it is in full bloom it makes hay of good quality for stock of all kinds, but especially for milch cows; and that, according to the testimony of experienced dairymen, cows that are taken from the native grasses and pastured on it will increase the milk, butter, and cheese product from 60 to 70 per cent.; and that, according to the opinion of the best 
sheep-growers, sheep grazed on it in a constant green condition will be entirely free from the diseases of the skin so prevalent in California.

One writer speaks of a farmer keeping four horses on an acre of it from the first of May till the first of October. The result of its cultivation in England is thus described:-

A field of elevon acres, sown with twenty pounds of seed to the acre, was first mowed, having attained proper growth; it was then sown with thirty bushels of soot per acre, after which it produced two other mowings. In the following year a part of the field was again manured, and the first mowing was made on the twenty-third of May. This supported thirty horses for seven weeks, with a small addition of oats; the second mowing supported twenty horses entirely for seven weeks; the third mowing supported twenty horses for six weeks with the addition of a small amount of oats. The next year the first mowing supported thirtyfive horses eight weeks with a small addition of oats; the second mowing supported nearly the same number for the same length of time. Again:-A field of eight acres of sandy soil, with dry, sandy loam for a sub-soil, after three years' growth (mowing beginning May 24th), gave entire support to thirty horses for six veeks, keeping them in good condition and health, with constant employment; the second mowing, which was commenced July $3 \mathrm{~d}$, fed

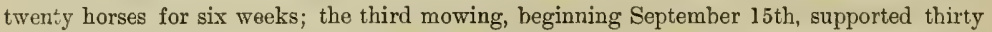
horses fourteen days, after which the autumnal feeding with sheep was equivalent in value to the expense of cleaning, etc.; in the previous spring, Mr. Reed, of California, states that a farm in Bakersville, in that State, containing one hundred and thirty-five acres, was let on shares; sixty acres were in alfalfa, five in vineyard or orchard, and the remaining seventy in timber or uncultivated. A part of the alfalfa was cut for hay, a part pastured by twenty cows, from which the milk was sold for fifty cents per gallon, and a part let for pasturage at $\$ 2.50$ per month per head. The proprietor reports that the net profits of the farm amounted to $\$ 3,500$, or nearly 26 per cent. of its value at $\$ 100$ per acre, with less than half in cultivation.

Mamuer of Cultivating Lucerne or Alfalfa. - In cultivating this plant it must be remembered that it is a native of a warm climate; hence, in northern climates it will be well to select those localities for sowing that are sheltered and warm. A southerly or southeasterly slope is desirable for the purpose. Cold, clayey sub-soils, that retain water, will utteriy exterminate it. It has a long root, hence the sub-soil must be of such a nature that it can be penetrated by it. It must have a deep, loamy soil, rich and free from weeds. The soil must be well pulverized and mellow, deep plowing being very essential. A want of such tillage has caused many a failure in the crop. Having prepared the ground properly, the seed is sown in drills of from fifteen to eighteen inches apart, in quantity of from sixteen to eighteen pounds to the acre, according to the nature of the soil. Frequently a much larger quantity is sown, but on a good soil well prepared the above quantity will be sufficient.

It is sometimes sown broadcast, but drills are preferred, as it admits of better cultivation in keeping down the weeds, which are apt to be, at first, very troublesome.

The soil to which alfalfa is adapted must contain a sufficient supply of lime, as will be seen by the following analysis of the ash of this plant by Sprengel: -

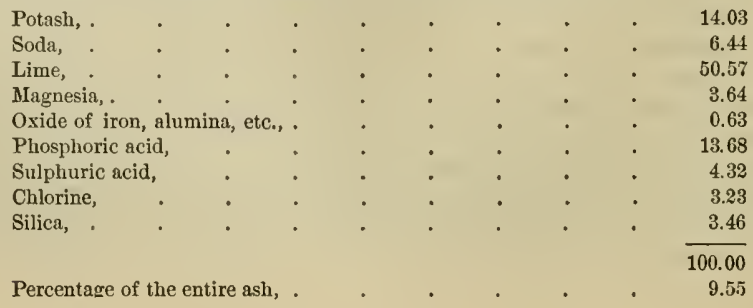


It will not, however, grow well in limestone lands where the lime lies in layers. It is a strong feeder, adapted only to rich soils, which must have their fertility kept up. It will soon run out all other plants in lands where it is sown. It will not thrive in regions where there are frequent rain-storms, clouds, and fogs, but will do best, other conditions being equal, in those localities that have the greatest number of cloudless days.

Some sow it with oats, barley, or peas, or, on light lands, with buckwheat; but it is better to sow it alone and in drills, that it may be cultivated frequently during the first year. It is sometimes sown broadcast, but this method is not generally recommended. When sown in this manner, however, from twenty to twenty.five pounds per acre will be required.

One of the ablest authorities on the cultivation of this plant says: - "If seeds are more than a year old, they germinate very imperfectly. They should be kidney-form, the plumper the better, and of a yellowish drab color; the browner they are, the less likely they are to germinate. I counted 10,432 seeds in an ounce, of which 544 seeds were defective; though this was from a very excellent sample, yet one seed in twenty was defective.

As soon as the plants are half an inch above the ground, the rows should be run through with a cultivator. All that now remains to be done is to keep the weeds down. It can be mowed four times a year, taking care to cut it always before it flowers, otherwise it becomes too woody. The next spring it should be sown with a coat of plaster, which answers quite as well for lucerne as it does for clover. It should receive a top-dressing of some kind every year, but it is better to change each year. Guano mixed with plaster is excellent; after three or four years a dressing of stable manure is desirable. A mulch of any kind of straw, late in the fall, will add much to the luxuriance of the crop. It will pay well to harrow it thoroughly in the spring; the deeper the harrow goes down the better, as there is no danger of injury to the roots. After the first year the plants will not require any other attention than vigorous harrowing and top-dressing in the spring. If this is done, it will last from ten to fifteen years, and be cut from three to five times per year, according to the quality of the soil.

An acre of it will furnish sufficient food for three or four cows during the soiling season, always, as before stated, taking care to cut it before it comes into bloom. Sheep should never be allowed to graze on lucerne, as they gnaw it off below the crown, and thus speedily kill it.

There is no known plant that will yield as much nutriment from a given acre, as can be furnished by lucerne. The seed is more easily threshed than that of clover, but the yield is less abundant."

Professor S. W. Johnson (in How Crops Grow) gives the following analysis of green lucerne:-

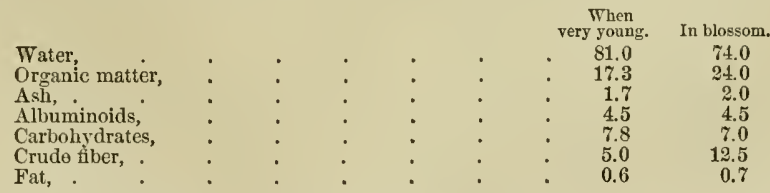

He gives the composition of the hay of lucerne as follows, from Professor Wolff's analy. sis, in 100 parts: -

Water,
Organic matter,
Ash,
Albuminoids,
Carbohydrates,
Crude fiber,
Fat,

$\begin{array}{ccc}\text { When young. } & \text { In blossom. } \\ \text { Lbs. } & \text { Lbs. } \\ & 16.7 & 16.7 \\ \text { : } & 74.6 & 76.9 \\ \text { : } & 8.7 & 6.4 \\ \text { : } & 19.7 & 14.4 \\ \text { : } & 32.9 & 22.5 \\ \text { : } & 22.0 & 40.0 \\ & 3.3 & 2.5\end{array}$


It has been found that alfalfa is well adapted to the soil and climate of the Southern States. Reports from Texas state that it is admirably adapted to the black prairie-soil of that region. It withstands well the drought of summer and cold of winter, keeping green the year round. Hon. E. G. Wall, Commissioner of State Board of Agriculture in Mississ. ippi, gives the following respecting its culture in that section:- "In well-prepared, rich, sandy loam, it succeeds remarkably, sending down its long roots many feet into the sub-soil, drawing up moisture from below; and thus will thrive where other plants are drooping from drought. In this respect it is far superior to clorer; for the latter, a suitable surface soil is of equal importance with the sub-soil, but for lucerne, a suitable sub-soil is absolutely necessary, as the roots are not fibrous. The enormous quantity of roots, penetrating the ground to the depth of sereral feet, prepares the land for increased production. It derives the larger portion of its sustenance from the atmosphere, and stores it up in its roots, so that as a fertil. izer it ranks high.

The soil is not only fertilized to the amount of several tons of roots, but it is mellowed from the mechanical displacement of the soil and the admixture of decajed vegetable matter.

The seeds of lucerne are yellow, when good; if brown they have receired too much heat, and if light, it indicates they were saved too green. The time of soming is the same with the other species of clorer, in the spring time. It should be sown in drills aud cultirated the first year, so as to keep down the weeds. It is easily smothered out by weeds, when it first comes up.

Then properly managed, the number of cattle which can be kept in good condition on an acre of lucerne, during the whole season, exceeds belief. It is no sooner mown than it pushes out fresh shoots, and wonderful as the growth of clover sometimes is, that of lucerne is much more rapid. In the dryest and most sultry weather, when every blade of grass droops for want of moisture, lucerne holds out its stem fresh and green as in the genial spring. The first year it is apt to be troubled by the presence of weeds, as it is slow in making a start; let the weeds be exterminated for that time, and afterwards no fears need be entertained on that subject, as it will take full possession of the ground."

Ifr. Read, of California, also says that alfalfa has two characteristics which especially adapt it to regions subject to long-continued hot weather. These are its very prolific and rapid growth, by which, after being cut, it rery quickly shields the surface by a thick, green coat, and the astonishing depth to which it sends down its long carrot-shaped root. In the Kern $\mathrm{Talley}$, Calo, the roots go down to the water-line, which is from twelve to twenty feet below the surface. It was found that where a freshet had exposed a perpendicular section of an alfalfa field, the roots extended down to the water-line, which was from twelve to twenty feet below the surface. In this country, the climate is generally farorable to its production from the extreme south to the latitude of Washington. It presents a remarkably luxuriant growth in the grounds of the Department of Agriculture. Farther north it thrives well only in favorable localities. It is reported as doing well in Nevada and Nebraska, also in Utah and Colorado Territories. It will not thrive in a compact clay soil, or any shallow soil with a hard-pan subsoil. It prefers a rich sandy loam, well drained, with a permeable subsoil. The seed requires a shallow covering, after deep and thorough pulverization of the soil. Broadcasting and drilling are both practiced, but if the land is foul, the latter mode is decidedly preferable, as it admits of careful culture until the crop is able to displace the weeds.

Although experiments have been repaatedly tried, with considerable success, to cultivate alfalfa in New England, yet we scarcely believe it will prove an entire success, as the minters are too severe. It being a native of a warm climate, it thrives best in a somewhat warmer temperature than New England could give it. The Southern and Southwestern states have in it an exceedingly valuable forage-plant, oring to its nutrition, its luxuriant and rapid growth, as well as the ease with which it may be cultirated when once started. And we are of the opinion that it will continue to be more bighly valued the longer it is cultivated. 
Sainfoin (Onobrychis sativa) is a native of England, and was doubtless introduced as an agricultural plant from France, having been long cultivated there as such before it was in England. It grows best on chalky soils and calcareous sands; it also luxuriantly grows on lime soils, even where lime rock is within four feet of the surface, and will not grow where there is no lime in the soil, hence its culture is chiefly confined to such localities. It occupies accordingly a very important position in agriculture in the rotation of crops, in all the chalk districts of England. It blossoms in spikes on long stalks, two or three feet high, the flowers being of a beautiful pink or flesh color. The blossoms appear in June and July. It is a valuable forage plant in localities with soils suited to its culture, and although it has not as yet been much cultivated in America, except in a small way, for the purpose of experiment, there are probably many of our soils in which it would thrive and prove a profitable crop.

When young and tender, sainfoin will not endure a severe winter, being sensitive to the cold, but after the second or third year it becomes more hardy. We know of no reason why, in some of the Southern states possessing soils suited to it, it should not prove a very valuable addition to our forage-plants.

Yanner of Cultivating Sainfoin.-As has been previously stated, it is a plant only suited to chalky soils and calcareous sands, where it thrives luxuriantly with proper cultivation. Referring to the chalky downs around Ilsley in Berks, England, Mr. Caird says that about a tenth part of the land is kept under sainfoin, in which it remains for four years, being each year cut for hay, of which it gives an excellent crop. A farmer having forty acres of sainfoin sows ten acres and breaks up ten acres annually, thus going regularly over the whole farm, the sainfoin not returning on the same field until a period of four years of rest from that crop. It is then plowed in the spring and sown with oats, the crop of which is generally excellent. The sainfoin is then sown at the rate of four bushels per acre, and is drilled in immediately after the oats have been sown, working the drill at right angles to its course when it deposited the grain. It is frequeutly pastured one or more years before being mowed. It is sometimes allowed to stand eight or ten years, but four years is considered better.

On soils suited to its cultivation, sainfoin will grow well for six or eight years; weeds and other plants will eventually drive it out, when it becomes necessary to plow the land. The roots are large and tough, which makes this a laborious process. The first year's growth is generally small, as the roots are then being formed, but after this period a fine crop will be secured, which well repays the trouble of its culture.

It is sown both broadcast and in drills, about four bushels of seed being required per acre for the former method, and three for the latter. A liberal supply of seed is thus allowed, as many, even new seeds, will fail to germinate. The soil should first be well pulverized and free from weeds.

It should be cut just as it comes into blossom, and is used both for soiling and for hay, chiefly for the latter. It is liable to be injured by rains during the curing process, as its stems are hollow. The aftermath is relished by horses, sheep, and cattle. The seed ripens in July, but as the lower part of the head matures so much earlier than the upper, and these seeds are larger and more plump, it is best to harvest them when the lower ones are fully ripe, as they will be liable to become shaken out and lost if the heads stand until the upper portion is ready to harvest.

Giant Sainfoin (Onobrychis sativa bifera).-There is little difference between this variety and the preceding, except this is darker in color and grows more rapidly, besides being somewhat larger and taller in the stalk, and the roots penetrate more deeply into the soil. Though it matures more rapidly than the common sainfoin, during the first growth, still the second crop of the giant variety is usually poor and straggling in most soils, with but little leaf, while the otber throws up a thick growth of leaves, abounding in nutriment after 
being cut, consequently this is regarded with less favor by agriculturists than the common variety.

Iiiduey Tetch (Anthyllis vulneraria).-This plant is said to grow in the poorest soil, and is found in various parts of Europe and Asia. It is much relished by cattle and sheep. It is grown as a forage-plant in France, Germany, and some parts of England, being culti. rated very much as clover is in some sections, that is, well harrowed into the oat or wheat stubble soon after the harvest, to be ready for feeding in the following spring. It is quite hardy and not easily exterminated by weeds, while as a forage-plant it possesses considerable value. It should be cut just as it comes into bloom. This plant has not been cultivated in the United States except by way of experiment, - and the results have not been sufficiently satisfactory to warrant its general cultivation for agricultural purposes.

Tetch or Tare (Vicia sativa).--Vetches of various kinds are cultivated as foragecrops quite extensively, in many parts of Europe. This variety is considered the most valuable by many. They are used green and much valued as food for horses, cattle, sheep, and even hogs. An acre of good vetches fed in a yard or stable to avoid waste, will, according to good authority, keep more horses than six acres of good pasturage. They also are valnable for milch-cows, both in increasing the flow of milk and enriching it. They can be sown in autumn or spring. The "winter vetches " should be sown in August if possible, in order to obtain a supply of good green food by the first of May, and so fill up the gap between the root-crops of the previous autumn and the summer food; whether for grazing or soiling, it is important, according to British agriculture, to have the crop ready for use at about that time. The later sowing in autumn will, of course, render the crop later the following spring. The land should be dry, well-sheltered, and enriched by deep plowing, and from twelve to fifteen loads of barn-yard manure to the acre plowed in. It should then be well harrowed. The seeds should be sown in drills, about a foot apart, about three and a half bushels per acre, and covered rather deep to admit of the roots developing before the top-growth takes place. As early in the spring as the state of the soil will admit, the crop should be hoed between the drills, and a top-dressing of soot or guano applied at the rate of forty bushels of the former per acre, or two hundred weight of the latter. The roller should then be used for the purpose of smoothing the surface and pressing down the plants that have been loosened by the frost.

By combining the winter and spring vetches, and making several sowings of each in its season at intervals of two or three weeks, it is practicable to have them fit for use from May to October and thus carry out a system of soiling by vetches alone, if desired. Some farmers mix a half bushel of wheat or rye to the seed per acre, but this is not considered by some as an advantage. Though cultivated to some extent in Canada, still, the dryer atmosphere of our country renders the growth less luxuriant and profitable than in England.

Spulry (Spergula arvensis). - This plant is sometimes used for forage to advantage on thin soils. It is cultivated extensively in Germany and some other portions of Europe for this purpose, also for the valuable oil and oil-cake its seeds produce.

It has been called "the clover of poor soils," and it is stated of it, that if sown in March. May, and July, and the three crops plowed in, to fit the poorest soil for clover production. It is a native of both America and Europe, and is found growing spontaneously in the Middle States. It is quite a hardy plant, and may be sown in the fall after a grain or early root crop has been harvested, and plowed in the following spring.

Three crops can be easily cultivated in a single season. Van Voght says, that by alter. nating these crops with rye, it will reclaim the worst sands, and yield nearly the same benefits if pastured off by cattle; while it adds materially to the advantages of other manures, if applied to the soil at the same time. It will grow on soils too poor for clover, but its roots do not descend as deep into the soil as clover or lupine. 
The Cow, or Field Pea.-This is much cultivated in the Southern States, and is more like a bean in appearance than a pea, and belongs to the leguminous or pulse family. The ease with which it can be cultivated, and its value as a forage-plant, as well as fertilizer of the soil, have given it a prominence in Southern agriculture. Two crops from two successive plantings can be produced in one season, as it grows very rapidly. Hon. H. M. Polk of Tennessee, in making a comparison between the field-pea and red clover, says:-

"The pea will thrive upon land too poor to grow clover.

It will produce a heavy and rich crop to be returned to the soil in a shorter period than any vegetable fertilizer known.

Two crops can be produced on the same ground in one year; whereas it requires two years for clover to give a hay crop, and good aftermath for turning under. In this time four crops of peas can be made.

The pea feeds but hightly upon, and hence leaves largely in the soil, those particular elements necessary to a succeeding grain crop, and the pea lay, in its decay, puts back largely into the soil those very elements required for a vigorous growth of the cereals.

There is no crop which is its equal for leaving the soil in the very best condition for a succeeding wheat crop.

It is the only crop raised in the South so rapid in its growth and perfection as to be made an intervening manurial crop between grain cut in the spring, and grain sowed in the fall, upon the same ground. And this alone makes the pea invaluable to Southern agriculture.

In our particular latitude, it flourishes equally with clover; and with two such renovators of the soil (aside from their value as food crops), no portion of the earth is equally blessed. North of us, the pea does not succeed.

It is admirably adapted to other crops, producing in the space between our corn-rows both a provision and a fertilizing crop, with positive benefit to the growing corn.

It aids in producing cheap beef, pork, milk, and butter. Withont the pea, pork could not be produced cheaply, where it costs so much to make corn.

It furnishes a double capacity for wintering stock, and with this a doubly-enlarged manure heap.

The large plantations of the South can only be restored by green crops turned under, united to a judicious system of rotation, looking to feeding the soil. This must be aided by all the manure manufactured on the plantation.

By its use, large addition is made to humus, upon which the tilth, as well as capacity of the soil for retaining moisture, so greatly depends.

As for the cultivation of the pea, one can scarcely go amiss. When two crops are intended for renovating, break the land, sow broadcast, and harrow in. Or drill in rows three feet apart, and plow out when a few inches high. When pods begin to ripen, if the crop is intended for manurial purposes, plow under with large two-horse plow, with a wellsharpened rolling coulter attached, or with chain passing from double-tree to beam of the blow to hold the vines down for facilitating covering. A roller passed over the vines before plowing under will assist the operation. Caustic lime should be sown upon the vines before plowing under to promote decay, and neutralize the large amount of vegetable acid covered into the soil. Select the pea which runs least. The vines are easiest covered into the soil. They are the black bunch-pea, and the speckle, or whipporwill-pea.

When planted in corn as a food crop, the bunch-pea ripens soonest; but the Carolina cow-pea, the clay-pea, or the black stock-pea are preferable, as they do not readily rot from wet weather, and will remain sound most of the winter. For early feeding of stock, plant whipporwill-pea by itself in separate enclosure from corn, where stock can be turned upon whenever desired.

Peas are often sowed upon the stubble after small grain is harvested. Flush up the 
ground, and sow either broadcast, or drill in furrow opened with shovel-plow, covering with scooter furrow on each side. Block off or run over lightly with harrow and board attached Again, they are drilled in every fourth furrow, when turning over the stubble, the succeeding furrow covering the peas. When either of these last modes of planting is adopted, the peas should receive one good plowing out when they are from four to six inches high.

When planted in corn (the corn should have been drilled in rows five feet apart), they should be step-dropped in a furrow equally distant from each corn-row, and covered with scooter, with harrow or with block. This should be last of May or in the first ten days of June. The only work they receive when planted in corn, is a shovel or sweep furrow run around them when the corn is being "laid by," unless there is much grass, when it becomes necessary to give them light hoeing. The crop might be said to be made almost without work when planted with corn; in fact, it is often so made by those planters who sow peas broadcast in their corn, and cover them with the last plowing given the corn.

There is much diversity of opinion as to the proper treatment of the vines in curing them for winter hay. And as much has been written upon the subject, the writer feels some diffidence in giving his own views. Suffice it to say, the great end to be attained is to cure the vnnes to the extent only of getting rid of a part of the succulent moisture in the vine, without burning up the leaves. When exposed to too much heat, the leaves fall very readily from the stems, and are lost.

When put up too green and too compactly, they heat, and when fermentation of the juices in the vine and unripe pods occurs, the hay is seriously damaged, if not completely spoiled. Mildewed hay of any kind is but poor food for stock, and when eaten is only taken from necessity to ward off starvation. Some planters house their pea-hay in open sheds, or loosely in barns, with rails so fixed as to prevent compacting. Others stack in the open air around poles having limbs from two to four feet long, to keep the mass of vines open to the air, and cover the top with grass.

There is a diversity of opinions as to the proper manner of curing and preserving this hay, but there is none as to the value of this rich food for all stock, and especially for the mileh-cow in increasing the quality and quantity of her milk."

Lupine (Lupinus).-This is the name given to plants constituting the large genus Lupinus, of the order Leguminosæ. Its generic name is derived from the Latin lupus, a wolf, in allusion to its ravenous appetite for certain alkaline constituents of soils, which it possesses to the extent that, if cultivated and carried off the land for several years in succession, it will exhaust the soil of alkaline properties; but where it is plowed under, or fed to cattle or sheep upon the land, its effect is beneficial in enriching it.

The lupines are distinguished from other cultivated leguminous plants by their strong branching habit of growth, and their handsome palmate leaves. One of the principal advautages of cultivating lupines is that they will thrive on very poor sandy gravels, and thin clays, greatly benefiting the latter. They also make excellent green manure, while the seeds, which are large when well soaked in water, make very good food for cattle. Sheep will thrive well on the green plants, which they eat with great relish.

The plants are easily injured by the frost. The lupine has been cultivated in Italy and France, and some other portions of Europe for ages. The yellow variety has there nearly superseded the white, on account of its being more hardy and better able to endure frost. Attempts have been made at different times to introduce the cultivation of this plant into the Southern States, which have not been very successful. We see no reason why it should not prove beneficial as a rotation crop in that section, on lands that are too exhausted to bear clover well. The seeds should not be planted until the season is sufficiently advanced for the grass to be quite green. From forty to sixty quarts of seed per acre are required, sowed broadcast, and harrowed in lightly. Care must be taken not to cover the seeds too deep, or 
they will fail to germinate. If the weather is showery and warm after sowing, they will soon make their appearance from the soil, but dry weather after sowing will necessitate a longer time for starting. The plant at first seems to expend most of its energies in root formation, and does not grow very fast, but, after the roots are well-formed, the growth of leaves and branches is more rapid.

It is very important that the seeds be perfectly ripe when sowed, imperfectly ripened seeds either failing to sprout, or producing sickly plants. The wild lupine of this country is found growing in many sections in sandy soil. The most numerous species of this plant in the United States are found west of the Rocky Mountains.

The cultivated lupine will grow well in all except calcareous soils, but seems to thrive best in a sandy soil.

Prickly Comfrey (Symphytum asperrimum).-This variety of comfrey was first introduced into England from Caucasus, as an ornamental plant. It grows luxuriantly, and is very hardy; so much so that it is difficult to eradicate it when once it has possession of the soil.

A few years since it engaged the attention of agriculturists as a forage plant, and much was said and written pro and con on the subject. Its cultivation has not, however, grown into general practice among farmers, although it is cultivated to a limited extent in some localities. It produces an immense amount of fodder per acre, while, being a deeply-rooted plant, it is not easily affected by drought or excessive moisture, and roots out effectually other plants when once established in the soil. It starts early in the spring and remains late. It is a large, coarse-leaved plant, remarkable for the prickly bristles with which it is covered. One farmer who favors its cultivation, says that he has cut 24 pounds of green fodder from one hill at the third cutting in the season, which would be an average of 53 tons per acre, the hills being three feet apart each way.

The advocates of its culture claim that it is especially adapted for the fattening of stock, and increasing the milk of cows; while the objectors claim that it is difficult to make stock eat it at all, as long as they can find anything else to eat. Hogs will eat it more readily than other stock.

Its culture is very simple. It cannot be grown from seed, and is propagated from cuttings. The best way to cultivate it, is to sprout the cuttings of the roots in a hot-bed, or by covering them with an inch of moist earth, and two or three inches of horse-manure. When sprouted, they may be planted in the field (which should be of rich, mellow soil, well plowed and harrowed), three feet apart each way, with plenty of well-pulverized manure under them. It should be kept well-cultivated at first to keep out the weeds, and will do better not to make any cuttings during the first year. After that, three cuttings per season can be made. In winter the roots ought to be well-dressed with manure. When once established, it is cultivated with little labor. It should not be allowed to flower. It requires about 3,000 sets of plants per acre, one pound of the roots making about 175 cuttings.

Having had no experience in its cultivation, we cannot speak from that knowledge, but from information gained from various sources, we doubt whether it will ever be a general favorite, as a forage plant, with the majority of farmers. 


\section{LIST OF GRASSES AND FORAGE PLANTS.}

$\mathrm{T}$

$\mathrm{HE}$ following table, giving the list of grasses and forage plants, with their common and botanical names, their time of blossoming, whether wild or cultivated, and their place of growth, may be of interest as well as practical benefit to many farmers. In giving the scientific names, the first word that occurs in parenthesis is the name of the grass; the second, that of the species; as, for instance, in herds-grass ( $\mathrm{Phleum}$ pratense), Phleum is the generic name, pratense the specific. A genus often contains many species."

\section{List of Grasses and Forage Plants.}

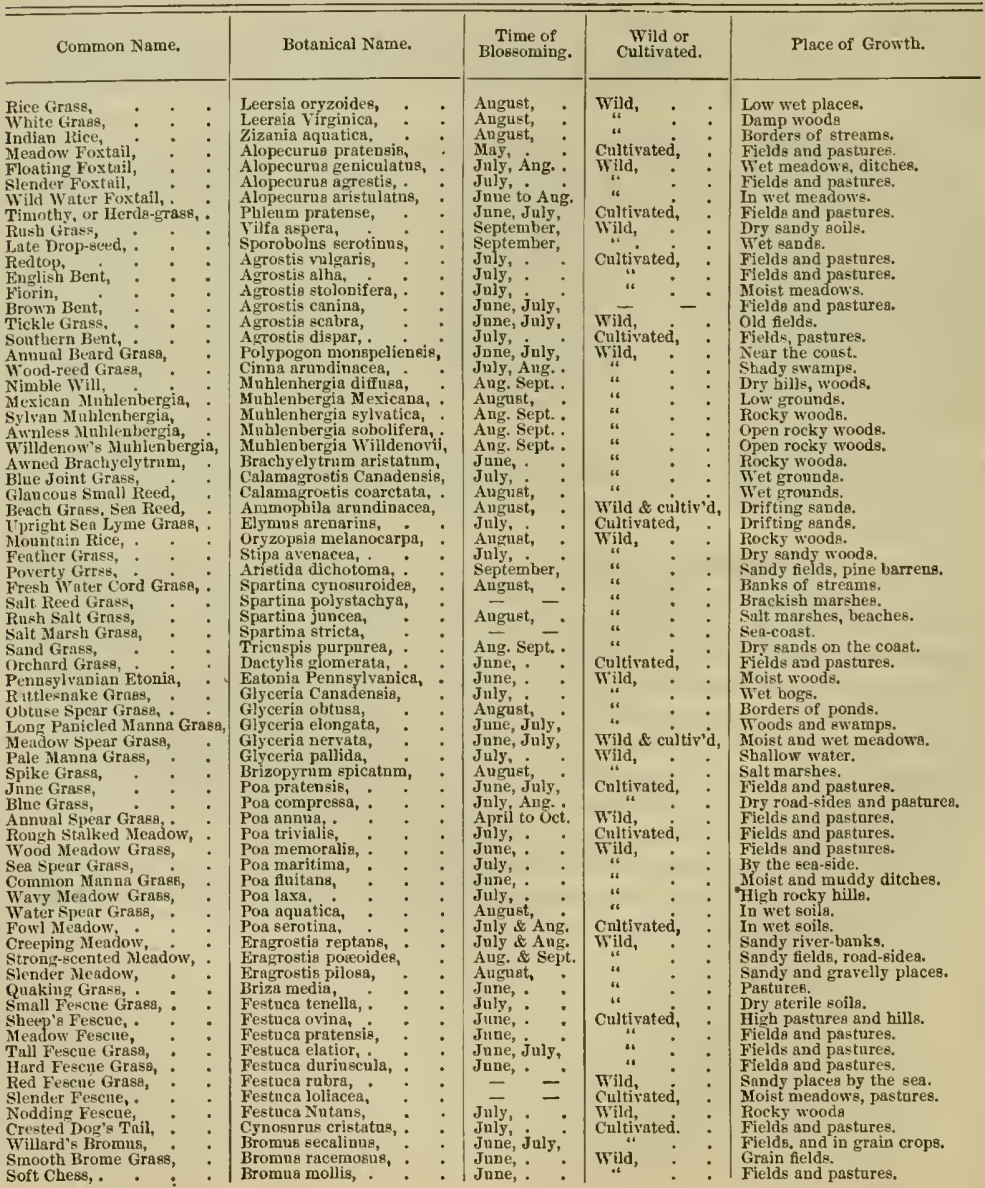


List of Grasses and Forage Plants.-Continued.

\begin{tabular}{|c|c|c|c|c|c|c|}
\hline Common Name. & & Botanical Name. & $\begin{array}{c}\text { Time of } \\
\text { Blossoming. }\end{array}$ & $\begin{array}{l}\text { Wild } \\
\text { Cultiv }\end{array}$ & & Place of Growth. \\
\hline 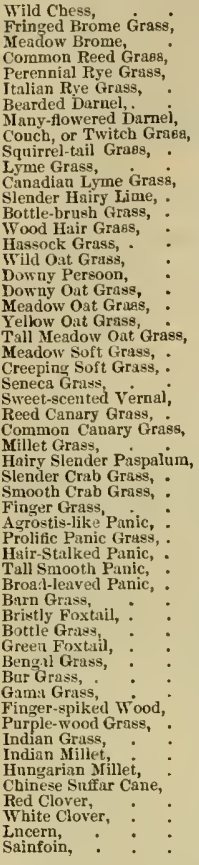 & $\dot{:}:$ & 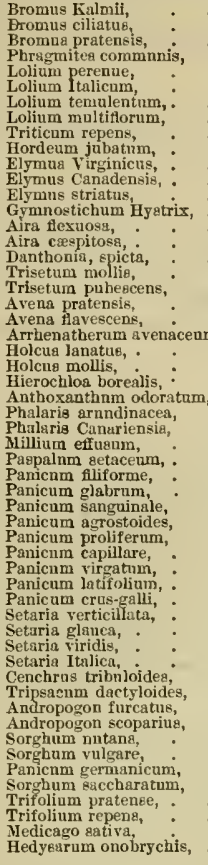 & 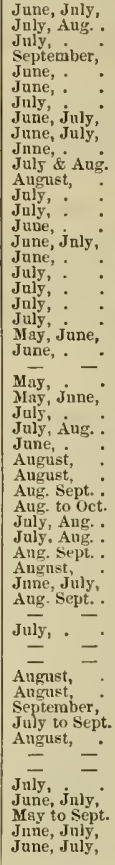 & 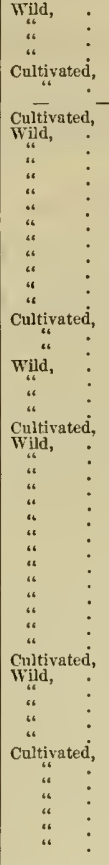 & . & 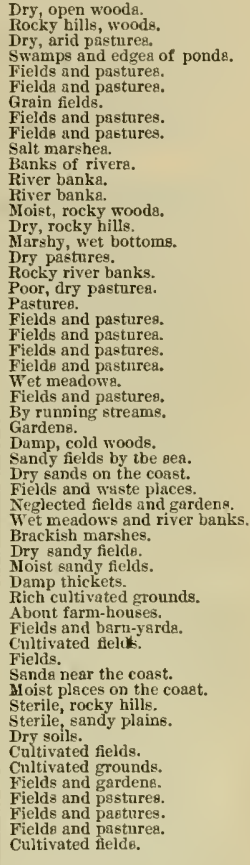 \\
\hline
\end{tabular}

\section{Glossary of Terms used in Describing Grasses.}

Acuminate-Extending into a long, tapering point.

Acute-Sharp-pointed.

Annual-Living through one season only.

Anther-The upper part of the stamen containing the pollen or fertilizing powder.

Awn-A bristle-like process proceeding from or attached to the glumes or palets of some grasses.

Biennial-Living through two seasons.

Boat-shaped-Concave within and convex without, as the glumes and palets of some flowers.

Bristles-Short, stiff hairs.

Bulbous-The base of the stem thickened so as to make a hard, roundish mass, as in timothy grass (Phleum pratense).

Cespitose-Growng in bunches or tufts.

Cauline-Relating to or growing from the stem or culm.

Citiale-Having the margin fringed with hairs. 
Culm-The stem or straw of a grass; when the stem creeps upon or under tho ground it is called a rhizoma.

Decumbent-Leaning on the ground at the lower part but rising at the top.

Digitute-Branching finger-like from a common center, as the spikes of Crab-grass (Panicum sanguinale).

Dicecious-The two sexes separated and growing on different plants, as in Buffalo-grass (Buchloë dactyloides).

Entire-Without notches on the margin.

Exserted-Protruded beyond the flower, as the stamens of grasses usually are when in bloom.

Fertile-Producing fruit.

Fibrous-Composed of thread-like fibers, as the roots of most grasses.

Floret-A little flower ; a pair of palets with the inclosed stamens and pistil. There may be many of these in a spikelet.

Glabrous_Smooth; destitute of hairs or roughness.

Glumes-The outer or lower pair of bracts or scales in a spikelet, and inclosing one or more, sometimes many, flowers or florets.

Hirsute-Rough-haired, bearded.

Indigenous-Growing naturally in a country.

Internode-The space between the nodes or joints.

Keel-A sharp ridge along the middle of a glume or palet resembling the keel of a boat.

Lamina or Blade-The extended part of a leaf, generally open and flat, but sometimes rolled inward longitudinally, when it is said to be involute.

Ligule-A small leaf-like appendage, usually thin and semi-transparent (membranaceous), found at the lower part of the leaf or at the top of the sheath. It is said to be entire when there are no divisions in its outline; bifid, when it is divided at the apex into two parts ; lacer. ated, when it is cut or divided on the margin ; truncated, when the upper part terminates abruptly in a transverse line, as if cut off.

Membranaceous-Thin and translucent, like a membrane.

Nerves-Rib-like elevations on the leaves, glumes, and palets.

Neutral Flower-One having neither stamens nor pistil.

Nodes-Knots in the culm where the leaves are given off.

Oblong-Longer than wide, with the sides nearly parallel.

Obtuse-Blunt-pointed.

Ovary - The portion of a flower containing the ovules or seeds.

Palet or Palea-The inner scales or bracts inclosing the stamens and pistil.

Panicle-The flowering part of the stem or culm of grasses, usually composed of a number of series or whorls of branches or rays, which are again divided into secondary branches. These may be short and close to the stem, or they may be loug and spreading.

Perennial-Living for more than two years ; indefinitely.

Pistit-The central organ of a fortile flower, usually consisting of an ovary, style, and stigma.

Pollen-The fertilizing powder contained in the anthers.

Pubescent-Covered with soft hairs.

Rachis-The nsme given to that kind of flowering branch where the flowers are arranged closely together on its sides without stalks or pedicils, as in Paspalum, and in the ultimate branches of the panicle.

Radical leaves-Those growing from the root.

Spikelet-The ultimate divisions of the panicles or flower-heads; they may be one-flowered, that is, a pair of glumes enveloping a single flower of a pair of palets (or sometimes one 
palet) with the inclosed stamens and pistil ; or they may be two or more flowered, there being but one pair of glumes to each spikelet, whether it be one or many-flowered.

Sheath-That part of the leaf which clasps the stem; it answers to the petiole or leaf. stalk.

Spike-When the flowers are sessile or without branches, as in Timothy grass (Phleum pratense).

Stamens-The organs of the flower which contain the pollen, consisting of the filament and the anthers.

Stigma-The extremity of the pistil which receives the pollen.

Wharl-A number of leaves or branches starting from one line on the stem.

Grouping of Grasses.-Grasses may be separated into five distinct groups, according to their marked peculiarities of growth:-

Finsr, we find the bush or jungle grasses, or such as are not inclined to grow with other species and form a close, matted turf or sward, such for example as the Tufted Hair Grass, (Aira caespitosa,) Meadow Oat Grass, (Avena pratensis,) Tall Fescue Grass, (Festuca elatior,) etc.

A few other grasses, if sown alone, will assume somewhat the same form in tufts or cushions, as Sheep's Fescure, (Festuca ovina,) Hard Fescue, (Festuca durinscula,) and Orchard Grass, (Dactylis glomerata.) This peculiarity in the growth of the last three grasses is prevented by close pasturing, rolling, and proper cultivation. These operations improve their natural tendencies, since, if left to themselves, they would be liable to assume the jungle growth, such as is often seen in poor, thin pasture soils, a close, fine, matted sward being attained only by careful cultivation.

Sccond in order, the aquatic or water grasses form another distinct group, among which may be found the Reed Canary Grass, (Phalaris arundinacea,) Common Reed Grass, (Arunda Phragmites,) Water Spear Grass (Poa aquatica,) Common Manna Grass (Poa fluitans,) Rice Grass, (Leersia oryzoides,) Floating Foxtail, (Alapecurus gernculatus,) Wild Rice, (Lizania aquatica.) These grasses grow mostly in water and are not cultivated generally as agricultural grasses with the exception perhaps of the first.

Wild rice grass is sometimes cultivated at the South, where it yields often very large crops. Floating Foxtail is also cultivated in Europe.

Third, Marsh or Salt Grasses, among which we find the Salt Reed Grass (Spartina polystachya,) Rush Salt Grass, (Spartina juncea,) Salt Marsh Grass, (Spartina stricta,) Black Grass, (Junous bulbosus,) Beach Grass, (Ammaphila arundinacea,) Goose Grass, (Poa maritima.)

FourrH in order, we have the field or pasture grasses. Under this head may be included a very large number of species. These grasses might be subdivided according to the soils and situations which they naturally affect; for though a grass may sometimes be found or placed in a soil which is not naturally fitted for it, yet no species will arrive at its most perfect development on a soil not well adapted to it. Among these might he mentioned as examples Timothy, (Phleum pratense,) Meadow Foxtail, (Alopecurus pratensis,) Common Spear Grass, (Poa pratensis,) Orchard Grass, (Dactylis glomerata,) Perennial Rye Grass, (Latium pernene,) Italian Rye Grass, (Lolium italicum,) Redtop, (Agrastis vulgaris,) Whitetop, (Agrastis alba,) Downy Oat Grass, (Avena pubescens,) Meadow Soft Grass, (Halcus lanatus,) Meadow Fescue, (Festuca pratensis,) Field Barley Grass, (Hordeum pratense,) Tall Oat Grass, (Arrhenatherum avenaccum, ) etc.

FIFrH in order may be classed the annual weeds, which, though proper grasses, are often very troublesome in cultivated grounds, either on account of their creeping, underground stems, or their rapid and luxuriant growth. Thrifty farming is a ceaseless struggle against these pests, and the farmer is generally careful to keep as clear as possible of them. Among 
these may be named Willard's Bromus, (Bromus secalinus,) Soft Brome Grass, (Bromus mollis,) Slender Foxtail, (Alopecurus agrestis,) Creeping Bent Grass, (Agrostis stolonifera,) Conch or Twitch Grass, (Triticum repens,) Rough Stalked Meadow Grass, (Poa trivialis,) Annual Meadow Grass, (Poa annua.)

Of these, the last three are not always considered as weeds, since they are sometimes sown as pasture grasses; but when they appear in cultirated grounds, in gravel walks and avenues, they are exceedingly troublesome and difficult to eradicate. Each of the groups previously indicated may be considerably enlarged by a consideration of the natural habits of grasses.

Many of the grasses which have been described possess but little value for the purposes of cultivation, it is true, but it should not be forgotten that they all have their uses, and these uses in the grand economy of nature are exceedingly important, however they may appear to our short-sighted vision. No plant comes up to the sunlight, or expands its beautiful leaves, that does not derive its support in part from the atmosphere, and even though its life be short, it adds materially in its decay to the vast mass of vegetable mould which covers the surface of the globe and forms the richness of the soil. This surface mould has been accumulating for ages in many localities; every plant that grew in ages past bringing down to us in a tangible form the riches with which the air that surrounded it was stored, which now lie waiting the farmers' use in meadows of exhaustless fertility, in swamps and bogs of vast, increasing utility in our agriculture, and in beds of peat, the value of which we have scarcely begun to appreciate. Thus, the grasses which are not cultivated for their direct nutritive qualities, are not without their value, and they deserve our careful study and attention.

Nutritive Talue of Grasses. - Different species of grass differ very materially in nutritive value, habits of growth, etc., as we have already considered, some being exceedingly nutritious, others possessing but little nutritive value; some yielding a luxuriant aftermath, while others can scarcely be said to produce any at all; some flourishing in elevated situations are best suited to the grazing of sheep, while others grow most luxuriantly on the low lands in marshes, and sustain the richest dairies, no soil being so sterile, no plain so barren, but that a grass can be found adapted to it.

Some varieties, indeed, will not endure a soil even of medium fertility, nor the application of any stimulating manure, but cling with astonishing tenacity to the drifting sands, while others prefer the heaviest clays or revel in the hot beds of ammonia. Some are grega. rious in their habits, requiring to be sown with other species, and if sown alone will linger along till the wild grasses spring up to their support; others are solitary, and if mixed with different species will either extirpate them, usurping to themselves the entire soil, or die and disappear. Nearly every species is distinguished for some peculiar quality, and most are deficient in some, comparatively few combining all the qualities desired by us in alternate field-crops, for pastures or permanent mowing, to such an extent as to justify a general cul. tivation. It is important, therefore, to learn the comparative nutritive value of each species thought to be worth cultivating.

This study is naturally attended with great difficulties, but sufficiently accurate researches have been made with a view of arriving at such positive results as would be entitled to full confidence. It is now very well established that the nutritive value of the food of an animal depends chiefiy upon the proportion of nitrogenous substances contained in it. Without doubt, the sugar which is found to be an ingredient of most vegetable substances at some periods of their growth, in some degree contributes to it also. The nitrogenous constitutents of any sub. stance, as grass or hay, for instance, may be determined with little difficulty and with great exactness, since it has been found by abundant research, that, when present, they are of nearly the same constitution, and do not vary in their combinations. The determination of the sugar is somewhat more difficult. 
The constituents of plants may be divided into two classes, one class embracing all those substances of which nitrogen or azote forms a part, and the other consisting of non-nitrogenous bodies. Gluten, albumen, gelatine, casein, legumen, and fibrin, belong to the former class, being nitrogenous substances, while starch, gum, sugar, woody fiber, mucilage, etc., are destitute of nitrogen, or non-nitrogenous.

Only a small quantity of nitrogen is found in vegetable substances, and it is derived, in part, at least, from the atmosphere, in the form of ammonia. On the other hand, nitrogenous substances form a large proportion of the constituents of the blood of animals, and appear in their whole system. As there is a constant warte in the animal, and a continual formation of new tissues, - as the whole body is constantly renewed through the agency of the blood which is converted into flesh and muscle, - there must be a never-failing supply of nourishment, and this nourishment for the higher animals is found, as already intimated, in the nitrogenous elements of plants.

For every ounce of nitrogen which the animal requires to sustain life and health, he must take into the stomach, in the shape of food, such a quantity of vegetable substances as will furnish him with an ounce of nitrogen. If we suppose one kind of hay to contain one ounce of nitrogen to the pound, and another to have only half as much, or only an ounce in two pounds, the pound which contains the ounce of nitrogen would go as far to nourish the animal — other things being equal — as the two pounds which contain only the same quantity of nitrogen. The importance of woody fiber to act mechanically in giving bulk to the food, is not, of course, to be overlooked.

Nor is this a mere deduction of theory. The experiment has frequently been made, and it is now fully established both by science and experience, that the greater the proportion of nitrogen which any vegetable contains, the smaller will be the quantity of that vegetable required to nourish the animal body, and the less nitrogen any vegetable contains, the greater will be the quantity of it required. Muscle and flesh are composed of nitrogenous principles, while fat is made up of non-nitrogenous matter. Every keeper of stock knows that to feed an animal on oil-cake alone, for instance, which is but slightly nitrogenous, might fatten him, but it would not give him strength of muscle or size; while if the same animal be kept on the cereal grains, as wheat or Indian corn, alone, his size rapidly increases, his muscular system develops, and he gains flesh without increasing his fat in proportion.

The non-nitrogenous substances are necessary for the production of fat to supply the animal body with heat, and thus they meet a want in the animal economy, although they do not contribute so directly to nourish and sustain the system. They are, therefore, important in the analyses of articles of food, though not so essential in determining merely their nutritive values.

From what has been said, the reader will very readily understand the following tables containing the results of the investigation of Prof. Way. The specimens of the various grasses on which his researches were made, were analyzed both in their green state as taken from the field, and after being dried at a temperature of $212^{\circ} \mathrm{Fahr}$., a point at which the moisure is found to be entirely expelled and evaporation ceases, and the importance of both determinations must be obvious on a moment's reflection.

The inquiries of Prof. Way were directed to ascertain

1. The proportion of water in each grass as taken from the field.

2. The proportion of albuminous or flesh-forming substances, including, without distinction, all the nitrogenous principles.

3. The proportion of oily or fatty matters which may be called fat-forming principles.

4. The proportion of elements of respiration, or heat-producing principles, among which are included starch, gum, sugar, pectic acid, etc.; all the non-nitrogenous substances, indeed, except fatty matters and woody fiber. 
5. The proportion of woody fiber.

6. The amount of mineral matter or ash.

The specimens were picked out, plant by plant, each specimen by itself, from fields in which they were growing naturally, or mixed in the ordinary mode of cultivation, and were not raised expressly for analysis.

The results of the analysis of the natural grasses in the green state, are arranged in the following table:-

TABLE I.-Analysis of Natural Grasses. (100 parts, as taken green from the field.)

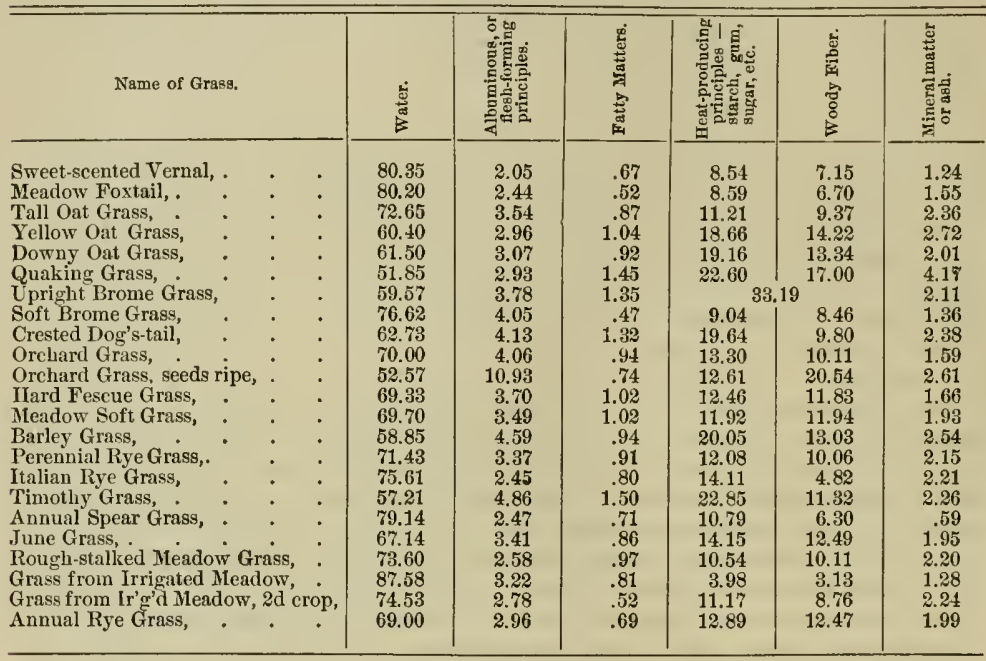

TABLE II.-Analysis of Artificial Grasses. (100 parts, as taken from the field.)

\begin{tabular}{|c|c|c|c|c|c|c|c|}
\hline Name of Plant. & & 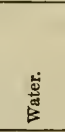 & 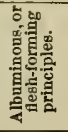 & 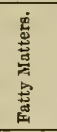 & 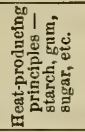 & 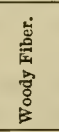 & 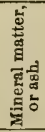 \\
\hline $\begin{array}{l}\text { Red Clover, } \\
\text { Perennial Clover, } \\
\text { Crimson Clover, : } \\
\text { Cow Grass, } \\
\text { Cow Grass, } 2 \text { d specimen, } \\
\text { Hop Trefoil, } \\
\text { White Clover, } \\
\text { Common Vetch, : } \\
\text { Sainfoin, } \\
\text { Liteern, or Alfalfa, } \\
\text { Black Medick, or Nonsuch, }\end{array}$ & 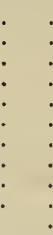 & $\begin{array}{l}81.01 \\
81.05 \\
82.14 \\
74.10 \\
77.57 \\
88.48 \\
79.71 \\
82.90 \\
76.64 \\
69.95 \\
76.80\end{array}$ & $\begin{array}{l}4.27 \\
3.64 \\
2.96 \\
6.30 \\
4.22 \\
3.39 \\
3.80 \\
4.04 \\
4.32 \\
3.83 \\
5.70\end{array}$ & $\begin{array}{r}.69 \\
.78 \\
.67 \\
.92 \\
1.07 \\
.77 \\
.89 \\
.52 \\
.70 \\
.82 \\
.94\end{array}$ & $\begin{array}{r}8.45 \\
8.04 \\
6.70 \\
9.42 \\
11.14 \\
7.25 \\
8.14 \\
6.75 \\
10.73 \\
13.62 \\
7.73\end{array}$ & $\begin{array}{l}3.76 \\
4.91 \\
5.78 \\
6.25 \\
4.23 \\
3.74 \\
5.38 \\
4.68 \\
5.77 \\
8.74 \\
6.32\end{array}$ & $\begin{array}{l}1.82 \\
1.58 \\
1.75 \\
3.01 \\
1.77 \\
1.37 \\
2.08 \\
1.11 \\
1.84 \\
3.04 \\
2.51\end{array}$ \\
\hline
\end{tabular}


TABLE III.-Analysis of Natural Grasses. (100 parts of the grass dried at $212^{\circ}$ Fahr.)

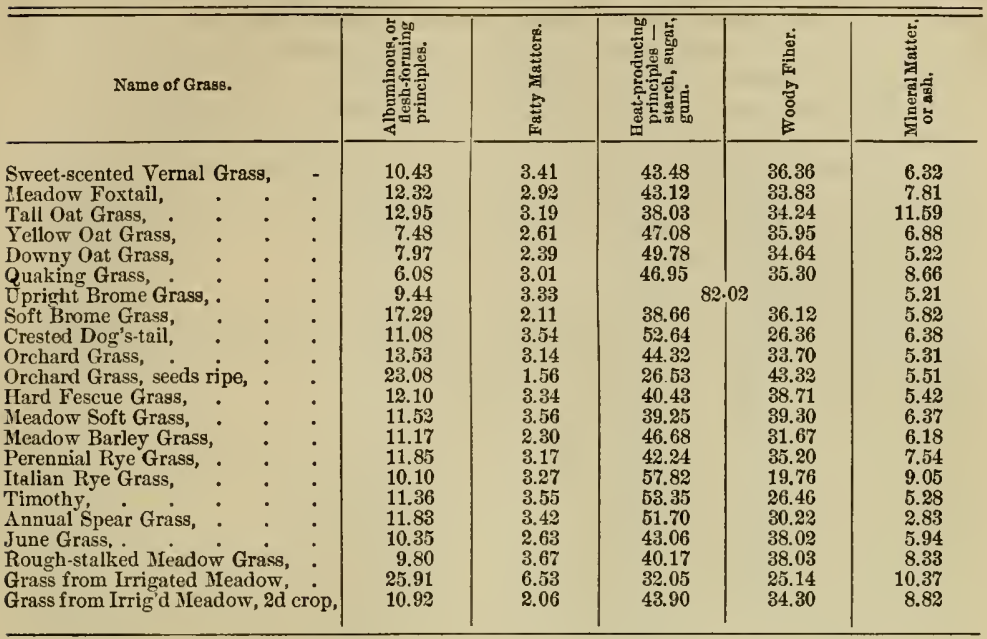

It will be seen that a great difference exists in the valuable constituents of the grasses analyzed in the previous table, ranging as follows :-

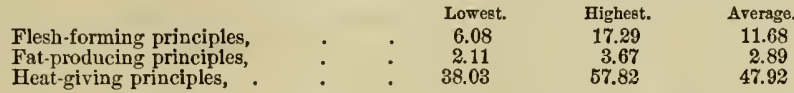

TABLE IV.-Analysis of Artificial Grasses. (In 100 parts of the grass dried at $212^{\circ}$ Fahr.)

\begin{tabular}{|c|c|c|c|c|c|c|}
\hline Name of Plant. & & 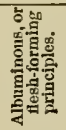 & 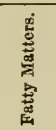 & 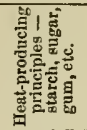 & 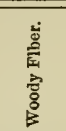 & 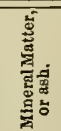 \\
\hline $\begin{array}{l}\text { Red Cbver, } \\
\text { Perennial Clover, } \\
\text { Crimson Clover, : } \\
\text { Cow Grass, } \\
\text { Cow Gras, 2d specimen, } \\
\text { Hop Trefoil, } \\
\text { White Clover, : } \\
\text { Common Vetch, : } \\
\text { Sainfoin, } \\
\text { Lucern, or Alfalfa, : } \\
\text { Black Medick, : }\end{array}$ & $\begin{array}{l}: \\
\vdots \\
\vdots\end{array}:$ & $\begin{array}{l}22.55 \\
19.18 \\
16.60 \\
24.33 \\
18.77 \\
20.48 \\
18.76 \\
23.61 \\
18.45 \\
12.76 \\
24.60\end{array}$ & $\begin{array}{l}3.67 \\
4.09 \\
3.73 \\
3.57 \\
4.77 \\
4.67 \\
4.38 \\
3.06 \\
3.01 \\
2.76 \\
4.06\end{array}$ & $\begin{array}{l}44.47 \\
42.42 \\
37.50 \\
36.36 \\
49.65 \\
43.86 \\
40.04 \\
39.45 \\
45.96 \\
40.16 \\
33.31\end{array}$ & $\begin{array}{l}19.75 \\
25.96 \\
32.39 \\
24.14 \\
18.84 \\
2.66 \\
26.53 \\
27.38 \\
24.71 \\
34.21 \\
27.19\end{array}$ & $\begin{array}{r}9.56 \\
8.35 \\
9.78 \\
11.60 \\
7.97 \\
8.33 \\
10.29 \\
6.50 \\
7.87 \\
10.11 \\
10.84\end{array}$ \\
\hline
\end{tabular}

A glance at the above table will show that the different principles in the artificial grasses vary to a great extent, as follows :

Flesh-forming principles,

Heat-giving principles, $\quad \cdot \quad \cdot \quad 33.31$

Lowest. Highest. Average

$\begin{array}{lll}12.76 & 24.60 & 18.68\end{array}$

$\begin{array}{lll}33.31 & 49.65 & 41.48\end{array}$ 
The difference in composition exhibited in the natural grasses of table III. are very marked, and, of course, the value of the grasses as compared with each other, must vary greatly. Still, the practical value of a grass depends somewhat upon circumstances which cannot be analyzed, such as the period at which it arrives at maturity, and the particular soil and location of the farmer. It might happen that a grass not in itself so rich in nutritive qualities as another, would be preferred on account of its coming to maturity just at the time when the farmer most needed it. But the particular value of this table is, that it shows the comparative nutritive qualities of the grasses, since all the specimens were collected and investigated in the same manner, at the same period of growth, - or as nearly as possible,when in the flower, so that whatever sources of error might exist to modify the results, they would naturally apply to all alike.

The grasses from the irrigated meadow consisted principally of June or Kentucky blue grass, rough-stalked meadow grass, perennial rye grass, meadow soft grass, barley grass, meadow oat grass, and a few other species, and it will be noticed that in combination they abound in flesh and fat-forming principles to a greater extent than we should be led to suppose from the composition of any one of them alone.

Our favorite timothy compares very favorably with the other grasses, containing a less percentage of useless matter as woody fiber than any other, except Italian rye grass and crested dog's-tail, a grass not common with us, and the irrigated grasses. In point of soluble, heat.producing principles, sugar, gum, and starch, it is surpassed by the Italian rye grass, but by no others. The analyses of this grass in its green and dry states in tables I. and III., fully justify the preference which we have long shown for the use of timothy; for, as taken from the field at the time of blossoming, it will be found to contain less water (table I.), a greater percentage of flesh and fat-forming principles, and less useless matter in the shape of woody fiber, than most of the other grasses. The deductions of science certainly correspond, in this case, with the results of practice.

A comparison of tables I. and III. with tables II. and IV., will show the comparative advantages of the use of the artificial grasses, in point of albuminous or flesh-forming princi. ples and fatty matters. The carbonaceous or heat-producing principles remain nearly the same throughout, while the percentage of waste matter or woody fiber is less than in the natural grasses. This is an important fact, worthy of the careful consideration of the farmer.

In the sixth column of table III. will be found the percentage of ash of each of the grasses analyzed. Table V. contains a still further analysis of this ash, which gives all the inorganic constituents which the plant derives from the soil and the manures furnished to it. It is important and suggestive to one who will examine it carefully, as indicating the kind of manure which in many cases it may be desirable to apply.

The first peculiarity which plainly appears from a glance at the ash analyses, is the very large percentage of silicates and potash contained in the natural grasses, and the very small comparative percentage of silica in the artificial grasses, the red and white clovers. The large percentage of lime and carbonic acid attract our attention in the latter. This table is exceed. ingly valuable, as suggesting the proper course of manuring for the most successful cultiva. tion of the various crops contained in it.

If now we look at the analysis of some of our common weeds (table VI.), we shall see how far superior the cultivated grasses are in nitrogenous or nutritive principles.

The albuminous principles are very much less than in either the natural or the artificial grasses. 
TABLE V.-Analysis of the Ash of some of the Natural and Artificial Grasses.

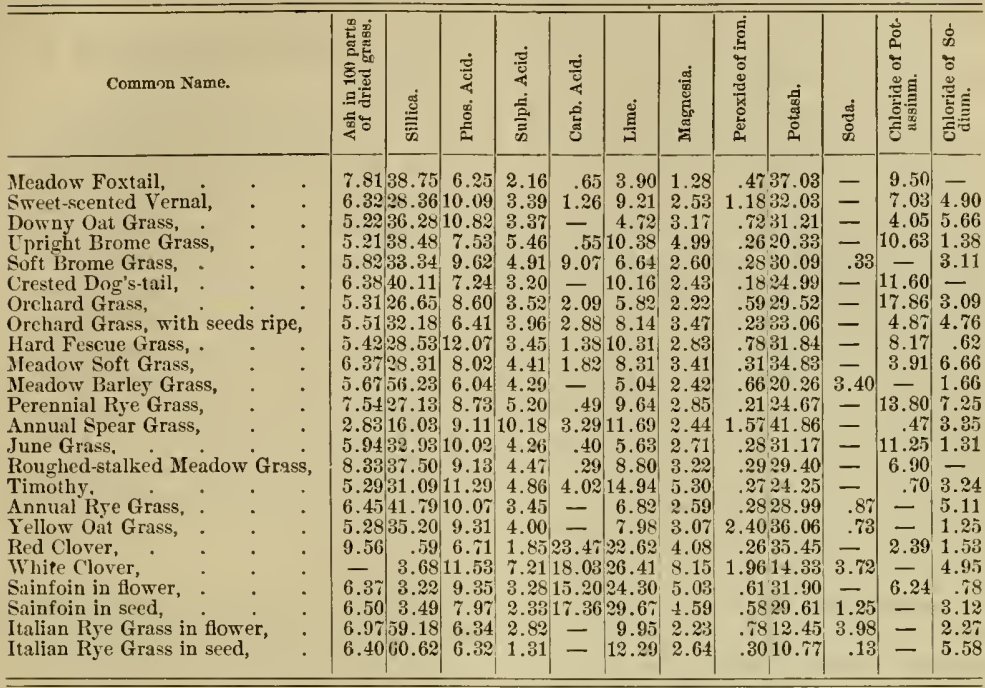

TABLE VI.-Analysis of Specimens of Weeds, as taken from the field, and when dried.

\begin{tabular}{|c|c|c|c|c|c|c|c|c|c|c|c|}
\hline & \multicolumn{4}{|c|}{ Name of Plant. } & 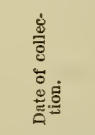 & $\begin{array}{l}\text { D. } \\
0\end{array}$ & 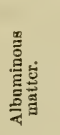 & 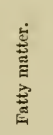 & 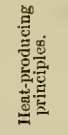 & 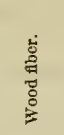 & 离 \\
\hline \multicolumn{5}{|c|}{ Ox-eye Daisy, (Crysanthemum leucanthemum,) } & June 23, & 71.85 & 2.12 & .999 & 12.64 & 10.51 & 1.86 \\
\hline \multirow{2}{*}{\multicolumn{2}{|c|}{$\begin{array}{l}\text { Iellow Buttercup, (Ran } \\
\text { Sorrel, (Rumex acetosa, }\end{array}$}} & cul & acms,) & 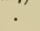 & June 13, & 88.15 & 1.18 & .507 & 6.26 & 3.00 & .91 \\
\hline & & . & . & . & July 4, & 75.37 & 1.90 & .545 & 7.62 & 13.04 & 1.51 \\
\hline Dried & SPECIMENS & $F T$ & SASYE. & & & & & & & & \\
\hline Ox-eje Daisy, & . & . & . & . & -- & - & 7.53 & 3.49 & 45.02 & 37.38 & 6.63 \\
\hline Buttereup, & . & . & . & . & $-\quad-$ & - & 9.98 & 4.28 & 52.69 & 25.34 & 7.71 \\
\hline Sorrel, . & . & - & . & . & $-\quad-$ & 一 & 7.71 & 2.19 & 46.82 & 37.16 & 6.12 \\
\hline
\end{tabular}

Time to Sow Grass Seed.-The universal practice in time of sowing grass seed was formerly in the spring; sometimes sowing it on the late snow, which, when melting, aided in settling the seed into the soil, thus obviating the necessity of harrowing. The action of the frost in leaving the ground, aided by the spring rains, also accomplished the same result when sown upon the soil after the snow had disappeared. But a change has taken place in the practice of this department of agriculture, within the last quarter of a century. It is now generally conceded by those best acquainted with the most desirable methods of practice in this respect, that the best time for sowing most varieties of grass seed, is from the middle of August to the middle of September, the precise time depending on the condition of the soil. This gives the grass a good start, and being early well-rooted in the soil, obviates the effect of drought, which spring sowing would not do, as there is more danger from summer drought than win. ter-killing. If for any reason it cannot be sown until the latter part of September, it would 
be better to defer it still later, even until the middle of November, as in that case the seed will not germinate so as to be injured by the severe winter cold, and will start earlier in the spring than it possibly could by an early spring sowing.

Clover and orchard grass may be considered exceptions to this rule generally, the best time for sowing these seeds being usually early in the spring.

Should the soil in August be very dry, it is better to wait till early September before sowing, but when the soil is favorable at the middle of August, that is the better time, as before stated. What we have said relative to August as the proper time for sowing, applies more particularly to the North In the extreme South, a month later would be a more desirable time. There can be no definte rule given relative to the precise time of seeding down grass land, since it will depend upon varions considerations, such as the latitude, the season, nature of soll, etc. The rule we bave given will be the best general rule, conditions being favorable. Sometimes it will be more convenient for a farmer to sow in the spring; in such a case, the soil should be very rich, moist, and mellow; or, if not particularly rich, it should have a generous supply of manure, that the seed may have a quick start, and luxu. riant growth; otherwise, the weeds will be liable to choke it, and it will not be sufficiently advanced to withstand the effect of the drouth in summer; the object being to enable it to obtain a strong hold upon life, and become well established in the soll before the hot, dry sea. son sets in. If sown when the ground is very dry, the seed will be bikely to be burned, or dried up and lost; for this reason the sowing should be delayed until the soil,-which had previously been prepared,-was well moistened by the early fall rains.

Selection of Seed.-It is of the utmost importance in the production of the grass crop, that not only the suitable varieties of seed should be selected, but that the seed should be fresh. Old seed that has been left over from year to year may be, and doubtless is, frequently mixed with the new, and put upon the market. This mixture is not easily detected, but the results will usually be seen in the failure in germination of the old seed. There is a great difference in the different varieties of plants with respect to the length of time the seeds will retain their vitality, some retainng it for several years, others only one or two years. The seeds of most of the grasses have been found to be of but very little value when they have been kept two or three years, hence the importance of procuring new, fresh seeds, and guarding against any mixture of the old and worthless with the new, as carefully as possible.

It is easy to tell whether the germinative power of grass or any other seed still remains, by the following simple method; and, if the buyer should be willing to try it, he might purchase only a small quantity at first, and afterwards obtain his full supply with more confidence, if the trial showed it to be good. Take two pieces of thick cloth, moisten them with water, and place them one upon the other in the bottom of a saucer. Place any number of seeds which it is desired to try, upon the cloth, spreading thin, so as not to allow them to cover or touch each other. Cover them over with a third piece of cloth similar to the others and moistened in the same manner. Then place the saucer in a moderately warm place. Sufficient water must be turned on from time to time to keep the three thicknesses of cioth moist, but great care must be taken not to use too much water, as this would destroy the seed. There should be only enough to moisten the cloths, and not enough to allow any to stand in the saucer. Danger from this source may be aroided in a great measure, however, by tip. ping up the saucer so as to permit any superfluous water in it to drain off. The cloth used for covering may be gently raised each day to watch the progress of the swelling or the moulding of the seeds. The good seed will be found to swell gradually, while the old or poor seed, which has lost its germinating power, will become mouldy in a very few days. In this way, also, any one can judge whether old seed is mixed with new. The latter will germinate much more quickly than the former. He can judge, besides, of the quantity which he must sow, since he can tell whether a half, or three-fourths, or the whole will bo likely to germi 
nate, and can regulate his sowing accordingly. The seeds of the clovers, if they are new and fresh, will show their germs on the third or fourth day; other seeds will take a little longer, but till they become coated with mould there is hope of their germinating. As soon as the mould appears it is decisive, and the seed that moulds is worthless.

Some farmers may think it too much trouble to test seeds in this manner. It will always, however, be well to save a sample of the seed sown, and if there is any difficulty in its germinating, it can be tested, and the real difficulty ascertained. Often the seed fails to start on account of its being covered too deep in the soil; in such a case, the fault is in the farmer, and not in the seed.

Mixture of Grass Seed.-In the mixture of seed for mowing lots, it is desirable to have a large variety, as it is a well-established fact in agriculture that a larger crop of hay will be produced from a given amount of land, other things being equal, than where but a single or few varieties are grown. It is also important that the varieties should be the best for the purpose, in order to secure hay of the best quality and quantity, and that they should be such as will be ready for mowing about the same time, - which is when they are in blos. som,-otherwise, if the earlier and later varieties are sown together, the early ripened may become dry, woody, and useless by being over-ripe, before the later varieties are ready to be cut. By this method, also, the fields of earlier grasses can be first mowed, and the hay properly cared for, before the later fields are matured, obviating the hurry and anxiety of the farmer respecting the loss or injury of certain crops of hay for lack of opportunity to secure it at the proper time.

For feeding to horses, at least a half of the time, many farmers prefer timothy which has been grown without the admixture of other varieties of grass; but for cows and sheep, a large variety is greatly to be preferred.

For permanent pastures, both early and late varieties are desirable in order to secure a con. tinuous growth throughout the season, the largest number of varieties, other things being equal, giving the best results. The following tables for mixtures have been obtained from various reliable agricultural authorities. Of course, the quantity of each variety can be varied, or the entire amount increased, as may appear judicious in view of the soil, climate, etc.

Light seeding will produce a larger and coarser growth of stalks with a tendency to weeds, while heavy seeding will produce finer stalks and nicer hay for cattle and sheep. Perhaps a medium in this, as well as in most other things, thus avoiding either extreme, is the better method and the one to be adopted in the majority of cases. Each mixture is in. tended for one acre of land.

\section{For Mowing Lands.}

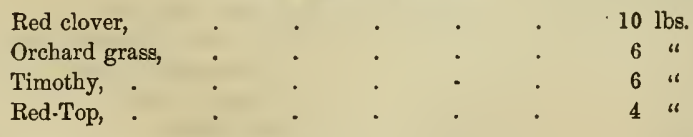

\section{For Permanent Pastures.}

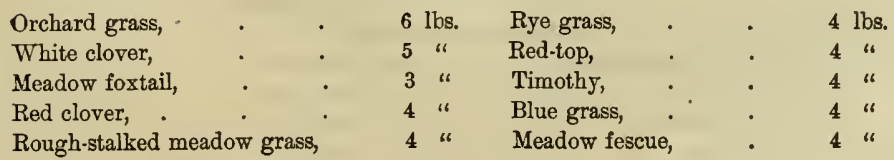


The following is also a good mixture for pastures, for both immediate and permanent use:-

\begin{tabular}{|c|c|c|c|c|c|c|}
\hline Red clover, & . & - & - & - & - & $6 \mathrm{lbs}$. \\
\hline Timothy, & . & • & . & . & . & 5 " \\
\hline Red-top, & - & ${ }^{\circ}$ & • & - & . & 5 \\
\hline Orchard grass, & - & . & . & . & . & 74 \\
\hline Alsike clover, & . & . & . & . & . & 34 \\
\hline White clover, & . & . & . & . & . & 3 " \\
\hline
\end{tabular}

\section{Hay and Pasture Combined.}

\begin{tabular}{|c|c|c|c|c|c|}
\hline Timothy, & . & . & 6 lbs. & Wood meadow grass, & 4 lbs. \\
\hline Orchard grass, & . & . & $6 " 1$ & White clover, & $4 "$ \\
\hline June grass, & - & . & 4 " & Rough-stalked meadow grass, & $2 "$ \\
\hline Rye grass, & . & . & $4 "$ & Perennial clover, & $2 "$ \\
\hline Sweet-scented ve & ernal, & . & $2 "$ & & \\
\hline
\end{tabular}

For the Southern States Mr. Howard recommends the following mixture:

\section{For Rotation and Improving the Soil.}

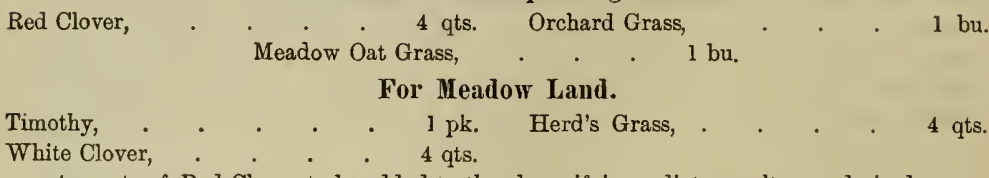

4 quarts of Red Clover to be added to the above if immediate results are desired.

For Sụmmer Pastures.

Bermuda Grass, Herd's Grass,
Red Clover, Natural Grasses,

\section{Crab Grass.}

\section{For Winter Pastures.}

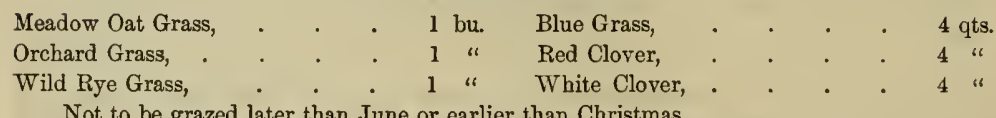

Not to be grazed later than June or earlier than Christmas.

In order to learn the best and most recent English methods of seeding down for pas. tures, a circular for this purpose, sent to the best grass-growers of the kingdom to ascertain their opinion and methods, resulted in some of the following returns.

From Durham-soil, a loam; portions of it light, with some clay.

\section{For Permanent Pastures, Per Acre.}

3 pks. perennial rye-grass.

13 lbs. white clover.

10 " trefoil.

4 " alsike.

6 " cow-grass.

3 " red clover.
$1 \mathrm{lb}$. crested dog's-tail.

1 “ cock's-foot (orchard grass).

1 " sweet-scented vernal.

1 " meadow foxtail.

1 " hard fescue.

1 “ smooth-stalked meadow grass (June grass).

In Cheshire the following mixture is used per acre:-

\footnotetext{
$\frac{1}{2}$ bush. cock's-foot (orchard grass).

$\frac{1}{2}$ " meadow foxtail.

$\frac{1}{3} \quad$ " perennial rye-grass.

$\frac{8}{4}$ " meadow-fescue.
}

$2 \frac{1}{2}$ Ibs. sweet vernal.
4 lbs. June grass.

3 " white clover.

2 " trefoil.

2 "Timothy.

$1 \frac{1}{2}$ " cow-grass. 
The practice on the estates of the Earl of Powis, on stiff soils, is to sow-

4 lbs. crested dog's-tail.

1 " sweet vernal,

3 " cock's-foot.

4 " tall fescue.

4 " meadow-fescue.

3 " rough-stalked meadow.
2 lbs. meadow foxtail.

4 "Timothy.

2 " alsike clover.

2 " white clover.

6 " perennial rye-grass.

5 " Italian rye-grass.

It will be seen by the three tables previously given that the English farmers are far ahead of us in respect to the varieties of grasses used in seeding down land for pastures, and we might gain from them lints, in this respect, to our own agricultural advantage. There are, in the Northern States, varieties that are maturing in succession for six months, of which we will mention a few of the most important. Beginning in April with the blossoming of spear-grass, we have in May the meadow fox-tail, sweet-scented vernal, and white clover (which latter we class among the grasses for convenience, thongh strictly it is not such, but a leguminous plant). The number of Jnne blossoming grasses is legion; this month being the graminivorous season for all grazing stock, as more varieties of grasses blossom dnring this month than any other, at the North. A few of the more important are, Timothy, the various species of fescues, orchard grass, June grass, rye-grass, and red clover. July brings the flowering of red top, foul-meadow and English-bent, while in August we have bluegrass, creeping-meadow and floating fox-tail, and in September hairy-panic, poverty grass and red grass, with many of those already mentioned, which continue blossoming from month to month

The following are some of the tables of mixture recommended by noted agriculturists in Scotland:

\section{For Permanent Pastures (Per Acre.)}

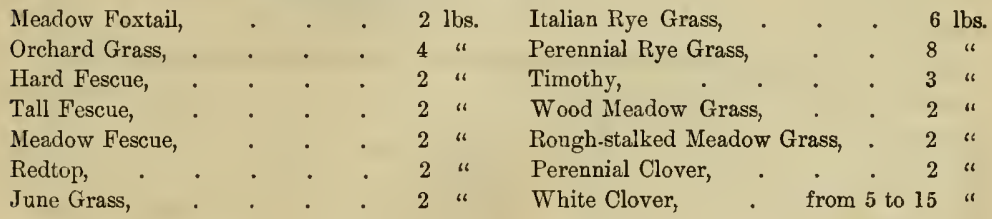

\section{For Permanent Lawns.}

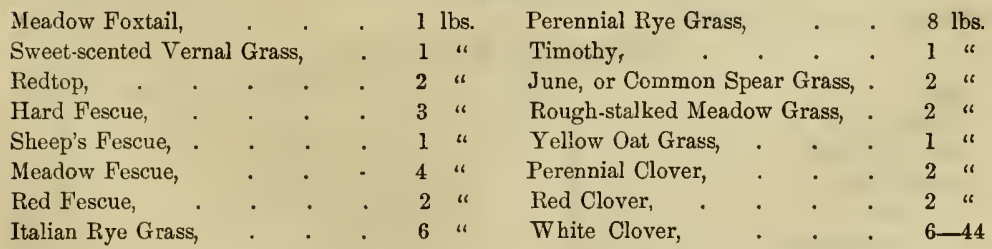

If the object is to make a permanent lawn, such as is frequently desirable around or near a farm-house, the above table will be found one of the best. It will resist the effects of our severe droughts better than those varieties commonly used for lawns. If it is desirable to omit anything from it, the red and perennial clovers, the yellow grass, and a part of the ryegrass could best be spared.

If a fine lawn is wanted where extra attention will be paid to rolling and mowing, the following mixture will do well: 
Fine Lawns Frequently Mown.

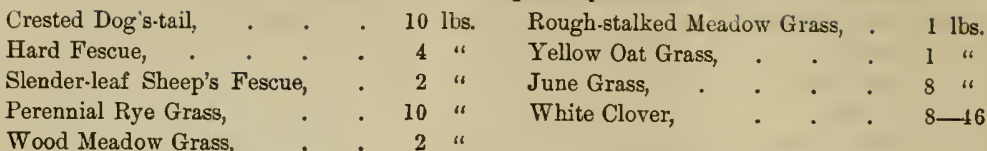

Hay and Pasture in Orchards and Shaded Places.

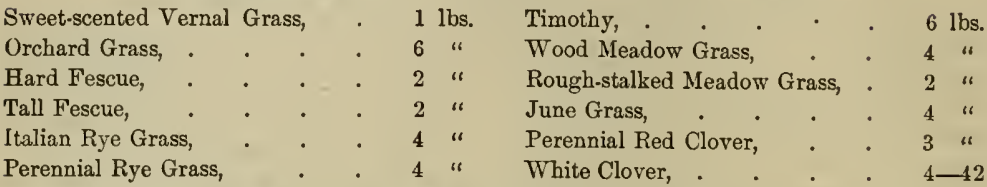

\section{Mixture for Ifowing on Light Sands.}

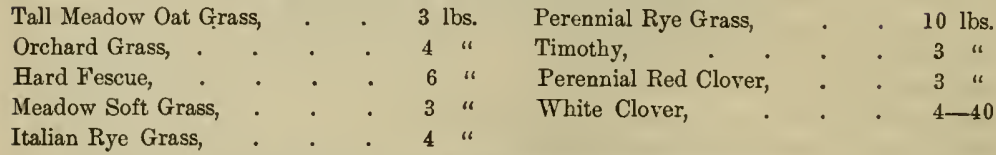

\section{Mixture for Reclaimed Peaty Lands.}

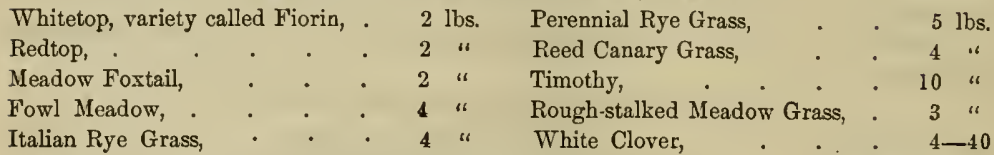

\section{Marshy Grounds, Liable to be Occasionally Orerflowed with Fresh Water.}

NAME OF GRASS. Peaty Soils, Alluvial Soils.

Fiorin, . . . . 4 lbs. 3 lbs.

Tall Fescue, . . . 3 " 3 "

Manna Grass, . . . 5 " 5 "

Reed Canary Grass, - 3 " 4 "
NAME OF GRASS.

Timothy,

Peaty Soils. Alluvial Soils,

The following will be found to be a valuable mixture when it is desired to corer rocky hills and poor soils with grass.

\section{Mixture for Rocky Hills.}

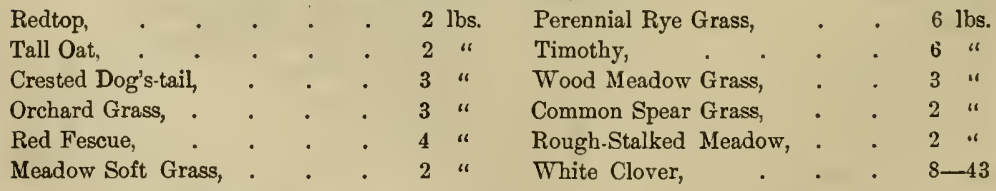

Sowing Grass Seed.-It has been the general practice with farmers of this country to sow grass seed with some kind of grain, preparing the land especially for the grain crop, and with the exception of covering it in the process of covering the grain, to make no provision for the crops of grass the land is expected to produce. Although by this means the land may be utilized to produce two crops at the same time, and labor saved, yet we think it far better and more economical in the end, to sow the grass seed separately, thus giving it a fair start and insuring a good crop; besides the practice of sowing together results in injury to both crops. 
By the usual method of sowing with grain, the grass crop suffers a great injury, by the grain taking possession of the fertilizing elements of the soil, and choking the growth of the grass, and also by shading it too much, and when the grain is cut, the grass is not only trampled and injured by the process of cutting, but is often dried up by the hot sun, being too tender to bear its scorching rays.

We do not therefore believe it a good policy, as a general rule, to sow grass seed with any grain crops. By so doing we rob the grass crop for the sake of grain, and the grass must in turn deprive the grain of some of the nutritive elements of the soil, to a limited extent; however, the grass crop is the one to suffer most by the practice.

Whenever grass is to be sown with any kind of grain, barley is the one to be preferred, but even this is very objectionable. Grass is an important product, and should be regarded as such in its cultivation. If we take a grain crop from our grass lands, the grass must of necessity be injured by it, although it may not be entirely killed by such treatment. In preparing the lands, it should be plowed, and given a good supply of manure or fertilizer of some kind, the quantity suited to the character of the soil; sometimes guano or grass fertilizer is sown upon the surface broadcast. The soil should then be pulverized with a harrow until it is mellow and fine, and the seed sown either by hand (as is still the old time practice on small farms in many sections) or by a machine, which is much better, since it will distribute the seed much more evenly over the surface than hand-sowing, besides greatly facilitating the labor. So many seeds fail to germinate, or are destroyed by birds and insects, that we favor a liberal quantity of seed sown, as well as a large variety, in order to secure not only a heavy growth, but hay of superior quality, for every farmer knows that hay is superior and finer in quality where the grass grows thick and compact in the sod. It is a common fault to cover grass seed too deeply. Mfost of our grass seeds germinate most surely when only covered one-fourth of an inch, and by actual experiment it has been ascertained that when covered an inch half the seeds fail.

Grass seed, being so very small, should be slightly covered; hence, if a common tooth harrow is used for the purpose of covering, as has frequently been the custom, the seed is buried too deeply in the soil. A common brush harrow is better for this purpose, to be followed by a good roller to press the soil down, which hastens the germination of the seed. Many farmers who are not in possession of a good roller use a simple wooden drag for this purpose, which is made similar to what is commonly called a "stone boat," the front being beveled up so as to avoid the uneven surface. From eight to ten feet long, and three and a half feet wide, are very good dimensions for such a constructed harrow. The inclined portion can be about a foot wide; this answers the purpose of a brush harrow and roller combined. It is a good practice in reclaiming old wornout meadows to give them a good coating of unfermented man. ure, and then turn the sod over. On this surface thus ploughed, a dressing of well-rotted manure or compost with ashes is spread, and thoroughly harrowed lengthwise the furrows. The seed is then sown and slightly harrowed in. followed with rolling; the decomposing manure, with the added fertilizing properties of the stubble and roots of the sod, will give a quick and luxuriant growth. Many farmers greatly improve their lands for mowing, when they do not wish to plough up and seed down, by furnishing a good top-dressing of manure in the fall or in early spring (fall is better) and then scattering grass or clover seed over the field. The seed thus sown will catch quickly and greatly improves the crop, if sown when the soil is moist, or just before a heavy rain.

Pasture lands are often improved by running a harrow over them in a manner to cut the turf slightly into small squares, an inch square being the most desirable size marked by the harrow lines. This should be done when the soil is rather moist. Upon the ground this prepared, sow a mixture of grass and clover seed of various kinds suited to both early and late growth, in order to insure a fresh, permanent pasturage through the season. Upon 


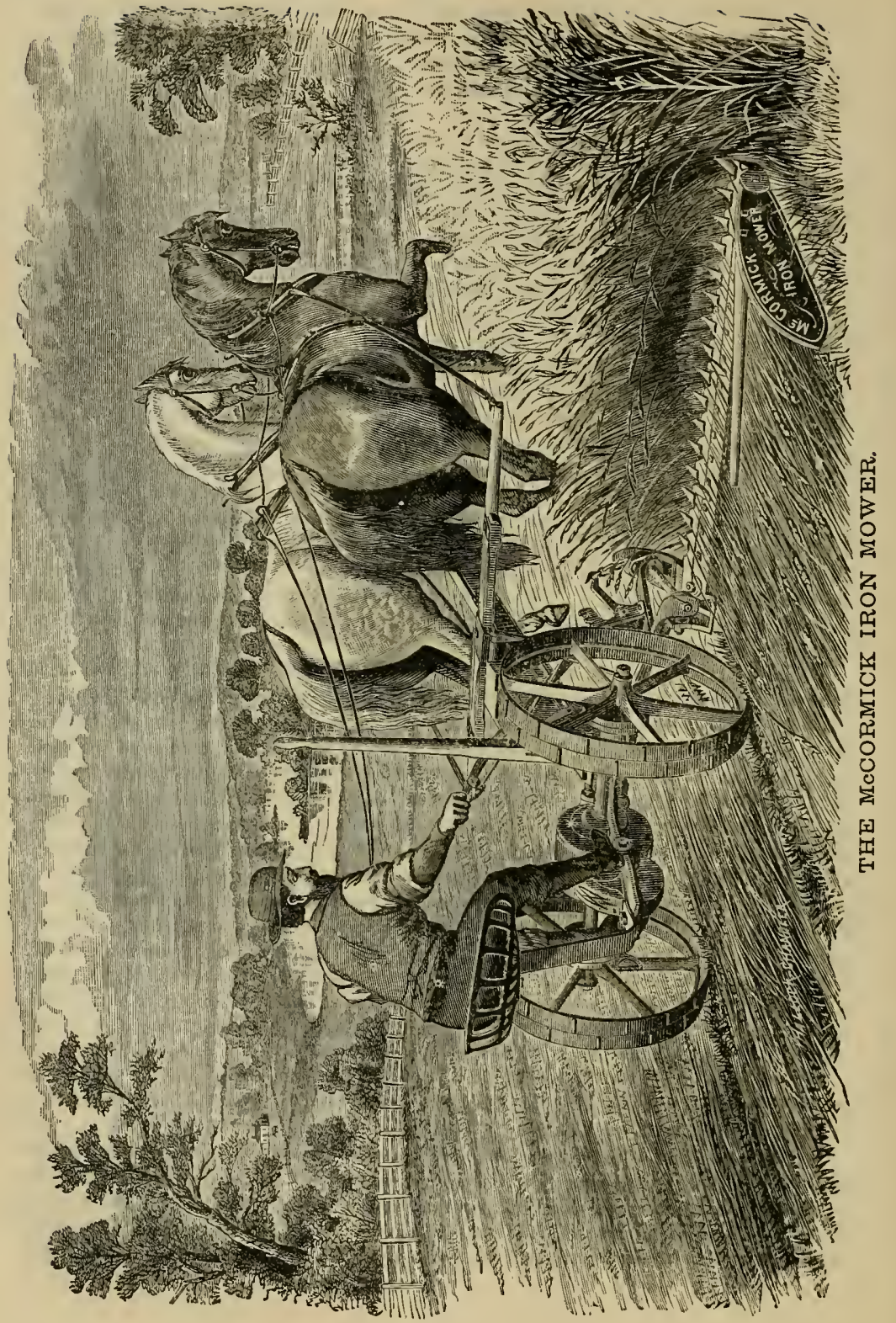


this put a litile top-dressing, and the seed thus sown will quickly germinate and greatly improve the grazing; though it should not be fed upon until it has had an opportunity to become deeply-rooted and well-established in the turf. It is very important, -and we would repeat it here to impress it more forcibly upon the minds of farmers, - that both mowing lands and pastures should occasionally have a dressing of manure or some other fertilizer. It will be economy in the end for farmers to put more manure on their grass lands, instead of putting it all upon their other crops to the neglect of the grass. If it is not profitable to cultivate corn or wheat without manure, neither is it profitable, nor should it be expected, to cultivate grass well without some assistance from fertilizers. The best time for applying the manure is in the fall, as it serves to protect the roots and give the grass an early start in the spring.

Time of Cutting and Method of Curing Hay.-As to the time of cutting grass for hay, there is still a diversity of opinions among the farmers of our country, although in the main, there bas been quita a change in favor of early cutting within the last twenty-five years. Some recommend cutting just before it comes into bloom, some when in perfect bloom, others when the blossoms have just fallen, others when the seed is in a milky state, and others still when the seeds are nearly ripe.

Two very important considerations are involved with respect to this subject. The first is, to cut it at the time it contains the greatest amount of nutriment; the second, to do it when it will involve the least injury to the aftergrowth. The question to be considered is, When is that time? With regard to the first, the opinion and practice of farmers bave greatly changed within the last quarter of a century. It was formerly the general opinion, that the best time for cutting most kinds of grasses, Timothy, and Orchard grass, for instance, was when the seed was nearly ripe and ready to shell; but experience and scientific analysis have proved that the time when grass contains the greatest amount of nutritive matter, such as starch, gum, and sugar, is not at this period, but at the period before the seed is formed, since most of these nutritive properties of the plant go to form the seed, leaving the woody fiber in the stalk, which serves to give bulk food, but not nourishment; hence, if not cut early, a great part of the nutriment of the stems and leaves is wasted. Experience and observation, together with the information afforded us by analysis, prove that the proper time for cutting nearly all kinds of grasses, is when they are in full bloom, or just as they are coming into bloom; opinions vary somewhat with respect to these stages of growth, but we believe the majority are in favor of the time of full bloom. Even a casual observer must have noticed that when cattle are grazing, they do not select the stalks of grass with ripened seed or withering blossoms. No, they pass these by and take the more tender grasses that have not arrived at this stage, and we think they must be better judges than we, as to which is the most desirable for food. Now, in making hay, our object is to have as little change as possible in the grass, and to preserve, as nearly as we can, the solid constituents, in the same state as when the grass was young and green, and if cut at the period of blossoming, there will be little of the woody fiber in its composition. If left until the seed is ripened, we find the seeds the storehouse of the starch, gum and sugar, as before stated, while the stalk becomes hard, wiry and about as nutritious as straw. Swale hay is almost worthless, if cut when the seeds are ripening. Clover should be cut also when in full bloom, and not when the head has become brown. It has been found by careful experiment that herds-grass, red-top and clover cut at the time as we have indicated, will produce more milk and butter than the same quantity of late-cut hay, even when fed with a certain quantity of grain daily, and that the same relative value will be found in making beef or feeding any farm stock; besides, no one can deny that the early-cut grass is most like green grass.

With respect to our second consideration, viz., the time of cutting that will best conduce to the aftergrowth, the same rule applies equally well. 
Any plant that is cropped at a certain time, before arriving at a state of maturity, will throw up a second growth more readily and be more vigorous than if cropped after it has matured. This is a law of nature that cannot escape the notice of any person, even the most unobserving. Now, if we apply this rule to grass-cutting, we shall not only have better hay, as we have already seen, but the aftergrowth will start more vigorously and grow more rapidly, which will not only protect the young and tender grass roots from the hot sun, but will furnish an aftermath or "rowen," - as it is often called, - which will be quite an addition to the hay crop already gathered, while a third crop will soon start and grow sufficient, before the frost comes, to secure the roots a protection through the winter; this protection aiding in an early growth in the spring. It is a fact that all experience corroborates, that grass will not only start earlier in the spring, after a protection, - whether of top-dressing or its own aftermath, - but will produce a greater amount of hay the following season than where no protection is thus given. Cưtting grass after the seeds have matured, leaves a dry stubble that is slow in starting again, and will look brown during the remaining part of the season, unless the soil is particularly rich or damp, while it will not produce as much hay in quantity or as good in quality in the following season, as the fields that are early cut. Thus we see that the present practice of early cutting is an improvement upon the old-time method, in securing not only a larger amount 'f hay, but hay of a hetter quality, and also leaves the land in a condition to furnish a larger crop of grass the following season.

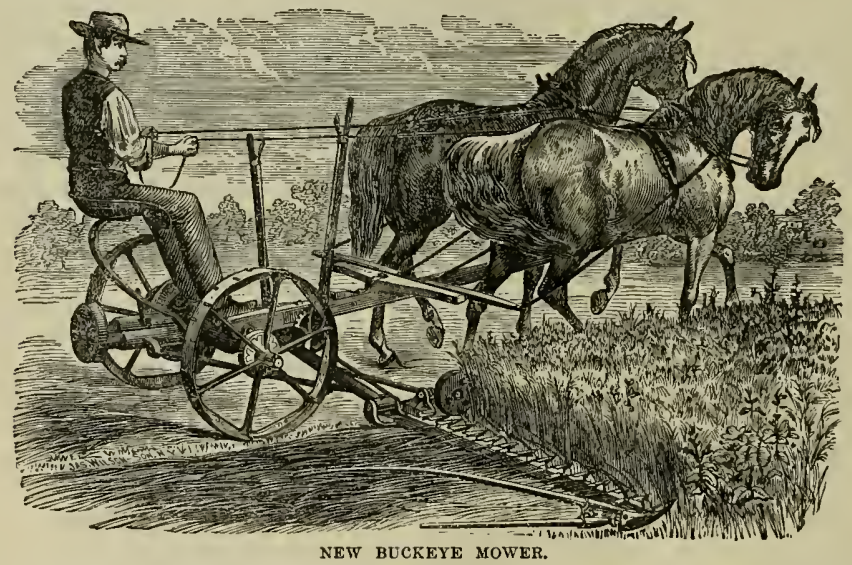

Respecting the cutting of grass, we believe the universal opinion among our leading farmers is, that it should not be cut until the dew is well dried off, as it will then cure much more readily. We think the practice of many farmers of overdrying their hay is as great an injury to its quality, as that of insufficient curing; in fact, we might safely say that farmers, as a general thing, lose more by drying their hay too much than by not drying it enough.

Even under the most favorable conditions with respect to the time of cutting, there must of necessity be considerable loss of the nutritive qualities of the grass in drying; the action of heat and moisture causing a loss of sugar and starch. This loss is dependent in a great measure upon the length of time it is exposed to the air and light; hence, grass cured with the least exposure to wind and sunlight, other conditions being equal, will be more nutritious, and contain less of woody fiber, than that which is overcured by long exposure to air and sun; and such hay is also more palatable to cattle, as is evidenced by their eating it more 
readily and showing a preference for it when given a choice of the two kinds. "Never dry hay so as to make it brittle when twisted in the hand," is a rule by which many of our best farmers are governed.

Hay should be housed on the day of cutting, if possible. Our experience in this respect goes to show that one good hay-day is sufficient for drying any kind of grass, unless it be, perhaps, wet meadow or swale hay, which requires more curing to keep well than most other varieties; however, many farmers have good success in keeping the latter in fine condition when put in the barn the same day of mowing. Of course much depends upon the kind of temperature, state of the atmosphere, etc., in curing any grass, even on a bright, sunny day. It is, therefore, very difficult to lay out any definite rule with respect to the time required to cure hay, as any farmer knows that on some days twenty-five minutes of sun will dry it more effectually than sixty minutes will at other times with a full sunshine. The wind has much to do with the drying process. In New England, the north or east wind will not assist the drying of hay much, while a west or southwest wind hastens it very perceptibly. Grass should never be dried any more than just to have it keep well in the mow, the greener and have it keep well the better, as it contains by far more nutrition and hence more value for stock food than that which is dried longer. Some experience in testing and experimenting will be necessary to determine just when this point of curing is reached. The extensive use of mowing-machines, and the degree of perfection that has been attained in their construction is such, that much of the hard labor attending haying in former times is obviated, besides facilitating the process of curing and getting it housed before the dew begins to fall. Of course, no good farmer will expose his hay to either dew or rain when it can be avoided, as the nutritive properties of grass are all soluble in either, and will be wasted by such exposure. Hay exposed to a long rain is almost as worthless as straw, since a large portion of its nutritive elements will have thus been extracted, leaving only the woody fiber.

Among the many fine mowing-machines at present in the market, that are so admirably adapted to the purpose of their construction, it is difficult to determine which should take the precedence in favor with farmers. The McCormick Iron Mower, manufactured in Chicago by the firm that bears its name, and the New Buckeye Mower by the Aultman \& Miller Co., Akron, Ohio, of both of which we give illustrations, may be named among the first-class implements of this kind, as they do most excellent work, and are strong and durable.

With all the modern facilities, the old adage, "Make hay while the sun shines," is as true now, and as important to be observed, as it was in the days of our forefathers. We do not advocate the practice of many farmers of cutting grass on clondy days, thus running the risk of rain to follow. The time thus gained in the haying process, although it may seem economy in that respect, is generally more than offset by damage or loss to the hay crop. One of our leading New England farmers, in eastern Massachusetts, gives his method of curing hay thus:-

"I am convinced that in most parts of New England our English hay should be cut and stored, if the weather allow, before the seed has set; commencing to cut with the mowingmachine as soon certainly as the dew is off. One man should be occupied trimming around the walls, trees, etc. At eleven o'clock commence turning with forks or tedder, and lose no time until mid-day, when by this process it will become wilted. In this way we can begin, if the grass has been properly tedded, to cart into the barn as soon as one o'clock, always taking care to have help enough to finish carting as early as half-past four or five o'clock, after which the dew has fallen and renders hay unfit to be packed. By this process we allow from three to four hours for the out-door curing of our crop.

We are always careful not to put hay into our barn that has any water in it, but never fear the sap if properly packed. When hay is taken from the cart or wagon it should not be rolled off, and then over and over on to the different parts of the mow, but it should be pitched on to the hay-mow, and evenly distributed over the mow in even forkfuls, and each forkful packed and trodden upon. In this way the 
mow becomes solid and closely packed, which is absolutely necessary for the preservation of the hay, always remembering that the greener the hay is put in the closer it must be packed. In rainy weather let the hay be trodden upon by the men. Remember to keep the barn closed as much as possible afterwards.

In order to secure rapid drying and facilitate the process, as well as to improve the quality of hay-since the quicker it is cured, the better the quality-a good hay tedder is indispensable, of which there are several varieties.

The following cuts represent valuable implements of this kind manufactured respectively by the Ames Plow Co., Joseph Breck and Sons, and the Whittemore Brothers, all of Boston, Mass.:

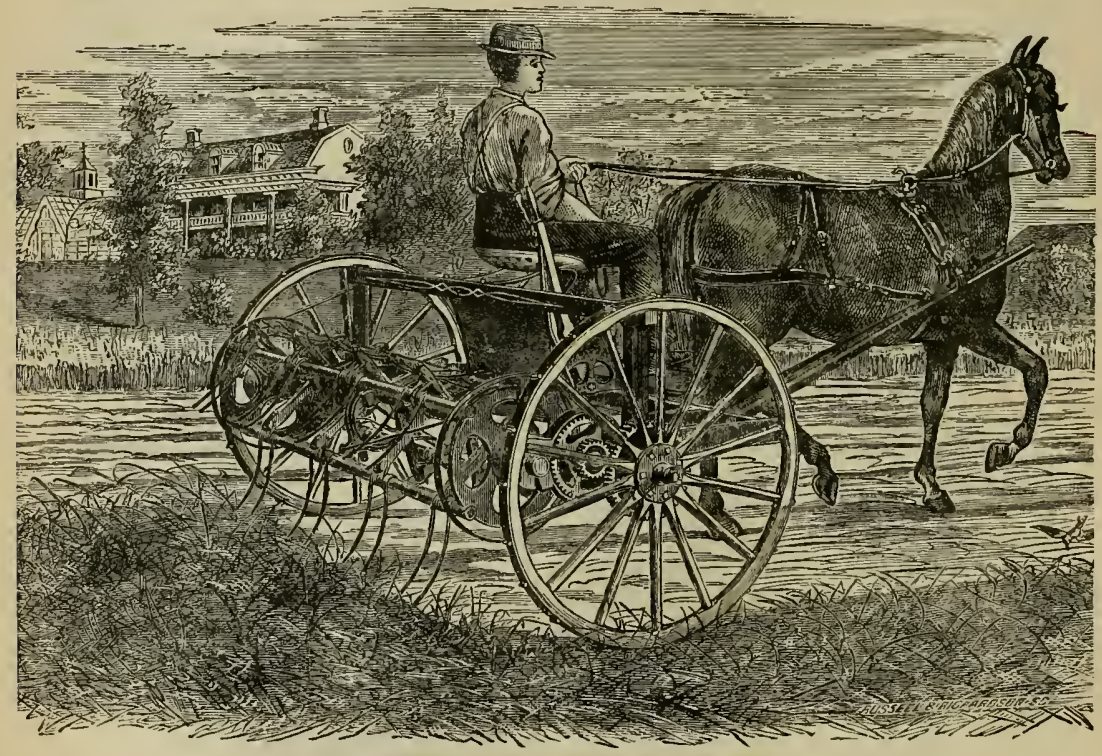

The American Garfield Hay Tedder.

The first two represented have been known to the farmers of the country generally for some time and their merits fully tested. The Mudgett tedder has been more recently introduced, but is very popular with those familiar with its working. Having never used the latter we are not able to express an opinion as to which is the best of these valuable implements, but from witnessing its operations we judge it not to be at all inferior to its predecessors.

A good tedder will toss the grass lightly, exposing it to the air and sun, and leave it in a loose mass upon the ground, under which circumstances it will dry rapidly, thus conducing to improvement in the quality of the hay, and consequently its increased value, since the sooner the curing process can be completed, the better the quality of the product. The improved agricultural implements of the present time, including mowing machines, tedders, horse-rakes, the pitching apparatus for loading and unloading hay, etc., not only facilitate the hay-curing process, and thus obviate loss or deterioration in quality by the grass becoming over-ripe before cutting, or exposure to rains after being cut, but also are a great saving of labor, 
time and expense, and the best of such machines are not only exceedingly useful, but are a necessity in the economy of every well-managed farm.

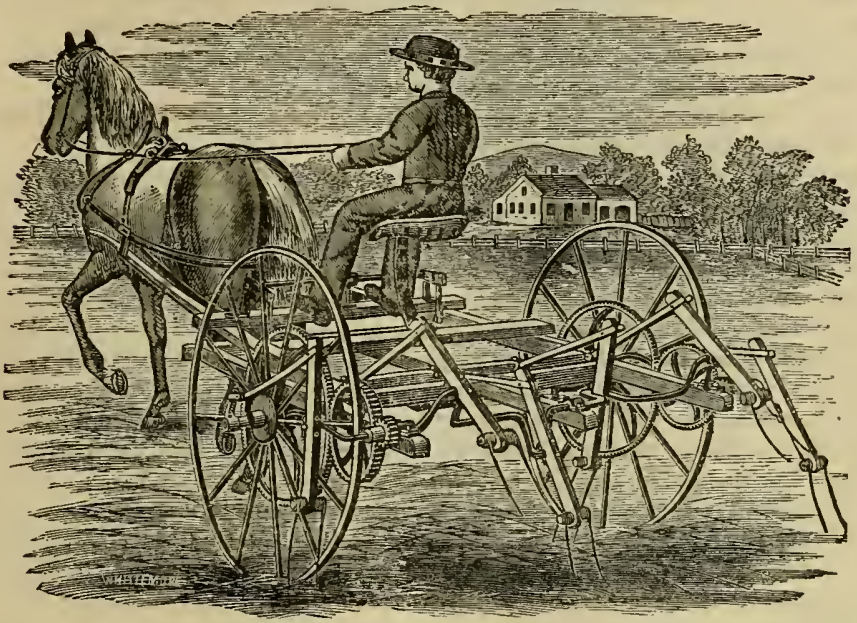

BOLLARD'S IMPROVED HAY TEDDER.

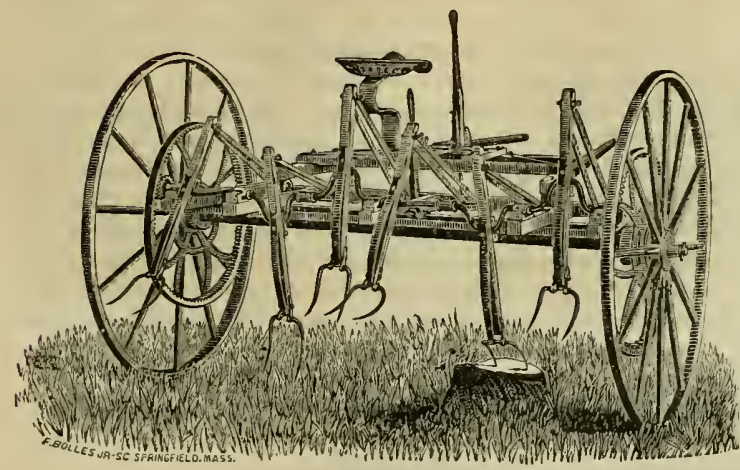

THE MODGETT TEDDER.

With respect to the height from the ground at which it is desirable to cut hay there is a diversity of opinions among farmers; some believing it is best to cut it as close as practicable, others varying from one and a half to four inches. Our experience and observation convince us that it depends much upon the soil, the season, and the kind of grass. That where the soil is dry and liable to be affected by the hot summer weather, as in upland mowing-lands, it should not be cut ciose, in order that the roots of the grass may have some protection against the scorching rays of the sun, but in low, wet lands close cutting could be practiced with less injury to the roots. As a general rule, the finer and more compact the grass, the closer it may be cut; while the heavier grass, timothy for instance, might be greatly injured by close cutting in hot, dry weather.

The Horse-rake has become a necessary acquisition to the agricultural implements of almost every farm however small The Thomas Sulky Rake, manufactured hy J. H. Thomas \& Sons, 
of Springfield, Ohio, and the Gleaner Hay and Grain Rake, manufactured by the Vermont Farm Machine Co., of Bellows Falls, Vt., are fine illustrations of this kind of machine. It is important that all mowing lands should be clean and free from everything objectionable to a mixture with the hay, such as dead stalks, fragments of brush, small sticks, \&c., since rakes of this kind collect everything from the surface; and the best time to secure this is in the spring before the grass has attained much growth.

A good horse hay-fork or loader, for loading hay on the cart, is a very desirable acquisition to the farm implements, and a great saving of labor and time.

Hay Caps.-As to the utility of hay caps there can be no question, since by them the farmer is enabled to protect his hay against the storms that frequently cause so much loss to this crop. Those who have been accustomed to their use value them highly. They can be easily made in the following manner: Procure common brown sheeting frorn fifty-four to sixty inches wide. This is torn into squares and the edges are turned down all around and sewed over a stout cord. Make a loop of the cord in each corner about six inches long, in which to insert skewers or hooks for fastening them to the ground. The cocks of hay should be made from five to six feet high, about four feet wide at the base and rather narrow at the top, and containing from 250 to 300 pounds of hay. If the sides are raked down so as to give the form described, the caps will shed the rain no matter how heavy or long-continued the showel. It is a good plan to give the cloth a good coating of boiled linseed oil, which will aid in throwing off the water. The caps are thrown over the tops of the hay-cocks and fastened to the ground at each corner by the loops, through which wire or wooden pins are inserted and forced into the ground. Te know a farmer who has had caps of this kind for his haying outfit for fourteen years, and they are still in use and in good condition.

Storage of $\mathrm{Hay}$ is a very important subject, and one in which many of our practical farmers take too little interest. The question arises, shall we put it into large, tight mows or on loose scaffolds, where the air is permitted to act upon it? If curing and keeping hay is in any respect analogous to the Chinese method of curing and storing the tea-plant, then the quicker it can be cured, and the tighter it can be stored, the better the quality of the hay. We believe that the less air there is permitted to enter the havmow, when properly cured, the better for the hay; in other words, hay that is compactly stored in large mows, other things being equal, is far superior to that having a free exposure to air. Though air is es. sential to curing hay, it is not essential to its storage. Every one knows that decay is a process of combustion, and combustion cannot occur where there is no air; hence, if the air is entirely excluded, no combustion or fermentation can take place, as is instanced in canned fruit, where the cans are hermetically sealed. There imperfectly cured hay is stored, it will generally be found that it is only those portions of the mow where air can circulate that heating and fermentation take place, and not down in the closely-packed portions where the air is excluded.

In feeding hay it is better to cut down the mow in sections, rather than feed from the entire surface, as in this way the exposure to air is less. The practice of many farmers of pitching the hay upon the barn-floor at night to remain there for the next morning's feeding to stock is not a good one, as much of the aroma of the hay is lost by exposure to air during the long interval of the night. It is the practice in some localities where the barn capacity is insufficient for hay storage, to stack the hay in the field. Though this may be a necessity sometimes, yet it should be avoided if possible, as hay that is housed is more nutritious and valuable than that exposed to the air and storms, as it must of necessity be in stacks.

Rather than stack his hay, it would be more economical for the farmer to procure a hay press, thus pressing it into bales, and by this method provide room for his hay in barns. Great saving of labor and time may be accomplished by the use of a Hay Carrier in the unloading, storage, and stacking of hay. The illustration of Clark's Hay Elevator and 


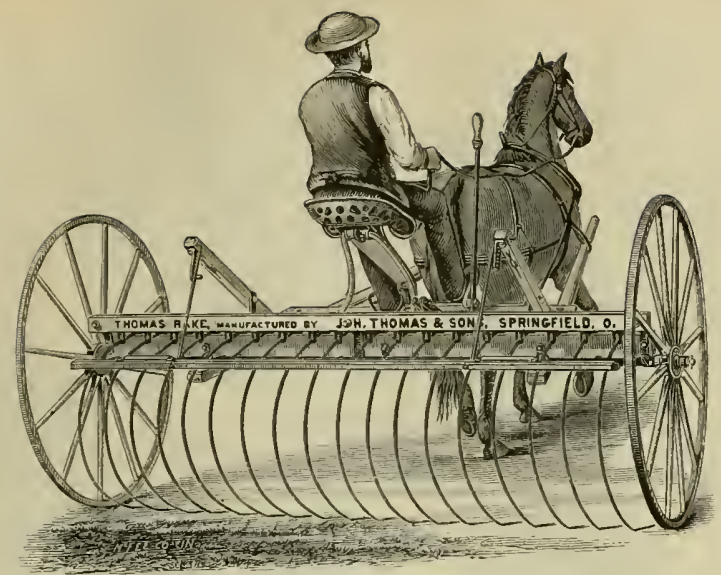

THE THOMAS SULKY RAKE.

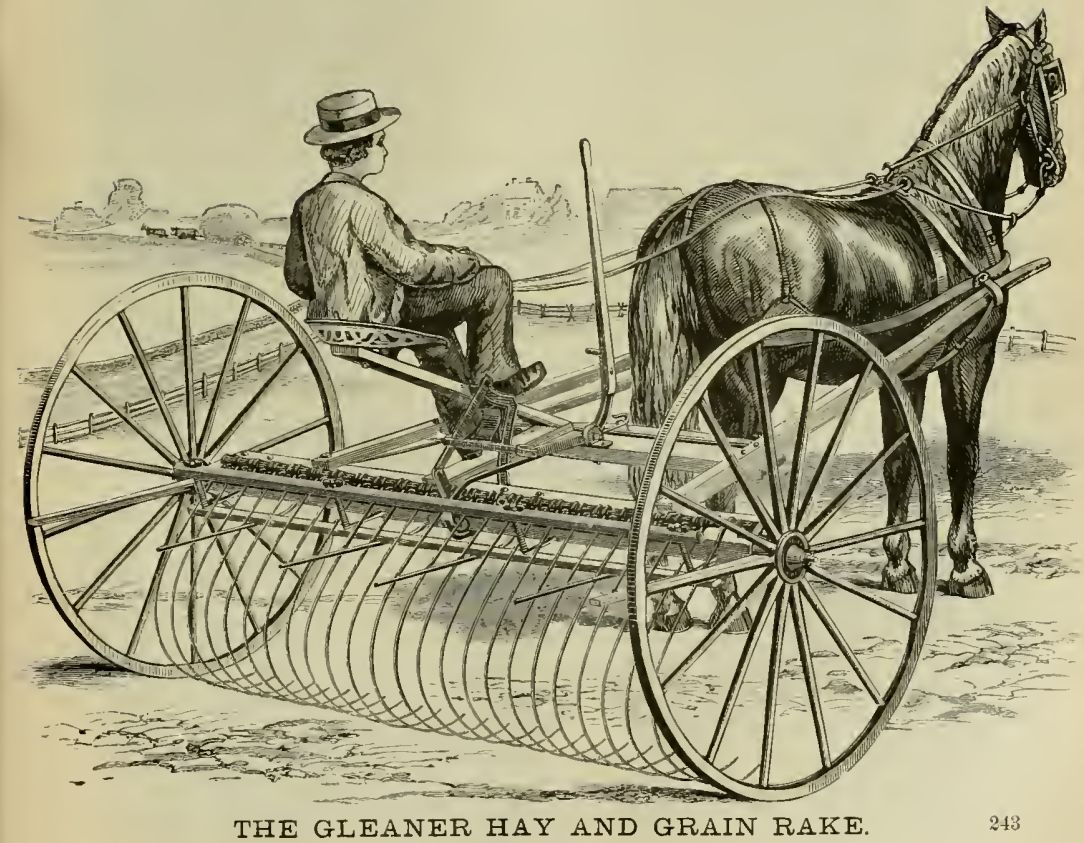




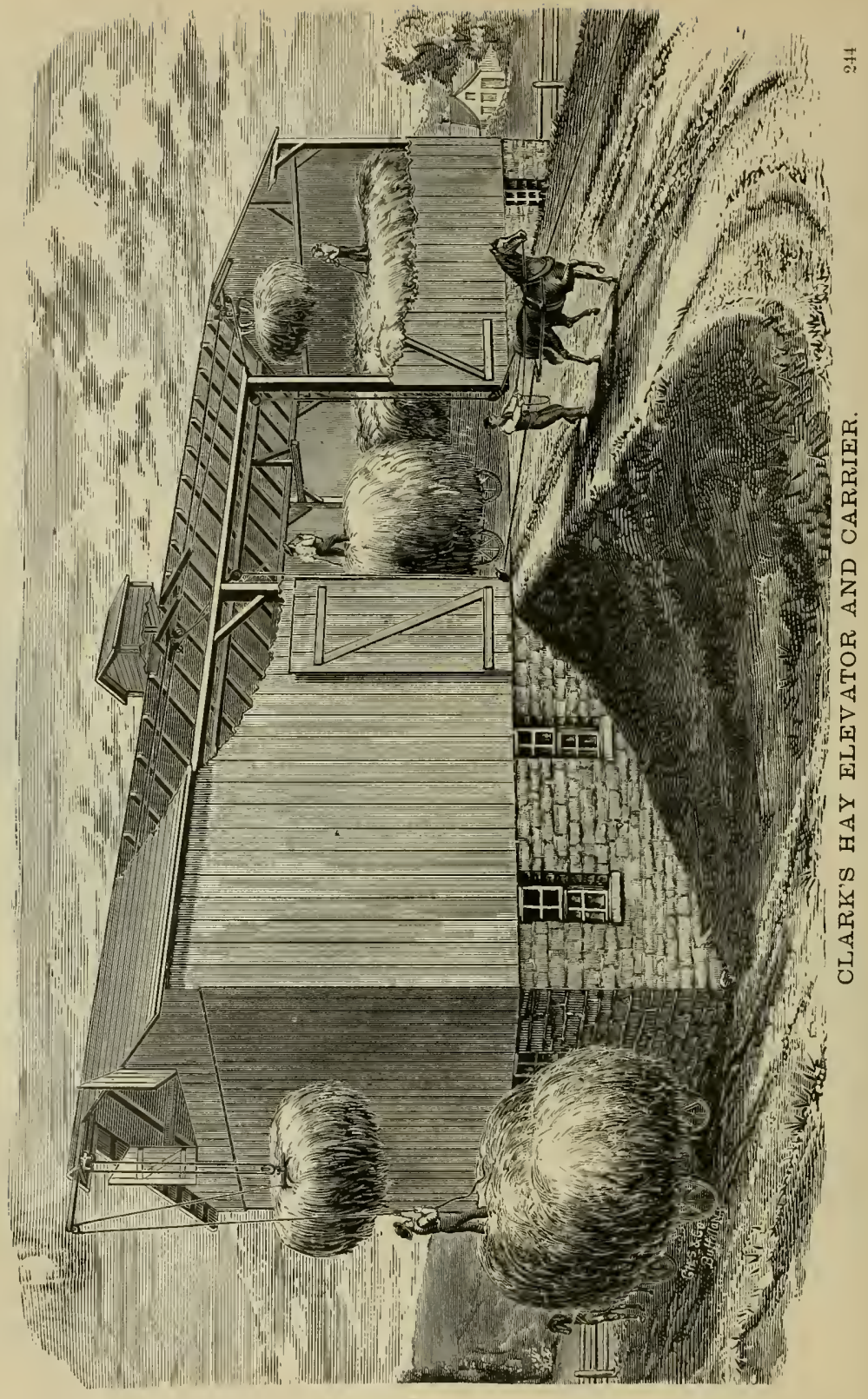


Carrier, manufactured by Clark \& Scott of Bridgewater, New York, represents a most admirable machine for this purpose. Any fork can be used with this carrier, which adjusts

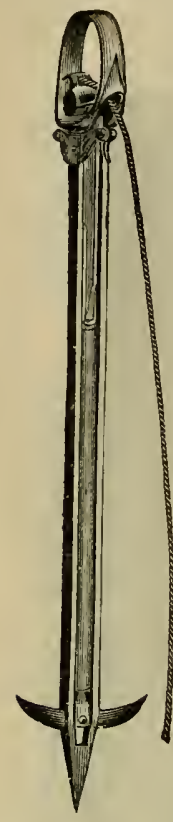
itself to any position, so that it makes no difference whether the load stands under the track or twenty feet away. It has been known to unload nineteen loads of hay, weighing over a ton each, in three hours, each load being pitched off at four forkfuls in from two to four minutes. An accurate and special test of its merits in point of time, was the unloading of 2,500 pounds of hay in a little less than two minutes.

There are so many valuable hay-forks in common use that it is difficult to tell which is best.

The following is an illustration of the Nellis Harpoon Horse Hay-Fork, from the firm of Joseph Breck \& Son, Boston, Mass. This hay-fork is simple and readily adjusted, durable and easily handled. It can be used for hay or straw, whether damp or dry, coarse or fine, and is a desirable adjunct to the haying machines. The figure at the right shows the fork with the blade closed for penetrating the hay; that at the left represents the same with blade open for lifting the hay. Grapple hay-forks are also extensively used.

Stacking Hay. - When necessary to stack hay (which should be avoided if possible), it should be done with the greatest care. The hay can remain in the cocks until quite a quantity is ready for the purpose. Before stacking, it should be turned over and that which has lain on the ground be exposed to the sun to dry out the accumulated moisture, and after ward taken to the place for stacking. For small stacks, poles are used at the center for support. The pole should be secured in the ground and be made to stand perpendicular. The hay is then packed as compactly around it as possible.

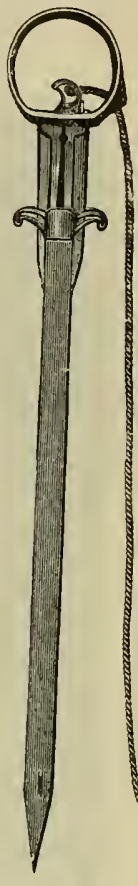

When finished, the top of the stack should be well covered with straw, carefully arranged to shed the rain. The Noyes Field Pitching Apparatus, manufactured by the Wind Engine \& Pump Company, Batavia, Mlinois, is an admirable arrangement for stacking large quantities of hay in a single stack, which can be done with great facility. Where this

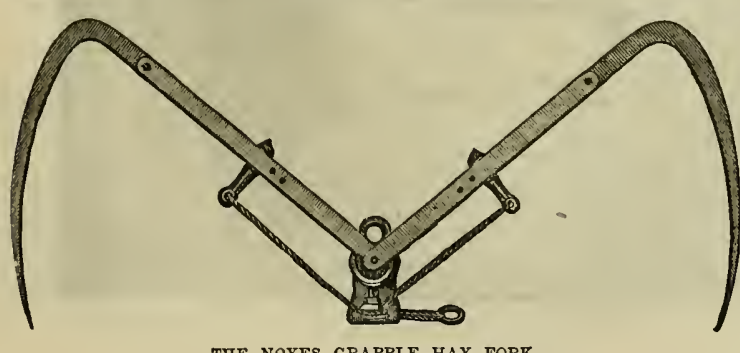

THE NOYES GRAPPLE HAY FORK, apparatus is used, stacks are frequently made so large that they do not require the pole in the center for support, the hay being packed hard in the middle of the stack, which together with its immense size, prevents its leaning. As has been previously stated,

baling hay is preferable to stacking, since it prevents waste and injury to the hay from exposure to the weather. 


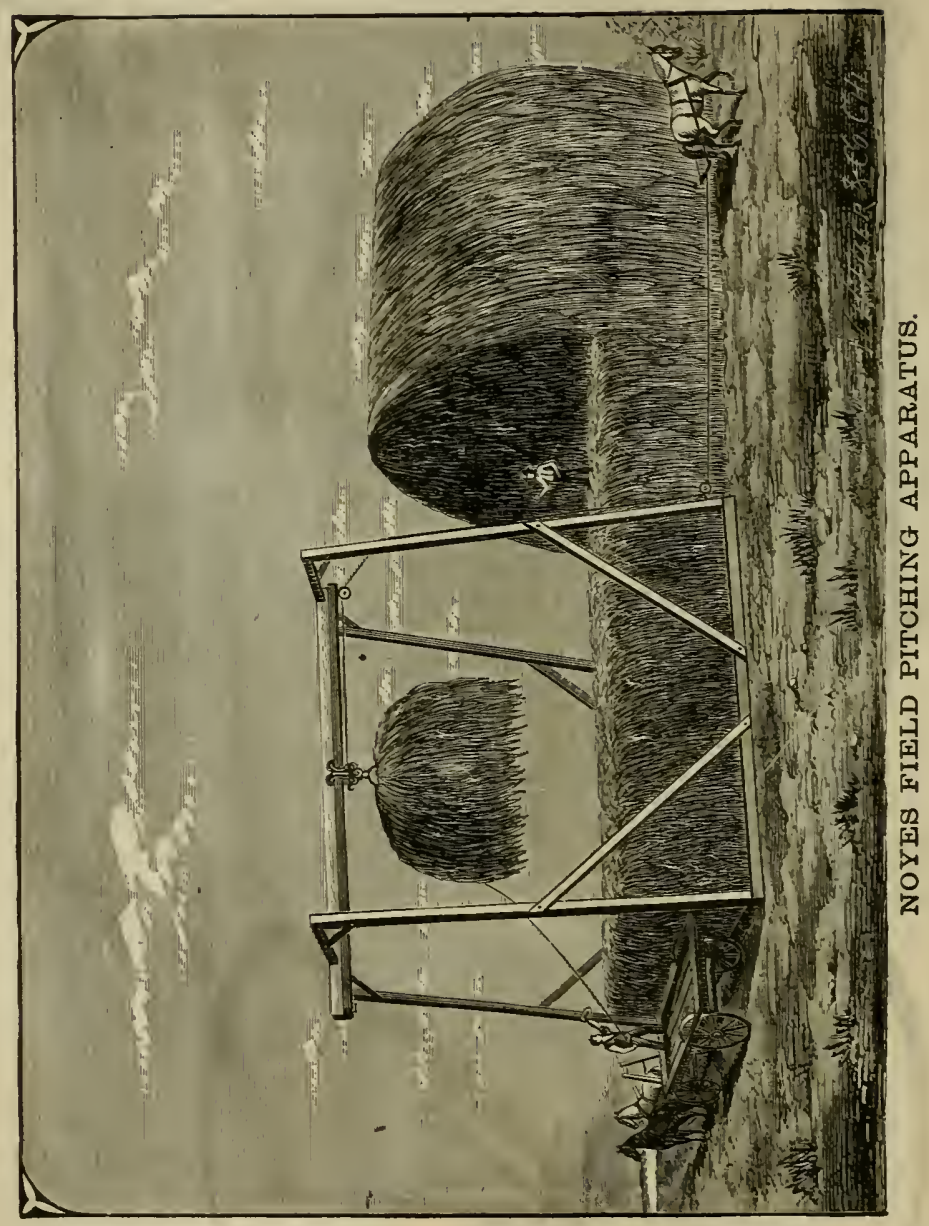


The cut at the left shows the carrier just after the pulley, to which the fork is attached, has run up on to the crooked arm, on which it rides. The carrier is now detached from the lug on the track, and has commenced to move off. On returning, the catch strikes the lug, the crooked arm is released, and the fork returns to the load.
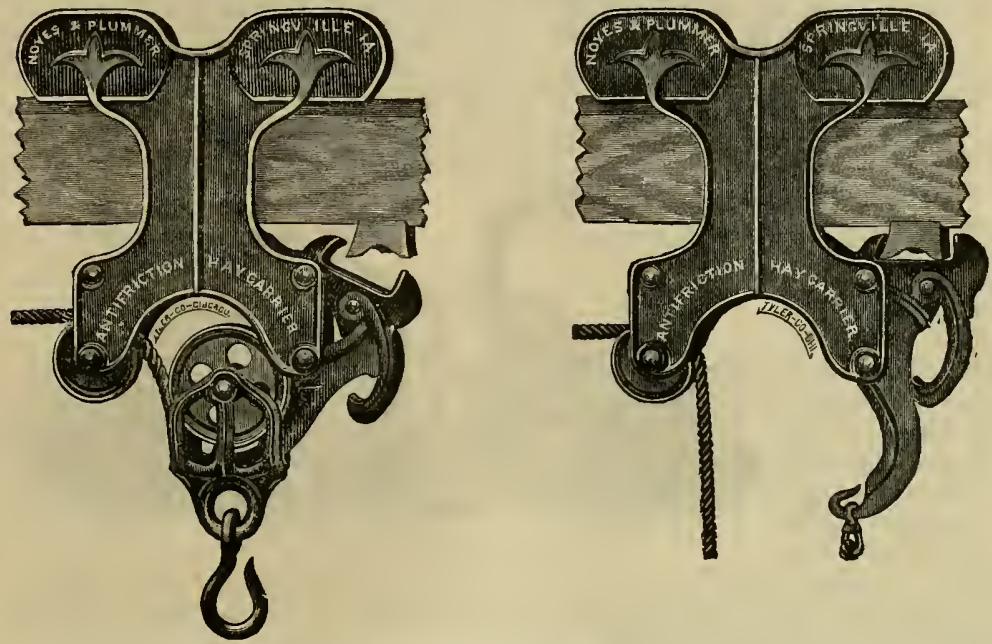

The cut at the right shows the carrier in position on the track over the load. It remains stationary until the pulley to which the fork is attached runs up on the arm, when it assumes the shape shown in the adjoining figure, and is released ready to move off. The continued pulling of the horse moves it along the track to its destination.

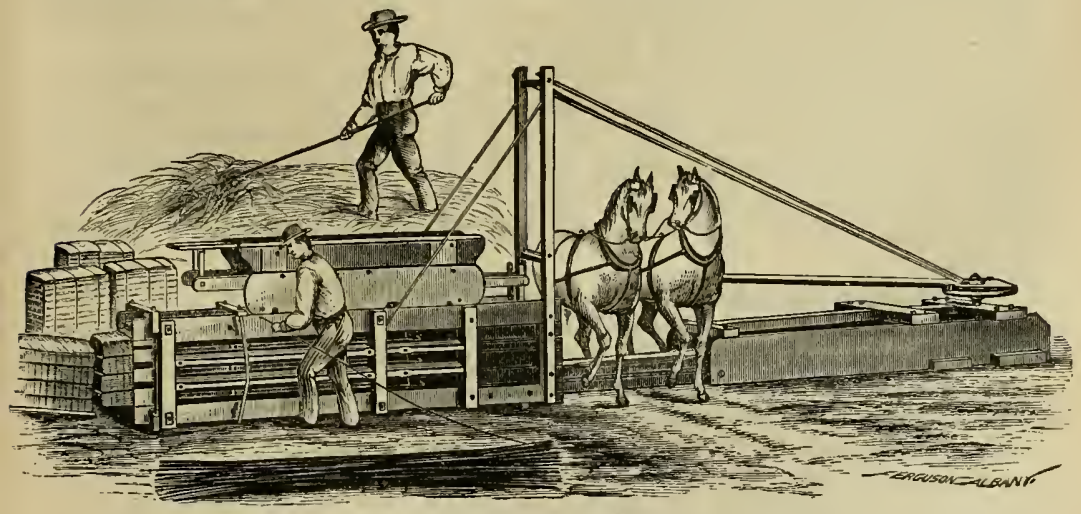

LEVER BALING PRESS.

Baling Hay.-There is great advantage, in an economical point of view, in baling hay. Where the farmer has insufficient room for storage, by baling his hay, and thus rendering it 
more compact, it may save him the necessity of building larger barns to make room for his crop, or stacking in the field, which latter would injure the quality of the hay more or less, however well it might be stacked.

The quality of the hay is also improved by baling, or rather by this means much of the aroma and consequent nutritive element of the hay is preserved which would otherwise be lost by contact with the air. By this process the bales are in a great measure rendered almost air-tight, except, of course, the external portions; besides, whenever hay is to be sent to a distant market, baling becomes a necessity to fit it for transportation.

The accompanying illustrations represent baling presses, manufactured by P. K. Dederick \& Co., Albany, N.Y. The first may be used with a one or two.horse power, requiring one man to pitch in the hay and an. other to tie the bales and assist the machine in storing them. This press also makes two pos. itive strokes, or presses two sections to each round of the horse, and the power is also doubled at the time

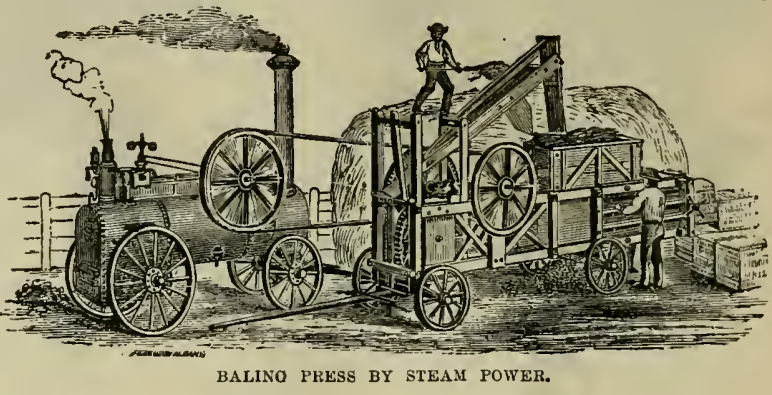
the work is done. The second is a press driven by a portable agricultural steam-engine, such as is ordinarily used for threshing grain, although the power of such an engine is sufficient for two or three presses of this kind.

Aftermath or Rowen, although not as nutritious as hay made from mature grass, and as it does not furnish that fibrin which mature grass furnishes, and consequently not desirable as food for horses and oxen, upon whose muscular system the great tax of labor is laid, still, for calves, sheep, and milch-cows, there is no forage crop that will surpass it. It approaches the nearest of anything to the green food produced by our pastures in summer for these animals, and for winter use furnishes a very desirable substitute.

By cutting the grass crop early, before the plant has become exhausted in seed produc. tion, the aftermath will have a longer time for growth before being cut, and thus a better crop secured, the great difficnlty with rowen having formerly been that it came so late in the season as to render its harvesting difficult, and hence it was often secured in an uncured condition. But with an early hay harvest, the second crop can be secured earlier, and can be cured mostly in cocks, which requires but little extra labor, and the former difficulty obviated.

Cultivating Grass Seed.-The first sowing of grass seed in this country, by our forefathers, was made from seeds of grasses collected from the barn-floor, and from under hay. stacks. This was sown with the chaff, they having no available means of separating such small seeds from the chaff. This method was practiced for a long time. Their next step towards progress in this branch of agriculture, we are told, was to sow a small quantity of seeds of those grasses they thought most desirable, upon the ground in which they had culti. vated their hoed crops. We of the present age have improved upon their method, but we are far behind the English in the variety of grasses cultivated: we should not only sow a greater variety than we now do, but more abundantly also. To secure the best results, the land should be well plowed and manured, the soil well pulverized, and pains taken to keep the land free from other grasses than the variety desired for producing the seed. When the 
seed is ripe, it will be known by the changed appearance of the heads of the grass. Care should be taken to cut it before it is so ripe that it will easily shell out from the heads, as much will thus be wasted. It should be cut when the dew is on, to prevent the loss of seed. After partially dryıng, it can be arranged in the small sheaves which should be placed where they can become perfectly cured, as it can remain unbound until wholly dry, but it will need to be handled with the greatest care to prevent the seed shelling out, as it falls very easily. It can be threshed with any implement used for threshing grain, and cleaned with a seedcleaner, or common fanning-mill with a fine screen. If the stalks are too green while in the stack, the hay will heat or ferment, and the vitality of the seed will be liable to be thus destroyed. This injury to the seed is not always apparent by a change in its color, conse. quently stale and worthless seed is often put into the market and sold for good. There is no certainty in this respect, except to test a sample before sowing the whole, and if these germi. nate well, there is a probability that the seed is good. The stalks producing the seed are woody and tough, yet by mixing with other food, and cutting, they may be utilized, but have not sufficient nutriment to be fed alone. Horses will eat them in this manner better than other stock.

After sowing the seed, and exercising care to prevent the introduction of other varieties not desired, it is a very easy matter to harvest a good crop of grass seed for several seasons, by simply a liberal supply of top-dressing. Many farmers are indifferent to extra pains in raising this product, and give it no more attention than the care necessary in harvesting it, but it will well repay any farmer to take special pains to produce the best crop of grass possible for this purpose, as the crop produced from such seed will be greatly superior to that where no such pains are taken to secure the best results. The more luxuriant the crop, the better the seed produced from it, being the general rule.

Fall Grazing of Mowing Lands. - The practice of turning the cattle on the mowing lands to graze after the aftermath has been cut, and the grass given a little time for subsequent growth, is very common, and although the testimony of nine farmers out of ten would probably be, that this practice is injurious to the grsss crop of the following year, yet they continue to do so, justifying the custom on the plea of necessity. This is especially true in New England, and we believe it to be one of the main causes of the deterioration of the grass crop there, the grass being often grazed so closely as to leave the roots exposed to the extreme cold of winter, which are thus frozen and killed; besides, where the soil is moist the roots are often pulled up by the grazing and an equal injury done in this way, while the tramp. ing hoofs of cattle upon the turf is an evil nearly as great. The roots need the protection of the grass for warmth through the winter in order to secure an early and vigorous start in the spring. Mowing lands are unquestionably better where never grazed, but when practiced, it should be early in the autumn, to provide for an after-growth for root protection during the winter, and to store up the elements of a thrifty growth in the following spring. Too close feeding is injurious at any time and should never be permitted, as it will cause the lands to deteriorate and the grass to run out in time. An experienced farmer in Massachusetts says: "It is now more than twenty years since I have allowed any kind of domestic animal to feed upon our mown land, and my opinion previously has been fully confirmed by my experience. It is a decided benefit to let the after-growth remain upon the land; it is a protection from summer's drought and winter's cold. Some of my neighbor's are following my example." And another: "I sometimes feed off my after-grass. When I do feed it off, I take good care to feed it early and leave a good growth to protect the roots of the grass from frost in winter. I think it an injury to feed; mowings will last longer not to be fed at all, and the land when broken up will produce a better crop of corn or potatoes than if fed."

Mowing lands should never be pastured in the spring, as the soil is so damp then that the roots of the grass are easily pulled up by the cattle, while their tramping hoofs are also 
very injurious to them at that season. Sheep are less injurious in grazing mowing lands than cattle, but we beheve the farmers generally would find it more profitable, in the end, to keep such lands entirely free from all grazing stock. The grass crop is one of great importance, since it lies at the very foundation of all prosperity and success in agriculture.

Grass is said to be "king among the crops of the earth," and more land devoted to its cultivation and more money value realized from it than any other product, not excepting eren cotton, which has been called, at the South, king. The human race existed for ages without cotton, but never without grass. The statistics of nations prove that grass is the most essential and remunerative of all crops. In view of its great importance, anything that will improve this crop and prevent its deterioration ought to receive the thoughtful attention and consideration of all farmers.

Rolling Mowing Lands and Pastures.-It is a very good practice to go over the mowing lands with a roller in the spring after the frost has left the ground, as by this means the roots that have been thrown out of the soil, by the action of the frost, are pressed into the soil again, where they will soon become firmly settled, and thus their drying up is prevented, as they otherwise would be liable to do. Experienced farmers have found this practice extremely valuable to their grass crop, and to amply repay for the labor attending it; some even value the result so highly, that they treat their pastures in the same way, whenever the surface is sufficiently even, and unobstructed by stones to admit of the use of the roller.

\section{MANURING MOWING LANDS.}

$\mathrm{I}^{1}$ is said that New England farmers are in the habit of robbing their grass lands to feed their hoed crops and arable lands; which has been in a great measure, we think, true until a few years past, since which time more attention has been giren to fertilizing, in various ways, lands designed for mowing. The improvement in this respect is however gradual, and even now there are many farmers who adhere to the old-time system of farming, and consider the grass crop of secondary importance, letting it take care of itself, year after year, with little or nothing given the land to meet the constant drain made upon it in producing its annual crop, while the manure made from it all goes toward the improrement of the other crops. Grass depends much upon the air in its material for growth, but also much upon the soil, and the elements it extracts from the soil in its growth should be returned to it, or exhaustion of soil will follow, and a consequent loss to the grass crop. No good farmer would think for a moment of cultivating his corn, or other hoed crops, without a liberal supply of manure, yet grass crops require the use of fertilizers just as much as any other, both for mowing lands and pasture, and unless such fertilizers are given the soil producing them as will restore the elements taken, there is no reason for expecting anything bat a failure in grass crops, the same as that of corn or any other cultivated crop, treated in the same manner. The analysis of hay shows over five per cent. of earthy matter, while dried clover yields from seven to nine per cent., yet we will readily see that where land produces at the rate of three tons per acre, this element is taken off to the amount of 300 pounds per acre per annum; what soil could long endure such a drainage without injury? The potash, soda and other salts requisite to the growth of the grass are thus taken from the soil. Grass land must be renewed with suitable fertitizers, or exhaustion must of necessity follow; there can be no other result. in the natural course of things. Commercial manures, such as ashes, lime, crushed bones, gypsum, and guano, are all good for the grass crop, gypsum being especially 
valuable to curver. To these should be added animal or vegetable manures. Some farmers apply these fertilizers in the fall, sume in the early spring when the grass just begins to start, others apply them just before the first rains fall after the grass has been mowed, that the rain may wasl the soluble matters into the roots; in such a case, the portions that remain un. dissolved are soon covered by the growing vegetation, and are absorbed more gradually. The best time for applying barn-yard manure is in the fall, since the frost will crumble the lumps and render them partially pulverized by the spring, while the manure serves to protect the roots through the winter, and its warmth aids in securing an early and vigorous start in the spring. The snow, in dissolving, aids in carrying the fertilizing elements down to the roots, which is a very great advantage that cannot be secured by applying it in the spring, while the soft soil will also cause the turf to be badly cut and injured by the wheels of manure spreaders or other implements for carting, if applied in the spring. The following is the opmion of Mr. Alex. Hyde, of Massachusetts, respecting the top-dressing of mowing lands:-"We have found a compost made of muck and leached ashes, in proportion of six or eight bushels of muck to one of ashes, an excellent fertilizer for grass. The wood ashes furnish the in. organic food in great variety, while the muck supplies the vegetable matter, and renders the soil light and porous, ready to absorb the gases of the air, and furnish them to the grasses as they may be demanded. In case the meadows are naturally cold and wet, abounding already in clay or peat, we should substitute, for the basis of the compost heap, sand instead of muck. Sand alone, scattered upon a peaty meadow, has a wonderful effect in warming the land and inducing the growth of sweet, nutritious heroage. Indeed, we have found that meadows, well drained, after they have been mucked for a series of years, are greatly benefited by a top-dressing of sand, or better still by a coating of alluvial soil which is to be found on the river-bank. This alluvium contains not only sand, but the disintegrated granules of the various rocks and soils that the river has brought down from miles above. We have used this alluvium lately in the compost designed for top.dressing, and have been much pleased with the result. When it cannot be obtained easily, the wash of the highway, or leaf-mold from the forest, answers a good purpose. The latter is particularly rich in all the elements of vegetable nutrition, and our forests can well spare some of it for the benefit of the meadows.

On a clover lay plaster operates most favorably. Spread broadcast early in the spring, at the rate of one hundred pounds to the acre, it increases this leguminous crop greatly. This great result from so small a quantity cannot be attributed solely to the increase of plantfood furnished by the two main elements of plaster, sulphur and lime, although, as clover contains both these elements, they doubtless contribute directly to its nourishment. But plaster is also a great absorbent, and its efficiency must in a measure be attributed to its power of retaining the ammonia of the air, and furnishing it to the clover and other plants as they may demand. Plaster alone cannot be relied upon to keep our meadows in heart for a series of years. Those who have seen its magical effects for a year or two, and have supposed that they could sell their hay and still keep up their meadows by spreading a little plaster upon them, have found themselves mistaken, and have complained that their fields became plaster-sick. The sickness was simply starvation for the want of a greater variety of food. Plaster, from its own elements, and by absorption from the air, can furnish only two of the many inorganic elements which enter into the composition of all our grasses. Clover is doubtless more benefited by its action than the other grasses, as the ash of clover shows over thirty per cent. of lime. Plaster is found to act with great efficiency in connection with wood-ashes, as they supply the inorganic elements in which plaster is deficient.

Farmers may differ about the action of plaster, but in the efficiency of barn-yard manure they are all agreed, and in the production of this, hay is the main reliance. Why should not more of this manure be applied to the reproduction of hay, and thus the law of action and reaction be carried out? The more hay, the more manure, and the more manure, the more hay." 
Many farmers of experience consider that liquid manure is the best application for grass lands, and recommend it where its application is not attended with too much expense and trouble

Salt is a valuable fertilizer One farmer of large experience says: "Salt is the only topdressing I have ever used on grass-land 1 have bought a car-load of it in a year; but some years I do not use more than fifty bushels ; this is refuse salt." The quantity used per acre depends much upon the nature of the soil ; from three to sixteen bushels being often used.

Before applying any kind of fertilizer it is always well to determine, if possible, by observation or experiment, what particular element of plant-food the soil is deficient in, before deciding which is the proper fertilizer for a given crop. The farmer must learn it for himself, and be governed accordingly. Barn-yard manure is supposed to contain all the elements of plant-food, and hence is one of the most valuable of fertilizers.

The use of manure frequently, in moderate quantities, is attended with better results than larger quantities at longer periods. When good results are not attained with repeated applications, it is often better to plough up the turf, and seed down.

Lands that are enriched by the overflow of water, such as the lands along a river-bank, require but little or no extra fertilizing, as the matter deposited in this manner is sufficient for that purpose.

\section{RENOVATING GRASS LANDS.}

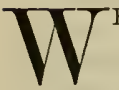

$\mathrm{E}$ have previously alluded to the great importance of feeding or top-dressing grasslands, hoth the mowing lands and pastures, that we may restore the fertilizing elements abstracted from the soil in producing the annual crop of hay, or by constant grazing, and thus not only improve them yearly, but prevent them from degenerating and becoming exhausted, -or, as farmers often express it, "run out " or "worn out." These top-dressings may be ashes, lime, guano, plaster, crushed bones, salt, or manure, etc.; but it occasionally happens that all top-dressing seems to fail of the desired effect, especially in mow. ing lands, which may be the result of grubs eating off the roots, or its having hecome turf-bound. In such cases, it is usually best to plow up the turf and re-seed with grass, after well pulver. izing the soil, and giving it an abundant supply of manure. Many farmers resort to a rotation of crops with good results.

Drainage is of the utmost importance to wet lands, and where this has been neglected, should be attended to at once. Many almost valueless lands, as far as grass production is concerned, will, with proper drainage, become the most valuable and productive. Clover is a wonderful recuperator of the soil, and when lands have become exhausted, will often restore the elements of fertility to the extent of entirely transforming it into the richest and most pro. ductive of land. We take the following extract from Dr. Harlan's work on "Farming witb Green Manures," which may prove a benefit to many farmers who wish to renovate their grass-lands without plowing :

"It is a very common practice among agricultural writers to advise all persons having large farms which are in a very poor condition to sell one-half or two-thirds of their land, and apply all the money they receive in manuring, and improving the balance of their property.

In some cases this may be the most prudent course to follow, but, as a general rule, I am opposed to this advice for two very good reasons :

First, you can get but very little per acre for your poor fields; and, secondly, if yor improve your property with judgment, you can enhance its value so rapidly, that in seven ol eight years it will be worth double or treble its former valuation. 
To begin your improvement, take the old field, about half a mile from the house, and which is now covered with thin yellow grass, and a mellow soil about one or two inches deep, produced by many years of exposure to the weather.

It has never been plowed since you knew it. And, I beg you, do not plow it now at the beginning of your efforts to make it better: Let me show you what a coating of fine mellow earth is worth upon the surface.

In Egypt the annual overflow of the Nile deposits on the land a thin stratum of very fine soil, which amounts to only four or five inches in a century. This yearly settling, which is only the twentieth of an inch in thickness, of almost impalpable dust, keeps the farms forever rich and productive. The Egyptians do not plow this precious coat under, but sow the seed on the moist ground as the waters subside, and then, if possible, they drive sheep and hogs or goats over it, to press the seed into the soil.

We should all learn a useful lesson from their example and experience. We should not plow down the only part which the air has enriched, by mingling and uniting with it for so many years, but early in the spring we should harrow as many acres of the old field as we can sow with clover seed, at one peck to the acre. After the seed is sown, we should roll the ground and sow one or two bushels of plaster per acre.

The principal roots of all plants must be near the surface, that they may feel the life-giving influence of air and moisture, or the soil must be loosened by nature or by tillage, that the atmosphere may penetrate even to the deepest fibers of vegetation. Hence the reason that plant-food acts so well upon the surface, and that all seeds germinate more quickly, more naturally, when covered by only one or two inches of soil. But these great truths must not be misunderstood. Though the soil must be loose, the finer the seed, the greater the necessity when planting or sowing of pressing, with the hand or foot or roller, the earth into close contact with the grain.

I remember a little incident which will illustrate this subject and fix it in the mind. An old-sea captain who lived in our neighborhood tried every year to raise for himself a little tobacco. He prepared a little patch of ground with the greatest care. The surface was as fine and rich and mellow as he could make it. Then he sowed the seed and raked it over once more very gently.

Yet, much to his surprise and vexation, only a few stalks grew each year. But, one spring, after the little bed had been sown with all the usual care, some fellow, to worry the old captain, went secretly on it and tramped and tramped, and danced and tramped it, till it was, to all appearance, as hard and solid as the most frequented public road. The poor old man gave him a seaman's blessing, whoever he might be, and left it to its fate. But on his next visit to it he was astounded to see the whole bed covered with vigorous plants of tobacco. It seemed that every seed had grown. He had a grand crop. After that he could always raise tobacco. He tramped the ground himself after the seed was sown.

Well, to return to our old field. If the clover should grow five or six inches high by the middle of August, give it a half or a whole bushel more of plaster per acre. The second year you must treat it in the same way, and if the clover is thin on the ground, sow more seed, and again roll it well. Do all this the third and fourth year, if necessary. After this, it will re-seed itself, provided you continue the plaster each year.

Here is a practical illustration of this plan, which I know to be a fact.

A person bought a very poor farm near the southern boundary of Pennsylvania, and tried to raise grain upon it in the usual way. But nothing grew large or strong enough to produce seed. Fortunately, he did not sacrifice the property by selling it at a very low figure, as many would have done. He sowed every acre of it with clover seed, and plastered it every year. For a living he followed the profession of an auctioneer.

About seven or eight, or more years, the clover grew upon his farm, undisturbed by 
plow or hoof of any kind. Then he concluded to try his hand again at farming. Many of his neighbors gathered to see the first plowing after so long a rest from tillage.

An old farmer who was present assured me that the soil turned over eight or nine inches deep, as black as your hat, and as mellow as an ash-heap.

More than fifty years have now passed since that occurrence, and the farm has the reputation of being rich and productive to the present day."

Many pasture-lands, especially in New England, have been so constantly grazed for years without renovating, - the general theory having been, that pasture-lands were manured suffciently by the animals feeding upon them, - that they have become worn out, the finer and nutritive grasses having gradually disappeared and the thin soil on which they subsisted covered with moss or worthless weeds, while many are overgrown with briars and bushes. Where a pasture is bound out and mossy, and the grass-roots unhealthy, it is a good practice to run a harrow over the surface, first in one direction and again in another, cutting it up into little squares. This process will loosen the soil, after which sow a mixture of grass-seed suited to pastures, with a little white or alsike clover, and then give it a light top-dressing of plaster or some other kind of fertilizer. Sheep are great renorators of the soil, and it is to be regretted that sheep husbandry is not more generally practiced in some portions of the country, especially in New England.

The editor of this work had an old pasture several years ago, which had been worn out by being fed by dairy cows, year after year for time out of mind. Bushes and briers had come in; huckleberry bushes, alders, mosses, and every sort of botanical specimen abounded, abundant enough to delight the heart of a botanist. It was the worst pasture, in that respect, that could be found. It was so rough and rocky that it could not be plowed, and the question was, what should be done with it? It contained about thirteen acres. The bushes were cut down, and then more than a hundred sheep were put in, which was a larger number, of course, than the pasture could possibly carry. But they were not expected to live entirely on what the pasture furnished, and could not, if it had been expected of them. Cotton-seed meal was bcught, at the rate of thirty-five or forty dollars per ton, and the sheep had their rations of this, - about a pint for each sheep at each feeding, - every morning and night, which they greatly relished, and which seemed to agree with them remarkably. In fact, they liked it so well that the moment the dispenser of their rations was seen by them in the pasture, they would come bounding to him from all directions, no matter how far away, call. ing in sheep language for their breakfast or supper, as the case might be.

The result was that they clcaned out absolutely every brier, and every sumac-bush in that pasture, besides many other shrubs, but not the huckleberry bushes. They could not be induced to eat these. They covered the pasture with manure. It was a delight to see the dress. ing they gave it. They went through the following winter in good condition, and the next spring twenty or thirty less sheep were put in the same pasture, and they went through the second year doing exceedingly well. The original plan was to run them three years in this pasture, which, if it had been carried out, would, without doubt, have entirely changed its character by that time, judging by the very great improvement made during the two years.

Mr. C. F. Hewes, of the State of New York, gives his experience in renovating his pasture lands, as follows:-

"My first experience with salt on grass was accidental, but it worked so well, I made it a practice. It so happened that after sowing on iny plowed ground all I desired, I had several barrels of salt left, and quite a quantity of unleached ashes, and as my pasture, which had never been plowed, and which I was quite averse to disturbing for many reasons, was badly "run out," producing little grass, but many weeds, and covered with moss, I determined to mix the salt and ashes, and spread them plentifully as far as they would go. Com. 
mencing on the farther side, I spread it over about half the lot. The result surprised me. The entire field was fully in view from my house, being on a gentle slope pitching towards the house. The great difference between the dark green color of the grass, abundant upon the ashed and salted part, and the color of the weeds and scanty supply of sickly grass upon the other, was as marked as between pink and scarlet, and when the vegetation upon the undressed part was burned up by the summer drouth, that upon the other retained its color, and grew luxuriantly until the hard frosts came. Upon close inspection, I found few traces of moss, all of which at last disappeared. I was so well pleased with the result, that the next spring I gave the other part the same kind of dressing, and so continued to dress (as far as I had ashes to do it with) every year, until the pasture was completely rejuvenated - "as good as new."

Due credit must, of course, be given to the ashes - one of the best fertilizers for that kind of soil (yellow loam) - but to the salt belongs the chief credit of resisting the drouth, exterminating the soil vermin, and "making latent fertility available."

Another farmer who has had experience and observation in England, says: - "Some farmers say, 'plough the field,' but in England, where old pastures are seldom broken up. I have known extraordinary results from top-dressing with crushed bones, more particularly on the large dairy farms in Cheshire. I am sorry I cannot give you the quantities. A neighbor of mine has harrowed an old, worn-out pasture, dressed with a liberal coating of barilla ashes, from six to seven cords per acre, and sowed white clover and rolled it. It came out a beautiful pasture. The brush harrow and roller applied to all grass land in the spring, will amply repay for the labor."

It is a well-known fact, that the permanent clay-soil pastures of Cheshire, in England, have been impoverished to the extent that it became necessary to renovate them with crushed - bones. The application of this fertilizer in a very short time so improved the lands, that it bronght up their value, in many cases, more than a hundred per cent. Milk contains considerable phosphate of lime, and bones, which are mostly of the same material, impart this element to the soil and consequently to the grass it produces. Ashes and salt are also of great value in improving pasture land, the results of which have been stated, the latter being especially adapted to soils in localities that are not supplied, from a nearness to the sea: Liquid manure is highly valuable to grass lands, but should be applied just after a rain, or just before the rain commences, that it may penetrate to the roots and not be lost by evapora. tion. It is better to apply a moderate quantity of fertilizer of any kind frequently, than a large quantity at long intervals. When applied at the time of being grazed by cattle, care should be taken to use only those that will not have an injurious effect if eaten. Very fine cattle have sometimes been poisoned in this manner. On lands that are not easily washed, this might be avoided by applying fertilizers late in the fall, or early in the spring, before the pastures are ready for grazing; or just before a heavy rain, would also answer the purpose as well. The rain would soon dissolve and carry the fertilizers down to the roots of the plants and into the soil.

The following table gives a summary of some of the results obtained by Mr. J. B. Lawes, at his noted Experiment Station, in Rothamstead, England, and extending over a period of twenty years, showing the produce per acre of hay on an average of the first ten years, of the second ten years, and the total period of twenty years. 


\section{Experiments with Different Manures on Permanent Meadow Land.}

The land has probably been laid down with grass for some centuries. No fresh seed has been arti. ficially sown within the last forty years certainly; nor is there record of any haring beeen sown since the grass was first laid down. The experiments commenced in 1856, at which time the character of the herbage appeared uniform over all the plots. Excepting as explained in the table and in the foot-notes, the same description of manure has been applied, year after year, 10 the same plot. (Area under experiment, about seren acres.)

\begin{tabular}{|c|c|c|c|c|c|c|c|}
\hline \multirow[b]{2}{*}{ Plota. } & \multirow[b]{2}{*}{ Manore, per Acre, per Annum. } & \multicolumn{6}{|c|}{ Produce of Hay per Acre, 20 Years, 1856-1875. } \\
\hline & & \multirow{2}{*}{$\begin{array}{c}\begin{array}{c}\text { First } \\
\text { Period. } \\
\text { Lbs. } \\
\mathbf{5 , 5 3 8}\end{array} \\
\end{array}$} & \multirow{2}{*}{$\begin{array}{c}\begin{array}{c}\text { Second } \\
\text { Period. } \\
\text { Lb8. }\end{array} \\
\\
4,348\end{array}$} & \multirow{2}{*}{$\begin{array}{c}\begin{array}{c}\text { Total } \\
\text { Periods. } \\
\text { Lbs. }\end{array} \\
\mathbf{4 , 8 2 4}\end{array}$} & \multicolumn{3}{|c|}{$\begin{array}{l}\text { Daration of } \\
\text { Periods. } \\
\text { Years. }\end{array}$} \\
\hline 1 & 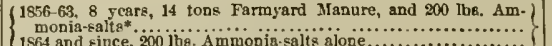 & & & & 8 & 12 & 20 \\
\hline 2 & 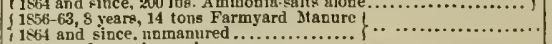 & 4,804 & 3,662 & 4.131 & 8 & 12 & 20 \\
\hline 3 & 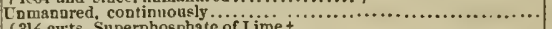 & 2.531 & 2.236 & 2.383 & 10 & 10 & 20 \\
\hline $4\left\{\begin{array}{l}1 \\
2\end{array}\right.$ & 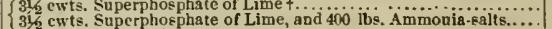 & $\begin{array}{l}2.732 \\
4.440\end{array}$ & $\begin{array}{l}2,384 \\
3,414\end{array}$ & $\begin{array}{l}2.527 \\
3.837\end{array}$ & 7 & $\begin{array}{l}10 \\
10\end{array}$ & 17 \\
\hline 5 & 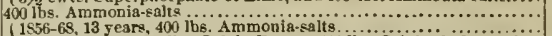 & 3,420 & $\begin{array}{l}3,414 \\
2,4 \pi\end{array}$ & 2.946 & 10 & 10 & 20 \\
\hline$\ddagger 6$ & $\left\{\begin{array}{l}\text { 1s69 and since, } 300 \mathrm{lbs} \text {. Sulph. Potass, } 100 \mathrm{lbs} \text {. Sulph. Soda, } 100 \mathrm{lba} . \\
\text { Sulphate Magnesia, and } 316 \text { crts. Supherphosphate. }\end{array}\right.$ & 3,425 & 3,502 & 3,452 & 13 & 7 & 20 \\
\hline 7 & 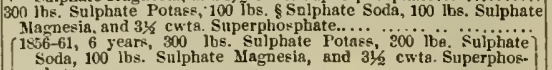 & 3,797 & 4,118 & 3.958 & 10 & 10 & 20 \\
\hline$\ddagger 8$ & 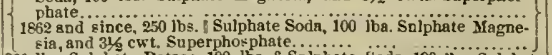 & 4,027 & 3,098 & 3,377 & 6 & 14 & 20 \\
\hline 9 & 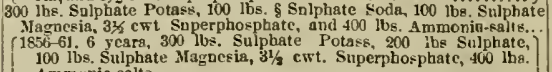 & 6,002 & 5,421 & 6.711 & 10 & 10 & 20 \\
\hline$\ddagger 10$ & 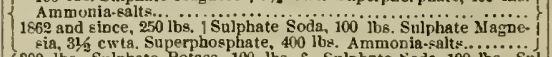 & 6,222 & 4,725 & 5,617 & 6 & 14 & 20 \\
\hline & $\begin{array}{l}300 \text { lbs. Sulphare Potass, } 100 \text { lbs. } 5 \text { Sulphate Soda, } 100 \text { lbs. Sul- } \\
\text { phate Magneaia, 36, cwta. Superphorphate, } 800 \text { lbe. } * * \text { Am- }\end{array}$ & & & & & & \\
\hline 11 & 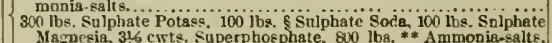 & 6,913 & 6,006 & 6,459 & 10 & 10 & 20 \\
\hline 12 & 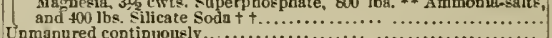 & & 6.909 & .993 & 10 & 10 & 20 \\
\hline 13 & 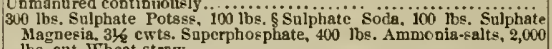 & 2,808 & 2,564 & $2,6 \div 6$ & 10 & 10 & 20 \\
\hline 14 & 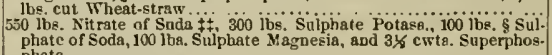 & 6,185 & $6,6 \pi 8$ & 6,432 & 10 & 10 & 20 \\
\hline $\begin{array}{l}15 \\
16\end{array}$ & 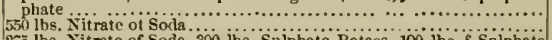 & $\begin{array}{l}5,944 \\
4,038\end{array}$ & $\begin{array}{l}6.777 \\
3,913\end{array}$ & $\begin{array}{l}6,406 \\
3,968\end{array}$ & 8 & $\begin{array}{l}10 \\
10\end{array}$ & $\begin{array}{l}18 \\
18\end{array}$ \\
\hline & $\begin{array}{l}2 \pi 5 \text { lbs. Nitrate of Soda, } 300 \text { lbs. Sulphate Potss, } 100 \mathrm{lbs} \text {. \& Sulphate } \\
\text { Soda, } 100 \mathrm{lbs} \text {. Snlphate Magnesia, and } 34 \mathrm{cwts} \text { Superphnsphate... }\end{array}$ & 0.1 & 0,0 & 5,210 & 8 & 10 & 8 \\
\hline $\begin{array}{l}17 \\
18\end{array}$ & 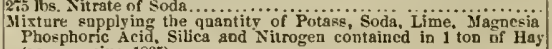 & & 3,755 & 3,791 & 8 & 10 & 18 \\
\hline 19 & 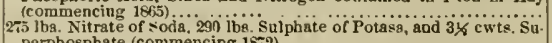 & 3,908 & 3,301 & 3,604 & 5 友 & 5.8 & 11 \\
\hline 20 & 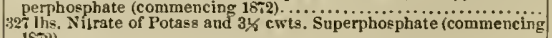 & & & 4,318 & 4 & . & .. \\
\hline & 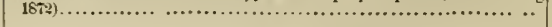 & $\cdots$ & $\cdots$ & 4,092 & 4 & .. & . \\
\hline
\end{tabular}

* Ammnnia valts" in all cases equal parte Sulphate and Mnriate of Ammonia of Commerce._- The "Superphosphate of Lime" is, in all cases, made from $200 \mathrm{lbs}$. Bonc-ash $150 \mathrm{lhs}$. Snlphuric Aeid Sp, gr, 1.7 (and water). besides the Ylanures specified, 2,00, lhs. Sawdust per acre per annum for the first even ycars, 1856-1862, but witbout effect.-

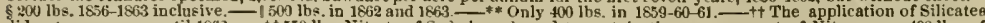
did not commence until 1862 . - $+\$ 550 \mathrm{lbs}$. Nitrate of Soda is reckoded to contain the same amount of Nitrogen as $400 \mathrm{lbs}$, of "Ammonia-eajts."

With regard to these experiments, Mr. Lawes says:-

"It will be observed that of the two unmanured acres, No. 3 and No. 12, one always gives rather more produce than the other, the average produce of one over the whole period of twenty years being 2,383 pounds, and of the other 2,686 pounds; for reasons which we have fully gone into in the original paper, we have considered it advisable to adopt the produce of Plot 3 instead of the mean of 3 and 12 as our standard of unmanured produce; 
but both show a decline in the second ten years as compared with the first. Experiment 2 received 14 tons of ordinary yard manure annually for the first eight years of the period, in all 112 tons per acre; but since the first eight years it has received no manure. During the eight years of the application of the dung, the increase of hay obtained on Plot 2 over the unmanured land was 2,139 pounds of hay per acre, and for the next six years after the application of the dung had ceased, the increase was much the same, being 2,147 pounds per acre; since then the produce has gradually declined, but even at the present time-seventeen years after the last application of dung-the produce is still several hundred pounds more than that of the unmanured land; and compared with the constituents contained in the dung, there is still a very large amount to be accounted for which has not been taken off by the increased produce.

In the two experiments 5 and 15 we have equal quantities of nitrogen supplied to the grass every year; in one, the nitrogen is in the form of ammonia combined with sulphuric and hydrochloric acid; in the other, it is in the form of nitric acid combined with soda. The ammonia experiment commenced two years earlier than the other, but in 1858-when the first application of the nitrate took place-the hay produced in the two experiments was nearly equal; siuce then the produce under the ammonia saits has rapidly declined, until it is now very little above that of the unmanured produce, while upon the experiment receiving the nitrate, the produce of the last ten years, as compared with the first eight years, has been the same.

This great superiority of the hay-producing properties of the nitrate over the ammonia - equal to about 1,000 pounds of hay per acre annually over the whole period-is due partly to the superior power of the nitrate to liberate and render available the stores of the soil, and partly to the supply of soda which it yields to the plant.

The property possessed by growing vegetation of using soda where there is a deficiency of potash in the soil, is very clearly established in several of these experiments.

The superiority of nitrate over ammonia is equally apparent when all the necessary minerals are employed in conjunction with these two substances, as is the case in Experiments 9 and 14. During the first period, which includes ten years with ammonia and only eight years with nitrate, the average produce is almost exactly even in the two experiments, being 6,000 pounds of hay per acre annually. In the same period of ten years, the produce of the ammonia plot had fallen from 6,000 pounds to 5,421 pounds; while the nitrate had risen to 6,777 pounds, thus again showing the superiority of the nitrate over the ammonia.

In Experiments 11 and 12, where much larger quantities of ammonia are used with the minerals, we have the largest growth of hay produced on any of the plots-the average in one case is close upon 7,000 pounds of hay per acre; and even this does not show the full effect of the manure, for since 1874 we have been taking two crops of hay annually, and the produce has amounted on the average to 10,000 pounds; and in 1877 it reached the amount of 12,344 pounds of hay per acre. Of this great weight of produce the soil has contributed about 8 or 9 per cent., and the remainder comes from the atmosphere.

In Plot 7 we have an experiment in which, from the commencement, no manure containing nitrogen has been placed upon the land; a very liberal supply of potash, soda, mag. nesia and phosphate of lime has been applied every year, and the average produce of the twenty years has been close upon 4,000 lbs. of hay per acre. It may be noticed also that the produce of the last ten years is rather the highest. Compared with the unmanured land, the produce upon Plot 7 is a little over $1,500 \mathrm{lbs}$, but it is nearly $1,800 \mathrm{lbs}$. below the increase given by the minerals combined with ammonia, and $2,448 \mathrm{lbs}$. below the minerals combined with the nitrate, annually.

In experiment 18 , which has only been carried on eleven years, up to 1875 , wo supply 
the exact quantity of minerals and nitrogen, which we estimate to be taken out of the soil by one ton of hay. It is not easy to find any basis for comparison in this case, as the fertility of the unmanured land had already been reduced by the removal of nine crops. If, however, we take the produce of the last ten years of the unmanured land for comparison, we find that we have only obtained 1,453 lbs. by materials which were competent to furnish $2,240 \mathrm{lbs}$. of hay; this is about two-thirds of what we should have obtained if all the manure had been taken up. The nitrogen contained in the increased produce amounts to $15 \mathrm{lbs}$, which is nearly one-half of that supplied in the manure. It may be mentioned also that there was a considerable decline in produce in the last ten years of the unmanured land, which we took for the basis of comparison.

I have now pointed out some of the most prominent of the experiments, and will conclude this part of the subject with a few remarks on the practical knowledge which may be derived from them.

In the remarks which I have made up to this point, I have purposely abstained from saying anything about the difference of the quality of the herbage produced by the different manures; as this point will be more appropriately treated when I have done with hay as an article of sale.

Whatever may be the case in New York, in Great Britain the value of hay depends upon its being of good color, and got in without injury from rain, than from any difference in its feeding qualities. Hay is sold in the London market by the load of eighteen trusses, weighing 56 pounds each; this is as nearly as possible the United States ton of 2,000 pounds. The price of a load of meadow hay is from $\$ 20$ to $\$ 25$, which does not differ much from the New York quotation of from $\$ 1$ to $\$ 1.25$ per 100 pounds.

The price of the most important ingredients in manures would not, I conclude, differ very much in the two localities. With regard to the effect produced by manures here and in the state of New York, possibly we may lose more of the soluble portion of our manures by drainage, while in New York, want of sufficient rain, combined with a higher tempera. ture, may prevent the crop from making use of all the elements of plant-food which are readily available.

Let us now endeavor to estimate the cost of the hay grown by the manure applied. In Plot 18 the exact ingredients contained in one ton of hay are applied as a manure. And as these ingredients are not specified in the table of manures, $I$ will give them here:

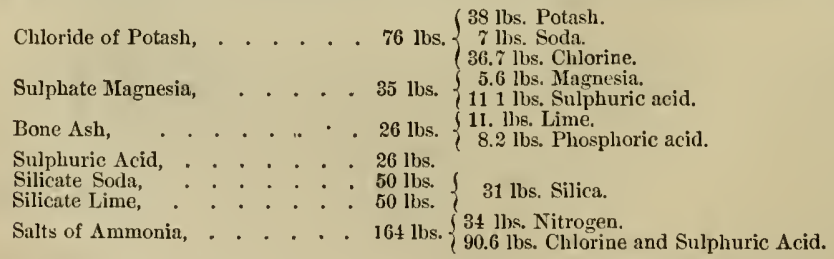

It is somewhat difficult to estimate the cost of this manures, but I do not think it could be bought and sown upon the land for much less than $\$ 16$; with a produce of 1,453 pounds of hay there would be little or no profit.

Plot 7. - The manures used in this experiment could be probably bought and spread upon the land at a cost of from $\$ 12$ io $\$ 13$; the increased produce amounts to 1,500 pounds of hay; there would, therefore, be a small profit upon this transaction. But it must be remembered that the success of a purely mineral manure depends upon the amount of nitrogen liberated from the store in the soil; and in the course of time this store will be reduced by the process of exhaustion to the same level as that which we now have in our arable land. 
Plot 14 received the same minerals as Plot 7 , but with the addition of 550 pounds of nitrate of soda, which, when placed upon the land, would probably cost about $\$ 20$; this, added to the cost of the manure of Plot 7 , would amount to $\$ 32$ or $\$ 33$ per acre. The increase of hay obtained by these manures was 4,000 pounds. It must, however, be observed that I have made no charge for the cost of labor attending the cutting and converting this increased produce into hay; I might also add that I have taken, as the basis for comparison, the lowest in produce of the two unmanured acres.

During the whole period of twenty years the 112 English or $125 \frac{1}{2}$ United States tons of dung had produced 35,000 pounds of increased produce of hay, which is equivalent to a pro. duce of about 280 pounds of hay to each ton of dung of $2,000 \mathrm{lbs}$; but the crops of the last six years prove that the efficacy of the dung is by no means yet exhausted. It is not in my power to place a value upon dung, as the cost turns entirely upon the carriage. I live 25 miles from London, and my farm is one mile from a station. The cost of dung by rail is 60 cents per English ton; delivery on my land costs an additional 42 cents-making altogether $\$ 1.02$.

The result of these experiments makes it somewhat doubtful whether hay can be grown profitably by means of artificial manures applied to permanent pasture. With us hay is generally grown near large towns, and the same conveyance which takes it to market brings back the manure at hittle or no cost.

Compared with its selling price, hay remores more of the soil constituents from the land than most of our other salable products. One hundred pounds of hay will remore nearly as much nitrogen and much more mineral matter than one hundred pounds of wheat. These considerations must all be studied when the question comes as to the profitable application of expensive manures. While, therefore, the evidence is somewhat against the use of artificial manures when hay is grown for sale, it by no means forbids their employment when grass land is used for the production of meat, milk, butter or cheese; and to illustrate this I will merely allude to one manure ingredient, viz.: potash. In the large crop of hay which we take from Plot 11 , we carry off annually 140 pounds of potash per acre; 1,000 pounds live weight of an ox or sheep contains about $1 \frac{1}{2}$ to $1 \frac{3}{4}$ pounds of potash. There are very few acres of land in the state of New York which will fatten one bullock per acre, and even if there were, the potash carried off would not amount to more than one pound. Of milk, 100 pounds weight contains a little over $\frac{1}{2}$ a pound of mineral matter, or about one-thirteenth part of what would be contained in 100 pounds of hay, while butter robs the land of noth. ing.

If land has been impoverished by the sale of hay, and hay is to be sold, dung is the cheapest manure to apply; but if land so impoverished is intended for the future to produce milk, meat, or other animal products, potash is sure to be wanting, and the best manure to apply will be either 200 pounds of sulphate or muriate of potash, or three times that quanti. ty of kanit salts, and, in addition to whicherer of these substances is selected, 200 pounds of superphosphate of lime and from 60 to 80 pounds of nitrate of soda.

If, however, the land has been impoverished merely by feeding stock, then the exhaus. tion will be more likely due to the absence of nitrogen and phosphate, and fertility must be restored by an application of these substances as manures.

Quantity rather than quality is the object to be attained when hay is the crop grown; but when animal products are produced from grass, the quality of the grasa is of very great im. portance. Quality of pasture is dependent upon the food in the soil: in land under grass there is a constant struggle going on between the various plants which constitute what we call a pasture. Upon my experimental ground, the pasture contained about fifty different species of plants when the experiments were commenced, and upon the unmanured ground these have been subject to but little change; but it is far otherwise upon the variously man. 
ured portions; if the food is abundant and good, the good grasses drive away all the weeds and bad grasses, and the ultimate result is a very simple herbage, consisting of not more than from fifteen to eighteen of the best species. The constant mowing, although it enables us to establish a great deal of valuable information respecting growth, is, at the same time, most destructive to the finer sorts of herbage; it cannot be expected, for instance, that much white clover will be found amongst grasses standing three feet high, and yielding 7,000 pounds of hay to the acre; with liberal manuring, therefore, there must be close feeding, and the coarse but highly nutritious cocksfoot and foxtail must not be allowed to smother the clover and trefoil.

Having once started a permanent pasture by means of a judicious mixture of artificial manures, the question arises whether it is more economical to keep up the fertility by a fresh application of artificial manures, or by the manure obtained by feeding stock upon the land with food grown in other localities? It is not easy to decide this point. I am myself inclined to think that the latter process is the most economical, and, in the conversion of arable into pasture land-upon which operation I have been engaged for the last ten years-I have trusted to the fertilizing properties of the manure from cotton-cake to enable me to accumulate the stock of fertility which, being exhausted by ages of arable culture, had to be replaced before the land could again become a pasture.

When hay, which is the product of arablel and, is grown for sale, it is by no means certain that the increase, obtained by the application of artificial manures, would repay the cost of the operation. Nitrate of soda, at the rate of 100 pounds per acre, applied in the spring, about a month before the crop began to grow actively, would probably give a considerable increase to a crop of timothy, but I cannot venture to give an opinion as to what would be the pecuniary result of the transaction.

Sunlight is cheaper than all artificial sources of light, and natural fertility is cheaper than any artificial compound; in the absence of sunlight we have recourse to purchased light; and as the natural fertility is exhausted from our soils we are driven to use fertility derived from other sources.

It is the object of science to investigate and explain the laws which regulate the growth of plants, rather than to enter upon the question of economy. In the present paper I have endeavored to unite, to a certain extent, science with practice, in the hope that the farmers of the United States, who take the trouble to read what I have said, may add something to their present stock of knowledge."

Old pastures that were formerly arable are often excellent lands on which to cultivate po. tatoes or corn, but the expense in many localities of fencing such plats is an item to be taken into account as to whether this would be a remunerative practice. In many sections where land is cheap and the soil poor, it may be best to let certain pasture-lands produce a growth of timber. By plowing with a strong plow, furrows can be turned from six to ten feet apart, and in these trenches may be sown the seeds of such varieties of trees as are desired, and also that are best adapted to the soil. As has been previously stated, sheep are excellent renovators of pasture-lands, and will destroy to an almost incredible degree the weeds and bushes. Hogs, when kept in large numbers, will also answer the same purpose by being penned in limited sections. For this purpose movable fences are necessary, and when they have rooted and exterminated the noxious vegetables in one part, remove them to another, and so on until the work is accomplished; but this is a rather slow method of clearing up a pasture.

Clearing land of worthless bushes and shrubs by hand is a laborious and expensive task. Hardhack can be killed by mowing off the shrubs annually for three years in succession, late in tlie summer. Alders are more difficult to exterminate, especially where the growth is large and dense, but they may be conquered by cutting and burning. Juniper can be 
1 N W 19.5 (2. 3.

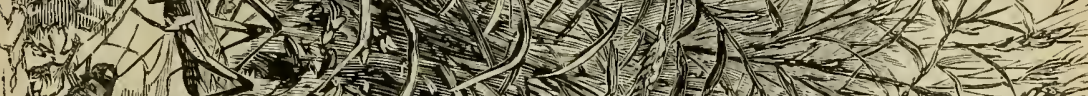

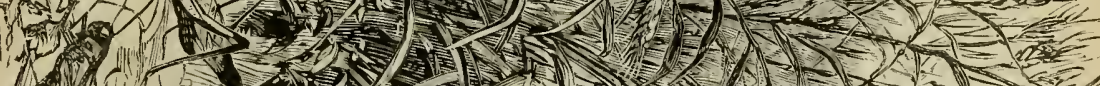

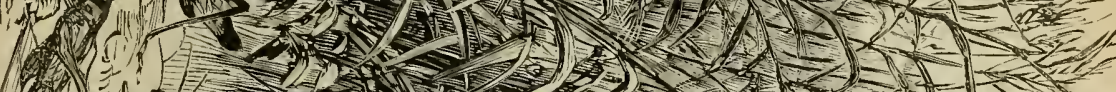
- 1 - 3 ( (1)

1 $15(1,1)$ $1<1,1-1$ a $3 x^{2}=$ (1)

\section{Nots} - $-1,12$

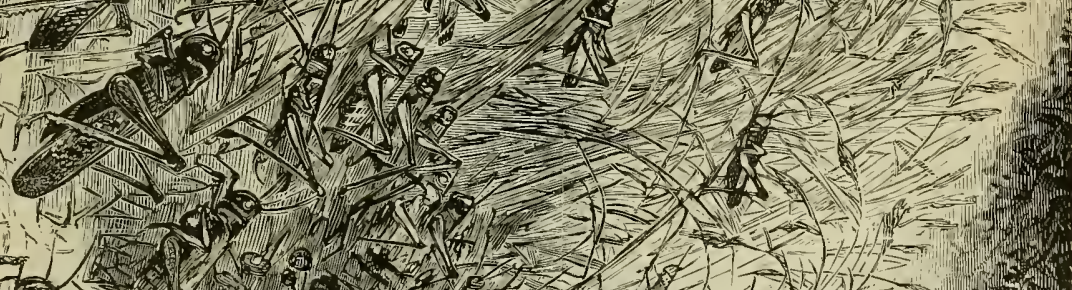
5.

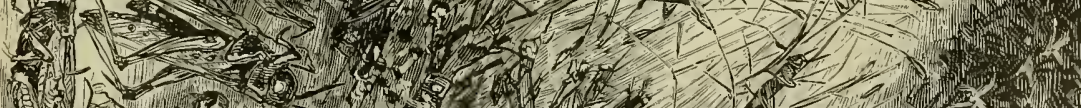

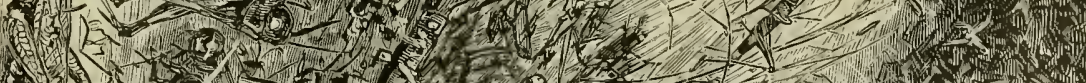

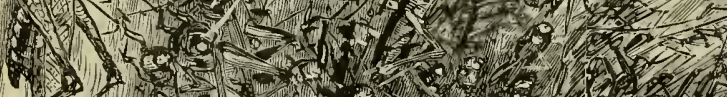

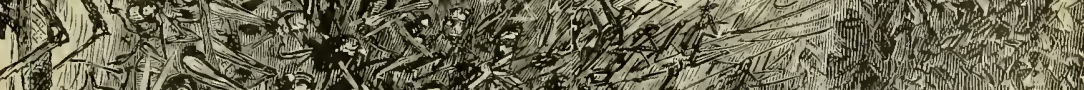

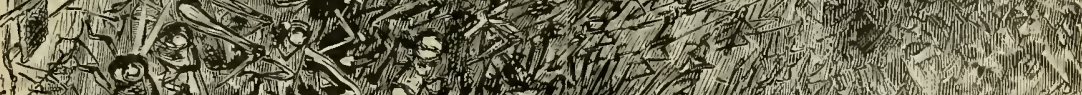
1. -

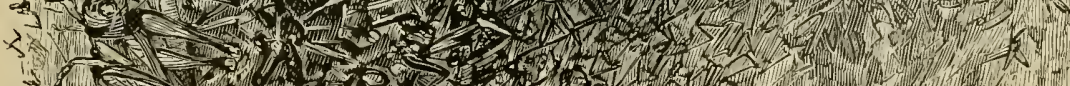
$\rightarrow-1$ - 


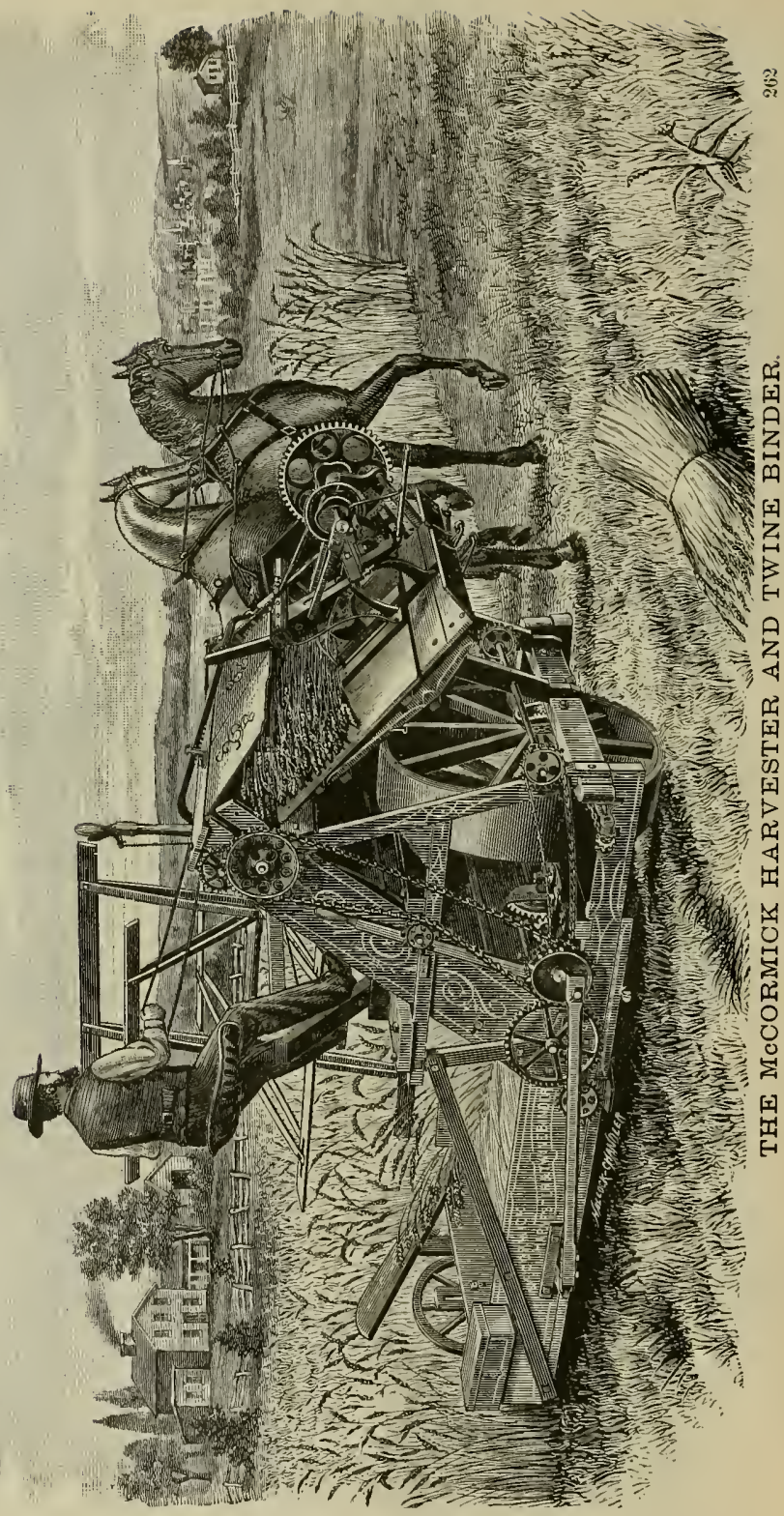


destroyed in the spring also, by fire, taking care to burn all the trailing branches. This destroys all the seeds, and is therefore a more effectual means of extermination than grubbing. Birches require several cuttings in August, each year, before they can be conquered.

In all cases, the surrounding circumstances will have to be taken into account in deciding what shall be done with such pastures, such as the nature of the soil, locality, etc. Some lands will not pay for the labor of renovating, and where this is the case, it will, of course, be the best way to let them alone; others will well repay the labor bestowed in vastly increas. ing the productive capacity and value of the land.

The soiling system is well worthy the attention of farmers having remote pastures or those of poor quality, and which would not repay the expense of renovating. The same might be said of the ensilage system in connection with a winter supply of green food for stock.

\section{CEREALS.}

$\mathrm{W}$

ITH the exception of buckwheat, cereal plants belong to the order Graminace (true grasses), but differ widely in their structure, character, and method of cultivation.

It is gratifying to note that during the years 1870 and 1880 , there was the unprecedented advance in the cereal production of the United States of about a hundred per cent. for all kinds taken together, while the increase of the protious decade, or between 1860 and 1870 , was but twelve per cent.

The increase in the cereal product between 1850 and 1860 , which was a period of noted agricultural growth, was forty.three per cent. The total number of acres under wheat cultivation in the United States in 1880 , was $35,487,065$, which yielded a crop of $459,591,093$ bushels. In 1870 the wheat crop of the country amounted to $287,745,626$ bushels. The increase of wheat-growing in the last decade is shown by a comparison with former enumera. tions, to be seventy-three per cent.; that between 1860 and 1870 , to be sixty-six per cent., and between 1850 and 1860 , sixty per cent.

We also find that Illinois shows the largest area under wheat, aggregating $3,218,963$ acres; next Iowa, with $3,049,347$ acres; Minnesota, with $3,046,821$; Indiana, 2,619,307; Ohio, 2,556,134; Missouri, 2,074,314; Wisconsin, 1,948,036; Kansas, 1,861,342; California, $1,837,322$; Michigan, 1,822,752, and that the six States of Illinois, Indiana, Ohio, Michigan, Minnesota, and Iowa, produce more than half the wheat of the whole nation.

The increase in the product of corn between 1870 and 1880 was one hundred and thirtythree per cent., the amount produced in 1870 being $760,844,549$ bushels, while that of 1880 reached $1,772,909,846$ bushels. In 1880 the number of acres in the United States devoted to the cultivation of corn was $62,326,932$, the State of Illinois leading in this product.

The increase in the production of oats during the last ten years has been about forty. five per cent; t that of barley, nearly fifty per cent.; that of rye only about seventeen per cent., while the minor cereal-buckwheat-has not increased in proportion to the advance in population-the total crop in 1870 being $9,821,72 \mathrm{I}$ bushels, while that of 1880 was 11,851 ,738 bushels.

It is also gratifying to note the increased interest in the cultivation of the cereals in the cotton-growing States since 1870-the increase in the corn product alone in that section being about forty per cent. From present indications the report of the next decade, or that of 1890 , may be expected to show a surprising advance on the above-mentioned rates of increase, whilo that which marks the close of the present century will doubtless exceed the most san. guine expectations, so immeasurably great are the resources and facilities of the country for the extension and development of this branch of agriculture. 


\section{WHEAT.}

$\mathrm{T}$ HIS is one of the most important and extensively-cultivated of the cereals, and next to maize, or Indian corn, is the most productive of all the grasses belonging to the genus Triticum. It has been cultivated for the food of man from the earliest ages, the Bible, as well as the Egyptian and Chinese records, substantiating this fact; and although it is not known in a wild state, most botanists incline to the belief that it had its origin in the central portions of Asia. Rice forms the principal sustenance of the rast population of India and China; corn and the various other grains also contribute to the support of multitudes of the human family; but wheat forms one of the principal articles of food of the most powerful and civilized nations of the world. It may be cultivated in a variety of soils, and will adapt itself to either cold or warm climates, but thrives best in the temperate zones, and in soils that are rich and rather heavy. The lowest mean temperature in which wheat will ripen is about $57.2^{\circ}$.

While the increased consumption of wheat in a country is an indication of an improved style of living among the general population, its extended culture is also an index of an improved agriculture, since it is only on soils naturally fertile, or that have been rendered so by careful cultivation, that it can be successfully grown.

Tarieties.-There are already many varieties and sub-rarieties of wheat, and the number is constantly being augmented by either the accidental discovery of new ones, or by cross-fertilization artificially brought about for this purpose. Some of these varieties are more hardy than others; but among the most popular kinds cultivated, some may be found that can be successfully grown in almost any section of the United States and the southern por. tion of British America.

The most common classification of wheat is that made by the time of its sowing, it being sown both in autumn and spring; hence the terms, "winter wheat" and "spring wheat." This distinction, however, often misleads, for while it is true that there are certain varieties best adapted for autumn and spring sowing respectively, it is also true that many kinds will admit of being sown at either season. The spring wheat has been obtained by means of a gradual change in the time of sowing. As a general rule, winter wheat, in localities adapted to its cultivation, gives a stronger growth of straw and larger yield of grain than the spring wheat, and the heads grow more erect and full, with heavier grains. Many of the varieties that give the most favorable results in cultivation at the South are not hardy enough for the climate of the Northern States, while many of the winter varieties are not sufficiently hardy for the extreme North. The selection of seed should always be adapted to the climate, and the more hardy varieties sown in the colder temperatures.

Spring varieties may biing a surer crop, and involve less risk of loss to the farmer, in localities where the winters are so long and serere that the crop sometimes "winter kills," though this may frequently be due to improper preparation of the land through lack of under. draining, or other causes; but is often due to want of hardiness in the wheat plant. The wheat-growers are constantly forced to seek new varieties to keep up the average yield.

Some writers attribute this frequent tendency to deterioration in quantity and quality to be a natural inclination to return to the original unimproved, uncultivated condition; but we believe, if the real canse were known, it would be found to be more in the deterioration and exhaustion of the soil, together with a lack of judicious care in selecting the very best seed of the crop for sowing, and that if farmers would restore those elements extracted from the soil, in the production of the harvested crop, in the form of proper fertilizers, before sowing the succeeding one, and select only the choicest and most perfect seeds for that sowing, the deterioration, so often the complaint of the farmers, would not be known. 
Among the many varieties of both winter and spring wheat, which have been most popular, may be mentioned the White Mediterranean, Red Nediterranean, Diehl, Fife, Silver Chaff, Treadwell, Fultz, German Amber, Clawson, White Russian, Champlain, Defiance, etc. Among the later spring vafieties above mentioned, are the Champlain, Defiance, and White Russian; those of the winter varieties are the Fultz and Clawson, although neither of the previously-mentioned are of so late an origin but that they have been tested for several years by cultivation. Besides these, the "Golden Grains" is a very hardy and productive variety, suited to either spring or fall sowing, and is one of the largest-grained wheats known. It grows with a beard until it begins to ripen, after which the beard falls off. It can be successfully cultivated as either winter or spring wheat in the Western States, but does best as a winter wheat in the Eastern section of the country.

Some kinds will do best in one section, and others in another, and we doubt whether any variety will ever be produced that will thrive equally well in all localities.

The soft, plump-berried varieties are frequently found the most productive, and for this reason are much in favor with those farmers who would naturally think more of quantity than quality. The flinty and glutinous varieties make the best flour, especially where that which is called the "new process" milling is employed, and consequently flinty varieties are more popular with the millers.

Wheat that is cut early makes not only whiter flour, but flour that contains more starch in proportion to the gluten than that made from wheat which is harvested after becoming fully ripe. The microscope reveals the fact that the starch and gluten are not formed in the grain at precisely the same period, the starch-cells in the interior of the grain-kernel being filled with starch-granules several days before the gluten-cells (which are next to the bran) are quite filled. For this reason, wheat that is allowed to get as rip 3 as possible, to avoid waste, makes better flour, because it contains more gluten, although it is not quite as white as from wheat that is early cut. The quality of the grain is somewhat modified by the soil ; if the soil be a moist clay and other conditions favorable, the berry will be plump and soft, while a dry sandy soil will produce a smaller but harder kernel, a better quality, but less in quantity.

The Defiance, Champlain, Silver Chaff, Treadwell, and White Russian are hardy varieties, and will thrive well at the North, while the Clawson, Fultz, as well as the German Amber, and several others, have proved varieties for successful cultivation at the South.

The Fife is very successfully cultivated in some portions of Canada; it has a hard, plump kernel of a reddish color, and is very productive and hardy.

The White Mediterranean is a spring variety; it is bearded, with large kernels, and yields well.

The Red Mediterranean is a winter variety, with heads bearded, ripening early. It is very glutinous and is quite popular in the Middle States, but it has one objectionable feature, and that is, in the straw not being stiff enough to bear up under a very heavy crop, and in consequence this grain is liable to become lodged in the field.

The Diehl is a winter variety, grain white; it is a bald wheat, prolific and hardy.

The Fultz is classed among winter wheats, and is beardless, of an amber color, and yields largely. The German Amber is also of a similar tint, and is quite a favorite in some of the Southern States.

The Clawson (sometimes known as Seneca) is a winter wheat; it is smooth and white, with red chaff, and is early and hardy, bearing a stiff straw and large crops, and is adapted to either the North or South.

The White Russian, as its name indicates, was imported from Russia. It is not exactly a white wheat, but of much lighter color than most of the other spring varieties. It is bald; with white chaff, the kernels being quite plump.

The Champlain is a bearded wheat with white chaff, and is quite free from rust or smut; the straw is strong and vigorous, and grows higher than most other varieties, with very large, full heads. 
The Defiance is also a vigorous, hardy wheat, with heavy straw and large, full heads. It is beardless, with white chaff and large kernels. The Silver Chaff and Treadwell are both hardy winter wheats.

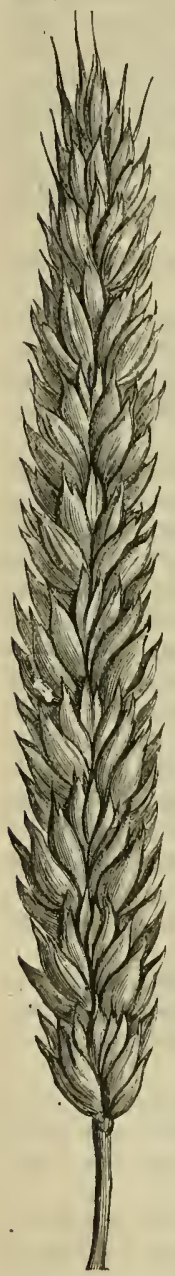

The Defiance.
To illustrate the manner in which different varieties are often produced, we will give the history of the origin of a few of the favorite wheats. In 1566, Mr. Gerret Clawson, living in Seneca County, N. Y., harvested a crop of wheat from mixed seed. Among the stubble of this crop he chanced to find a head of wheat that appeared to him to possess uncommon excellence, and he decided to sow it separately and note the result. He did so, and thus gave to the agricultural world the popular "Clawson" variety.

Mr. C. G. Pringle, of Charlotte, Vt., who is a practical hybridizer, has not only produced new and val. uable varieties of potatoes, but in a non-wheat producing State has also originated two very valuable varieties of that kind of grain. This gentleman commenced the work in 1870 , by the cross-fertilization of a few ovules in a head of the Black Sea variety with pollen from the Gold Drop, sometimes known as Siberian. He selected the former because it was one of the most hardy varieties then in cultivation, and the latter on account of the superior quality of its flour, hoping to unite the hardiness of

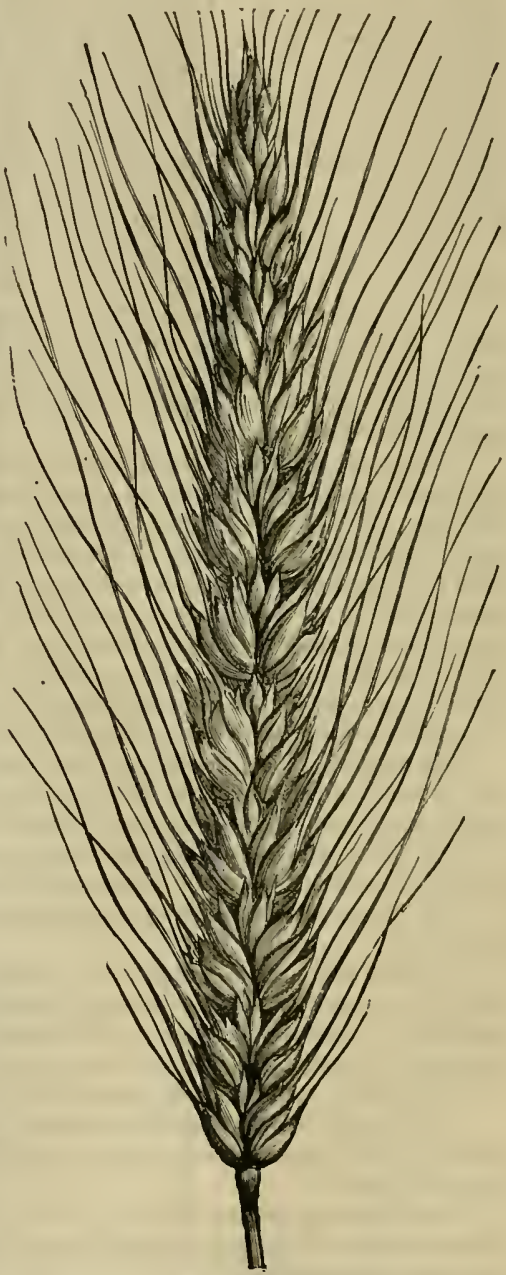

The Champlans. of this cross was about a half dozen grains, which were sown the following spring, and cultured with great care; the result was several plants showing uniformity of character, intermediate between the Black Sea and Gold Drop. This product was nearly beardless, like 
the Gold Drop, with chaff of a reddish tinge like the Black Sea, whille the kernels were larger, plumper, and of a lighter color than the latter, and resembling the Gold Drop, the plant being very vigorous and the heads of superior length, supposed to be due in part to the crossing and part to superior cultivation. This seed was sown the second spring in drills apart froin other varieties, and, to the surprise of the careful hybridizer, the plants appeared of every grade between the two parent varieties, but, by selecting a few of the most promising heads of similar characteristics, and continuing these experiments for four years, he succeeded in producing several varieties of a fixed character, the most marked and valuable of which was the "Champlain."

In 1871, Mr. Pringle began another cross between the common Club wheat and one of the varieties from the Pacific coast, which, after similar experiments and selection, resulted in the fine type of the Defiance. These two varieties, hybridized by Mr. Pringle, were brought out through the energetic firm of B. K Bliss \& Sons of New York city, and their merits soon made known to the farmers generally We learn from Rees that several of the formerly popular English wheats were produced by a gentleman at Bradfield, who, walking one day in his wheat-field, was impressed with the variety of colors which the different blossoms of the grain assumed, and, after careful examination, concluded that these different bues were signs of certain specific differences in the character of the wheat, and accordingly selected the heads of colors unlike, and marked eleven distinct varieties. When these were ripe, he gathered them, and planted them separately the following year. The same characteristic differences continued to be seen in the product, and after another year's effort in experimenting, three new and valuable varieties were the result, which were more productive and earlier to ripen than any known previous to that time.

It is stated by good authority, that American wheat contains more gluten than English, and that produced in the Southern States, more than that in the Northern; and as gluten is an element which imparts to flour the quality that is termed by bakers "strength," and which enables it to absorb a large quantity of water when made into bread, consequently in an equal number of pounds of flour possessing a greater and less per cent. of gluten, the quantity pos. sessing the largest per cent. of this material will make proportionally the largest quantity of bread; hence, it has been asserted that while fourteen pounds of American flour will make twenty-one and a half pounds of bread, the same quantity of English flour will make only eighteen and a half pounds. Of course, different varieties of wheat will differ in the proportion of gluten furnished, but, as a general rule, other considerations being equal, the dryer or warmer the climate in which the grain is raised, the greater is the evaporation, and the more condensed is the flour of the grain, and consequently the more moisture it is capable of absorbing. There is considerable difference in the productive, as well as other qualities of the many varieties of wheat, and it is, of course, well for the farmer to endeavor to select the best that is adapted to his soil and climate; but the kind to be sown is not more essential than the thorougn preparation of the land to receive it, since no variety, however good, would thrive except the soil be first well prepared. The important question as to how may the increase of wheat per acre be accomplished may therefore be answered in the following manner: by better tillage, by improving the soil, and by sowing better seed.

Preparation of Soil for Wheat.-There is scarcely any crop that requires more thorough and careful preparation of soil for successful results than wheat, yet having once obtained a vigorous start, it grows very rapidly under favoring circumstances, although it is not as hardy as some other of the cultivated grains. It is partial to a very fertile soil, its cultivation usually being most successful in rich clays or heavy loams, although many light soils, when in proper condition, will produce a good yield. Lands for wheat should never be too wet, and when an excess of moisture exists it should be obviated by a good system of drainage, for unless this is done, the crop suffers by being heaved out by the frosts in win. 
ter, and "winter-killed," or chilled, or dwarfed, in the spring and early summer, clay lands being especially liable to this difficulty. The beneficial effects of the best tillage on lands of the most superior quality will be counteracted by a surplus of water; hence, the proper amount of moisture in the soil should receive the first attention. The land should be so well drained that no water will stand in the furrows. The field to be appropriated to wheat cul. ture should be well plowed, at least twice, and afterward thoroughly harrowed to reduce it to as mellow and pulverized a condition as possible, as this will serve to unlock the fertility of the soil, and put it in a state to best sustain the growing plant; it should then be well rolled to be as firm as possible before putting in the seed. The old adage, that "Tillage is manure," is a very true one, and one especially adapted to the culture of wheat. If, after being thus well prepared, a rain happens to fall in sufficient quantity to form a crust upon the surface, it should be harrowed and rolled again in the same manner before drilling or sowing the grain. By the thorough use of the harrow and roller, the best pulverization of the soil can be secured, and a sufficient density given it without packing it too closely, and also leaving it sufficiently porous for the access of air and water. Many of our most successful wheatgrowers plow the land, letting it lie for several weeks or even months, thus giving the heat and air an opportunity to act upon the soil, working it occasionally with the harrow and rol. ler, until a complete pulverization is secured. The summer fallow is regarded with much favor in some localities. A good depth of soil is indispensable to a large crop, as the wheat plant has two sets of roots, the first set springing from the seed and extending downward; the second extending laterally from the first joint and lying near the surface of the ground. A difference of opinion exists respecting the depth of plowing for wheat, some advocating only three or four inches, others a considerably greater depth; we are of the opinion that for most soils, from four to five inches is the proper depth, where the land has previously been deeply cultivated for other crops; much, however, depends upon the nature of the soil.

In clearing up new lands, such as those previonsly occupied by a forest, where the trees have been chopped and the soil been burned over, as is the practice in some of the nnsettled portions of the country, a fair wheat crop can often be obtained with bnt slight labor in preparing the soil, since the proper plant-food for the seed is found in the decaying vegetable matter of the soil, and the ashes of the débris burned in clearing; hence, a good crop of wheat is often grown upon the inverted sod with but little further preparation; but for old lands, the most thorough preparation is necessary. Early plowing is always desirable for wheat crops. If wheat is sown upon oat-stubble, it is best to plow the stubble as soon as the crop is off, and before the ground becomes too hard to plow well. The scattered oats will sprout, but may be destroyed by harrowing thoroughly and repeatedly.

Fertilization of Soil for Wheat.-Some soils are so rich naturally, that they will require bnt little or no addition to their fertility to secure a large yield of wheat, but these are the exceptions rather than the rule, and even most of these will, after a few years' crop. ping, become sufficiently exhausted to necessitate the use of fertilizers in the form of plant. food. This sustenance should altways be in a condition for immediate use; hence, coarse manures, that cannot be readily taken up by the delicate-fceding wheat-plant, will not meet the demand, or be of any assistance in sustaining its life until sufficient time has passed for them to become assimilated to its use throngh atmospheric and other agencies, which often requires two or three years.

A crop of wheat, if fertilized by coarse barn-yard manure, for instance, might starve before the plant-food that lay within the soil would be in a condition to nourish it. Wherever barn-yard manure is used, therefore, in the culture of wheat, it is essential that it be pulver. ized very fine; when applied in this condition, it is highly valuable.

A heavy crop of clover, which has received a good dressing of lime, is sometimes plowed under with very good results; but this should be done several weeks before sowing the 
wheat, that it may have sufficient time to decay and be in condition to stimulate the growth of the crop. Lime has long been regarded as an important aid in the growth of wheat, and in preventing rust, also in obviating an undue growth of straw and assisting in filling out the grain.

For some very old lands which show a tendency to exhaustion, lime and salt are often used with good effect. Mr. J. B. Lawes of Rothamsted, England, recommends Peruvian guano as the best manure for the wheat crop in English soil, to be sown broadcast and harrowed in before sowing the seed; he also mentions that if mixed with twice its weight of common salt or ashes, a more equal distribution through the soil is attained. Whatever the fertilizer used, the soil should be made very rich, and the material for making it so should be put near the surface, for if buried too deep in the soil, it will be beyond the reach of the delicate roots of the wheat-plant, and hence of no material aid in its sustenance. It is a common complaint with farmers in many sections, that the lands that formerly produced large crops of wheat now yield only about half that quantity. This is due to the exhaustion of soil, attending constant cropping without the use of fertllizers to return to the lands, thus drained of their fertility, an equivalent; hence, their former fertility cannot be restored without the application of an abundance of plant-food.

In England, the problem of wheat production seems to have approached a very satisfactory solution, the average product of this grain sixty years ago having been only sixteen bushels per acre; now the average is thirty bushels, and many farmers harvest regularly an average of from forty-eight to sixty bushels per acre.

Mr. George Cowan of England states, that when on a visit to Mr. Mackenzie's noted farm in Manitoba, that gentleman informed him that his average yield of wheat the previous year was forty-one bushels per acre, and, the year preceding, thirty-six bushels, and that his oat crop the previous year yielded an average of eighty-eight bushels per acre. When the farmers of the United States can attain an equal average in the production of these grains, the pursuit of agriculture will be attended with more satisfaction and profit than at present. We believe this can be accomplished by proper tillage, and a liberal supply of the right kind of fertilizing element.

Mr. Fust of Quebec says, in relation to this subject: "It is my firm belief that the real reason why our wheat crops only yield half as much as the English crops is that in England they utilize sheep as grain.growers, while we only consider them as wool and mutton makers."

The great value of sheep as fertilizers is elsewhere treated in this work, and therefore does not require repetition here, but we fully concur in the above-expressed opinion, and believe, if our farmers would utilize sheep for enriching the soil, they would realize a decided increase in their yearly crops of wheat.

In the experiments of Lawes and Gilbert, of England, a repeated cropping of the same lands yearly, for thirty years, gave an average of $16 \frac{3}{4}$ bushels of wheat per acre, with mineral manures alone, while the unmanured gave 14 bushels per acre during that time. The addi. tion of sulphate of ammonia, with the same repeated cropping of the same land, brought the average up to 36 bushels per acre. With the leguminous crops it was the reverse, the experiments proving them to be most benefited by mineral manures. They also found, by repeated experiments, that cereals were most helped by nitrogen, next by phosphates, and very little by potash.

For wheat, we would advocate the use of artificial fertilizers, in preference to farm manure, for the reason that farm manure is rarely applied in a mechanical condition to be readily assimilated by the wheat-plant, while the former responds very quickly, and is in a condition to be readily taken up by the growing plants. This will, of course, involve some outlay, but the increase of the crop will well repay for the expenditure, experiments frequently proving that the judicious application of ten dollars' worth of the proper fertilizers 
often brings a return of from twenty to thirty dollars' worth of grain. Too great importance cannot be placed upon finely pulverizing the soil, and having the fertilizing element near the surface, where it can be within the reach of the plant when it first gets started. Some farmers apply the fertilizers with the drill at the time of sowing the grain; when this is done, it is better to mix the fertilizers with about twice the quantity of earth, or to apply it broadcast and harrow it in, taking care that it is not covered too deeply; either of these methods prevents injury to the seed that might result by having the strong chemical fertilizers coming in direct contact with it, which would have a tendency to injure the germ.

Ground bone or superphosphate of lime is a very valuable and available fertilizer for wheat; it also hastens its maturity from one to two weeks in many soils. It should be lightly harrowed in, and never mixed deeply with the soil. From two hundred and fifty to four hundred pounds or more per acre should be applied, according to the nature of the soil. It is estimated by good authority that twenty-five bushels of wheat, with straw, takes from an acre of ground 51.85 pounds of ammonia, 33.70 of potash, and 26.10 of phosphoric acid, which is equal to about 57 pounds of bone phosphate lime.

Selection of Seed.-In order to secure the best results in the culture of wheat, it is not only important that the soil be sufficiently fertile and thoroughly prepared for the reception of the seed, but that the seed upon which the future crop is dependent should be of the very best quality, perfectly sound, fully developed in kernel, well ripened, and entirely free from any imperfections of any kind. The natural law of "like producing like" is as arbitrary in the culture of grains as in the propagation of animals, or any other department of nature, and the deterioration of the wheat crop so common in some sections is largely due to carelessness or indifference in the selection of seed. The best soil, and most careful cultivation will not produce a good crop from inferior seed. The difficulty and time required for sepa. rating the largest and most perfect kernels for sowing. is probably the reason why so much inferior seed is sown. Many years ago, before the grading fanning-mills and separators were invented, farmers were obliged to resort to various devices for separating the best kernels for this purpose. One method was to dip up from the pile at one end of the barn-floor a small quantity of wheat in a saucer, and throw it upon the floor at the other end as far as possible; by this means the heaviest grains would go farther than the light ones, and when a sufficient quantity had accumulated at the farthest end of the whole mass, the best seed was secured.

A nother method was to put up a wire sieve several feet long, giving it a sufficient slant to have the grain, when poured in at the highest end, pass over its surface; by gently shaking this sieve, the small grains would drop through, and the large ones go on over the opposite end. Others selected the perfect heads from the bundles of wheat and shelled them by hand, which was a long and labored process to obtain a quantity of seed. The grading-mill saves much of this labor and time, but it is by no means without objection, since many imperfect seeds find their way among the selected ones, though it is perhaps the best practicable method where very large quantities are required for sowing. Where practicable, the selection of the hest heads, with a further grading by sifting out all the smaller seeds, will give a better selection, and by carefully cultivating these, and permitting no weeds to grow among the wheat, yearly repeating this process, combined with the best cultivation, will not only prevent deterioration in the quality and quantity of the crop, but improve it in all respects.

One of the best means of securing the choicest grains for seed is to take only those growing in the lower half of the head, these being invariably larger and more plump than those of the upper half. This process of selection, of course, involves much labor and time, but it might be accomplished easily where only a small amount of seed were required, for a choice plot or for experiment in growing for seed. Some very successful wheat-growers save all the grain for seed that shells out itself in handhing the sheaves, since the largest and heaviest kernels 
will shell out the most readily. A recent writer on this subject says that by this means of selection he has not only largely increased his average crop of wheat, but the heads are much longer and heavier, and the kernels larger. Mr. H. Stewart says, in this connection:-

"The question occurs how the habit of growing long full ears is to be fixed on a variety. I answer, by selection and constant cultivation, and producing a pedigreed variety. Every farmer might do this for himself, hut few will; therefore there is a large and profitable business for farmers, to grow seed and make a special thing of it; not only by producing new varieties by crossing, but by cultivating the best of what we now have and improving them in prolificness. If by enriching the soil we can produce only half the standard number of ears, and by selection of seed gain an average length of five inches of ear, we have fifty bushels of wheat per acre, and can then afford to snap our fingers at bonanza farming."

As to the varieties of seed, upon the selection of which so much depends, no definite rule can be given, since some kinds are best adapted to one locality, and others to another; careful and repeated experiments will alone determine their adaption to certain soils and temperatures. The mention of some of the most popular and leading varieties, with their characteristics and general adaptation already given, will aid the farmer somewhat in making his choice for experiment on his own particular lands.

Time of Sowing Spring Wheat.-This will, of course, depend on the latitude and season; but, as a general rule for any climate, we find that the earlier in the season spring wheat can be safely sown, the better for the crop. The best way is to partially prepare the soil in the autumn by plowing and reducing it as far as practicable, to be followed by another plowing and harrowing in the spring, as early in the season as possible, without having the soil too wet; and if properly drained, as all wheat-fields should be, either naturally or artificially, this can usually be accomplished in time to give the wheat-plants an early start. Spring wheat should always be sown in any locality as early as the weather and condition of the soil will admit, which in different latitudes will differ with the season, some seasons in the same latitudes being much earlier than others.

Time of Sowing Winter Wheat.-Winter wheat should be sown in time to give it a good start before the ground freezes, which will, of course, be modified by the latitude. The usual time at the North is from the the 10th to nearly the last of September, although many farmers consider it expedient to have it sown at or hefore the 15th of September, in order to give it time to root well before the frost makes its appearance. The time of sowing at the South is from the middle of October to the middle of November, although in some sections it is sometimes delayed until the early part of December; but this, we think, rather late for any wheat-growing section, and involves some risk to the crop. The appearance of the Hessian-fly sometimes modifies the time of sowing winter wheat, it generally making its appearance about the first of September, and if sown at this time may be liable to its attack. If it is sown sufficiently early in any section to secure a good, strong root to the wheat-plant before the ground freezes in the fall, an earlier growth in the spring is secured, and, consequently, an earlier ripened crop for harvest.

Quantity of Wheat Sown to the Acre.-There has been much discussion upon the subject of thick and thin sowing of wheat, many of the advocates of both methods, as is usually the case on all subjects, being extravagant in praise of their own favorite method, and often erring in carrying out their ideas to the extreme, some insisting that but a few quarts per acre is all that is essential for producing a good crop, and that all seed sowed in excess of that quantity is mere waste. Others would sow so thick that the growth of the plant would be well nigh impossible, for want of room. We believe the medium is generally the safer ground on most suljects, and this especially.

When the largest and most perfect kernels are separated from all others for sowing, as 
they always should be, a less quantity will be required than where many of the grains are imperfect and will fail of germination; hence, a difference should be observed in the kind of wheat to be sown. A heavy sowing will generally produce lighter straw and heads, as all plants when crowded thickly are more slender in growth. There is also a tendency in wheat and most of the other cereals to tiller, or throw out new shoots for future growth, which seems to be an effort in nature to cover the entire ground occupied. Thick sowing will generally prevent this in a great measure; however, if sown too thick, there is not sufficient room for the growth of the plants, and not only is lighter straw the result, but, we think, it also modifies in a measure the size of the kernel, as well as that of the heads, and this manner of sowing will soon deteriorate the quality of the wheat. Light sowing produces a strong, vigorous growth, with large, well-filled heads. We would, therefore, advise a medinm in quantity sown, and avoid eitber extreme. Mr. H. Stewart says, in giving the result of his experience and observation.-

"It is a fact that a field of good wheat, whatever may be its yield, bears a pretty constant number of ears per acre, and this standard number is about $1,250,000$, or 250 to the square yard, or 28 to the square foot. This wonld bring the ears over the field to within $2 \frac{1}{2}$ inches of each other. Now, every farmer may know that this is possible, for it is easy to grow one plant on a square foot with 28 ears on it. The $1,250,000$ grains are equal to two bushels, so that the amount of crop depends npon the number of grains to each ear. This number varies greatly, both from the length of the ear and its contents in grains. If the ears average 20 grains, the product should be 40 bushels. I have found that good wheat generally earries about ten grains to the inch of ear, but that ears vary in regard to the compactness with which the grain is packed in them. I have found only 50 grains in an ear of Clawson wheat 7 inches long, and 40 grains in an ear of Treadwell 4 inches long, and the same in an ear of Red Mediterranean, and several others. The desirable point to be reached, then, is to produce long, well-filled ears. I do not think this is possible, except with thinner sowing than is usual. Two bushels of plnmp seed will contain 1,250,000 grains, but in not one case in a hundred or in a thousand will the crop produce one ear for each grain sown, or one ear for every $2 \frac{1}{2}$ inches each way. I once sowed a field of 13 acres with Treadwell wheat, in strips, from one bushel of seed to the acre up to two bushels; there being 5 strips, increasing by a peck of seed from one side, crossing these strips with others that were dressed with superphosphate of lime at the rate of 100 to 300 pounds per acre, there being 5 strips also, increasing by 50 pounds of fertilizers. It was all manured with 20 loads of good manure and of even character. The whole field when threshed averaged 25 bushels per acre.

The strip with 4 pecks of seed was the best of all; the ears on this averaged 7 inches in length, and one ear was picked out that was nearly 9 inches long, and had 92 grains in it. On this corner of the field the seed was much thinned out by a neighbor's pigeons, and several stools had each 30 ears. The opposite plat produced ears not much over 2 inches long, and the straw was quite thin; the ears were smaller and thinner all along this strip of the field. The best were on the strip most thinly seeded. At a careful estimate, taking the grains in average ears, the best of the field produced over 40 bushels per acre, and the poorest not more than 15. The length of ears was so conspicuous on the thin-seeded portion, which was next a public road, that many neighbors stopped and gathered liberal bunches for their own use without the formality of asking for them.

I consider this to have been a proper and useful experiment from which one could gather definite knowledge, and better than those made on small plats, because it was made in regular farm-work. It would have been more certain had the crop from each square of about half an acre been kept separate and measured, but my object was attained as well by noting the size of the ears and their contents in grains. It proved two things, one being the advantage of one bushel of seed per acre, and the other was, the usefulness of 300 pounds per acre of superphosphate against the other quantities." 
The quantity of seed to be sown to the acre is a question of great importance to the farmer, since it influences to so great an extent the quantity and quality of the product; there are, however, two important considerations in connection with it, as has previously been intimated, the first being the anticipated productiveness or quality of the soil, and the second the yield of the variety to be sown.

The following table shows the number of grains upon a square foot, yard, and acre at certain quantities :

\section{Grains per \&q. foot.}

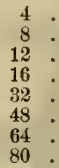

Grains per sq. yard.
$36 \quad$ :
$72 \quad:$
108 :
144
288
$432 \quad:$
576
$720 \quad:$

Grains per acre. 174,240-1 peck. $348,480-2$ pecks. $522,720-3$ pecks. $696,960-1$ bushel. $1,393,920-2$ bushels. $2,090,880-3$ bushels. $2,787,840-4$ bushels. $3,428,800-5$ bushels.

By marking a square foot of space upon a board or paper, and then dividing it into four equal squares, and placing a grain of wheat in the center of each square, it will readily be seen how much space will be appropriated to each grain of wheat when sown at the rate of one peck to the acre. By placing two grains in each square, twice this quantity, or a half bushel per acre, would be indicated ; four grains, one bushel per acre, and so on. Some grains may fail to germinate, therefore due allowance should be made for this in deciding upon the quantity to be sown. "Divide a square foot of surface into sixteen squares, each of which contains nine superficial inches, and place a grain in the center of each square, and it will give one bushel of wheat to the acre. If any person will examine his winter wheat, he will find that if the plant have a vigorous growth, each seed fully stooled covers more space than it would find in the area of nine inches. Put two grains of oats to each square, and it will give two bushels to the acre. Make three to each square, and there will be three bushels of seed to the acre.

English wheat-growers seed more heavily than those of this country, as a general practice. Such a crowding of the land with the plants as will have a tendency to prevent a proper development of the heads should of course be avoided, but the ground should be well occupied when active growth commences. In sowing winter wheat, a noted English authority says : "The best results are secured by using two bushels per acre for the sowing made early in October, and by increasing this quantity at the rate of half a peck per week, until three bushels is reached, which may be held as a maximum. Less than this should not be used from the middle of November to the end of the season." This quantity would be quite in excess of that used by American farmers generally.

As a general rule, the quantity to be sown will be from one to one and a half bushels per acre if drilled, and from one and three-fourths to two bushels if broadcast; this to be modi. fied according to circumstances, such as quality of soil, productiveness of variety sown, etc.

Sowing.-This may be done either broadcast or in drills, the latter being by far the better and improved method, and will, in all probability, soon supersede broadcast sowing. where it has not already, wherever the land is in suitable condition to admit of it. Its advantage over broadcast sowing consists in distributing and covering the seed more evenly, and putting it in at proper distances; this method also requires less seed, and admits of after-cultivation, insuring a stronger and heavier growth of grain, besides the grain grows more uniformly, the heads being all about of a height, and ripening about the same time. It is comparatively of recent date that farmers have learned that after-cultivation will pay in the wheat-field, and that there is nothing of greater promise to graingrowers in this direction than the stirring of the soil between the rows of wheat and killing the weeds that obstruct and retard its growth. Cultivation is, of course, only possible where the grain is in regular rows, and regularity of sowing is only 
attained by the use of the drill, hence, the invention of the wheat hoe or wheat cultivator follows, as almost a necessity, that of the drill, which is merely a seed sower upon a large scale. A box contains the seed which is delivered by tubes, generally eight in number, at the desired distance apart for the drills, and which can be arranged to distribute a larger or less number of seeds in a given place. The difference in the various styles consists mainly in the arrangements for opening the soil, the covering of the drills, and the regular supply of

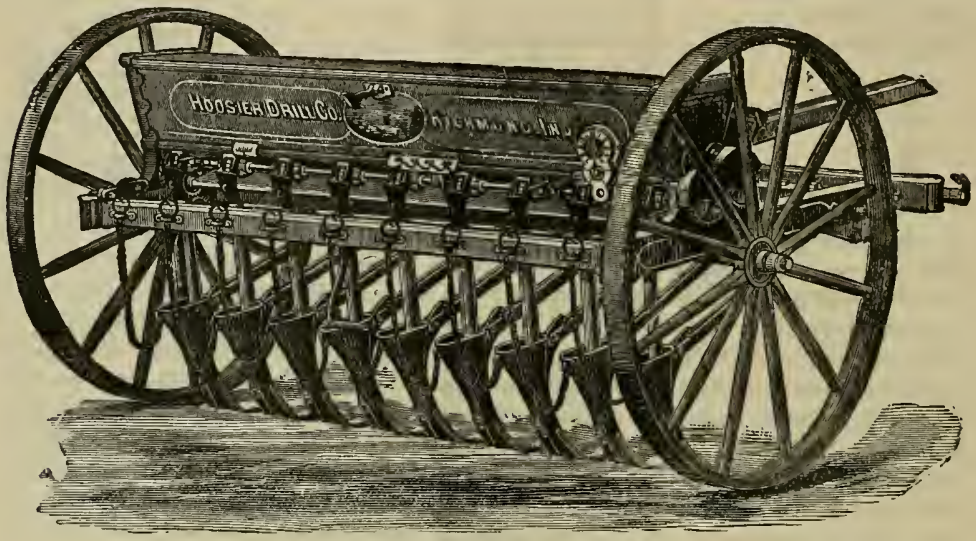

IMPROVED HOOSIER GRAIN DRILL.

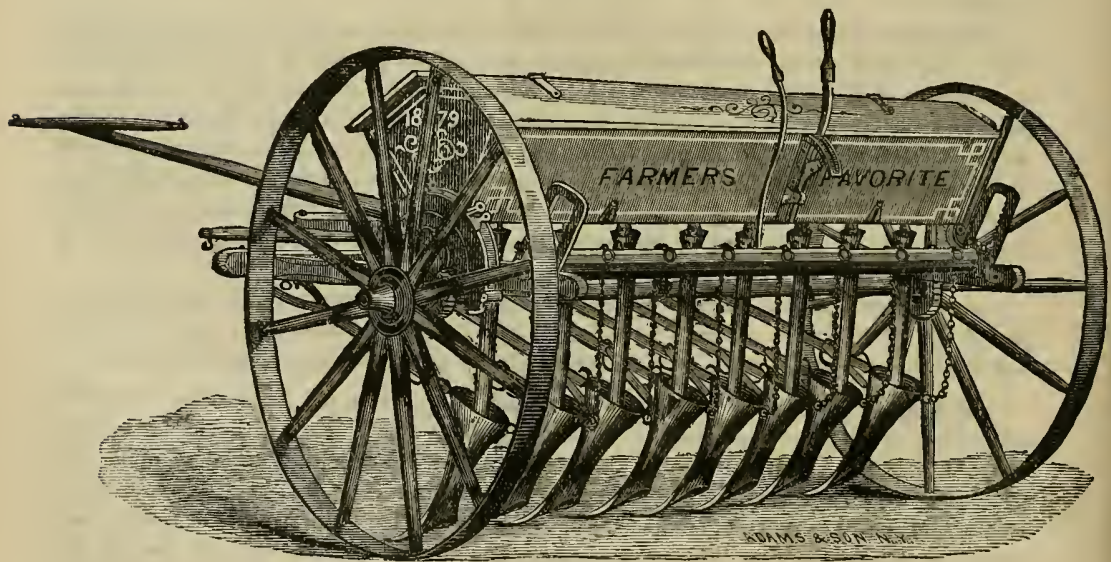

THE FARMER'S FAVORITE.

the seed. They also have an attachment for sowing concentrated fertilizers with the grain, when desired, and can be used for sowing corn, grass and clover seed, and all otler kinds of grains and similar seeds, by means of an adjustable feeder. The first. illustration of these machines represents the improved Hoosier Grain Drill, manufactured by Deere, Mansur \& Co., St. Louis, Mo. This machine will sow all kinds of small seed in any quantity desired without 
any change of gear wheels, and may be adjusted to deposit the grains either twelve, sixteen, or twenty inches apart, to suit the requirements of various soils and varieties of seed. It lias a land measurer or surveyor attached, that measures and registers the number of acres gone over in drilling. The second represents a similar implement of great excellence, manufactured by Bickford \& Huffman, Macedon, New York, which will plant from fifteen to twenty-five acres per day. In whatever manner the seed is sown, the soil must be rich, mellow, and finely pulverized. When sown broadcast, it is either harrowed into the soil or worked in with a cultivator. After being covered, the earth should always be well pressed down upon the seed with the roller, as this facilitates germination. Wheat that is drilled, comes up as soon as that sown broadcast and harrowed, and grows more vigorously, yielding larger crops, besides being less liable to winter-kill; it also withstands drouth better, and the roots having a firmer hold, the grain is not so liable to be blown down and lodged in the field. It is stated by good authority, that the Hessian fly is less liable to injure wheat that is in drills than that sown broadcast. Thaer, in his " English Agriculture," refers to the benefits derived from drill culture, but overlooks in a great measure the influence of the tillage which it introduces. He writes thus :-

"Besides, the advantages from drill-sowing do not, as some imagine, consist solely in the saving of seed which is thus effected, but in the increased amount of produce, which, under this system, the land may be made to bear. The fact of this increase is demonstrated by a thousand experiments, and no doubt can longer be entertained on the subject, even by the most virulent opponents of the' system. No general estimate as to the average amount of this increase can be given, as most of the comparative experiments made for the purpose of determining it have been attended with different results. In many of them the wheat thus sown yielded one-third more than that which was sown broadcast. According to another experiment, it only yielded one-fifth more, and another only one tenth. The variation in these results was, in a great measure, created by the nature and condition of the soil. The richer, deeper, and more free from stones and weeds the soil is, the greater will be the advantages arising from drill-sowing; while on poor, shallow land, the benefit will be but trifling."

Where soil is not naturally very fertile, it should be made so artificially before appropriating it to the culture of wheat, as this crop will never be abundant on a poor or shallow soil. Some farmers whose land is exposed to cold, sweeping winds make a practice of sowing from two to three pecks of oats per acre with their winter wheat as a protection, since the oats will grow up much more rapidly than the wheat, and will help to shade and protect it from winds and the cold. When frosts come; the oats die, but in falling they cover and shield the wheat, a protection to the roots and leaves which remains through the winter, while the little that is extracted from the soil by their growth, is returned to it by their decay. By this means the wheat crop is less liable to winter-kill, and, being kept warm through the cold weather, has an earlier start and more vigorous growth in the spring. The different varieties of wheat will be sure to mix if planted near each other, and this mixture will, almost without exception, be deleterious to the grain; hence, if different varieties are to be sown on the same or contiguous farms, they should be in distant fields, that the different kinds may be kept pure and unmixed. An occasional change of seed should be practiced, as this will increase the vigor and productiveness of the plants, deterioration being sure to follow the use of the same seed for several successive years on the same soil.

The Depth of Corering Wheat will depend somewhat upon the nature of the soil; a heavy, moist soil requiring less depth generally than one that is light and dry. From two to two and a half inches is the usual depth, although some very dry soils may require three inches, and some that are very moist and mellow may not require more than one and a half, but this would be the exception; we should say that about two and a half inches would be 


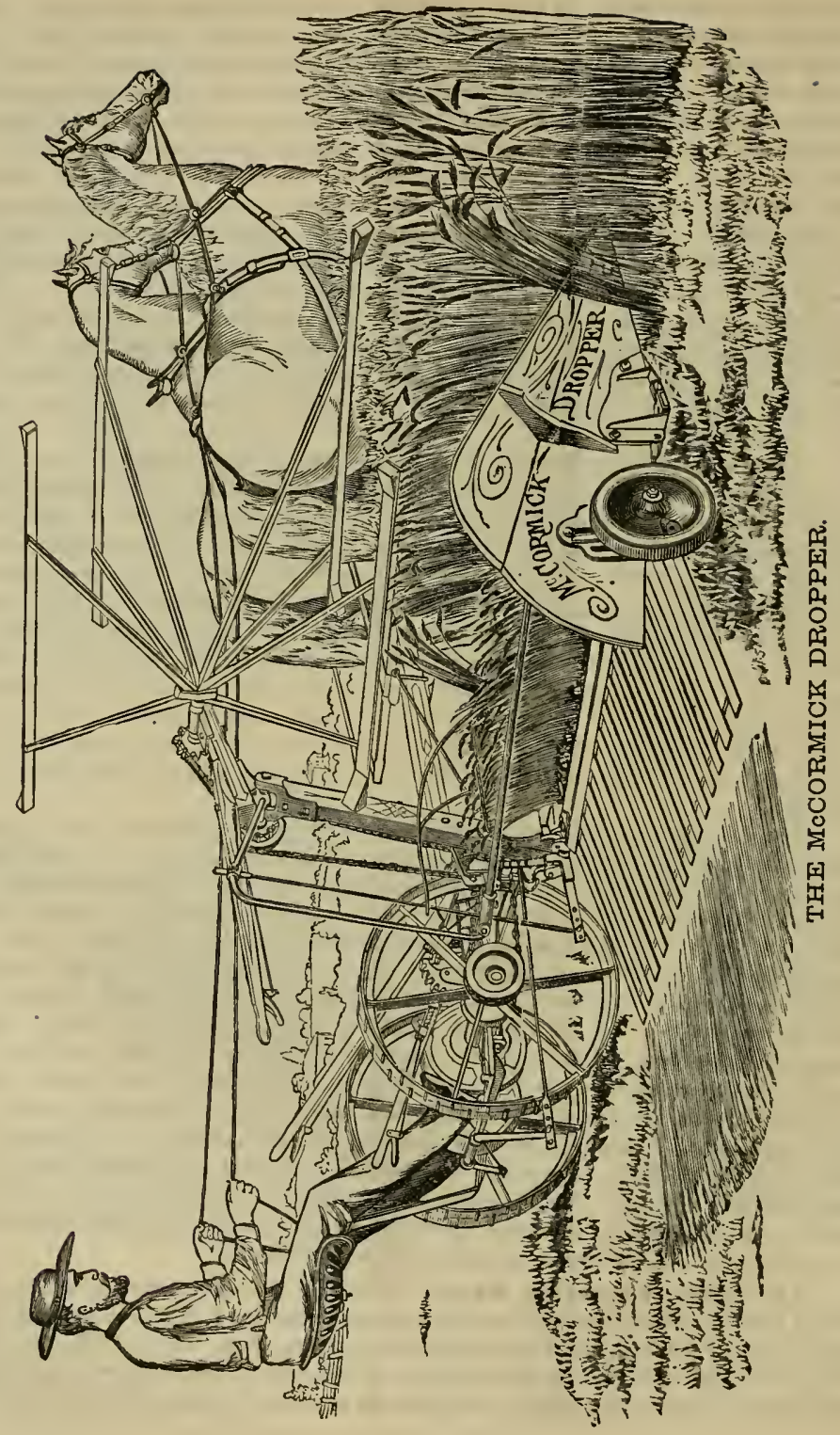


the average depth for most soils. A recent experiment, by a well known agriculturist, on the depth at which wheat should be covered, resulted as follows. of fifty grains deposited at the - depth of eight inches, only two came up and these formed no heads; at a depth of seven inches, one-fourth came up, but formed no heads. Ten out of fifty came up when covered five inches deep, but had defective heads. At four inches covering there were a few perfect heads, but the majority were objectionable. Of those covered three inches, all came up, but the best yield was from those covered only two inches deep. We regret to state that in these experiments the conditions of the soil, or its quality, was not stated, nor the state of the season, but from the results obtained, we infer that the soil was moist, and that the season afforded the average amount of rain.

After-Culture of Wheat.- It has been found by repeated experiments that loosening the soil about the roots of the growing wheat-plants adds materially to the crops, when done at the proper time and in the proper manner; hence, when wheat is sown broadcast, harrowing in the spring is often resorted to, and although it may result in the loss of some of the plants, this process is thought to produce such an increased and rapid growth. combined with the tillering which it produces, that the benefits derived more than compensate for the loss, tillering being desired when it occurs so that the heads will ripen about the same time. In England the practice of drilling wheat and hoeing between the rows or drills has been generally followed for several years, and the increase in the amount thus produced seems to well repay for the additional labor. The practice of cultivating the growing crop has been adopted only to a limited extent in this country, but will probably become in a few years the common method, especially where wheat is sown in drills. Some of the wheat hoes and cultivators that have recently been invented have proved very efficient and valuable - implements for this purpose. These should be employed to loosen the soil and exterminate the weeds, but care should be used not to break or disturb the roots of the growing plants. The former practice in England of hoeing drilled wheat by hand, involved much time and labor. This process would not, of course, be practicable in this country on farms where its extensive culture is carried on. It is a good plan to go over a field of winter wheat with the roller in the spring, in order to press back those roots of the plant that have been thrown out by the action of the frost. This should not be done, however, until the frost is entirely out of the ground.

Harresting. - The time for harvesting wheat is when the part of the stalks near the ground has turned yellow, and the interior of the kernel has passed from a milk state into a harder consistency, sometimes denominated the "dough state," which is when it is easily compressed between the thumb and finger. If cut before this time, repeated experiments have proven that the kernels of grain will shrink and give light weight and reduced measure, although the straw will be more valuable for fodder, as a portion of the nutriment that goes to perfect the formation of the kernel at that time still remains in the stalk. If the wheat stands later than this period before harvesting, the grain will be liable to waste from shelling out in the field, and the straw will become quite hard and less valuable for use as fodder. Where only a few acres of wheat are raised, it can be cut by hand, but large fields require the use of the reaper. Cutting by hand is a slow and laborious process, and where the field is large, much of the grain will become over-ripe and shell out with a consequent loss; therefore, it is always best to hire a reaping machine where one is owned on the premises.

The improved reapers and binders of the present time, that will reap and hind from six. teen to eighteen acres of any kind of grain per day, with the aid of only one man and a pair of horses, show the advancement made in agriculture since the period, comparatively but a few years remote, when all the grain harvested was cut by hand. The McCormick reaper and twine-binder of which we have previously given an illustration represents one of these 
improved machines at work in the field, showing the facility and ease with which the grain is cut and bound. The dropper, which is a machine that combines both mower and reaper, is adapted to the wants of those farmers who raise considerable grass, and small crops of grain, and who do not need separate machines for each kind of cutting. These machines are manufactured by the McCormick Harvesting Machine Company, Chicago, Illinois.

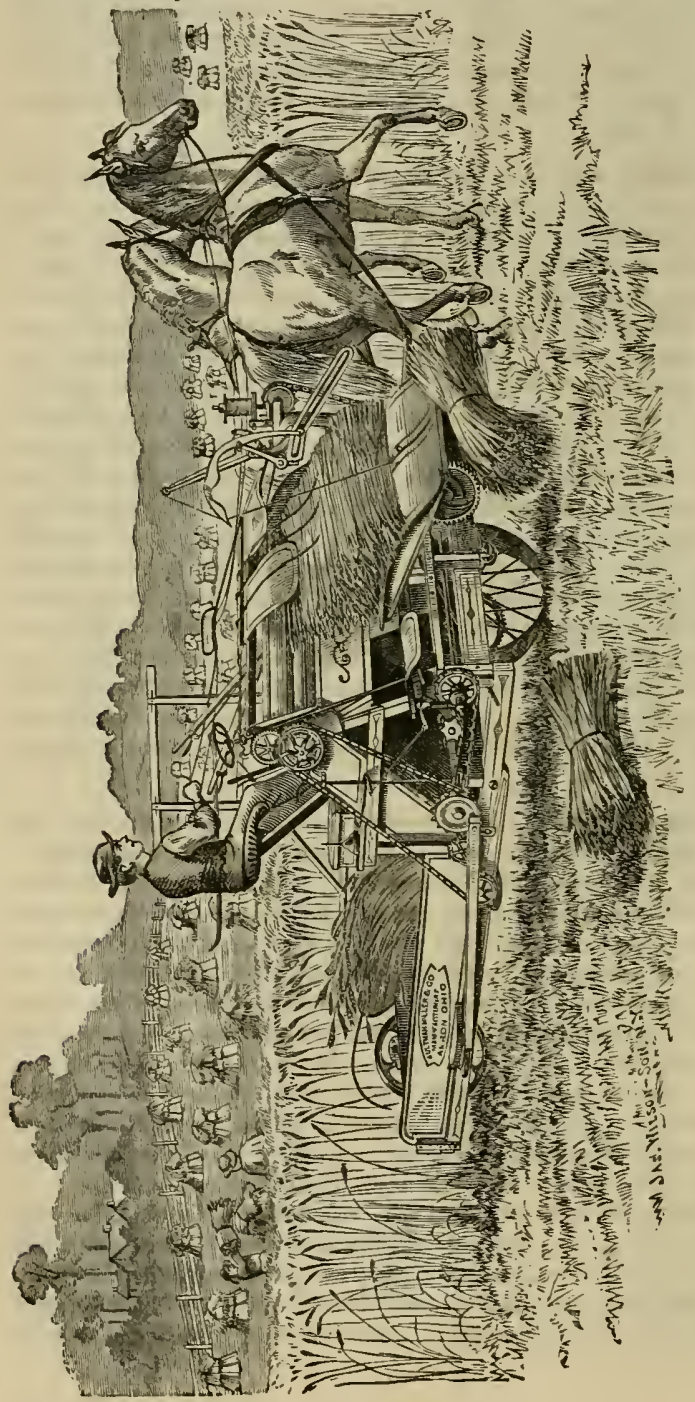

The self-binding harvester and New Buckeye table rake represent ma. chines manufactured by Aultman, Miller \& Co., Akron, Ohio. The small surface of straw covered by the twine in binding does not prevent the air from passing through the sheaf readily, hence it will dry much sooner than the straw-bound bundle.

Twine is preferred to wire in binding, since the wire in the threshing-machine is injurious to the straw, and only straw that is to be burned should have pieces of wire scattered tbrough it; besides it requires considerable time and labor to remove the wire bands fast enough for the thresher, difficulties which are all obviated by the use of twine.

The Table Rake does ralnable serrice as a harrester, both in stand. ing and in lodged or tangled grain.

In California a machine called the header is used in harvesting wheat, wbich cuts off merely the heads of the grain, leaving the straw standing in the field. It is admirably adapted to that locality as will be seen by the follow. ing from the pen of 
Col. Marshall P. Wilder: "In favorable seasons the yield of wheat in California is about twentyfive bushels to the acre. Instances, are not uncommon where in new and very fertile loca.

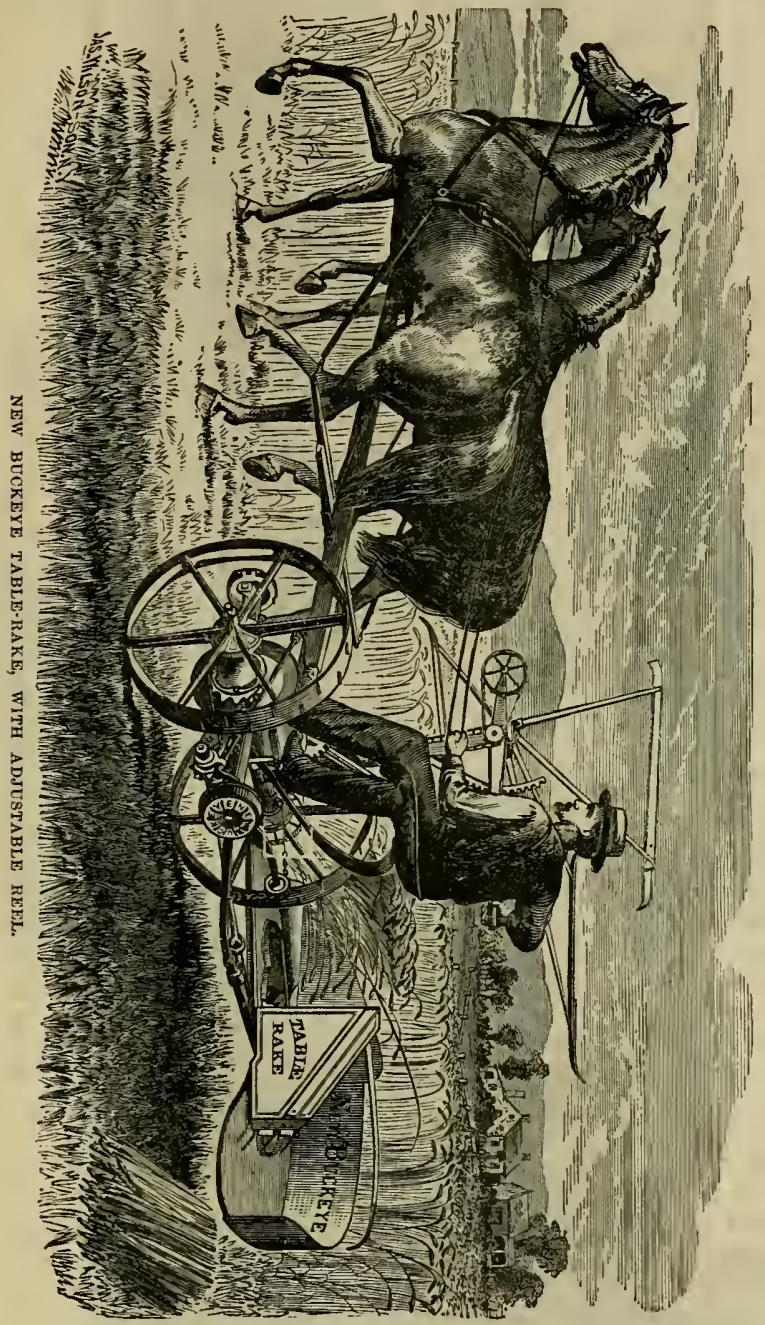
tions it has reached fifty and even sixty, and seventy bushels per acre. The seed is large, plump, white, and so well ripened by the high temperature, that it may be stored in bulk for months without danger of sweating or injury, and in fact often requires moistening before it is ground.

The quality of the California wheat is world-wide renowned for its weight, strength and whiteness. Some of the districts, such as Alameda, Santa Clara and San Mateo, produce the finest wheat in the world; and the quality of the whole state av. erages better than that of the states this side of the $\mathrm{Ne}$ vadas. As there is no rain in the summer, the grain crops are left standing in the fields for weeks after they are ripe. Much of the grain is harvested by a machine called the header, which pass. es through the field cutting a swath fifteen feet wide, taking off the heads eight inches long, throwing them into a wagon by its side, at the rate of an acre in less than an hour. The crops are generally threshed by a steam machine brought into the field. This machine requires two horses, two men, and two 
horse-forks to feed it, and we were told, threshed 800 to 1,000 bushels of wheat and put in bags per day. These bags remain in the open field piled up in rows until taken away by teams to the railroad stations. We saw acres of bags piled up five bags high awaiting shipment. On many of the large farms, the plowing is done by gangplows, six abreast, and drawn by ten or twelve horses. A sower is attached to the plow, and in this way nine or ten acres of grain may be put in the ground in a day. And have you ever thought of the importance of laborsaving machines, as applied to the arts of husbandry? Without the modern inventions, the crops of our country could not be harvested, its prosperity would be paralyzed, and a partial famine would soon ensue. How wonderful the improvements in our own day! Some of us remember the old wooden plow of our boyhood, for which we often drove the team afield, and which with much hard labor could be made to turn the furrows for only about one acre per day. Com. parethis with the

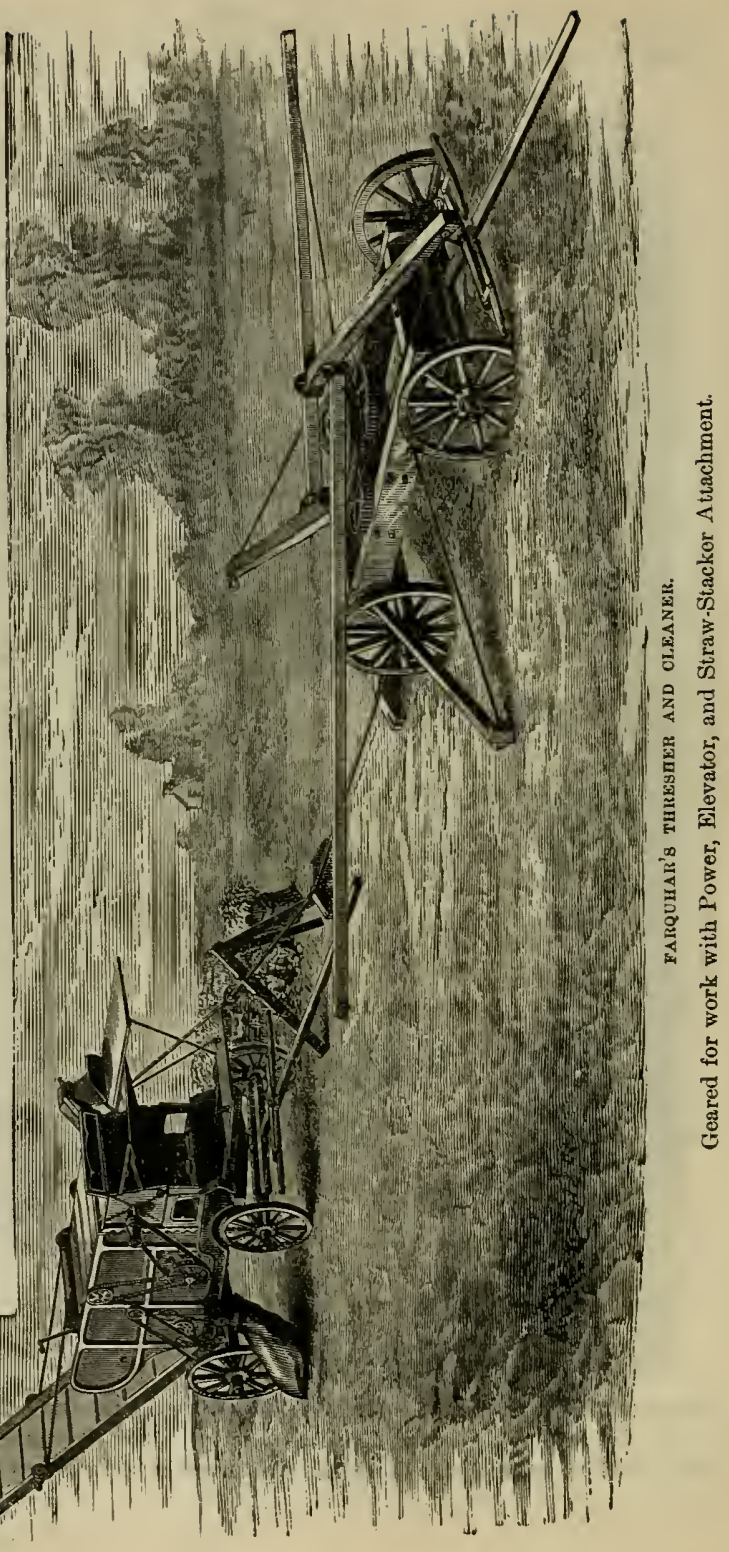


The preceding cuts represent machines manufactured by A. B. Farquhar, York, Pa. The first shows the thresher at work. The second represents the same machine geared, with power ready for work, and with the elevator and straw.stacker attached. These machines thresh the grain, separate it from the straw, and clean and bag it ready for market.

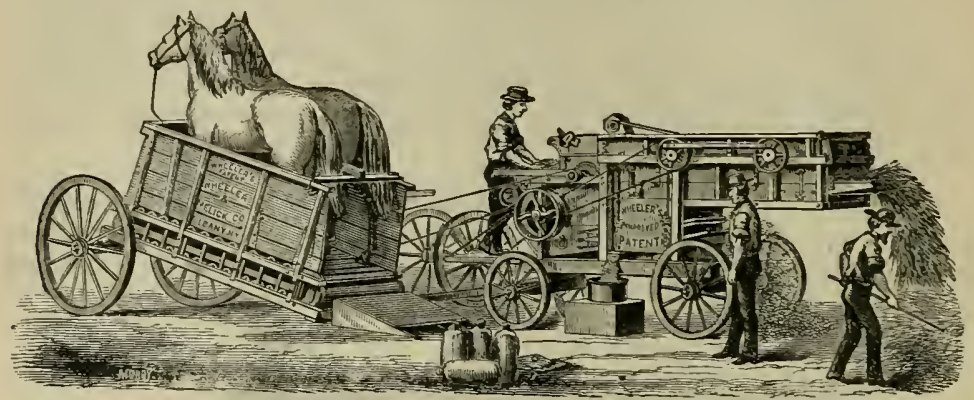

WHEELER'S THRESHER AND CLEAXER.

The above cut represents a very good thresher, manufactured by the Wheeler and Meelick Co., Albany, N. Y. It is a two horse power machine and is a thresher and cleaner combined.

Diseases and Insects of Wheat.-The diseases and enemies of wheat are more numerous than those of any other cereal, and have sometimes, in certain localities, nearly or wholly cut off the entire crop. The most destructive diseases of wheat, and the ones which the farmer has most frequently to contend with, are smut and rust. The destructive insects are numerous, the principal being the chinch bug and Hessian fly.

Smut is a disease of grains in which the kemels assume a dark brown or black appearance, or are converted into masses of blackish powder. It is caused by parasitic fungi which are propagated by spores, and which absorb the nutritive juices of the stalks or heads to which they are attached. There are many varieties of this minute plant growth or fungus, hence, that which attacks wheat (Tilletia caries), is of a different species from that of oats, rye, or that which produces distortions of the ears of corn. It usually first attacks the weaker grains, hence, it is essential that only the largest and most perfectly-dereloped seed should be sown, and also those obtained from a field unaffected by the disease. The usual remedy is to soak the seed in very strong and quite hot brine before sowing, stirring it well, and skimming off all the imperfect kernels that rise to the top, afterwards mixing it thoroughly with slaked lime, and sow as quickly as possible. This process seems to destroy the germ of the fungi, and is one of the best remedies known. Another method to cleanse the seed is to soak it in a tub for two or three hours in a solution made of four ounces of sulphate of copper to a gallon of water, or the grain may be put in a basket on an elevation, under which a board may be placed in a sloping condition with a tub under the end to catch the solution as it is poured over the grain and filters through. A flannel cloth should be placed over the tub to strain out the smut that washes from the grain. When it ceases to drip, the contents of the tub can again be turned into the basket over the grain, and the pro. cess repeated until it is thoroughly cleansed, after which it should be spread on the barn-floor and dried by being mixed with a little lime.

Rust is also produced by the growth of microscopic regetation, and is most common in wet, hot weather. Winter wheat is more liable to be attacked by it than spring wheat, and 
some varieties are more readily affected than others. Like smut, it is more liable to attack the weaker plants, which is an added argument in favor of always sowing the best seed, since these will produce the most hardy plants, and consequently crops less liable to disease of any kind. It affects the stalks of wheat while the grain is forming, the minute plants making horizontal ridges along the stalks of the wheat, which are of a russet orange tint. It is supposed that the spores, or seeds of the fungus, are constantly in the air, and when they fall upon the stalks and leaves of some plants, quickly germinate, and subsist upon their sap. Rusts of different species affect the leaves of many plants; wheat, oats, the bean, strawberry, raspberry, pear, apple, quince, hawthorn, mountain-ash, oak, beet, cabbage, clover, fern, flax, barberry, rose, sorrel, thistle, and many others, both wild and cultivated. It is a question if one kind will not affect a plant of another variety; it is known that some rusts infect other plants than their own native sort.

There seems to be no remedy for this disease when it once makes its appearance in the field, and the grain should be harvested as soon as admissible. The only remedy is in prevention, by selecting the most hardy varieties, and sowing the most perfectly-formed and ri. pened seed of such varieties. Sowing on elevated lands where the air has a free circulation, and the abundant use of fertilizers that contain salt, lime, or gypsum is thought by some to - be in a great measure a preventive. The evil may be largely remedied by treating the seed before sowing as directed for smut. This process has a tendency to destroy the germ of the disease. The use of a solution of bluestone in the proportion of one-fourth of a pound to a bushel of wheat, is recommended by Prof. Pendleton for both rust and smut.

Chincli Bug. - The insects that are destructive to wheat are very numerous and formidable, embracing many varieties of flies, weevils, bugs, worms, caterpillars, \&c., the most destructive of which are the Chinch-bug and the Hessian-fly. The Chinch-bug, (Blissus cucopterus Say) is one of the most formidable and destructive pests with which the farmer has to contend, as it attacks not only the wheat crop, but also indian corn and the various kinds of other grains, besides grass, garden vegetables, \&c. This bug is about three-twentieths of an inch in length and has white fore-wings, each having a black spot in the middle of its edge; the body being usually black. The wingless young are at first red, with a white band on the back, and, aside from this latter characteristic, somewhat resemble the bed.bug. It is slow in motion, and possesses no other weapon of destruction than its tiny beak, with which it attacks the tender parts of the plants, sucking the juices, apparently poisoning the part that is bitten; yet although small, it makes up by numbers for the lack of individual de. structive capacity, being very prolific. This insect is not uncommon in New England and other parts of the eastern portion of the United States, but there its ravages are not as conspicuous as in the extensive grain-growing sections, the valley of the Mississippi having suf. fered extensively from its ravages. The female lays her eggs in the ground, about five hundred in number, and there are often two broods of the larvæ in a single year. The first brood begins its attack upon the wheat crop generally about the middle of June, and does not always disappear before the middle of August. The second brood appears in Autumn. In the year 1864 about one-half of the corn and three-fourths of the wheat crops in many sections were destroyed by this pest, the estimate in loss to the country being about a hundred millions of dollars. After that they seemed to nearly disappear for a time. They are now quite common again, and very troublesome in certain sections. Prof. Cyrus Thomas states that two successive dry years are necessary to the development of them in large and injurious numbers, and that wet weather has a tendency to destroy both eggs and larvæ. Various remedies are resorted to in order to accomplish the destruction of these insects. As they seek hiding places in the stubble, weeds, corn-stalks, and all the rubbish that can accumulate in a field, many are destroyed by clearing the field of everything, including weeds and grass of the fence corners, by burning, as soon as the grain is taken from it. Another practice is to 

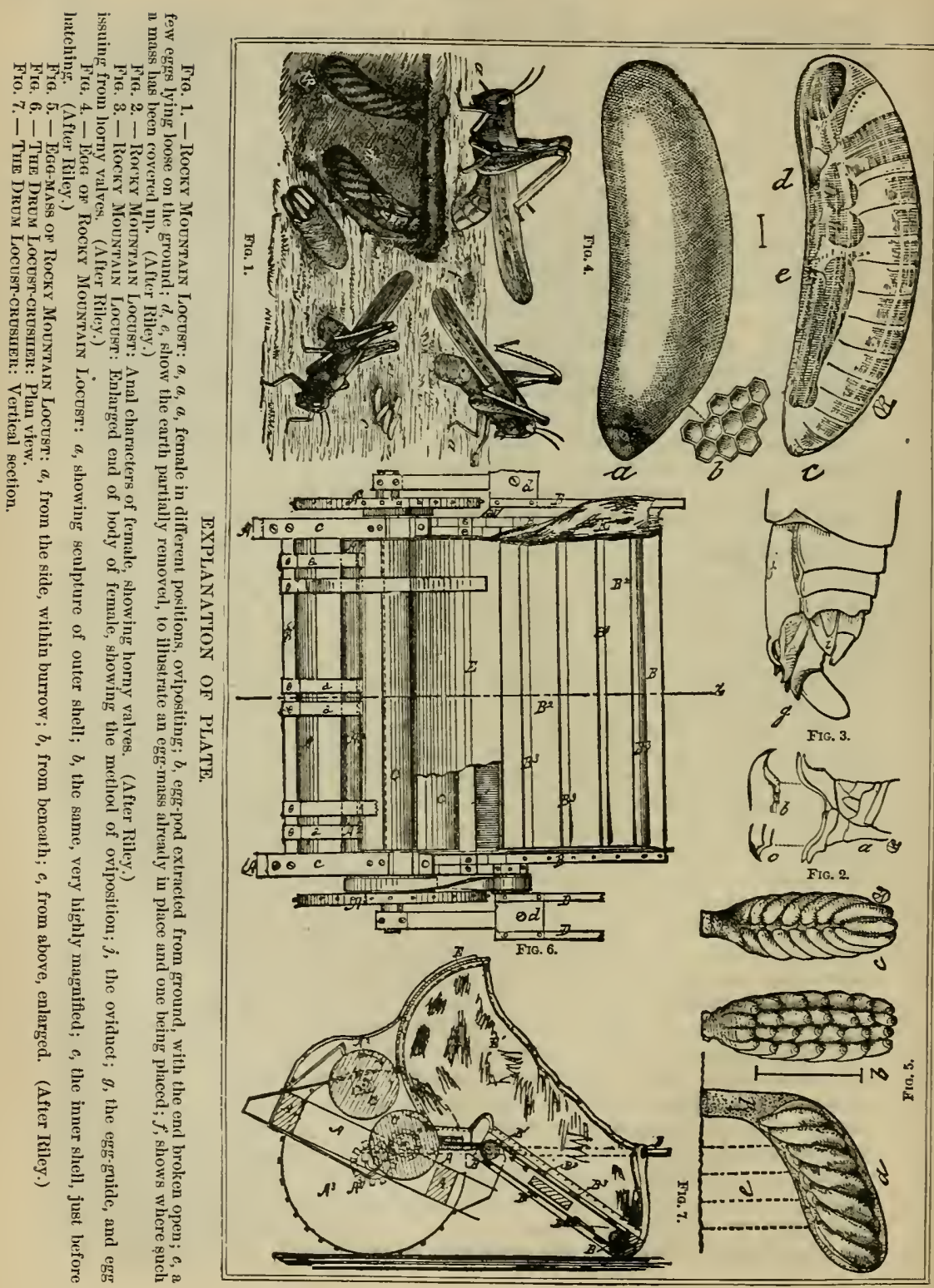
plow under very deep all the stubble of the small grain as soon as cleared of other rubbish by burning. Rolling the field as soon as the grain is sown, and again when it is four or five inches high, destroys many of the eggs.

There seems to be no effectual remedy for exterminating these pests, and the best method is to make the land very fertile and give the crop an early and vigorous growth before they make their appearance, that it may be able to better withstand the attacks, and be less injured by it. Prof. C. V. Riley says:-

"Not to mention the different means to be employed in counteracting the ravages of this insect, a diversified agriculture is undoubtedly one of the most effectual. It must necessarily follow that the more extensive any given crop is cultivated to the exclusion of other crops, the more will the peculiar insects which depredate upon it become unduly and injuriously abundant. The chinch bug is confined in its depredations to the grasses and cereals. Alternate your timothy, wheat, barley, corn, etc., upon which it flourishes, with any of the numerous crops on which it cannot flourish, and you very materially affect its power for harm. A crop of corn or wheat grown on a piece of land entirely free from chinch bugs will not suffer to the same extent as a crop grown on land where the insects have been breed. ing and harboring. This fact is becoming partially recognized, and already hemp, flax, and castor beans are to some extent cultivated in localities where they have not hitherto received much attention. But there are many other valuable root and forage plants that may yet be introduced and grown as field crops; and if the late calamities only awaken the farmers of this country to a full realization of the importance of greater diversification in their culture, the lesson will not be too dearly bought.

Of root crops that would escape the ravages of the winged insects, and which would grow in ordinary seasons, and furnish excellent food for stock, may be mentioned turnips, ruta bagas, mangel wurzel, carrots (especially the large Belgian), parsnips and beets. Of tubers that are not as profitable but of which it would be well to plant small quantities in locust districts, for the reason that they grow with such ease, and are less likely to be injured by the insects, the Chinese Yam, Jerusalem Artichoke (Helianthus tuberosus), and the Chufa (Cyperous esculentus), are worthy of trial."

Hessian Fly. - This insect is so called because it is supposed to have been introduced into this country from Germany, by the Hessian troops, in their straw, during the year 1776 , at which time the British army, then occupying Staten Island, received large re-enforcements of Hessians under General De Heister. It is very destructive to wheat, and produces two or three broods each year. It is found in the winter wheat from late in the autumn through the winter, until about the middle of April, the larvæ of this brood being found between the stalk and sheathing-base of the leaf of the young grain near the root, and slightly beneath the ground. The second brood attacks the wheat in the late spring and summer, and are an inch or two above the surface of the ground at the lower joints of the stalk.

"In the ordinary course of nature, therefore," says Fitch, "our crops of winter wheat are liable to two attacks of the Hessian fly, one generation reared at its roots producing another, which occupies the lower joints of the stalks. Thus the larvæ and pupæ are present in it almost continually, from the time the tender young blades appear above the ground in autumn till the grain ripens and is harvested the next summer Our spring wheat, on the other hand, can rear but one brood of these insects; they consequently resort to it but little, if at all. Nor can the Hessian fly sustain itself except in districts where winter wheat is cultivated, in which for it to nestle during the autumn and winter."

The first brood usually appears in April and May, the second in August and the early part of September, while a third sometimes appears in October. There is no doubt that, as is the case with all other insects, the varying degrees of heat and cold will accelerate or retard the various transformations of the Hessian fly. In from four to eight days after the 
fly has deposited her eggs upon the leaves of the growing plant, they hatch and the maggots or larve work down the leaf between the sheath and stem to a joint in the stalk, and attach themselves, feeding upon the juices of the plant, which soon shows unmistakable signs of their presence by turning yellow, and a swelling of the stalk. Sometimes the joint of the plant becomes so weakened, that it breaks off at the point of their depredations. In five or six weeks they enter a semi-pupa, or what is termed the "flaxseed state," from which they change into the pupa, and then become perfect insects.

The remedies are those suggested for the chinch bug, burning all the stubble and refuse of the field in the autumn, being the most effectual; however, this necessitates also the destruction of the parasites of the fly, which destroy large numbers of them. Some recom. mend late sowing as a remedy to avoid the evil, since if deferred until the fly has laid its eggs, the wheat escapes the first brood; but this is objectionable in respect to the crop not being thus able to obtain a good start before the frost comes, and will consequently be liable to winter kill. Pasturing the crop with sheep for a day or two, or mowing off the tops of the plants and feeding to stock in order to destroy the eggs, together with the use of the roller, are means often employed, but, as we have recommended as a remedy for the chinch bug, and as has previously been stated, we consider burning the stubble the most effectual remedy aside from that of giving the best chance to the crop to obtain a vigorous growth before these pests make their appearance.

Prof. Cook, of the Michigan Agricultural College, states that when the season approaches, the flies will lay their eggs upon the first suitable plant they can find, and recommends sowing a narrow strip of wheat around each field in August or early in September, and defer as long as the season will permit, sowing the wheat from which the crop is to be obtained. By this means, the wheat sown in the strip will make its appearance first, and most of the eggs of the fly will be laid upon it, after which period it should be plowed under very deeply, that the eggs may be destroyed, and the later wheat then sowed. The outer strip may then be included also. This method, if successfully carried out, will destroy the fly and protect the wheat at the same time. These insect pests come and go in occasional and consecutive years, and will sometimes disappear altogether for a longer or shorter period, when they will again suddenly make their appearance. There are several kinds of destructive parasites of the Hessian fly, the combined attacks of which are supposed by entomologists to destroy about nine-tenths of all that are hatched. The most important of these is the Chalcid fourwinged fly (Semiotellus destructor), which destroys what is termed the "flaxseed "; there is also another important parasite that does much injury to the eggs. An application of unleached ashes in damp weather is sometimes beneficial in destroying the larvae, and lime and soot are used with the same effect, but lime should be applied with care, as it is liable to injure the plant. The sowing of hardy varieties is advisable as a protection against most of the dis. eases or insect enemies to which the wheat crop is subject. Grasshoppers or locusts are often very destructive to the cereal crops of the West, coming as they do in such swarms as to destroy every green thing, and leaving no appearance of vegetation in their track. We know of no better remedy for this evil than the prevention of the wanton destruction of birds, which feed upon such insects. 


\section{RYE.}

T HIS crop is more extensively grown in the Eastern and Middle States than in any other section of the country. It is very hardy and more easily cultivated than any of the other smaller grains, uniess it be oats; however, it will thrive where oats will not, requiring less fertility, a sandy soil and cold climate being best adapted to it. It is often produced in the Western States on fields that have become partially exhausted from the con. stant cropping of wheat, large crops being obtained on lands that would utterly fail in the wheat crop in time of harvest. When we take into consideration the cheapness with which rye can be produced, and its various uses, we shall find that there is scarcely any grain that yields so large a return for what is expended. Besides its various uses as grain, the straw is valuable for many purposes, such as for cut-feed for either horses, cows, or any other stock, which are all fond of it, and it is often raised for this purpose principally, and cut while in the blossom. It is soft, clean, and free from dust, and quite nutritious when cut in the blossoming period of its growth, is rarely injured by extreme cold winters, and grows so quickly in the spring that drouth rarely affects it; it is valuable to supplement the hay crop in case the latter is for any reason short. There is nothing so cheap and convenient for use on the farm, such as for bands to tie corn-shocks and bind fodder, etc., and will bring as high a price in many markets as the grain or the best timothy hay. It is also used in the manufac. ture of paper.

Rye is valuable for soiling, producing forage late in autumn and early in the spring; for this purpose it should be sown at the rate of from three to four bushels per acre, to produce a heavy growth; when not too closely fed, and on good soil, it will often produce a crop of grain also. Such feed is very valuable in the spring for cows and other stack, as it comes on early when they need such a change after the long winter with its attendant dry fodder. A small field of rye for this purpose, located at a convenient distance from the barn, will be found a profitable investment to most farmers. When clover fails to catch, as it sometimes will, a field of rye will be found a good substitute, since it will grow so rapidly that it will be ready for pasturing stock at least two weeks earlier than clover. Some farmers sow timothy seed with the rye in the fall, which will come on and produce good feed when the rye has become too old for the purpose, and thus good pasturage will be secured all summer. Rye is a valuable manure when plowed under lightly in the fall.

It is also a well-known preventive of washing on rolling lands, where the soil is easily gullied out by water, as its roots grow very firmly and compact, and will hold and protect the young grass until a sward is formed. We have omitted to state anything concerning its bread-making properties. In this respect it is second only to wheat, and when grown continuously on sandy soil especially adapted to it, will produce flour nearly as white as some brands of wheat; hence, such grain has gained the name of "White Rye," which is only the result of culture, and not a difference in variety, for if this same seed be sown on a rich, loamy soil, the flour produced will be of a darker color, and when repeated crops are produced on the same soil, will finally become as dark in color as that of the original grain first used upon the sandy soil. The unbolted flour has a peculiar flavor not found in that finely bolted, and is much used in connection with corn-meal, in making the brown bread so commonly found in New England cooking, as well as many of the other States in the eastern section of the country. Many farmers use the rye-field for fall pasturage of stock, especially sheep, young calves, and cows, and claim that a benefit to the crop is attained through the trampling of their hoofs and the grazing of the flocks, a more luxuriant growth in the spring being the result. As we have never practiced this method of treatment, we cannot speak from experience as to results, but would not think it safe to pasture rye except the soil were quite dry and the growth unusually luxuriant and heavy, and even then only by such animals as sheep 
or calves, and not allow it to be grazed very closely, as there should be a sufficient growth of the leaves of the plant to afford a protection to the roots during the winter.

Varieties of Rye. - These consist of the spring and winter grain, the former being only a modification of the latter, produced,-as in the case of wheat,-by continuous late sowing in the fall for several years until the result is a grain that can be successfully sown in the spring. The spring variety can be easily transformed into the winter by reversing the process, and sowing continuously earlier each year, until the usual time of sowing winter rye is reached.

Soil for Rye.-Although rye can be successfully grown on a great variety of soils, yet it is of the finest and best quality when produced on a dry, sandy one, where few, if any, other grains can be cultivated with equal advantage. By this, we do not mean the poorest soil that can be found, and which contains but little of the elements of plant-food, or that rye can be successfully grown with but slight preparation of the land, and the constant cropping of the same fields with it, and no manure supplied to return the nutritive properties extracted by the successive crops. A rotation is better for any kind of crops, since the same, repeated year by year, without any equivalent return to the soil of the plant-food extracted, will drain it more surely and quickly than different crops succeeding each other, as different plants require different proportions of the elements of plant nutrition that the soil furnishes.

The better the soil is adapted to any plant, the better its preparation for the seed and the after cultivation, the better the crop, as a general rule, and while we do not think those farmers do wisely who make a practice of giving their rye the poorest soil, with little or no manure, and the least preparation and care of any crop, still they will secure a better harvest of this grain under such unfavorable circumstances than almost any other.

The most profitable agriculture is that where the soil and cultivation is especially adapted to what is intended to be produced, and no farmer has a right even to expect a good harvest without giving the crop to be cultivated a fair chance of realizing his expectations.

We have seen a good growth of rye on a sandy soil that would produce scarcely any. thing else, but the soil was fairly enriched before the seed was sown. Clay is not favorable to its cultivation, especially a heavy, undrained clay, and it will never do well in a wet soiI of any kind. A clay loam will produce a fine growth of straw, but the grain will not be as good as that grown on a sandy soil, the latter producing a more plump kernel of better quality than the former. A rich loam will produce a larger quantity of grain than sandy soil, but of less value. Rye is a strong feeder, and will extract about the last element of soluble plant growth from the soil; hence, land that has become so exhausted that it cannot yield rye, is very poor indeed, and will require a long period of rest, or a large amount of manure of some kind to cause it to produce anything. It is more hardy than wheat and not as liable to lodge with heavy growth. Almost any kind of fertilizer is beneficial,-finely-pulverized farm manure, that from a well-prepared compost heap, ashes, or chemical fertilizers having been found valuable in its cultivation. Green manures are also beneficial. Rye frequently follows corn in cultivation with little or no additional fertilizers.

The color of the grain, as well as its quality for making flour, is greatly modified by the character of the soil that produces it.

Sowing and Harvesting Rye.-The land should be plowed for rye to a moderate depth, and pulverized with the harrow, the manure to be lightly harrowed in before the seed is sown. When winter rye is grown, the best time for sowing it is between the middle of August and the last of September, although many farmers delay it until the last of October. When sown early it has a better chance to tiller out and cover the ground before cold weather than that sown later, also a better start in the spring; consequently late sowing is not desirable. The poorer the soil, the earlier the seed should be sown, and, as a general rule, when 
sown early, a less quantity of seed will be required. Winter rye is to be preferred to the spring variety, but, when the latter is sown, it should be put into the ground as early as prac. ticable, that is, as soon as the ground is in a suitable condition to receive it, in order to give

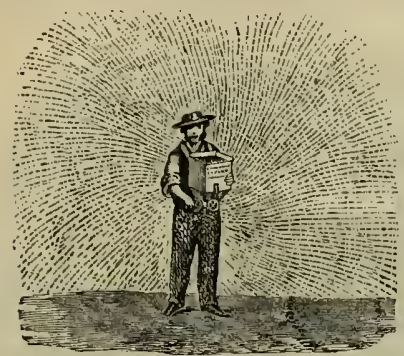

CAHOON'S BROADCAST SEED-SOWER.

it an early growth. The quantity of seed to be sown depends upon the character of the soil and the time of sowing. On light lands, and if sown carly, from three pecks to a bushel per acre is the usual quantity when sown broadcast. On good soil, and when sown rather late, from one and a half to two bushels per acre may be required. When sown principally for green soiling fodder, or pasturage, where a luxuriant and heavy growth is desirable, from three to four bushels per acre may be sown, according to the nature of the soil. When drilled in, only about a bushel per acre will be required for ordinary land.

Small fields of rye are still sometimes sown by the old-time method,-by hand,--but the broadcast seed-sowers and drills have, in a great measure, superseded that practice, since they facilitate labor and distribute the seed so much

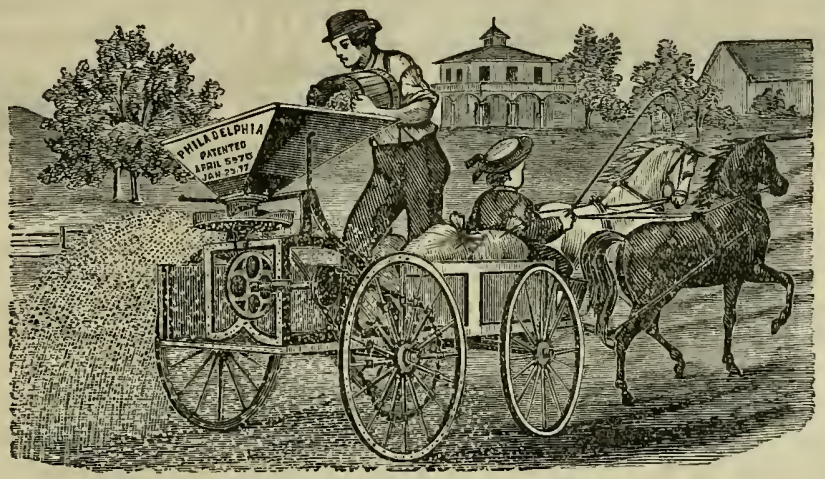

PHILADELPHIA BROADCAST SEED-SOWER.

more evenly than can be done by hand. Where largely cultivated, and the surface of the ground will admit, it is usually drilled in the same as wheat. In the use of the seed-sowers, the quantity of seed per acre can be regulated the same as with drills, the heavier the seed the farther it can be thrown by the sower. A hand machine will sow from four to six acres per hour; a horse machine, from twelve to fifteen.

The two preceding cuts represent machines of this kind for sowing broadcast all kinds of small seeds and grains, as wcll as the various chemical fertilizers, such as bone-dust, plaster, pulverized poultry manure, phosphates, etc. The former is manufactured by the Goodell Co., Antrim, N. H., the latter by Benson, Maule \& Co., Philarelphia, Pa.

The following figure shows a machine of different kind for the same purpose, which is attached to a horse-rake by removing the teeth from the latter, as shown in the cut, thus com. bining two farm machines in one. It is manufactured by Wheeler \& Melick Co., Albany, N Y. 
Rye should be covered with about the same depth of soil as wheat, and should also be pressed in with the roller. This crop is sometimes sown among the standing corn before the latter ripens, and is covered by the use of a cultivator or hoe, which gives the corn a late hoeing and the rye an early sowing, as well as a moist and sheltered locality at a period in the

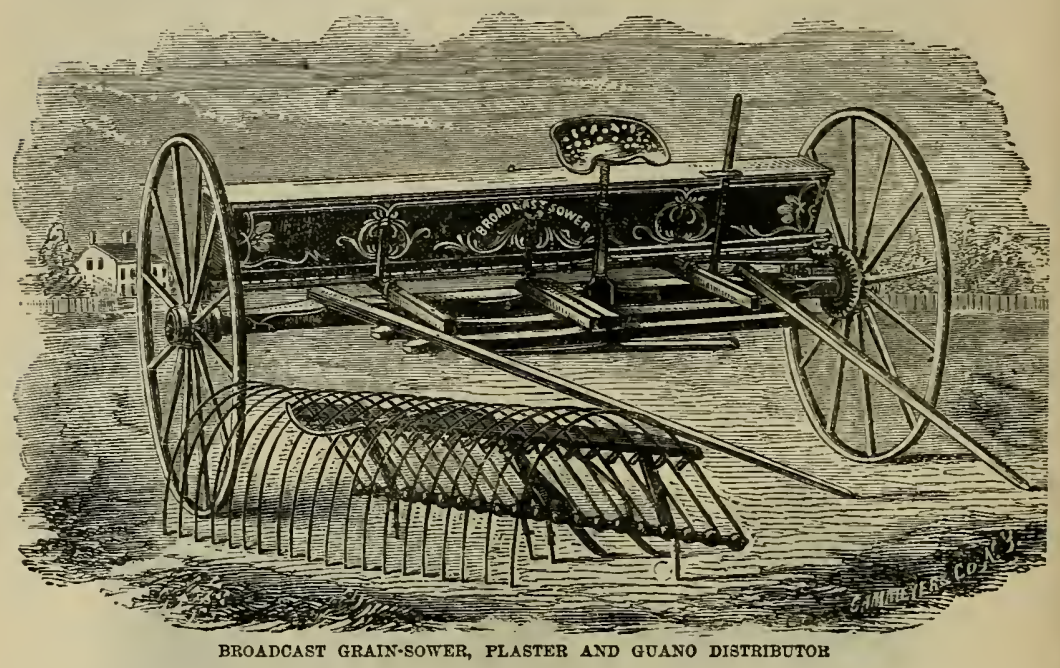

season when drought or the heat of the sun might be a check to its growth. This practice involves extra labor in carrying the corn to a considerable distance in harvesting it, or the necessity of stacking it upon the rye, and we are inclined to the opinion that the derived advantage from it is not sufficient to compensate for the extra amount of labor it requires,

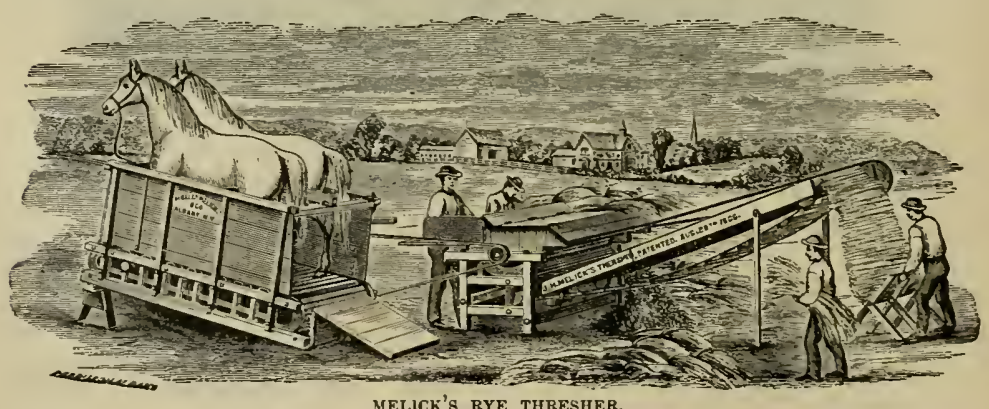

and that harvesting the corn as early as it is ready to be cut, and stacking in another field and sowing the rye as soon as possible when the field is cleared, is a better one.

No after cultivation is needed for rye, except perhaps the use of the roller after it is well started, which is thought by many to be very beneficial. 
When the color of the straw changes, and the kernel passes out of the milk state, as is the case with wheat, it is ready to be cut, which may be done with the reaper and self-binder, or it may be cradled by hand, though, of course, the latter is a very slow and laborious process, and can only be practicable where there is a small quantity to be harvested. When quite free from weeds, the grain will usually be sufficiently dry, in a day or two of clear weather, to be put under shelter; but if the weather be wet and many weeds have been harvested with it, it is better to set the bundles up in stacks of from ten to twelve each, until dry. The practice of some farmers of leaving it in the field until they are ready to thresh it, is not one to be recommended, as it is far better to have it securely under cover free from exposure to rains, being often seriously injured in this manner. It is threshed the same as wheat, although in some localities a special rye-thresher is used, which separates the grain from the straw, without injury to the latter, and which is considered an advantage in sections where the straw is used for making paper, or where it is put up in bales for shipment.

The previous cut represents such a thresher, manufactured by the Wheeler \& Melick Co., Albany, N. Y., and is said to be an excellent machine for the purpose.

Diseases of Rye.-The diseases and casualties to which rye is subject are fewer than those of wheat, the principal being ergot, or what is sometimes termed cockspur. Like rust and smut, this is a fungoid growth or parasitic plant, most common in a hot, wet season. It grows in the heads of rye, causing them to turn black and send out long spurs, which are considered quite poisonous for man or beast, when eaten in sufficient quantity, and are also supposed to be the cause of serious disease, several fatal epidemics in Europe having been traceable to the use of rye wheu thus affected.

The sloughing of the hoofs and horns of cattle has been attributed to ergot eaten with - grass and grain. Emaciation and debility, sometimes resulting in the death of animals, is often occasioned by it. It also has a tendency to produce miscarriage in pregnant animals. The oil extracted from these spurs or fungoid growths is sometimes used in medical practice. When this condition of the grain exists, it is commonly called "spurred rye," and was long supposed to be occasioned by diseased kernels of the grain; but microscopic examination has caused the present prevalent opinion, that it grows originally from the ovary of the grain, that the spores of the plant are taken up by the roots of the rye, and that they germinate there, having been deposited in that part of the grain by the sap.

The spurs grown on the rye-heads are generally of a violet black or dark purple externally, and are whitish, with a pink tinge within. They grow from a third of an inch to one and a half inches in length, and have an unpleasant rancid taste. The mills for grinding are so arranged that most of this poisonous substance is separated from the grain by the grinding process, and largely prevented from mixing with the flour; still, there is risk in using rye that is affected by ergot. There is no remedy known, when it attacks the grain, hence prevention is the only means to be used in avoiding it. If possible, the seed sown should be from a field unaffected by it, and only the largest and most perfect grains selected for the purpose. Before sowing, it is a good plan to put the seed into hot brine, stirring thoroughly and skimming off all that rise to the surface, and afterward to spread it and dust with lime. This is the best preventive of the evil known. Rust, like that which attacks wheat, is also known to rye, and when thus affected it should be harvested as soon as possible. 


\section{OATS.}

$\mathrm{T}$ HE oat plant is one of the most hardy and thrifty of grains, and is a native of cold climates, although it will adapt itself to a wide range of latitude and a great variety of soils. It, however, succeeds best in the northern part of the temperate zone, being decidedly a plant of that section, but does not reach as far north as barley. It can be grown in a southern latitude where the summers are long and the temperature very warm, but it does not flourish as well in such localities, and is apt to degenerate under such conditions very rapidly.

As with most of the other cereals, there are many varieties and sub-varieties of oats, the difference in the latter being due mainly to difference in the soil and climate. While new kinds are being frequently introduced, some of which prove very valuable and fully merit the praises they receive from the extensive advertisements given them, others are found by repeated experiments and considerable expense to the farmer, to be failures, as far as their adaptation to general cultivation is concerned. Oats that are imported from Norway and Scotland and a similar latitude, are quite heavy and have a plump kernel, also those from Canada, but if sown in a latitude considerably farther south, the product, with the cultivation they at present receive, sopn degenerates to the common local standard in quahty. We beheve this deterioration is mainly due to the indifferent treatment the oat receives in cultiva. tion almost everywhere in this country, and that if proper care were exercised in the selection of the seed, and preparations of the soil to receive it, it might be prerented. The grain sown should be the most plump and perfect kernels from a crop cultivated on good soil and unaffected by disease of any kind, such as rust or smut. We see no reason why oats may not be cultivated here and kept up to their original standard of excellence, or even improved upon, as well as any other crop, when properly treated. The general idea prevalent among farmers is, that oats can grow on almost any soil, and with but little preparation, the poorest land and most indifferent culture being accordingly given them. Were it not a very hardy plant, it would utterly fail under such treatment. With some pains taken in the preparation of the soil, and a suitable supply of manure given, a profitable crop is almost always a certainty.

This grain has obtained the reputation of being very exhaustive to soils upon which it is grown, which we are inclined to think is unmerited, and that if the soil before sowing received the same amount of fertilizing properties given to many other crops, it would be found to leave that soil after the harrest more fertile than many crops that have not this reputation. It is a strong and vigorous plant, and being such will sometimes smother and too heavily shade smaller and weaker plants that may he grown with it,- grass, for instance.

It is, as has been previously stated, a rery good practice, and one adopted by many farmers, to sow oats with winter wheat where the field lies in an exposed and cold locality. By this means, the oats soon spring up and grow sufficient to give protection to the wheat during the winter, their leaves, when killed by the frost, forming a good corering for the wheat crop, and "winter-killing" is prevented, while an earher start is also secured in the spring. The amount of fertility extracted from the soil by the growth of the oats is returned by furnishing, in this way, a slight dressing to the surface by the decay of their leaves and stalks.

Oats are cultivated principally in England as provender for horses. In Scotland they are extensively produced, more than half the grain crop of that country consisting of this product.

The cultivated oat (Avena sativa) is an annual, although the genus contains several perennial species. It is supposed to be a development of the wild oat (Avena fatua) that is found in Europe, and is regarded as a weed also in California, where it grows wild orer extensive tracts of land, and yields a good quality of hay. The wild oat differs from the cultivated variety by having the inner palet and the grain covered with hair, and the outer palet provided with a long awn. Experiments made at the Royal Agricultural College, England, by Prof. 
J. Buckman, prove that this seed when gathered ripe and sown the following spring will produce a grain differing very materially from the original, and when subjected to further cultivation, a grain is produced bearing a very strong resemblance to many of the varieties now cultivated. Oats vary in weight per bushel according to the variety, some of the large and heavy-grained variety weighing from forty to fifty pounds per bushel. American oats vary generally from thirty to thirty-five pounds per bushel. The average yield of oats per acre in the United States is from thirty to thirty-five bushels. When the soil is of good quality and well-prepared and the season favorable, the crop may be made to be largely in excess of that amount.

As a proof of what may be accomplished with this crop on thin soil, a thin, rocky drift soil at the Massachusetts Agricultural College was sown, and produced without manure, fifteen bushels per acre. With a suitable supply of manure, the yield was sixty-two bushels per acre. The same principle holds true respecting sowing seed that is free from smaller and blighted grains, and that which has not been thus separated. At the Agricultural College at the Wisconsin University the experiment was tried by Prof. Daniels which resulted in giving a yield of $48 \frac{1}{4}$ bushels per acre to the cleaned seed, and $43 \frac{3}{4}$ bushels per acre to the uncleaned. As a general result, we think the difference would be considerably greater than these figures show.

Oats are one of the very best crops cultivated for feeding working animals, and especially as provender for the horse, they are superior to all other grains. In Scotland, oatmeal forms a very important item in the diet of the peasantry, as well as that of the more wealthy classes also, to a certain extent, to which fact has been attributed the robust health, strength and welldeveloped forms and muscles characteristic of that nationality. More than half the annual grain crop of Scotland consists of oats. In this country the use of oatmeal as an article of diet has been more common for a few years past, and if the taste of the American people could be cultivated more generally in the direction of regarding it a dish to tolerate, (if not as a palatable one,) - there is no doubt that it would do much towards increasing their strength and vigor of constitution.

We once heard a gentleman remark, while ordering a dish of oatmeal at a hotel, that he did not bike the food, - on the contrary it was distasteful to him, but he ate it from principle, - out of a sense of duty, or "as a sort of penance for his sins," hoping to receive good to the body thereby, - which is certainly a novel theory on diet. For invalids and young children, it is very nutritious and strengthening when properly prepared. Oat straw makes quite good feed for farm animals when cut fine and mixed with other materials. When cut while in blossom and cured like hay, it makes excellent fodder for cows that are giving milk, or for sheep ; especially, pregnant ewes or those with young lambs. When stirred into water oatmeal makes a very healthful beverage for laborers in hot weather, and obviates the evil effects of drinking too much water under such circumstances.

Varieties of Oats.-Among the numerous varieties of this grain, we shall mention some of the long.established and leading ones, together with a few of those of more recent origin that have become established in agriculture, or that give promise of success. The White or common oat is quite extensively cultivated in England and Scotland, as well as in this country, and is known by its white husk and kernel, the latter of which is of medium size, the grain weighing from thirty-two to thirty-five pounds per bushel.

The Black oat is characterized by a black husk and its adaptability to poor soils, doing better on such lands than many of the other varieties. The Red oat has a brownish red husk. ripens early and is suited to a late climate; the grains are quite firmly attached. The Potato oat has quite large and plump grains, which are rather thick-skinned and white, and are double and sometimes treble, the straw being quite long. English authorities state that this oat is more extensively cultivated in the south of Scotland, and north of England than any other, and that this, with the Hopetown, Sandy, and Early-Angus are chiefly cultivated on the best class of soils, in those sections, these commanding the highest 
prices in the English market, the weight per bushel being from forty-two to forty-six pounds, and yielding generally from fifty to sixty bushels per acre.

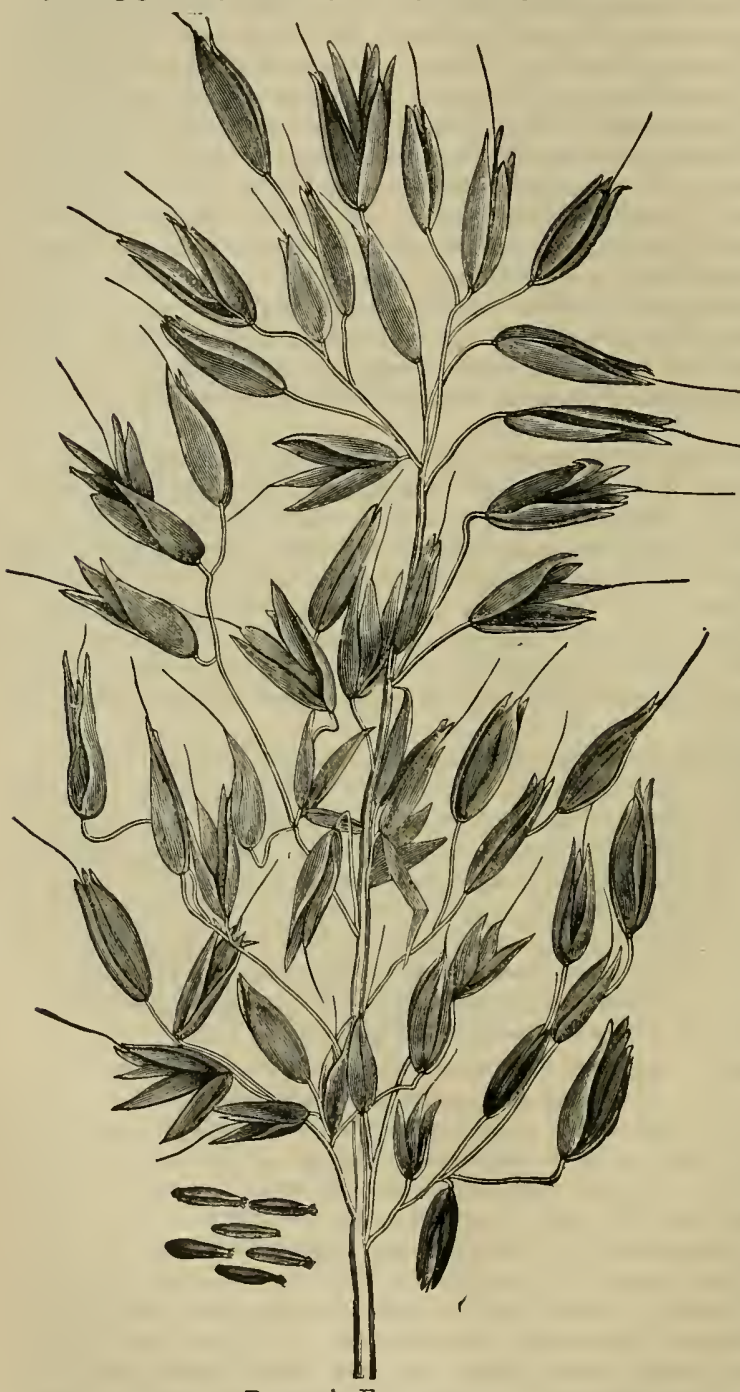

Pringle's Excelsior.
The Poland oat has a thick, white husk, short, white kernels, and short, stiff straw. It is very prolific and does best in warm, dry soils. The Black Poland has a large, black kernel, and will sometimes weigh from forty-five to fifty pounds per bushel.

The Friezland or Dutch oat has a plump, white grain, mostly double, and thin skinned, resembling the Poland somewhat, but with longer straw, The White Norway and New Brunswick White arc old and popular varieties. The Black Tar tarean is esteemed in England for its large produce and adapta. tion to inferior soils, but is rather coarse in quality. The White Tartarean is late and prolific, and said to do well in southern latitudes. Many of the above mentioned are more extensively cultivated in England and Scotland than this country, some of the heavier varieties showing considerable deterioration when grown in our drier climate, and requiring a frequent importation of seed to

keep up the standard. The White Russian is comparatively a late variety, that in some localities gives very satisfactory results. 
Among others of the somewhat recent kinds that have proven very successful, are the Probsteir, Excelsior, Challenge and Pringle's Excelsior, a comparatively new variety hybridized from the common Chinese Hulless and the Excelsior, which is a variety of the common oat of great vigor. The grains of this variety are nearly twice the size of the Chinese, and Hulless. It is very hardy and prolific, and the straw quite strong in growth. The inserted cut of this variety, copied by permission from the catalogue of B. K. Bliss \& Sons of New York city, is a very correct representation. The Surprise is an oat of very good quality, and gives general satisfaction to those familiar with it. As to which variety is best for general use, is yet an undecided question, some preferring one and others another. With this crop, as most others, much depends upon the soil, climate and the cultivation given it. We are of the opinion that of the two classes, the white varieties are generally preferable to the black.

A hardy kind, known as Winter Oats, is now being cultivated to a considerable extent, and has proven very successful in certain localities; but we doubt whether it will bear the rigors of the New England winters, or those of a latitude much above $37^{\circ}$ or $38^{\circ}$, although a valuable kind for a more genial climate. We believe it has thus far proven a decided success in the South, where spring oats frequently fail on account of rust. A rather deep sowing will make the plants more vigorous, and serve to keep the roots warmer during the cold season. Time and pains-taking may eventually result in producing a winter variety sufficiently hardy for even northern New England and the Canadas.

Winter oats are said to be larger and the grain heavier and more suited to milling purposes than most of the spring varieties. The common White oat has been more extensively cultivated in the United States than any other, but new varieties have for a few years past been introduced, and many of them proven, for certain sections, far superior to the old in quality and productiveness. By testing new varieties, or depending upon reliable experiments of others in this respect, the farmer can soon ascertain which are the most desirable kinds and best adapted to his own soil and climate, as some will thrive best in one locality and others in another, the soil, climate and kind of cultivation affecting materially the product, both in quality and quantity.

Cultivation.-Oats will thrive well on almost any land that is well drained or naturally dry, a wet soil being very injurious to the plants, and almost sure to result in killing them. Of course, the better the soil, as a general rule, the better the crop, but oats will give a fair remuneration for their culture on quite poor soil and with but little care in preparation, although we should not advise any farmer to practice this style of agriculture for any crop, for we believe it most profitable and the best economy to endeavor to obtain the best results always, and adapt the cultivation to that end. They are sometimes sown on inverted turf, but this practice is not to be recommended, as they do best on pulverized soil. They will generally thrive well to follow potatoes, corn, or any of the well-manured, hoed crops. Un. less the soil is naturally quite fertile it should be enriched by some kind of manure, which may be a well-prepared compost harrowed in, guano, or any of the concentrated fertilizers in use at the present time, but no green barn-yard manure should be used. Many of the special fertilizers are valuable. Salt, sown broadcast over the land at the rate of from two to six bushels per acre, is highly recommended by some as a fertilizer, and as also useful in destroy. ing insects injurious to the oat crop. It is useful on lands upon which grain would be liable to lodge, and should always be used when sown on the sod.

The soil for oats should be plowed to a medium depth and well pulverized; we believe it a general rule, with but few exceptions, that all small seeds and grain require a good seedbed and their "food cut fine." In a moist season, oats will do well on a sandy soil, but we believe that the best yields are made on a fine clay loam with good drainage and the seed sown early. We believe in sowing as early as practicable, as in most sections a better harvest is the result, but not until the soil is dry and mellow. In some localities mildew is quite sure 
to affect the stalks and grain if put into the ground late in the season. While bright straw and a heavy berry will usually result from early sowing, those sown late are almost always light and of poor quality.

Oats may be sown broadcast or with a drill, some preferring one method and others the other, each having their respective advantages, but we believe the drill is omore generally preferred on large farms where this crop is extensively cultivated. Where the sowing is broadcast a seed-sower should be used, as it distributes the grain more uniformly and much more rapidly than the old laborious practice of hand-sowing. Seed sown with a drill are covered at a more uniform depth, which can be regulated at will, and where they will come in contact with the moist earth, which will cause a quicker germination; consequently they will come up sooner and much more evenly than when sown broadcast, and there is no danger of any ir. regularity from skipping or lapping spaces, if the wind blows during the operation of getting the seed into the ground; therefore, a heavier crop per acre is generally the result of the use of the drill. There is also more opportunity for the circulation of air among the plants, besides less liability of lodging. When sown broadcast, the seed should be lightly harrowed in, and whatever the method of sowing, the field should be rolled, unless the land is quite wet and inclined to bake or harden; this presses the soil upon the seed and causes it to germinate more quickly.

In some portions of the South, oats are sown in the fall, like winter wheat, which gives them an early start in the spring. There is also a winter variety that is cultivated quite successfully in latitudes as high as the mountainous portions of Tennessee, and by repeated efforts in acclimatizing and hybridizing, we doubt not that a winter variety will yet be produced that can be successfully cultivated considerably farther north. The quantity of seed sown varies from one to four bushels per acre. When the sowing is early, about two bushels per acre, for most soils, is a fair quantity. If the sowing be rather late or the soil poor, three or four bushels may be required. The richer the soil and the more early the sowing, the less the quantity of seed, as a general rule. English authorities speak of some. times sowing six or more bushels per acre, but this is quite beyond the average rate. As we have previously stated, for most soils, we believe two bushels will produce a larger crop, with the grain of better quality, than filling the soil with a surplus of seed, which must result in crowded stalks and small heads of grain, with inferior sized kernels.

The seed should always be well cleansed by running it through a fanning-mill, or, what is better, a sieve of such size as to sift out all the inferior and small kernels, as well as all seeds of weeds, \&c., leaving only the largest and best grain for the sowing. Oats and all other kinds of small grain are benefited by after cultivation. When sown in drilss, a wheat hoe is very beneficial for this purpose, or, if such a machine is not available, a light harrowing when the plants are from four to six inches high is generally followed with good results. Upon smooth land, the Thomas Harrow is very good for this purpose, but if the land be rough and full of corn stubble or other similar obstructions, there would be danger of tear. ing out and destroying many of the plants by this process.

Greater vigor and productiveness is secured to oats, as to wheat and most othercrops, by occasionally changing the seed, although we do not think it necessary to make such a change very often. Many of the best agricultural writers in England assert that in making this change it should always, if possible, be from an earlier climate and better soil; others assert with equal confidence, that while this is true respecting high-lying lands which represent poorer soil and a colder climate, yet with a good soil and fair climate the best results are obtained with seed from a later locality. We are inclined to the latter opinion, from our own experience and observation, and believe that new seed-oats should always be bronght from a northerly locality, as they are heavier and larger grained than those of a southerly climate. Many farmers carefully cultivate their grain for seed on a small field apart from 
the main crop, giving it the best soil and fertilizers in order to keep up the highest quality of the variety unimpaired, or to improve upon it, if possible. This method is almost invariabiy atterded with good results.

Har resting. - Oats should be harvested when the lower part of the stalk has turned yellow; the plant then ceases to draw nutriment from the soil, and the grain has passed from the milk state and may be easily compressed between the thumb and finger. If cut at this period the straw is better for fodder and other purposes, and the grain more plump than later; but if the cutting be delayed, the kernels will be liable to shell out, and considerable loss to the crop may be sustained, besides the quality of the straw impaired.

Small fields of oats may be cut with a cradle, and when badly lodged a scythe is often used for the purpose, but for large fields a reaper seems to be a necessity; those that both reap and bind are of course the best, since they economize labor most. It is very important that the oats should be well dried before being put away, if not threshed in the field, as they will be very liable to heat badly and the grain become discolored. Exposture to rain and dew will also have a tendency to discolor the grain, and lessen its value for market purposes.

It is very important that oats be cut in dry weather, and the bundles put in small stacks for a day or two, and exposed to the sun, that they may become entirely free from moisture. When cut by a cradle or a reaper that is not self-binding, they may dry in the swath before being bound. Nuch of the labor of handling bundles and stacking them away is obviated by threshing the grain in the field with a machine that also cleanses and bags it ready for market. When rain chances to fall upon the crop, it should be thoroughly dried as soon as possible.

Diseases of 0ats. - There are fewer diseases and insects that affect oats than most grains, it being seldom injured by rust or smut. Whenever it is attacked by either, it is usually in very warm, damp weather. The wire-worm has at times been very destructive in some localities, and is most liable to be troublesome when fresh sod is used for sowing. We would not advise the use of fresh sod for this crop, but whenever such a course is pursued it would be well to turn it over late in the fall just before the ground freezes, which leaves these insect pests without a retreat from the frost. The use of salt as a fertilizer, and also lime, has a tendency to extirpate them from the soil. When rust or smut are seen in a field, it is best to have the crop cut as soon as possible. The brine-wash for the seed does not seem to have the same effect in destroying the germ of the fungus in oats as with wheat and rye, owing to the hard, thick covering of the grain.

\section{BARLEY.}

$\mathrm{T}$ HIS is one of the most widely distributed of grains. It was an important article of food in remote antiquity, and commonly cultivated by the Hebrews, Greeks, and Romans. In Europe it is at present a very important crop, ranking in Great Britain next to wheat both in an agricultural and commercial point of view. It is used for bread in many portions of Northern Europe, and especially in the lowlands of Scotland, where unleavened cakes or "bannocks o" barley meal" constitute an important item in the diet of the peasantry. It is also used in preparing the "barley broth," so much esteemed by the Scotch nationality. It is appropriated, however, both in this country and Europe quite as much to the manufacture of malt liquors as any other purpose, although to a certain extent for fattening live-stock, especially swine. It were better for both countries if its cultivation were entirely discarded, rather than it should be perverted to the former use, to the incalculable injury it does to multitudes of human beings, and we hope no honest farmer who wishes 
well to his fellow-man will ever raise this grain for such a purpose, however remunerative such a course may be.

There are three leading varieties of this grain, viz., the two, four, and six-rowed barley, besides several sub-varieties, which are the result of modifications of the soil, climate, and cultivation. Those most commonly cultivated in this country are the two-rowed and six-rowed; the former can be raised in land less rich than the latter, and is thought to be the most reliable for a moderate yield; but if the soil is just suited to it, the season and other conditions favorable, the six-rowed barley will yield the most, while a failure of the latter crop is usually more complete a failure than the two-rowed. The six. rowed ripens a few days earlier than the other variety, and requires harvesting in many localities at the time wheat does, which is thought to be something of a disadvantage to extensive wheat-growers, as no crop suffers more from neglect in harvesting just at the time it is ready for cutting than barley. The two-rowed has the largest grains, the six-rowed next, and the four-rowed the smallest.

The early ripening of barley renders it a good fallow crop to precede wheat, as it enables the farmer to plow two or three weeks earlier than he could after oats, and get the land in much better condition than would be possible where the plowing was late. Like some other cereals, certain kinds of this grain have been so modified that they are regarded as winter and spring varieties. The winter varieties are not as hardy as winter wheat, consequently we would not advise their cultivation north of $38^{\circ}$ or $39^{\circ}$. New varieties may be produced by crossing, as with wheat and other grains. A species sometimes known as "German rice" is much valued and cultivated in Germany. The straw of barley is very hard and coarse, and of little value as food for stock.

Cultivation.- - Barley thrives best in a warm, friable soil, rather rich, but less so than for wheat, and neither too light nor too heavy. It is important that the land be quite uniform in fertility, or the grain will ripen unevenly, to the deterioration of the crop. It should never be sown on a cold or wet soil of any kind, a well-drained loam being the best. This will admit of early preparation-as it is very essential that it should be sown earlyand such land will perfect a good crop, if the season is at all favorable. It should by no means ever be sown on a very sandy soil, or any that is easily affected by drouth, for if the land becomes quite dry at the critical period of forming the head, both the quality and quantity will be greatly diminished. It will, however, endure more heat and drouth at this period than either wheat or oats. The soil should be one that is free from weeds, or what is termed a "clean soil," and the tilth both fine and deep.

Weeds are a great detriment to this crop; consequently no green farm manure should be used for the land, and all vegetable or animal matter of any kind should first be well decomposed and of the nature of humus before being applied.

Commercial fertilizers are of special value to barley, and salt is frequently used with good results. The latter is thought to promote the growth of the plant and basten the full development of the grain. It is well to put in the seed when the land is rather dry, and to be deposited quite deep; if covered to the depth of four or five inches, or twice the ordinary depth of sowing wheat, so much the better, in most soils. Good crops are often obtained from an inverted sod, a clover sod being the best.

It may follow in rotation corn, potatoes, turnips, or any of the hoed crops, but should never follow wheat, rye, or oats, nor should two crops succeed each other, except the land be very rich, and even then the practice is objectionable, as the quality of the grain is liable to deteriorate, an evil to which barley is more subject than any other of the cereals, hence, it will not bear careless or indifferent cultivation, and a frequent change of seed is desirable. With spring varieties, early sowing and a finely-pulverized soil are very essential to success with barley, while the winter grain will require a good start before cold weather. The seed 
may be distributed broadeast or sown in drills, but in either case should be covered deeper than wheat, rye, or any of the other cereals. It should be followed with the roller, a procesis which some farmers repeat when the plants are four or five inches high, if the ground is dry and not very compact. This is thought to give support to the roots and increase the vigor of the stalks, as well as to destroy insects.

The quantity of seed requisite will vary according to the nature of the soil, from two to three bushels per acre being the usual allowance when sown broadcast; when drilled, about a third less. When sown early, or on light and mellow soils, a less quantity is generally used than for rich land or late sowing, yet many of our most successful farmers use only from one and a half to two bushels per acre, according to the nature of the soil. A less quantity than formerly is at present sown in the cultivation of most grains. The Hessian fly, smut, and rust are sometimes liable to attack this crop, but not as frequently or to the extent that they do wheat.

Harvesting.-The harvesting of barley should be done seasonably, or as soon as the grain is fully ripe, since it is more liable to shell out in the field and waste than any other grain; but, on the contrary, if cut too early, and before being fully matured, the kernel will shrivel. The thin-skinned grain, which is considered the hest, will sometimes have this slightly shriveled appearance when fully matured, owing to the thin coating of the kernel, but the distinction between this and the immature seed can easily be seen. The husk or coating of some varieties of barley is too stiff and harsh to shrink, and will often look smooth when the contents of the berry are considerably shrunken, and can only be detected by carefully cutting open and examining a few grains. When harvested at the right time, it is much brighter than when allowed to stand longer in the field, as it will then assume a dull, brown color, which will often affect the market price of the grain. It should be well dried in order to prevent heating after being stacked or packed away in the harn. It is a good plan to put the bundles in small stacks for a day or two to be exposed to the sun, the same as wheat, but they will require careful handling not to shell out the grain, and all exposure to rain should be avoided.

\section{BUCKWHEAT.}

$\mathrm{T}$ HIS cereal is a native of Central Asia and the basin of the Volga, and has been known and cultivated for ages in different portions of the civilized world. In this country and Europe the flour is used as an article of food for human consumption, being employed mostly in making cakes, for which it seems especially adapted, and which are considered quite a luxury in many portions of the country, where "buckwheat cakes" with maple syrup have attained considerable celebrity as a supplementary breakfast-dish, to be enjoyed during the coolest season of the year. When properly ground, the flour is as white as that of the best quality of rye. In grinding, the outer covering of the grain is entirely removed. Buckwheat is a valuable food for poultry, and when mixed with corn is much used in fattening swine. It will also fit up a horse quickly with a fine sleek coat. When well cured, the straw makes good fodder for sheep and cattle, and when spread upon land will rapidly decay, and make a valuable fertilizer. Corn should never succeed this crop in rotation, as it rarely does well when this plan is followed. It is often employed as a fertilizer, its rapid growth admitting of turning under two crops in a single season.

It is of less value than clover for this purpose, but will grow on soils that will not pro. duce clovar; therefore is employed in renovating very poor lands. It will thrive on the hard. 
est and toughest soil, and for this reason is often used in subduing wild lands, being sown on the inverted turf, with but slight preparation, which it will penetrate and pulverize, leaving it in a mellow and fine mechanical condition.

As a green manure for rye, it is considered by most farmers quite valuable. If sown during the second week in July, it will be ready to turn under in the early part of September.

Buckwheat is considered by many farmers a very exhaustive crop, and yet the land that will not produce a single crop of corn without manure, will yield several crops of buckwheat. It will also grow and thrive on poor soil that will scarcely give any yield of either corn, wheat, or barley, and do this for several years in succession without seeming to diminish in any large degree the productive capacity of the land; on the contrary, we have seen rich soil that seemed to be much injured by a single crop of this grain. It is in these respects a very peculiar plant. The theory that it "poisons the soil," as we have heard some farmers assert, is absurd. It is proven by chemical analysis that it requires, as the elements of plant-food, considerable potash and a moderate amount of nitrogen and phosphoric acid. We do not think this crop, when compared with others, is really an exhaustive one to soils generally. $\Lambda$ good crop yields about forty bushels, and often more, per acre.

Cultivation.-Although buckwheat will grow and produce a fair crop on poor soil, with little or no manure, and often with the most careless preparation, yet it yields the most remunerative crop only on those that are fertile and properly prepared. Sandy loams are quite a favorite with this grain, especially such as have long been kept in pasture, or in mow: ing lands, but require plowing, although it will do well on a great variety of soils. Wet lauds are unfavorable to its cultivation. Chemical fertilizers are admirably suited to it, also barn-yard manure, when well composted. Fresh manure is injurious, and fills the crop with weeds. Wood ashes are also valuable in its cultivation. If too large a quantity of manure is used, the straw is liable to lodge.

Beside the common variety, or dark-colored grain, there is another of a light gray color, known as the "silver-hulled buckwheat," which in many respects is considered superior to the former. The corners of this grain are less prominent than in the ordinary variety; it is more plump and heavy, the husks thinner, involving less waste in grinding, while the flour is thought to be whiter and more nutritious. It is said to be more prolific than the common variety in soils suited to it, but more tender, and to thrive best on high, rolling lands in good condition, and to require better soil than the common kind generally, or, rather, that it will not produce a good crop on certain poor soils that result favorably to the common variety.

The period of sowing this grain will vary according to the latitude, as it is necessary to give the crop an opportunity to ripen before the time of frost. In some of the extreme Northern States, it will be necessary to sow it as early as the middle of Nay; in others of a warmer climate, it may be delayed until the first of July; in some of the Southern States, it is sown in August. It is generally sown broadcast and harrowed in, but sometimes in drills. When the soil is well-pulverized, it is benefited by the use of the roller. As the plant branches out and covers considerable ground, many farmers sow comparatively but a small quantity of seed, and we have known some, who make a specialty of it, to use even but a peck per acre with good results; it is sown in quantities varying from this to two pecks and a bushel and a quarter per acre. It ripens very unevenly, and, if allowed to remain without harvesting, will continue to blossom during the entire season, but the largest crop will be from the first blossoms, and it should be cut when these, the earliest seeds, are perfectly ripe.

Many farmers are particular to cut it while the dew is on, to prevent the grain shelling out. We subjoin a few items on the culture of this grain from the pen of an intelligent and practical farmer of Western New York:-

"Neglect too often attends the cultivation of the land intended for buckwheat, and the 
poorest and worst.conditioned land is usually given this erop. Besides, from the lateness of putting it in, the land becomes grassy, and it cannot be plowed well without the grass mak. ing its appearance to the detriment of the crop, with no benefit to the land. Now, instead of this treatment, the buckwheat field, worked after the spring's sowing, affords a chance to im. prove the land and insure a good crop-decidedly a paying one, if the season is favorable. Buckwheat is somewhat peculiar, as with a favoring season and a heavy growth, the income is sometimes surprising. I have known a single crop pay for the purchase of the land at $\$ 40$ per acre. The land was plowed early enough to rot the sod, and re-plowed just before sow. ing. If put in good condition, it does not need to be very rich. What it needs is ripe and uniform fertility; it does not want wet soil turned up and exposed to the hot sun, as I have often seen, thus injuring rather than benefiting the land. Instead, buckwheat should be made the means (as there is a chance for it) to reclaim or improve the soil. It can be made to equal, if not exceed, the effect of summer fallow, and with less expense-the crop so densely shading the ground, and from the start, that not a weed or a grass-blade can survive; all is smothered by the exclusion of air and light, the ground kept comparatively moist and mellow, and the texture thus improved. The farmer who, therefore, does not avail himself of advantages here offered, misses his opportunity; unless he is among the fortunate ones who have their land all improved. Even then buckwheat is a benfit to the soil, as there is less expense of cultivation, and the weeds are effectually kept at bay. It also adds to the variety of crops.

The time for sowing buckwheat here is from the 26 th of June to the 4 th of July. Half a bushel per acre is seed enough, as on rich ground it stools out well. This is better than to have it closer and denser. It should be branching, giving thus more room for blossoms, and affording protection against the direct rays of the sun, the plant thus being in a measure self-protecting, favoring the lower or partially hidden seeds, which sometimes are to be depended upon for the crop, the more exposed or outside being blasted. Instead, therefore, of giving it careless treatment, buckwheat requires careful management. The selection of soil is also of some importance, a sandy loam being better than clay. A stiff clay must be brought into a friable condition before it is fit to grow this crop successfully. Sod or green crops turned under, or coarse manure worked in, rotting and mixing the soil and manure well, will do it. Avoid all wet soil for buckwheat, unless first drained. We have here a real ren. ovator of the soil, and get a good crop besides. Sometimes, however, the crop gets blasted. When this is the case, turn it down. Instead of being a misfortune, it will be found to be a benefit, affording a large quantity of good material, decaying readily, and having a good effect upon the soil."

It should be thoroughly dried before being put away under cover, as it will be liable to heat; it is, therefore, customary to set it up in the field in small stooks for this purpose. It should always be harvested before a frost, and it is well to thresh it as soon as dry, as this practice obviates the liability of its shelling.

In localities where both the common and silver-hulled buckwheat are cultivated, it is very difficult to retain the latter seed pure and unmixed, as the buckwheat field is such a favorite pasture with the honey-bees that cross-fertilization with the common variety will constantly occur from the pollen carried by these little workers from one field to another. 


\section{CORN.}

7 HIS is the most abundant of the cereals, as well as one of the most important agricultural products cultivated in the United States. The corn-plant belongs to the natural order-Graminæe or grasses-and is indigenous to America, where it formed the principal food of the Indians, and for which reason it received the name of Indian corn.

History states that Columbus found it cultivated quite extensively by the natives of Hispaniola, while it was found in the possession of all the native tribes in portions of the coutinent subsequently visited by explorers.

Their implements of agriculture were, of course, of the rudest kind, the hoe often being constructed from the scapula of the moose, a thin, sharp stone, or a crooked stick; still with these imperfect utensils, and although they had but a few varieties of the grain, compared with those of the present time, we learn from undoubted sources that they cultivated several distinct varieties that answer in description to those with which we are familiar, such as the Mandan, King Philip, Sioux, Squaw, Tuscarora, and probably sweet corn.

In Nov. 25, 1620, some of the Pilgrims found corn that had been buried in the earth, which, according to the description given, consisted of "some yellow, and some red, and others mixt with blue." We also read of the "white and yellow," and those of which "the graine be big."

Cartier, in 1555, describes the maize at Hochelaga-the Indian name for Montreal- "as great, and somewhat bigger than small peason." Thus, numerous instances might be men. tioned to show that several varieties were in cultivation at that period. Corn is still found growing in a wild state in many portions of the continent, and with each grain of the ear covered with glumes or husks, that long cultivation has eradicated. It is supposed to be a native of the table-lands of Mexico or Peru. Its cultivation was introduced from this con. tinent to Southern Europe, Asia, and Northern Africa, where it thrived and spread rapidly; it is not, however, grown in England to any extent, as the summer beat of that climate is not sufficient to favor its successful production.

It will grow in any portion of the United States, and readily adapts itself to different soiis and climates, but thrives best between the thirty-sixth and forty-second degrees of lati. tude, as it attains its most perfect development under a hot summer sun, growing rapidly and ripening early, which renders it peculiarly adapted for this portion of the northern latitudes, where the summers are short and warm, however severe the winters may be. In high northern latitudes, close attention to its culture is necessary, on account of the difficulty of ripen. ing it. The value of the corn crops annually produced in this country may, perhaps, be justly estimated in a financial point of view as amounting to a certain number of millions of dollars, but, in another sense, its value to this country is inestimable, when we consider the effect of its cultivation in hastening its settlement and the continuance of its prosperity, entering, as it does, into the food of all classes of people, and also greatiy cheapening and in. creasing the product of beef, pork, and mutton, besides the many other uses to which it is appropriated, and the increased wealth of the nation resulting from the annual exportations of this product.

Immense as is the production of this staple, the average yield of corn in this country at the present time is far below what it might, and ought to be, and what with a little more care in cultivation might easily be attained. The average yield in the United States for the en. tire number of acres cultivated does not at present exceed thirty bushels per acre, while with good culture it might be made to reach from sixty to a hundred bushels or more, and thus the amount now produced be more than doubled, with its attendant benefits to the nation and to the farmer individually. The farmers of this country are gradually learning more of the 
possibilities of this crop, and that there is more in its careful cultivation than was formerly supposed. There is no doubt that exaggerated statements have often been made relative to the amount harvested, and which have caused farmers to look upon reports of unusually large yields with distrust; yet we know from authentic sources, that under favoring circum. stances of soil, climate, skillful cultivation, and a season suited to its perfect development, immense and almost incredible yields have been produced, which prove the possibilities of the crop when properly managed, and which should lead corn-growers to consider the question as to whether their present method of culture is the most profitable one that can be adopted, and whether more care in enriching the soil and tilling it would not in the end be more economic and profitable.

Dr. E. L. Sturtevant of Massachusetts, states that he has obtained, from one acre, one hundred and twenty-three bushels of shelled corn; Mr. Henry Stewart of New Jersey says: "I have grown a crop equal to one hundred and fifty. bushels and forty-eight pounds per acre of shelled corn on a potato-stubble, and one hundred and eighty-nine bushels of shelled corn on two acres of oat-stubble."

Mr. E. S. Carman, editor of "The Rural New-Yorker," recently raised upon his Long Island farm one hundred and thirty-four bushels and a half of shelled corn on seven-eighths of an acre, and one hundred and fifty.nine bushels and one-third on another whole acre. Other reports equally reliable give the yield a hundred and thirty-eight and a hundred and forty-six bushels per acre, while we know of frequent yields from eighty to a hundred bushels. We have seen the published assertion, that in one of the extensively corn-growing States, the immense yield of two hundred bushels of shelled corn have been grown per acre, with exceptionally good cultivation, which may or may not be correct, as the statement has never been authentically verified. We do not give it as a fact; however, it would be well for our farmers, if more of them would aim to attain that result, and thus approach nearer to it than they now do. The majority of our best farmers have found it difficult to reach a yield of ninety or a hundred bushels per acre, but by making the standard of yield high, those whose lands are suited to the cultivation of corn will find that higher results can be reached than is commonly supposed.

The kind and quality of seed will largely affect the yield, while other conditions, such as soil, culture, \&c., will modify it still further. The height to which the full-grown plant attains varies with different varieties and soils, sometimes reaching the luxuriant growth of eighteen feet on the rich soil of some of the Western prairies, and those of a similar character. The color of the kernel depends upon that of the hull and the oil it contains. In the white varieties they are both transparent; in yellow corn the hull is transparent and the oil yellow; the red and blue kinds owe their color to the hue of the hull or covering. In some varieties of corn there is a larger amount of oil than in others, some being nearly destitute of it. Oil is useful in protecting the grain, when germinating in the soil, from being too rapidly decomposed in long-continued wet weather, and in imparting nourishment to the young plants. It also has a tendency to keep meal from souring as readily as it otherwise would, that ground from corn having the least oil becoming musty, or sour, soonest. The Tuscarora corn contains very little, if any, oil, and the meal made from it will keep sweet but a short time. In the manufacturing of whisky, during the process of fermentation, the oil separates from the corn and rises to the surface, and is taken off and used for various purposes. According to good authority, rice-corn contains the most oil, pop-corn next, Canada corn ranks third, and broom-corn fourth; and as the lowest varient types are the small rice-corn and pop-corn, and the highest some of the most valuable improved varieties, it would seem that the effect of careful cultivation is to increase the starchy properties of the grain to the lessening of the fatty contents.

As an article of food for man, it is a very healthful and nutritive diet, containing, as it 
does, the elements required for the development and support of the animal system. The analysis of corn, as given by Palson, is as follows:-

\begin{tabular}{|c|c|c|c|c|c|c|c|c|c|c|}
\hline Gluteu, etc., & - & - & - & - & - & - & . & . & . & 8.8 \\
\hline Starch, & . & . & . & • & . & . & & . & - & 54.4 \\
\hline Gum and sugar, & . & . & . & . & . & . & & . & . & 2.7 \\
\hline Oil, $\quad$. & . & . & . & . & . & . & & . & . & 4.6 \\
\hline Bran, etc., & . & . & . & . & - & . & & . & . & 15.8 \\
\hline Ash, & . & . & . & 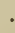 & . & . & . & . & . & 1.7 \\
\hline Water, & . & . & . & . & . & . & . & . & . & 12.1 \\
\hline
\end{tabular}

Although there is a slight difference in the proportionate ingredients that enter into the grains of different varieties, yet the above analysis would represent a fair average of the different kinds cultivated.

To show the difference in some of these varieties, as well as the difference in the properties of water contained in the meal or kernel of that thoroughly or imperfectly cured, we in. sert the following analyses recently made at the Connecticut Experiment Station, by Prof. S. W. Johnson:-

Analyses of Maize.

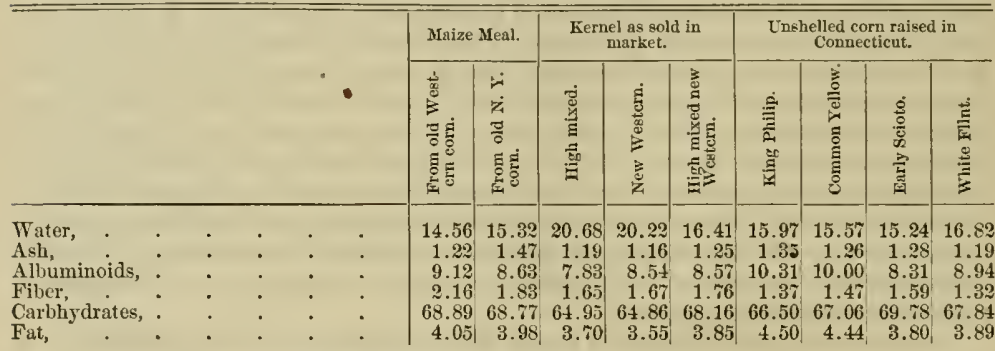

\section{COMPOSITION RECKONED ON DRY SUBSTANCE.}

\begin{tabular}{|c|c|c|c|c|c|c|c|c|c|c|c|c|c|c|}
\hline A. & & & & & & 3 & 4 & 1.50 & 45 & 1.50 & 1.60 & 1.49 & 1.51 & \\
\hline All & & & & & & 10. & 10.19 & 9. & 10.70 & 10.25 & $12.2 \pi$ & 11.88 & 0 & 10 \\
\hline & 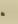 & - & & & & 2. & 2.16 & 2.08 & 2.09 & 2.10 & 1.62 & & 7 & \\
\hline Car & 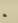 & 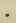 & & & & 80.63 & 81.21 & 81.88 & 81.31 & 81.55 & 79.15 & 79.62 & 82.24 & 81 \\
\hline & & & & . & . & 4.74 & 4.70 & 4.66 & 4.45 & 4.45 & 5.36 & 5.27 & 4 & \\
\hline
\end{tabular}

\section{Dieestible Nutrients in air-DRy substance.}

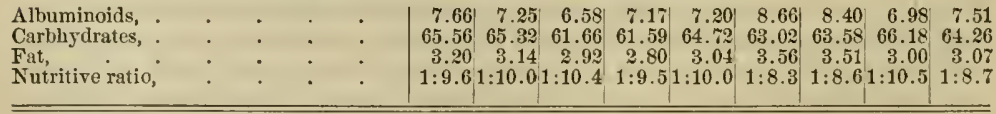

These analyses were made upon the different specimens as they reached the Experiment Station and before any of the moisture was lost by subsequent drying. Dr. Jenkins found, in summing up the results of sixty-three analyses of corn, that the amount of water ranged from six to fifteen per cent., the average being ten and a half per cent. Dr. Goessman found, in eleven analyses, the average per cent. of water to be ten. Other analyses, by Prof. John. son, from meal offered in the market, resulted in a quantity of water from twelve to over twenty-one per cent., and he concludes that all together prove that the quantity of water in corn kernel or meal, as found in the markets generally, may range from thirteen to nearly twenty-two per cent. of the entire weight; that well-cured corn and meal may contain from 
fifteen to seventeen per cent, and that new corn or meal are quite likely to contain as much as twenty or twenty-one per cent. of water. It will be readily seen, therefore, that it is a matter of some importance to the farmer, in large transactions, whether corn and corn-meal be bought in a slightly, or thoroughly cured state. There is quite a difference between new and old corn in the amount of moisture contained, as will be seeu by the analyses previously given, and which, if known, is not always appreciated.

Varieties. - There is no other of the cereals which presents so many varieties as corn or maize, although there are comparatively but few which are distinct in general cultivation. But hittle effort was formerly made to keep the different kinds pure; more care, is, however, being exercised within a few years in this respect than formerly, and as new varieties are constantly being introduced and tested, the merits of the leading ones will doubtless soon be more extensively appreciated, resulting in the more general practice of pure-bred varieties of this grain. The height attained by the plant varies, according to soil and kind, from that of the little shrubby stalk of three feet, to the beautiful and luxuriant growth of eighteen, while in color and form, we have white, yellow, red, striated, brown, black, purple, blue, etc., either in tiny compact ears with small, flat grains, or with rounded little kernels closely wedged and resembling grains of rice, and very appropriately called rice-corn, or all the intermediate varieties between these and the magnificently-formed proportions of ear and kernel which characterize that produced by some of the Western States, where it attains, in size, its most perfect development. Some of the cobs are red, others white, irrespective of the colcr of the grain, while the ears vary in length from one inch to iffteen inches or more, with proportionate variance in diameter and size of grain.

In many of the corn-growing sections, the only recognized distinction is in the color of the grain, "white corn" or "yellow corn," according to the color of the kernel, being the only distinguishing terms in use to designate the kinds generally cultivated. Although the lower types hybridize inore readily than the more valuable, improved varieties, still the latter are so liable to mix with different kinds grown in their vicinity, and also adapt themselves to different soils and climates, varying to a certain extent with almost every degree of latitude and longitude, that it is somewhat difficult to retain the original type unmixed and unchanged.

As an instance of the changes produced by climate,-when some of the small varieties which ripen early in the extreme Northern States are cultivated a few years in the Southern, where the summers are so much longer than at the North, they will gradually change, by ripening later, and with a much larger growth of ear and kernel. This change goes on from year to year, until the plant has perfectly adapted itself to its new location, where it remains a fixed variety, unless it should be moved again North, when it will gradually assume its former type.

The small "flint" corn which has from eight to twelve rows is, as a general rule, found cultivated mostly in the New England States, and the northern portion of New York, as well as that of one or two of the most northern of the Western States, these being sections where it is necessary to cultivate the early-ripening kinds, while in the South and most southerly of the Western States the "dent" or "gourd-seed" varieties are at present more extensively grown than any other.

Among the varieties of the "flint" that are very popular and generally considered among the best are the "Holden," which is a small eight-rowed sort, and quite productive, the Yellow Canada, of similar type, Kingsbury's Excelsior, an early twelve-rowed kind of larger growth than either of the preceding, and the Early Compton, a fine and prolific variety, which is a seedling from the Dutton, and resembles it somewhat in appearance. The kernels are of medium size and bright yellow; ears eight.rowed and quite long; ripens early. Besides these, we have the well-known New England Golden, the White Flint, Early Dutton, 
and Early Scioto, all of which are long-established and valuable varieties. Therc are many kinds of the large Southern and Western corn, among which are the White Gourd Seed, Early Galena, Southern Big Yellow, Illinois Yellow, Long John, Queen of the Prairie, Chester County Mammoth, Evans and Proctor, all of which are largely cultivated. The last three varieties are of extremely large growth, both in stalks and grain, and thrive best on very rich soil. There are many sub-varieties intermediate between the larger and smaller kinds, which are known and valued in certain localities, and which seem especially adapted to those sections, intermediate between which the early-ripening and the larger and later varieties are grown. These are considerably larger than the early sorts, but smaller than the large gourd. seed varieties. The "Connecticut Valley corn" and its numerous variations are examples of this species, and are much more extensively grown in Connecticut and Massachusetts than any other. It is very prolific, ripens in about four months from the time the seed is planted, while its quality is superior to the gourd-seed kinds. The Improved King Philip or Rhode Island is of a similar type, and has long been one of the leading kinds. The White Prolific and Waushakum Thoroughbred are recent varieties of great promise. The Sweet corn, with its many types, forms a very palatable and nutritious article of diet, when properly prepared, and one which in this country is very popular.

It is almost needless to particularize the valuable and popular varieties, as new ones are constantly being introduced, and the farmer will find no difficulty in selecting such as, with proper cultivation, will give good results. At a recent meeting of the Massachusetts Board of Agriculture, the following description of the model or ideal ear of corn was given by the committee:

"It has an ear medium in size as regards the diameter; the kerne's are deep; the cob is small at the butt; and the ear holds its bigness towards the point until very near the tapering. off. It should be capped over; and the kernels should hold their bigness towards the point and at the butt run out straight, and not crinkle."

In the selection of ears for planting purposes, it will be well for the farmer to have an "ideal ear" in mind as far as the main points are concerned. Whatever the kind cultivated, it should be adapted to the soil and climate. Only the early-ripening varieties should be grown in the extreme northern section of the country, as the later ones will not have time to fully mature and ripen during the short summer. Many of the early kinds will ripen in ninety days after planting.

Selection of Seed. - The quality and quantity of the crop is largely modified by the quality of seed sown, and whatever the variety, only the most perfectly developed and ripened seed should be selected for this purpose. Corn taken from the bin at random for planting, as is the custom, with many farmers can never give in result that of careful selection. Some of our choicest kinds have been brought to their present high standard by selecting only the earliest and largest ears from the most prolific and thrifty stalks, and by careful cultivation for several years in succession. The selection can best be made in the field before the crop is gathered, as it affords a letter opportunity of comparison. By careful selection and cultivation of seed for a few years, astonishing results will be produced in the improvement of the crop. Dr. Sturtevant says:-

"To the seed must we look first, in order to secure improvement, in order to gain the maximum crop at the least expense. It is not enough to select the ear; this process has done something; but alone, this is not sufficient. We must pay attention to the past history of our seed; we must breed our corn; we must secure prolific ancestry, and use the seeds from fecund parents.

My attention was forcibly called to the importance of the seed in 1875 , the seed used appearing to the eye of equally good quality, but from different sources. The field was of like history, was manured alike with burn yard manure, and was cultivated as one field. 


\begin{tabular}{|c|c|c|c|c|c|}
\hline & . & & & & $\begin{array}{c}\text { Bushels } \\
\text { Shelled corn. }\end{array}$ \\
\hline Seed-corn A yielded with manure, & ${ }^{\circ}$ & . & - & - & 110 \\
\hline Seed-corn A yiclded without manure, & - & . & . & . & 68 \\
\hline Seed-corn B yielded with manure, & . & . & . & . & 55 \\
\hline
\end{tabular}

Here the better seed yielded, without manure, more than the inferior seed with manure; and under equivalent circumstances the better seed yielded just double the crop of the inferior.

After thinking over this result, which seemed to me surprising, I concluded to attempt the forming of a seed-corn of prolific habit. I became aware that the appearance of the ear was but a secondary consideration, the past eropping of the seed being the primary. The summary of my procedure is this: as corn has an hereditary character, I secured prolific male parentage by carefully castrating the tassels from the barren stalks in my seed-growing field, and then selected the best ears of the crop for next year's seeding. The results have been marked. I have secured prolificacy, uniformity of ear, and a heavy corn-grain."

Thoroughbred types are as essential in the production of plants of a high order and the consequent improvement of crops as in breeding live-stock, and a carefully cultivated, pure-bred variety of corn is as much superior to an indifferently cultivated mixed kind, in transmitting its good qualities to its product, as a thoroughbred animal is in this respect superior to a grade.

Some farmers cultivate a special small field or plot for seed with great care, and then select only the best of this crop for planting. This is a practice to be commended. The plot should always be planted at a distance from any other in the field, whether of the same variety or otherwise, as if near and of the same kind, even, there will be a liability of some of the ears being fertilized by the pollen of inferior plants or of the suckers, the product of which will result in inferior or infertile plants. Imperfect or barren corn-plants, - those bearing no ears, -are as injurious in their effect in a seed-plot as an inferior male among choice, thor. ough-bred live-stock; they perpetuate their kind. Consequently the corn-grower should not permit a barren corn-plant to cast its pollen in his field. The perfect corn-plant bears a staminate (the tassel) called the male flower, and a pistillate or female flower called "the silk"; the imperfect plant has therefore the tassel or blossom, but no silk, and will produce no grain ; but if permitted to stand in the field with the other plants, it will cast its pollen upon some of those plants and fertilize them, and the crop produced from the seed thus fertilized would be liable to be barren plants, and the yield of the crop greatly deteriorated. By going through the field and cutting off the blossoms or tassels of all plants that have no ears formed or silk, the difficulty is obviated, for such blossoms will not then be left to fertilize other plants. Many planters go so far as to cnt down every such stalk entire. This should be done before the tassels are fully out or able to shed their pollen. In this manner the imperfect seed will soon be bred out, and the prolific qualities of the crop be largely increased. By selecting for seed ears from a stalk bearing two or more, the yield of the product of such seed will be greater than from stalks bearing only one ear, as the prolific tendency will become in a short time fixed, and a characteristic of the variety. From such ears should be selected those that are uniform in every way as compared with each other, having small cobs, the kernels compactly placed on the cob in straight rows, and uniform in shape and size throughout. If a different variety be grown near a seed-plot it will be impossible to maintain the seed pure, as the pollen will be carried even quite a distance in the air, or by insects that collect on the blossoms, and this explains why sometimes an occasional ear or a few kernels of an ear of another kind will be found mixed in the product of the pure seed.

It is not uncommon to find occasionally a few kernels of red, blue, or sweet corn mixed in this way with an entirely different variety. Mr. J. E. Read says respecting the distance to which pollen may be carried and its affecting adjacent or distant fields : 
"The habit of planting several different kinds of corn upon one farm is not at all to be commended. It is injurious to the farmer's own interest, and makes it still more difficult than it otherwise would be for his neighbors to grow the kind which they desire to produce. The distance to which pollen is carried is not certainly known, but it is probably greater than farmers generally suppose. We once found a bright red ear in a field of yellow corn, and there was not another red ear on the farm, and but few red kernels could be found on other ears. There was no red corn grown on the adjoining farm, but heyond that was a farm upon which there was a field of this colored variety. The red ear must hare been fertilized by pollen from this distant field. There have been other strongly marked cases, and they prove that it is unsafe to rely upon the purity of seed-corn which is grown without being separated from any other kind by a much longer distance than is usually thought necessary."

Some farmers put a strip of broom-corn or sorghum a few rods wide close to a field of corn to prevent the pollen of its blossoms from reaching the plot of seed-corn that may not be sufficiently far away to be otherwise protected.

When a separate plot is cultirated for seed, great care should be used in the preparation of the soil and after cultivation, and all the feeble and inferior plants be thinned out when grown sufficiently high to be detected. In saving the seed for planting, the small and imperfectly-dereloped kernels at the small end of the ear should always be discarded. Prof. P. A. Chadbourne says : "In order to raise good, prolific seed-corn it will be necessary to plant the best seed that can be procured, and, before the corn tassels out or produces pollen, to go along the rows and cut out every mean, miserable stalk, so that every ear shall stand on a proper stalk, and shall be fertilized by pollen that has come from a strong, healthy, corn-pro. ducing stalk."

Preparation of Seed. - In order to hasten the germination of seed and the growth of the young plants, many farmers soak the corn in warm water a day or two before planting. A solution of saltpetre or copperas is sometimes recommended and said to accomplish the same result, as well as to protect the corn against the attacks of birds, squirrels, mice, and worms for a time; but there is a liability of the germinating. power of the seed being destroyed or injured in this way, by getting the solution a little too strong of these sub. stances, while, unless rather strong, it has no effect in keeping these little mischievous depredators at bay.

Coating the kernels with coal-tar, or common tar, will generally prevent hirds from pulling up the plants, or attacks from any other of the common enemies of this crop that are such an annoyance to the farmer. When coal-tar is used the seed should first be soaked a few hours in warm water; after this is drained off, a very little tar stirred into it will he sufficient to cover the kernels, a pint of tar being sufficient for two or three bushels of seed-corn.

When common tar is used, a pint of boiling tar will he required for a half-bushel of seed, stirring the corn briskly as it is turned in, until every kernel is coated with it. Whether coal or common tar be used, rolling the seed in plaster, bone-dust, ashes or fine soil-dust will prevent either kind from being troublesome in planting when a drill or corn-planter is used, and it can be done with these machines the same as before it was thus treated, except they must be regulated to give the seed a little more room for outlet, otherwise the grain will not drop freely. Before the tar is applied, care should be taken to soak the seed thoroughly, as the coating will have a tendency to keep noisture from the seed and prevent germination.

Various other preventives are often recommended, but we know of nothing as sure in its results as that above recommended.

Most birds are a help to the farmer in destroying insects and worms that are injurious to his crops, and the few that sometimes pull up the corn-plants will generally do more good than harm in exterminating these pests; hence it is far better to make the seed distasteful to them by the use of tar or other means, rather than kill them, as they are really the farmer's friends in the protection of his products. 
Soil for Corn and its Preparation. - Corn thrives best in a rich, mellow soil, one that is warm and rather dry, although it will grow in almost any kind, being a plant of extraordinary aptitudes. It should, however, be sufficiently moist to make rapid growth, but not wet. A rich loam is best, and if it be of a dark color so as to absorb the heat of the sun, it will be so much the warmer and better adapted to the purpose. Corn will not do as well on wet lands or those that are very coid, like some of the strong clays, hence soils that are wet should be well drained for this crop, and cold soils should be cultivated in ridges and be planted with an early-ripening kind, in order to mature well. Although it thrives best on soils especially adapted to it, yet land that will produce almost any cultivated crop can be so carefully fitted that, with a selection of the variety suited to it and the climate, a good crop of corn can be produced. An old sod plowed under, and especially a clover lay, when properly prepared, makes an excellent seed-bed for this crop, but it should always have a good supply of manure in addition, either farm manure or chemical fertilizers, for soil can scarcely be made too rich with the proper kind of fertilizers for this crop. "Breeding first, and then feeding," is the old maxim of the stockman, which, when applied to cultivated crops, means quite as much as in its application to animals, for the selection of the seed is the breeding, and the choice of soil, the fertilizers and the cultivation are the feeding. With the corn crop gener ally the largest yields have always been where the feeding has been of the right kinds of plant-food, and the most liberally supplied.

A well-balanced and uniform fertility, such as is found in sod that has all the elements of plant.growth, in the best available proportions, or lands inundated by rivers, are what give successful results.

The corn-plant is vigorous in growth, and like all animals that are such, is a strong feeder, and will appropriate almost any kind of available food. It is said to take the place among vegetable products that the hog does among domestic animals respecting the food it con. sumes,-requiring a large amount, yet not over-particular as to the kind, or quality.

Manure from the barn-yard (well decomposed), the hog-pen, sheep-yard, poultry-yard, or privy-vault, are all used with good effect on this crop, but they should be spread broadcast and well mixed with the soil. The wide-spreading roots of the plant will be sure to find it if it is in the soil, and not so deeply buried as to be beyoud their reach. When manure is applied simply in the hill or drill, the plant makes an early and rapid growth, producing an extension of the roots, which soon go beyond the fertilized portion in search of food, and find but little support, hence its growth is retarded at this important period, preparatory to the formation and maturition of the grain. Sometimes an early growth of stalks and leaves is made at the expense of the grain in this way.

When scattered broadcast and well-mixed with the soil, this difficulty is obviated. The danger of over-manuring with strong fertilizers generally, especially those rich in free ammonia, is that they have their effect mainly in the early part of the season, when a large supply is also needed in the latter part, to aid in the formation and growth of the grain. There is however little danger of the crop having a surplus of fertility, the common error with farmers being in not suplying a sufficient amount. Sod, by its gradual decomposition, is admirably adapted to this crop, as it furnishes a constant supply of plant.growth, and a rich element late in the season, when it is required for the perfection of the kernel and ear. It has been proven beyond a doubt by numerous careful experiments that the corn-plant requires ammonia in its early stages and nitrates in later growth. Stable-manure should be well pulverized before being applied, and the soil be made mellow in preparation for the seed, as it cannot be readily assimilated by the plants unless in proper condition to be taken up by the tender roots. An animal might starve with an abundance of food attainable, unless that food were first masticated and reduced to a condition to be taken into and digested by the stomach; on the same principle, plants may starve when the food to be appropriated by them is not in a digestible and available condition to give them the necessary nourishment. 
Plaster, for soils deficient in lime, is beneficial to corn, and wood-ashes are also excellent. Chemical fertilizers are especially valuable for this crop, and are very reliable in securing successful results, some of the largest crops having been grown by their application. They are always soluble and available, hence, are a good substitute for barn-yard manure. They should always be near the surface, consequently should be lightly harrowed in, in order to be within reach of the roots of the young plants. It may be taken for granted, as a general principle in raising corn, that unless the soil is exceptionally ricl, like that in some sections of Manitoba, for instance, where fertilizers are unnecessary, a large crop cannot be produced without a liberal application of well-balanced manures or specially-prepared fertilizers suited to the soil and crop; old soils require it. It should also be borne in mind that richly. manured soil is more cheaply and easily worked, and large crops are more cheaply produced from it than from lands that are less biberally fertilized; in brief, that it pays the farmer better to work one acre of good rich soil, than two or more of poor soil. The quantity of fertilizers to be applied will, of course, vary with different soils, some requiring more thau others. The quality of the seed used should also largely modify the quantity applied. Where the seed to be planted is very prolific, and capable of producing from eighty to a hundred bushels per acre, a larger application of fertilizers will be required than where the seed used is able to produce about half that amount.

Where the soil is thoroughly cultivated, a larger amount can also be profitably used than where only an indifferent cultivation is practiced. Dr. Sturtevant states that in his own practice he finds five cords per acre of manure that has not been diluted with other substances to be sufficient; but that the larger portion of his fields are enriched with artificial fertilizers, which are applied according to the past history of the field, the quantity to be used being varied according to circumstances. Mr. Stewart says that in producing a crop equal to one hundred and fifty bushels and forty-eight pounds of shelled corn per acre, he used artificial fertilizers alone (Mapes' Complete Corn Manure) at the rate of six hundred pounds per acre. The quantity of artificial fertilizers applied when used without other manure varies from three hundred to six or seven hundred pounds per acre, according to the condition of the soil and the crop to be grown.

When barn-yard mauure is used in considerable quantity, it is noticeable that its effects are seen for three or four years afterward, and each succeeding crop that follows in rotation receives benefit from the fertility that remains in the soil. This is not because there was more manure in the land than the corn crop could appropriate to its own use, but because much of the manure that was applied was not in a suitable condition to be taken up by the crop during the first year, and it required considerable time for it to become reduced to that condition. The corn crop probably took all of the nourishment from the soil that was ready for it to receive during the first year, and would have taken much more if it had been placed there for its use. It being, then, a fact, that only a portion of barn-yard manure applied to land will be in a condition for the crop to appropriate to its growth during the first year, a liberal supply of it should always be given on this account, or some commercial fertilizer that is quickly taken up by plants should be used in connection with it.

Some farmers feed their soils as they do their animals, in a stinted manner, thinking they are thereby practicing economy, and receive corresponding results; others feed liberaliy and receive in return a liberal compensation. Some of our most successful farmers use forty or more common cart-loads of manure or compost per acre; other consider from eight to twelve sufficient; hence, the one obtains a fine crop, while the other only a moderate yield. We know of no crop that responds more freely to liberal feeding than corn, although it is a patient plant, and will often give very fair results with the most indifferent treatment.

The manner in which manure is applied affects in a measure its efficiency. When placed only in the hill, as was formerly the custom, the roots soon grow beyond it, and are not 
uniformly nourished, as we have previously stated; when applied broadcast, a more uniform fertility is secured with greater benefit to the crops. Many farmers apply the manure broadcast and harrow it in well, afterward using commercial fertilizers of some kind in the hills or drills, according to the method of planting; this secures an early start to the plants and increases the formation of root-fibers. Most of the corn-planters and drills have the means of distributing commercial fertilizers near the seed at the time of sowing. It is customary in some localities to spread the manure broadcast upon the sod before plowing, and then turn it under with the plow. We do not recommend this practice, as it buries the manure too deeply in the soil, and under the sod where it will remain too far away from the reach of the young plants at the time they require its nourishment to secure a vigorous start; besides, much of it might be lost altogether by leaching into the subsoil. The most successful results will attend the application nearer the surface, by plowing first, and harrowing in the manure afterward. Even with shallow plowing, the manure should lie above the sod in the soil.

When the soil has been previously cultivated for other crops, a shallow plowing in of manure is not, of course, objectionable. Chemical fertilizers, except those made especially for use in hills and drills, are generally sowed broadcast and lightly harrowed in. It is not well for the seed to come in direct contact with the manure or strong chemical fertilizers of any kind, as the germ of the seed is liable to be injured by them; to avoid this difficulty it should always be slightly covered or mixed with a little soil when applied in hills or drills. Shallow plowing for most soils is best for corn, as the most fertile portion is that which has been exposed to atmospheric influences, and where the rains and sun can exert the most power; consequently, as a general rule, the richest soil is near the surface. For most soils, unless they are very deep and permeable, plowing to the depth of five or six inches is sufficient for lands that have been eultivated, shallow plowing with deep pulverization being the most desirable.

Planting. - The time for planting differs according to the latitude and character of the season. It is said that the old Indian rule was, "When the oak-leaves are grown to the size of a squirrel's foot, it is time to plant corn." When the season is ordinarily early, and not too rainy, the time of planting this grain in the extreme Northern States, or in Canada, is in the early part of June; in Massachusetts and States of a similar climate, from the 10th to the 20 th of May; in the Middle States and those of the same latitude, from the first to the middle of May; in the Southern States of a latitude and climate similar to that of Virginia, from the middle to the last of April; in sections farther South it is done in March. In some seasons the planting can be done a week or two earlier than the usual time, while in others, when unusually cold or wet, it will of necessity be delayed as much later or longer. It can also be done considerably earlier on high than on low lands. It is always best to delay planting until the weather is favorable, as corn will not grow until the ground is dry and warm, and if planted when cold and wet the seed will be liable to decay in the ground before germinating; still, all farmers realize the necessity of getting corn started as early as practicable, and it should not be delayed later than necessary for the welfare of the crop. Early planting, when the season will admit, will give better results than that which is late; besides, it can be cultivated earlier, at a period when the farmer has more time to attend to it than when planted later, and is less liable to be disturbed by birds and insects, or injured by diseases of any kind when planted early. Early planting is especially desirable in those sections subject to early frosts in autumn, as both the grain and fodder are injured by it; hence, the earliest planting that the season will allow is always to be preferred.

Hill and Drill Planting.-Corn-planting may be done by planting-machines or drills, and the seed may be deposited either in hills or drills, some preferring the old method of hill-planting, while others the newer and more recently adopted one of drilling. When bill-planting is in squares, the corn may be cultivated both ways, which stirs the soil more 
than when it can be done only in one direction. When the corn is planted in drills, the plants can be more uniformly distributed over the ground, giving the sun and air free access to them, while there is less crowding. The advocates of drill-planting claim that these benefits more than offset those of hill-culture. The advocates of hill-culture, on the other hand, claim that an increased yield is obtained by their favorite method. Both methods have their especial advantages, and by experimenting for himself the farmer can determine which he prefers; we are inclined to favor drilling. The best planting-machines can be so adjusted as to drop the seed either in hills or drills, as desired, as well as a common drilling-machine. They will open the furrow, drop the seed at regular intervals, and in proper quantity, in suraight rows or drills, which admits of after cultivation by machinery, cover the seed at the proper depth as well as could be done by hand, roll it down, and measure and mark off at the same time the distance for the next row or drill to be planted. In this manner a single. rowed machine, witl one man and a horse, will plant from seven to ten acres of corn per day. With a double-rowed machine, planting two rows at a time, about $t$ wice this amount can be planted, or from fifteen to eighteen acres a day; these machines doing the work as rapidly as a horse can walk. Some of the smaller ones can be worked by hand, if desired.

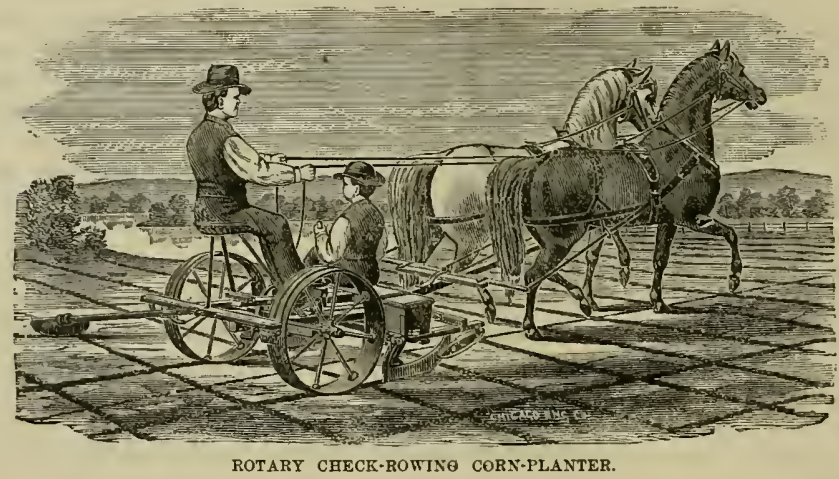

The Check-Rowing Planter, manufactured by the New York Plow Co., New York City, is an excellent machine for this purpose.

Whether the corn be planted in hills or drills, it is well to give it sufficient room for growth, that the plants may not be crowded and the air and sun excluded. The rows should also be far enough apart to admit of the movements of the horse in the use of the cultivator or horse-hoe. Such culture results in larger ears, that are better flled out, and consequently a larger crop. The smaller varieties will admit of closer planting than those of a larger growth. Corn is planted in drills from eighteen inches to three feet apart, with drills from three to four feet apart, according to the fertility of the soil, its moisture, and the variety to be grown. When planted in hills, opinions and practice vary with respect to the distance between and the number of stalks to be grown in a hill. The distance between the plantings should depend upon the number of stalks to be grown together, the kind of soil, and the variety to be cultivated. Where only one stalk is grown in a hill, such cultivation will admit of closer planting than where three or four stalks occupy a similar space; besides, a rich soil will admit of more plants to a given surface than a light one, while a variety of corn of large growth will require more room to thrive well than a smaller variety. We think the general fault with farmers is in limiting the space too much and crowding the plants too closely 
together, and that the largest varieties should never have more than three or four stalks to the hill, and, in such a case, the distance between the rows should never be less than four feet. Where only one or two stalks are grown in a hill, the distance could, of course, be lessened.

Dr. Sturtevant of Massachusetts, gives his opinion as follows: "Distance for planting must be governed by the necessities of the culture, and also regulated by the fertility and other agricultural conditions of the field. In a droughty field it is well to plant sparingly, as the corn-plant is a great evaporator of water, and many plants call for more water than do few plants. Water which may not be sufficient to supply 3,500 hills to the acre may be ample to supply 2,700 hills. It also requires more fertility in the land to carry a thick planting through to maturity than would be used in a thin planting. The character of the plant must also be considered, as a large, strong-growing variety will require greater space for development than will a small-growing variety.

The same principles which led me, on Waushakum Farm, to plant 6,223 hills (in drills) per acre, might lead the farmer in the South or West to plant 2,722 hills. The like reasoning and experience which leads me to use four kernels in a place might lead other farmers to use one kernel, or five; in a word, there can be no unvarying rule. We should plant in the way best fitted to meet the circumstances of our position.

I plant in drills three feet and a half, hills in drills two feet apart, four kernels in a place. This gives 6,223 hills, or 24,892 kernels, per acre. In careful experiment I have found increase of crop to follow increased thickness of planting up to 45,000 kernels per acre; but the necessities of farm culture and profit confine me to the number used, viz., 25,000 .

The depth of planting must necessarily vary with conditions. In the extreme southwest the Indians punch a hole eighteen inches deep with a stick hardened in the fire, and, enclosing the kernel of corn in a ball of moist mud, they drop it to the bottom and punch it down with a stick. They do this in order to get moisture, and in that climate the temperature is suffi. ciently high at that depth of earth; they also secure apposition of the earth with the seed. We must secure the like conditions; we must have moisture, heat, and contact of earth with the seed. In this region about an inch and a half to two inches fulfills these conditions in part, and pressure with the hoe or the machine completes. This statement illustrates how the same principles may be secured in different ways, and brings out the facts of the essen. tials which are to be secured.

The suminary of rules for securing large and profitable crops of corn seems to be: -

1. Seed of a prolific variety.

2. Shallow plowing and deep pulverization.

3. Surface-manuring, and sufficient, but not overmuch, manure.

4. Thick planting; early planting.

5. Thorough and judicious cultivation, carried out with horse labor.

To be avoided:-

1. Poor or unknown seed.

2. Insufficient or overmuch manure.

3. Hand labor.

The seed should be well covered, about two inches being a depth suited to a moderately moist, mellow soil; a sandy soil requiring more depth than clay. It is always well to drop one or two more kernels in a hill than would be necessary for growth, in order to provide for the kernels that may not germinate or produce healthy stalks, and when the plants are sufficiently high, to make a selection of the feeblest, to pull up the latter, and leave only the thrifty and healthy plants to mature. 
Cultivation.-Frequent and careful culture are essential to the highest success with the corn crop. Air and water are necessary to the growth of plants by not only contributing to their sustenance, but also by aiding in reducing organic matter to its mineral elements and a soluble condition, and by effecting a disintegrating and solvent action on the mineral particles that the soil contains; consequently the more the surface of the soil is broken and stirred, the more moisture is absorbed by it, and the more the circulation of air is effected within it. Among some of the large crops of corn that we have had authentic proof of having been harvested from one acre, was one of a hundred and fifty-one bushels, when shelled, and this was cultivated once a week up to the time when the ears were forming, and after the silk had been fertilized; we are also credibly informed that at this advanced period a dressing of special corn fertilizer was worked into the soil; this latter being done to promote the growih of the ears and grain, and as an experiment, on the principle that these should be encouraged in growth, rather than the stalks and leaves, after the latter had attained a certain desirable size. A requisite amount of leaf-growth is essential to the perfect growth and maturity of the grain, but, beyond this requirement, an excessive amount of leaf-growth is not of importance or desirable.

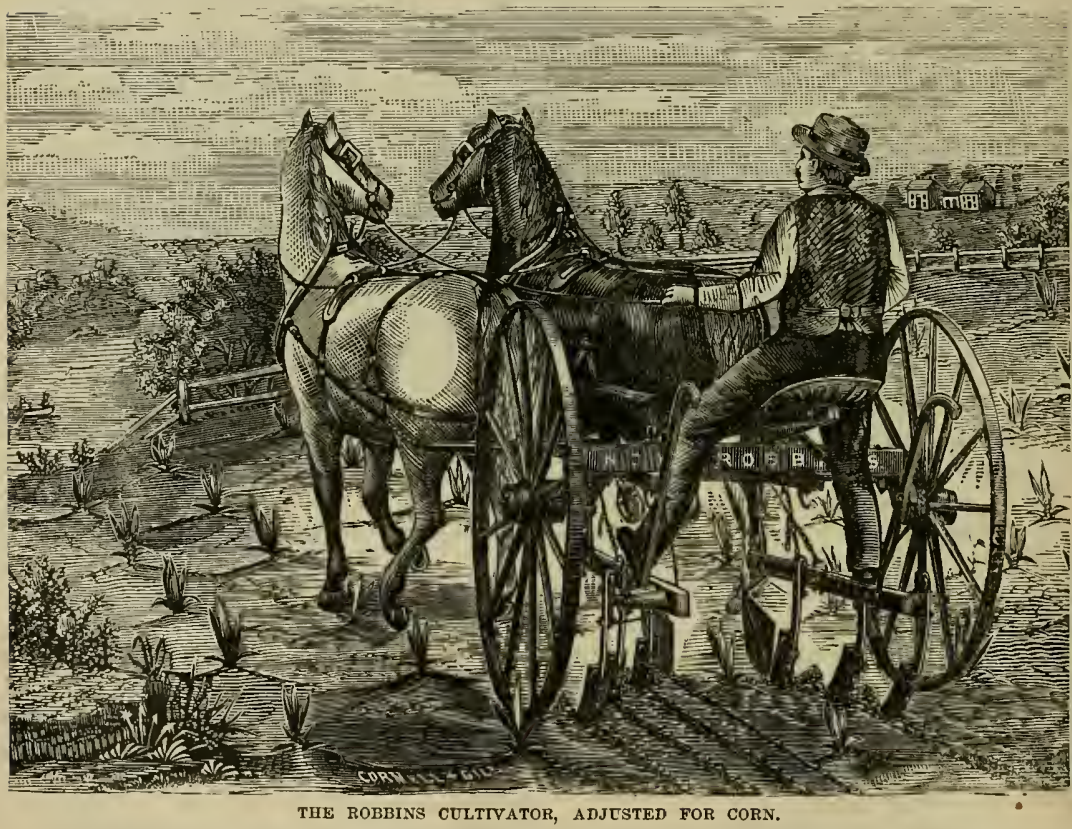

If we can, by frequent stirring of the soil, aid the plant in the production of more grain, by promoting the growth of larger and more perfect ears, and preventing abortive or false ears, or vacant spaces in the ears, we shall achieve in this department of agriculture what will prove of immense value and profit in the production of this crop, and the labor expended will prove a paying investment. Frequent stirring of the soil also hastens maturity Mr. Stewart says, that in the cultivation of sweet corn for market he has hastencd the maturity of the crop several days by weekly cultivation. It is not necessary to wait for weeds, but do it for the 
benefit resulting to the crop. It too often happens that corn receives but little cultivation, the most of it being required in the most busy season with the farmer, when other farm-work demands attention, hence the corn-field is liable to be neglected.

With only the two or three workings of the soil that the corn crop usually receives, of ten hastily performed, there will be but little benefit to the crop, while the weeds will soon be rivals for the occupancy of the field. We think every farmer will find it more profitable and better economy to cultivate his corn crop more thoroughly and frequently than is the usual practice. And here I would say a word with respect to the use of improved agricultu. ral machines in the performance of farm labor. The Western farmer, as a general rule, has a more fertile soil to cultivate than the Eastern farmer; it is consequently more easily tilled, and he resorts more commonly to the use of labor-saving machines than the latter does,_such as the sulky plow, drill, corn-planter, reaper and binder, corn-harvester, etc.,--his larger farm and more extensive crops requiring it; but his lands are not as thoroughly cultivated as those of the Eastern farmer. On the other hand, the Eastern farmer is still too often seen laboriously making his cross furrows with a plow, dropping the seed by hand, making the hills with a hoe, and performing much of the after-cultivation with the plow and hand-hoe. If you ask lim why he does not resort to labor-saving machines, he will probably answer that his farm is small and does not necessitate such implements, or that much of his land would require considerable labor and expense in removing stones and other obstructions to admit of the use of such machines. Now, we would say to the Western farmer, a more thorough cultivation of your lands would insure to you a proportionately larger profit in the increase of your crops, with less exhaustion to your soil. To the Eastern farmer we would say, if the labor, time, and expense involved in your present method of agriculture were expended in preparing your lands for and the use of labor-saving machines, you would find it a more profitable and economical method of agriculture in every respect, involving less severe labor, less time, less expense, and better crops; for hand-work is not only laborious, and comparatively slow in execution, but expensive. By the use of machines for plowing, planting and cultivating, all crops can not only be more easily, quickly, and consequently more frequently cultivated, but with less labor and expense.

Hilling and Level Culture.- Whether the culture should be level or hilled, will depend much on the character of the soil. Where the lavd is dry, it is better to keep the surface as level as possible. This admits of more uniform warmth and moisture, consequently hastens maturity, and leaves the surface in the best condition after the crop is harvested, and may be seeded down more easily to grass or clov. er, if desired; besides, hilling does not save the plants from being blown over by a severe storm, for the small

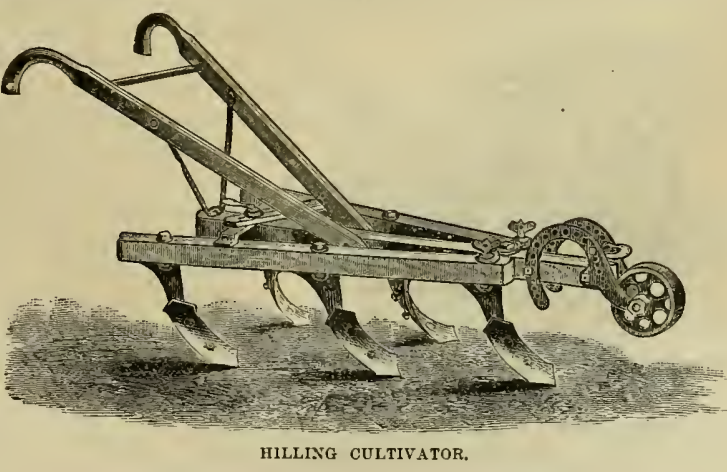
support thus given is of little effect when compared with the force of the wind upon the broad leaves. On wet lands, or when coarse manure is used in the hills, it may be necessary to make the hills higher, but in all other cases, level cultivation is to be preferred. Shallow 
cultivation is always beneficial to corn; deep cultivation is always attended with some risk, on account of breaking or disturbing the roots of the plant, which extend to a greater distance than is commonly supposed; therefore we would not recommend the plow for corn-culture; it goes

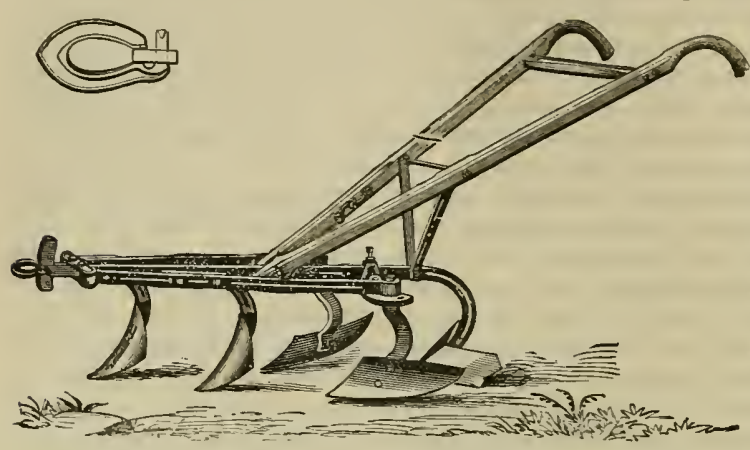
too deep and breaks the roots. Farmers frequently have a mistaken idea with re. spect to the character of the roots of the cornplant. It was a com. monly-received opinion many years ago, that the roots of trees and plants extended from the base of the stems, only as far as the bran. ches extended e a c h THE PLANET, JR., HORSE-HOE. way from the trunk or stems above. It re. quires but a little observation to become acquainted with the fact that all trees throw out roots on each side as long as the entire height of the tree, and frequently to a much greater distance; the same is true of many plants. Says a recent authentic writer on this sub. ject:-

"Te have made some recent examinations of the roots of beans, potatoes, and of corn. Roots of beans within four weeks of the time of planting the seeds, and when the plants were only five inches high, were found which had extended a foot and a half from the stem; and potatoes planted the first of May, in rows three feet apart, were found to have pushed their fibrous roots by the middle of June so as to occupy the entire space between the rows. Very careful examination was of course required to ascertain these facts, and careless observers would be likely to overlook them entirely.

The roots of corn do not extend so far as those of beans and potatoes during the early weeks of their growth; but early in summer, before the corn plants were six inches high,

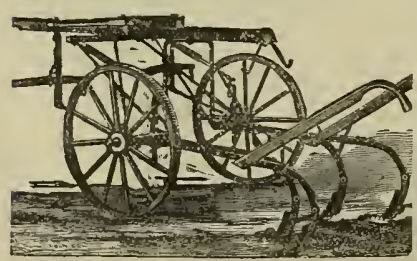

THE "NEERE SPRENG" CULTIVATOR. they were found to have horizontal roots in some instances a foot in length, making a circle of fibers two feet in diameter, or four times the height of the plants. When the corn had reached twelve or fifteen inches, the roots had met between the three-feet rows, and while yet the plants had not attained more than one-tenth their final weight or bulk. Long before the ears form, the entire ground between the rows is occupied with their long and slender fibers.

Observing farmers have long since discorered that much hilling of the corn crop is injurious to its growth, and lessens its product. But they generally assign wrong reasons. One of our best practical writers lately stated that hilling does harm by throwing the water of falling rains away from the roots of the plant-evidently supposing that they are crowded in a little mass a few inches in length at the foot of the stalks, instcad of actually spreading far and wide. As heavy rains soak the ground alike over the field, the hilling would make little difference; or if it did, the water would be as freely thrown on the roots between the rows as in them."

The writer then refers 10 other erroneous methods of reasoning and practice sometimes 
followed, which consist of throwing the soil away from the plants for a time to admit the rays of the sun to the roots, and further says:-

"This mode is just the reverse of hilling, and if performed to any depth would be as likely to cause injury by uncovering or breaking the roots, as burying them unnaturally deep in high hilling would occasion harm. Both modes are founded on the supposition of short roots, and both deprive the plants of the benefit of the broad, even, mellow bed of earth for the roots to extend in at all distances from the plants, where there is little or no hilling."

Shallow culture, is, therefore, to be preferred for corn, stirring the soil only on the surface, but very frequently. This is of the greatest importance to the crop in dry weather, and after rains, when the surface has become hardened and dry. Some farmers go over the field with the harrow before the corn makes its appearance from the ground, in order to destroy the young weeds when in the germ and before they have taken hold of the soil; this may be well if the field has been plowed a week or two before planting, and has the effect of cleaning the soil of weeds. After the corn is well up and has taken root, it should be cultivated frequently for the purpose of checking the weeds and promoting the growth of the crop. If it could be cultivated as often as once a week until after it has tasseled out, it would be of great benefit to the crop. The old-time custom of hand-hoeing, still practiced to a considerable extent in some sections, especially New England, is unprofitable and expensive, and should be superseded by the use of the horse-hoe or cultivator, which will be of more effectual benefit to the crop, with less labor, time, and expense. There are various kinds of these implements: The Robins Cultivator, manufactured by Luther \& Sumner, Grand Rapids, Michigan, is an excellent implement. Also the Hilling Cultivator, made by the New York Plow Company, New York City; the Planet Jr. Horse-Hoe, by Joseph Breck \& Sons, Boston, Mass, and the Deere Spring Cultivator, Deere \& Co., Moline, Illinois, all of which are valuable in the cultivation of corn and other farm crops that require shallow cultivation. Corn requires a large amount of heat, and frequent showers; still, it will endure quite an extended drouth with less injury than most crops. Cold and heavy rains are injurious in the early stages of its growth. The necessity of having good, prolific seed for the corn crop, a soil highly enriched and thoroughly prepared, absence of weeds among the plants, with frequent and careful cultivation, are important items in this department of agriculture that are being more fully appreciated and observed by the most successful farmers of the present time than formerly, and which, when observed by farmers generally, will in a few years result in an astonishing increase in the pro. duction of this crop. The old custom of "topping" the corn, which consisted of cutting off the tassel and leaf-growth above the ear, is now nearly obsolete, it having been ascertained that a valuable amount of nutriment is evaporated by the atmosphere and lost by this means, while the grain is not benefited by the process, which is a laborious and expensive one; but if the plants are cut at the roots before the grain is fully ripened, the sap remaining in the stalks will continue for a time to nourish the grain, and, to a certain extent, carry on the ripening process. The culture of corn for soiling and ensilage will be considered in another department of this work.

Har vesting. - This should be done before frost makes its appearance, as the grain and fodder will both be seriously injured from exposure to it, if standing. In New England, early frosts are trouble.

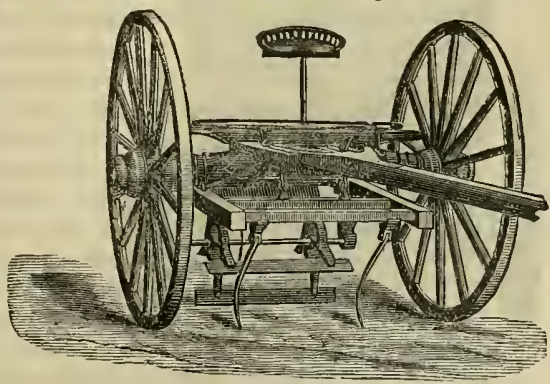

URIE STALK-CUTTER. some, and generally necessitate securing the crop as soon as it is ready, which is when the 
kernels are well glazed. In sections where this danger does not exist, it may stand in the

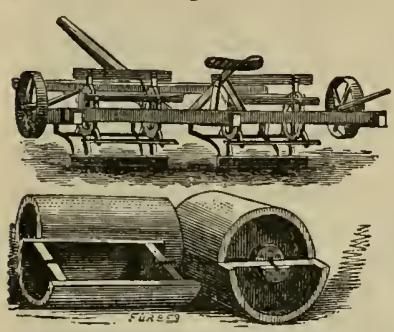

EUREKA DOUBLE-ROW STALK-CUTTER. field until fully ripe, if desired. When the stalks and leaves are desired for fodder, the crop should be har. vested as soon as the grain is fully ripe, as it wili contain more nutriment, and the grain be just as valuable, as when left standing in the field longer.

Various methods are employed in harvesting corn. The common custom in the Eastern States is to cut the stalks near the roots, and bind them in bundles, setting them up in stacks of from eight to ten bundles each, where they remain until well dried. When well cured, the bundles are taken to the barn, or husked in the field; the stalks and leaves all being saved for fodder.

It was formerly the custom among Western farmers, and is at present to a certain extent,

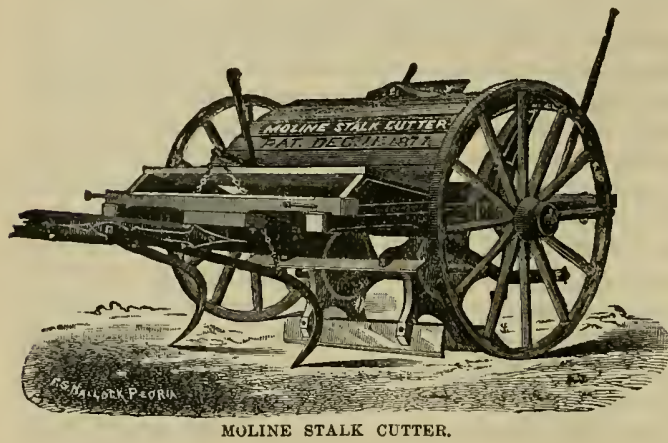
of gathering the ears of corn in the field and leaving the stalks to be trampled by cat. tle, or to be plowed in. They were also sometimes burned. Sometimes the grain and stalks were permitted to stand in the field till required for feeding cattle or other stock, when they were fed in an adjoining enclosure as wanted. Many of the Western farmers are, however, beginning to make use of the leaves and stalks for fodder,

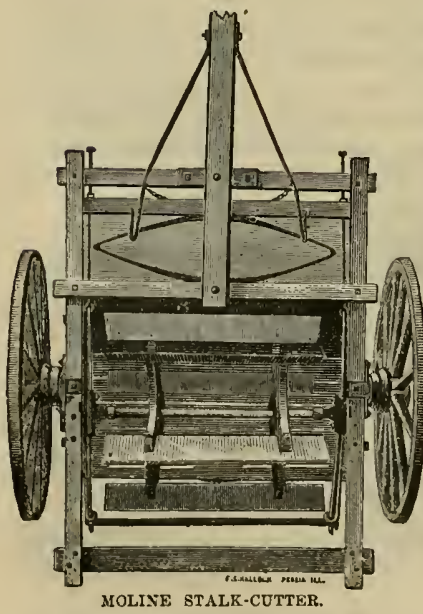
and realize its value for this purpose. It is better to cut and plow them into the land than to burn them. when not wanted for fodder, as when decomposed they furnish something towards the nutriment of the future crop. Small fields of corn in many sections are still cut by hand, a good average day's work in cutting and stacking from drills, being about half an acre a day per man; but where the grain is raised extensively, a cornstalk-cutter is necessary. These machines cut the stalks within a few inches of the root, which can readily be plowed under. The double-rowed cutters are said to cut from sixteen to eighteen acres of corn in a day. The Uric and Eureka stalk-cutters are manufactured by the Eureka Manufacturing Company, Rock Falls, Illinois. The latter has a roller attachment for cutting up the stalks and getting them out of the way of the plow, as is seen in the cut.

The Advance Cultivator, manufactured by Deere, Mansur \& Co., St. Louis, Mo., has also a stalk.cutter, which can be readily attached to that implement

when desired, thus combining two machines in.one. 
Machines for both cutting and husking the corn at the same time have been invented, but are not yet in "general use. As soon as the stalks are thoroughly dry, the corn should be husked and stored in dry bins or cribs, that are proof against rats and other vermin, and the fodder put under cover or stacked at a convenient distance from the feeding-yards. Machines for husking are a great saving of time and labor.

Corn should not be shelled until perfectly dry. It will shrink in weight and measure for several months after husking. this shrinkage being generally more in the cob than the grain in proportion to the weight of the two, still there will be considerable loss in the weight of the grain from the time of harvesting until spring, even when comparatively ripe when harvested.

In order to have some reliable data in regard to the comparative weight of corn in the fall and in the spring, which is an item of no little importance to the farmer and dealer in corn, we give the following result of experiments made at the chemical laboratory of the Illin. ois Industrial University, under the supervision of M. A. Scovill, Professor of Agricultural Chemistry.

The experiments were made with the following six varieties of corn grown on the University farm: Mammouth, Thomas, Murdock, Geneseo, Wright's Gold, and Cameron.

In order to secure an average result ten ears of corn of each variety were employed. The corn was gathered on October 6th, and carefully weighed. It was then properly labeled, and the whole placed in a room, which was entirely cut off from any artificial source of heat. On November 6th the corn was weighed, with the following results:-

Loss of weight of corn from Oct. 6th to Now. 6ith, in 100 parts.

\begin{tabular}{c|c|c|c|c|c}
\hline Cameron. & Wright's Gold. & Geneseo. & Murdock. & Thomas. & Mammouth. \\
\cline { 1 - 5 } & 17.5 & 8.2 & 12.2 & 17.5 & 25.5 \\
\hline
\end{tabular}

After weighing the corn, it was put back into the room as before, and allowed to remain until Nov. 29th, when it was weighed again.

Loss of weight of corn from Oct. 6th to Nov. 29th, in 100 parts.

\begin{tabular}{c|c|c|c|c|c}
\hline Cameron. & Wright's Gold. & Geneseo. & Murdock. & Thomas. & Mammouth. \\
\cline { 1 - 4 } & 21 & 10.7 & 14.3 & 21.2 & 30.8 \\
\hline
\end{tabular}

Finally the corn was allowed to remain in the room until February 28 th, when it was again weighed, with the following results:-

Loss of weight of corn from Oct. 6th to Feb. 28th, in 100 parts.

\begin{tabular}{c|c|c|c|c|c}
\hline Cameron. & Wright's Gold. & Geneseo. & Murdock. & Thomas. & Mammouth. \\
\cline { 1 - 5 } & 22.5 & 17.4 & 16.8 & 22.8 & 33 \\
\hline
\end{tabular}

By the last figures, it will be seen that the loss in weight from October 6th to February 2 sth by drying, was, on the average, a little more than twenty-one per cent., and that the shrinkage was less during the last month of the experiment than the preceding. A similar experiment in determining the amount of loss in bulk by shrinkage, and which extended 
from November until the following July, incicated a loss in that respect of about eighteen per cent.

The farmer who makes an early sale of his corn will consequently have larger weight and bulk of this grain at that time than at a later period, while those who store their grain for later sale should govern their prices accordingly.

The influence of moist and dry weather is also very great in determining the weight of corn. During those seasons when severe drouths are experienced, the corn in the ear will frequently be found more dry by the middle or last of September than it is in moist stasons by the early part of winter. It was found by careful experiment after a certain moist autumn, that corn which was weighed in the ear on the first of January had lost twenty per cent., or one-fiftl of its weight, by the following October. Under such circumstances, old corn at a dollar per bushel would have been cheaper to the purchaser than the new at the rate of eighty cents per bushel.

The proportion of weight that the cob bears to the grain will generally average about one-eighth. Corn stover, or the corn-plant after the ripening and removal of the grain, should be put under cover as early as possible, for if allowed to stand in the field exposed to the rains, much of the nutriment will be washed out. Its value for feeding stock is estimated to be about half that of good hay.

When corn is husked by hand, much time and labor may be saved by providing the best facilities for performing the work. A husking-bench or rack will be found a great convenience, and is easily and cheaply constructed. This may be done by the use of two small benches, or horses, for supports at either end of the rack, which are connected by means of two pieces of wood about two by three inches, and ten or twelve feet long. Small crossboards laid across at the ends will serve as seats for the huskers, which can be moved up or back as desired. The boards for seats should have cleats on the under side, so as to admit of their being moved forward or backward without dropping.

When enough stalks are husked to form a bundle, they may be bound without the huskers leaving their seats, and thrown one side. The long pieces that form the sides of the rack should be two feet or a little more apart, and fully two feet high, or rather the benches at the ends should be this height; if lower than this, they are not as easy to rise from. This rack may be used in the field, and may be easily carried from one shock to another by two men, one at each end to take hold of the projecting ends, and for this reason it should be made as light as consistent with the strength necessary for the support required of it. It is stated by those using a husking-rack of this kind. that its convenience is such that an ordinary workman will husk one-fourth more in a day than when he is compelled to reach down, while standing, for every ear of corn that he husks, or if he is compelled to take any position on the ground to get at his work.

Shelling and Grinding Corn.-Corn should not be shelled until well dried. Machines for performing this work are needed on all farms where corn is cultivated to any extent. For small farms the hand-machine will answer all purposes, but for large farms, where great quantities of grain are used, either steam, wind, or horse-power may be utilized; this can be easily done where a farm-engine or wind-mill are in requisition for farm use, while every farmer has horse-power at his command. With these machines, the farmer can shell his corn at such times as is most convenient for him without extra expense or assistance, as this can be performed when other work is not pressing.

There are various shellers in common use; the best not only separate the cobs from the shelled grain, but are supplied with a fan that blows all the dust and chaff out of the corn, making it perfectly clean.

There are also machines that will shell the corn clean, either with the husks on or after they have been taken off, which save the labor of husking, and prove quite valuable in large corn-growing sections. 
The first two illustrations of cornshellers represent excellent machines, - made by the Sandwich Manufacturing Co., Sandwich, Illinois - the first being a small hand-machine, adapted to small farms; the latter larger, and better adapted to farms where a large amount of grain is utilized, and can be used as a hand-sheller or with other power, as desired. The third sheller represented shows a machine mounted and ready for use that will save the labor of husking, as it shells, equally clean, either husked or unhusked corn. It is manufactured by Kingsland, Ferguson $\&$ Co., St. Louis, Mo., and proves very valuable as a labor-saving implement where large quantities of corn are raised.

It is a much more economical method of feeding, to first have the corn ground, rather than give it to stock in the ear; this has not only been proved by many careful experiments, but by the experience of the majority of our best farmers. Mr. Brown, Professor of Agriculture at the Guelph Farm, Prov-

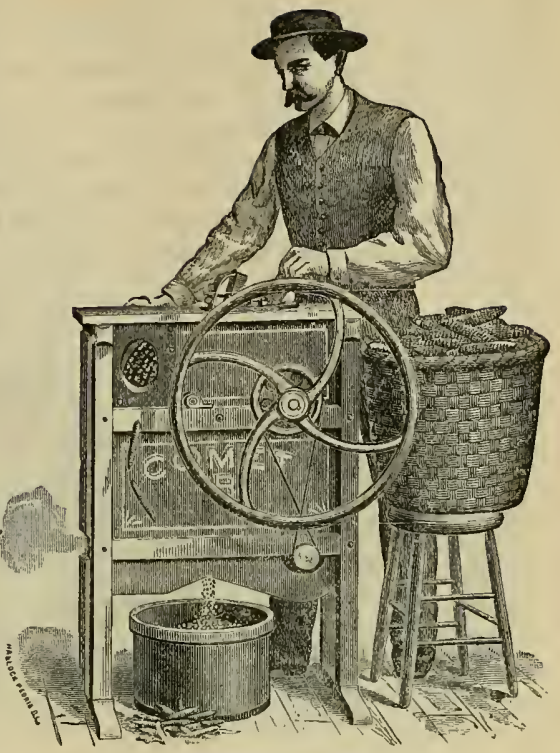

RIGHT-HAND COMET.

ince of Ontario, has recently been making a variety of "valuable experiments; among others he has ascertained that apparently about one-fifth of the ground corn passes through the stomach and alimentary canal of cattle undigested. If this be true, a much larger proportion of unground corn must pass undigested and be lost. Besides, when fed in the ear, much of the corn is wasted by being trampled under the feet of the animals while eating.

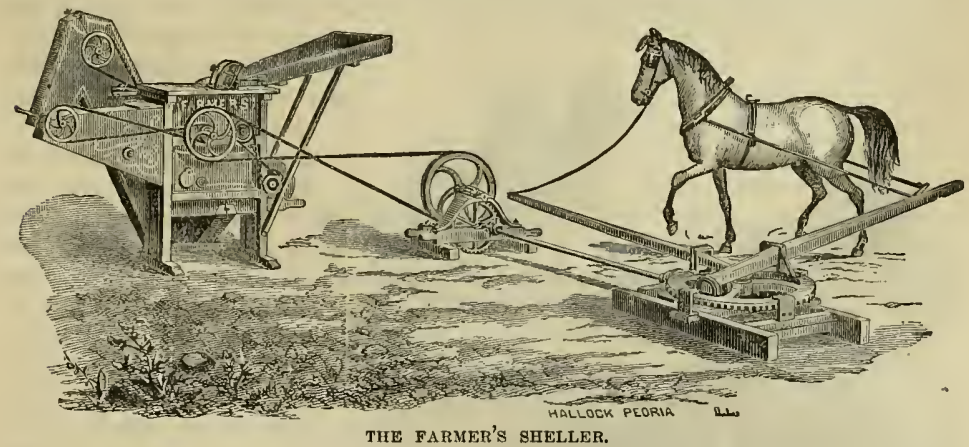

Many farmers grind both corn and cob together, as it saves shelling, and the cob is sup. posed to contain some nutriment. ' It is also sometimes ground before being husked, the thin dry husk being easily reduced by the grinding process. It contains the elements of animal food to a linited, extent, and saves husking, where the cob is to be ground. A difference of 
opinion, however, exists respecting the beneficial or injurious effects of the ground cob on the animal system; some regarding it as possessing sufficient nutriment to make it profitable for grinding, others regarding it of no value as food, and as injurious to young stock, it haring a tendency to cause irritation of the digestive organs; while for fattening animals so large a quantity is needed that the stomach will be unduly distended by it. In order to fatten animals rapidly very nutritious food is required, and not simply bulk. As we have never made use of cob-meal, we cannot speak from experience, but should consider cobs as more valuable for manure than food, since they contain quite a proportion of potash, provided they could be reduced to a suitable manure to be returned to the land.

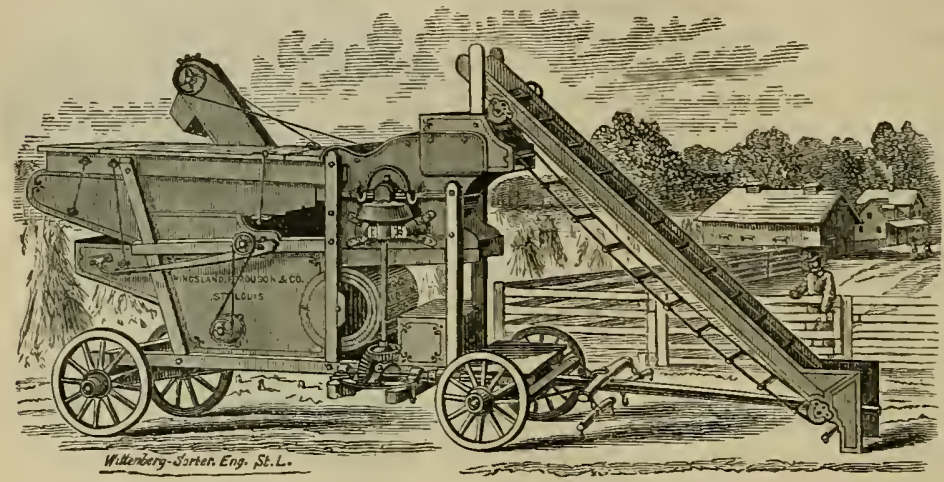

SHELLER FOR EITHER HUSKED OR TNHCSKED CORN.

In order that the farmer may be able to grind his own grain, and have it done at his own convenience, farm mills for grinding corn and other kinds of grain are not only very desirable, but in many sections of the country where farming is conducted on a large scale, they are regarded as an indispensable farm implement; especially are they so on those extensive farms devoted mainly to grain-growing and live-stock, and under such conditions it is not only a necessity and convenience, but the practice of economy as well, and in most

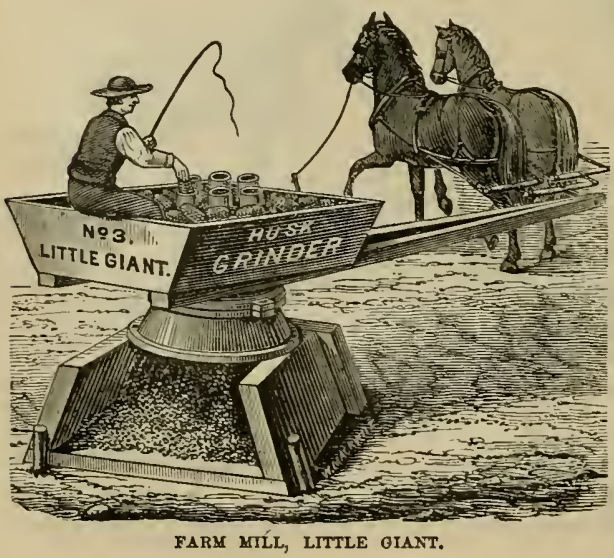
cases, although attended with some expense at first. such a mill will pay for itself in a short time. The power used for grinding may be the same as for other farm work of a similar kind, viz., the farm engine, windmill, or horse-power.

The two accompanying illus. trations of farm mills, from the manufactory of Deere, Mansur \& Co., St. Louis, Mo., represent implements that will grind all kinds of grain, coarse or fine; corn can be ground either shelled or in the ear, husked or unhusked. The first represented is provided with large feed-boxes, tubes for the unhusked corn, and a seat for the driver, who does the feeding. In 
grinding shell corn, wheat, or other grain, it is only necessary to remove the tubes and fill the feed-box, which will hold from eight to ten bushels.

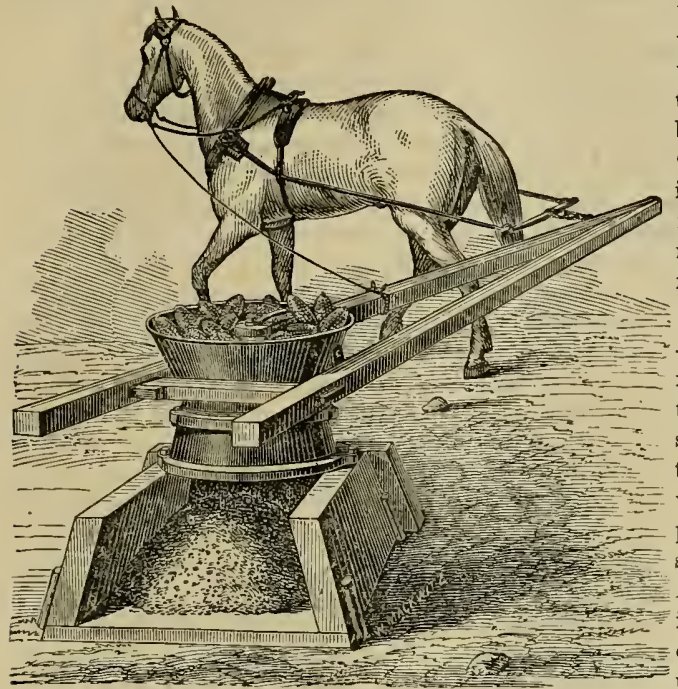

LARGE GIANT.
A one-horse mili of this kind will grind from twelve to fifteen bushels per hour; where two horses are used, from fifteen to eighteen hushels can be ground in that time. The "Large Giant" has a grind. ing capacity for about twentyfive bushels per hour. Such milis are very durable, and not liable to get out of repair.

Diseases of Corn, etc. -The diseases of corn are neither very numerous nor $\mathrm{ex}$. tensive in their results. In some seasons, especially those that are warm and wet, smut will sometimes make its ap. pearance and destroy a considerable number of the ears. Almost every farmer is famil. iar with its appearance to a certain extent, and its results upon the crop. It does not always attack the grain, as it

appears on the tassel, stalk, and even upon the leaves. Wherever it makes its appearance, there is an unnatural growth of a spongy nature, an increased size or swelling of the part affected, which at a later period is broken open, showing the smut, which consists of microscopically small grains-the spores or reproductive bodies of the corn-smut fungus. These grain smuts are minute parasitic plants, the portion of which corresponding to the root of other plants lives in the tissues of the corn, causing the abnormal growth.

How or when the smut-plant begins its work upon the corn is not known, various theories being entertained respecting it, some supposing that the spores are planted with the grain, and germinate and grow as the plant increases in size; others, that these spores float in the air, and, coming in contact with the corn, germinate and grow upon the surface, sending their roots through the tissues of the corn-stems and leaves. Whatever may be its mode of growth or origin, it is apparent that wherever it makes its appearance, it robs the plant upon which it grows of nourishment for its own support, and produces, after a time, a num. ber of spores by means of which the smut-plant is perpetuated. This disease, or abnormal growth, is most frequently confined to the grains, but may affect other parts of the plant. When the grains are affected, they become at first of an unusual size, and gradually turn dark in color, and soft; finally, the covering breaks, and a dark, dry, dusty powder is seen.

The results of eating such grain are often very serious, sometimes producing abortion, and even death, among cows, the medicinal effect being the same as that of ergot, or "spurred rye." Its effect is otherwise deleterious upon the animal system, and such grain should never be fed to live-stock of any kind. It is more apt to appear upon broken stalks and leaves than others, and to be most prevalent when the weather is warm and moist, or what is termed "muggy." Salt sown upon the soil before planting is suggested by some as a preventive of this disease, which is not yet fully understood by the best scientific agriculturists. 
The quickest and best method of ridding a field of smutted corn that we have ever tried, is to pass through it as soon as this disease first makes its appearance, and gather all the parts affected and burn them. By this means, countless millions of spores that would otherwise ripen to spread the disease, are destroyed, and the means of its perpetuation prevented.

Corn should not be planted upon the same field more than two years in succession, and if a judicious rotation of crops is observed, together with careful cultivation and a fertile soil, a good crop will generally be secured in spite of insects or disease of any kind. It is always best to avoid the spreading of disease as far as possible, and this is one way to accomplish it. If the soil is too wet, producing a surplus of water about the roots, corn will not have a healthy growth; the leaves will turn yellow and have an unthrifty appearance, and the growth will be slow. Such land should be drained, if devoted to corn culture.

The chinch-bug, wire-worm, cut-worm, corn or ball-worm, and various other insects, are sometimes very troublesome and destructive to the corn crop in certain localities. The wireworm attacks the corn before it comes up, by eating into the kernel before it has germinated; it also eats the roots and penetrates that portion of the stem of the young plant that is covered by the earth. The failure of seed to come up is often due to the attacks of this pest, sereral of them often being found at work upon a single kernel. The cut-worms, called in Europe surface-grubs, attack both roots and stalks, often cutting the stalks entirely off, and are frequently found as high as the ear on the stalk, sometimes continuing their depredations until the corn is nearly ripe. They are generally most troublesome on sod-land.

There are various means resorted to by farmers for the purpose of getting rid of these pests, such as burning all the stalks and stubble left in the field after harvest, fall plowing, etc. Prof. Cyrus Thomas, State Entomologist of Illinois, in referring to the wire-worms, says:

"I am decidedly of the opinion that starving them out by refraining from planting for a year, and repeatedly stirring the ground, is the only sure remedy. One great advantage of this plan is that it not only tends to destroy these worms, but also the white-grubs, cut-worms, and numerous other species that reside in the ground in the larva state, and are not easily reached by topical applications or other means. It is not difficult for farmers to test this remedy on a sinall scale where these insects are troublesome, and it is certainly worthy of a trial. Where they are very numerous, and the nature of the soil will justify it, it would be mell to spread unslaked lime over the surface and turn it under as deeply as possible before rain falls upon it. If this could be done consistently with the nature of the crop to be grown, very eariy in the spring, this would probably be the best time; otherwise it should be done late in the fall. I am not aware that this remedy has been tried-lime has again and again been used, and"generally without favorable results-but the use of unslaked lime in the manner indicated, and in as large quantities as the land will bear, has not, so far as I can learn, been tried. To be of any value it must be applied freely, and well turned under.

According to Curtis, when the wire-worm reaches its full size it descends a considerable depth in the earth, where it forms an oval cell of the surrounding particles of soil, not lining it with silk; it then casts its skin again and becomes a pupa or chrysalis, generally, in England, at the end of July or beginning of August; the time here is probably a few weeks earlier. The chrysalis is long and narrow, bearing a strong resemblance to the perfect insect, but is of a paler color, usually yellowish or yellowish white. They remain in the pupa state, so far as observed, from two to three weeks, but it is probable some species remain in this state over the following winter.

The same author, in the summary with which he closes his article on these worms in his 'Farm Insects,' makes the following statements in reference to their habits and the remedies used to counteract them :-

'Very hazardous to re-sow where they have destroyed a crop unless the soil be plowed repeatedly. 
They are most to be dreaded in dry seasons, yet they cannot exist withont some moisture.

Affected plants are known by the dying off of the outer leaves.

They cut into the stem above the roots and sometimes separate the stalk.

Gardens suffer exceedingly; lettuce often falls a sacrifice to them.

On light lands they do most mischief from the beginning of March to June.

Lower parts of fields bordering on marshes most infested.

Rye-grass most dangerous with clover, for encouraging wire-worms.

Gravelly and sandy soils most infested; strong loam and clay most free from them.

Wheat sown in dry weather most likely to suffer.

By constantly disturbing the insects it is probable they may be driven from a locality.

A summer fallow and burning the rubbish is recommended after clover and grasses; it zills the eggs and starves the worms; but fallows must be kept very clear from grasses and weeds. Nothing more dangerous than to leave strips and patches of grass or lays in plowed fields.

Feeding land close with sheep will prevent the eggs from being laid. Folding oxen and sheep in the spring may also keep the beetles from coming ont of the earth. Harrowing and hard rolling in March and April strongly recommended. Top-dressings of lime useful before rolling.

Domestic fowls and numerots sniall birds eat a large number of them.'

Fall plowing has been found to be very beneficial; this exposes them to the frost, which will have a tendency to destroy vast numbers of them. A Western farmer gives his experience as follows:-

'Last fall I commenced to plow a stubble-field of twelve acres; I had it abont half done when winter set in, consequently the other half was not plowed till spring. The part broken in the spring was at least one-fourth destroyed by the wire and grub-worms while the other part was scarcely touched.'"

Coating the seed with tar, as has previously been suggested under "Preparation of Seed," has also a very good effect in checking the evil.

The corn-worm or ball-worm does considerable damage to the ears of corn in the latitude of southern Illinois, also to both cotton and corn in the cotton-growing sections. It attacks corn in the ear, eating first the silk, and as it increases in size afterwards, the kernels, sometimes ruining whole fields of corn. Fields that are early planted are less liable to be attacked by it than the late. Fall plowing is also a good remedy, and in fact the most effectual means of destroying these insects, as the larvæ are brought to the surface and frozen. Many farmers build bonfires near the corn-fields in the evening in snmmer at the time the insects that lay the eggs producing these various pests are most active, and by which means many are attracted to the fire by the light, and destroyed.

Birds are sometimes troublesome, especially crows and blackbirds, which will frequently pull up the young corn-plants in order to obtain the kernels. Blackbirds and birds of several kinds will also sometimes do injury to the grain in the ear. Notwithstanding these facts, we would say to the farmer, spare the birds! The vast numbers of insects they destroy daily is a great benefit to the farmer, and, if the birds are diminished in numbers, the insects will increase. It is better to frighten the birds away from the corn-fields at the time they may do the most mischief, and permit them to live to aid the farmer in exterminating these minnte pests-worms and insects-that cannot be frightened or driven away so easily. Millions of dollars' worth in products are annually destroyed in this country through insects, and birds are the only enemies able to contend against them vigoronsly. They are the noted caterpillarkillers and insect-destroyers generally. Crows will destroy not only insects, but mice, young rats, lizards, small snakes, caterpillars, and other vermin, and are valuable scavengers on the farm. Coating the soed with tar, as has been previously suggested, is a good remedy. 
Newspapers opened and spread. about a field here and there, and kept in place by a stone, will sometimes keep them away, or a rattling windmill erected in the field. Twine put up about the field on elevated sticks at the time of planting will usually frighten crows, although blackbirds are more courageous and saucy, and not as easily intimidated. Small pieces of polished tin or looking-glass suspended from a bent pole by a string in a manner to reflect light from the sun, are usually effectual in frightening most birds; also a piece of cloth that will flutter in the wind like a flag. A blackbird or crow might be sacrificed for the purpose of frightening his companions; by being hung in the field suspended from a pole where it can be readily seen it usually produces the desired effect. We hope no farmer will be so cruel and inhuman as to hang the poor bird up alive, and leave it to suffer and die in that condition. We have seen this cruelty practiced, and are of the opinion that a man that would do so mean and cruel an act to any helpless creature deserves to have all of his corn pulled up, and we think the whole race of birds would be entirely justified in avenging in this manner such an atrocity perpetrated upon their species! All remedies for kceping the birds away should be resorted to as soon as the corn is planted, if not before, for if they once get a taste of it, it will be difficult to frighten them afterwards. New devices will have to be resorted to occasionally, as what will prove effectual one season may not another. Earlyplanted fields are less liable to be attacked by birds than late, a fact that is well worth the farmer's attention.

\section{RICE.}

$\mathrm{T}$ HE cultivation of rice in this country has not been one of its flourishing or permanently prosperous interests, being limited, as it is, and always has been, to a comparatively small area. Its culture was formerly confined principally to the sea islands and lowlying shores of South Carolina and Georgia, where it is now quite extensively produced, as well as in Louisiana, Mississippi, and several of the other Southern States. According to some authorities, there are two principal species of this grain, of which there are many varieties; one, commonly known as the lowland rice, (Oryza satica,) which grows on wet land, and is cultivated with excessive irrigation; the other, (Oryza mutica, ) a species adapted to a dry or mountainous region, of which the product of Ceylon and Java furnish an example, and which will not flourish with long inundation, thriving best in a dry soil. Other authorities regard the two mentioned as different varieties of one species, somewhat modified by a difference of soil, climate, and mode of culture.

Rice forms one of the principal articles of diet of the inhabitants of China and nearly the entire southern portion of Asia, as well as the numerous islands of the Pacific and Indian oceans, and a large portion of Africa. It is extensively cultivated, also, in Southern Europe, and, in fact, to a certain extent in most of the tropical and semi-tropical countries. A species of this grain, called "cargo" or "Bengal rice," is a favorite with the Orientals. The kernel is large and sweet, but rather coarse and of a red color. The East India rice is white, but small-grained. The variety known as the "Carolina rice," which combines the merits of the others, being large-grained, sweet, and white, is considered superior to all others.

According to Ransay's History of South Carolina, rice was introduced into that state in 1694, by a vessel from Madagascar, which, in distress, sought refuge in Charleston harbor. The captain of this vessel, having subsequently formed the acquaintance of a resident of Charleston,-Mr. Landgrave Smith,-presented him with a small sack of rough rice. This gentleman had it planted in his garden, and the plants yielding abundantly soon attracted the attention of his neighboring planters, among whom he distributed the seed, who in turn 
extended its dissemination along the rivers farther towards the interior, until, in a few years it became, by careful culture, one of the principal cultivated crops of the colony.

In some of the southern localities the cultivation of rice is regarded as more profitable than that of the sugar-cane. In the swamps of South Carolina and Louisiana and the rice lands of lower Mississippi it yields very largs crops, and is cultivated with comparatively little labor; but the great difficulty in its production is that the localities in which it thrives best are generally very damp, inducing malarial fevers, and is detrimental to health generally.

Considerable difficulty is also experienced by planters in selecting seed free from the "volunteer" or red rice, which is the product of the self-sown grain left in the soil after har. vest, and which springs up the following year, and grows with the cultivated crop, the plants being indistinguishable from them. The grain of this self-sown product will after a few years become loose in its attachment to the stalk, and consequently easily shells out, so that it is almost impossible to eradicate it from a soil when it once gains a position there. Its seed is also red and coarse, requiring considerable care in milling to clear the harvested crop from it. To show the effect of climate and soil on rice, a few years since several samples of seed were selected from the South Carolina crops, and sent to India to be sown in the rice fields of that country. Specimens of the product of that seed were suisequently sent to the planters who furnished it, who were surprised to find the grain so changed that it could scarcely be recognized as the same variety. The heaviest grain is usually grown in the most northerly of the rice-growing sections. Rice, as an article of food, contains but very little oil, and a large proportion of starch.

Cultivation.-The lands best adapted to rice-culture, or that produce the largest grain, are those swamps and rush.lands lying adjacent to tide-water rivers between $29^{\circ}$ and $30^{\circ}$ north latitude. The plantations for this purpose should be located above the junction of salt and fresh water, because rice, being a fresh-water plant, is fatally injured by salt water in any stage of its growth. These lands are of rich alluvial soil, and by being easily irrigated are admirably adapted to the culture of this grain. Inland swamps are also valuable for this purpose; they are reclaimed by means of drainage, and the water reserved for irrigating purposes, in such a manner that it may be turned on or off at will. Tide-river plantations are protected by means of embankments or levees, which have been described by Mr. Augustus Favean of South Carolina, as follows: "These levees are made high and strong enough to effectually bar out the water of the river. Smaller embankments, called check-banks, subdivide that portion of the plantation lying between the main river embankment and the highland into squares or fields, generally from fifteen to twenty acres in area. These squares are all subdivided again into beds or lands, of twenty-five or thirty feet width, by a system of main ditches and quarter drains. Canals, from twelve to thirty feet wide and four or five feet deep, are sometimes cut from the river embankment, through the centre of the plantation, to the high land, for the purpose of introducing or draining off the water to or from those fields situated far back from the river. These canals also form a very conspicuous feature in the harvest. scene, as they serve as a medium of navigation for the large flat-boats which convey the rice to the stack-yard in quantities of eight or ten acre's yield at a load; and as rice usually yields from two to three tons of straw per acre, the value of this immense water-carriage can be easily conceived.

Flood-gates or trunks having doors at both ends are buried in the embankments on the river, as well as in the canal embankments and the check-banks, those at the outlet of canals being so constructed as to permit the flat-boats to pass into the river. By means of these flood. gates or trunks the whole system of irrigation is carried on under the complete control of the planter, and the lands are flooded or drained at will."

These lands, being enriched by annual alluvial deposits from the river, do not require deep plowing, four or five inches being generally sufficient, after which it is thoroughly har- 
rowed in order to pulverize it as finely as possible for the reception of the seed; thorough harrowing also breaks up the sod and exposes the roots and seeds of grasses and weeds to the action of the sun, which will be a great benefit in the after-cultivation of the crop. The seed is sown either broadcast or in drills, usually the latter, at the rate of from two to three bushels per acre. Two methods are employed, some preferring covering the grain in the soil two or three inches, others regarding the open-trench system as preferable, where the grain is left uncovered and the water immediately turned on very gradually until the rice adheres to the soil, after which it is slowly flooded to the depth of twelve or eighteen inches. When the grain is covered by the soil in sowing, the water is immediately turned on to the above depth, and remains until the rice is well germinated, after which the water is drawn off and kept out until the plant has two leaves. When the land is well flooded, all the rubbish and drift that rises to the surface is taken out with rakes in order that it may not settle upon the field when the water is drawn off. The sowing is generally performed in March, although it is sometimes done as late as the fifteenth of May and even later. Germination of the seed is hastened by soaking the grain in water a few hours before sowing.

When the rice has two leaves of growth the water is again let on to the same depth as before, entirely submerging the plants, and remains at this depth from a week to ten days; this gives a vigorous growth to the grain, and has a tendency to destroy the grass and weeds. Mr. Flavean further says that "the planter should be governed by the weather in regard to the length of time the plants remain thus submerged; if warm, seven days, if cool, ten; after which the water is drawn gradually off, until a general "verdure" is seen floating all over the field. At this point the water is stopped and a mark set upon the gate as a "gaugemark." To this gauge the water is rigidly held for sixty or sixty-five days from the day it first came on the field. It sometimes happens that, during this flow, the crop takes a check and stops growing. In this event to take off the water is fatal, as it will produce "foxed rice; " but it must be held firmly to the gauge, and in a few days the plant will throw out new roots and recommence growing. If the maggot attack it in this flow, the water is drawn off for a day or two and replaced. And where water is abundant and easily handled, the maggot can generally be avoided by beginning, about the thirtieth day, to change the water once a week. To do so skillfully, both gates must be simultaneously opened at the "young flood." The stale water will thus rush out and fresh water come immediately back with the rising tide to float the rice leaves and prevent them sticking to the ground in their fall. If the maggot gets serious, the field has to be dried nolens volens. The maggot is a tiny white worm, which is generated by stale water, and attacks the roots of the plant, causing serious injury to the crop. The presence of the maggot may always be suspected by the stiff and unthrifty appearance of the field.

If the land is fertile, at the end of the sixty-day flow it will be found, on drawing off the water, that the rice has attained a vigorous growth of about three feet, and is well stocked with tillers, while also, if the field is level, and the harrowing and pulverizing was thoroughly attended to before planting, no grass will be seen, nothing but rice and the clean soil beneath. The field is kept dry now for about fifteen or twenty days, or until the land dries off nicely and the rice takes its second growth. And if there be no 'grass' it ought not to be disturbed with the hoe, as the laborers, at this stage, often do more harm than good. This, how. ever, does not apply to 'cat-tails' and 'volunteers,' which should, of course, be carefully pulled up by the roots, and sheafed and carried to the banks, to be disposed of by the hot sun.

At the end of fifteen or twenty days, as above mentioned, the water is returned to the field as deep as the rice and banks can bear, never, however, topping the fork of the former. This water, where circumstances permit, is changed every week or two, by letting it off on one tide and taking it back on the next, and increasing the gauge with the growth 
of the rice, When the heads of the rice are well filled and the last few grains at the bottom are in the dough, it is fit to cut, and as little delay is permitted as possible, as the rice now 'over-ripens' very rapidly, and shatters in proportion during the harvest. The water may be drawn off the field from three to five days before cutting the grain, and the land will be in better condition for harvesting."

The methods of culture differ somewhat with different localities; some planters harrow in the grain and inundate it until it germinates, after which the water is withdrawn, and is not let on again until the plants are about four inches high. The water is then let on again until all but the top leaves are covered. The crop is then kept inundated until about two weeks before harrest, when it is again withdrawn in order that the stalks may harden preparatory to harvest.

The method practiced in Northern Italy is to sow the seed in April, previous to which it is soaked a day or two in water to hasten germination. After sowing, the ground is irrigated to the depth of about two inches. The rice comes up through the water, which is then drawn off to give the plant strength, and after several days it is let on again. This mode is said to be more liable to mildew the rice than the inethods practiced in this country. Aquatic grasses and weeds are apt to be troublesome in some soils, and should be destroyed as fast as they make their appearance. The chief business of the cultivator is to do this, and to keep the water from stagnating and as fresh as possible, which may easily be done by turning it on at one point and drawing it off at another at the same time. Then grown on dry land in the upland sections, its culture is similar to that of other small grain. It should be planted in shallow drills two and a half or three feet apart, and the soil kept free from weeds by the use of the cultivator or horse-hoe. When cultivated in this manner the crop is inferior to that grown by means of irrigation, both in quantity and quality. In the latitude of Georgia it is sometimes planted as late as June; its straw makes good forage.

Unlike other grain, rice can be kept several years in a warm climate without its quality deteriorating, by simply winnowing it semi-annually, to prevent the weevil and other insects from injuring it. Rice does not impoverish land upon which it is grown, like many crops, and is often used in upland sections to precede corn in rotation. It adapts itself to the soil. climate, and mode of cultivation, but, like most other grains, the seed will degenerate if not frequently changed and the best specimens always selected for sowing.

After the land has been properly prepared for growing rice, by ditching; embankments, leveling, etc., a single individual can cultivate an immense crop, and with comparatively little labor. The desideratum in this product is to have the grains as nearly of a uniform size as possible.

Harresting.-The proper stage of maturity for cutting rice is indicated not only by the grain being well filled out in the heads, and a few kernels at the bottom of the head being in a dough state, but also by the straws turning yellow. The metlod of harvesting is similar to that of other grain, such as wheat or oats, except it is generally cut from twelve to eighteen inches from the ground, according to its growth, which is usually from four to six feet high. It is laid upon the stubble for a day or two to dry, which is generally accomplished in twenty. four hours in good weather, after which it is bound into bundles and placed in small stacks. It is never well to bind it when the dew is on the straw. As soon as sufficiently dry it may be threshed in the field, or stacked to await a convenient time for doing it. In order to prevent the germination of the "volunteer" rice, as soon as the crop harvested is taken from the land, the field should be flooded quite shallow, so as to promote fermentation; it should be dried again every ten or twelve days for a day or two at a time. By continuing this process until the ground freeze-, most of this foul seed will be destroyed.

Thxeshing and Milling.-Rice has a much larger and more harsh beard than wheat, which is a prolongation of the outer hull of the grain; but, unlike wheat, the threshing pro- 
cess does not separate the inner hull or covering from the grain, consequently special treatment is required for this purpose. It may be threshed and winnowed by steam, horse, or any of the powers used on a farm. At this stage the grain is called "rough rice," and is thus shipped to market from the plantation in cargoes, or first subjected to the milling pro. cess that removes the inner hull. The machinery for this purpose is too expensive to be adjusted to plantations generally, hence it becomes in a great measure the business of large manufactories. This hulling process cleanses the grain of the inner covering, and usually separates it into three grades; the first grade or whole kernels, the second grade or broken rice, and the third grade or flour of rice, or as sometimes graded, "whole," "middling," and "small rice." It is estimated that a little more than half comes out from this process in first grade or prime rice, the remainder being broken or small rice, flour of rice, and chaff. With good land, properly irrigated and cultivated, reliable authorities estimate the average produce of this crop to be from forty to fifty bushels of clean rice per acre, and often the yield has far exceeded this estimate. With suitable conditions, rice is one of the most profitable crops that can be cultivated.

\section{ROOTS AND ESCULENT TUBERS.}

$\mathrm{R}^{\circ}$ OOT crops, which form so large a proportion of the agricultural products of the English farmer, have not received that attention in this country, generally, that their real value would seem to warrant, but are gradually gaining favor, being cultivated more extensively at present as food for stock than formerly. There are reasons why the English farmer cultivates roots so extensively, aside from the real value of the crops. The climate of the country is especially adapted to the cultivation of roots, while it is not adapted to the successful cultivation of corn. On the contrary, the climate of the United States is not as well adapted as that of England to the cultivation of roots, while it is most admirably adapted to that of corn. Yet, notwithstanding these considerations, there are many reasons why the farmers of our country should devote more attention to the cultivation of roots than has formerly been the custom.

Various experiments have proved the fact that, although roots are not desirable as an exclusive or principal article of food for animals, yet when used in connection with grain, meal, hay, or other substances, animals will thrive better, fatten more readily, and keep in a more healthy condition than when roots are entirely discarded from their food. It is also a fact, well known to all dairymen, that more milk of a better quality can be obtained from cows fed with a liberal supply of roots during the winter, than when hay, meal, and bran only are used, and that butter made from such milk will not only be of better flavor and color, but more in quantity. Stock should have some kind of green food during the long winters, and roots are admirably adapted to this purpose, taking the place of grass in a measure, where ensilage is not used on the farm. Roots are easily cultivated, and large quantities can be grown on comparatively small areas of land. In England, turnips form the most important root crop in cultivation, being used for feeding stock, and also as a clearing and fallow crop in the regular rotation for the soil.

Potatoes are the most important root crop in the United States. Green manure should never be applied to any kind of root crop, as it not only injures the quality, but will cause potatoes to grow irregular and deep-eyed, turnips, beets, parsnips, etc., to have forked and illshaped roots, and onions to be coarse in texture and thick-necked.

Indian corn is a good crop to precede any root crop, and by heavily manuring the land for it, and supplying a good dressing of composted manure to the land the fall previous to culti. vating the roots, or commercial fertilizers well harrowed in about the time of sowing the latter, good results will generally be secured. 


\section{POTATOES.}

HE potato has become the most extensively cultivated and valuable of esculent tubers, both in this and foreign countries, although when first introduced as an article of food it was regarded with disfavor or indifference by the majority, and won its way gradually into popular esteem, until it has become an important article of diet with all classes, from the opulent to the very poor, and is one of the leading crops cultivated.

It is a native of this continent, being found in a wild state in the elevated tropical valleys of Mexico, Peru, Chili, the Argentine Republic, and the island of Chiloe, where it closely resembles the cultivated product, except that the tubers of the former are much smaller, they rarely being more than an inch in diameter, and the flavor very unpalatable; careful cultiva. tion having not only greatly increased the size, but the edible properties in a proportionate degree. It is supposed to have been introduced into Virginia from Florida by the Spanish explorers, and was carried to Spain and Italy from Peru early in the sixteenth century. Its introduction into Great Britain from Virginia was, according to some authorities, in the year 1565 , by Sir John Hawkins, and according to others, about the year 1586, by Sir Walter Raleigh. Houghton, in his "Collections on Husbandry and Trade," gives the somewhat amusing description and history of this product:-

"The potato is a biciferous herb with esculent roots, bearing winged leaves and a bell flower. This I have been informed was brought first out of Virginia by Sir Walter Raleigh; and he stopped at Ireland, some was planted there, where it thrived very well, and to good purpose; for in their succeeding wars, when all the corn about the ground was destroyed, this supported them; for the soldiers, unless they had dug up all the ground where they grew, and almost sifted it, could not extirpate them; from whence they were brought to Lancashire, where they are very numerous, and now they begin to spread all the kingdom over. They are a pleasant food, boiled or roasted and eaten with butter and sugar. There is a sort brought from Spain that are of a longer form, and are more luscious than ours; they are much set by, and sold for sixpence or eightpence the pound."

The potato was at first regarded in Europe as a delicacy, but it was not until near the middle of the eighteenth century that it acquired any real importance there outside of Ireland. It was also unknown in New England until the early part of the eighteenth century, when it is supposed to have been introduced there from Ireland. It is sometimes called a root, which is an erroneous term, as the roots are entirely distinct from these tubers, or underground stems. It is ailied to several powerful narcotics, such as tobacco, henbane, and bella. donna, and other common esculents, viz., the tomato and egg-plant. According to the best authority, the strong, bitter principle of the potato is more or less poisonous, and is aggravated by the light, such as exposure to the sun by having the soil long removed from it in the hill, and which causes the portions of the tubers thus exposed to turn green. All such parts should be removed before cooking. The potato consists (aside from water) almost entirely of starch, the analysis of it giving about seventy-five per cent. of water and twenty-five per cent. of dry nutritive matter; it is, therefore, deficient in nitrogen, and not adapted for an exclusive article of diet. Its composition shows it to be designed as an accompaniment of meat, instead of a substitute for it, and all nations now using it appropriate it to this purpose.

The proportions of its constituents vary according to the different stages of ripeness and different varieties. The more mature, the less the quantity of water, some of the richer varieties furnishing as high as thirty-two per cent. of dry nutritive matter, the latter consisting mostly of starch, with a small proportion of sugar, gum, cellular fiber, fatty matter, mineral matter, etc.

Aside from its use as food for the human family and all domestic animals, it is largely employed in the manufacture of starch, as well as alcohol. Sugar has even been made from 
it, but not very extensively, owing to its being more cheaply made from other products. Previous to the appearance of the disease known as the potato-rot, which occurred in 1845 , and destroyed nearly the entire product, this was a very productive crop and was cultivated with hittle expense, and extensively used in fattening all kinds of stock; but since that period, the varieties then most esteemed have been replaced by others, and the yield has largely decreased. The cost of production has also proportionately advanced.

About the time of this decline in the crop, Rev. Chauncy E. Goodrich, of Utica, N. Y. imported a number of wild varieties directly from South America, and proceeded to raise seedlings by crossing them with various kinds then in use, and from which a vast number of varieties were produced, some of which were very popular, one of the principal being the Garnet Chili, from which many of our most valuable kinds have descended. This gentleman devoted more time, zeal, and skill in propagating the potato than any other man. He carefully studied and experimented with regard to it for sixteen years. In 1848 he imported from South America, the home of this plant, some of the native tubers, and from these commenced his experiments in producing new varieties. He succeeded in originating in all about fifteen thousand seedlings, which he divided into seventy-four distinct families. After testing these seedlings for four or five years, he selected the best, rejecting such as did not reach his expectations in yield, habits of growth, health, edible qualities, etc. Mr. Goodrich died in the midst of his useful experiments, but not until he had been successful in establish. ing in public favor several varieties, among which were the Garnet Chili, Early Goodrich, Cuzco, Gleason, and Harrison varieties, which for a long time were the leading ones in cultivation in the country, being of fine quality, very productive, and healthy.

While American varieties often succeed fairly well in England, the English varieties, as a general rule, are not adapted to our soil and climate. Frequent experiments have resulted in a large proportion of the tubers being affected with rot at the time of digging. The climate of the British Isles seems more favorable to the development of the potato-rot than our own, which makes it a matter of great importance there, as well as with us, that such varieties as will resist this destructive malady be procured, as far as practicable, for planting. The potato crop is one for which there is always a demand in the market, and in which an increasing interest is being taken at present by agriculturists in producing constantly new and improved varieties.

Varieties.-In the early period of the cultivation of the potato, it was regarded as a species of vegetable having no distinct varieties. For the introduction of different varieties we are indebted to the market-gardeners near Manchester, England, who, being encouraged by the demand for this product, vied. with each other in endeavoring to produce the best and earliest kinds for market. They did this by marking the plants that blossomed earlicst, saving and sowing their seeds, and again securing the earliest from their product, until they finally obtained varieties which were two months earlier than those previously cultivated. They also preserved the seed of the most farinaceous and best flavored, most productive and best shaped tubers, and in this manner improved both edible and productive qualities, as well as early maturity.

The varieties at the present time are almost innumerable; differing in form, size, color, texture, smoothness of surface, flavor, time of ripening, productiveness, hardiness, etc., those being most preferred that are the most farinaceous, fine textured, delicate flavored, and have a smooth surface, combined with the other desirable qualities, such as vigor of growth, hardiness, productiveness, and freedom from disease. A potato that is termed "soggy," that is, watery and deficient in farinaceous properties, is one of the most undesirable articles of food, and however productive or hardy such a variety may be, it is unprofitable as far as the market value is concerned, except as food for live stock, and even then such kinds are not as nutritious for this purpose as those that are light and flaky when cooked. A deep eyed potato is also objectionable. 
Some varieties thrive best in one locality and kind of soil, and others in another, there being nearly as much difference in this respect in the several kinds, as in those of apples, pears, or any of the varieties of fruit. The early kinds are distinguished from the late by early blossoming and decay of the tops.

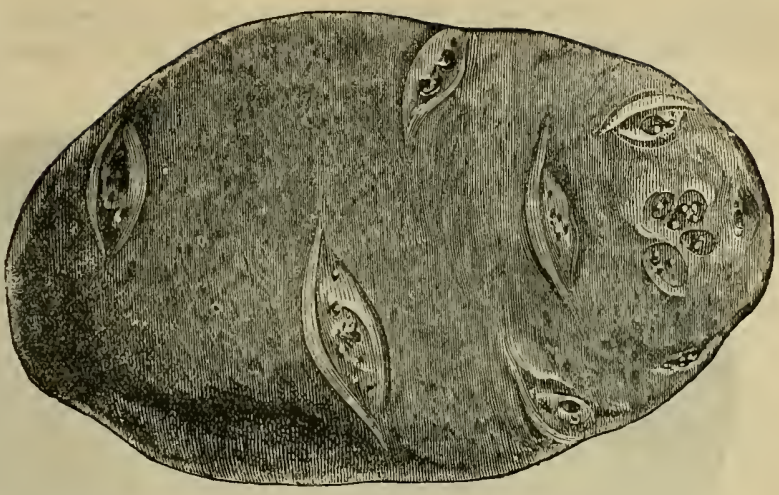

SNOWFLAKE.

Among the many valuable kinds at present cultivated, it is difficult to particularize those of especial and superior merit; besides, new and choice varieties are constantly being introduced, and it often happens, as has been previously stated, that certain varieties will be most

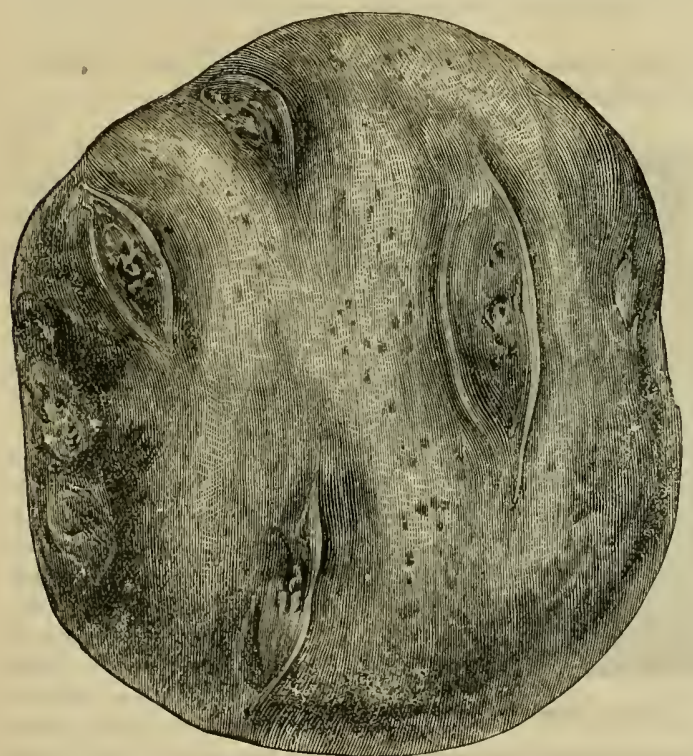

Improved Peachblot.

popular and produce the best results in certain localities, the soil being especially adapted to their perfect development, while others will produce similar results in a locality and character of soil in which the former might not prove as satisfactory; however, different varieties have their special qualities and characteristics by which they are distinguished, and which are in a measure unaf. fected by soil or climate, although in a general sense largely modified by both. Many of the old favorites are still culti. vated in some sections. Some of the varieties that are quite popular in many localities are the Early Peach Blow, Early Rose, Tuate Rose, Ruby, Snowflake, Improved Peach Blow, Late Beauty of Hebron, Late Snowflake, Early Ohio, Mammoth Pearl, Silver Skin, White Star, The American Giant, Extra Early Vermont, Manhattan, Alpha, Magnum Bonum, and Compton's Surprise, all of which possess fino 
qualities, and are quite prolific, the two latter being especially productive. Our illustrations representing some of the different varieties of potatoes and the diagram for cutting to a

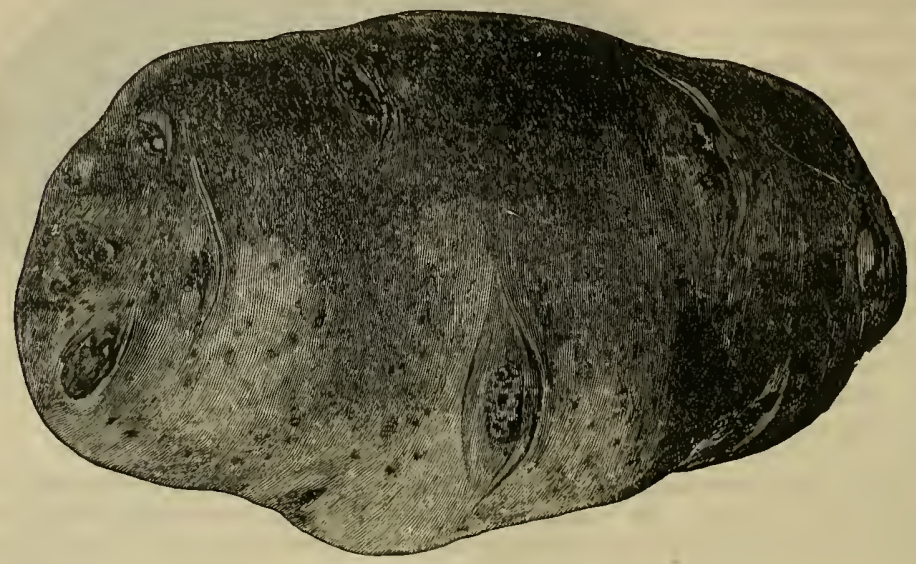

Late Beauty of Hebron.

single eye, were obtained through the courtesy of the well known firm of B. K. Bliss \& Sons, New York city, being copied by permission from their catalogue.

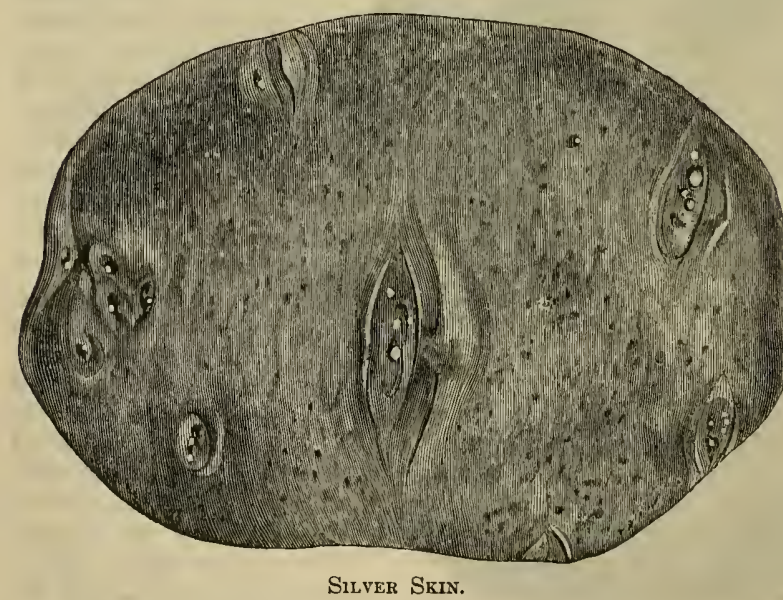

\section{Soil and its preparation.}

Although good crops of potatoes may be grown on a great variety of soils, that best adapted to this product is a rich, sandy loam, neither too wet nor too dry; should there be a clay subsoil, it will require drain. ing to make it sufficiently dry. Clay soils are unfavorable to the health of the potato in a wet season, since

they closely envelop the tubers and bury them so compactly that they prevent the access of air, light, and heat, so essential to their perfect development. Should the surface of the unripe potato be kept constantly wet, as it would be in a clay soil in a wet season, this alone will be sufficient to induce decay, although the vines might be perfectly healthy. A wet soil of anv kind is injurious to the quality of the product, it being generally coarse, watory, and 
deficient in farinaceous quality. A cool, moist soil will produce larger potatoes, but in a northerly chmate a warmer soil is to be preferred, as being less liable to injure the quality.

A calcareous soil yields a potato of fine quality, and can generally be depended upon for the production of a good crop, and when such soil contains but little lime or plaster, this should be used in fertilizing. Early potatoes will mature much quicker in a warm, light soil than a heavy one, and also present a brighter and cleaner appearance when dug. Old sodland, especially clover sod, is excellent for this crop. It should be turned under in the fall (August or September being the best time), and lightly plowed and harrowed in the spring. Lands should never be plowed while wet and heavy, as it is an injury to the soil, and is labor lost. Mr. A. Hyde, of Massachusetts, says:-

"The inverted sod of an old pasture is one of the best for potatoes. With a little wellrotted compost harrowed in thoroughly upon such a sod, to give the plants a good start, we have raiscd good crops upon comparatively poor soil. An old pasture contains much vegetable matter, and the tubers delight in the mellow bed which such a soil affords, and come out in the fall clean and healthy. We have also raised good potatoes in a mucky soil apparently having little but vegetable matter in it. This can only be done in a dry season. In a wet summer the muck retains too much water, and has the same influence on the tubers as compact clay. Leached ashes sliould also be put in the hill with potatoes, when planted on muck, to furnish the inorganic matter in which muck is deficient. A compost made of muck and leached ashes is one of the best possible manures for the potato. The muck makes the soil porous, and furnishes a bed in which the potato delights as much as our mothers formerly did in a feather-bed. Sufficient potash is left in the leached ashes to furnish this essential ingredient of the potato.

Sand soils are often as much too open to atmospheric influences as clay soils are closed against them. Sand both cools and heats too rapidly, and feels the sudden changes of temperature which are so trying to the potato. Still, on poor, sandy soils good crops of potatoes can be raised by the aid of muck and ashes. The perfect drainage and slow growth secure this result. The seed should be planted deeply and cultivated on a level, so that the tubers may be less affected by the sudden changes of temperature. We have known potatoes to rot as badly on sand as on clay when planted superficially and hilled up in contracted hills."

One of the leading agricultural writers of the "Country Gentleman" expresses the following facts and opinions respecting potato culture:-

"From such facts as have come to my notice, I am led to the conclusion that when potatoes, and, indeed, all, or nearly all vegetables, and perhaps the cereals, which have originated in cool climates, are grown under a warmer latitude than where they make the strongest and best development, they require a soil very much stronger in the inorganic elements of plantfood, such as potash, lime, magnesia, and phosphoric acid, and demand also to be grown wider apart, one from the other, because, perhaps, the extra stimulating forces of a hot climate shorten the period of growth, and there must not only be room enough for the feeding roots, but the soil must be full of plant-food. Going South, one notices that nearly all common northern vegetables have a tendency to grow above ground, and I was told that unless the soil is heavily manured with stable manure, it is nearly impossible to get cabbages to head, turnips to bottom, or potatoes to come to full growth. In the truck patches about Mobile, where cabbages are planted out in November and potatoes in February, an experi. enced grower informed me that his success with cabbages and potatoes was just in proportion to the amount of stable manure and cotton-seed meal he used in the case of the cabbages, and potash in the form of the ash of cotton-seed hulls, in an incredible quantity, was absolutely essential to success with the potato crop. Perhaps the absence of vegetable gardens in the South, which so forcibly strikes the northern observer, is quite as much due to the want of manure (on account of the limited quantity of stock of all kinds, which is seldom yarded and rarely stabled) as to any indisposition to do the work of successful truck_patching. 
Potash in the soil is not only essential to the vine growth of the potato, but to its quality, since the mealiness so much sought after results from the presence of starch in the tuber, not a grain of which can be formed in the absence of this salt. In cool climates, the growth and ripening of the potato extend over some months, and the processes of the elaboration of starch occupy many weeks; hence it will be found that a poor soil, in a high northern latitude, will grow better potatoes and more of them than a richer one farther south. When potatoes are grown on the worn, sandy first and second bottoms of the Mississippi River, north of Cairo and as far north as St. Lonis, the crops are not unfrequently tolerably large ones; but the tuhers are apt to be soft, watery, and waxy, showing that while there is still enough potash present in the soil to answer the purpose of vine and tuber growth, there is not enough to admit of the changing of the cellulose into starch, in the brief space of the intensely hot summer season allowed for this process. But the growers for the St. Louis market manure highly with material obtained in the city stables, and the crop, though never comparable to northern.grown tubers in quality, is a fairly good one. Probably the extraordinary fine quality of Peerless potatoes I have eaten, grown for the northern spring market in the winter vegetable gardens in the vicinity of New Orleans, owed that excellence to the very liberal use of cotton-seed meal and ash, in connection with stable manure, as in the case of those in the suburbs of Mobile.

Probably the best form of potash for potatoes is in that of wood-ashes, leached or unleached, - the former being of more than half the value of the latter,- while soft coal-ashes are by no means to be despised, especially in the western country, where more or less wood is burned in connection with coal. Indeed, for vegetables of any kind, coal-ashes, whether hard or soft, are to be sought after, since they are always valuable as an amendment to the soil, and possibly though they may contain no plant-food, they do contain ingredients which render certain forms of insoluble plant.food soluble.

The lesson to be learned is that potash, and an abundance of it, is essential to successful potato-growing; that its best form is wood-ashes, not forgetting soft coal-ashes and wellrotted stable manure, and that as one goes south he must increase the quantity of potash and other inorganic elements of plant-food, in order that the starch in the tuber may be quickly elaborated."

Virgin soil with little or no manure will produce the best quality of potatoes, as a general rule.

Whatever the character of the soil, it should be plowed deeply, and thoroughly pulverized with the harrow before planting. A deep, mellow soil is, according to the best authority, better adapted to resist the extremes of moisture and drought than any other.

When barn-yard manure is used, it should be well decomposed, or composted, fresh manure being objectionable for potatoes, as it causes them to grow ill-shaped, with deep eyes, and gives them a strong, unpleasant flavor.

Poultry manure, wood-ashes, and plaster, mixed in equal proportions, make an excellent fertilizer for this crop. Superphosphate of lime also gives very good results, as well as marl, bone-dust, guano, and similar fertilizers, and on wet soils are very beneficial in rendering them more dry, as they are of an absorbent nature.

As has been previously stated, potash is highly beneficial to this crop, and may be applied in the form of wood-ashes, which should be placed in the hill in planting.

As a general rule, fall plowing is to be preferred. unless the soil is very friable. When sod is used for the crop, or heavy land with a hard-pan or clayey subsoil, it should be plowed rather deep in the fall, and harrowed and plowed again in the spring as soon as it is dry enough to be friable.

If the manure is of a fine texture, it should be spread on and harrowed in, but if coarse manure is used, it can be spread on before the spring plowing or put in the hill or furrow. 
Some prefer to spread a part broadcast, and the remainder to be applied either in the hill or drill, according to the method of planting. Chemical fertilizers are often used in this manner in connection with yard manure, the former deposited near the seed, and the latter spread broadcast and harrowed in. Chemical fertilizers should, however, be mixed with the soil, for if applied directly to the seed, they will be liable to destroy the eyes or young sprouts.

New land is commonly considered the best for producing large crops that are free from disease. This may be due to the fact that new land contains more potash than that long cultivated, which is an element necessary to the perfect development of the potato.

Many of the chemical fertilizers made especially for this crop, of which superphosphates are the principal basis, often prove highly beneficial in producing large yields of the best quality.

Potatoes do not require as much fertility as corn, but still thrive best with an abundance of it, and in as nearly a mineralized condition as may be,-well composted, ready to be easily taken up as plant-food.

The quantity should be suited to the nature.of the soil, new lands requiring less manure than those that have been long in cultivation.

If the soil is manured too heavily, and the growth over rapid, many of the varieties will be hollow in the center of the tuber, or, as is commonly termed, "hollow-hearted." A long, slow growth is better than a forced, hasty maturity, giving a better quality as well as a larger crop that can be better preserved, being less liable to disease.

Some farmers use from eighteen to twenty two-horse loads of good yard-manure per acre, spread broadcast, and from seven to eight hundred pounds of chemical fertilizers in the hills in addition, and consider this the most desirable amount for this crop; others use twice this quantity of manure with the artificial fertilizers. The quantity of fertility supplied to this crop should be modified in a great measure by the character of the soil.

Selecting and Cutting Potatoes for Planting.-The varieties chosen for cultivation, should be those possessing fine, edible, and productive qualities, and also those best adapted to the soil. The most perfect specimens of tubers should be selected for planting, that is, those of medium size, not too large or too small, and which are as nearly perfect in size and condition as possible, carefully avoiding any that have the least appearance of disease. Since it is the law of nature that "like produces like," if diseased potatoes be planted, the crop will be very liable to be not only a small one, but an unsound, diseased one as well; or, if disease does not make its appearance with the maturity of the crop, it will be liable to at a later period, and necessitate quite a loss before the following spring.

There is still quite a diversity of opinions among farmers as to whether it is better to plant pieces of the largest potatoes, or whole small ones; also if pieces be used, which is the best end of the tuber for planting, and the best size. There has also been quite a variety of opinions as to whether the perfectly ripe tuber should be used, or those not fully ripened, some claiming that the latter are more vigorous and early in sending out shoots.

Repeated and careful experiments, by some of our most successful agriculturists, as well as long practice, go far in establishing the opinion, that potatoes of a medium size are best for planting, and that pieces are better than the whole tuber for this purpose, although good crops are often obtained from small whole ones; that those perfectly ripe are to be preferred to those not fully ripened, and that the eyes from one part of the potato are as prolific as those of another part.

Some, however, according to the old custom, still make a practice of planting whole potatoes, one large or two small ones to a hill; on the other hand, Mr. MI. J. Wheeler of Massachusetts, says that the best crop of potatoes he ever produced came from sprouts taken off the potato. Cutting to a single eye is at present quite extensively practiced, and is a method highly recommended by some of our best authorities on this subject. Mr. B. K. 
Bliss says: - "Cutting is one of the most important subjects to be considered in the propagation of potatoes, and there is such a diversity of opinions regarding the manner and method of doing it, that many pages could be filled in giving the different experiences of the professors in this art. While we do not attempt to decide this question to the satisfaction of every one, we shall give our own views, and claim that in our method an enormous quantity of the tubers now annually planted may be thrown into the market, causing a reduction in the prices charged for this common and necessary crop.

Without discussing the respective merits of planting whole potatoes, or half a dozen pieces in a hill, each piece containing three or four eyes, we shall state what has been proven by so many cultivators, that two good eyes are ample for one hili, and the yield of large, marketable potatoes is larger than when more are planted."

This practice will seem a small allowance for a field crop by those who have been accustomed to use a larger quantity; still, we know it has many advocates, and that too much seed is usually planted, which results in crowding in the hills, and producing a large number of very small tubers, which are nearly worthless, except as food for farm animals, too much seed being as detrimental to the crop as too little.

Mr. Terry, of Summit county, Ohio, gives the result of his method of planting a single eye, as follows: - "For seed I use only the very choicest specimens, of perfect shape, in size varying from medium to large, having large, strong eyes.

These potatoes are cut to one eye. At the seed end one eye is left on, and the others cut off and thrown away. The most expert and careful hands are set at this job. One piece is dropped every 12 to 14 inches in the drill, and the drills are made 32 inches apart, for such varieties as Clark's No. 1, Early Rose, Beauty of Hebron, and Snowflake. The cutting and dropping are done the same day, and, if the sun shines, the coverer follows the droppers closely. Such seed, cut to one eye and put in as I have described, will produce fewer potatoes to the hill usually, but, with moderately favorable weather and good care, they will be large and fine, and of No. 1 quality. On this point I will give a little of my experience, to show whether it pays to take all this trouble or not.

A few years ago potatoes were very low in the market to which I go. Early in July I took a sample of large, fine ones, and spent all day in trying to get an order for a load at 40 cents. At last A decided to buy a load, they were 'such beauties.' The market was full of ordinary potatoes, and at no time did they sell for more than 25 cents. After this I had to work night and day to supply the demand, and made a fair profit, whereas at 25 cents I should have lost money.

Two years ago the season was favorable, and my potatoes were very large and fine. I took my first load (30 bushels from 16 square rods) to a very particular customer. There were bushels of potatoes in it that would weigh one pound each. I had nearly 2,000 bushels of those potatoes, and within three days they were all spoken for at my price. I have passed men drawing their potatoes home (small, dirty-looking things), unable to get a bid, at a time when I was over-working men and teams to supply the demand, and they would say: "Terry, you are the luckiest man I ever knew.' There was no luck about it; I simply did the best I could to raise a choice article and get it to the market in nice shape. The way is open to all. Luck rarely comes to those who do not strive for it. It pays on the farm, as well as elsewhere, to do everything the very best we know how, and in such a manner as to secure the very best possible results."

The quantity of seed will vary somewhat with the soil and variety, and we would advise each farmer to experiment for himself, which can be very easily done by marking off some small portion of his potato-field for this purpose, first preparing it so that it will be of uniform fertility. By planting one row of hills with a single eye, another with two eyes, another with three, then with four, etc., on the same field, giving each the same cultivation 
and carefully measuring the product of each separate row, each farmer could easily settle this question to his own individual satisfaction, providing the soil of each of the different plant- ings were of about equal fertility. Some of the old works on agriculture recommend from six to eight eyes to a hill, but repeated and careful experiments have proven this to be altogether too large a quantity.

The following cut will show how a potato can readily be cut to a single eye and each eye be supported by an equal amount of flesh, which will be sufficient to start it into a healthy and vigorous growth. Many may consider this method of cutting too troublesome where the common varieties are used, but by it only about half the usual amount of tubers noed be used for planting; and to those who wish to obtain a large crop from a small quantity of choice potatoes this method will prove quite valuable.

This is done by holding the stem.end down, keeping it in a perpendicular position throughout the cutting. With a thin-bladed sharp knife the first eye nearest the stem-end can be removed by placing the knife equally distant between it and the eye next above it in the rota. tion, sloping the cut towards the inden. ture at the stem-end, and taking the flesh of the tuber with it ; then turn the potato around until the next eye appears - which will be in a line a little above the former-and remove it in the same manner ; and thus continue until all the eyes have been removed. It will be noticed that after three or four eyes have thus been removed the bottom part of the tuber will have something of a pyramidal form, also that each piece will be similar in form, the eye near the central portion of the flesh of the tuber which is to start it into growth and give it sup. port. The cut on the right shows what remains of the potato after all but the small eyes, sometimes called the "seed end," have been removed, and the dotted lines represent how to separate these in a similar manner.

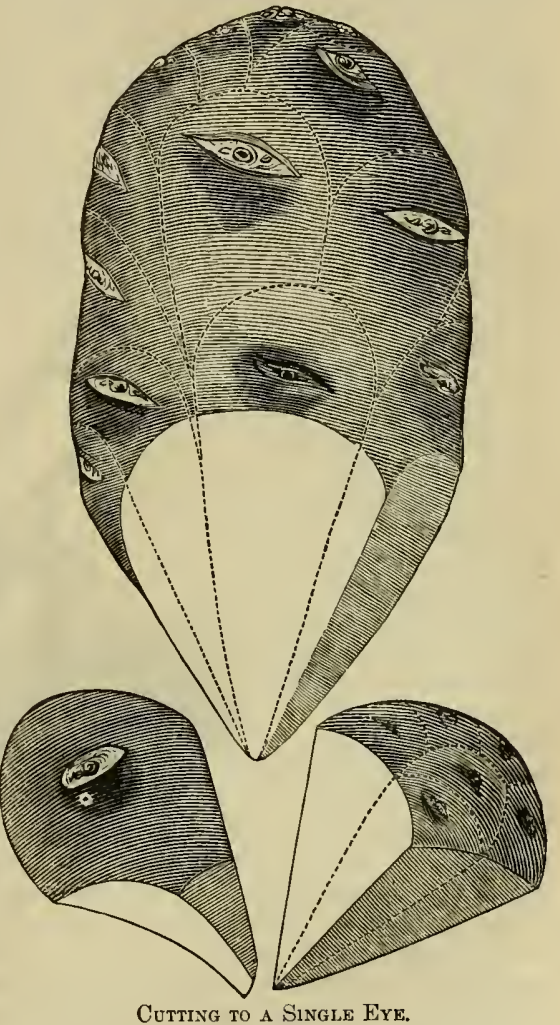

Planting.-A fertile soil, ploughed rather deep, and finely pulverized, in order to render it as mellow as possible, gives the most successful results in growing potatoes. Deep tillage, fine pulverization, with good drainage, will give a light friable soil, and allow the excessive moisture of an unusually wet season to pass readily through it, leaving an increased fertility for the benefit of the crop; while if the season be an unusually dry one, abundant facts substantiate the truth of the statement, that such conditions render the soil better able to withstand drought, however contradictory and paradoxical it may seem. Potatoes may bo planted either in hills or drills, some preferring the former method and others the latter. Hill-planting admits of a more thorough stirring of the soil in after cultivation, as it permits the cultivator 
or horse-hoe to run in both directions, while experiments prove that the yield of botl methods is about the same, the drills, perhaps, giving slightly the larger crop. If planted in drills and the land is sloping, the drills should be made to run in the direction of the slope, so as to admit of partial drainage, and prevent washing in heavy showers. If drill planting is decided upon, and the planting is to be done by hand, the rows should be marked with a plow suited to the purpose, making small furrows about four inches deep, the furrows about three feet apart. If planted in hills, the furrows should be marked across the field at the same distance, so that the marking will be in squares.

When chemical fertilizers are used, they shonld not come in direct contact with the seed, but should be mixed with four or five times their bulk of soil before being put in the hills or drills, or should be slightly covered with soil before dropping the seed. The seed should then be dropped, two or three pieces to the hill, or if in drills, a single piece every ten or twelve inches, after which the covering can be done by a plow or hand hoe. Planting is done to a considerable extent in many localities by a potato planter.

These machines mark the rows, cut the potatoes for dropping, drop and cover the seed at one operation. The following cut represents one of these implements, - manufactured by Nash \& Brother, New York City. The hopper will hold about a bushel of potatoes, and the knife is so arranged that it will be almost impossible to cut pieces without one or more eyes in them. As a general rule, the planting should be done early in the season. Farmers frequently delay this until so late that the crop does not have sufficient time to mature. It was formerly the custom to plant potatoes after the corn planting was com. pleted, but our best farm. ers now have the planting of this crop precede that of corn. Even the lateripening varieties should be planted as early as the ground will admit. Mr.

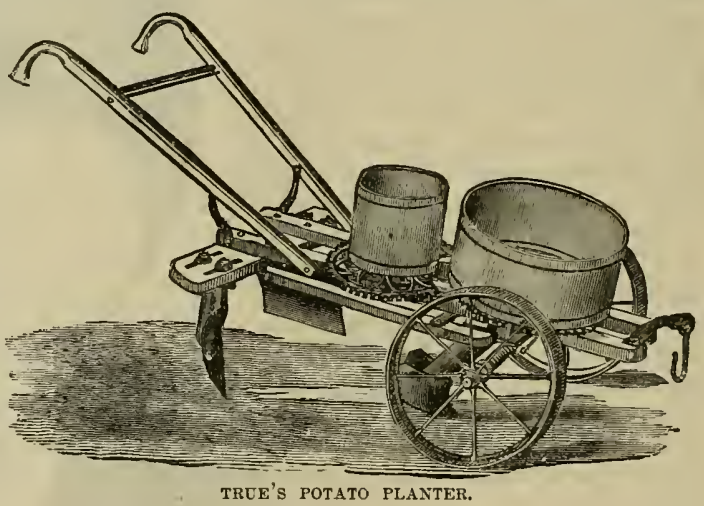
H. Stewart, of New Jersey, says, as the result of his experience:- "I presume that the precise meaning of the word late, as applied to any soil product, is that it has a longer period of growth than the early kinds. Thus early peas, potatoes, or corn may mature in forty or fifty, seventy or eighty days respectively, while late sorts may take fifteen or twenty days longer to mature. But practice does not conform to this natural characteristic, and it seems to be based rather on a supposition that these later maturing kinds require to be planted late.

A neighbor expressed surprise to see me planting Evergreen sweet corn on the same day with Early Concord, and that Early Vermont and Late Rose potatoes should go into the ground the same week, and each kind be treated precisely alike as regards planting. But that is my way, and I think I am right. Last year I had an excellent crop of Peachblows, a kind which I plant for my own use, on account of their superior quality, and the seed was put in the ground the day after Early Rose and Snowflake. The Peachblow was green in November after having been in the ground more than six months, and in yield surpassed greatly the Early Rose.

Do we give the late varieties, when we plant them so late as is usually done, sufficient 
time for their full growth? I think not, and I have the impression that it is to this imma. turity of growth that the failure of some excellent varieties to yield may be due. I have also found that a long season of growth with moderate manuring will produce sounder potatoes than when the maturity of growth has been forced by more copious use of manures. Heavily manured late potatoes-and early ones too, of some kinds-are apt to be hollow-hearted; plainly an effect of over-rapid growtl. These grow to a great size, bnt like overgrown roots, are unsound and of poor quality. Slow, sound growth is consistent with excellence of quality and moderate size. With a long season of growth, there will be a full production of moderate-sized tubers, with no small ones, and this long season can only be secured by early planting. Therefore, I would plant late potatoes as soon as the early ones were in the ground, and I would plant them deep. Deep planting encourages a large growth of roots, and this produces a strong, aerial vegetation, which gives great vigor to the plants, and greatly assists the formation of tubers. Eight inches is not too deep planting for late potatoes, or for early ones either, but especially for late ones, which have then an opportunity for making a substantial growth, and a large space of soil to forage in."

The above accords with our own experience, and we believe that if those farmers who are accustomed to plant their potatoes late would plant as early as the season and condition of the soil would admit, they would not only have a larger crop of more uniform-sized tubers, but of better quality, also.

It is the custom with many farmers to put a good-sized forkful of manure on each hill after the seed is well covered, or, if in drills, cover the tops of the drills with manure. This economizes the use of manure, and tends to keep the moisture about the roots and young potatoes, which greatly facilitates their growth before drouth can affect the plants. Their progress is in this way further facilitated by the leaching of the manure by the rains, which sends the plant nutriment directly to the rootlets. The manure will become covered with soil in the after cultivation. Deep planting is also desirable, since it affords protection to the roots in time of drouth, furnishes them a cool, moist bed, and promotes a stronger and more vigorous growth of plants as well as that of tubers. Besides, the potatoes are less liable to become washed or uncovered by rains and ruined in this way. It will be noticed by a little attention to the subject, that diseased potatoes are frequently found near the surface of the soil, and that those planted rather deep are less liable to disease. Deep planting also admits of a more thorough use of the harrow in destroying weeds, and thus facilitates the after-culture, as the field can be harrowed two or three times without disturbing the seed or the young shoots, and the weeds at first kept easily in check. We should prefer a deptl of six or more inches to that of any less. By planting as early as the soil and season will admit, and to a suitable depth, the many evils resulting from late and shallow planting will be avoided.

An intelligent farmer in Fort Plain, N. Y., gives the result of his test in planting potatoes at different depths thus:- "Selecting the soundest seed I could find, I planted some in the usual way, covering 2 or 3 inches deep; others 6 and 8 inches. The first were a failure - a few small potatoes in a hill and of poor quality. This agreed with the general crop of the neighborhood. It was a matter of gratification - and to the neighbors a surprise - to see the fine, bright tubers that came up when the deep-planted were dug - which was the middle of June; those covered six inches ripening earlier, as they also came up earlier after planting. They were clean and sound, and when cooked were white, mealy, and sweet, with no rank, unhealthy odor."

Deep planting necessitates working the soil correspondingly deep, with the manure well and deeply mixed with it. If the seed lies on a cold, hard subsoil, failure will be the result, since the roots cannot penetrate it, or subsist upon it There should be a few inches of rich, mellow soil below the seed for the roots to feed upon. The objection to planting too much seed is, as we have previously stated, an over-crowding in the hill, and the crop will in consequence 
be a large supply of very small tubers that will be nearly worthless, and but very few large ones. The same objection holds true in planting whole potatoes, there wiil be too many stems for the space occupied, and therefore not room for the proper development of the tubers. The objection to selecting the largest potatoes for seed is, that when such a course is practised there is a tendency to reproduce large, overgrown tubers of a coarse texture and less delicate flavor than those of a medium size; besides they are liable to be hollow-hearted.

The following cut shows the product of a single eye to the hill of the Compton's Surprise, a very prolific variety. If each farmer would experiment for himself, as we have previously suggested, planting different rows with a quantity of seed varying from one, two, and three or more eyes, and carefully noting the product of each row, this question could easily be settled individually in one season. $\Lambda$ s far as our own practice is concerned, we should never plant over two or three eyes to the hill. A diversity of opinion also exists respecting hilling and level culture, each method having zealous advocates. Hilling would be the most desirable on clay lands or those with a tendency to retain undue moisture, as the soil would thus

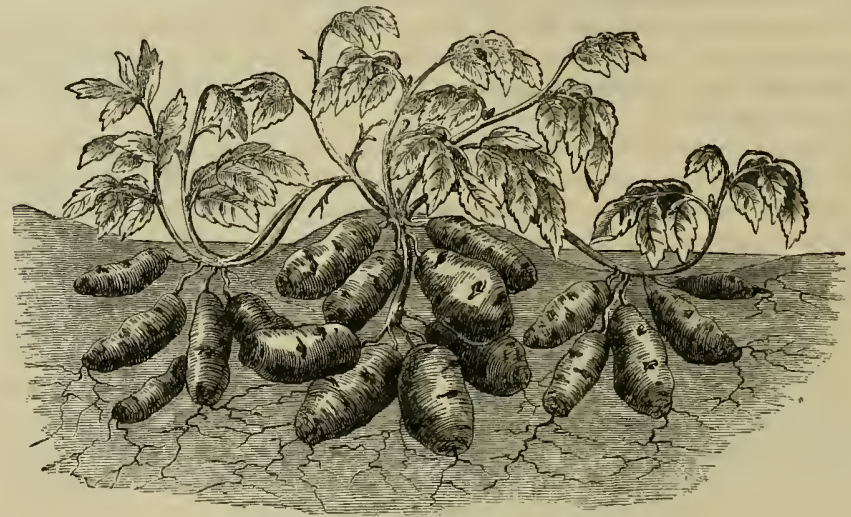

Compton's Surprise Potatoes.

Showing the growin from a single eye in the grounds of F' Seiter, Verona, New Jersey. who raised $384 \mathrm{lbs}$ from one th of the tubers, wilh ordanary culture.

be better drained. Level culture usually gives good results on rather dry soils, and is gener. ally less affected by drought or changes of temperature. Where hilling is practiced, it must not be done after the roots have been well established, or there is any appearance of blossoms, as it will then leave the fine roots exposed to the sun, and cause a new set of tubers to start, thus proving a great injury to the crop. We believe hilling is the more general practice with this crop; whether this be from a choice of the two methods resulting from careful experiment, or from long habit principally, we are not able to positively state, but it is to be presumed that, as but few farmers make any distinction in the culture of wet and dry soils, the practice is due mainly to established habit.

It is said that the natural life of a variety of the potato will average from fourteen to twenty years, under the most favorable circumstances, bence the necessity of getting new seedlings from the best, most vigorous, and prolific varieties. When propagated from the tubers in the usual manner the potato is quite liable to deteriorate or revert to its original condition, and one of the fruitful causes of this deterioration is a continual planting upon the same soil, without a change of seed; therefore a rotation of crops and change of seed is as necessary with this product as others. It has also been found that a dry season will not have the effect to produce a dry and mealy quality of the tubers, but generally the reverse. 
In order to produce an early crop, the seed should be planted just as soon in the spring as the ground can be put in proper condition; the earliest kinds can be marketed in from sixty. five to seventy days from the time of planting, if the season bo as favorable as the average. Seed potatoes should not be exposed to the cold before planting, as they will not produce healthy vines under such circumstances, even if the cold is not sufficient to change their outward appearance. The severe cold seems to lessen the vitality and vigor of the embryo sprouts, or buds of the tubers. Whenever there is a prospect of frost, after the shoots make their appearance from the soil, a little light earth thrown hastily over them will furnish ample protection from injury, and will not be detrimental to the crop, as they will in a few days push

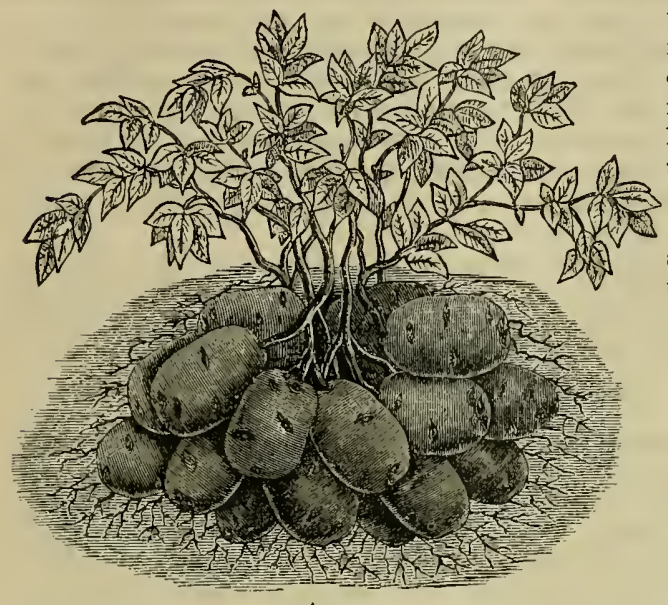

ALPHA.

themselves out of it. Some varieties require more space for growth than others, not only on account of difference in size, but in root extension, having rootlets that rander in the soil much farther than others.

The two preceding figures show the habit of growth of the Compton's Surprise, and the Alpha-one of the earliest varieties known-which illustrate this point. When the seed is changed, as it should be occasionally, it is better to obtain it trom a northern locality rather than a southern, as a product from the former, having a shorter season in which to mature, will have established this habit, and ripen earlier than from the latter source, which would mature more slowly. Prof. Goodale says that his observations and experiments lead him to believe that an important advantage results from such change of seed, and as an instance, he gives it his opinion that farmers in New Jersey would add one-third to their product by planting for seed, potatoes that were grown in Maine. Different varieties should be planted separately, otherwise they will become mixed and the value of the crop greatly deteriorated.

Forcing Potatoes.-When an unusually early start is desirable for a crop, the growth of the young plants can be greatly facilitated by selecting whole and perfectly sound tubers, of medium size, of some early variety, and placing them quite close together in a moderately heated bed especially prepared for them, and composed of either light loam or partially decayed leaves, or both combined. Mr. Bliss recommends this to be done three or four weeks previous to the time of planting. The tubers will, by this time, have become sufficiently well sprouted to be set out in the field, which should have a warm soil, well fortilized and pulverized. The potatoes should then be carefully cut for planting, especial pains being required not to injure or break the young shoots, and also to give each sprout a proper amount of the tuber for support, according to previous directions. Plant the pieces thus started from three to four inches deep, having first placed a little horse-manure in the bottom of each hill to secure warmth and furnish fertility to the young plants. By this process a much earlier crop can be produced than the ordinary method of planting, and although attended with a littlo extra trouble, will be valuable to the farmer who wishes to raise au unusually early crop for market or family use. Another way of accomplishing the same result with comparatively little 
trouble, is to put a few well-selected, smooth tubers of medium size in a warm room in the latter part of winter or early in the spring, and placed where they can have the light of the sun and artificial heat of the room. This should be done long before there are any indications of the soil being ready to work. The sprouts will soon begin to start and will continue to expand and grow vigorously, though slowly, producing very desirable plants for the future crop. As soon as the soil is dry and warm enough for planting, these tubers should be carefully cut the same as for ordınary planting, taking care not to break off the sprouts. The pieces should be set out with care as above directed, and if properly manageu will sur. prise the grower by the early maturity of the crop.

Cultivation.-Frequent stirring of the soil and freedom from weeds are indispensable to a large yield of potatoes. It is a good plan to plant rather deep, and give the soil one or two good harrowings before the young plants make their appearance from the ground, in order to cheek the weeds. Even after they are well started and are from two to three inches high, many farmers use the harrow with little or no injury to the plants, as they are then well. rooted and have a firm hold upon the soil, and if a few become broken off, the new shoots will grow very rapidly to take their places. By these repeated harrowings in the early stages of growth, the after cultivation is made much more easy, as it effectually checks the weeds at this period and prevents their getting a hold upon the soil.

When the plants are well up, the cultivator or horse-hoe can be used to good advantage. If the planting is in hills, the ground should be stirred between them by running the cultivator in both directions, which can be brought quite close to the plants without injury ; if planted in drills it can be used only in one direction, between the rows. This stirring of the soil should be frequent, in order to check the weeds and keep the ground mellow and open to atmospheric influences.

After the plants have made their appearance, some farmers make a practice of top-dressing with compost, chip manure, or a little plaster and ashes in which a small proportion of salt has been mixed; this stimulates the growth of the plants, and the latter has also a tendency to keep off for a time injurious insects. The hilling horse-hoe is often used for this purpose. Some farmers follow the practice of harrowing the whole surface, as has previously been suggested, regardless of rows, a week or more before the shoots come up, and then drag with a smoothing harrow just before they break the ground, which will kill all the weeds that are then up, using it once or twice after they are above ground. After this, the cultivator is run between the rows two or three times before the plants blossom, after which they will require no further cultivation, providing the weeds have previously been properly kept down.

No culture should be given after the blossoms begin to appear, as it will cause the setting of a new lot of tubers and thus prove a great injury to the crop. The use of the handhoe may be necessary in potato culture, in order to destroy a few weeds that are nearest the plants and may be so located as not to be accessible to the cultivator or horse-hoe. It was formerly a custom among some farmers to pick off all the buds before they opened in blossoms, in order to improve the crop, but the slight gain thus obtained does not repay the labor, and the practice is now obsolete.

How to Raise from Cuttiugs. - An ordinary hot-bed is usually employed for the purpose of starting the tubers. These should be cut lengthwise in halves and planted with the flat or cut side down upon the soil. They are to be kept dry until the cut part has become healed and the sprouts begin to start. When the sprouts are grown about four inches they may be cut off from half to three-fourths of an inch above the eye, and set in the soil of the hot-bed an inch or more in depth. They should be kept shaded from the sun, and the soil moistened until they are well rooted and the leaves begin to develop, when they can be trans. planted to a well-prepared field, taking care not to injure the roots in so doing; and to press 
the soil around the bottom of the stem to retain moisture about the roots until they have become established in their new home. A cloudy day, or near evening, is the best time for doing this, as the hot sun will otherwise be liable to wither and destroy many of the young plants. They can be set in drills or hills the same as tubers are planted. These plants are generally quite vigorous, after getting well started, and will grow rapidly. The pieces of potato from which these cuttings or slips are taken will continue to send out shoots to an almost incredible extent, which can be removed in the same manner as the first as soon as they are long enough for the purpose. In order to increase the crop still more, the first cuttings may themselves furnish slips when they are eight or ten inches high, by having their tops removed about four inches, and planted in the same manner as the first. Sprouts will soon start from the base of the lower leaves of the first set, when this method is practiced. This is a laborious mode of propagating, but may be employed with advantage and profit where new and highpriced varieties are being introduced. No cuttings should be planted after the middle of July in a northerly climate, as they will not have sufficient time to mature before cold weather. It is stated that a pound of tubers, - which will contain about four of medium size,- -will furnish by three cuttings a total of about two thousand slips or plants to be set out.

Two Crops of Potatoes a Year. - The following method of producing two crops of potatoes in one season is recommended by B. K. Bliss \& Sons, of New York, and will prove of advantage to those who may wish to increase their potato product in this manner, which, whether regularly practiced or not, may be desirable in certain circumstances, such as for the propagation of a sufficient supply of seed potatoes of some choice kinds for the succeeding year.

"Take good, sound, early potatoes and cut into single eyes (as shown in the cut previously described). Allow these pieces to dry for a day or two, and then plant as early as the ground can be worked (a slight frost will not injure the potato after being well planted). With ordi. narily favorable weather the new crop of tubers will mature in from eight to ten weeks. As soon as they are ripe, dig them, and after remaining a day or two in some dry and warm place proceed to cut them to a single eye as before. Place the pieces thus obtained in pans or boxes containing dry plaster or gypsum. This absorbs the moisture, which would otherwise greatly check the growth if it did not destroy the sets entirely. Allow them to remain in the plaster for ten or twelve days, or until the eyes commence to start, when they are to be taken out and planted as before. In the latitude of New York this is only applicable to early varieties, like the famous Early Rose, or Extra Early Vermont, which are of quick growth, and early maturity ; but in many parts of the South, where the growing season is long, it may be practiced indiscriminately upon all varieties. A gentleman raised two crops of Early Rose, a short time since, in this vicinity, the two crops yielding an aggregate weight of twenty. five hundred pounds. He planted his pound, cut into single eyes, early in March, and dug his first crop about the middle of May. These were then treated as above described and planted the 10th of June, and the second crop dug the 1st of September. The yield from the one pound at the first digging was fifty pounds, and the second crop of this increase was twenty-five hundred pounds, or over forty bushels. This method is within the reach of all, and there is no extra expense incurred for hot-bed sashes or any other forcing requisites."

Hybridizing Potatoes. - Many of our best varieties of potatoes were produced by hybridization, which usually results in a larger number of good kinds than by planting the seed of the seed-balls at random. It is accomplished by selecting for this purpose a few of the most perfect flowers from the most thrifty and vigorous stalks, and removing all the other flowers except those to be hybridized; the anthers (or those parts of the stamens containing the pollen or fertilizing dust,) of the blossoms to be impregnated are carefully cut out with a pair of sharp scissors. This should be done just before they commence to cast their pollen. 
Then when the flowers are free from dew or moisture of any kind, shake the blossom containing the pollen of the variety that is selected for crossing with the latter over it, being particular to do this when the anthers of the flower used in crossing are ready to discharge their pollen freely. The blossom will thus be impregnated, after which a piece of thin muslin or fine lace should be carefully tied over the flower to prevent bees or other insects from introducing pollen from other flowers. When the seed-ball matures, its seeds will contain the germ of varieties partaking in a greater or less degree of the characteristics of both parents. Blossoms of all plants can be hybridized in this manner.

The covering of the flower can be removed after a few days. It should be left to ripen undisturbed, and when fully ripe can be removed from the stalk. This seed should be planted and the product carefully saved. The tubers will be about the size of walnuts the first year, though sometimes of considerably larger size. When perfected in size and other qualities, which requires three seasons, their real value as varieties can be ascertained, and if any are found of sufficient worth to be propagated they should be planted apart from others to prevent mixing.

How to Raise Seedlings. - It is generally known that all new varieties of potatoes are produced by hybridizing or crossing different varieties as previously described, or by planting the seed-balls at random and selecting from the product. It may rarely happen that seed thus sown will produce the same variety of that which produced it; we have known such instances, but they are very rare indeed; the general rule being that there will be nearly as many different varieties resulting from the product of one such seed-ball as there are seeds within it.

A good way for starting the seedlings is to save the well-ripened seed of some good variety, and plant it in the early spring, in boxes that may be well drained in order to pre. vent an excess of moisture. The soil best adapted is a sandy loam. Sow the seed on the surface rather sparingly, and sift fine soil over it to the depth of one-third of an inch; water but little, but just enough to keep the seed a little moist, and hasten germination. Set the boxes in a place where the soil will receive warmth and light from the sun. When the young plants are three or four inches high they can be transplanted directly to the field, taking pains not to disturb the roots in removing. It is always best to take up a little of the soil with each root. These should be set in a soil well pulverized, and properly prepared for a seed-bed, in order to thrive well. They may be set in drills, one plant in a place about a foot apart, the drills about three and a half feet apart, or in hills the same as in planting pieces of tubers. Instead of sowing the seed in boxes, some very successful growers sow in beds prepared in the open ground, generally selecting a partially shaded place.

If sown in rows, they should be about ten inches apart. When sown in the open ground a little more depth will be required in covering. a half-inch of soil not being too much. When the tubers are harvested, they will not usually be much larger than walnuts. They should be carefully stored in a cool place until the next spring, when they may be planted the same as any mature variety. Three years are generally required to fully mature the seedlings or to ascertain their true valuc, and not more than one or two varieties will be commonly found among the product that will be considered worth propagating; sometimes none; however, if only one valuable kind can thus be produced, it amply repays for all the trouble.

Harrestiug.-The decay of the potato.vines indicates the maturity of the tubers; and the crop should be harvested soon after. The tops should, however, be mainly dead before this is done, as the ripening process goes on until this period, and if dug before they are perfectly mature, it will greatly deteriorate the good qualities of the product, while, if they are allowed to remain a long time in the soil after ripening. they will be liable to decay. Very late harvesting in some localities might result in a loss of the crop through freezing. 
Potatoes may be thrown out of the ground by various implements - the horse potato. diggers being the best. The plow, hand-hoe, and potato-hook are also used; but the former often injures many of the tubers, and buries others in the soil, while digging by hand is a slow and laborious process. Where large crops are raised, a potato-digger is almost a necessity.

The following cuts represent different kinds of these implements, the former manufactured by A. Speer \& Sons, Pittsburg, Pa., the latter, Joseph Breck \& Sons, Boston, Mass.

When properly worked, a good potato.digger will pay for itself several times over in a single season, where a farmer has a large crop to harvest. Their use is a great saving of labor. The digging should always be performed when the soil is dry. Mr. A. Hyde of Massachusetts, of whom we have made previous

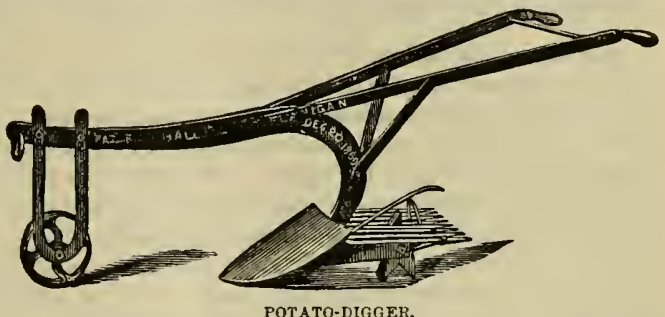
reference in connection with this crop, gives the following sensible advice on harvesting potatoes:-

The time of digging must depend upon circumstances. If the crop is designed for winter and spring use, and the soil is dry, we should prefer to let the pota. toes lie in the ground till the weather is cool enough to allow them to be immediately stored in the cellar. But if the soil is moist and the crop shows a tendency to rot, it should be dug as soon as mature, and placed on some dry knoll, scattering with every half dozen bushels a quart of fresh-slacked lime. Over the pile the potato vines may be thrown, and over the whole a

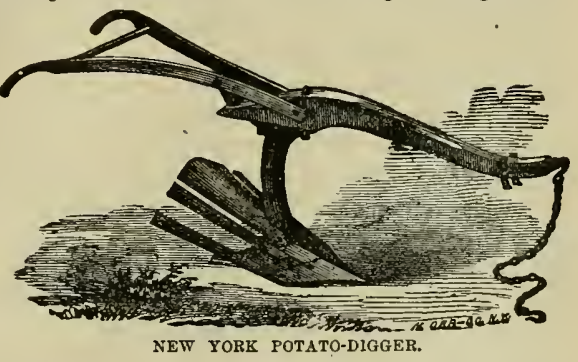
few inches of dry soil in a conical form, making a pit much like the charcoal-pit. The lime cliecks the tendency to rot, and we have never known potatoes thus treated to fail of keeping well. Some recommend charcoal dust instead of lime, and we presume it is useful, as it is an antiseptic; but we cannot recommend it from personal experience. When the weather becomes cool the potatoes can be removed to the cellar or taken to market.

By all means dig in dry weather, and store the potatoes away as dry as possible, with but little exposure to the sun. The skin of the potato is of a corky nature, impervious to water, and designed to keep external moisture from the potato and the internal moisture from evaporation, and, if too long exposed to wet, will sometimes rot, when the tuber must perish. A well-ripened potato, put up dry in the fall, will lose little weight during the winter, its skin preventing evaporation as effectually as does the tight cork of a bottle. In the warm weather of summer, the starch is converted into sugar, and slowly evaporates through the pores of the skin.

All cutting and bruising of potatoes must be carefully avoided. They must be treated as things of life, and not like the stones which can be tossed about without sensation. Every cut and every bruise increases the tendency to decay. The potato may not be quite as sensitive as the apple, may stand more hard whacks; but still, every bruise breaks the cellular 
tissue and puts the vitality of the tuber to a hard test. The digging must not be entrusted to careless boys, or the potatoes will look sadly hacked."

The practice, common in some localities, of leaving the crop exposed, for several hours after digging, to the injurious effects of the strong light and heat of the sun, is very detrimental, and should be aroided. It injures the quality of the tubers; therefore, if the harresting is not performed on a cloudy day so that they may lie exposed on the ground without harm, it is better to gather them into small heaps, with some of the tops spread over them, until the moisture of the surface be dry, when they are ready to be stored.

Storing. - The usual method of storing potatoes is to put them in large bins in a cellar. It is important that the cellar be not too damp or too warm, a cool, dry one being the best for keeping them in a healthy condition until spring. It is a good plan to keep the winaows of the cellar open until late in the autumn, and thus maintain as cool a temperature as possible without freezing the tubers. The potatoes should always be dry when put into the bins. It is a well-known fact that a washed potato never keeps in as good a condition as one that is simply dried, just as it is dug from the soil. When they are wet from a sudden shower, or any other cause, before being put under cover, many farmers put a little airslacked lime orer them in storing them away. This has a tendency to keep them dry and prevent decay. It is common in some sections to bury potatoes in the ground in large pits or trenches prepared for the purpose.

A dry lill-side with a northern exposure, or under a shade, is to be preferred, as it will preserve them from the effects of the sun as spring approaches. When a trench is dug for the purpose. it should be cut up and down the hill to prevent any trouble from water getting to them, thus securing good drainage. It is better to make several pits rather than have too large a quantity stored together, as they will in this way keep better; about fifteen or eighteen bushels to a pit being sufficient. A trench is usually filled in sections, beginning at the bottom or lower end of the trench; from fifteen to trenty bushels may be put in to the required depth; at the upper end of the pile a few bundles of straw and some dirt are placed, and another section made in the same manner until the trench is filled. Straw should be placed over them in covering to the depth of five or six inches, followed with a suffcient depth of soil to prevent them from freezing. It is best to corer with the straw and boards simply for a few dass, to prevent injury from rain, until they are well dried, after which the soil may be added. and the covering complete.

If not covered sufficient to prevent injury from frost, the tubers will be ruined, while if corered too deep they will be liable to decay; consequenily, more or less loss is usually sustained with this method of storage. A narrow ditch, or a few furrors plored on each side of the trench, will aid in drainage to prevent a surpius of water. This is especially necessary on clayey soils, and is a saier practice for those of any kind.

A large cellar or pit, constructed similar to a silo for ensilage, with walls of concrete, brick, or stone, a cover of planks and earth or other material, and a door at one side or end, would be very valuable, and much better than the above-mentioned pits for keeping potatoes, as well as all kinds of roots and even fruit; and where large farms are cultivated, requiring storage-room for immense crops, it will well repay any farmer for constructing such a cellar near his farm-buildings for this purpose.

Such potatoes as are designed for seed should be put in dry bins or barrels in a cool place, where they will be kept dry and at a low temperature, but will not be in danger of being frozen. A corering of dry sand will have a tendency to keep them better than an exposure to the atmosphere.

Diseases, etc.-The most destructive disease to which the potato is subject is the rot, while the insect that effects the greatest infury to the crop is the Colorado Beetle, commonly 
designated the potato-bug. Other difficulties are occasionally met by the farmer in raising this erop, such as the scab, and attacks from the grub, but these are slight when comparcd with the effects of the former.

Potato Rot.-In 1845 the potato rot was very destructive to the crops in the United States and a large portion of Europe. In Ireland especially, where the potato constitutes the principal article of food, great distress was experienced at this time: This disease was so violent in its attacks, in many sections, that whole fields often turued black and rotten in the course of a few hours. In other localities its progress was more gradual, but none the less effective. This disease has made its appearance several times since 1845 , but never with as much severity and the occasion of such extensive loss as at that period; in fact, it is liable to occur to a limited extent in almost any damp season, being caused by a parasitic fungus which attacks the plant, and to which a damp and rather warm season, or such a condition of the atmosphere as is commonly termed "muggy," is best adapted.

This disease is still but imperfectly understood, and there is no effectual remedy known when it once makes its appearance. The stems and leaves of all diseased plants should always be destroyed, to prevent its spreading. By the use of lime in the hills and dusting the growing plants occasionally with it. as well as a similar use of either salt, charcoal dust or gypsum, crops have been known to escape its attacks in localities where other fields were destroyed by it. Avoiding the use of fresh stable manure is also advised as a preventive. It has long been known that a fresh sod that has remained untilled for several years is also less liable to its attacks, and that early-planted fields are not as apt to be infected as those later planted. When a field is slightly attacked, sometimes the preventive measure of mowing off the tops of the plants will prove effectual in checking it. It has also been found that those crops cultivated by the use of commercial fertilizers are less liable to this disease than where farm manures and composts of various kinds are applied to enrich the soil.

Potato Bug, or Colorado Beetle.-This insect has become the scourge of the potato crop, and the greatest pest known to the farmer in the cultivation of this product, which is one of such vast importance in the agriculture of our own and foreign countries. Its destructive powers are so great, and its dissemination has been so extensive since 1860 , that few insects have attracted more attention during that period. In the year 1819 the noted entomologist, Mr. Thomas Say of Philadelphia, accompanied the United States government exploring expedition to the Northwest T'erritories, he being at that time the zoologist of the expedition. While on this tour of exploration numerous species of beetles were found on the upper Missouri near the base of the Rocky Mountains, among them the Doryphosa 10-lineata, which has since become commonly known as the Colorado Beetle, and which was first described by him in 1824. It gradually extended its course eastward, and in 1859 we first learn of its invading the potato fields in Nebraska. In 1861 it becomes known in Iowa, being assisted in its course by railroads, doubtless, as they were often found in and on the cars of the trains that had passed an infested district. They traveled eastward in a direct line over fifteen hundred miles in sixteen years, and became spread over an area of about 1,500,000 square miles during that time, until now its ravages are extended throughout the entire country, and there seems to be no method known of wholly exterminating them, Paris green being the most effectual remedy thus far tried for checking their increase. With this insect, as with many others, it is the larva that is the most destructive to plants.

Prof. C. V. Riley says of it: "This insect bibernates in the perfect or beetle state under old rubbish or in sheltered situations of whatever kind, but normally in the ground, generally but a few inches beneath the surface, but exceptionally at a depth of three feet. As vegetation starts in the spring, it issues forth from the ground, and long before potatoes are up, or even planted, it may be seen flying on genial days in search of food and company, the rose-red underwings 
. contrasting prettily with the yellow and black of the clytra. It will frequently work into a sprouting hill of potatoes, as these are raising the soil, and feed upon the tender sprouts and tubers; and as soon as the plant shows itself the female begins to lay her oval orange eggs in clusters of from ten to forty, each attached by one end to the under side of a leaf, or to a stem. With favorable weather, there hatches in the course of a week from each egg a small, dark, Venetian-red hunch-backed larva, which becomes paler and acquires a double row of lateral black spots as it advances towards full growth. This period arrives in about three weeks from hatching, and the larva finally burrows into the ground, where, within a simple earthen cavity, it becomes a pupa, and finally a beetle in from seven to ten days; the whole cycle of its transformation from the egg to the beetle requiring rarely more than a month." Mr. J. C. Tachë, in his pamphlet entitled "La Mouche, ou la Chrysomele des Patates," says respecting the number of eggs deposited:

"The eggs are deposited in rows and by groups, of which the number most frequently ranges from ten to forty; but groups have often been observed of all degrees of numerical value. In the course of numerous experiments which I have made with insects kept separate, I have seen groups of all numbers, from a deposit composed of a single egg, up to one of 122 eggs, laid without quitting the spot, by a female kept in close seclusion."

From two to four broods are hatched and perfected during the season, according to the locality and length of the season, the last brood going into the ground in a perfect beetle state, to lie dormant during the winter, reappearing as soon as the ground becomes warm enough in the spring to revive them. Each female is said to lay from five to ten hundred eggs during the season; therefore, if in the spring, when they first make their appearance from the ground, some practical method of destroying them could be effected, large numbers prospective could be disposed of in every female bug that should be killed. Destroying by hand the first beetles and eggs that make their appearance on the young plants is often resorted to, but this is a slow and laborious process; it will well repay, however, in the check it may give early in the season. Machines for horse and hand power have been used to a con. siderable extent in some sections after the plants have attained considerable growth, but it is better to prevent their depredations upon the crop before this period, if possible, as much injury may be done the tender plants when they first make their appearance from the ground.

Paris green, (arsenite of copper,) is the most effectual remedy yet known in exterminating these pests; but it must be used with the utmost caution, being a deadly poison. Nothing in which it has been placed should ever be used for any other purpose, and it should be kept from all animals, as when mixed with water and carelessly left where horses or other animals could have access to it, it has often been drank by them, and valuable stock lost in this manner. When used in the powder or in water, animals gaining access to the field would be very liable to be poisoned by cropping the vines or other herbage containing it.

Paris green may be applied either in a dry or liquid state; each method has its peculiar advantages as well as disadvantages. When used in the powder, it is usually applied when the dew is on the vines, or after a shower. The advantage of this method over the liquid is in its adhering better to the leaves and stalks; in the absence of heavy rains it retains its power longer than when applied in a liquid form. The advantage of the liquid application consists in the facility with which it is applied, and the less danger attending its use. Like many other substances of general commerce, Paris green is frequently adulterated and its effects proportionately diminished; hence, there are many grades of the poison, the pure article being of course more effective than any of its adulterated forms and requiring a less quantity to accomplish the results intended. When the pure article is used, a tablespoonful of the powder to three gallons of water is the usual quantity. Some also mix a little molasses in the solution to render it sticky and cause it to adhere to the plants. This poison is not readily soluble in water, and will sink to the bottom; therefore it must be freçuently stirred 
to secure a uniform distribution over the field. It may be applied with a common wateringpot, if performed by hand, or a small brush-broom with a handle sufficiently long to keep the hands protected from the water. By passing down the rows with a pail of the solution, and frequently inserting the broom and sprinkling the plants and insects, taking both rows right and left in passing, the labor will be made more easy than when only one row is sprinkled at a time, as this process reduces the labor of traversing over the whole field, by one half.

Several machines for sprinkling with horse-power have been devised and are in use in some sections. The fields should be sprinkled as often as the larvæ make their appear. ance in order to hold them in check. When the powder is used, it is generally mixed with thirty-five or forty parts of some dilutant, like lime, ashes, or flour; the latter is considered the best by many, as causing it to adhere to the leaves and stalks best, while lime is highly recommended by others as being an aid in exterminating the insect as well as a fertilizer and invigorator to the vines. Many farmers use a considerably less proportionate quantity of the poison, and consider one pound of Paris green sufficient for seventy-five pounds of the lime or other mixture; much depends upon the quantity sifted upon each plant. We prefer the liquid form as being most safe and more easily accomplished.

There is great danger of inhaling the powder, when applied dry. Various dusters have been invented and are employed when this method is practiced. Some farmers make a duster for their fields by puncturing a tin box in the bottom and attaching it to a long handle, and with this sift the powder over the plants. Applying it in this manner would be dangerous when there was a breeze, unless the operator kept carefully to windward, as there would be a liability of inhaling the poison or getting it into the eyes, either of which would produce serious results.

TVe would recommend the liquid form of application as preferable to the latter. A heavy shower will wash off much of the poison when either methods are practiced. It requires but a very small quantity to kill these insects. Mr. Taché, the French author, to whom previous reference has been made, recommends even a smaller quantity than Anerican writers; he says: -

"In the application by the liquid method, which I think should be generally adopted to the exclusion of any other, a teaspoonful (level, not heaped), is sufficient, that is, about a hundred and ten grains (quarter of an ounce) of Paris green, of superior quality, to an ordi. nary pail, holding from two to two and a half gallons of water. A pound of Paris green is sufficient, therefore, for sixty-four pails of water, or about one hundred and forty gallons. Should the Paris green be of inferior quality, or adulterated, a greater quantity, proportioned to the reduced value, would be necessary."

We are reluctant to recommend the use of any poison so dangerous in its results if accidentally or carelessly used, and we hope the time is not far distant when some equally effective but harmless substance may be found to take its place for this purpose. Many of the harmless insect-powders have been tested with unsatisfactory results. London purple is cheaper than Paris green, and may be used as a substitute, but its effects are not as speedy as the Paris green; therefore the latter is to be preferred.

A field that has been once attacked by this insect will be liable to continue to be so, as they have a habit of remaining where they once obtain a foothold. Concert of action in exterminating them is also necessary among farmers, as they will spread rapidly to contiguous fields or those in the same vicinity. This insect, like all others, has its natural enemies that aid in checking its increase, and if the farmer would befriend and encourage these, they would be of great benefit to him in largely reducing, if not wholly exterminating the pest. Among these enemies of the potato beetle, Prof. Riley mentions the various species of birds, and states that the rose-breasted grosbeck often entirely clears a potato field of these insects. The quail is also useful in this direction, as well as the crow, and many other birds. Chick- 
ens can also be taught to acquire a taste for them, although they will not always eat them at first, and in many sections have proved very valuable to the farmer in this way. Mr. Ware, of Massachusetts, says:-

"One way of protecting the potato-rines from potato-bugs is to train a flock of Light Brahma fowls to eat them. The fowls can easily be trained to work down the rows by scattering a little corn in them. Twenty fowls will usually protect an acre of potatoes."

An Iowa farmer gives his method of raising potatoes, as follows:-

"Plant them in good, rich soil, close to the house or barnyard where the chickens run, the more chickens the better; hoe them as soon as they are out of the ground, then plow them at least once a week till they are in bloom. The chickens will keep the bugs off if the latter are not too numerous. If there are more bugs than the chickens can consume, knock them off the vines just before the plow and plow them under every day till they disappear. In this way I have raised plenty, while my neighbor's crops were all destroyed."

Among the reptiles, the toad is a valuable assistant, while of quadrupeds the skunk is known to feed upon these bugs. Some species of spiders make prey of them, but still larger numbers are destroyed by insects of a similar class to its own. These are very numerous, and our limits will admit of a mention of only a few of the more important specified by Prof. Riley, among which are the fiery ground beetle (Calossoma calidum) and those of its order, the convergent ladybird (Hippodamia concergens), the fifteen-spotted ladybird (1/ysia fifteen punctata) - a larger variety than the former-the spined soldier bug (Arma spinosa), the many. banded robber (Harpector cinctus), and the ring-banded soldier bug (Perillus circumcinctus). Besides these a trichina-fly, which resembles the common house fly, destroys vast numbers by laying its eggs on the larvæ, which, when hatched, enter the body and feeding upon it, thus destroy it.

When the potato beetles are collected in large quantities, precaution should be used in burning or scalding them, as the oil of their bodies is very poisonous, and the inhaling of steam or vapor from them has been known not only to severely poison, but to cause death. Early planted fields are not as liable to be injured by the beetle as those planted later.

Scably Potatoes. - It is supposed that the scab on potatoes is produced by mites or minute animals which burrow in the skin of the young tubers, causing blisters and subsequently leaving holes or pits which give them that peculiar rough appearancewen fully matured. The use of lime or ashes in the hill is thought by some to be a preventire, but these will prove ineffectual in some soils. Land on which potatoes have not been raised for several years is less liable to this evil, than where a less period has interrened, while new soils will generally prove an entire exemption from it. The exclusive use of commercial fertilizers is also said to be an infallible remedy.

How to Tell a Good Potato. - But few persons are able to judge of the quality of potatoes without cooking them, as it is often the case that the external appearance may be deceptive and cannot be wholly relied upon; hence, a few reliable hints for testing may prove valuable to such as are not already familiar with them.

Take a sound potato, and, paying no attention to its outward appearance, divide it into two pieces with your knife and examine the exposed surfaces. If there is so much water or "juice" that seemingly a slight pressure would cause it to fall off in drops, you may be sure it will be "soggy" after it is boiled.

The following are the requisite qualities for a good potato which must appear when one is cut in two: For color, a yellowish white is desirable; if it is a deep yellow the potato will not cook well; there must be a considerable amount of moisture, but not too much; rub the two pieces together and a white froth will appear around the edges and upon the two surfaces; this signifies the presence of starch, and the more starch, and consequently froth. 
the better the potato, while the less there is, the poorer it will cook. The strength of the starchy element can be tested by releasing the hold upon one piece of the potato, and if it still clings to the other, this in itself is a very good sign. These are the tests generally made by experts, and which are usually reliable, a failure being the exception rather than the rule.

Sweet Potatoes. - The sweet potato is a native of Southern Asia, and was formerly considered an exclusively Southern product; but repeated experiments demonstrated to Northern farmers a few years since that it might be grown in almost any of the Northern States with very fair success. It is now quite commonly cultivated in many sections North, although the quality of this product is not generally considered quite equal to that of some of the Southern or Middle States, and Northern growers are oblized to take better care of the crop than those of a southern latitude in order to insure success, and to have the tubers mature before the frost makes its appearance.

With the exception of precautionary measures against frost, the general methods of cultivation for both North and South are essentially the same. This crop is an article of food as common in every Southern household, white or black, rich or poor, as the Irish potato is at the North, or rice is in China, while throughout the entire country it is regarded as a very delicious and valuable vegetable. It was stated some time since on the authority of Dr. Voelcker, who analyzed this potato sent him by Colonel Ott, of Virginia, that the starch obtained from it was proven to be more valuable than that from the Irish potato. It seems surprising that a product of so much value as the sweet potato should be so little known in Europe. In some localities the yield is surprisingly large, it having been known to reach as high as four hundred bushels per acre, varying, of course, according to the soil, climate, season, and cultivation; the average yield, with fair culture, being about a hundred and twenty-five or fifty bushels per acre.

Varieties.-There are comparatively but few varieties of the sweet potato. A yellow variety, known as the Nansemond, is generally preferred and the most extensively cultivated at the South. A large light-yellow variety, called in some sections the "yam," is more productive and early, and also keeps well, but does not equal the former in quality. For Northern culture, the Early Peabody is generally regarded as the best, being of good quality, large size, hardy, and quite productive. Those raised North, though fair in quality, do not equal those of Southern cultivation in this respect.

Cultivation.- - Like the Irish potato, the sweet variety may be propagated from either tubers or sprouts, but the latter is the more common and better method. They may be grown upon a variety of soils, but one that is dry, warm, and sandy will give a product of the best quality. A sandy loam will give very good results both in quality and quantity. The ground should be plowed to a moderate depth, about six or seven inches being sufficient, and well pulverized with the harrow. In the Northern States this may be done about the first of May, although the plants should not be set out in that latitude until the last of May or first of June, as they are quite tender and might be injured by the cold, being easily chilled, which will stunt their growth. In a latitude farther South an earlier transplanting would be admissible. The fertilizers used are horse manure, or any other from the farmyard. Lime is also valuable as a fertilizer, and likewise aids in decomposing the vegetable matter in the soil, and rendering it available for plant-food.

After the soil has been well pulverized, the field should be marked out with a plow for drills or hills, according to the method of culture. If for hills, the furrows should run each way across the field, from three and a half to four feet apart, marking it off in squares, running the plow twice in each furrow to make high hills or ridges. If for drills, the furrows will run only in one direction, which should be north and south, to secure the most warmth. 
Manure should be well pulverized and liberally applied in the hill or furrows, which should be covered in the drill with a plow by turning the furrow on each side so that it will fall upon and cover the manure. Where hills are used, the manure can be covered by hand with a hoe. The drill culture is preferable, as it does not necessitate hand labor in covering the manure. Both bills and drills should be made high and their tops smooth and compact by spatting down with a hand-hoe, in order to be in a good condition to receive the plants. The ridges should be a foot or more high, and not less than a foot wide at the base; the top may be five or six inches wide. Some planters think a close, hard bottom to the ridge induces the tubers to grow more plump, or less long and slim, and for this purpose sometimes put strips of sod in the bottom of the ridges, where only a few are grown, as in the garden, for instance.

Plants for setting out may be obtained of those who have them for sale, or they may be grown for that purpose. The usual method of securing sprouts or slips for planting is to grow them in a hot-bed or cold-frame, and, when four or five inches high, separate them from the tuber and set them out in the field. Stable manure, to the depth of two or three inches, should be placed at the bottom of the bed, covered with two or three inches of sandy loam or sand. The seed potatoes should be cut lengthwise, and placed in the soil with the cut side down. They may be placed quite near each other; without touching, and covered to the depth of two or three inches. They should be kept rather warm and moist, but great care will be required in order to secure the proper degree of both heat and moisture, as an excess of either will rot the tubers, while if there is not a sufficiency of either they will be liable to be affected with the black-rot.

Excessive heat or dryness will also prevent a healthy growth of sprouts. In cool latitudes they should be protected at night by a covering of boards or straw, where not otherwise covered by glass, but they should have an abundance of air and sunshine during the day.

When the sprouts are four or five inches high, they may be separated carefully from the tubers, one at a time, with the thumb and finger, so as not to disturb the potato, which, if uninjured, will send up other shoots in a short time. These sprouts or shoots may then be set out in the field, the preparation of which we have already given. The sprouts are placed from twenty inches to two feet apart, and should be set out on a cloudy day, or towards erening. The hot-bed should be started from the first to the last of April, depending much upon the locality, the last week of May or first week of June being a good time for setting out the slips in the latitude of Massachusetts, while an earlier period may be suited to a more southerly section; the latitude, climate, and season always being taken into consideration with respect to the time of starting the growth of the crop. In the latitude of Florida, they are set out from April to August, and mature from July to November. A bushel of good sound potatoes, when properly managed, will yield from twelve to fifteen hundred sprouts at the first drawing, and about three-fourths of that number at the second.

The tubers selected for planting should be of medium size and rather smooth, all diseased or injured ones to be carefully excluded in making the selection, as they will be liable to propagate a diseased crop. If thrifty slips are secured they will grow very rapidly, and the high hills or ridges should not be leveled in after-cultivation. It is better to pull the weeds quite near the plants by hand, as the use of the hoe may injure the tubers that lie near the surface. The cultivator or horse-hoe should be run between the rows to exterminate the weeds, which should be kept out of the field, as they will greatly injure the crop. After the vines have attained a length of two or three feet, they will take root at many of the joints, and wherever they do so, a new set of tubers will start, which will not attain any size, and will greatly injure the crop, especially at the North, as they will not have time to mature, and will exhaust the productive power of the plants to such an extent that those in the hills will not be perfected in growth. These vines should therefore be loosened from the soil and pre 
vented from rooting. This may be done by hand or with a wide fork, which should be used with care in order not to injure the vines. This should be done several times during the season. When the tubers are sufficiently matured, or when the frost has killed the vines, the crop may be harvested. They should never be left in the soil when it is sufficiently cold to freeze the ground, as it will ruin the crop. Cold, wet weather has a tendency also to deteriorate the quality. The principal essentials to the successful culture of this crop at the North are generally conceded to be liberal manuring, ridge culture, freedom from weeds, and frequent loosening of the vines to prevent them from taking root.

The following, from the pen of Mr. James G. Tinsley, of Virginia, an experienced and successful sweet-potato grower, will give the method of cultivating this crop in that section of the country, which differs in some minor respects from that already described:-

"Sprouting the sweet potatoes in the bed is the first step to be taken. In this latitude the best time to put the beds down is the 1st of April, as the slips cannot, with safety, be put out before the 10th of May; this gives ample time to get them large enough.

First, dig out a pit nine inches deep, five feet broad, and as long as you may desire it, say fifteen feet, as that is a convenient-sized bed; then, to keep moles and ground-rats out, plank up the sides by nailing the plank to stakes driven in the ground, raising the plank on the north side, say one foot above the ground, and, on the south side, six inches above ground; then slant the short sides of the bed that run from north to south, so that the cover, that is usually of plank or slabs, will make a tight fit; now throw the dirt that you have thrown out of the pit back against the plank tbat is above ground. The object in having the lower side to the south is to have a better exposure to the sun. The next step is to fill this bed so as to generate a moderate heat to sprout the potatoes. There is as much danger of too much, as too little beat.

I will now try to describe the simplest and cheapest way, and one that is used almost universally in this section. First put oak leaves in the bed, watering and trampling them well, and continue to do it until there are six inches of leaves well moistened and trampled. Now, upon these leaves, put three inches of fresh stable manure, after it has been well packed in the bed-not putting as much as three inches in the middle of the bed, as the heat is always greater in the center than on the sides; upon this manure put three inches of mold from the woods, or light dirt if you cannot procure the mold; on this put the potatoes as thick as you can without letting them touch each other; after you have completed the layer of potatoes then cover with mold or dirt two inches.

Now you have finished the business of putting down the potatoes to get sprouts. Examine your bed every day to see whether you have too much or too little heat; if you want to increase the heat, it is a very good plan to cover the dirt on the top of the bed with three or four inches of pine tags, as that helps to keep in the heat. Raise the covers every warm, clear day about nine o'clock, remove the pine tags and put them in the sun, and let the beds be exposed to the sun until about four o'clock in the evening, when the tags must be again put on them and the covers let down. Continue to use the pine tags until sprouts are about to come through the ground, then dispense with them altogether. When you find you have too much heat on your bed, the best thing you can do will be to drive a short stake in the center of the bed through to the bottom and shake around sufficiently to make a vent for the heat to escape. After the plants come up, continue to cover them as long as there is danger of frost; then remove the covers, as it is necessary to barden them before setting out. In drawing the plants, be very particular not to mash or trample the bed, and draw the slips one at a time, drawing them sideways to keep from pulling up the potato. After the plants have been drawn, water the bed well and continue to keep the ground moist as long as you wish to raise plants, as nothing makes them grow faster than for them to be well watered.

We usually try to set out all we can in the month of May-set out after that time the 
crop is not generally remunerative. The proper soil is a light sandy oue, or any land that is well impregnated with sand. Stable manure is the best fertilizer, and after that cow-pen manure. In this section mold from the woods and pine tags are used in large quantities, the same land being often put in potatoes. I never have been able to make good sweet potatoes with guano or artificial fertilizers alone; but it is necessary to supply coarse manure of some kind to mix with it. My plan is, to drill all the stable and cow-pen manure I can spare for my potatoes, and by that means it goes much farther. Three feet is the best distance for the rows to be apart, and I am accustomed to list on the manure that I have drilled in the rows, throwing up the list as high as I can with a single plow, putting two furrows together. I make it a rule never to list in a day more than I can set that day, as the plants live better in a fresh soil.

The distance apart for the plants in the row is twenty inches, and it is best to set them deep in the ground, as, if they should be cut off by cut-worms or anything else, they will be more apt to come out again. The evening is the best time for setting out, and after a good rain in Nay, you can set usually for four or five evenings. In June the sun is so hot it is very difficult to get plants to live without a good season. In the cultivation of potatoes the secret of success is never to let them get grassy, but work them as soon as a crust forms on the ground. If they get grassy, it is impossible to remove the grass without injuring the potato roots; and it is easier to work them three times when there is no grass, than once when they are grassy. You must always see that the hoes do not cut into the hill, but merely scrape the ground around the plant and then pull up a little dirt to it.

Now, by my plan of horse cultivation, I save a great deal of hoe work. First, throw out in about ten days after setting out the plants the little balk that was left in throwing up the list, and try to get the dirt as high as you can on the list, so as to smother out any grass that might start to grow on the list where the potato-plant is. Before this dirt that $I$ have thrown up by this plowing commences to put up grass, I run a cotton-scraper, (which is attached to Watt's A and B plow,) as close as I can to the potato-plants, throwing the dirt from them-trying not to let it cut more than half an inch deep. A good plowman can run the point of the scraper in less than an inch of the potatoes.

If the vines have run any, of course I have to send a man ahead to throw the vines in every alternate balk, and the scraper has to first run all through the patch on one side of the list, and then have the vines thrown back on that side that has been worked and run to the other side. The last working with the plow is to throw all this dirt the scraper has pulled away from the list back to it, moving the vines out of the way just as you did for the scraper, and plowing one side of the list all through the patch, and then come back and plow the other side in the same way, trying to make this fresh dirt meet in the middle of the list. Let your hands come on behind and see that no vines are covered up, as nothing lessens the size of the potatoes in the hill more than to have the vines covered with dirt."

Harresting. - A slight frost will kill the vines of sweet potatoes. When ready for harvesting,-which should be before the cold is sufficient to affect the tubers,-the vines should be cut off quite near the ground; this may be done with a scythe or a sharp cornknife, the former being the easier method. In some sections a sharp hoe is used for this purpose, but it is very objectionable, since the tubers lie so near the surfaco that they will be liable to be injured by being cut during the process. If a few potatoes are desired for household purposes before the crop is fully matured, they can be obtained by carefully detaching them and replacing the soil. This can be done by running the finger down beside the vine uutil a large tuber is met, when it can be taken out, leaving the smaller ones to grow. Sweet potatoes should always be dug when the soil is dry, and in a clear day. It is better to dig them in the forenoon that they may have a warm sun in which to dry. After the vines have been cut, they should be taken out of the way so as not to interfere with the digging, which 
may be performed in various ways. A plow is frequently used for throwing the potatoes out of the ground, but they are liable to be cut or bruised in this way; besides, many will be buried in the soil. A good potato-digger is the best implement we know of for this purpose, where large quantities are to he harvested, as the crop is less injured by its use than by the plow, and the labor is greatly facilitated. Where extreme care is observed, and only a small crop is to be harvested, a six-tined fork is often used for lifting them out of the soil.

The tubers should be handled very carefully, as even slight bruises or cuts will cause them to decay very quickly. When first taken from the soil, they should be left on the ground two or three hours fully exposed to the sun and air to dry. They are then sometimes sorted in the field, when desired for ready market, and put into barrels or boxes for that purpose, taking care not to injure them by bruising or otherwise.

Storing.-When intended for winter storage, sweet potatoes are usually spread in a warm, dry, sheltered, and airy place for a week or two, that the moisture from the surface of the tubers may be ahsorbed, after which it is a good plan to pack them carefully in dry sand, in boxes or barrels, rejecting all that are not perfectly sound, or that have been cut or bruised in the least. They will keep best when packed bke eggs, or so placed that each tuber will be separated from others and entirely surrounded by the sand, which must always be verydry. After being well packed they should be put away in a rather warm, dry place, as they are very easily chilled and are entirely worthless if once touched by the frost; but if put in to a place where the temperature is too warm, the dry rot will be liable to attack them. Moisture or cold are equally detrimental, and the principal things to be avoided in storage.

Some care will be required to have the temperature kept uniform and other conditions just suited to their perfect preservation. It is always best to pack them without transferring from one place to another more than necessary, as the more they are moved about the more liable they are to get bruised, and consequently the less liable to keep well.

Where sand suited to the purpose is not convenient of access, chaff, fine-cut straw, or fine shavings are frequently used for packing instead, but dry sand is the best material we know of for this purpose. In some sections where this crop is largely produced, store-houses are built especially for this crop, the walls sometimes being made double and filled with sawdust or earth, where they are stored in shallow bins or boxes without being packed in any. thing ; but with this method careful attention is always necessary in order to maintain a roper degree of temperature and sufficient ventilation, as well as to also remove any unsound ones as soon as they begin to decay.

The Yam. - This is an esculent tuber cultivated to a certain extent in this country, but is not as highly esteemed as the sweet potato. It is extensively grown in the East and West Indies, as well as other tropical countries. There are a number of different varieties, which vary in quality, size, productiveness, and hardiness. The yam can be propagated by small tubers produced by covering the vines with earth and severing them near the angles of each pair of leaves after they have taken root, or by planting small pieces of the root.

The Chinese Yam was introduced into this country from France in 1855, in the form of small tubers ahout the size of peas, which had been obtained by covering vines with earth as above described. It is similar in appearance, though smaller than the yam of the Indies, and thrives in both the Northern and Southern States. It has a climbing vine with heart-shaped leaves, and clusters of small white flowers.

The Chinese yam requires two seasons to mature when produced from the small bulbs, but a crop suitable for cooking purposes can be obtained the first season when the propagation is from the roots, which are club-shaped. When fully matured and cooked, it is dry and farinaceous, and much resembles in taste the common potato. The flesh is also very white when cooked. The land in which it thrives best is that of a deep, rich soil, which should be 
well pulverized and plowed very deep, as the roots have a tendency to penetrate perpendicu. larly into the ground, growing from two to three feet or more in length, the larger end always being deepest in the soil. This is the great objection to its culture, as considerable digging is required to obtain them.

The vines may either be made to climb poles like some kinds of beans, or may creep on the ground. They may be planted in the spring as soon as the season is sufficiently advanced to preclude the danger of frost, placing them from eight to twelve inches apart, and may be cultivated in a manner similar to that of the sweet potato or yam of the Southern States, except it should be left undisturbed in the ground from one year to another until desired for use. The principal growth of the roots takes place in the autumn; therefore, when used the first season, they should not be dng until November. They will not be injured by frosts, even in New England, but will live through the winter, and when once started, will continue to grow and spread with as much rapidity as artichokes, requiring very little care. They will not, however, be injured in quality and become strong and woody like the roots of the parsnip, and some other plants, after the first year's growth. It is well to give them a covering of straw, leaves, or spruce boughs for the winter, in those sections where the climate is vory severe, which covering should be removed early in the spring. It is a very nutritions and healthful article of food, but will never be valued as highly in this country as the sweet or common potato, although it might prove quite a good substitute for either, in case there were a necessity for a substitute, by a failure of the former crops. They may be roasted or boiled, possessing something of the rice flavor as well as that of the Irish potato. The vines of the Chinese yam make a very pretty climbing plant, and suitable for screens, arbors, or coverings for unsightly places. They will not blossom until the roots are two years old, after that the flowering is very abundant, the blossoms having a fragrance similar to that of cinna. mon. A few tubers planted near a door or window, with the vines trained over or about it, make a very desirable ornament.

\section{TURNIPS. .}

$\mathrm{T}$ $\mathrm{HE}$ value of the turnip product of this country is not to be compared with that of England, where it forms one of the most important of field crops, as food for stock, and where they are also commonly fed to sheep from the fields, which are enclosed with hurdles or movable fences. There is, however, scarcely a farmer in this country but that has his turnip-field, although usually limited when compared with the extent of land appropriated to many other crops. The nutritive properties of the turnip, when compared with some other root crops, are not great, on account of the large proportion of water in their composition, which is about ninety per cent.; but they are valuable when fed with other substances. The turnip is found growing wild in Europe and Northern Asia, but cultivation has improved it almost beyond recognition as the same species of plant.

The common flat turnip was introduced into the United States by our English ancestors, and has ever since that time been regarded as a palatable vegetable for the table. In this country, turnips are used for feeding sheep, more than other stock, carrots and mangel-wurtzels being generally considered more valuable food for cows and horses. Turnips are a crop that can be raised with comparatively little labor and in large bulk, the average yield of many sec. tions being from 800 to 1,000 bushels per acre, and hence in this respect are an economical crop for those farmers having lands and stock suited to their cultivation and use. They are, however, considered rather cold food for severe winter weather, and when given to pregnant ewes in this season, should be mixed or fed in connection with something having a tendency to produce warmth, such as corn or meal. 
To give an idea of the comparative nutritive value of some of the leading root-crops, we append the following table of analyses from the work of Drs. Voelcker and Lankester :-

TABLE OF NUTRITIVE VALUE OF ROOTS.

\begin{tabular}{|c|c|c|c|c|c|c|c|c|}
\hline & & & & Water. & Flesb Formers. & Fat Formers. & Woody Fiber. & Asb. \\
\hline Sugar Beets, & . & . & - & 81.05 & 1.00 & 15.40 & 1.03 & .80 \\
\hline Mangel-W urtzels, & . & . & . & 87.78 & 1.54 & 8.60 & 1.12 & .96 \\
\hline Ruta-bagas, & . & - & , & 89.40 & 1.44 & 5.93 & 2.54 & .62 \\
\hline Yellow Aberdeen & Turnip, & & . & 90.57 & 1.80 & 4.64 & 2.34 & .65 \\
\hline Large Globe Turni & ip, & . & • & 90.43 & 1.14 & 2.96 & 2.00 & 1.02 \\
\hline Carrot, . & . & . & . & 85.00 & 1.50 & 10.80 & 1.70 & 1.00 \\
\hline
\end{tabular}

Varieties. - There are many varieties of both the white and yellow turnip, while the ruta-baga, Swedish or Russian turnip,--also known as the "French turnip,"-form a distinct class. The following are some of the best white-flesh varieties : Early Flat Dutch, Early Snow-Ball, White-Top Strap-leaved, Red-Top Strap-leaved, Purple-Top Globe, White Globe, Long White Jersey, Large White Norfolk, Long White Tankard, and Long White or Cow Horn. The first two are of medium size, quick growth, tender and juicy when young, but if overgrown or long kept, will become spongy and inferior in quality. Both the strap-leaved varieties are early, of medium size, and nearly round in form ; they have but few leaves, which somewhat resemble the horse-radish in shape. They are essentially alike, except the Red-Top is either red or purple above ground, while the other is pure white. They are now taking the place of the old Early Dutch varieties. The Purple-Top Globe is a fine market variety and keeps well.

The White Globe attains a large size and is better adapted to stock.feeding than table purposes, being too coarse in texture for the latter. The same might be said of the Norfolk, which is a large English variety, and valuable for field culture and feeding stock. The Long White Jersey and Long White Tankard are both good early varieties. The Long White or Cow Horn, attains a large size, and is in shape similar to the carrot; it grows half out of the ground with tops small and spreading, is sweet and tender and valuable for general cultivation. It should be harvested before severe frosts, or its quality will be liable to be injured, being exposed to frost more than those covered wholly by the soil. Some of the leading yellow varieties are the Yellow Malta, Early Yellow Dutch, Early Yellow Finland, Aberdeen Purple-Top, Robertson's Golden Ball, and Large Yellow Globe. The first three are early, with small or medium-sized globular roots, and are excellent garden varieties for summer and autumn use. The Yellow Aberdeen is globular, hardy, and solid, and valuable for general cultivation. Robertson's Golden Ball is globular, of rich flavor, and has unrivalled keeping qualities; it is valuable for both culinary and stock purposes. The Large Yellow Globe has a large globular root of pale yellow, and is one of the best for general use ; it will keep hard and brittle until late in the spring. The Ruta-baga is close-grained, very hard, and will endure considerable cold without injury, but is not in perfection for table use until nearly spring. It is much richer and sweeter than the common turnip, and will keep much longer in good condition.

Among the choice varieties of the ruta-baga are the Skirving's Purple-Top, Laing's Improved, the Bloomsdale, Carter's Imperial Swede, Sutton's Champion Swede, Improved American, Sweet German, and Long White French. The first, by its quick vegetation, generally escapes the ravages of the enemy of the turnip, the fly, and is of strong growth and well adapted to field culture. Laing's Improved is a very handsome variety, of globe shape and fine quality. Carter's Imperial Swede is a new English variety, highly recommended for both its quality and for producing extraordinarily large crops. The Bloomsdale is an improved purple-top variety and is regarded with much favor. Sutton's Champion Swede is also an English variety, and is said to be the hardiest, heaviest cropping, and one of the most nutri. 
tious kinds known. The Improved American is largely grown in this country both for table and stock-feeding purposes, and is of fine quality and keeps well until summer. The Sweet German and Long White French are both sweet-lavored and fine-grained and valuable for table purposes ; they are quite similar in all respects, the latter being a variety of the former. In good soil and under favorable circumstances, the Sweet German will grow very smooth and regular in form, but under unfavorable conditions it often grows very uneven; they both require earlier sowing than other varieties of turnips.

Cultiration.- Turnips can be grown on almost any kind of soil, from sand down to heavy clay and muck, but the common English turnip does best on a light sandy, or gravelly loam, well-manured with superphosphates, bone-dust, or guano, and ashes applied at the time of sowing the seed. Ruta-bagas thrive best on a heavy soil made very rich. Land that has been newly cleared and burnt over, and old pasture ground plowed two or three times during the summer, and enriched with superphosphates, bone-dust, or guano and ashes, as above recommended, will produce the smoothest and sweetest turnips. When farm manure is applied, it must be thoroughly decomposed, and made very fine, besides being well-mixed with the soil. Fresh stable manure should never be used for turnips, as it injures the quality of the crop for table use, giving them a strong, unpleasant flavor; besides, they are more liable to be eaten by worms when farm manure is used than when commercial fertilizers are applied; neither will the bulbs grow as regular in form, or as smooth.

Land for turnips should be plowed to a moderate depth in the spring, about the time of plowing for corn, and kept free from weeds by an occasional harrowing. Just before sowing the seed, it should be plowed again and thoroughly harrowed, that the soil may be wellpulverized and moist, to induce an early germination of the seed, and rapid growth.

The fertilizers used should be applied broadcast, and well-mixed with the harrow. A rapid growtl is of importance in order to get the plants beyond the danger of injury by insects as soon as possible. The sowing may be broadcast or in drills, though the largest crops are obtained by the latter method, which admits of after.culture, and which is a great benefit to the crop, while the former does not.

From one and a half to two pounds of seed will be required per acre for broadcast sowing, and from one to one and a half sowed in drills. This is a larger quantity than would be required providing all the seeds should germinate, and the plants thrive; but as some will not, and many plants will be destroyed by the turnip-fly, or other insects, it is safe to sow liberally. One-half inch is a sufficient covering for the seed.

It is best to put in the seed just before a rain, as this will afford a better escape from the fly, and induce a quick germination and growth.

For the fall and principal crop of the English or common turnip, the sowing at the North may be from the middle of July to the last of August; at the South, it may be delayed until later, according to the latitude. If in drills, the rows should be from sixteen to eighteen inches apart. When the plants are well started, or when the rough leaf is fairly developed, they should be thinned out to about six or eight inches apart. This may be done with a small hoe the first time, cutting out rows so as to leave small tufts of four or five plants at regular intervals. After the plants rally from this operation, the surplus ones may be removed, leaving the strongest and most vigorous plant of each tuft for growth. This may be done by hand, but it involves considerable labor in a stooping posture, which, to say the least, is very uncomfortable. This labor can, with little practice, be very easily performed with a hand-hoe; it is accomplished by an alternate thrusting and drawing motion of the hoe, a little practice of which will enable the operator to perform so successfully that double plants or vacant spaces will be very rare in the rows. The single plants should be from eight to ten linches apart after the last thinning process.

The weeds should be kept down with a cultivator or horse-hoe, and the soil stirred in 
this manner several times during the season, which cultivation should be completed before the leaves attain a very large growth. A hand-hoe will be required for use between the plants. Shortly after the plants are out of the ground, they should receive a sprinkling of lime-dust early in the morning, when wet with dew, to prevent the attacks of the turnip-fly. This application, repeated a few times with three or four days intervening, will prove an efficient safeguard. After putting out the rough leaf, they are safe from the attack of the fly. Many farmers give the soil a top-dressing of plaster and wood-ashes immediately after sowing the seed.

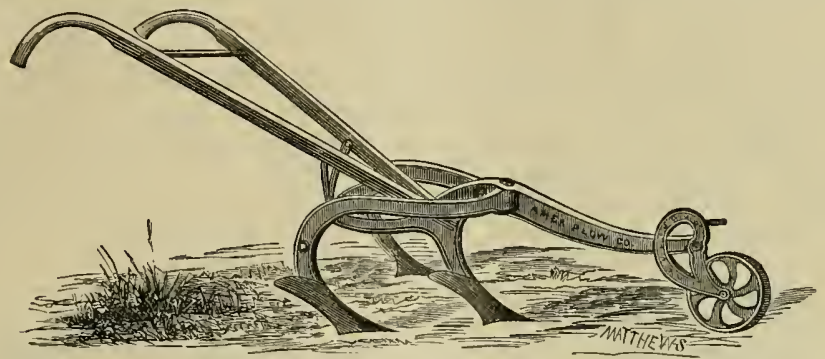

THE BOSTON HORSE-HOE.

The above cut represents the Boston horse-hoe, which is extensively used in the cultivation of corn and all kinds of roots. The rear plows or moulds are reversible, so as to throw the earth to or from the plants, as desired. It can also be contracted to fifteen inches in width, or expanded to thirty-six inches, and is gauged to work any depth from three to seven inches.

Cultivation of Spriug Turuips.-For an early crop, the seed should be sown as soon in the spring as the ground will admit of preparation, and should be well-pulverized and enriched, as previously recommended.

The Early White Dutch, or Strap-leaved varieties, are very good for this purpose. They should be sown in drills, and kept perfectly free from weeds, the culture given to be the same as that for the fall crop.

Mr. B. K. Bliss advises that, when the roots begin to enlarge, the earth should be brushed away about them to the depth of a half-inch or more, and a light dressing of wood-ashes given them. He says this is the surest mode of obtaining fair and smooth spring turnips in old gardens, where they are almost certain to grow wormy, if the earth is allowed to remain in contact with the roots. It is important to get them started very early, so that they may have time to grow of sufficient size before the very hot weather, when they will soon become tough and strong.

They may be started much earlier by sowing in a hot-bed, or cold-frame, and transplanting in a wet day, when they have attain ad to five or six leaves. They should have the earth pressed close about the roots in setting out, and be watered well for a few days after. A fine crop of spring turnips may be raised by the ordinary method on fresl land, or land that has not been subjected to cultivation for several years.

Cultivation of Ruta-Bagas, or Swedish Turnips.-Heavy soils are best adapted to this crop, and the land should be rather dry and rich. It frequently follows corn or wheat in rotation, but roots of the best quality are obtained from fresh soils, being less liable to worms or insect enemies. Very good crops are obtained from freshly-turned sod. The quality of fertilizing material to be applied will depend upon the nature cf the soil, but they 
will require a richer soil than the common turnip, although the same kind of fertilizers. The seed should be sown from the 20th of June to the 1st July. Drill.culture is the usual mode, although some set them in hills. The drills should be at a distance of two feet, and the plants either thinned out or transplanted ten inches apart. It is customary in many sections to sow the seed in a bed and transplant afterward. The after-culture is similar to that of the common turnip previously given.

The following method of cultivating the Swede turnip is given by a gentlemen of Ontario: "This crop is one which only a few farmers cau raise to perfection; but for these few, it is not a difficult matter. When once its requirements and manner of cultivating the soil are understood, it is an easy matter to raise a crop of turnips. Neither does the land chosen for the crop need to be free from weeds, or in a high state of fertility. In this township- rick. ering, Ont., noted for its large number of stock-breeders, and the excellent quality of its stock-every farmer has his turnip-field ( $\mathrm{I}$ know of only one exception) sowed generally at the rate of 5 acres on a 100-acre farm. The field chosen is next to the fallow, the weediest on the farm. It is first plowed, well-harrowed and rolled; then between fifteen and twenty loads of manure to the acre are drawn on. By loads, I mean as much as two good farm. horses can, without straining, draw on a wagon. Then it is plowed twice more, at intervals of a week between each plowing, giving at least three harrowings, and a rolling between each plowing. This may be thought to be a great deal of work, but as the destruction of weeds is one of the objects, this object is, as regards thistles, thoroughly reached by the repeated plowings and harrowings, especially if it be a dry season. This large quantity of work, also, both pulverizes and mixes well the manures with the soil, and renders available all the fer. tility, a great part of which would otherwise lie dormant.

The field is then ridged up, either with a double mould.board plow made for the purpose, or with a common plow, and the seed is sowed as fast as ridged, so that it is sure to be placed in the yet moist earth. The sowing is done by a drill, sowing two rows at once. Generally two pounds to the acre are put in, as the young plants do better when a little crowded. The seed must not be sowed until after the 15th of June, as the fly will eat the young plants as fast as they appear if sown earlier than that date. After the plants are large enough to hoe, a hoe about ten inches long and two wide, made for the purpose, is used, and the soil, together with the supernumerary plants, is pushed away from the plant left. The plants, after thinning, are from ten to twelve inches apart, and lying on their sides. The rows must first be scuffed as closely as possible. After this, one or two scufflings and another hoeing are all that are required.

The last week in October and first week in November is the harvesting season. Two rows are pulled at once. The knife being held in the right hand, the turnip is pulled with the left, cleaned and topped, then thrown into the furrow at the right, while the tops are thrown to the left; thus four rows are thrown into one, leaving, after pulling, just room for a wagon or cart to be driven between the rows. September and October is the growing season. Frosts that do not freeze the ground solid have no effect on the Swede turnip. By this manner of cultivation crops averaging from three to nine hundred bushels per acre are grown, often yielding as high as a thousand bushels, while the land is left in the best condi. tion, rich and free from weeds."

Harresting.-Turnips should be gathered before severe frosts set in, although on those sections of the country not affected to any extent by frost they are often, when on dry soils, left on the field during the winter; the quality is, however, improved by gathering, as they are liable to grow rank in flavor and wormy by remaining in the ground so long. The tops are most easily cut with a sharp hoe before pulling the turnips, a common hoe ground sharp being used for the purpose. It is better to cut off the tops about an inch from the bulb if possible, as they will keep better than when cut close. The roots may afterwards be pulled 
with a chain harrow, a hoe, or by hand. The following method of harvesting turnips is given by a leading farmer in the State of New York: -

"The one thing that makes labor in the root-field so unpalatable to Americans is the constant stooping that it nearly always involves. I obviate this almost entirely in harvesting by using the hoe, ground sharp. With this in your hands, begin at the outside row, and as you follow it down, cut the top clean from each turnip with the blade, striking right or left, as is most convenient. The impetus given to the top will carry it about half way to the adjoining row. Returning in this, you strike in the same direction, and so proceed. After a few minutes practice, two or three tops may be cut with one blow, and almost any one can "top" as fast as he ordinarily walks. After the field has been thus "topped," it will present this appearance: Two rows of turnips will alternate with each row of tops. In pulling the roots, strike the blade of the hoe back of the turnip, and with a quick jerk, pull it toward the adjoining row, pulled or unpulled. The blade of the hoe cuts many of the lateral roots, thus rendering the task of pulling comparatively easy. After topping and pulling, a row of turnips will alternate with a row of tops; and in hauling, the wagon should be driven between these rows of turnips. I have myself topped and purled by this method four hundred and five hundred bushels in ten hours." The tops are worth gathering as food for stock.

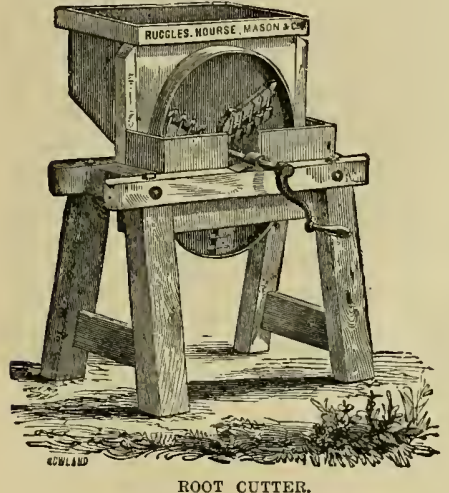

Storing. - Turnips require a cool, dark place for storage. When raised only in small quantities they may be stored in a cellar, either in bins or upon the ground. They will keep better to have but a few bushels in a place, and cover slightly with earth or sand. Heat is detrimental, but they will bear considerable cold without in jury.

They may be preserved in very good condition in trenches; in fact, all root crops will keep better in trenches than in a common cellar, and come out in the spring almost as fresh as when put in, providing the trenches are properly managed. They should not, however, be piled deep in such pits. Trenches from two to two and a half feet wide and two and a half feet deep, and as long as required for the quantity to be stored, are the best for this purpose. The turnips should be put in to the depth of only a foot and then covered with the earth that was thrown out in digging. It is better to have the soil for the pits quite dry, and the land a little sloping, to admit of drainage. Should the weather be very severe, a covering of stable manure thrown upon the tops of the trenches will prevent freezing. A root-cellar, made similar to a silo, and which has been previously recommended for the storage of potatoes, 
is one of the best means of storage for turnips; they are, however, somewhat expensive in construction, but when once made, are serviceable for many years. It is customary in some sections to lay them upon the dry ground, in a locality slightly sloping to the south, in long piles like hay-windrows, the piles about three feet through; they are then covered with straw about six or eight inches, and earth eight or ten inches, leaving a straw ventilator every ten or twelve feet. Another method is to put them in large round piles of from thirty to forty bushels each, and cover in the same manner. These methods involve considerable labor and some risk in freezing in extreme cold weather, which are obviated in a properly-constructed root-cellar.

Turnips and ruta-bagas are best fed to cattle and sheep in an uncooked state; they should always be first properly cut before being fed to stock of any kind. They should also never be fed to cattle on the ground in a yard or field, but always in the manger, where they can eat them more quietly without danger of getting choked or hooked by others, and where they can also get their proper share, as a few in every herd-are the "master spirits," and will drive and fight all others when they have the opportunity.

Raising Turnip Seed. - For growing seed, the best turnips should be selected from the crop in the fall, and placed apart from the others where they will be well preserved, leaving an inch or so of the tops on the bulbs. As soon as the ground will admit in the spring, these should be set out in land that has been plowed and pulverized, setting in rows that are three or four feet apart, and from eighteen to twenty inches apart in the rows. The setting should be carefully done, making provision for the end root, and covering the bulb entirely with the soil, leaving only the sprouts at the top out of the ground. The plants should be kept free from weeds, which will involve the use of the hand-hoe; or if a large quantity of seed is to be grown, the cultivator or horse-hoe may be serviceable for this purpose. When the plants are about blossoming, it is a good plan to bring the earth up around the stalks in order to give them support and keep them in an upright position. When a majority of the pods have become dry, the stalks should be cut and put in some safe place to dry. It is well to cut them when the dew is on them or just after a shower, as the pods will open very easily, and the seed shell out; careful handling will be necessary, even with this precaution, to prevent the loss of seed. Different kinds should never be set near each other, as they will be liable to become mixed, and the distinct varieties thus lost. When the pods have become dry, the seed may be easily shelled out and cleaned, the same as clover or grass seed.

\section{BEETS.}

$\mathbf{T}$ HE beet may be used as an esculent in all stages of its growth, and is universally culti. vated in some of its varieties, of which there are many. There are also a few varieties grown specially for stock-feeding purposes.

Beside these uses, large quantities are annually utilized in Europe, and to a limited extent in this country, in the manufacture of sugar, but whether beet sugar will ever become a profitable product in America has not yet been sufficiently demonstrated to place the question beyond a doubt, in the minds of many of our leading agriculturists. The different varieties vary in size, form, and color, but more especially in the latter respect, although the color does not seem to affect their quality. They are deep red, purple, pink, yellow, white, or mottled with varieties of shades. The culture of all varieties is similar. It is estimated that an acre of this crop can be grown as cheaply as the same area appropriated to potato culture, but that the yield will be four or five times that of the latter, in bushels.

The abundance of the crop will, however, depend much upon the soil and method of 
culture. Beets are one of the cheapest, most productive, and nutritious roots that can be raised for cattle, sheep, and swine, and are especially valuable as food for milch-cows, and breeding-ewes.

Varieties.-There are so many varieties of the beet, some of which are of a local character, that it is almost useless to attempt to enumerate them. The following, however, are some of the most popular among the leading varieties generally cultivated. The Early Bassano, originally from Italy, is one of the earliest varieties known. The root is flat and light red in color, the flesh being white, encircled with rose color. It is tender and juicy. When designed for winter use, it should be suwn late, as early-grown varieties will not keep through the winter. Other standard varieties are Bastian's Extra Early Red, Bastian's Half-long Blood Beet, the Egyptian Blood Turnip, Early Blood Turnip, Early Yellow Turnip, or Orange, Long Blood Red, Lamb's Improved Imperial Sugar, and the French Sugar-Beet. The last two mentioned are especially valuable as containing a larger percentage of sugar than the ordinary varieties, and may be used for table purposes, feeding stock, or sugar manufacture. The French variety is cultivated extensively in France for the latter purpose. The Swiss Chard, sometimes called "Sea-Kale Beet," is cultivated for its leaves, which are used as spinach.

Cultivation.-A fair crop of beets may be grown from a variety of soils; very fine yields being generally obtained from one of a rich, strong, and partially clayey nature, but that upon which it thrives best, and which is most suited to its successful culture, is a rich but rather light loam, which has been well manured the previous season.

Where this cannot be had conveniently, manure that has been thoroughly composted may be used, as fresh yard-manure injures the quality of the crop and causes the roots to grow tough and forked, while smooth, straight and medium-sized roots are the most desirable. Unless well-fermented farm manure can be had for the purpose, guano may be successfully used instead; bone-dust or superphosphate will also answer the same purpose. Some farmers apply a light dressing of guano after the plants are up, in addition to previous application of fertilizers to the land.

The soil should always be well pulverized for this crop, and is best rendered so by a fall plowing, to be followed by another plowing and harrowing in the spring; the manure to be well harrowed in. For an early crop, the seed should be sown as soon as the ground can be worked in the spring; but for winter use, the sowing should be delayed until June or the early part of July. The sowing should be in drills from fifteen to eighteen inches apart, and covered about an inch deep with friable soil. A dry day is best for this purpose, and if the seed has been soaked in tepid water for twenty-four hours previous, it will germinate much sooner.

Sowing in a hot-bed or cold-frame, and transplanting, is sometimes resorted to by those desiring an extra early small crop. An ounce of seed will sow a drill of one hundred feet in length, and from four to five pounds is sufficient for an acre. When the plants are from two to three inches in height, they should be thinned out from seven to nine inches apart with a hoe, the same as turnips, taking care not to injure or cut the leaves of those left for growth, leaving the plants in little tufts of two or three. When these have rallied from the thinning process, another thinning should be given, leaving only one plant in a place. All vacant spaces should also be filled by transplanting. 'The wheel-hoe, or a similar implement, should be frequently used to keep down the weeds, and the surface be well stirred about the roots of the young plants, which, when last thinned, should be from two to twelve inches apart. Cultivation should be continued until the leaves nearly cover the ground. When the extra long varieties are grown, the manure may be covered to a depth of four or five inches at the second plowing, and the drills made a little farther apart than for the smaller kinds. In all after-culture, care should be exercised to prevent breaking the leaves of the plants. 

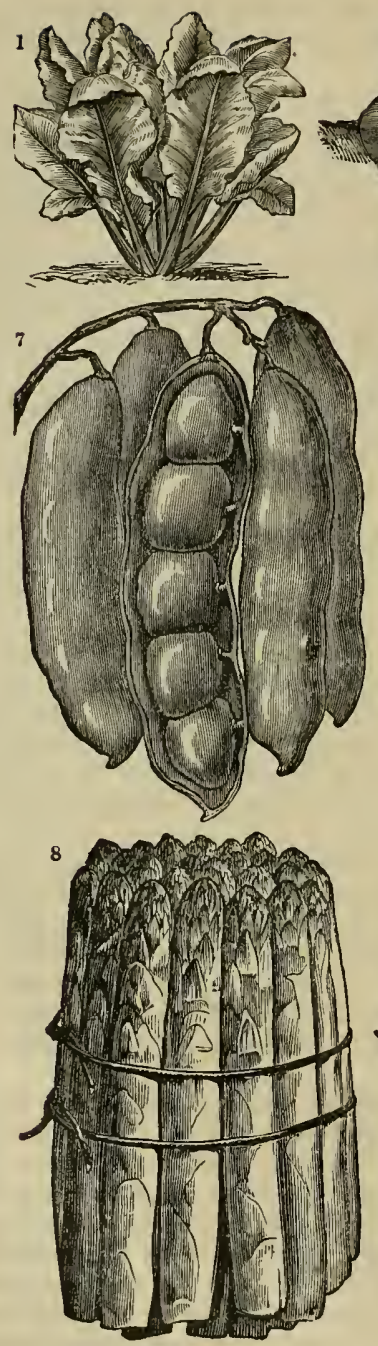

1. Swies Chard Bert.

1. SWIES CHARD BEET. 3. BABTIAN'B HALF-LONO BLOOD BEET. 3. Bagtian's Haif-Lono Blood BE
4. Eotptua Blood TuRnip BeEt.

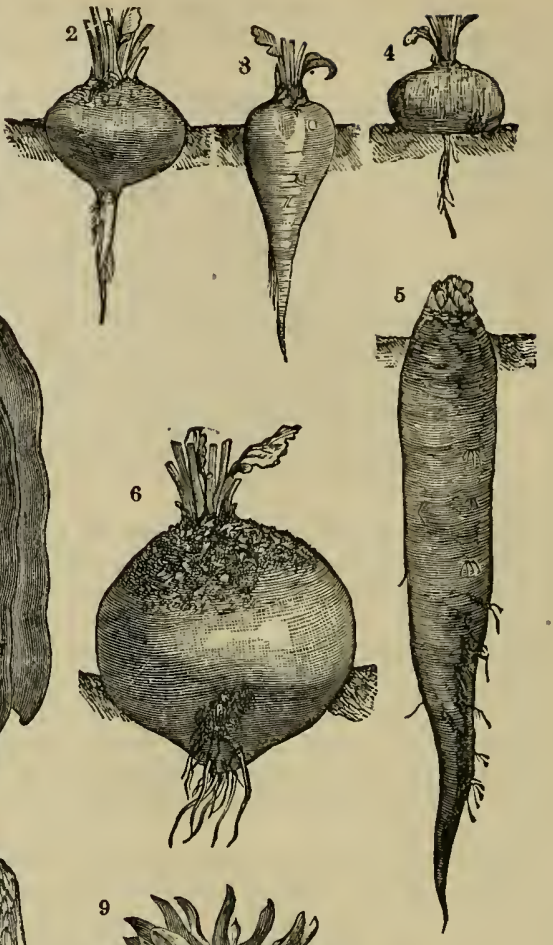

$$
\text { Copied by permission from Catalogue of H. A. Dreer, Philadelphia, Pa. }
$$

5. Improved Lovo Broad Re Bem.

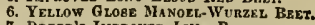

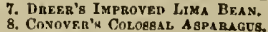


The winter crop should be harvested before severe frosts, the last of October being the usual time in the Northern States. Beets are subject to but few diseases and but slight injuries from insects.

Mangel Wurzels.-This is a large, conrse-textured variety of beet, which is extensively cultivated for feeding stock. The roots grow to a very large size, but are similar in their constituent properties to the common beet,-although of coarser texture. There are several varieties of the Mangel Wurzel, among the most desirable of which are the Long Red Mangel, which is sometimes, when young, used for table purposes; the Mammoth Long Red, which, as its name indicates, grows to a very large size, a single root weighing fifty pounds having been exhibited not long since at the Smithfield Club Cattle-Show; it is also of very good quality. The Yellow Globe, Red Globe, and the Ovoid, both Yellow and Red, are also good varieties.

In the feeding of Mangel Wurzels, only a small quantity should be given at first, as they will have a tendency to irritate the bowels if fed too liberally, until the stock is accustomed to the food, or if fed in the early part of the winter, before the ripening process is completed, and the starch they contain is converted into sugar. Where turnips are used for stock food, it is better to feed those first, and reserve the mangolds until the middle or latter part of the winter, when they are more nutritious, and will not be as liable to have an irritating or diuretic effect upon the system of animals. From twenty-five to thirty tons per acre is the average yield, although under favoring circumstances as many as eighty tons to the acre have been produced, the yield depending much upon the variety, soil, etc. They will keep well until the middle of the summer, if properly stored.

Culture.-The soil and its preparation for Mangel Wurżel should be similar to that for the ordinary beet, except that land intended for the former crop should be plowed and finely pulverized long enough before sowing the seed to admit of its settling down a little firmly before the seed is deposited, which should be from the middle of April to the middle of May, the quantity required being four or five pounds per acre. The seed should be sown about two inches apart, in drills that are from two to two and a balf feet apart, in order to admit of the use of horse-power in the cultivation of the crop. If the soil be light and the weather dry, the roller should be used to press the earth down upon the seed and hasten its germina. tion. When the plants are up about two and a half inches, they should be thinned out to twelve or fifteen inches apart, and the after-culture be similar to that of the common beet. In cultivating, as much care as possible should be used to prevent breaking the leaves. Two or three hundred weight of salt per acre mixed with the manure, is thought by many growers to be of great advantage to the crop.

Harvesting and Storage.-Beets should be harvested before there is danger of injury from frosts. The tops should be cut to within one inch of the bulb, and the small roots remain on such as are intended for late keeping. In pulling and cleaning the roots, care should be used not to wound or cut off any of the large fibers, as it injures the quality and induces a tendency to decay; neither should they ever be allowed to wilt, for having once become wilted or shriveled, they will never recover their firm and brittle texture. In stacking them, they will keep better if the crowns are placed outward. They may be stored in a cool cellar and slightly covered with dry earth, or in piles of from twenty to thirty bushels each, and covered with sufficient straw and earth to keep out the frost, as recom. mended for ruta bagas. 


\section{CARROTS.}

THE carrot is one of the most valuable roots for feeding stock, besides being quite hardy and easy of cultivation. It is of especial value for feeding horses and cattle. Milch-cows will give an increased flow of milk, with rich, yellow cream, when fed upon it. While horses can be kept in good condition with a considerably less quantity of oats than the usual allowance when about twenty pounds of carrots are given them daily; they also promote a healthy condition of animals and give a sleek appearance to the coat. Larger crops can be obtained on light soil than of turnips, and with less exhaustion; in fact, if the land is well manured, carrots can be grown upon the same soil for several successive years without deterioration to the crop or soil. They can be kept to an advanced period in the spring when properly stored. Care should be exercised in saving the seed from only the best selected routs, as there is a tendency in this crop to degenerate, if the seed is not of the best quality.

The plant is a native of the East, and in its wild state is a small root, sticky and strong. flavored, which cultivation has changed almost beyond recognition.

The leaves of the carrot were considered so beautiful during the reign of Charles I, that they were worn for ornaments, in place of feathers, by the ladies of England. They are eaten by stock, either green or dry.

Varieties.-There are several varieties of the carrot, which differ with respect to size, form, color, qualities, and period of ripening. The following are considered the best for field culture and stock feeding: the Improved Long Orange, Long Red Altringham, Half-long Scarlet, Danvers (an intermediate variety), Orange Belgian, and the Large White Belgian. The last two are a large variety, grown in this country exclusively for stock; they are less fine in texture than the other varieties, although the yield is larger. The roots grow high out of the ground, which renders the harvesting of the crop quite easy. The Large White Belgian is much cultivated in France for soups and seasonings as well as for stock.

Among the varieties for garden culture are many of the above mentioned, together with the Earliest French Farcing, a small root prized for its earliness and superior flavor; the Early Scarlet Horn, an early variety of deep orange color and abrupt termination of root; Early Short Horn, and Early Half-long Carentan. The carrot is said to be next to the potato in nutritive value.

Cultivation.-The soil best suited to the cultivation of carrots is a light, deep, sandy loam, or one of a peaty character, although very fair crops can be obtained from almost any that is neither too wet nor too dry, and that is made very rich. Farm-yard manure should not be applied until after it has been well fermented, as it will, if fresh, induce the growth of forked and ill-shaped roots of inferior quality; therefore it is best to select land that has been heavily manured the previous season, or if that is not practicable, most of the manure should be applied in the fall. The plowing should be very deep, and the soil finely pulverized and free from stones, but most of the manure should be near the surface, within reach of the young plants in the early stages of their growth. The manure, as well as the soil, should be made very fine. Bone-dust, guano, or superphosphate may be used as a substitute, or to sup. plement farm manure for this purpose. Artificial manures are often applied after the plants are out of the ground. It is well to plow the land for this crop early in the spring, and again just before sowing the seed, in order to secure a thorough pulverization of the soil and the destruction of the weeds. The best crops are generally secured where the soil is deeply trenched and ridged, as long before sowing as possible.

The early varieties may be sown as soon as the ground is in a working condition in the spring, but the main crop should be sown in the early part of May. The seed should be 
sown in drills from fifteen to twenty inches apart, the larger varieties requiring more space than the smaller. If the seed of the early varieties is put in early in the spring, the crop will be liable to ripen too soon and not keep as well during the winter as those sown later in the season; but if the sowing be delayed too late, the young plants, being rather weak when they first come up, will be liable to be dried and withered in the hot sun. The seed should be covered evenly to the depth of about an inch. If soaked a day or two in tepid water before sowing, and mixed immediately after in dry plaster or ashes, the plants will come up much sooner, and the first weeding will thus be much easier. As soon as the plants are sufficiently strong, they should be thinned out so as to leave about six inches between each plant, if of the smaller kinds, or eight inches of the larger varieties. The ground should be kept clear of weeds, but deep stirring of the soil should be avoided, as it would have a ten. dency to injure the roots, and cause them to grow forked and irregular. There are various weeders, and both horse and hand hoes admirably adapted to this purpose.

Cultivating Seed.-It is very im. portant to secure seed of the previous year's growth, for if older, their germination cannot be depended upon with any degree of certainty. An ounce of seed

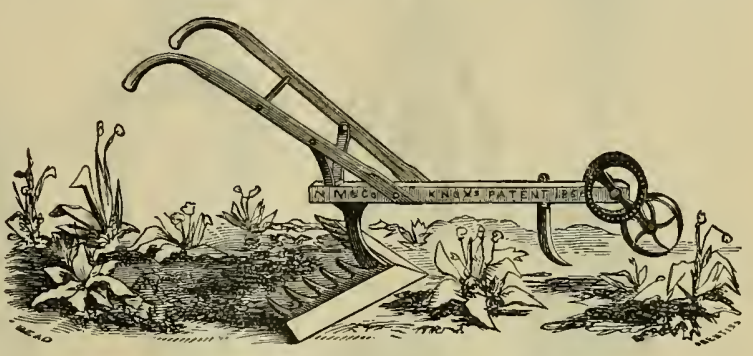

KNOX'S CARROT AND COTTON TEEDER.

will sow a hundred and fifty feet of drill, and from two to three pounds will be sufficient for an acre. In securing seed for carrots, it is important that the best roots be selected for the purpose-those of good size, form, and color. These should be set out as early as the ground will admit, without danger from frost, in land deeply plowed and properly fertilized. The roots should be well covered, and if they are very long, holes should be made for them in the soil with an iron bar. The rows should be from three to four feet apart, and the roots from one and a half to two feet apart in the row. They should have an occasional hoeing and be kept free from weeds. When the branches become dry and yellow and the seed turns brown, the heads may be cut and put in a dry place. The heads will ripen unevenly, and will require cutting at different times.

Harvesting and Storing.-The crop should be gathered before bcing injured by the frost, and in dry weather when the roots will come out of the ground dry and clean. They may be harvested with facility by running a plow on one side of the rows, after which they can be easily removed by hand; but care should be taken not to injure or bruise them in any way, as it will cause them to decay. The tops should be cut close to the crown to prevent sprouting in winter. They should lie a day in the sun, and become well dried and a little wilted before storing. They may be deposited in small heaps in a cool cellar and covered with dry sand, or stored in trenches, the same as other roots. Carrots are apt to sweat and heat when packed away in large quantities. They will keep best in a dry place and one that is sufficiently cool to avoid freezing, dampness and heat being detrimental to their good preservation. 

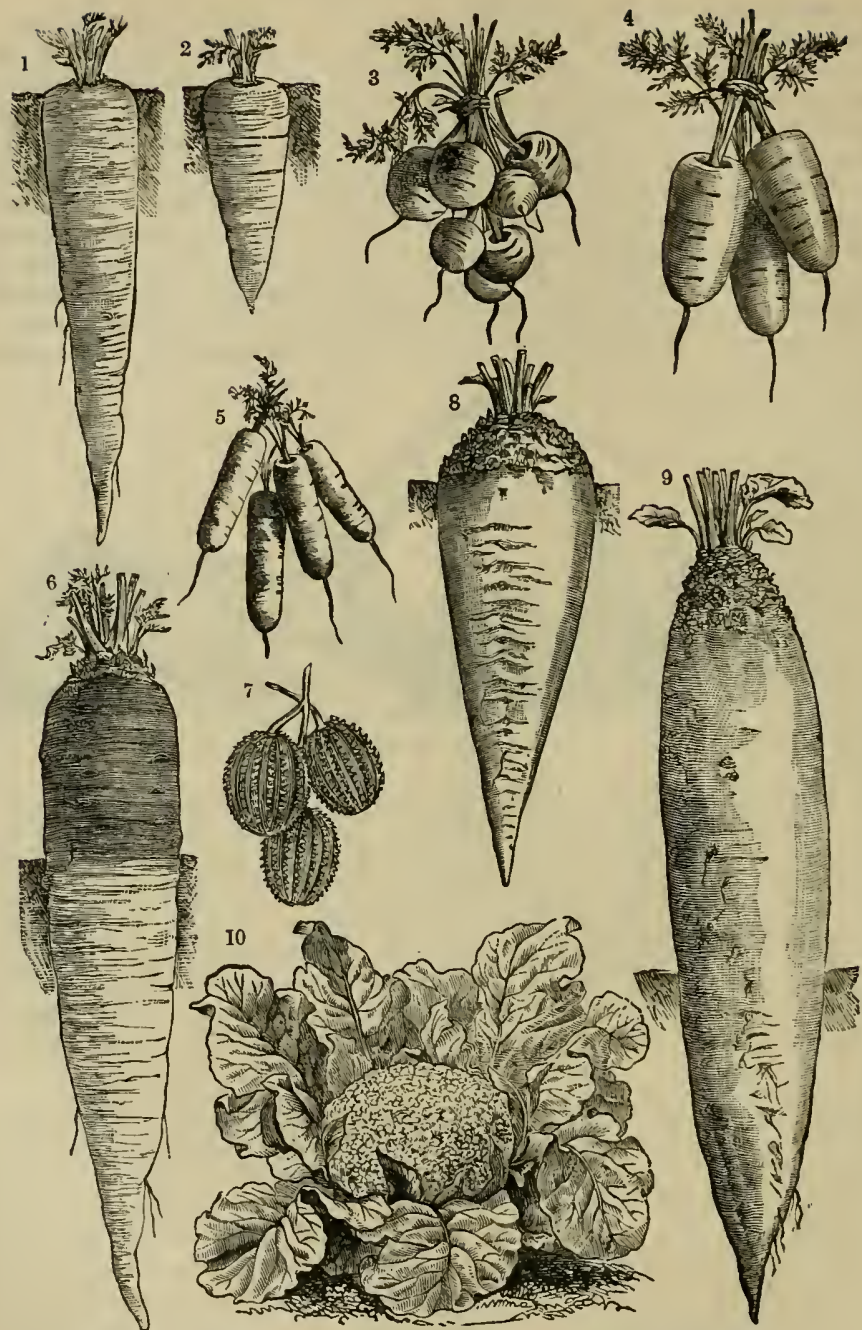

1. Improved lono oranos Carrot.

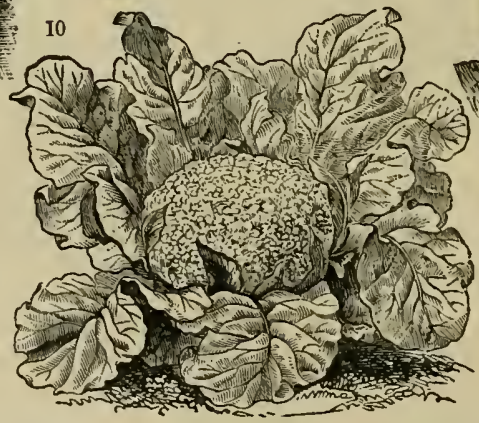

2. EARLY HALF-LONO SCARLET CARROT.

1. Early scarlet Hork Carhot.

6. Lakoe Watti Bzlousn Carrot.

7. SMALL GaERKIN, OB BURE CtCengra,

8. IMPROYED IMPEBIAL SUOAB BEET.

9. LOYO REO MLNOELWTRzRz BEBT.

10. Levozmand'a Shont-Stem Caultriokem

6. Farit Hatrolovo Carentan Carkot.

Copied by perwission from Catalogue of H. A. Dreer, Philadelphia, Pa, 


\section{PARSNIPS.}

$\mathrm{T}$

HE parsnip is a delicious table vegetable, especially in the early spring; besides, it is excellent for feeding stock, being nearly equal to the carrot in nutritive value. It is relished by cattle, sheep, and swine, but is thought to be not as good for horses as carrots. The leaves furnish fodder for cattle whether green or dry. In its wild state the roots are fibrous, small, and strong flavored, but cultivation has changed it into a root of fine texture, good size, and most delicate flavor.

Varieties.-There are but few varieties of the parsnip; the principal are the Early Round French, an early variety of delicate flavor, the Long Smooth, or Hollow Crown, with long, smooth roots, tender and sweet, the tops of which are small with a red tinge at the crown, which rises from the center surrounded by a slight depression. It is very hardy and keeps through the winter well without any protection. The Student is a new variety of fine qtality, improved from the wild parsnip by Prof. Buckman of the Royal Agricultural College, Cireucester, England, which has met with much favor.

Cultivatiou.-The soil for parsnips should be heavier than that for carrots, this crop thriving best in a deep, rich soil, and, unlike the latter, favors that in which clay predominates. The soil for all root crops should be deeply tilled, and finely pulverized. The culture given should be similar to that for carrots. All fresh manure for fertilizing should be avoided, being injurious to the crop, and, as is recommended for carrots, the best lands are those that have been heavily manured the previous season. When farm manure well fer. mented is used, it should be made very fine. The seed should be sown in drills from fifteen to eighteen inches apart, scattered sparingly, and covered from half an inch to an inch with finely pulverized soil. Soaking the seed a day or two in tepid water before planting will cause them to germinate more quickly. The sowing should be early in the season, as they require considerable time to mature, and will bear the frost well. When the plants are about two or three inches high, they should be thinned out, leaving them from six to eight inches apart. The soil should be kept free from weeds, and frequently stirred. Keeping them in the ground during the winter improves them. If the roots are small in the autumn, and a light snow falls before the ground freezes, they will frequently continue to grow, and sometimes nearly double their size before spring. They should be taken out of the ground before commencing a spring growth, for if not, the quality of the crop will be greatly injured. A few may be harvested and stored like other roots in the fall, for use during cold weather. An ounce of seed will sow about two hundred feet in drills, and from four to five pounds will be required to the acre. The seed is cultivated the same as for carrots.

\section{ARTICHOKES.}

$\mathrm{T}$ HIS is a crop that produces an immense yield with comparatively little culture, and, when once established in the soil, will continue to grow abundantly for successive years, with little care. The quality of the tubers is inferior to that of the potato, which they resemble, but as this crop yields so abundantly, growing freely on poor soils and with but little attention, it is a profitable one for culture. The tubers are used for feeding stock. especially swine, which will fatten upon them when freely fed, or allowed to do their own harvesting by being turned into a field, and thus considerable labor in gathering the crop saved. In such cases, enough will be left in the ground to reproduce for successive seasons. They are much in favor with the pork-producers of the West. Milch cows, sheep, and in 
fact all kinds of stock, seem to thrive when fed upon them, which proves that they contain considerable nutritice matter, as well as the following analysis of it by $\mathrm{Wolff}$ and Knop, comparing its nutritive value with the Irish potato, from which it will be seen that the artichoke has as much flesh-forming material as the potato, and nearly as much heat and fat-producing material.

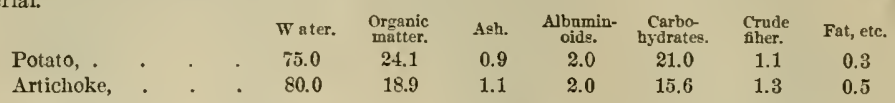

The tubers are sometimes used for the table, when pickled, or for slicing in vinegar like the cucumber They are usually fed to stock raw, but are said to be more nutritious when boiled. The tops, when cut and cured as hay, furnish a fodder that is relished by cattle and sheep. It is rather a difficult crop to exterminate from the soil when once established in a locality. This can be done, however, by plowing the land when it is about a foot high, or by mowing them in the latter part of August.

Varieties.-There are but few varieties of the artichoke. The Jerusalem is quite extensively grown, and one of the oldest, although some of the newer ones, such as the Improved White French and the Red Brazilian, are generally considered more productive - and superior in quality. The yield varies with the soil and variety, but it is always large, even on inferior land, while the reports from some sections give the astonishing product of from eighteen hundred to two thousand bushels per acre. On good land, from a thousand to twelve hundred bushels per acre are not uncommon. Dr. Pollard, former Commissioner of Agriculture of Virginia, refers to this crop as follows:-

"Mr. A. C. Williams; of Ohio, says:- 'The keep of my hogs in warm weather is blue grass and Brazilian artichokes. Forty head of hogs and their pigs may be kept without other food on an acre of artichokes from the time the frost is out of the ground until first of June (about two months), and from September to October, until the ground is again frozen.' We suppose this means that the hogs are to be taken off first of June and the artichokes permitted to grow and form fresh tubers until the fall. They fill the ground so thickly with their tubers that it would be almost impossible for the hogs to get them all in the two months, and thus bear seed enough for the summer's growth. I am prepared to believe this statement of Mr. Williams from an experiment I made with them this year. The variety I planted was the 'White French;' procured from the United States Agricultural Department. I could not procure the 'Red Brazilian,' which is preferred in the West. In spite of the drought, I think each hill would average half a peck. They are not all yet taken up. Two hills selected as having large stalks yielded one and a half pecks. At half a peck to the hill, and planted three feet by one foot, the usual distance, the yield would be upwards of 1,800 bushels. A neighbor of mine, from one hill of the common artichoke heavily manured, produced a measured peck-upward of 3,600 bushels to the acre."

There is an entirely different plant, known as artichoke, (Cynara scolymus,) a native of Barbary and Southern Europe, which is cultivated for its flower heads, which, in their imma. ture state, are boiled and eaten as food. The bottom of the heads are very fleshy, which is the edible part. There are two principal varieties of this plant, the Large Globe, producing large globular heads of a dusky purple color, and which is considered the best for general culture, and the Large Paris, with large oval heads, a kind much cultivated and esteemed by the French,

Planting, Cnltiration, ete.-The artichoke will grow on almost any kind of land. and in shady localities, but does best on a light, rich soil, with open exposure. It is very hardy, and will endure severe cold without injury, living through the winters in the ground in any part of the United States. 
To grow them with the best success, the soil should be rich, and plowed rather deeply, followed by harrowing. They are usually planted in hills, though sometimes in drills, but as they grow and spread so rapidly, the former is the better mode. The ground should be prepared as for potatoes, although, if moderately fertile, a good crop can be produced without any manure. The hills should be about three feet apart. About three bushels of the tubers will be required per acre, planting the small ones whole, and the larger ones in pieces containing one or two eyes, with only about two of such pieces to a hill. A potato-planter may be used for this purpose, or they may be dropped by hand and covered with a light plow or hand-hoe, the covering to be about as deep as that for potatoes. The planting should be done as early as the ground will admit. They should be kept free from weeds by the use of the cultivator or hoe.

Soon after blossoming, which is in August, the tubers begin to form, and continue to grow rapidly until the frost kills the stalks, or they are cut down.

It is said that the tubers can be largely increased in size by pinching off the tops just previous to flowering. If the crop is harvested, instead of being left in the ground during the winter, it will be ready for gathering as soon as the tops have withered. They may be stored the same as potatoes or turnips.

Chufas.-This crop is cultivated mostly in the Southern States for its tubers, which are used principally as food for swine and poultry. They have a sweet, nut-like flavor, and possess considerable nutrition. They are difficult to eradicate from the soil, very productive, and easily cultivated.

Cultivation.-This crop is propagated by means of the tubers. It will thrive on almost any soil, a sandy loam being the best. The land should be plowed to a moderate depth, and harrowed. On rich land, good crops can be produced without much manure being applied, but where the soil is not naturally fertile, a good supply of manure should be given it. The planting should be done about the time of corn planting, in drills from two to two and a half feet apart, the tubers being about a foot apart in the row.

The tubers should be dropped singly and covered with about two inches of soil. The weeds should be kept down, and the soil occasionally stirred with a horse-hoe or cultivator, the same as for potatoes. It is a plant that is best adapted to a Southern climate, although it will grow in much cooler temperatures, and as far north as Massachusetts.

Ridge Cnlture for Root Crops.-Ridge culture has some special advantages in growing root crops, especially where the soil is heavy and rather wet; but it will fail if the manure is coarse, and not well mingled with the soil. It is often resorted to when lands are insufficiently drained, thus securing the advantage of drainage.

The use of coarse manure in a wet season will not affect the crop, however, to the extent that it will if the season is unusually dry, as plants will always suffer in time of drought, if large pieces of manure are left under them. In sections possessing light soil and subject to severe drought, ridging would not be advisable, providing the whole surface be well manured, and the soil deeply pulverized before planting.

The usual method of ridging is to first get the soil in a fine, mellow condition, and then plow the land into furrows, the distance apart varying according to the kind of crop to be grown. The common practice is to turn two furrows together from opposite directions, and plant upon the top of the ridges. The manure in such cases is sometimes applied broadcast, and harrowed into the soil bofore ridging, and sometimes placed in the furrows and covered with the soil. The former method requires more manure, but less labor in preparing; the latter economizes manure, but involves extra plowing to throw the soil over the manure, and change the furrow where it was deposited into a ridge. This method is as follows: After pulverizing the soil and throwing it into ridges with the plow, finely-pulverized manure is 
thrown into each furrow, partially filling it. The plow is then run through the center of each ridge, throwing the soil over the manure, and forming ridges over it. A light roller or drag-plank drawn over the field will sinooth down the ridges, and fit them for the seed, which may be put in with a drill.

The ridge method of culture, as well as all other modes for root crops, requires a deep, clean soil, finely-pulverized, and after-culture sufficient to exterminate the weeds. A cuitivator can be set sufficiently narrow for this purpose, or the wheel-hoe may be used.

The ridge system of cultivation requires more labor in preparing the field for seed, and, usually, a larger area of land than the common method.

How to Coustruct Ventilators for Root-Pits. - In storing vegetables in pits, it is necessary to provide some means of permitting the vapor and moisture from them to escape without admitting the rain or frost, as either would cause a speedy decay of the roots. The American Cultivator recommends the following simple and sensible methods of ventilating root-pits, which may prove of value to many farmers who make use of such modes of storage for these crops:

"In pitting roots, abundant openings should be provided for the escape of the heated air and moisture, produced by the ripening and fermentation of the roots. All moist veg. etable matter, when packed closely in a heap or mass, will begin to heat and ferment in the course of a few hours. The heat produces considerable vapor, which must pass off, and in its escape carry off the heat with it, else the temperature will increase, and, before long, decomposition will begin, and the mass will rot. Danger of this is averted, both by having the roots free from outside moisture when pitted, and by facilitating the escape of that which they give off. This is usually done by leaving open spaces every few feet at the top of the pits, into which bundles of straw, or drain-tiles, are placed, to serre as flues. But it is well to open a ventilator from the bottom of the heap, into which the hot and moist air may escape from ail sides. This may be done by tying a number of rails together (the more crooked the rails, the better, as they will provide more spaces), and placing a bundle in the pits at about every six feet of their length. The top of each bundle projects a few inches, and this may be protected by a cap of straw. This cap should be tied firmly to the top of the rails, to secure it from winds, and to shed rain. If small bunches of straw were fixed in the bottom of the pit, half-way between the ventilators, so long as the weather is not too cold, they would greatly facilitate the cooling and drying of the roots. When frosts are looked for, these bottom air-holes should be securely closed, and then the roots can take care of themselves."

\section{ONIONS.}

$\mathrm{T}$ $\mathrm{HE}$ onion is an important culinary vegetable. It was cultivated in Egypt and Asia ages ago, and has been introduced from thence into nearly all civilized countries. As an article of food, it is healthful, nutritious, and also often used for its medicinal properties. There are numerous varieties which may be largely modified by the influence of soil and climate.

Some localities are especially noted for the culture of this crop, such as Wethersfield, Conn., Danvers, Mass., and the Bermuda Islands, where the soil seems especially adapted to its most successful production.

The supply of onions found in our city markets is produced, to a large extent, by market gardeners, although many farmers make this crop a specialty, and find it a very profitable one. Onions may be grown'from sets or bulbs, or from seed. 
In the southern latitude, onions cannot be grown from native seed in one season, as the hot weather prematurely checks the growth of the plant, when southern-grown seed is sown, and the tops die down before the bulbs have matured, or attained a sufficient size for use; consequently, these small bulbs, or "sets," as they are called, require another season's growth in order to become of desirable size and good quality. They are gathered in the usual man. ner the first season, and set out the following spring to complete their growth. It is stated from good authority, that northern-grown seed, with favorable conditions, will mature goodsized bulbs at the South during the first season. We have never tested this assertion, but know that such a result cannot be attained with seed ripened in a southern climate.

The most favorable region for raising onions, is that north of $40^{\circ}$. South of this parallel, the summers are too dry and hot for their successful culture; hence, as previously stated, a second season's growth is required where native seed is sown. The method of culture by setting out bulbs is practiced at the North when an early crop is desired, which will be ready for the market in June.

Varieties.-The varieties of the onion are quite numerous, some of which are much more productive and desirable in quality than others.

Among those raised from seed are as follows: the Early Red, which is of medium size, quite productive, and mild in flavor; the Early Cracker, which is much earlier than the Large Yellow, and of good quality; the White Portugal, delicate-flavored, of medium size, but not as productive as some varieties, and not as good to keep, being liable to gather moisture and decay early unless spread very thin in storing; the Danvers Yellow, a fine variety, which originated in Danvers, Mass.; it is a favorite in the Boston markets, and is considered one of the best for general cultivation at the North, being very vigorous and productive; ripens early and keeps well. It is rather large, straw-colored, and mild in flavor. Another very popular variety, and which is the staple for the New York market, is the Large Red Wethersfield, which is fine-grained, productive, of large size, and keeps well. It requires a long season for growth, and ripens in September.

There are many other kinds that are favorites in certain localities, but the above-mentioned are the most extensively-cultivated and popular varieties.

Among the Italian varieties which are of very fine size and quality, and which are said to be particularly adapted for cultivation in the Southern States, are the following: the Marzagole, which grows to a large size, and matures early; if sown in the autumn in warm cli. mates it will be ready for use in March; the Large Italian Red Tripoli, of fine flavor, and grows very large; the Giant Rocca of Naples, a variety of large globular form, light brow in skin, and delicate flavor; the Giant White Tripoli, a large white Italian variety of quick growth, and nearly flat. Another variety remarkable for its keeping qualities, as well as the rapidity of its growth, is the New Queen. If sown in March, it will, under favoring conditions, produce bulbs from one to two inches in diameter early in the summer, which will keep sound until the following summer; or, if sown in July, the crop will be ready to harvest late in the autumn, and remain sound until the following autumn.

Top or Button onions are a variety that is propagated from little bulbs which grow on the top of the flower stalk, sometimes to the number of ten or a dozen, growing in the same manner and place on the stalk, as the ordinary seeds, but are small, distinct bulbs, which are transplanted the following spring. They are coarse in quality, and do not keep as well as some of the varieties raised from seed, but are quite early, of mild flavor, and easily cultivated, which constitutes their principal merit, instead of fine quality.

The Potato Onion is a marked variety, producing bulbs in the soil, which are offsets growing near the root in a manner similar to the growth of top onions at the top of the flower-stalk. It is easily cultivated, but of inferior quality.

The Shallot is similar to the Potato Onion, growing in clusters something like the garlic. 
The tops and bulbs are sometimes both eaten. It nerer attains a very large size, but when the little, irregular bulbs are set out early in the spring. will be ready for market several weeks before seed-sown onions are matured. In quality they are superior to the Potato Onion, and also keep well.

The Rareripe is another form of this product differing from the sets, from the fact of their being mature onions which hare been kept through the winter, and which, if set out in the spring and prevented from going to seed, will increase in size and be ready for market early. It is customary with farmers to sometimes select the small mature onions of the crop and set them out early in the spring for a larger growth, which is secured by keeping the stalks cut down to where they begin to enlarge. They will be ready for market early, but will not be of very fine quality.

Sets, are immature onions set out for larger growth and maturity.

Cultiration from Seed. - A deep, rich, loamy soil is best adapted to the culture of onions; it should, however, be neither too heavy nor too light, and of medium moisture, the extremes of either wet or dry being equally objectionable. Land with a slight declivity, well drained, should be selected, and it should be neither stony or poor in any respect. A sandy or gravelly loam well manured will yield fine crops; some other soils will also yield fairly well. Where very large bulbs are desired, a deep, rich loam, rather strong, is neces. sary; the growth in such a soil will also be more rapid, and there will be less liahility to the attacks of the onion-fly or maggot than on a light, dry, sandy soil. Unlike most crops, onions can be cultirated for sereral years in succession on the same field, and seem to do better by this method of culture, while it is much less labor than to follow a rotation and change every year or two, they being one of the rare exceptions to the theory of rotation of crops. Weeds are detrimental to this crop, consequently their successful production necessi. tates a clean soil. For this reason, carrots or celery are very good preparatory crops to grow on land previous to its being occupied by onions. Near large cities, it is quite a common practice to have every sixth row in the onion field occupied with celery. After remor. ing the onions, the celery is banked up with the soil for blanching, thus giving a second crop from the same land, which is generally about as profitable as the first. Land designed for onions should be plowed late in the fall, just before winter sets in; this will insure the destruction of the wire-worms, and a more thorough pulrerization of soil by the aid of the frosts. The plowing should not be very deep, four or fire inches being sufficient, and if the land is a slight declivity, the plowing should be across in such a manner as to prevent the rains washing the soil by running down the furrows. Stable manure, hog manure, and right soil are beneficial to this crop, also wood ashes, the sulphate of potash, land plaster, and many of the other commercial fertilizers. Stable manure should always be well rotted or fermented for onions, and very finely pulrerized. A heary dressing of manure is essential to a good crop, from twelve to fifteen cords per acre being none too much; in addition to this, from forty to fifty bushels of unleached wood ashes, and about four hundred pounds of plaster are desirable. Where the manure is not to be had for this purpose, about six hundred pounds of superphosphates will answer as a substitute, to which the ashes and plaster should be added.

As a general practice, those interested in the cultivation of onions do not enrich the soil sufficiently to make it a profitable crop, or rather, by enriching it more, the profits would be much larger. We believe this is why so many in their first attempts in growing onions fail, as with proper fertility and care they are one of the surest of crops. We have known of several who have cultivated onions from eight to fifteen years in succession, and have never experienced a single failure during all that period, which is more than can be said of many crops.

Some farmers spread the manure in the fall immediately after plowing, and harrow it 
in, or where this is not conveniently done, they work it over during the winter occasionally, to make it very fine, and plow it under in the spring about three inches, after which the ground is rolled with a heavy roller. Others prefer to apply the manure very fine, only two or three days before sowing the seed. Commercial fertilizers, wood ashes, and plaster should always be applied about that time, or within a short time of sowing. If the manure is spread in the fall, the land should be plowed to the depth of three inches and harrowed thoroughly in the spring just before sowing,-a disc barrow being best for this purpose,after which the ground should be rolled. Rolling crushes the clods and lumps and thus aids in pulverizing the soil. When land is plowed in the fall, this process should be repeated in the spring.

All stones should be removed, and the finer the soil can be made, the better. After the soil is made as fine as possible with the harrow and roller, the surface should be carefully raked with a fine-toothed hand-rake to further remove all the small lumps of earth and stones that may yet remain. It requires considerable labor and pains-taking to prepare a field for onion culture, but it is of the utmost importance that it be done well, as the yield of the crop will depend in a great measure upon the manner in which it is prepared; besides, if thoroughly done the first season, much labor will be saved in the growing of subsequent crops of the same kind which may follow in succession for several years with advantage, if the land continues to be heavily manured each year.

It is very important that the seed sown should be of the very best quality and that grown the preceding year. It cannot be of the best quality unless the best and largest bulbs are selected for producing it, and it is fully matured before being gathered, besides being well dried and cleaned.

Some farmers are indifferent in this respect, and often raise seed from inferior, illformed bulbs, but such seed will not only produce a crop of inferior quality, but will make a difierence in many bushels of the product, in quantity per acre. It is also equally essential that the seed be fresh and of the preceding year's growth, as old seed will not germi. nate well, and is very unreliable. It is stated by good authority that of seed two years old less than half will ordinarily germinate, while that three years old will scarcely germinate at all; consequently it is always safer and the best economy to secure fresh seed for planting, and never to risk the old, as planting over is an injury to the crop, besides involving a great deal of extra labor, all of which may be obviated by the use of fresh seed of the best quality.

The sowing for large crops should always be done by a machine, as hand-sowing is not only slow and very laborious, but results in the loss of considerable of the seed, and cannot be done as uniformly as by a small machine that will drill it in uniformly and cover it evenly. There are several garden seed-sowers for this purpose in the market that can be procured at slight expense, and will do the work much better and easier than can be done by hand. A seed-sower, hand-cultivator, and weeder combined will be the best implement of the kind for the purpose, as it includes three machines in one.

The main crop should be sown as early in the spring as the ground is in working condition, since it is best to secure as large a growth as possible, before the usual drouths of August. The seed should be deposited in drills from twelve to sixteen inches apart, the latter width being the most desirable for large or medium-sized bulbs. It should be sown evenly and rather sparingly, and covered a half inch in depth. About four seeds an inch is considered the desirable distance in sowing. Most of the seed-drills cover the seed and press the soil down upon it, but where this is not done by the machine, it is well to go over the ground with a light roller after the seed is covered, which has a tendency to quicken the germination of the seed. Four pounds of seed are sufficient for an acre, where the drills are about sixteen inches apart. Should the drills be nearer, a bittle more may be required. 
An ounce of seed will sow a bed four and a half feet by thirty feet. It is important that the seed be sown early, as the bulbs will all be more liable to mature well and there will be no "scullions," as they are called, which are merely unripe onions.

It is customary to weed this crop three or four times during the season. As soon as the plants make their appearance, a wheel-hoe or cultivator should be used between the rows to check the weeds which will always start vigorously in any soil newly worked over, and which will ruin the crop, if not kept down. If this cultivation is delayed until the weeds get well started, it will require a great amount of labor to exterminate them, and which more prompt attention might obviate. If this cultivation be done once a week or oftener for the first few weeks, the weeding in the drills by hand will not be very laborious; besides, as onions do not shade the ground much, and the soil must of necessity be very rich, weeds in an onion field will grow very fast and be sure to ruin the crop unless prompt and vigorous attention be given to keep them constantly in check. They must be exterminated as soon as they make their appearance in the rows. This is commonly done by hand, and is hard work, as it involves the getting down of the operator upon his hands and knees. If a good weeder or cultivator is used between the rows, and so operated as to work close to the drills, there will be but few weeds between the plants to be pulled out. There are various little weeding hoes invented for this purpose, to take the place of hand-weeding, but many farmers prefer the latter as being more effectual in results.

An experienced farmer, who has cultivated onions for over ten years successfully, says that a useful tool to weed with, where the ground is hard, can be made from an old lioe, by cutting off the sides of the blade until there is a strip left an inch and a half in width; after. wards grind the sides and edge of this miniature hoe, sharp, and put in a handle ten inches long. This may be used very successfully where the soil is rather hard, but where it is soft, - fingers can do more rapid and efficient work. If the fingers get tender and sore, rubber finger-stalls, or fingers cut from an old kid glove, are a great protection.

After the first weeding, many consider it beneficial to give the crop a light dressing of wood-ashes. Two or three other hand.weedings with frequent use of cultivator or weeder between the rows, will be required before the crop will be ready for harvesting. All sickly and diseased-looking plants should be pulled up and thrown off the field from time to time. It is a crop that cannot be neglected and fair results follow ; the work must all be thoroughly and promptly performed, if a good crop is to be obtained. When the bulbs begin to ripen, the tops will fall over to the ground; for a short time after this period the bulbs will grow very fast, therefore they should not be harvested too early.

When the tops are nearly all fallen over to the ground, and are dry where they join the bulb, they are ready for pulling. Sometimes it happens that the crop ripens unevenly. When this is the case, the usual practice is to roll something light, such as an empty flour barrel, over the rows in order to bend down the tops of the green upright stalks, which will hasten the ripening process. If onions remain in the ground too long after being ready to be pulled, they will commence a second growth by sprouting again, which will ruin the crop. Should the maggot make its appearance, as it often does, to the great injury of the crop, a little plas. ter, guano, or unleached wood ashes scattered along the rows of plants will be an aid in its extermination.

Cultivation of Sets. - It is quite common in the Southern States to grow onions from sets, also in the Northern, when a very early crop is desired; but the main product is produced in the latter section by seed-sowing. Southern-grown sets are to be preferred to those of a northern latitude.

To produce sets, the land should be made moderately fertile, be very finely pulverized, and made smooth and even, the same as for the reception of seed for the seed-crop. The seed should be drilled in rows nine or ten inches apart, the drills to be broad, to secure a large 
number of bulbs to the drill, sowing the seed thickly, which will require five or six times the amount for the mature crop from seed. They should be kept free from weeds, and when the tops indicate that they are ready for gathering, should be pulled and stored through the win. ter in a cool, dry place, well ventilated. To insure their keeping well, they should be spread very thinly, otherwise they will gather moisture and be liable to decay. In the following spring these little bulbs may be set out as early as the weather will admit in rich land, finely pulverized and mellow. They should be placed in drills from ten to twelve inches apart, the bulbs being about six inches apart and lightly covered with the soil. Sufficient cultivation should be given them to keep out the weeds. When they are well ripened they should be pulled, dried, and stored.

The Cultivation of Top Onions is similar to that from sets. The tops, containing the small bulbs, are gathered when ripe and stored in a cool, dry place. In the spring they are set out and the culture given similar to that recommended for onion "sets."

Cultivation of the Potato Onion and Shallot. - The bulbs should be planted in rich soil in drills about fourteen inches apart, the bulbs to be ten or twelve inches apart in the rows. The planting should be as early in the season as the soil can be worked, the largest bulbs to be selected, the crowns of which should be set just below the surface of the ground. They should be kept clear from weeds, and a little earth gathered about them from time to time in the process of after-cultivation. The harvesting should be as soon as the tops are dead.

The Culture of Rareripes is not to be recommended very highly, as they are of por quality generally, but as small onions can be economically used for the purpose of a larger growth, many farmers resort to this expedient to find an early and ready market for them. As we have previously stated, this is a form of the onion that differs from the sets for which they are often mistaken, by being small mature onions. These are generally selected from the crop, and set out the following spring for a larger growth, as early as the condition of the ground will admit. Sometimes large onions that are badly sprouted are treated in the same way. They will soon begin to send up seed-stalks, which should be cut off just below where the stalk begins to enlarge, and as soon as the swelled appearance of the stalk is noticed. By this method, and keeping the soil free from weeds, the bulbs will grow quite fast and will be ready for an early market. They require a little more room than sets, when the bulbs are larger than the former.

Cultirating Onion Seed. - As the weight of the onion crop depends largely upon the quality of the seed used to produce it, it is very important that the utmost care should be exercised in the selection of bulbs and the subsequent attention given them in growing the seed. Many prefer to buy this seed of a reliable dealer or some one who raises it, in whom they have confidence. To the latter we would say-unless you are able to grow that of the very best quality, it will be better to buy seed of such quality than to depend upon your own skill in this respect, for it is only the first-class seed that will give a first-class crop, and it is not every farmer that can raise seed of that quality without consid. erable experience. It is of great importance that a careful selection of bulbs be made, that is, a selection of the form and size that it is desired to produce in the future crops. These bulbs should be set out very early in the season. in rich land and be well cultivated. They should be hoed repeatedly, with all weeds kept in check, and the earth kept heaped up about the stalks for protection. A frame-work of slats or a stake and strings should be used for the support of the stalks and heavy seed-heads, otherwise they will be !iable to break or so bend over that the seed will either be injured or entirely ruined.

When the szed stalks indicate maturity by turning yellow near the ground, and the seed. cells begin to open, the tops or heads should be cut, leaving about six or eight inches of the 
stalks attached to them to aid in perfecting the ripening process. These should be spread thinly in a warm, dry place, and frequently stirred, that the drying may be uniform, and moulding prevented. When thoroughly dried, the seed should be shelled and cleaned through a fine seive. It is well to afterwards winnow it in order to get out as many of the imperfect seeds as possible. It is customary for some seed.growers to separate the imperfect from the sound seed by putting the whole in water, and after stirring it thoroughly, skim off all that rise to the surface, after which all the seed that sinks to the bottom is taken out and carefully dried. If this practice is to be followed, we would not recommend it in the autumn, but rather in the spring. just before sowing; then there would be no danger of loss of seed from molding, through excess of inoisture, and imperfect drying. The drying should be in a warm place, but not near a fire. After the seed is thoroughly dried, it should be stored in a dry place, where it will not be liable to gather moisture. The seed used should be fresh every year, it being of little value the second year.

Harvesting and Storing. - When most of the tops are fallen over and look dead, the onions are ready to be harvested. The usual method is to pull them by hand, throwing the product of six or eight rows together into a windrow to dry, but this is a laborious process. When very ripe they may be raked out with a common hand rake. It would be well if some machine were invented for cutting the tops first, after which they could easily be raked out. An implement of some kind for running under the rows to cut the roots aids materially in the harvesting prócess, as the bulbs can then be very readily raked out. They should be left on the ground until the tops are all dead and the bulbs feel hard and solid; this sometimes requires two weeks or more; during the time they should be raked over with a hay rake every two or three days, especially after every rain, in order to hasten the drying process. When the tops are well cured and the bulbs hard and solid, they may be taken off the field in a dry day, and stored on the floor in a dry shed or barn, tops and all, if not spread over three feet deep. Careless handling: causing cuts or bruises, will result in a loss, as such bulbs will decay in a short time.

Where the crop is not to be sold for use at once, care must be taken that the tops are dry close to the bulbs, as nearly the whole top will sometimes seem to be dry when the neck is green, and if cut in this state the bulb will soon decay. In order to keep well, therefore, the tops must be entirely dry and dead before cutting. When not convenient to cut at this stage, and they are not intended for immediatesale, the cutting is often delayed until winter. This may be done with a sharp knife, but the best implement for doing it is an old pair of sheep shears, or a pair of common shears with the blade cut off nearly half-way down. The larger ones may then be separated from the small ones, and are ready for market, or storage until spring. The smallest, or those about the size of a small walnut, will sometimes bring a fair price for use as pickles.

Whether it will pay the grower best to sell the crop in the fall, or to store them for sale in the spring, will depend upon a combination of circumstances, such as the state of the market, the locality, etc. This crop sometimes brings a very high price in the spring when they are scarce in the market; still there is considerable risk in keeping them, as they are bable to decay badly during some seasons. When they are very plenty, and the prices are low in the spring, they will not prove as profitable to the grower to be kept over as a fall sale.

When designed for winter sales, they are generally kept in a cold, dark cellar in barrels or bins, after the cold weather sets in. If the barrels in which they are stored have pieces cut out of their sides with a hatchet, thus giving better ventilation, they will be liable to keep better. The barrels should be covered at the heads to exclude the light, in order to prevent sprouting, and all bins used for storage should have the light excluded by some kind of covering that will not keep out the air, and cause them to gather moisture and become heated. Good ventilation and a cool, dry temperature are essential to their keeping well. 
They will bear about as much frost as apples, without injury; in fact, when intended for spring sales it is quite a common practice to freeze them, and then cover with hay or other material to prevent their thawing, keeping them well covered and frozen until a few days before selling. Once freezing them does no injury, but repeated freezing and thawing is ruinous.

Some farmers who cultivate this crop extensively, have a cold, airy building constructed, especially adapted to the purpose of storage, with bins of lattice work where they are stored, about three feet deep, and covered well to keep them from thawing until spring, or where they are spread in piles and covered with hay and other material for the same purpose. When wanted for sale, they should be uncovered and allowed to thaw, and then spread a few days to dry; they are then ready for market. Much labor could be saved in the spring, if, as soon as the crop is taken off the field in the fall, all the decayed onions and tops, as well as weeds, are cleared off, and the ground be well harrowed. The harrowing should be repeated in a few weeks. This will cause the germination and subsequent destruction of any weed seeds that may remain in the soil. Just before winter sets in, the field should be plowed, in order that the frost may assist in mellowing and pulverizing the soil for the next year's crop, which plowing also causes the destruction of worms and insect eggs by freezing. From four to six hundred bushels per acre is considered a fair crop of onions, but we have known of authenticated cases where a thousand and even twelve hundred bushels per acre have, in rare instances of peculiarly favorable soil, season, and culture, been grown from an acre. 


\section{MISCELLANEOUS AGRICULTURAL PRODUCTS.}

\section{COTTON.}

$\mathrm{C}$ OTTON is one of the most valuable agricultural products of the country, and has for many years been one of its principal exports; in fact, the plant which will supply the material for one of our leading industries, as well as for the clothing of all nations of the globe, should claim a rank second to none in the vegetable kingdom, except it be that which supplies the necessary sustenance of life to the human family in the form of food. It is a plant indigenous to all inter-tropical regions, and can be cultivated with success in no other. Its culture antedates the commencement of the Christian era, the first mention of it of which we have any record being by Herodotus, about 450 B. C. He says of it, "There is a plant in India which produces wool finer and better than that of sheep, of which the Indians make their clothes." Pliny, late in the first eentury, says, "There grows a shrub called Gossypium or Xylon, in Upper Egypt, producing a stuff from which the white garments of the priests are made." In the Chinese records, no mention is made of cotton until about two hundred years before the Christian era, from which time to the sixth century cotton cloth was regarded as something exceedingly rare and precious, and was paid in tribute, or offered in presents to the emperors; it is also recorded, as an incident of importance of the Emperor Ou-ti, who ascended the throne in 502 , that he had a robe of cotton.

Columbus found cotton in the possession of the natives of Hispaniola, and the plant was found growing wild in the Mississippi valley and Texas by the early explorers of the country. It was first cultivated by the early settlers as an ornamental garden plant, and it was not until 1739 , according to reliable authority, that it was first exported, this exportation consisting of but one bag of the product, from Savannah, Ga. It is an incident of record, that in the year 1784, an American ship having on board eight bags of cotton, bound for Liverpool, was seized on the ground that so much cotton could not have been produced in the United States! In the conquest of Mexico, in 1519, it is stated that Cortes received garments of cotton as presents from the natives of Yucatan, as well as cotton cloths for coverings to his huts. Specimens of cotton fabrics have also been taken from the ancient Peruvian tombs. In the year 1785 the cultivation of the short-staple cotton was commenced in the United States, and in ten years from that period one million pounds were exported from Charleston, Sonth Carolina. Since that time the immense increase in this product in the Southern States, and the revenue brought to that section from its exportation (the latter amounting to a hundred and fifty millions of dollars annually), has been mainly due to inventions of improved machinery, the principal of which was the saw cotton gin, a machine for separating the cotton from the seeds, which adhere to the fiber quite tenaciously, and which was invented by Mr. Eli Whitney, of Massachusetts, in 1793. Although it brought the inventor but small profit, and a vast amount of troublesome litigation, this invention resulted in great benefit to the human race, by immensely increasing the cotton industry of the whole world. Previous to this invention, the separating of the cotton seed from the fiber was mainly performed by hand, being a slow and expensive process. Mr. Whitney's machine cleaned a thousand pounds in the same time that one pound could be cleaned by hand, and consequently very soon caused a revolution in the cultivation and manufacture of the cotton product.

The United States stands first among the countries of the world in the amount and value of its cotton products, British India ranking next, but the fiber is inferior to that of this 
conntry. American seed and American plants have been introduced from time to time into India, but without the desired result, as the fiber after a little while deteriorates, loses its elasticity, and becomes short, dry, and harsh, with a low rate of yield. Egypt is quite prominent in cotton production, both in quality and amount, the best from that source taking a high rank, and second only to the famous Sea Island product, which is universally considered the best.

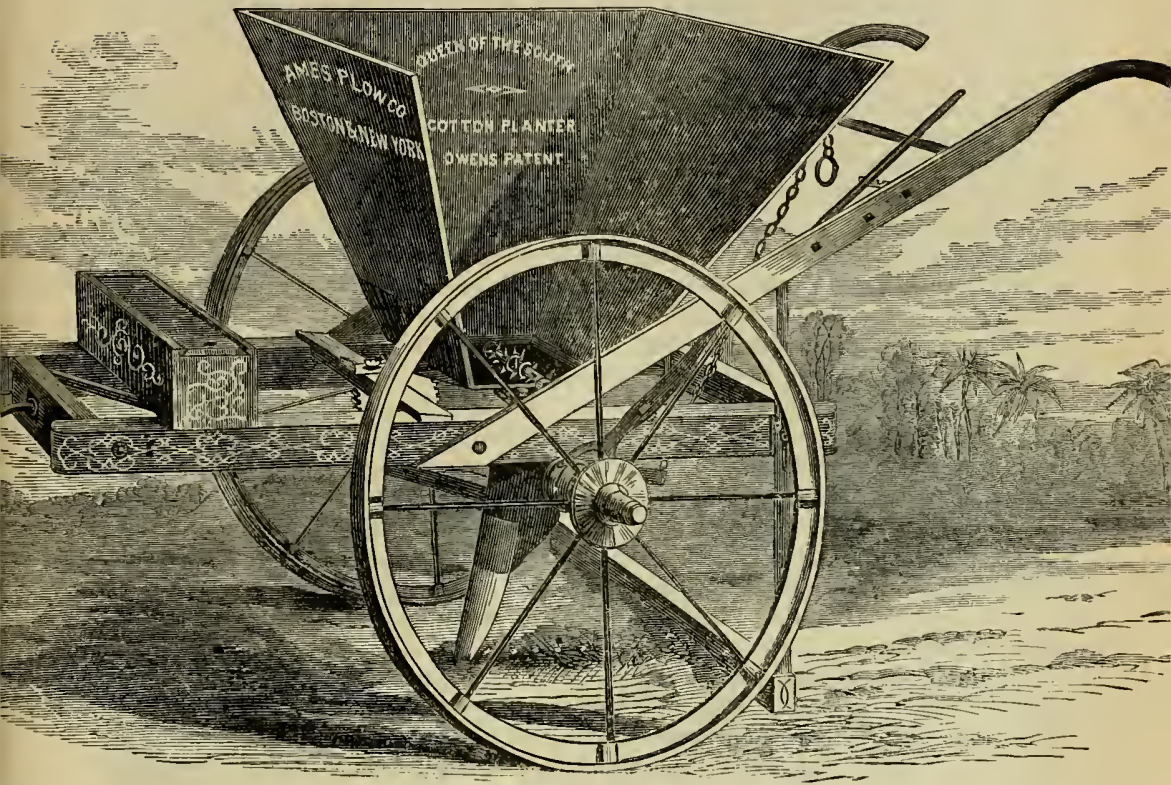

QUEEN OF THE SOLTH COTTON PLANTER.

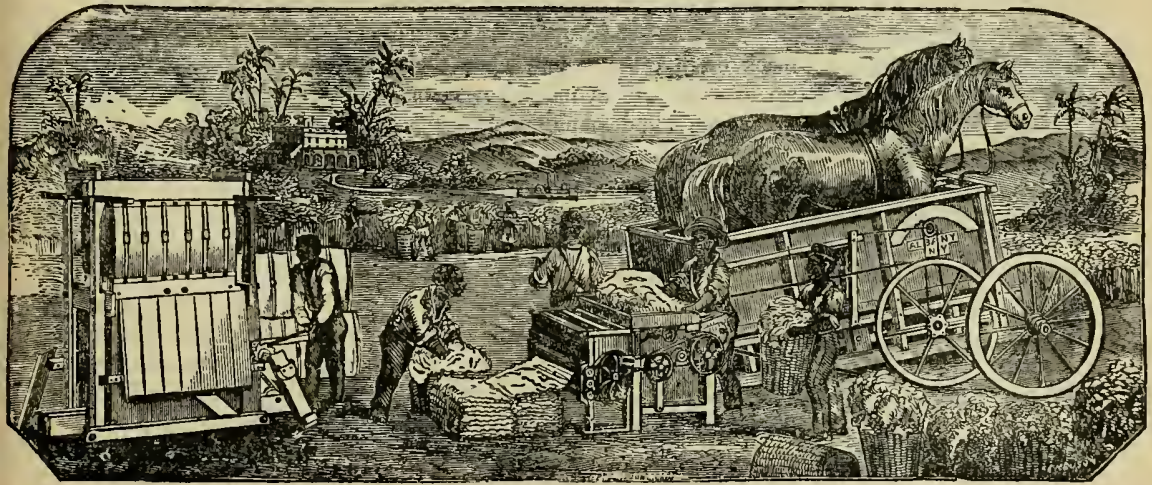

DEDERICK COTTON PRESS. 
The constant cropping which the cotton fields of the Southern States formerly received, with little or no fertility restored, together with poor cultivation, have cansed the soil in many parts of that section to become nearly exhansted, and large areas that were once rich cotton lands, are now regarded as unproductive, but with improved methods of agriculture, and an intelligent use of fertilizers suited to the necessities of the soil and crops, this evil may in the future be remedied and avoided. The adoption of a more thorough system of agricnl. ture at the South within the past few years, together with the establishing of cotton mills and other industries, have been the means of increasing and improving the cotton product of the country, with at present a still brighter outlook for the future in this respect.

The poems and romances of the Chinese literature of the seventh century are much given to the description and celebration of the beauty of the cotton flower. The blossoms are indeed very attractive, and a field of cotton in full bloom is a beautiful sight. Though varying slightly with different varieties, the flower of the cotton plant resembles that of the althea in size and shape. The color of the variety most commonly cultivated in this country is, on first opening, a creamy white, changing to a light yellow the second day, and a purplish red on the third, when it drops. The bolls are egg-shaped, varying in size, one the size of a goose-egg being considered extraordinarily large. As they ripen or mature, the outer covering turns brown and opens generally into four parts, the white fiber bursting out of its enclosure, so that a cotton-field divested of its leaves resembles a field of white roses. The leaves are a deep glossy green. By planting the seed in boxes, in order to secure an early start, and transplanting as soon as the weather will admit, cotton plants could easily be grown in Northern gardens as an ornament, their beanty exceeding many of the popnlar varieties of cultivated flowers. Besides the extensive use of the cotton fiber in the manufacture of fabrics, and afterwards of paper, the seed is used largely in the manufacture of oil, that which remains after expressing the oil being valuable as food for stock, and also as a fertilizer of the soil. In Brazil and some other cotton-growing countries, the leaves of the cotton plant are thought to have important medicinal qualities. The root is used to a limited extent in medicine.

Varieties of Cotton.-There are several varieties of the cotton plant, although botanists differ with respect to the exact number of species. Withont going into a discussion of the subject, as to whether there are five, seven, ten or more species of this plant, bnt leaving that question for the botanists to settle among themselves, we shall mention only the most exten. sively cultivated kinds that furnish the great cotton product of the world. The two principal divisions of the cotton product are those of the Old and New World, or what are known as the Indian and American cottons, the difference in the botanical characteristics being slight, but sufficiently marked to distingnish the one from the other, the seed of the eastern plant, or that of India, never being black or divested of lint, while the curvature at the base of the leaf lobes is composed of two opposite curves, instead of being heart-shaped like that of the Western Continent. The cottons most in demand are those of the United States, as we have previously stated, while British India is second in this respect. The principal cotton crop, or that most extensively grown, is of the short-staple variety, as the best quality of the longstapled or Sea Island cotton can be grown successfully only in certain localities, hence its cultivation is necessarily limited to a comparatively small territory. The cottons most in demand are the Sea Island and the New Orleans or Upland varieties. The former receives its name from its being grown mainly upon the low-lying islands near the coast of Georgia, Florida, and South Carolina, where the soil, climate, and peculiarly favoring conditions render them especially adapted to the production of this variety, which surpasses all others in the length, strength, and beauty of its staple, its fiber being very long and silky. It is used for making the best quality of sewing thread. for mixing with silk, etc., and brings the highest price in the market. The seed of the Sea Island or long-stapled variety is black and smooth, with a little of the cotton fiber adhering to the small end. The variety known as the Georgian Upland cotton is the result of cultivating the Sea Island cotton on the uplands of Georgia; it is also sometimes known as "Boweds." 
Of the New Orleans or Upland cotton, there are two principal varieties, each of which have several sub-varieties. One of these has green seeds and is quite hardy, the other has grayish seeds and a more flexible, silky staple, but is slightly less hardy than the former. Both are quite prolific.

The New Orleans and Boweds cottons constitute the great bulk of the production of the country, and are known in European markets as "American cottons." There are a number of forms of the Hirsute or Orleans type, one of which is the Cuba Vine, "a large and showy plant," as it is described by one writer; another is a plant bearing yellow or brown-stapled cotton, known as the "Nankeen," from which nankeen cloths are made, and still another type known as "Bourbon" cotton, but they are neither of them considered as profitable for culture as most of the other varieties. Some of the long-stapled cottons of the Sea Island type have been so widely scattered over the cotton zone of the world, and in consequence have become so modified and diversified in characteristics, that they have been classed as different and distinct species, among which are the Peruvian and Brazilian cottons; the latter being remarkable for its peculiar arrangement of seeds, eight or ten of which adhere closely together in a compact, slightly oval form, which has given it the name in some localities of "kidney cotton."

In some portions of India the cotton plant becomes a tree (Gossypium arboreum), and bears dark green leaves and reddish purple blossoms, the product being a silky fibre. It is there regarded as sacred, and grown about the temples. Attempts made to improve its cultivation by hybridizing have thus far been unsuccessful. Though there are many varieties and subvarieties of the cotton plant grown in this country, some of which seem best adapted to one locality and soil, and others to another,-thus giving rise to a local popularity more or less extensive, yet doubtless not a few of these could be traced to the same source, the difference being mainly changes produced by climate and cultivation, which have caused them to become fixed in certain characteristics, until they differ so widely from the original plant, that they seem new types or distinct varieties.

Among the long-established varieties of the widest popularity are the Dixon, which has a fibre of medium length and is quite hardy and prolific; the Peeler, producing a fibre of unusual length and fineness, but not quite as prolific as the former; Boyd's Prolific, of medium length of staple and quite productive. Other varieties that have been much in favor in certain sections are the Early Simpson, McClenden Prolific, Boyd's Prolific, Petit Gulf, Johnston, and Hurlong's Improved. Of the last two, the former has been cultivated more extensively west, than east of the Mississippi, while the latter is more frequently found in the region from Alabama to Texas. The Mammoth Prolific has also been quite popular in some sections. Beside those already mentioned, varions other kinds of greater or less popularity are grown in certain sections, and new varieties are being introduced from time to time, and their respective merits tested.

New and improved varieties will doubtless be obtained by careful and patient experiments in hybridizing, the same as from other cultivated crops, while the older sorts may have their quality. of staple and productive capacity more nearly perfected by improving the fertility of the soil, as well as the general method of cultivation and the adoption of a judicrous system of rotation, which will prevent the exhaustion of soil that has formerly characterized the production of this crop at the South.

The Cotton Growing Belt.-Although the extremes of the cotton belt, or the section where, according to the most authentic sources of information, cotton can be grown with profit, are included between the twenty-eighth and thirty.eighth degrees north latitude; yet it is possible, with favoring conditions of season and locality, to cultivate it considerably farther north of this limit, the crop having been fairly successful as far north as Missouri and Delaware in favorable seasons. 
In the period of the early settlement of Central Illinois, or between the years $181 \mathrm{~s}$ and 1828 , cotton was in that locality a common crop, not for exportation, but simply for home consumption, to be used in connection with wool in manufacturing clothing for the settlers. But since that period, the experiments made to cultivate it in that section have generally proved a failure. This difference is probably not so much due to a change in climate or in the length of the seasons as from the fact that the early settlers of Illinois confined their corn and cotton culture to the timber fields, which were sheltered and warm; therefore the crops would mature earlier than in unsheltered localities in the same climate. Cotton requires a warm climate to be successfully grown, and in such a temperature can be cultivated with less expense and no danger of failure from causes which render its culture beyond its natural habitat attended with considerable risk-an early frost or backward season often resulting in a partial or complete failure of the crop in such sections.

We doubt if its culture north of North Carolina and Tennessee will be attended with as much profit as that of other crops better adapted to the latitude. It can, it is true, be grown considerably beyond this limit, but the question to be settled in the mind of the planter is, Will it be as profitable a crop to cultivate as some others better adapted to that locality?

Prof. Pendleton, of Georgia, gives as his opinion, that north of the thirty-fourth degree of latitude the seasons are not sufficiently long to give uniform success with the cotton plant, while south of the thirty-first degree the seasons are so wet, and insects so abundant, that results will be quite uncertain, and the cost of its culture be also greatly increased. While there may be some few exceptions, such as in warm and sheltered localities, where an earlier growth and quicker maturity of the crop can be secured, yet, in the main, the belt between the two above-mentioned degrees of latitude will be found to be the most favorable region for the successful cultivation of the cotton plant.

The following table from the Census Report for 1880 shows the total cotton production in each of the cotton-growing States during the previous year (1879), the average product per acre, etc., the names of the States being arranged in accordance with the order of their rank in the amount produced.

Table I.-Showing Total Colton Production and Average Product Per Acre.

\begin{tabular}{|c|c|c|c|c|c|c|c|c|c|c|c|c|c|}
\hline & \multirow{3}{*}{$\begin{array}{l}\text { States in order of } \\
\text { production. }\end{array}$} & \multicolumn{3}{|c|}{ POPULATION. } & \multicolumn{2}{|c|}{$\begin{array}{c}\text { COTTON } \\
\text { PRODUCTION. }\end{array}$} & \multirow{2}{*}{ 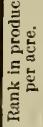 } & \multicolumn{4}{|c|}{$\begin{array}{l}\text { AVERAGE PRODUCT } \\
\text { PER ACRE, IN LBS. }\end{array}$} & \multicolumn{2}{|c|}{ TOTAL, IN TONS. } \\
\hline & & Total. & White. & Colored. & Acres. & Bales. & & 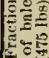 & 密 & 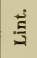 & 总密 & Lint. & $\begin{array}{l}\text { Cotton- } \\
\text { seed. }\end{array}$ \\
\hline & & $\mathbf{1}$ & 2 & 3 & 4 & $\mathbf{5}$ & & 6 & 7 & 8 & 9 & 10 & 11 \\
\hline & Total, ......... & $16,808,664$ & $11,024,123$ & $5,781,541$ & $14,441,993$ & $5,73 \pi, 25 \pi$ & . & 0.40 & 507 & 189 & 878 & $1,362,599$ & $2,725,197$ \\
\hline 1 & *Mississippi, ...... & $1,131,592$ & 479,371 & 652,221 & $2,093,330$ & 955,808 & 8 & 0.46 & 651 & 217 & 434 & 227,001 & 454,009 \\
\hline 2 & Georgia,........ & $1,542,180$ & 816,906 & 725,274 & $2,617,138$ & 814,411 & 12 & 0.31 & 414 & 148 & 296 & $193,43 \pi$ & 386,859 \\
\hline 3 & *Texas, .......... & $1,592,574$ & $1,197,499$ & $395,0 \div 6$ & 2.173 .732 & 803,642 & 11 & 0.37 & 528 & 176 & 352 & $190,8+i 5$ & 381,730 \\
\hline 4 & Alabama,........ & $1,262,50115$ & $66^{\circ}, 185$ & 600,320 & 2.330 .086 & 6999.654 & 13 & 0.30 & 429 & 143 & 286 & 166,168 & 332,336 \\
\hline 6 & Arkansas,........ & 802,525 & 591,531 & 210,994 & $1,042,976$ & 608,256 & 3 & 0.58 & 831 & $2 \pi$ & 554 & 144.461 & 285,92 \\
\hline 6 & South Carolina,.. & $9.5,5 \%$ & 391,105 & 604,472 & $1,364.249$ & $52 \cdot 2,5 \cdot 48$ & 10 & 0,38 & 546 & 182 & 364 & 124,105 & 248.210 \\
\hline 7 & Louisiana, . .... & 939,946 & 454,954 & 484,492 & 864,787 & 508,569 & 2 & 0.59 & 837 & 279 & 558 & 120.785 & $2 \$ 1.570$ \\
\hline 8 & North Carolina,.. & $1,349,750$ & 867.212 & 532,508 & 893,153 & 289,598 & 9 & 0.44 & 621 & 217 & 414 & 92.530 & 185.059 \\
\hline 9 & *Tennessee, ...... & $1,542,4153$ & $1,139,120$ & 403,343 & 792,549 & 330,644 & 7 & 0.46 & 651 & 217 & 434 & 78,528 & $15 \pi, 056$ \\
\hline 10 & Florida,......... & 269,493 & 142,605 & 126.888 & 245,595 & 54.997 & 14 & 0.22 & 318 & 106 & 212 & 18,062 & 26,124 \\
\hline 11 & *Missouri,..... ... & $2,168,804$ & $2,023,568$ & 145,236 & 32,711 & 19,733 & 1 & 0.60 & $8 k 1$ & 287 & 574 & 4,687 & $9,8,3$ \\
\hline 12 & Indian Territory, & & & & 35,000 & 17,000 & 5 & 0.49 & 693 & 231 & 462 & 4,037 & 8,075 \\
\hline 13 & Virginia, ........ & $1,512,5,5$ & $\$ 80,858$ & 631,7 & 24,000 & 11,000 & 6 & 0.46 & 654 & 218 & 436 & 2,612 & 6.225 \\
\hline 14 & Kentucky, ..... & $1,648,690$ & $1,3,7,179$ & 271,511 & 2,667 & 1,367 & 4 & 0.51 & 729 & 243 & 486 & 325 & 649 \\
\hline
\end{tabular}

* The popnlation figures are still subject to possible correction.

The first three columns give the population of the several States, divided according to color. The acreage and total production are given in the next two columns. Column 6 gives the ratio between the figures of columns 4 and 5 in decimal fractions of a bale produced per acre. 
The average weight of a bale of cotton is estimated at 475 pounds. Upon this basis, and upon the commonly-accepted average proportion of one part of lint to two of seed in the "seed-cotton" as it comes from the field, are based the data given in coluunns 7 to 11 . Of these, the first three give the weight in pounds, severally, of seed-cotton, of lint or fiber, and of cotton seed produced per acre, on the average, in each State, while columns 10 and 11 give the weight in tons of the total product of each of the States, in lint and cotton-seed.

It will be seen by a comparison of the total population of the States of the cotton-belt proper, from North and South Carolina to Texas, that in all but two cases there is an approximation to the proportion of one bale to every two persons, or rather one-half a bale to each inhabitant. These exceptional States are Mississippi and Arkansas, in which the ratio is from two-thirds to more than three-fourths of a bale per head. As will be seen, Mississippi stands first in the total production of cotton, while sixth in population among the cotton States, making its product to .84 , or more than eight-tenths of a bale per head. It appears that the high production of this State is due to the great fertility of the soil together with the circumstance that the culture of this product is the leading pursuit of the population. In Washington County, Miss., the product per acre averaged .87 of a bale or 413 pounds of lint, which is equal to 1,239 pounds of seed-cotton per acre. E. W. Hilgard, Professor of Agriculture of the University of California, says, with respect to the above table:-

"Even with the imperfect tillage and incomplete picking of the cotton crop now prevailing in the Yazoo bottom, Miss., the present average product per acre is over three-fourths of a bale; and estimating the lands reclaimable by simple exclusion of the Mississippi overflow at three million acres, the annual production could thus readily be raised to 2,250,000 bales, without any change in the methods of culture, in the Yazoo bottom alone. With improved cultivation the production could easily be brought up to $5,000,000$ bales, and thus, with a similar improvement in the culture of the uplands, it is evident that the State of Mississippi alone could produce the entire crop now grown in the United States. So far from this being an over-estimate, this statement does not adequately state the possibilities within reach of careful culture. Fully 1,000 pounds of lint have repeatedly been picked from an acre of the 'buckshot' soil of the Yazoo bottom.

Georgia stands second in total production; but examination shows the causes that place the State so near to the highest in position to be widely different from those in Mississippi. With half a million more inhabitants than Mississippi, the cotton product of Georgia is a little over half a bale (.53) per head, and the average product per acre is but two-thirds of that of Mississippi. A detailed examination of the soils of Georgia shows that her area of what in Mississippi are considered first and second-class soils, is very limited; far more so than is the case in the neighboring State of Alabama. Yet Georgia stands ahead of Alabama in the average cotton product per acre, and is only a trifle behind in production per capita. In other words, the high position of Georgia is due, not to natural advantages, but to better cultivation of the soil, the use of fertilizers, and the thrift of an industrious population.

The geographical position of Alabama between the States standing at the head of the list gives double interest to the question regarding the cause of her position in the same, wlich would be the third place, but for the enormous area of Texas, where the sparse population has thus far picked the best lands. Alabama is a newer State than Georgia, and there reach into it from Mississippi the two belts of rich prairie lands, which terminate short of the Chattahoochie. Northern Alabama is almost identical in its agricultural features with Northern Georgia; and we should therefore expect to find a much more marked difference in favor of Alabama than is shown in the figures above quoted. The inference seems irresistible, that while Mississippi is still partly within the period of the first flush of fertility, and Georgia has reached the stage when the use of fortilizers is renovating her fields, the soils of Alabama have passed the first stage, and her population has not yet realized the necessity of sustaining the soil's powers by fertilization. 
Cotton culture in Florida is chiefly confined to the northern part of the State, lying adjacent to Georgia. This is mostly pine land, and is cultivated without manure; hence the low product of less than a quarter of a bale per acre. Notwithstanding this, there has been a respectable increase in production since 1870 , though not so large as that of the population; a circumstance doubtless due to the prominent position which the culture of tropical fruits has assumed during the past decade, and to which most of the new-comers have given their attention. No cotton is returned from the portion of the State lying south of Tampa Bay, and but little from the coasts, as well as from the extreme western part. The cotton-growing counties show an average product of 0.26 , or a little over a quarter of a bale per inhabitant.

Tennessee presents the striking fact of a total production less than half of that of Alabama, but with an average product per acre one-half greater; equal even to that of Mississippi. The cause of this state of things becomes apparent when we circumscribe the regions of production in accordance with the natural divisions of the State. It then appears that the portion of Tennessee lying east of the "central basin,"* from the eastern highland rim to the line of North Carolina, and comprising about one-third of the area of the State, produces only about one per cent. of the total amount of cotton; while eighty-four per cent. of this total is produced in the country lying between the Tennessee and Mississippi rivers. More than this, within this region, the average production per inhabitant is 0.52 of a bale, and a little less ( 0.49 of a bale) per acre; while the arerage for the entire State, per inhabitant, is only 0.21 of a bale. Again, of the above 84 per cent., 70 belongs to the two tiers of counties lying nearest to the Mississippi river. Of these, only a small portion is bottom-land of the Mississippi river; the greater part by far being gently rolling uplands ("table-lands "), such as form a large body in Nortbwestern Mississippi, also, and extend, gradually narrow. ing, as far south as Baton Rouge, Louisiana.

It thus appears that the cotton production of Tennessee is concentrated upon a comparatively small area of highly-productive land, the rest being devoted preferably to grain, tobacco, grasses, and other industries, to which the soils and climates are more specially adapted; while in the other cotton-growing States, cotton is very generally grown, as a matter of course, regardless of other cultures, of which the partial pursuit, at least, would in the end be more profitable than exclusive cotton-planting.

Arkansas produces its 608,256 bales on somewhat over a million of acres; making the average product per acre slightly higher than that of Louisiana, viz., 0.58 , and 0.76 of a bale per inhabitant. A cursory examination shows that by far the greater portion of the cotton produced comes from the eastern and southern portions of the State, which contains a large proportion of bottom lands; while in the extreme northern and northwestern counties but lit. tle cotton is grown. The form of the returns makes it difficult to segregate the production of the uplands and lowlands in this case; but the product, per acre, of Chicot connty stands second to the highest on the list, and it is safe to assume that, on detailed discussion, the average production of uplands and lowlands will be found to be, resfectively, about the same as in Louisiana. In both States, alike, the use of fertilizers in the large-scale production of cotton, may be regarded as wholly insignificant in its influence on the general result.

In the case of Louisiana, as in that of Tennessee, a considerable portion (about one. fourth) of the State is devoted mainly to other cultures than that of cotton; the sugar-cane gaining precedence in the lowland country lying south of the mouth of Red River, in which only about 6 per cent. of the total amount of cotton is produced, but at the average rate of 0.80 bale per acre. Nearly the same, or a slightly higher average per acre, is obtained in the alluvial lands north and west of the mouth of Red River, and in the Red River valley itself. The small parish of East Carroll, in the northeast corner of the State, has the highest aver-

* The "central basin" includes the valleys of the Cumberland, Duck, and Elk rivers, with tributaries. 
age product per acre of any county in the cotton States ( 0.95 of a bale), and stands second in total production within the State. It will be noted that East Carroll corners upon Washing. ton county, Mississippi, and adjoins Chicot county, Arkansas; both representing maxima of production in their respective States. We have here, apparently, the center of maximum cotton production on natural soils in the United States, and probably in the world.

The average product per acre in the uplands of Louisiana is approximately half of that of the lowlands $(0.41)$; and as the average for the State is 0.59 , it follows that somewhat more than half the acreage in cotton belongs to the uplands, while the lowlands yield nearly two-thirds of the entire amount. This predominance of lowland cotton explains the higher average product per acre in Louisiana, as compared with Mississippi, where less than onethird of the cotton production comes from the Yazoo bottom-lands. Within the cotton-growing region proper, the average production is approximately 0.95 of a bale per inhabitant; but as this figure excludes the entire population of the city of New Orleans, so largely interested in cotton, it is not fairly comparable with the proportion existing in other States. If one-half of the population of the city be taken as mainly interested in cotton, the per capita proportion would stand 0.80 bale.

The great State of Texas stands third in the list of total production, while first in population, among the cotton States. The fact shown by the figures of acreage and total production, viz., that in the average product per acre it stands eleventh in rank $(0.37)$, will be a surprise to most persons, and is doubtless in part to be accounted for as an accident of the season, the year 1879 having been an unusually dry one, and therefore especially unfavorable to a country in which so large a proportion of the staple is grown on upland soils. Among these, the heavy black prairie soils, so highly productive in favorable seasons, are notoriously the first to suffer from drought. It is probable that in ordinary seasons the average product per acre in Texas would approach more nearly that of Mississippi or South Carolina.

A discussion of the returns shows that 52 per cent. of the cotton product of Texas is grown in the northeastern portion of the State, north of the thirty-second parallel and east of the ninety-eighth meridian, and that within this region the production is highest in the counties adjoining Red river, the product averaging 0.54 bale per acre. Southward of the thirtysecond parallel the average yield is 0.34 bale per acre. The coast counties produce but little cotton; inland, between Red river and San Antonio, about 35 per cent. of the total product is grown on black prairie land, the average product per acre on such land being (in 1879) 0.34 bale per acre. A comparison of the returns of the present census with those of the preceding one shows that within the last decade the region of cotton production has extended 75 miles to westward. On the south but very little cotton is grown south and west of the Nueces river.

Compared to the area of fertile lands susceptible of cotton culture, the present cotton acreage of Texas is almost insignificant.

The cases of the two Carolinas, with respect to cotton production, are nearly alike, and may as well be considered together. In both States the average cotton product per acre is high as compared with that of Georgia and Alabama, and, in the case of North Carolina, approaches that of Mississippi itself. Without entering into details on the subject of the distribution of cotton production in these States, it may be broadly stated that the culture of cotton is reported to have greatly extended of late, even up the slopes of the Blue Ridge itself. Among the causes leading to this gratifying result, reports received show that the use of fertilizers, and, with it, better methods of culture, are foremost.

In other words, these two members of the original union of thirteen States have been first to place cotton culture upon a permanent foundation, by adopting a system of regular returns to the soil; and the high product per acre, as compared with Georgia and Alabama on the one hand, and with Mississippi on the other, exhibits tellingly the tide-wave advancing westward, 
the ebb of the first native fertility in Alabama and Florida, the rising tide of restored productiveness in the Carolinas, with Georgia on the westward slope of the wave, on which it is rising and showing distinctly a higher product per acre in its eastern than in its western portion, where the use of fertilizers is much less extended.

The concentration of cotton culture upon the most fertile lands, already so apparent in Tennessee, becomes even more so in Mlissouri, the most northerly region of large-scale cotton production.

It appears that Missouri stands at the head of the list for cotton-product per acre culti. vated in that crop, and it seems singular that this should be the case at the extreme northern limit of cotton culture. But the anomaly disappears when we locate the area of production; when it becomes apparent that it embraces almost exclusively the highly-fertilized lowlands lying at the head of the great St. Francis' bottom in the southeast corner of the State. Their product per acre must, therefore, be compared with that of others of a similar character, that of the Yazoo bottom, for example. Here the average product ranges between .80 and .86 of a bale per acre, to offset the .67 shown by the Missouri cotton area.

Assuming the soils to be similar in average fertility in either region, the difference is manifestly due to the comparatively short season for the development of the cotton plant in the latitude of the Missouri cotton region; and for the same reason cotton is there grown only on those lands where high fertility insures the most rapid development.

The cotton production of Kentucky pertains, in the main, to what has been appropriately styled the "penumbral" region of that industry. The bulk is produced in the counties lying adjacent to Western Tennessee and to the Mississippi river, the latter embracing portions of the rich bottom, with an average product per acre of from .48 to .56 of a bale. Eastward the cotton is grown in small patches, mostly for home consumption. Such small tracts being well cultivated, the product per acre is comparatively high, even so as to reach the average of the counties bordering on the Mississippi river, doubtless through the use of manure. Data, regarding the production of cotton in Virginia, are at this time too scanty to warrant a discussion. It appears, however, that there also cotton production has increased materially during the last decade, owing, doubtless, to the use of fertilizers.

Selection of Seed.-In the production of cotton, whatever the variety of seed planted, it makes a vast difference with the quality and yield of the crop, whether the seed was the product of a succession of carefully-cultivated products, or from those of slovenly and indifferent cultivation.

This is a well-known principle in vegetable physiology. It is also equally true that favorable conditions, such as good soil and proper cultivation, will secure a marked improvement in the quality of the seed of any plant-principles which the most intelligent and successful cultivators of the soil have strictly regarded. If any are indifferent in this respect, and have been accustomed to regard "one seed as good as another," we feel sure they would change that opinion by trying the experiment of planting only the very best seed produced by the most careful cultivation for a short time, and noting the result.

We are well aware that impositions have been practiced in this respect, and exorbitant prices have been obtained for what were claimed to be new and valuable varieties of cotton, which, when planted and matured, proved to be a product of either very ordinary or inferior quality.

Experience of this kind would have a tendency to produce skepticism with respect to new varieties highly recommended, -the natural result of fraudulent practice; but it must be borne in mind that the spurious and worthless are to be often found in almost every product of agricultural art, capable of being adulterated-a fact to be greatly deplored; but this does not prove the non-existence of that which is truly valuable.

When not grown on the same plantation, seed should be obtained only from the most 
reliable sources, since quite as much depends upon the quality of the seed, in the result of the future crop, as the soil and cultivation afterwards received.

Not only will the unfavorable conditions of soil, cultivation, etc., cause a deterioration in the quality of cotton-seed-the same as with other plants-but there is also an equal necessity of an occasional change of seed ; the constant use of the same seed year after year produced from the same soil having a tendency to deteriorate the quality of the crop, even with the best cultivation.

Careless and indifferent cultivation is, however, one of the surest causes of deterioration; hence, it is of the utmost importance that not only the best variety of seed be secured for the crop, but that it be such as was produced on good soil, and under the most favorable condi. tions of cultivation.

It is a principle in nature that "like produces like," and with the cotton, as well as with all crops, the best seed of the variety most adapted to the condition of culture in which the crop is to be grown will produce the most remunerative results. By careful and improved systematic culture, the cotton crop of the South will doubtless in a few years be double that of its present or former average yield on a given area. An improved agricultural system. the proper amount and kind of fertilizers, together with the use of modern agricultural implements which have been and are being introduced, will develop the agricultural resources of this section of our country, and the possibilities of the cotton crop, to almost an incredible extent. There is no portion of the civilized world, and no equal extent in the cotton-growing zone, so peculiarly adapted to the culture of cotton as some portions of the Southern States, especially those of the Gulf coast; there being found the rare combinations of large areas, with soil of requisite quality, the temperature required, together with the degree of humidity necessary; all of which are so essential to its perfect development and most successful culture.

Soil, and its Preparation. - The cotton plant is less exacting than most crops with respect to soil. If properly managed, it will thrive well on a variety of soils and on those of a widely different character.

It will also admit of being re-planted on the same lands for a greater number of succes. sive years than almost any other crop, although a judicious rotation produces the best results. For upland cottons, the best soils are generally conceded to be a rich loam, a clayey loam, and silicious soil with a clay subsoil.

This crop, with good culture, is also very productive on sandy soils underlaid by sand. stone or limestone rocks, and the rich alluvial deposits of bottom lands. With respect to the wide range of soils suited to its successful cultivation, we quote the following, translated from an eminent French authority, Guide Pratique de la Culture du Coton, par le Dr. Adrien Sicard, Secrétaire General de la Societé d' Horticulture, etc., of Paris.

"If we exclude lands composed of compact clays, with a soil of no depth and an imper. meable subsoil, we shall have eliminated from the vast variety of them, all soils which will not produce cotton. Indeed, recent studies and investigations have proved that, with the use of an appropriate fertilizer and proper cultivation, where the climate admits of the maturity of the bolls, cotton can be more or less profitably grown on all kinds and qualities of land. Thus, on the rocky sides of the mountains of Hindostan, in Africa, and on the dry hills of the West India islands, in soils which are too light and thin to produce any other crop, cultivators grow and make good crops of cotton. Further, cotton is so indifferent to the quality of land producing it that it grows almost equally as well on the rich loams of Egypt, in the pure clays of Syria, in the sands of Arabia, and on the volcanic fields of Sicily; while on the sandy second beaches of Georgia and the Carolinas the best cotton in the world is produced."

Soils deficient in the mineral element are not suited to the successful production of this 25 
crop, neither those in which there is a large amount of undecomposed regetable material. Cotton has a long tapering root, which runs perpendicularly and deeply into the soil in the early stages of its growth, and afterward sends out laterals, which furnish the small feeding roots of the plant. It is thought by some that the crop is increased in regions that are subject to frost, by decreasing the length of the root and thus increasing the laterals which furnish the necessary plant-food, for hastening the growth and maturity of the crop. A good authority says with reference to this subject:-

"We have reason to think that the radical system of the annual cotton plant gains more if we diminish the length of the tap root than if we increase it, and as evidence of this we offer the enormous crop produced on lands where the soil is very thin, but of excellent quality. Such are those about Sierra Leone, where cotton is grown on a soil of a few inches in thickness only, all beneath being solid, level rock. Here the tap root exists, as elsewhere, but the moment it encounters the rock its growth is arrested, and thereafter the laterals push out prodigiously, and gather nourishment enough to produce great yields of the staple."

This peculiar structure of the plant explains the reason for its early maturity and enor. mous yield, on certain very thin soils, overlying a rocky or impenetrable subsoil; it also shows the necessity of underdraining soils with a stiff clay subsoil that may be penetrated by the root, as a surplus of moisture about the roots is very injurious to the crop. When this cannot be conveniently done, open ditches may be the best remedy to avoid the evil. Where neither can be accomplished, ridge culture with furrows for draining between the rows, though not a very efficient mode, will prove to a certain extent beneficial. A proper system of underdraining is to be recommended as preferable to all other means of removing surplus water from lands, whatever the crop to be cultivated.

Underdraining has not only a tendency to carry off the surplus water from the soil, but permits it to remain sufficiently moist by the retention of the heavy dews, so common in the cotton-growing section, and also renders the subsoil better fitted for the long roots to penetrate it, thus obviating the evil effects of drought; hence, the cotton crop is greatly benefited by it in both wet and dry seasons, having a tendency to promote a greater uniformity of moisture, so essential to the perfect development of the plants.

In upland sections, which may be badly washed by heary rains, thus removing much of the fertilizing elements of the manure that has been applied, planters often resort to what is termed circle-ditching, or circle plowing, for the cotton crop.

By this method, the lowest point is found for an outlet of the water. From this point a ditch is made with a bank of earth firmly laid on the lower side to prevent washing. The ditch is then made to gradually wind around the hill, maintaining a slightly uniform and gradual descent. This ditch is usually made by plowing two or three furrows so as to lap over each other, and then cleaning out the last one with a hoe or trench-plow. In plowing such a field, the furrows should follow the curves of the ridge thus formed, never disturbing it, and the cotton should also be planted in rows following the same direction. By this means, many of the upland sections that would otherwise be injured by the heavy rains, are made to produce fine crops, and all evils from washing prevented.

A rotation with other crops is essential to the most successful results in cotton production. Cotton for two years is often followed by corn, sweet potatoes, and wheat or oats. Cotton followed by oats in January, where the climate will admit of it,-followed by cow.peas immediately after, a part of which may be cut for hay, and the remainder plowed under for green manure,-followed the next season with corn, and this crop again by cotton, is a very good rotation for most soils. Corn, oats, potatoes, and cow-peas are generally considered by planters to be the best crops for a rotation with cotton. The plowing should be rather deep, and the soil well pulverized, since the cotton-plant has a long root that penetrates down into the earth quite a distance, sometimes into the subsoil. Care should, however, be observed in 
plowing not to throw up the subsoil to the surface, and bury the surface soil beneath, as it is only in very rare instances that the subsoil is of such a nature as to benefit the surface or agricultural soil by being mixed with it; the soil on the surface that has been exposed to the atmospheric influences and mixed with decomposed vegetable material generally being the richest, and hence possessing the largest amount of plant-food.

The stalks of the previous crop are usually disposed of by being cleared off the field and burned before plowing, but sometimes they are broken down and plowed under for fertility to the soil, which they will furnish to a certain extent by their decay.

The best method is to plow in the fall, thus giving the soil all the benefits resulting from the action of the frost, while many of the eggs and larvæ of noxious insects will also be liable to be destroyed by this means. This should be followed by plowing again in the spring and thoroughly harrowing before planting.

The land for cotton is generally plowed in ridges, these ridges being made two or three weeks before the time of planting, in order to give the soil time to settle well before the seed is deposited. Just before planting, they are thoroughly harrowed to loosen the upper portion of the soil, after which the middle of the ridge is opened for the seed by the cotton. planter, drill, or other implement for the purpose. The ridges are so arranged that the rows of plants shall be from three to six feet apart, according to the nature of the soil, and each row stand upon a narrow elevation with a furrow between for the water. The richer the land, as a general rule, the farther apart should the rows be planted. On very poor soils, this distance is sometimes not more than two and a half feet; on very rich soils it sometimes exceeds six feet.

When stable manure or compost is used for fertilizing, it is generally placed in a furrow, and the plow used to throw the soil over it, thus leaving it covered in the middle of the ridge, where the roots of the plant can soon reach it. Where commercial fertilizers are used, they may be mixed with four or five times their bulk of dry soil, and be deposited in the hill at the time of planting, or placed in the hill and slightly covered with soil before planting. Chemical fertilizers should never come in direct contact with the seed, as they are very liable to injure the germ, being very strong and penetrating.

Various kinds of fertilizers are used for the cotton crop. It has been the practice in many sections of the South to produce this crop with little or no fertility added to the land, and hence exhaustion of soil has followed, with its attendant evil consequences. The pro. duction of the lint alone would not cause so very great an amount of drainage of the fertilizing elements of the land, but when we add to this the elements necessary for the production of the roots, stalks, leaves, and seed of the plants, it is not surprising that soils thus treated should become exhausted; the only surprise is, that they do not fail in this respect sooner than they do. It has been found by experiment that, when the seed is removed from the soil, the exhaustion is about twenty-five times as rapid as when the lint alone is taken, and fully equal to that caused by the production of corn or wheat. Hence the necessity of returning to the cotton-growing lands a substitute in the form of fertilizers of some kind, for what has been removed by the crop. Cotton-seed composted with other materials makes a valuable fertilizer for this purpose. Common stable manure is also a complete fertilizer, and is especially valuable to this crop. A compost of cotton-seed and stable manure, mixed with lime or plaster, is a very effective fertilizer. Commercial fertilizers are also very beneficial.

The most important mineral element removed from the soil by the cotton crop, and which must be supplied by the cultivator, is claimed by agricultural chemists to be phos. phoric acid. Potash and lime are also removed to a certain extent, but in smaller quantities. Phosphate of lime is also highly recommended for this crop.

Gypsum, bone-dust, and guano, as well as wood-ashes, have also been found very bene- 
ficial. There is probably nothing better than barn-yard manure made from grain.fed ani. mals, since this contains all the elements of fertility, and is to be recommended where it can be obtained in sufficiently large quantities for the purpose.

The local resources of the cotton-growing belt for the fertilization of the soil for this crop have been pointed out by a former Commissioner of Agriculture as follows, and shown to be abundant, accessible, and inexpensive:-

"Every farmer should rely mainly upon his stock for manures; hogs should be fattened upon field-peas; cattle and horses should be penned at night in deeply-littered yards. Accretions to the manure-pile may be made from a great variety of sources, including all decaying vegetable and animal matter, waste and wash from the kitchen, muck from the swamps, and pine straw or leaves from the forest.

There are many special fertilizers in this section ample for a perpetual supply of all possible drain upon the resources of the soil. The coast-line from Virginia to Texas, including all the sounds, inlets, bays, and estuaries, has an aggregate extent of thousands of miles, and every mile can furnish abundant stores of fish and sea-weed for manuring adjacent fields. Oyster-shell lime is also plenty and cheap in the tide-water regions.

No mineral manure is more abundant than marl, which is found in the whole tide-water section of the Atlantic coast, in the Mississippi Valley, and in Texas. It underlies wide belts at various depths, often very near the surface; it is in many localities easily obtained in large quantities; and its value, though variable, is undoubted for application for soils needing lime. Gypsum can be obtained from native beds at no great distance from any locality in the South. Lime is abundant in the mountain valleys from Virginia to Northern Alabama; and the 'rotten-limestone' formations of Alabama and Mississippi are unsurpassed for fertility.

All these home resources should be used in bringing up the average cotton yield per acre, and obtaining from half of the present acreage all of the fiber needed, leaving free a sufficient area to produce the bread, the fruits, the vegetables, the beef and mutton necessary for the home population, and a surplus of the lighter products for exportation."

Green manuring with cow-peas, oats, or clover will also be found valuable in restoring the fertility of partially exhausted lands, and a good fertilizer for the cotton crop, when permitted to lie long enough after being turned under to become thoroughly decomposed.

The quantity of fertilizers to be applied will depend upon various conditions, such as the nature of the soil and its productive capacity, and whether the seed is removed or not. Where the seed is returned to the soil, a less quantity of other fertilizers will be necessary.

The difficulty is generally in using too small an amount of fertilizers rather than too large, although, of course, a sufficient amount might be used to be deleterious to the crop. As a general rule, however, the richer the soil, the better the crop.

Cultivation.-When the soil has been well prepared to receive the seed, by ridging and then harrowing, the planting may be done with a drill or cotton-planter that opens the furrow, deposits the seed, and covers it at a uniform depth. This method is much to be preferred to hand-planting, since it is a saving of time and labor, and the work is done better than could be performed by hand, the seed being dropped more evenly and covered at a moro uniform depth, while the rows will be made more regularly, admitting of a better use of the cultivator, horse-hoe, or other suitable implements, in the after-culture of the crop.

When hand-planting is practiced, the ridges are opened with a light plow, or some implement that makes a small drill into which the seed is dropped, being afterwards covered by harrowing. Soaking the seed a day or two in tepid water, and rolling it in gypsum or wood. ashes before planting, will hasten germination, and cause it to come up sooner than when planted dry.

The quantity of seed necessary will depend upon the quality of the land, poor land requiring a larger amount than rich soils. From two to four bushels per acre are used. It 
is well to have an abundance of seed planted, since the enemies of the cotton-plant are so numerous and destructive that many of the young plants will be liable to be destroyed. If all the seed germinates, there will, of course, be a large surplus of plants, which must be removed by thinning. The drills should be straight, in order to facilitate after-culture. The distance apart of the hills varies from ten inches to three feet. The larger the distance apart, the larger the number of plants in a hill, as a general rule. The quality of the soil must also be taken into account, since the richer the soil, the larger and more thrifty the growth of the plants, and consequently the more room they will require.

Care must also be used not to cover the seed too deeply in the soil, as the crop will be injured by this means. In such cases, some of the seed will fail to germinate; others will produce but feeble and sickly plants. It should be well covered, however, from an inch to an inch and a half being the usual depth, sandy soil requiring a greater depth than rich, heavy loams or clays. The soil should be pressed down upon the seed to hasten germination, and the work thoroughly done. The time of planting will vary in different localities. In some sections, the planting is done as early as the middle of March, in other's it is delayed

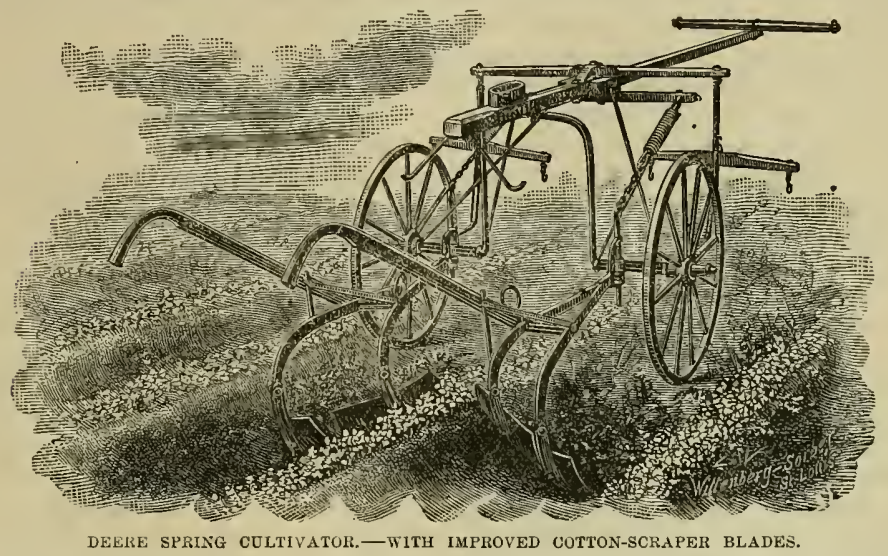

until the middle or 20th of May. While, with favoring conditions, an early planting insures an early harvest, yet there is danger of injury to the crop if planted before the land is sufficiently warm. The seed will not germinate if the soil is too cold and wet; they will, on the contrary, be liable to rot in the ground, while with a proper temperature, even with rather late planting, they will soon start and become well established in the soil.

Poor soils, however, necessitate an earlier planting than rich lands that are naturally rich, or have been made so by being heavily manured. One of the advantages of late planting on rich soil consists in the saving of labor, since if such lands are planted early, large quantities of grass and weeds will spring up and feed upon the soil, extracting much of its fertilizing properties, consequently injuring the cotton crop, and also requiring considerable labor to exterminate them. As the plant grows slowly on poor soil, a late planting, on such lands would not be advisable, as it would not have time to fully mature. The time before the young shoots make their appearance varies according to the time of planting, and the weather. Late-planted seed will start sooner than the extremely early, in consequence of the greater warmth of the soil, the time varying from five to fourteen days or more. With favorable temperature they will usually come up in from five to seven days. If the weather 
is unusually dry at the time of late planting, the seed will sometimes remain in the ground three or four weeks before starting, and when a rain comes will soon make a good staud.

The first cultivation should be given when the plants are well out of the ground, in order to check the growth of grass and weeds. The soil should also be kept light and mellow, which greatly facilitates the growth of the plants. The implements formerly used for this purpose, and at present to a certain extent, are the hand-hoe, sweep, harrow, plow, etc. In many sections, better implements, such as the horse-hoe and different varieties of cultivators, are being used quite extensively.

The Deere Spring Cultivator with cotton-scraper blades, manufactured by Deere, Mansur \& Co., St. Louis, Mo., is said to be a valuable implement in the saving of labor and the thoroughness with which it does the work. Many of the cotton-plows are also very valuable for this purpose.

When the plant has attained its third or fourth leaf, the thinning process is performed, together with the use of the hoe or cultivator. This should be done with care, the finest plants to be saved and the surplus ones removed. The distance apart at which the plants are thinned varies with the nature of the soil; rich lands requiring that the distance be greater than those of less fertility. In soils of medium fertility, the distance is from eighteen to twenty-four inches apart, three or four plants being left in a hill at the first thinning, which number is generally reduced, in after-cultivation, to one or two. After the thinning process, the fresh earth should be drawn around the roots of the young plants for support, and all weeds and grass kept carefully excluded from the hills. The after-culture should always be shallow, to prevent injury to the roots, especially in the use of the plow. The hand-hoe may be necessary in removing weeds growing quite near the plants or between the hills, but much of the labor of after-culture may be performed by other farm implements suited to the pur. pose, and involving less labor.

Plowing is frequently beneficial in hastening the growth and maturity of the plants. Care should be used, however, not to bruise or cut them, as they are very tender, and any such injury will affect their productiveness. The after-cultivation should be sufficiently frequent to exclude the grass and weeds, and keep the surface light and porous. Good culture is one of the great essentials in the cultivation of cotton, and a frequent stirring of the soil is necessary in order to keep out the weeds and permit the entrance of a sufficient amount of air and moisture.

The use of the sulky plows and cultivators, together with other improved agricultural implements, wherever introduced, have been of immense advantage to the planter in the cultivation of this product, not only in the saving of labor, but in securing better cultivation, and consequently larger crops and profits.

Harvesting.-Cotton generally grows in this country to the beight of from two to four feet, different varieties varying somewhat in this respect. The height it attains is also largely modified by the quality of the soil, the richest soils producing the most luxuriant growths. Unlike corn and other farm crops, cotton, being a perennial, which in some parts of the tropics becomes a small tree, continues to bud and blossom continuously, thus producing new flowers and fruit until the plant is killed by frost. In this country it is killed by the frost each winter, and its cultivation for a long period has fixed a tendency in the plant to produce lint and seeds, rather than wood. This habit of continued blossoming and ripening of its bolls until the black frost comes, necessitates several pickings or gatherings of the crop during the season. The plant generally opens its first blossoms from the middle of May to the first of June, according to the time of planting, the season, etc. The bolls turn brown and open in from four to six weeks after the flowers appear. Some planters have their fields picked regularly four or five times during the season, others but once or twice. The most energetic and systematic growers usually have the crop gathered as often as a sufficient 
amount is open and ready to be harvested, the rule being with many, to commence picking as soon as one laborer can pick a hundred pounds per day.

The ripening of the bolls is indicated by their turning a dark brown, and expanding, which causes the cotton to protrude. The picking is performed by hand. Heavy rains at this season sometimes cause considerable loss in the crop. It may be ginned as the picking progresses, or be first dried, and afterwards ginned. One of the most successful planters at the South says that it should first be dried until the seed will crack when pressed between the teeth, being frequently turned over and stirred so as to facilitate the drying process; after which it should be packed away to remain until ready for the ginning process, or it may be ginned as soon as dried. The same authority also says:-

"Having all things ready for picking cotton, I commence as usual, early, as soon as the hands can gather even twenty pounds each. This is advisable, not only in saving a portion of that from being destroyed, if rains should fall, which often do at this season (about the middle of August), but for another reason; passing through the cotton has a tendency to open out to sun and air the limbs that have interlocked across the rows, and hastens the early opening. On low grounds, especially, much loss is incurred in some seasons from the want of the sun to proluce an expansion of the fiber within the boll, so as to cause it to open.

If the cotton seed is not wanted, pack the seed-cotton away into the house, to remain until a gentle heat is discovered, or until sufficient for ginning; after it has heated until it has a feeling of warmth to the hand, and looks as if pressed together, open out and scatter it to cool. The cotton will gin faster, have a softer feel, is not so brittle, therefore not so liable to break by rapidity of gin, and has a creamy color. The wool has imbibed a part of the oil that has exuded by warmth of seed, and is in fact restored to the original color; for the oil. being vegetalle, is dissipated by sun and air, and the color by moisture (of rain and dews) and light. I have known of a number of sales made of this description of cotton, and even those who are most strenuous against the heating, admit it bore a better price."

The cotton should never be left on the field after being picked, and thus exposed to the weather, as is the practice in some sections. Such a course not only involves loss by an injury to the quality of the cotton, but large quanti. ties of the product often rot and becomes entirely worth-

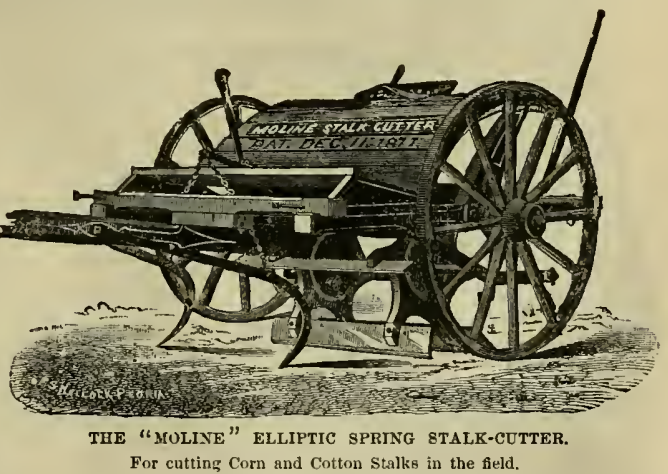
less by such exposure. When not ginned at once, it should be housed, and thus protected from the heavy dews and rains. After cotton is all harvested the stalks are usually left standing in the field until the time of plowing for the succeeding crop, when they are either pulled up and burned, or broken down and plowed under.

An easier method of clearing the field is to cut them with a stalk-cutter, when they can quickly be disposed of by burning, or other methods, as the judgment and circumstances of the planter may dictate. Various implements of this kind are in use for both corn and cotton fields, and are a great saving of labor. The above illustration represents an implement manufactured by Deere, Mansur \& Co., St. Louis, Mo. It may be used with one or two horses, as desired. 


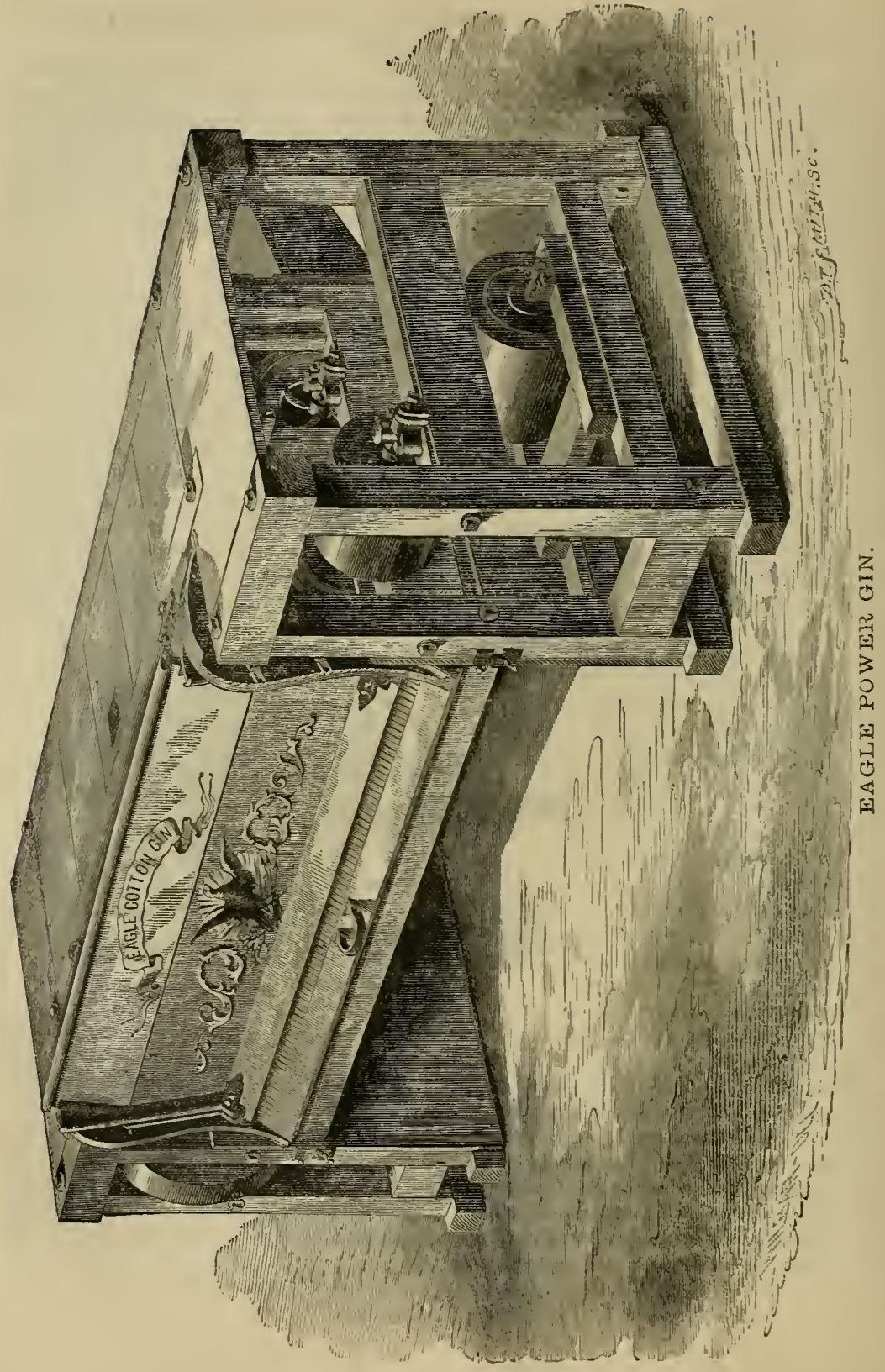


Ginning.-It is very important to the cotton planter's interest that this valuable product be well prepared for the market, as great loss has often been sustained by sending the cotton off in an imperfectly-cleaned condition, owing to a defect in the ginning process, either by the incompetency of those who performed this labor, or the use of poor machines for the purpose. In order to have it well ginned, good implements must be used, with competent supervision, and the cotton be in proper condition for the process. It should never be ginned when damp, neither should the process be unduly hurried. The gin must always be kept in good order, and used only under the direction of one who thoroughly understands the business. When used by a careless, indifferent person, or one ignorant of its mechanical construction, the work will probably not only be very poorly done by forcing the gin, and feeding in large compact masses, but the machine will also be liable to be greatly injured by such a course. In some sections of the South, we believe, it is the practice of a few planters to combine in the ownership of a gin-house. By this means the preparation of the cotton product for market is attended with less expense per individual; the best machines may be brought into requisition at a comparatively small cost to each, while the building could be centrally located with reference to the situations of the plantations of the several owners, so that there need be but little inconvenience in conveying the cotton to it for the purpose. Such an arrangement, to be generally adopted, would doubtless be attended with great benefit in the cultivation of this crop, since it enables many who are not able to build expensive ginhouses and supply them with first-class machines to cultivate this crop with greater profit.

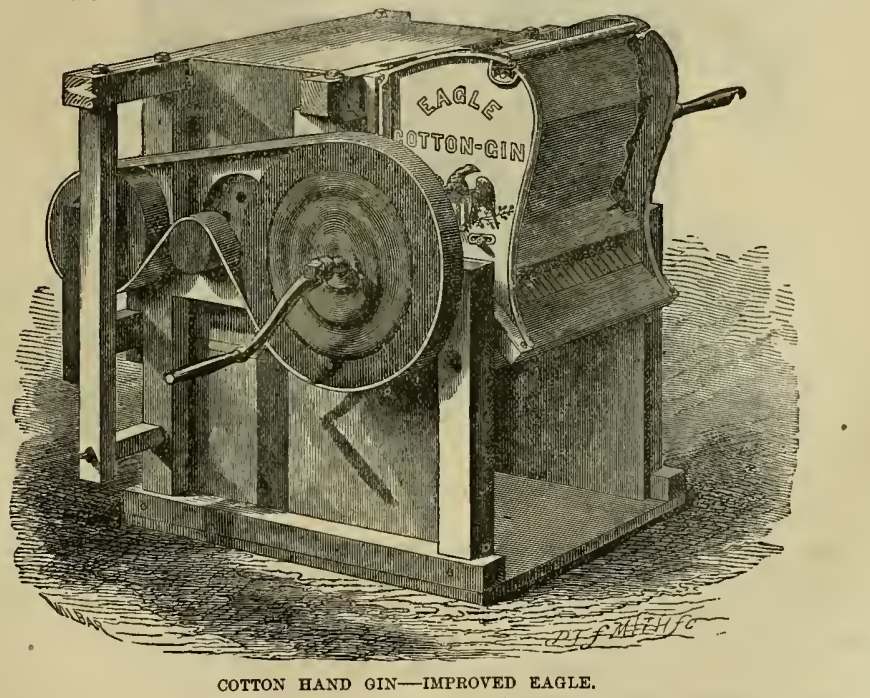

The original apparatus for freeing the cotton fiber from the seeds consisted of two rollers revolving in cpposite directions, between which the cotton was passed. The gin invented by Mr. Eli Whitney, in 1793, consisted of a hopper, one side of which was constructed of parallel wires, between which circular saws were made to revolve, the teeth of which drew the fiber of the cotton through the wires, leaving the seeds behind. Great improvements in gins hare of course been effected since that time, and at present the best of these implements seem almost perfect in their work. Many excellent machines of this kind are in the market, as well as some very inferior ones. The foregoing cuts represent the Eagle gin, manufactured 
by the Eagle Cotton Gin Co., Bridgewater, Mass., and is an implement the value of which is too well known to require comment. The power gins are similar in construction, though made much larger than the hand gin, and can be used with either steam or horse-power, steam being generally preferred. The machine should always be set firm and level, anc kept well oiled. Before using it, the operator should see that all the belts and bolts are tight, and that every part is in proper working order, being particular to notice that the screen cylinder and compression roller turn freely. The machine should always be started before putting in the cotton; the quantity of cotton from the feeder to the gin to be regulated by arrangements for that purpose. It will be some minutes after putting in the cotton before any machine will do its best work.

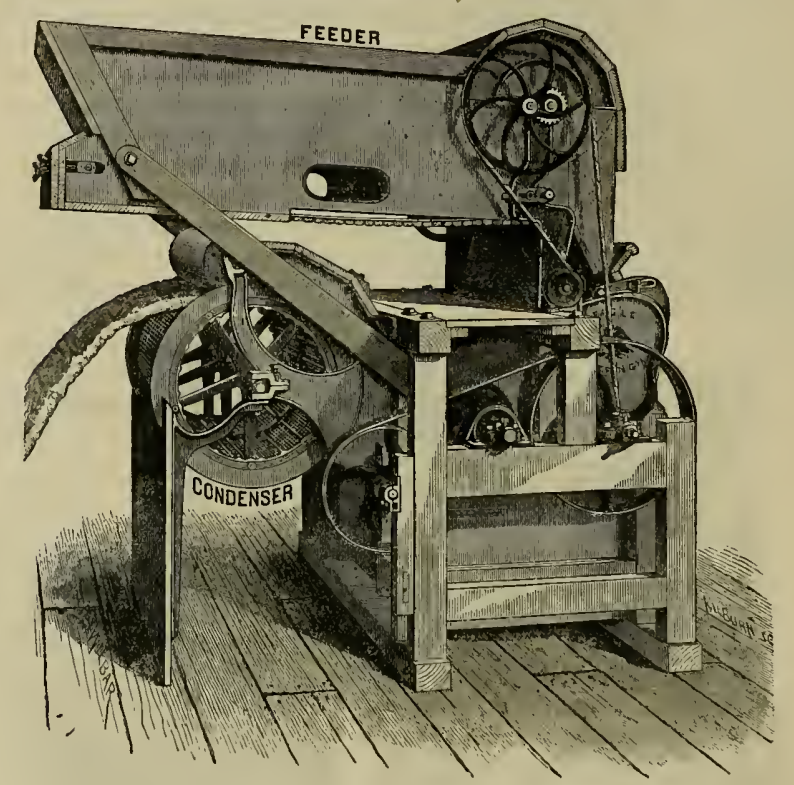

EAQLE COTTON GIN, WITH FEEDER AND CONDENSER ATTACHED.

After the ginning process, the cotton should be pressed into bales ready for market. This may be done by eitlier steam or horse-power, and requires comparatively but little time and labor. The press of Dederick \& Co., of Albany, N. Y., represented on a previous page, illustrates this process. When the bales are ready for market, there still remains for the planter to decide whether to make an immediate sale, or hold the product for an advance in the market-price. This question must be settled according to his knowledge of the state of the market at home and abroad, the prospect for the future, and his own judgment, guided by experience and observation in the past, together with his financial condition at the time. If confident that the price will be no lower, and that it will advance sufficient to repay for withholding it from the market for a time, it may be well to delay the sale. But, as a general rule in the disposal of crops, where a fair price can be obtained, and there are no positive indications of an advance, it is better to sell, than to depend upon uncertainty, and run the risk of the price being still lower. It would be very poor policy for any man to borrow 
money and pay the interest, rather than sell a product for a fair consideration, unless he had very good reasons for supposing that he could safely depend upon a sufficiently large increase in its market price to make such a course profitable.

The bales are usually made to weigh from 450 to 500 pounds. The lint, after the ginning process, is less than a third of the gross weight of the seed-cotton; the amount of seed in the cotton being about seventy per cent. of the entire crop gathered, or the gross weight.

Uses of Cotton Seed.-A few years ago cotton seed was considered almost worthless, no particular use being made of the surplus quantity that remained after planting. At present it is regarded as a valuable fertilizer; particularly when used in composts. Cotionseed meal is also one of the most useful kinds of food for farm stock, when judiciously fed to them. It is regarded by some as possessing twice the nutritive properties of corn-meal, and, being so rich, must be fed in small quantities.

About two pounds per day, with other fodder, are generally considered sufficient for a milch-cow, and from two to four ounces for a calf. It should always be given mixed with other food, or in connection with it. It should never be fed to swine, except with bran or roots. It is stated by good authority that when used in the proportion of one pound to half a peck of boiled potatoes, it makes a good food for fattening pigs.

Horses do well on it if not given more than two pounds per day with meal or oats; but if fed too generously with it, it will have an injurious effect upon the system, inducing inflammation of the bowels and kidneys. Sheep will also thrive on it, when fed in connection with hay or roots. Analysis proves that a ton of cotton-seed meal contains 38 pounds of potash, 56 pounds of phosphoric acid, and 78 pounds of nitrogen, which shows its value as a fertilizer, and as an element in manure when fed to stock.

During 19 years of continuous cropping from the same land, Mr. Lawes of Rothamsted applied on adjoining plots, annually -

1000 lbs. rape cake (with 300 lbs. mineral superphosphate), with $50 \mathrm{lbs}$. nitrogen.

$200 \mathrm{lbs}$. ammonia salts (with $300 \mathrm{lbs}$. superphosphate), with $41 \mathrm{lbs}$. nitrogen.

$275 \mathrm{lbs}$. nitrate of soda (with $300 \mathrm{lbs}$. superphosphate), with $41 \mathrm{lbs}$. nitrogen.

And in each instance harvested annually about 48 .bushels of barley and 28 cwts. of straw, containing together 56 pounds of nitrogen.

The oil expressed from the seed of cotton is also valuable for various purposes. The seed is very rich in oil, and will usually yield from fifteen to twenty per cent. of its own weight. After expressing the oil, the cake is used for food for cattle and other farm stock. The seed should never be fed whole or raw to animals, as this practice will frequently be attended with fatal results, besides much waste will be occasioned by it. It should either be ground into meal, or boiled before being fed. The former is the better and easier method of disposing of it. In order to utilize cotton seed to the fullest extent for food, since the harsh hulls are injurious to the stomach and intestines of animals, and also retard the decomposition of the seed when used as a fertilizer, a huller of some kind is found very serviceable in removing the hulls or outer covering of the seeds. These machines may be used by hand, horse, or steam power; also to advantage in grinding and cracking corn or peas. The illustration represents a hand-machine of this kind manufactured by David Kahnweiler, New York city, and is said to hull from three to four bushels per hour; the power hullers for larger plantations hulling a much larger amount, according to the size.

Rank of American Cotton, etc.-As has previously been stated, the United States stands first among the countries of the world in the amount and quality of its cotton products, the famous Sea-Island cotton taking first rank, since it surpasses all other varieties in the length, strength, and beauty of its fiber, it being very long and exceedingly soft and silky. The fact of the superiority of our cotton product was signally demonstrated at the Cotton 
Exposition at Atlanta in 1881, where specimens from different sections of the cotton-growing belt of the whole glohe were exhibited, the best that Egypt, Persia, India, Russia, Mexico, and other cottor-growing countries could produce, being brought in close comparison with our own; which comparison substantiated the fact beyond question, that, whether taken in part or as a whole, the cotton produced in the United States is so superior to all others, that it gives to our country a practical monopoly of the cotton markets of the world. The superior excellence of the quality of the product, together with the extent of the cotton belt and the facilities for cultivating cotton that the Southern States afford, give to that section of our country opportunities of no small import, and augur well for the future development of its

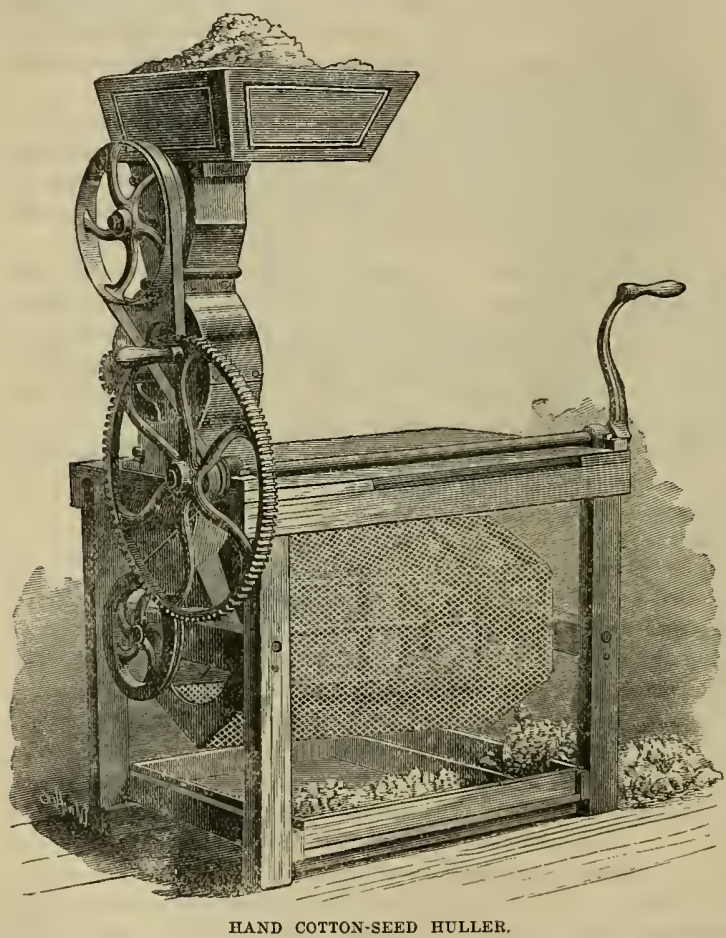
resuurces, which have never yet been fully tested.

When we remem. ber that, in the year 1830 , the crop of cotton produced in this country reached only 1,000 , 000 bales, that the larg. est amount ever pro. duced prior to 1860 was only a little over 4,500 , 000 bales, and that the crop of 1881 was about $2,000,000$ bales in ex. cess of the latter number, we can easily see that the possibilities of the cotton production of the United States have not yet been reached, and that the future outlook is indeed promising. The heavy increase in the cotton crop during the last few years is mainly attributable to the increase of the cotton acreage by the use of suitable fertilizers; thousands of acres of land formerly supposed to be beyond the possible limit of the cotton belt having been made into the best of cotton lands by being artificially enriched, thus hastening the maturity of the crop, as well as the increased product, while partially exhausted lands have been restored by the same means.

Prof. Morehead, President of the Mississippi Valley Cotton-Planters' Association, states that the entire cotton crop of the year 1881 might have been easily raised in fourteen counties bordering along the Mississippi river. The capacity of the South for cotton production seems almost limitless, and when we consider the enormous demand for cotton goods from all the different parts of the world, and that this is the principal quarter of the globe best adapted to furnish the supply and meet the demand, there is truly a great inducement to Southern 
planters to avail themselves of every improvement and facility within their power for the cultivation of this valuable product.

- Enemies and Diseases of the Cotton Plant. - The enemies of the cotton plant are numerous and often very destructive. The cut-worm is generally the first to make an attack, sometimes eating off the tender shoots just after making their appearance from the soil. A top-dressing of wood ashes, or plaster mixed with the earth around the roots, will sometimes check the evil. It is noticeable that where commercial fertilizers are used in the soil, this pest is less common.

Aphides, or Cotton-Lice, are sometimes very damaging to the crop, as they attack the plant in the early stages of its growth, sucking the juices from the tender stalks and leaves, causing them to turn yellow and drop off. These insects are very small, but at the same time are very prolific, and when numerous are quite damaging to the crop. They are generally most troublesome when the spring has been wet and cold.

They have many enemies that prey upon them, among which the most important are the ants and birds. Paris green is often used with good effect, both in powder and dissolved in water, as recommended in a previous part of this work for the potato-beetle. Great care should be exercised in its use, as it is a deadly poison. It is a fact worthy of notice that all insect pests are less troublesome in localities where birds are numerous, and their presence should always be encouraged.

The Caterpillar, or Cotton-Worm. - This pest is sometimes called the "cottoncaterpillar," but is more generally termed the "cotton-worm," in contradistinction to the "boll-worm." It is one of the most destructive enemies of the cotton crop with which the planter has to contend. It is stated that in the year 18 13 , this caterpillar, or worm, caused by its depredations an estimated damage of about twenty millions of dollars, in the course of two weeks. According to the best authority, it is indigenous to South America, and is an introduced insect in the United States. The first appearance of it in this country, now on record, was in 1793. This period was followed by an extremely disastrous invasion in 1804, and from that time until the present, there has never been a season in which the cotton worm has not done more or less damage to the cotton product in some portions of the Southern States.

The worm is hatched from an egg deposited by the female moth. The four states or stages of its development are given as follows by Prof. C. V. Riley, and will enable any one unacquainted with any or all of them to recognize the species in any phase of its growth, and to distinguish it from other insects; besides, a correct knowledge of its habits will enable the planter to better defend his crop against its depredations.

"The Egg. - In this stage of its existence, the cotton-worm is known to but few people, both its color and size shielding it from observation. Every planter should, however, not only become familiar with its appearance, but know just where to look for it. With this knowledge, time may be gained, the loss of which in the application of remedies may result disastrously.

The egg is $0.6 \mathrm{~mm}$. wide, circular, much flattened and ribbed. Of bright bluish-green or sea-green when first laid, it contrasts sufficiently with the warmer green of the leaf to be easily detected, even by the naked eye, when practiced. It is laid singly, and fastened with such firmness as not to be easily removed without injury. It is laid by preference during early summer on the under side of the larger and lower leaves, and seldom more than three or four are found on one leaf.

In confinement, and exceptionally in nature, it will be laid on the upper surface of the leaf, or on any other exposed part of the plant. In autumn, more particularly, the upper leaves receive a due share of the eggs, and I have counted as many as forty-nine eggs pand 
egg-shells on a single leaf. With development the color becomes more dingy, or pale yellowish, frequently with brownish borders or a green curve, due to the coiled embryo, which may be seen through the transparent shell. The young worm or larva eats its way out through an irregular hole on one side, usually during the morning, ere the dew is dissipated, and from three to four days after oviposition. This is the average time elapsing between the laying of the egg and the hatching of the worm therefrom in ordinary midsummer weather, but the time varies with the temperature, and a much longer period is required in spring and late autumn.

All eggs perish that are unhatched when overtaken by frost, as is not infrequently the case. The vacated and glistening shell is more readily noticed upon the green background than the unhatched egg.

Humidity seems to favor hatching. Aphides or plant-lice are quite often mistaken for the eggs of this insect, while the "Mealy bug" (Dactylopius adonidum), a species of Aleurodes, the eggs of the lady-birds (Coccinellidee), those of the lace-wings (Chrysopa), and even a minute snail, not uncommon on the cotton plant, are likewise so mistaken.

The Worm or Larca. - This is familiar to every planter. Varying greatly in ground. color, it is characterized by the particular position of the black piliferous spots upon the head and upon the body; by the white ring which surrounds each of the latter; by its pure white subdorsal lines and by its elongate and slender form. It is semi-looper, the first pair of prolegs being very much reduced in size and seldom used, and the second pair, though longer, only about half as long as the succeeding pair.

The worm molts five times during growth and changes appearance but little after the first molt. Exceptionally only four molts are suffered.

The newly.hatched worm measures $1.6 \mathrm{~mm}$., is of a uniform pale dingy yellow, with polished black, slightly elevated spots, each bearing a short pale hair. Before the skin is shed, the color often becomes slightly greenish, and sometimes inclined to orange. After the first molt the piliferous spots are more conspicuous, the hairs from them longer and black, and the characteristic markings appear, though less distinctly than after the second; but from this time on, the prevailing color is exceedingly variable, being either of various shades of pale or pea-green, or more or less intensely black along the back.

The normal number of larval molts is five. This is the number which I have observed during the autumn months, while in the midsummer, when the development is more rapid, I have on several occasions traced but four. The term of larval existence varies from one to three weeks. There is a very general behief among planters that the first worms of the season are pale and the later ones dark, and while light and dark worms may always be found together in spring, summer, or fall, it is true that the green ones predominate earlier in the season and the dark ones later.

Immediately after molting, the body is pale and without marking,-a rule with all molting animals. After the earlier molts, the cast-off skin, which remains more or less fully stretched, is sometimes eaten. Some of the peculiarities of the habits of the worm deserve mention here because of their practical bearing.

Until after the second molt, it always remains on the under side of the leaf, feeding upon the parenchyma, and leaving untouched the coarser veins, stomata and upper skin or epidermis. The leaves where they are thus feeding present a blotched appearance, the semitransparent epidermis becoming pale yellowish, and these blotches are, as a rule, at once distinguishable from other somewhat similar ones made by a few other insects.

After the worm begins to eat entirely through the leaf, which is usually before the third molt, but sometimes later, it instinctively ascends toward the top of the plant and feeds on the more tender foliage, "ragging" it, to use the expressive language of the planter.

- It can let itself down by a web from the moment of birth, but can also fling itself from 
one part of the plant to another in a manner quite characteristic. The fling or jump is made by bending the fore and raised part of the body to one side and then suddenly jerking it to the opposite side, relaxing meanwhile the three hind pairs of legs by which it held to the plant. This is a quite common mode of motion when disturbed, and the normal way of getting from one plant to another. The maximum distance which a worm can thus jump in a horizontal direction is about two feet, and it almost invariably alights on its legs. During chilly weather in autumn this motion is feeble and can be easily watched. When not feed. ing, the worm either rests stretched straight on some part of the plant, or may be seen swaying its fore-body from side to side, holding the while by the hind prolegs.

Though preferring the foliage, it will, when hard pushed, eat every exposed part of the plant, even barking and girdling the stems. In feeding on the bolls, however, it does not bore like the boll-worm (Heliothis armigera), but eats the external parts as well as their contents. It cannot thrive on any other plant than cotton, and is evidently confined in its diet to the different species of the particular genus Gossypium. At all events, there is yet no satisfactory evidence to the contrary, all experiments made confirming the belief.

As one correspondent naively puts it, "the worms feed only on cotton and one another," the cannibalistic propensity being freely indulged, when the occasion presents. It is a common remark that the presence of the worm is easier detected by smell than by sight. The planter says that he can "smell the worm." There is a peculiar ordor arising from the excrement, but particularly from the gnawed and mutilated leaves, that gives rise to this saying; but where the worms are numerous and large enough to render it obvious, there they have already existed several days, perhaps weeks, in smaller numbers.

When numerous enough to utterly defoliate a field before they have attained full growth, the worms will travel in all directions on the ground, and they have been exceptionally known to collect together and travel in vast bodies in their search for fresh food.

The Chrysalis.-Having obtained full growth, the worm, in the language of the planter, "webs up." forming for protection a more or less perfect cocoon, usually within the fold or roll of a leaf, sparsely lined with silken meshes. Here it contracts and thickens, the distinctive marks are nearly obliterated, and the green color acquires a verdigris hue. Witlin twenty-four hours in midsummer, the skin splits just back of the head and is gradually worked to the end of the forming chrysalis, now soft and green, but acquiring in the course of an hour or more a brown color and firmer consistence. This chrysalis state lasts, on an average, about a week in hot weather, but may extend to thrice that time, with lower temperature. Where necessity obliges, the worm will spin upon any other plant, or in any situation that offers shelter.

In confinement, it will make a cocoon on the ground, covering and disguising the same with particles of earth, or it will even transform on the ground without silk or shelter. Such cases rarely, if ever, occur in a state of nature, but when the worms are very numerous in a field, the chrysalides frequently have their leafy protection eaten away, so that many of them either hang by the few hooks at the extremity, or fall to the ground.

In no case, however, does the worm burrow in the ground as does the boll worm, or could the moth issue from the chrysalis were the latter accidentally buried even an inch beneath the surface.

The Moth, or Imago.-The moth measures from $1 \frac{1}{8}$ to $1 \frac{1}{2}$ inches from tip to tip of wings when these are expanded. Its general color, above, is ohivaceous, more or less effectually subdued by lilaceous or purple hues, and often having a clay yellow or faintly golden cast. The under side is more gray with nacreous reflections.

The markings that more particularly characterize and distinguish it from other North American moths are certain undulating vinous or carmine lines across the front wings, a dark oval spot near their disc containing pale scales which usually form a double pupil (the 
basal or inner one the smallest and whitest), and three white specks dividing the space between this dark spot and the shoulder in about three equal parts.

The habits of this moth can only be studied at night, as, like almost all the rest of its family, it is nocturnal. During the day it simply starts up when disturbed, and darts by swift and low flight to some other sheltered spot a few yards, or perhaps rods, away. After sunset, however, it may be seen leisurely hovering about, either bent on the perpetuation of its kind, or feeding upon whatever sweets it can get, whether from the cotton or from other sources. It is very strong and swift of wing, and capable, when the necessity arises, of fly. ing long distances. In alighting upon the plant, it generally turns its head downward, and when it rests, the wings are but shallowly roofed. the front ones closed along the back and fully hiding the hind ones. In this respect it may always be distinguished from the parent of the boll worm, which rests with the front wings partly open and not entirely covering the hind ones.

The female begins to lay her eggs in from two to four days after issuing from the chry. salis, the time varying with the different generations and according to temperature. In experiments which I have made with moths confined in vivaria, eggs have sometimes been laid thirty-six hours after issuing, and the moths have continued laying for twenty-one nights, the number laid each night ranging from 4 to 45 .

Examination of the ovaries of females at different seasons shows a much greater prolificacy than belongs to most moths, as the number of well-developed ova may reach 500 , and of potential ova half as many more. In confinement, it is difficult to obtain from one female more than 300 eggs, but that fully double this number are produced in the field dur. ing the height of the season there can be little doubt, while the average number may be esti. mated at about 400 .

The natural food of the moth is the sweet exudation from the glands upon the mid-rib of the leaf and at the base of each lobe of the involucre of the cotton plant. Nevertheless it is attracted to all kinds of sweets, and in most parts of the South it finds a bountiful supply in the exudation from the spikes of Paspalum love, a tolerably common grass, but particularly in that copiously secreted by glands at the apex of the peduncle, just above the pods of the cow-pea (Dolichos). In the spring of the year, as Judge Bailey, of Marion, Ala., has observed, it may often be seen in the evening feeding in numbers, first from the blossoms of the Chicasaw plum, and subsequently from those of the peach, Chinese quince, mock orange (Cerasus Carolinensis), the early apples, and blackthorn. Later in the season, when the glands above mentioned begin to exude and the tree blossoms are no more, the moths do not seem to be attracted by other nectar-storing flowers, since observations during the past two years by myself and assistants have resulted in finding but one species of verbena (Verbena aubletic L.) frequented, even where both moths and all sorts of flowers were abundant. But frnits of all kinds, as they ripen, are resorted to, and figs, apples, peaches, plums, apricots, grapes, persimmons, and even melons are often greatly injured.

Carefully examined, the tongue is seen to be armed along its terminal half with stout and sharp spines projecting forward from the upper surface and increasing in density toward the tip, which is beset with them on all sides. It is by means of this spinous tip of the tongue that the moth works a hole in these fruits, and is thus enabled to absorb the more liquid portions. Apple pomace is especially attractive to them.

The time elapsing from one generation to another varies according to temperature, and therefore according to season. There is increasing activity and acceleration in development from the first appearance till July, and thenceforth decreasing activity, and retardation in development till frost. Thus in midsummer the whole cycle of individual life, from the hatching to procreation, may occupy less than three weeks; while in spring and late autumn it may occupy twice that time. Taking the whole season through, however, the time from the eggs of one generation to that of another will average about a month. 
Wet weather favors the development of the worms. These pests have many natural enemies to assist in reducing their numbers, foremost among which are the birds and ants. Bats devour large numbers of the moths, while all domestic birds, chickens, turkeys, guinea. fowls, and geese will feed upon them.

Reptiles, such as the tree frog, will eat them, while the skunk, opossum, and raccoon are said to devour them. The former, however, will break down the plants in so doing, and neither of these animals, or hogs, can be of much assistance in their extermination, except where the worms are in such large numbers as to travel the ground from field to field.

Various insects, such as certain species of spiders, wasps, beetles, bugs, flies, crickets, etc., prey upon the cotton worm, while several parasites are known to be quite effectual in their destruction.

Some of the means of obviating the evil may be found in the mode of cultivation, among which are the planting of the most thrifty and vigorous varieties, those that will pro. duce the strongest plants, also mature soonest, such as seed from the more northerly sections. Early planting is also advised, together with frequent cultivation, in order to hasten the maturity of the plant and secure a portion of the crop beyond the reach of harm from the more destructive broods that make their appearance in July and August. Frequent cultiva. tion also has a tendency to knock off and distribute the worms. It is also thought that some varieties of cotton are more subject to attacks than others, the long staple being found to be more injured than the short staple, when planted side by side. Topping the cotton is sometimes resorted to in hastening the maturity, and also to destroy the worms that feed upon the tender part of the plant, but this practice occasions considerable labor and often with very little good resulting from it.

Various implements for brushing or knocking the worms from off the plants to the ground and crushing and killing them have been devised, but the most effectual mechanical means for their extermination has been found in the use of Paris green, althongh arsenic, London purple, pyrethrum powder, etc., have been employed with greater or less benefit. More or less danger attends the use of all poisons, by inhalation, or getting them upon the hands, while these applications possess the further disadvantage of kilhing the enemies of the worm, as well as the worm itself. Birds that eat the worms thus poisoned will be poisoned hy them; also the various insects which destroy the worm.

But Paris green is the best remedy thus far tested, although attended with disadvantages, and we hope the time is not far distant when some better method will be devised. It may be applied either dissolved in water or in powder. When used in powder it is usually mixed with flour, plaster, or wood ashes, as this is a more convenient and economical practice; besiles, the pure powder is liable to injure the cotton plant. For the proportions of such a mixture, quantity to be applied, methods of application, etc., in order to avoid repetition, we refer the reader to directions previously given in the use of Paris green in connection with the destruction of the Colorado beetle, or potato-bug, in the cultivation of the Irish potato.

The Boll-Worm. - This is also a very destructive enemy of the cotton plant, which works upon the small bolls or buds, causing them to fall off, and thus seriously diminishing their rate of production.

It feeds upon many plants beside cotton, and is known by many different names in different countries and sections of a country, according to the plant upon which it feeds most. For instance, throughout the cotton-growing States it is known by the name of the "bullworm"; in the Western States, and other localities where it infests the corn crop, it is called the "corn-worm." In many of the Southern States it is said to be known in the early part of the season as the "corn-bud worm." When found upon tomato plants it is termed the "tomato-worm," etc., it heing the same species-wherever found. It was not known for many years that the cotton-boll worm and the corn-worm were the same, but the fact has now 
become fully established. They eat into the stalks and ears of corn, the same as into the cotton bolls; also into the green as well as ripe tomatoes, causing them to rot. They will also eat the leaves of plants when they can find nothing better. It will bore into the pcds of the garden pea, devouring the entire contents of one pod before leaving for another. The heads of hemp are not exempt from its depredations, but often suffer from its attacks.

The eggs are laid upon all parts of the plant, but, according to authentic sources, usually in cotton upon the outside of the outer calyx, or young boll. One authority says respecting the place in which the moth deposits her eggs: - "On one cotton plant I found eleven eggs, which were distributed in the following manner: one on the outer calyx of the boll, two on the stalks, and eight on the leaves."

The duration of the egg before hatching varies with the season, as it does with the cotton-worm. The young larvæ feed upon the part of the plant upon which they are hatched, for a longer or shorter time, but eventually make their way to the flower-bud or boll, into which they eat. They are sometimes seen in the full flower. A description of these worms is given in the Report of the Department of Agriculture, as follows:-

"As the boll-worms increase in size, a most wonderful diversity of color and marking becomes apparent. In color, different individuals will vary from a brilliant green to a deep pink or a dark brown, exhibiting almost every conceivable intermediate stage, and from an immaculate, unstriped specimen to one with regular spots and many stripes. The green worms are more common than those of any other color; but those of varying shades of pink or brown are so abundant as to make it impossible to fix upon a type. Early in the season the prevailing color is green. A common variety is light green in color. Running from the first ring back of the head to the posterior end of the body on each side is a broad whitish line; just above is a broad dusky line; down the center of the back is another dusky line, or stripe, as it should preferably be called; this dorsal stripe has a narrow white line down its center, and it is bordered on each side by a narrow white line. Between the dusky dorsal and lateral stripes run four or five very faint, wavy, longitudinal, white lines, so faint as not to interfere with the general color of the body. Each body-ring has eight black spots, which, upon being examined with a lens, are seen to be tubercles, each with a stiff hair upon its tip. These spots are arranged in two transverse rows of four, the spots in the front row being slightly closer together than those in the back row; the outer spot of the back row is small and placed nearer the front row.

Of these features the most constant seems to be the whitish stripe on each side. When the boll-worm is brown, these stripes assume a yellowish hue. Another pretty constant feature is the relative position of the tubercles just described. They are not always of a contrasting color to the rest of the back, and hence cannot always be spoken of as spots. When they are not discernible as spots, however, an examination with the lens shows them still present as tubercles, each surmounted by a hair. This point affords apparently a good and reliable means of distinguishing the young boll-worm from the young cotton-worm, which otherwise might prove a matter of difficulty during the earlier stages and in the early part of the year, before black cotton-worms are to be found. In the cotton-worm the two middle spots of each of the two rows of four are of the same distance apart, so as to form the four corners of a rectangle. In the boll-worm, however, the two middle spots of the hind row are more widely separated than the corresponding spots of the front row. This distinction may be recognized at a glance when the eye has become accustomed to it. The dusky dorsal stripe is often wanting, as also are the dusky lateral stripes, and, as just stated, the spots are often indiscernible."

The general color of the body and upper wings of the moth that lays the eggs varies from a light gray, tinged with olive green, to a rich yellow gray or tawny color. The most prominent feature in the marking of the boll-worm moth is a broad black band on the back 
wings. When at rest this moth does not fold its wings like the Aletia or cotton-worm moth, but keeps them partly open and slightly raised. It is difficult to determine the number of broods of this worm, in a single season; but, from careful observations recently made, there are supposed to be at least five. They, however, retire from the field sooner than the cottonworm, that usually sends out another brood after the boll worm progenitors have left for winter quarters. The same remedies with respect to the destruction of the cotton-worm are applicable to the boll-worm, their natural enemies of birds and insects being the same, while the use of Paris green is also available to a certain extent. As the worms enter the bolls, however, they are more difficult to reach by applications of this kind; yet those hatched upon the stalks and leaves that have not migrated to that portion of the plant may be destroyed by this means. Planting rows of corn occasionally through the field, late in the season, is highly recommended by some, as the moth that lays the eggs prefers corn, and will be liable to choose it instead of cotton.

Fires built in the early evening at the edge of the cotton-field will attract many of the moths, and large numbers are frequently destroyed in this way. Too much cannot be said, however, in favor of the protection of birds of all kinds that feed upon insects, for they are the best friends and aids of the farmer in destroying injurious insects of all kinds.

There are other insects, besides those already mentioned, that attack the cotton-plant, but their injuries are slight in comparison with the former, and the same remedies for their extermination are equally applicable.

Diseases of the Cotton-Plant. - These are not numerous, though often resulting in considerable injury to the crop. There is a species of rust that sometimes proves very disastrous in certain seasons, which is quite similar in its cause and effects to that which proves so destructive to the wheai crop at the North. This disease is occasioned by parasitical plants which attach themselves to the stalk, from which they absorb the sap and thus weaken its vitality and productive power.

Weak plants are more liable to be attacked by this disease than those that are strong and thrifty, and it is also more liable to occur on coarse, sandy, or ill-drained soils than others. Potash is considered a good remedy; consequently the use of those fertilizers that contain the element of potash is highly beneficial. A proper system of drainage is also highly essential on wet lands. Liberal manuring, careful and frequent culture, together with the planting of the most vigorous and hardy varieties, combined with what has been previously suggested, will prove an efficient remedy in most cases.

Blight and other kindred diseases are supposed to be traced to the same general cause, which is a lack of some essential element of plant-food in the soil. By supplying a liberal amount of plant-food through fertilizers of some kind, removing the surplus water by a proper system of drainage, and giving the growing plants frequent cultivation, these evils may be largely, if not wholly, avoided.

Rotting of the bolls is generally attributed to long-continued wet weather. When the plants are crowded so as to form a very dense foliage, excluding the air and sunlight, the bolls will also rot and much of the crop will be lost by this means. Planting farther apart would remedy the evil when the latter is the cause. Breaking off some of the limbs, causing a better circulation of air and admitting the sunlight, will generally also prove effectual. Sometimes a mule is driven between the rows for this purpose. Shedding is attributed to very dry weather being followed by wet; hence, in seasons rather wet, this evil does not appear, but they are the periods when the aphides, boll, and cotton-worm are the most destructive. 


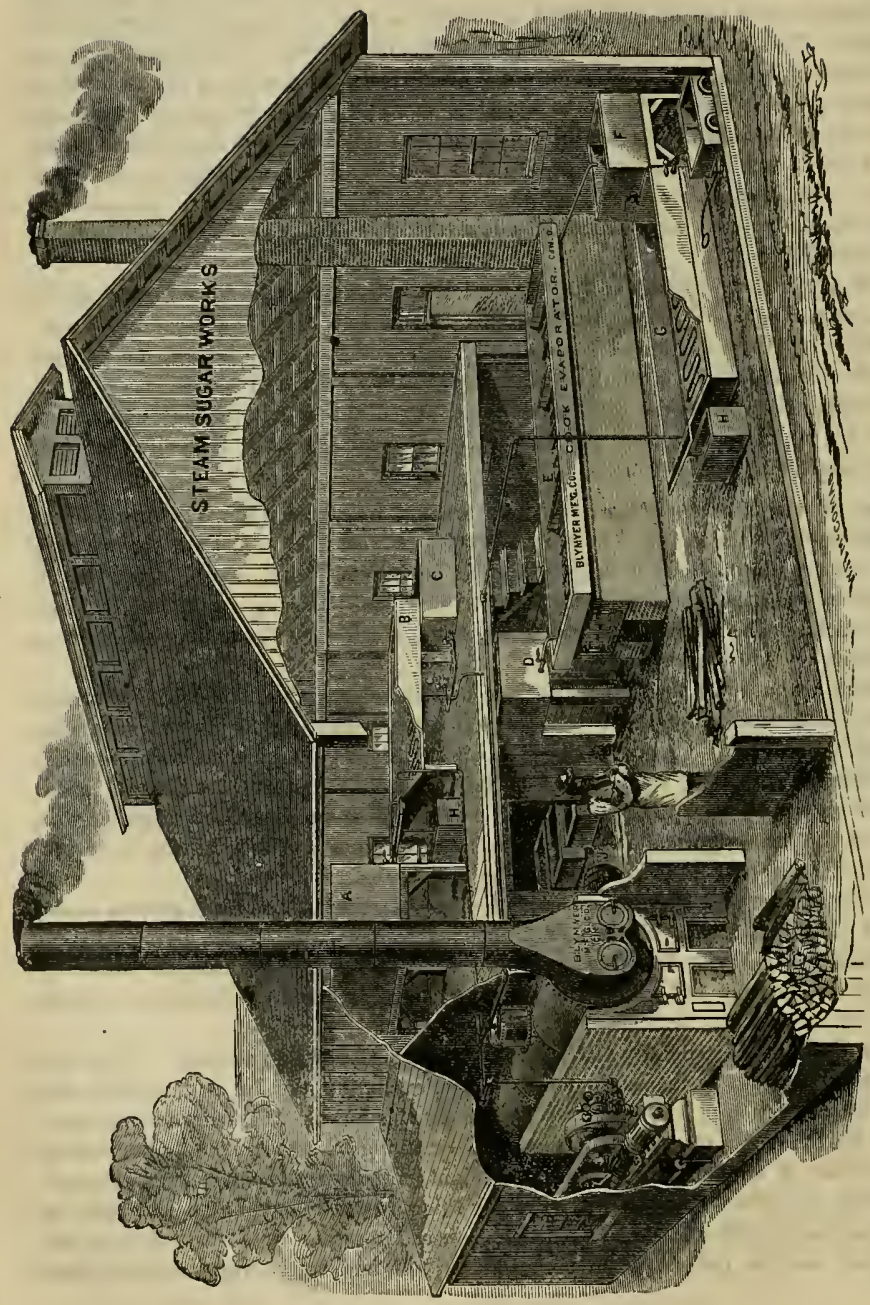

हैं

क्ष क्ष

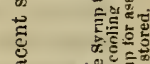

总

sô sे हैं

胥

5 हुํำ

ॠ रिक्ष के

4 $\quad$ स

प

來

क छ

है

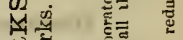

मि

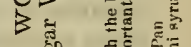

मि क्ञ हैं

b घ हैँ

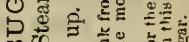

U क

५宅

4 ส

पर ट है है

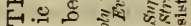

U2 ह 올

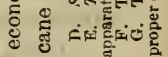

ॠ

。

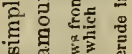

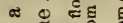

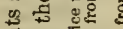

호

它

월.

ox

.5

สำ

80 원

की

\% क्षे

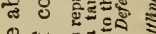

है 4艹

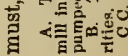




\section{SUGAR.}

$\mathrm{T}$ HE sugar product of the country is one of immense and growing importance, as well as the various industries connected with its production. With the increase of this staple and also of population, there has been for several years an increased proportionate consumption of sugar per individual. In addition to the consumption of the home product, the fact of there being over a hundred and seventy-five millions of dollars annually paid for imported sugar aud molasses by the people of this country, proves that the cultivation of the sugar cane is not as extensive here as it might and should be. The one State of Louisiana, with its resources well developed, might easily be made to furnish this supply, and the amount of money thus expended on the importations be retained at home to benefit our own nation. The largest importations of sugar into this country have for several years been made from the island of Cuba, which produces about six hundred and fifty thousand tons of sugar per year, a greater part of which is taken by the United States, where it is refined and then put upon the market. The importations from other countries, although in the aggregate amounting to considerable, are each small in comparison with those from Cuba. The introduction of sugar-cane into this country is said to have been in the year 1751 by some French Jesuits, who brought it from San Domingo, and planted it on the present site of New Orleans. The accounts of the progress of the manufacture of sugar under Spanish rule are somewhat conflicting. It was first manufactured in this country about the year 1764, but it was not until between 1794 and 1800, at a period when the revolution in San Domingo sent many planters to Louisiana, that the cultivation of this product became of marked importance. Sugar-cane belongs to the family of Gramines or grasses, and is supposed to be a native of Southeastern Asia. Its growth is from eight to twenty feet in height, according to variety and conditions of soil, climate, etc., having a solid stem from an inch to an inch and a half in thickness, which is jointed from every three to six inches. The leaves are from three to four feet long, and about two inches in breadth, and fall off with the ripening of the plant. Louisiana is the great sugar-producing state, although Florida, and all the other Gulf coast states, yield it in considerable quantities. The coast counties of Texas are especially adapted to this product, where its cultivation is being rapidly extended. Sugar may be made from a variety of substances, but the principal sources of supply are the sugar-cane, the sorgo-plant. the beet-root, and the maple tree; although efforts have recently been made to utilize maize for this purpose, the enterprise has not, thus far, proved sufficiently profitable to be recommended.

Varieties of Sugar-Cane.-There are many varieties of sugar-cane, some of which differ so widely from others, - such as the Chinese for instance,-as to be thought by many to be distinct species. The Otaheite or Tahiti cane is extensively cultivated, and is taller, more hardy, larger jointed, quicker in growth, and more productive in sugar than the Creole variety, while its juice, being more easily crystallized than the latter, together with its large yield and quick maturity, render it very popular. The Ribbon cane, thought by many to be the best kind cultivated, and the Bourbon belong to this variety. The Creole, also known as Madeira or common sugar-cane, grows to the greatest height, and has thin stems closely jointed. It will grow on moist soil at the altitude of, 5,000 feet above the sea. Another variety, known as the Batavia or purple violet, is covered with purple stripes, grows from eight to ten feet high, and is characterized by a resinous or waxy substance about the joints. The Chinese cane, Sorgo or Sorghum, also known as Northern cane, is a very hardy variety, and can be grown in nearly all parts of the country, the cuitivation of which will be treated separately, and following that of the southern sugar-cane or tropical product. There are various other varieties cultivated to a limited extent, but the above-mentioned are the prin. cipal or leading ones known generally in this branch of agriculture. 
The Batavia, or purple-violet cane, is a native of Java, and is often used in some localities as a border for other canes, to defend them from cattle. New varieties of the cane may be produced in the same manner as those from other agricultural products, and, although the kinds most cultivated are many of them very fine, there is no doubt that great improvements will be made, from time to time, in this direction, by the careful experiments of those skilled in this department of agriculture.

The Ribbon cane (Saccharum officinarum) is a genuine tropical plant, of which there are many varieties. It is regarded with great favor, and is the most extensively cultivated in this country. It will not thrive in a cool climate, and is easily injured by the frost. It is a perennial plant, but the stalks will die down each year from the effects of the frost, if not cut before it makes its appearance.

In Northern Louisiana the plant will not flower, and in the southern part of the State it will not mature its seed. Even in the West Indies the seed ripens but indifferently well. Consequeutly, the production of the tropical sugar-cane in the United States is restricted to a comparatively narrow belt of country, mostly bordering on the Gulf of Mexico. The northern limit of its profitable production in this country is estimated by those who have given the subject considerable attention, to be the thirty-second degree of latitude. Like corn and sorghum, sugar-cane belongs to the family of Graminæ, or grasses.

Analysis of Sugar-Cane.-The analysis of the Otaheite cane, by Payen, gives the following result: Water, 71.04 per cent.; cane sugar, 18 per cent.; cellulose, lignine, pectine, and pectic acid, 9.56 per cent.; cerosine wax, fats, resins, etc, 0.37 per cent.; soluble salts, 0.16 per cent.; insoluble salts, 0.12 per cent.; silica, 0.2 per cent. Other analyses give glucose from two to three per cent. The smallest quantity of glucose is usually found in the lower and more perfectly-ripened portion of the stalk, while the larger proportion is found in the upper and green end, which is protected from the rays of the sun by the leaves.

The analysis of the ashes of the cane is as follows: Silica, 46.46 per cent.; phosphoric acid, 8.23 per cent.; sulphuric acid, 4.65 per cent.; lime, 8.91 per cent.; magnesia, 4.5 per cent.; potassa, 10.63 per cent.; chloride of potassium, 7.41 per cent.; chloride of sodium, 9.21 per cent.

Cultivation.-An exceedingly warm climate, and a soil of deep, rich, moist loam, are the best conditions suited to the production of sugar-cane. A surplus of water is very detrimental to the crop, therefore the land should be well drained, either naturally or artificially. The system of culture, as previously followed in this country, has not been of that character which would produce the best results; hence, great losses have been sustained by the planter, both on account of the agricultural methods practiced in its production, and the mechanical appliances employed in extracting the saccharine matter from the cane.

The successive planting of the same cane, together with a lack of proper cultivation and sufficient manure, have resulted in greatly deteriorating the quality and yield of the sugar product. The establishing of an improved system of cultivation, - of which there are at present strong indications, - and improvement in the quality of the seed, through importations from other latitudes, will result in larger profits and a surer test of the possibilities of this crop than have ever yet been attained.

A proper system of drainage is too often overlooked in the cultivation of the sugar.cane. There are many low, marshy tracts, at present entirely unoccupied for any agricultural purposes, that by a proper system of drainage would make rich and highly-productive sugarlands; while there are sugar-plantations in cultivation which, with improved drainage, could be made to produce nearly twice the present amount.

The soil for cane should be deeply plowed, and well pulverized. It should also be kept well fertilized to prevent exhaustion, by supplying those elements of plant-food that are essen- 
tial to the growth of the crop. Chemical fertilizers-such as lime, gypsum, and the superphosphates-are used with good effect, but animal manures, or those containing a large pro. portion of nitrogen, when too liberally applied, are thought to produce plants, the juices of which are less rich in sugar, and contain more impurities than the former. When stable manure is used, it should be well fermented, either before its application, or by being plowed in a sufficiently long time before planting the cuttings, in order to admit of its becoming perfectly decomposed in the soil. All fertilizers used should be well worked into the soil with the harrow or cultivator.

The propagation of the cane is effected by cuttings; the cuttings for the purpose being produced by planting the seed. The seed is of necessity imported, since it does not become perfectly ripe in the sugar-producing belt of this country. It is obtained principally from Otaheite. It is of the utmost importance that the propagation, either by cuttings or seed, should be from the very best cane. Such cane should be not only the best varieties that can be obtained, but the strongest and most healthy plants of such varieties. Any neglect or carelessness in this respect will affect very materially the quality and yield of the crop, and may thus cause a serious loss to the planter.

The cuttings for planting are taken from the main stalks, and are generally from fifteen to twenty inches in length. They are planted in trenches or drills, and so placed that the eyes, which are on the opposite sides of alternate joints, should be on the sides. These stalks are placed quite near each other in the row, so as to furnish plants when grown that will be from six to eight inches apart. Sometimes a double row of stalks are planted, but, unless the trenches be sufficiently far apart, the plants will be liable to be crowded. The stalks are cov. ered to the depth of from two to three inches of soil. Agricultural implements for covering and pressing the soil upon the cuttings are a great saving of time and labor.

The planting may be either in the spring or fall. Different varieties should not be planted together or near each other, as there is a great tendency to mix in this species of plants, which is an injury to the product, pure varieties being the most desirable. The shoots which the cuttings send up will be large enough to cut in from ten to fourteen months after planting. After cutting, a new growth of shoots will spring up from the roots called "rattoons," which produce the next crop, and so on; this process being repeated to the extent that a single planting will supply cane for several years. In Louisiana, on account of con. stant deterioration, it is necessary to re-plant from cuttings every third or fourth year, onefourth of the land devoted to sugar-culture being employed for the propagation of the cuttings from which the cane on the other three-fourths is grown.

In the West Indies, one planting will last from five to ten years, while in some sections it has been known to supply cane for nearly twenty years before the old ruots died out. Such cases are, however, rare. The cane will flower in from twelve to twenty months after plant. ing. The cuttings should always be planted in rows at a sufficient distance apart to admit of space for growth and cultivation. The cultivator should be used frequently in order to keep out the weeds and grass, and to promote the growth of the cane by keeping the surface mellow. The last cultivation given before harvesting is generally in the early part of June. At this time, the soil is brought up around the roots and stalks of the plants, to the depth of from three to four inches. It is then left to ripen.

The sugar.cane beetle is the most destructive enemy of this product known. The borer is also quite destructive in some sections. Various methods have been employed to extermi. nate these pests, but none have proved very effectual.

Harvesting. - The cane commences to ripen at the bottom of the stalk, the ripening process extending upwards at the rate of about one inch per day. When sufficiently ripe for harvesting, which will be indicated by its appearance, the tops of the plants are first cut down as far as the leaves are dry. The leaves are then all pulled off from the stalk while standing in the field, the stalks cut close to the ground, and taken at once to the sugar-mill. 
The cutting of the cane should be as close to the ground as possible, since the lowest joints of the stalks are richest in saccharine or sugar-producing matter; besides, the sprouts or rattoons will grow more vigorously when the old stalks are cut close than when left stand. ing above the surface of the ground. The best results are obtained from cane when it is worked up within twenty-four hours after being cut in the field. It has sometimes been the practice, when not convenient to do this, to leave the tops on the stalks, and cut and pile them in the field in long rows, to the height of three or four feet, the tops of the cane from one hill being thrown over the ends of another, and so on, putting the stalks from three or four rows together. It has frequently been kept three or four weeks in this way before taking it to the mill, and without any apparent infury from frost, unless it be unusually severe. This practice, however, is not to be recommended, as it has been found that the product is in this way injured. In such cases, the tops should be cut off just before expressing the juice. It is better to have the cane taken to the mill as soon as cut, if practicable.

Implements for cutting the crop have been invented which greatly facilitate the method of hand-labor. It should always be harvested before the frost makes its appearance. The tops and leaves may be used for feeding stock, utilized in the compost-heap, or left on the ground for manure. The tops should never be planted, as this will result in deterioration of the crop. Only the best part of the stalks of thrifty plants should ever be used for cuttings.

Maunfacturing Sugar.- The inefficiency of machinery for expressing the juice of

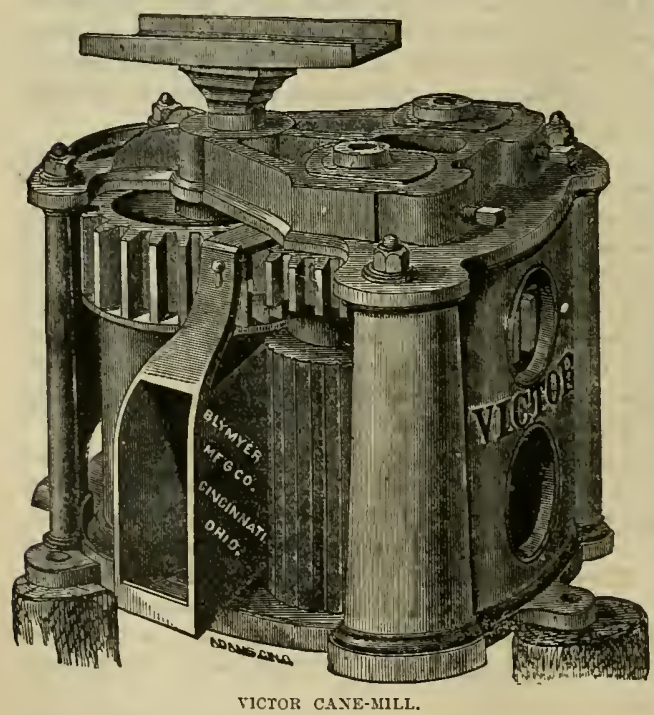
the sugar-cane has been the source of great loss to the planter, and a serious detriment to the profitable production of this crop. The object of the mill is to extract the juice from the cane. This is done by crushing it, which requires a machine of great power, and afterwards expressing the juice by heavy pressure. It has been estimtead by those who have inrestigated the subject, that about forty per cent. of the sugar which bas been cultivated during the past few years in this country has been wasted on account of a lack of sufficiently strong mills for extracting the juice of the cane. Mills that will perform the best service must of necessity be made heavy and strong, a powerful pressure being required, and are consequently quite expensive. However, the quantity of sugar saved by the use of a good mill will soon be sufficient to repay the extra expense of procuring it. It is a good plan for a few planters to purchase, in company, a complete set of the best machinery for the manufacturing of their sugar. By this means, - - a practice highly recommended by some of the most able agricultural writers of the South, - the use of the best implement can be secured to each member of the company at a comparatively slight expense. The sugar product is a remunerative one when properly managed, and the necessary implements for its cultivation and manufacture are enployed. There are many valuable mills in the market, for both steam and horse-power. 


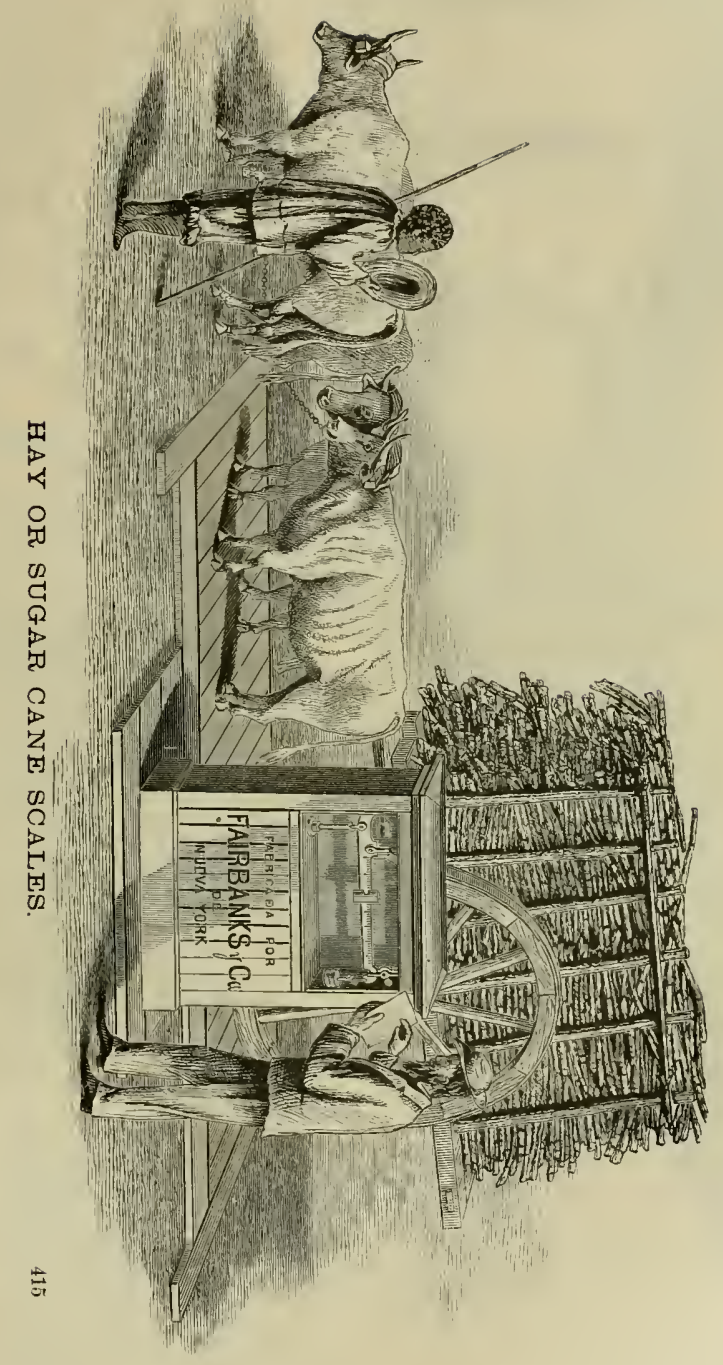




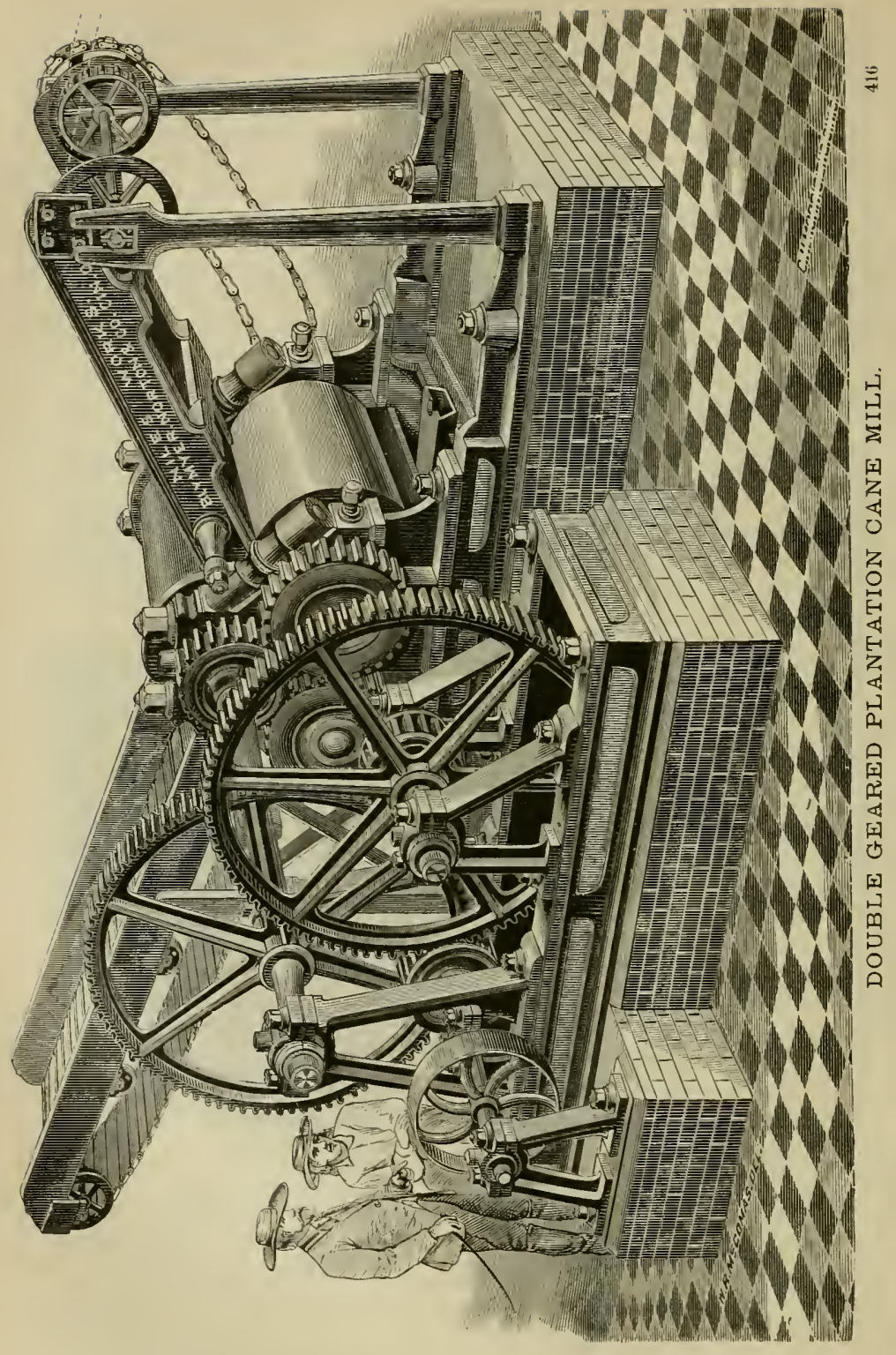


The illustrations of sugar-manufacturing implements which we insert were made from photographs of those made by the Blymyer Manufacturing Co., of Cincinnati, Ohio, and are good representations of this class of machines.

Extracting the Juice.-The preparation for the mill consists in cutting off the tops of the stalks, and removing the leaves. It is always best to have the juice of the cane expressed from the stalks as soon as possible after harvesting it, as it has been found that a change commences at the base of the stalk very soon after cutting, and gradually progresse.s upward. The juice freshly-expressed from the cane should not remain in contact with the air for more than an hour; even twenty minutes will sometimes be sufficient to produce a change. In crushing the cane, care should be used not to have the mills work at too high a rate of speed. A high authority on this subject says, that very good results may be obtained by the use of rollers which dovelop a surface of four or five yards in length per minute, so that a roller of two feet in diameter should only make from two to two and a half revolutions per minute, in order to extract generally the largest amount of juice from a given weight of cane, an increase in the capacity of the mill being obtained by increasing the length of the rollers, rather than the velocity. It is estimated that the fresh cane contains

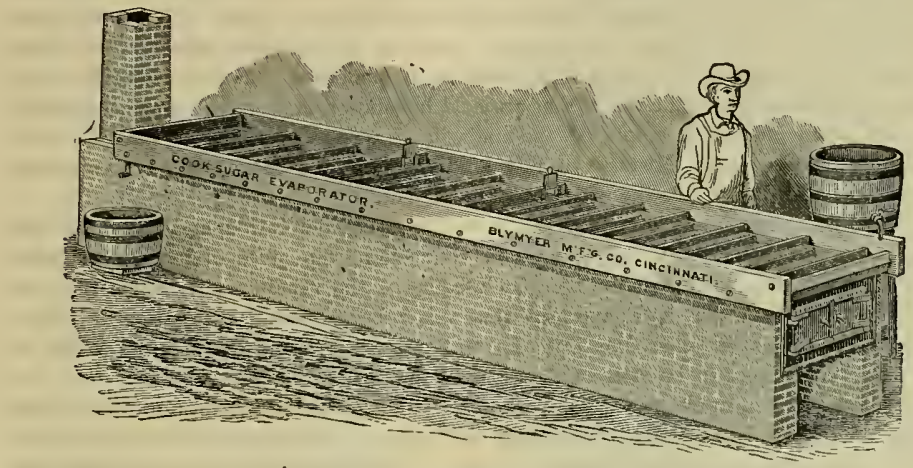

COOK'S SUGAR EVAPORATOR.-FURNACE AND PAN.

from eighty-two to eighty-five per cent of its weight of saccharine juice. Most of the ordinary mills extract from fifty to sixty per cent. of the weight of the stalk, hence there is the loss of about forty-one per cent. of the juice where only fifty per cent. of the weight is extracted. It will sometimes repay the labor of expressing the crushed cane, by the increased yield of the juice thus obtained.

Defecation or Clarification of the Juice.-Owing to albumen and other nitrogenous substances contained in the juice of the cane, the process of fermentation soon commences if it is left exposed to the air after being expressed from the stalks. The mill should be placed upon an elevation in such a manner that the juice, as it is received from it by the pipe that conducts it into the defecating tank, may pass from thence into the evaporator. A strainer of wire gauze, or coarse cloth, is placed at the outlet of the receiving-tank, to separate the juice from any fragments of cane, etc., that it may contain. As it leaves the mill, it is of a yellowish-green color, opaque, and frothy.

The more quickly this juice of the cane can be converted into sugar, after being expressed from the stalks, the better the result. As soon. therefore, as it comes from the mill, it must be freed from all impurities. The quality of the sugar will depend largely upon 
the skill with which this is accomplished. On account of the acids and other matter contained in it, as has been previously stated, cane-juice will commence to deteriorate as soon as exposed to the air. The result of this deterioration is to destroy crystallization, and convert true cane-sugar into grape-sugar, or glucose. It should, therefore, not be retained in the reservoir, but be carried at once from the mill to the boiling.apparatus. The tendency of the juice to fermentation by contact with the air may be arrested by sulphur fumigation. Syrup and sugar thus treated are lighter in color and usually superior in quality. This is not, however, a necessary practice in the process of sugar-making.

The first important treatment of the juice as it leaves the mill, is defecation, or the sepa. ration of it from all the impurities which it may contain. The strainer only removes the coarse foreign substances, but the acids and other impurities, which it holds in solution, can be freed from it only by chemical action and heat. The most common metlod of accom. plishing this is by the use of lime. It must, however, be used with care and skill. It must be pure and fresh, and never used in its caustic state, but carefully slaked and washed in boiling water to remove the potash it may contain, lime itself being but slightly soluble in hot water. When properly prepared, it will be of the consistency of thin cream, or milk of lime. The exact quantity to be used will depend upon the amount of acid in the juice, and can only be determined by a person experienced in the business, or tests with litmus paper. From one to four pounds of lime are generally used to five hundred gallons of the juice, according to the quality of the latter. It must be remembered that the use of lime is to separate those substances in the liquid that are held in solution in it, which prevent crystallization, but unless judicionsly employed, the aim will be defeated, and the crystallization will be impaired or prevented. Mistakes of this kind made in the early stages of the process cannot be successfully remedied by subsequent trcatrent.

Next to lime, heat is an essential agent in the process of defecation, and this should also be employed judiciously, both being used in connection, the juice of the cane running directly from the mill into the defecator. On account of the rapidity with which the juice changes on exposure to air, it is important that this process should progress as rapidly as possible. As soon as the juice runs into the tank, the heat should be applied. When the entire quantity of liquid to be defecated has risen to the temperature of about $180^{\circ}$, or a degree of heat just endurable by the hand when immersed in it, the preparation of lime may be added, and stirred in thoroughly to become well mixed. The heat should then be brought up to the boiling-point, but not permitted to boil. When this point is reached, the heat should be shut off at once, and with a large skimmer or other appliances for the purpose, the thick, dark-colored scum that has formed should be quickly removed. The liquid may then be drawn off into another tank, and the sediment at the bottom strained to remove the impurities. Various methods are employed in clarifying the juice; sometimes a portion only of the quantity of lime is added to the liquid at first, and the contents heated and skimmed, after which the process is repeated before evaporating. After the removal of the scum, the liquid will be clear, and of a lighter color than before.

Eraporation.-This is conducted in various ways. The three principal methods of concentrating or evaporating the juice of the cane are, first: by a direct application of fire only, as in the use of kettles, common pans, and some kinds of evaporators. The second is by the use of both fire and steam, where fire evaporating-pans and steam defecating and finisling-pans are employed. The third is by the use of steam alone, as in the ordinary steam trains, or the stearn trains with vacuum-pans. While the steam train is considered complete in itself, vacuum-pans are to be preferred, especially on the larger plantations.

The oldest method of evaporating the juice of cane consists of a series of open kettles, four or five in number, varying in size and arranged in a row in an arch over a fire. This was called a "kettle train." The kettles were made of iron or copper, and varied in size, in 
order that, as the juice became reduced in quantity, it would still be sufficient to fill the kettles, and prevent any portion of the metal from becoming overheated. By this means the - defecation is very imperfect, owing to the scum being constantly mingled with the juice, and prevented from separating by the constant boiling, while the dipping from the first kettle to the second, then from the second to the third, and so on to the last, hinders the crystallization of the syrup, also darkens both syrup and sugar by the prolonged boiling and imperfect separation of it from the impurities it contained. It also required considerable fuel in proportion to the amount of labor performed. A great improvement on the "kettle train "is the use of flatbottom pans arranged with compartments, and - an apparatus for skimming automatically, also a strike-pan.

The use of steam for evaporating is preferable to fire, as it admits of a more uniform heat, or a heat that can be better controlled, and is at the same time more expeditious. There is, also, no danger from burning, the heating surface being immersed in the liquid, through pipes, while it economizes fuel and labor. One of the most reliable authorities on sugar-making states as follows, respecting the use of steam in this connection:-

"The most perfect method of sugar-making

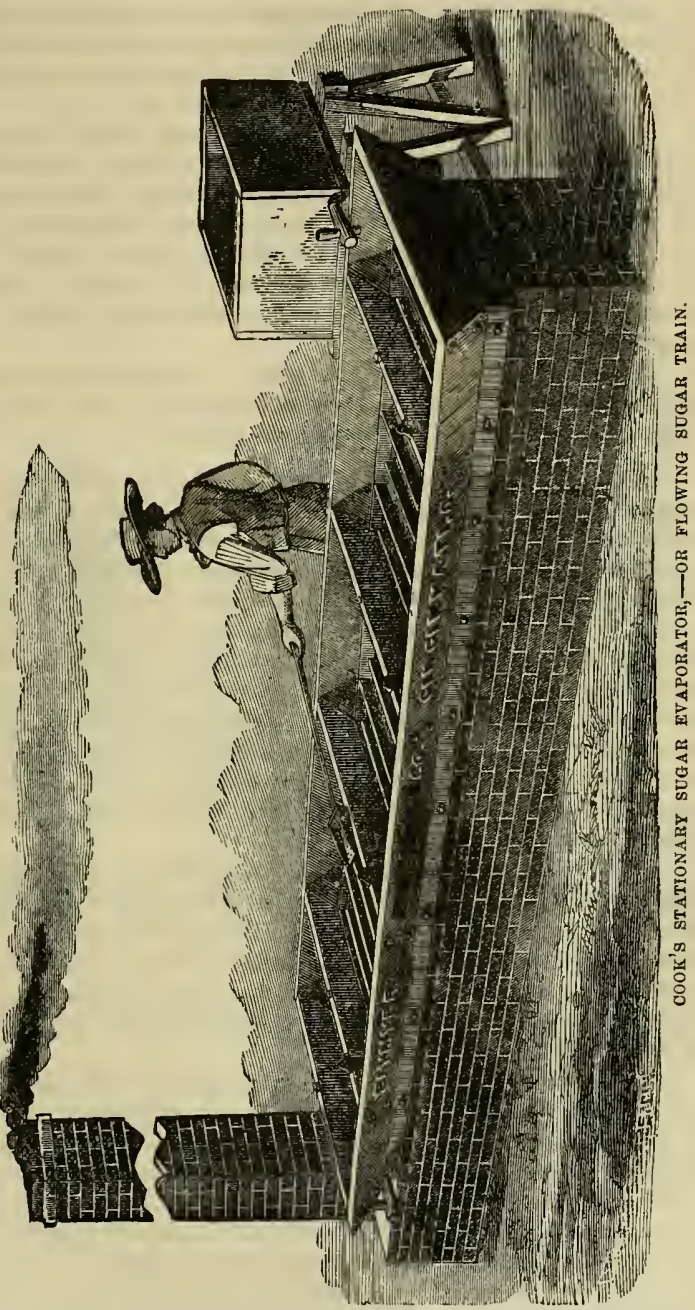
is found in the connected steam train. This consists of a series of vessels of different sizes, arranged in order, and all supplied within with steam-heating pipes, connected by branches with a main pipe from the boiler. 
This places the successive operations of defecating, concentrating, and finishing by steam under the immediate and convenient control of the sugar-maker. The heat is readily increased or diminished or withdrawn from either vessel at pleasure.

As sugar-making by a connected steam train is a continuous as well as rapid process, it is important that proper arrangement and proportion of all the parts be provided, including, also, the even and reliable working of the mill, so that the continuity of the operations may be harmoniously and effectively preserved to the end.

Whilst beyond question steam is the best agent used in boiling cane-juice, and the connected steam train the most perfect and simple apparatus, the effectiveness of the latter may be seriously impaired or totally destroyed by ignorant management, or improper proportions of vessels and pipes. In these, experience and skill are absolutely necessary.

If the planter has an engine and boiler of sufficient capacity to run his mill, and plenty of steam to spare, he can use steam profitably for working up even a small crop, either with steam pans entire, or with steam pans for defecating and finishing, and a fire evaporator for concentrating."

The chief objection to the open pans in the evaporating process is in the high temperature required to boil sugar solutions in the open air, which converts much of it into glucose. Vacuum pans, therefore, are greatly to be preferred.

Crystallization. - After a perfect cleansing or defecation of the juice, and its rapid evaporation to the sugar point, the next step in the process is to secure crystallization. When this condition is reached is a somewhat nice point to determine, for one not experienced in the business. The common test is, that when the liquid has reached that degree of concentration that a drop of it, placed between the thumb and fore-finger and pressed, may be drawn into a thread which has a granular appearance, it is an indication that the evaporation is complete, and it is ready to be transferred to the cooler. This shonld be large and shallow. As the sugar gradually cools and crystallizes, it should be occasionally stirred to make the granulation as uniform as possible. A small quantity of granulated sugar, mixed into the syrup as it cools, will hasten the crystallization; hence, by putting a little into the first that is drawn off into the coolers, and permitting some of the sugar to remain attached to the bottom and sides, as they are emptied each time, to be again refilled, will materially aid in this process.

After the sugar has crystallized in the coolers, it must be separated from the molasses, or syrup, that does not granulate. This is done by various methods of draining. Large tanks, tubs, or boxes, perforated at the bottom, and provided with movable plugs, or false bottoms of slats covered with coarse sacking, for straining out the liquid portion, are sometimes used; but the best method is the centrifugal machine, which rapidly expels the molasses from the sugar by force, leaving the latter quite dry. This is a great improvement in sugar making and refining.

There are various kinds of centrifugal machines. They are constructed with a cylinder, the walls of which are formed of finely perforated metal. This is supported, and with proper appliances, is made to revolve with great rapidity, from a thousand to eighteen hundred times a minute. The sugar is placed inside the cylinder, and the machine set in motion, which causes the sugar to form a lining of uniform thickness on the interior, while the molasses is driven through the perforated metal and caught in the drum that surrounds it. The machine is then stopped, and the sugar scraped out. By this means the separation of the sugar and molasses may be easily accomplished in a few minutes, while it might require days by the former draining process. The yield of sugar varies from half a ton to two and a half or three tons per acre, according to the variety of the cane, methods of culture, soil, season, and the efficiency of the machinery employed in its manufacture. The 
loss estimated through the imperfect manner of extracting the juice and the inversion of the sugar into glucose is enormous.

In the manufacture of sugar, it is highly important that the mill, tanks, and everything used in connection with it should be kept perfectly clean. The bagasse or crushed cane, after the juice has been expressed, furnishes a nutritious fodder for stock. It is also used in the manufacture of paper.

Sorgo and Imphee Sugar-Cane. - Sorgo or Sorghum is commonly known as the Chinese sugar-cane, also Northern cane; the former name having reference to its origin, the latter to its adaptation to the Northern States, in contradistinction from the tropical or Southern cane. It is a native of China, where it has been cultivated from time immemorial. Its first appearance in Europe was in 1851, when it was introduced into France. In 1854 some of the seed was sent to the United States, which was disseminated, and the culture of sorghum soon became general.
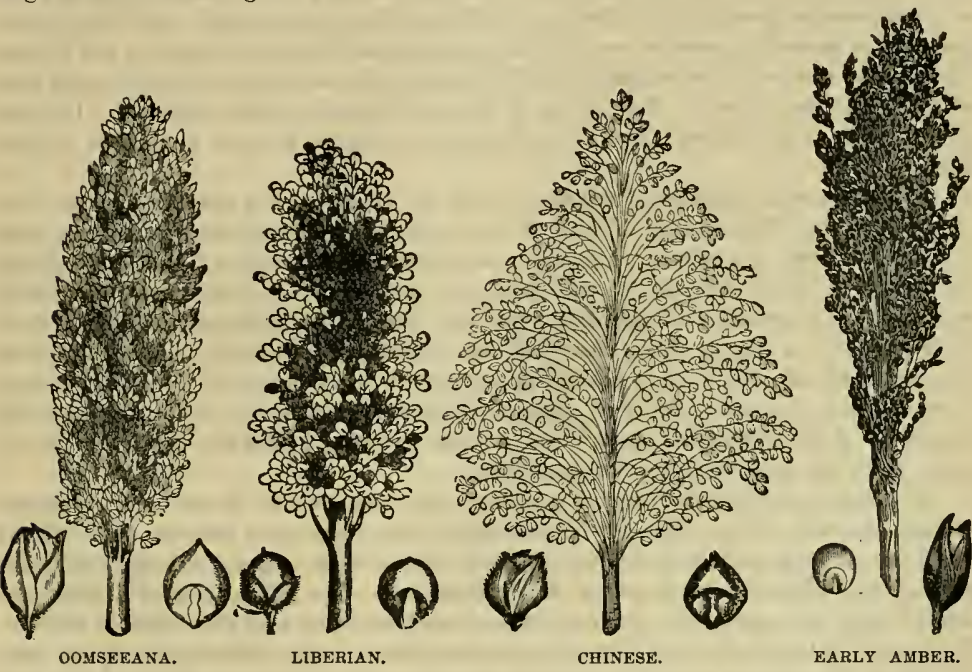

Imphee, or African cane, was introduced into France from the southeastern coast of Africa in 1851. Thus the sorghum and imphee reached France about the same time from different quarters of the globe, and from thence were introduced into this country.

Though resembling sorgo, the African cane is not generally considered quite as hardy and therefore not as well adapted to cultivation in the higher latitudes as the former. It is, however, valuable in those sections suited to its culture, while it has been employed in the production of a large number of hybrids between that and the Chinese cane, some of which are quite popular, and seem to unite the leading characteristics of both species.

The seed heads of the imphees are generally more compact than the sorgo. The principal varieties of imphees known in this country are the Liberian, Neeazana, and Oomseeana. Of these, the Liberian has thus far been most successfully cultivated. The natives from whence it was introduced,-the Zulu-Kaffirs, - are said to regard the Neeazana with most favor, as being the sweetest of all their varieties. The Liberian is a heavy producer, and quite free from disease, it being almost unknown to smut, mildew, or blight of any kind. The 
Neeazana yields a good quality of syrup of light color, but is said to have deteriorated in the extreme South. The Oomseeana is a fine variety, and in some sections is given the preference. It produces more sugar in proportion than syrup, while the varieties previously mentioned produce a larger proportion of syrup than sugar. The sorgo matures about two weeks earlier than the imphees, but the latter require cutting when in a greener state than the former, so that the harvesting season is about the same for each.

The color of the Oomseeana seed when fully matured is a dark brown; that of the Liberian a deep red, while the Neeazana is of a light cream or wood color. The seed of the Chinese cane or sorgo, when fully ripe, is of a dark purple, or black color; that of the Early Amber is also dark.

The Chinese cane grows to the height of from twelve to sixteen feet or more. The stalks are more slender than those of corn, and not so thick as those of the imphee. The time required for the perfect development of the plant varies with the climate, soil, and season, but averages generally, with favoring conditions, about five months. The Early Amber presents, in general appearance, some of the characteristics of both the sorghum and imphee. This variety receives its name from the early ripening of its seeds and the bright amber color of the syrup made from it. This is one of the most popular kinds cultivated. It grows to the height of the Chinese variety, but is thought to be richer in sugar production than the latter.

There are many varieties of sorghum which are cultivated to a certain extent, all more or less valuable. The chemical division of the Agricultural Department has recently made numerous analyses of sorghum, embracing nearly forty varieties, with a view of determining the period at which the juice of each particular variety contained the most crystallizable sugar that could profitably be separated. For this purpose, the analyses were begun at an early stage of the growth of the plant, and were repeated from time to time till later in the autumn, which afforded the best possible opportunity of ascertaining the comparative value of each variety, the practicability of sugar production from sorghum, aside from the actual separation of the crystallized sugar itself in manufacturing it, and also the time when the plant yields the greatest amount of cane-sugar.

These analyses extended over a period of time from July to November, showing the amount, not only of sugar contained in each variety, but of glucose at the same time. It was found that after the middle of August, the Early Amber, when properly managed, will make as much crystallizable sugar as any of the tropical cane. The full amount of sucrose was reached early in August, and continued without much variation until the middle of October. The glucose in this variety was found to be greatest early in July, diminishing rapidly until the first of August, and slightly after this period, the amount of glucose diminishing as that of sucrose increased. The White Liberian variety reached its full amount of sugar-production by the middle of August, and continued until November. Among the later varieties, some produced only about one-half or two-thirds of their full amount of sucrose by the middle of August, their full development not being reached until the middle of October.

From the above facts, it is apparent that in the Northern section adapted to the culture of sorghum, the advantages gained in growing the Farly Amber are in its early maturity, and the long period during which the manufacture of the sugar and syrup may be continued. These analyses proved that the amount of sugar afforded by the different varieties did not vary largely, being from twelve to fifteen per cent. of the juice. The superior value of the different kinds consists, therefore, mainly in the amount or weight of cane that can be pro. duced on an acre, and their adaptation, with respect to the period of ripening, to the latitude in which they are to be grown. Some of the late varieties may be admirably suited to the South, but the earlier ones are to be recommended for Northern cultivation.

With respect, then, to the value of the different canes, other conditions being equal, 
that variety is best for any locality which matures earliest and remains longest in a working condition. An improved Early Amber, known as the Minnesota Early Amber, is much cultivated in the Western States. The improvement of this variety was secured by selecting seed from the finest crops of Northern growth, and sending it to a Southern latitude to be grown, on the principle that when cultivated in a high latitude for several consecutive years, canes will naturally degenerate. The result was an improved variety with respect to the weight of cane per acre, and the amount of saccharine matter produced.

As an agricultural product, sorghum has become within the past few years one of continually increased importance in this country, especially in the Western States, owing to the improved facilities for manufacturing sugar which are at present attainable, a lack of which caused so many farmers to abandon its culture shortly after its introduction into the United States.

A large proportion of the sorgo grown in this country has formerly been manufactured into syrup, but at present sugar is being made to a considerable extent. When the most approved methods of crystallization are better understood, and the use of better mechanical appliances in the various processes of sugar-making are more generally employed, we see no reason why the cultivation of sorghum in this country may not prove very profitable to the farmers, and highly beneficial to the nation generally, since sugar is an article of food in great demand; and if the home product could be largely increased, the necessity of sending millions of dollars annually abroad for the imported article would be obviated, and the enor. mous sums thus expended be retained at home. Besides, if farmers produced this crop only in sufficient quantities for home consumption, it would prove an economical and profitable enterprise in a small way, and they would also be sure of being provided with a pure, unadulterated article, which they cannot be assured of in purchasing the vast quantities of glucose sugar and other vile compounds with which the markets are crowded.

As with the tropical sugar-cane, the leaves and tops of the stalks of sorghum, as well as the bagasse, or refuse cane from the mill, furnish excellent fodder, whlle the seed is valuable as food for domestic animals, its nutritive value being but little less than that of corn.

Soil and its Preparation.- Sorgo may be cultivated on any soil suited to the successful production of wheat or corn. The best for the purpose, however, is a light sandy loam. It has been found that the quality of the juice of the plant is largely affected by the soil upon which the crop is grown; for instance, while black, heavy soil will produce a vigorous growth of the cane, the juice of such plants will not be as rich in saccharine matter as those grown upon a light sandy loam. Sandy uplands fertilized with well-composted farmmanure, chemical fertilizers, lime, plaster, or wood-ashes, will give exceedingly good results. Wet lands are not adapted to this crop, and should first be thoroughly drained before attempting its culture. Clay soil will produce a product of good quality, but the yield will not be large. New lands, if of the proper quality, will produce well, but fresh manure will give unsatisfactory results; hence, when yard-manure is used, it should always be first well fermented. A compost from the pig-sty is exceedingly valuable in the cultivation of sorghum.

The soil should be made rich in order to produce a rapid growth, that the crop may mature before there is any danger from frost. Vegetable or yard-manure plowed in, in the fall, will greatly improve clay lands for this crop.

The tillage for sorgo should be deep, and the soil well pulverized. When the land is plowed and thrown into ridges in the fall, it will be in a better condition for working early in the spring. As a general rule, the land for sorghum should be prepared in the same manner as for corn. In some sections, where the land is inclined to be too wet, ridge-culture is practiced; but in such cases draining will give better results.

Planting. - It is of the highest importance that the seed for planting should be of the best quality. That produced by the most thrifty plants of the most desirable variety should 
be obtained. Seed that has been grown in a somewhat warmer climate than that in which it is to be planted is best for the Northern States. It should also be from a pure variety and unmixed with other plants. It is a well-known fact that sugar-canes and similar plants, such as corn, millet, broom-corn, etc., will readily mix when planted with or near each other, greatly to the deterioration of the product; hence the importance of obtaining good seed. It is the only safe way to procure it from some well-known and reliable source.

Soaking the seed in tepid water from twelve to twenty-four hours before planting will canse it to germinate sooner. It may also be tested by putting a little of the seed in some earth and keeping it in a warm place, or by putting it between two layers of thin muslin saturated with water, and placing it in damp soil for a few days, watering slightly occasion. ally. If it germinates readily by either of these methods, it is quite probable that it will do so when planted in the field. The planting should be done as early as the ground is sufficiently warm, and there is no danger from frost. The planting is generally in hills, drill-culture not usually being considered so satisfactory in results as hills, since the latter admits of cultivation in both directions. The rows should be about three feet apart, and the hills two feet apart in the rows. It is well to put plenty of seed in each hill to make allowance for such as may not germinate, and afterwards thin out the plants, leaving from six to eight of the most vigorous ones to grow. From twenty to twenty-five seeds to the hill would be sufficient. Care should be used not to cover the seed too deep, as it would be liable to rot in the ground, if the weather should be cool and wet. For early planting, a half inch of covering will be sufficient. When the planting is late in the season, and the ground warm and dry, it will be well to cover to the depth of an inch.

Cultiration.-When sorghum-plants first make their appearance from the ground they much resemble broom-corn, or a species of very coarse grass. The growth in the first stages is very slow, the plants being feeble, but in a little time, with a suitable soil, it will be quite rapid. As soon as the plants are up, the cultivator should be used in destroying the weeds. It is very essential for the welfare of the crop that the soil be kept clean of grass and weeds during the early period of its growth, for as its progress in growth is then slow, it will soon be choked with the fast-growing weeds, if neglected. This is the period when the crop is said to be "made," and the culture it then receives has more influence in determining the yield than that subsequently given. When the stalks are five or six inches high, the plants should be thinned out in the hills, leaving from six to eight of the best. Careful culture is essential until the plants are about three feet high, after which it will in a great measure take care of itself. If the cultivator is used afterwards, it should be run very near the surface, as deep tillage would be liable to injure the roots, and thus injure the stalks. The culture throughout is similar to that of corn, except it does not extend as late in the season.

Harvesting.-The time for harvesting sorghum is when it has reached that degree of maturity that the seed cannot be crushed between the thumb and finger. At this period the saccharine matter is in the greatest quantity in the stalks, and the glucose in the least. If cut before this period, there will be much waste, and the juice will be of inferior quality. The cane should never be touched by the frost. The time for cutting imphees, or African cane, is earlier than that of sorghum, and may be when the seed is in the milk state. Some planters claim that it may be worked up at any time from the period of the falling of the flowers until the seed is ripe.

It is better to have the cane ground at once as soon as harvested. When this is done, it may be topped in the field, cutting off one or two joints, and the leaves stripped off, after which it may be cut and taken to the mill. When not worked up at once, the tops and leaves should remain on until ready for grinding, and the stalks stacked in the field like corn. By leaving the tops and leaves on the stalks in such cases, cane can be kept three or four weeks without much injury. It should not, when stacked, be exposed to rain or frost. The 
tops should be laid in small piles to dry. The seed should be shelled and used as food for stock. It may be threshed like wheat, or treated the same as broom-coru seed. Sheep will eat it on the heads without shelling, but for other stock it is best when shelled and ground. The sorgo-plant ratoons or tillers the same as the tropical cane, and at the South will thus produce two crops in a year, but at the North only one crop will have tirne during the season to mature.

Mannfacturing Syrup and Sugar from Sorghum.-In preparing the cane for the mill, the stalks are tied into bundles about eight inches in diameter. When not intended for immediate use, the bundles should be piled across each other in such a manner as to admit a free circulation of air, being placed where they will be kept perfectly clean. It is best, however, as previously stated, to grind it at once, if practicable, as soon as harvested. Where the leaves are left on the stalks in grinding, there will be a greater waste of juice, since they will absorb it more than would be at first supposed. In grinding, the mill should be fed by the butt end of the cane entering first. The largest portion of the sorgo product in this country is converted into syrup, but sugar is made to a certain extent, more being made at present than formerly.

The different processes of mannfacturing syrup from sorgo, consisting of the expressing of the juice, the defecation or cleansing it from impurities, and the concentration, are essentially the same as those already given in the manufacture of sugar from the tropical sugarcane; the processes for syrup being complete before the period of crystallization is reached.

Sugar may also be manufactured from sorgo, the same as from the tropical cane. The principal difficulty attending this process has been in the crystallization. In order to better secure granulation or crystallization, the chemical division of the Department of Agriculture, after numerous experiments, recommended the following special treatment:-

"Sorghum syrups should be reduced to a density that, after a lapse of from twenty-four to forty-eight hours, when kept in a warm room, it will become an almost solid mass of sugar. It requires then a special mode of treatment, the crystals being fine and held together by only a small quantity of molasses. When in this condition, the mass is to be thrown into a large tub or mixing-vessel, and a small quantity (about one-tenth of its volume) of a fair, thin syrup, prepared from sorghum.juice of a density of about $30^{\circ}$ Baumé; when cold, it is to be poured upon it and thoroughly incorporated in it by means of a wooden stirrer.

An iron mixing-mill constructed somewhat like the feed-hopper of a centrifugal sugardrainer, with a revolving shaft in its center, set with long projecting teeth, may be employed in regular work. This will bring it to the semi-fluid state, if the room in which the operation has been performed has been kept heated. The syrup dilutes the uncrystallized sugar sufficiently to render it mobile, and does not dissolve the cane-sugar. The mass may then be drained in a centrifugal, the inner drum of which is very clearly but minutely perforated, and running at the highest rate of speed.

Another method which may be followed is similar to that employed in some sugar factories to extract the juice from the pulp of the beet and also to separate the saccharine matter left in the scum.

A number of linen and coarse muslin sacks are provided, of any convenient size, but their length should be about two and one-half times their width, say twenty by fifty inches; each sack is to be about one-third filled with this sugary mixture, folded once on itself in the middle, and flattened by placing it upon a table upon a sheet-iron plate with rounded corners, a little larger on every side than the partially-flattened half of the sack and its contents, the loose half being folded under. The open end of the sack may be folded twice, if necessary. The plate and sack are then to be placed within a frame on the bed of a powerful screw. press, and a series of such sacks and interleaved plates laid neatly one upon another, being turned in opposite directions, and subjected to pressure, gradually applied at first, to avoid 
rupture of the sacks, and afterwards with sufficient power to remore all the syrup and leare the sugar nearly dry. This fine dry sugar is then to be transferred without further drying to a heating-vessel, and about one-tenth of its weight of pure water mixed with it. Here it is to be heated very gradually, with frequent stirring, to diffuse the heat through the mass, and when it has partially re-melted, and it is in the liquefied state, it is to be poured fiually into the crystallizing-boxes in a room heated to about $90^{\circ} \mathrm{F}$., where it will form a solid mass of crystals as soon as it becomes cool. The result is a very coarse-grained, beautiful sugar of a high grade. If properly prepared, it will be almost white, and the immediate yield is almost double that which may be secured in any other way without re-boiling.

The sugar prepared from sorghum in this way has the additional advantage of not being contaminated with the secondary products usually formed by re-boiling. The final crystallization is attended with no risk, is easily and cheaply done, and in quality, with due care, should rank nearly or quite equal to vacuum sugar. The very small quantity of syrup left in contact with the crystals will drain off from the crystallizers, and, being almost free from glucose, will crystallize gradually if exposed in broad trays at the temperature of the room. If the production of sugar of a softer and more open grain is desired, it can readily be accomplished by a mode of treatment almost identical with the "stirring off " process adopted by maple-sugar producers, but with better results. As soon as the half-liquefied sugary mass, produced as already mentioned, has been poured in the crystalliziug-bores, it should be stirred with a broad oar-shaped wooden instrument, without interruption, until it is cool and the sugar has become dry."

For the successful manufacture of syrup or sugar, the best appliances, in the form of good mills and evaporators, are highly essential. There sorgo is commonly grown, it would be well for a few farmers. living in the same vicinity, to own machinery for this purpose in company, thus securing the use of first-class implements at a comparatively small expense. Or a single party, having sufficient capital, and other favoring conditions, might manufacture the product for the farmers of the neighborhood, for a specified remuneration.

Sugar-Beet.- Various efforts have been made in this country within the last fifteen or twenty years to establish the heet-sugar industry, which enterprise has not thus far proved as successful as was anticipated. The general reason for the want of success in this direction is in the cost of the production. Sugar of the best quality has been made in these experiments, but the high price of labor has failed to reuder its manufacture profitable. The lack of proper appliances and skillful knowledge of the business have also been obstacles in the way of its success. That the manufacture of beet sugar will eventually prove a successful enterprise in this country there is no reason to doubt, when a better knowledge of the art, combined with improved machinery, and other methods employed, shall so reduce the expense as to render it a source of profit to both the grower and manufacturer of the product.

In Europe, large quantities of sugar are made from the beet-root. More than forty per cent. of the estimated sugar product of the world is derived from this source. The beet can be grown both North and South, but in the latter section it is not as well adapted for culture as the tropical cane, since it will deteriorate in saccharine matter in a warm climate. About the forty-fifth degree of latitude seems to be the southern limit of its successful culture for sugar production. It is, however, a very hardy plant, and can be successfully grown, even in the extreme North.

There are many varieties of beet used in the manufacture of sugar. Only the whitefleshed kinds are employed for this purpose; those that are firm, brittle. perfectly solid, and emit a cracking sound when cut, are generally the best in quality. The beet for sugar man. ufacture shonld also be smooth, rather long, with tapering roots, or pear-shaped, and finegrained; not growing above the surface of the ground.

The variety known as the White Silesian seems to be most in favor with manufacturers 
generally, and the best adapted to sugar production. The French, Siberian, Imperial, and many other varieties are used to a greater or less extent for this purpose; but the Silesian, although not as rich in saccharine matter as some others, being a vigorous grower, will produce more sugar to the acre, and is therefore more extensively cultivated in France and Germany, than any other kind.

Cultivation, Harresting, Storing, etc.-The most approved methods of cultivating, harvesting, and storing beets have been previously given in the department treating of Roots and Esculent Tubers, and as the cultivation of the crop used for sugar production is very much the same as that for other varieties of beets, a repetition will be unnecessary in this connection. The soil should not contain a large proportion of mineral salts, neither be too wet, or of a clayey nature. An excess in nitrogen, as well as the general effect of soil and manure on this crop, will be seen by the following, which is an abstract of experiments made at the Paris Academy of Sciences, with a view of examining the influences of soil and manures on the size and saccharine quality of sugar-beet:

Holes were bored in the sides and bottoms of a large number of tubs, and in each was placed a layer of gravel, to allow water of irrigation to drain off. The tubs then received various kinds of artificial soils-pure sand, limestone, and clay, exempt from potash. Beet. seed was sown in May, procured from the Aisue, where it produces roots containing 11 to 13 per cent. of sugar. The manures employed, either separately or mixed, were sulphate of ammonia, nitrate of potash, nitrate of soda, chlorides of potash and soda, superphosphate of lime, guano, rasped horn, and stable-manure. Irrigation was generally with the ordinary water of Paris, but sometimes with distilled water, in order to avoid the salts of the common water, and sometimes with water containing chemical manures. The leading points in the results were as follows: Roots are capable of attaining a weight of 1.4 pounds to 1.6 pounds in artificial soils wholly devoid of humus, provided they are regularly watered and supplied with chemical manures; while, in the absence of such manure, if the artificial soil be merely watered with distilled water, the roots will hardly attain an ounce in weight. Nitrogen, in the form of nitrate of soda or of potash, or of sulphate of ammoma, etc., favors increase of size of root, but is liable to injure the saccharine quality of the crop. Beets growing in a well-manured soil, and having combined nitrogen within reach, tend to form albuminous substance at the expense of the sugar. Beet-roots containing less than 10 per cent. of sugar are often found to contain twice as much nitrogen as those which have a saccharine richness of 15 to 16 per cent. On the same principle, in certain soils, beet-roots will exhibit but little sugar, not because the soil has become impoverished, but because, on the contrary, it is too rich in nitrogen. Beet-roots grown in artificial soils, and watered with chemical manures, are found to reach as high as 18 per cent.

In order to obtain mature roots, which is a matter of great importance,-since the full amount of saccharine matter is only obtained when perfectly matured,---the best cultivators of the sugar-beet plant early, and avoid stimulating manures, which would have a tendency to produce roots of undue size and late maturity.

Deep plowing is essential for this crop, in order to give the large roots room to develop entirely beneath the surface of the soil. Shallow plowing will cause them to grow partly above ground, which injures the quality for sugar production. The portion grown in this manner not only contains no sugar, but furnishes acids which will lessen the quantity of sugar contained in the other portion of the root. The harvesting should always be done before the appearance of the frost.

The leaves should also be cut close to the crown, when grown for the manufacture of sugar, while if any portion of the beet grows above ground, that should also be cut off with the leaves. This being done, the roots may be taken at once to the factory. Bruises and wounds by careless handling should be avoided, as this induces fermentation, and deteriorates 
the quantity and quality of the sugar. When roots are to be kept awhile before being worked up, it will also cause them to decay. Beets for this purpose may also be preserved in pits, or stored in various ways, the same as other root crops, but it is generally better for the farmer to dispose of them at once to the manufacturer, thus avoiding the labor and loss that may attend the storage. They are sometimes frozen and kept in that condition until wanted for use, but this practice is not to be recommended, since there is a liability of loss occasioned by their thawing out by a change of weather before being used; besides, the freezing process lias a tendency to deteriorate the sugar product.

The Ilaunfacture of Sugar from Beets is a complicated process, a detailed description of which would require more space than the limits of this work-devoted as it is to such a variety of subjects-will admit; we shall, therefore, in this connection, be able to give, as it were, only an outline of the process, while we would refer those of our readers who desire more specific directions and information with regard to it to some treatise of standard authority, devoted exclusively to this subject.

The first operation in sugar-making from beets is to cleanse the roots thoroughly from all particles of soil that may adhere to them. This is effected by various means, the most common being a revolving drum of open iron or wood.work submerged in water. The next step in the process is to extract the juice.

This may be done also by various methods. Sometimes the cleansed roots are reduced to a pulp by a rotary rasping-machine, which revolves with great velocity, after which the pulp is then put in bags or sacks, and subjected to a powerful pressure, thus expressing the juice, or it may be macerated in water, thus extracting the saccharine matter.

Another method is in exhausting the pulp by means of a centrifugal machine, the largest portion of the juice being thrown out by its rapid revolutions, after which water is applied and the remainder of the juice washed out in the same manner.

Diffusion is another process, and one which has been extensively practiced in many of the beet-sugar manufactories of Europe. The beets are by this method cut into thin, narrow strips by a cutting-machine which revolves with great rapidity. Water is then used, in con. nection with a diffusion battery, in extracting the juice.

In some batteries for this purpose, the cylinders are so connected by tubes that the liquids can be passed from any one of them to another, the same liquid being used repeatedly for different quantities of the beet, thus increasing its richness, and economizing in the evaporation. In others, the ribbons, or thin slices of the beet-root, are made to pass through a current of water which is moving in an opposite direction, and which will have a tendency to wash out a large portion of the sugar element of the juice. Various appliances have been employed on this principle, differing in their methods of construction and operation.

The advantage of diffusion over other methods consists mainly in obtaining a purer quality of juice than by other processes, since the cells of the beet or cane are not fractured, and the albuminoids and other substances, injurious to the sugar product, are not extracted.

The next process after extracting the juice, is to clarify or cleanse it of all impurities contained within it, and which would injure the quality of the product, and prevent crystallization. This is accomplished by heat and the use of lime, a process similar to that of defecating the juice of cane; after which the excess of lime and other substances contained in it are precipitated, and the caustic alkalies carbonated by the use of carbonic acid gas. Various other means, some of them of a complicated nature, may also be employed to accomplish this purpose. Filtration is the next step in the process of sugar manufacturing. This is accomplished by the use of bone-black, which is the charcoal of the bones of animals.

The hot juice of the beets is made to pass through a long column of pulverized bone. black, wilich removes the coloring matter contained in it; also, purifies it from other sub. stances contained in it which are injurious to the sugar. It is next evaporated to a thin 
syrup in vacuum pans, and subjected to a second process of filtering through bone-black, when it is again evaporated to the crystallization point and cooled. The sugar is next separated from the liquid by different processes. The centrifugal machine-the same as that used for cane sugar-is the best for this purpose. This liquid is again boiled, and a second. class quality of sugar is obtained in the same manner, and so on, a fourth, and even fifth, grade of sugar being produced in successive order.

The molasses that remains is so charged with salts and other impurities that it cannot be used for food. It is distilled for making alcohol, that which remains being converted into crude potash. The pulp furnishes a rich and sweet food for stock. It is good for milch cows, cattle and pigs, and is a valuable auxiliary for fattening animals.

Maple Sugar.-Large quantities of sugar are made in this country from the sap of the maple tree. It is naturally of a light-brown color, and has a peculiar moisture and deli. cious aromatic flavor that is not possessed by the sugar produced from any other source. When it has been subjected to the refining process, it loses much of this flavor, and resembles more that of the cane product; in fact, when refined, there is scarcely any difference between the sugar of the maple, tropical cane, sorgo, or beet.

The maple-sugar industry is one that is constantly improving, not so much in the quan. tity, as the quality of the product. It will probably never compete with cane sugar for the general purposes to which the sugar product is applied, but for special use it is the most deli. cious of all the manufactured sweets.

The syrup, when properly made, is, in the opinion of many, much to be preferred to the finest quality of honey, while it is more healthful as an article of food, and does not cloy the taste as honey does. There is, however, as much difference in the different grades of maple sugar, as found in the market, as in those of butter, owing to the different management it receives from the makers, care and judicious handling being essential to the production of a first-class article. Two varieties of the maple tree are principally used for the manufacture of sugar,-the rock or sugar maple, and the black maple.

The sugar maple grows abundantly in the Northern States, especially in Northern New England, and in New York, Ohio, Michigan, Wisconsin, and Canada.

It is a beautiful tree, and, aside from its value in sugar production, should be planted more frequently in some localities than it is. As an ornamental shade tree, we know of none to be preferred. Its dark-green foliage is changed into gorgeous tints in autumn, presenting a striking contrast with that of other forest trees, while the stateliness and uniformity of its growth are not among the least of its attractions.

It is also very hardy and will grow in a diversity of soils and climates. In the more southerly latitudes it will not, however, yield as much sugar as at the North, a sufficient degree of frost being required for this purpose.

As the business of sugar-making comes at a season when there is little other farm work that could be done, farmers having maple trees on their lands will find it an advantage to appropriate them to this use, as the sugar thus obtained will amply repay the labor of manufacturing it, and the proportionately small quantity of sap extracted does no harm to the trees, not even those about the house for shade and ornamental purposes.

Obtaining Sap.-The season for making maple sugar and syrup is early in the spring, or as soon as the frost begins to leave the ground, the bright sunny days and frosty nights causing a free and rapid circulation of the sap. This may be from the latter part of February to the early part of April, according to the latitude and season. The tapping of the trees should be done promptly as early as the season will admit, since the first sap is the richest in saccharine matter, and the most free from other substances that deteriorate the quality of the sugar. It should never be done, however, before the sap runs freely, and when so cold that it 
will freeze in the buckets before being gathered. Freezing injures the quality of the syrup or sugar; besides, the trees, buckets, spouts, etc., are also liable to be injured.

The largest trees are the best for sugar production, those from twenty-five to thirty years old giving the best yield. Trees a foot in diameter may be made to yield something, but they should never have their sap extracted before attaining that size.

The usual method of tapping trees is to bore holes a half or three-quarters of an inch in diameter on the sunny side of the tree, fifteen or eighteen inches from the ground and about five or six inches apart. These holes should never be more than three at a time, and frequently but two in number, according to the size of the tree. Care should be used not to bore too far into the alburnum, or white bark, and never through it, the entire depth of the hole not often much exceeding an inch. The boring should incline downward slightly, in order to permit the sap to pass off readily. Wooden spouts are sometimes inserted in these boles to conduct the sap into buckets placed to receire it. These may be placed upon the ground or hung upon the spouts. These spouts are usually made of elder or sumach, and are eight or ten inches in length. Another method is to drive galvanized-iron or tin spouts into the bark below the hole to collect and conduct the sap to the buckets.

What is called "boxing" the trees is practiced in some localities, which consists of cutting a narrow channel three or four inches in length, slanting upward, and about an inch deep, and inserting an iron spout into the bark below to collect the sap. This method is, however, not to be recommended, as it injures the tree. Whaterer the method of extracting the sap, care should be used not to make the incision too deep, or the opening large. Taking out large pieces of the bark and wood with an axe is, as has been justly termed, a barbarous and slovenly practice, and will soon result in killing the trees. Mr. W. J. Chamberlain recommends the galvanized-iron spout for obtaining sap. His suggestions on maple-sugarmaking are highly valuable, which we quote, as follows:

"By repeated and continued trial of the galvanized-iron spout, side by side with various wooden and tin spouts, I am fully convinced that it sours the sap least of any, and gives the largest yield. The first merit is more important than the other, for sour sap will nerer make good syrup. The buckets should always be tin, soldered inside and out at every seam. They will not rust inside in many years, and should never be painted there, as that makes them more rough and more liable to sour. Painting the outside, however, will help preserre the bucket. For our Ohio climate (and I am inclined to think it true as a rule everywhere), the buckets should invariably be corered tight. A hole just below the wire rim slips over the notch of the spout, and a board a foot square is laid on top, and excludes rain, snow, dirt, and insects, and prevents the sap freezing except in extreme cold, or souring by the sun's heat, except in very warm weather. I know of no one thing more essential to the production of the best grade of syrup than corers. They should be planed and painted, and it is a great help in gathering to have one side painted, say, red and the other white. All are placed red side up, for instance, in tapping, and then all are reversed at each gathering. If a tree is missed, the color of the cover shows it at a long distance. So none need be missed, and two trips need never be taken to the same tree in doubt whether its sap has been gath. ered.

The gathering should begin as soon as the tapping is done. The former should be finished by noon if possible. Otherwise one force of hands should continue this, and another force should begin soon enough to overtake before dark the force that is tapping. Sap should never stand over night in the buckets, if it can be avoided, but should be gathered as late as possible before dark, and boiled as soon and as rapidly as possible. It begins to deteriorate almost as soon as it leaves the tree, especially if it is very warm, or, on the other hand, if it freezes and thaws.

The following gathering-cask seems best adapted of anything for the work to be done. It 
is simply a cask five feet long and about two feet in diameter, fastened firmly to a "boat sled," large end behind, the front end a little the highest, so that when the sled stands level the sap will all flow from a faucet in the rear, through a tin conductor with funnel-shaped "head," down the slope into the store-trough below.

The sap need never be lifted but once, or dipped or rolled up skids in barrels at all. It is poured down into the gathering-pail from the bucket, which hangs at the tree and is not removed from the spout in emptying. It must be lifted a little and poured into the tunnel of the gathering cask, and that is all. After that, by taking advantage of a slope, it will run into store-trough and thence into boiler without further labor.

Location of Sugar-House.-The sugar maple seldom grows spontaneously, except on ground that is somewhat rolling, and in almost every sugar-camp, can be found side-hill advantages in a sufficiently central location. If the slope is not as steep as that represented in the cut, a longer conducting tube must of course be used, so that the gathering cask can stand far enough off up the slope to bring it to the required level. In hilly New England, there is usually no trouble on this point, but even there I have seen sugar-houses on level ground near a fine slope, of which they took no advantage. And in Ohio, until within a few years ago, such was the common custom. The store-trough stood on a level with the "arch,"

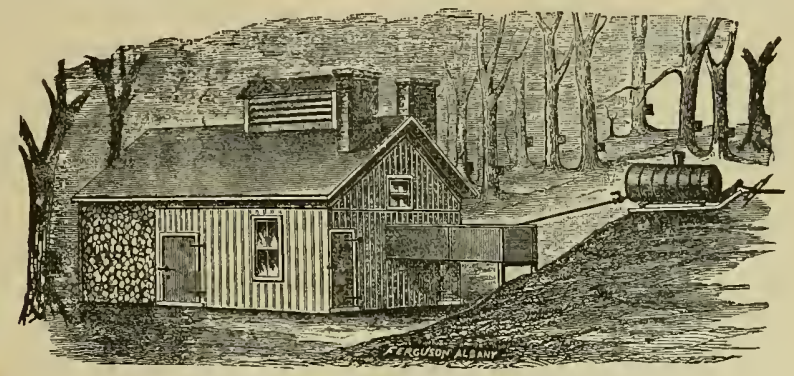

MAPLE-SUGAR-MAKING-VIEW OF SUGAR-HOUSE.

the barrels were laboriously rolled up two skids and emptied into the trough, and then the sap was lifted, pailful after pailful, and poured into the kettles or pans. Men are strangely slow in learning to take advantage of gravitation and the other forces of nature, even when she seems daily to thrust them before our very eyes."

The accompanying illustration represents the method of collecting the sap and conducting it to the sugar-house, as previously described.

Respecting the circumstances which affect the flow of sap from the sugar maple,-careful experiment and observation, by daily weighing the sap from several trees, and a comparison with the meteorological observations of the same period, have furnished sufficient proof to establish the fact that while the general flow corresponds with the season, yet the daily and hourly flow will vary with the weather; that the most favorable weather is that which is either steadily or severely cold, or uniformly warm and foggy; while the best weather for procuring sap are those days that are warm and bright at noon, but are preceded by freezing nights. The variations of temperature most likely to affect the flow of maple sap are supposed to occur when the ground is covered with snow, since the heat of the sun during the day cannot then moderate the cool temperature of the night. The explanation of the effect of these alternations seems to be that the outer tissues of the tree are partially emptied of the sap contained within them by the contracting influence of the cold, it being forced by this 
means into the heart of the trunk and large roots. During this time ajsorption coatinues to go on under ground the same as before, and thus, when the outer layers of the wood are separated by the heat of the sun, the sap rushes back into the cells and tissues, and flows in abundance. The observations of Biot in France on the poplars, and Nevins, in Ireland, on the eim, would seem to prove the above theory correct.

Maple-Sugar-Haking.-In making sugar one of the first essentials is, in keeping the sap and everything used in connection with it perfectly clean. Exposure to the air will readily deteriorate the quality of the sap, hence it must be evaporated as soon as possible after leaving the tree. This is necessary to secure the best quality of sugar and syrup, even when everything is kept perfectly clean; but where there is negligence an 1 carelessness in keeping the ressels and tauks cleau, the sap will of course sour much sooner, and an inferior grade of the product be the result.

Mr. Chamberlain, the above authority quoted, recommends scalding all the buckets used in collecting sap once a week, while the casks for storing, evaporators, etc., require it much oftener. He says: -

"Perfect cleanliness and sweetness of ressels and sap, is another essential. The Termont climate is better, but in Ohio, as a rule, I find I must scald all buckets about once a week, and store-troughs, evaporators, etc., much oftener. It costs a good deal, but pays in the product. With a cask of hot water and a team, two men will scald well 1200 buckets at the trees in a day, and there is almost always a rest between 'runs' as long as that each week. Our climate, too, requires that the sap be stored out doors. The trough runs into the house just far enough to feed the sap into the heaters, but not to sour that in the store. trough by the heat of the fire. The store-trough, or troughs, should have close-fitting corers, to protect from rain, sun, and freezing by night. In Vermont the cold is so great at night that it is common to store the sap in the sugar-house."

Where the maple sugar is made in large quantities, the evaporators used in making sorghim and other cane-sugar may be used to best advantage. These are constructed on the principle of a rapid evaporation, secured by a shallow depth of sap moring slowly orer hot and cool spaces alternating with each other, the cool spaces being secured by the projection of the pan beyond the fire on each side. The best work with these evaporators will be secured by keeping the sap as shallow in the pans as will be safe to prerent burning. The pans of course must be shallow, with a large heating surface. This will require constant watchfulness on the part of the person having charge. Where small quantities of sugar are made, kettles are used for boiling, but the evaporators require less labor, and are much more easily managed.

The sap should be kept boiling over a steady fire, and replenished with a fresh supply as it is eraporated. As soon as it reaches the boiling point, scum and other impurities will rise to the surface, and must be removed at once; all that arises during the boiling process must also be carefully removed. If a large quantity of the fresh sap is added at irregular intervals, the boiling is checked for a time, and irregularity is the result. Where eraporators haviug every arrangement for receiving a constant and steady supply of sap are not used, some arrangement should be made to have a reservoir of sap placed in such a manner that it can supply the boiling-pan or kettle with a small, steady flow of sap from a faucet. A little observation and experiment will enable the operator to regulate the supply in accordance with the degree of evaporation. After boiling from eight to twelve hours, keeping the quantity in the evaporator or kettle about the same, by a regular supply of fresh sap, the contents will be reduced to about the consistency of honey or thin syrup. It should then he strained while hot into a clean tube or other vessel, in order to remove as much of the sedj. ment as possible. A piece of clean white flannel is better for this purpose than cotton or linen. It should then stand long enough to become well settled. When the sediment 
remaining is well settled, the syrup should be boiled again - after first separating it from the sediment at the bottom - until it is sufficiently evaporated for granulating. During this process great care is necessary to secure a steady and constant boiling, and at the same time prevent the boiling-over. The sugar is sometimes clarified by the use of milk or the whites of eggs, or both combined, it being mixed with the syrup after it has been strained, and when it is just hot cnough to endure the hand for a moment, or before it is scalding hot, before boiling. As soon as it reaches the boiling point, a scum rises to the surface, which must be immediately skimmed off, and never allowed to boil into the syrup and thus mix with it. For this purpose, to a hundred pounds of sugar, five or six eggs beaten to a froth and added to a quart of new milk, and the whole mixed with the syrup, is the usual proportion. Some sugar-makers object to the use of clarifiers, as useless. If everything is kept perfectly clean in the whole process of sugar-making, it will not be necessary to clarify it in any way. Everything having a tendency to stain or discolor the syrup should be avoided. Rusty iron should on no account ever be permitted to come in contact with it. Even an iron spoon, used in stirring off the sugar, will often give it a dark color.

To determine when the syrup has become reduced to the granulating point, it may be tested by putting a small quantity on snow. If it cools to a waxy consistency, it is not sufficiently boiled to produce sugar hard enough for cakes, but it may do for tubs. For making hard sugar, the syrup must be evaporated until, when tested in this manner, it will be brittle when cold. Another test is to stir a small quantity of the syrup in a dish, and if it granulates with a moderate amount of stirring, it will make soft sugar, suitable for putting in tubs; but if, when applying a drop between the thumb and finger, and pressing, a granulated thread is formed, it will be hard and may be put into cakes. Rapid stirring a few moments before turning into the molds will make the sugar finer grained.

If the molds are first dampened with water before putting in the sugar, the cakes can be taken out more easily. Sugar may be made in a small way, where only a few trees are had for the purpose, by boiling the sap over the kitchen range in vessels of tin, or porcelain. lined iron kettles; but a sugar-house is a necessity where sap is obtained in considerable quantities.

Maple sugar retains its peculiar flavor best when kept from exposure to the air. The flavor of the syrup is best retained by canning or putting in jugs while hot, and sealing, the same as canning fruit. The cans or jugs should always be put in a cool, dry place, and never in a damp part of the cellar, or on the damp cellar-bottom, as this will cause it to mold. Before canning the syrup, the vessels in which it is placed should be made perfectly clean and sweet. If kept in too warm a temperature, or exposed to the air for some time, maple syrup will readily ferment, unless boiled down very thick. The sap will continue to run as long as the nights are frosty, the sugar scason lasting only a few weeks; but that obtained towards the last will not be of as good quality as that which is first produced.

Yield of Maple Trees. - The amount of sugar which can be produced from maple trees varies, some sap being richer in saccharine matter than others, while the amount of sap produced from trees of apparently the same size will vary somewhat. The amount of sap produced from the same trees also varies according to the season. The average amount of sugar contained in the sap is estimated to be from two to two and a half per cent., although some trees will produce more and others less than this estimate. A recent report from several large sugar orchards in Vermont gives the average amount of sugar per tree at two and nine-thirteenths pounds, the best average being three and two-thirds pounds; the lowest, one and three-fifths pounds. Eleven hundred and fifty trees in Canterbury, Vermont, yielded in one season 618 barrels of sap, or 19,776 gallons, from which 4,000 pounds of sugar were obtained. This is about one pound of sugar to five gallons of sap, or three and a half ounces of sugar per gallon, which is equivalent to three and a half pounds per tree. There are 
instances of extraordinary yield that might be cited; for example, it is stated upon good authority, that a tree in Leverett, Massachusetts, produced in one season the almost incredible amount of 1,400 pounds of sap, which probably contained fully forty pounds of sugas. A sugar orchard in Eaton county, Michigan, is said to have yielded 950 pounds of sugar, or on the average of nine and a half pounds per tree. Another orchard in Vermont is said to have yielded at the rate of six pounds per tree in one season. But these are the rare exceptions; as a general rule, the average yield will be found to be about two and a half or three pounds per tree, during the season.

\section{TOBACCO.}

$\mathrm{T}$ OBACCO has become one of the leading products in many parts of the country, while it is cultivated to a greater or less extent in nearly all sections. And not only this, but there is a continued increase in its production, as shown by the Census Report of 18s0, which was eighty per cent. in excess of that of 1870 . The abovementioned report contains the following summary respecting this product in the different States:-

"Fifteen States produce, now as in 1870, more than ninety-nine per cent. of the tobacco of the United States, though it is reported in twenty-two other States and six T'erritories. Of these fifteen, only Missouri, Illinois, Indiana, and Massachusetts producewless than in 1870. Kentucky occupies the first position, producing thirty-six per cent. of the total product of the country. Virginia holds the second place; Pennsylvania has advanced from the twelfth to the third; Wisconsin from the fifteenth to the tenth; and North Carolina, Connecticut, and New York have each gained one point in the rank of tobacco States. Those that have retrograded in relative production are Massachusetts, Maryland, West Virginia, Indiana, Illinois, Missouri, and Tennessee."

We do not propose, in this connection, to treat much of the evil effects of the use of tobacco upon the mind or physical system, or the principle involved in its culture, but rather to give the best methods in its cultivation, as practiced by the most successful agriculturists at the present time.

Tobacco is a native of America, and was introduced to the civilized world soon after the discovery of this continent. Columbus found it cultivated by the Indians in 1492, they using it as a means of producing intoxication by smoking it in clay pipes. Its culture was undertaken by the settlers of Virginia from the very settlement of the colony, where it soon became not only an important staple, but the principal currency of the colony. It is recorded that in the year 1615 the gardens, fields, and even streets of Jamestown were planted with tobacco. During the first century of communication between the New World and the Old, there seems to have been but little demand for this product by the latter, but after this period we find it entering quite extensively into the trade of the colonies with the mother country, which proves that its consumption was becoming more general.

History states that James I of England wrote a "Counterblast to Tobacco," which was intended to do away with this evil altogether, and that Pope Urban VIII issued an edict, excommunicating those who should use tobacco in churches, while Amurath IV of Turkey, the Grand Duke of Muscovy, and the Emperor of Persia, all prohibited the use of tobacco in their several dominions during the seventeenth century. Although thus violently opposed and denounced as useless and injurious, its use continued to make rapid progress, until at the present time it is known to almost the whole world. 
The price it commanded was a great inducement to the early settlers in favor of its cultivation, the market value in 1617 being from thirty-seven to seventy-five cents per pound, according to quality. The amount of tobacco produced by the colonists in 1622 was 60,000 pounds, which was more than doubled during the next twenty years. The total product in the United States in 1880 was nearly $500,000,000$ pounds, being the yield of 638,841 acres.

The evil effects of its use are attributed mainly to nicotine, a deadly poison which it contains, and which, when taken in sufficient quantities, will produce convulsions, followed by paralysis and death, as has been proved by numerous experiments on animals, it being one of the most powerful nerve-poisons known. The word "nicotine," as applied to this plant, is said by Torrey to have becn derived from John Nicot, embassador from France to Portugal in 1560, who introduced this weed into Europe. The word "tobacco" is supposed to have been derived from a place in Yucatan called by that name. The quantity of nicotine contained in tobacco varies greatly with different varieties and modes of culture, according to the best authority, as has been found by careful analysis of the plant; that produced on heavily-manured lands, as a general rule, containing a larger per cent. than that cultivated with a less quantity. The highest per cent. of nicotine found in about forty different specimens of the cured product, representing different varieties, different types of the same variety, with different methods of cultivation and curing, was 5.81 ; lowest, 0.63 ; only two specimens of the whole number being found below one per cent., and more than one-third above four per cent. By the analyses of Posselt and Riemann, 10,000 parts of fresh leaves contained six of nicotine, one of nicotianine, 287 of bitter extractive, 174 of gum, 26.7 of green resin, 26 of albumen, 104.8 of a substance analogous to gluten, 51 of malic acid, 12 of malate of ammonia, 20.6 of potash salts, 40.6 of lime salts, 8.8 of silica, 496.9 of lignine, and 88.28 parts of water. The odorous or volatile principle of tobacco is supposed to be due to the nicotainine it contains. The ash clement is exceedingly large, as will be seen by burning the leaves. One hundred parts of the ash of tobacco, according to the analyses of Fresenius and Will, contains 30.67 of potash, 33.36 of lime (together with a little magnesia), 5.95 common salt, 6.03 phosphates, 18.39 silica, and 5.60 gypsum. The superior kinds con. tain the largest amount of potash, that of inferior quality the largest amount of lime.

Much labor is involved in the culture of tobacco, and it is also liable to many injuries, either from insect enemies, severe storms, or early frosts. A fine field of tobacco is often utterly ruined when nearly ready to cut, by a hail-storm of only a few moments' duration, while a strong wind will frequently do great damage by breaking off the leaves and blowing over the plants. An untimely frost, just at the period of harvesting, has been known to not only nip the leaves of the entire crop, but with them the "bud of hope" in many a farmer's heart, respecting a field which perhaps during the entire season had been his pride, and upon which he had bestowed much careful labor. It is a very exhausting crop, and requires frequent and large applications of manure in order to cultivate it without impoverishing the soil. Some of the most fertile lands of the country have been in this manner injured by its growth; some of the exhausted lands of Virginia, and many of the New England tobacco farms, for instance, being examples.

Farmers are too apt to rob other farm crops of the manure which should be appropriated to them, for the purpose of supplying their tobacco-fields, while, if sufficient manure is pur. chased to supply all the crops, considerable expense would be involved which might not be warranted by the profits of the farm. Sometimes the market is overstocked with tobacco, and the farmer cannot find a ready sale at a fair price, and is obliged to keep it over another year or two before disposing of it-the capital invested in it thus lying idle and bringing no return. In many sections, however, notwithstanding the risk of its culture, it has thus far, in the main, averaged one of the best-paying crops produced, when regarded simply from a business point of view, and the exhaustion of soil either prevented by liberal manuring, or not being taken into the account. 
Varieties.-There are numerous varieties and sub-varieties of tobacco, the latter of which have been produced principally by the differences in soil, climate, and cultivation. The varieties most cultivated in the United States are the Connecticut seed-leaf, Havana, and Vurginia. The Connecticut seed-leaf is considered best adapted to the Northern section, that with its varieties being grown most extensively there, although the Havana and other varieties are cultivated to a certain extent. It is also well suited to the Middle States. It is usually known in the different Northern States under some local name, which has given rise to so many types of the seed-leaf varieties. The kinds most generally preferred in the Southern States are the Havana and Virginia, although a number of other varieties are also grown. The White Burley is a variety that is quite popular in the Ohio river counties, and some other sections.

In all the various types of the seed-Ieaf varieties, the form of the leaf has much to do respecting its value as a cigar-wrapper, these varieties and the Havana being used mainly for this purpose. The leaf that is designed for fine-cut, chewing tobacco, or fillers, may be either broad or narrow, long or short, the form of the leaf making no difference with regard to its use. Formerly the broadest leaves were preferred by most buyers for cigar-wrappers, but recently the long narrow leaf is preferred by many, the preference being about equally divided between the two. The latter is thought to cut more wrappers to the leaf and case, and yields more leaves per plant than the broad, while the former usually gives a somewhat heavier product per acre. The texture of the two varieties is about the same. The broad, spreading leaves are very brittle, and are apt to break easily in working among them. Whether the broad or narrow leaf be cultivated, there will be quite a distinction between the same variety grown in different localities; the nature of the soil, method of culture, etc., making a great difference in the quality and texture of the leaf. Some years ago a number of farmers in the Connecticut valley obtained seed from Ohio, known as the "Ohio broad-leaf," and tested it in various localities in that section. The result was a broad-leaved type much superior in texture and quality than the original plant, and which was known in many sections as the Connecticut broad-leaf. The variety known as the White Burley is said to have originated in the following manner:-

A farmer by the name of Marshall Slack, living in Higginsport, Ohio, was engaged in the culture of what is known as the Red Burley tobacco, and noticed one day in his seed-bed three or four plants differing from the others. The leaves were much lighter colored, in fact almost white. Thinking that they looked sickly, and that some insect was working at the roots, thus producing the peculiar appearance, he pulled one of the plants up and examined it carefully. Finding nothing the matter, but rather that it seemed perfectly healthy, he permitted the others to grow, and in due time set them out, giving them the same culture as the rest of the crop. These plants throughout all the stages of their growth and cultivation maintained their peculiar color of stalk and leaf, and through curiosity one of the plants was allowed to ripen its seed. This seed was planted the following year, and all the plants came up white. Its cultivation extended rntil it became quite popular, and thus a different type was originated. It is a vigorous grower, and seems admirably adapted to limestone sections.

By such accidental means, hybridizing, better culture, together with a perfect adaptation of soil, new varieties may be produced and the old kinds improved, forming new types, the same as with all other plants. There is no crop that has a wider diversity of quality than tobacco, and none that will respond more quickly to extra skill, labor, and management in its cultivation.

Soil.-Tobacco is an exhansting crop, and requires a rich, warm soil. In fact, we know of no plant that is so largely influenced by the character of the soil as tobacco. A decp, mellow soil will always secure the best results; one that is naturally rich or made so by the application of the proper kind of fertilizers. A light, loamy sand is one of the very best 
adapted to it; alluvial lands that are well drained and fertile are also good. It will not thrive on soils containing a surplus of water, however rich or whatever their character, and such lands should never be devoted to this crop until they have been thoroughly under-drained. Soils containing a large amount of potash and lime, either naturally or by application, are the best suited to it. It will not thrive upon such lands as are denominated by farmers generally as "sour," unless the quality be first remedied by the application of salt or lime, and previous cultivation. On such lands it will generally attain about one-fourth of its growth, and then seem to remain stationary for a time, the leaves assuming meanwhile a yellowish tinge. The crop in such cases will prove almost worthless-what there is of it-the stalks being hard and the quality of the leaf very poor.

On certain clays that are heavy and strong, it will be difficult at first to get the plants to live when transplanted from the seed-bed; but after getting well started, or in common farmer-phrase getting a "stand," such soils will often produce the very best crops, the growth being strong, heavy, and of fine quality. A Virginia farmer of many years' experience says:

"On very stiff, strong, red-clay land, the trouble is to get the plants to live. After being well set and started, however, such soils will make the strongest and heaviest sort, rich and weighty; but I have planted and re-planted such soils seven or eight times, and then only obtained a poor stand of irregular growth, and a large proportion of green tobacco was the result. Let your soil be of a loamy nature, with clay foundation, rich, and in 'good heart,' if old land. A good natural body is absolutely necessary for heavy, dark tobacco. Neither chemical manures, or even any other kind, will make the genuine, first-class article, unless the land already possesses 'body.' Guano alone will give it size and push its growth; but when that is said, all is said.

If the land is fresh, just cleared, or is being cleared for tobacco, white oak, hickory, beech, and walnut growth are sure indications of soil well suited to the growth of tobacco, in regions adapted to its cultivation."

It is not well to cultivate tobacco on sod land, but it should rather follow a crop that has been heavily manured, and kept free from weeds. The Connecticut River valley product, so noted for its fine quality, especially of cigar tobaccos, is grown on a rich, deep, clayey loam, with a sufficient amount of sand to make it permanently arable. This is overlying, for the most part, the new red sandstone. And we might add, that there is scarcely any section of the Middle States which yields regularly, year after year, such abundant crops of various kinds of farm products as this locality.

Preparation of Plant-Beds. - An abundance of strong, healthy plants is one of the essentials for a good crop of tobacco, and in order that such plants may be obtained, it is necessary that the seed.bed be properly prepared, and suitable attention given it until the young plants have attained a sufficient growth for transplanting. In the cultivation of tobacco, he who fails to secure an early and abundant supply of strong, healthy plants will find it very difficult to repair the loss at a later period, if it be not utterly impossible. On the contrary, the farmer who, at the proper time for setting out the plants, finds himself sup. plied with an abundance of them, of suitable size and vigor, may consider one important step taken towards a successful crop. Many are accustomed to take their risk, and depend upon their neighbors in this respect, but this is very poor policy. unless the order for the supply be previously arranged, since no one would be willing to limit the amount required for his own crop, and would take for such use the largest and strongest plants, leaving the smaller and weaker ones of the bed, while the best opportunities for setting are also often lost by the delay in obtaining them. The soil of the bed for sowing must be very rich and pul verized extremely fine; the finer, the better.

It is a good practice, and one followed by some of the most successful cultivators of tobacco, to burn a pile of brush and leaves on the bed before sowing. This will have a 
tendency to kill the seed of weeds in the soil, while the ashes will prove beneficial as a fertil. izer, potash being one of the best known for this crop. In such cases, however, the soil should not become charred by the burning.

The locality of the bed should be in some warm, sheltered spot, protected from cold winds, with a southeasterly exposure, in order to secure the warmth of the sun. The land should be neither too wet nor too dry. It is well to partially prepare the bed in the fall, reducing the surface to a fine tilth, and applying lime, ashes, and well-fermented barn-yard manure very finely pulverized. In the spring, the bed should be forked over or hoed, and raked off, in order to remove any of the coarser lumps. Where a hot-bed is used, the soil will, of course, be warmer, causing the seed to germinate quicker. In some sections of the South, the ground selected for the seed.bed is in the woods, where the soil is rich with decomposed leaves and other vegetable matter. A planter in Virginia says: -

"The ground selected should be in the woods (original growth), and in the thickest part of them, with a gentle slope (sufficient to drain well) to the south or east; soil of a dark allu. vial nature, such as would be used in filling flower-pots; not wet or sour, but with sufficient moisture to endure the miaday sun. Cut away all growth of every kind that will draw or shade the land, and nearly all that is near enough to shade the plants; a little shade is beneficial. It is best to have gathered the wood to be used in burning some time before, in order to have it seasoned; it burns much better when it has been stacked up and dried a little, and you can get along much faster, and will not have any trouble in keeping up the fire when it is moved. From a half hour, to one hour, is sufficient for the fire to remain in one place, when it should be moved, which is done by the use of a common wood-book or sapling which has a prong, and the prong cut off eight or ten inches from the trunk. The long row of wood should be laid on 'skids' before the fire is started. Under no circumstances must the earth be parched, or charred.

The object in burning is simply to kill the vitality of small seeds of grass, weeds, etc., and to secure a nice preparation, with the benefit, too, of the ashes as a manure. When the fire is moved, the coals should be pushed along with the fire; leave nothing on the bed but ashes; then hoe the ground over six or eight inches deep, but be very careful not to turn up the soil. Continue to chop the ground over until the soil is pulverized as fine as you would prepare a hot-bed for cabbages, or tomatoes. Rake it over nicely and sow, as a fertilizer, Peruvian guano, and rake it in with a fine hand-rake. I like it much better than any of the home-made manures, which are flled with all kinds of seeds that come up earlier than the tobacco, and guano is not so forcing as stable manure, which is so generally used, and causes the seed to sprout, and be killed by the cold frosts of February or March. One is certainly sared a good deal of hand-weeding by the use of guano. Half a bushel is sufficient for four hundred yards."

The seed-bed should be well drained. This may be accomplished by having the bed raised above the surrounding level, or by making ditches along each side, for, while moisture is necessary. an excess is as injurious as a lack of water.

Poultry manure, and that from the pig-sty and barn-yard are good for this purpose, either used alone, or in connection with commercial fertilizers, but their use involves more or less trouble with weeds, especially the latter two. Te have found but little trouble with weeds when commercial fertilizers were used exclusively for fertilizing, and the beds had been previously prepared by burning brush and leaves over them, as above recommended.

Sowing and Weeding. - The time of sowing tobacco differs in different localities. As the seed does not germinate quickly, and the plants at first are also of slow growth, tobacco should be sown as early as the soil will admit of being thoroughly worked, and warm enough for the seed to germinate. It is customary with many farmers, especially at the North, to sprout the seed before sowing in the bed, as the plants will thus be started 
earlier, and considerable labor in weeding will be obviated. In the Northern States this is generally done about the last of March, or the first of April. At the South it is done from the first of January to the fifteenth of March, according to locality, the warmer climate admitting, of course, of the earliest sowing.

The best material for sprouting is well-rotted wood. Apple-tree wood is the best for the purpose, although anything that will keep the seed damp will answer the purpose. The szed is mixed through the rotten pulverized wood, and put in a pan or box and set in a warm place near the stove until sprouted. It should be occasionally stirred, to insure sprouting evenly. After it has become well sprouted, it is scattered over the bed, usually being left on the surface. Sometimes boards put on the bed for a few days after sowing will hasten germination by keeping the soil damp, and pressing it upon the seed.

When seed is not sprouted before sowing, it is generally mixed with fine earth, sand, or wood-ashes, as it is so very small that it will not otherwise be as liable to be sown evenly, and the plants come up uniformly. Great care should be used to scatter it as evenly as possible. Scattering it both ways secures the best distribution. Where the seed is good, but little is required. One tablespoonful, that will germinate readily, will be sufficient for setting five or six acres of land, providing the plants should thrive well.

However, as considerable of the seed may not come up, and as many of the young plants are liable to be destroyed, it will be well to allow a larger proportion of seed, as it is always well to sow enough to make allowance for such contingencies, and have a liberal supply of good-sized, healthy plants at the time of setting.

The rule with some farmers is, to sow in the proportion of a tablespoonful of seed to sixteen feet square of surface. If the spoon is heaped a little, this will give about four hundred seeds to the square inch, and if one mature, healthy plant is produced from every three seeds, a bed sixteen feet square will supply thirty thousand plants, which is sufficient to plant about seven acres at the ordinary distance.

Some scatter the seed over the bed, and rake it in very lightly, but there is danger of covering it too deep in the soil by this process unless great care is used. It is a good plan to scatter it on the surface of the bed and roll it very lightly with a hand-roller, thus pressing it into the soil; but the soil must not be made too compact by this process.

During the first two or three weeks after sowing, the seed-bed should receive daily attention. The soil must be kept constantly moist (not wet) on the surface, or the newly.germinated seed lying on or near the surface of the ground will be destroyed. Even an hour of hot sun, when the seed-bed is dry, will sometımes kill the young and tender sprouts or plants, and, if left to careless or indifferent care, there would be danger of loss in this respect, or from the other extreme of keeping the soil too wet. When glass is used, there is still greater danger of sun-burning. It is a good plan to cover the bed with fine brush to protect it from frost and the hot sun. Sometimes straw or chaff, free from grain, is used for a covering, but there is always more or less danger of injuring the plants by removing it in such cases. At the South, pine tags are much used for this purpose, which are not removed, since they aid in maintaining a sufficient amount of moisture. The tags are generally used in connection with a covering of light brush, the latter being removed when the leaves are about an inch long.

After the plants are up, a good top-dressing may be made of well-fermented, pulverized horse manure, or a mixture of equal parts of stable manure and wood-ashes, plaster and vegetable mold, to which four pounds of soot and sulphur are added to four bushels of the mixture. This mixture will be a sufficient quantity for any ordinary bed, and, if applied in judicious quantity every ten days, it will not only secure a rapid growth of plants, but prevent the attacks of insects. When late sowing is practiced, the plants will require stimulating to be sufficiently grown at the time of transplanting. A narrow bed is more conven- 
ient to manage than a wide one, and several small beds are more easily cultivated than one large one. Dr. Thomas Pollard, recent Commissioner of Agricul ture in Virginia, recommends the following method in the care of seed.beds: -

"The sowing of the beds should rather be too thick than too thin, at least a tablespoonful for erery fifty square yards, sowing both ways to insure regularity, and then later sow a teaspoonful over the same surface. After the plants get four leaves, they should be forced by application once or twice a week (some say even three times a week) of some good fertilizer, or better, dry pulverized stable manure from horses fed on fodder and grain, to avoid grass seed, to which may be added some gypsum. The 'fly,' or flea bug, must be looked for and combated by liberal applications of manures, to force the plants out of its way, and by plaster, in which rags saturated with kerosene oil have lain for some hours, and by every . means which have been found to be available. Plenty of plants is the foundation for a good crop. Better have a hundred thousand too many, than ten thousand too few. And it is important to have them early. A forward crop is generally best, even if the late heavy dews are not secured to the plant."

To obviate the danger of cooling the soil of the seed-bed, tepid water should be used for sprinkling the surface. Light and frequent waterings are preferable to a more thorough wetting, as would be the case when applied but once or twice a day. The aim is to keep the soil warm and moist, but not to saturate it with water. Liquid manure is very good for stimulating the growth of plants after they have become well started. This can be made by placing a sack of hen manure or guano in the barrel containing the water for sprinkling the bed. Spronting the seeds before sowing will hasten the plants from a week to ten days; but where they are not first sprouted, the plants will not generally appear until about three weeks after sowing.

The seed-bed should be kept free from weeds, the weeding beginning as soon as the weeds are large enough to pull. This is a laborious process, especially where the seed-bed has not previously received the attention requisite for destroying the seed of weeds in the soil. The weeding should not be slighted, but attended to thoroughly, as often as necessary. The use of commercial fertilizers, and burning over the bed previously, will save much of the labor of weeding. When glass is used for beds, a portion of the sash should be raised each day when the sun shines hot, in order to give ventilation and prevent the plants from being wurned. The glass should also be entirely removed a few days before the plants are large enough to set, in order to toughen them sufficiently before transplanting them in the field. On very cool nights, when there is danger from frost, or the plants being chilled, the bed should be protected by a covering of some kind, such as boards, old blankets, or pieces of carpeting.

The usual rule is to sow two hundred square yards for every ten thousand plants wanted. It must always be remembered, also, that unless the soil has been made previously very rich, the plants will not have sufficient to feed upon, consequently will not grow, but will assume a yellow, sickly appearance. A board frame surrounding the bed, fitting it closely, answers a very good purpose for keeping off intruders, where glass is not used. It also is very convenient for supporting and holding a cloth covering, that may be necessary to protect against the frost or insects. This covering may be cheap, thin, unbleached cotton, and costs much less than glass, and many consider it equally good. This is very efficacious in keeping out the tobacco fly. Some other plant-seed, such as radish, could be sown outside for the fly to work upon, in connection with the covering, thus securing a double protection.

A recent writer gives his method of covering the beds with cloth, as follows: -

"When your bed is rolled, put up, edgewise, eight to ten-inch plank all around the edges of the bed, and on the end planks put gable ends cut from planks a foot wide, then a ridge- 
pole like that for any roof. This gives a frame for a covering of muslin of the thinnest and cheapest kind, and in convenient shape to remove and replace, as you choose, for watering, manuring, etc. But if you have liquid manure, you may water and manure at once by sprinkling the cover well with a common sprinkling-pot with the nozzle-holes enlarged. After the plants are well started, if there are no flies, or if the plants are big enough to defy them, in good weather the cover ought to be removed at 3 to $4 o^{\prime}$ clock P. M., and replaced at 10 A. M. for several days, to gradually harden the plarts to the exposure, and then removed altogether, to harden them for transplanting. Plants may be brought to maturity in this way much sooner than the usual time in open beds."

In ordinary open beds, with proper care, the plants will be ready for transplanting in from six to eight weeks after sowing.

Preparation of Soil, Fertilizers, ete.-Lands for tobacco should be plowed in the fall, that the frost may aid in pulverizing the soil, also in the destruction of the worms, insect eggs, and larvæ that it may contain.

It should also have two plowings, or their equivalents, in the spring. The first plowing should be early in the spring, which should be to a shallow depth. When the manure to be applied is not well pulverized, it will be well to spread it over the land before the plowing. When it is well decomposed and pulverized, it can be applied just before the second plowing, which is about the time the plants are to be set. It is best to spread fertilizers broadcast for tobacco. After the second spring plowing, a thorough harrowing will greatly aid in reducing the pulverization of the soil. The roller may also be very useful in aiding the pulverization by crushing the lumps. Very much depends upon reducing the soil to a fine condition, and the labor bestowed here will be amply repaid in the results of the crop, the aim being to render the surface as fine as possible.

Any well-decomposed manure will do for tobacco, but of course the better the quality of the manure, the better will be the crop. Manure finely pulverized is much the best, since it can be more readily taken up by the roots of the young plants than that of a coarser texture. Commercial fertilizers can be used in connection with farm manure with good effect. Many of the special fertilizers can also be used in the same manner. In the use of commercial fertilizers, however, much depends upon the season, a moderate amount of rain giving often remarkably good results, while during a dry season their effect seems almost entirely lost. There is probably nothing better for tobacco than farm manure of the best quality, where it can be obtained in sufficient quantities. Where a sufficient supply cannot be obtained, it may be used in part, and commercial fertilizers be applied in connection to make up the deficiency.

We have never seen better crops grown than those produced by a liberal application of barn-yard manure, well rotted and finely pulverized. For heavy clay soils there seems to be no substitute for it, since it not only supplies the requisite fertility, but renders the otherwise compact soil loose and pliable.

A good clover crop plowed under green, and allowed to become thoroughly decomposed before setting the plants, together with a liberal application of manure, is thought by some to be the best preparation for tobacco that a soil can have. Next in value to clover, for green manure, a heavy growth of common peas might be chosen.

Poultry manure, pulverized and mixed with plaster, is excellent for tobacco. A farmer in Central New York, who has cultivated tobacco for many years, and has tried many experiments with different kinds of fertilizers, recommends this as superior to anything with which he is acquainted. Fertilizers containing the elements of lime and potash will always be found of value to this crop, since they enter largely into the composition of the plant. Wood-ashes are always adapted to tobacco, since they supply a large proportion of potash. Manure containing an abundance of ammonia, in connection with lime, will always be found highly beneficial also. 
When level culture is practiced, the land should be marked both ways with a small plow adapted to the purpose. The rows should be at least three feet apart, and the marks for the hills from two to two and a half feet. Much depends upon the nature of the soil and the variety to be grown. A rich soil will require a greater distance than one moderately fertile, while a variety of large, spreading growth will also require more room than one of smaller growth. Many farmers make the rows the distance of three and a half feet by two and a half, while on very rich soil the practice in some sections is to extend the distance to even four feet by three and a half. There should only be sufficient distance for the plants to spread well and secure a vigorous growth, any waste of space being a waste of manure used to enrich the soil, as well as the labor in preparing it; besides, if the plants are too far apart, they are more liable to be blown over or broken by the wind. On the other hand, if crowded, the growth will be more slender and the plants will not mature as perfectly, or produce as heavy a crop. Where the furrows made by the plow cross each other, small hills should be made with a hoe, and the top patted down, indicating the place where the plants are to be

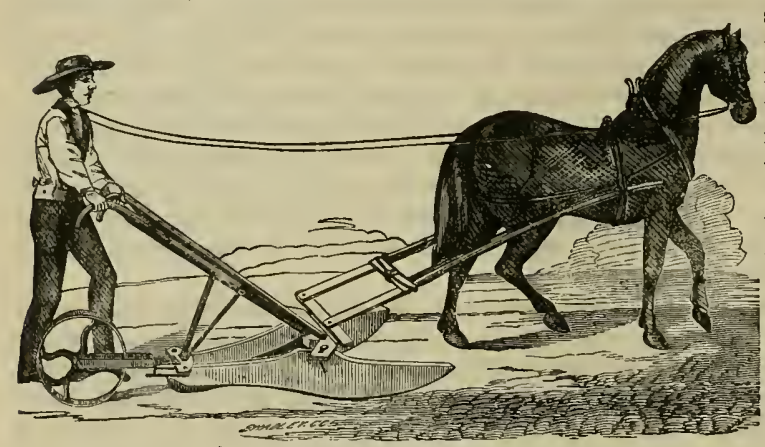

GOSLEE'S TOBACCO-RIDOER AND POTATO-COVERER. set. Where ridge-cul. ture is practiced, the ridges may be thrown up by lapping the fur. rows of the plow, or, what is much better, a tobacco-ridger may be used for making the ridges and marking the place for the plants. The wings of the machine gather the earth into a ridge, together with the fertil. izers that are spread broadcast. The smoothing-plate that the machine rides on smooths the ridges, and the wheels with the points partly make the holes for the plants, and space them off. The accompanying illustration represents a tobacco-ridger manufactured by Belcher \& Taylor, Chicopee Falls, Mass., and may be also used in the cotton and potato fields with good effect. A corn-marker is sometimes used for marking off the ground. In these marks a cultivator having reversible shares is run, with the shares turned inward and the front one removed, which forms the ridge. A light roller follows, having cleats nailed across at equal and sufficient distances apart to mark the distances for setting the plants. This preparation for setting tobacco should be made just before the plants are ready for transplanting.

Trausplanting. - The tobacco crop requires between four and five months' growth, from the time the seed is sown until the time of cutting, and nearly half of this time is confined to the seed-bed; hence the necessity of much care to secure the best possible condition of the plants at the time of transplanting.

The best size for setting is when the leaves are from an inch and a half to two inches in length, or, as one planter expresses it, "about the size of a dessert-spoon." The time for setting, in the latitude of New York, is in the month of June, although some set them about the 24th of May. This is, however, quite early. From the 1st to the 20th of June is generally considered the best time, when the season will admit. In a warmer latitude the transplanting will be comparatively earlier, according to the climate. If set too early, the plants will be liable to become chilled, and will not thrive as well; while if delayed too late, 
the crop will not have sufficient time to mature. The medium time is best, which is that already given. If transplanted at the proper time, rapid growth and quick maturity - other conditions being favorable - will be the natural result, which saves labor and expense in cultivation, while such crops will be larger and of better quality than those of slower growth.

The usual time for transplanting is just after a rain, when the soil is moist; otherwise the plants require watering, which involves considerable extra labor. If at such a time, however, the sun comes out hot immediately after, a large percentage of the plants will be hable to wither beyond recovery. A cloudy day and damp soil are to be preferred, but it is better to set the plants at the proper time rather than delay too late for desired conditions. If plants are set when the soil is saturated with water, followed by a hot sun, the earth will harden and bake around the roots and prevent their growth; hence a damp soll is to be preferred to one containing a surplus of water. Never set plants when the soil is of the consistency of mud. Great care should be observed in removing the plants from the seed. bed, that the roots may not be broken. To avoid this, the bed should be saturated with water. It is well to loosen the earth with a trowel and pick up the plants separately.

No careless person should be allowed to perform this work, for much injury to the crop might result from mutilating the plants in any way. The practice of crowding a large number of plants into a basket to be taken to the field is a very poor one, resulting in the bruising and breaking of the roots and leaves. The young plants should be kept straight after being taken from the seed-bed, with their roots together, and placed in shallow boxes or pans to be taken to the field, and not a sufficient number placed together to admit of crushing, keeping what little soil adheres to their roots from being loosened. They should also be protected from the hot sun on the way to the field. Many plants will sometimes become wilted before setting, through carelessness in this respect. One person should drop the plants ahead in the row, one plant to each hill, and the setter follow. In setting, the plant should be taken in the right hand, and a hole made in the center of the hill with the left forefinger for the roots, which should be deep enough to take them in without bending to the same level they occupied in the seed-bed. The earth should then be pressed firmly around the roots with both hands. The pressure should be sufficient to close the hole in the soil at the bottom, as well as at the top. Care should be used not to set the plant too deep, or press the bud of unopened leaves in making the soil compact. The roots should, however, be well covered, and have a sufficient depth of soil. It is also a good plan to set an extra plant every rod or two, which may be used to fill vacancies that will be found in the after cultivation. Such plants may be taken up with a small quantity of soil attached to the roots, and reset without injury.

As the cut-worm will destroy some of the young plants, and others will wither, the ground should be carefully examined and reset every few days, until a good stand is secured. It is important that the setting of the plants be well done. When a hot sun succeeds the transplanting, shading the plants with a handful of grass, a large leaf, or a piece of paper, for a day or two, will generally prevent them wilting as badly as they otherwise might.

If the ground is quite dry at the time of setting, water must be turned upon the hills before putting in the plants. In such cases, it is well to press the soil around the plant in a slightly concave or basin-shaped form, in order to hold the water that it may be necessary to afterwards apply. Such plants should be watered rather liberally just at night, and may require this repeated for three or four days. Such extra care requires considerable labor, but it pays well in the end. Negligence at this period can never be wholly made up in the after culture. The best time for transplanting, when the weather is warm, is on a cloudy day, or just at night, accompanied with watering.

Cultiration.- A few days after setting, or when the plants have secured a good stand, the first cultivation should be given. This generally consists of a very light stirring of the soil with a hand-hoe, simply breaking the crust that has formed, and killing the weeds. 
It is very important that the soil at all stages be kept free from weeds. Tobacco is a sensitive plant, and will not grow well in connection with anything else. Weeds or grass near the roots, though of small size, will affect the weight and growth to an almost incredible degree. Neither will it attain perfection if crowded or shaded in any way. It must have the soil all to itself, and a hot sun. As quick growth is essential in order to produce a good quality of leaf, the aim of the cultivator should be to push the growth of the young plants as much as possible, after they have been started in the field. Next to extremely fertile soil, nothing will secure this result so effectually as frequent cultivation, not only to keep the field free from weerls, but to stir the surface and prevent the soil from becoming hard and compact, and thus lessen the danger of injury by drouth. It is a well-known fact that the best preventive of injury from drouth is to keep the surface of the soil mellow around the growing plants. The cultivator or horse-hoe should be used frequently, to keep the soil clean and mellow, hand-hoeing being required three or four times during the season, in order to loosen the soil near the plants and bring the earth up around the roots. The extra plants not required to reset should be taken out when there is no further demand for them. When the leaves are large, they become easily broken, and all, such culture must then be abandoned; but, until this period arrives, the cultivation should be thorough and frequent.

Enemies of the Tobacco Plant.-Although there are several enemies of the tobacco plant, more or less injurious, the principal ones are the cut-worm, and the large green worm called the "tobacco-worm." The first commits its depredations on the young plants soon after they are set out, by eating off the stalks. Their presence can easily be determined by going among the plants early in the morning, and noting whether there are any small heaps of fresh earth, or small, round holes near the hills. If these are found, they are a sure indication of the enemy.

Where plants are found with a portion or all of their leaves gone, this is another sure indication of the cut-worm's presence. Ashes in the hill will sometimes destroy these pests, but they are difficult to eradicate. It will pay to search for them in the hill and kill them, where they are very troublesome. All hills that have had the plants destroyed in this manner should be supplied by re-setting, but the worm should first be dug out and killed, or each plant that occupies the spot will be destroyed in turn.

The tobacco-worm is hatched from an egg deposited by a moth. The egg is laid generally upon the under side of the leaf, and, when first hatched,-_which is in about six days, the worm is so small that it would scarcely be noticed by one unfamiliar with its habits. It grows very rapidly, and proves one of the most destructive of enemies.

It begins to eat the leaf on the under side; hence, is not at first easily detected. A small hole through the leaf is the first indication of its depredations. They increase in size so rapidly, and are so destructive, that if left unmolested for a few days the entire leaf would be destroyed. When full grown, the worm will eat nearly an entire leaf of large size in a single day. Their size and length at this period is nearly that of the forefinger of a medium. sized man.

There are two sets or broods of these worms during the season-the first appearing when the plants are about half grown, and the others when the tobacco is almost ripe. It is particularly important that the first brood be destroyed, for if they are not they become transformed into the moth, which lays the eggs for the second brood, each moth laying about two hundred eggs.

The "yellow.jacket" is said to be a valuable aid in destroying these worms. The "ich. neumon-fly" is a parasite of this worm, and destroys many. Dr. Thomas Pollard of Virginia, says that, in worming, care must be used not to destroy the worms covered with a white film or net-like substance, as it is the cocoon of this parasite, and that in sections where this fly is met, tobacco-worms may be found filled with the larvæ of the fly, looking like little bits of 
cotton, half of their bodies being inserted in the worm and the other half projecting, having eaten their way out thus far. The worm in such cases will be found in a half torpid state, undergoing a slow but certain death.

The flea-beetle has made its appearance from time to time in some sections, to the detriment of this crop, but only to a limited extent, and in certain sections.

Worming.-Unless the worms that feed upon the leaves of tobacco are destroyed, the crop will be liable to be, or, at least, rendered so worthless as not to pay for the labor bestowed in its cultivation. The surest remedy is hand-picking, and this must be sufficiently frequent to prevent injury to the leaves. The field should be gone over every few days, if possible, during the period of worming, as many will escape notice, and the eggs will continue to hatch. By a careful examination of the under side of the leaves, the eggs may often be seen and destroyed before the worms are hatched. They are about the size of a mustard seed, of a light greenish cast, and semi-transparent.

Flocks of turkeys are said to be valuable in destroying the worms. Bonfires built near the field at the edge of evening, at the time of the appearance of the moth that lays the eggs, will be the means of destroying large numbers, since they will be attracted to the fire by its light.

Major Rayland, of Virginia, says: "Dissolve an ounce of the cobalt of the shops in a pint and a half of water and mix it with molasses or other syrup, bottle it and drop it through a quill into the heart of the blossom of the jimson-weed. It should be done about sundown, and the poisoned flowers pulled off next day, otherwise the plants will be destroyed. It has been authentically stated that this weed, so treated, planted around the edge of the tobacco-lot, and here and there through the patch, will prevent, to a great extent, the ravages of the worm."

Topping.-This consists in breaking off the tops of the stalks of tobacco, and is done in order to increase the size and value of the lower leaves; also, to prevent the plant from seeding, and to hasten its maturity. The best time for doing this is generally considered to be just before the flower-buds open, or, rather, just as soon as the blossom-bud at the top of the stalk is formed. When there has been a partial failure of the first setting of plants, rendering the growth uneven, it will sometimes be necessary to go over the field twice for this purpose, topping at first only the earliest plants, and the remainder a few days later. It is, however, desirable to top all the plants at the same time, if possible, in order to secure uniformity in the maturity of the crop.

The condition of the plant will indicate how great a length of the stalk is to be removed in this process. The general rule is, to take off all the leaves that are less than six inches in length, or just above the last well-developed leaf. When quite late in the season, it may be necessary to go a little lower than this, and take off a few of the larger leaves in order to have the crop mature before the appearance of frosts.

In some of the Southern States, priming is practiced, which consists of removing the first or lower leaves of the stalk. The arguments in favor of priming, as given, are-that the lower leaves harbor worms, making the worming process more laborious, requiring so much of the stooping posture, while the saving of those leaves is often a sufficient temptation to the producer to prepare them for sale, where they add to the worthless product which is cumbering the market. Other advantages of priming that are presented are-that the removal of the lowest leaves permits additional nourishment to be supplied to the other leaves, and admits of a better circulation of air, with additional light and heat, which are so essential to plant life. The old adage with many of the Southern planters is, "Prime high and top low," while some do not prime at all. It is not practiced to any extent at the North. Of course, all plants intended for producing seed for a future crop should never be topped, but be permitted to blossom, and ripen their seed. 
Suckering.-A few days after topping, the suckers, or young shoots, will start from the axils of the upper leaves. These should be broken off close to the stalk as soon as they are at least three or four inches long. If a short end is left in breaking, it will be liable to tear the leaves in subsequent handling; besides, the water from rain or dew will produce decay of the portion left, causing the leaf to look sickly and sometimes fall. If the suckers are permitted to grow, they will greatly injure the crop, as they will take just so much growth and nutriment from the leaves, which constitute the product.

In about ten or twelve days after suckering, the crop will generally be ready to cut. Just before cutting, however, the suckers should all be broken from the stalk again, as they will grow fast. The worms should also all be removed, none being allowed to remain on the plants when harvested. Working among the growing plants when they are wet from rain or dew will sometimes cause rust upon the leaves, and should be avoided. Some varieties of tobacco will send out suckers faster than others.

Cutting.-Tobacco should be cut when fully mature. If left to stand beyond this period and over-ripen, the leaf will not, when cured, be as soft and silky, and hence not as well adapted for wrappers. Longer standing will, however, give a somewhat heavier pro. duct, but the weight will be gained at the expense of the quality.

The time for cutting is determined by the appearance of the leaf, which changes its color to a somewhat yellowish cast, and becomes slightly spotted or marbled, especially when looked at towards the light. Sometimes this appearance seems a faint mottling of the leaves with spots of a lighter green. As a general rule, it will be ready to cut in about ten or twelve days after topping, according to the weather. When the weather is hot and dry, the ripening will proceed more rapidly than when cool or wet. Another indication of ripeness is in the feeling of the leaves, which will seem thicker than they did a few days before, and will crack when folded and lightly pressed between the thumb and finger. The leaves will also become rustling and stiff. It is somewhat difficult for an inexperienced person to determine when tobacco is fully ripe, the experienced eye being the only sure guide; but the above rules can be relied upon as the most definite that can be given. Over-ripening deteriorates the quality of the crop; therefore it is best to commence harvesting as soon as the signs of ripeness appear, as the last of the crop that is cut will be liable to become too ripe when left later, if a large crop is to be gathered, since it ripens very fast. The juices in the stems of that first cut will perfect the ripening process.

It is very important that the crop be gathered before cold weather, as the least appear. ance of frost will ruin every leaf that it touches; hence, while it is well to have the crop sufficiently ripe before cutting, it had better be harvested before it is quite ripe, rather than run the risk of losing the entire crop by frost.

Various implements are used for cutting tobacco. A tobacco-hatchet is one of the best we have ever used for this purpose. A cornstalk-cutter is also very good, while a butcher. knife may be used to good advantage. Some prefer a small hand-saw. In cutting, the plant should be bent over with the left hand, and the stalk cut close to the ground with a blow from the hatchet, or a drawing cut with a knife.

Very heavy tobacco is left for a time to wilt before being carried from the field, as the leaves will be less liable to become bruised or torn by subsequent handling. but light varieties may be carried directly from the field with less liability of being injured.

Tobacco should not be cut when wet with dew or rain, or in the middle of a very hot day. Care must be taken not to let it remain in the field long enough after cutting to become sunburnt. Rain should also be guarded against. If any is left cut in the fiold during the night, it should be laid in heaps of a dozen or more plants in a place. If tobacco is thus left in piles more than twelve or fourteen hours, there is danger from heating.

As soon as the plants are sufficiently wilted after being cut, so that the leaves will not be 
easily broken by the handling, they should be taken to a tobacco house or barn and hung up to cure. The best method is to put it on laths or poles in the field ready for hanging, as this requires less handling. It is customary with some tobacco growers to load the plants, after they have become sufficiently wilted, into a cart and put them on laths afterwards in the shed or barn, but the best method is to do this part of the labor in the field, if practicable.

Curing. - The usual method of curing tobacco is to hang the plants on lath, on which they are put by means of a large steel needle made with a socket at one end, in order to fit the end of the lath. The needle, after being fastened to the end of the lath, is pushed through the lower part of the stalk, and thus the lath is forced through. From six to ten plants can be put upon a common lath in this manner, according to the size. The laths on which the plants have been strung are put into a cart or wagon arranged for the purpose. Some are particular to have each end of the lath supported, that the plants may hang down without being broken or crushed. This requires more labor, as a less quantity can be carried at a time, but the plants are less liable to injury by this means.

The laths are placed upon slats or crossbeams arranged for the purpose in the tobacco. house or barn, where the plants are suspended for drying. Where large quantities of tobacco are produced, a tobacco-house is indispensable. Such a building has beams and joints in sev. eral tiers, arranged to support the laths or poles on which the plants are hung. A bout onehalf of the boards of such a building are hung on hinges, so that they can be opened like a door, in order to better admit the air and light. Where the air and light are thus admitted, the laths can be placed nearer together than where the circulation of air is less free. About ten or twelve inches apart is the usual distance of placing the laths from which the plants are suspended. In a common barn or close shed, considerable more room will be required. Very large plants will also require more room than smaller ones.

After hanging the plants for curing, the doors should be kept open in pleasant weather to admit the air and light, which are so essential to perfect the process. During damp or rainy weather the building should be kept closed to exclude the dampness, and after the curing process is completed the building should be kept closed constantly.

When tobacco dries rapidly, the tendency will be to produce a light color. When it dries slowly, the tendency will be in the opposite direction. The great difficulty in curing tobacco is to so govern the ventilation as to secure slow drying, and obviate what is termed "pole-burning," or pole-sweating- This latter difficulty is most liable to occur in hot, muggy weather, when there is little air stirring, and during the first three weeks of the curing process.

In very dry, windy weather the plants might dry too rapidly if the means of ventilating were all employed, and it may sometimes be found desirable in such weather to keep the doors closed entirely for whole days together. The judgmeut of the person having charge of the crop at this stage will be called into requisition, the treatment given depending upon the condition of the tobacco at the time. Pole-burning may be largely avoided by hanging the plants further apart.

It is the nature of the plant, irrespective of weather, to begin to sweat a little a few days after hanging, the moisture standing in drops on the stems and leaves. When this moisture dries off well, there is little danger from pole-burning. At this period there should be a free circulation of drying air through the plants. Pole-burning is most liable to occur during the first three weeks after the tobacco is cut. If the air is then damp and heavy with moisture, the plants will not dry off well, but will ferment or "burn." The best method is to exciude the dampness in wet weather, and admit the dry air in clear weather, taking care not to crowd the plants in hanging. The leaves should also be well shaken out so as not to cling to the stem. Fully ripe tobacco is less liable to pole-burn than that which is cut greener, but such tobacco is considered less desirable for wrappers. In all the process of curing, great caro 
should be observed not to bruise or tear the leaves, or break them off the stalk. Where poles are used for hanging tobacco, the plants are generally tied to them with twine, which is wound around the plant stalks securely, and then around the pole in successive order; the use of laths is considered the better method, as it is attended with less labor.

There are various other methods of curing practiced in some sections, such as "suncuring," "open-fire-curing," "flue curing," etc., but we consider the process already described to be very much superior to any other.

Stripping and Assorting Tobacco.-Tobacco should never be taken down until properly cured. This period is when the leaf stem will yield no juice by wringing, although still pliable and damp. There may be an occasional green or "fat" leaf, which will never cure, but these should be very rare exceptions.

The time for stripping is in the late fall or winter, during warm, wet weather, which will render the leaves pliable, and easily handled without being torn or broken. In order to keep the plants moist, they are taken from the laths and laid in small piles upon the floor. The leaves are then stripped from the stalk one at a time, taking care not to tear them, and keeping each one straight. The leaves are assorted according to quality, a crop often making six or eight different grades, the grade differing in numbers according to the custom of the locality, or demand of the market. The leaves of the same quality and size are kept separate from others. All torn or worm-eaten leaves, and those otherwise injured, should be kept by themselves. The manner of assorting tobacco differs in different localities. The following method is given by a tobacco-grower of large experience in central New York:

"Taking tobacco from the poles, stripping the leaves from the stalks, assorting them into the respective grades, and packing the 'hands' or 'hanks' into boxes, constitute the chief occupation of the tobacco-grower during winter. The plants should not be taken from the poles until the leaves have become soft and pliable so that they may be readily pressed together in the hand without breaking them, when brought into this condition by warm, damp weather. After being taken from the poles, the stalks should be stripped of their leaves and the latter tied in bundles weighing from six to ten pounds, and then carefully packed in 'ranks,' with the butts of the leaves extending outward and their tips lapping together in the center. In order to preserve the moisture in the leaves, the ranks must be kept covered upon the top and ends, but the butts of the leaves or the sides of the ranks should remain open, and exposed to the air so as to give the greenness at the butts an opportunity to become thoroughly cured.

Next comes the work of 'assorting.' For this a tight, warm room is required, such as may be made comfortable by an ordinary stove in the most inclement weather. This assorting room should be roomy, well lighted, and provided with tables suitable for the work. A skylight is preferable to side windows, as from the former the light coming from above shines directly upon the leaves of the tobacco, and not in the eyes of the assorter, thus enabling him to better judge of the qualities of tobacco. Every year the dealers and manufacturers of seed-leaf tobacco demand of growers more care in the manipulation of their crops. Formerly it was customary to assort the leaves into two grades; now we are frequently requested to assort into six or more qualities, which requires no small amount of labor and care to perform the work correctly.

But it is unquestionably for the grower's interest to put his crop in the best possible shape, and to handle it in the most systematic and painstaking manner. For this reason I advise making at least five grades or qualities of leaf; namely, first or A A wrappers, second or A wrappers, scrub or rough wrappers, binders and fillers. Some buyers demand that the leaves of wrappers shall be assorted as regards length as well as quality. Then first or A A wrappers are divided into two lengths, making long $\mathrm{A} A$ and short $\mathrm{A} \Lambda$, etc.

It is a difficult matter to describe the exact methods of assorting tobacco in a short arti- 
cle like this. I can simply state that the best leaves, as regards general quality, are placed in the first grade of wrappers, those not quite so good in the second grade, and those still poorer in the third grade or rough wrapper class, and so on down until the 'trash' or poorer part of the crop is reached, which grade is called fillers. To perform the work of assorting correctly requires some experience. No article can be written upon the subject which will enable a person who never performed the work to do it properly. The leaves, when assorted into respective grades, are tied in small hands or 'hanks,' containing from twelve to eighteen leaves each, according to the quality. We usually place twelve or fourteen leaves in a hand of wrappers, about sixteen in those of binders, and twenty in fillers. The tying should be done neatly, so that the hand, when finished, will present a neat and workmanlike appearance. Small, pliable leaves should be used for this purpose, which should be selected by the assorters. We usually employ small boys for tying the hand. A small boy can tie for two to assort.

Great care needs to be exercised while handling the tobacco, to keep it from drying out. The ranks should be kept covereo at all times, and the room wherein the assorting is done should be kept in a damp condition by placing a vessel of water upon the heating-stove, the steam from which will impregnate the air and keep it moist. Ordinarily, tobacco, when assorted, should be re-ranked to give the butts of the leaves opportunity to become thoroughly cured before the tobacco is cased. Guide-boards should be placed at the proper distance apart to admit of ranking between, the butts of the hands being placed against the boards. When the rank is completed the guide-boards should be removed, and the top and ends of the rank carefully protected with boards or a like material to prevent drying of the tobacco. In this shape tobacco should remain until ready for casing."

In assorting, all the green or "fat" leaves should be thrown out, as they are worthless for anything except as a fertilizer. The casing or packing is done either by the buyer or producer, but generally by the latter.

Packing.-Tobacco is gererally packed in boxes, the usual size being from three and a half to four feet long, two and a half wide, and two and a half deep. It should be packed quite closely, the man who does it being in the box and pressing it down with his knees. When the box is even full, the contents can be crowded still more compactly by means of a lever, and follower that will just fit the inside of the box, after which more tobacco can be put in. Close packing prevents undue drying, thus maintaining the desirable degree of moisture for handling. When packed as full as the box will admit, the top should be nailed on firmly. About three hundred pounds can be packed in a box of the above description. In some parts of the South and West, hogsheads are used for packing tobacco, which will hold, when closely pressed, from fifteen to twenty hundred pounds. Major Rayland, of Virginia, recommends the half-hogshead for the purpose of packing, the grades to be kept apart, or when necessary to pack two or more grades together, a layer of paper may be placed between. The smaller boxes are much more convenient for handling in transferring from one place to another. Boxes for this purpose can be much more cheaply obtained from the manufacturer than can be made by the farmer.

Cultivating Tobaceo Seed.-When seed is to be raised, the best and most thrifty plants should be selected. These should be carefully cultivated, and left to grow naturally without topping. From four to six good plants will produce, on the average, a full half pint of seed. The worms should be kept from these plants, and the suckers removed as they make their appearance, the soil frequently stirred, and all weeds carefully excluded. At the time of cutting the crop, from one-third to a half of the leaves should be taken off. This will have a tendency to hasten the maturity of the seeds.

When the pods have turned dark brown, the heads should be cut off and hung in a dry 
place to become cured. When thoroughly dry, it is a good plan to look the heads over carefully, and with a pair of scissors clip off all the smaller or imperfect pods, leaving only the largest and best-looking ones, which are found at the crowns and ends of the best shouts. Those cut off should be thrown away, being unfit for seed. The others may be shelled and cleaned with a fine sieve, and put away in a tin box in a dry place until needed for sowing the next season. Before shelling, the heads should be handled with care, as they sift out easily, and the contents of the best pods might thus be lost.

\section{COFFEE.}

$\mathrm{C}$ OFFEE is a tropical product, a native of Western Africa and Abyssinia, and has become naturalized in many other countries. In its natural or wild state, the coffeetree attains a height of from twelve to twenty feet, and bears but few branches. In cultivation, the tree is topped to from six to ten feet from the ground, the branches beginning quite near the root, which, after being topped, gives it the general form of a pyramid. The leaves are oblong-ovate, from four to five inches in length, are opposite on the branch, evergreen, of a rich dark-green hue, thick and glossy.

The flowers are clustered in the axils of the leaves. They are small, white, and very fragrant. When ripe, the pod or seed-vessel is of a dark-red color, and contains two cells, in each of which is a seed, called the coffee-bean, or berry.

Coffee is an important plant in the agriculture of those inter-tropical countries suited to its cultivation, as its use is almost universal, and the demand for the product great, while the section of the globe to which its successful culture is restricted is limited, when compared with that of many other products. It is stated by reliable authority, that the annual con. sumption of coffee in the United States is greater than that of any other country, being six times the amount of that consumed by some of the European countries. Germany and France rank second, or next to the United States in this respect.

Brazil, at present, is the largest coffee-producing country in the world. The product is, however, inferior to the Java and Mocha, but is often sold in the market under the name of these varieties, which are thus counterfeited. The regions in which the latter are cultivated are so very limited that it would be impossible for them to supply but a comparatively small fractional part of the quantity that is sold under those names, Brazil and other South American countries producing quite a large proportion of this product. The leaves of the coffeetree are said to be used by the inhabitants of Sumatra, instead of the berry, and are prepared for use by drying. Chicory root, dandelion root, carrots, Indian corn, rye, peas, etc., are often used as substitutes for coffee after being roasted.

Varieties.-The varieties of coffee are quite numerous. The Mocha, which comes from A rabia, is considered by many to be superior to all others. There is so much of the counterfeit in market under that name at present that it is difficult to find the real article. It is known by the beans or seeds being of a small size, and of a greenish-gray color. Java, or East India coffee, has beans of a larye size and yellow hue. Jamaica has beans somewhat smaller than the Java, and greenish in color. Bourbon coffee has yellow beans of a light shade. Surinam coffee has the largest beans. The Rio, Ceylon, Maracaibo, etc., are varieties slightly differing, and though good, are not generally considered equal to the Mocha or Java. The product known as the Male Berry differs from others in the form and size of the bean, it being quite round and small, instead of flat like most other coffees. These round beans will be found in the product of any kind of coffee in the proportion of about one to every twenty or twenty. 
five, and are said to be the product of the new shoots or wood of the previous year's growth. They are found singly in the pod, the old wood producing flat berries with two in each pod. The Male Berries are selected from the flat beans and sold in market as a separate product. There are many other varieties and sub-varieties besides those already mentioned, produced by a difference in climate, soil, and cultivation.

Cultivation.-The climate suited to the successful cultivation of coffee is tropical. It should be so warm as to be secure against frosts in winter. Hence, in this country it can be profitably grown only in the extreme South. That it can be grown in Manatee county, in Florida, has been fully proven by experiment extending over a period of several years, and we believe there are other localities of about the same latitude in which it will thrive equally well, and that planters in such sections will do well to try the experiment. At Togartyville, Florida, coffee-seed from Cardova, Mexico, was planted, which, after four years' cultivation, resulted in trees that were full of berries of different sizes, and in all stages of growth, some of which were ripe, others half-grown, together with the blossoms and buds. At this age, one of the trees was six feet high, contained eighty branches, and measured sixteen feet around the tips of the branches, and three inches around the trunk. The berries hung in clusters of five and six, from one and a half to two inches apart. Trees will also bear when three years old, under good cultivation, and continue to be productive for twenty years. In regions subject to occasional slight frosts it is well to protect the trees in winter with pine branches. Setting the coffee-plants among banana-trees is sometimes practiced, as a protection against cold winds. There is at present a small coffee-plantation at the previously-mentioned town in Florida, located near the mouth of the Manatee river, which is in a thriving condition, and has been pronounced by experts in coffee-culture in Mexico and India, a decided success.

Coffee, like tobacco, owes much of its difference in quality to the climate and soil upon which it is grown. It requires a very rich soil and warm climate. It thrives best in a soil that is rather moist, but not wet. In very dry sections artificial irrigation is practiced before the fruit begins to ripen, the supply being cut off at the ripening period to improve the quality of the product. Coffee plantations are generally laid out in quadrangles, the trees being from eight to ten feet apart each way in rows. The ground should be kept free from grass and weeds, and the trees pruned or topped so that they will not exceed the height of from six to ten feet. The trees will continue to bloom for eight months in the year, which results in the fruit ripening very irregularly. In the West Indies and Brazil there are three annual gatherings of the crop.

Gathering.-The fruit is picked by hand from the branches of the plant, and spread on the floor or large mats in the sun to dry. It is frequently raked over or turned, in order to dry evenly. When thoroughly dried, the outer pulp of the fruit, together with the case that encloses the seeds, are removed by being crushed between heavy rollers, which causes the seed to shell out. This seed, or the coffee-bean, is then separated from the refuse by winnowing. It is then ready to be packed for market.

The care with which it is cultivated and prepared for market, makes a great difference in the price of the product. 
TEA.

$\mathrm{M}$

ANY and repeated experiments in this country have fully demonstrated the fact that tea can be successfully produced in the southern portion of the United States. These experiments have extended over a period of more than twenty years-those of the past few years resulting so satisfactorily as to warrant the belief that the time is not far distant when American soil, and American industry, will supply the demand of our people for this product.

It may be some time before tea will be cultivated here on a very large scale, but we are

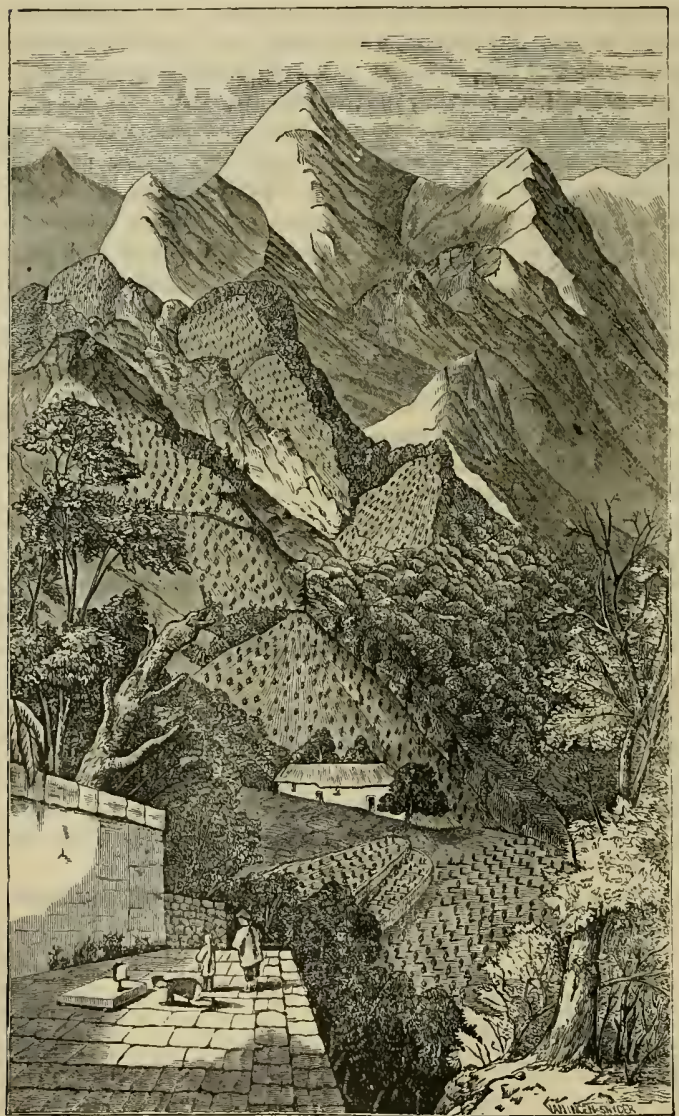

SCENES IN A RLACK-TEA DISTRICT IN BOHEA.

in diameter. In this country, however, it grows as a shrub. three years, and continues to be productive about twelve years. confident that it can be produced by the farmer and gardener, on a small scale, at a much less cost than the imported article involves, while there will be the addi. tional advantage of having a pure, unadulterated article for use. Many of the imported teas are, when placed upon the family table, a decoction of various poisonous materials used in their manufacture, special dyes and chemical substances being applied to change their appearance, and give them the desired color.

In Liberty Co., Georgia, there is, according to the best authority, a tea-plantation owned by Mr. John Jackson, which embraces nearly forty acres, and is occupied by a hundred and sixty thousand tea-plants. Tea has been cultivated in various portions of the South in a small way, for many years. The tea-plant has been found growing wild in the mountainous regions of Assam and Yunnan, and this has led to the opinion that it is a native of this section.

In its wild state, it grows in the form of a tree, sometimes attaining the height of thirty feet or more, and its trunk measuring ten inches It bears at the age of two or 


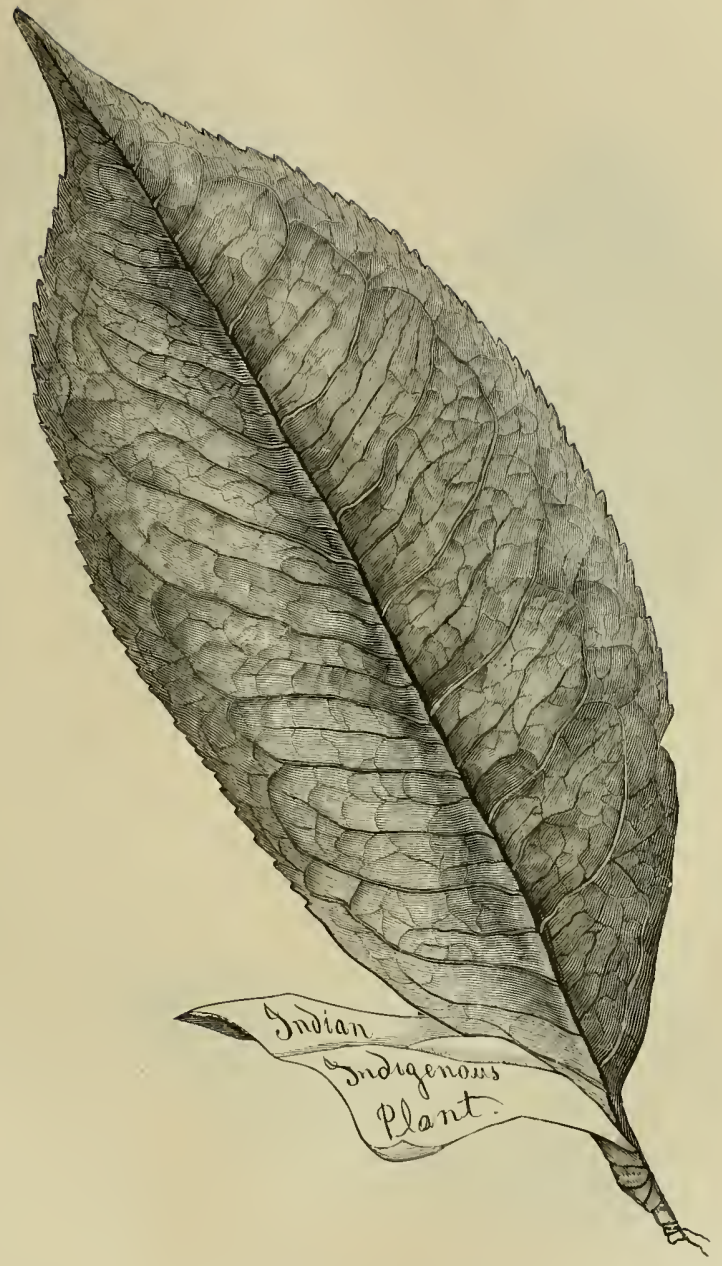

LEAF OF TEA. 


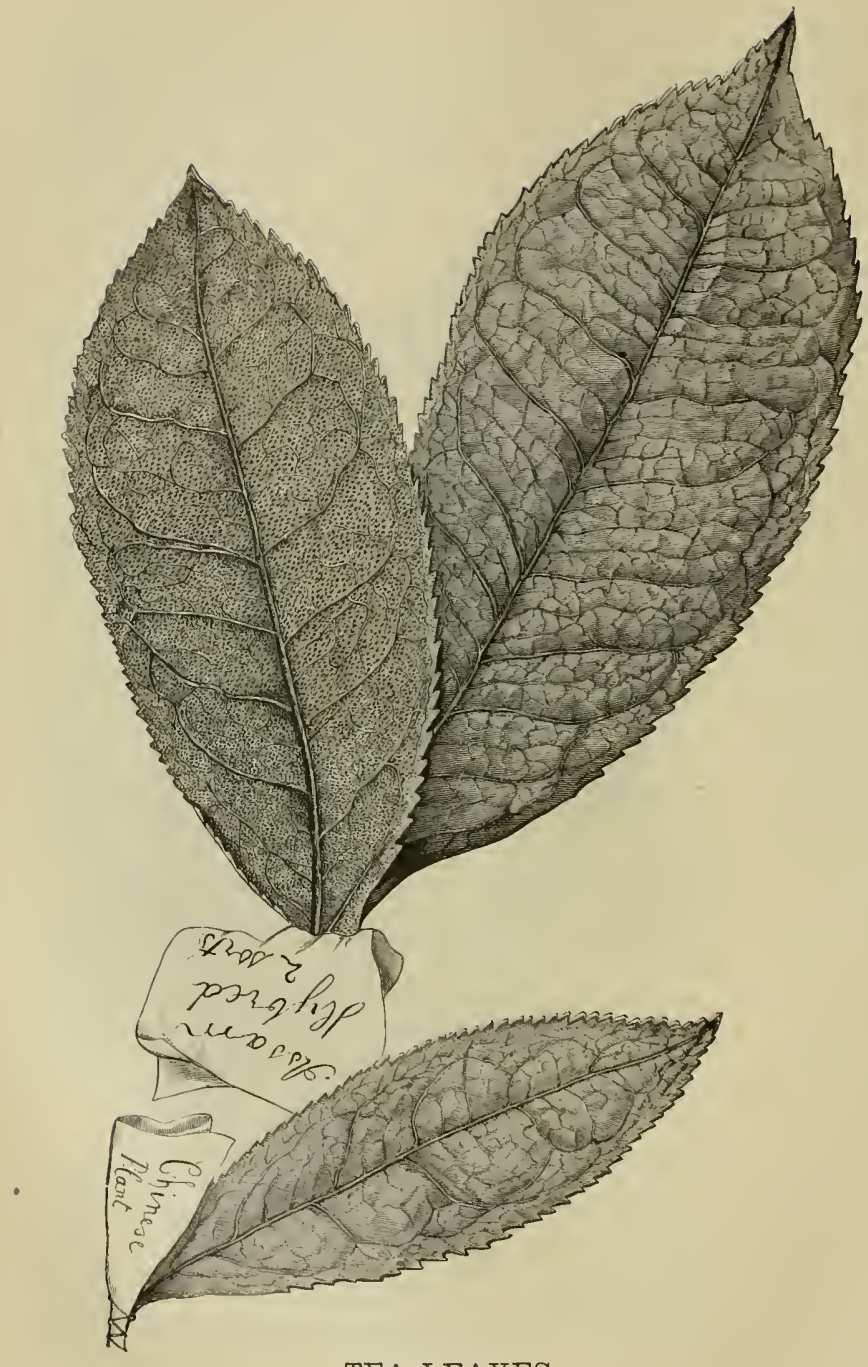

TEA LEAVES. 
The average product of a single plant is about six ounces, which is gathered at three different times during the season. Sometimes there is a fourth gleaning, but the quality is - inferior to that previously obtained. The flowers open early in the spring, are rather small, pure white, and slightly odorous. They appear at the axils of the branches, on short stalks, usually solitary, but sometimes two or three together. The flowers are described as having five or six sepals supporting the blossoms, which fall off after the flower has expanded, and leave from six to nine petals surrounding a large number of yellow stamens that are joined together at their bases, forming a kind of floral coronal. The seeds are enclosed in a hard, smooth capsule, somewhat triangular in form, which is divided in the interior into from two to five cells, each containing a firm, white, and slightly oily seed, of a peculiarly bitter flavor. These seeds vary in size from that of a pea to a good-sized hazel-nut. The seed ripens, according to the climate, from October to January. The stem is bushy, with many branches. The foliage is dense, and the wood of a light color, hard, and close grained. It is an evergreen, and quite an ornamental plant. The leaves are rather large when full-grown, and of a somewhat elliptic form, - as will be seen by the illustration,-glossy and smooth, serrated except at the base, and of a dark green color.

Varieties.-The difference in the various brands of tea, as they appear in the market, is due more to the artificial manipulation of the product, and the condition of the leaves when gathered, than the difference in variety.

Many of the names applied to teas are descriptive of the locality or country that produce them. such as the Java tea, Japan tea, etc., while according to the condition of the leaf when gathered, we have the Bohea tea from leaves fully grown, and gathered after the regular harvest, which is of the coarsest quality; the gunpowder tea, made from the small, curled leaves that are young and tender, while the black and green teas are produced from the same plant, their difference in color being due to the different process in curing and manufacturing.

Cultivation.-Tea is quite a hardy plant, and capable of adapting itself to a great variety of soils and climates. In Japan it is cultivated successfully as far north as forty-three degrees, where, during the winter, the ground is sometimes frozen six inches deep for weeks. The plant will also bear an ordinary drought well in summer. In Java, the conditions are the reverse, the temperature being very warm, while in China it thrives equally well under a tropical sun, and in higher latitudes where snow and ice often cover the leaves. Tea is cultivated most extensively in China and Japan, and, next to these countries, in East Bengal in the provinces of Assam and Cochar.

The tea-plant will thrive well on a variety of soils, but those that contain much water are injurious; consequently the land selected for tea culture should be well drained, either naturally or artificially, and not liable to overflow. Almost any kind of soil, not too wet, and of medium fertility, will answer the purpose, that which is very rich not being absolutely essential to its successful culture. Any soil must, however, be kept well cultivated, and free from weeds. A rich, sandy loam in the vicinity of small streams is generally regarded as the best.

The plants are grown from seed which somewhat resemble hazel-nuts, as previously described. The good seed can be separated from those that are worthless by soaking them in warm water about twelve hours. Those which sink to the bottom should be planted, and those that rise to the surface should be discarded. They require some time to germinate, and start soonest if put in a cool, moist place, well shaded. They are sometimes sown where the plant is intended to grow, three or four being dropped near together, and covered to the depth of two or three inches; but the more common practice is to plant the seed, one in a place, in a nursery, and transplant afterward.

They can be transplanted without injury when from six months to a year old, if care is 
taken not to break the long roots, and a small portion of earth is permitted to adhere to them in taking them up. The land should be well tilled, and thoroughly pulverized before sowing the seed or transplanting.

The roots of the young plants are quite sensitive to the hot sun, consequently they require considerable shading during the first year. This may be done by boughs of pine, or other foliage furnishing a good shade. Mr. Jackson, previously referred to, states that plants under shade give the highest yield, but those that have been mulched are not far behind in this respect. The mulched plants probably produce the most shoots, but the sun hardens them quicker than those grown in the shade. The best time for transplanting is in April or May, the plants being set at a distance of about four feet each way.

Then they are about two years old, they are cut down to about eighteen inches. This pruning is usually done in January. The plants soon throw out new shoots, which are permitted to grow until they uttain considerable size before the picking commences. When two or three feet high, the shrubs produce flowers and seeds.

Picking.-Plants thrive best if the picking is delayed until they are nearly three years old, when they will be from four to five feet high. In the following December, after pruning, which is when the plant is three years old,- a second pruning is given, which is more carefully performed than the first, since it gives form to the bush that is to furnish the future crops.

In picking, care is necessary in order that only about two-thirds of the leaf be taken, the other third being left to protect the new-forming buds from injury. The tea that is made from the young and tender leaves first gathered is of the best quality.

The usual number of tea-pickings during the year is three, but sometimes a fourth is given; this, however, will be of inferior quality. The yield varies with the size of the plants. From six to eight good plants, with proper care, would be sufficient, with the average yield, to supply an ordinary family with tea for a year.

The method and time of picking varies in different countries. In Japan it generally commences in April after the heavy rains, and continues through Nay and June, the first three leaves of every stalk or stem being picked, while in India the first six leaves are taken.

Curing. - The most difficult process in the management of the tea product is the curing; involving much time and labor, combined with skillful practice. The methods adopted in old tea-growing countries, have not yet been closely followed in the United States, but when their art is more perfectly understood, and the best machinery for the purpose has been devised and employed, we have no doubt that the product of this country will fully equal, if not surpass, that of the best, pure, imported teas.

Some who have cultivated tea to a considerable extent for home consumption, simply heat the leaves slightly in an oren, and spread them in a dry place to perfect the curing process. Others practice a much more elaborate method, which consists of carefully assorting the leaves, spreading them on a table as soon as gathered, where they remain about twelve or fourteen hours, or until they are sufficiently wilted. They are then rubbed with the hands until they are soft and pliable, and left in small heaps for two or three hours, when they are put into a Dutch-oven, and, with a moderate fire, carefully roasted. During the roasting process, which lasts four or five minutes, the leaves are constantly stirred to prevent their being burned, or unevenly cured. They are then taken out and rubbed and rolled on a table as before. Then they are spread in the sun for a short time to dry, being frequently stirred, after which they are again roasted and rolled. This process is followed by putting them about an inch thick in a wire sieve, and holding them over the hot coals, being con. stantly stirred, when they are taken out and again subjected to the rubbing and rolling. This is repeated until the leaves are of a dark color. They are then put in a basket and 


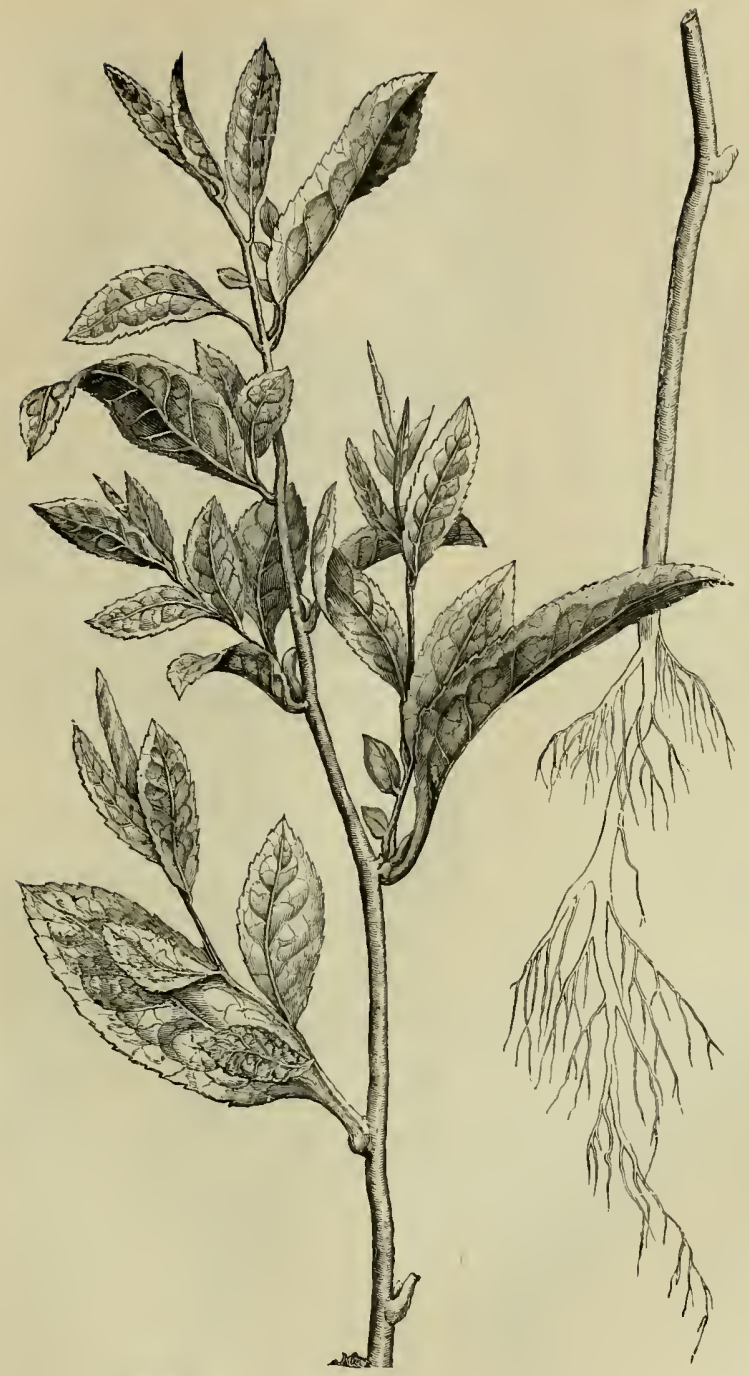

YOUNG TEA PLANT AND ROOT. 


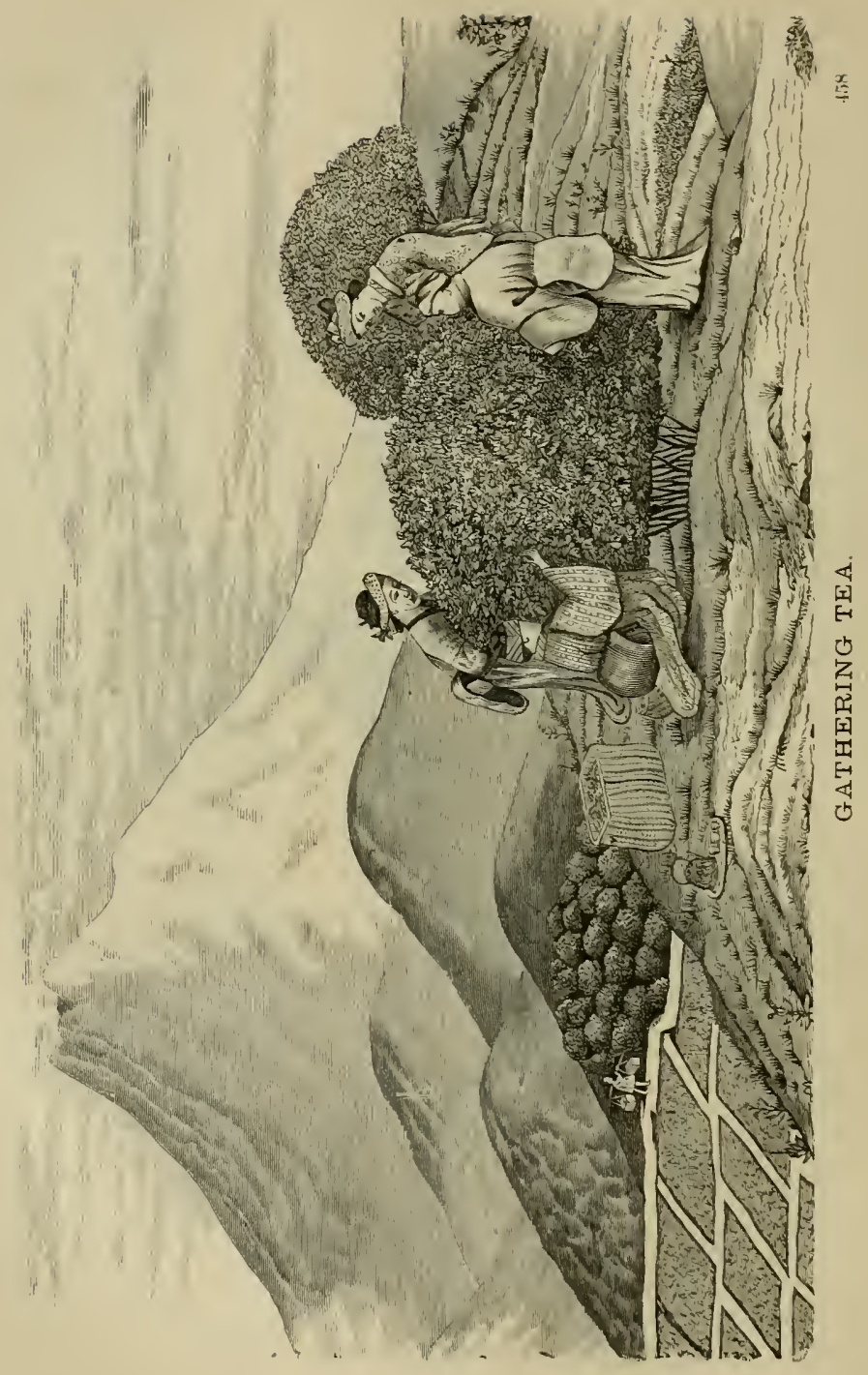


hung over the coals and stirred until the leaves are quite dry and black, when they are ready to be packed in tight boxes or jars.

The thorough manipulation thus given is said to greatly improve the quality, and pro. duces a fine flavor that could not otherwise be obtained. This method of cyring produces the black tea. The process described, combined with squeezing the leaves when they are moist and hot, gives them the peculiar twist that characterizes them when dry.

When the leaves become sufficiently crisp to be easily broken by the thumb and finger, the tea is considered ready for packing. It is generally packed while warm, care being ifed that the box be perfectly dry.

It was formerly supposed that green and black teas were produced by different varieties of the plant, but it is now generally admitted that the color is due to the different manner in which the leaf is cured.

Green tea, instead of being first exposed to the air for several hours, is put over the fire almost as soon as picked, and heated a few moments, after which it is cured the same as black tea. This process imparts a greenish hue to the leaves. The black color being the result of longer exposure to the air and heat, green tea can be changed into black, but the black tea cannot be turned into green, without adulterating it by coloring.

The method of curing tea differs, somewhat, in different countries, and in different sections of the same country. The following is translated from a Japanese work, showing the method practiced in that country:-

"The leaves are carried in from the field, and by means of sieves the two small bracts attached to every stem and broken or fragmental leaves, must be separated from the good and whole leaves. The old leaves, sticks, etc., should also be carefully separated from the good leaves. It is always the best way to prepare the leaves on the same day they are picked; for if kept through the night, their quality is somewhat impaired; if two nights be allowed, they will lose much of their flavor; therefore, the quantity to be picked must be calculated according to the number of hands and heaters (or hoiro, a utensil made of thick paper, with frames, for the purpose of heating the leaves). The fire-place must be built large enough for a boiler about two feet in diameter; fill this boiler with eight-tenths of water, and boil it until it reaches two hundred and twelve degrees. When the steam rises, a square piece of cedar board with a large hole in the center is fixed on the boiler. On this board, and around the outer edge of the circle, is placed a circular mat, made of rice straw, to prevent the steam from escaping, and on this mat is placed the steamer.

Then ahout half a pound of the green leaves are put in the steamer and covered. After thirty seconds the cover is taken off and the leaves are stirred up by means of small wooden sticks, made of Paulownia imperialis. The same process is repeated thirty seconds afterward. The leaves soon become adhesive, and have a tendency somewhat to cling to the sticks, and this is a sign that the steaming is done. This is the time to take them aside and put them in a cooler place, and this is done by turning the box upside down, as the steamer, which is on the bottom of the box, will come out at the upper part. Then spread the leaves, cooling them with fans, and after they become cool enough, put them into baskets, and get them ready to be sent to the heating department.

In heating, a place must be arranged three feet wide, six feet long, and about three feet high, plastered inside and out with mud. Burn in the furnace about twenty pounds of oak-wood charcoal. When the fire becomes hot, put in two or three bundles of straw in order to make the heat softer; then put iron bars across the furnace and the copper-wire nets over the bars, and spread the heater (of thick paper) which is made to fit the place. Four pounds of the steamed leaves may then be scattered on the paper; rub them very softly with both hands; winnow or throw them very lightly, and stir them. This ought to be skillfully performed, so that the proper color and flavor may be secured. Then they must be taken 
aside at the moment when they are almost dry. When the day's work is over, take the fire out from the furnace, and prepare as was done before; then scatter the leaves which were heated during the day, drying them in this way during the night. At this time about twenty-four pounds may be spread over, but it requires great experience to heat them in this way. The softer heat is preferred to the greater heat. The quantity of the best tea which may be prepared by one laborer per day is about thirty pounds on an average; and the quantity of the inferior quality, from twenty-eight to thirty-seven pounds. About one pound and three-fourths of tea is generally made out of eight pounds and five ounces of the green leaves.

For the finishing process a sieve should be used, in which the dried tea is to be softly rubbed by the palms of the hands, separating the tea from the stems. The next process is to separate the tea from dust, sticks, stems, etc., by winnowing; and if this is difficult to do, put them on a stand and sort them into two or three classes, and then use a finer sieve. After this has been done five or six times, separate the larger leaves from the others, and so on with the finer leaves."

Adulteration of Tea.-Pure Japan tea, in its natural state, has a long twisted leaf, and is of a brownish green color. Much of the imported teas are adulterated in order to increase their weight, and improve their color, gloss, etc., inferior grades of tea often being made to have the appearance of those of the best quality. These adulterations consist of leaves of other plants being mixed with the tea; the mixing of exhausted tea-leaves; silica, metallic iron, prussian blue, indigo, turmeric, kaolin, etc., many of the adulterations used being very poisonous. It is a significant fact, that the "Celestials" themselves will never use these colored teas, but prepare them for the palates and stomachs of their American and European neighbors; those for their own use being always of the purest and finest quality.

\section{PEANUTS.}

WHE peanut, known also in different sections as the ground-nut, ground-pea, goobes, pinda, etc., is a leguminous plant, and a native of Africa. In this country its cul. ture is, therefore, best adapted to the Southern States, although it may be successfully cultivated much farther north than was formerly supposed. It has been grown as far north as Massachusetts, in a small way, but we doubt whether for market purposes it would prove a profitable crop in a much higher latitude than that of Virginia.

In the United States, the peanut is most extensively cultivated in Virginia, Tennessee, and North Carolina, although it is grown to a certain extent in many other sections. This product, in the State of Virginia alone, in one year recently amounted to about one million and three hundred thousand bushels.

The blossoms and vine of this plant resemble somewhat the common pea-the latter growing quite rapidly and thickly overspreading the surface of the soil in which they are planted. After flowering, the pods form and penetrate into the ground to a sufficient depth to be well covered, where they remain and ripen.

In $A$ frica and South America, the peanut has long been cultivated as an article of food. In this country it has become a product of considerable market value, and is also largely employed in the manufacture of oil, of which the kernel yields over twenty per cent. They were formerly cultivated largely at the South for the purpose of fattening swine, but at present are used in this manner only to a limited extent. The oil expressed from the kernel is valuable for many purposes, but mainly for mixing with olive oil. When not earthed up, it is said that the vines will yield a fodder scarcely inferior to the best clover. 
Cultivation. - This crop is easily cultivated in a climate favorable to its growth, while, when well-manured, the yield is generally large in proportion to that of many other products. The peanut is a plant not very exacting with respect to soil, if it be sufficiently dry, although a rich soil is to be preferred. It thrives best in a sandy loam, which should be kept well stirred, in order to be in a condition to permit the pods to enter it. A light-colored soil is desirable where the crop is designed for market, as the color of the soil affects very materially the color of the pods, and consequently the market price, although there may be no real difference in the quality of the nut produced by such soils and those of a darker color.

The stain of red clays and other dark soils cannot be removed from the pods even by washing; while nuts with clean, light pods will bring considerably more per bushel than those of a darker hue. Lime is one of the best fertilizers for this crop, but it should be used in conneotion with oompost or stable-manure. The soil should be plowed to the depth of about four or five inahés, and thoroughly harrowed, in order to render it as mellow as possible. A practical oultivator of this crop in Virginia gives the following method:-

"In choosing a site for planting, reference should be had to the crop of the previous year. Peanuts require a clean soil. They will follow any hoed crop to advantage, with the exception, perhaps, of sweet potatoes. Corn land is generally preferred. In tide-water Virginia, much of the land was heavily marled in former years, and when this is the case, an important, and perhaps the chief, requisite to success has been already provided.

The peanut will not fruit well except in a calcareous soil. The vines may grow with the greatest luxuriance, covering the whole ground, but, in the absence of lime or marl, the pods do not fill; they turn out to be nothing more than what is popularly called pops. If, then, the land has not been previously marled or limed, it will be necessary to apply, say, a hundred and fifty bushels of marl, or fifty bushels of lime, to the acre. It is applied in either of several ways, according to the convenience of the planter, and with about equally good effect. If there is any choice, spreading broadcast is perhaps the best, to be done before the land is plowed, in which case the quantity should be about fifty hushels per acre. A favorite mode, where a large surface is to be planted, is to strew the lime in the furrow over which the bed is to be raised for planting. In this case, a less quantity will answer by reason of its being more concentrated--say twenty bushels. Other planters, again, who are hurried in their work, spread the lime over the beds after the crop is planted, at the rate of about thirty bushels per acre. Either mode is attended with good success, but wherever it is practicable to have a choice of land that has been sufficiently marled or limed in former years, and preserved by judicious culture, the best results are found to follow. In such cases the yield not unfrequently reaches a hundred bushels to the acre. The product ranges from the quantity stated down to twenty-five or thirty bushels to the acre, according to the skill, or want of skill, in the planter, a fair average of the whole being estimated at fifty bushels.

Few persons make peanuts part of a system of rotation, but the preëminent success of a gentleman who has followed the plan is worthy of special reference. The lot intended for peanuts the following year is seeded to stock peas, and the vines plowed in some time in September. The vines contain a great deal of vegetable matter that becomes thoronghly decomposed by the time of planting the crop. When the season for planting is at hand, the ground is re-plowed and laid off, and ten bushels of lime and a hundred and fifty to a hundred and seventy-five pounds of superphosphate strewn in the furrows to be ridged over. The year following peanuts, the land is planted in sweet-potatoes, with a liberal dressing of stablemanure. The third year it is laid down in stock peas again, to be followed by peanuts, as before, always repeating the lime and superphosphate.

Having selected the ground, it is plowed in March or April to a depth not exceeding four or five inches. The advantages of shallow culture will be apparent from the fact that the peduncles continue to penetrate the earth until a firm bed is reached on which to deposit 
the nut; and the still further fact of increased facility afforded in harvesting, as will appear when we come to treat of that branch of the subject. From the 10th to the 20th of May is the time for planting. If the land is thin and needs manuring, open the furrows three feet apart and strew in a hundred to a hundred and twenty-five pounds of Peruvian guano, or from a huudred and fifty to two hundred pounds of superphosphate of lime. The former is generally used because of the greater certainty of getting a pure article, but nothing can be better than the latter when well prepared.

The furrow is then to be ridged over, and the whole surface thrown into three-feet beds, which should be reduced to within two or three inches of the general level of the field. Then mark off the rows, and at distances of eighteen inches plant two seeds, corering them from an inch to an inch and a half deep-not more.

A matter of primary importance is to provide seeds of a good •quality for planting, and, in order to be assured of their excellence, the planter should either raise them himself, or buy them of a person on whose fidelity he can rely. If, after the vines are dry and are lying in the field, they should be exposed to frosty weather, the germinating principle would be destroyed or impaired. As a merchantable article, howerer, their value is not affected. Neither should the nuts become the least heated or mouldy, nor should they be pieked off the vines while wet, or before they are thoroughly cured. It is obvious, therefore, that the most careful attention is requisite in this matter. Prerious to planting, the pods should be carefully shelled, and every faulty bean thrown out; not even the membrane enclosing the seed should be ruptured. It takes about two bushels of peanuts in the pod to plant an acre.

In ten days or two weeks, according to the weather, the soung plants begin to come up. As it is very important to get a good start, the missing hills should be re-planted at the earliest moment. It is the custom of some planters to put an extra quantity of seed in every fourth or ffth row, to furnish plants for transplanting, if needed; if not needed, they can bo thinned out. As soon as the grass makes its appearance, give a light plowing, throwing the earth from the vines, and following with the hoe, thoroughly remoring all the grass and weeds from the row. Plow again as soon as the grass reappears, this time using a double shovel or cultivator, and the hoe as before directed.

If the season should prove to be very wet, a third working may be necessary, making use of the cultivator and hoe again. Next comes the time for laying by, the vines haring by this time extended nearly half way across the space between the rows. This is done by run. ning a mould-board once in the middle between the rows and drawing the earth up to the rows with a hoe, care being taken not to cover the vines and to disturb their position as little as possible, as the fruit will now be forming. It will te necessary, also, to guard against making the bed too high. When there is grass in the row, it must be pulled up by hand. Soon after this the vines will corer the whole ground and repress every other growth, unless it may be a chance weed that escaped notice at the former working.

In some sections it is customary, as soon as the vines show blossoms, to corer them with about an inch of soil taken from between the rows, this covering to extend over the entire plant, except the tips of the runners, which are left peeping out of the ground. It is, how. ever, more common to leave them uncovered, according to the method previously recommended.

Harresting Peanuts. - The time for harvesting the orop is from the 15th to the 30 th of October, immediately after the first frost. When the crop is forward, or when it is an object to get a portion of it early in market, the operation may be commenced in the latter part of September, but the longer the vines continue to grow, the greater will be the number of sound pods. Select a time when the weather is settled and favorable, and with threepronged hoes loosen the vines along the rows. Hands follow the digger, pull up the vines, shake the dirt from them, and leare them in the same place. In dry weather they will be sufficiently cured in two days to be shocked. 
Showery weather, though it may somewhat delay the curing, does no injury. One of the advantages of shallow culture becomes apparent in harvesting. When the fruit is deposited only a few inches below the surface, the vine is detached from its position with little or no loss; when the depth is greater, the stems are liable to be broken off. In shocking, provide stakes seven feet long, made sharp at both ends; then lay two fence-rails on the ground as a foundation, but with supports underneath to afford free access to the air. The stakes are stuck in the ground at convenient intervals between the rails, the stacks built up around them and finished off by a cap of straw to shed the rain. The diameter of the stack is made to conform to the spread of a single vine. After remaining about two weeks in the stack, the picking should be begun, taking off noue but the matured pods. These are to be carried to the barn, and prepared for market by finishing the drying process and then fanning and cleaning. The most tedious part of the work is the picking.

An expert discriminates at a glance between the mature and immature pods, but cannot pick more than two and a half or three bushels per day. A machine to perform the operation would be a most valuable invention. Unless the management in the barn is carefully conducted, there is great danger where there is much of a bulk that the peas will become heated and mouldy. The condition in which the early deliveries are often received at market renders this caution quite necessary. In fact, there is as much slovenliness in the handling of this crop as there is in regard to any other, perhaps more, for the reason that so many inexperienced persons engage in the culture every year. Until the pods are thoroughly seasoned, the bulk should be frequently stirred and turned over. A certain classification in respect to quality is as applicable to peanuts as any other agricultural product. The descriptive terms in general use are "fancy," "prime," "ordinary," "inferior"; but these are not so definite as to admit of no intermediate grades. Assuming prime to be the standard, and that the prime are two dollars and seventy-five cents per bushel, then the inferior would be worth a dollar or a dollar and a half, the ordinary two dollars or two dollars and a half, and the fancy three dollars.

There are two very distinct varieties of peanuts, known respectively by the names of Virginia and the Carolina or African. The diversity between them, however, does not amount to a specific difference, the chief characteristic being that the one has a large pod and bean, and the other a small one. The Virginia is cultivated almost exclusively for eating, while the Carolina is principally used for the manufacture of oil, which cannot be distinguished from olive oil, and is accordingly sold as such.

After the crop has been harvested, the swine should be turned on to the ground and allowed to eat what remain in the soil. Some planters prefer flat culture, but the ridge method is generally regarded as the best.

Preparing for Market.-Assorting and preparing this crop for market is often done in establishments for this purpose, as follows: The peanuts are placed in a large cylinder, from which they enter another with revolving brushes, where each nut receives fifteen feet of a brushing before it becomes free. Then they are dropped on an endless belt passing along at the rate of four miles an hour. On each side of this belt are stationed girls whose duty is to pick out, with a quick motion of the hand, all the inferior-looking nuts, allowing only the best to pass the crucible, those that do pass dropping into bags placed for their reception. As fast as the bags are filled, they are sewed up and branded as first quahity. Those that are selected out from these are again assorted and the best of them branded as second quality. These are generally as good in quality as the first, but do not compare with them in shape and color.

Those that remain are again assorted, and constitute the third grade, and so on. The last that remain from the various cullings are shelled by a machine for the purpose, and winnowed, after which they are bagged and supplied to confectioners for the purpose of making taffy, or peanut candy. Even the shells are utilized, as they furnish excellent bedding for horses. 


\section{INDIGO.}

THE indigo plant was formerly cultivated in the Southern States to a considerable extent. The decline in its cultivation in this country was probably due to the intro. duction of the cotton-gin, and the consequent greater profits arising from the cultivation of cotton.

It is easily cultivated, and one of the most profitable crops of agriculture in Hindoostan. Whether it will ever again be grown to any extent in the United States is a question yet to be determined. The market demand for the product, and the price it commands, are such as to warrant the opinion that, with sufficient capital and judicious management, its cultivation might be made a profitable industry in those sections to which it is adapted, and especially in many of the Gulf States.

There are several varieties of the indigo plant growing wild in the United States. The East and West Indies, India and Central America supply, at present, most of the indigo of commerce.

There are several varieties in cultivation, differing in the quality and quantity of the product they supply. The coloring matter is furnished by the leares of the plant.

Cultivation, etc.-Indigo requires a rich, but dry soil, and a warm climate. After being once fairly started, the plants will sprout annually from the roots for four or five years; consequently its cultivation is much less difficult than that of many other crops.

The soils generally regarded as the best for this product, are rich, sandy loams, although it will grow on those of moderate fertility, if not too wet, a surplus of moisture being very objectionable. Hence, it thrives best in a climate naturally dry, and in sections where there is not a large amount of rain during the season.

The soil should be made friable by the thorough use of the plow and harrow. The fer. tilizers used are supposed to exert a considerable influence on the amount and quantity of the coloring element of the plant. Lime, ashes, poudrette, and barn-yard manure are all valua. ble, but the latter should always be well fermented or composted before being applied, or used abundantly in connection with a previous crop of some other kind. Barn-yard manure and lime combined make a good fertilizer. Weeds and grass are very injurious to the crop, and should be exterminated as fast as they make their appearance.

The seed should not be sown until the ground is quite warm. In the latitude of Central Georgia, from the first to the tenth of April would be sufficiently early. The seed will germinate best when the soil is moist; therefore it is a good time to sow it just after a rain. If sown when the soil is very dry, it heats, and the germ is destroyed.

The seed is generally mixed with ashes or sand, and sown in shallow drills from twelve to fourteen inches apart. About four quarts of seed is the average allowance for an acre. It should be kept free from grass and weeds, in all stages of its growth; this is especially important when it first comes up. The weeding may be done with cultivator, horse-hoe, or weeders suited to the purpose, a little hand-weeding sometimes being necessary in the rows.

When the plants are an inch or two high, the soil should be loosened about the roots. The first cutting should be when the plant first puts out the flower buds. If permitted to blossom, the leaves are more hard and dry, and the indigo produced will be of inferior quality. The cuttings should always be in wet weather, for, if harvested in dry weather, the plants will not send up another growth. When necessary to cut it in dry weather, it should be done late in the afternoon, that the roots may have the benefit of shade and dew before being exposed to the sun. It is a plant that is very sensitive to the hot sun immediately after being cut. The first crop is ready for cutting in two or three months, and, with favorable weather, a subsequent cutting may occur in six or seven weeks. 
It is stated that in Georgia, two cuttings have bren known to yield an average of sixty pounds of indigo per acre. In some countries the seed is sown only once in seven or eight years, and two crops usually obtained per year.

Preparing Indigo for Market.-The process of extracting the coloring matter from the plant differs somewhat in different countries, although similar. In Bengal, the plant is used both in a green and dry state, but, as a general rule, the green leaves are preferred. Three vats are so arranged at different levels that the contents of the first can be drawn into the second, and that of the second again transferred to the third.

The plants are placed in the upper vat in sufficient quantity to well cover the bottom, and sufficient water added to cover them to the depth of three or four inches, where they are left to ferment. The fermenting process usually commences soon after, and is completed in abont eighteen or twenty hours. During this time, the plants swell and give out a coloring matter that changes the water at first to a yellow, then dark green, with a blue scum. After all the coloring matter-sometimes called the "grain "-is thus extracted, the liquid is drawn off into the second vat, where it is beaten or churned for about an hour and a half. This is done to expose it to the action of the air, or, rather, to introduce oxygen, which changes the color from a green to a dark blue. During this process, a large number of air bubbles or gas escape from the liquid.

In India, from eight to ten men are sometimes employed to perform the beating with oars or paddles. Dashers and paddle-wheels are a better substitute for hand labor. After being sufficiently beaten, the indigo separates from the water and settles at the bottom of the tank. A little lime-water added will sometimes hasten the process, but it is apt to injure the quality of the indigo. When the grains are settled, the water is drawn off, and the contents remaining, strained and boiled. The surplus moisture is evaporated by drying, and when of the proper consistency for cutting, it is eut into little cubes, and dried, preparatory to packing for market.

Small quantities of indigo are often manufactured for domestic purposes by putting the plants, when fresh cut, in a barrel or other large receptacle for water, and then fill up with soft water, sufficient to have all the plants well covered to the depth of three or four inches. It might be well to put weights on to keep the plants well covered, and prevent their rising to the surface.

When the water assumes a dark green, and a bluish scum rises on the surface, the plants may be taken out and squeezed or wrung with the hands, in order to extract all the coloring matter that may remain in them. The water must then be churned for some time. A basket with a suitable handle for raising it up and down is very good for this purpose. To test the liquid, in order to ascertain whether it has been churned enough, take out a spoonful and add to it a few drops of very strong lye. If it then curdles, or the grains separate, it is churned sufficiently. At this stage, the process is hastened by adding a little strong lye or lime-water to the contents of the barrel, but care must be used not to put in too much, as it will injure the indigo. It may then be left to settle. In churning, the foam that rises must not be wasted, as it contains some of the best elements of the coloring matter. A little oil sprinkled over the surface of the water will make the foam subside.

When the indigo is well settled in the bottom of the barrel, the water may be drawn off by boring holes in the side, as far from the bottom as the quantity of the coloring matter will admit. What remains should then be strained, to take out the impurities, and again permitted to settle, draining off the clear water as before. It can then be put in a shallow pan or dish, and the water evaporated in the sun. 


\section{FLAX.}

$\mathrm{F}$ LAX has been known and cultivated from time immemorial, being one of the oldest agricultural plants of which history furuishes us any record. It was one of the leading crops in this country, until cotton supplanted it by the introduction of the cot. ton-gin, which made the cultivation of that product so much more profitable, and attended with less labor. But it is somewhat surprising that the culture of flax should decline in this country as rapidly as it has during the last twenty years, while other nations find it so profitable. About a century ago, flax and wool were the principal materials from which the common fabrics of the American people were made, and large quantities of flax were spun and woven by American women for the bedding and clothing of their families. Much labor was involved under the old system, both in its cultivation and manufacture, but under the new system, with inproved machinery, its culture is comparatively easy.

An increased interest in the cultivation and manufacture of this product in the United States, would not only largely extend our mechanical industries, but result in retaining at home vast sums of money that are now annually sent to foreign countries for the linen which can, at present, be obtained nowhere else.

In addition to the great importance of its fiber, the seed of flax is of much value for the oil it supplies, and the food it furnishes to farm stock-especially cattle. What is termed "oil-cake," consists of the seeds after the oil has been expressed, and is an excellent article of food for young calves and lambs, as well as other farm stock.

The plant is an annual of quick growth, and attains the height of from one to three feet. The leaves grow alternate upon the stems and branches, which are straight and slender. The flowers are blue, about an inch in diameter, and contain five petals. The petals drop within a few hours after the flowers open, and the seed-vessels grow rapidly, being nearly round in form. The fiber is long and silky. In this country, flax is at present grown principally for the seed, but little attention being paid to the quality of the fiber.

The varieties of flax are not sufficiently marked to require special notice.

Cnltivation for Fiber.-Flax will grow in almost every variety of climate from the torrid to the frigid zone. Its successful culture requires a good soil, well tilled. A dry, deep, loamy soil is one of the best for this product, although any other of sufficient fertility that is not too wet will answer the purpose. Lands intended for the culture of flax and containing a surplus of moisture should therefore be first thoroughly underdrained. The under. draining should be done a year or two before the flax crop is grown, in order to render it sufficiently dry. It is a somewhat exhausting crop, and should not be grown on the same soil oftener than once in five or six years. A good wheat soil is generally considered a good one for flax. The land should also be well enriched for previous crops before this is repeated. Fresh manure is very objectionable as a fertilizer, owing to the quantity of woed and grass seeds it contains. When stable manure is used, it should be either applied liberally to the crop that precedes it, or should first be well composted. Salt, guano, ashes, and plaster are also valuable. Lime is thought to be injurious to the quality of the fibre. Salt and ashes are generally applied at the time of sowing the seed. When the plants have nearly attained their growth, a sprinkling of plaster is often highly beneficial, not only as a fertilizer, but it also destroys worms and insects that feed upon the tender plants.

The best rotation for the fiber is thought to be after wheat, where the soil is quite fertile; but where the soil is of moderate fertility, potatoes are considered a good crop to precede flax. A corn crop that has been heavily manured also leaves the soil in a good condition for it. Clean culture should always precede it, in order to obviate the danger of troulle with weeds. 
The plowing preparatory to receiving the seed should be rather deep, as the roots are long, often penetrating the soil to a distance equaling one-half the length of the stalk. A thorough harrowing should follow, to reduce the soil to a fine tilth. The ground, after harrowing, should be left finely pulverized and level. This tillage should be early in the season, and the ground left a week or two before sowing, in order to give the weed seeds that are in the soil time to germinate. At the end of this period, the land should be again thoroughly harrowed, in order to destroy the weeds and fit it for the reception of the seed for the crop.

One of the great essentials in the culture of flax is to keep the soil free from weeds and grass, a clean soil being more necessary for this crop than most others. The general rule for flax-growing is, a rich, dry soil, finely and deeply pulverized, and free from weeds, and a rotation that shall not repeat its growth on the same land oftener than once in five or six years.

Some roll the ground to consolidate and level the soil, and then lightly harrow again before sowing the seed. This renders the surface of the land more level. The seed that is plump, shining, and heavy should be selected for sowing.

It should be thoroughly cleansed from all seeds of weeds, as this will save much trouble in the after-culture. This may be done with a sieve or a seed-cleaning machine. Seed that has not been produced on the soil is better, as a general rule. The quantity of seed to be sown depends upon the purpose for which the crop is cultivated.

When fiber is the object of the crop, a thick sowing is required. With thick sowing, the stalks will be long and slender, with only one or two seed capsules at the top; hence the fiber will be long and of a fine quality, and there will be but a comparatively small quantity of seed; consequently it is better to sow too thick than too thin. With thin sowing the plants have large branches of coarse growth, which produce many seed bolls that will be well filled with seed, but the fiber will be of a coarse, inferior quality. Therefore when fiber is the object to be attained in the crop, and the soil is rich, from one and, a half to two bushels or more per acre may be sown. Some farmers use three bushels per acre. Where the soil is very rich, and the seed of the best quality, a less quantity will be required than where the soil is of moderate fertility, or rather light, and the seed of second or third rate quality. There is a great difference in seed. Where inferior seed is used, there will be many that will not germinate at all, consequently a larger quantity will be required, to make allowance for those that fail in this respect.

The seed is sown broadcast. It was formerly the custom to sow the seed by hand; but this practice generally resulted in uneven sowing; the seed is of such a nature that it slips through the fingers easily, hence the quantity is not so well regulated; besides, this is a slow process where large crops are to be grown.

A broadcast seed-sower is the best for this purpose, after which it should be highly harrowed or brushed in, and rolled, in order to press the soil upon the seed, causing it to germinate more quickly. The time for sowing the seed is when the soil is warm. The usual time in the Middle States is from the 15th of April to the lst of May. In harrowing in the seed, it is well to go over the field twice, once up and down, and then across at right angles, as this mixes it more evenly in the soil, obviates the little drills made by the teeth of the harrow, and prevents uneven growth and ripening. If properly harrowed and rolled, the seed will be covered to the depth of about an inch. Seed should always be sown when the soil is rather warm, and not too wet. Care must be taken not to roll when the land is so wet that the earth will adhere to the roller. If the soil has been properly prepared and made clean of weed seeds, there will be little trouble with weeds after sowing the crop.

When weeds make their appearance, however, they must be carefully pulled by hand so as not to injure the young plants. The first weeding should be when the plants are three or four inches high. Boys are often employed to perform this work, and either go barefoot or 
wear shoes with thin soles and without nails, in order to prevent the plants from being crushed and broken, as they are quite tender at this period. It is well for the weeders to face the wind during the process, so that the plants that becoine bent in the operation may be blown upright again. When plauts are bent only one way they usually recorer their position again; but if crushed or twisted by the carelessness of the weeders, they scarcely ever rise. Once weeding is sufficient, and it should always be done when the soil is moist. When the weeding has been delayed until the plants are seven or eight inches high, it is better to let this process be omitted, as the injury to the plants will be greater than that to the weeds. It is important that the weeds be taken out at the right time, that the crop may get the advantage of them in the occupancy of the land. Some farmers are particular not to have a team upon the land after sowing: hence perform the after-labor of harrowing or brushing in the seed by hand. This crop has few insect enemies, and with careful culture, and a favorable season, will almost invariably bring good results.

Cultiration for Seed.- When the object of the crop is mainly the production of flax seed, although the preparation of the soil should be the same as when grown for the fiber, the quantity of seed sown should be much less than that for the fiber product, for reasons which have previously been given,_namely, that thick sowing produces plants with tall, straight stalks, fine fiber, and but a small quantity of seed, while thin sowing results in plants of coarser growth, with spreading branches and a larger product of seed bolls. The bolls will also be better filled, and the seed more plump and of a larger size.

The principal difference in the cultivation for the two objects for which flax is grown is, therefore, in the quantity of seed sown, and the time of harresting; the methods of treat. ment aside from these, being essentially the same.

When the crop is grown for the seed principally, from half a bushel to a bushel of seed per acre will be sufficient; the quautity depending somewhat upon the nature and condition of the soil, and the quality of the seed. Only the best quality of seed should be sown, for that of a poor quality will invariably give unsatisfactory results, howerer good the soil or cultivation given.

Harresting. - The time when flax is ready to be barvested is one of the important considerations in connection with its cultivation. If harvested too soon, the fiber will be very fine; but there will be great waste in the after-preparation for the market. If the harvesting be delayed too late, there will be a larger quantity of fiber, but it will be of a coarse quality. The proper time to gather the crop for fiber is when the seed is beginning to turn slightly brown, and the lower balf of the stalks are turned yellow. If harvested when flowering, the fiber will be exceedingly fine, but the waste will be so great as to render it unprofitable.

When grown for seed, it should not be harvested until the seeds are quite brown and ripe. If cut before they are sufficiently ripened, the unripe seed will not afterward mature.

In order to obtain a good weight, and seed of the best quality, the crop should not be gathered until the seeds are full and plump, as well as brown. The former method of harvest. ing flax was to pull it by hand, which was a very laborious process, besides requiring a great deal of time. At present, reapers and binders are employed for this purpose where it is culti. vated on a large scale. Machines for pulling have also been used to a limited extent, but the reapers are preferred for general use. The common grain cradle and scythe are also used, while some still conform to the old-time practice of pulling by hand. When the fiber is the important part of the crop, and reapers and binders are employed, it should be cut as close to the ground as possible, care being taken to have the knives of the reaper in good condition. It will then cut as easily as wheat. If bound by the reaper, it should be put in small stacks to dry. When properly dried, it may be put in a large stack, or stored under cover, which latter is best. It is very important that it be thoroughly cured before storing. When cradled or 
pulled by hand, it may be either dried in the sun before binding, or bound at once. If cut with a scythe, the swath should be thrown towards the standing flax: in this way the stalks are all left leaning against the flax, and may be gathered and laid in gavels to dry or be bound into sheaves.

When the ground has been thoroughly drained, and is uniformly level, and the sowing even, the stalks will generally all be of about the same length. Where pulling is practiced in harvesting, considerable care is taken to shake off the soil that adheres to the roots, and to keep them even at the root end.

When the seed is the main object, it is not necessary to cut the stalks lower than to secure the seed-bolls, the stalks that are left being plowed under to enrich the soil. It should be bound at once and put in small stacks to dry. Care should be taken to have the seedbolls well cured before stacking or storing. If stacked in the field, the stack should be made solid, with the heads in and the stalks' ends out, keeping the center filled and a little crowded. The stack should be covered with hay or straw and allowed to remain until it has gone through the usual sweating process, which will generally be in about two weeks. Sometimes the sweating process is omitted, and the bundles are loosely stored until the seed is threshed.

Preparing the Fiber. - There are various methods of preparing the fiber of flax for use. The object to be attained is the separation of the bark containing the fiber from the woody stem. This is accomplished by a rotting process, which dissolves or destroys the gluey or mucilaginous substance which holds the fibers together and to the stalk, and renders them easily separated.

The usual practice, after the heads have been removed, is to submerge the bundles of stalks in soft water, being weighted with stones or other heavy material to keep them well covered. The length of time required to complete this process varies, according to the temperature, from four to fourteen days or more. Water containing iron, or other mineral matter, would be liable to stain the fiber, as well as hinder the process of rotting.

It is important to know just when the process is completed, for if left remaining too long in the water, the fiber will become weakened, and will break and tangle in the after-management. If not sufficiently rotted, it will break up with the stalk. When it has been continued just long enough, the woody part of the stalk may be easily separated from the fibrous portion, and the fibers themselves from each other.

Sometimes this process is accomplished by dew-rotting, which consists of spreading the stalks upon the grass and exposing them to the dew, turning them occasionally to render the process uniform; but the method previously described is greatly to be preferred. Following this, is the process of "breaking," which consists of freeing the fiber from the woody portion of the stalk; this is followel by scutching or swingling, which is that of getting out the small particles of bark and stalk adhering; and this again by hatcheling or combing, which strengthens the fibers, separates the fine from the coarse, and frees them from all tangled or broken pieces.

These various methods of preparing flax for use were once all performed on the farm, but at present a large portion of the product is sold after the bolls or heads have been removed, and these various processes are performed by the manufacturers of linen goods.

Preparation of Seed for Market. - In preparing flax-seed for market, the bolls are first thoroughly dried and threshed, after which the seed is cleaned by a winnowing or seed-cleaning machine. The threshing is generally performed with a machine where large crops are produced, as hand-threshing, or any other means of opening the bolls would be a verý slow and laborious process.

Where small quantities are grown, the seed is sometimes shelled by drawing the heads of flax through a comb for the purpose, containing sharp iron teeth; also, by breaking them 
over a large stone or block, or by the use of the flail. The latter is the best method of handshelling, but a good threshing-machine is necessary where large crops are cultivated. The process of removing the heads with the comb, as previously described, is called rippling. After being threshed from the heads, the seed should be winnowed or otherwise cleaned, to separate it from the seeds of weeds and other foreign material. Flax is at present cultivated in this country principally for the seed.

\section{HEMP.}

$\mathrm{T}$ HE cultivation of hemp for its fiber was formerly practiced to a certain extent in some of the Western States, where it proved a profitable crop; but as the labor of harvesting it is much greater than that of most products, and it is also very exhausting to the soil, especially when the seed is ripened, it has never been a favorite crop with farmers. It can be more profitably cultivated in other countries, where labor is cheaper than it is in our own. The fiber of hemp is similar to that of flax, although it is much stronger and coarser. Like flax, hemp is cultivated both for its fiber and seed, the latter of which is used for feed. ing stock, and also for the oil which it furnishes. It is generally considered more wholesome and nutritious when cooked, before being fed to animals. The ripening of the seed causes more exhaustion to the soil than the production of the fiber.

Hemp belongs to the nettle family, having the fruit-bearing and sterile flowers upon different plants. The stalk is rougl and angular, and, like flax, branches considerably when growing alone, or with thin sowing, yet is quite straight when crowded. Hemp will grow almost as rapidly as Indian corn, and will attain the height of ten or twelve feet in a rich soil, but the average height is from six to seven feet. It generally blossoms in June, if sown sufficiently early, and ripens its seed in August.

The demand for hemp in this country exceeds, at present, the supply of the home product, and we doubt if it ever becomes a leading staple in the United States. The culture of hemp, like that of flax, while drawing largely from the soil, imparts nothing to it; hence, large quantities of plant.food are extracted, which require that a rotation of other crops shall intervene before its cultivation shall be repeated on the same soil.

Cultiration. - A deep, mellow soil of more than moderate fertility is required for hemp. Tell-prepared sward land of a clayey nature is excellent for the production of a good quality of fiber. When such soil is used, the ground should be plowed in the autumn and again in the spring, before sowing the seed. The soil for hemp should be moist, but not wet. It is necessary that the land be carefully prepared, and the soil well pulverized.

The seed should not be sown until the ground is warm and rather dry; still, it is well to get it started as early as practicable. The sowing is sometimes delayed until the middle of June, but this is rather late to obtain the best results from the crop. From the middle of April to the middle of May is better. It is necessary that the seed be fresh, that which is old not usually germinating satisfactorily. In England, from two to three bushels of seed per acre is frequently used; but in that country it does not generally attain the height that it does in our own. In this country it is the practice to sow from five to six pecks per acre, scattered broadcast over the field, and harrowed in. When drilling is practical, a less quan. tity will be required, from three pecks to a bushel being sufficient.

The ground should always be rolled after sowing the seed, to hasten its germination. It is covered to about the same depth as flax. When the soil is moist at the time of sowing, it will not be necessary to cover as deeply as when it is quite dry. In the latter case, it will 
be well to soak the seed about twenty-four hours in tepid water before sowing, to hasten germination. After the seed is in the ground, the crop requires but little or no attention until harvested. Being a strong, thrifty plant, of rapid growth, it will generally soon smother the weeds and take care of itself, unless the land is very weedy, and the seed does not start well.

Cut-worms are often troublesome, but if the blackbirds and crows are encouraged on the hemp-field, they will do valuable service for the farmer in exterminating them. When hemp is cultivated for seed, it requires a richer soil than when grown for the fiber alone. It is frequently cultivated on good corn ground, well manured. In England the culture for seed and fiber is the same, but in this country, when seed is the object, the culture is similar to that of corn, the hills being from three and a half to four feet apart, according to the fertility of the land. In planting, a dozen or more seeds are deposited in each hill. When the crop is first hoed, the plants are thinned out to five or six in a hill. During the subsequent cultivation given, this number should he reduced to three or four to the hill. The cultivation Bhould be sufficient to keep down the weeds.

Hemp belongs to that peculiar class of vegetable growths having the fruit-bearing, or what is termed female blossoms, on one plant, and the sterile, or male plants, on another. The difference between the two is determined when they are sufficiently developed to make preparation to blossom. The latter, which are known by their getting ready for blossoming, are then generally nearly all cut out, a few only being left to furnish pollen for the fertilization for the seed crop. The rule is to leave one of the best male plants for this purpose in every other hill, of every other row. When these male plants have blossomed, and shed their pollen (the yellow dust that falls from them when shaken), they are then cut out to leave more room for the other plants to grow.

Harresting. - The time of harvesting hemp that is grown for the fiber principally, is when the blossoms begin to turn yellow and drop. This will be in about three or three and a half months after sowing, according to the soil, season, and other conditions. If it should stand a week or more later than this period, no serious detriment will result to the crop, the only objection being that the process of rotting will not be as uniform, and the breaking will be a little more difficult, the stalks being more woody and hard.

Formerly, this crop was pulled by hand, but eutting is now almost universally practiced. The harvesting of this crop is the most difficult part in its management and the chief objection to its culture, being a slow, laborious task. When the hemp does not grow more than seven feet high, it may be cut with a heavy scythe, or a strong cradle, such as that used for grain, except it must be much stronger, as the stalks are hard and tough.

By this method of harvesting, it is estimated that one man may cut an acre per day. A good machine, sufficiently strong for cutting, would greatly lessen the labor and encourage its more general cultivation. When it attains the height of more than seven feet, a stronger implement is required, which is generally a kind of hook, similar to a bush-scythe, or sickle. Only about half an acre per day is considered a good day's work for one man with one of these implements. After two or three days' drying, it is bound and stacked.

The seed-hemp is cut before the heads are sufficiently dry for the seed to shell out of itself and be wasted. It is stacked in the field until dry, and then threshed out, after which it is carefully winnowed, and spread in thin layers in a warm, airy place to dry. Unless thoroughly dried before being stored, it will heat badly, and the quality thus become greatly injured.

After-Management. - When the weather becomes suffciently cool in the autumn, the hemp is either spread out on the ground to be rotted by the dew, or it may be put in vats or pools of water for this purpose. When dew-rotting is practiced, it must be occasionally 
turned. When put in water, it should be kept constantly submerged with weights of some kind. It will require from ten to twenty days to perfect the process, according to the temperature.

When the fiber separates readily from the stalk, the process of rotting is completed. It is then dried and stacked, after which it is broken and freed from the boon and waste material, and packed in bales ready for market.

\section{JUTE.}

$\mathrm{T}$ HAT jute can be profitably cultivated in this country, has been abundantly proved by the numerous experiments that have been made during the last ten jears, and which have given most satisfactory results when the climate and soil selected for the purpose were those to which it is naturally adapted.

The plant is a native of the East Indies, and will prove a most raluable addition to the agricultural products of the extreme Southern States. The great demand for jute in this country, as well as the price it commands, and the ease with which it may be grown, are strong inducements in favor of its cultivation in those sections to which it is adapted. If its cultivation could become sufficiently extensive to meet the demands of the American market for this product, millions of dollars would be annually retained at home, that are now sent abroad. At present, India is the main source of supply, and has been for a long time.

A recent estimate of the annual value of the jute importation, was nearly ten millions of dollars, while the demand is constantly increasing. Jute is an annual plant of the genus Corchorus capsularis. It grows to the height of from ten to fifteen feet, the stalks being from a half inch to an inch in diameter. They are rery straight, and branch only at the top. The leaves are about six inches long, two inches wide, and sharply serrated. The bark, like that of the flax and hemp, is fibrous, the fiber being more soft and silky than that of either of these products, also more fine in quality and of greater length. It is sometimes woven with silk and cotton in the manufacture of certain kinds of dress goods. It is also used in the manufacture of cheap carpets and almost every form of coarse textile fabrics, such as gunny, a kind of cloth used in baling cotton, bagging, coffee-sacks, rice bags, etc. It wis formeriy used in making ropes, but has been found too easily affected by water to be desirable for this purpose, hemp being preferred.

The ends of the stems of the jute-plant are used in making paper of a coarse quality, as well as the old sacks and bags made from the coarser jute fiber. The stems are used in the manufacture of coarse baskets, etc., almost every part of the plant being appropriated to some useful porpose, but, of course, the fiber is the object sought in its cultivation. It is said that cotton fields surrounded by jute.plants are exempt from the ravages of the cotton-worm.

Cultivation. - Jute requires a warm climate, and a fertile soil. A moist, allurial soil, - - not too wet, - or a sandy clay seems best adapted to it. Many of the Southern States, especially the Gulf States, are admirably suited to its cultivation. California has also produced jute successfully for several years. The land should be well prepared for the seed by being thoroughly plowed and harrowed, and the soil, if not sufficiently rich, should be made so by the application of manure or other fertilizers. The seed may be sown broadcast, or in drills. When the former method is practiced, from thirty to thirty-five pounds per acre are required; the latter from fifteen to twenty pounds. When sown broadcast, the seed should be harrowed in. In either case, the field should be rolled after the seed is deposited. The plant is of strong and vigorous growth, and will require but little cultivation. 
Plants for the production of seed should be grown in a separate field, and only the best seed sown. These should be sown in drills, and receive careful cultivation. Drilling, for the production of fiber, is preferred to broadcast sowing, as it admits of after-cultivation. On rich soil, and with other favoring conditions, the amount of yield in fiber is enormous.

The seeds will generally be ripened in four or five weeks after the blossoms have become fully developed. Whether for the production of the fiber or seed, it may be'sown in March or April, and the blossoms will make their appearance in July or August.

Harresting. - The best time for barvesting jute is when it begins to blossom, as the fiber is then of a better quality than when the plant is older. It is cut with a mower or reaper. The albumen in the plant is said to render it easier to cut than dry wheat.

The fiber is separated from the stalk by submerging in water until it separates readily. It is then prepared for use in a manner similar to that in which flax is prepared, except the stalks are not afterward dried and broken. The seed should be harvested when well ripened, and be thoroughly dried before being stored.

\section{PEAS.}

$\mathrm{T}$ $\mathrm{HE}$ cultivation of peas, as a field crop, is practiced to a considerable extent in some sections of the United States, but more especially in Canada and England, where they are regarded as an important product, and are much used as food for domestic animals. Next to the bean, the pea is ranked as one of the most important of the pulse family.

Sheep and swine are fond of them; also poultry. Their nutritive value is considerably greater than that of corn, and as food for swine, seven bushels of peas are said to be equal to ten bushels of corn. A meal of ground peas is considered better for swine than feeding them whole. This should be mixed with milk or water, and used in connection with other food. A mixture of peas and corn-meal is thought by some farmers to be better than the pea.meal alone.

When cut and cured as hay, the vines make a good fodder for cattle and sheep, or they make an excellent crop for soiling during the summer, and may be cut from the time they are half-grown, until they are fully ripe. The pea-weavil is very destructive to this crop in many portions of the Southern States, but is not troublesome in Canada and the Northern States, except in a ferv localities.

There are numerous varieties suited to both garden and field culture. The earliest kinds are generally not of as heavy growth in vines, or as productive in peas, as those that mature a little later. The varieties most cultivated as field-crops at the north, are the Marrow. fat and the small yellow-pea, which is largely grown in Canada. The former is a rather late variety of large growth, and requires a rich soil. The latter can be successfully grown on light land.

The cow-pea is much cultivated at the South, where it is greatly valued for fodder and the ripe seeds, which furnish food for stock; also as a fertilizer when turned under green, being considered next in value to red clover for this purpose. It will grow on soil too poor to produce clover, and hence may be used to advantage where clover cannot. This plant, however, much more closely resembles the bean than the common pea.

The cultivation of the "Pindar" or ground-pea, has been given under the heading of Peanuts, and does not require repetition in this connection. 
Cultiration and Harresting. - Peas will grow on almost any soil, but will produce the largest crops on lands that are rich. No stable manure should ever be used for this crop except it has been previously fermented or rotted, either by being composted or plowed under for a previous crop. When well-rotted, it is one of the very best fertilizers for peas, but when used before the fermenting process has taken place, it has a tendency to increase the growth of the vines, and diminish the quantity and quality of the product.

The ground shonld be well pulverized by plowing and harrowing before sowing the seed. The peas are sown both broadcast and in drills. When the former method is practiced, from two to three bushels are required per acre; for drill-planting, from five to six pecks per acre are generally used. They should be covered to the depth of three or four inches; couseqnently a light plow or cultivator is better for this purpose than a harrow. In either case, the ground should be afterwards rolled to press the soil upon the seed and make the surface even. An even surface renders the processeof harvesting much easier than where the ground is left in ridges.

Peas are not easily injured by the frost, consequently may be sown early in the spring and as soon as the ground is dry. When sown later, or in dry weather, they should be covered deeper than when it is done early in the season. Some farmers cover to the depth of five inches. A deep covering enables the crop to better withstand a drouth. Early sowing admits of harvesting the crop in time to plow the land for wheat. Soaking the seed for twenty-four hours in tepid water before sowing, hastens germination.

It was formerly the practice with farmers to sow peas with oats; but this is not to be recommended, as the two crops do not ripen at the same time, and the oat stalks are not sufficiently strong to support the vines. They are sometimes sown in corn-fields, by being drilled in between the rows of corn. They are by this method liable to be unduly shaded by the heavier growth of corn.

When a succession of crops for soiling are wanted through the season, peas may be sown in April as soon as the ground is dry, and at intervals every three or four weeks. At the North, the earliest varieties are sown in April or May, and the later in June, the latter being the more general practice, unless more than one crop is desired. When sown in drills, the soil may be worked with the horse-hoe or cultivator soon after they are out of the ground.

When cultivated for green fodder, they can be cut at any time after being half-grown until the pods are ripe. When peas are the object of cultivation, they should not be harvested until nearly ripe. They may then be cut with a scythe, dried in the field, and afterwards threshed. The hauln or straw furnishes excellent fodder for sheep and cattle. When fully ripe so that their roots may be easily pulled, they are sometimes harvested with a horse-rake, and left in heaps to dry. The cow-pea may be harvested in the same manner; but when the growth is very heavy, an iron-tooth harrow is sometimes used. Swine and cattle are frequently turned into the field to eat the crop, but this practice is not to be recommended unless it is designed to turn it under as a fertilizer, as quite as much would be wasted as eaten, by being trampled into the ground. 


\section{BEANS.}

$\mathrm{B}$ EANS, as a field crop, are cultivated considerably in this country, but not to the extent that they are in Europe. They are an important product, and should be more generally grown by farmers than they are at present. As an article of food for man and farm stock, they are very nutritious. Sheep are very fond of them, and will also eat the straw or the dried vines and pods. It should never be fed to them, however, when mouldy or musty. Beans are also one of the best known substitutes for a meat diet. They are easily cultivated, are not comparatively an exhaustive crop, and may be grown on very poor land. On soils of less than medium fertility, and with but little cultivation, large crops may be produced, and they are a product which will always bring a fair price in the market. As has previously been stated, beans will grow and produce crops on very poor soil, but will prove more remunerative when grown on one of moderate fertility, and to which they are best adapted.

The two general varieties of beans are the dwarf or bush bean, requiring no support for their vines, and the pole-bean or climbing varieties, requiring support. Between these are intermediate varieties. The pole-bean is never cultivated as a field crop. The varieties of the field-bean are not large, and there is probably not much difference in their profit, except it be from local circumstances, where certain varieties may be more in demand in the market than others. As a general rule, the large seeded varieties are less reliable and require a longer season to mature than the small. As the sultivation of the garden varieties will be given in another department, they will not be mentioned here.

The kinds most generally grown as a field crop are the small white, the kidney, and the marrow. The first-mentioned is an early variety, very small, and yields well. The second is of medium size and somewhat better quality, but not as early or productive. The marrow is a large. round bean of quite good quality, rather late in maturing, and of fair yield. The small white bean is most generally cultivated as a field crop. There are other varieties of local popularity. In the vicinity of Boston, an early kind, known as the "yellow-eyed bean," is extensively cultivated. It is of medium size and white, except a slight yellow ring about the eye. The plant branches out quite high, is a strong and vigorous grower, and the pods are kept at a greater distance from the soil than most of the other bush varieties. They are, therefore, less liable to be injured by the wet. The English field-bean, called also the "horsebean," is rarely cultivated in the United States, as the climate here is too dry and warm, but is grown to a considerable extent in Canada.

Cultivation.-A light, warm, dry soil, or that of a sandy nature is best adapted to the cultivation of beans. On soils that are heavy or highly manured the tendency is to produce a heavy growth of vines and foliage, rather than the seed, whereas a comparatively light soil of the proper quality will promote greater productiveness and earlier maturity. Stablemanure is objectionable as a fertilizer for this reason. Superphosphate of lime is highly recommended for this crop.

The land should be plowed some weeks in advance of planting, in order to give the seed of weeds an opportunity to make their appearance, when they can be destroyed by the use of the harrow or cultivator, and be afterwards kept in subjection with much less labor. The soil should be finely pulverized before the seed is deposited. Wet lands are objectionable for this crop, but if necessary to use soil containing a surplus of moisture for this purpose, the surface should be ridged in order to secure better drainage. The best results are to be obtained on a warm, dry soil.

The proper time for planting depends upon the season. While peas will bear considerble frost, beans, being of inter-tropical origin, are very tender and are easily killed by the 
slightest frost; therefore it is never safe to plant them until all danger in his respect is past. As they grow very rapidly, the planting may be delayed beyond the point of danger, and they will then have sufficient time to mature before the frost makes its appearance in the fall. About the first week in June, at the North, is the common time of planting, unless the season is unusually early. If planted when the soil is cold and wet, the seed will be liable to rot; it should therefore be done when the ground is warm. If the ground be very dry at the time of planting, germination will be greatly hastened by soaking the seed in tepid water from twelve to twenty-four hours beforehand. They may be planted either in hills or drills. The distance apart in the hills and drills should be regulated by the variety cultivated, since some kinds require more room for growth than others, the plants being larger and the branches wider-spreading. For plants of moderate growth, the distance between the rows should not be less than two and a half or three feet, and that of the hills not less than from one to two feet. Drills should be the same distance apart as the rows, and care taken not to sow the seed too thickly. From six to eight plants to a hill is sufficient. The covering should be to the depth of two inches, and the soil pressed down upon the seed. On soil free from weeds, they have been known to produce largely, when sown broadcast, but this method is not to be recommended, as it will not admit of after-cultivation. The crop should be kept free from weeds with the horse-hoe or cultivator.

Early ripening is desirable when other crops are to succeed field-beans during the same season. When there is a tendency to produce a luxuriant growth of vines to the detriment of the seed-product, it is well to cut off the ends of the vines once or twice during the season. This has the effect of hastening the maturity of the crop and increasing the product. Like all other crops, the use of good seed is of the greatest importance. Late-grown seed usually gives the best results, and it should be from a crop which grew more to seed than vines. Beans are sometimes planted in connection with corn, but it is a practice that cannot be generally recommended.

Cultivation of Horse-Beans. - The culture for the variety of field-bean known as the English field or "horse-bean," differs somewhat from the common field-bean. The method practiced in Canada, is given by a farmer in that province as follows:-

"The horse-bean thrives well in this section, the yield on my farm generally being from forty to fifty bushels per acre. It requires good, heavy land, prepared the same as for potatoes, either well manured in the fall and plowed in, or else the manure is put into the drills in the spring. We spread the manure well in the bottom of the drills and then sow the beans on the top of the manure, two and a half bushels to the acre, and cover six or eight inches deep with the plow. I make the drills twenty-eight inches apart so that there is room to work them and keep them clean like potatoes. A few days before they come up I take the saddle-harrow and harrow down the top of the drills, and when they are about ten inches high a furrow is thrown away from the drills. With a drill-harrow, $I$ then level the piece down, and then hoe and weed them. They are then moulded up like potatoes. They will usually grow from four to five feet high and quite thick and bushy. They completely kill Canada thistles. I think the reason is that the beans are so thick that they smother the thistles."

Harresting.-Beans should always be harvested before the appearance of frost. It is better to gather the crop before fully ripe than to permit it to be injured in this manner. When there is no danger from frost, and the land is not wanted for other purposes, it will not be necessary to harvest them nntil the pods are turned yellow. In some sections the plants are pulled by hand, the dirt shaken from the roots, and the vines thrown into small heaps or spread on the ground to dry. In other localities, a small iron rake is used for the purpose, or if the vines are partially green at the time of barvesting, they are sometimes 
mown. The harvesting should be done in dry weather, as exposure to wet will cause many of the pods to mould and rot.

If the vines are nearly dry at the time of gathering, two or three days' exposure to the warm sun will dry them sufficientily for storing in the barn. When drying, it is a good plan to turn them over occasionally, that the curing process may be uniform. As beans heat and mould very readily, it is important that they be thoroughly dried before storing. When the vines are rather green, they are sometimes stacked in the field, around stakes, with the roots in the center, and secured at the top with a cord or a wisp of straw. In two or three weeks of good weather, they will be sufficiently dry to take to the barn, after being spread out with a few hours' exposure to the sun.

If not threshed at once, it is well to spread the vines on the floor or scaffolds as thinly as the storage room will admit, to avoid all danger of moulding. They should be threshed out from the pods, and winnowed by a fanning-mill, after which they should be spread thinly in a cool, dry place until thoroughly dry. It is well to cull out imperfect specimens, as a few discolored beans will injure the appearance of the whole quantity, however good it may be, and will be liable to reduce the selling-price.

Castor Beans. - The castor bean is a tropical plant, hence, cannot be successfully cultivated at the North. It is frequently seen growing there, however, as an ornamental plant, its stately appearance and large, broad leaves of a purplish hue rendering it very attractive. It is a perennial in warm climates, and sometimes attains the height of thirty feet, and will live for several years. In regions subject to a frost it becomes an annual, and grows to the height of from three to ten feet. It is a native of Southern Asia and Northern A frica, and has been naturalized in Southern Europe and other warm climates. In the West Indies it grows with great luxuriance. It has been cultivated to a limited extent as a field crop as far north as $40^{\circ}$; but the climate of the Southern States is best adapted to it.

In Texas and Southern Florida it strongly shows its perennial tendency. A castorplant is said to have been grown in a garden in Galveston, the stem of which attained seven inches in diameter. This plant continued to yield seven or eight years. A hundred bushels of beans have been raised per acre in localities adapted to its culture in Texas. In the southern portion of the Middle States, and States bordering on the Ohio river, it grows to the height of five or six feet, and yields from twenty to thirty bushels per acre. The seeds or beans are oval and quite large. Their value consists in the oil which they yield. When bruised and subjected to a great pressure, the yield will be nearly a gallon per bushel of coldpressed oil, which is much superior to that obtained by boiling, the latter being of dark color.

When pure, the oil is of a light yellow color, but when inferior in quality it has a greenish and sometimes a dark yellowish tinge. Exposure to the sun's rays bleaches it to a certain extent. It is used in medicine as a cathartic. It is also much used in lubricating machinery, carriage wheels, leather, etc. In Hindoostan, it is quite extensively employed for burning in lamps.

Cultivation.-The cultivation of the castor bean can only be made profitable where the climate and soil are adapted to it, and also in localities near a mill for extracting the oil.

The very best seed should always be obtained for the crop. That of the large variety grown in the West Indies, should be planted, and not the small, light-colored bean that has been grown in some of the Northern sections. The soil best adapted to the castor-plant is a rich, sandy loam, although any dry, fertile soil will answer the purpose. The surface should be well-pulverized, and the seed planted in hills, in rows five or six feet apart. Three or four seeds are deposited in a hill, to make allowance for those that may not germinate, and for such of the young plants as may be destroyed by the cut-worm, which is sometimes very troublesome. When the plants are six or seven inches high, they are thinned to one plant. The cultivation afterward given should be similar to that for Indian corn. 
The seeds will begin to ripen generally in August, according to the latitude, and continue to do so until frost makes its appearance. A place for spreading the seeds should be prepared about the time of their ripening. This is sometimes a spot of dry ground, cleared and beaten hard, and sometimes a low scaffold for spreading the pods. When the pods on the spikes begin to crack, they should be cut, and spread in the sun to dry, in the place previously prepared. They should be spread thinly, in order to dry well. There will be several gatherings of the crop, as the pods mature and ripen. The heat of the sun causes the pods to open, and the beans to shell out. In warm weather, two or three days will generally be sufficient to dry them.

When all the beans are out of the pods, the latter are raked off and the beans gathered and cleaned by a fanning-mill, or seed-cleaner, with a suitable screen. They are then spread in a cool, dry place to become thoroughly dried before being stored. It is well to occasion ally turn them during the drying process.

\section{BROOM CORN.}

$\mathrm{T}$ HIS plant is a variety of the sorghum, and a native of the East Indies. It is cultivated for its branching panicles principally, which are used in the manufacture of brooms, etc., although it is grown to a limited extent as a forage plant. This family of plants are all more or less rich in saccharine matter, but as this variety has been cultivated for a long period with direct reference to the production of the brush or seed-bearing stems, and their improvement in length and fineness, without regard to its sugar-producing properties, its appearance is very different from that of the common sorghum or Northern sugarcane.

The introduction of broom-corn into this country, as a cultivated plant, is attributed to Dr. Franklin, who, finding a seed on a whisk broom that had been imported, planted it, and produced seed from the plant that was the result of the experiment. From this, originated its culture in the United States.

The production of broom-corn was formerly quite limited, being confined almost exclu. sively to New England, but it has now become an extensive industry in some of the Western States, where the climate and soil seem admirably adapted to it, and where it proves, in many localities, a very valuable crop.

At the extreme Nerth, the season is rather too short to admit of the perfect maturity and ripening of the seed. In many of the Western States the seed is not permitted to ripen, the crop being harvested before this period, in order to produce a finer quality of the brush product. In such cases, a more southerly latitude must be depended upon for seed in sowing.

The average product of brush per acre is about five hundred pounds, although with the best soil and cultivation, rare instances are known of the crop amounting to nine hundred or a thousand pounds per acre. As there seems to be no substitute for the brush, and there is always a demand for it, and it is a crop that can be easily cultivated, it is a desirable and profitable one for the farmer to raise, in those sections to which it is well suited.

When mixed with oats, the seed makes very good food for horses. It is also valuable for poultry, especially chickens after they are a week or two old, and when fed in connection with other food. It is much more nutritious when ground sufficiently fine to break up the tough husk. When ground with corn, in the proportion of three bushels of broom-corn to one of corn, it makes very good fattening food for cattle, sheep, or swine. This proportion also makes a good ration for milk-production in winter. 
It is always well to mix it with some of the common grains. When mixed with corn, it is said to be as nutritious as an equal quantity of oats. Sheep will masticate the whole seeds better than cattle, because they will grind it finer. Still, when not ground, it is always best to cook the seed, as it is thus rendered soft and more digestible.

Soil and its Preparation.-The best soil for the cultivation of bropm.corn is that to which Indian corn is best adapted. It should be rich and mellow, free from weeds, and not subject to early or late frosts. Lands for this crop should never contain a surplus of water. Such as have a subsoil retentive of water, should be under-drained. The best river bottoms are well adapted to it, providing the situation gives them a good natural drainage. Its culture is not, however, confined to any particular kind of soil. Any soil that is dry and of a verage fertility, will yield a fair crop.

Unless the land is naturally quite rich, fertilizers of some kind are essential. Well-fer. mented manure of any kind is good, that from the barn-yard, pig-sty, or sheep-pen being valuable for this crop. Guano, plaster, wood-ashes, and lime, also give good results, but generally not quite equal to the former.

Soil should be made as clean and free from weeds as possible, as the young plants seem weak and feeble at first, and are consequently easily choked by the weeds. A clean soil also admits of less labor in the after-culture.

As with common corn, different cultivators adopt different methods of preparing the land. Whatever the method of preparing the soil, the surface should be finely pulverized by plowing and harrowing before planting. The manure is generally applied broadcast; in addition to this, a small quentity of manure applied to the hills or drills before planting would serve to give the young plants a vigorous start. The preparation of the soil should in all respects be similar to that made for corn.

Planting.-Broom-corn may be planted either in hills or drills. Hills are generally preferred as a matter of convenience in the after-culture, as they admit of running the cultivator both ways.

The rows may be from three and a half to four feet apart, according to the variety to be grown. Dwarf varieties are sometimes planted three feet apart, but four feet is sufficiently near for the large kinds. The hills may be about two and a half or three feet apart, according to the variety grown, although some prefer to have them the same distance as the rows.

In the cultivation of this crop, it is very essential that the seed be of the best quality and thoroughly ripened. Seed of inferior quality, or that imperfectly ripened, will either fail to germinate or produce weak, sickly plants.

As many of the seeds often fail to germinate, even when of average good quality, the usual practice is to plant a liberal quantity to the hill, and afterwards thin out the plants to five or six, leaving only the strongest and most thrifty looking ones. From fifteen to twenty seeds to the hill are sufficient. If too large a quantity is planted, the young plants will be crowded, and as they are naturally at first very weak, overcrowding at this period would render them more so, and prove injurious to the crop.

When the seed is sound and well-ripened, two quarts will be sufficient for an acre, this being the usual allowance. If much of it is imperfect, a much larger quantity will be required. For drill planting, a somewhat larger amount will be required than for hills. The planting should be done when the soil is warm and dry, and all danger of frost is passed; about the time of planting corn, or a little later.

When the seed has been thoroughly cleaned of the stems or pedicles adhering to them, it can be planted by any of the common seed-drills that will plant seed of small size. Corn planting machines and wheat-drills are used for this purpose. When only a few acres are to be planted, dropping the seed by hand-although a slow process-is often practiced in some localities. A small garden seed-planter is much to be preferred to planting by hand. 
The seed should not be covered much more than about an inch deep, unless the soil is light and dry. In such a case, the covering should be a little deeper. If the seed is put in as soon as the ground is prepared, the moisture of the soil will serve to lasten germination.

Thick planting produces the finest and toughest brush. Old soil long in cultivation is also thought to give a better quality of brush than new, fresh soil.

Cultiration.-As soon as the plants make their appearance, the cultivator should be run between the rows to loosen the soil and check the growth of weeds. This, should be frequently done during the early stages of growth, as stirring the soil promotes the growth of the plants, and holds the weeds in check, which are exceedingly injurious to the young plants. If the weeds are allowed to once get the start of the crop, it will prove very detrimental, and require the plants a long time to recover the effect, if they ever do.

It is well to begin the stirring of the soil before the plants are up, and consequently before the weeds appear, and when they are germinating in the earth; but in such cases it will be necessary to use considerable care, or the seed of the crop may be disturbed. The practice of some farmers of allowing the weeds to grow several inches before they are exterminated, is not only very injurious to the crop, but more than quadruples the labor of their destruction afterwards. Those farmers who have learned the great economy of the practice of killing weeds before they come up, with respect to corn, will appreciate the importance of this practice as applied to broom corn.

This constant stirring of the surface will make a great difference in the yield of the crop. The various horse hoes and cultivators in agricultural use may be employed for the purpose.

The thinning should be done when the plants are three or four inches high, leaving five or six of the most thrifty ones in a hill; or, if drill-culture is practiced, the stalks should be left three or four inches apart. The thinning is a very laborious job, and the most to be dreaded of any part of the labor attending the cultivation of this crop. The general rule with the best cultivators is to keep the whole surface of the soil clean of weeds from the very beginning, and use the cultivator for this purpose once a week, as long as the size of the plants will permit. Different varieties of broom-corn are very liable to mix when planted near together; consequently, when growing seed for future crops, they should be planted entirely separate and in fields remote from other varieties, that the seed may be kept pure. The same is true if it is planted near any of the varieties of the sorghum or Chinese sugarcane, with which it will readily mix.

Harvesting. - The period of harvesting will depend somewhat upon the use to be made of the crop. When the brush only is desired, broom-corn can be cut much earlier than when the seed is one of the objects sought. In the former case, the harvesting may be performed as soon as the blossoms begin to fall. The quality of the brush is better at this period than if the cutting be delayed till later. The brush then will be of a green tint, and fine and tough in quality.

A few days later, when the seed is in a milk state, or is approaching maturity, the brush will assume a reddish shade, and lose somewhat of its weight, being in quality inferior to the green-tinted product, and accordingly commanding a less price in the market.

When the grower wishes to secure a good quality of seed and does not care for a very fine quality of brush, the harvesting should not be done until after the seeds are fully matured. The quality of the seed that is planted influences largely the character of the crop; consequently, care should be taken that all seed designed for planting should be perfectly mature before harvesting. Broom-corn should always be harvested before frost makes its appearance.

Before harvesting the larger or tall-growing varieties, it is customary to break down the stalks, turning the tops of the stalks of two rows towards each other, thus leaving a vacant 
swace for the harvester to work in cutting. This is done for the sake of convenience, as, if left standing, the height of the stalk at the place of cutting would make it a very laborious practice, some of the large varieties on rich soil attaining a height of from fifteen to eighteen feet. From ten to twelve feet is, however, a good height. The stalks are generally brolsen at the height of two and a half or three feet from the ground, those of two rows being turned towards each other diagonally in such a way that the stalks of one row will cross those of the other with the tops projecting about a foot on each side. The crop is then said to be "tabled." Each two rows of the field are thus broken over, leaving a vacant space for passing between the rows and cutting the brush, which is usually done immediately after the crop is broken.

Dwarf varieties do not require this process, but are usually harvested by the stalk containing the brush being pulled out from the sheath by which it is surrounded. This is a more difficult operation than cutting.

After being "tabled" as previously described, the brush should be cut so as to leave only eight or ten inches of the stalk below it, and no leaves. These should be laid in small bundles upon the tables as cut, and then gathered and put under shelter. It is the practice in some sections to leave the brush exposed to the sun two or three days to cure; but this is not to be recommended, as this will have a tendency to give it a reddish tinge, even though cut when quite green. It also injures the quality, rendering it less tough and more easily broken. Exposure to rain is also injurious. Therefore it should be gathered and put under shelter as soon as a sufficient quantity is cut.

Curing, etc. - When only small quantities of broom-corn are produced, it may be cured in any sheltered place, such as in a shed-loft or on a barn-scaffold, where it will be exempt from exposure to sun and rain. But where extensive crops are grown, a house especially adapted for curing the crop becomes a necessity. The interior of such buildings have a large number of racks prepared, upon which the brush is spread very thinly to dry. The racks are made with narrow strips of boards alternating with open spaces, in order to admit the air and hasten the curing process. These rack-shelves should be from eight to ten inches apart, so as to permit a free circulation of air. The building should have every con. venience for ventilation, but the ventilators should always be closed in stormy or damp weather.

When the brush is harvested green, the seed is generally separated from it before curing. This may be done in various ways. Machines made for this purpose, with revolving cylinder, or similar to a threshing machine, are much used where large crops are cultivated. They can be worked by hand, foot, horse, or steam power, according to the size. It can also be done by holding the heads to the cylinder of a threshing-machine, and not permitting the brush to be drawn in. Where small quantities are cultivated, a hetchel made for the purpose, or a long-tcothed curry-comb are frequently used.

When secd is immature, it is of little value as food for stock, and may be best utililized in the compost heap. Broom-corn seed is very liable to heat, and should be spread thinly on a scaffold or floor to become perfectly dry before being stored. Rats and mice should be kept from the brush when curing, as they will destroy and waste large quantities. 


\section{HOPS.}

$\mathrm{H}^{\circ}$ OPS grow wild in the northern portion of the United States and Europe. There seems to be but one botanical species of this plant, but numerous varieties have been produced from this by long cultiration. The root is perennial, but the rines are killed by the frost every autumn, new shoots starting up from the roots in the spring. It is a climbing vine, with rough leaves and stems, grows rapidly, and always twines around its support from left to right, or following the course of the sun. In its wild state, it often climbs high up among the branches of tall trees, and when cultivated requires tall poles or other substitutes for this purpose. Different varieties, however, differ in this respect, some requiring longer supports than others.

Hops have become an agricultural product of considerable importance in many countries. They are chiefly used in the manufacture of beer, although to a certain extent for domestic purposes, such as the making of yeast, and for their medicinal properties, etc. The portions of the hop-flower which enter into the composition of beer are the seeds and the yellow matter which surrounds the outer covering of the seeds, and the base of the petals. This yellow, glutenous material emits a very pleasant aroma, and has an exceedingly bitter taste. It is also quite volatile, and unless packed rery closely as soon as the hops are dried, this principle is in a great measure lost. For this reason, hops that have been kept more than a year are of considerable less value than new. The new product is essential to the manufacture of beer of the best quality. This bitter principle is of more delicate flavor in sone varieties than others, and in proportion as the seed is developed. In selecting varieties to plant, three considerations should be taken into account; these are the delicacy of flavor, yield of the crop, and time of maturing. As a general rule, the strongest growers are the largest yielders, but have not that delicacy of flavor that characterizes the smaller and less prolific varieties. Early maturity is desirable in localities subject to early frosts. On farms where hops are extensively cultivated, it is well to plant varieties maturing at different times, as the picking season will be long. By this means the early varieties may be harvested first, and the later varieties will not become over-ripe before being gathered.

Varieties.-In England the Farnham, Canterbury whitebines, and Goldings are most extensively cultivated. These are strong-growing, prolific vines, that require poles from fourteen to twenty feet long. The grape varieties, of which there are several, are smaller, and are so called because their blossoms, or the hops, grow in clusters. These require poles of from ten to fourteen feet in length. In this country some of the grape rarieties are quite extensively grown; although many of the large varieties, embracing those of greater or less local popularity, are also cultivated.

The hop is not only grown as a field crop, but it may be made to subserve the double purpose of utility and ornament, by being trained on supports for ornamental purposes. There are few plants that have a better foliage, or that cling more tenaciously to any support provided for them. When in full blossom, it is certainly a graceful plant, while its pleasant aroma also renders it attractive. When grown for ornamental purposes, there are many ways in which it may be trained that are suited to render it graceful and truly beautiful, the use of the straight, upright pole for this purpose being anything but graceful or artistic. For the country home, they make a very pretty arch when trained to span the walks in the kitchen garden, and may be mixed with other vines, such as the clematis or woodbine. A rustic trellis, with arch made for their support, will thus be transformed into a beautiful ornament for the home surroundings, and, if desired. may be utilized to supply the family with all the hops necessary for domestic use. They also add a beautiful feature to gardens, by planting them where they can run wild over old fruit-trees, trunks of dead trees, o: even 
summer-houses or other outbuildings, since, when once planted, they last for many years, requiring no care except to train the vines a little to their supports when first starting in the spring. They will also grow in almost any ordinary garden soil. Hops cannot be profitably grown south of latitude 40 .

Cultivation. - Where there is sufficient depth of good soil, not too rich or too wet, hops, with proper cultivation, may be continued almost indefintely. If, however, the soil be shallow, with a hard, impenetrable sub-soil, or is cold and wet, the roots will soon die out, and the enterprise prove a failure. If the land be too rich, the product will be vines, ratier than fruit, a small, pale-green hop being the result, rather than the large, fully-matured, yellow bur, which is the product of a less growth of vine, and consequently grown with less shade and more air and sun. Hops have long roots, whose nature is to penetrate deeply into the earth; therefore it is quite as necessary that the sub-soil be of the proper quality to be thus penetrated on the surface-soil. If the sub-soil be saturated with water, or of a hard, compact nature, the roots cannot enter it, and the plants cannot therefore thrive. All soils intended for hop-culture should be either naturally or artificially drained. The former is better, but when the latter is employed, tile-draining is essential.

Hops, like corn, require a warm, mellow, and aerated soil. Any soil that will produce a good crop of corn, will also produce hops successfully. They are an exhausting crop, and lands on which they are grown require to have manures rich in nitrogen applied from time to time, in order to maintain their fertility. A deep, rich, well-drained, alluvial soil, in a locality well protected from sweeping winds, is generally considered the best for hops. A sufficient quantity of air is essential, but strong winds are very injurious. Wet soils, hot suns, and strong winds, in conjunction, are fatal to the hop product.

High land, if the soil be good, is to be preferred to low land, especially if protected by hills or trees, and not so exposed to the winds that they will be bare in winter. The soil should be deep and friable, containing a sufficient amount of humus but not a surplus of moisture, and yet one that will withstand drouth. If tenacious clay soils are used for this product, they should be well drained.

When the land designed for planting hops has been long in use for other crops, it should be well dressed with compost or an abundant supply of fresh manure, previously plowed in for other crops and largely retained in the soil. Fresh farm manure should be avoided, as it has a tendency to produce a heavy growth of vines to the detriment of the crop. Light loams or gravelly soils, when made sufficiently rich with fertilizers, will produce good results, but a naturally fertile soil is much better. A gentle slope to the south is desirable, but this should also be where there is a free circulation of air, yet protected from injurious winds. When land is strong, it should be cleared before planting. The plowing should be quite deep. The best time for doing it is in the fall. Well-composted farm manure is used to advantage for this crop; also lime and phosphates, on some soils.

The surface of the land should be well pulverized, and rendered as mellow as possible. It should then be marked off in squares at a distance of about eight feet, the rows to be in a straight line. The distance between the hills varies, according to the nature of the soil and the variety to be cultivated, some kinds growing much more luxuriant than others. It is well to avoid crowding, for if planted too closely together, it will have the same effect upon the product that close planting has upon the yield of corn. There should be a sufficient amount of space between the hills for sun and air. A lack of these essentials will result in more vines than blossoms, and there will be a deficiency not only in weight, but in the quality of the bur. A soil that is too rich, as well as an unusually wet season, will also give the same result. A light growth of vine will often produce a surprisingly large growth of bur's of good quality in proportion to the growth of the vine; hence, it is not advisable to force the growth of the plants too much. 
When the hop-seeds are planted, the result will be a variety of kinds, the same as with potato or fruit-seed; therefore the plants should be multiplied by planting slips from a wellknown and desirable variety. The underground vines, or sprouts from the old plants, are used in planting. Each piece should be from six to ten inches long and contain, at least, two or three eyes, one for root formation and one or two for the vine. About two bushels of these sets will be required for planting an acre. The hops, like many other plants, produce the male and female blossoms on different plants. The blossom of the former grows in a loose panicle, while the latter is compact in form, like the cone of some rarieties of the pine tree.

In obtaining slips for setting a hop.field, those from the male and female plants should be kept separate. Only about eight or ten of the male plants will be required for an acre. These should be planted in separate hills, and evenly distributed over the field. In setting a field, it was formerly the custom to put six plants in a hill, but four are now considered a more desirable number, as it involves less crowding with all the attendant evil results. Four good slips should be put in a hill, and covered with two or three inches of finely-pulverized soil.

Some growers put a fork.full of well-composted manure in each hill before planting the slip. Others make the land sufficiently rich without it. Whether this practice will be advisable or not, will depend upon the nature of the soil and the preparation it has previously received. If the land is quite dry, hill-manuring may be omitted, if the soil is sufficiently fertile to warrant it. If it is inclined to be too wet, manuring in the hill will remedy the evil in a measure. Level culture is considered best in this country, as a general rule, as the drouths to which the soil is subjected is detrimental to the hop-plants, but in the moist climate of England, hilling is more generally practiced. In planting, therefore, the land should be left as nearly level as possible. During the first season of growth, potatoes or corn are sometimes planted between the rows of hops, and the cultivation given is sufficient to keep down the weeds. With some growers the poles are not set, and no effort is made to obtain a crop until the second season. Others set the poles the first season, and secure a small crop.

No fresh manure should ever be used for hops. In place of manuring the hills, many growers put a quantity of richer soil in each hill before planting, in order to secure a good start. In planting, the sets should be spread out carefully, and the fine, damp soil be pressed down upon them. Where poles are set the first season, they may be only from eight to ten feet long, and one pole will be sufficient for each hill. The poles should be sharpened at the end, and set one foot in the ground. They may be made of various hinds of wood. Cedar is generally considered the best for the purpose. The land should then be kept free from weeds. The length of the permanent poles will depend upon the soil and growth of the variety planted. Where the soil is fertile and the strong-growing varieties are planted, poles from twenty to thirty feet long may be necessary; but where plants of smaller growth are cultivated, shorter poles may be set. Two poles are required for each hill, to give sufficient sun and air to the plants.

They should be set firmly in the ground, in order not to be blown down by the wind. As they are often thus blown down or broken off, to the injury of the crop, horizontal yards are preferred by many hop growers. This method renders the labor of harvesting much easier. It is also much more economical than the use of long poles, and the plants generally grow better. With this method, one stake is set to each hill. These stakes should be sawed from eight to ten feet long, an inch and a quarter square, and be covered with coal tar.

A row of stakes are also set around the field at a distance of eight feet from each hill. The tops of all the stakes are thus connected with strong twine that has been well tarred. In those hills of the field occupied by male plants, long poles should be set. This is for the purpose of facilitating the distribution of the pollen from their blossoms orer the whole field. 
When the vines attain the length of two or three feet, they should be tied to the poles to prevent being blown and injured by the wind; also to assist them in clinging to the supports, care being taken to twine them the natural way, which is in the course of the sun. They will need such assistance occasionally, in the progress of their growth, and when the horizontal method of culture is practiced, the vines, after growing beyond the length of the stakes, will need to he assisted in turning and twining around the strings.

In order to attain the best success in hop-growing, the culture should he similar to that of corn. Frequent stirring of the soil, and the extermination of all weeds are essential. The best growers of hops cultivate the crop about once a week during the season, when the weather will admit. The essentials for the best success with this crop are, a deep mellow soil, perfect drainage, continued cultivation of the crop from spring to fall, a sufficient supply of the fertilizing element, and a plenty of space between the hills for sun and air. The hop is a tender plant and cannot thrive under neglect or hard treatment of any kind.

When early harvested, it will bleed equal to the grape vine, and with as much injury to the plants. Exposure to cold in winter will frequently kill it. If forced too much in growth, its vitality will be exhausted and mildew be the result. If the weeds and grass are kept out of the field, the moisture and shade which are so injurious will be obviated to a great extent, unless the plants are set too closely together. A uniform, healtliy growth, with light and air, are the requisites necessary to perfect the development of the vine and bur.

Deep culture to the soil after the plants are set should be avoided, as it tears the roots. Shallow and frequent working of the surface is the better practice, and the finer and more mellow the soil is kept, the hetter for the crop.

In the autumn, a shovelful of well-fermented manure should be thrown upon each hill. This serves to keep the roots warm during the winter, and the rains and melting snows will carry the fertilizing elements from it to the soil and roots. This is carefully removed in the spring, and the roots, stalks, and vines trimmed off, after which the cultivation should be frequent, as previously directed, in order to keep down the weeds, and maintain a mellow surface. The ordinary corn cultivators are generally used for this purpose.

Harvesting. - The season for picking hops generally commences the last week in August, and continues several weeks. Some varieties mature earlier than others. The time of picking is denoted by the seed turning hard and purple. If the picking commences before the hop is ripe, the plant bleeds profusely and may be either greatly injured or killed by this means. When the horizontal method is practiced, the picking may be done from the vines while they are supported by the cords, or the cords may be loosened from the stakes to bring them within easy reach of the pickers.

When poles are used for support, the vines are cut from one to three feet from the ground, and the poles pulled up and laid over large bins or boxes around which the pickers gather, dropping the hops into the bin as they are picked. Care is necessary not to permit leaves, stems, or other waste material to fall into the bin during the process. When the bins are full, the hops are put into large boxes and taken to the kiln for curing.

Drying. - When small quantities of hops are cultivated, they can be dried by being spread in a store room, or other suitable place, and stirred often enough to prevent heating; but when extensively grown, a kiln for this purpose will be necessary. A kiln is a building constructed for the purpose of drying the hops by a quicker process than can be secured without artificial heat. There are numerous patent methods of constructing kilns and curing. The hop are generally spread to a depth of from twelve to fourteen inches, where they are subjected to a heat of sufficient temperature to become thoroughly cured in from twelve to twenty hours.

The hops are sufficiently, cured when they will crumble two-thirds to pieces when han. 
dled and the stems are dry. It is very important that hops be well dried. They should not be left exposed to the air after being cured, as the volatile principle will thus be lost. When properly dried, they should be packed closely and put in a dry place.

\section{MUSTARD.}

$\mathrm{T}^{\mathrm{H}}$ HE mustard family includes many valuable plants, such as the cabbage, turnip, rad. ish, horse-radish, water-cress, nasturtium, etc. The varieties of mustard cultivated as a field crop and garden plant for the seed are two,-the white and the black or browu. The black or brown mustard has a small globular seed of a dark brown color, and light yellow within. The white variety has large seed of a light color. The flour mustard of commerce consists of the mixed ground seed of both varieties. The black variety is the strongest. The ground mustard is a popular condiment for table use, and was known to the ancients. It is also of great value for medicinal purposes, and is used in cases of poisoning. A heaped teaspoonful or more of the ground mustard, mixed with a turnbler of water and drank, acts as a prompt non-nauseating emetic, and may be used with the greatest success and safety in cases of poisoning. This is a fact that every adult member of the household should know, as cases of accidental poisoning are liable to occur in any family, and it is soinething that is always at hand and may be used promptly in time to save life in such cases, when waiting for a physician might prove fatal. We once witnessed its wonderful efficacy in saving life when a person was apparently in a dying condition from the effects of an overdose of veratrum viride. It cannot be too highly recommended in such cases. When moistened with vinegar or water (the former is best), and applied to the skin, it is a most powerful counter-irritant, and will relieve internal pains and sprains.

The mustard plant furnishes a valuable crop for green food for cattle or sheep. It is also used for plowing under as a green manure. In England from twenty to thirty bushels of seed per acre are frequently grown. The principal objection to its growth is, that it is difficult to eradicate from a soil where it has once been cultivated, the seed that is self-sown in harvesting, producing tronblesome weeds for future crops. The wild mustard is an exceedingly noxious weed in some portions of the West. The best means of ridding a soil from mustard where it has been previously cultivated, is to permit the plants to grow until they are in blossom, and then plow them under.

Cultivation.-A rich, loamy, moist soil, reduced to a fine tilth, is the most desirable for mustard, although it will grow well on any soil of medium fertility, if it is not too wet or cold. A warm soil is essential. It may be sown either broadcast or in drills. The latter method admits of after-culture, which is an advantage over the former. When the latter is practiced, the drills should be about two feet apart, for convenience in cultivation. Care should be taken not to sow the seed too thickly. A peck to the acre is required for broadcast sowing; for drills, about six quarts is sufficient. The ground should be kept free from weeds and frequently.stirred. When a majority of the plants have ripened their seed,which may be indicated by the pods turning brown or yellow,--the crop should be carefully cut with a reaper or scythe. It may be set in small stacks for a day or two in the field to dry, or, if so ripe when cut that the seeds shell out in handling, it should be taken at once to the store-house. It should always, when taken from the field, be carefully laid in a cart with a light covering of cloth over the bottom and sides to prevent the seed from wasting. It should be taken to a store-room, where it may remain until ready to be threshed. It should be handled with the greatest care, as it is very liable to shell out and a good share of the crop will thus be wasted.

The quality of the seed is liable to be injured by wet weather about the time of harvesting. As soon as it is perfectly dry, it may be threshed and cleaned ready for market. 



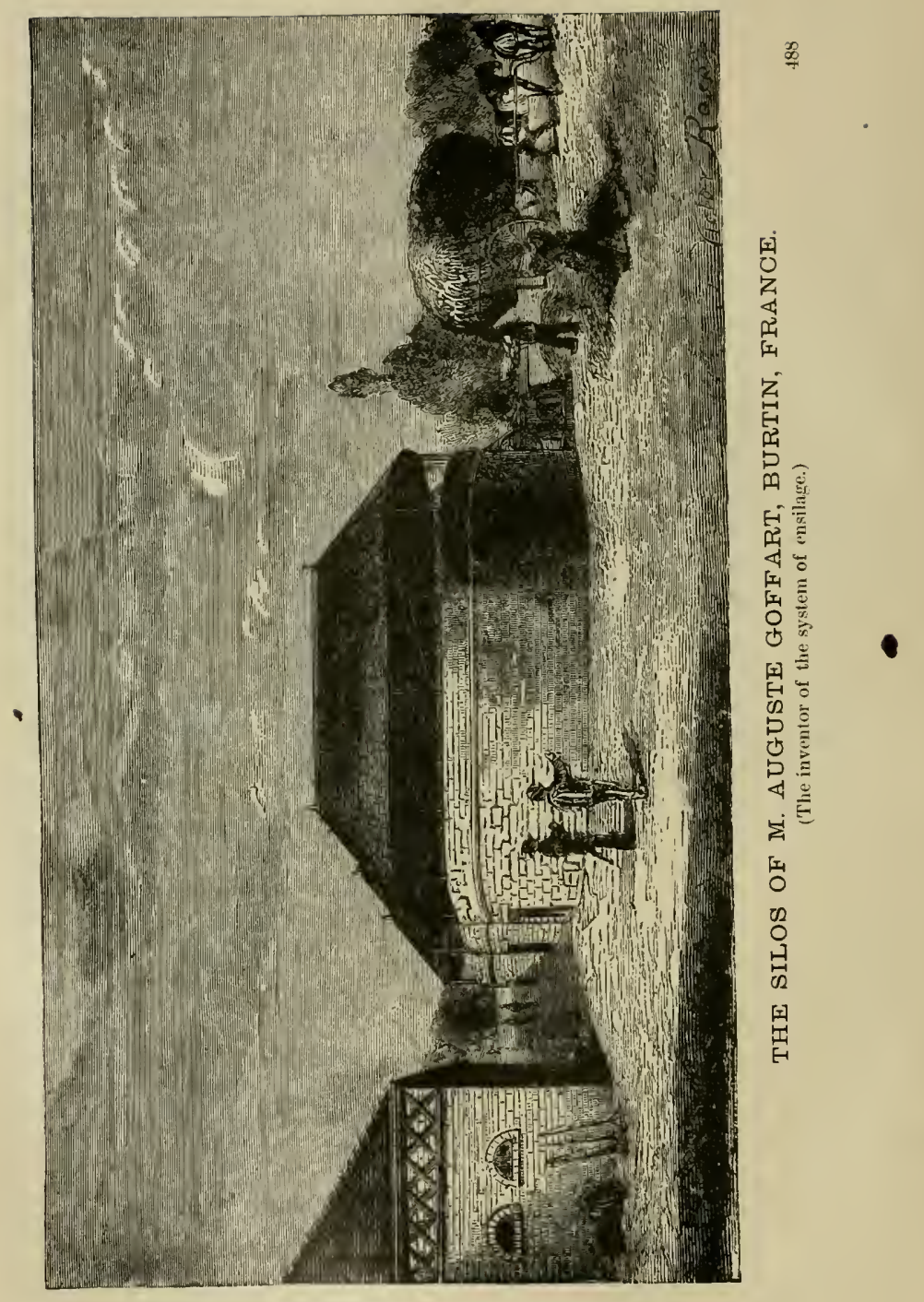




\section{ENSILAGE.}

$\mathrm{T}$ HE system of ensilage, which has attracted so much attention for a few years past both in this country and Europe, is one of vast importance in the agricultural world, and of practical interest to every farmer, since it bids fair to supplant in part, if not wholly, the present system of hay-making, or preserving fodder by drying,-a process not only laborious, but expensive, while it involves at the same time considerable loss of valuable nutriment by desiccation, or the drying process. One has but to enter a meadow or mowing field where the new mown hay is being thus cured, and inhale the fragrant odors that are so invigorating and pleasant, to be convinced of the fact that some of the best elements of the grass are by this process being wasted by passing into the atmosphere. Thus much is lost by the curing process, even under the most favorable circumstances, while great loss is often sustained through rains, heavy dews, and unfavorable weather at the time of gathering this crop,so important to the farmer. Now, any improvement in the method of preserving vegetation, which not only involves less labor, time, and expense, but furnishes better and more nutritious food for the stock, - keeping cattle in as good condition through the winter, and causing milch cows to produce as much milk and of as good quality as when supplied with the best pasturage in summer,-is well worthy the attention and interest of all lovers of improvement and laudable prosperity. The advocates of the ensilage system who have experimented most, and consequently have had the most experience with regard to it, make this claim in behalf of its adoption into common practice. And not only this, but that it furnishes the opportunity in time of an abundance to lay by a supply for the time of drought or other failure of the forage crops. By this process, also, a mnch larger number of cattle can be supported upon a civen extent of land, and thus the land of any farm utilized to better advantage, since the yield of the nutritive matter in the stalks of cereals is much greater per acre than that of any of the grasses. By maintaining a larger number of cattle, the quantity of mannre produced on the farm is proportionately greater, which affords the farmer a better opportunity of enriching his lands and keeping them in a higher state of cultivation, obviously resulting in larger crops, with consequent larger profits for his labor. Ensilage is a French word which means " the act of compressing into pits, trenches, or compartments called silos." It is also used to denote the green crops thus preserved in silos. Silo is a word used to denote a "compartment used for storing green fodder in an air-tight manner." A Silo may be wholly above the ground, an excavation, or partly below and partly above the surface of the ground. Great credit is due Monsieur Auguste Goffart for not only discovering, or inventing, the system of ensilage, but for his patient and persevering efforts in bringing it so nearly to perfection as it is at present, having labored assiduously for over twenty-five years before arriving at desired results. The following from $\mathrm{M}$. Goffart's writings, as translated by $\mathrm{Mr}$. J. B. Brown, gives a brief history of that gentleman's experiments:-

"In 1850 I made some experiments in the ensilage of wheat at Versailles, since which time the preservation of fodder has become my favorite occupation. In $1852 \mathrm{I}$ constructed four underground silos, with masonry, and connected them, each having a capacity of two cubic metres; these silos I have filled and emptied several thousand times. Maize, Jerusalem artichokes, beets, sorgho, turnips, potatoes, and straw I have experimented upon with more or less success. Some years ago I had in the antumn more than eighty horned cattle, and my hay crop would not have permitted me to support ten. One should be an agriculturist of Sologne to know what such a trouble means. In rich countries, when the hay crop fails, it means that instead of harvesting 5,000 or 6,000 kilogrammes to the hectare. there are only 3,000 or 4,000 , but in Sologne it means that there is no crop at all. In such difficulties the enterprising cultivator must use more intelligence and more industry. "What the man is

Noтะ. $-\mathrm{A}$ hectare is about $2 \frac{1}{2}$ acres. A kilogramme is about $2 \frac{1}{\mathrm{~b}}$ lbs. A voirdupoise. 
worth,' is an old proverb, but I will improve upon it by saying that the man should be worth more, as the land is worth less.

I got safely through that year by having 50,000 bundles of wheat, rye, and oat straw. I cut them up, and with 35 kilogrammes of rye flour, which I fermented each day in large tubs, and in which I soaked the straw, I obtained food that was softened by fermentation, which my cattle ate freely and digested easily. Thus I reached the following spring without being obliged to sell my cattle at a low price. I must acknowledge, however, that at the end of the winter they were in a sorry condition, but the first grass quickly restored them, and I was not compelled to replace them at a high price in the spring; and now the two years of scarcity which my fellow-agriculturists have passed through have been for me-owing to my silos-years of unprecedented plenty. What I have done can be done by thousands of others, and my earnest desire, my sole ambition, is to enable them to imitate me as soon as possible. Until 1872 I only expected from my limited ensilages the means of prolonging for three weeks, or at most a month, the use of maize, so desirable a food for my cattle. To that end I made many experiments. I have mixed my cut maize with various proportions of straw, in order to find which would give the best result. I have made silos without cover, burying the ensilage under bundles of straw, then with earth (never sand). I have filled my four silos with every possible mixture, which would sooner have put me upon the road to a positive success, if I had not been too easily alarmed by shight alterations on the surface, and which I caused to extend all through by too frequent examinations.

In $1873 \mathrm{I}$ had a real success, due mainly to accident; and it is to be recognized that chance nearly always plays an important part in the happiest discoveries.

Until this time I had hardly believed that the preservation of green maize for a long time was possible, and I had very little confidence. I hesitated a while, and should have probably hesitated a good while longer if I had not been in a measure compelled to do something. The year 1873 had been exceptionally favorable for the culture of maize. At Burtin the crop was enormous. After having fed my cattle abundantly until October, besides having all that they could eat while green till December, I found that I had more than 170,000 kilogrammes that would be lost if I could not keep it, at least till the following March. I went resolutely to work, and I have described elsewhere the means that I used and the result that I obtained. The difficulties were greater than one would believe, on account of the lack of faith of my employees. One day I had to leave my workmen for a while, but my return was sooner than they expected. The work had ceased, of course. They were talking together, and I overheard my foreman say to the workmen, 'This work that we are doing is all foolishness, M. Goffart had better throw his maize into the dung-heap at once, because that is where it will go to at last.' I said nothing, but redoubled my watchfulness, knowing how little zeal I could expect from people so convinced of the uselessness of their labor."

In his earlier experiments, M. Goffart, after making his silos air and water tight at the bottom and sides with cement, put in the maize, cut in pieces of three or four centimeters long, with a mixture of cut straw, and pressing it down, placed on the top a layer of straw, cut in length about ten centimetres; over this a layer of loam beaten with care to prevent all contact with the air outside. When fissures appeared in the earth, they were iminediately closed with care; but on opening the silo, a vacuum was always found between the fodder and earth covering, showing that the mass had settled and fermentation had set in; consequently the ensilage could be preserved but a short time. After many experiments he found that by cutting the fodder into very short lengths (about one centimetre), ${ }^{*}$ and mixing but about one-tenth part straw, or leaving the straw out entirely, and placing upon the mass a continuous pressure, that would settle with the fodder (which will always settle in any silo to a

*A centimetre is about $4-10$ th inch. 
certain extent), thus entirely excluding the air, the preservation of the fodder was a com. plete success, and it could be this preserved for any length of time.

This continued pressure is a heavily weighted plank covering, which will follow the material as it settles, and thus maintain a uniform pressure. M. Goffart says: "I pile on the cover of my silo when it is filled, four or five hundred kilogrammes of stones or blocks of wood per square metre of surface.* By my first processes (covering without much weight) I obtained only a temporary and incomplete preservation; with my last, I obtained a preservation indefinite and absolute." Thus, after many failures, and partial successes, and years of study and experiment, was the problem at length solved, and it is now no longer a theory, but a demonstrated fact, that green crops can be preserved in this manner with their valuable nutritive elements unimpaired, which is the real object of the ensilage system.

Mr. Brown says respecting M. Goffart's achievement: "No doubt many farmers in this country have an idea, especially since the canning process has become so popular, that vegetation might be preserved in a similar way. Some have gone so far as to pack some green stuff in barrels by way of experiment; some have piled stalks in heaps and covered with dirt. When the heat and moisture of the season have favored vegetation and produced larger crops than could be consumed before frost destroys it, many a farmer has doubtless revolved the problem in his mind.

Apple pomace, turnips, cabbage, and different kinds of leaves seasoned with celery, grape leaves, leaves of beets, beets themselves, pulp of beets, beets from sugar-factories, some of these have been preserved in pits from time immemorial in Europe for feeding cows and goats. Necessity has so long compelled the efforts of human beings, that we may find precedents in almost every line of improvement. But all experienced men who know the great difference that separates a happy suggestion, or even a successful attempt, from a practice well enough confirmed to become the base of a regular business, will admit that these proceedings do not destroy the merit of any man who, like Monsieur Auguste Goffart, has accomplished a continued success. In 1876 the French Government rewarded him with the prize so dear to every Frenchman, the Decoration of the Legion of Honor."

How to Build a Silo.-Various methods of preserving different crops in a green state in such a manner that their nutritive value will remain unimpaired have been resorted to, some of which have proved exceedingly satisfactory, while others have resulted either wholly or partially in failure. Although the system of ensilage is still in a comparatively experimental stage, and the experience and experiments of the future will suggest and develop improvements upon the present standard attained, enough has already been demon. strated by this system to show that its success is no longer a subject of doubt or conjecture, but an established fact. The methods thus far that have resulted most satisfactorily are by the use of the silo and trenches, the former being most generally preferred. In giving directions respecting the manner of building a silo, it may be of special interest to the farmer to receive this instruction directly from some of the pioneers in the ensilage system, who have thoroughly tested silos of different construction, and are therefore prepared to speak from experience. We have, therefore, adopted this method, in the main, in connection with the subject, as well as in giving the experiments which have led to successful results.

Mr. Potter's Method of Constructing Brick Silos.-Mr. O. B. Potter, of New York, the first person to try the ensilage system on a large scale in this country, furnishes us for this work the following description of his manner of constructing silos:-

"The process of preserving fodder in pits is exceedingly simple and easily practiced. The conditions of success are these:-

First.-The preserving-pits must be wholly air-tight, so that when sealed, the air cannot come in contact with the food preserved.

\footnotetext{
*A metre is about three feet and $3 \frac{1}{8}$ inches.
} 
Second.-The pits should be of such form and dimensions as will best facilitate the settling and compacting of the food into a solid mass, and when opened for feeding will expose as small a part of the surface to the atmosphere as practicable.

Third.-The fodder must be cut green when in the best condition or in bloom, passed immediately through the cutting-machine to reduce it to uniform short lengths of not more than one inch, and at once be deposited and trod firmly into the pit, sufficient salt being used to render it palatable, but no more. As fermentation, which will commence at once, proceeds and the mass settles, the cutting and treading in of fresh fodder must be continued at intervals of thirty-six to forty-eight hours (depending upon the rapidity with which fermentation and settling proceeds) until settling has ceased, and no more can be trod into the pit.

Fourth.-The pit, as soon as completely filled and settling has ceased, must be securely sealed to exclude the air wholly and arrest fermentation, and must be kept so sealed until opened for use. My own practice is as follows:-

I make my pits of hard brick, with twelve-inch perpendicular walls, well laid in cement with smooth joints. If the ground is sandy or gravelly, the outside of the wall next the earth is covered with a coat of cement, or the walls are filled in behind with clay or clayey earth to prevent the passage of the air through them. The bottoms are also laid with brick upon the flat in cement. The pits are made from eight to ten feet wide, from six. teen to twenty feet long, and about fifteen feet deep. The deeper the pits, the more they will contain in proportion to measurement, owing to greater density of the contents from the weight of the mass above. In all cases where practicable, pits should be made at least twenty feet deep. The walls are made so smooth upon their inner sides as to offer no obstacle to the settling or compacting of the food by friction of the sides. These pits are made either open at the top and covered with a roof, or arched over, and covered underground, with two necks to each coming up to within one foot of the surface of the ground, through which they are filled, and the necks then sealed with earth. This last construction I have found most convenient in connection with basement stables, to which the food is carried or wheeled by a passage from the pits through the foundation walls of the stable. In this construction I make one pit parallel with this foundation wall, and from the side of this pit most distant from the stable, other rows of pits are made at right angles with and connecting with this, and with each other by doorways.

It will be seen from this construction, that as many tiers of pits may be made, end to end, at right angles to the first or entrance pit, as may be required and space allow, and that after the contents of this first or entrance pit are fed out, each of the other rows of pits may be opened and fed out, one pit at a time, and that only the surface of the food at the end of the one pit which is being fed will at any time be exposed to the air until the whole are fed out-and this without opening or disturbing the necks of the pits above, which remain sealed. Any other form of construction of pits which answers the conditions may be used. Pits or wells open only at the top, either round, elliptical, or rectangular, may be used, the food being put in and taken out through the top only. Such pits would have one adrantage, that successive croppings might be put in the same pit, one above the other, each being sealed with a layer of earth when put in. Where sufficient depth cannot be got above water, pits may be made partly above and partly below the surface, the earth excavated being used to make a broad and firm embankment around them to their tops.

It is important that the pits be so constructed and located, that the fodder as drawn from the field may be deposited conveniently at or over the top of the pits, and the cutting-machine may be so placed that the fodder when cut will fall as readily as possible into the pits. The fodder when green, being very heavy, it is quite important to avoid handling it unnecessarily."

Mr. Potter objects to silos built above ground, having tested those built partly above and partly below ground, and those constructed entirely below ground thoroughly, and gives the 
latter a decided preference. He states that that portion of the ensilage contained in the part of the silo above ground was found spoiled on several occasions, while he has experienced no such difficulty with the silos constructed entirely under ground.

Mr. Stewart's Method of Constructing Concrete Silos.-Mr. E. W. Stewart, of Lake View, N. Y., gives his method of builing a concrete silo as follows:-

"For a silo twelve feet by twenty feet (or longer) and fourteen feet deep,-which would hold seventy-two tons, or sufficient for ten cows six months with full rations, - the concrete wall should be fourteen inches thick at the bottom and ten inches thick at the top of the side walls, with the bevel on the outside of the wall, and the end walls twelve inches thick top and bottom, the inside being perpendicular and smooth, so that the plank covering may settle with the ensilage. The concrete wall is stronger than ordinary stone wall, and for this short silo fourteen inches at bottom is thick enough. It is not best to go any deeper in the earth than can be well drained, and a trench should be cut on the outside of the wall, six to ten inches deep all around, to carry off all water that may reach this depth. If the land around the silo is nearly level, it is best to go only so deep that the bottom of the wall will be below the frost.

Having excavated the earth as deep as the wall is to go, fifteen feet wide and twentythree feet long, then set the standards for the boxes to form the concrete walls in. It will require twenty standards three by six inches, fifteen feet long (if the walls are to be fourteen feet high), of straight-grained timber. Those standards intended for the inside of the wall should be joined straight on one edge, so that the wall may be made very straight and plumb on the inside. There will be three standards upon each long side-one at each corner and one in the middle.

The outer edges of these inside standards will be 11 feet 9 inches apart, and as the boxing plank are $I_{\frac{1}{2}}$ inches thick, this will bring the walls just 12 feet apart. The outside standards will be opposite the inside ones, and just three inches farther apart than the wall is thick, so that when the planks are placed inside, it forms a box 14 inches wide at the bottom, and the bevel, or slant, on the outside of the wall is made by bringing the outside standard 4 inches nearer the inside standard at the top.

The end standards will be parallel with each other and 15 inches apart. These standards are held together by nailing a lath under the bottom end, and a bracket across the top end, holding the side standards 17 inches apart at the bottom, and 13 inches at the top. Then when the standards are set up, and the inside standard plumbed very carefully, and both stay-lathed to hold them firmly in position, and the standards placed all around the proposed silo, it is all ready for fitting in the boxing-planks.

These boxing-planks should be straight-grained hemlock or pine, 14 inches wide, $1 \frac{1}{2}$ inches thick, and may be the whole length of each side and end, or, if more convenient, the sides may be two planks long, and the outside end plank will require to be $14 \frac{1}{2}$ feet long, but they may run by the ends of the side planks.

The outside of the ends must be plumb, so that the outside plank of the long sides can be raised, but for the end walls, being shorter, 12 inches thick is enough for strength, and has the same material per foot of surface. When these boxing-planks are placed, there will be a continuous box, 14 inches on the sides and 12 inches on the ends, around the silo.

Water-lime concrete is the only concrete suitable for silos, as it requires a strong; airtight, smooth wall, and one that can stand moisture to some extent. This kind of wall is easily made air-tight, and is built cheaper than an ordinary stone-wall. It is only necessary to use water-lime or cement enough to completely coat the particles of sand, so as to cement them together, and this becomes a cement to fill in spaces among large gravel or between stones. The cement is made by mixing one part of water-lime with four of fine sand, while dry, so that the lime and sand can be evenly mixed. Then work it into mortar, and if you 
have coarse gravel and no stone, you may put in five or six parts of gravel, and this will be sufficient to cement all together. The gravel is best mixed in the mortar-bed, but it must be used at once, as such mortar sets in a few minutes after wetting. But if you have rough stones of any kind, cobble or flat stones, they can be worked into the wall to good advantage, and save coment. When stones are to be worked in, put one or two inches of thin mortar in the wall box, then bed into this mortar a layer of stone, leeping the stone back a half inch from the boxing.plank, so that the cement may be tamped all around the stone, leaving a smooth surface on both sides of the wall. This cement is a poorer conductor of heat, cold, and moisture than stone. A properly built concrete wall never shows frost on the inside. In many parts of the country, thin, flat, irregular stones ase found in abundance, and these are well adapted to concrete walls, it requiring only a thin layer of concrete mortar between them, and the wall becomes solid in a few days. But with these flat stones, it is better not to bring them quite to the boxing-plank, but to let the concrete come over the edges so as to form a smooth surface.

When this concrete wall is laid with stone, sand, and lime, as stated, so large a proportion of stone may be worked in, that the water-lime will be only one-tenth of the wall, and the same when the wall is made of sand and coarse gravel; so that, to find the amount of waterlime required, count one barrel to 40 cubic feet of wall to be built. If water-lime is very expensive, and you have flat stones, no matter how irregular, you may use quicklime after you get one foot higher than the earth will come against it. One of quicklime to five of sand will make an excellent mortar to lay these stones in, doing the work in all respects as above stated. The concrete should be well tamped into the boxes, filling all crevices between the stones, and solid against the planks. Water-lime will set hard cnough so that these box. ing-planks can be raised 12 inches every day. That is, if you fill the box all around the silo in one day, the next morning you may raise the boxing-planks where you began the day before; and as you fill, raise section after section of planks till you get around again. This you may repeat each day till the wall is completed, provided the mortar sets in the usual time. But if quicklime is used, this sets slower, and will take two or three days to become strong enough to raise the plank. It will be noted that the planks are 14 inches wide, but are raised only 12 inches, which leaves a lap of 2 inches on the wall below, keeping the sides of the wall smooth and even. The proposed silo wall will have $952 \mathrm{cubic}$ feet in it, and requires 22 barrels of water-lime, of the Akron or Rosendale brand. This lime in many places will cost from $\$ 1$ to $\$ 1.25$ per barrel, or $\$ 22$ to $\$ 27.50$. The only other cost of the wall is the labor, which can be done by common laborers. The standards can be set by any one who can use a level and plumb. When the walls are completed, take a seasoned board as wide as the wall is thick, tar one side and turn the tarred side down upon the wall. This will prevent the moisture from rotting the plate rim placed on top of the wall.

The roof placed over this silo must be elevated some 3 feet above the plates, so as to give head-room for filling the silo full. This may be done by framing short posts into the timber on top of the wall, and placing light plates on these, upon which the roof is to stand. It will be seen that this silo can be built, by many farmers, with only a small expenditure for water-lime, shingles, and nails, all the rest of the materials being from their own farms. The bottom of the silo is cemented to prevent moisture from rising from below.

I bel teve the silo is to be generally used in the future for storing green food for winter feeding."

Dr. Bailey's Silo.-Dr. J. M. Bailey of Billerica, Mass, says: "Having resolved to try the experiment thoroughly, I broke ground on the 17th of July, selecting a side-hill, and locating the silo so that the corner joined the northeast corner of my barn. I excavated on the west side and south end, seven feet deep, and put in a solid stone-wall on the west side, 44 feet long and 12 feet high. This was built of very heavy stone, and in the most substan. 
tial manner. I afterwards graded up on this side to the top of the wall, making a level spot to set an engine and ensilage-cutter upon; also to drive upon to deposit the corn-fodder as it came from the fields on dump-carts. It took 13 days' work of a stone-mason, $43 \frac{3}{4}$ days' work of laborers, and $28 \frac{1}{2}$ days' work for one horse, to excavate and build the stone wall and foundations for the silo.

On the tenth day of August I commenced building the silo walls. These are 15 inches thick, built of concrete in the following manner:

First, $3 \times 4$ joists are set up at each of the angles, and also at intervals of about eight feet on each side of the walls. These scantling are placed eight inches apart, spruce plank 12 inches wide and $1 \frac{1}{2}$ inches thick, are set up on the inside of the scantling, which leaves 15 inches between the planks as the thickness of the walls.

We are now ready to commence building the silo walls. The concrete is made by mix. ing one barrel of Newark, Rosendale, or Akron cement, with three barrels of plastering sand and four barrels of clean gravel. This is thoroughly mixed together while dry. It is then wet and thoroughly mixed again, making a very thin mortar.

About three inches in depth of this mixture is put in between the planks; then stone of all sizes and shapes are packed and bedded in this layer of concrete, after which another layer of concrete is poured in on top of this layer of stones, and the operation is repeated until the space between the planks all round each silo is filled; then the planks are raised about ten inches, and the space filled with concrete and stones as before, until the walls are at the desired height. The best way is to have a sufficient number of hands to just raise the wall the width of the plank each day. Time was pressing with me, however, and I sometimes raised the plank two and three times in one day, the concrete 'setting' so that I was able to do so safely. But I do not recommend this haste, as the walls will not be as smooth as they would be if the cement had all night to 'set' in before the planks were raised. A $4 \times 12$ sill was bedded on the wall in the last layer of concrete. This sill was made of $2 \times 12$ inch spruce plank nailed together. Upon these sills a building was placed with posts five feet high, the beams on the top of these posts being thoroughly braced to the posts, thus firmly tying the whole structure together.

In sections of the country where clean sand, gravel, or stone is not easily obtained, silo walls may be constructed of brick in the usual manner of brick buildings. To put up the concrete walls and bed the sills, together with grading the upper side, where the cutting of the fodder is done, took of the foreman $28 \frac{1}{2}$ days; work of laborers, 149 days, and 34 days' work of one horse. Butting up the frame to hold the plank, took two carpenters two days. It required 124 barrels of cement, costing $\$ 1.25$ per barrel at Lowell.

The teaming of the cement and lumber is included in the above account of horses and laborers. My silos (capacity about 800,000 pounds) cost me about $\$ 500$. In other words, silos will cost about a dollar and a quarter for each ton's capacity. Large ones will cost less, small ones more. Silos may be built of stone pointed with cement mortar, and plastered on the inside, or of brick, or concrete. Whichever material is the cheapest and most convenient in any locality is the best to use there. Brick will cost more than the concrete. Concrete walls cost about ten cents per cubic foot. As a general rule, silos should be built rectangular in form, the width being about one-third the length, and the height about two-fifths of the length, and, if possible, should be sunk about one-half below the surface of the ground.

If there is a side-hill near the stables, so that the surface of the earth will come nearly to the top of the walls at one end of the silos, it will be found very convenient in filling the silos, in weighing the ensilage, and in removing the weights as it is fed out.

These walls must be built sufficiently strong to withstand, when empty, the pressure of the earth inward, as well as the pressure outward, caused by the settling of the ensilage under 
the weights placed upon it. Where it is not convenient to get stone for weights, heavy logs of wood may be used, sawed in pieces about three feet in length, and placed on end all over the planks which cover the ensilage-three feet of wood being about equal in weight to one foot of stone. Bags or boxes of earth may be used as weights. M. Goffart recommends that the corners be rounded; I find that cutting them off answers the purpose as well, and is less expensive." I find upon opening the silo, that the ensilage is preserved as well, and settled as evenly in these corners as elsewhere; also, that the preservation is just as perfect close to the walls, as in the center, showing that a concrete wall is more impervious to air than a brick one."

Monsieur Goffart's Silos. - The description of Monsieur Goffart's silos, as given by himself, is translated as follows: - "With my new stables at Burton finished, I shall be able to house one hundred horned cattle. I have just finished three united silos, which form a part of the plan of my new stable. The form of the silo exercises a great influence on the results. It should avoid all angles, and should offer the least possible resistance to the packing down of the ensilage. The elliptic silo with vertical walls is the best form, both for use and durability. It is important to have them as large as possible compatible with the conditions of easy and economical use. The preservation of the ensilage in small silos is always less perfect than in large ones. No matter how much care is used, and how much weight is applied, I have always found the portion which is farthest from the walls to be the best preserved, and that close to the walls there is always some alteration, not serious, but which it is important to reduce as much as possible. Small receptacles offer proportionately much more surface for contact. A rectangular silo, for example, of one metre each way, containing one cubic metre, presents five square metres of contact surface, while one of ten cubic metres, with 1000 cubic metres of contents, presents only 500 square metres of contact surface, diminishing nine-tenths the evil indicated.

But I do not advise silos of such dimensions as this. At the commencement of my experiments I recommended small silos, in order that when opened they might be quickly consumed before they became a prey to the slow combustion which the contact with the air produced, with as small an entrance as possible for the air, of which the first effect was to raise the temperature, and then produce fermentation - first alcoholic, next acetic, and then putrid.

But the day that I discovered the new process of a movable, weighted covering, so that I was able to maintain in the mass a continuous density whereby the penetration of air became impossible, I abandoned the small silos. Since then I have made them as large as possible, and they are only limited by the economy of the different operations of ensilage. My new stables are a square of twenty-four metres on each side, divided into two compartments, each of which has a central passage between two rows of stalls. These passages are connected with the silos by a small railway, which makes it convenient to bring the feed before each animal. The maize and the other ensilaged fodder is carried in willow baskets, all of the same size, which are frequently weighed, in order to keep account of the weight of the rations given to each lot of cattle. My silos are elliptic in form, with perpendicular walls as smooth as possible inside, five metres wide and the same in height. Should I modify them in any way in future, it would be only to increase the height. My farm at Burtin presents exceptional difficulties for building silos. Everywhere the water is met at one metre below the surface, and as I want to sink my silos nearly two metres, because the part below the ground preserves in summer more moisture than that part above the ground, I am obliged to first dig a ditch lower than the excavation all around it, and then to cement the lower part, which causes a considerable expense.

I put concrete on the bottom, and upon this I build the vertical walls of the thickness of two bricks (45 centimetres) to the top of the ground. Above the ground I reduce the thickness to one brick and a half (about 34 centimetres). 
I coat the walls with Portland cement sufficient to insure their perfect impermeability. My triple silos have cost me 4176 francs, and their total capacity 812.45 cubic metres, about 5 francs 14 centimes per cubic metre. I intend next year to raise the walls of my silos another metre, so that their capacity will be about a thousand cubic metres. I postpone till that time my decision as to a special cover for them.

Most agriculturists are more favored in the profile of their soil; many of them have a hillside in the neighborhood of their barn, in which they can open silos that will always be dry, and in some places can dispense with masonry by having solid rock. Those who wish to imitate me will have less hesitation when they know that Burtin is a particularly bad place for building silos, and that they can obtain the same results with much less outlay.

In making use of such large silos as these, it is necessary, of course to have a cutting-machine with a six horse power engine at least, and an elevator to raise the cut fodder over the walls of the silos. I

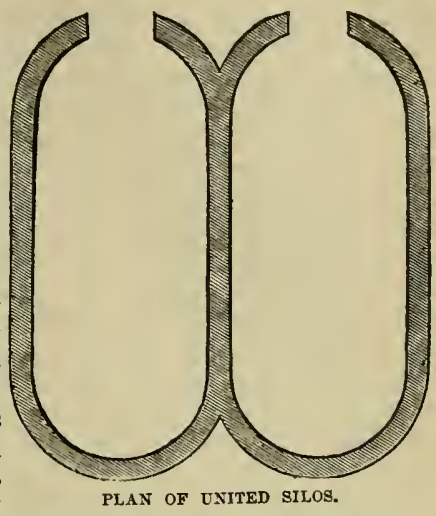
estimate that with these instruments one silo can be filled in three days, at most, without difficulty. This rapidity is necessary in order to assure the success of the ensilage. When the elevator and cutter are combined in the same machine, the process will be simplified."

Earth Silos. - The practice of depositing the ensilage in trenches dug in the ground, with a slight covering of some material that will keep the earth from coming in direct contact with the cut fodder, followed by a covering of earth, or earth and other weights combined, is preferred by some to a well-constructed silo. The arguments offered by the adherents of this system of preserving ensilage are that it obviates the expense and labor of building silos, while the preservation of the ensilage is as complete as that stored in silos.

At the recent ensilage congress assembled in New York city, in which several hundred farmers and others prominently interested in the subject of ensilage participated, Mr. Francis Morris of Maryland - one of the ensilage pioneers of this country - stated that ensilage was a very simple thing, but farmers generally seemed to think there was something mysterious about it, because the words "ensilage" and "silo" were new to them; but it was, in fact, a very simple thing. He dug his trenches in the ground and did not line them with brick or cement at all, but used, when filling, a covering of felt, with the earth above for a weight. Mr. C. W. Mills of New Jersey, also well known in his connection with the system of ensilage, spoke on the same occasion in favor of trenches, and claimed that all that was essential in lining such trenches was that there should be a slight wooden frame- not necessarily air. tight - which would prevent the spread of the ensilage crop when the weight was placed on the top, the pressure being about three hundred pounds to the square foot; and that the expensive silos of concrete, brick, and stone, the cost of which had frightened so many farm. ers, were unnecessary. Thus we see that the practice of those who have adopted the ensilage system, varies very materially in the manner of storing the fodder, as well as the expense attending it. We should suppose that one of the chief objections to the earth silo would be the difficulty of keeping the ensilage unmixed with water and earth, which are obviated in properly constructed silos.

Mr. Morris' System of Ensilage. - The following description of the earth silos used by Mr. Francis Morris is given by Mr. J. B. Brown, to whose courtesy we are also 
indebted for the illustration of M. Goffart's silos: - "The earth silo has been used by Mr. Francis Morris more than by anyone else in this country. His soil in Maryland consists of clay for a foot or two and a kind of rotten rock beneath. He uses oxen and a scraper, and makes a trench or pit $5 \frac{1}{2}$ feet deep, $7 \frac{1}{2}$ feet wide on the bottom, and 11 feet wide on the top, and any length desired. A width of 11 feet on top prevents danger of arching. At this slope the sides remain firm, and he does not plaster the face. The surface water is drained from it. In filling it, the sides are lined with straw standing, so that the ensilage will slip down well. The bottom is floored with plank, the top rounded up and covered with a thin layer of long straw, the thinner the better; above that a sheet of tarred roofing felt, and above that the earth is piled on two feet deep. The cut stalks are pounded in, and rolled with a heavy roller frequently at first. Vigilance is the price of safety with an earth silo. He has recently built more of them, all radiating from a center where the cutter stands, so that he can fill all without moving his machinery. He can cut ten tons per hour with a sixhorse engine. The stalks are hauled from the field in advance in the morning, in order to keep the machine going. He still uses two masonry silos in the stone barn, which were the first pits built for the purpose in this country; those, however, are also covered with dirt and compressed until they have ceased to settle. Mr. Morris thinks very much of ensilage as a forerunner of great wheat crops. He says: - "Clover, with its long roots, drawing sustenance from the sub-soil, when plowed under, and barnyard manure in abundance, will keep land strong for wheat and other exhaustive crops, such as cannot now be raised profitably in the Eastern States.

I made use of Indian corn as winter food for stock as long ago as the winter of 1876 , and $I$ have used it in each succeeding winter with great success. The earlier it is sown the better; its growth is more rapid and luxuriant in May and June than in July and Augusta bushel of corn to the acre, in drills twelve to fifteen inches apart. The crop should be worked twice, and, when in tassel, should be cut by a mowing machine, carried from the field in wagons to the feed-cutter, cut up in pieces of about three-quarters of an inch, and put into the silo prepared for it. The trench should have a shed over it, or a shed thatched with straw. Water should be kept from the cut-up maize, as it would doubtless injure the quality, if not destroy it.

Maize or Indian corn requires from forty-five to sixty days to ripen it into tassel, and therefore it can be safely sown up to the 15th of July. If the land is in good condition, it will yield twenty tons to the acre; it requires a ton a month for each cow, and all animals will improve and do well upon it. By the use of superphosphates the crop can be doubled, but this is a matter subject to the will of the farmer. Ten acres of maize will feed thirty cows during the season that they cannot feed out of doors, and will furnish a quantity of manure to give a wheat or corn crop. The advantage of this crop is so great that it must change the agriculture of every corn-growing country.

Cattle and sheep will be raised on every farm to an extent heretofore not thought of. Wheat, to-day, by all our best farmers, is followed by clover, the clover is cut and made into hay, and this is fed to the stock. Maize will take the place of clover-hay, and the clover will be grazed off the land, and the animals will return it to the land better prepared to act as a manure than if the clover was cut, made into hay, carried to the barn, and then fed to the stock. The advantage of grazing clover off the land is very great, as it at once returns to it all that the clover takes from the sod.

We recommend every farmer who reads these suggestions to sow an acre of land with corn or maize-if you have no drill, sow it broadcast, and when in tassel, use any old mowing machine you may have to cut it down, and then if you have no feed-cutter, buy or borrow one and cut up the fodder, as ordered-bury it in the ground, and when winter comes feed your stock upon it, and when you try it once you will never be without it again. I have 
used it for four seasons, every time with complete success, and I know that it multiplied the value of our land three or four times over."

Mr. Mills' System of Ensilage. - At the first meeting of the Ensilage Congress in January, 1882, Mr. C. W. Mills stated as follows: "In 1876 I tried an experiment, but failed from lack of knowledge. I tried to mix the southern corn with our species of corn indigenous to the State of New Jersey, but I made a mistake. When one kind was ripe enough to cut, the other had only just begun to tassel. Some of the Southern corn grew higher than this room. My neighbors would say, 'Is that the two-year-old corn?' 'Is it going to come out next year?' 'There is another city farmer come down to show us how to do things.' I got pretty well nettled, and I determined to put it out of sight, any way. I came to the conclusion that if I could exclude the air, I could keep it. To produce fermentation, three things are required, air, warmth, and moisture. Take away any one of these and there is no fermentation, consequently no decomposition.

I placed it in pits whole, and covered it over with boards and straw. I then covered the top with dirt. It settled until the top was a little below the surface of the ground. My neighbors would say, 'What are you doing?' I would reply, 'I am making manure.'

When I opened one of the pits and commenced to take it out it seemed to be packed and glued together. The color was richer than any samples I have seen here, and I do not think I have ever seen a sample of ensilage to compare with it. To get it out, I had to rig up a derrick, put on a double purchase, and pull it out with a team of powerful horses by the use of grappling irons. The cattle would smell the stuff from quite a distance, and were wild for it. It was eaten up very clean. Then I found I had a good thing, and I doubled my stock to eat up this ensilage.

Now about the matter of preservation. If you do not exclude the air, but keep it dowu to a low temperature, it will keep quite well. Though incipient fermentation may have set in, it does not seriously injure it, but I prefer the exclusion of the air, as far as possible. I think I can keep the air out by pressure. Let us have the matter as simple as possible, whether we exclude the air by pressure, or by hermetically sealing, which is more particularly Mr. Potter's plan. I maintain that pressure excludes the air the most effectually.

I have two large masonry silos each 40 feet in length, 20 feet deep, and $12 \frac{1}{2}$ feet wide. There is a wooden extension above each silo of 15 feet, making the whole depth 35 feet. I shall never build another such silo. If I should wish to increase my capacity, I should build on the surface, notwithstanding I have fine facilities for putting in silos. I. am satisfied that the less contact there is with the ensilage, the better it will keep. Last winter both of my silos were full. My west wall was exposed for about eight feet deep. I had taken out part of the other end, and left it standing with nothing intervening; I had a pressure of about 300 pounds to the square foot. The part left standing with nothing intervening between it and the weather, kept perfectly, say for two or three weeks, in the coldest and bitterest weather we had last winter, while the ensilage on the other end was spoiled for some three or four feet, next the wall.

If I should build another receptacle for my fodder I should build it of wood, entirely ahove ground. My system is unique and different from that of M. Goffart. I depend entirely upon a sufficient, uniform, and continuous pressure with no let up from the time it is put on until the fodder is taken out for use.

The principle of ensilage is nothing more nor less than the preservation of green fodder from the action of the oxygen of the atmosphere. I cut it up no shorter than is necessary to have it pack well in the silo; I allow no tramping. If you bruise the succulent stalk, the juice will exude, and air will take its place in the cellular tissue, the very thing to he avoided.

I would not use rye for the silo at all, on account of the ergot. I prefer oats to corn, but give me perennial grasses above them all. When I get my farm rich enough, I propose 
to raise nothing but the perennial grasses. I cut my corn after tasselling, when it is full of saccharine matter. I use the Southern horse-tooth corn, getting my seed from one section, from one particular State. I plant about eight bushels to the acre, and I plant by hand."

It will be seen by extracts previously given that, while the methods of preserving ensilage differ somewhat with adherents of the system, they all agree with respect to the value of the fodder thus preserved.

The Most Profitable Crops to Ensilage. - As has been previously stated, all kinds of forage crops may be preserved in accordance with the system of ensilage; still some

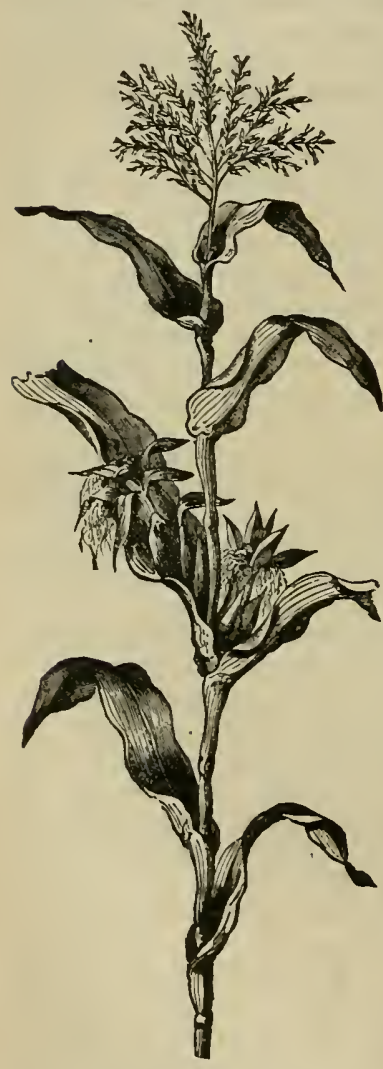

SOUTHERN SEED CORN. kinds are better suited and more profitable to be preserved in this manner than others. Mr. O. B. Potter states as the result of his experience in preserving corn, rye, grasses, clover, sorghum, etc., that in many respects red clover is most admirably adapted for ensilage, since it settles in the most compact mass, and thus meets the principal requisite of the process, which is to exclude the air and retain the moisture, and that he prefers sorghum to corn for this purpose. That sorghum will produce more milk and flesh to the acre than corn, but will also exhaust the soil more in its production than the latter, and also requires longer to mature.

Corn has been used thus far more extensively for ensilage than any other crop, while perhaps clover, and sorghum or millet would rank next in this respect.

The varieties of corn generally used for this purpose are those of the largest and most thrifty growth, such as the Southern Seed Corn, or some of the large varieties of sweet corn.

The Southern Seed Corn has an abundance of foliage, heavy stalks, and is very sweet and nutritious. It does not mature in the Northern States, but reaches full height and tassels in that climate.

The cow-pea is a very valuable food for ensilage, but cannot be raised as easily as fodder-corn in all parts of the country.

Broom-corn, sorghum, millet of various kinds, oats, rye, clover, the various kinds of grasses, etc., can also be preserved in this manner, but as has been previously mentioned, corn has thus far been given the preference generally.

Method of Cultirating Maize for Ensilage. -Maize or corn for ensilage may be sown either broad. cast or in drills, but the drill method is to be preferred, since it admits of after-culture, produces a heavier growth, and is less liable to be blown down by the wind. The soil should be rich and well pulverized. It is a fact well known to all farmers that the richest soil will produce but a partial crop where this necessary part of agriculture is neglected. The usual method is to plant in drills three or three and a half feet apart, the seed from four to six inches apart in the drills.

Different methods are preferred by different farmers, some of which differ very 
materially from others. Mr. J B. Brown claims that the best method of planting corn for easilage, whereby the largest crop can be secured with the least labor, is to make two drills four inches apart, with spaces of twenty-eight inches, cultivating while small with a light smoothing-harrow, and afterwards with the plow and cultivator; the grains in the drills to be from three to four inches apart, and the drills to be made North and South. By this means the sun and air strike both sides. The varieties of largest growth will, of course, require more space than the smaller kinds. The planting can be done with any kind of grain-drill or corn-planter. The cut below represents the Ensilage Corn-planter, manufactured by the New York Plow Co. It is a one-horse machine, which opens the drills, drops the seed and covers it.

After planting, the ground should be rolled to press the soil upon it, in order to insure quick germination. Care should be used not to drill the seed in too thick, as the growth will not then be as heavy. About three pecks per acre of the Southern Horse-tooth corn is suffici. ent, and gives more nutritive stalks than thicker sowing. As a general rule, the richer the soil, the less seed is required. The seed should be planted the last of May or first of June, according to the season. It should be kept free from

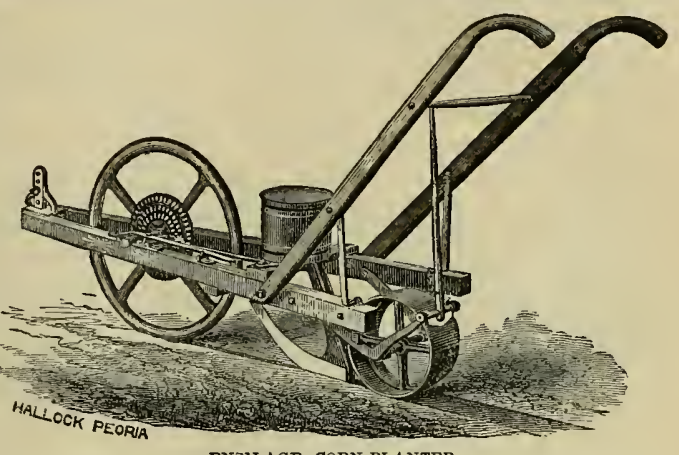
ENSIILAGE CORN-PLANTER. weeds and grass, and the surface frequently stirred to promote rapid growth; the afterculture being the same as that for the ordinary corn crop.

Time for Harvesting Maize for Ensilage.-The best time for cutting maize for ensilage is when the stalks are fully matured, being then full of saccharine matter and consequently containing the greatest amount of nutriment. This stage is indicated by full blossoming. If the harvesting is delayed beyond the proper point of maturity, the crop is deteriorated, since the plant does not remain juicy and tender more then ten days or two w eeks after blossoming. If cut later than this, it is also not as liable to be preserved as well in the silo. Whenever a change takes place in the leaves, like fading or turning yellow, it shows that the air has entered the cells and fermentation has begun, and when cut in this condition the ensilage will invariably become sour. This practice of leaving the maize stand. ing too long before cutting for the silo, will be the common error of beginners in the system of ensilage, and much loss will in consequence be the result. But by proper attention and care in what may seem at first to be minor matters and of little importance, there is no reason why the first experiments in this method of preserving green fodder may not be attended with the most satisfactory results.

The corn-cutter is the best machine for cutting the corn fodder in the field preparatory to ensilaging it. Some use an old mowing-machine, others corn-knives. Dr. Bailey recommends corn-knives for this purpose, on the ground that the labor can be performed cheaper than by a mowing-machine, since when thus harvested it can be laid in bunches, rendering it easy to load, the extra cost of cutting being thus more than made up by the facility with which the crop can be loaded, and taken to the silo for cutting. 
How to Cut the Maize for the Silo.-Various and repeated experiments have proven the fact that the finer the ensilage is cut, the better the preservation in the silo, other conditions being equal. Since it is essential to the perfect preservation of green fodder that all fermentation must be prevented, and this cannot be prevented without entirely excluding the air (as air furnishes the means of combustion or fermentation) this can best be accomplished by having the maize cut in very short pieces, as it requires less room and packs more densely in the silo, leaving less space for the air to occupy.

More time and labor will, of course, be required to cut the fodder four or five-tenths of an inch in length, than to cut it three or four inches, but its good preservation when thus cut fully compensates for all the labor required.

Many of the large ensilage cutters, when run by steam power, will make from twenty. five hundred to three thousand cuts per minute, thus cutting as fast as two men can spread it in the silo. The great secret of success in ensilage, as thus far indicated by experience, is in the exclusion of air, which, as previously specified, is secured by short cutting, and dense packing. M. Goffart says on this subject:-

"The fineness to which the maize is cut at the moment of ensilation, is extremely important in view of good preservation. Cut in disks of only one centimetre thick, the maize packs better in the silo, it occupies less space, and takes the form and consistency of a species of pulp, leaving in its mass the least possible amount of air. In proportion as the length is increased, the preservation becomes less perfect, and finishes by being entirely defective. Last year a cultivator of the valley of the Loire took from me the dimensions of my elliptic silo, and reproduced it exactly on his own farm. He filled it in the autumn, and when he opened it during the winter, he took out a poorly-preserved product, which his beasts only eat with repugnance. Greatly disappointed, he brought to me a sample of his maize that he had cut in lengths of five to six centimetres, instead of one or two at most, as I had advised him. I recognized at once the cause of his failure, and asked him why, contrary to my advice, he had cut it so long. He replied, 'I was not able to procure a steam engine which I expected to use, and I had to use a horse-power; the work did not get along fast enough, and in order to hasten it, I decided to cut it in such long pieces.' He was surprised at the excellent preservation of the maize at Burtin, and he carried home a hundred kilogrammes; his cattle were thus enabled to appreciate the difference. I cite this fact because it contains a valuable lesson.

At the beginning of my ensilages $I$ had as principal resource for the sustenance of my stock a great quantity of wheat, oat, and rye straw, etc. In order to induce my cattle to eat it, I mixed all that I could with my maize and my green cut rye, but I was not slow to notice that this mixture kept much less time, as the proportion of straw was greater. A fiftieth in volume, or a tenth in weight, was the maximuin of what the maize could carry without being exposed to an early alteration; when I increased this quantity, the time that it kept always diminished, and at last did not exceed forty-eight hours. I attribute this to the fact that the straw, being very dry, absorbs from the maize too much of its water. The moist condition of the ensilages, instead of being a cause of deterioration, is, on the contrary, to a certain extent, indispensable to the good preservation of the whole matter.

Maize in its normal condition contains about eighty-five per cent. of water; when the addition of dry straw has caused the mixture to decline to an average holding less than seventy-five per cent., the good preservation is much compromised, and quickly becomes impossible if we try to go below it. Besides the too great dehydratation that the presence of the straw may cause, it also offers another serious inconvenience, especially rye straw. This straw when cut forms a great quantity of little tubes, the envelopes of which resist decomposition for a long time; these tubes inclose an appreciable 
quantity of air which is the most dangerous enemy of ensilage. Oat straw, or others of softer texture, are less objectionable in this respect than rye straw. While I used at first the short straw from my threshing, always troublesome on account of the room that it occu. pied, henceforth I shall bury my fodder almost without any mixture. When maize has been cut before the frost, and arrives in good condition at the cutting machine and then at the silo, it will not yield water easily, even when it is submitted to a considerable pressure. But it is not the same when the fodder is too old, and has been exposed to the rains and frosts at the end of autumn."

Crops designed for ensilage should be cut and packed into the silo as soon as possible after gathering, as any wilting or drying by exposure to air or sun, even for a short time after being gathered, is very injurious, and liable to produce fermentation in the silo. The Cycle Ensilage Cutter, manufactured by the New York Plow Co., and of which we give an illus-

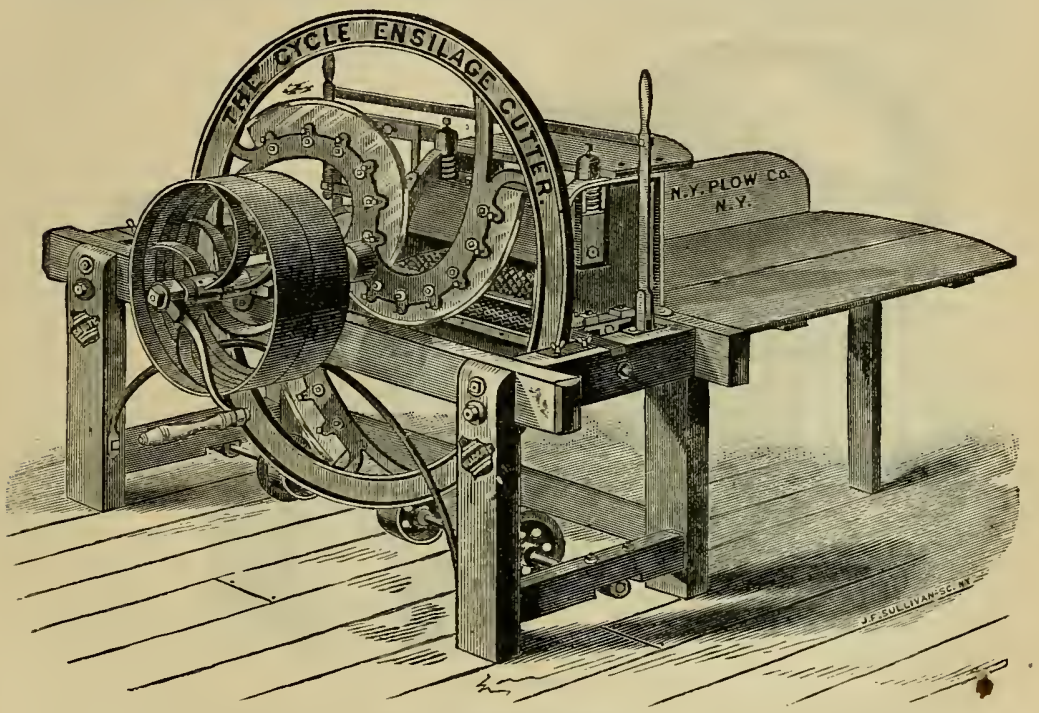

CYCLE ENSILAGE CETTER.

tration, can be run by either horse or steam power. It combines three knives with a long draw. ing cut, so leading each other that the cut is continuous and steady without jar. There is an aperture for dropping out stones before reaching the rollers, and another after passing the rollers, so that there is very little danger of any reaching the knives. It is well however to keep the stalks as free from stones as possible, as breaking a cutter while filling a silo is a serious accident.

The system of ensilage requires a large cutter to make it profitable and safe where the silos are of large capacity, in order that they may be filled sufficiently fast to prevent heating.

The cost of cutting fodder four-tenths of an inch, with a large machine and sufficient power, is estimated by reliable authority to be not more than twenty-five cents per ton. The following cut represents a somewhat smaller machine than the former, manufactured by the Whittemore Brothers, Boston, Mass. It cuts from one-eighth to two and a half inches long. The length of the knives is from fifteen to twenty inches. It cuts rapidly, and requires but a small amount of power. 
It will be seen by what has been previously stated that it is of the highest importance to cut the fodder for ensilage very fine. Some cut to the length of one inch, but a half or fourtenths of an inch is greatly to be preferred, and although it requires more time and labor, it is a safer practice, insuring a better preservation.

How to Fill a Silo. - The following directions for filling a silo are given by Monsiever Goffart: "It is necessary to procure, either by purchase or rental, a motive-power and a powerful feed-cutter. Large farms are generally provided with these machines, but the arerage farmer will have to hire. It may be that the traveling contractors for threshing will become contractors for cutting maize for ensilage, with a machine that possesses sufficient weight to be solid, and is also portable. Filling the silo should be done as rapidly as possible, and the layer of maize should be kept level all the time. The greater the compression, the better will be the preservation. The packing along the walls (which should be as smooth as possi. ble,) should be attended to carefully.

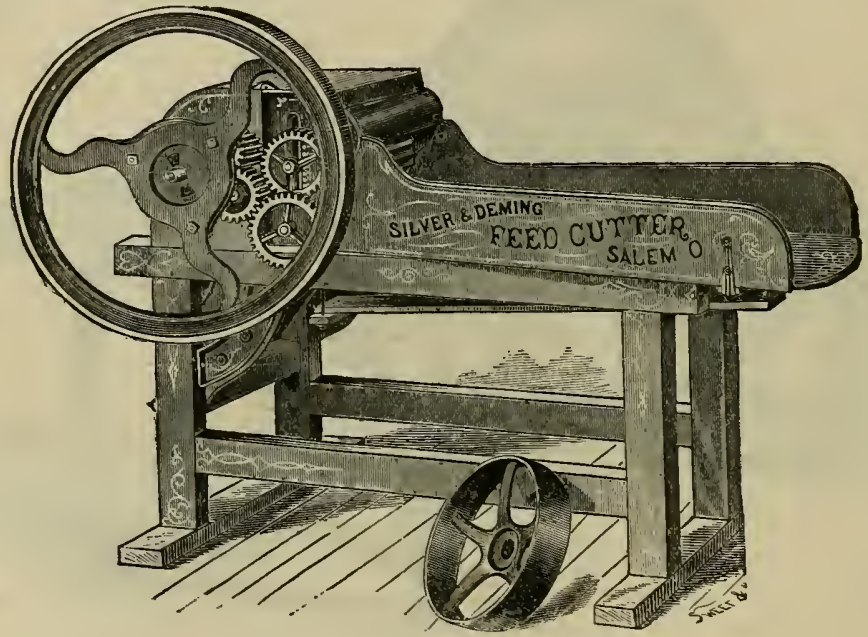

SILTER \& DEMING ENSILAGE CUTTER.

When the silo is filled to the top and carefully leveled, spread along the surface short straw four or five centimeters thick, then place on top of this boards fitting close together. These should be put across the silo in order that when it is being fed out they may be taken off one by one, as the silo is cut down vertically. Upon this flooring there should be piled abundance of weight, such as stones, bricks, logs of wood, or old bags filled with dirt, etc. At Burtin I have abandoned using loose earth as a means of compression, as it infiltrates into the ensilage, and adhering to the walls, a vacuum forms as the maize settles away, which is destructive.

Any ridge on the silos is objectionable, as the ensilage cannot be sufficiently compressed, and the dry rot soon attacks it and communicates to the material below. As to using salt in the silos, it is not very important, and I often omit it without any bad result; but I believe the moderate use of salt is favorable to the health of animals, and I sometimes mix in my ensilage one kilogramme to a cubic metre of maize, the average weight of which, after being packed, is 812 kilogrammes. 
When the ensilage is fed out, it should be exposed to the air fifteen or twenty hours, in order that the alcoholic fermentation may commence. The proper time depends upon the temperature, but if kept longer than this, the fermentation becomes excessive and injurious. The spontaneous heat which is produced in the feed should never exceed 35 or 40 degrees (R.). Two years ago I had no silos at my farm at Gouillon, and I carried every other day from Burtin what was necessary. From the second day the heat exceeded these limits, aud the alcoholic vapor abundantly emitted indicated the serious loss that was going on. The acetic acid was not slow to join the party. In the north of France the best pulp that is fed in winter is nearly always quite sour. It is to this circumstance that I attribute the poor quality of milk and butter obtained from the animals kept on this food.

It is indispensable to superimpose four or five hundred kilogrammes per square metre of heavy materials upon the covering or movable planks of the filled silos. I meet here the most important question-that which I have had the most trouble to solve, and which I have only really solved quite recently. When a silo has been filled, it does not answer only to prevent the external air from penetrating it ; is necessary at once to seek means for expelling the mass of air that it encloses between its disks and in its cells. It is here that the heavy materials with which I load my silos become important; it is necessary that the air inclosed in the silo should find between the joints of the covering planks an outlet; it is necessary that a strong compression should compel this air to pass out quickly and to quit the place where it would cause most serious damage if it remained. It is necessary that this powerful compression should continue during several months, because the tramping of the workmen is insufficient, for the follow. ing reasons: At the moment when the green maize is cut, it is all alive, and is so elastic that it reacts forcibly against the momentary pressure of the feet of the workmen. It is not the same several days or weeks there-

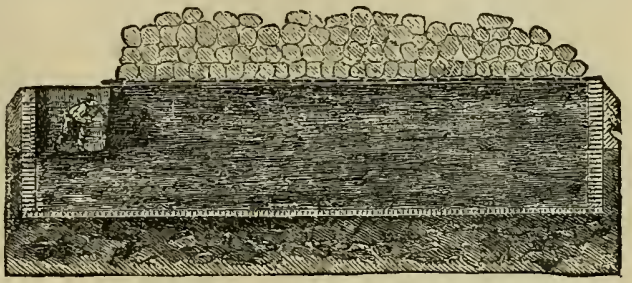

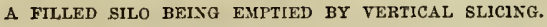
after, but its elasticity diminishes, or, in other words, its compressibility increases in considerable proportions; it is then that the heavy superimposed materials follow the maize down in its softened condition, continuing to press it in proportion as its compactness increases, and brings it to that state of density that is necessary in order to put it out of reach of all alteration."

Some farmers who have had experience in the ensilage system advise mixing clover, and grass in which clover predominates in the same silo with fodder corn, millet, or sorghum; the clover becoming, after slight fermentation, a putty-like mass which renders the whole amount more compact and weighty, excluding the air, while at the same time it improves the quality of fodder. Farther experiments will doubtless greatly improve this system, the greater number of experiments made thus far having been with fodder corn.

As has been previously stated, great care is necessary in order to exclude as much of the air as possible, and to distribute the fodder evenly in the silo. Some object to tramping down the mass in order to compress it, for the reason that any portion of the stalk subjected to pressure will cause the juice to exude, and when this pressure is removed the air immediately rushes into the cells of the plant taking its place, which results in fermentation. A little salt, sufficient to render the food palatable, is sometimes sprinkled into the silo during the, process of filling; this is not, however, necessary for preserving it.

After being filled sufficiently full, the plank covering should be put on across the silo so 
that a small portion can be uncovered at one time in opening, thus exposing to the atmosphere only what is taken out, which should be cut down vertically. A little space is usually left between thesp planks for the escape of air from the silo as the mass settles. Every facility should also be made for the covering to move so freely that it will meet with no obstacles on the sides of the silo, but follow the whole mass as it settles, thus excluding all air, by a uniform pressure. The weights for compressing the ensilage should be evenly distributed on the covering in order that the pressure may be equal in all parts of the silo. This will gradually settle until the average shrinkage in the silo will equal about one-third the original bulk.

Mr. Francis Moulton thus describes Mr. Mills' method of filling a silo: "The corn is harvested in the latter part of September, when the stalks are fully matured-not dead, but whilst still green in color-and filled with saccharine matter. This stage of growth is indicated by full tasseling and the beginning to ear. The harvesting is done in the ordinary old-fashioned way, by hand, with a stalk-knife, and a sufficient labor force is employed to fill a pit of 300 tons capacity within three days, because it is essential to gather and cut the corn and put it into the silo, and get it under uniform and continuous pressure within the shortest possible time, in order to save all the food properties. Mr. Mills uses two corn-cutters, one cutting one-half inch, and the other an inch length, of a coinbined capacity of 100 tons per day, using steam-power for the purpose.

He has two silos or pits, each 40 feet long, 13 feet wide, and 20 feet deep, located in the center of his barn, the walls of which are constructed of a concrete of stone and cement two feet thick, the sides and ends parallel, and the bottom well cemented. Upon the walls, flush with the inside of them, a structure of ordinary boards is built, fifteen feet high, which serves as a feeder to the pit, and which, when both are filled, will compensate for the shrinkage of the mass by compression. When the pit and feeder are filled, the surface is leveled, and sectional covers four feet in width, and in length one inch shorter than the width of the pit, are placed upon it, upon which are placed 50 tons of grain in bags (making five tons to each section) evenly distributed. Mr. Mills uses grain for weight because it is convenient. Anything else that can be uniformly distributed would answer. It takes about ten days for the whole mass to compress sufficiently for the covers to be on a level with the top of the pit, and then the feeder can be taken down. Mr. Mills allows no tramping or mussing of the mass, for the reason that he finds that any portion of the succulent stalks subjected to pressure will cause the juice to exude, and immediately upon relieving the pressure the air takes its place in the cellular tissue of the plant, and fermentation results in the body of the mass. The one inch of space between the cover and the sides of the pit is left for the escape of air and ambient moisture; the uniform and continuous pressure forces out the atmosphere and gases -which may have accumulated while preparing the mass for pressure-through the half-inch opening between the cover and the sides, and keeping them out until the pressure is removed.

When this fodder is to be used for the cattle, the bags of grain from the first section of the cover are removed and their contents ground for use, while the fodder lying immediately underneath them is being fed. The fodder is taken down perpendicularly to the bottom of the pit. Thus one section after another is fed until the whole is exhausted."

Removing Fodder from Silos.-When the fodder in the silo is needed for use, the weight and cover from the first section should be removed, exposing as little of the contents of the silo as possible to the action of the air. The fodder is then taken out perpendicularly to the bottom of the pit.

While one section is being used, the pressure on the remaining ones should continue to be the same, which practice will exclude the air as effectually, and thus fermentation be as completely obviated as though each section were in a separate pit. One section after another is thus fed out until the silo is empty. 
How to Feed Ensilage.-The quantity of ensilage fed to stock will, of course, vary with different individuals, the same as with other kinds of food; hence, in determining this question by comparing the results of experiments made by leading ensilagists of the country, an approximate estimate only can be obtained. Ensilage is generally fed in connection with bran, meal, shorts, or some other article of nitrogenous food. While some feed all the ensilage that the stock will eat, aside from other food given in connection with it, others practice the better method of allowing a certain amount per day to each animal. The amount of ensilage estimated as a full ration, is one and a half cubic feet, or about seventy pounds per day for an $0 x$, cow, or other large animal, or one cubic foot per week for a sheep; the amount of food required for a sheep being, as a general rule, about one-sixth of that required for a cow. Others use from twenty-five to a hundred pounds of ensilage per day, according to the quantity of other food used in connection with it. Mr. Mills formerly fed sixty pounds of ensilage per day with three quarts of grain, but has recently reduced the amount of ensilage to about one-half that quantity, with the same amount of grain. Dr. Bailey states that from his experience he considers ensilage to be worth one-half as much as the same weight of the best timothy hay; but that he would not, however, exchange ensilage for hay and give two tons of it for one of hay. He says:-

"My method of feeding is as follows: I remove from the silo 50 pounds of ensilage (about one cubic foot) for each grown animal daily, mixing one pound of oil-meal and wheat bran to every 10 pounds of ensilage. I have a large box standing upon the barn floor, in which I mix it and let it stand about twenty-four hours before feeding. By that time it is quite warm; the grain addition has had time to become soft, and its digestibility is undoubtedly increased to a greater degree. There is in every 50 pounds of ensilage about 40 pounds of water,-nearly all the animal requires. It is a great advantage to have this amount of water warm when taken into the stomach. There has been no labor or fuel expended in warming it, which is quite an item. When animals are allowed to drink ice-cold water in winter, there is quite a large percentage of the food which would produce fat consumed in raising the temperature of the water they drink, from freezing cold to blood heat."

In experimenting on the value of ensilage when fed alone, the same writer says:-

"On the 29th day of April I weighed two thoroughbred two-year-old Jersey bulls. Rossmore weighed 960 pounds; from then until the second day of June, I fed him 40 pounds of ensilage daily, and nothing else. The other, Hero, weighed at the same time 890 pounds; he was fed 40 pounds of ensilage and 3 pounds of wheat bran daily, and nothing more. On the second day of June I weighed them both again. Rossmore weighed 960 pounds, having neither gained nor lost. Hero weighed 943 pounds, having gained in the 34 days 53 pounds. Both times they were weighed in the morning after eating their breakfast, and before drinking.

I do not pretend that 50 to 60 pounds of ensilage is sufficient to keep a cow in full flow of milik. She should have in addition to the ensilage, four to six pounds of wheat bran or its equivalent in some other nitrogenous food. But I do say that the 50 or 60 pounds of ensilage will keep her better than all the timothy hay she will eat."

$\mathrm{Mr}$. Potter expresses as his opinion, that where clover is properly preserved by ensilage, a piece about six inches cube- - wing to its compactness-is sufficient for a good ration for a cow.

Mr. E. M. Washburn, of Massachusetts, feeds about 70 pounds of ensilage, 5 pounds of hay, and 3 of grain to each animal per day. It might be well for each farmer who adopts the system to experiment for himself as to the amount of ensilage necessary for a fair ration. By this means he can establish his own method, and adapt it to the requirements of his herd, and other conditions. 
Ensilage Adapted to the Southern States.-There seems to be no reason why the agricultural interests of the South may not be particularly benefited by this system, which has been proven to be as practicable in that climate as at the North. If any doubts arise with reference to this subject, it may be well to remember the fact, that the climate of that part of France where Monsieur Goffart has been so very successful in preserving ensilage in silos, is about the temperature of Virginia, Kentucky, Missouri, etc., and if cut at the proper time and ensilaged with care, there is no reason to doubt that this system will prove a success throughout the entire Southern section. By means of this new departure in Southern agri. culture, one of the great needs in the culture of the soil in nearly all the Southern Statesfarm manure-may be obtained at a low cost. This may be accomplished by providing an inexpensive food in abundance for stock, whereby a large number of farm animals can be kept, where now there are but few.

Stock-raising and profitable farming cannot well be separated in any country, or section of a country, where the soil will not produce spontaneously, and where a good supply of either commercial fertilizers or farm manures are required to produce a moderate crop. Corn. fodder, sorghum, millet, and other crops used in ensilaging can be grown to perfection in the Southern States. The varieties of corn used for fodder at the South are of heavy growth, with leaves long and broad, the stalks when in full bloom being peculiarly sweet and nutritious, and are also the kinds most used for this purpose at the North, while the cow-pea, growing as it does in the greatest luxuriance at the South, would be admirably adapted for the silo, if cut while in full bloom, with the pods just forming. Being fully equal in nutritive value to clover, an acre of rich land will produce about twice the amount that it would of clover.

If the silo is a desideratum at the North, where grasses of the best quality, and clover grow in abundance, how much more essential is it at the South, where the best grasses do not thrive well, or require frequent renewing. With ensilage as a new departure in Southern agriculture, the South may become a stock-producing section, with all the attendant beneficial results, and the resources of this part of the country, as well as those of the North, thus more perfectly developed.

Analysis of Corn when Cut in a Green State.-By summarizing various analyses made of the different parts of the corn-plants, as given in Mr. Brown's translation of M. Goffart's work on Ensilage, it is found that the ear with cob and stem, forms about onefifth of the whole plant, either green or dry. That the leaves contain over forty per cent. of the solid material of the whole plant, also three-fourths of the mineral element of the plant. That the stalk, leaves, and tassel contain nearly three times as much nutritive value as the ear, taken when the ear is in the milk. That the ear and cob contain but little more than two-thirds as much sugar as the leares, and less than one-sixth as much as the entire plant; also a little more than one-fourth as much as the stalk. That the leaves contain one-fourth more phosphoric acid than the ear, and that the latter contains but thirty-two per cent. of the entire amount of this element in the whole plant. That the ears contain only six per cent. of the entire sulphuric acid of the plant, and but eighteen per cent. of the chlorine. That the leaves contain more than half as much potash as the ears, the stalks nearly as much as the ears, and that the ear, cob, and stem contain but about forty-two per cent. of the potash in the entire plant. Iron was not found in the ears, while but a small amount of silica was present there. When corn is preserved by the ensilage process, all these nutritive elements are retained as food, and the waste that attends the drying, storing, and feeding of the dried product is entirely avoided. 
Analysis of Ensilage.-The following is an analysis of ensilaged corn fodder, by Prof. C. A. Goessmann, the sample analyzed being taken from the silo of Dr. J. W. Bailey, of Massachusetts:-

Moisture at $212^{\circ}-220^{\circ}$ Fahrenheit,

Dry matter left,

This dry matter consists of :-

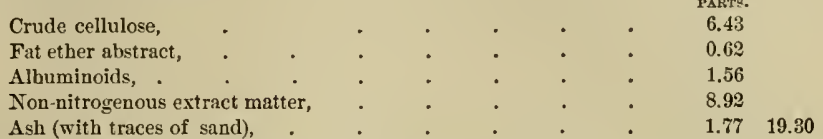

By way of comparison, we also give an average analysis of the corn-plant in the milk by the same authority:-

\begin{tabular}{|c|c|c|c|c|c|c|c|c|}
\hline Moisture at 212 & $2^{\circ}-220^{\circ}$ & Fahrenheit, & • & • & . & . & - & $\begin{array}{l}\text { PER CENT. } \\
85.04\end{array}$ \\
\hline Dry matter, & . & . & • & - & . & • & - & $\begin{array}{l}14.96100 .00 \\
\text { PARTT. }\end{array}$ \\
\hline Ash, & . & - & . & - & - & . & . & 0.82 \\
\hline Albuminoids, & . & . & . & • & . & . & . & 0.86 \\
\hline Fat, & . & . & . & . & . & . & . & 0.26 \\
\hline Crude cellulose & & . & . & . & . & . & . & 4.53 \\
\hline Non-nitrogenor & us extra & active matter, & . & . & . & . & . & $8.49 \quad 14.96$ \\
\hline
\end{tabular}

Adrantages of the Ensilage System.--In summing up the benefits to be derived from the ensilage system, some of which have been alluded to in previous pages, we will place first (since farmers generally are such an incessantly laborious class) economy in time and labor, although this, in a monetary point of view, may not appropriately take the first rank. When once a farmer has his silo well built, it will last for years with little or no expense in repairs, while the labor and expense attending the preservation of his forage crops by ensilage are but slight when compared with that of cutting, drying, and securely housing the hay crop, to say nothing of the anxiety in successfully accomplishing this result on account of unfavorable weather, or the loss often sustained by rains and cloudy weather.

Another important advantage of this system, is in preventing the loss of nutritive matter necessarily sustained by the drying process in converting the green forage into hay, all the succulent and nutritious juices of the food being preserved by the ensilage process.

It is a well-known fact that hay-fed cows always produce milk and butter of an inferior quality and light color, and that cows fed upon the same kind of grass before it has been converted into hay, produce milk and butter of excellent quality, the butter being of a golden tint and delicious flavor. The question naturally arises as to what causes this difference. If the only difference consists in drying out the water contained in the green grass by changing it into hay, or in other words, simply concentrating the nutritive juices of the grasses, there would be no loss of nutriment. Consequently rations of hay and water in quantity propor. tionate to that of the rations of grass before it was dried, would give the same results as grass in producing butter. The fact that dry hay and water when fed to cows do not produce the same results as are secured by feeding green grass, is but one of the many proofs that there is great loss of nutritive matter in the drying process.

Now if this same grass, or an equivalent fodder, can be preserved in a green state as nearly as possible to that which is grazed in summer, without the loss of nutriment, it follows that, aside from the labor and expense attending the drying process, there is not only an economy in preventing any loss of the nutritive element by drying, but better milk and butter are also furnished by the cows feeding upon the ensilaged fodder. Again, as foddercorn can be cultivated so much easier, and in such larger crops per acre than grass, the ensil- 
age of that food will prove more economical, since a much larger number of cattle can be kept on the same farm than before, while with the increased supply of manure thus obtained, the farmer can enrich his soil to the extent of making it much more available, and consequently profitable, than it has ever previously been. With less labor, and a consequent saving of time, there follows, as a natural consequence, more time for reading and self. improvement to the farmer, taking from his life much of the toil and drudgery that has so long characterized it, with a like benefit to the farmer's wife, whose lot is often the harder of the two, she working early and late to accomplish each day's duties, with the added burden of more hired help upon the farm to care for during the haying-season.

This new departure, then, means to the farmer less labor, less expense, better stock, an increased capacity on the farm for keeping stock, a larger supply of yard-manure, better crops, increased fertility and productiveness of the land, preserving the nutritive quality of the forage that has previously been wasted in the drying process, a better income from the farm. more home comforts, more time for reading, and the means of intellectual improvement. These are some of the advantages that experience and experiment in this new departure of agriculture seem to promise, and which we trust will be secured to the farmers of our country after the test of a few years, which will be necessary to secure its general adoption.

\section{Opinions and Experiments from Various Anthentic Sources Respecting} the Ensilage System.-Among the many favorable opinions current respecting the ensilage system, we have space to insert but a few, and these are from sources that would seem to be a sufficient guarantee for their reliability. It is often a fact that the advocates of a new and favorite system are too enthusiastic in their praise of the same, and place an exag. gerated estimate upon its merits. Although this may or may not be true with reference to some of the advocates of the ensilage system, we know that there are those among them, and by far the larger number, who are strictly practical men, and whose judgment and conclu. sions, as derived from actual experience, can be accepted without discount. In a letter to Mr. J. B. Brown, Monsieur Goffart writes that the cost of the maize he was then feeding was ten cents per 225 pounds, and the crop was 36 tons per acre. He says:-

"The longer experience I have, the more I am convinced that this method of feeding cattle is destined to render the greatest service to the agricultural interest. Frum October, 1878 , to October, 1879, I fed the one hundred animals in my stable exclusirely upon ensilaged maize during winter, and concurrently with fresh maize during the season when I had it. The animals have always enjoyed the most excellent health, and I can testify that they had more appetite for ensilaged maize than for fresh food, whatever kind it might be. The cows fed with fresh maize give milk of excellent quality, and the butter is of exquisite taste; fed upon ensilaged maize, the milk is still very good, and its quantity undiminished, but the butter, while being still of excellent quality, is not quite so fine. But it should be remembered that, whatever is the food of the cows in winter, butter is neter quite so good as that which is made during the fine weather (la belle saison)."

In regard to the cost of this fodder, he says:-

"Taking six per cent. of the weight of the animal for its daily ration, I arrive at an expense of 3.6 cents per day to feed an animal of 1,400 pounds. I do not know any kind of food that costs so little as my ensilaged maize, and it was a bad year for raising it, owing to the wet, and labor was unusually expensive, on account of the high price of wine."

Mr. C. W. Mills claims that the system of ensilage will revolutionize the present system of farming. He says: "Last summer my pasturage run short, and being out of ensilage, I cut oats when in blossom from five acres and put that in the silo, and subjected it to pressure. It sustained my milch cows (about 80 in number) for a space of six weeks. I had no trouble except in contact with the stone wall. I kept last year one hundred and trenty head of horned cattle and twelve horses, from October 15th to May 15th upon the product of twelve 
acres, without any hay or straw. I fed three quarts of grain per day. I gave about sixty pounds of ensilage per day, and it was a great mistake; I am now feeding but thirty, and two or three quarts of grain per day.

I plauted my corn in hedges about thirty-two inches apart, and about six inches wide, planting forty or fifty kernels to the running foot, and I got a wonderful growth. My land is not rich. It has been abused for the last hundred years."

Mr. O. B. Potter's statement respecting ensilage is as follows: "I have been feeding out of silos for five years, and I never have any trouble. I commence and cut it down from the top with a hay-knife, and usually cut off enough at one cutting to sustain the animals one day ahead. The cattle eat it up very clean.

I had one result which may interest farmers. " Last spring I was milking fifty-six cows, and sending fifteen cans of milk to the city every day. They were being fed upon ensilage entirely, with two quarts of barley meal. About the middle of June I turned them out where the grass was from six to twelve inches high, with the best of water and shade. The result was that the yield decreased from fifteen cans to eleven, and they did not begin to gain until I put them back on ensilage again in the fall.

I think a great gain will be found in feeding different kinds of ensilage at the same time. My cattle do not do as well on corn alone as when it is varied, with clover or mixed grass."

Mr. Hood, of Dutchess County, New York, says: "My silos, of which I have four, are about sixteen feet square and twenty-one feet deep. We opened one on December 1st, and found it was perfectly green and sweet. We were then feeding hay, and six quarts of feed to each cow. We stopped the hay and commenced on ensilage, and fed four quarts of feed and twenty-five pounds of ensilage to each cow. The milk gained rapidly, and is still gaining. The top portion of the ensilage is slightly moulded, but so slightly that the cattle did not seem to object to it."

Colonel J. W. Wolcott gives the result of his experiments in the dairy as follows :-

"The result of my experience is that the heavier the weight, the better the ensilage will keep. As to the quality of the cream I can say that by feeding fifty pounds of ensilaged maize, and one quart of cotton-seed meal, the increase over the amount when feeding English hay, and six quarts of corn meal averaged twenty-five per cent. in milk, and ten per cent. in butter from the milk, which is a total gain in butter of thirty-seven and a balf per cent. The butter brings the highest market price in Boston markets."

Mr. E. M. Washburn, of Lenox, Mass., thus gives the result of his experience with ensilage: "Our crops the present season consisted of 192 tons of corn and 91 of millet, counting 40 cubic feet of compressed ensilage one ton. The whole cost of the corn in the silo, aside from manure, $\$ 1.33$ per ton. The corn was raised in drills $3 \frac{1}{2}$ feet apart, three stalks to the foot. Variety, Southern white. The whole cost per ton for millet in the silo, aside from fertihzer, was $\$ 1.03$. The corn was cut by hand in the field, and the millet with a reaper. Wages for men $\$ 1.50$, for team and man, $\$ 4.00$ per day.

In one silo was put 135 tons of corn, in the other 50 of corn and 90 of millet. The silo that was filled with corn has been all fed, and the preservation was found perfect throughout the entire mass.

Our herd, to which we have fed this ensilage, consists of 40 Holsteins, and have been fed an average of $\mathbf{7 0}$ pounds of ensilage, 5 pounds of hay and 3 of grain, to each animal per day. This has fed them 87 days, or 135 tons of ensilage, $8 \frac{1}{2}$ tons of hay, $5 \frac{1}{1}$ tons of grain has fed one animal 3,480 days or 116 months, at a cost for ensilage of $\$ 178.45$, for hay $\$ 127.50$, for grain $\$ 157.50$, total $\$ 463.45$, or $13 \frac{1}{3}$ cents per day for each animal. Upon this feed my stock have steadily gained in flesh, and the younger ones have made a satisfactory growth, the returns in milk have been greater than ever before in the winter. The 
quality of the milk has improved from that made while the cows were in pasture, showing au improvement of over ten degrees by the lactometer. The butter is fully equal in color, flavor, and in every way to that made from the same cows in September, while at pasture.

In feeding our ensilage last season, we found a decided superiority in the millet over the corn, though it was so mixed that we could make no accurate trial. Analysis shows that the feeding value of millet compares to that of corn as 30 to 17 , when both are cut at the most suitable state, and clover and rye vary but little in value from millet, so that should the millet cost to raise and ensilage twice as much as corn, it still would be about as profitable in the end. There is much said at present by scientists and others about the great loss in ensilage by fermentation, the sugar, glucose, and starch, almost wholly disappearing, leaving all the water and fiber and a small amount of alcohol, with some other nearly valueless constituent, making, in their estimation, a food of about the value of fine sawdust. Now, when I go into my neighbor's stables, and see the amount of dried corn fodder that, it requires to feed his cattle and see the amount that is wasted and refused by the stock, when I see their condi. tion and amount of milk they yield, and compare it all with our experience with ensilage, I think it would not take a very wise man to decide which is the better method.

Another great point made by many farmers, is the great cost to build silos. Now, my silos that will hold about 375 tons, have cost me less than $\$ 460$, and I certainly would like to see the barn that will hold the equivalent of this three hundred and seventy-five tons of ensilage, in hay or dried corn fodder, that was built for the same money. I have yet to learn of the first man that has built a silo and not proved it a success. With me ensilage has proved a success, in its relative first cost as compared with hay or dried corn fodder, in its comparative value in feeding, and in its results in the production of milk, butter, and beef."

Numerous other authorities might be cited in favor of this system if necessary, but enough has been given to prove the preserving of fodder by ensilage a success thus far in practice, and to render it safe to predict for it a general adoption by the farners of the country before many years shall have passed away. Although a long time may elapse before it will entirely supersede the curing of hay by the long-practiced method, yet the present indi. cations are that it is destined to do so eventually.

\section{GO0D SEED.}

$\mathrm{I}$ F there is any one thing that will cause the agricultural products of the soil to deteriorate more than another, it is in the use of inferior seed. The custom of some farmers, of selecting seed at random, and of using successively, year after year, seed grown upon the same soil, or that produced by chance, or with little or no cultivation, or perhaps seed that has lost its vitality from being kept over for several seasons, is, to say the least, not an economic one.

The poor quality of seed and light yield of crops may often be justly attributed to the care. lessness or indifference of farmers and gardeners with respect to that which they plant. With the best soil and the most skilled cultivation, poor seed cannot, in the nature of things, give good results; hence, unless the seed planted be of proper quality, the labor and fertilizers bestowed upon the crop are in a great measure lost.

The average yield of ordinary crops is much below what it ought to be, or would be, pro. viding more pains were taken in the selection and cultivation of the seed that produced them. Plants for seed raising should not only have the best possible culture, but they should 
be the most vigorous plants selected from the best variety for this purpose. Even then, only the most perfectly developed and matured seed of such plants should be preserved for planting.

The effect of cultivation and careful selection of seed may be seen by comparing any one of our agricultural products with the original plant in its wild state, such as the potato, for instance, which, when growing wild, has scarcely any tubers at all, they being very small and of an exceedingly strong, unpleasant flavor, its product being principally the balls growing at the top of the vines for the production of the seed. The long period of cultivation given this plant, the constant aim being to develop the size and quality of the tubers, has resulted in changing its appearance almost beyond recognition, when compared with its original progenitor. We shall find it thus with respect to all cultivated plants. Our many and choice varieties of fruit are the result of long and patient labor in improving upon the wild types, the flavor, size, color, period of ripening, adaptation to climate and soil, etc., all being taken into account in the process of development towards the ideal standard which the grower has in mind, and at which he is constantly aiming, the time required to reach the desired results sometimes requiring many years, or even a life-time.

Choice of Plants for Producing Seed.-In no department of agriculture is more skill and good judgment essential than in the raising or selection of seed. It is well known, as has been previously stated, that all variations of agricultural products have been produced by a long course of cultivation and reproduction from the seed, in many cases changing a seemingly worthless, bitter weed into a valuable, edible plant. In this cultured state, which has a tendency to develop the highest possibilities of the plant, it is in an artificial condition, as it were, and there is a constant tendency to revert or return to its original wild state ; therefore there is required, on the part of the cultivator, much care in the selection and grow. ing of the seed, in order to counteract this tendency.

In the production of seed, the choice of plants is of the highest importance. All plants of the most approved varieties will not produce the same quality of seed. Other conditions being equal, the general rule of nature, that "like produces like," is as true when applied to the vegetable as the animal kingdom; and the principles of reproduction that apply to the one, will also apply equally well to the other. No farmer would expect to obtain a pure-bred animal from a mongrel and inferior herd, or a healthy progeny from a weak and diseased animal of a pure breed. Such a result would of necessity be an anomaly in nature. It is equally true that seed of the best quality cannot be produced from inferior plants, whether they be of an inferior kind, or the weak and sickly plants of a choice variety. The process of forming or establishing a distinct breed of animals that will transmit fixed characteristics, is a slow and difficult one. However fine the individual animal may be, there is that tendency, which is everywhere seen in nature, to revert to the original type, and the bopes of the breeder will often be blighted by the occasional sports and reversions of this kind, that will prove serious obstacles in his way. Hence it is necessary to select the most perfect types of ani. mals, those having those qualities that are desirable for transmission, for perfecting or maintaining the distinctive characteristics of the breed. The same skill is essential in producing and im. proving varieties of plants, and since the larger portion of the profits of the general farmer are obtained from the quality and yield of his plant growths, the labor and expense involved in the raising or purchasing of the very best seed will be amply repaid in the results of the crop. In the first place, then, farmers should take pains to raise only the best varieties of crops. It costs no more labor, time, or money to produce a good variety of vegetable growth, than a poor one, unless it be at first in the purchase of choice and rare varieties of seed when making a change in this respect. A tree, grape vine, crop of potatoes, or grain, yielding an inferior product, will extract as much nutriment from the soil, and require as much care and attention from the grower, as those that yield the choicest products of their 
kind. There is, therefore, economy in always selecting the best varieties for cultivation, those yielding the best in quality, and the largest amount in quantity, since this course is the most profitable and satisfactory. Farmers should, therefore, take pains to keep themselves informed with respect to the most desirable and improved varieties, and not be left in the background with the cultivators of a past age. We do not mean by this that farmers should discard a good and reliable variety for a new and untried one, simply because some speculative seed-seller loudly trumpets its merits through their special agents, or the medium of exaggerated advertisements. Too many impositions have already been perpetrated upon the farmers of this country in palming off a worthless or inferior article at an exorbitant price; but when the merits of a really valuable product have been thoroughly tested, and proven to be superior in all respects to the old, it will then be found more profitable to discard the old and adopt the new. We do not intend by this to cast any reflections npon seed-men and gardeners in general. We believe the majority of those engaged in this business to be strictly reliable and honorable in all their business relations. But in this business, as well as all others under the sun, there are impostors, and they have, by their false dealings, shaken the faith of many previously unsuspecting victims, and created a prejudice against adopting the new and untried that is not easily overcome.

There are those in this business who know nothing whatever of producing or improving what they offer to the public, but buy up, at a low price, old and worthless or inferior stock that can be found in the market, and then offer it for sale at an exorbitant price, under the claim of rare and special merit. All such impostors should be classed with counterfeiters of all kinds, including the manufacturers of oleomargarine and other food adulterers, and swin. dlers generally, and be dealt with according to the penalties of the law. Buying only of well known and reliable parties, will be a safeguard against such frauds.

Planting a little seed for one or two seasons in a small plot by itself, sufficiently far from all others to prevent mixing, and giving it good cultivation, will be a safe way to test a new variety before planting a large crop, where a knowledge of the experience of reliable parties in planting it cannot be readily obtained.

Next in importance to obtaining the best variety for seed-production, is that of selecting the most healthy and vigorous plants for this purpose, and these should be grown apart from all others.

All plants will mix more or less readily with others, when planted with, or sufficiently near other varieties to permit of fertilization from the pollen of their blossoms. Maize or common corn, broom corn, sorghum, millet, will all readily mix with each other, as well as with different varieties of the same species. This mixing or hybridizing will, of course, greatly deteriorate the quality of a choice variety, and should be carefully avoided. The distance to which the pollen may be conveyed by the wind, or by bees or other insects, is almost incredible. It will often happen that in a field of corn, an occasional red, mottled, or blue ear will occur among those of a choice variety of the yellow or white type, the origin of which can be accounted for only in this way. When such cases occur, it will usually be found that in an adjoining field or farm, or at a distance even more remote, the variety that is represented by this mixture will be found to have been grown during the season. T'he dust of the pollen of blossoms, being exceedingly fine, can be carried quite a distance by the wind. This is seen in the culture of hops, where five or six hills of male plants, being distributed at equal distances over an acre in a hop field, and supplied with tall poles for climbing, by which the fertilizing process may be facilitated, will have a sufficient amount of pol. len conveyed over the blossoms of the field by the wind to fertilize the entire acre of plants. Bees are another fruitful cause of the mixing of varieties, their iufluence extending even beyond that of the wind, by carrying the pollen that adheres to their wings and bodies to dis. tant fields while going from one flower to another in pursuit of honey. We therefore see 
the necessity of growing all plants designed for seed-productions apart from all others, that it may be kept as pure as possible. It would be well for farmers living in the same neighborhood, or adjoining farms, to agree to plant the same varieties for this reason. When different varieties are grown on the same farm, such as pop corn, sweet corn, and the kind constituting the regular corn product, these should be planted as far apart as practiable.

Special Cultivation Essential. - Plants for producing seed should not only be planted apart from all others, but should receive special cultivation, in order to maintain their high standard and prevent deteriorating; also, to improve, if possible, upon the variety. The benefits of cultivation have already been referred to in regard to all agricultural products, in comparing the improved and long-cultivated plants with the wild types from which they originated. This difierence is equally striking in horticulture. No one but a botanist would recognize the small and single blossom of the wild rose, or the coarse flower of the wild dahlia from the tablelands of Mexico, with its single row of petals, as kindred to the beautiful and rare products of the skillful gardener's art; and yet this great difference is all due to cultivation, combined with careful selection. When a better class of plants are to be developed, as those possessing special characteristics in a more marked degree than has been attained, care must always be exercised in selecting those that possess the desired qualities most prominently, whether it be for special color, fragrance, beauty of form, size, or other qualities. It is only by this means that certain characteristics can be perpetuated and more fully established. In the cultivation of seed, the locality selected should not only be apart from all other plants with which it may become mixed, but the locality chosen should be that to which it is well adapted, whether with regard to the soil, degree of heat and cold, sunlight and shade, shelter from winds or other influences adverse to its proper development.

The soil should be well tilled, and of the degree of fertility adapted to the plant that is grown, while frequent and careful culture should be given of such a nature as is also suited to its most perfect growth and maturity. During the progress of the growth of the plants, all injurious insects should be kept from them, and the weak and sickly plants carefully thinned out, leaving only the most healthy and vigorous ones to mature and ripen their seed. All foreign growths, such as weeds and grass, should be kept down, and the surface of the soil frequently stirred to increase the growth and productions of the plants. Those plants that produce suckers, such as corn, for instance, should have these inferior growths removed as they make their appearance, for if allowed to blossom and shed their pollen, they will thus fertilize the fine ears of corn as readily as the blossoms of the most perfectly developed stalks. This will be sure to deteriorate the quality of the product; too much importance cannot therefore be placed upon their removal before blossoming. It often happens that when planting even the largest and finest looking ears, where this precaution has not been taken, the farmer will be greatly disappointed in the result, the crop produced being in every respect an inferior one. This is caused by the kernels of the fine, large ears having been fertilized by the blossoms of one of these inferior growths, and deterioration naturally follows. If every farmer would take such precautions, the average corn crop of the country would be greatly increased.

Harresting and Storing. - It is very essential that seed should be harvested before the appearance of the frost, and if possible, when sufficiently ripe, before a long rain storm. If allowed to remain exposed several days to a rain, some kinds of seed might be seriously injured by sprouting, while others might become shelled out and wasted. All seed should be well ripened before gathering. It should not, however, be allowed to remain in the field after becoming sufficiently ripe for harvesting. A slight neglect in this respect will, with some kinds of seed, often cause the loss of nearly the entire crop.

Some plants ripen their seed very unevenly. With such, the time for harvesting will be 
when the largest portion of the best seed is ripened. In harvesting, care will be necessary in handling certain varieties, to prevent shelling out and loss. After gathering, it should, as a general rule, be well dried before shelling; this is usually done in a cool, dry, airy room. In shelling, the most perfectly developed seed from the most vigorous and productive plants only should be saved for planting. Partially ripened or imperfect seed may germinate, but it will invariably produce inferior plants. All seed should be thoroughly dried before being stored; otherwise it will be liable to mould, and its quality be greatly impaired or rendered entirely worthless. In storing, a perfectly dry place should be selected. If allowed to gather dampuess while being stored, even though perfectly cured, its vitality will be ruined. Occasional dampness and drying are equally injurious. It should also never be exposed to extreme heat, or greatly varying degrees of heat and cold, but will best be preserved by being kept perfectly dry, and at a uniformly cool temperature.

Qnalities Desirable in Seed. - In order to attain the most satisfactory results, the seed which is to produce the crop should possess certain essential qualities. These are vitality, vigor, and productiveness. In order to possess vitality, the seed should be grown from vigorous, healthy plants, and properly matured before being harvested. It is equally essential that it be well dried and protected, in storing, from dampness and other adverse influences. It is also very essential that it be fresh. The length of time that seed will retain its vitality varies with different varieties, but, as a general rule, nost seed will deteriorate after the first year, some kinds, such as onion seed, and some of the varieties of grasses, being almost worthless if kept over to the second or third year. The seed of melons, cucumbers, wheat, corn, etc., will usually retain their vitality for several years; but although such old seed may be made to germinate and grow, fresh seed of any kind is always the best, and possesses the greatest amount of vitality and vigor. It is always well to test seed before planting, by planting a small quantity or otherwise germinating it, the length of time required to produce germination being a good test of its vitality and vigor.

Seed that will produce a vigorous growth is also highly essential. Some seed may have sufficient vitality to germinate, but not sufficient to produce vigorous, healthy plants. Productiveness in a plant is also as fully essential as vigor, and these qualities should always be combined in the plant that is to be instrumental in perpetuating its kind. There are some plants that in certain soils will grow rank and thrifty, but yield little else than stalks, or vines, and foliage. Seed from such growths will never give good results. It is only the vigorous, and at the same time the most productive plants which will produce a satisfactory crop. In using old seed, many will fail to germinate at all, but such as do germinate will produce plants having less vitality than those from the new, consequently are liable to be feeble and less productive, and their use will be attended with serious loss to the farmer. For all crops, without exception, whether for the farm or garden, we would recommend the planting of fresh seed of the purest and best quality.

\section{TIMBER CULTURE.}

$\mathrm{T}$ HE preservation and extension of our forests is a subject of vast importance to the interests of the country. The great value of timber for the various purposes to which its use is applied-for many of which no substitute could be found - and the yearly diminishing of the timber resources of our continent, are the cause of grave apprehensions among the leading minds of the present age, lest the needless and reckless waste that has so rapidly increased the scarcity of timber within the past few years, will cause the utter ruin 
and annihilation of our noble forests, unless some means are taken to check this waste and restore them by planting trees. The European governments long ago recognized the destruction of their forests as an evil, and took active measures to counteract it by planting and cultivating trees, having special officers appointed to supervise the work. The care of forests receive so much attention by most of the governments in Europe that schools have been established where young men are educated with special reference to this branch of pub. lic service.

In this country an increased interest is being taken in forestry, and we hope the time is not far distant when more active measures than have yet been adopted will be taken in regard to this subject. There are, in all sections of the country, tracts of waste or poor land that might be devoted to timber culture most successfully and profitably. If each farmer, even, would take measures to plant trees upon his own lands, that could most profitably be devoted to the purpose, and encourage others to do so by example, much could be done in this direction for future generations, as well as the present; for while some trees are of slow growth and require a long time to reach maturity, others grow very rapidly, and will in a few years attain a considerable size.

Various Influences Exerted by Forests.-The ruthless and wanton destruction of the magnificent forests which once characterized our land, is to be greatly deplored, not only on account of the reckless waste of valuable timber, and the barbarous practice that depletes the face of the country of one of its most beautiful and attractive features, but also on account of the deleterious effects of their removal upon the climate, vegetation, and healthfulness of the country. The climatic infinence exerted by trees, and the results that follow their removal are subjects of great interest; and while there does not seem to be a perfect unanimity of opinion among scientists in this respect, there is no doubt that not only the dis. tribution of the amount of rain-fall is greatly infiuenced by the removal of the forests, but also the climate and healthfulness of a country, as well as the soil, and consequently its agricultural interests.

We believe it was Humboldt who said that, in felling trees which cover the crowns and slopes of mountains, man in all climates is evidently bringing upon future generations two calamities at once-a want of fuel and a scarcity of water. He might also have added, that he who plants trees and protects them from destruction confers blessings upon future generations, and for his philanthropy is worthy their gratitude. A certain poet has said, with much truth, as well as considerable sarcasm:-

Give fools their gold, and knaves their power,

Let fortume's bubbles rise and fall;

Who sows a field or trains a flower,

Or plants a tree, is more than all."

For our own part, aside from all practical and utilitarian views of the subject, we never could see the ax of the woodman on its mission of destruction, cutting into the very heart and life of a noble tree, without a pang of pain penetrating our own soul, and an impulsive desire to implore-

$$
\text { "Oh, woodman, spare that tree!" }
$$

It may be a foolish superstition, but we have never been able to quite banish the impression, that almost took the form of a certainty and belief in our childhood days, that every noble tree possessed an indwelling spirit, and it was this that made them so companionable and sympathetic; that whenever the ax penetrated into its life, a shudder of pain ran through that noble trunk with each cruel blow, and when at last it fell, we could almost in imagination hear the groan that accompanied the severing of that spirit from its loved habitation.

Trees have a double mission-utility and ornament; and while we would not underrate the former, we would not ignore the latter; for whatever is beautiful in this world should be 
cherished and appreciated on account of its ennobling influence, and the happiness it imparts, and is consequently, in one sense, quite as important as that which is only useful. He who sees only with practical eyes, fails of much of the happiness in living, hence his life is incomplete and imperfect.

Influence of Forests on Rain-fall.- While it is doubtless true that the average amount of rain-fall in a country during a period of several years will not largely rary, whether the land be diversified with extensive forests or not, yet the uniformity of the distribution of the rain is largely influenced by this feature. Where a considerable portion of the land is covered with timber, the trees are instrumental in intercepting the clouds, and the rain falls frequently and in refreshing showers, thus favoring the growth of vegetation and purifying the air; but in regions where there is a scarcity of trees, the rain-storms are less frequent and more heavy, being often accompanied with violent winds. A large portion of the water that thus falls upon the earth runs into the rivers and seas without proving of much benefit to the land, and frequently doing considerable damage. Hence, in sections subject to such violeut storms, the soil becomes dry and parched in summer during the long and unequal intervals of rain-falls, while there is not that benefit received from them generally that is derived in forest regions.

In this manner forests are instrumental in preventing both floods and drouths, and are consequently of great benefit in an agricultural point of view. Even the rivers owe their origin to the streams that have their birth in the forest-covered mountains and hills. We know it to be a fact, that where the forests have been cut down, the brooks and rivulets that for years had been a marked feature of the region, either become nearly exhausted or disappear altogether.

Extensive regions entirely destitute of forests have but little or no rain, and often become deserts, with scarcely a vestige of vegetation. This is due not so much on account of the lack of fertilizing elements in the soil, as the absence of water. Many previously desert sections have by the means of artificial irrigation been transformed into some of the most fertile lands with luxuriant growth of vegetation of all kinds. In regions thus transformed, there is always this noticeable peculiarity, namely, that as soon as the growth of trees and vegetation is secured by artificial irrigation, then the rain becomes frequent and falls in gentle showers, enriching the soil and refreshing vegetation. Utah, previously referred to in connection with the subject of irrigation, furnishes a remarkable instance of this kind.

Influence of Forests on Climate, etc.-Forests are a protection against the force of bleak winds in winter, and therefore render the climate warmer in cold weather, and modify in a measure the sudden changes of temperature. They also render it more cool in summer by preventing the rapid evaporation from soil thus shaded, that would otherwise be caused by the hot rays of the sun, while their power for retaining moisture enables them to become the source of springs and small streams which are always found there. The more extensive our forests, the more uniform our temperature, and the more we are exempt from the extremes of both heat and cold. The modifying influences of forests will readily explain the reason why, in sections that were formerly protected by ihem, but which are now denuded of them, the climate is more severe, the springs later, and it is impossible to cultivate certain delicate plants and trees that before this period could be grown with ease. Thirty years ago or more peaches were a profitable crop in many of the Northern States where now they can scarcely be grown at all, owing to the tendency of the trees to winter-kill. Trees are also healthful in their influence, and act as purifiers of the atmosphere. We quote the following from Hon. George B. Emersou on this subject:-

"By planting your fields with trees, you not only make your homes more pleasant, but you make them healthier, and you make your own domain more valuable. Here is a fact 
which every individual ought to know. It is a most important fact in the relations of the vegetable world to mankind. Every tree is a purifier of the atmosphere. There are on the leaves of every tree literally millions of hittle openings, large enough for the particles of air to enter, and into which they do enter. When a breeze passes over a forest, or over a single tree, the particles of air enter these little openings in the leaves, and there the leaves part with their carbonic acid, which is so unwholesome to breathe; they part with their nitrogen, and with everything else, really, which is not perfectly wholesome, and they pour into the air pure oxygen. The forest thus completely purifies the air that blows through it; it takes from it everything that is poisonous or even injurious to man, and throws out to us pure oxygen, or that mixture of oxygen which is best for us to breathe.

There are regions in Italy which anciently were very wholesome and pleasant places.to live in, but which, for the last one or two hundred years, have been growing more and more unhealthy, until, a few years ago, they were considered pestiferous and unsafe for anybody to live in, and those who dwelt there took care to go away at certain seasons of the year. This was in a part of Italy nearest the sea, which is thence called Maremma. Within a few years trees have been planted in various places in that region, and the effect has been to restore the original purity of the atmosphere. Large forests have been planted in some places, and the region has in consequence become perfectly healthy, so that the ancient towns and villages which had been deserted are again repeopled. The same thing has been found in various other parts of Europe, and also in this country. In Washington, a gentleman who had paid some attention to this subject, said: 'Here is a region which the soldiery have occupied, and found very unhealthy. If you will give me leave, I will plant it with sunflowers.' He planted a great number, several rows, and the effect was immediate. The very next season that region, protected by the sunflowers, became healthy. The sunflowers have been continued, or something else put in their places-trees and plants of various kinds; and that region is now one of the most healthy in Washington. The lives of hundreds of our soldiers and others who are obliged to live in Washington, have undonbtedly been saved by that device of the sunflowers. So, I say, you may render every farm more healthy, as well as more pleasant, by planting trees."

Although trees planted near a house beautify it and render it more healthful, yet they should not be planted so near as to intercept the sun's rays, and thus shade it. A house from which the sunshine is excluded will soon become unhealthful. They should be planted neer, yet always sufficiently removed to prevent shading.

Varieties of Trees for Planting. - Great benefit may be derived to the timber resources of the country by not only planting trees, but in preventing the needless destruction of those that remain, especially young and immature trees. When necessary to cut timber, there should be selected for this purpose only the mature trees, leaving the young and vigorous ones for future growth.

Tree-planting has, however, become a necessity, as a means of restoring the forests, and the sooner and more thoroughly this is done, the better.

The questions naturally arise with regard to the varieties best adapted for the purpose, when, where, and how to plant them. With respect to the first, much will of course depend upon the locality and climate. As a general rule, the most valuable timber trees adapted to the climate should be planted. Most of the forest trees thrive best when they are mixed with others that differ widely from them in character.

A natural forest is always mixed, and Nature's rule in this respect is the safe one to follow. Different varieties probably extract different elements from the soil, the same as different kinds of agricultural plants, and this is doubtless the reason for it. That a tree may be useful for general planting, it is necessary that it should grow without culture over a large belt of country, thus possessing the power to thrive and adapt itself to various condi- 
tions of climate; also that it should grow rapidly, and that its timber may be used for either general or special purposes.

In making a selection, therefore, for forest-planting, the timber value, rapidity of growth, and adaptation to climate should be considered. Some timber is, of course, much more valuable than others, but nearly all kinds may be applied to some useful purpose.

There is no tree more beautiful than the sugar-maple, and it is a very valuable one as well. It is adapted to a wide extent of territory, will thrive in a variety of soils, and should be planted extensively in regions suited to its growth. The ash, beech, elm, and walnut are all valuable trees, as well as the European larch, the oak, locust, chestnut, cedar, and pine of their several varieties, many of which will thrive both North and South.

For the Western States, the hardy variety of the catalpa tree is very desirable. as it grows rapidly and furnishes a very durable wood. The yellow cottonwood is also valu. able for that section, and is said to make a saw-log or rail-cut sooner than almost any other tree, while the osage orange, white elm, black walnut, maple, locust, willow, silver and Lombardy poplars, are all suited to the soil and climate, besides several of those previously mentioned.

The Kentucky coffee-tree is also highly recommended for forest-planting. It grows quite as rapidly as many other choice varieties of trees, and its timber is also valuable. With regard to the locality to which it is adapted, Prof. C. S. Sargent, Director of the Arboretum of Harvard University, says:-

"Of its power of adapting itself to very different climatic conditions, there can be no question. Few trees range over a wider area of the United States. It grows from Canada and Western New York, to Wisconsin, and south to Kentucky and Tennessee. It extends across the Mlissouri into Nebraska; is common in Missouri and Arkansas, and not rare in Kansas and the Indian Territory. It is not often found in New England, where, however, it is perfectly hardy, nor in the Atlantic or Gulf States. The Kentucky Coffee Tree grows always in rich, strong soil, generally along river valleys, and reaches, probably, its largest size in that of the Lower Ohio. It is not a particularly fast-growing tree, nor does it increase more slowly than other North American trees, while it produces a wood equally strong and heavy."

At the South, the many varieties of pine, cedar, and oak flourish and make valuable timber; also the maple, ash, hickory, persimmon, linden, magnolia, cypress, bay, etc.

We have a great variety of beautiful and valuable timber trees, many of which are adapted to a wide range of climate and soil. Some increase in size very rapidly, while others are of a slower growth. Hon, G. B. Emerson states that in his opinion, we have the most beauti. ful trees in the world; that in visiting Europe for the purpose of studying the forests there, be was convinced that there were more valuable trees growing naturally in our forests, than can be found in any portion of Middle or Northern Europe.

Age and Size of Trees. - The age to which trees live, differs greatly with different species. While many are short-lived, and decay in a few years, others will maintain their vigor for thousands of years. The elm has been known to live three hundred and fifty years; the chestnut nearly twice as long; the cedar from seven to eight hundred years; the oak from a thousand to fifteen hundred, while some of the California giant trees have been estimated to be at least five thousand years old. The old Charter Oak, at Hartford, Conn., was estimated to be about nine hundred and fifty years old, and measured nine feet in diameter, at a distance of four feet from the ground.

Some of the gigantic trees of California, (the Siquia gigantea), a species of mammoth cedar, have attained the height of four hundred and fifty feet, with a circumference of one hundred and twenty feet or more near the ground. The largest of these trees now standing is said to be three hundred seventy-six feet in height, and about one hundred and six feet in circumference. 


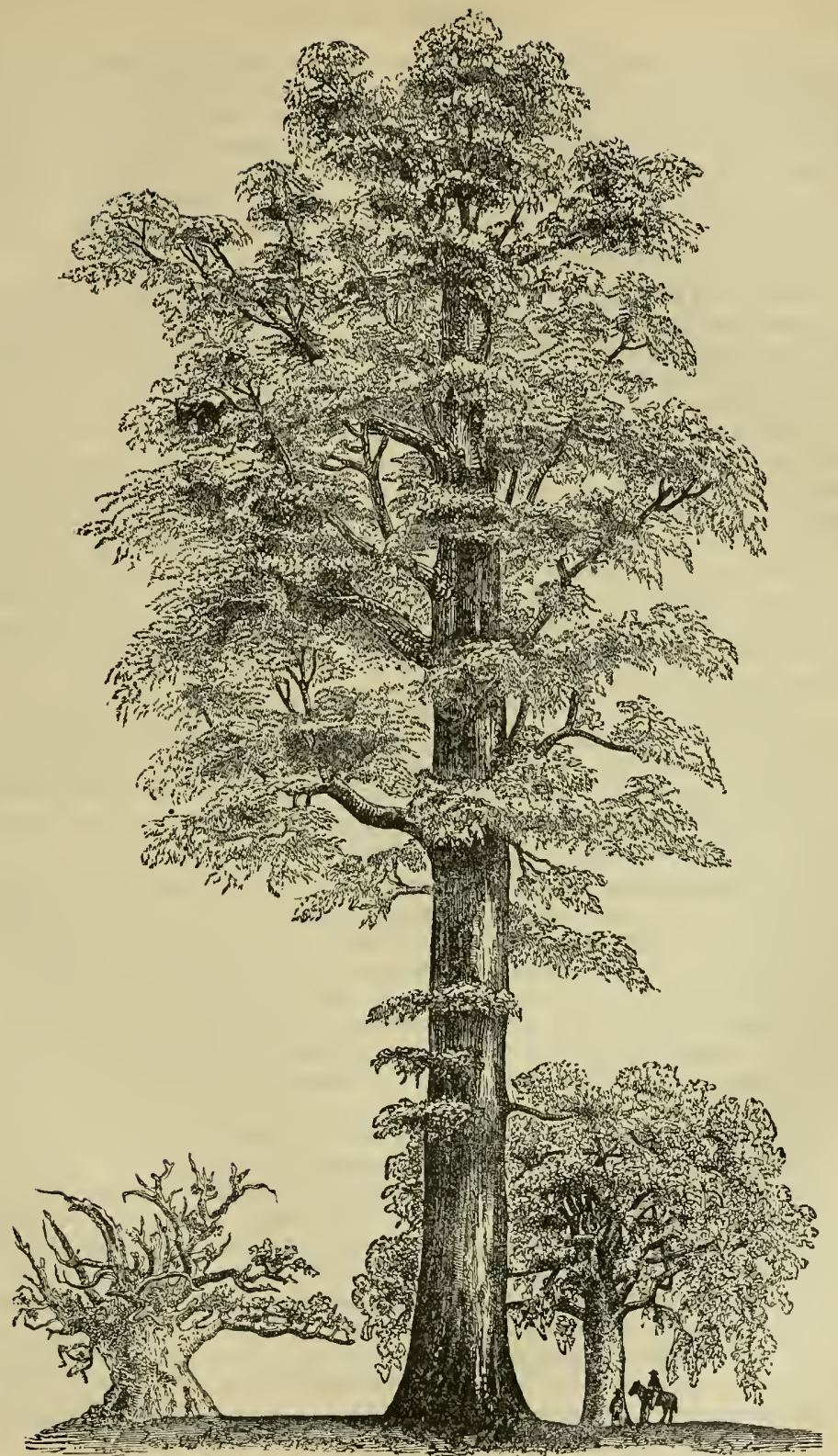

SEQUOIA GIGANTEA (GIANT REDWOOD).

(290 feet high, and 96 in circumference. See page 522.) 
The tree of this species, of which we give an illustration, stands two hundred and ninety feet in height, and ninety-six feet in circumference. It has been broken off at a considerable distance from the top, as will be seen by the size at the point broken. By an accurate estimate the tree before being broken must have been about four hundred and fifty feet from the base, and higher than any now standing. The tree represented at the left in the illustration, is a gigantic English oak that measured forty-eight feet in circumference. The one at the right, the old Boston Elm. They are both drawn on the same scale as the redwood, and show by comparison, the immense size of the latter.

Where to Plant Trees. - Trees for shade should be planted almost everywhere, or rather, anywhere that they may be either ornamental or useful; on the lawn around dwell. ing-houses, about other farm-buildings, by the road-side, in groups in the pastures to serve as shade for stock during the sultry summer weather, in fact, in any place that can be rendered more attractive, comfortable, or healthful by their presence.

Where to plant for timber-culture is also an important question. As a general rule, we would say, plant on land not well suited to cultivation. There are tracts of what are now almost useless lands, in almost every section, both North and South, that the planting of trees would not only render more healthful, but would greatly improve the soil, besides producing valuable timber. On every farm there are lands which are not as desirable for the cultivation of the common agricultural crops as others, and which would be admirably adapted to this purpose. A hillside is a good location. There are many such lands that if cultivated, would soon be washed to the extent that they would become nearly barren, which if planted with trees and sown with grass suited to the purpose, would become both a source of proft and ornament to the farm.

It is well to plant a belt of trees for shelter on the north and west sides of orchards, or exposed fields, for the cultivation of crops. It has been stated by good authority, that if one: fifth of the prairie land in the Western States were covered with foresis, they would produce as much wheat as when the entire land was sown, or rather, the warmth and protection secured by the forest would increase the wheat crop to such an extent, that its present yield would be produced on four-fifths of the land now devoted to it.

Tree-Planting from Seeds. - Trees may be planted from the seed, in the place they are intended to grow, or may be planted in a nursery or garden, and transplanted when three or four years old. Transplanting is the better method, since it secures more uniformity, and obviates the evils attending the too common practice of sowing the seed too thickly.

The following are some of the most common causes of failure in producing timber trees from seed: the use of poor seed, or that which has become too old or dry to germinate; insufficient preparation of the soil; covering the seed too deep, and lack of sufficient shade for the young plants. It is important that the seed be gathered at the right time, that is, when it is fully ripe.

The following directions on tree-planting from seed, taken from the columns of the Country Gentleman, will be found valuable and interesting: -

"In planting such large fleshy seeds as chestnuts, acorns, horse-chestnuts, and beechnuts, the most common cause of want of success, is allowing them to get too dry. As soon as they ripen and fall, therefore, which is usually about the middle of autumn or soon after, they should be gathered and kept slightly moist and fresh till they germinate. They might be planted at once, and the surface of the ground protected from drying winds by moss or evergreen branches were it not for mice, which show much skill in finding everything of the kind. A light covering of straw is still more certain to attract them. The safest way, therefore, is to pack them in damp sand or slightly damp moss, and place them in a cold cellar or other cool place till early spring planting. As they sprout very early they need not be planted 
deep, they will have moisture enough until they have thrown down their roots into the soil, if buried with only an inch of earth. The hickory and walnut are to be treated similarly, except that somewhat more care is necessary to prevent drying, as the thick shells serve as partial protection. But after the exterior covering of either chestnuts or walnuts have dried so long that they become hard and impervious to moisture from without, it will be useless to plant them.

The maples are of two classes-those which ripen their seed the first of summer, like the red and the silver maples, and those which do not ripen till October, as the black and the sugar maple. The former will supply well-matured seeds three weeks after the leaves have expanded, and as they soon lose the power of germinating, they should be planted at once in finely pulverized soil, not over an inch deep-if moist enough, half an inch would be betterand if hot, dry weather follows, they should be partially shaded from the sun's rays. But seeds of the sugar maple, maturing in October, may be kept in damp sand in a cool place and planted early in spring; or if properly protected, as above mentioned for nuts, they may be planted in autumn.

All the elms ripen seeds quite early in the season, and if sown shallow at once in fine mellow soil, they will make a good growth, and be a foot high in autumn. White ash seeds, which mature early in October, may be treated like the seeds of the sugar maple. The same treatment may be given to the tulip tree and the basswood. The catalpa ripens plenty of seeds in its long pods, and these are easily kept, and planted the next spring; they grow freely. The birches have small seeds ripening in summer or autumn, and when sown the following spring require good care, as the fine earth must be thinly sifted over them, and kept sufficiently moist to insure germination; and the young plants may need the protection of shade under a hot sun. It may be cheapest for the inexperienced to buy the young plants by the thousand of nurserymen.

Seeds of the common locust and the honey-locust will keep several years if quite dry. The common locust seeds require scalding to make them germinate. Put a quantity in a pint or quart basim; pour on boiling water and let it cool. In a few hours a part will be found swollen to double size. Pick these out and plant them at once an inch deep and they will grow. Repeat the process successively on the remainder until all are swollen and planted. Without this scalding process, the seeds will remain for years unchanged in the soil. The honey-locust does not require this treatment. The poplars and willows grow freely from cuttings, and are rarely raised from seed.

Conifers require more skillful management than deciduous trees, and it is commonly cheaper to buy the young trees, or seedlings, of nurserymen. A few suggestions are, however, offered to those who would attempt the experiment. The larch is perhaps the easiest to raise, but the seeds should be fresh and good, as they will not keep a year. The cones of the white pine drop their seeds about the first of October, and they must be gathered in time to secure them. The Norway spruce (now so largely grown of bearing size) matures its cones late in November, and they must be saved before the seeds fall out. The same may be said of the native black spruce. The seeds of conifers often require a month to germinate and come up. They must have the soil finely and thinly sifted over them, and the young plants always require more or less shading.

The berries of the red cedar are to be gathered late in autumn, mixed with an equal bulk of moist sand, and planted at once, or early the next spring. Most of them will grow the second year. We have always found them to succeed best by washing the pulp from the berries, although it is usually not regarded necessary by nurserymen.

The seeds of most evergreens being quite small, a great number of plants may be raised from a small quantity, if the seeds are good and fresh, and most of them grow. A pound of seed of the white pine contains 20,000 ; of the Scotch pine, 69,000 ; of the Norway spruce, 
58,000; of the hemlock, 100,000; of the European larch, 60,000 to 75,000, and of the Amer. ican arbor vitæ, 320,000 . It will usually be much cheaper to buy evergreen seeds than to collect them, but the latter mode will be sure to secure them fresh. Many other seeds of trees may be gathered advantageously by those who desire to make plantations, and in this way fine collections of young trees are obtained at small expenditure."

For maple, birch, and seeds of a similar kind, it will be well to delay planting until spring, in very cold latitudes. The soil should not be too retentive of moisture, for any variety. The planting should not be done in wet weather on naturally wet soils, as the seed would be liable to rot before germinating.

All trees require considerable shade in the early stages of growth. The natural protection of the mother tree and the forest are best suited to their growth. A hot sun will often kill many young trees, especially the evergreen species, while all varieties require more or less protection by shade when young. When planting in a nursery, a high hedge of thick growth, or a high, tight board-fence will be necessary, if no other shade is afforded for their protection.

Several years since, a gentleman in Massachusetts experimented in tree-planting, selecting for his location a very high bill containing, in all, about twenty-five acres. This section was bare, without a single tree. At the top where it was bleak and cold, and the soil extremely poor, he planted as elsewhere, at first, trees of many kinds, but they did not thrive. He then obtained, at considerable expense and trouble, some of the hardiest varieties of the pine, spruce, and hemlock, from a more northerly latitude, and from abroad, and planted these. Being varieties that would thrive almost anywhere, they grew and flourished. When they had made a good growth, he planted close by the side of each, that shelter might be thus afforded, some other variety such as the oak, maple, or hickory, that had previously failed to grow there. This experiment proved a perfect success, and to-day, owing to the efforts of this man, that whole hill is covered from summit to base with a fine growth of a variety of forest trees. Many forests bave been grown in this manner, and it would be well if more would follow examples of this kind.

Hon. Geo. B. Emerson, previously referred to, expresses as his opinion, that every one of our native trees may be propagated by seed. He says: "I have tried so many of them that I have no hesitation in saying that every one of our native trees may be propagated with perfect certainty, if you only know how to take care of them. A tree speaks for itself, generally, as to the time the seed should be planted. When a seed falls to the ground, it falls to produce another tree. When, therefore, the seeds are ripe and fall to the ground, that is the time to sow them. As to the mode of sowing them, you can sow them in the field, just as you do a crop of corn, taking care not to cover them too deep, and taking care to have them protected. I have heard of a man (and I was very sorry not to go and see him) who had sown a quantity of seeds in a field of rye, and the rye protected those little trees that sprang up perfectly, and the owner let the rye stay till the next spring, to protect them. Here is a hint that the trees give us of how they ought to be planted. The oak tree lets its acorns fall on the ground, and there they take root. The same thing is true of almost all our trees; not all; they grow best under their mother's care, under her protection. In Germany I went to a great forest school, thirty miles from Berlin, and the superintendent escorted me about and showed me how they planted their oaks. I found a magnificent for. est of several hundred acres that has been given up to this forest school, that the young men may have an opportunity to study the tree in every position and in every condition. I found the place that he had selected to plant oaks was a little opening among oak trees, - a noble forest, with high trees on every side. The seed is planted in this spot, where the trees pro. tect them from the sun, except for half an hour or an hour in the hottest part of the day; they are partially protected almost the whole time. That is a mattex of very great import. 


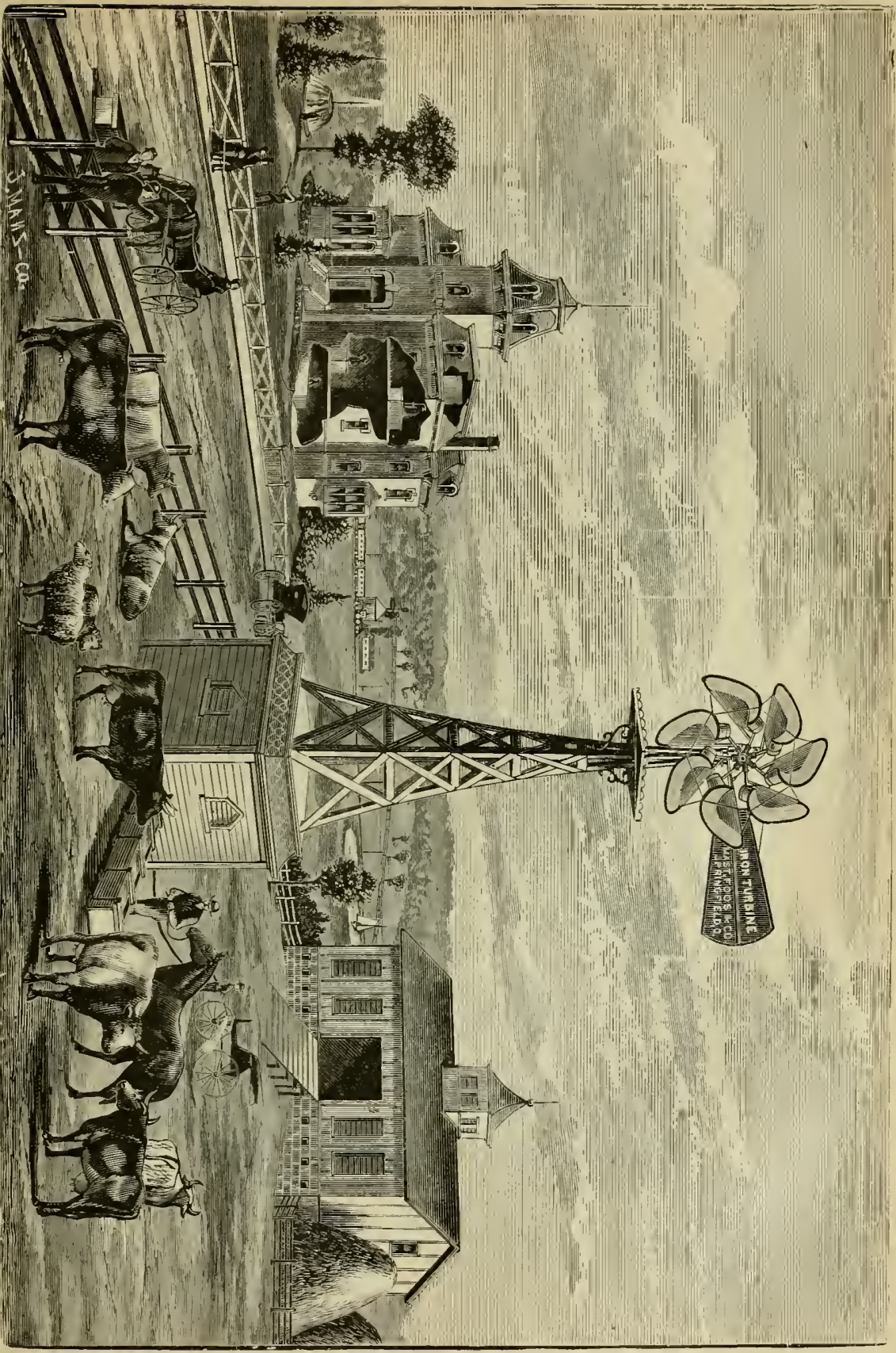




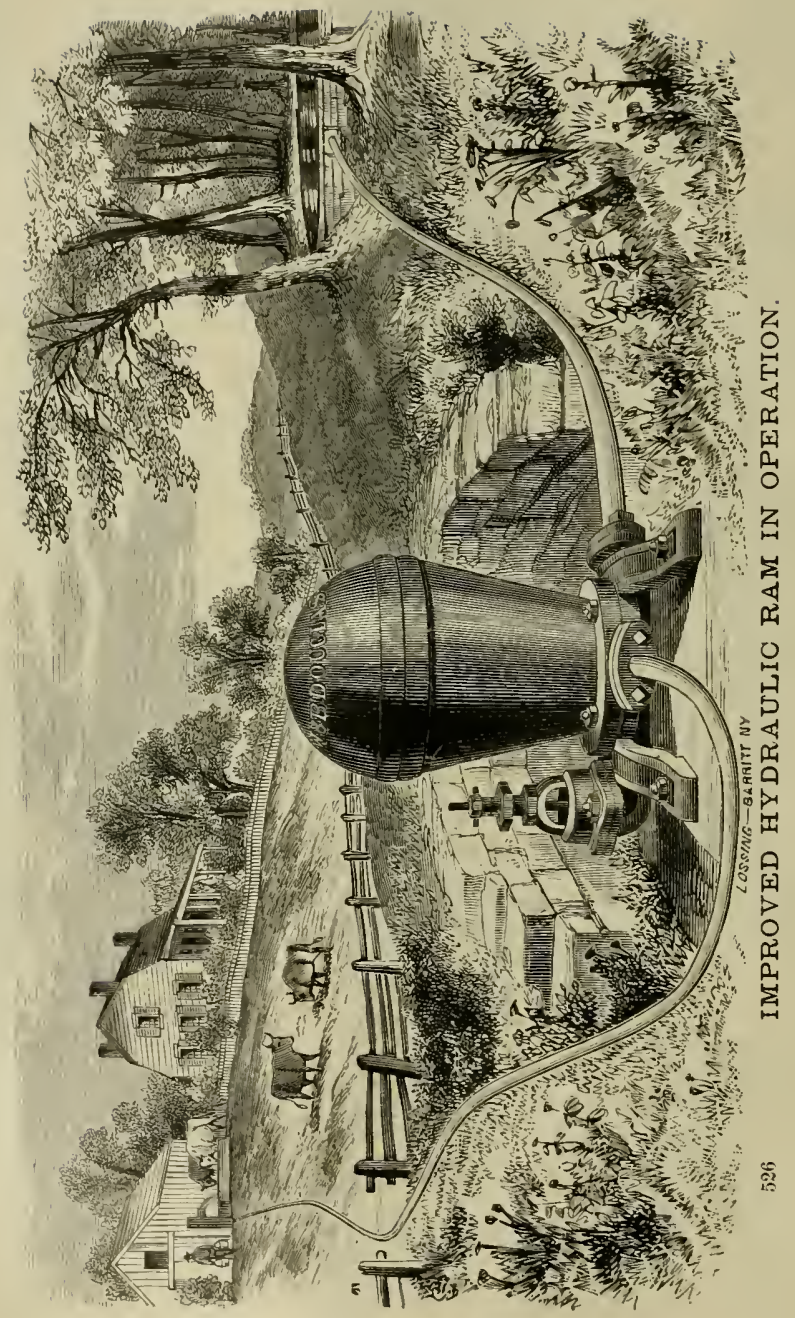


ance, very much greater than it is commonly considered. If you are going to plant seeds, the fruit of trees, in a place which is not protected, it will be always advisable to have a hedge or a fence built up between them and the sun, so that they shall not be exposed to the full heat of summer."

Transplanting Trees.-At the North, trees are transplanted, both in the autumn and spring. Both practices have their advocates, who use strong arguments in favor of their respective theories. As a general rule, we should recommend that it be done in the spring, just before the leaf buds begin to swell. When performed in the antumn, it should be late in the season, after all the leaves have fallen. At the South, an intermediate time is most favorable, which is in mid-winter.

The soil for the reception of the roots should be finely pulverized, and the opening in the earth be made sufficiently large to accommodate the size and extension of the roots. If the work be carelessly performed, the growth of the tree will be liable to be checked considerably, or it may die altogether. Many valuable trees are annually lost by indifference or carelessness in transplanting. A cloudy day, or towards night, is the best time; or, better still, just preceding a rain. Wetting the soil freely about the roots is a good plan while setting, if there is no immediate prospect of rain.

All mutilated portions of the roots should be trimmed off, the tree set to the same depth in the soil that it was before being taken up, and the soil carefully pressed upon the roots. Tender trees may require some shading for a few days after transplanting, until the roots become well established in the soil.

If the tree transplanted has attained a considerable size, it will be well to protect it from being blown about by the wind, by driving a stake near the trunk, to which the latter may be tied.

The bark of the tree should be protected from rubbing by a piece of cloth or other substance being wrapped around before tying. In transplanting young trees for a forest, a suffcient allowance of space should be made for the size of the tree when grown, if it is not intended to thin out at different stages of their growth. Where the latter is the object, the planting can be much closer, and, when partially grown, a portion of the timber can be removed, leaving the remainder to fully mature. By this means, a larger product can be secured from an equal area of land.

\section{WATER SUPPLY FOR THE FARM.}

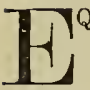
QUALLY important as the proper amount of pure air for the health and comfort of both man and the lower animals, is an adequate supply of pure water; yet how frequently do we find the farm supply not only limited in quantity or inconvenient of access, but also often poor in quality, and containing the elements of poison and disease, which, though they may not be apparent for a time, will eventually be the cause of most disastrous results. This is not only true of country farms, but in many, and we might say most of our large cities the water supplied for drinking purposes and cooking is not as pure as it should be for the health and welfare of the inhabitants, and too little interest and concern is manifested by the majority of the consumers in remedying this evil. A city frequently depends for water upon a river, which a few miles above receives the sewage of another city, or town, or the refuse of tanneries, paper manufactories, woolen mills, chemical works, or other manufacturing establishments, which deposit in it substances which render it foul and unwholesome Even some of the cleanest spring waters are often frequently impure, 
since they contain, in a soluble form, more or less of the elements of the soil, and are fre. quently largely charged with mineral and other substances. The character of all spring water, therefore, depends upon the character of the soil through which it has passed before it issues from the ground in the form of a spring, the impurities often not being perceptible, either to sight, taste, or smell. It should, therefore, as a general rule, be filtered before using. As ordinary wells are supplied partly by springs, and partly by surface drainage, it is highly important that they be located where they will not be contaminated by cesspools, privy raults, barn-yards, kitchen refuse of any kind, or anything that shall have a tendency to render the water foul. In many instances of the most malignant types of diphtheria, fevers, and kindred diseases of blood poisoning, often resulting in death, the real cause of the disease has been traceable to the contamination of water through some of the above-mentioned or similar sources, from which the deadly poison has emanated. And yet the usual custom is to ignore all such causes of the evil, and regard such affictions in families as inscrutable dispen. sations of an All. WV ise Providence, when, if a little more intelligent supervision of the sanitary conditions of the premises had been exercised, the evil might have been avoided. We believe there is more attributed to the Supreme Being, in the suffering and sorrow of this world, than we have any right to suppose that source responsible for, and that if we traced the responsibility of it more frequently to its proper source, we should find it in our own ignorance and neglect.

Doubtless the Supreme Being could avert the evil effects of all ill that we bring upon ourselves, if He chose thus to do; but He does not generally do it; and if we take poison into our stomachs, either through ignorance or design, nature is generally permitted to take its course, and the natural results follow, as a consequence. It is often astonishing to see how indifferent people are respecting such common sanitary conditions. We do not believe there is more than one farmer in a hundred that regards water of a poor quality on his prem. ises, any thing more than an inconvenience, simply, the ninety-nine of the number consider. ing a sufficient supply of pure water a fortunate circumstance, but failing to realize the danger involved in the use of that which is not pure.

Prof. Chandler of New York says, that in many cases, from the proximity of cesspools and privy vaults, the water becomes contaminated with fitered sewage-matters which, while they scarcely affect the taste or smell of the water, have, nevertheless, the power to create the most deadly disturbances in the persons who use the water, and that in the neighborhood of grave-yards the water of wells is often found, on analysis, to be impregnated with animal matters from the recently filled graves. Jules Lefort states, that as long ago as 1808 , it was decreed in France that no one should dig a well within one hundred metres of any cemetery. In driven wells, the water is not exempt from contamination, the same as other wells, except in cases where there is near the surface a bed of clay or "hard pan "impervious to water; when such a stratum is penetrated by the tube, and the water is drawn from beneath it, the well is somewhat protected from surface drainage. The water from small, stagnant ponds is very impure, though that from large ponds or lakes is often purer than the water of some springs, as well as also being softer. When used in stationary or locomotive boilers, impure water produces incrustations which often form a complete lining. In those sections of the country where the water is quite hard, from the presence of lime salts, many housekeepers have noticed the same effect upon the inside of the tea kettles, that are in constant nse, and are obliged occasionally to remove it in some way. It is stated by reliable authority that as much as 1,300 pounds of such calcareous matter in the form of incrustations, have been taken from the boiler of a single locomotive on the New York Central Rail Road. Boiler explo. sions are sometimes occasioned by such incrustations, on account of the metal becoming very much overheated, causing the scale of the calcareous matter to crack, and permitting the water to coine in contact with the hot metal, which produces immediately such a surplus of steam, that the boiler is burst. 
Injurious Effects of Metallic Pipes.-Water is frequently rendered impure and unsafe for use by the metallic tubes through which it is conducted. In many cases even pure water dissolves the metal, such as copper and lead, the mineral being readily detected in the water, by analysis. Cases of sickness are known to have occurred, caused by water being drawn through copper pumps; but lead poisoning is of more frequent occurrence, since lead pipes are most commonly used for conducting water. This metal is most easily dissolved by water, and is also the most poisonous, the quantity thus dissolred often causing paralysis or palsy, and even death. Lead should therefore never be used for conducting water that is to be drank by man, or any living creature. Its use for this purpose is positively dangerous, and any one who will give the subject due consideration cannot fail of being convinced of the fact. Any farmer therefore, having not only the health of his family in view, but also of his farm animals, will provide other means than lead pipes for conveying water to them. Many instances are known of cattle failing to do well where the cause was traceable directly to the use of lead pipes in conveying water to them. Besides, the beef produced by cattle having metallic poison in their veins cannot be healthful.

For farm purposes, some writers recommend wooden pipes of good material carefully laid. These are safe, as far as metallic poisons are concerned, but their chief objection is that they are not very durable. Iron pipes are often recommended, but they are very apt to make the water rusty, galvanized iron is considered unsafe on account of the zinc which is so readily taken up by the water, rendering it as objectionable, on account of being poisonous as lead, for this purpose, many cases of zinc poisoning having occurred from its use not long since in New England. Brass is also equally objectionable. Gutta percha is not durable, and glass and porcelain, which have been tried to a certain extent, do not possess the flexibility and softness which is requisite for bending, cutting, and fitting pipes.

After various experiments with different materials as a substitute for lead, a pipe has been invented which consists of lead for the outside, encasing a block-tin pipe, or in other words, a tin-lined lead pipe, which is found to answer the purpose best, since the water comes in contact only with the tin surface, which does not render the water impure. Prof. Chandler states that the water in the tin-lined pipe, when tested side by side with that from the ordinary lead pipe, from which the water would take up from one-tenth to two-tenths of a grain of lead per gallon, was not perceptibly affected by remaining for a considerable length of time in contact with the tin surface. In using this pipe, of course, one of solid tin or some other material that would not affect the water, should be used to be extended under the water in the well or tank, as the lead on the outside of the tin-lined pipe would be objectionable, if coming in contact with the water. Doubtless a better substitute than this kind of pipe may yet be found.

Springs, Brooks, etc.-The farmer who has a good spring from which to supply his house and farm buildings with pure, running water, has a treasure indeed. Or if he has a good well for a water supply, he is fortunate; but he should be especially careful that it does not become contaminated by surface drainage, or poisonous substances that may enter it by percolating through the soil, in a manner so gradual and subtle as not to be detected except by its disastrous effects.

Great care should be taken also to keep a spring which supplies water to the premises, clean and free from everything that would render it impure. A good strong fence should be built around the spring to keep off cattle and all intruders. A fine grate or strainer should also be placed over the end of the pipe, to prevent obstructions from entering. The water shoula flow into a tank or reservoir near the spring, from which pipes should connect it to the buildings. The spring should also be frequently examined by the owner, and kept as free from impurities as possible. If the spring is higher than the buildings to which the water is to be conveyed, it will be a very easy matter to take it there, as water will readily run down hill. 
If the buildings are on a higher point than the spring, the water can be forced up by a hydraulic ram or by means of a windmill, either of which are not expensive, and, if properly cared for, are quite durable.

A clear, running brook that never dries up in summer is of immense value on a farm. Ponds of pure, fresh water are also to be highly prized, but ponds of stagnant water are seriously objectionable in any locality, rendering it extremely unhealthy for both man and beast. The importance of having a good supply of pure water for stock of all kinds is very properly set forth by the late Alexander Hyde, whose pen has given so much valuable agricultural instruction to the world:-

"All farmers recognize the necessity of good food for their stock, but the value of pure, wholesome water is not so generally appreciated. It bas been said that green, succulent grass is both food and drink for sheep in the summer, and in the winter, when they exercise and perspire but little, snow will furnish them all the drink they need. Sheep may possibly live under such management, but they cannot thrive, and if they could talk they would tell a sad tale of suffering. Snow is a miserable substitute for water. Sheep will eat it if they can get nothing else, but it is so cold that it partially paralyzes the mouth, throat, and stomach, and when reaction takes place, it is accompanied with more or less fever, and the health of the animal is impaired. Precisely what the action of snow is upon the sheep we can learn by trying it as a substitute for drink for ourselves. The first feeling may be one of refreshment to the parched tongue, but it is followed by a sensation of numbness, and soon after heat and increased craving for drink.

Sheep can stand such cold treatment better than cattle and horses, but it is poor econorny, to say nothing of its cruelty. If they can have convenient access to pure water, they drink a dozen times a day, and often leave the feeding-rack to go to the watering-trough. As for cattle and horses, no one pretends that they can get along without daily rations of water. Many, however, compel their cattle to go to such a distance from the stables for drink that, in a cold, blustering day, the beasts hesitate whether to expose themselves to the winds and snow or suffer with thirst. Generally they muster up courage enough to start off for the brook or spring once a day at least, but sometimes have to be goaded into going even once. If water is brought into the yards where cattle have free range, they will drink three or four times each day, and this is much better for them than to stuff themselves once or twice, till they are as round as a snow-ball, and almost as cold.

Drink early and drink often, is a much better maxim for stock, than its paraphrase is for voters. The health and thrift of an animal depend about as much upon its drink, as upon its food. Water is so common and so cheap in most parts of our country that, hike air, its virtues are not sufficiently prized. A Northern farmer needs to go upon the prairies of the Southwest to fully appreciate the value of the springs and rivulets that ooze out from the hills and flow through the valleys of his well-watered section. This abundance of pure water is no small compensation to the New England farmer for the want of the deep and easily. tilled soil of the prairies, and the wonder is that he does not better improve his facilities for irrigating his land and watering his stock. A Texan stock-breeder, owning a ranch of 5,000 acres and a flock of 4,000 sheep, recently told me that the great drawback to the success of his business is the want of water. Here at the North, when almost every farmer possesses a spring, the water of which, at small expense, can be brought to the barn, comparatively few farmers avail themselves of the blessing. Such neglect shows ignorance or shiftlessness, possibly a mixture of the two. So much has been said of the value of pure air, that the community are generally alive to the importance of securing good ventilation both in the house and the barn, but pure water is equally essential with good air. True, both man and beast can go without water longer than they can without air, but the suffering for the want of it is more intense than that from the want of food. Shipwrecked and starving sailors 
testify that their first want is water, and that a parched tongue is more intolerable than a gnawing stomach. Our farm stock would bear the same testimony could they speak.

In order to appreciate the value of water for stock, we must remember that it constitutes, on the average, three-fourths of the weight of the animal, and it furnishes largely the elements, oxygen and hydrogen, of the bodily structure, that it is essential for softening solid foods, so as to facilitate mastication, digestion, and the passage of waste material through the body, and, finally, by its evaporation from the surface, it keeps the body cool and healthy. Fever always ensues when perspiration is checked. The estimated amount of water which an average man throws off each day is eighty-three ounces-forty in the urine and fæces, and forty through his lungs and skin. The average ox requires a much larger allowance than this, and a cow that gives twenty quarts of milk a day must have at least eighteen quarts of water in some form from which to manufacture this milk, for its analysis shows about ninety per cent. of water.

The objection most commonly urged against bringing water to the barn for the convenience of stock is the expense, but where there are large herds or flocks, the benefit in one year will compensate for the outlay. Most farmers have logs suitable for making the aqueduct, but if not, lead and iron pipe are so cheap in these days, that it pays even to borrow the money and invest it in a water privilege. A three-quarter inch iron pipe can now be bought for five or six cents a foot, and there is no better material for constructing an aqueduct. Logs rot and lead corrodes, but iron, if it does rust a little, furnishes no poison. In a constantly-filled pipe there is not rust enough to make a good tonic, so that iron is probably the best conductor of water. After a log becomes rotten, the water conveyed through it must be more or less contaminated, and what the farmer wants for his stock is what he wants for himself and family-the pure article.

If the farm is so unfortunately situated that no spring or brook can be tapped that will flow to the barn, then a water-ram or wind-mill can be used that will overcome gravity. Windmills have long done service in Europe, and they are getting to be quite common in this country. Their advantage over water-rams is that they can be utilized not only for pumping water, but for threshing and grinding grain, sawing wood, and any other farm purpose for which power is required. A neighbor has two or three in operation on his farm, and they are labor-saving machines. Wind in a cheap power with which to furmish water."

We believe with the above-mentioned writer, that all animals should have free access to an abundant supply of good water, and it will well repay any farmer in the improved condition of his stock to incur the necessary expense of procuring it for them, since it is one of the first essentials of successful farming. We regret to say that many farmers still adhere to the shiftless and unprofitable practice of failing to provide sufficient water for their stock, and cblige them to drink from holes cut in the ice from some frog.pond or brook, often driving them long distances, and exposing them to the cold weather and danger of having their legs broken by slipping on the ice, or of being booked by others of the drove while crowding about such drinking places. This is not only a very inconvenient way of managing stock, but it is extravagant and detrimental to success. About eighty per cent. of all flesh being water, animals cannot thrive well without a sufficient supply of it. Clean water abundantly supplied to cows in the barn-yard, where they can have frequent access to it, will prove a paying investment and cheapen the production of milk.

Cisterns.-Aside from wells and springs for a water supply, another very cheap and easy means of furnishing this necessary element is in utilizing the rain that falls upon the roofs of buildings, and collecting it in a cistern for present and future use. If cisterns are made of ample size (as they always should be), a permanent and sufficient quantity of water can be obtained in this manner, and with so little trouble or expense that no farmer need be put to any inconvenience in procuring good water for his house or barn. We would not recom. 
mend this water for drinking purposes for the family, as many writers do, though it is used in many of the lime sections of the country for this purpose, but we must say that when properly filtered (as it always should be), we prefer it to that of many wells and springs that we have tested. It is excellent for the various household purposes, such as washing, being very soft, which renders that necessary operation in every household a much easier task than when performed with the hard water from many wells or springs, and does not require much more than half the amount of soap that hard water does. Aside from the convenience of having cistern-water for use,-where other supply of water is uncertain, or rain-water is used, -it is much better for the premises to have it collected, than running into the cellars and about the buildings, besides, eaves-troughs and conductors are a great protection to the yard manure when supplied to a barn, and prevent many dollars' worth, yearly, from washing away, or leaching into the soil; hence, when a barn is supplied with a good capacious cistern, two very desirable benefits result from this means of water supply. Cisterns or tanks are constructed in various ways; sometimes of wood, lined with lead, zinc, cement, or of slate, in which latter case the sides and bottoms are grooved and cemented, to prevent leakage. The usual and better method is to construct a cistern of stone or brick, and cement it at the bottom and sides. When stoues are used for this purpose, small ones of nearly a uniform size are selected, but brick is to be preferred. When lead or zinc are used for lining a cistern, the water is unfit for drinking purposes, either being poisonous.

In a compact clay soil, cisterns are often made for stock purposes, by making an excavation of sufficient size, and laying a thick coating of cement over the entire inner surface, from which reservoir the water is to be pumped into troughs for the stock. Water for household purposes should always be filtered. Cisterns of this kind should be made in two apartments, so as to contain water filtered ready for use in the one, while the other receives it from the pipes. In cementing the interior of a cistern, great care should be used in making it perfectly water-tight; hence, the sides and bottoms should be properly joined, and the cement well prepared before applying. The most desirable form for a cistern is circular, though they are made in various forms. A recent agricultural writer describes that of his barn as being a long brick arch, running under the earth drive-way, with a capacity for holding 500 barrels. This cistern runs a foot deeper than the basement floor of the barn, and as near the top and ends of the bank as is safe, in view of frost. The size will depend upon the amount of water to be used, and the extent of surface of the roofs to furnish the supply. It is better to have a cistern larger than necessary, rather than too small. A large cistern does not cost as much, in proportion to its size, as a small one.

A circular cistern five and a half feet in diameter will hold, for every ten inches in depth, a little more than one hundred and forty-eight and a half gallons.

According to the best. scientific authority, the average amount of rain in the United States is about three feet per year, or at the rate of about three inches per month.

Upon this average, every inch of rain-fall upon each ten feet square of the roof will amount to a little more than two barrels, and a little more than seventy-two barrels a year (based upon the calculation of three feet of rain); therefore, a barn of only ordinary dimen. sions would supply a large amount of water during the season, when rains are heavy or frequent.

The cistern should be arched over with brick, leaving an opening for a trap-door at the top large enough to admit a man when necessary to clean out the interior. This opening should be kept covered with a closely-fitting trap-door. It should be kept constantly closed, not only to exclude rats and other vermin, but because of being very dangerous for children, who, through the carelessness of parents or servants, are frequently drowned in such places. After a long season of dry weather, roofs of buildings are usually quite dusty; therefore it is better to have pipes connecting the eaves with the cistern so arranged that the water can 
be turned off for a few moments after it commences raining, to prevent the dirty water entering it, after which, the roofs becoming sufficiently washed, the pipes can be re-adjusted to supply the cistern.

Table of Contents for Circular Cisterns.-The following table of contents may be of assistance to those designing to build cisterns. For each foot in depth, the contents of circular cisterns is as follows:-

A cistern 5 feet in diameter will hold for every foot in depth, .

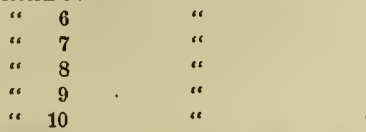

"

A cistern $3 \frac{1}{2}$ feet diameter will hold for every 10 inches in depth,

$\begin{array}{cl}\text { “ } & 4 \\ \text { “ } & 4 \frac{1}{2} \\ \text { “ } & 5 \\ \text { “ } & 5 \frac{1}{2} \\ \text { “ } & 8\end{array}$

"
"
"
"

"

4.66 barrels.
6.71 "
9.13
11.93 “
15.10 “
18.65 "
59 gallons.
78 “
99 “
122 “
148 “
176 "
$310 ~$

By the above table, it will be seen that a cistern five feet in diameter and six feet deep will hold nearly twenty-eight barrels; eight feet in diameter, and ten feet deep, 119.3 barrels, and so on.

Rule for finding the contents of any circular cistern in wine gallons, the diameter and depth being known-

1. Find the square of one-half of the diameter, and multiply the product by $3 \frac{1}{7}$, which will give the area of the bottom of the cistern-nearly.

2. Multiply the product thus obtained by the number of feet in depth, which will give the cubic contents in feet.

3. Multiply this last product by 1,728 (the number of cubic inches in a foot) which will give the number of cubic inches.

4. Divide the result thus obtained by 231 (the number of cubic inches in a wine gallon), which will give the number of gallons in a cistern.

By dividing the number of gallons in capacity by the number of gallons in a barrel, we have the capacity of the cistern given in barrels.

Filters for Cisterus.-In making cisterns, it is very essential that arrangements be furnished for filtering the water as it is received from the pipes of the roof. By the filter. ing process, all the suspended impurities, such as mud, animal and vegetable substances, etc., are removed from the water.

The usual method is to divide the cistern into two equal compartments by a wall of brick, which is open at the bottom to the height of six or eight inches. One of these compartments is for receiving the water from the roof, and the other for containing it when it is filtered, ready for use.

In the bottom of the one for receiving the water, alternate layers of pounded charcoal, sand, and gravel are placed to a little more than the depth of the bottom opening between the partitions. In the filtered department it is filled to the same height as the former, with clean sand and gravel. Since the water passes through all these layers in reaching the filtered department, it will be comparatively pure by this process. Of course, the pipe from the roof enters the former compartment, and that of the pump the latter. In making such a cistern, it would be well to build the wall forming the partition between the two compart. ments with a curve, so as to act as an arch towards the unfiltered portion,or side that is always fullest, setting the ends frmly into the sides of the cistern. This form will counteract the 
pressure of the water from the receiving side, and the tendency to break down the middle wall, which would be liable to occur, if empty, in a heavy storm, providing the wall was built straight, since the water would enter so much faster than it could filter through. It will be necessary to occasionally clean out the cistern, and substitute new filtering material, in order to keep the water pure, as the sand, gravel, etc., will in a few months become filled with the refuse substances taken from the water.

Another mothod of filtering, is to build the brick wall forming the partition, to the bottom of the cistern, leaving no opening whatever between the two. In such cases the water filters through the bricks, the bricks making a perfect filter, as is stated by those who have used cisterns made in this way; but we should prefer the former mode, providing proper care be taken to keep the filtering material clean.

Wells. - The common source of water supply among farmers is in the ordinary well, which is, with but comparatively few exceptions, a permanent and unfailing one, even in time of drouth. A well of good, pure water is something to be prized by anyone possessing it, containing, as it does, the elements of comfort and health; for pure, cold water is truly a luxury, aside from all sanitary considerations. We have said so much at the beginning of this subject of water supply respecting the necessity of keeping wells free from surface drainage, sewage contaminations, etc., that it will be unnecessary to repeat here; but we wish to impress upon the minds of our readers that pure water is essential to health and impure water is incompatible with health, either in man or beast, and one of the principal sources of disease among mankind; also, that the decomposition of animal matter in water, such as the animal excreta from privy vaults, or barn.yards, are by far the most objectionable and dangerous to the system of all impurities, often engendering the most fatal diseascs. The decomposition of vegetable matters is highly dangerous, acting as a poison upon the blood, but not to the extent that animal substances are. It is also well to remember that these poisonous substances will percolate through the soil for a long and sometimes almost an incredible distance before being deposited in a well, and that water thus contaminated will often be considered of the finest flavor and quality by those who drink it, the evil not being detected by taste or smell. We have in mind a well of this kind in a certain city not far from New York, which was located in one of the public parks. It was celebrated for the delicious coolness of its water, as well as excellent flavor, which was clear and sparkling. In warm weather people would often go out of their way quite a distance to get a drink of it. Some of this water was finally analyzed by a competent chemist, and found to contain a substance that could be traceable to no other source but urine, either human or animal, and the well was ordered closed by the city authorities. The difficulty was afterwards found to be in the leakage of a city sewage pipe that passed several feet from the well. We might give many similar instances, but state this simply to prove how easy it is for persons to be deceived respecting the quality of water, in taste, sight, or smell, and how very important it is to take special care in preventing any contaminating substances whatever from entering a well. It is a good plan to have the earth around a well brought up in the form of a mound, to prevent surface water from running into it. A well should never be located in a low spot of ground to receive such drainage. The higher the ground for the well the better, other conditions being favorable.

A cellar, or a locality near to a cellar, is also, for this reason, an objectionable place for digging a well. It is not uncommon to see, in the country, a well for household purposes located but a few feet from the barn-yard, so that in a wet season the drainage must of necessity enter it. Wells should also be frequently examined, and occasionally cleaned out, that the sediment, soil, and other substances that may have found an entrance there may be removed.

In digging wells, it is better to go to a sufficient depth to furnish an abundant supply of 
water, that in time of drouth it may not be as bable to fail; besides, much trouble and expense is attended with deepening a well that has once been completed. Farmers sometimes have a well for stock purposes, located in or very near a barn-yard. In such cases great care should be taken that no drainage from the yard entered it. All water from the yard should be conducted in another direction, the earth about the well be made higher than other portions of the yard, and every precaution possible used to keep the water pure, since cattle cannot thrive without pure water, and the milk of cows that drink water contaminated with the drainage of the yard cannot be healthful or palatable.

The danger of this drainage is lessened when the sub-soil is of a clayey nature, being in a measure impervious to water; but when the sub-soil is of a gravelly or sandy character, there would be danger of its leaching through the soil into the well, even with all necessary precautions observed on the surface. Absorbents to take up the liquid manure will obviate the evil in a measure, when a sufficient quantity is used for that purpose. The digging of a well is, of course, attended with considerable labor and expense; but, when once properly dug, it becomes a permanent source of water supply, and the convenience of having such a source to depend upon in all seasons well repays, even in one year, all that is expended in obtain. ing it.

Driven, or Tube Wells, sometimes called American wells, are an ingenious and easy method of obtaining water. They are made by driving a small iron tube, which has a perforated hollow conical point of steel, into the ground until it strikes a subterranean spring. By applying a hand-pump to the orifice, a supply of water is generally obtained sufficient for all practical purposes, and often obviates the necessity of common wells, the digging of which is atteuded with more labor and expense. In some localities these wells are a perfect success, and, when they prove so, are one of the cheapest means of water supply. They are not, however, as permanent and durable as wells that are dug in the usual way, since the iron tube will, in time, rust out; but they will last many years, and when they prove a success, will well repay the slight expense incurred in making. As they sometimes prove a failure, it is well for the farmer to always have a good supply of water guaranteed by the agent before arranging the terms, that the risk may not be his own. No one should pay for such a well unless it supplies water, and as agents are always desirous of custom, they will generally take the risk themselves.

In many of the hilly and mountainous portions of Europe, water for irrigating the land and supplying the domestic animals, as well as for household purposes, is obtained by driving a tube horizontally into the side of a hill or mountain until it reaches some spring, which furnishes a constant flow. The water from such springs is generally very cold, and of good quality.

Artesian Wells. - The use of artesian wells is becoming quite common in some portions of the country, both for irrigation and other uses, and especially in some of the arid regions of the West, where both surface and spring water is frequently not only very scarce, but is so impregnated with certain mineral substances as to render it unfit for drinking purposes. In California, artesian wells are used quite extensively for irrigating the lands, but are comparatively rare in many sections of the United States. They are, however, quite common in New York, Pennsylvania, Virginia, Alabama, and Mississippi, some of them throwing up oil, some mineral water, and others pure water. The numerous oil wells of Western Pennsylvania, West Virginia, and vicinity are principally remarkable for the large supply of petroleum which they furnish.

Among the deepest artesian wells in this country are two in St. Louis, Mo. The first was bored to the depth of 2199 feet, and discharged $\mathbf{7 5}$ gallons of water per minute. The second was sunk to the depth of 3843.5 feet, the last 40 feet being in solid granite. The 
diameter of these wells is four and a half inches, and both furnish water impregnated with saline sulphur. The water from the first has a temperature of $73.4^{\circ} \mathrm{F}$., the second $150^{\circ} \mathrm{F}$. At Louisville, Kentucky, there is an artesian well three inches in diameter and 2086 feet deep. At Charleston, South Carolina, there is one sunk to the depth of 1250 feet, which discharges water ten feet above the surface at the rate of 1200 gallons per hour. At Chicago there are two artesian wells, one of which is 700 feet and the other 1000 feet deep. These wells are five inches in diameter, and supply the city with 800,000 gallons of pure water daily. One of the deepest artesian wells in the United States is that at the State House in Columbus, Obio, which is $2775 \frac{1}{3}$ feet, but the water is salt, and does not rise above the surface.

Among the most noted of these wells in Europe is that of Grenelle, in the vicinity of Paris. It is about 1800 feet deep, and exerts a pressure equal to a rise of 812 feet above the surface (in pipes), and discharges about half a million of gallons per day. This water is very pure, and is supplied to the portion of the city in which it is located; but as its temperature is $82^{\circ} \mathrm{F}$., it requires cooling before being used for drinking purposes. It is used also for warming the hospitals at Grenelle.

Artesian wells are of ancient origin, there being unmistakable traces of them in Lombardy, Asia Minor, Persia, China, Egypt, Algeria, and even in the great desert of Sahara. A well within the old Carthusian convent at Lillers, bored in the year 1126, still flows with undiminished force. They derive their name, however, from Artois in France (the ancient Artesium), where they have been used for a long period.

They are usually from three to six inches in diameter, though sometimes even larger, but never exceeding twelve inches, and are made by boring into the earth until the required depth is reached. This depth varies in different localities, depending upon the geologic structure and general surface conformation. When a subterranean sheet of water is reached, which is confined between two inclined, impervious strata of clay or rock, one above and the other below, and so formed that the pressure of the water is brought to bear against the part penetrated by the boring instruments, an artesian well is formed, which furnishes constantly. flowing water. In this respect they differ from the common well, since they are simply artificial springs, which bring water to the surface from a great depth, often throwing it to a greater or less height above the surface of the ground.

They are bored either by hand, horse, or steam power, the latter being the most effective in facilitating the labor; various kinds of augurs, chisels, and other implements being required for boring through the different layers of rock, clay, etc. A great improvement has been made within a few years by the invention of the diamond-pointed drill. It consists of a long steel bar, the end of which may be either conical, concave, or annular, and is firmly set with diamonds, so as to present cutting edges to the rock when turned right-handed. It is generally worked by steam or compressed air, and by its use the hardest granite may be bored at the rate of several inches per minute; this instrument is also extensively used in mining. Tubing is generally necessary to prevent the well from caving in at the sides, to exclude undesirable veins of water, or the entrance of quicksand, the latter of which is said to be the most troublesome source of difficulty in such wells. The tubing is sometimes of wood, but generally of iron; bronze and copper also being used to a certain extent. Of course, the making of such wells is attended with considerable expense; but in those regions which are deficient in other sources of water supply, it often becomes a necessity, and when we con. sider their durability and the great benefits to be derived from their use in such localities, they are comparatively cheap. We know of some farmers and fruit-growers in California that have three or four artesian wells on their lands, which are exceedingly productive, but which would be almost worthless for agricultural purposes without them. 
Hydraulic Rams. - For the purpose of forcing water to a height greater than that of the source from which it is obtained, this simple and inexpensive power is admirably adapted. To those having no spring above the level of the house, but one below it, that they wish to utilize, this little machine will be found useful in bringing the water to the desired locality. It can be made to force about one-eighth of the water that flows through it up a steep hill and through a pipe a long distance, taking it even to a reservoir in the attic of the house, if desired. Water discharged from the constantly running pipes of some underdrained lands is sometimes utilized and conveyed to barns by this means.

Wind-mills, however, are at present more commonly employed for this purpose. They are inexpensive, and with good care will often last many years. A recent writer says that he has used the hydraulic ram for supplying his house with water from a spring for more than thirty years, and during that time has only had two rams, the one he is now using being apparently good for many years to come. He says:-

"Repairs have cost me, about once in eighteen months or two years, five or ten cents for a leather valve inside the air chamber, and once in about six or eight years a new brass plunger valve, costing about five dollars."

The illustration which we insert of an improved implement of this kind, by Joseph Beck \& Sons, Boston, is a good representation of this little machine in operation.

A correspondent of the Scientific American gives the following directions for increasing the power and capacity of hydraulic rams: "Drill or file a small hole, say one-thirty-second of an inch in diameter, in the supply pipe about a foot above the place where it enters the ram. At every stroke of the ram a small stream will be discharged from this orifice. This at first sight would seem to decrease the power rather than augment it, but when the reaction takes place in the pipe there seems to be a small quantity of air sucked in, and this air is probably liberated from the water when it reaches the air chamber, thus increasing the pressure. At least this seems to be the most feasible explanation. Certain I am that I have repeatedly tried this plan, and find it to increase materially the power of the ram."

Wind-mills.-These have for a long time been employed as a motive power for the various purposes of farm use in many portions of Europe. In this country their use has been, until recently, confined almost exclusively to California and some of the other Western States. They are at present, however, being introduced to other sections, where they prove very cheap and efficient aids in various ways, such as pumping water, grinding grain, threshing, sawing wood, shelling corn, cutting hay, straw, or other fodder for cattle, churning, turning a grindstone, etc., including nearly all that can be done by hand or horse-power.

In many portions of Europe they are used both for draining and irrigating lands. They are especially useful for pumping water, or any similar work that does not require the con. stant attention of a person. They require neither food nor fuel, work constantly except in calms, and are self-regulating. They can run during the night as well as day, performing such labor as does not require attention, such as pumping water, grinding grain, etc.; con. sequently this will make up for loss of time in calms. But they are at the present time so perfected in their construction as to run with ease is very light winds.

By the use of a windmill, water can be furnished at any desired place, and by making a tank sixteen feet long, eight feet wide, and two feet deep, a reservoir of nearly sixty barrels can be kept filled by a mill, which will be a supply for occasional calms. The principal objection to wind-mills has hitherto been their liability to get out of order, or blow down in a heavy gale, and in not supplying a constant power. But these objections have been in a great measure overcome by improvements that have been made, combining strength and lightness, with an adaptability to utilize the lightest winds, and to be self-adjusting in a severe storm, so as to prevent injury. They are without doubt the cheapest power the farmer can employ on the farm. 


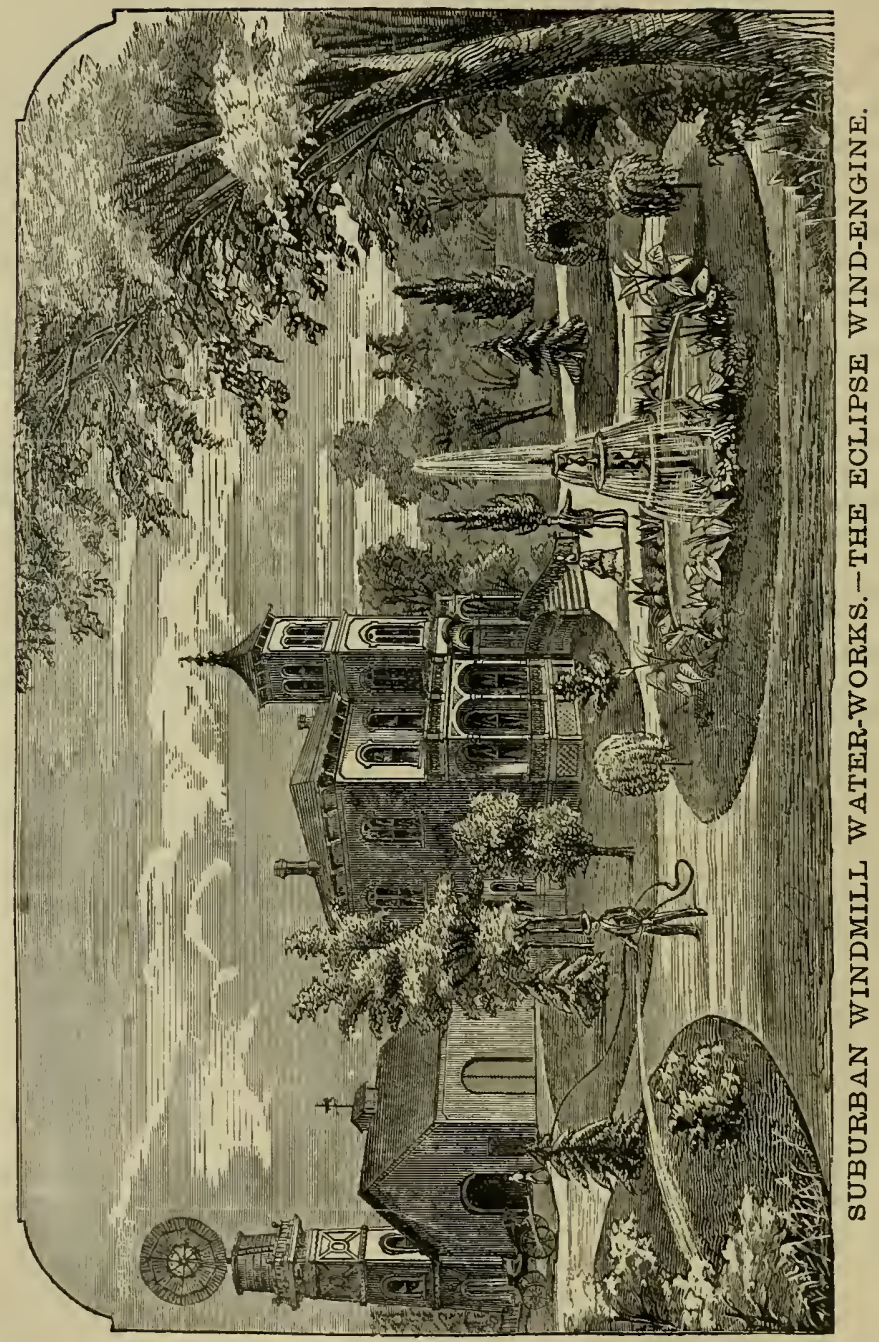


President Barnard of Columbia College, New York, says: "They adapt themselves admirably to the circumstances of sparse settlements, in prairie districts, and low alluvial regions where streams are few and sluggish, where fuel is costly, and where the population, chiefly engaged in the cultivation of the soil and living in comparative isolation from each other, find the conversion of their grains into flour and meal for domestic use a serious tax upon both their time and means. To such, it would be very useful for the elevation of water for drainage, for grinding, and many of the other exigencies of rural life."

And not only are they adapted to such localities as President Barnard has described, but

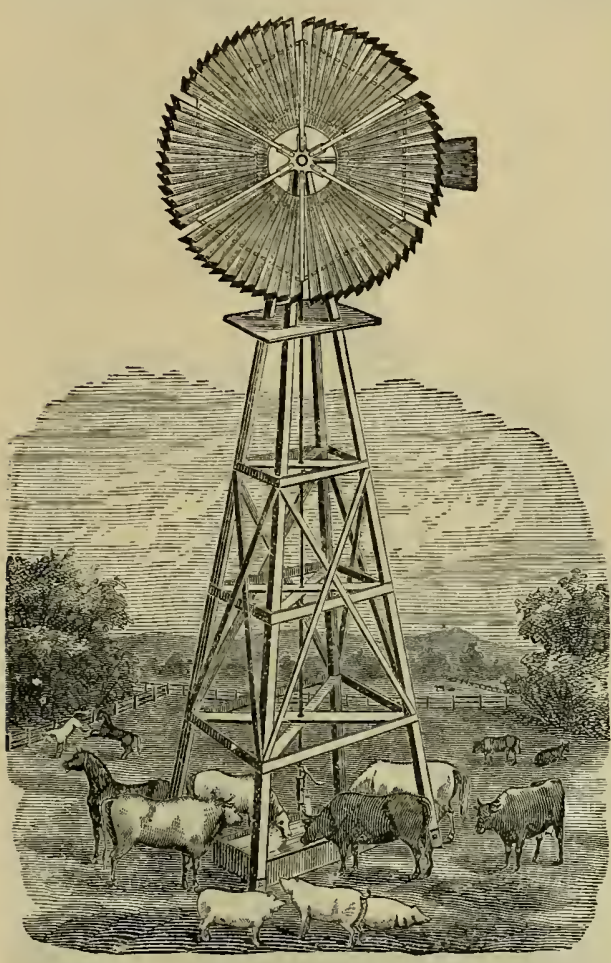

IN MOTION. they can be finished with such taste as not to be an inappropriate attachment to the elegant suburban buildings of any country gentleman, by means of which a cheap water supply can be obtained for the house, garden, stable, fountain, fish-pond, lake, and various other uses.

The water can thus be brought from well, spring, or river, at almost any distance, and delivered in the house, barn, or elevated tank in a tower, from which it may be drawn for any desired purpose. Towers can be very ornamentally constructed for the double purpose of water supply and observatory in the upper portion, while studios or readingrooms can be made on the first and second stories of the tower. They are made to run quietly, with no annoyance of noise or rattle of any kind, while a boy can easily keep one in order.

The foregoing illustrations of the Iron Turbine and the Eclipse wind-mills, by Mart, Foos, \& Co., Springfield, Ohio, and the Eclipse Wind-Engine Co., Beloit, Wisconsin, respectively, show admirably the construction and working of such farm machines that are becoming at present so common in many sections.

The above cut represents a front view of the latter wind-mill, when facing full in the wind. On the right appears the small side vane (the rudder in the rear not shown), which is attached to the same casting as the wheel, and against which the wind, as it increases to a gale, blows, and acting as a lever, takes the wheel around to the side of the rudder out of the way of the wind, in the same way that a door, if not latched, blows open and presents no resistance to the wind.

The figure on the following page represents the mill at rest, when pulled out of the wind or closed by a storm, no part of which presents a resistance to the wind, except the edge of 
the wheel and tail, consequently there is very little strain upon it even in the severest gale. It regulates itself in turning out of position by simply raising a weight, which brings the wheel back again when the wind dies away, on the same principle that a gate will shut itself when hung with a weight. When the utility of wind-mills becomes better appreciated by the farmers of the country, they cannot fail of being used quite extensively in the varions employments pertaining to the farm.

Use of Siphon in Water Supply.-The use of the siphon has long been known and applied to many valuable purposes. It consists of a bent tube, one portion of which is longer than the other, and can be used in conducting liquids from one place to another, when the place of discharge is lower than that from which it is taken, and the air from the tube is exhausted. In this manner, liquids may be conducted from a higher to a lower level, over an obstacle, provided the height be not more than that of the fluid column the atmosphere can support above the higher level, like drawing the entire contents of one barrel into another when on nearly the same level, by inserting the siphon into the vent of each. The siphon can be used to great advantage in conducting water to a house or barn from a well or spring, when they are located a little higher than the building they are intended to supply. This is the cheapest and simplest agent for transferring water, where the circumstances will admit of its use. When once properly arranged, there is nothing to get out of repair, hence, is a permanent water supply.

A gentleman in Carlisle, Iowa, has recently succeeded in supplying his stock-yards with water from his well by the use of a siphon. He dug a well in front

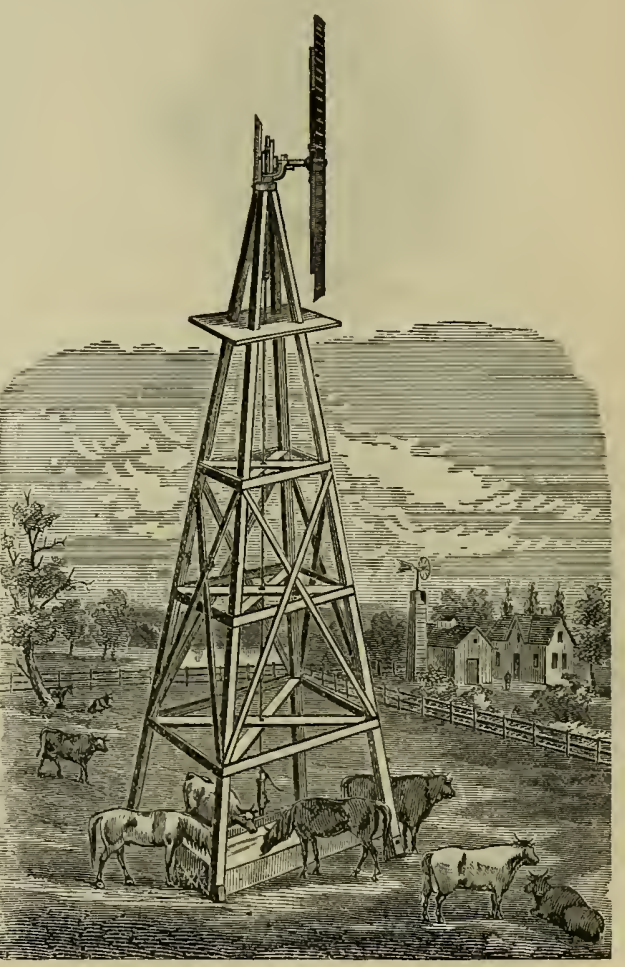

AT REST.

of his barn, and struck a large vein of water at ten feet. By putting in a half-inch lead pipe from the water up over the top of the well, and extending it two hundred feet down a hill to the corner of a stock-lot, a continuous stream, supplying about nine hundred gallons every twenty-four hours, was secured.

Mr. E. L. Lawrence, chief director of the Illinois Industrial University, gives the following result of an experinient made not long since on the farm connected with that institution: "The subject of obtaining water for stock being one of vast interest to the stock-growers of the West, I propose to give the result of an experiment made on the farm of the Illinois 
Industrial University, with a view of obtaining a pure and permanent supply in a more economical way than by the usual manner of a wind-mill.

- At one side of a lot, recently seeded and used as a pasture-field, is a well nine feet deep, with a supply of water varying from three to four and one-half feet. On making a survey, I found that, by going 700 feet, and making an excavation of three feet, a point could be reached one foot lower than the bottom of the well. This passed over an elevation some five feet higher than the surface of the ground at the well, and would give a maximum fall of about six feet, when the tank I proposed to construct was empty and the well full; and I estimated an average fall of three feet. By calculation I estimated that by the use of the siphon-using a one-half inch gas-pipe, placed two and one-half feet below the surface, thus giving a total rise of six feet, and a fall as stated above-a sufficient amount of water would be discharged to supply fifty head of cattle.

In July last this plan was carried out and put in operation, and now has been going three months, and I can safely say that it is a perfect success, as we have pumped no water from that date to the present.

To prevent mud and water in the space excavated, eight by twelve feet was planked over at the side of the tank, with tile laid under the plank, connecting with a previously-laid drain, one hundred feet farther down the slope; and a gas-pipe from the top of the tank was run through the plank and into the drain, to carry off any overflow of water. Later, a float was arranged to stop the flow when the tank was filled, thus preventing any waste of water.

As in these times the cost of everything is a large item, I will give it in this case:-

Tank, holding 12 barrels, . . . . . . . $\$ 9.75$

700 feet $\frac{1}{2}$-inch gas-pipe $@ 3$ cents, . . . . . . $\quad 21.00$

Digging and filling 700 feet of ditch, . . . . $\quad$. 9.00

Excavating for tank, fitting pipe, etc., . . . . $\quad 15.00$

Total, . . . . . . $\$ 54.75$

To remove the air from the pipe, an old thrown-away iron pump was used; and this is left in position to force out any sediment that may settle in the pipe. A common cistern. pump, such as is usually used in sinks, costing about three dollars, would be sufficient for this.

Great care must be taken in the use of the siphon to exhaust all the air from the pipe, and to so arrange the end inserted in the well or spring, that sand or mud will not be liable to get into it and obstruct the water.

The water will sometimes become obstructed in the siphon on account of air getting into it. All running water contains more or less air. This air will naturally accumulate at the upper end of the siphon, and when the pipe becomes filled with it, the water stops running. When this occurs, the only thing that remains to be done is, of course, to remove the air. A vertical branch may be inserted in the top of the siphon (the highest point in the bend), having one or two stop-cocks. If two are used, a space of a few inches of tube should be left between the two. When air accumulates in the bend of the siphon, open the lower stop-cock, and let the air up into the tube above it, which should previously be filled with water. When the air fills the tube close the lower stop-cock, and open the upper one, and pour in water to fill the tube again. By repeating this process the air can all be removed. Care should be taken, in inserting the branch, that it does not extend into the tube to impede the current of the water. It should only extend to the upper face of the inside of the tube. This branch should have hay, leaves, sawdust or some other material packed about it, in order to be kept from freezing in winter. When a single stop-cock or a screw-cap is used for the same purpose, the two ends of the siphon will need to be plugged up, after which water should be poured through the branch. When the air has escaped, close the branch, unstop first the upper and then the lower end of the siphon. A stop-cock at either end of the siphon will prove a more ready and easy means of opening and closing it. 


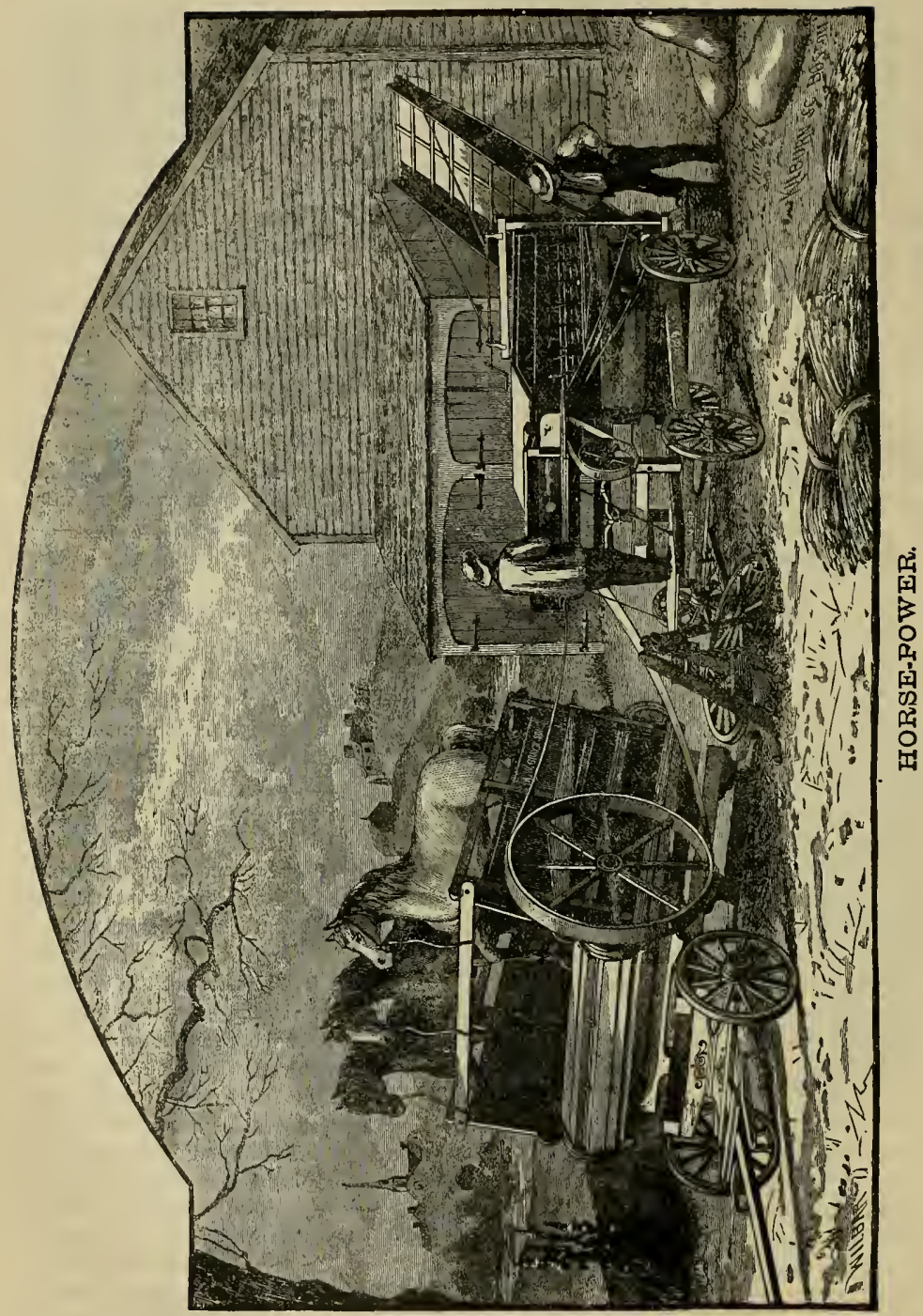




\section{MOTIVE POWERS FOR FARMS.}

A GOOD motive power is a great convenienc: for both large and small farms, as well as often a necessity. Such powers are valuable for pumping water, churning, sawing wood, grinding grain, cutting feed for stock, threshing, shelling corn, and various other farm purposes. The principal kinds in common use are horse-power, wind, and steam.

Dog and sheep powers are often employed for light purposes, such as churning, etc. As steam-engines are expensive, and water-power rarely available on a farm, the powers that can be employed for an ordinary-sized farm at a limited expense are either horse-power or that furnished by a windmill, either of which will do valuable service and economize in the expense of labor, some farmers finding it more profitable to use the former, and others the latter.

Horse-Power.-As horses are kept for use on most farms, they furnish a very convenient power for many purposes, while they possess the advantage of being a power that can be transferred to any locality on the farm or off from it, as desired, and also one

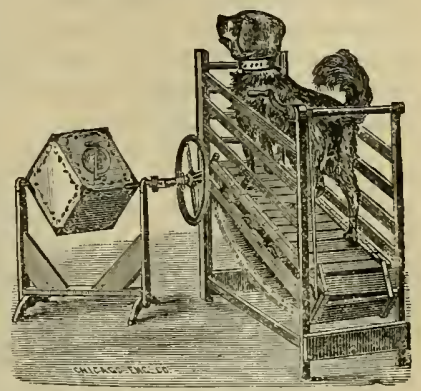

DOQ-POWER. that comes within the means of most farmers. A horse-power is easily regulated, and readily attached to any machine that is desired for use. It is also a very inexpensive power, and can be used at any time, while wind is variable and can be used only at certain times. Each power has its own especial advantages over others. A wind-engine, once located, requires no further expense but to merely keep it in order, which is slight, while it is the only available power that can be used in pumping water in distant fields. Steam-power can be either stationary or movable, but for small farms is too expensive to be made profitable, while on very large farms it becomes a necessity. The power to be used on any farm must always be adapted to the amount and kind of work to be performed.

The machinery for horse-power is generally so constructed as to be adapted to the use of one, two, or three horses, as desired, and according to the amount of power required. That represented on the previous page is by the well-known firm of A. W. Gray's Sons, Middletown Springs, Vermont. There is an endless platform on which the horses walk. This platform is made of pieces of plank of suitable length fastened together on the under side by a wrought-iron gear connected by cast-steel rods, which also serve as axles for rollers, and move with the platform, running on a cast-iron and steel track.

Wind-Power.-In some portions of the country, especially the Western States, wind. mills have for some time been used quite extensively for furnishing power for many farm purposes. They are becoming more common in other sections, as has been stated in previous pages (See Windullus, page 537), and are one of the most economic powers known. Wind is variable, it is true, and cannot always be relied upon, and a wind-mill is confined to a cer. tain locality, yet the power it furnishes is one that never tires, and when the winds are favorable it can work both night and day, often unattended, performing such labor as pump. ing water for distant pastures or household use, grinding grain, etc. For the former purpose it has no equal, and by having a large tank provided, a supply of water can be constantly kept on hand in all seasons for use when there is not sufficient wind for running it, while it can be so regulated as to be self-adjusting, ceasing to pump when the tank is full and commencing again when it requires filling. 
Steam-Power.-For large farms, such as many of the extensive ones in the Western States and other portions of the country, steam can be used to great advantage as a motire power, and a steam-engine becomes one of the necessary farm implements. Steam-engines for such purposes may be stationary or portable, each having their peculiar advantages; the latter for most uses being, of course, the most desirable.

The past few years lave been conspicuously marked by the extensive increase in this country in the use of steam-power in connection with agriculture, and numerous manufactories of farm engines have sprung up throughout the different sections, showing the largely. increasing demand for such power. The introduction of steam-engines on farms has been greatly facilitated by their cheaper and simpler form, the various improvements made, besides greater ease in their management.

The smaller and iower-priced engines of two or three horse-power may be used for cutting or grinding feed, pumping water, sawing wood, driving small threshing-machines, and churning in large dairies, while the larger ones are extensively employed for large threshingmachines, for itinerant work, or for extensive farms. We learn from reliable sources that twenty or more large agricultural engines are in use by Mr. Dalrymple in his operations in wheat farming on his extensive and noted farm, commonly known as the "Dalrymple Farm," in Dakota, and which contains about a hundred thousand acres. A good stationary engine for farm use can be procured at a comparatively low price, and in those portions of the country where fuel is cheap and abundant they may be used quite extensively as a motive power for driving various farm machines. Of course, it is essential to safety and the successful use of an engine, that the person having it in charge should perfectly understand the business, and use it with discretion and care, to prevent accident.

Engines may be made nearly self-regulating, and thus less liable to explosions; but in any case, if the manager of it is careless or indifferent, the machine might be soon ruined, and its owner meet with a heavy loss in consequence. Efficient and careful management, in any department of the farm, pays best and brings in the end the most satisfactory results. An engine may also be utilized in steaming food for stock, cooking roots, etc., when desired.

Steam-power has been used, to a considerable extent, as is commonly known, for plowing in England, for several years, but to only a limited extent in our own country; yet $\pi \theta$ predict that the time is not far distant when most of the plowing will be done by steam on the larger farms, such as are found on the extensive prairies of the West, or on the large plantations in many portions of the South. The testimony of large landholders in England who have used steam-power extensively is, that it is less expensive than horse-power; but, of course, it would not prove as economical for a small farm.

In many localities where the farms are not sufficiently large to warrant each farmer in the purchase of an engine or horse-power, the plan of mutual ownership can be profitably adopted. By this means, a few farmers doing a small business, whose lands are near or adjacent, may purchase the machine in company, and thus, at a comparatively slight expense, each may derive nearly as much benefit from it as though owning it entirely.

In other cases, a single individual may own a machine and make it very profitable in going from farm to farm doing itinerant work in threshing, etc., for his neighbors. When moring on the road, a self-propelling engine is usually guided by a pair of horses, as shown on the following pages, which represent engines manufactured by C. \& G. Cooper \& Co., Mit. Vernon, Obio. It is much cheaper and more convenient to guide or steer an engine on the road by one or two horses, than by any mechanical means; besides, the horses are a reserved force which may be employed to take the engine along in case anything should occur to render such assistance necessary, as, for instance, provided any part of the machinery should get out of repair on the way to the field. 


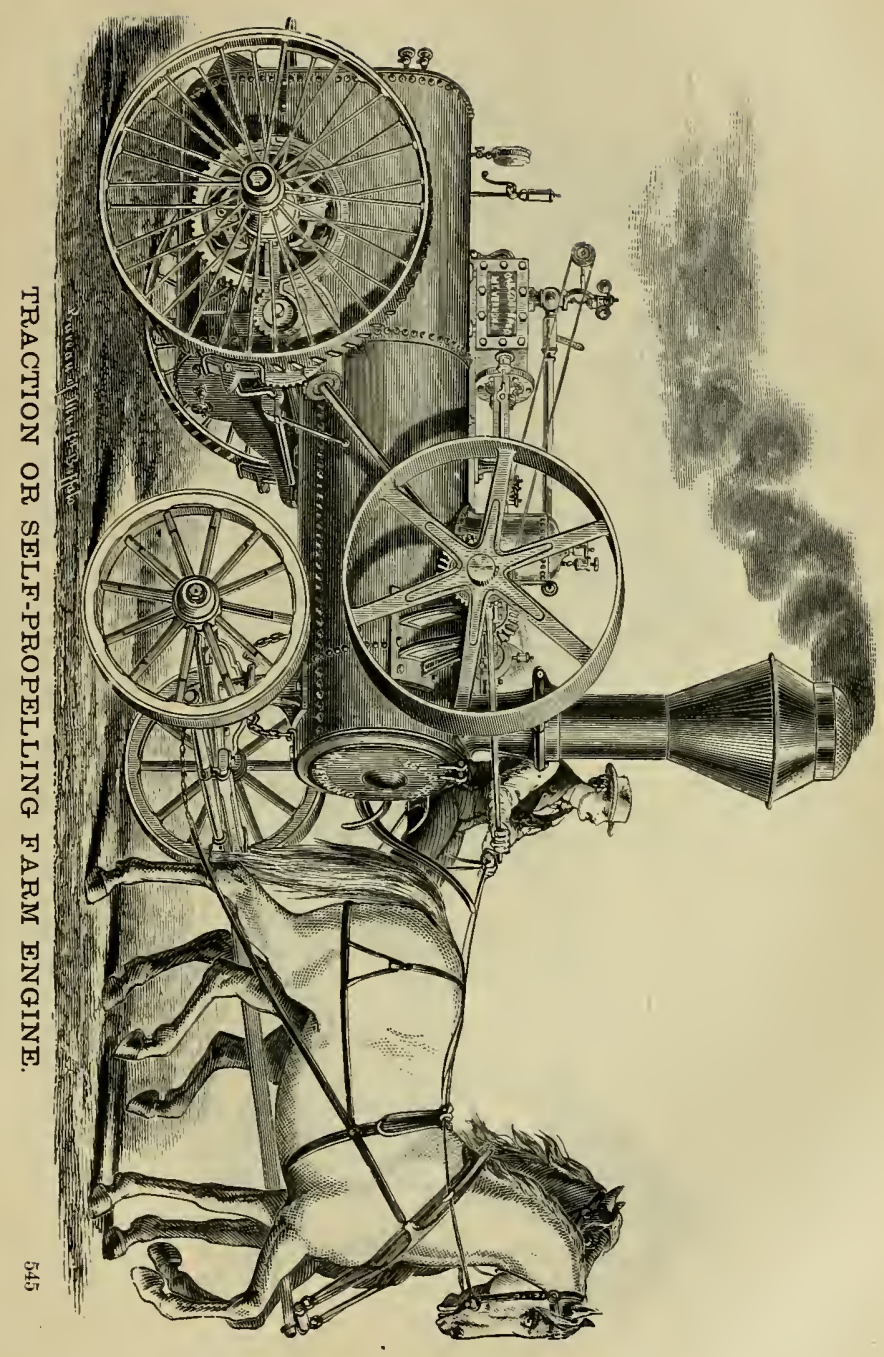




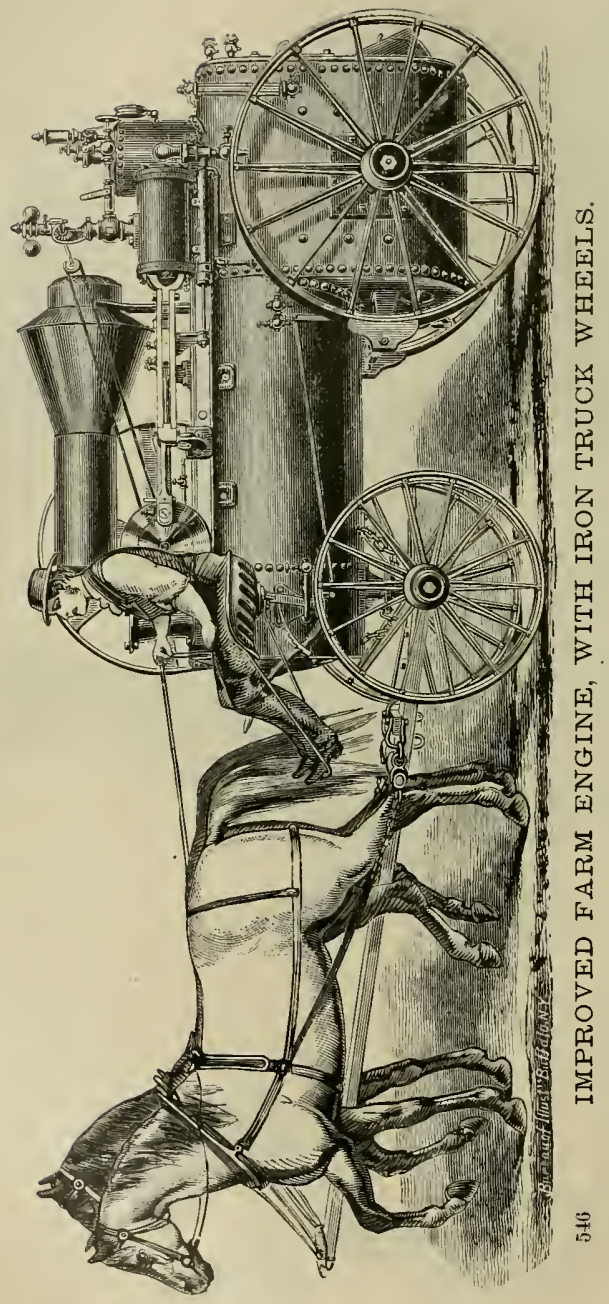




\section{FARM TEAMS.}

$\mathrm{A}$

TEAM of some kind is indispensable to every farm, however small. And since it costs no more to maintain a good team than a poor one, the former will always be found the most profitable. Whatever the kind of team employed, the animals selected for the purpose should be intelligent, tractable, gentle, strong and healthy. We will also add, that if to these qualities we add comeliness in form and color, we increase their value very materially, for no one is so blind to the sense of beauty as not to prefer to see about them animals attractive in appearance rather than those that are unsightly in color, ugly in form, ungainly, and awkward in movements, however desirable they may be in other respects. Some farmers seem to think that the ability to perform the work required is all that is essential in an animal for farm use, utility being the chief and only object sought; but inasmuch as comeli. ness is to be preferred to deformity and unsightliness in everything else, we see no reason why it should not apply equally well here. We remember an old farmer in New England that, in our boyhood days, used for several years on his farm a pair of the most unattractive oxen we ever saw, and yet the owner was always descanting upon their rare merits for work. One was a brindle with a wall-eye and short lopped horns, the other a red-and-white spotted animal, with wide branching horns and a hollow back, the sight of the two together being about as good a combination of ugliness as could well be imagined. We recall another honest old farmer who drove for years a horse so ill-looking that it was enough to give one the nightmare whenever the beast made its appearance, and yet it was a valuable animal for work. Great improvements have been made with respect to farm teams, as with everything else pertaining to agriculture, since that period, and yet we frequently see too much indifference manifested by some farmers in this respect. Let us not only have good and valuable teams for service on the farm, but let us have those that are pleasing to look upon, as well. The principal animals used for the purpose of farm labor are the horse, mule, and ox.

The horse is usually considered the best for all general purposes, and is employed more extensively for farm work than either the mule or ox. For most uses the horse or mule is better than the ox; still, there are places where an ox-team can work to much better advant. age than either of the former. Horses are employed more extensively in New England for farm work than they were from twenty to thirty years ago, and seem to be gradually taking the place that the ox-team formerly occupied there; but in many sections oxen are still preferred.

The mule is an exceedingly strong animal, and possesses remarkable powers of endurance; besides, it is easily kept, and is very long-lived. It is sure-footed and, when worked singly, will often be preferred to a horse for some kinds of labor. They are also quicker than oxen on the road, and for this purpose are greatly preferred to the latter. It is, however, difficult to keep them in an enclosure, as they will leap any ordinary fence. When kept on a farm, it will be almost a necessity to keep them stabled for this reason, when not at work. Mules are used quite extensively in connection with horses, both in the Southern and Western States. Oxen are very useful for heavy kinds of work that require strength, patience, and docility, such as plowing rough lands, pulling out stumps, drawing off heavy stones, etc. For plowing on rough, stony lands or on steep billsides, they have no superior. They are slow travelers when compared with the horse or mule; still, they are generally very patient, docile, and reliable, "as patient as an ox" being an old and trite maxim.

Where teaming is to be done to a considerable extent, horses or mules would do better service than oxen, since the latter are so slow in getting over the road and consequently require so much time. Horses are more expensive to keep than either mules or oxen, and the cost of purchasing a good team of horses is considerably more than that of a good ox or mule team. 
The kind of team that is best for the farmer to keep will depend entirely upon his circumstances, and the character of the land he is to till. On some farms oxen, as we have seen, would prove the most inefficient team the farmer could employ, while on others they would prove the most efficient and profitable.

Horses and mules are indispensable in the extensive grain-growing sections of the coun try, where a delay of a few days in the sowing or harvesting of the crop might be attended with great loss. On a small farm, where the farmer cannot afford the expense of keeping horses or mules, a good ox-team may perhaps be found most desirable, all things being considered.

The expense of keeping a pair of horses or mules shod during the year will exceed that of oxen. The necessary outfit of harnesses, wagons, etc., for a horse or mule team will also be considerably more than that required for the ox-team, while the feeding expense of horses and mules is greater than that of oxen. The horse and mule will depreciate in value as they grow older, until they finally become worthless, while an ox may be readily fattened and converted into beef, bringing a good price in the market.

On the other hand, one horse is almost a necessity, even on a small farm, for use in travel, and it might be that some farmers would be so circumstanced that it would be more profitable for them to keep an extra horse to complete the farm-team than to keep in addi. tion a pair of oxen. Each farmer must consider his own individual circumstances and decide accordingly, as to what team is best adapted to his use.

The number and kind of teams should always be suited to the kind and amount of work to be performed. On large farms. where a large amount and variety of labor is to be performed, a large number of teams will be required. It will not be economy, however, to keep more animals for this purpose than are needed on the farm.

Whatever the kind of animals used for farm-teams, they should always' be humanely and kindly treated. They are the farmer's patient and willing servants, constantly doing his bidding in performing tasks for him, wearing their lives out day after day uncomplainingly in his service, and as such are entitled to kind consideration and care. He who abuses such faithful servants, or permits those in his employ to do so, is guilty of base cruelty, and does not deserve to be benefited by their labors. There is no farm animal but that can be best managed by kindness, while with some, such as a high-spirited, sensitive horse, blows and harsh words are often absolutely ruinous, and always greatly lessen the money value of the animal thus treated.

We have known many valuable horses to have been rendered vicious and worthless by such means. Very few animals are naturally vicious, the majority of those that are so having been made thus by improper management. To some animals harsh words are almost as abusive as blows and kicks. The man who abuses in any way the innocent creatures in his power deserves to change places with them, and learn from experience the suffering caused by such treatment. Aside from the humane consideration, animals kindly treated are much more valuable and profitable to their owners, being more tractable and reliable; therefore for this reason, if for no other (although the humane principle should be the leading one), every farmer should feel personally responsible for the treatment his animals receive. 


\section{ROADS AND ROAD-MAKING.}

$\mathrm{T}$ HE importance of having good roads cannot well be over-estimated; and since the subject of roads and road-making is one that pertains to the personal interests of every farmer, either directly or indirectly, and roads of country towns are made and kept in repair principally by farmers, it may not be inappropriate to give a few practical suggestions relative to it in an agricultural work designed especially for farmers.

In so doing, we give, as most applicable to the purpose, the larger portion of an address on this subject, delivered before the Massachusetts Agricultural Society not long since, by the editor of this work, omitting those parts that may be mainly of special local interest:

It would be easy to show the importance of this subject by referring to the intimate con. nection which exists between the value of land, the general prosperity of the community, and the condition of its roads. Every farmer, every owner of real estate, has a vital interest in the perfection of the roads which lead to it, and especially the roads which lead from it to the natural market. And this interest is a pecuniary one, not based on considerations of comfort merely, but coming home to the pocket in the shape of the wear and tear of teams and vehicles, and the amount of draught required to move a load, always dependent, to a very large extent, on the character and condition of the surface of the road, and in the enhanced value of a farm lying on a thoroughly good bighway. So true is this, that it is no exaggeration to say that there can be no better test of the progress of civilization in a community than the condition of its roads. This close connection of the prosperity of the people and the perfection of the means of communication between one community and another, is so well understood in Europe that good roads may be said to be the rule there, and poor ones the exception-just the reverse of what we see everywhere in this country. Nor do we think it too much to say that the poorest road we saw in Switzerland, Germany, and France is better than the best of our own.

It is safe to say that the common roads, though the original cost, mile for mile, may have been much less, are vastly more important to the population of the State than the railroads. It would be difficult to do without railroads, to be sure, and no doubt put us to untold inconvenience, especially as we have become accustomed to their use; but it would be quite impossible to do without common roads. They are indispensable to a civilized community, and may be reckoned among the necessities of life.

Location of Roads, etc.-In proceeding to treat upon this subject, we shall have but little to say in regard to the location of our roads, since, bad as they are in many cases, their line was long since determined, and new roads to be laid out will always bear a very small proportion to the old ones. It ought to be borne in mind hereafter, however, when any new road is proposed, that any reasonable amount of money spent in procuring surveys by the most competent engineers, will be the best investment that can be made, rather than to run the location by "guess-work," and perhaps eventually involve a vastly larger expense in building and grading, than a well.considered location would have cost.

Our roads are neither laid out properly nor constructed as roads ought to be. The first settlers pushed off into the forest to seek new lands, and naturally built in elevated situations to avoid the miasms of swamps, and for the purposes of protection or greater safety. Their ways were foot-paths or bridle-paths cut through the woods, till they could clear up and make passable roads, and when a town finally grew up, the roads were naturally made to accommodate its individual citizens. Of course it could not be expected that they would be laid out in accordance with any fixed principles, or with any reference to the wants of a more advanced civilization. The greatest good of the greatest number was sacrificed to individual interest, or the convenience or caprice of a single person. It is time now that we had some- 
thing better. And let us not boast of our times till we have better means of communication. It is a principle perfectly well established among engineers, that roads should be so substantially constructed that the cost of maintenance shall be reduced to the minimum. The fundamental principles of formation and construction should be studied and understood by every road-builder, and observed in all localities, though they may not admit of so complete application iu a thinly-settled district as in the vicinity of a city where the travel is greater, and the means of construction more abundant.

One of these general principles is that the nearer the location of the road approximates to a straight and level line, the better; but a straight line which does not at the same time admit of a level profile will ofteu lose the advantage of being the shortest distance between two places. It did not occur to the projectors of our earlier roads and of our turnpikes, that the handle of a pail or a kettle is no longer when it lies at rest in a horizontal position than when held upright. They did not know that while a horse on a level is as strong as five men, on a steep hill he is not so strong as three; for three men with a hundred pounds each will ascend a hill faster than a horse with three hundred pounds. Straightness of line should always be sacrificed to obtain a level or to avoid a steep and heavy grade.

A road curving around a hill will often be no longer than a straight one over it, for this latter is called straight only because its curvature is less apparent to the eye, and compared with a horizonal plane it is decidedly crooked. And after all, the difference in length of a straight and slightly curved or winding road is small, for taking two places ten miles apart with a road curving so that you could nowhere see more than a quarter of a mile of it at once, and its length would exceed a perfectly straight road between the two places by only a hundred and fifty yards.

It has been laid down as a general rule that you may increase the length of a road to avoid a hill to twenty times the height that is to be saved by such increase; that is, to save a hill a hundred feet high, it is better to go two thousand feet around it, ald even then you'll find "the longest way round the shortest way home." We see, therefore, that straightness, though very desirable when it can be had, is by no means the highest characteristic of a good road. It is far more important that it should be level, for unless we have a level surface, a large part of the strength of the team must be spent in raising the load up the hill, in addi. tion to the friction to be overcome. To draw a load up an incline, the resistance of the force of gravity is as great an addition to the whole weight of the load, as the height of the incline added to its length, so that an incline of one foot in twenty requires the team to lift up by main strength one-twentieth of the whole weight in addition to overcoming the friction caused by the entire load.

But leaving the location and the construction of new roads, as coming more properly within the province of the professional road engineer, we call attention to a few of the more striking and common mistakes in the mending and care of country roads, faults which seem to arise from a want of knowledge of the first principles of road-making on the part of those intrusted with the supervision of the highways.

Form of the Road-Bed.-Over a gravelly and hilly country, and over a flat country with a stiff or clayey soil, no one would hesitate to say that the road-bed should be raised above the level of the sides, and crowned sufficiently to shed the water; but the error, astonishingly prevalent, is to finish them in a convex curve forming an arc of a circle with the center raised a foot, and often eighteen inches or more, and the curvature at the sides so abrupt as to make it dangerous to turn out on meeting a carriage, and always giving the driver a feeling of insecurity. We do not refer to the elevation above the surrounding land, but simply to the shape of the road-bed, the elevation of the center above the sides, or what might be called the "transverse profile" of the traveled part of the road itself.

To show that this is no uncommon occurrence, we may mention that the county commis- 
sioners of one of our large counties, only two or three years ago, in making the specifications for a road only twenty feet wide, required that the road should "crown" in the center no less than eighteen inches, or one and one-half in ten, and no amount of reasoning could lead them to reduce this enormous convexity. Is it not time for the law to step in and define what the transverse profile of a road ought to be for a given width?

Let us see the results of this serious error. The convexity is so great that the center of the road is the only place where a carriage stands upright. The travel, therefore, ciings to the middle of the road, wearing one path for the horse, and two ruts for the wheels, thus wearing the road down very unevenly.

The water, therefore, invariably stands on the middle of the road, while it is constantly washing away the sides. A road ought to be formed so as to indnce travel over all parts of it. But with this great convexity, whenever a carriage is compelled to turn to the sides, it causes great additional wear on account of sliding down the sides, while by this sliding tendency, being at right angles to the line of draught, the labor of the horse and the wear of wheels is very greatly increased. The evil of too great convexity is manifold, and a vastly better form is that of two inclined planes meeting at the center, with the angle of junction at the top slightly romnded by a curve. Of course the exact inclination will depend much on the character of the surface, and the width of the road. A very rough and bad surface will require a greater incline than a hard smooth face, but no road should ever be allowed to be so rough as to require a transverse inclination greater than one in twenty, which, for a road. bed twenty feet wide, would make the center six inches ligher than the sides.

With broken stone or a hard nnyielding surface, a proper medium of one in twenty. four is adopted, or half an inch to a foot. Telford, the most successful and noted road-builder of England, adopted one in thirty, or six inches curve in a road thirty feet wide, and MacAdam fixed one upon one in thirty-six, and sometimes as slight as one in sixty, or only three inches crown in a thirty-feet road.

The transverse slope should increase with the longitudinal inclination, and should always a little exceed it in order to prevent water from running down the length of the road to gully it out, but it must be apparent that no practicable amount of crowning or convexity would serve to carry the water from the slightest rut, not even if it were only an inch deep. And hence MacAdam testified before a committee of Parliament, saying: - "I consider a road should be as flat as possible with regard to allowing the water to run off it at all. I have generally made roads three inches higher in the center than at the sides, when they are eighteen feet wide." Now, a dirt or even a gravel road may require a little greater inclination than the solid surface of broken stone, but if the road is so neglected as to have a soft or loamy surface, no amount of convexity will shed the water, and a very convex surface will invariably hold the more water.

It should be constantly borne in mind that any convexity at all is a necessary evil, and that the less that it can be made, and accomplish its object, the better for the travel.

Removing Obstructions from Roads.-Analogous to the previously-mentioned fault is the practice, often seen on a wide road and through a village street, of dumping down along the center of the highway a kind of winrow of material, whether loam or gravel, eight or ten feet wide and from six inches to a foot thick, and sometimes more in the middle, designed to form a crown to the road. If you ask what that is for, you will be likely to be told, that it will all flatten down in a few months, and that it is the best way to drain the water off. You will observe that it drives the teams off to one side, often corn. pelling them to cut up the grass along the gutters. It requires no argument to show that this is all wrong, both in principle and in fact, for this mass of stuff acts more like a sponge than like a duck's back, and you can never expect to make a permanently good road by leaving the surface in that way. 
And this leads to another most common defect, which arises from the custom of semiannual repairs, and that is the neglect to pick up and remove the small loose stones that are constantly working up through the improperly-applied material to lie on the surface, to hammer up the road-bed at every blow of the wheel, and to endanger life and limb. Hard, firm rocks projecting above the surface are bad enough, and cause the resistance of collision; but other inequalities, loose round stones and other loose materials, striking against the wheels, are far worse, for they cause great loss of momentum and waste of the power of draught, for the carriage has to be lifted up over them by the leverage of the wheels. Any town that fails to remove such obstacles promptly and often, ought at least to be reported to the Society for the Prevention of Cruelty to Animals. No money can be better invested than in frequently removing the loose stones always to be found in a badily.constructed road, and in snow ploughs for a prompt removal of snow in winter.

Drainage for Roads.-Perhaps the most common defect to be observed in the methods of making repairs upon roads is the total want of any proper attention to the drainage. You will see whole miles of roadway perfectly water-logged in spring, making it very difficult for light carriages to pass over them, and for heavily-loaded teams quite impos. sible. The treatment for such sections requires to be radical.

They need to be reconstructed, and it requires quite as much skill and judgment to reconstruct properly roads that have been badly built, as it does to make good roads in the first place, and probably more. But drainage is one of the things that can be carried out in the course of repairing without any very serious outlay over and above what it would have cost to have drained them properly in the original construction of the roads.

There is no one point in which our highways are so lamentably defective as in being wet at the foundation. They nced thorongh drainage as the first step to any possible improvement in their permanent condition, and thorough drainage alone will in many cases make a good road out of a bad one, while without it no amount of labor will result in pcrmanent improvement.

In many cases, hundreds of cartloads of gravel will be dumped in to fill up a sinking slough, when perhaps half the money spent in drainage wonld have remedied the evil.

As a general rule, there ought to be two independent systems of drainage for most com. mon roads, one to control the surface water by means of side ditches and culverts wherever needed, and another to drain the foundation on which the surface or shell of the road-bed rests. For this latter, under-drains are most serviceable and properly-laid tile-drains on the whole the cheapest, because they are most durable and effective, and, being laid below the frost, they continue to operate when the surface is frozen, and allow the road to settle when the frost comes out of the ground in spring without an entire breaking up of the surface coving, to the infinite inconvenience of the public. Of course, this work, wherever it is done, is in the nature of a permanent improvement, and could not generally be undertaken by a small and poor town on all its roads at once, but by taking a portion, or the worst portions, from year to year, and doing them well, the roads in such a town would, in the course of a few years, begin to wear an entirely different aspect.

Catch-Waters, or Bars.-One of the very common errors in the manner of constructing catch-waters or bars on steep grades, and one which often causes the traveller no little inconvenience, is to make them too high, and crossing the road often diagonally, so that the wheels strike them at different times with a shock sometimes sufficient to unseat the driver. If raised too high, also, they become dangerous for the horse. They should be made in the shape of an inverted $\mathrm{V}$, with the point directed up the ascent, so as to divide the water. 
Repairing Roads.-Another very serious mistake in mending our roads, or rather in attempting to mend them, is to plow up the side ditches and throw the material, sods, sand and manure, which the rains have washed off into them, back into the center of the drive-way. Absurd as this practice appears, it is quite too common in our country roads, and that, too, in many cases where good road-material is easily accessible. The consequence of it is, that the first rains convert this loose organic material, vastly better for a top-dressing for grass than for the surface of a road, into a perfect slough of mud, and a hard rain washes it back into the ditch. In a dry season this material becomes a perfect bed of dust, annoying to the traveler, destructive to vehicles, and about as bad as the mud itself. No strength of language is adequate to do justice to the iniquity of this bad practice, and the surveyor who allows it ought to be complained of as an enemy to society.

Nothing is more certain, nothing better established by the experience of engineers and of practical men, than that a solid and unyielding foundation is one of the first requisites for a good road. And yet, to throw such material as sods and sand and loam into the road from the sides, even if it is designed to cover it with a coating of gravel, is utterly destructive to the foundation of the road. All such stuff should be carefully thrown out of the road-bed, as the first and most important step in laying the foundation. The loose stones that have from time to time been picked from the surface and thrown aside to be an eyesore to every inan of taste who travels there, constitute an infinitely better road-material than the soil on which they lie. Sods and turf are often deceptively tough, and they seem "so handy" to fill a hole or a rut with, that they are used for the purpose without considering that they rapidly decay and work down into soft mud. But some go to the other extreme and fill up the deep ruts with stones, put in and covered up in such a way as to conceal them at first, but so that they never wear uniformly with the rest of the road, but appear in hard ridges and bumps.

And here we must condemn the promiscuous use of the plow and the scraper in repairing roads. Common as they are, they should never be used in crowning up a road from the sides, and perhaps the only place where they should ever be tolerated on the road is in loosening and removing the tops of hills to reduce the grade by taking off the gravel, for their work, though large in quantity, is very poor in quality, and, in fact, destructive to the condition of the road; for the one breaks up the surface and the shoulders of the road which time and travel may have solidified, while the scraper drags up from the side ditches the soft alluvial matter previously washed into them, and leaves it upon the road, the very place of all others where it is never wanted and never should be allowed.

We shall find, on inquiry, that the most conmon reason given as to why this vegetable matter is used is, that there is no suitable material handy. In some cases, like sandy locations and where long stretches of country occur, destitute of rocks and gravel, there is, no doubt, some shadow of reason in this excuse; but we have often heard it where plenty of good gravel could be had, within a hundred rods, almost for the carting. And how easy it would be, in most sections, to remedy the difficulty by employing men by the year, to be always on hand to keep up the roads and to keep an abundance of material, crushed rocks, screened gravel, etc., on hand for un in various parts of the town.

Would it not be better economy for some towns to invest a few hundred dollars in a good stone-crusher and a heavy roller, to be kept on the town-farm for use on the roads, than to pay men a dollar or two dollars a day to stand out their road-tax, leaning upon their hoehandles upon the road? There are stone-crushers that will crush a ton of boulders an hour with a ten-horse-power engine, with the help of three or four men to throw the stones into the hopper and clear away the fragments.

Crushed stone forms one of the best materials for a road-bed, being firm and unyielding, and, at the same time, such as is easily kept in repair. A heavy roller, for solidifying the 
material and evening the surface, is essential in the proper construction and repairing of good roads.

Objections to the Common System Relative to the Construction and Care of Roads. - One of the striking evils of the present system, sanctioned by the statutes of many States for the building and care of roads, is the want of uniformity. One town takes a pride in its roads, spends money freely, adopts a progressive plan of operations, and really secures very passable highways; and if all the adjoining towns would do the same the traveler on a long line of main road could get along very well. But the next town, perhaps, shirks its duty to the public, works out its highway tax by labor (a plan most skillfully devised to accomplish nothing), does as little as possible to enable it to just graze within the letter of the law, and the great public has to suffer accordingly.

Now, we will see what will be the result. There may be a long stretch of road over which a team could easily carry a ton, or perhaps two tons. But in some part of the line over which the traveler has to pass, there is a long, steep, and rocky hill, up which the team can draw only a half or a quarter part as much as it can easily draw on a level, hard, and unyielding surface. The consequence is that on account of this one steep incline, or it may be more, the load can be only one-quarter or one-half as much as could have been easily drawn, but for such an obstacle. The teamster, therefore, loses a large part of the advantage of the good portion of the road, because he must reduce his load to what can be carried up the one or two miserable hills which he must chimb before reaching the end of his journey.

We have, therefore, the general proposition, that steep ascents, being always injurious, become especially so when they occur on a long road which is comparatively level. In such a case, it becomes vastly more important to avoid or lessen the slope, or else to perfect its surface. But it lies in a town which does not care enough about its roads to improve them by reducing the hill, and the whole community has to suffer. If it costs the teamster more to transport produce, both producers and consumers of that produce are obliged eventually to pay that cost.

And why should the condition of our great highways, which constitute so very important an element of the wealth, the comfort, and the safety of the whole public, be allowed to depend on the short-sighted views of economy, or perhaps the indolence or indifference of every small town through which the roads may happen to pass?

Another great objection to the present system is that it allows towns to elect a multitude of surveyors without reference to their competency, who cannot by any possibility manage the money appropriated with that degree of economy, comprehensive foresight, and wisdom of one thoroughly competent and skillful road engineer or superintendent.

The town meeting comes, and the people are called to vote for surveyors of highways, often without any previous consultation as to the competency of men to fill the position, perhaps by nomination on the spur of the moment, and many are chosen who have no adequate conception of the manner of performing the responsible duty assigned to them. Each has a certain district allotted to him, and not unfrequently, having an idea of fixing the road near his own place, he takes measures to procure the appointment for the special purpose of working on the road near home. The object is to do just enough to prevent the road from breaking his neck - an object altogether too selfish to admit of a proper regard to the public good. What better illustration could there be of the old adage that "what is everybody's business is nobody's?" Instead of doing a work which, of all others, has its times and seasons fixed by natural laws, they do it "when it comes handy," after the spring work is over, or at any other leisure time that will most suit their own convenience. There are, there can be, no continuous repairs. "A stitch in time" has no application here. The fact that a dollar judiciously spent in repairs in April, or when the frost is coming out of the ground, is worth more than two in June or July, and more than three, or even five; at a later date, is of no significance where this plan is adopted. 
Now, if this mode of management affected only the town which adopted and persisted in it, the evil would be of comparatively small consequence; but the main roads through a town are often great thoroughfares between other important points, so that the whole community suffers, to a greater or less extent, for the want of an efficient head to do the thinking and the planning for the roads in such a town.

The worst feature of the whole is, that no amount of ability or faithfulness displayed in the performance of the duties of a surveyor will insure his continuance in office over one year. If he does his duty by making a good road, he will be quite sure to lose the position. All his experience, study, and observation will be lost to the public when another takes his turn to undo what the former has done, and begins his apprenticeship at the expense of the public, and of the condition of the road itself. In other occupations, an apprenticeship, often of some years, used to be thought requisite to authorize a man to set up business; but a surveyor, the moment he is chosen, is presumed to be fit to direct works which often require much scientific attainment, great skill, and intelligence.

Besides, the hasty appointment of surveyors, and the assignment of districts to each, with a specific amount of money to spend, leads to another kind of wastefulness. Some districts may have money to spare from the want of any knowledge or inclination to put it into permanent improvements, while others have too little. In one district, teams will often be standing idle with a surplus of men, while, perhaps, in another there is a want of both. How can you expect any harmony of action with twenty or thirty men to do the work of one first-class, competent superintendent?

And again, that part of the present plan recognized by the law by which the taxes are or may be worked out, is altogether out of date. It is unsound in principle, as Gillespie says, wasteful in practice, and altogether unsatisfactory in its results; a remnant of the times of feudal vassalage, when the tenure of land required the farmer to make the roads passable for the troops of the lord of the manor. And how absurd it appears, on a moment's reflection. Men who may be skillful enough in their own occupations are taken for the performance of work of which oftentimes they know absolutely nothing. A good plowman is not necessarily a good watchmaker, and yet to build a good road requires more thought, more skill, more scientific knowledge than to make a good watch, for the latter is an operation chiefly mechanical, while the former often demands the highest engineering attainment, and to spend money with the greatest degree of economy, even in repairing a common road, requires much judgment, knowledge of materials, and practical experience in using and applying them. And yet the law presumes that every man is competent to build a road 1

Is it any wonder that there is a universal complaint of its utter inefficiency? Is it any wonder that we have to wade through mud and mire in the spring and through dust in the summer, stumbling over rocks, with the endless wear and tear of carriages, horses and teams, and that we suffer the discomforts and annoyances which traveling over such roads implies? These are only a few of the defects of the system, yet many others might be enumerated in this connection.

Now, how shall they be remedied or removed? It would seem that the change ought to be radical, that the medicine could hardly be too strong to meet so serious a case of disease; but lest the general sentiment of the community should not be found educated up to such a treatment, we will allude to one or two milder, half-way measures at first, which would clearly be an improvement upon the present state of things, and then say what seems to us to be required to effect a complete change in the present system.

At first the law might require that the whole supervision of roads should be vested in the board of selectmen, who should be obliged to appoint a thoroughly competent superintendent, who should hold his office for a term of years, not less than three, and perhaps not more thas. five, subject to removal only for good cause shown, to whom should be committed the entire 
responsibility of the repairs of roads, and who should have a sufficient force of workmen con. stantly employed to make permanent improvements, and to keep up the roads. Nothing is better or more clearly proved by the experience of the past, than that the plan of annual or semi-annual repairs is totally inadequate to keep up the roads, though it is undoubtedly the most expensive and wasteful of the public money.

It might be well, also, to require by law that at least one-half of the money raised should be devoted to making permanent improvements, using the balance each year to keep up such parts of the ways as could stand the delay, picking out loose stones and otherwise keeping them in a passable condition till their turn came for a more perfect treatment.

This would be one plan. Another would be to authorize or require the towns to elect a road-master, under whose direction all the surveyors for the year, whatever the number might be, should work, and to whom alone they should be responsible after their election by the town. He should also be elected for a term of years with a liberal salary, to be fixed either by the law or by the town at the time of his election. He should be required to give his personal attention to all the important alterations or repairs of the highways, and generally direct the time and manner of the performance of all labor done on the roads by the surveyors or those under their employ, reporting in writing at the annual town meeting with a statement of what had been done, and suggesting the requirements of the road for the future.

Another plan would be to authorize the towns to elect a board of perhaps three commis. sioners, in the same manner as school committees, who should hold their office for a term of years, to whom should be committed the whole supervision of the roads, and who, so far as the construction, laying out, and repair of roads go, should hold the position already suggested with reference to the selectmen. Being chosen with special reference to fitness for the position, they might be more competent than any board of selectmen chosen for other and more general duties.

Another still better plan, perhaps, would be to authorize or require the towns to appoint a skillful road engineer, with all powers, rights, and duties suggested for the superintendent, and which are now exercised by the highway surveyors. He should be required to perform all the duties relating to laying out, altering, and repairing the highways, which now devolve upon the selectmen. His plans might be subject to the approval of the board of selectmen, if thought best, or be submitted to the town for acceptance.

Still another plan would be to require each town to appoint an inspector of roads, to act in concert with two, three, or more similar inspectors from adjoining towns, and also three agents in each town to make the repairs of roads, one to have the entire charge of repairs on the main roads, for instance, and the other two to have control of needed repairs on cross. roads, all the roads being divided, perhaps, for convenience, into first and second-class.

The three, four, or five inspectors so appointed should be required to pass over the main roads in company with the agents, and point out to them in detail the manner in which the roads should be repaired. To save time and money, the inspector for each town might have the supervision of the cross-roads in his own town, and the same direction over the agents having charge of those roads which the board of inspectors had over the agent having charge of the main roads. After the repairs are made, it should be the duty of the inspectors to pass over the roads and see that the work has been properly done, with the power of acceptance or rejection, according to circumstances. These inspectors might be chosen by the towns or appointed by the selectmen, and in case an agent proved himself to be incompetent, the inspectors should be required to report him to the selectmen, who should be empowered to discharge him and appoint a temporary agent in his place.

These are a few of the simple changes that might be made to secure greater efficiency, the labor-tax of the commutation system being entirely abolished in either case. They are at best only half-way measures, and liable to some of the objections of the present system, 


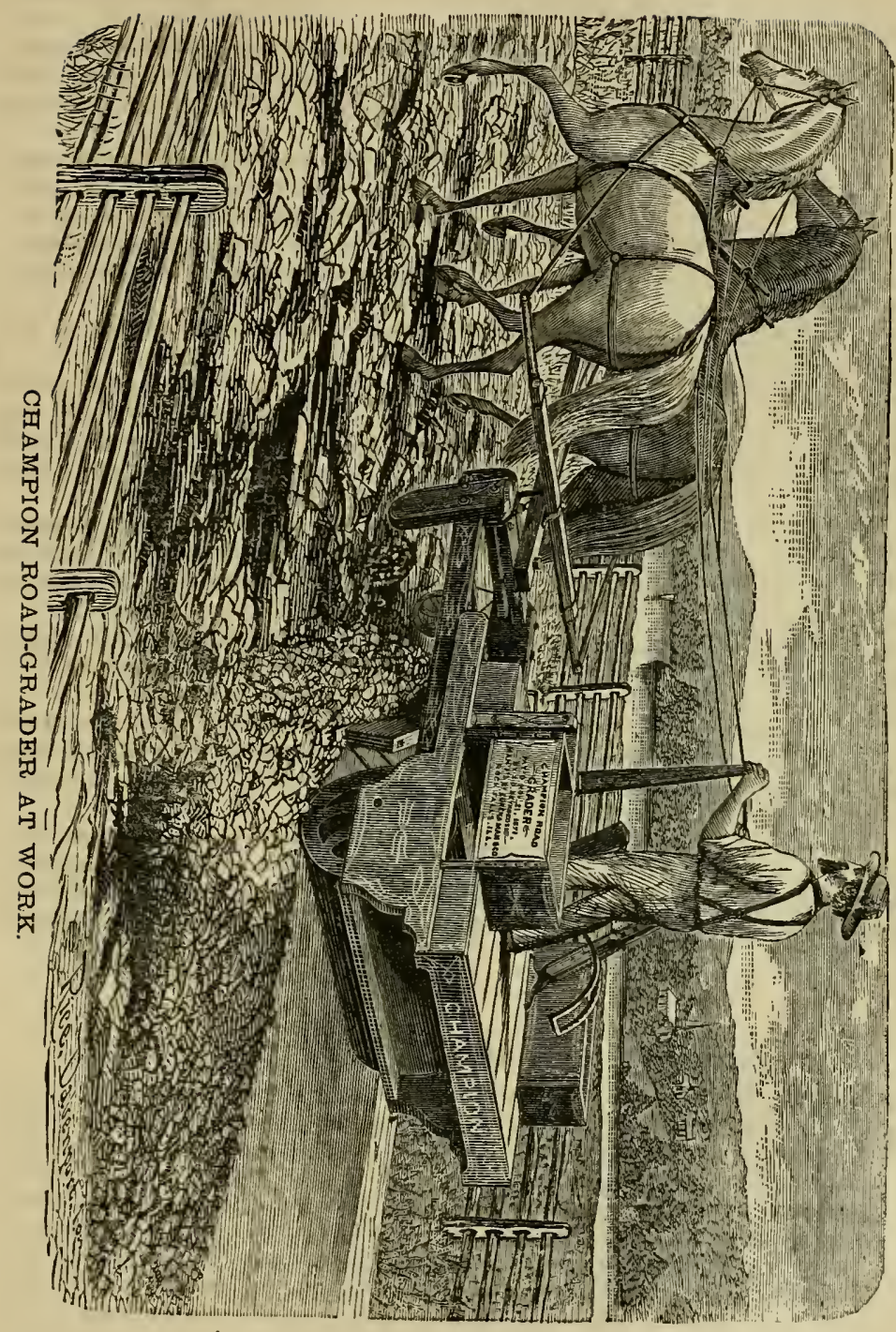


such as iocal prejudices and interests, and political strife and rivalry in the election or choice of the officers suggested, by which the best interests of the public might in some cases be sacrificed to party intrigues.

It will be noticed that this plan insures a constant oversight over all the roads, and this, after the roads are once properly constructed, is unquestionably the best economy, and costs less, in a series of years, than that of semi-annual repairs. It is the only way, in fact, by which a road can be kept constantly in good condition.

Now, after all, as we have stated, the plans which have been suggested are what might be called only half-way measures, which might be adopted as modifications of the present system, with the understanding that the fundamental principle which underlies them all, and which is based on the truest and most far-sighted economy, is "to sacrifice a portion of the resources of the road, or the money raised for roads, to insure the good and judicious employ. ment of the remainder."

A far better plan, it seems to me, is that suggested in one of the prize essays on this subject, published by the Massachusetts Board of Agriculture:-

"For the efficient and economical maintenance of the public roads, it is essential that there be a uniform system of management common to the whole State. The first step towards a complete reform of system would be the creation of a State department of roads and bridges, to have general charge of all the roads, to arrange and direct the carrying out of the details, and generally to look to the effective working of the system.

The chief of the department should be a practical civil engineer, thoroughly conversant with the art of road-making. For the purposes of proper supervision, the State might be divided into districts, say by counties, and these again into sub-districts, larger or smaller, as might be found expedient.

There should be a resident engineer or superintendent for each district, to have charge and oversight of the roads and bridges within his district, and to be held accountable to the chief of the department.

He will ascertain the condition of the roads in his district, determine what improvements are to be made, and in what order, decide upon the kinds and amount of work to be done, estimate the sums needed to carry it on, and at stated periods report the same, with all the matters pertaining to his office, to the chief of the department.

For each sub-district there will be required an assistant-engineer or road-master, subordinate to the resident of the district, to manage the working details within the limits assigned. As the improvements progress, these sub-districts may be enlarged and the number of subordinates reduced, so that each and all shall always have work enough to keep them occupied."

\section{FARII ROADS.}

$\mathrm{R}$ OADS leading from one part of the farm to another are a great convenience, and the benefits derived from them amply repay for the labor and expense of their construction. Those farmers who do not have them are apt to drive their teams all over their lands wherever they have occasion to go, the result of which is that the mowing and grain fields are thus badly cut up by wheel-tracks, and the treading of the team. Where heavy loads are to be drawn, it is much easier for a team to have a firm, substantial road on which to draw them, and much more labor can be accomplished in a given time by this means; besides, when the soil is softened by the rains, it is difficult to draw heavy loads, and hence, aside from the injury done the lands, much more labor and time will be required in transferring produce and other material from one point to another, without good 
roads. A team will draw a much heavier load on a good, hard road than on turf-land or simply a cart-path, which soon becomes worn into ruts by the wheels. These ruts not only render the drawing more difficult, but furnish a place for standing water, after every rain, which is soon converted into mud. Such paths are especially troublesome in the spring when the ground is wet and soft.

Although constructing roads on the farm will require some labor and expense, the benefits derived will soon more than compensate for it all, and when once properly made, a very little labor will be required to keep them in repair from year to year.

The manner in which the roads on a farm should be laid out, will depend upon the size of the farm, its locality, and the relative locality of different fields, and that of the farm-build. ings, especially the barn. As a general rule, there should be one good road, which, with its branches, shall lead from each field to the barn.

Farm roads should be constructed in a manner similar to those for public use, the harder and firmer the road-bed is made the better. Where the land is naturally dry, their construction is very easy, but where the land is wet, considerable labor will be required, since drainage will be essential. The ground for the road should be plowed, and the bed so graded that it will be elevated a little above the level of the surrounding land, and the middle of it slightly higher than the sides, in order to admit of the water running off easily.

After properly grading, it will be greatly improved by the use of a roller. Where the land is nearly level a furrow should be plowed on either side for drainage, and to prevent the road from being washed by heavy storms. Coarse gravel makes an excellent bed for a road, and when it is convenient of access will on most soils well repay for the labor and expense of procuring it for this purpose. On lands that are naturally wet and retentive of water, the construction of roads is more difficult.

The soil should first be thoroughly underdrained, after which the earth should be thrown out of the road-bed to about the depth of two feet, and the trench thus made filled with small stones or coarse gravel, over which the earth is placed. Care should be used to grade it in such a manner that the center of the road shall be somewhat more elevated than at the sides. Ample provisions should also be made for drainage at the sides. For this purpose a trench filled with stones, tiles, or a deep open furrow may be used. Where the soil is of such a nature that it is easily washed, the furrow will need to be opened frequently to prevent its filling up with sediment, where only the latter is employed for drainage.

The Champion Road-Grader is an implement much used in many of the Western States in road-making, and it is said to prove a very effeient and valuable machine for this purpose. It is made by the Eureka Manufacturing Company, Rock Falls, Illinois; when properly adjusted it will grade the road-bed as desired, and roll it down, rendering it compact and hard.

Farm roads need not be expensive in construction, since they are not in constant use like public roads, but they should be made sufficiently substantial for all practical purposes. Where the ditches at the side for drainage are shallow enough to admit of teams passing, which will not frequently occur inside a farm, - the road need not be made over ten or twelve feet wide. Roads should always be kept in good repair. It is much cheaper and more profitable to provide good roads than poor ones, whether for public or private use. A small amount of labor, properly employed, will serve to keep the road-bed smooth and in condition to admit of the water passing off readily, thus enabling it to become more and more compact by use, while, on the other hand, if neglected, a small defect soon becomes a serious one; the surface gets worn and torn up, and places are formed for the water to accumulate. The soil becomes soaked and softened, and gullies are formed which grow deeper by use, soon rendering it either very inconvenient or unsafe for passage.

Many serious accidents might be avoided, and expense and time saved by keeping roads 
in a better condition than they commonly are. Horses often receive injuries that render them lame for life through such negligence, to say notbing of the danger to the life and limb of the driver, while the wear and breakage of wagons and carts are no small item in this connection. Roads when not properly cared for will also become so worn by constant use, that the middle of the bed will be concave instead of convex, where the water readily collects and often makes a channel lengthwise until it finds an outlet, cutting gullies in its course. A single rain-storm will frequently cause great damage to a road when in this condition, while if but a small proportion of the amount necessary to expend in properly repairing it had been devoted to keeping it in good condition at first, time and money would have been saved. Good roads on a farm, as well as in the locality of it, are not only a very great convenience, but increase the value of land. Such farms will always find a more ready and remunerative sale, other conditions being equal, than those that are not thus provided with proper uneans of transit from one point to another.

\section{FARM FENCES.}

$\mathrm{T}^{\circ}$ 0 what extent farm fences are a necessity, is a question that is at present attracting considerable attention from agriculturists. That an immense amount of needless expenditure in time and money are employed in making and keeping in repair fences that are not only of no practical benefit to the farm, but which, if dispensed with, would greatly improve in appearance the lands which they enclose, cannot be denied. In no country on the whole globe is there such an enormous fence-tax as in the United States. In fact, it might be stated in a general sense, that the custom of entirely encircling farms and separating one owner's lands from another by fences, is an innovation, and one that is pecu. liar to this country.

In Germany, England, Italy, and many other countries of the Old World, long under cultivation, and containing a dense population, fences for the pyrpose of marking the terminus of land, or as lines of demarkation, are rarely seen. In this country, especially in many portions of New England, farms in many instances are divided and subdivided by lines of fences that enclose small areas, and which must have cost, in the aggregate, nearly as much if not more to construct, than the entire farms, in some cases, will now sell for. Frequently these lines of division have little or no regularity, and seem to be entirely without any established plan or system.

Under the usages and customs of a former generation, who, in settling their country, first cleared a field and then fenced and cultivated it, permitting the farm stock to run in the uncleared portions, this system of fencing was inaugurated. But the necessities of that remote period are not the necessities of to-day, and the customs and usages of that generation are not essential to the present.

While fences on a farm seem, to a certain extent, to be a necessity, yet, according to the former custom of fencing, they are far more extensive than is essential, except, perbaps, in the prairie regions; and that whicb is spent in making and keeping them in repair might much more profitably be appropriated to other purposes.

Surplus fences are not only expensive and unprofitable, but they are an incumbrance to the land. Many of them are so constructed that they take up valuable land that might otherwise be under cultivation, such as the stone walls common in many portions of New England, the zigzag or Virginia fence, etc. Besides, the general appearance of a farm that is not divided by numerous lines of fences is much more in conformity with good taste. 
Fences furnish a place for weeds to grow and ripen their seed, which are scattered by the wind over the land; they also are a safe harbor for mice, rats, and other pests.

Unnecessary fences on a farm, are, therefore, not only a useless expense, and an obstruction to cultivation, but a disfigurement to the landscape, and a harbor for weeds and vermin; and if the amount of unproductive labor which they incur were reduced, great benefit would be derived, since by so doing, the productiveness of the farm would be practically increased, without any increased outlay in labor, money, or draft upon the fertility of the soil. In some parts of New England, the old division and highway fences have been removed, which has greatly increased the general attractiveness of such farms, as well as the towns in which they are located. The owners have by this means improved the general appearance of their farms, and reduced the expense of maintaining them.

In almost every State in New England there are examples of such improvements, which are gradually extending. Among the many places made especially attractive by this means might be mentioned Cheneyville, in South Manchester, Conn.; also, Amherst, Mass., and vicinity. Very few fences are also seen in some of the river valleys, where the annual inundations would sweep them away.

To what extent farm fences are essential will depend somewhat upon different conditions and circumstances. The crops must, of course, be protected. This protection may be furnished by fencing the farm animals in, appropriating a certain portion of the farm to this purpose, or by enclosing the cultivated fields by fences, and thus fencing them out. Where the law requires every owner of farm-stock to keep his animals on his own land, or to be responsible for all damage done by them, highway fences will not be necessary, since the owners, being made liable for such damage, will be careful to keep them confined to their own premises. The fences required in such sections, therefore, are those that will keep each owner's animals confined on his own premises, and not such as shall fence out those of his neighbors; consequently, pasture fences, and fences about some of the farm buildings, will be all the permanent ones that are essential. Where soiling is commonly practiced, even pasture fences could be dispensed with.

In those sections where the statute law and custom require a highway and division fence, a boundary fence will be essential, but the law and custom may be changed, and if farmers use their influence in securing the enactment and enforcement of stringent laws in respect to restraining stock, this may be accomplished, and highway fences be rendered unnecessary.

Farm animals that are not under the direct charge of a keeper, who is responsible for the injury they may do, should never be tolerated in the highway. Such toleration would be detrimental to the best agricultural interests of the country. If certain portions of the farm, aside from the common pastures, are desired to be used as pasturage at any time, such as mowing-lands for a season in the fall, movable fences may be used, similar to the hurdle fences, so extensively employed in England. As a general rule, the permanent fences necessary, except where boundary and division fences are required, will be those for the pasture, and around farm buildings, such as barns and sheds. A temporary fence can occasion. ally be used when necessary, which will secure the convenience of a permanent one without its disadvantages, and for this purpose a movable fence, as previously recommended, is the most desirable.

In England and other portions of Europe, when cattle and sheep are pastured where there are no fences, a shepherd is employed to take charge of them, who, with the assistance of a well-trained dog, will keep large flocks and berds under perfect control, and as strictly confined to prescribed limits as though there were fences for this purpose. This practice of employing shepherds is based upon the principle that it is less expensive to take care of the herds than to keep up the fences. 
Remoring Fences.-We would recommend to all farmers the maintaining only of such fences on the farm as are absolutely necessary for the safety of the crops, and the confinement of the stock, and the removal of all such as are not essential. Unnecessary fences on a farm are an expensive nuisance.

In England, where hedges have been used quite extensively during the past, they have to a certain extent, during the last decade, been uidergoing a process of extermination, and there are at present thousands of miles of hedges less than there were formerly. In this country, as previously stated, in those sections where needless fences have been removed, a great improvement has been effected in the general appearance of the farms, as well as the convenience of cultivation and the reduction of the expenses of the farm. In the removal of useless fences, but little labor, comparatively, will be required for those constructed of light materials, such as rails or boards. The removal of the stone-walls, however, that are found in many parts of New England, would involve much labor and expense, and in some instances it is questionable whether their removal would pay for all the expenditure of time and labor that would be required to accomplish the object. This would depend upon the locality of the wall, the improvement and convenience secured by its removal, and the use to which the stones could be appropriated, or the facility with which they might be gotten out of the way. Many of these walls have been built for a century or more, and have been kept in repair from generation to generation. They were appropriated to this use, originally, partly because the fences were considered a necessity, and partly as a means of getting rid of the stones by which the land was encumbered. What to do with the stones, in removing such fences, would be the question to be considered. The best and most practical way of disposing of them is to use them where they will be a benefit in drainage. On nearly every farm there are wet lands that require drainage, and by using these incumbrances of the land for this purpose, acres of new or virgin soil may be secured for cultivation that might otherwise be nearly worthless for agricultural purposes.

Ravines and swales may also be filled with stones, while many may be utilized for the foundation of farm buildings, and thus in one way and another they can be disposed of in a manner that will increase the value of the farm.

Mr. Starr, the former proprietor of the famous Echo Farm, settled the perplexing question of what to do with the stones in clearing his fields of them, in a manner that may be of advantage to some other farmers to imitate who have this difficulty to meet.

Selecting an untillable spot in a field in which there were one or two natural mound-like hillocks, a large pile of stones was made, consisting of several hundred loads, and, as an experiment, this pile was covered with tussocks of coarse swamp-grass, which are hard of decomposition. These were inverted, covering the stones. On this foundation, a light dress. ing of soil was placed, and grass-seed sown.

This experiment proved a success; the grass soon grew over this artificial mound, which appeared to bear the protracted droughts even better than the natural ones, while the first showers made them conspicuously green.

From time to time these mounds have been extended and multiplied, and in all cases proved a success. The object was not to form new land, but to dispose of the stones. Whenever practicable, natural depressions may be made use of for depositing stones. By such means the surplus stones may

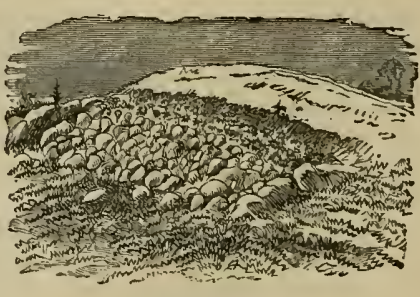

STONE HEAPS. be gotten rid of without being an encumbrance to the land, or marring its appearance, and also without being a place in which noxious weeds, briars, and shrubs will find refuge. 
Rail Fence.-The kind of fences used on a farm will vary according to circumstances, the most available material being generally employed in each section. As the country becomes older, and the material for fencing purposes becomes more scarce and expensive, the question 2s to the most economical and durable fence to construct becomes a more important one to determine. The first settlers of the country, finding timber and stone abundant, made use of these principally in the construction of fences. Hence, the rail-fence and stone-wall became the most common at that time. In newly-settled portions, where timber is plenty, the common rail-fence, or what is termed the zigzag or Virginia fence, is quite extensively employed, owing to the material being cheap,-often an incumbrance in clearing up new lands,-and the rails being easily split.

This style of fence has been very appropriately termed by a recent writer, "the relic of a lavish era of unlimited forestry," the counterpart of which is seen in no other country, it being typical of Yankeeland. Cedar is most commonly used for making rails, although bemlock, chestnut, and other kinds of timber may be employed for this purpose.

In making a rail-fence, wooden blocks are preferred to stones for supports at the corners, as the stones will soon sink into the ground and become of no use whatever. Blocks will decay in time, but they may be replaced by others. The stakes used should always be large enough to give sufficient strength and support to the fence. It will be a practice of economy also to make them long enough to be re-sharpened and used again when the ends decay. Long stakes projecting at the corners, however, give a fence an unsightly appearance. A more symmetrical and neater-looking fence. besides being equally strong, can be made by putting two upright stakes, one on either side of the angle formed in crossing the rails, and securing them by a plank in which holes are made of sufficient size and distance apart to admit of being slipped over these posts to hold them securely after all but one or two of the top rails have been laid. The upper rails are then put on to hold the plank firmly in place. Annealed wire of large size may be wound around the stakes to hold them in place, instead of the use of the plank, if desired. The objections to the common rail-fence are, the large amount of timber necessary for its construction, the ease with which it may be thrown down by stock, or blown over by the strong winds, and the amount of land it occupies. Where timber is abundant, land plenty, and saw-mills not easily accessible, some kind of a rail-fence may prove the most profitable; but, as a general rule, with ordinary facilities for obtaining other material, and where land is valuable, some other style of fence is to be preferred.

Post and Rail-Fences.-Considerable less timber will be required for this than the common rail, or Virginia fence, and it also occupies only about one-third the land required for the latter, besides being more substantial. It is, however, a more expensive fence to make, since considerable labor and time are involved in digging the holes for the posts, and in making holes in the posts for the rails, and fitting the latter to them. The posts are liable to be thrown out by the frost, and also to decay, requiring to be occasionally reset. Some kinds of timber will, however, last much longer than others, and if the most durable kinds are used, a fence of this style, when once properly built, will last several years with but few repairs. The posts should be pointed at the end, and set from two and a half to three feet in the ground. The hole should be made quite large, and the post placed in the center and surrounded by very small stones, which should be pounded in firmly around the post, as the hole is filled up. This will prevent heaving, and the post will also last much longer than if surrounded with earth.

Nailing a piece of board about two feet long to one side of the post near the bottom, and another piece on the opposite side a little higher, and packing the earth firmly down, is also a good preventive against heaving. A post set in this manner will generally rise and fall with the earth, and hence will not be liable to be thrown out. Of course, the hole in such cases must be dug large enough to admit of the extra width required by the boards 
ihat are nailed to the post. The best timber for posts, with respect to durability, is red zedar, yellow locust, black walnut, white oak, and chestnut. The bark should always be :emoved from posts before setting.

Preserving Fence Posts.-Various methods have been employed for the preservation of posts, some of which have proven very efficient in retarding the progress of decay. There is a great difference, however, not only in the durability of different kinds of timber, but in the manner upon which different soils act upon wood, it generally decaying very slowly in a compact clay, but rapidly in silicious sands and gravelly soils. Placing the post in the ground in a position which is the reverse of that of its natural growth, or the upper end downward, will render it more durable. Charring the part that is buried, or covering it with coal tar, as well as imbedding it in ashes or clay, is thought to add to its durability.

Soaking the posts in a solution of blue vitriol, in the proportion of one pound of vitriol to forty pounds of water, is highly recommended by some as a means of preserving all kinds of timber that is exposed to the weather or moisture. If the timber is dry it should be soaked ten days; if green, six days will be sufficient.

But one of the best preservatives with which we are acquainted is to completely saturate that part of the post to be placed in the ground, with kerosene or common coal oil, and afterward covering it with tar.

A farmer in Mississippi gives the following statement with respect to this method of treatment:

"Ten years ago I built a grapery at the end of the house, as a screen against the western sun, using sawed pine posts. Anticipating the difficulty of ever replacing these posts after they became covered with vines, I took the extra precaution of completely saturating the lower ends with kerosene-common coal oil-before applying the tar. These posts are now perfectly firm and almost as sound as they were when put in. All other pine posts set at that date have entirely rotted and perished. The result of this experiment so thoroughly impressed me with the value of coal oil as a preservative of timber under ground, that I now use it on all posts in building, afterwards covering with hot coal tar.

"I add this, however, which I think will doubtless prove of great value: I bore a $\frac{1}{2}$ to $\frac{3}{4}$ inch hole in the post near the ground, slanting downward and reaching beyond the center; this is to be filled with kerosene from time to time-perhaps once in three or four years will answer. I feel sure that insects very greatly hasten the decay of timber, to say the least; and kerosene being repellant to them, makes it a valuable application at any point where they are likely to do mischief."

Dr. Z. H. Mason of Florida, also recommends the use of kerosene oil for this purpose, and says that in that State it has proved a very effectual preservative of wood against decay from moisture and the attacks of the white ant, known in that section as the wood louse, which is very destructive to timber. Timber suitable for posts becoming scarce, by this means almost any kind may be used for the purpose, and rendered quite durable, thus saving a large amount of money, time, and labor.

Post and Board Fence.-This fence presents a much better appearance than that made of posts and rails, and in sections where timber can be easily converted into boards, it is cheaper than the latter. The posts should be placed from seven to nine feet apart. The boards, if about six inches wide, should be placed about the same distance apart and firmly nailed to the posts. If substantially made, such a fence always looks well, and will last a long time with few repairs. Before setting the posts the ends to be placed in the earth should be saturated with kerosene oil and covered with coal tar to increase their durability. Precaution should be taken also, in setting, against their being thrown out by the frost, according to directions given in connection with the post-and-rail fence. 
Stone Walls.-The stone wall is very common in New England, where it has been used to subserve a double purpose, namely that of a fence, and as a place of deposit for the stones that have been taken from the land. When well built, they form a very permanent and substantial fence, but in order to be well built an immense amount of labor is required; hence they are a very expensive fence. When improperly built, they are anything but substantial, and an old, dilapidated stone wall is one of the most unsightly objects that can be found on a farm, being an index of negligence and shiftlessness that is not pleasant to look upon, and one that is far from being complimentary to the owner.

When well made, a stone wall forms an impassable barrier for horses and cattle, but will not confine sheep or hogs unless it is surmounted by a rail or similar obstruction. The frost will often throw it down by upheaving the earth, while a loose stone, being displaced, will often result in a wide gap; hence, aside from the expense of building, stone walls require considerable care and repairing.

It is a fence that also takes up a great deal of land that might otherwise be under cultivation, while its removal, when desired, involves a vast amount of labor and expense. It would be far better for the farmer to dispose of the surplus stones by burying them where they will be useful in underdraining the land, or in filling up ravines, and construct cheaper fences of a lighter material.

The following method of building a stone wall is taken from "Facts for Farmers," and may be useful to such as are not skilled in this respect, and wish to obtain the knowledge of what we believe is destined to become, before many generations, one of the "lost arts."

"Have the surface soil removed so that the foundation stones will rest on firm earth. Contiguous foundation stones should be as nearly as possible equal in size, and large enough to extend the full width of the wall, and every foundation stone firmly bedded in the ground. If boulders, or stones of uneven form are used, always plant the roughest side downward, or at least so as to bave a flat side up to lay the next course upon. If your wall is built of a double line of stones, whatever their shape, it should frequently be bound across with flat stones or wooden ties made of split pieces of cedar, chestnut, white oak, ash, or any durable tough wood, from half an inch to one inch thick, two to four inches wide.

'Break joints!' should be rung in the ears of a young wall-builder incessantly, until he would do it instinctively every time he laid a stone into the wall. You can tell at a glance as you ride along the road, whether the wall was built by a workman, by the way the stones break joints. You may sometimes see them so placed that a joint extends from top to bottom. That wall was built by a cheat or bungler, probably both.

Beware of a jobber who is continually chinking small stones into the joints of the face of his wall and filling up the interior with stones thrown in as carelessly as you would fill up a hole in the ground. If you find your jobber working this way, discharge him peremptorily. He is both a cheat and bungler.

If your wall is built double, cap it with a course of even-sized stones, so as to give it a uniform appearance. If the stones are generally flat, cap your wall with flat stones of even thickness and of a width greater than the wall. This not only helps the appearance, but sheds off water, which is often the means of destroying badly-built walls, by running down inside and freezing so as to bulge out the two lines of stones with which the sides had been faced up and not bound together.

Good walls are sometimes built of very bad stones by using cross-binders of wood in the lower courses, and then near the top laying two boards, each about one fourth the width of the thickness of the wall along the line, and upon those building up the remaining height. These boards will last many years and serve to hold cobble-stone together quite firmly.

It is a better plan, however, we believe, not to build the wall as high by a foot, and take the strips of board designed for binders in the wall, and nail them to small posts built in so as to give sufficient height for the fence. 
A very common fence in some sections is built of cobble-stone about two feet high, topped with two bars inserted in posts, or with strips of boards nailed on."

Hedges.-In many portions of Europe, especially in Great Britain, hedges have been long used to a considerable extent, hedge-rows having formerly been a characteristic feature of rural life in England; but they are now beginning to be regarded there as objectionable, and in many parts of the country are being exterminated from the soil. In this country hedges have been used to a limited extent, but more especially in sections where timber is scarce. For lack of anything better for fencing, it may sometimes be well to make use of them for boundary fences, although there are many objections to introducing them generally. They require in some localities considerable care to secure a strong and uniform growth, and to keep them well pruned and trimmed, and also occupy a great deal of land, and harbor noxlous weeds, furnishing lurking places for enemies of the field-crop, orchard, or garden. In some climates their growth is so rapid that they require constant care in pruning. while their overgrowth is a serious evil, and when once in possession of the soil they are difficult to eradicate.

Another objection to hedges is that when the land is cultivated, the roots not only interfere with tillage, but they rob the cultivated crop of plant-food. There are, however, many places where they might be introduced without interfering with cultivation, such as around pastures, orchards, etc.

For making hedges, some quick-growing, hardy shrub, armed with thorns or spurs, is generally used, although other shrubs and even trees are employed to a certain extent. The arbor-vitae, cedar, hemlock, buckthorn, pine, yellow willow, osage orange, honey locust, cotton-wood, barberry, and privet have all been employed to a certain extent. In England, the hawthorn has been used for this purpose for centuries. At the South, the Cherokee rose is quite extensively employed as an ornamental hedge. The osage orange and honey locust are perhaps used more than any other; the former in the Middle, Southern, and Southwestern States, it being liable to winter-kill in the extreme Northern section. The latter will thrive in all parts. The willow is well adapted to wet localities, but its rapid growth necessitates frequent trimming, which is a serious objection. Evergreen hedges are liable to injury from drouth or extreme heat and cold, but are very attractive in appearance when properly cared for and trimmed. The arbor-vitae (Thuja occilentalis) makes one of the most beautiful hedges that can be found at the North. It is hardy and flourishes in any sol that is not too dry, branches out thickly from the ground, and is of comparatively slow growth. It is much used as an ornamental hedge. The principal objection to the osage orange is its vigorous, rapid growth, and the exhaustion it occasions to the soil within reach of its long roots.

Planting and Trimming Hedges. - In the successful use of hedges for fences, much depends upon a proper selection of the plant for the purpose, its adaptation to the climate and locality, as well as the subsequent cultivation and pruning it receives. The best authorities on hedge-setting advise that, on soils naturally damp or retentive of moisture, the plants be set upon a slight elevation or raised surface from five to eight feet wide. The reasons given for this practice are, that the hedge will be more liable to escape winter-killing, from the fact that the roots will then be above the level of saturation; that the setting can be performed earlier in the season than where the ground is not ridged; that the roots of the young plants will strike down obliquely, instead of extending horizontally, as on level land, and will also attain a more fibrous growth; that the plants, thus having a more uniform con. dition of soil with regard to moisture, will attain a more uniform growth, and will generally be rexempt from gaps and thin places made by partial winter-killing, and which requre considerable labor and care in replacing. In setting the hedge, the soil may be thrown up 
by repeated plowings, and the top afterward leveled by the use of a revolving harrow or a roller.

When the hedge is to be set on sod-land, two shallow furrows are generally opened, the sod removed, and the soil made as mellow as possible to quite a depth. In order to set the hedge in a straight line, a cord should be stretched from one point to another, and be marked with red or some contrasting color, to show the place where the plants are to be set. In setting, the soil should be firmly pressed about the roots. The osage orange, when planted at a distance of five or six feet apart, will furnish a good, substantial fence in from three to five years. The willow is very hardy, and will grow readily from branches of the trees cut in suitable length and inserted in the ground.

During the first year the hedge should be cultivated with the ordinary corn-cultivator, to keep out the weeds and loosen the soil, which will aid much in establishing a good growth. Subsequent cultivation may be performed with the plow, always throwing a light furrow towards the hedge. When the hedge has become strong enough to restrain stock, and it is desirable to check, in a measure, its growth, this can be accomplished by running a pruningplow or similar implement along the sides of the ridge, or, if on level ground, at a suitable distance from the hedge, and thus cutting off the ends of the roots, which will check the growth of the plants without killing them, and keep the roots within prescribed limits. Otherwise they will be liable to occupy too large a portion of the soil, and appropriate to their growth the nutriment that should be taken by the growing crops.

When hedges become, in time, thinned at the bottom, they will require renewing in this part to remedy the evil. This is done in different ways, sometimes temporarily by inserting branches cut froin the over-grown or thicker portions of the hedge, and by layering. This latter method reduces the height of the fence greatly, causing the new growth to be made near the ground. It is more successfully practiced in damp, than dry soils. Some kinds of hedge require more frequent trimming than others. They should be cut when the sprouts are green and soft, as it requires less labor at this period of growth. The osage orange, and hedges of equally rapid growth, should be trimmed at least twice a year, and many think it a saving of labor to cut them even three times, thus preventing the wood from getting hard and of large growtl. For the cutting of the green or annual branches, a strong and sharp grasssickle will answer every purpose, which, with a little practice, can be readily accomplished.

Embankments, with ditches on one or both sides, are used for fencing in some parts of England, but have not been employed to any extent in this country. A large open ditch is dug on the division line, and the earth taken from it is thrown up, forming a high embankment. A double ditch, one on either side of the ridge, is a more effectual barrier. Sometimes a hedge is planted on the top of the embankment. On very wet lands, such ditches will serve for drainage purposes, but we doubt whether they could be profitably employed as a fence.

Portable Fences. - Portable fences of various kinds are a great convenience on the farm, since they furnish all the advantages of a permanent fence, without the disadvantages. They can also be made during the winter, when other farm work is not pressing. In Canada, where timber is abundant and cheap, a portable fence much in use is constructed with supports something in the form of the letter $\mathrm{A}$, the pieces joined at the top forming an acute angle, with the bar near the bottom, and three boards nailed at equal distances on one side of these supports. This fence requires considerable material, but it is cheap and durable, is easily constructed, and when once made requires no extra labor in putting up. Another very convenient portable fence may be made as follows: The supports are made of inch boards, crossing near the top, but allowing the ends to project a sufficient length to furnish a support for the upper board of the panels forming the fence, the support being cut out at 


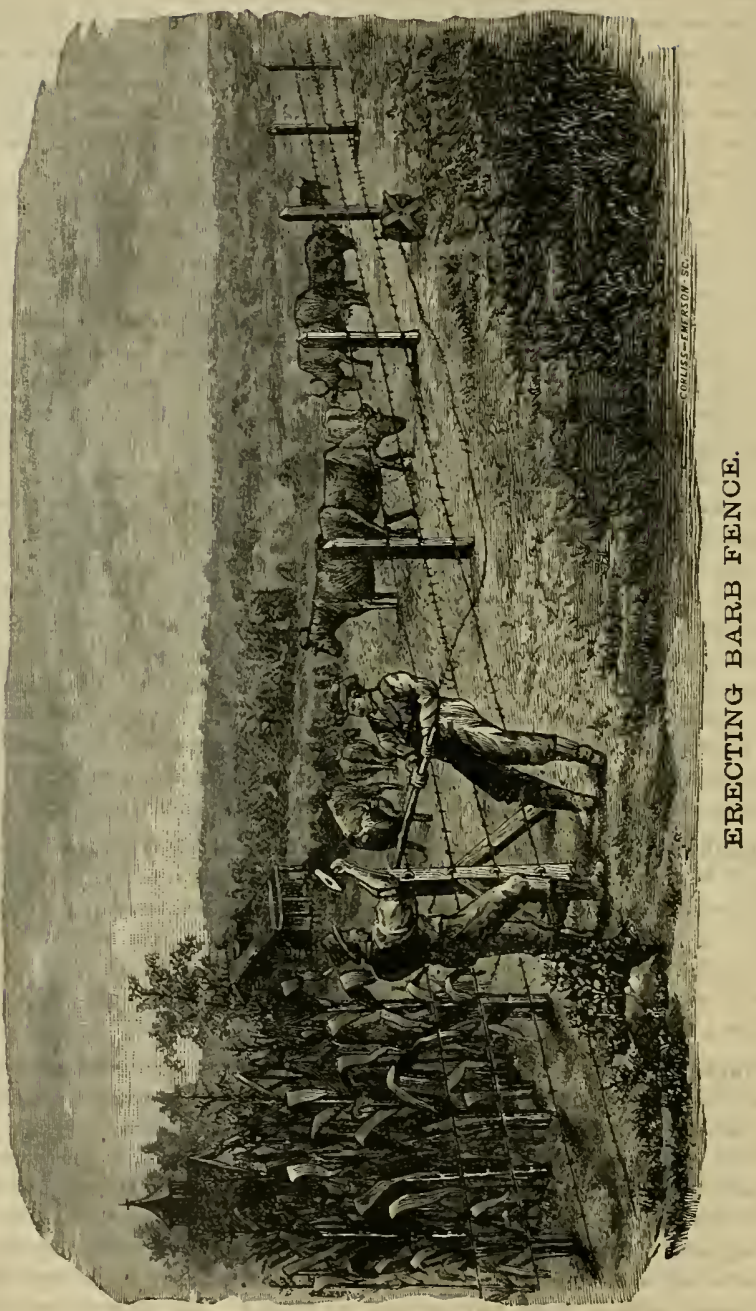


the upper angle thus formed, to admit of the top board of the panels fitting it well. $\mathrm{A}$ board is nailed across the support near the bottom at such a distance that it may be inserted in the space between the two lower boards of the panel, the lower side of which is cut out to fit them in such a way that they cannot easily be pushed out of place. The panels, or main part of the fence, may be made of inch boards about sixteen feet in length and of any width desired, and placed nearer together towards the bottom than the top, if designed to confine small animals, such as young pigs or lambs. These panels may be made by placing the sixteen-feet boards at the desired distance apart (about four of them being required for a panel, if the boards are about six inches in width), and nailing a board across the middle and near either end, to hold them in place, leaving the ends of the boards to project from six to eight inches. This forms one complete panel. When properly constructed and set up, the bottom of the top board of each panel rests on the top of the support, and the bottom board of the panel goes under the cross piece of the support. By digging a little for the posts of the supports, it forms a very secure fence. This fence can be opened at any place, simply by raising up the ends of two panels.

By the exercise of a little ingenuity, various kinds of portable fences may be constructed at slight expense that will prove of great utility on the farm, and a desirable substitute for many of the permanent fences that are now in use. They are especially useful where it is desired to feed different portions of a field crop, such as clover, turnips, peas, etc., in successive order by stock.

Flood Fence. - It is frequently desirable to construct a fence across the bed of a stream that is subject to floods after heavy rains, or to put across sloughs when too wide for a flood-gate. The following, from the American Agriculturist, describes two methods of making a very convenient and easily-constructed flood-fence: "Logs on which the fence rests are the trunks of straight trees about eighteen inches in diameter, which are hewed on two sides; posts are morticed in each of these logs, and on them planks are firmly nailed. The logs are then linked together with inch iron rods, and the first one connected by means of a long link to a tree, or post firmly set in the ground upon the banks of the stream. The links must all work freely. When high water occurs, the fence is washed around and left on the bank; after the water has subsided sufficiently, the logs may be dragged back to their places by means of a horse hitched to a staple in the end of the log."

Again: "Posts are driven or otherwise put down from three to four feet, with the tops about one foot above ground. The other posts, that the planks are nailed to, are bolted to the top of the inserted posts, and a wire is placed over the tops. The ends of the panel that connect with the post on the bank are slightly nailed with cross strips near the top, so as to be easily broken loose when the flood comes. There are also temporary braces bearing upstream, put in to prevent the fence from falling, but are easily washed out when the fence falls down stream, and logs and other obstructions pass by readily. As soon as the flood goes down, the fence is easily raised, a panel at a time, to its proper place."

Wire Fence.-The post and wire fence is quite extensively used at present, and seems likely to become in time the common fence of the country, especially in those sections where timber is scarce. At first the plain single wire was employed for fencing purposes, but failed tc give entire satisfaction, being defective in some important respects. The single wire contracted in cold and expanded in heat, causing the fence to get out of repair easily, while it did not repel unruly animals. Improvements were effected which have resulted in the use of barbed wire; the object being to secure durability, strength, and a protection against all intrusion from animals, or trespassers of any kind. That most commonly employed is made by the Washburn \& Moen Co., Worcester, Mass., and consists of two twisted steel wires, one of them having inserted at short intervals of space a firmly.twisted barb. 
By being twisted, greater strength is secured, and the wire is better able to resist the effects of changes of temperature. The barbs in such fences should, as nearly as possible, be just long enough to repel infringing animals without being capable of inflicting a serious injury. In erecting a fence of this kind, the number of strands to be used must depend upon the special object to be accomplished. Two strands, about twenty-one inches from the ground and from each other, will turn horses, cattle, cows, and young stock. Three strands, the lowest being placed twelve inches from the ground, the second twenty-three inches, and the third forty-two inches from the ground will, of course, be better and make a more substantial fence.

Four strands are most commonly used, while even five are frequently employed when

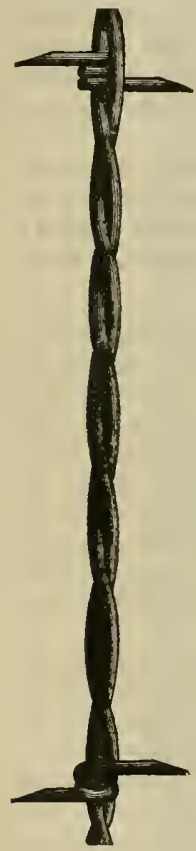

BARB WIRE.

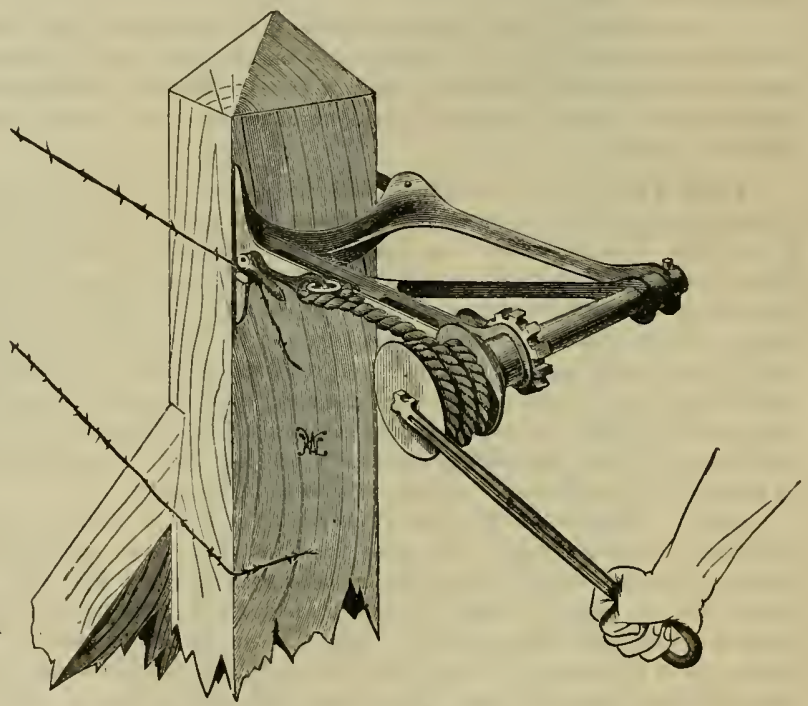

USE OF STRETCHER.

some special object is desired, such as excluding dogs, pigs, poultry, and other small animals. In such cases the lower strands are placed nearer the ground and to each other than the upper ones. In constructing a four-strand fence, the strand nearest the ground might be about five inches from it, the next twelve inches, the third twenty-two inches, and the fourth forty-eight inches from the ground. This would give a fence four feet high. In erecting a wire fence, the main posts may be about fifty feet from the corner posts and from each other. They should be firmly set in the ground, so as to prevent being thrown out by the frost. They should always be sharpened at the top, or left wedge-shaped, better protection against intrusion being by this means secured. After setting the posts, they should be marked where each line of wire is to cross. The first end of a spool of the wire is then fastened firmly to the first corner post with staples and carried quite a distance, allowing it to unwind as the 
spool proceeds. Draw the line from the starting-point to the proposed post as straight as possible with the hands, and then apply a stretcher and strain it tightly to its place. While the line is thus under strain staple it firmly to each intermediate post between the point of starting and the stretcher. Repeat from this point and so on. It is a good plan to brace

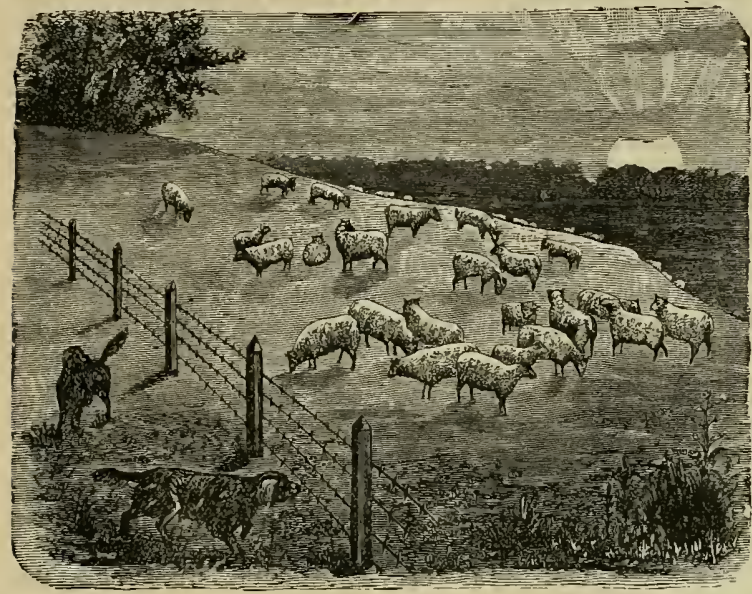
the corner posts each way with joists beveled to fit the post at the place of contact, and firmly spiked as high on the post as the upper line of fenc. ing, running from this point at an angle of $45^{\circ}$ into the ground below frost, and kept in place at the bottom by a flat stone or other substantial material. The posts on each side of a gate or pair of bars should be of the same size as the corner posts, and be braced in the same way. It is important that the wire be strained rigidly in place when being put up; in fact, there is little danger of straining it too tightly, as it is very strong, and being composed of two strands twisted together, it readily adjusts itself to different temperatures.

The arguments in favor of the barbed wire fence are its strength, durability, the ease with which it may be erected, its cheapness, and the complete protection it affords from all intrusion. It requires fewer posts than any other fence, as the posts can be placed at quite a distance apart; it is easily kept in repair, and cannot be destroyed by fire. It is also valuable where snow-drifts are an incumbrance, since it forms no barrier to the strong winter winds; the snow cannot accumulate into drifts, as it does where fences that offer obstructions are used.

The principal argument against the use of the barbed wire fence is that animals are sometimes injured by it, and it has for this reason been termed by some "a cruel fence." We think this objection could readily be met and entirely obviated by. placing a narrow board or pole above the top wire from post to post, which will render the fence easily seen by the animals, and thus prevent all such accidents. Whatever the kind of fence used, when designed to be of a permanent character, it should always be made of good material and be substantially built. It is cheaper in the end to build a good fence that will require but few repairs in a long time, than a poor one that requires a constant rebuilding by way of repairs. When posts are used, the timber selected should be of the most durable kind, and well set. For a post-and-board fence, heavy nails should always be employed, and enough of them put in to render the work strong and substantial. 


\section{WASTES AND WANTS OF THE FARM.}

$\mathrm{H}$

OW to make business pay, or in other words, how to secure the largest profits from the money, labor, and time invested, is the study and aim of all business men. This is no less the case with the farmer, than with those engaged in other avocations. In fact, we beheve farmers, as a general rule, labor harder to secure their profits than any other class. Many of them, occupying small farms, too often find at the close of the year that with all their toil, working early and late, they have fallen short of their expectations in results, and beyond defraying the common expenses of the farm and household, financially they are but little, if any, in advance of the previous year. Such a result, we believe, is not the fault of the business, but of the one who manages or mismanages it, and that when properly conducted, farming may be made as profitable an avocation as any other.

Such failures may be attributed largely to the wastes and wants of the farm, and should this subject receive the consideration wbich its importance demands, we believe much larger profits could be secured with less labor, by the majority of farmers, than are now realized by the present common practice.

Waste of Manure, etc. - Farmers are proverbially economical in the use of money. They labor hard for it, and hence realize its value, and are loth to part with it. This economy in the spending of money is often carried so far that it borders upon penuriousness, and we frequently hear it remarked, that farmers, as a class, are exacting and close-calculating in their dealings with others. No doubt this accusation is often unjustly founded, but however this may be, there are many things in respect to which the average farmer is too prodigal, the saving of which he does not seem to realize will aid very materially in augmenting the profits of the farm, and which, if rightly appropriated, is equivalent to money earned; since the resources of the farm will thereby be increased without additional outlay.

One of the common wastes of the farm is that of manure. The great need of the farms in the older-settled portious of the country is more manure, and the problem for the farmers of those sections generally to solve, is how to obtain an adequate supply.

Owing to the lack of a sufficient amount of farm manure, many farmers are obliged to depend upon superphosphates and other commercial fertilizers to supplement the quantity required, the expense of which reduces largely the profits resulting from the crops. Although this lack of manure is generally admitted, yet, inconsistent as it may seem, there are very few farmers but that permit a large proportion of the manure made by the farm stock to be wasted. It is a common practice with farmers to permit the liquid manure to be lost. How few barns in the country are provided with means for utilizing this valuable fertilizerl And yet it is claimed by,chemists, that the liquid manure of cattle is of as much value as the solid.

Prof. Dana states that "The quantity of liquid manure produced by one cow annually is equal to fertilizing one and a quarter acres of ground, producing effects as durable as do the solid evacuations. A cord of loam saturated with urine is equal to a cord of the best rotted manure. *** If the liquid and solid evacuations, including the litter, are kept separate, and the liquid is soaked up by the loam, it has been found they will manure land in proportion, by bulk, of seven liquid to six solid, while their actual value is as two to one."

Other noted authorities might be cited, but we have treated of this subject so thoroughly in connection with fertilizers, that a repetition is not necessary here.

The fact has been sufficiently established by experiment and chemical analysis, from the best authorities, that the farmer who makes no provision for saving the liquid manure made by his stock loses fully one-half the value of the manure they supply, while he who utilizes both the liquid and solid excrement, has, therefore, double the amount of manure every year than he otherwise would have. 
To permit one-half of the manturial resources of the farm to be wasted is certainly not good economy, when with a little extra care it might easily all be saved. Furthermore, to spend money in buying commercial fertilizers to make up the deficiency of this waste, is not good management, when the expenditure might so easily be obviated. In order to save the liquid manure, various methods are employed, such as barn-cellars, etc. These are very con. venient, but not absolutely essential for this purpose. By the use of proper absorbents for the bedding of the stock, which will take up all the liquids, they may thus be saved. Suitable material for this purpose may be easily procured, such as dry loam, dry swamp-muck, fine sand, road-dust, chaff, dry leaves, etc.

Another wasteful practice is in throwing the solid manure out of the stable-windows and permitting it to lie exposed to the rains and hot sun until wanted for use upon the land, where the most valuable fertilizing elements are either washed out or evaporated. Manure thus exposed loses fully one-half its value. Thus we see that those farmers who permit the liquid manure of their stock to be wasted,-which as previously stated is computed to be one-half ol the entire quantity, - and are in the habit of allowing the solid manure to be exposcd to the weather,-thus permitting one-half or more of that to be wasted,-lose fully three-fourths of the entire amount of manure made by their stock. Dr. Voelcker found by careful experiment, that five tons of fresh manure, after having been spread in the yard and exposed to the weather from November $3 \mathrm{~d}$ until the following August 23d, lost nearly two-thirds of its actual value as a fertilizer. Now, if farmers would take pains to prevent this waste of manure, by the use of absorbents for the liquid, and shelter for the solid excrement, either in barn-cellars or sheds, a good supply of mauure would be had where now it is limited, thus requiring less expense for the purchase of commercial manures, and an increased fertility of the soil with no additional expense, together with better crops, and consequently larger profits.

Another waste common on the farm that might be appropriately mentioned in this connection, is in exhausting the resources of the soil by constant cropping, without a sufficient supply of manure. By this means valuable lands in some sections have become almost worthless, and farms that once were very productive, yielding for a time large profits, have become either entirely unproductive, or can hardly be made to prodnce sufficient to pay the expense of tillage. This might all have been avoided by the judicious use of supplying a sufficient amount of plant-food adapted to the crops to be cultivated, and thus valuable lands saved from deterioration, and running to waste. Very few farmers would think of spending to no purpose, or wasting the amount of money which such injudicious management involves, yet they will permit such wastes to go on upon their farms year after year with the utmost indif. ference.

Lack of Economy in Labor. - While farmers, as a general rule, are a very hard. laboring class, working more hours to the day than those engaged in almost any other avoca. tion, yet it is too often the case that the time and labor expended do not result in the profits that they otherwise would, owing to the various ways in which there is a lack of economy in utilizing the labor thus expended. The neglect to perform work at the proper time is a source of waste on the farm, as well as in every other business. If the plowing be done for the crops when the soil is dry and in a suitable condition for tillage, instead of being saturated with water, much needless labor will be saved both to the farmer and team.

Planting at the proper season, and usin $\zeta$ good seed for that purpose, will economize labor and bring profits that cannot be obtained by planting too early and thus permitting the frost to kill plants, necessitating a second planting, or planting so late that only a partial crop can be procured from the labor expended. The planting of poor seed is labor wasted. The farmer who takes measures to kill the weeds in his cultivated fields when they first make their appearance and can easily be exterminated, instead of permitting them to grow until they have secured a partial or complete possession of the soil, will save not only much 
hard labor in destroying them afterwards, but will prevent the injury and loss to the crop which would be caused by negligence in this respect.

The same principle holds true with regard to harvesting crops, or any other of the duties pertaining to farm labor. We have seen farmers neglect to provide suitable drainage for lands until the heavy rains came and saturated the soil with water, and when in this condition they did, with twice or thrice the amount of labor that would otherwise be required, what should have been done in a dryer season. Carting dirt from one point to another when the amount of water in it would constitute nearly, if not quite, one-half its weight, instead of doing this when the soil was dry, is another of the many methods of a waste of labor. Some persons seem to possess the faculty of always doing things in the hardest possible way, through negligence and careless management, and in order to secure certain results will perform many times the amount of labor that would be required by others practicing a thorough and systematic method.

Slovenly Management.-The slovenly management seen on some farms causes one to wonder how, under such a system, or, rather, with such a lack of system, any profit whatever could be made, and yet we know farmers who have made quite large profits by farm. ing in just this manner. Their work would be done at nearly all times out of season; the repairing of fences would be neglected, and much time be spent in the most busy and important part of the season in looking up the stock that had for this reason strayed away. Dilapidated farm buildings were permitted year after year to become more dilapidated; drains filled up and were left to take care of themselves. Wood necessary for the household would never be provided in large quantities beforehand, but be picked up from time to time on the farm, as the supply became exhausted, in the shape of broken rails, old rotten stumps, or trimmings from the apple orchard, which would have to be burnt in a green state.

The poultry would have no warm quarters provided, but be obliged to find a roosting. place in the trees or other out-of-the-way places.

Pigs wallowed, and even swam in the accumulations of undrained pens and yards. The harvesting of some of the crops would be delayed so late as to be nearly ruined by the frost, and every thing on the farm would seem to go in a hap-hazard way.

Now, if any profits whatever can be made by such methods, how much more profitable will farming prove under a thorough and perfect system of management.

Doing Work Over-Nicely.--In striking a contrast with the former, might be men. tioned those who spend too much time and labor in performing their work. They do not possess what is called in hackneyed phrase, "the knack to turn off work," and much time and labor is spent in doing unnecessary things, or that labor which will prove of no real ben. efit to themselves or any one else. They either spend much time in doing things that are not at all essential, and thus waste time and labor, or they are over-nice and particular in the per. formance of necessary work.

They perhaps hoe their crops with the utmost precision, by hand, when a horse-hoe or cultivator would do the work just as well, and with the expenditure of much less labor and time. Or they may insist on carefully mowing their fields with a scythe, instead of using a mowing-machine, because they regard the former method as doing the work better, and leaving the surface more even than the latter. Such persons seem to fail to perceive that labor and time are equivalent to money, and that all ncedless waste of either is a loss in money value. Work should always be done thoroughly and well, but an unnecessary expenditure of time and labor is a waste, and cannot properly come under the system of good management.

Use of Poor Tools.-Another example of false economy is seen in the use of poor tools, or those which are not adapted to the purpose designed. The employment of heavy and cumbersome implements when lighter ones would do the work just as well, and perhaps 
better, is the wasting of a certain amount of labor for either the man who handles them, or the team that draws them. It is, therefore, poor economy to furnish implements that are ill adapted to perform the work with facility to hired help, since the full value of the money expended for such labor is not realized in return.

It is not good management to use inefficient tools, when by a little extra expenditure for suitable ones, from a third to a fourth more labor might be performed in the same time.

The farmer who persists in using the old-fashioned cradle in harvesting his grain, instead of a good reaper, involves the waste of both labor and grain, since with a good reapingmachine that implement and horses could easily and quickly perform what would require a great amount of labor and time when done by hand. In order to economize labor and time, the best implements and methods should always be employed in every department of farm labor.

Borrowing Tools.-The practice of borrowing farm tools is one that is quite too common in some sections; more especially in those localities where small farms are cultivated. To such an extent is this practice followed by some farmers, that the larger portion of the work performed on their farms is done with borrowed implements. Such borrowers usually apply to their neighbors for favors with some such remark as, "Are you going to use your cart to-day?" or, "Are you using your horse-rake?" seeming to take it for granted that if not in use by the rightful owner, it is their right to appropriate all such farm implements to their own benefit. It is not infrequent that farm tools, by constant lending, are worn and broken more by the borrowers than by the owner. The impertinence of such borrowers is often truly astonishing. We know of one man who positively refused to return a borrowed tool until he was through with it himself, when it was sent for by the owner. The man who will constantly depend upon his neighbors for those things that are essential upon every well-regulated farm, must indeed possess a mean and selfish disposition, and but little of the true spirit of manliness. But setting aside the meanness of the practice, it is certainly not a good policy to spend time and run the risk of disappointment in hunting up tools on neighboring farms, that should be always at hand and ready for use. It is generally the case that those who have so little self. respect as to be constantly dependent upon others, have at the same time too little honor and principle to return the articles borrowed in proper time; hence, those who are frequently being applied to for such favors have generally, in addition to the annoyance occasioned by the breakage and wearing out of their tools by others, the additional one of looking up and bringing back the borrowed articles.

Although we believe in acts of neighborly kindness, and in accommodating others to a reasonable extent, yet such constant borrowers should be taught the lesson of honesty and self-dependence by either a charge of a stipulated price for the use and breakage of tools, or by a prompt refusal to loan.

Lack of Care of Farm Implements.-In addition to slovenly management gen. erally on the farm, might be mentioned the neglect to take proper care of farm implements, such neglect often causing greater injury to them than all the wear in performing the work for which they are adapted.

There are some farmers who are very careful respecting their expenditures of money, talk of hard times and the necessity of practicing economy, never purchasing anything except what they consider absolutely essential, but who will leave valuable farm machines out in the open field, exposed to the weather for months, without a thought of the waste thus incurred. Plows, harrows, mowing-machines, horse-rakes, reapers, carts, wagons, etc., are left where their use in work happens to stop for the season, and there they remain exposed to the rains, snows, and hot sun until they are wanted the following season, when they are then looked up for that purpose. It is unnecessary to state that such negligence will cause more injury to 
farm implements than their constant use would during that period, and the farmer who fails to provide a place of shelter for his tools, has a very poor idea of the proper economies con. nected with his business, as well as but little system or method in managing it. Western farmers are generally more negligent in this respect than Eastern, but such mismanagement is quite too commonly seen in all sections of the country.

Repairing Tools.-Failing to repair tools at the proper time is also a great waste. The old maxim with regard to "a stitch in time," is as true in relation to farming as any other business. Some farmers never think of repairing a wagon, or harness, until it absolutely breaks down or gives out altogether, and even then make a temporary repair in a bungling manner, to make the broken parts hold together for the time being, at the imminent risk to life and limb thus involved. Neither wagons nor harnesses are scarcely, if ever, oiled, the friction thus produced on the axles of the former causing them to wear out much faster than they otherwise would. The tire of the wheels gets loose, and is never reset, until by long rattling-which would be sufficient to signal the approach of such vehicles at a long distance -they finally come off, and repairing becomes an imperative necessity. Harnesses get dry and stiff for lack of oil, soon break in consequence, and are tied together with tow strings, or leather strips made after the manner of the old-fashioned cobbler's shoe-strings, and are thus used until they can be used no longer, and a new one is bought, to receive the same kind of care. It seems strange that farmers who are often economical, and even penurious with respect to spending money for other things, should permit such wastes, when with a little care it might be obviated.

A reaper should never rattle when at work, and if by taking hold of a shaft it can be shaken, it shows that the boxes need filling. This may be done by almost any farmer himself, or it will cost but a few dollars to have it done at a machine-shop. A few pounds of old type when melted will serve for the material. In the use of all farm machines, they should be kept in good repair, with every nut and rivet tight. They should also be kept well oiled where the latter is essential.

A few moments spent in replacing a lost bolt, tightening a loosened screw, or making any such slight repairs, will often save much labor and expense in making more extensive ones that may be required through neglect at first. Serious accidents to rider and team may also not unfrequently be avoided by attending to such slight repairs in season.

Insufficient Help. - The number of laborers on the farm should always be adapted to the amount of work to be performed. With ideas of false economy, some farmers employ only about one-half the hired help that is necessary in order to perform the work in the proper time and manner, and endeavor, by working this force to the utmost, early and late, to be able to secure the accomplishment of the work for the season, at a much less expense than would ordinarily be involved in accomplishing it. By this course, much of the work is delayed beyond the proper time of doing it, and many of the crops become seriously injured. The cultivation and harvesting is in many instances delayed, weeds obtain the mastery of the soil, on account of other duties pressing, that there are not sufficient hands to perform at the proper time, crops become over-ripe, the grain shells out in the field, and the whole machinery of the farming system seems elogged. The loss occasioned by such management will usually far exceed the expense of employing a suitable number of hands; while hired help who feel that their employer is endeavoring to over-reach them by exacting more from them in service than is just, for the compensation received, will not take that interest in the work, or generally perform it as well, as when they feel that they are fairly treated. Besides, aside from motives of policy, which should be secondary, any course that is based upon such selfish motives is not founded upon honor, or the principle of right, and the man who is not willing to give a just and fair equivalent for what he receives, is not an honest man, and is not 
entitled to the respect of others. The employer who treats his help fairly and reasonably in all respects is the one who will, as a general rule, secure the best results from their service.

Poor Teams.-Labor and time are often wasted by the use of inefficient teams in per. forming the farm work. Perhaps oxen are used where liorses would be more serviceable, or the reverse - teams that might be good in themselves, but not adapted to the kind of work or the place. For performing certain work, such as plowing, two men may, perhaps, be employed where one man with the proper team would do as well, and the labor of one man appropriated to other purposes, thus saving the labor of one extra hand.

In other cases, weak, fractious, lame, or otherwise inefficient animals, may be employed, which results in slow progress and work poorly done, when a good team of the right kind would have performed the work better and perhaps in one-half the time. Besides, it costs no more to maintain a good team than a poor one, and the labor done by the former is so much more satisfactory, that it is far better economy to always keep good teams, although the first cost of purchasing such would be more than that of an inferior one. Good teams are the cheapest in the end.

Inferior Stock.-The same might be said of the stock bred upon the farm, or animals selected for the dairy, as of poor teams. Under a mistaken idea of economy, the lowest-priced animals are used, when the product of the best cows for the dairy and thoroughbred stock for breeding purposes would more than double the profits, besides soon paying for the extra expense in purchasing. Where milk is the principal object, a milking breed should be selected; if beef is the leading consideration, a beef breed should be chosen. The aninals should be adapted to the purpose for which they are to be used. False economy, and a lack of the proper knowledge in selecting, breeding, and feeding animals, is one great cause why this department of farming does not prove as remunerative as it otherwise would.

Since it requires as much labor and expense to maintain inferior stock as the best, it is a waste of labor and money as well as loss to the farmer to maintain and breed the former, when so much larger profits could be secured by keeping the latter. There is too much indifference manifested by farmers generally with respect to this subject. Great improvement could be made in the poorest flocks and herds in a few years, by the use of thoroughbred sires of suitable breeds, and farmer3 generally who have not previously given their attention to the subject, will find that they can advance their interests and profits very materially by this means. The same principle holds true with respect to feeding and handling all kinds of farm animals. Generous feeding, good care, and kind treatment, have quite as much influence, if not more, in regulating the profits which result, as the breed. A poor animal or farm implement is costly at any price, while the best are eventually the cheapest.

Inconrenience of Farm Buildings, etc. - Another cause of waste of time and labor may be found in the fact that the farm buildings are not conveniently arranged or the farms properly laid out.

On some farms the buildings have every convenience with respect to construction and location; there are suitable places for the storage of crops and tools, and the shelter of stock. Hence the labor is all utilized to some purpose, and there is no unnecessary waste in this respect. Where soiling is practiced, the crops grown for this purpose are near the barn, where they may easily be cut as wanted and fed to the stock, while the yards and sheds are supplied with an abundance of pure, fresh water for watering all the farm animals. The cultivated fields are conveniently located for carting manure and for tillage, and everything seems arranged to help on the farm work, instead of hindering it. On the other hand, some farms seem so arranged as to require double the amount of labor and time in accomplishing the same results. The buildings are not convenient in location or arrangement, and there is not sufficient room for the storage of anything. No water is provided in the yards, and the 
stock must be driven quite a distance twice a day in winter to a field, and the ice broken in order to water them. This involves much trouble and time, with a liability to injury to some of the animals from slipping on the ice, or hooking each other, aside from the exposure occasioned on severely cold or stormy days. The soiling crops are grown at a distance from the barn, involving the necessity of the use of a team every time anything is cut for feeding, while all the arrangements on the farm seem calculated to impede rather than assist the progress of the farm-work. Much of the labor expended in such cases would have been unnecessary under a better system of arrangement, and is a constant expenditure of labor and time that brings no real compensation in return.

Better Knowledge of Farming, and Less Drudgery.-Money, time, and labor are often wasted by farmers, from a lack of knowledge of the nature and requirements of the soil and plants cultivated.

A vast amount of labor is frequently expended in attempts to prodnce crops from soils which are not adapted to those particular kinds of plant-growths, or which lack some of the essential elements of plant-food. Failing to inform themselves with respect to the improved agricultural methods of the present time, many farmers do not profit by that which has been gained by years of experience and observation; hence, they are a generation or more behind the age in which they are living. Knowledge is power in every branch and department of business, and the farmer who possesses the best knowledge of his business is the best capacitated to make that business a success.

The hands should serve the head, and the farmer that has the best agricultural knowledge, combined with the mental ability to successfully plan and execute the most thorongh system, will not be obliged to make his life a mere drudgery of toil from morning till night, day after day, and year after year. The devising of the best plans and methods should be the first consideration, and their execution secondary. He who drudges on, withont any system or method, will never be anything but a. mere drudge, or attain to auything but a meager success. Farmers, as a class, should spend more time in informing themselves in their business, by reading the best agricultural books and papers, attending farmer's' clubs, etc., and also more time in devising the best methods for all kinds of farm-work, based upon the knowledge thus obtained. By such means, more hear-work, and fewer hours of labor with the hands, will secure far better results than are now commonly obtained.

Farmers should also acquaint themselves with the best and uniformly cheapest rates of transporting their crops to market. This consideration will be called in question in deciding what crops to raise. Farmers, as a class, also need a better understanding of business principles to enable them to buy and sell to the best advantage,- the time, manner, and rates for the different products of the farm all having due consideration. They need, in this connection, to deal more directly with the consumer and manufacturer in disposing of their products, and in purchasing implements, clothing, etc., and less with the "middle-men," whose commissions largely modify the receipts and expenditures of the farmer in such cases. With better information on agricultural subjects, there will be better tillage, the use of better seed for crops, better farm-stock, better planning and systematizing, less hard labor, and better profits. Constant and severe toil incapacitates the mind for the best thought of which it is capable; there will be neither the energy nor time for it.

A certain amount of rest from hard labor is, therefore, a paying investment, as the hands will then be made to serve the brain to the best advantage, and there will be less drudgery and more knowledge and skill in conducting the business, while larger profits other conditions being equal - will be the result.

Improved Farm Implements Essential.-The improvement made in farm implements during the last quarter of a century is truly astonishing, as well as the influence 
of their use upon agriculture. During that period they have been substituted in a great measure in place of hand-labor on the farm, which has resulted in vastly increasing the agricultural resources of the country, since by their use labor can be performed much cheaper, faster, and better than by hand. They are a great convenience on small farms, and absolutely indispensable on large ones, where extensive crops could not be cultivated without them.

Take the reaper, for instance, the introduction of which into the grain-fields has added many millions of dollars to the annual harvests, by rendering it possible to secure the entire product, and also by admitting of a largely-increased area of grain culture. The same might be said of improvements in harrows, cultivators, plows, and all other farm machinery.

The use of suitable farm implements also enables a farmer of small means to conduct a much larger business than he otherwise could where only hand-labor was employed, thus cheapening the cost of production.

Since labor can be performed so much more easily and cheaply by the use of improved machinery, it is the practice of economy to employ them whenever practicable. There are, however, many farmers who, with ideas of false economy, still persist in depending mainly upon hand-labor, while the fow implements that may be in use upon their farms are of the most inferior kind.

Where the amount of crops cultivated will warrant the purchase of the best machines, they will soon pay for themselves many times over, in the amount of labor saved and the increased facilities thus afforded for cultivating larger and better crops. The use of machines also saves time, labor, and health. By their aid the farmer can raise larger crops with less physical labor, and consequently less risk to health. As a general rule, farmers look older, according to their years, than almost any other class of individuals. This is due to the severe toil to which they subject themselves, and the constant over-taxing of their strength. We do not wish to be understood to convey the idea that we do not consider farming a healthy business. On the contrary, we believe it the most healthful of all occupations when judiciously followed. But every good is liable to perversion, and we believe the majority of farmers either injure their health, or hasten old age, and break down in the latter part of life quicker than they ought, through the excessive hard labor that they impose upon themselves. If machines can be employed to perform the necessary farm-work, and thus prevent a waste of strength and health, how much better to make use of this means than to make life a drudgery, with all the attendant evil effects.

Instead of feeling that he cannot afford such aids, the farmer who has a correct idea of economy will feel that he cannot do without them. In connection with the use of better farm implements, which, by admitting of better tillage and increased facilities of cultivation and harvesting, will enable the farmer to produce larger crops, might be mentioned the economy of so fertilizing the soil, combined with improved methods of tillage, that much larger crops may be produced from the same area. By this means labor will be saved, and comparatively larger profits be secured. English agriculture, where a dense population requires that the cultivation of the soil be such as to secure the highest results from a limited area, might be mentioned as an example of this method. Where land is abundant even, this principle will hold true to a certain extent, since about the same amount of labor will be required for the cultivation and harvesting of a light or a heavy crop.

The land must be plowed and harrowed, the grain sowed and reaped, and if, by a little extra care in preparing the soil, a third or one-half larger crop can be produced, it will well repay for the extra labor and expense bestowed.

A leading aim with the farmer should be to raise the largest amount of farm products with the least expense. To half till a field of ten acres, and obtain but three or four hundred bushels of corn, when that amount could be raised by cultivating properly four or five acres, would be poor economy, sínce the same result could be reached with balf the amount of 
plowing and other necessary labor in cultivating and harvesting. Besides, the well-cultivated four or five acres would be left rich and mellow, and could be much more easily worked for subsequent crops than the hard, poorly-tilled soil of the ten acres.

It is the best economy for the farmer never to cultivate more land than can be done in the most thorough manner. Anything beyond this, as a general rule, will result in a waste of labor, and prove unsatisfactory in the end.

Systematic Management.-One of the great wants pertaining to farming generally is a more perfect system of management,-a system that will not only admit of the various kinds of work on the farm being done in the proper time, but in the most profitable manner. Time and attention should be given in ascertaining the best methods of conducting the busi. ness, and then, having decided upon and adopted a certain plan or system, it should be car. ried out thoroughly.

Experiments in this manner may sometimes result in changes in certain respects, and new and improved methods will be adopted, from time to time, if the farmer is progressive-as he should be-in order to keep abreast of the times and the age in which he lives; still, he should conduct his farming in a methodical manner, whatever that system may be, and never permit the business to manage itself, as is too frequently the case.

A well-developed system will enable the farmer to pursue an even, uniform plan of operations, and have a tendency to obviate the frequent shifting from one department to another, with no permanency in any one of them, which practice reduces farming to a continuous routine of profitless experiments and ventures. Not that we would condemn experimenting in a judicious way. Every progressive farmer will not only experiment for himself, but will profit by the intelligent experiments of others; but experiments should be the exception and not the rule for general practice in farming, and should always be made judiciously, and with an intelligent understanding of the nature of the things to be experimented with.

A lack of system is one of the fruitful causes of failure in all kinds of employments, and especially so in farming. To be a successful farmer requires a wide range of knowledge, and methodical practice. He must not only know when and how to cultivate the various crops, the soil to which they are best adapted, but how, when, and where to dispose of them in the most profitable manner, how to purchase the necessary farm supplies to the best advantage, what crops and farm-stock are the most profitable for him to raise, etc.

In order to ascertain definitely with respect to the comparative profits of different branches of farm industry, and the real state of his business, every farmer should keep a correct account of all the receipts and expenditures. It is only in this way that an accurate knowledge of the profits or losses of the farm can be obtained. Such an account is also often convenient for reference, as furnishing important data that can be obtained in no other way. Keeping such an account has also a tendency towards systematic practice in every department, and cannot fail of much profit to the farmer in all respects.

More Capital in Farming.-It frequently is the case that the limited success of the farmer is due to a lack of sufficient capital. It may be the young farmer just starting in life, who has invested nearly all of his money in purchasing a farm, and has but little left with which to supply the necessary outfit. Hence, in procuring a team, farm implements, stock, etc., he is from necessity obliged to run in debt or purchase those that are inferior; or, if of first quality, not the number that is needed on the farm sufficient to make the business as profitable as it should be. To be limited in capital, or to incur a heavy debt, are both a great drawback in successful farming, and many a young man has to contend with this evil year after year before being freed from it. As a general rule, it will be better to purchase less land at first, and add to it from time to time as means will permit, and retain a sufficient amount of capital to furnish and stock it well, rather than to expend nearly all in land and be embar. rassed in managing it for lack of means to render the labor bestowed profitable. 
There may, however, be exceptions; for instance, desirable lands that can be purchased at a low rate may be so located as to soon largely increase in value, which, if lying even unimproved for a few years, will bring in selling many times the original expenditure; but we refer more particularly to farms purchased with a view of cultivating the whole, or larger portion of them.

Again, with ideas of false economy, many farmers who possess an abundance of means for cultivating their lands in the most successful manner, will limit their expenses to the lowest possible figure, permitting their farm buildings to run down for lack of repairs and improvements, using antiquated and unsuitable machines in doing the work, in order that they may put the receipts of the farm into the bank, bonds, or other securities. By investing such receipts in the improvement of lands, farm buildings, farm implements, and the farm business generally, might in many cases perhaps double the profits resulting from the former course. Extravagance and waste should always be avoided, but it must be remembered that it requires capital to make capital, and the farmer who invests a sufficient amount of money in his business to maintain and continue all laudable improvements, has the best oppor. tunities, other conditions being equal, to make that business a success. 


\section{FARMS AND FARM BUILDINGS.}

\section{CHOICE OF FARMS.}

$\mathrm{T}$ HERE is something in the ownership of land that gives independence to a man's character. It is in itself an honor, and has connected with it a kind of reflex influ. ence that does not seem to be associated with other possessions. The retention of the old family homestead and farm by a long line of ancestry for successive generations, is one of the interesting features of the older-settled portions of the country, and is in many respects a desideratum, whether we regard it in the practical light of an investment, or of a pardonable pride, as the basis of a sentiment of family honor and respectability that is to be associated with the name and inheritance. Among the many changes of ownership of lands, it is pleasant to see, as we do occasionally, altbough too rarely, an instance of this kind. While the few among the multitude of farmers have this privilege of retaining the old home, the majority, in establishing themselves in business, either from necessity or choice, locate elsewhere.

In selecting a farm, there are many things of importance to be taken into consideration. The advantages and disadvantages are to be carefully weighed, and the sum of each taken into the account. The location, quality of soil, size, the purposes to which it is to be appro. priated, etc., all are to be judiciously considered, and that decision given which seems to favor the highest possibilities of success. But it must be remembered that absolute perfection, either with regard to mankind or locality, will never be found on the face of the whole earth. We must therefore not expect it, and take things as we find them, making a choice of such as seem to us, by the use of our best judgment, to contain the most good and the fewest erils. No location can be found but what will have its disadvantages as well as its peculiar advantages, and, according to the great natural law of compensation, the sum of the one will in a measure offset that of the other.

Whether mixed agriculture or special be the object, the facilities for transportation and nearness to market must necessarily be considered in selecting a farm, as well as the adaptability of the soil and climate to the crops to be produced. If tropical products, for example, are to be raised, the farmer must make choice of lands where the soil and climate are best adapted to such crop. If grain is to be the specialty, some of the Western States will afford the best facilities. If the object be sheep husbandry on a large scale, a ranch in the great West is most assuredly the place to be chosen. If it be the care of large herds of cattle, it should be a ranch in some of the far Western States or Texas. But if the farmer wishes for himself and family the peculiar advantages to be derived from a home in the older-settled portions of the country, some State in the Eastern section should doubtless be his choice.

If he desires a locality remote from other inhabitants, he must forego the educational, social, and other privileges that a nearness to a city or largely-populated section will furnish; while, if the choice be in a densely-populated region, he must of necessity give up the peculiar advantages of retirement, and accept the objectionable features that such a place may pos. sess. In making a selection of locality, the farmer should never overlook the health, com- 
fort, happiness, and general welfare of his family. These should always be primary, and the money-profits of the business a secondary consideration; for, while money-making is one of the great desideratums with most men, it is not the chief good in life, neither does it constitute the sum total of earthly happiness, as many, by their lives, seem to regard it. Success and happiness in life do not depend so much upon the location and business, as the character of the individual. As a general principle, the man makes the business, and not the business the man. Success is what we make it, and the man of the right stamp, who is honorable in his dealings, energetic, capable, systematic, practical, and thoroughly business-like, will be successful wherever he may be located, or in whatever laudable business he undertakes. And those farmers who are generally dissatisfied with their condition, and imagine that they may be greatly benefited by a change of place, will find, in the majority of cases, that the fault is more in themselves than in their surroundings, and that the adoption of a better system and improved methods will produce better results than a change of locality. For this reason a change should never be made without due deliberation, and an honest endeavor to ascertain the true reason for a lack of success that has thus far characterized their business. Of course, there are exceptions to this, as to all rules, but we think it will prove applicable to most cases.

The desertion of the old homestead is too frequently a mistake with young men. If properly managed, money can be made there as well as elsewhere; while other considerations of importance are involved, such as the influence of the association connected with the old home on successive generations, their tendency to restrain from evil and promote the good in a desire to hand down the family name with honor from one generation to another, etc. In many of the sparsely-settled portions of New England the old homesteads are passing into the hands of foreigners, and the state of society, as well as the lands in those sections, is deteriorating. For the sake of the general good of the community in such localities, enough of the native population should remain to sustain our institutions and maintain that intelligent enterprise and progress which is characteristic of American people, and which shall be a credit to the country at large.

Advantages of Large Farms.-The size of a farm should always be adapted to the purposes to which it is to be devoted, but it will also be largely influenced by the location. In the Eastern section, where there is a dense population, where taxes are high, and land sells for a comparatively large price, smaller farms will be found more profitable than at the West and South, where land is plenty and can be bought at much lower rates, and also where extensive use can be made of improved agricultural implements. As a general rule, the number of men who are capacitated to successfully manage extensive farms is small compared with the number represented by those who can make profitable the management of farms of small or medium size. Sorne men have special talents for successfully engaging in large enterprises.

They possess the ability not only to originate the most perfect and systematic plans, but to successfully execute them as well. But these are in the minority, and it will be found in the majority of cases, that the management of small farms, or those of moderate size, will in the aggregate, prove most successful. Large farms, however, possess many advantages over small ones. While the former will, as a general rule, cost less in proportion to their size than small ones, they furnish a larger proportionate area of tillable soil from which an income can be obtained. The insurance, taxes, repairs, and other expenses will also be larger in proportion to the investment on a small than large farm; hence, there is a larger proportion of unproductive property in the former than the latter.

Large farms require a smaller proportionate amount of capital to be invested in build. ings. The same principle holds true with respect to fencing.

The expense of furnishing farming implements on a small farm is very much higher in 
proportion to the amount produced, than on a large one, while the cultivation, being performed on a larger scale of operation, can be done with much more facility, and under a more perfect system of management on the latter.

A farm of seventy-five or a hundred acres will require nearly as many kinds of farm implements for performing the work, as one of five hundred acres, and they will cost about as much when purchased, as those for the larger farm. The principal difference will be, that the owner of the five hundred acres will have several times as much profit from their more extensive use, as the owner of the one hundred acres; hence, a much larger per cent. of profit on the investment. In fact, on very extensive farms, where a number of the same kind of machines are required, such as sulky or gang plows, harrows, reapers, etc., these implements can be purchased at a large discount, making the expense for this purpose on the large farms very much less in proportion to small ones. The same is true to a certain extent with respect to teams for the farm. In disposing of produce where mixed farming is practiced, the time and labor spent in taking to market the surplus products of a small farm are about the same as for a large one, with the exception of a difference in handling in loading and unloading, and the heavier cartage.

While the business of a large farm can be managed to better advantage, and those who are adapted to it can make it more profitable than that of a small farm, still there is almays more risk attending it. The losses are greater in cases of failure. On cheap lands, and until a section becomes densely settled, larger profits will be found generally in cultivating large areas devoted more particularly to special crops. When mell populated, the land becomes more uniformly dirided, the farms, as a consequence, are reduced in size, and a more thorongh cultivation is given. The natural result of continued cultivation of large areas devoted to special crops, is to produce exhaustion, while smaller farms under a more thorough system, combined with proper rotation, will not only retain, but increase their fertility.

Advantages of Small Farms, etc. - While large farms possess many advantages over small ones, yet the latter also admit of some especial advantages orer the former. In the Western sections, where bonanza farms are under cultivation, corn and other grains bring a much less price than in the East, where its cultivation is necessarily limited; hence, the Eastern farmer receives comparatively much larger returns for his crops, and therefore it is essential that the Western farmer should cultivate more land and secure larger products in order to make even the same profits that result from the crops raised by the Eastern farmer.

Small farms always require less capital invested, and a man of moderate means can establish himself on such a farm without incurring a heavy debt, the interest of which would be con. stantly consuming his profits, while the mortgages would prove the source of a continuous "nightmare" if he were a man of energy and business capacity. They also require less hired help, less expenditure in supplying with suitable teams, etc., while there is less care and anxiety, as well as less risk attending their management.

A small farm admits of more thorough culture, and, if properly tilled, can be k'ept in a higher state of fertility than a large one, and be made to produce a larger crop in proportion to the area cultivated. While there are some farmers who could increase the size of their farms with profit, yet by far too many own more land than they can properly manage, or their capital will warrant, and are what might be called "land-poor." As a general rule, it is not profitable for the farmer to hold unproductive property. He should own no more tillable land than he can properly cultivate, and should add to his farm from time to time as his resources will admit. An authoritative writer on agriculture says, respecting capital in farming:- "Were I asked to point out the best-paying farms of this country, I should seek them not where land is cheap and agriculture conducted on a rast scale, but upon the outskirts of some metropolis, among the market-gardens, the secrets of whose success is hidden only by the shades of night, when cart-load upon cart-load, the waste of city consump- 
tion, is conveyed back to the outlying farms; and thus while the world is sleeping, is supplied as to a growing school-boy, such vitalizing gain as more than balances the daily loss. Nowhere better than such sections of city-surrounding country, can the system of high farming be carried to perfection. The nearness of the consumer furnishes a ready market for vegetable products which, from their perishable nature, demand immediate consumption, while unrivalled facilities for obtaining fertilizers leave a margin for profits which can scarcely be equalled in places more remote."

As a general rule, the money-value of land, the size of the farm, and the kind of products to be raised, are determined by the distance and importance of the nearest markets. As an instance showing the profitable revenue derived from a small farm, we cite that given at a recent farmer's meeting in New England, by Richard Van Deusen of Shaker Village, who stated that he cultivated twenty four acres, and was not satisfied unless he made each acre return him a hundred dollars of clean profit. He raised a variety of products, in order that the harvesting could extend over a longer period. He read from his diary a list of the crops raised and sold the previous year, as follows: - " 4,300 pounds of red strap-leaf turnip-seed, $\$ 1,032 ; 915$ pounds of onion-seed, $\$ 2,297$; potato onions, one-fourth of an acre, $\$ 175$; early potatoes, $\$ 125 ; 40$ rods of sage, $\$ 80 ; 7$ acres of golden wax-beans (blasted), $\$ 117$; threefourths of an acre of cabbage for seed, $\$ 180 ; 4 \frac{1}{2}$ acres of cucumber-seed (20 cents a pound), $\$ 1,725 ; 1$ acre of peaches, $\$ 304 ; 4$ acres of pears (with two crops of hay on the same land), $\$ 100$; one-fourth of an acre of asparagus, $\$ 75$. Of the last from three to five bushels were cut a day and freely used by the Shaker family. In all, $\$ 5,282$ worth of produce was sold from twenty-four acres, or at the rate of $\$ 520$ an acre. His expenses were $\$ 1,212$, and the chief items were $\$ 841$ for labor, and $\$ 317$ for manure."

The fifty-acre farm of Artemus Fisher, near Keota, Iowa, is described, together with its management and success, by the Keota Eagle, as follows:-

"He keeps one team of horses, three first-class cows, and a nice little drove of the best hogs. He milks his cows for the creamery, and they made him nearly $\$ 200$ last year. Will, perhaps, do better this year. He will sell $\$ 500$ worth of hogs this year, and have 30 stockers to keep over. He has $\$ 200$ worth of flax-seed to sell; has an abundance of hay and grain to keep his stock in first-class condition during the coming winter. He keeps everything in the best order about his farm; his cows revel in clover up to their eyes; he attends to feeding, watering, and milking as regularly as the clock strikes; hence he gets the best results with the least possible feed. He keeps his stock under cover in the winter, and never allows any animal to shiver in the fence-comers. He has a barn that is a model of convenience and economy. It is snow-proof, and as warm as the old-style kitchen. He has a first-class selection of fruit-not a large orchard, but a choice selection of the varieties that thrive and bear the best in this locality. Everything about the farm bears marks of intelligence, thrift, and economy. Besides making a living for himself and wife, he will sell at least $\$ 800$ worth of stuff off his fifty acres this year, and not be exceeding that of former years. There is no rush or hurrah about the work on this model farm. Everything goes off quietly and regularly. The expenses are very small, and the gains sure."

Thus we see, that rightly-managed, and their highest possibilities tested, even very small farms may be made quite profitable.

In those sections where the farms are generally small, it is a good practice for a few of the proprietors to coöperate in the purchase of expensive farm-machines or choice stock, thus largely reducing the outlay to each, and increasing proportionately the profits, while it permits each individual interested in the ownership to obtain the benefits resulting, at a comparatively slight expense. Whatever the size of the farm, the capital should never be all invested in land. Thre should always be a sufficient amount of working capital left in the hands of the owner and manager of the farm to thoroughly till it, and no land that is capable of being tilled should be permitted to remain unproductive. 


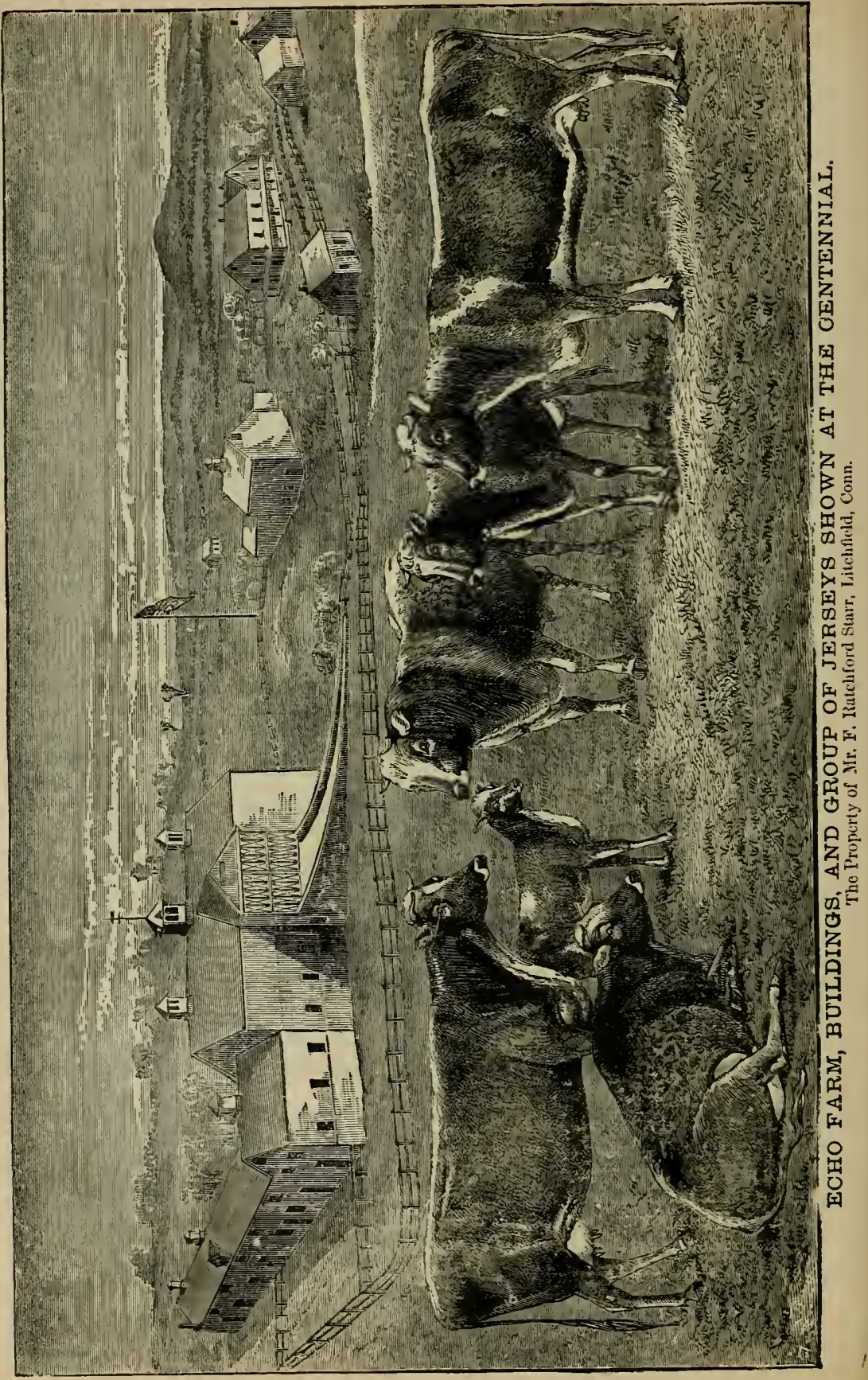




\section{MODEL FARMS.}

$\mathrm{T}$

HERE are many farmers who are strongly prejudiced against scientific farming, or, what they term "book-farming," regarding it as pertaining more to a mere theory than utility in practice. They consider all who favor it as visionaries, - the old methods as supreme, and all attempts towards an innovation as fanciful and unprofitable schemes. It will invariably be found that those who are the most strongly opposed to scien. tific agriculture are the most ignorant concerning it. To be sure, mere scientific knowledge is not by any means all that is necessary in successful farming. Theory is important as far as it goes, but the art is fully as essential; and when we have the two combined, viz., the science and art of agriculture, or, in other words, when we have the skill to put in most successful practice the knowledge that science bestows, it is then that we have a complete union, and the highest standard attained in agricultural achievements.

But what is scientific agriculture of which we hear so much, and-unfortunately-see so little? Science, literally, means knowledge, and when used in connection with agriculture, it means no less.

Scientific agriculture, therefore, means the employment of that knowledge obtained from Nature's vast laboratory, - of her forces, and her laws, - in the practical art of farming.

But why should we not make use of the aid which science gives? Why refuse any assistance that will give us a more perfect knowledge of the elements with which we have to deal? It is a fact that there is no business whatever that requires such a varied and accurate knowledge and observation of Nature's laws, as farming; neither is there any business that calls into exercise more frequently that faculty that is usually denominated "practical common sense."

No one can deny but that the manufacturer is enabled to make vastly larger profits by the aid of science, and conforming his labors to scientific principles, and why not the farmer as well?

We do not claim that farming can be reduced to a science as exact as that of mathematics or mechanics, for there are too many varying influences over which the farmer has no control, to admit of it, - such as the heat and cold, rain and sunshine, etc.,_-but we do claim that the highest attainments in this direction are reached only through the knowledge imparted by science. Those farmers, therefore, who reduce their practice to the most intelligent system, will, other conditions being equal, be the most successful. The best methods, when properly put in practice, will always be found the most profitable. To show some of the improved methods of scientific farming, and the results of such practice, we give a descrip. tion of what might be called a few of the model farms of the country. It will be noticed that in every instance of improved farming, the most careful attention is paid to cleanliness in every respect, including pure air, pure water, clean food of proper quality and quantity, etc.; the health of the animals being taken into consideration, as well as the quality of the breed.

Fcho Farm.-This noted farm, formerly the property of F. R. Starr, but more recently that of the Echo Farm Company, is located about a mile eastward of Litchfield. The description given of it by a visitor is as follows: "This farm, with its group of neat build. ings and patriotic flagstaff, crowns a hill some sixty feet higher than the ridge upon which the village is situated, making it nearly 1,300 feet above the sea-level. Mr. Starr, having impaired his health by too close confinement to business, after spending some time in trav. eling both in this country and in Europe, was attracted to this spot on account of its salubriousness and its proximity to the pleasant village of Litchfield. His original purchase of sixty-six acres was made merely with a view of securing a summer residence, and without 
the most remote idea of ever engaging in farming or stock-raising. The land around the house needed to be cleared and laid out, natural features of beauty and interest were to be preserved and improved, and this gave him pleasant employment. The work grew in inter. est and importance under his hands, and finally so absorbed his attention that he may almost be said to have become infatuated with the love of it.

It is hardly to be supposed that an active business man, having both capital arid enthu. siasm to apply to farming, could find employment for his faculties on a grass farm of sixty. six acres. Field after field was cleared of stone, new walls were laid, old ones removed, and very soon the work done made it obvious that soon there would be little more to do. Therefore adjoining properties were purchased and added to the farm, so that now 'Echo Farm' contains nearly 400 acres, which lie mostly in one compact body. A great part of this is under thorough tillage, and that which is not even now laid down to grass, both for mowing and for pasture, is rapidly being prepared for these uses. Miles of massive stone walls enclosing lawn-like meadows, pastures thoroughly cleared of stones, and laid off in great regularity,

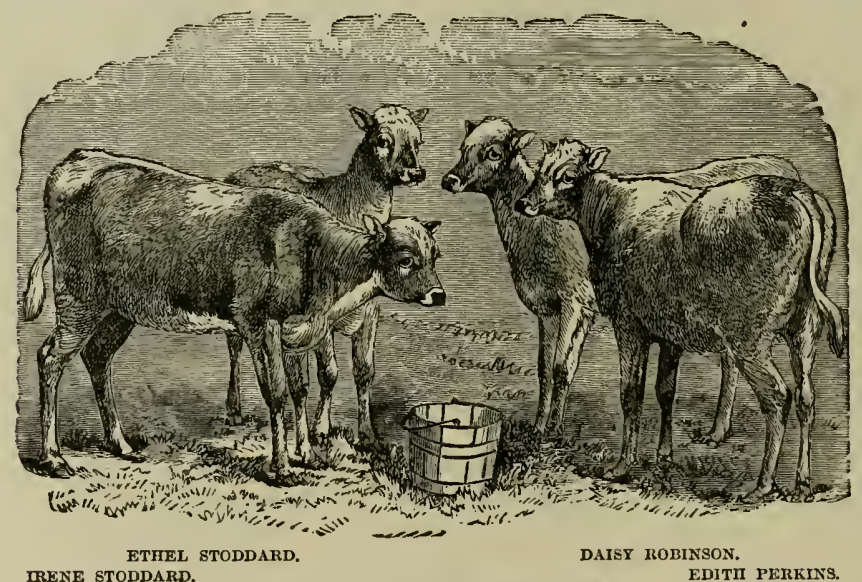

IRENE STODDARD.

GROUP OF YEARLING JERSEY HEIFEKS.

define its boundaries, while its rolling and cultivated surface is diversified by patches of woodland.

The introduction of an improved system of agriculture, and the application of the familiar principles of business to farming, necessitated new buildings in addition to those bought with the several farms now united. In 1873, therefore, Mr. Starr erected a barn $66 \times 25$ feet; in 1874 , a barn $100 \times 40$, and in 1875 , a barn $191 \times 35$; and in 1876 , these were not only all in use, but full.

In the perspective view of the buildings, the conspicuous building is what is called 'the '74 barn,' entered by the double approach or driveway, and adjoining this, upon the left, is 'the '75 barn,' while 'the ' 73 barn' occupies a similar position upon the right of the main, or '74 barn, but is not attaclied to it. These three buildings form three sides of the barnyard. They are of pine, upon massive granite foundations, for the most part two feet wide, laid in cement, rising from a base three feet wide laid in grouting, while under-drains carry off any water which might otherwise reach the foundations. In fact, the masonry of the foundation-walls, and of the massive 30 -foot approach, is like that of a fortification. The latter 
curves around a quarter of a circle, the granite walls being three feet thick, laid in cement, and some of the stones not less than eight feet in length. A height of twelve feet is gained, and the space between the walls (thirty feet) being filled in with stones solidly packed and topped with gravel, a solid roadway of uniform ascent is secured.

The principal storage-room for hay is the main floor of the ' 74 barn. Through the center of this the double threshing-floor crosses, so that several teams and wagons bringing in hay may enter and discharge their loads independently of one another. When the mows are nearly full, one of the floors is closed, and forms a big bay, which may itself be filled to the ridge-pole.

I have never before met with this contrivance of a double floor, and like it very much. There is storage-room in this loft for over 150 tons of hay.

The cow-stable is immediately beneath, occupying the full size of the building, $100 \times 40$ feet, 11 feet high. It is lighted by 18 large double-sashed windows, the sashes being hung by weights, open at top and bottom. Having the object in view, which has since been so

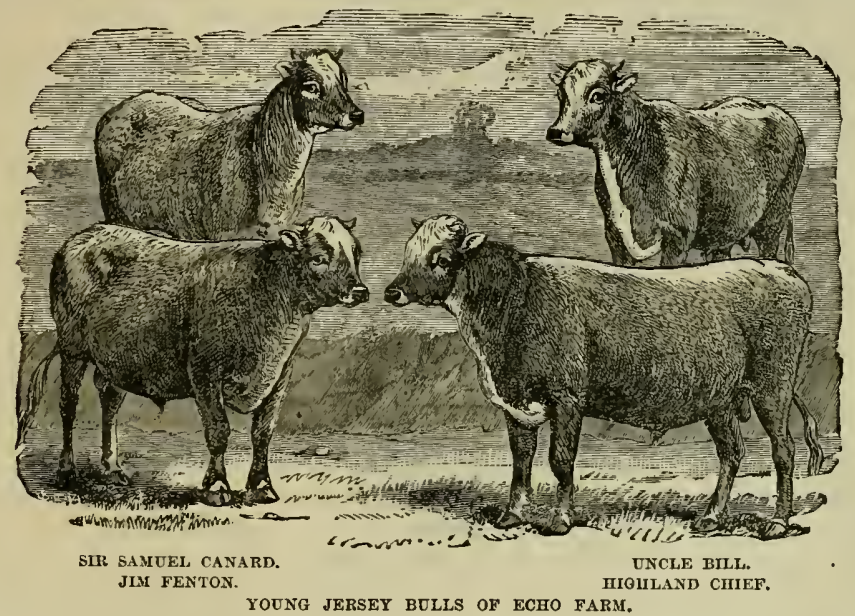

successfully accomplished, namely, the production, both in winter and summer, of as perfect butter as possible, the accommodations for the cows are absolutely luxurious as regards freedom, purity of air, comfortable warmth and abundant light, while for ease of attendance and convenience of inspection-that the foreman and the proprietor may at a glance see if every man has done his duty--they could not be surpassed. There are stalls here for forty-eight cows, and a simple calculation $(100 \times 40 \times 11=44,000 \div 4 S=917)$ shows that each cow has, making a liberal allowance for fixtures, posts, etc., not less than 900 cubic feet of air space, which is more than twice as much as is ordinarily considered ample. Through the center lengthwise there is a ten-foot passage-way between the two rows of stalls, and, crossing at the center of the floor, another passage-way of eight feet in width. These separate the floor into four divisions of twelve stalls each, and by a very convenient arrangement, by means of a few bars which are kept hanging upon wooden pins against the posts, and which, when in use, fit into sockets and slots, the cows of each section are turned loose to go at will into the yard for water and exercise, through a door at the south end of the cross passage, or through the main entrance at west end, to pasture. There is, besides, a broad passage behind the 
cows, and conveniently wide ones across each end, so that each section is sarrounded by a passage- way.

The stalls are nearly five feet wide-too wide, 1 think; the mangers or feeding-troughs about two feet wide, and the slightly sloping platform upon which the cows stand, $5 \frac{1}{2}$ feet. At the rear is a gutter which holds the manure and conducts the liquid to traps placed at convenient intervals in the gutter, through which traps the manure is dropped into the capacious, nine-foot cellar, 135 feet in length by 40 in width.

It will be noticed that the double drive-way, before alluded to, does not extend up to the barn proper, but stops some 12 feet short, which space is bridged over by what is called the 'dormer,' and forms a room below $12 \times 30$ feet, which is used as a wardrobe and dressingroom. The men call it the 'Parlor,' not because they meet here to talk, but, I take it, because it is the culminating point of the all-pervading neatness and cleanliness which distinguishes this establishment. Here are closets for the men's clothes, and for the brushes, brooms, cards, and other tools; wash-basin, towels, a looking-glass and toilet articles; and here, morning and evening, before going to milk, the milkers wash their hands and faces, comb and brush their hair, and make such changes of apparel as may be necessary. I am free to say that the majority of farmers in this country, which may indeed be justly proud of the civ. ilized cleanliness which prevails among its rural population, come to the table with less atten. tion to personal tidiness than is here both the rule and the practice.

At milking-time, though the barns are often, one may say, full of visitors, all are excluded, and the milkers have the floor to themselves. Spring balances hang at convenient points, and the milk of each cow is weighed as soon as it is drawn, the weight set down by each milker upon his own slate, and when he has done, the slate is delivered to the dairy. maid.

At the eastern end of this stable is a room $35 \times 40-$ the corner room, and part of the "'75 barn" in which there are eight large box-stalls on two sides, with space for several more. This is used as a lying-in ward, or for cows which may be injured in any way. The long loft in this building, which occupies the greater part of the same floor, is filled with hay, straw, and bedding, sufficient for the stock beneath. A portion is used for storing duplicate implements and machines, wlich, consisting of churns, butter-workers, a hay-cutter, and sun. dry others, are kept ready for use in case of an accident, that no delay may occur - an admirable provision. Another portion, opening out of the lying-in room, is occupied as a grain and meal room. A passage-way, four feet in width, extends through to the southern end of this floor upon the western side. The manure-cellar, before referred to, is continued under the room in which the box-stalls are, but the rest of the basement is otherwise occupied. An inclined plane, well cleated, makes a passage-way for animals up and down when it is necessary to transfer them. The ox-stable is at the left of this, on the ground floor; while on the right are the bull-stables.

Every farmer, and not less every householder, can appreciate the inestimable comfort to man and beast of a never-failing supply of pure water. Without it the barns would have been ill-placed and ill-planned. So important a matter, of course, received its due consideration. Upon the hill, above the barn, not far from the position chosen by the artist for his sketch, and at a distance of some 500 feet from the barns, several springs are collected into a reservoir. This is stoned about, roofed over, and connected by iron pipes with the dairy, and with the barns, throughout which it is distributed by pipes. There are troughs, or sinks, with discharge-pipes leading to the drains, located in convenient places, in the stables and other rooms, and spacious drinking troughs in the yards.

Mr. Starr dwelis with force upon the importance of giving the animals the regular exercise of a walk for their drink, and of their being watered twice a day, and, in carrying out his view, the cows of each section of the mair floor are turned out for an hour or more 
every day. They get this exercise and fresh air regularly, even in the coldest weather, except during severe storms.

An abundance of pure air has been provided for in every apartment in which live-stock of any kind is kept. The high ceilings (11 feet) of the cow-stable, and of the stables in the basement of the ''75 barn,' have already been mentioned. Two large ventilating trunks go from the cellar to the ventilators in the roof; others, with slides at floor and ceiling, pass up independently from the stables and cow.floor, but ad. joining those from the cellar; besides, everywhere there are windows hung by weights, which not only admit light and sunshine, making the stables light even to the innermost corners, but giving in summer, or sultry weather, all the fresh air any cow can need. The result is, that no temporary neglect or carelessness can cause bad odors, or vitiated air to prevail. And what is even of greater importance,-if in. fectious disease should occur, the rapid change of air which is obtained night and day offers the greatest security against its spread. The system of feeding that is followed is very simple: mixed bran and meal are fed upon cut feed twice a day. One good meal of roots is given by themselves. Mangels and sugar-beets are the only rnots used, and the cows have besides all the dry hay they will eat.

Everything is of the sweetest and purest character - the hay, fragrant; the few corn. stalks used, thoroughly cured and free from mustiness; the corn-meal, corn and oats, and bran (50-pound stuff), of the best quality. Thus, nothing

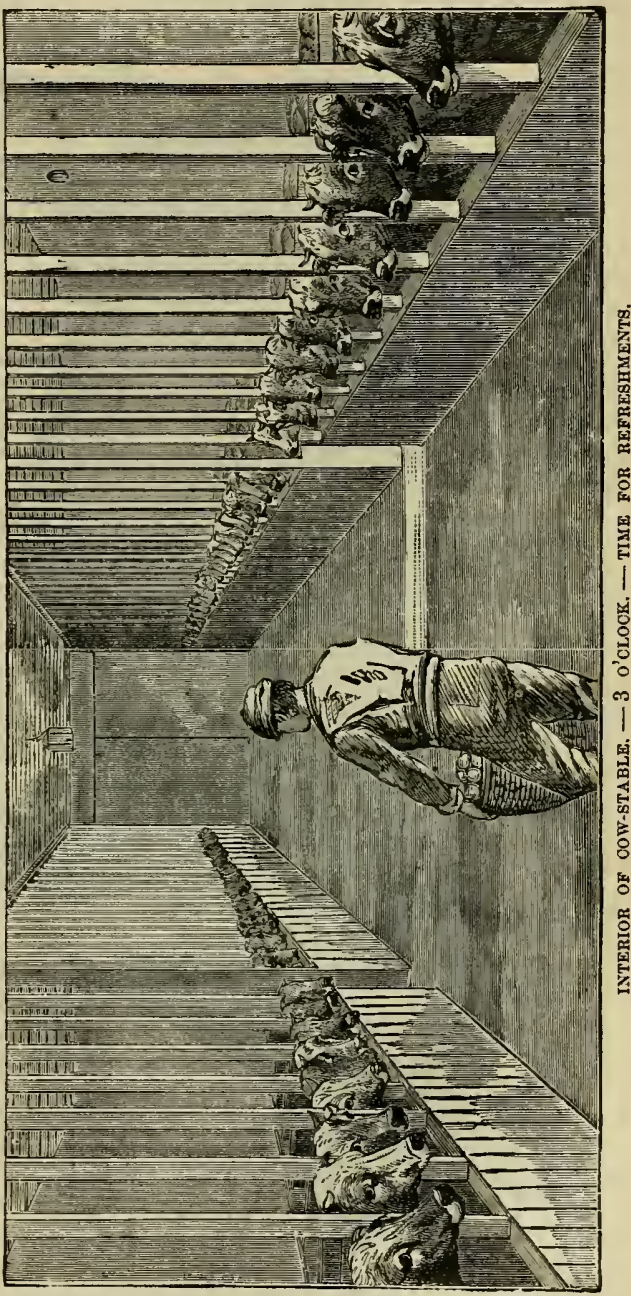
can be eaten by the cows in the stable which can affect the milk unfavorably, and everything contributes to its high flavor and excellence. The proportions between mangels and meal, vary according to the abundance of the former. When a full crop of roots is harvested, the quantity fed is profitably increased. 


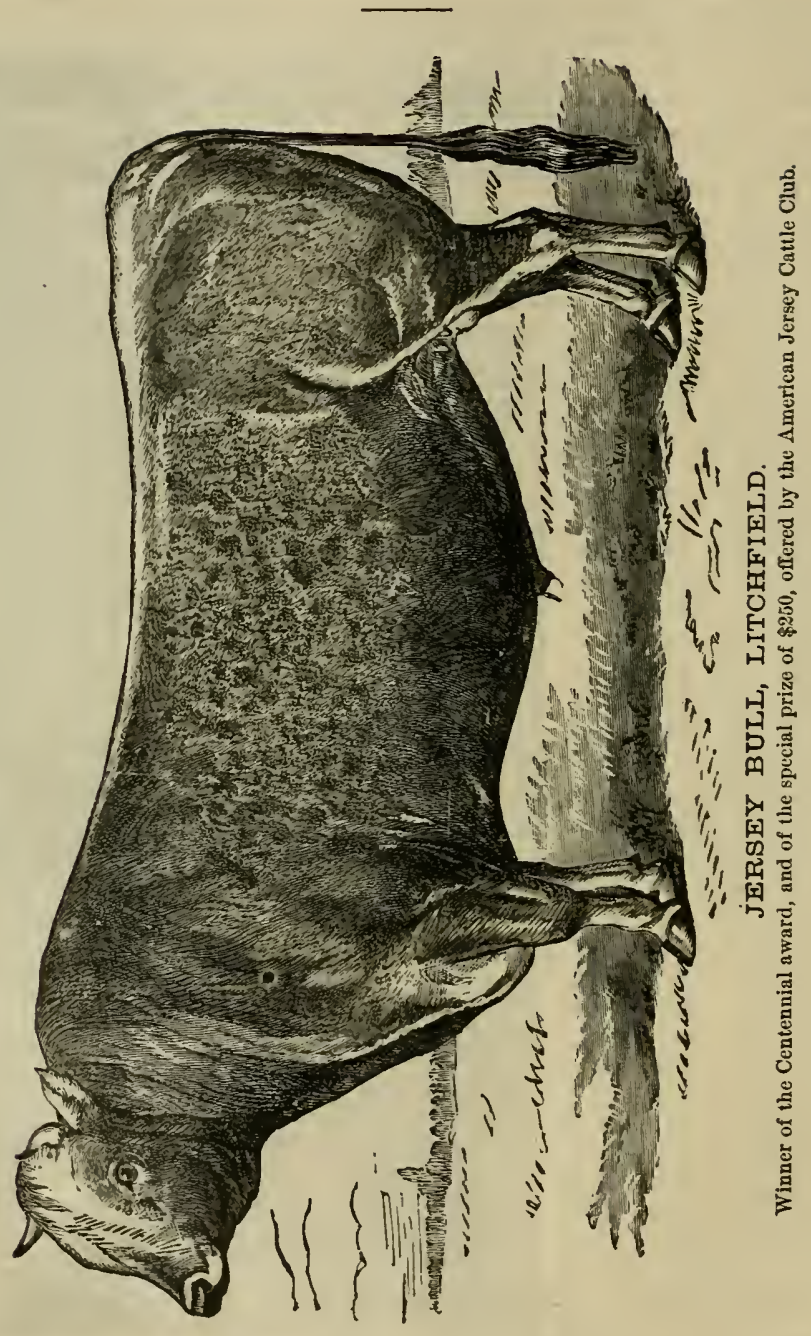


Stable Management. - It is some one's duty to look to every individual cow, calf, and bull every hour through the night, from dark to dawn, and at stated intervals during the day. This gives regular employment to one night-watchman, and to three regular dayhands. The stable-men clean out the stalls, cut, mix, and give the feed, clean the cows, sweep out the stables, turn the cows out to water, feed the calves, etc., under the supervision of an efficient foreman, who, indeed, supervises all the work of the farm, working with the men wherever his labor will do the most good, but in all things following the directions of the proprietor, who takes the responsibility of ordering everything of importance. It is an inflexible rule, that all the animals shall be treated kindly and gently. No shouting, hallooing, or alarming demonstrations are made; hence they grow up docile and gentle, and the bulls, old and young, have so far proved no exception.

All the animals are kept clean; the cows brushed or carded daily; their stalls not only cleaned out, but swept out and sanded. The temperature of the stables is regulated by the ventilation in cold weather, and thermometers are hung where they may be conveniently inspected. During the winter it is intended not to allow the temperature to sink below the freezing point; but a temperature of $40^{\circ}$ is considered desirable.

Regularity is a marked feature of the management. Every important daily duty in and about the farm, barns, and dairy has its appointed time. A large clock is centrally placed, and all hands are held responsible for accurate punctuality. Not only are things done like clock-work, but if anything goes wrong by day or night, the proprietor, if at home, may be at once communicated with by telephone. A signal given at the lower stable, the upper stable, or at the foreman's dwelling, notifies the proprietor at once that his presence or that of the foreman is needed. From his office the proprietor can also summon the foreman, gardener, coachman, or others, at any moment. This is an arrangement which is not only a great security against accidents of many kinds, but a great convenience For instance, if anything seems to the night watchman to be going on wrong in the lying-in stalls, in the horse-stable, or elsewhere - anything that he cannot himself manage - he has only to leave the animal long enough to give the signal, and he is sure of help within a few minutes.

The Dairy, etc. - Each milkman has his slate to record the weight of the milk given by each cow. With the last pails of milk, these slates are brought to the dairy and delivered to the tidy dairy-woman.

The milk is set in deep cans in summer, which stand in running water, and are thus kept at an equable temperature; but in winter the old-fashioned shallow pans are used; no seamed ones, however - but those struck or stamped from a single sheet. It is poured from the strainer milkpails, having the usual form, and a fine brass wire-cloth strainer at

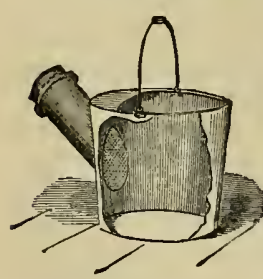

TRIPLE STRAINER-PAIL. the spout, into a large pail, which is a triple strainer - an Echo Farm invention, and one worthy of being extensively copied. The spout of this pail is a four inch cylinder. There is a fine brass wire-gauge strainer placed over the spout inside the pail. Over the outer end of the spout a hoop of tin is fitted loosely; and by means of this, two thicknesses of muslin are fastened like a drum-head over the end. The milk is poured into the pans, or deep cans, from the pail,

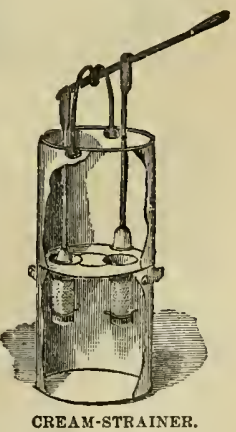

and I must say it seemed as though it was carrying the cleanly hobby rather far, to strain the milk virtually four times; but after seeing the milk of some fifty cows strained, and making a thorough personal examination, I was fully convinced of the wisdom of the course adopted, and that it was in keeping with the other rigid and excellent rules of the farm. 
After the evening's milk is recelvod, the dairy-woman fills out her return for the day. To do this, she has blanks which give the names of the cows, just as they stand in the barn, numbered as the stalls are, from 1 to 48 , with those in other stables under $a$ and $b$. There are two columns ruled for the morning's and evening's milkings, and the weight of milk given by each cow is recorded, and the whole footed up; then the disposition of the whole is noted on the same paper. The dairy is charged its quota, after whatever is necessary is taken out for the house, for the families of the men, and for the young calves. This report is filed daily, and at the end of the month each cow is credited with all she has given, and at the end of the year the grand totals are footed up. So complete is this system, and so thoroughly adhered to, that it takes but a few hours of simple figuring, on the 31 st of December, to exhibit a full statement for the 365 previous days.

The advantage, in large dairies, of straining or filtering the cream is well understood. A thorough homogeneousness is secured, whereby the butter 'comes' quicker and is more
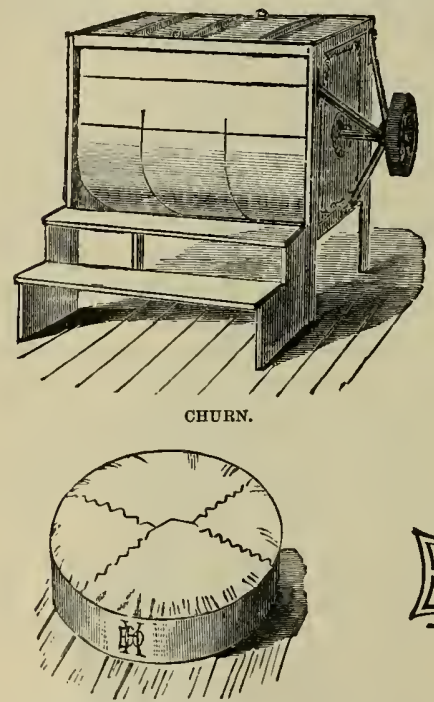

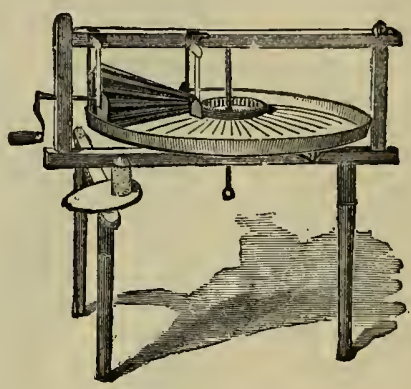

BUTTER-WORKER.

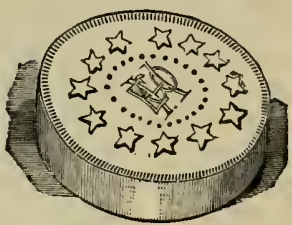

thoroughly removed at one operation. If the cream lacks this quality, only a part of the butter, sometimes, will come, while a second or continued churning will be needed to secure the remainder; besides, specks from the windows, or open doors, are liable to fall in, which ought $t o$ be removed. For these reasons the cream is strained, and it is no easy operation to strain thick cream from flat pans. On this farm an apparatus is used, which is found in some of the best dairies of Pennsylvania, and which is represented on the previous page. Two vessels are here shown-one, a simple receiver or the strained cream; the other, the strainer. This is a cylinder like a large can, of heavy tin, having above a heavy, fixed, rigid iron bail, with a socket in the middle for a pump-handle to work in at right angles to the bail, while below, in the bottom, are two tubes about six inches long, and two and a half inches in uiameter. These have their sides perforated with small holes.

The bottoms are solid and locked on, so that they may be removed for cleansing the apparatus. The mouths of the tubes are conical, that the cream may run in quickly, and 
not only for that, but so that the pluggers, which are worked by the pump-handle, and alternately lifted out and plugged in, forcing the cream through, may, without other guide, drop directly into their proper places. The cream, thus rapidly and thoroughly strained, and all parts mingled, is then ready for churning.

The churning is performed by horse-power. After being carefully worked, the butter is put up in half-pound prints for shipment. These are of the usual circular form about an inch and a half high, each having a neat device upon the top which consists of the monogram 'Echo' in the center, surrounded by twelve stars, each star indicating a month of the year; and as they are represented in a circle, and of equal magnitude and brilliancy, the indication is obvious that the quality and quantity of the butter do not vary the year round.

As soon as the butter is moulded, each print is wrapped in clean muslin and placed in a neat, white paste-board box.

For final packing for shipment, the prints, each held in the little paper box, to keep them from being defaced or otherwise injured, are packed in a wooden box, and sent from the dairy every Tuesday and Friday morning by express, and are delivered at the residences of custo. mers on the evenings of those days without the butter being handled."

We have thus given in detail some of the main features of the methods practiced on this noted farm, simply to show the perfect system to which such a business may be reduced.

To give some idea of the benefits derived from keeping thoroughbred stock, and giving them good care and kind treatment, we will briefly state that Filbert, the cow represented standing at the left in the illustration of the centennial group, was sold by the proprietor in 1879 for fifteen hundred dollars. She gave in July of that year, $809 \mathrm{lbs}$. of rich milk, and in August $949 \frac{1}{2} \mathrm{lbs}$. The records of many of the others of the herd are very high, although not quite as good as that given. The Jersey Bull Litchfeld was also winner of the Centen. nial award, and of the special prize of $\$ 250$ offered by the American Jersey Cattle Club.

That such a system "pays," may be inferred from the fact that in the same connection new dairy buildings have recently been completed about three miles from the original farm, with apparatus for bottling five thousand quarts of milk daily, which, with the facilities afforded by Echo Farm of 2,500 quarts, enables the entire establishment to send 7,500 quart bottles of pure milk to New York each day.

Deerfoot Farm.-This farm is located in Southborough, Mass., and is the property of Mr. Edward Burnett. The description of it given by Dr. E. L. Sturtevant in a recent Department Report, being the best we have seen, we take extracts from that authority for our pres. ent purpose:

"Perlaps it is safe to say that there is no farm in America which can present so much that is novel and useful to the observer, as Deerfoot Farm.

It is not amatour farming that is to be seen here, but real 'fancy' farming, the use of intensive conditions, the employment of abundance of labor, and the availing practically of erery new idea adapted to the conditions that promise improved profits.

This farm covers about 300 acres, of which some 100 are tillable. Its specialties are fancy pork, gilt-edged butter and cream, family milk, skim-milk, and buttermilk.

To meet these requirements, much money has been expended for conveniences, and the farm partakes in its management of the character of a factory. The swine are grown on the place, or to order, are slaughtcred as pig-pork, and are presented for sale in small, neat, and attractive packages, which include 'Deerfoot family pork,' 'Deerfoot hams,' 'Deerfoot bacon,' 'Deerfoot jowls,' 'Deerfoot pigs' feet,' 'Deerfoot sausages,' 'Deerfoot lard,' etc. From tlie pens in the piggery, through the slaughter-room and packing-rooms to the market, there is the most precise cleanliness, and the wise use of all the advantages that well-constructed machin. ery, moved by steam-power, can offer. In 1879 the number of pigs slaughtered was about 1,500 , of an average weight of 175 pounds, the extreme weights of carcass being 140 and 250 poinds. 
We, however, do not propose to describe this farm and this farming in detail, but to confine ourselves to the presentation of the dairy branch, which in like manner is worthy of attention from its development and from the novelty of its processes, for here are in use the only centrifugal milk machines; on other than an experimental scale, in America, and the skilled thought of the experimenter and the machinist have combined to produce the results best fitted for the handling, care, and manufacture of the milk.

The foundation idea which underlies this kind of farming is, that there is a large discriminating public, who desire to purchase the best articles of the class, and who are willing to pay an increased price in order to secure perfection and uniformity of supply on their tables. Hence an expenditure may be justified in order to secure purity and cleanliness of product, attractiveness of packages, and such a sameness of quality that the brand stamped thereon shall justify confidence.

There are two sources of supply for the milk, the home herd, and that furnished by the neighboring farms. The milk of the morning and the evening is kept separate. The morning's milk from the horne herd is poured from the milk cans into a large cooler, and is thence, after being cooled, bottled for market as new milk. In summer it is shipped at 7 P. M. The cooler which receives this portion is a large metal cylindrical vat of the capacity of 150 gallons. Within this is suspended a box containing ice, and attached to a lever, so that motion can be communicated to it in case the cooling is desired to be hastened, or a sort of propeller which keeps the milk in movement. As soon as the temperature is reduced to $50^{\circ}$ the nilk is drawn in successive portions into a pail, and thence poured into the bottles, which, after being corked securely, are transferred in the frames to the water refrigerator, as it may be called, where they remain until shipment.

The milk-tank, with its cooler, which receives the milk from the upper floor, is suspended at a convenient height on the elevator, and by means of a faucet delivers the milk into the pail which is used to fill the bottles. The bottles are handled in wire frames which hold twenty, and these frames are transferred to the water-refrigerator, where they rest on a wire grating, which is raised and lowered by means of machinery, thus conveniently lowering the filled and tightly-corked bottles under the ice-water, and raising them again to the surface for handling.

These bottles are of the Cohansey pattern, and are of the capacity of one quart. The cover is secured by wire clamps, which, by compressing against an intervening rubber, form a tight joint. These bottles are delivered to the customer each morning, and at the same time the empty ones are returned to the farm, where, after a thorough cleansing, they are again filled for use.

The upper story is on a level with the ground in the rear. Under a shed is the delivery, each can of milk being weighed at the scales, and the weights charged off. The cans are then moved into the delivery-room, and the milk is emptied into the tank within the refrigerator-room, thence to pass by a pipe into the centrifugal machine below, or is poured into the tank for fresh milk delivery, as described.

The empty cans, after being cleansed over steam jets in the shed, are stored in the de. livery-room until again put into requisition.

The cans used are of the capacity of 20,30 , and 40 quarts, and bave large covers, which spring into place, and strong handles.

The next room is the wash-room. The tanks are furnished with cold water through faucets, and also with steam-pipes, through which steam is admitted to the water in the tanks to warm it. Movable draining-trays, or slatted tables on casters, receive the bottles after the cleansing in the hot-water tanks. Into this room opens the stairs from the lower floor, and other doors lead to the storage-refrigerators, and the churning-refrigerator-room.

In the storage-refrigerator-room are kept the cream, the butter awaiting delivery, and the 
milk in the tank, which supplies the centrifugal machines below. In the churning-refrigerator, the cream is churned by power in a barrel-churn, and the butter is worked and pressed into form for the market.

Passing into the centrifugal-room on the lower floor, we find three centrifngal machines, over each of which is a pipe connecting with the milk-tank in the refrigerator-room overhead, and three tanks in the floor which receive the skim-milk in cans, and where the cans remain until shipped. In these tanks of water a block of ice is kept floating.

Centrifugal Machines.-Two styles of centrifugal machines are in use, one a selfdelivery, the others intermittent deliveries. We shail describe the first as machine No. 1, and the second as machine No. 2. The machines being put into motion, the fancet of the pipe connecting with the milk-tank is opened, and each machine receives its charge. After running about fifteen minutes, the cream has collected on the interior wall of the milk, and then, in No. 1, the faucet is again turned, and the admitted milk displaces a thin stratum of cream, which is collected. At the same time the skim-milk escapes through small valvular openings in the bottom. A small cup occupies the axis, and from which a pipe extends towards the circumference. This receives the milk as it falls from the pipe and conveys it toward the circumference and away from the cream wall. Collected in this apparatus, the milk is carried to the pipe or outflow. The skim-milk, passing into the surrounding frame, is likewise conveyed by a pipe into the receptacle placed to receive it.

Machine No. 2 is of a different construction. After the cream has collected to form the interior wall, a pipe-scoop is brought into contact with the revolving surface, and the cream is forced along the pipe and conveyed to a pail placed near for its reception. After the cream is removed, a like quantity of milk is added from the faucet, and this displaces the cream which has escaped removal on account of its position to the point where the scoop works. In a few moments the cream is thrown off through the scoop-pipe, and then the skim-milk is removed in the same way, when a new charge of milk is admitted. This process takes place about three times an hour. The pails of cream are now removed to the refrigerator-room, upper floor, while the cans of skim-milk are transferred to the ice-water tanks on the lower floor. In one experiment, watched by myself, so as to secure the ordinary conditions, 172 pounds of milk, in machine No. 2, yielded 21 pounds of cream, such as is bottled for market, or 12 per cent. of its weight. Machine No. 3 is similar, except being slightly larger than machine No. 2, and requires no separate description."

Care of the Cows.-The cows on this farm are milked with great regularity at 5 o'clock in the morning and 5 P.M., eight or nine cows being considered sufficient for one milker. They are driven to their stalls to be milked and for passing the night, but are pastured during the day in summer. Sand is used for bedding, and the stables are kept very clean, being frequently whitewashed. The cows are regularly carded each day.

The method of feeding is thus given by Mr. Burnett:- "The essentials to produce good results are good cows, good feed, regularity, cleanliness about the stables and dairy, and a thermometer. I will give my own method of feeding, and in so doing those dairymen who aim at quantity will realize that we are shooting at different targets, for with me quantity is secondary, quality being the greatest desideratum. Our finest butter is obtained in early summer, when the pastures are sending forth their early, sweet, succulent grasses, and we depend entirely upon them; but when these begin to fail, about mid-summer, I begin to feed wilted clover and a small quantity of grain, increasing as the season advances, unless the pastures are unnsually good. I cut all my grass early, beginning by the 3 Ist of June, and generally get a good second crop, thus trying to have an abundance of rowen hay. When in winter quarters, I begin feeding at about 5.30 in the morning with hay, a little jag or wisp at a time, not so much but what the cows will eat up clean. Then, after milking, they are 
given grain, from three to six quarts, according to the cow, consisting of two parts of Indian meal and one of shorts or bran; or feeding entirely on ordinary cobbage (corn and cob ground together). After this, more hay, which lasts until about 9 A.M. I begin again at 3 P.M with a littie hay, followed by roots (mangolds) cut fine, a bushel being divided between three cows; then more hay again, which lasts them until about 6.30 P.M.

I maintain that if more shorts are fed than are necessary to counteract the heating quality and condensed richness of the corn meal, it deteriorates the butter. During last Narch I saw this illustrated, being called upon in Boston to examine some butter from one of the first dairies in the State, and which was troubling the dealer who sold it. He said it was negatively good, nothing could be said against it, yet bitle could be said in its favor. It seemed to lack that fine nutty flavor so necessary to fresh butter that commands over forty cents per pound. I said at once, on tasting it, 'Too much shorts and not enough corn meal. He answered, 'Just what I thought, but didn't dare say so until it was confrmed.' In less than ten days the butter from that diary was improved."

"Mountain-Side Farm."-This noted farm is the property of one of New York's leading merchants and citizens, Mr. T. A. Havemeyer, and bies in Bergen County, Northern New Jersey, at the base of the Ramapo Mountains. In a very elaborate article in the Journal of The American Agricultural Association. Mr. Francis D. Moulton, President of the International Dairy Fair Association, has given a detailed description of this farm and its adrairable system of management, a few extracts only of which we shall find space for insertion, and which will serve to give the reader some general idea of the completeness and perfection to which this system is carried:-

"The mountain range, curving in broken lines on either side, presents a grand and striking setting to a charming picture, the whole aflording a most picturesque and beautiful view. Through the farm from North to South flows the Ramapo River, an attractive feature of the landscape, and an eminently valuable and practical adjunct to the farm.

'Mountain.Side' comprises six hundred acres, three hundred of which lie in the valley and are under cultivation, and the remainder on the sides of the mountains, which at this point rise to the height of about six hundred feet. The natural herbage of this latter portion affords excellent pasturage for sheep and cattle. The soil of the bottom-land is a gravelly loam, and under the advanced system applied and the admirable methods adopted, it is being brought to a high condition of fertility. Of the cultivated land, this year, about one hundred acres are in pasturage, ninety acres in hay, fifty acres in fodder-corn, and fifteen in rye. Three acres are in peas, three in vetches and barley together, and three acres in potatoes.

Mr. Havemeyer's private stable is fitted up with box-stalls, and contains room for a large number of carriages. Extending from it is a wing fifty feet long, from which reaches out another wing at right angles, 150 feet long, containing in all ten stalls, forming a parallelo. gram, and leaving an open airy court.yard in the center, by which means the ventilation is made perfect. The stable has constant attention, and is kept scrupulously clean. The barn, dairy, and silos are comprised in one building forming the letter $\mathrm{T}$. The barn proper stands East and West, and contains the cattle-floor, hay-loft, feed-bins, and manure-cellars. The south wing contains the ice-house, dairy, engine-room, and quarters for the dairyman. In the north wing are the silos. The length of the barn-floor from East to West is 263 feet; its width 44 feet; the length from North to South, including the dairy and silos, is 263 feet, the south end being 31 feet wide, and the north end, or silos, 40 feet wide.

Beneath the barn, on each side, and directly under the cattle, are the manure-cellars, each 14 feet in width and 180 in length. All the droppings from the animals pass into these, and earth is carted in daily to absorb the moisture. Horse or ox-carts can be driven to all parts of these cellars to haul out the manure. Under the center of the barn, and sur. rounded by the manure-beds, is a room for keeping roots, 150 feet long and 15 feet wide, 


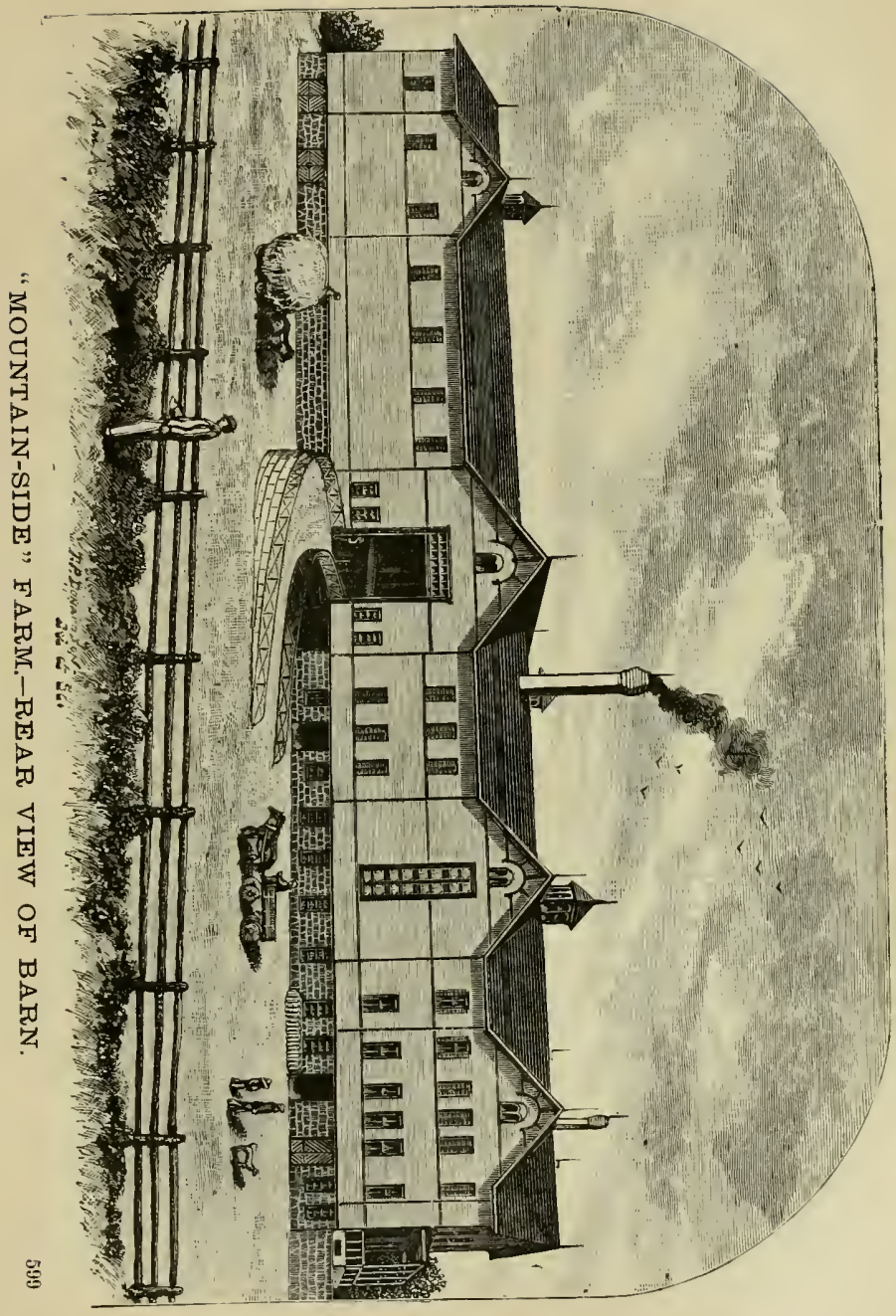




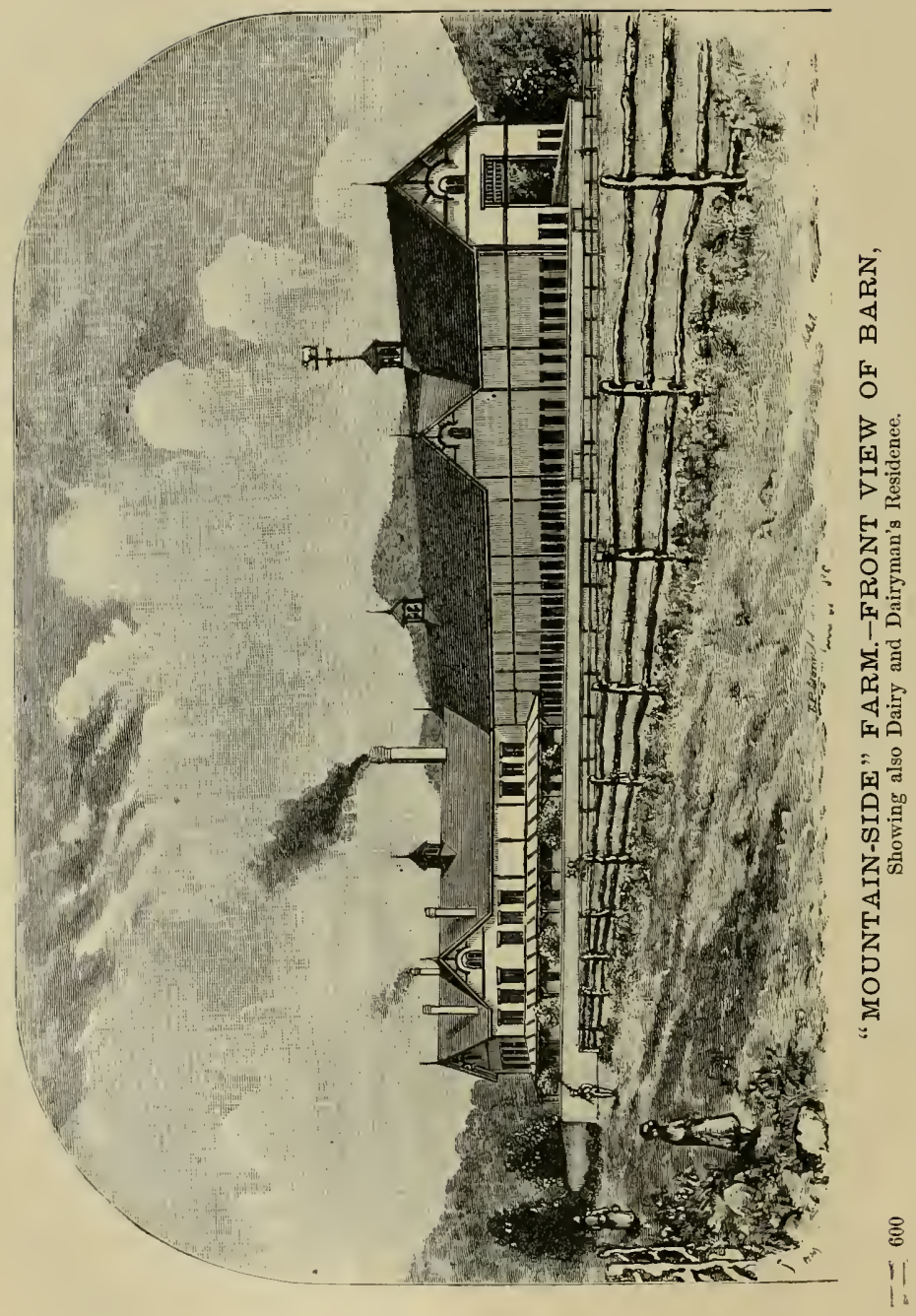


with stone walls and cemented sides and bottom. This root-cellar is thoroughly ventilated by flues of its own, quite disconnected from that of the manure-cellar, and for the cattle-floor above. At the extreme west end of the barn-cellar, between the two cartways, is built a cistern of cemented work, 53 feet long, 15 feet wide, and 12 feet deep, having a capacity of 35,000 gallons of water, which is supplied from the river. From this great cistern, into which also the water from the barn-roofs can be carried at will, a steam-pump raises water to two large tanks high up in each end of the main barn, and from these tanks, giving an excellent head, water is supplied by a system of pipes to all parts of the various farm buildings. Back of this cistern stands the gas-machine, which supplies the house and all the buildings with hight. Entering the main floor of the barn, one is struck with the immensity of its size, its cleanhiness, absence of odors, and its exact adaptation to the purposes it is designed to subserve.

Besides the main entrance at either end, the barn is entered by seven doors on each side, six feet by nine. It has eighty-four windows, five by two and a half feet, and, together with the distance from the barn-floor to the peak of the roof, and the passage through the main doors from East to West, has the best system of ventilation and lighting that could be devised. Both in winter and summer the air here is pure and almost free from odor; a fact which affords the best assurance that the condition of health of the animals and the purity of their products are sedulously maintained.

Among the hogs are seventy-six of the Yorkshire breed and some Chester Whites. One hundred head of Southdown sheep are also kept on the farm, which shear about five pounds per fleece. There are twenty working-horses, comprising as fine a lot of agricultural and draft horses as can be found. They are kept for the work of the farm, besides one yoke of Hereford oxen. The work-horses are kept in box-stalls, untied. Mr. Mayer (the superintendent) says that he wants his horses to sleep as comfortably as his men, and finds that it pays to afford them such rest as a box-stall, well-littered, gives them. They do more work and keep easier. Whilst all the horses are high-spirited and well-bred, they are made kind and docile by care and gentle treatment."

The dairy herd on this farm are all of the best Jersey breed, and the stable-room accommodates two hundred head.

Care of Cattle, etc.-The same writer describes the management of the dairy animals as follows: -

"In the care of his cattle, the same thoughtful attention and judgment are exhibited ou Mr. Havemeyer's farm which are noticeable in the details of every other department. The feeding.place in front of the cows is upon the floor, without any other arrangement, in orcler that the cattle can obtain their feed clean, and that no particles shall get into corners to sour and injure it. Here there is noticeable an entire absence of feed-boxes, and all fixtures. The cattle are watered by means of a trough, which can be raised and lowered at will, and is sup. plied from the tanks above. When it is desired to water them, the troughs are lowered, and when not in use they are raised to the top of the stall. This is quite the best system which I have ever examined for this purpose. Every cow is cleaned daily with a curry-comb and brush, the same as a horse. They are treated with absolute kindness and gentleness.

A daily record is kept of the milk-yield of every cow. The calf is taken from the cow when three days old, the cow being tied up in her place in the stall. The milk, perfectly sweet, is heated up to 90 degrees and fed at this heat, which is the same as the original temperature when taken from the cow. The calf is given milk, at first four quarts every day in three feedings, morning, noon, and night, increasing the quantity as the calf grows. It is kept in a stall until ten days old, and then turned out in the morning to obtain the benefit of the sun. At a month old it is turned into the pasture. Until four months old it is fed with milk, at the end of that period being given some ground oats. Each calf is kept 


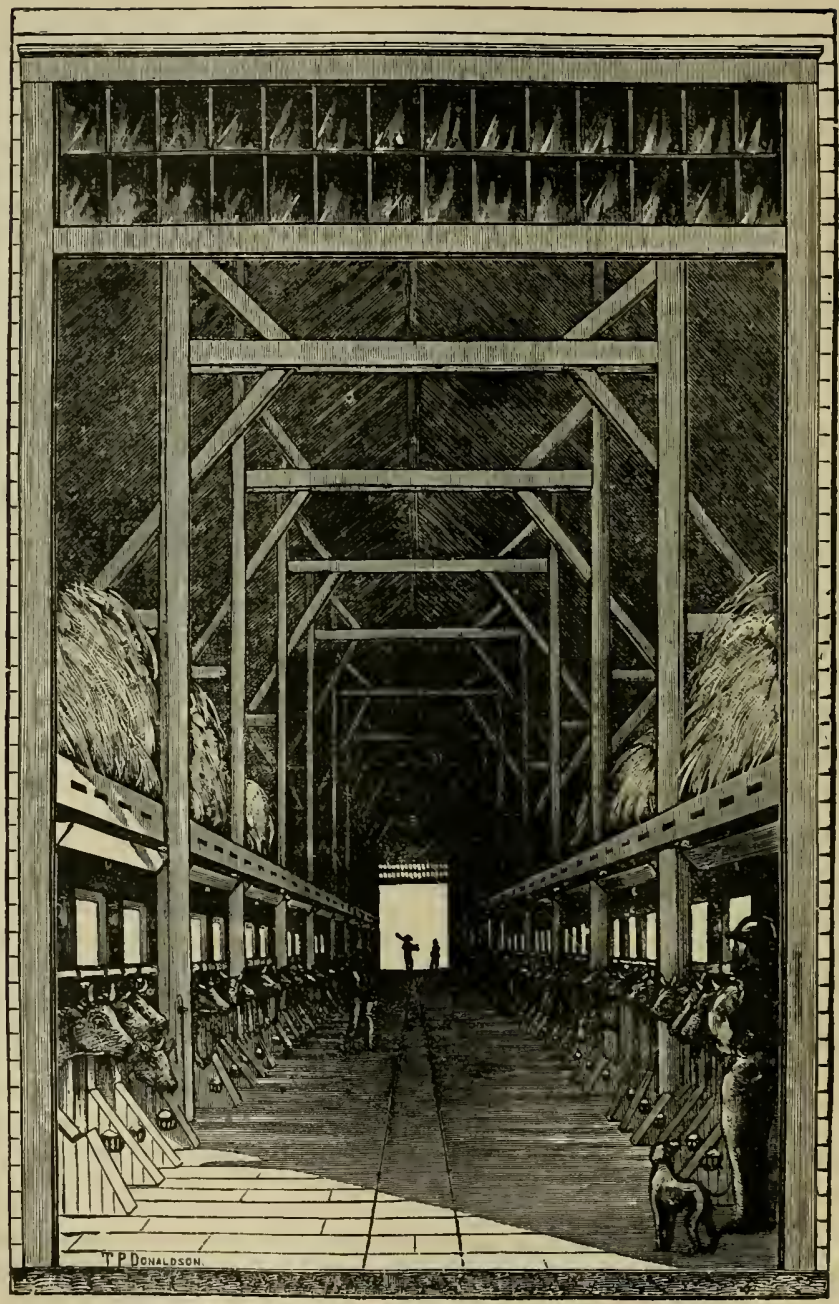

"MOUNTAIN SIDE" FARM. Interior of Barn (Looking West). 
separate, to prevent it from sucking the others, until it is three or four months old. After the cow has calved, she is fed with meal, ground oats, and corn, four quarts per day, with all the hay or grass she will eat. The milk is used after the third day, if the cow is in perfect health. Cows are allowed to go into the yard every day, winter or summer, two or three hours for exercise. They are kept in separate stalls in the winter. Each animal gets half a bushel of feed.

A month before calving, all meal is taken away from the cows, and they are fed on three quarts of ground oats per day in two feeds, with all the hay or ground feed they want. They are put in box-stalls ten days before calving, in order to get accustomed to the place and that they may feel perfectly at home. This system is found to prevent milk-fever. Mr. Mayer, who has had the care of cattle for many years, has never had a case of milk-fever. This system also enables the cow to clean herself in six hours, her bowels being kept open. The stalls are cleaned immediately after the birth of the calf, and the cow removed at once to another stall, the first one being purified by using chloride of lime.

"Lorillard Stock Farm." - The committee of the Burhington County, Now Jersey, Agricultural Society on farms, farm buildings, crops, and reclaimed land, recently submitted their report, which concludes as follows:-

"The next and last farm visited was that of Pierre Lorillard's stock farm, near Jobs. town, about 1,100 acres of land, and comprising every convenience and requirement of a complete farm. The mansion is a handsome three-storied semi-gothic building; near by is the coach-house, with accommodations for 24 horses, and the farm-stables, with accommodations for 40 horses. In the center of the yard belonging to this establishment is an enormous food-bin capable of holding 9,000 bushels of grain at a time, and from which the food is drawn by shoots near the bottom. A broad and well-kept road leads from the mansion to the farm-buildings, cattle-yards, and a barn holding 400 tons of hay. The stock kept here numbers about 1,200 heads, as follows, viz.: 300 horses, 300 cattle, 400 hogs, and 200 sheep. To prepare food for this large number requires a steam-engine of twenty-horse power, which runs five mills for grinding feed, shelling corn, cutting stalks and coarse fodder, and steam. ing the food in several large vats, from which the cooked food is taken in box-cars which run on railways between the long range of cattle-stalls where the Ayrshires, Jerseys, and Durhams are fed, each animal being marked through the ear with a strip of tin stamped with its number to correspond with the number in the herd-record made at the time of its birth, so that the pedigree can readily be traced through sire and dam to its proper source; another railway leads through the front of a long range of hog-pens 320 feet long by 18 feet wide, each pen having a yard paved with brick 11 by 14 feet between the eating department and the open yard, leading to a trough of water running through the lower end of each yard, and in all 340 feet in length.

The system of ventilation and drainage is so perfect and complete that there is no unpleasant smell or offensive odor to be detected, and the whole family of Suffolks, Essex, Berkshires, and Jersey Reds seemed to enjoy their situation in perfect health and comfort. There are four horse-barns, 100 feet square, on the outside, located distant from each other; the stalls open to the yard inside, which is lowest in the middle where the sewer is placed to carry off the drainage under the ground.

In the brobd-mare stables there are comfortable stables for 100 horses; the colt-stables surround a circular track where the colts can be exercised under shelter, one-eighth of a mile in circumference. A much larger barn is now being constructed than any yet completed, requiring 560,000 feet of lumber. The building is 343 by 100 feet, eaves-posts 20 feet, inner box 28 feet, two wings 100 by 123 feet each; in the center there is 98 by 95 feet supported by truss-work, nearly all to be covered with glass. Three cisterns, 25 feet in diameter by 16 feet deep, three apartments in each, the middle filled with charcoal, through which the water 
is filtered before being drawn for use. But it is in vain to attempt to describe the grandeur of these magnificent edifices in the few moments devoted to them.

For the best-arranged and most convenient set of farm buildings the premium is awarded to Pierre Lorillard.

There have been laid on this farm, fifty-one miles of underdraining and nine miles of open ditches, mostly discharging into the Ananicken creek, a branch of the Assiscunk, which passes through the farm, which have reclaimed what was, a few years since, worthless swamp and bog, but is now beautiful meadow, thickly set with fine grass and rich pasture, and is the most successful experiment in reclaiming marshy land shown to the committee, and for which they award the premium.

For the most profitably-cultivated crop, the profits of which shall exceed one hundred dollars per acre, they award the premium to D. E. Howatt, farmer for P. Lorillard, for $6 \frac{8}{4}$ acres of carrots, yielding by estimate (after pulling a few) 400 bushels per acre, making 2,700 bushels, which at 40 cents per bushel is $\$ 1,080$; expense of seed, drilling, hoeing, cultivating, thinning, and six per cent. interest on the land, rated at $\$ 150$ per acre, $\$ 212$; leaving net profit on $6 \frac{3}{4}$ acres, $\$ 868$.

The attention of the committee was called to a field of 35 acres of good corn recently cut and stacked up, and the ground (a sandy loam) was then being seeded with wheat. They estimated the crop at 50 bushels per acre, making 1,750 bushels, which at 50 cents per bushel gives $\$ 875$; the whole cost of plowing, planting, and cultivating (rating the teams at $\$ 2.50$ per day, laboring men $\$ 1.13$ per day, boys 75 cents). amounting in all to $\$ 242$, which, taken from the value of the crop, leaves a profit of $\$ 633$ on 35 acres; being an average of $\$ 18$ per acre.

They thought the above crop worthy of notice, as showing that farming when properly managed will pay, even in these dull, hard times.

Beautiful driveways, arched, gravelled, smooth and firm as a good turnpike-road, lead to the different departments, buildings, and enclosures, and are lined with elegant fences made of the best material; pickets and slats are largely of locust, posts six to seven feet high, all painted, or coated with tar; they are clean, straight, erect, and free from any foul growth, and for which the premium is awarded to P. Lorillard for the best and cleanest-kept fences on a farm of not less than one hundred acres.

The premium offered by the society for the best and most profitably-cultivated farm of not less than fifty acres is not disposed of, as the committee are not able to arrive at the immense value of the hundreds of thoroughbreds annually produced here, the records of some of which are of world-wide fame; but as far as their knowledge extends, they can say that this establishment is the largest, best, and most conveniently arranged in all its departments for the purposes intended, to be found anywhere."

"Hillhurst." - The proprietor of this farm, Mr. H. Cochrane, of Compton, Canada, has gained an enviable reputation both in this country and England for the breeding of choice stock, principally short-horns. The farm contains 1,100 acres, 300 of which are in constant cultivation, and 300 in permanent pasture. The rotation followed is: first oats, second roots, third wheat or barley sown down with timothy and clover, then grass; the first two years being always cut for hay, of which the second season produces invariably the most. The soil is a dry loam, considerably stony, but advantage has been taken of the stones in building substantial stone-walls and in constructing dikes along the road which intersects the farm. The surface is undulating, somewhat hilly, the buildings extensive and commodious, providing ample accommodations for a large stud of horses, a fine herd of short-horns, and a magnificent flock of Shropshire sheep. The produce of one cow has brought Mr. Cochrane $£ 27,000$. Mr. J. Sparrow, of Woodlands Farm, Bath, England, one of the Tenant Farmers Delegates for the Dominion of Canada, reports the following concerning "Hillhurst:" 
About a mile farther and we came to Mr. Cochrane's farm, situated on one of the ranges of hills that abound in this part of the country; the hills seem as fertile as the plains-1ndeed, the apple trees thrive much better on the hills than in the plains. We drove in through a fine gateway. He has a pretty villa-shaped house, the lawn being on our left-hand and the conservatory and garden on our right; then through another gate and we came upon the barns, stables, cattle sheds, and other buildings, around a large yard. The farm is called 'Hillhurst,' and some of the cattle take their name from it. It was purchased by Mr. Cochrane about 15 years ago, and contains about 1,100 acres. Mr. Cochrane received us, and we inspected his cattle, sheep, pigs, etc. The cattle need no comment from me. They are well known, and show what can be done in this country. I took a note of some of the animals. A dark roan short-horn cow, 10th Duchess of Airdrie, is a magnificent creature, and was purchased by Mr. Cochrane from England, at a cost of 2,300 guineas, but has given him good returns. In the autumn of 1877 he sent a consignment of 32 head of cattle to England, where they were sold by Mr. Thornton for $£ 16,325$ s. Two realized respectively 4,100 guineas and 4,300 guineas, the latter price being paid by the Earl of Bective for the 5th Duchess of Hillhurst, and the former by Mr. Loder for the 3d Duchess of Hillhurst. These two cows were descended from the celebrated cow, 10th Duchess of Airdrie. Her last calf, a splendid creature, dark roan, calved April 6, 1880-weight, 500 lbs.; sire, 3d Duke of Oneida. He has many other fine animals, particularly two bulls, one a dark roan, Duke of Oneida, nine years, and a dark red, Duke of Oxford, five years.

Mr. Cochrane is about to start breeding in the Northwest Territories, and is importing a stock of Herefords as a foundation for his herd. I was surprised to find this valuable herd grazing on the pastures, and but little high feeding indulged in. The most remarkable feature of the herd is the good health maintained. The Swedes and mangel on the farm are very good. He said he had just threshed some of his wheat, which yielded nearly 30 bushels per acre."

"Long View Farm" is the name applied to the farm of Mr. T. S. Grant of Enfield, Conn., and shows what may be accomplished under adverse conditions.

The New England Homestead gives an interesting account of this farm, from which we give a few extracts: "Here is a farm of 120 acres, nearly all of which are under cultivation. In 1872 it kept 16 cows and two heifers; two years later it cut only 40 tons of hay, and only about 40 acres were under cultivation. Since then more than half of the farm has been reclaimed and brought up so that the hay crop this year, without counting the rowen crop, will exceed 140 tons, and will winter over 100 head of neat stock and horses. This enormous increase in the yield of the farm has been caused by the reclamation of worn. out pasiures and inaccessible swamps, and now the whole farm, in a magnificent stretch, has been brought into two adjoining fields by the removal of all the division fences, and it is the aim of the proprietor to make the whole farm produce just as much grass as possible, this being the crop that he is striving after. Other crops, like oats, corn, and tobacco, are raised, but only as a means of stocking down to grass.

System of Management.-As all the pasture lands (except a few acres for young stock) have been improved, the question naturally arises, What does Mr. Grant do with his 50 and more cows in the summer? They are all kept in the barn the year round, except for about six weeks or so, from the middle of August to the 28th of September, during which time they are turned out on fall feed, of which there is an abundance. During the soiling season, they are fed on green feed and hay, commencing with rye in May, and following this with clover and fodder corn. This can be made to last as long as desired by different plantings, but by the time the first planting is gone the fall feed is ready to turn into, and this continues till the last of September. Sometimes after this there will be green feed in the 
shape of late fodder corn or rowen clover, but usually the winter feed commences about this time, and is kept up according to a regular system. The constant soiling of so many cows produces an abundance of stable manure, and this is disposed of every spring and fall. In. deed, it is to the soiling system that is due in a large measure the great improvement which has taken place on the farm. Six years ago only about 400 loads of manure were made. Two years later the soiling system was adopted entirely, and last year the stock made 1,500 loads of strong manure. It is perhaps needless to add, that with this amount of stable manure but little use is made of commercial fertilizers.

The system of feeding is a most reasonable one, and one that shows up well in practice. The herd, nearly all thoroughbred or high-grade Jerseys, occupy the whole of the first floor of the barn, in several parallel rows of stalls. Each cow has her herd number, which is marked on a metallic tag in her right ear. Over each stall is a corresponding number, together with the animal's name. A record is kept of each cow in a book for the purpose, which gives the amount of milk for every milking during the year, the percentage of cream, the time of service, and calving, together with the herd number of the progeny at each calving. Knowing from this each cow's capacity for milk, she is fed accordingly, with the idea always in view of making each cow produce the greatest possible quantity of milk. Mr. Grant believes in steaming certain kinds of fodder, having found that corn fodder, and oat straw, when steamed with one-quarter hay, makes a most excellent feed, which the cows will eat up clean. Meal is fed in winter, but in summer green feed takes its place. The grain fed is corn meal and wheat bran, the latter being scalded before feeding. The advantage of feed. ing the corn meal and bran together is that, while the former is necessary to give richness to the milk, if fed beyond a certain amount it goes to fat rather than to milk, while if bran is fed with the meal the feed will go to milk; and in this way every cow can be fed according to her capacity for producing milk.

The feed is, in winter, as follows: One man gives each cow a feed of steamed fodder, another follows with a feed of bran, giving to each cow such an amount as is required. A feed of meal follows on the same plan. This feeding is all under the supervision of one man, who keeps each cow's record, and knows just what each cow will bear. The stables, meanwhile, are cleared, and clean sand put under the cows; the cows are cleaned, their udders washed if necessary, and everything made ready for milking. And here it is in order to remark that the same scrupulous care with regard to cleanliness is observed in every opera. tion, from the milk-pail to the butter package. Before milking, every milker must go to the wash-room adjoining the stable and wash and comb. While milking, each one is provided with a sponge. and if his hands become soiled or wet, they must be wiped dry on the sponge. Covered milking-pails are used, in which the milk is strained as it passes in, the strainer being cleaned several times during a milking. Each cow's milk is measured separately by a very simple means. A large tin pail is fitted with a glass in one side, across which, at regular intervals, short horizontal wires are soldered, which indicate, in quarts and pints, the contents. As soon as the milk of any cow is measured, the amount is set down opposite her number, on a blackboard hung in the stable for the purpose, from which the figures are transferred daily to the record book. From this the milk is again strained and carried to the dairy, where it is strained a third time into the pail in which it is set. After milking, the cows are fed with dry hay, and again at night the same process is repeated.

At the dairy the milk is set in deep pails, eight inches in diameter, ten quarts to a pail. Adjoining is a 'spring-house,' in which a large well about 12 or 15 fcet in diameter is sunk, and supplied with an abundance of water from living springs at no great depth below the surface. A curb surrounds the well, and over this the setting pails are hung in the water, which is always kept at about $50^{\circ}$, this being about the natural temperature in summer, and in winter the temperature is brought up to this point by steam pipes which run into the well. 

608.

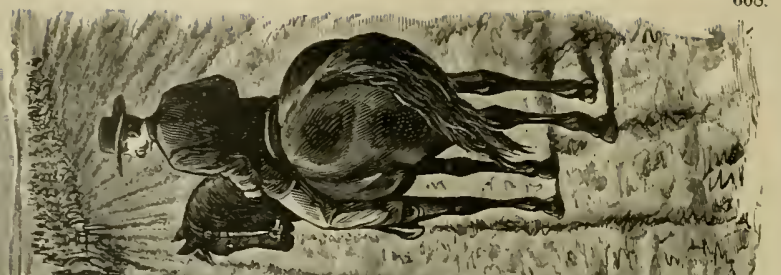

2
0
$1-1$
4
4
$1-1$
0
0
7
7

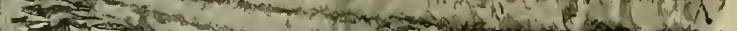
-

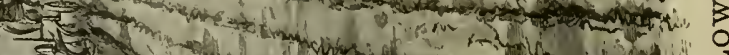

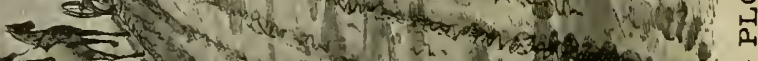

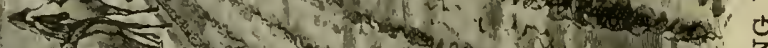

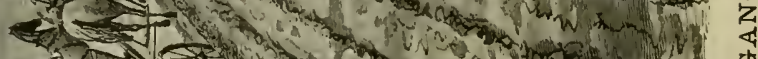
4 Af

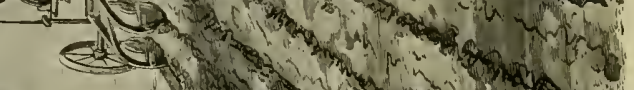

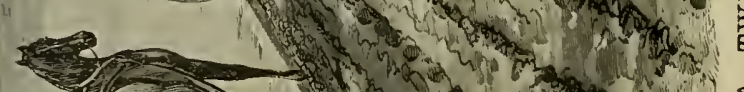

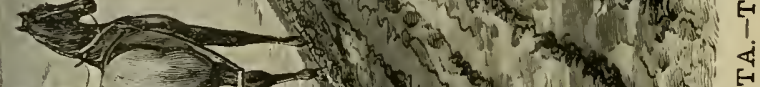

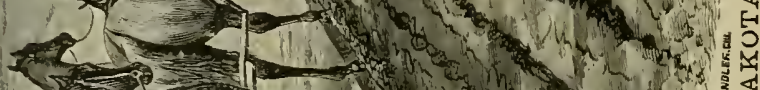

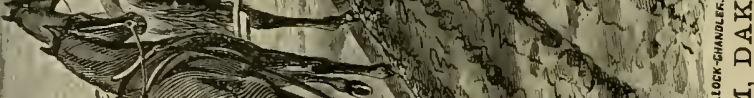

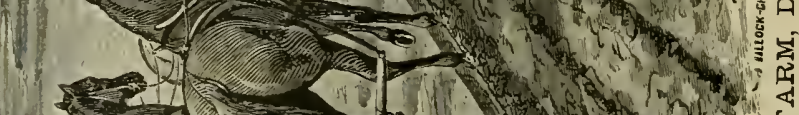

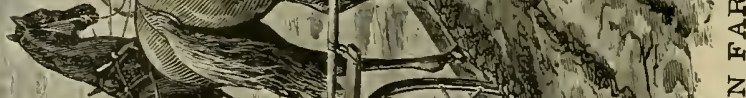

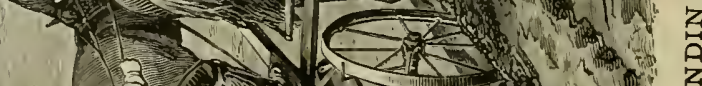
(for)

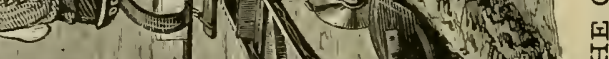
$\longrightarrow-1$. (1)

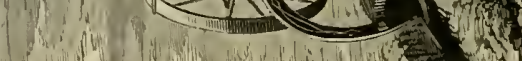
괵

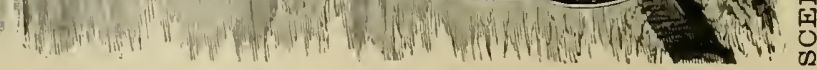


The milk is set thus for 24 hours, and the cream taken off with a deep skimmer shaped like an inverted cone, which can be sunk in the milk withont displacing the cream. About two quarts of cream are taken from each pail. The cream is churned at the temperature of $59^{\circ}$ in summer and $62^{\circ}$ in winter. The churn used is of the old-fashioned dasher pattern, and is made of a barrel, the dasher being worked by steam. The buttermilk is drawn off as soon as the butter comes, while it is yet in the granular form, and worked in fresh, cold water. The salt is then added, three-fourths of an ounce of salt to the pound, and by the time the salt is worked in thoroughly the butter is all 'gathered.' It is then set away to cool, after which it is worked in an old-fashioned lever butter-worker, weighed, molded and printed in a common mold, with the Long View Dairy imprint, and after being wrapped in muslin, each print is packed in a neat box of white pasteboard."

It is surprising that so few farmers among the many, adopt the improved methods, and with a well.defined system, rigidly enforced, endeavor to rise above the drudgery that char. acterizes farm life as generally practiced. Capital is not the only essential. A man with a small capital and few acres of land can practice as complete a system, as far as it goes, as one with immense wealth, like the proprietors of some of the farms previously described. Small farms, properly managed, can be made very profitable, as we have already seen, and with better planning, involving more brain-work and less physical labor, the average farmer can make the results of his labors bring him by far more profitable returns than have ever been secured by following the old way of no system in particular, and letting things manage themselves.

\section{LARGE FARMS OF THE COUNTRY.}

$\mathrm{T}$ HE people of the United States naturally take a considerable interest in the bonanza farms of the country, which contain the great wheat-fields of the world, as it were, whose thousands upon thousands of acres of waving grain seem to beckon to the destitute and starving millions of the old countries to come and find plenty; and it is not at all surprising if a degree of pardonable pride, together with a feeling akin to that of proprietary right, were entertained personally, concerning them, for is it not "our " country, and are not all of the products "our" harvests, and "our" crops?

The following interview is said to have taken place between a Western farmer, who owned a large farm in Dakota, and some of his Eastern acquaintances, whom he wished to impress with the idea of the immensity of the farms in his section:-

"We own some big farms up there, gentlemen. A friend of mine owns one which he had to give a mortgage on, and I give you my word, the mortgage was due on one end before they could get it recorded on the other. You see, it was laid off in counties."

There was a murmur of astonishment, and the Dakota man continued:-

"The worst of it is, it breaks up families so. Two years ago I saw a whole family prostrated with grief; women crying, children wailing, and dogs barking. One of my men had his camp-trunk packed on seven four-mule teams, and he was bidding everybody good. bye."

"Where was he going?" inquired a listener.

"He was going half-way across the farm to feed the pigs," replied the Dakota man.

"Did he ever get back to his family?"

"It isn't time yet," replied the Dakota gentleman. "Up there we send young married couples to milk the cows, and their children bring home the milk. We don't count by acres; we count by townships and counties. My yield was $\$ 68,000,000$ on wheat alone, and I am thinking of breaking up a hittle patch of eighty to one hundred more counties this season." 
A Texas man, happening to be in the group, and not wishing to be outdone in statement, replied: "That's purty good as to size, but 'way down South, where I come from, we can't raise pumpkins at all."

"Why not?" was asked.

"Because the vines grow so fast that the pumpkins wear out dragging along the ground!" was the reply.

The description of some of the famous large farms of the country might seem to those who were not acquainted with the real facts, as being almost equal in extravagance of statement to the above; and, indeed, when we consider the almost unlimited resources of the country for production, and the possibilities of those yet undeveloped, we are lost in the computation of the immensity of the prospect.

The Dalrymple Farm. - This noted farm - the largest in the world - consists of from seventy thousand to a hundred thousand acres, all of which are under the supervision of Mr. Oliver Dalrymple, the celebrated wheat-grower of the Red River Valley. It now embraces the Case, Cheney, Alton, and Grandin farms - the latter alone containing 40,000 acres, and is located on the west bank of the Red River, about 25 miles north-west of Fargo, Dakota. This immense farm is managed on strictly business principles - as, indeed, all successful farming must be, whether on a large or small scale - and is under the direct supervision of Mr. Dalrymple, who, from his office, can communicate by telephone at any moment with each of his agents in charge of the different sections. It is said to contain nearly 40,000 acres under cultivation, more than two-thirds of which are in wheat, the remainder being in oats and barley, while new land is being constantly broken to increase the acreage under cultivation.

The reapers employed are the self-binding harvesters, of which there are 125 used in the harvesting of the entire crop, each requiring three horses or mules, and reaping twelve acres or more per day. The grain is threshed in the field, the threshers being operated by steam, and, like all the other kinds of work, is done with the utmost system. The estimated yield is about 18 bushels per acre of wheat and 90 of oats, the wheat crop alone averaging about 432,000 bushels, which is about 900 car-loads, or 45 train-loads of 20 cars per train. This immense crop is generally expected to net 60 cents or more per bushel at the farm.

Mr. James Biggar of England, one of the delegates sent to Canada recently to report on that country as a field for the settlement of agriculturists, paid a visit to a department of this farm, of which he writes as follows: -

"Next day we drove over the Dalrymple farm. On this division they had about 12,000 acres in crop last year, yielding on an average about 19 bushels per acre. We first saw on the horizon a dark bine which, as we approached, proved to be a gang of thirteen double plows, each drawn by four horses, and turning 2 furrows, 15 inches each in width, and 3 to 4 inches deep, going after each other on a furrow a mile long. On another section, seven double and six single plows were at work, and on another eleven double plows drawn by four mules each. The horses were similar to second-class 'bus horses and showed signs of work; but the mules were in fine condition, and seemed to stand the work much better than horses. The sight was one not easy to be forgotten. In surveying the vast, unbroken prairie there was a sense of loneliness and a doubt of its value as an agricultural subject, but the rich, black soil being turned up, the strong, clean stubble of the former crop, and the fact of its suitability for cropping being thus practically demonstrated, dispelled the idea of wildness, and brought back a fecling of admiration for the enterprise and system of that style of farming.

There are four or five steadings on the farm, with excellent accommodation for men and horses. The implements were also put past in capital order. In one shed we saw fourteen self-binders and four or five steam threshers. In another, nineteen seed-drills and a pile of 
harrows; in others, spare parts for reapers, plows, etc.; and a row of wagons outside. Each of these donble plows travels from 18 to 20 miles a day, and turns over about 5 acres daily. The crop is cut down by self-binding reapers, cutting down 12 acres a day, and attended by a driver and two stokers. It is threshed out in the field, the straw burned, and the wheat taken straight to the cars on a special siding. Each machine threshes about 1000 bushels daily. Wages for ploughmen are $\$ 18$ to $\$ 20$ a month and board. We were told that analyses of the sub-soil showed that it contained all the elements necessary for growing wheat, should the surface soil become exhausted, but the latter is expected to last many years. In returning to the station we saw the train approach quite half an hour before it reached us, as the track is perfectly straight for 50 miles west. On our way back to Glyndon we saw extensive prairie fires raging to the northwards, but they were fortunately extinguished by heavy rains during the night. We met the rest of the party at the station next morning, and proceeded by rail to Winnipeg, which we reached in 18 hours."

Another section of the Dalrymple farm, known as the Grandin Farm, is thus described by another writer: -

"The lands were purchased from the railroad company in 1875 and 1876, the firstmentioned tract to be devoted to wheat-raising, and the second to stock. With the exception of the small belt of timber-lands along the Red and Goose rivers, both tracts are beantiful expanses of the richest lands in this noted valley, the surface a gently undulating prairie, soil a rich alluvial deposit, with clay sub-soil of unlimited capacity for production of grass and grain. The first breaking on the wheat farm was in 1876, 2600 acres, and the season of 1878 was the second year of its operation. The writer was on the place, and from its efficient agent, J. R. Hogan, got the following facts.

There was under cultivation in 1878 as follows: In wheat, 4,000 acres; yield, 80,000 bushels (not having been all shipped at the time, part of the yield was estimated by measurement in the bins, but the quality of the grain being extra No. 1, it will overrun this amount by weight). Oats, 330 acres, 14,025 bushels; average, $42 \frac{1}{2}$ per acre. Barley, 79 acres, 5,701 bushels; average, $72 \frac{1}{5}$ per acre. Turnips, 3,000 bushels from 53 acres. Potatoes, $13 \frac{3}{8}$ acres, 2,000 bushels; average, $149 \frac{1}{2}$ per acre. There were $8 \frac{1}{2}$ acres of beans not then threshed. Hay to the amount of 2,000 tons had been cut and stacked. New breaking, 1,750 acres, making for cultivation in $\mathbf{1 8 7 9}$ some 6,265 acres. Of implements to carry on the operations, we counted 79 plows, 55 harrows, 24 seeders, 28 self-binding harvesters, 6 steam threshers, 40 wagons, with full supply of all other necessary articles. Dnring harvest and threshing time the roll exhibited 235 men, with some 25 hired teams in addition to the 109 head of horses and mules owned on the farm. The farm is divided into three parts, and the buildings for each subdivision, so far as erected, are as follows:

Division No. 1. - Headquarters; has a dwelling 32 by 32 feet, one and one-half stories; office and store-house, 25 by 50 feet, one story; granary No. 1, 56 by 60 feet; two and onehalf stories; blacksmith shop, 16 by 26 feet; pig-pen, 14 by 60 feet; lodging-house, 16 by 32 feet, two stories; stable, 50 by 60 feet, 20 feet posts, with one-story wing, 28 by 60 feet; steam feed-mill, 16 by 40 feet, two stories, with wing 18 by 26 feet; wheat elevator, 18 by 40 feet, two stories, with fine shed for storing engines and threshers, some 75 feet long.

Division No. 2. - Dwelling, 32 by 32 feet, one and one-half stories; stable, 56 by 60 feet, two stories; granary No. 2, 56 by 60 feet, two and one-half stories; wheat elevator, 20 by 40 feet, two stories.

Division No. 3. - Dwelling, 32 by 32 feet, one and one-half stories; stable, 30 by 60 feet, two stories; total stabling capacity now, 190 horses; granary capacity, 65,000 bushels.

These buildings are of the most substantial character, plain but symmetrical, and of pleasing style of architecture, built with reference to all the conveniences necessary to make them of the greatest possible benefit in their various uses. All the buildings are supplied 
with water-pipes, all furnished from three windmills at the river, pumping into 250 and 1200-barrel tanks, through one and one-half miles of pipe. Among other modern, and, on such an immense farm, necessary conveniences, we find four miles of telegraph (telephone) wire, leading from the headquarters office to the offices of the different superintendents on the three sub-divisions.

The railroad station, Fargo, from which the products of the farm are shipped, being on the Red River, this river is used as the channel of communication, and, with a view to cheap carriage for the present, as well as future products, the proprietors have built, and now run their own steamboat, with four barges, and at Fargo have their own elevator, with 50,000 bushels capacity, upon a plan that can be enlarged to any needed wants, besides their own freight warehouse. By the aid of the facilities thus made use of, wheat from the farm is landed in the Duluth elevators at eighteen cents per bushel. The steamboat proved a good investment, independent of the benefits arising from the cheapness of transportation of the farm crop, for when not engaged in their own work, it was employed to a profit in regular river trade between Fargo and Winnipeg, Manitoba.

The first crop, in 1S77, from 2,600 acres of wheat, was 62,660 bushels; in 1878, as before stated, from 4,000 acres, was 80.000 bushels, the first an average of $24 \frac{1}{10}$ bushels per acre, the second 20 bushels per acre. Prices realized, net, $187 i, 95$ cents; from 1878 crop not less than 85 cents. Shipment is made direct to the Buffalo or New York markets, saving all the cost of middle-men in handling, which in these quantities is in itself a good profit.

The superintendent adds- 'From the above you can draw ycur own conclusions; we are well satisfied with zesults so far, and will push enlargement as rapidly as we can. We neither know nor care what other people expect to realize from wheat-farming; at the figures given you, the percentage of gain is so far better than any income we can expect from Eastern investment, that we will not be discouraged even if we occasionally meet with loss of an entire crop; the margins on ordinary average, to say nothing of good years, is large enough to carry a good many losses." "

The net profits, as estimated by the superintendent of this section was, according to the above authority, $\$ 2.50$ per acre, and the cost of producing the crop about $\$ 8.50$ per acre.

Good business management must, of course, be one of the principal factors in the success of such extensive farming, while it is equally dependent upon the soil and climate being peculiarly adapted to wheat culture. Our space will not admit of an equally extended description of the Case, Cheney, and Alton farms, which, as has been preriously stated, form different portions of the famous Dalrymple farm; but sufficient data has already been giren to illustrate the perfect system and magnitude of the enterprise.

Quinn Farm, Glenu Farm, etc.-Dr. Quinn, of California, is reputed to own fiftyfire thousand acres of rich grain land, forty-five thousand of which are in cultivation. A leading California paper states that the proprietor, who is one of California's millionaires, keeps twenty ships busy transporting his wheat to England, and that one continuous furrow on this farm is seventeen miles in length.

A San Francisco correspondent of a leading Philadelphia journal writes as follows respecting the Glenn farm, etc.:- "The largest wheat-producer in California is Dr. H. J. Glenn. He was formerly from Monroe County, Missouri. He is a man of great enterprise and energy. His ranch lies in Colusa County, and comprises 60,000 acres, nearly all arable land. He has this year 45,000 acres in wheat, which, at a low calculation, will produce 900,000 bushels. His wheat will sell for $S 5$ cents per bushel, or $\$ 765,000$.

Another farm in California, owned by Mr. F. A. Schaeffer, of Hamilton, Butt County, is described as containing several ranches, one ranch numbering 2,223 acres of choice land, 240 acres of which are devoted to barley, and 1,700 acres to wheat. The average height of the barley in one season is given as four feet and three inches, the heads being remarkably heavy, 
and the kernels larger than usual; the estimate of yield being sixty bushels per acre. The average estimate yield of the entire wheat crop was twenty-five bushels per acre, while 600 acres of it produced, on the average, thirty-five bushels per acre.

Three other ranches are also owned by this gentleman, one located three miles from the former, and containing 455 acres, 200 of which were in wheat, and another of 1,250 acres, on Butte creek, 550 acres of which were in wheat, and 700 acres in grass, which would aver. age two tons of hay per acre. The third, known as the Feather River Ranch, contains a tract of 700 acres of wheat. The same authority continues:-

"Mr. Schaeffer is one of our pioneer ranchers, having settled upon his home-place in 1855. At that time he was poor, but by dint of great perseverance he has accumulated a competency, and to-day possesses one of the best farms in Northern California. Farming upon the scale that he does necessarily requires the use of numerous horses, to accommodate which he has upon the several places eight large barns, as follows: At the home, three barns, withacap a city for 100 head; Butte Creek, two, with stalls for 40 head; Feather River, three, capable of accommodating 60 head. At this season of the year he has only 20 men employed; during plowing-time he works from 35 to 40 men, and when harvesting begins from 50 to 65 hands are numbered in the force. Parks Bros., of Marysville, are interested with $\mathrm{Mr}$. Schaeffer in Feather River Ranch, but aside from that he is alone. His system of farming is to plow deep, sow plenteously, drain low lands, keep everything moving, have machinery in good order, take good care of stock, and prevent the little leaks so common among the California ranchers."

Some of the other large farms that might be mentioned, and which serve as samples of their kind, are the following:

\begin{tabular}{|c|c|c|c|c|c|c|c|c|}
\hline \multicolumn{3}{|c|}{ G. R. Scofield \& Bro., Cass Co., } & . & . & & • & $\begin{array}{c}\text { Acres in Farm. } \\
3,840\end{array}$ & $\begin{array}{c}\text { Acres in Wheat. } \\
\quad 1,280\end{array}$ \\
\hline J. B. Raymond, & & & . & . & - & . & 6,000 & 2,400 \\
\hline W. H. Wright, & & & . & . & . & & 3,500 & 2,200 \\
\hline J. B. Chapin, Cas & s, Traill & nd Barn & & $\cdot$ & . & • & 9,600 & 1,200 \\
\hline Clapp $\&$ IcCraw & Cass an & Traill, & . & . & . & & 2,500 & 1,100 \\
\hline J. W. Jorrow, C & ass Co., & . & . & . & . & . & 5,500 & 1,625 \\
\hline A. C. Batchelor, & " & . & 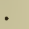 & . & - & . & 2,569 & 1,350 \\
\hline Col. Huntington, & “ & . & 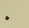 & . & . & • & 2,500 & 1,250 \\
\hline George C. Howe, & " & . & & $\cdot$ & - & & 2,600 & 2,500 \\
\hline A. Leech \& Sons, & “ & . & • & . & . & $\cdot$ & 3,800 & 3,500 \\
\hline
\end{tabular}

When we consider the gigantic scale on which farming may be carried on in the United States, and that this is due mainly to the use of improved agricultural implements, we are enabled to realize more fully the great advancement made in agriculture during the past half century, and the possibilities of this country in this all-important industry for the future.

\section{FARM BUILDINGS.}

A $\mathrm{S}$ the degree of civilization attained by a people can be very accurately determined by the manner in which they till the soil, and the implements used for that purpose, so in like manner can the standard of civilization be measured by the kind of houses they construct for themselves and their domestic animals. The lowest types of the human family live in holes dug in the ground, burrowing like some kinds of animals, while savages of a little higher order than these construct rude huts, that are a slight improvement upon the former habitations. As intelligence and civilization increases, the style of architecture improves in a proportionate degree, until the habitations of the most intelligent nations of the earth are characterized by thrift, refinement, and taste; wealth, intelligence, and culture being as unmistakably evidenced by the style of architecture of such a nation, as by the customs and manners of the inhabitants themselves. 


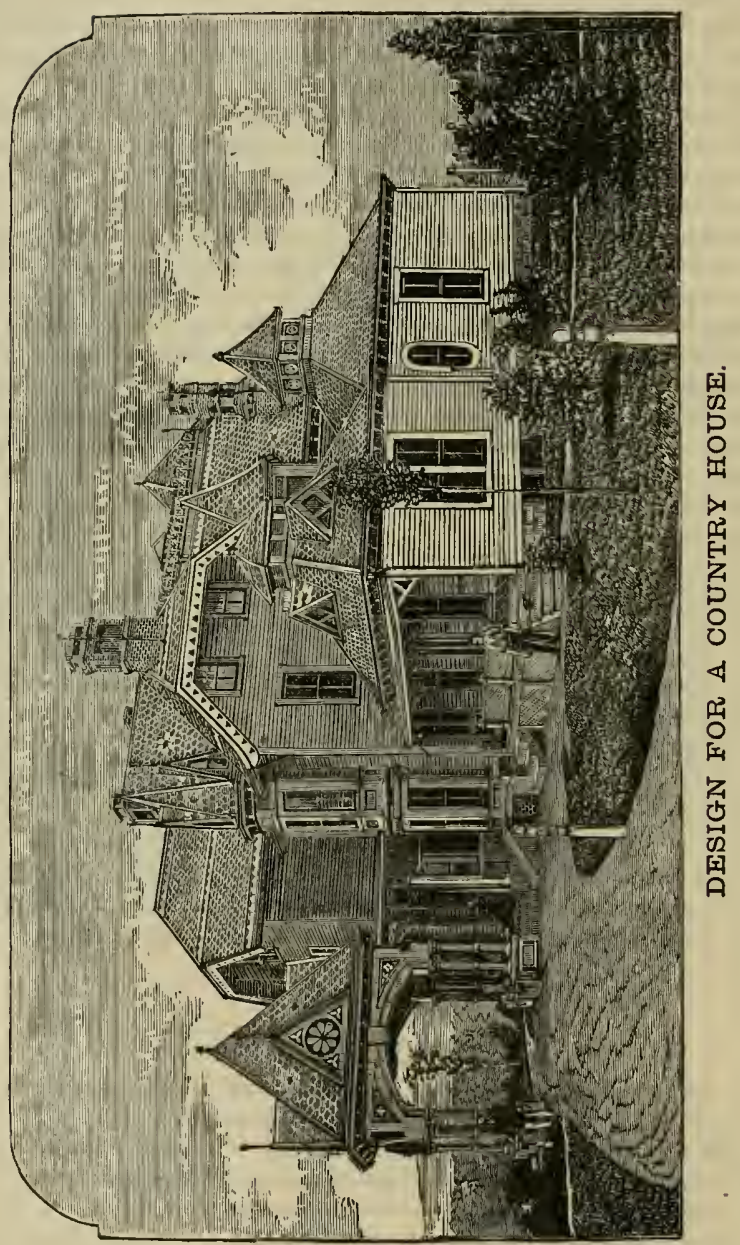


What is true of nations, is equally true of individuals, and the condition of the farm and farm buildings will, as a general rule, be an index of the thrift, enterprise, and refinement of the owner. Although there has been a great improvement in the construction of farm buildings of all kinds during the last quarter of a century, not only in the number adapted to the various purposes of farm use, but in the convenience and style of their construction, still, there is in many sections a great deficiency in these respects, which occasions much incon. venience and loss.

Buildings Necessary on the Farm, etc.-The number of buildings required on the farm will depend upon a variety of circumstances, such as the size of the farm, the purposes to which it is best adapted, the special departments of business to be conducted, the productiveness of the land, etc. A large farm will require more and larger buildings than a small one. Inasmuch as the family should have the first consideration above that of the domestic animals, of course the house should receive the first attention, and be considered the most important of all the farm buildings. It should have the most care and money spent upon it in rendering it healthful, pleasant, and attractive, while the barn should be secondary in this respect, although we know of some farmers who seem to regard their horses as first in importance, and wife and children as sccondary, and who will be at great expense of money, time, and labor for the welfare of their stock, providing fine, comfortable barns, in which they seem to take much pride, while they are at the same time careless and indifferent with respects to the wants of the family, and provide for them a home comparatively much inferior in convenience, comfort, and general architectural construction to the barn. We are glad to know that this class of farmers is in a small minority, and yet such are by far too numerous, and wherever found will always be characterized by a lack of the essentials of true manliness and intelligence that are characteristic of farmers generally.

In many sections of the country it is necessary to provide shelter for the stock during a portion of the year, hence a barn is essential for this purpose, as well as for the storage of their food. Where large numbers and a variety of animals are kept, a stock-barn will be necessary for this purpose, aside from that for the crops constituting their food. In those parts of the country where cereals are cultivated to any extent, a granary will be essential for the safe storage of such crops. A hog-house will be indispensable on a farm where swine are kept, while the poultry-house, wagon-house, wood-house, or place of storage for fuel of any kind, store-house, tool-house, and repair-shop will be found equally necessary. Sometimes one building may be made to answer the purpose of two or three combined, on small farms, such as the wagon-house, tool-house, wood-house, etc., being different departments of the same building.

Aside from those already mentioned, the ice-house will be found a great convenience on every farm, and the source of supplying a luxury which, when once enjoyed for a season, will be regarded as a necessity. The expense and labor attending it is slight, compared with the benefits that may be received. On a dairy farm an ice-house is very necessary, as is also a milk-house, in those sections where there are no creameries or cheese-factories in the vicinity. These will not only prove a great convenience, but will contribute largely to the profits of such farms by improving the quality of the dairy products. Where tobacco or other special crops are exclusively cultivated, buildings adapted to the purpose will also be required.

If to the above, the farmer is so circumstanced that he can add a small conservatory, not as a necessity, but as a luxury, and a means of increasing the educating and refining influ. ences of the home, as well as adding to its attractions, the money and labor thus expended would be found, wherever such a course is practicable, to be a profitable investment.

In the construction of all farm buildings, convenience and good taste should have due consideration. It costs but little more, at first, to construct a building that is convenient and tasty, than one that is lacking in these respects, while convenient buildings will prove the 
cheapest in the end, by the vast amount of labor saved, that is always involved where build. ings are wanting in this essential. Of course, good judgment and taste are necessary in securing such results, and while the one who plans the structure may possess the former, he may not the latter, for it is not every one who is endowed with sufficient taste to plan the artistic arrangement of a building, as will be seen by the attempts in this kind of art, exemplified by many of the buildings commonly seen upon the farm. In the construction of farm buildings, health, comfort, and convenience should have the first consideration, being of primary importance, while beauty of design and ornamentation, though of secondary importance, should not be entirely overlooked.

Repairing and Painting Buildings, etc. - In the architectural study of farm buildings, a recent writer has divided them into two classes, viz. : - "Those already built, and those which are to be built"; in other words, the old, and the prospective new. As the former are by far the most numerous, we will consider them first.

The importance of keeping farm buildings in good repair should not be overlooked by any farmer who has regard not only to the general thrifty and orderly appearance of his surroundings, but to economy as well. On every well-regulated farm, frequent repairs in buildings become a necessity in securing their preservation, and unless these necessary repairs are made in season, and thoroughly performed, the expense of repairing will be largely increased, and permanent injury to the buildings often be the result. If a leak in the roof of a building, or elsewhere, is promptly stopped, no injury is occasioned by it; but if neg. lected month after month the frame-work of the building will be liable to decay, and become after a little time so seriously injured as to be worthless. When buildings need painting, the sooner the new coat of paint is applied, the better. If too long neglected, the surface becomes rough by exposure to the weather, which will render the painting more difficult to perform, and also require much more paint to cover the surface well; hence, promptness in such cases is an economy in both the expense of labor and material.

Besides the economy of keeping buildings in good repair, their neat and orderly appear. ance is no small argument in favor of such management. Good work, promptly performed, is in all respects the cheapest. In painting buildings, two objects are secured, viz.: ornamentation and durability. Paint adds to the beauty of the buildings, and also tends to preserve the wood to which it is applied. Buildings that are kept well painted have a neat, attractive appearance, are an indication of the culture, refinement, and prosperity of the owner, and render home more pleasant to the family circle. Unpainted buildings have a dingy, neglected appearance, and will require a new covering of wood-work much sooner than those that are kept well painted.

In answer to the question, whether it will pay for the farmer of small means, and many expenses, to incur the additional expense of keeping his buildings well painted, we would say, that it depends upon various circumstances; if mere money value in benefits resulting from having well-painted buildings is considered, it will depend upon whether it will cost more to procure the paint necessary to preserve the wood-work than to newly cover the buildings when they need it.

In some sections, where lumber is cheap, the paint would be the most expensive; in others, where timber is scarce, the cost of timber and labor of re-covering would prove the most expensive. But the money value of things is not the only consideration to be involved. The attractions that may be added to the home by the outlay of keeping the farm buildings well painted, and in good repair, and the pleasure and satisfaction afforded the family, besides the refining and educating influence of pleasant surroundings, are considerations which it would be difficult to weigh by a money standard.

If farmers would take more pains to make their homes attractive and pleasant, and farm. life something better than the hard drudgery that it too commonly is, there would be more 
respect and love entertained for farming, as an occupation, by farmers' children, and less complaint by farmers generally of being left to till the farm alone, in their old age. If farmers wish their sons to be attached to the farm-home, and farm-life, they must make that farm-home and farm-life sufficiently attractive to induce some of their boys to stay; and how can they make a better beginning than to commence right at home and first make the farm buildings neat and attractive? WVe are sorry for the farmer's son or daughter who feels ashamed to say to a stranger friend, "This is my home"; while it is refreshing to see a kind of pardonable pride manifested by children for their home. Home should be the dearest and most attractive spot on earth to husband, wife, and children, - attractive to both heart and eye, and in ordcr to make it such, there is more responsibility involved individually in connection with each member of the household, than mere sentimentalism might include.

The home should be made attractive, - beautified. Money thus spent is capital well invested, and will bring larger returns to the farmer and his descendants, in real happiness, comfort, and elevating influences generally, than almost any other investment that could be made; and to those farmers who can make such a course practicable, we would say, spare no pains or reasonable expense in making your homes as attractive as possible.

The expense of painting may be greatly reduced by the farmer being able to perform the labor of applying the paint himself, instead of paying a professional painter to do the work for him. This was formerly in a great measure impracticable, on account of the lack of knowledge of the proper method of mixing paint; but the difficulty is now obviated by the use of what are called "mixed paints," which have for several years been in general use. These may be found in the market mixed in the proper proportions, ready for use.

Like white lead, and nearly everything else that is marketable, and capable of being adulterated, unadulterated paints may be difficult to find; still, there are some brands that are such, and are also cheap and durable, and when they can be procured, it will be quite a saving of expense for the farmer of limited means to purchase them, and do the painting himself.

Procuring a well.known brand that has previously given perfect satisfaction will always be the safer way. In order to do the work of painting well and rapidly, a certain amount of practice will be essential.

The surface to be painted should be clean; hence, when it has become soiled, like some portions of the interior wood-work of the house, for instance, it should first be thoroughly washed and dried. If the surface is rough, it should be smoothed before applying the paint. Sandpaper is frequently used for this purpose. For the outside of buildings, which do not ordinarily require such nice work as the inside, less preparation of this kind will be necessary. The nail-holes and large cracks should, however, all be filled before applying the paint, and the dust brushed off. Where the outside of the building has become weatherworn from long neglect, it will be well to apply one or two coats of cheap oil before painting; since, if the paint were put on without this previous preparation, the oil of the paint would be liable to penetrate the wood, causing it and the lead to separate, and the latter to fall off in scales. The best time for painting is in the spring or fall, when the weather is dry and sunny, and neither very warm nor very cold.

The implements for painting are a good brush, a tin pail to hold the paint, and a good strong ladder to which the pail is attached by a hook. We might also add that a small brush-broom for brushing off dust, etc., will be very convenient where the surface requires it. In painting, the brush should be made to work in the direction in which the grain of the wood runs, covering the entire surface well, and working the paint into the small cracks. Special pains should also be taken to brush over the laps in the wood-work smoothly; care. lessness in this respect failing to give the work a uniform and neat appearance. Care should be used not to put on too much paint at a time, but just sufficient to cover the surface well. 
Two coats of properly-prepared paint will generally be sufficient where the work is well done; but on buildings long neglected, sometimes a third coat will be necessary.

The choice of color in respect to farm buildings has much to do with their general appearance. This is a matter of taste, since one color of paint would prove as good a pro. tection to the wood as another, where durability is the principal object sought in painting. Fismerly, white was the prevailing color for farm-houses, and a country village, except houses entirely "innocent " of paint, presented an unpleasant glare of light in a bright, sunny day. This practice also gave a similarity to the buildings that is not in conformity with good taste. At present there is a desirable change in this respect, and we now frequently see the variety of colors that are more pleasing to the eye, and less cffensive to the taste.

In the choice of colors and shades suited to the purpose, there is much laticude, and the opportunity for the display of a cultivated taste and skill in producing a harmony cf colors, together with an agreeable and striking contrast. The most pleasing effect is generally produced by painting the body of the house one color, and the cornices, corner-boards, casings, and ornamental work another, considerably darker. The farm-house and cther buildings rnay be painted the same as the house, or other colors may be chosen for the other farm buildings. We prefer the former style; but it is merely a matter of taste. Where different colurs are chosen for the out buildings, they should always be such as will harmonize wi:h that of the house.

The Farm-House.-The location of the farm-house should never be chosen without due deliberation, as it has much to do with the health and comfort of the household, as well as the pleasantness of the home surroundings. The site chosen for the new house should always be on dry soil. A damp cellar is one of the most objectionable features of a house, and the fruitful cause of various ills. Many incurable diseases, besides deaths. that may have been regarded by the members of the family as the dispensation of an overruling providence, might be directly traceable to this source. Unless the site chosen be on a naturally dry soil, it should be made perfectly dry by carefully and thoroughly underdraining. A low, marshy locality should be avoided by all means, the air of such places being filled with dampness and malarial disease.

Hon. Alex. Hyde, Mass., says with reference to this subject:- "A prairie farmer once said to us, 'I would give a thousand dollars for one of your New England gravel knolls on which to build my house'; but here, where dry knolls abound, they are too often neglected in selecting a buildiug site. The excuse for locating farm buildings in low, damp places is a desire to avoid bleak winds; but the pure, dry air, cold though it may be, which plays about the summit of a bill is not half so much to be dreaded as the damp, malarious atmosphere of the more sheltered valley. The fogs which infest the low lands are more chilling and pernicious in their influence than the dry winds of the hill. We have often noticed in riding over our hills and through our valleys on a summer or autumn evening, that while the air on the high lands might be brisk, it was warm and dry compared to that in the valley. As we have descended into the latter, the transition was as marked as on going from an airy chamber into a damp cellar. It is not necessary that the valley should be marshy in order to perceive this difference in the dryness of the air. We have often noticed it in descending from the hills into the valleys of the Connecticut and Housatonic, where the land of the valley was a dry alluvial.

It is a mistake to suppose that the hill is colder than the valley. Every farmer must have noticed that the late frosts of spring and the early frosts of autumn do more damage on the lowlands than the highlands, and the thermometer of a cold, still night shows a lower degree of temperature in the lowlands. The valley may furnish a shelter from the winds, but not from the cold. Cultivate, therefore, the valleys, but place your farm buildings on the hills, where an equally good shelter from the winds can be secured by clusters of white pines or other evergreen trees planted on the windward side of the buildings. 
As a second suggestion, we say, locate farm buildings where the sun will shine the most hours of the day and the most days of the year. The value of sunlight, both for man and beast, has never been fully appreciated. There are life, health, and elasticity of spirits in sun. shine. Show me a woman that has worked for years in a dark, gloomy cellar-kitchen, and in all probability you'll show me one, the corners of whose mouth are turned down, whose constitution is impaired, and who has lost all buoyancy of feeling. Show me an ox that is stalled in a dark cellar-stable, and yarded on the north side of a barn, and I will show you one whose eye is dull, hide inelastic, hair bristling, and step heavy. Physicians tell us that patients located on the south or sunny side of hospitals are more likely to be cured than those on the north side, and heliopathy is as much in fashion as hydropathy once was. What the exhilarating and invigorating effects of a sun-bath are we can conceive from the change that comes over our feelings and powers when the sun shines out clearly after having been hidden for a long time behind the clouds.

Very nearly allied to the location of the house where the family may enjoy the full benefit of the sun's rays is our next suggestion, that the house be not surrounded by too many shade trees. A tree is 'a thing of beauty and a joy forever,' and we would by no means discard all trees around the farmer's premises; but it is possible to have too much of a good thing. A house without any shade trees looks naked, and is naked. A few well-located elms, maples, mountain ashes, and white pines, add much to the beauty and comfort of home, but no one should live in a forest. Musquitoes may live and thrive in such a dense shade, but man finds his true development where air and light find free access. We never desire to see so many trees around a house that the grass will not make a velvety turf on the lawn. Beautiful as are trees, and exquisite as are the forms and colorings of flowers, there is nothing that pleases the eye more, day after day, than a well-kept lawn. A stately elm here, and a cluster of evergreens there, adorn and protect a rural home far better than a perfect swamp of trees.

We cannot dismiss the trees without alluding to the protection from winds and the healthful influences which evergreens rightly planted furnish in this cold climate. Clusters of balsams and white pines placed between the house and barn, and pig-pen, ward off all noxious effluvia from the latter, and if there is any swamp near the premises, the same trees, with their millions of leaflets, will absorb or turn aside the spores of disease which are constantly exhaling from decaying vegetable matter. Planted on the north of the house and garden, which is generally the windward side, evergreens not only protect from the cold winds, but they fill the air with a most healthful balsam."

The southern slope of a hillside is a desirable site for a house, as it furnishes the opportunity for the enjoyment of the full sunlight during most of the day. The northern side of the road should also be chosen if practicable. The house should also be located near the highway. We have seen houses in the country located in such a manner, and so far from the road, that it would be almost impossible for the inmates to catch a glimpse of what was passing. on the highway, and with the exception of going from the premises, or the receiving of visits from friends, they would seem almost as much isolated from the outer world as though they were behind prison bars. The farmer and his sons would not be as much affected by the unpleasant location of the farm-house as the wife and daughters, since their business calls them away into the fields and broad sunlight so large a portion of the time; but it does very materially affect the health and happiness of those com. pelled to spend the most part of their time in such a location.

The house should be so located and planned that the rooms most occupied in the daily tasks of the home duties should be upon the sunny and most pleasant side of the building, commanding the best view of the highway and neighboring farm-houses. To persons possessing certain temperaments, the isolation and retirement which some localities in the 
country impose, is a serious cause of nervousness and morbidness, and it has been stated by some of the highest medical authorities, that much of the insanity among farmers' wiveswhich is more frequent, in proportion, than among almost any other class of persons-may be directly traceable to excessive hard labor and this isolation and monotony in life. With nothing to divert from the dull and monotonous routine of labor, day after day, and year after year, the mind is apt to prey upon itself with the consequent evil effects.

Our surroundings have much to do in making up the sum of happiness in life, and no. thing that contributes to it, even in the least, should be overlooked.

Country life is, of necessity, devoid of much of the variety which the village or city afford, but it need not be rendered doubly isolated and the home a lonely hermitage for that reason. The most pleasant location possible should be chosen for the home, at a convenient and desirable proximity to the public road, on a slight elevation if practicable. In sections where the land is low and nearly level, a slight elevation can be made artificially by carting earth and building up the surface. This involves considerable labor, but will well repay in some locations by the better drainage thus secured, as well as improving the appearance of the grounds.

Influence of the Dwelling upon Character. - In the construction of all farm buildings, they should be adapted to the purposes for which they are intended. As the house is designed for the protection, comfort, health, and happiness of the household, it should be constructed in a manner suited to subserve these purposes; hence, it should be convenient, roomy, and of sufficient size to meet the wants of the family. It should be well lighted and ventilated, pleasant and tasty in arrangement and design.

It should be borne in mind that "home" is not merely a place of shelter from the storms and cold of winter, and the heat of summer - a place in which to sleep securely at night, and labor by day; it is all this, and something vastly more. It is a place where the children receive their first and most lasting impressions, those that go far in molding and forming the character of the man and woman in after life. A tasty, orderly home has a refining, educating influence upon its inmates, while an unattractive, gloomy-looking, and poorly-furnished house has an influence which is the reverse from elevating. Where there is nothing to cultivate a refined taste, and there is necessitated a constant association with things that are meager and mean, the mind naturally is warped in the same direction. A pleasant home will not only prove an attraction to the children of the owner, keeping them from places that are debasing in their influences, but will also attract better associates for them, who will come and visit where they find the same refining and pleasant surroundings to which they are accustomed in their own homes.

Things that may seem small in themselves are often vastly large in their influence, and determine the whole course of many a human life. We are apt to speak of "destiny" in life, and regard it as something mysterious and inevitable - an indefinable power that determines the fate of mortals, and over which they have no control. But the fact is, our destiny is in our own hands, and is what we make it; consequently, our own lives, and the lives of those depending upon us, are, in a great measure, what we make them.

We are more or less influenced by our surroundings, and too little attention and importance is generally given to this fact in the construction and furnishing of our houses. But some farmers will say: "Such talk is all very well for those that have plenty of money and can afford to have nice homes, but we are not able to make our homes tasty and attractive; we are poor, and we and our children must work for a living. We have neither the means nor time to bestow in beautifying our homes, and the idea of farmers of such limited means, that they can scarcely make a living from their farms, embellishing their homes, is all nonsense!"

To be sure, "bread" is, indeed, the "staff of life," and the material wants must receive 
the first attention; it is better, if we cannot have but one, to have the body properly fed and clothed, than to have a beautiful home. But without pleasant surroundings, life is but half a life, and how few realize at what slight expense a home may be made tasty and attractivel How few understand how pleasant and enjoyable life on the farm in the country can be made, and at what small expenditures the rural home may be rendered convenient, tasteful, and really beautifull For the exercise of good taste and ingenuity does not necessarily imply extravagance. We have seen houses in the city furnished with the most wanton extravagance, where money was lavished almost without limit, and yet they were not beautiful, because there was no taste displayed in the selection and arrangement. To be sure, everything was expensive and rich, but there was a lack of harmony and good taste that offended the eye, as a discordant note in a strain of music offends the ear.

On the other hand, we have entered many a rural country home - a small, bird's-nest kind of cottage, perhaps - where everything seemed so neat, tasteful, and perfectly adapted to the place and surroundings, that it possessed a charm and attraction that rendered the term "beautiful" not an inappropriate one to apply to it; and yet, perhaps, many of the furnishings and ornamentations were rustic carvings, or other work performed by the father or boys of the household, on winter evenings or rainy days, when not employed on the farm, while the wife and daughters had beautified each niche and nook within, with specimens of their decorative art and handiwork, and without, with trailing vines, blooming shrubs, and flowers, in a manner that only a certain quality of feminine taste and ingenuity can devise and execute.

Wealth does not always furnish a tasty, or a happy home, although it may possess the means of doing this, while the lack of a competence need not necessarily prevent the posses. sion of a home that is tasteful and attractive, the abode of contentment and happiness.

Height of Buildings. - In the construction of all buildings; adaptation to the purposes designed, as has been previously stated, should be an important consideration. The area of surface covered by a building is not the only standard by which to judge of the capacity. It requires no extensive knowledge of mathematics to perceive - and it may be needless to state - that a building two stories high will contain twice the capacity of one covering the same area that is only one story in height, and that although the two-story building will cost more to erect than that of one story, the former is comparatively cheaper, since twice the capacity is secured, with but a comparatively small proportionate increase in cost. It costs no more to cover or lay the foundation of a high building than a low one, other conditions being equal, while the increase in cost of the extra height is small in proportion to the benefits derived from the increased capacity; therefore, high buildings are proportionately cheaper than low ones. It will be well to bear this in mind in the building of barns for storage, granaries, etc.

In the construction of farm-houses, the height should be proportionate to the surface of ground covered by the building, in order to look well, while the convenience and other benefits derived from having plenty of room should also be taken into consideration. A small, narrow house, built so high that it seems in danger of being blown over by the first strong wind, represents anything but taste in its style of architecture, while a house covering a large area, and so low that it gives the impression to the beholder that the builder was obliged to cut short his work for lack of means or material, is nearly as objectionable, although not quite as much so as the former. A farm-house should, at least, be two stories high. Sleeping-rooms, on the second or third floor even, are much to be preferred to those on the first floor. Such rooms are more healthy, as well as pleasant, since they are more airy and farther removed from the exhalations of the cellar and dampness of the ground. They also afford a finer view of the surrounding scenery.

In some of the newly-settled sections of the country, where building materials are 
scarce, small, low farm-houses of a single story are very common, but as the owners become more prosperous and population increases, these give place to the more convenient and roomy structures found in the older-settled portions. Too much room is not desired in a house, but there should be sufficient to meet the wants of the entire household, and a surplus is to be preferred to a lack in this respect, if we were to make a choice of two evils. A house that is a story and a half high is but little better than that of a single story, while it will cost nearly as much as a two-story house. The rooms of a dwelling-house should be sufficiently high to be airy, and admit of good ventilation. Low rooms are unhealthy, and should be avoided.

Extra room in buildings for storage, etc., can often be secured at slight expense, by making the building a little higher than the original purpose requires; as, for instance, the wagon-house, tool-house, or wood-house, may be made, by this means, to furnish room over. head for the storage of small crops, or the spreading of those that require drying.

Plans for Farm-Houses. - Before erecting a new house, it will be very essential for the farmer not only to have a well-defined plan, which will secure for himself and family all the conveniences and comforts practicable, but also to carefuily estimate the expense that will be incurred, and whether he can safely and without injury to his financial prosperity withdraw from his business the amount of money necessary for the purpose. In some cases it may be found better to occupy the old house two or three years longer, until the financial status of the farmer is such that he can safely invest in the enterprise of building the new; for while a neat and attractive house to live in is a very desirable thing, still it is better to occupy one that may be old and time-worn, and even shabby in appearance, than to be driven into bankruptcy by the erection of a new one before being really able to do so. On the other side of the question, farmers, as a general rule, are very cautious and quite too apt to go to the opposite extreme of delaying the enjoyment of the new beyond their means, instead of taking them in advance. And too many of them subject themselves and families to the deprivation of many things that might and should be enjoyed.

Life is short at the longest, and if a few years of it may be made brighter for the household by occupying a neat and commodious house, why not brighten these few years for such members as soon as may be, instead of delaying it and involving a loss to all, and, perhaps, until it shall be too late for some loved one ever to enjoy it?

A house, in order to be comfortable and pleasant, need not necessarily be very expensive. A neat and tasty cottage, relieved by slight ornamentation of the severe plainness so frequently characterizing country homes, would be very suitable for the purpose. The few plans which are here presented will be found to come within the range of uost farm. houses, both as to size and expense, and also valuable, as suggesting improvements in design in the general style of architecture, as well as convenience of arrangement, and will prove of practical value to those contemplating building or remodeling. For the first four designs represented in this department we are indebted to W. T. Comstock, publisher and successor to Bicknell \& Comstock, of New York city, being copied by permission from the specimenbook of One Hundred Architectural Designs.

The first-a design for a country house-represents a large handsome building of six. teen rooms of superior accommodations. It was remodeled two or three years after being built, to its present style. The materials are of wood, the frame sheathed and felted, the roof slated, and the interior finished in a tasteful and appropriate manner. At the time of being remodeled, a gas-house was built, with fixtures for lighting the house. This design represents the most expensive dwelling which we insert-the estimated cost of erecting being about $\$ 10,000$. The archway over the carriage drive-way adds very much to the appearance of the house, besides being a great convenience. The lower floor contains a parlor, diningroom, library, hall, sitting-room, two pantries, laundry, kitchen, and a dinner-service room. As a country-seat for a city gentleman, or a house for the farmer of atnple means, such a building is admirably adapted. 
TuE Veysey Homestead Cottage was recently erected in the village of Tenafly, N. J., at a cost of about $\$ 2,000$. This sum includes a large school-room which communicates with the

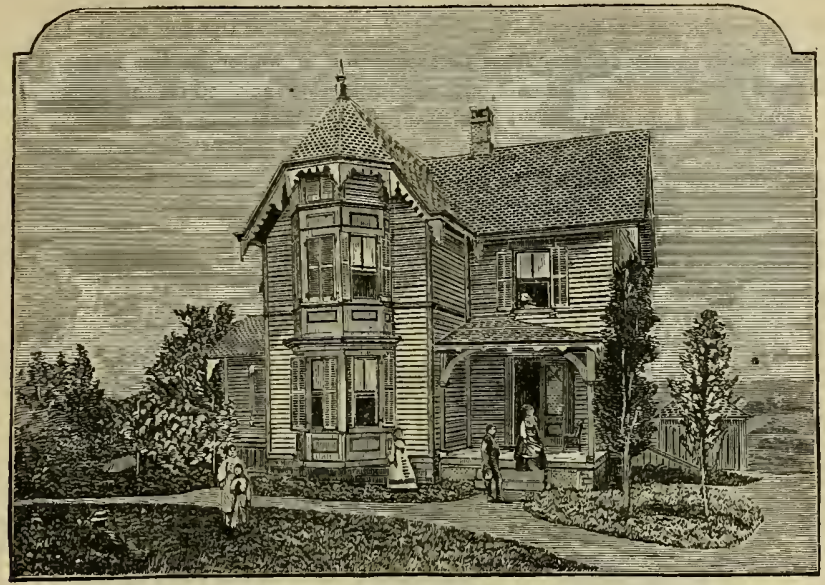

THE VEYSEY HOMESTEAD COTTAGE.

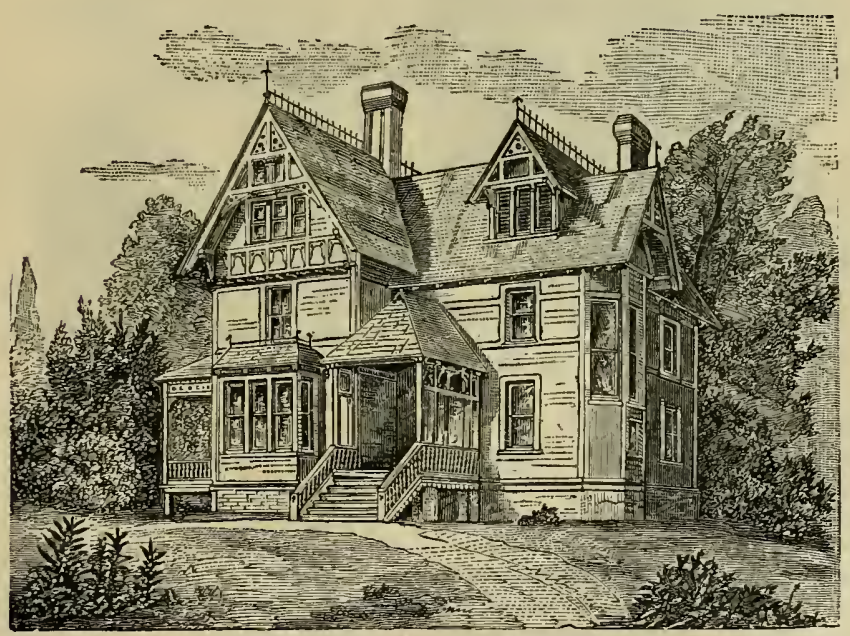

A PICTURESQUE COUNTRY VILLA.

dining.room, and four finished attic rooms. It is a very commodious, tasty house and one that would be an ornament to any country town or village. The owner of this house used the very best material of their several kinds. By using material less expensive such a cottage could, of course, be built at considerably less expense. The above is an illustration of a 
somewhat more elaborate and expensive house, which contains a conservatory in front of the first landing of the stairway in the octagon end at the right, and a dressing-room below. The library is in the rear of the main hall, and at the right of the back hall, which includes the back stairway. The parlor occupies the front of the house at the left of the hall, with the dining-room, pantry, store-room, china-closet. and dumb waiter in the rear. The plan is designed for a kitchen in the basement, although it can be included on the principal floor, if desired. The second floor contains three bed-rooms, a bath-room, and five closets. Three rooms may also be included in the attic. The estimated cost of such a building will vary according to the price of material at the time of building, but may be estimated at from about $\$ 3,000$ to $\$ 3,500$.

The following represents a small cottage suited to a family of two or three persons. Such a building could be erected at a cost of from $\$ 800$ to $\$ 1,000$, according to the locality and style of finish.

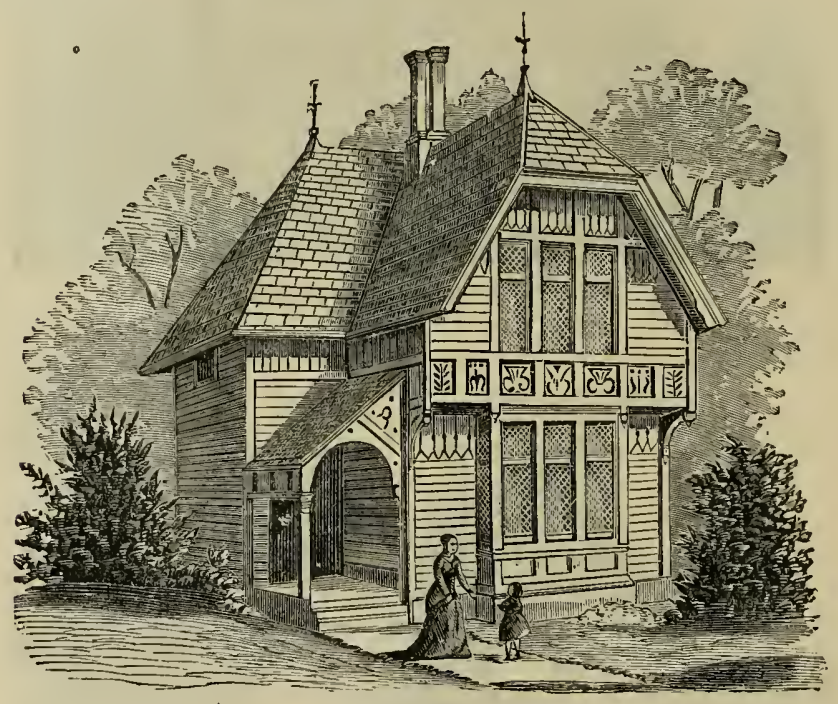

AN ORNAMENTAL COTTAGE.

The ground and chamber floor plan of the English cottage that follows, as well as those of the rural cottage on page 627 , will be readily understood by a careful examination; hence they require no extended explanation

Although designs are a great assistance in suggesting a plan for a house, still if a person contemplating building could examine several houses which seem to be well-suited to his purpose, he could obtain more real information and satisfactory knowledge respecting a plan than from any diagram on paper that could be given. Combining the best things contained in several designs will often prove very satisfactory. It will be noticed that in some of the plaus given, provision for a dairy-farm has been made by including a milk or cheese room.

In those sections where there are no creameries or cheese factories at which to dispose of the milk, a milk-house, located a short distance in the rear of the dwelling-house, could be erected for that purpose, or a suitable room in the house appropriated to that use; but 
tho former is the better method. Every plan that might be proposed would of course be subject to modifications, according to the location and wants of the family.

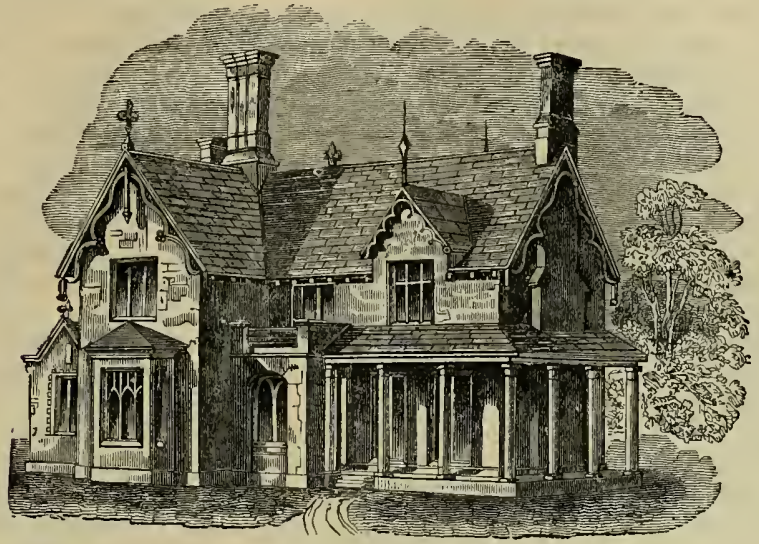

ENGLISH COTTAGE.
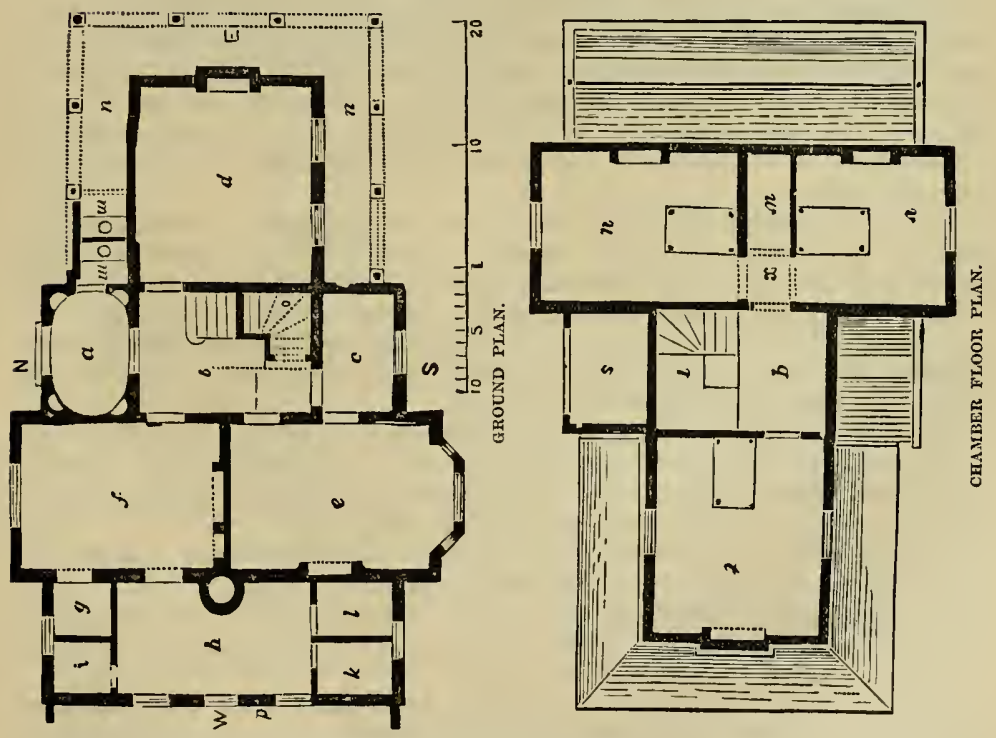

As a general rule, however, there are certain things that will apply equally well to all farm-houses-large or small. They should be in structure, types of stability, and completeness, adapted to their surroundings, not excessively ornamental, but sufficiently so to be 
tasteful and attractive, and to avoid that severity of plainness that characterizes country houses generally.

Every farm-house should have a bright, sunny and pleasant kitchen. We mention this room first, because it is where the majority of farmers' wives spend much of their life in performing the daily tasks for their household. Therefore this room should be located in the most pleasant and cheerful part of the house. Next in importance should be the sittingroom, where the family spend their evenings, and the wife and daughters perform the sewing for the family in the afternoons, when the general housework for the day is done, and where, in the long, winter evenings, the hours should be diversified with reading aloud by some member of the family, or enlivened by music, while old garments are being rejuvenated, or new ones made by skillful hands. This room can also be used as a library if necessary, for all farmers should have something of a library-the more books the better, if of the right kind.

In many country houses the kitchen is large, and serves the purpose of both cook and dining-room. A better arrangement than this would be, to have the kitchen made just large enough to serve the purpose of cook-room and general house-work, with a good-sized diningroom leading from it.

Opening into the sitting-room there should be a good-sized bedroom, which may serve, when occasion requires, for a "sick-room." This room should be well lighted, and located on a sunuy side of the house; it should contain an open fire-place or grate for heating in cold weather, and also for purposes of better ventilation. A parlor is a great convenience, since it is not always desirable to introduce callers into the sitting-room. But we do not approve of a parlor, as such, or as is generally found in most dwellings, which is a room set apart for the "best things"-things regarded too choice and sacred for the common use of the family - the best carpet, furniture, pictures, books, \&c. and into which the family rarely enter, except to entertain visitors. Nothing should be too good or choice for the enjoyment of the family circle every day, and nothing should be used by the family too ignoble and poor to be seen by visitors. The parlor should be a place to be freely enjoyed, when desired, by the entire household, a kind of second family room, to be appropriated as con. venience requires.

In every farm-house there should always be one room set apart for the children, to be used as a play-room by them at will. It should be large and airy, and located on a sunny side of the house. The pantry should be of good size, and conveniently arranged adjacent to the kitchen, in order to avoid unnecessary steps in doing the work.

The cellar-door and door leading to the wood-house should also open into the kitchen. A wash-room or laundry, and a room for the storage of groceries, should also be on this floor, unless such arrangements are made in the basement.

If cheese and butter are made on the farm, separate rooms will be required for the milk and the storage of cheese, either in the dwelling-house or in a small dairy-house suited to the purpose. The dining-room should contain a china-closet. Another closet in this room for the storage of other things would also be a great convenience.

Every bedroom and chamber should also contain a good-sized closet. Closets are of great utility in a dwelling, and there can scarcely be too many of them.

In planning such conveniences for a house, a woman's judgment and ingenuity will generally be more suggestive and reliable than a man's, as her business pertains more to such matters; hence the farmer should always consult his wife and daughter in this respect.

Perhaps the reader may have heard of the wealthy gentleman who established and liberally endowed a now popular institution for the education of young ladies, and that in the large and costly edifice erected for the purpose, and planned by the donor, the young ladies who became pupils found, to their utter consternation, not a single closet from attic to basement ! 


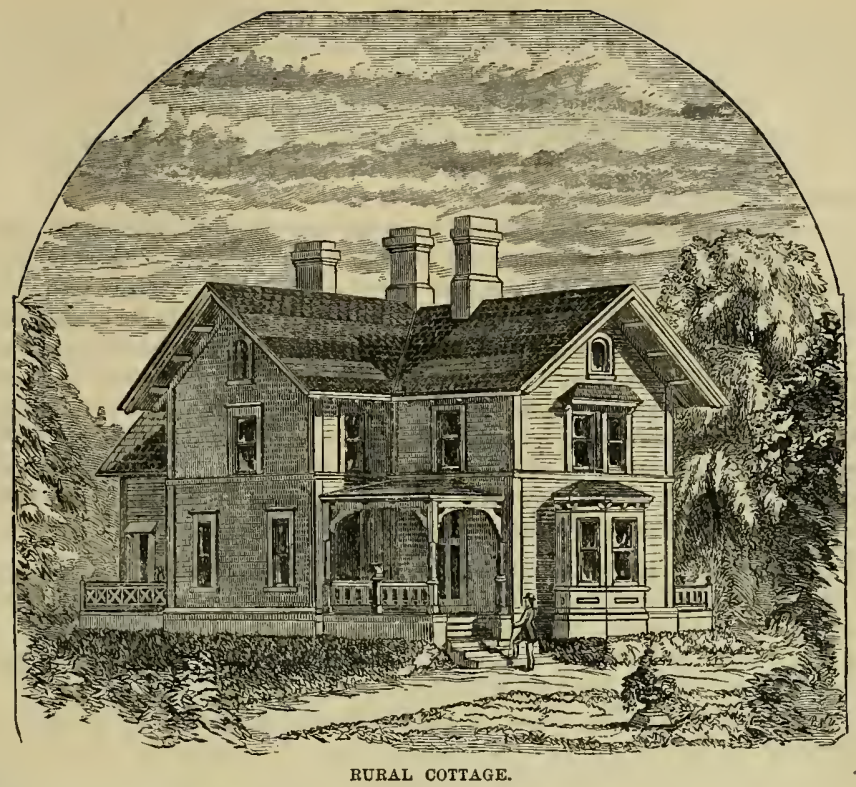

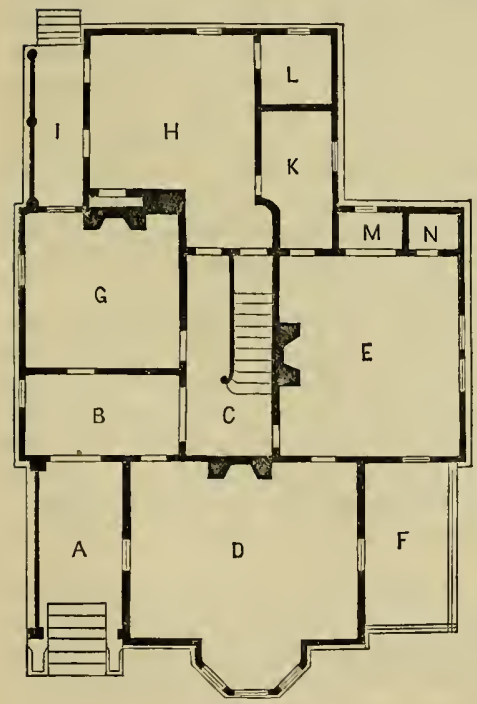

GROUND PLAX.

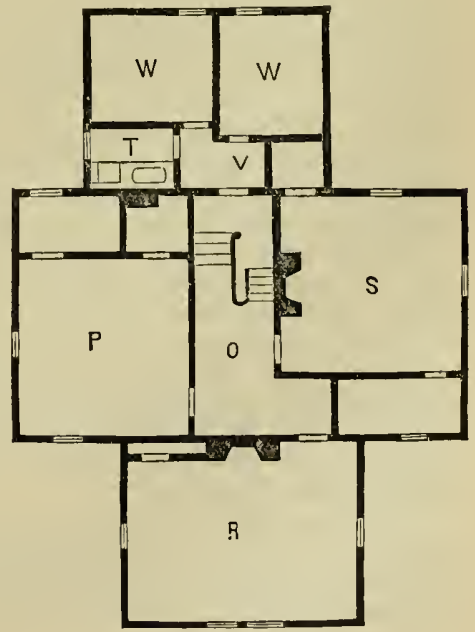

CHAJBER-FLOOR PLAN. 
On being consulted on the subject, the liberal donor replied that the young ladies would find two hooks on the back of the door of each room, one for the "every-day dress," and the other for the "best,dress," and that he did not consider a closet necessary ! Had a woman's advice been consulted and followed in this respect, it is quite certain that the above plan, which seemed so admirable to the designer, would have been considerably modified. The sleeping-rooms of a dwelling should be large and airy, with ample means provided for good ventilation-the latter is very essential.

In constructing a house the aim should be to render it healthful, comfortable, and convenient. These are the first essentials; but since so much time is to be spent in it by the family, it should also be in good taste, with pleasant surroundings. We have seen dwellings so unskillfully planned, that it would require three or four times the number of steps in doing the work that another would, which was designed with a view to convenience. Farmers' wives, as a general rule, are over-worked, and suffer in health and spirits from the excessive labor they perform; hence they show age earlier and break down sooner than almost any other class. But to take unnecessary steps and perform labor that might just as well have been avoided is a great waste of time and strength-a loss that brings no recompense in return. Life and health are too sacred and precious to be thus wasted, and too much pains cannot be taken to secure convenience and labor-saving in planning a house.

Warmth of Dwellings. - In those sections of the country where the climate is uni. formly. warm, a protection against cold in the erection of buildings would not seem important, but it becomes a very essential consideration in this connection in the Northern States, where, during nearly half of the year, the temperature is low, and strong winds force the wintry air into every crack and crevice, thus causing the cold to seem doubly intense.

A cold house is not only exceedingly uncomfortable to live in, but is also equally unhealthy, and we believe many of the numerous cases of consumption to be found in the country towns of the Northern States might be traceable to this source. Unless the utmost care is taken in building a home to render it perfectly tight and secure, it cannot be made comfortable for the inmates in severe weather by any method of heating. We have heen in houses where in extremely cold weather the temperature of the rooms would be much too warm, while it would be impossible to keep the feet comfortably warm, on account of the cold current of air that was constantly finding entrance through the cracks of the floor or about the casing. Ventilation is a good thing for a dwelling, but health and comfort require certain prescribed methods and rules for accomplishing it, and we venture to say that a country house was never yet built so tight and warm as to render it unbealthful on this account, although they are generally unhealthful through lack of suitable ventilation. In building, the greatest care should be used to render the walls impenetrable to the cold winds.

Brick or stone houses are generally warmer than wood, as the walls are uniformly tighter, but wood houses are pleasanter, and can be rendered warm by being suitably con. structed. A sufficient amount of the proper kind of material should be used for the covering, and it should be well put on. Brick is commonly recommended for filling the spaces between the inner and outer walls, except the places required for the posts, studs, and braces. . These serve the double purpose of warmth and that of excluding the entrance of rats and mice between the walls. Old bricks are as good as new for this use. The more recent practice in architecture of adding an inner lining of felt or heavy paper in covering the walls, aids very materially in securing warmth.

All the work about the building should be well done. The walls should be nicely plas. tered, and the casings and all the wood-work well fitted. The lumber employed for the inside finishing should all of it be well seasoned, and kiln-dried before being used. Unless this precaution is taken, the wood-work will be liable to shrink and crack, making it look very badly. Very nice houses are frequently greatly marred from this cause. One of the 
luxuries found in the majority of city dwellings, and but rarely in the country, is the facility for warming the building throughout. A good furnace for this purpose costs less than steam apparatus, besides, we think it more desirable, if at the same cost, and although attended with some expense in procuring and supplying with a sufficient amount of coal for the purpose, we believe many farmers would find that the comfort thus afforded the household would amply repay for the additional outlay.

Windows.-The importance of an abundance of sunlight in a house can scarcely be over-estimated. Light is a promoter of health, comfort, and cheerfulness. Plants will not attain a healthy growth in a darkened room, neither will individuals that are deprived of the sunlight be strong and healthy. It is a well-krown fact, as has been previously stated, and the testimony of prominent physiciars that invalids occupying the sunny side of a hospital are more liable to recover than those occupying the side where the sunlight scarcely ever enters. Everyone feels more or less the depressing influcnce of a succession of cloudy days, an influence that is only dispelled when the bright sunlight again floods the earth with beauty. There is too much indifference or carelessness with respect to admitting the sunlight into our dwellings, or rather it is the common custom to carefully exclude the sunlight; and for fear of its fading the "best" carpets or the upholstering of the furniture, shades are carefully pulled down, blinds tightly closed, until scarcely a ray of light is permitted to enter. The result is that such rooms become damp, musty, and unhealthy, and were a perfectly healthy person compelled to be confined within them for a stfficient length of time they would deteriorate both in health and spirits. For ecme diseases, sun-baths have been found to be one of the most successful remedies.

Every dwelling, as well as kuïding for sheltering animals, should be constructed with a view to admitting the sunlight, and thase parts of the house most occupied by the family should be located on the sumiest side of the house. Ample provision should be made for windows, both in number and size, in planning a house. When glass was first introduced into use for windows, it was very expensive, consequently small windows were a necessity; but at the present period glass has become one of the cheapest of building materials.

Very small windows do not look well in any house, whether large or small, while windows that are too large for the size of the building look almost as badly. We think windows should be made as large as may be without being disproportionate to the size of the house, and as many of them be used as the dimensions of the house will admit, and at the same time keep within the limits of good taste. If it is found that a number of large-sized windows in a house furnish the opportunity for the cold to enter about the casings during wintry weather, this evil can be easily remedied by the use of double windows on the north and west sides of the building, or throughout during the cold weather. This might be done at a comparatively small expense, and a home once supplied, is permanently furnished in this respect. Such windows could be taken out in summer and be easily replaced for winter use; by this means, other conditions being favorable, a house can be made very warm and comfortable.

Small-sized panes look cheap, and are not in good taste; they are now seldom seen, except in very old buildings. Very large panes are more expensive than those of medium size, and if broken are not as easily replaced. For a farm-house, medium-sized panes seem most appropriate. A good quality of glass should be procured, and the sashes should be made of suitable material, well put together, and painted on both sides. The windows should be made to lower at the top, as well as raise at the bottom, for the purposes of ventilation, and rendering the rooms cooler in summer. They can also be washed easier. The best arrangement for this purpose is a cord, weight, and pulley, by which means the window may be lowered at the top or raised at the bottom at any distance, and held in place without any kind of fastening. 
Each window should be supplied with a gool patent spring fastener to keep out intruders. The best for this purpose are those that are furnished with a little spring, which lolds the catch and prerents its being pushed back from the outside by any mesns, such as running a knife-blade or thin piece of steel or iron hetween the upper and lower sash. Baywindows, properly located, add much to the exterior and interior appearance of a dwelling, and are just as much in conformity with good taste on a farm-house, as that of a village or city dwelling.

Do0rs.-The arrangement of the doors of a dwelling should be such as to secure comfort and convenience, and sare unnecessary steps in the honsehold rork. Their location should be so planned as to gire ready access to all parts of the house, and at the eame time not interfere with each other. They should also be of sufficient size. Narrow doors are a great inconvenience, and should never be tzed in any building. In moving large articles, such as furniture, from one room to another, a narrow door involves the risk of injury to both the mood-work of the house as well as the furniture, as the battered condition of many doorways fully prove. In farm-houses generally the doors are much too fow in number and too narrow.

Double sliding doors for certain rooms are a great convenience, since they admit of throwing two apartments into one when desired, furnishing a large, cool, airy room in hot weather, or ample accommodations for a large gathering of friends on social occasions. A sitting-room and parlor, or dining-room and sitting-room, can thus be easily converted into one large apartment, rendering the interior of the house more pleasant, convenient, and comfortable at certain seasons.

The most common and convenient door-fastenings are those of the combined catch and lock style, with a knob on each side of the door. For perfect security, the outside doors should be provided with a peculiar spring.lock which cannot be easily tampered with. The common lock can easily be fitted with an ordinary key, or the bolt readily forced back by other means, hence, it is not a very good protection. Strong inside bolts for the entrancedoors of a house are also a good security against intruders.

Floors. - The supports for the floors of a house should be strong and durable, and the floors of good material, well seasoned and carefully put dorn. It would not probably be of fre. quent occurrence that the strength of a floor of a farm-house would be fully tested, but there are occasions, such as social gatherings, etc., when a large company might be collected in a single apartment, and we have personal knowledge of serious accidents being occasioned by the floor giving way in old or improperly.constructed buildings, at such times. A great strain might be brought upon a floor in this way, and it is well to provide beforehand for any such contingency, by having the floors well supported in constructing a house, or, if the house is already erected, extra supports can easily and at slight expense be placed under the floor, and thus additional strength imparted.

In laying the floor, if the timber is not well seasoned, it will shrink. leaving large cracks between the boards, which will furnish a safe harbor for moths and bugs under the carpet; or, if uncarpeted, a place for the dust to collect, besides giving a room an unsightly appear. ance. In rooms that are not carpeted, a floor of alternate strips of light and dark wood looks very nicely. The boards should all be of uniform width, not exceeding four inches, although for a small room three inches is to be preferred. Black walnut and ash are the best materials, but chestnut or yellow pine is sometimes used instead of ash for this purpose. Such floors should be thorouginly oiled before being used. Finishing the interior of such rooms with the same kinds of wood is also in very good taste; for instance. having the doors of black wal. nut, and the wood.work about them of ash and black walnut, the lighter wood coming hetwcen the door and the outer casing, etc., and the light and dark alternating in the other 
portions of the wood-work. As black walnut is somewhat expensive, other wood stained to imitate that color might be used for the doors and casings at much less expense, and would answer the purpose very well; but for the floor, the walnut should be used.

Stairs. - The steep, narrow stairrays that are so commonly seen in old country houses the not only a great inconvenience, but positively dangerous, especially for children, while they are also a very difficult means of transit from one floor to another, for the older members of the household.

The stairs of a building should always be located where they will be convenient of access, since in doing the work of the household it is necessary that they should be frequently used. They should be made broad, of slow elevation, and easy of ascent in every respect. They should also be furnished with a good stout railing on the side opposite the wall. Whatever means may be used in eccnomizing room in a house, it should never be employed in respect to the stairs. Sucl a course will always be found to be very poor economy in the end. To be obliged to carry furniture or anything heavy or cumbersome up or down a steep, narrow stairway, or to be obliged to go up or down such stairs many times a day, is no easy task. It is not a matter of surprise tliat so many farmers' wives grow old so early, or become broken down in health, when we consider the vast amount of hard labor they perform, together with the cxtra labor to which they are so often obliged to submit, througl the inconvenient arrangement of the homes they occupy. Straight, broad stairs of slow elevation are the most desirable, but winding stairs economize room, and aro to be preferred to the steep, narrow ones so commonly seen in farm-houses.

Ronfs. - The style of the roof will depend mainly upon the general architectural plan of the building, althongh some styles of architecture are not as arbitrary in this respect as others, and the taste of the owner can be brought into exercise as to choice between a steep or moderately sloping roof. A flat roof is objectionable, especially in those latitudes where a large amount of snow falls. On such rnofs the snow is liable to accumulate, and requires a considerable amount of care and labor in removing during the winter. Various materials are used for coveriug roofs, wood, slate, and tin being the most common. The preparations in which tar is used are considered objectionable, since they are more liable to take fire, and conmunicate it more rapidly than others.

Slate and tin are durable and the best preservatives against fire, although there are certain preparations and paints that are very good in this respect, which are used to some extent for covering shingles. The best paints for this purpose are such as will be fire-proof and at the same tume preserve the shingles. Shingles have been in use for roof coverings for $\mathbf{a}$ long time, and are still extensively employed, although in some sections slate and tin have taken their place in a grei.t measure. The principal kinds of wood used in the manufacture of slingles are ceclar, spruce, and pine. Many others are, however, employed to a certain extent. In order to make a durable roof of shingles, they should be perfectly dry when put on. If thase containing the least amount of sap are used, the roof will soon be liable to leak, since they will warp and shrmk in the hot sun, thus furnishing abundant opportunity for the rain to find an entrance.

Tin roots are durable, but require considerable care in making, in order to obviate leakage. They should always be kept well painted to prevent rusting. Slate, when well laid, makes a very durable roof. Such a roof must first be covered with boards so tight as to prevent the entrance of rain and snow in a strong wind; besides, the rafters must be so very strong that any amount of snow that may accumulate on the roof shall not by its weight cause it to settle in the least. If it does settle, the slates, being very brittle, will break and come off. Steep roofs, on which the snow cannot lodge, are to be preferred where slate is used. 
Chimneys. - The hearth-stone, around which our forcfathers gathered, is now only known in poetry and song; and the mammoth chimney up which the bright flames leaped and roared, while the hours were beguiled with pleasant household chats or neighborly sociability. interspersed with such refreshments as corn popped in the ashes, rosy.cheeked apples, ancl sweet cider, is among the obsolete things.

Instead of the old-fashioned fire-place, we now have the steam radiator, the furnace. register, the air-tight stove, and kitchen-range, while the only suggestion that is furnished us of the cheeriness of that old time-honored institution is the open grate, at present too rarely seen, and typical of the past. Instead of one large chimney for the house, as was formerly the custom, we now have smaller ones and more of them in number. These should always be conveniently located, of small size, and well built. By such arrangement, a better draught is secured, much less stove-pipe is required, and the general appearance of the house improved, while there is also much less danger from fire, owing to less pipe being required. In build. ing, the utmost care should be taken to have the chimneys properly constructed, and the foundations substantial. Defective flues are the frequent cause of destructire fires, and only the most skilled workmen should be employed on this part of the building. The danger from falling sparks is diminished, and a better draught secured, by having the chimneys built to a good height above the roof.

Mantels. - Mantels add much to the appearance and convenience of the interior arrangement of a house. They impart a more cozy and better finished look to a room, while they are very useful for arranging the ornamental, as well as some of the more useful things pertaining to the house. Marble is, of course, the nicest material for this purpose, but it is quite expensive in some sections. Very pretty mantels may be made of wood, and, when tasteful in design and neatly finished, look quite as suitable for a farm-house as those of more expensive material.

Closets.-In building a house, we would recommend by all means that there be plenty of closet-room. Closets and cupboards can scarcely be too numerous, and only those housekeepers who have been limited in such conveniences can realize the great inconvenience caused by a lack in this respect. Places of storage for small things, such as closets, cupboards, and drawers, will always be found useful, and save many needless steps daily, in the performance of the house-work, that would otherwise have to be taken. In planning a house, there should be a closet or clothes-press in every chamber, and at least one on the lower floor for general use in hanging coats, hats, etc. Besides these, there should be a china-closet in the dining. room, with drawers underneath the shelves for the table-linen, and another closet for the storage of various things for table use. If, in completing the plan for a house, any little nooks or space can be found, aside from the closets already planned, finish them into a closet or cupboard; they will never come amiss, but will all be utilized.

. Piazzas.-Porches and piazzas add much to the attractive appearance of dwellings externally, and render them more pleasant to live in. A good piazza will make the rooms of a house that adjoin it more oool in summer by its shade, while it will be a comfortable and pleasant place in which to spend leisure hours, such as the noon time and evenings, in warm and pleasant weather. There is an objection to their being so built as to exclude the sunlight from the house too much; still, a narrow piazza is not as convenient, neither does it look as well as a wide one. To avoid this difficulty, an awning is frequently used instead of a roof for covering or extending a piazza located on the south side of a building. By this means shade may be had when needed, and. by its remoral, the sunlight admitted at other times. A veranda located on the north side of the house furnishes a most cool and comfortable place in hot weather, while the objection that might be raised respecting a southern location, of excluding the sunlight in cooler weather, would be obriated. 
Eave Troughs.-No house is complete without being supplied with eave-troughs for conducting the water from the roof. By their use, rain-water may be secured, if desired, for various purposes, such as for cisterns, or watering-troughs. In sections where the well water is hard, this is a very easy means of securing soft water for washing and other household pur. poses. Where barn-roofs are sufficiently large, cisterns may in this manner often be kept well supplied with water for watering stock.

Wherever eave-troughs are used, the water should either be condncted into cisterns, or some other place away from the house, to permit its reaching the cellar. Without them, the constant dripping from the eaves in a rain-storm washes the soil and spoils the turf close to the building, while the water is very apt to find its way into the cellar, making it very damp and unhealthy. It is rare to find a dry cellar where they are not employed. It is also very unpleasant to go in and out of a house when it rains, and have the surplus water caught by the roof come flooding down upon one, as it will from buldings that are not supplied with them.

Blinds. - These add much to the pleasant appearance of a house, both externally and internally. While they are not absolutely a necessity, still a dwelling seems bare and unfinished without them. They make the house much cooler in summer, warmer in winter; are a protection to the windows, admit of regulating the intensity of light in a room better than could be accomplished by any other means, and also aid in keeping out flies and other insects in summer.

A house seems much better furnished and more home-like with them, and they add a kind of completeness and finish to a building that is not secured in any other way. The best blinds are the cheapest in the end, and should always be procured. They should be well fitted and hung, and provided with strong and suitable fastenings to make them secure, whether open or shut.

Lightning-Rods.-A good lightning-rod, well put up, is a great protection to a building against lightning, while a poor one, or a good one that is improperly put up, is a source of danger. In procuring a rod, only the very best should be selected, and a persou who thoroughly understands the business be employed to put it up.

The size of the rod should be nearly uniform throughout, and not less than three-fourths of an inch in diameter. A larger size is to be preferred to a smaller. The number of points, and the height to which they should rise, will depend upon the size of the building. In case of a small house, a single point may be sufficient, providing it be raised sufficiently high above the roof. The usual rule to be observed in such cases is, that the point should at least be elevated half the distance to which its protection is intended to extend, or, in other words, the protection secured will be extended over the area of a circle of which twice the elevation of thə point is one-half the diameter. Thus, if the ridge of the roof be forty feet in length, the point should have an elevation of ten feet, which is one-half the radius of the circle of protection; if the ridge be ffty feet in length, the elevation of the point should be twelve and a half feet, and so on. Where there are several points on the same building, it would be safer to have them a little nearer each other than this distance, and they must always have a perfect connection with each other. The more direct the course of the rod is to the earth, the better, and acute angles by bending it in its course to the ground should be avoided.

The lower end of the rod should be placed sufficiently deep in the earth to always be in contact with moist soil. This is very essential, and if it could terminate in an underground spring of water, or beneath the surface of the water of an old well located at a sufficient distance from the house, so much the better. But it should never terminate in a cistern. This would be a serious mistake, because the water contained in it is insulated from the earth by the lining of cement. When the building is covered with a metallic roof, it should be con. 
nected with the lightning-rod, or the lightning might take the course of the pipes which con. vey the water of the eave-troughs to the ground, instead of the rod. Worthless rods have been extensively sold by unprincipled agents in different parts of the country, and hence a prejudice has been created in many instances against the use of lightning-rods; but a counterfeit article does not necessarily prove the genuine to be of no value. Many worthless rods have also been devised, and patented by persons entirely ignorant of the principles of electricity.

Prof. Joseph Henry, of the Smithsonian Institute, recommends that the rod be round, or rather cylindrical, because electricity repels itself, and tends to escape into neighboring bodies from points or sharp edges; hence, flat or twisted rods are for this reason imperfect conductors, as they tend to give off lateral sparks from the sharp edges during the passage of the discharge, which might in some cases set fire to very combustible materials.

Safest Position During a Thunder-Storm.-With respect to the safest position during a thunder-storm, especially in a house not well protected by a lightning-rod, the best locality is generally conceded to be in the middle of the room, and a horizontal, rather than a vertical position.

Windows, whether open or shut, should be avoided, also chimneys, but in a house not properly protected by rods, no place can be considered as entirely safe. Trees in the open air should also be avoided, as, the trunk of a tree being a bad conductor of the electrical fluid, the discharge will leave it and pass through the body of a man or animal that might be near it.

Tentilation.-The necessity of an abundant supply of pure air in maintaining a healthy physical condition, is too little understood, or, if understood, too commonly disregarded by the great majority of people.

Even the most highly educated classes, who are perfectly familiar with the laws which regulate sanitary conditions, are frequently careless and indifferent with respect to ventila. tion. Hence, we have dwellings, churches, public halls, school buildings, factories, steamboats, cars, etc., so constructed that it is impossible to furnish an adequate supply of pure air for those occupying them, and they consequently become places where the blood is poisoned by the inhalation of vitiated air, and various forms of disease are thereby engendered. We believe the majority of diseases to which human life is subject (and we might also add that of many of the domestic animals) are due to improper ventilation. This seems strange, when pure air is so free and abundant, and we have but to permit this gift of heaven to reach us with its life and health-giving influences. But it is nevertheless a fact that instead of admitting this necessary element, we shut it out of our houses, exclude it from our presence, and breathe in its place poisonous gases freighted with the elements of disease and death.

The majority of persons would refuse to eat food or drink water that they knew to be unclean, or to wear clothing that was soiled and untidy, and yet they will breathe over and over again air that has been rendered impure, either by its having been exhaled from their own or other persons' lungs, without the least thought of its uncleanliness, or the evils that may result.

Consumption, typhoid fever, scarlet fever, diphtheria, and many other diseases are fre. quently cassed by breathing impure air. When there is such gross carelessness and indifference with respect to these sanitary conditions, it is no wonder that such diseases are so prev. alent, and their victims so numerous.

It is stated by no less an authority than Dr. Leeds, that it is as easy to prevent consump. tion by the use of pure air, as it is to prevent drunkenness by the use of water. We have known of many cases of incipient consumption, or consumption in its first stages, as well as fevers and other diseases, having been cured by the patients being constantly supplied with 
an abundance of pure air. It is often astonishing to see how rapidly a person will recover as soon as his strength will admit of his getting out to spend a considerable time in the open air. It has been found by experiment that an adult man gives off, in breathing, from six to seventenths of a cubic foot of carbonic acid in an hour while awake, and from five to six-tenths of a cubic foot when asleep. Also that he inbales at least twenty cubic inches of air at each breath, which, allowing twenty respirations per minute, is equal to fourteen cubic feet of air passing through the lungs per bour.

The air that is expelled from the lungs in breathing contains from 4 to 5 per cent. or more of carbonic acid, and is saturated with moisture from the lungs. Besides the vapor given off by the lungs, there is also that which escapes through the pores of the skin, which is estimated to be in an adult person equal to from $\frac{1}{12}$ to $\frac{1}{6}$ of a pound per hour, which also escapes into the surrounding air. These vapors, thus escaping from the body through breathing and perspiration, contain substances which are injurious, if taken into the system again, and which are necessary to be removed at first, in order to maintain a bealthy condition. One of the functions or uses of breathing and perspiration is to remove them from the body. We can, therefore, easily perceive how soon the air of a small and perfectly tight room would become vitiated by even the presence of one individual, and also how important it is that the effete waste matter once thrown off from the system, should not be taken into it again. This can only be prevented by proper ventilation, which shall provide a sufficient supply of pure air to be inhaled, instead of that which has been thus poisoned.

In the construction of a house, one of the most important things to be considered is providing suitable means for its proper ventilation. To permit of a suitable amount of fresh air being introduced into a room, and thoroughly distributed without producing draughts upon the occupants, should be the object in planning for this purpose. Volumes might be written on the different methods that might be employed in ventilating buildings and the arguments given to maintain them, but our space will admit of only a few general suggestions with respect to the subject.

No change of air can be obtained in an apartment except when the inside air is either warmer or colder than the air outside, or in other words, except there is a difference of temperature between the indoor and outdoor air. For this reason open windows will not prove an effectual means of ventilation unless the air in the room is warmer or colder than the atmosphere without. The old-fashioned fire-place furnished to our ancestors an admirable means of ventilation, since it permitted the impure air to pass up the chimney, while pure air could be admitted by windows or doors, or what would be better, through a tube or pipe suitably arranged for the purpose of conveying it into the apartment. For this reason open grates and stoves similarly constructed furnish better facilities for ventilating a room than close, air-tight stoves. A considerable portion of warm air will, of course, also by this means escape up the chimney, but the benefits to be secured by the improved condition of air in the room will more than repay the extra expense of heating.

The windows of a house should all be so arranged that they can be lowered at the top, as well as raised at the bottom. Doors and windows should be freely opened in a house during the summer, in order to permit the pure air from without to have free circulation through the building. This cannot be well secured unless the windows or doors on opposite sides of the room be opened, thus furnishing the means for the impure air to escape and the pure air to enter. If no means are provided for the escape of the air already in the apartment the outside air cannot be admitted, for nothing can ever be more than full, and we cannot force air into a room already full. Windows should also be opened every few hours during the winter, if no other means of ventilation are provided.

The firt in every ordinary stove furnishes the means for the escape of some of the air of the room by the draught produced, while fresh air from without finds entrance from about 
the windows and doors; but this is far from being sufficient for ventilating purposes. Chim. ney-flues or other means of ventilating should be employed. Chimney-flues, in order to be effectual as ventilators, must have the air within them warmer than that in the apartment, otherwise they cease to act. In arranging for ventilation, whaterer the system practiced, two things are essential, viz., the providing of suitable inlets for the admission of fresh air, and equally capacious outlets for the escape of the impure air.

The opening for the introduction of fresh air for ventilating purposes may be either above or below the place of outlet for the foul air. It is a good plan to build a ventilatingflue close to the chimney, by which means the air within the flue will be warmed, causing a good draught. It is important that the air admitted for ventilation should always be pure. The custom that is frequently followed, of supplying the air to a furnace or other heater directly from the cellar of a house is very dangerous, since the bad air of the cellar is thus diffused throughout the house. Even cemented cellars are not an exception to this rule. The air for such purposes should be obtained from outside, and always be pure and fresh.

As a general practice, people suffer more from bad ventilation in their sleeping-rooms than elsewhere. Too much fear is entertained from breathing what is called "night air," hence, windows are closed, or only slight openings made for ventilating purposes, and the air of the room breathed over and over again, and the blood poisoned by the process. During the day there is no air for us to breathe but day air, and there is just as certainly no other air for us to breathe during the night but "night air," consequently there is no other alternative for us but either to cease breathing at all during the night or breathe "night air." We must therefore take that which is ahready in the house, and to a greater or less degree impure, or that which is pure from without; and how much better to have the pure air from without.

The air of a sleeping-room should be just as pure in the morning, after the room has been occupied all night, as the outdoor air, and yet how few sleeping-apartments could be found under such sanitary conditions. Some writer has called sleeping-apartments, as generally managed, "charnel-houses," suitable only to die in, which, unfortunately, is quite true in a majority of cases.

The farmer and lis sons, being out in the field during the day, suffer less from poor ven. tilation than the wife and daughters, who are confined more within doors. If farmers geuerally, either in constructing new dwellings or repairing old ones, would pay more attention to securing the means of proper ventilation, and the best use of such facilities were employed by their families, there would be less need of ductors and their potions, and the figures of the mortuary records of the country would be greatly reduced.

Cellars.-A dry, well-ventilated cellar is essential to the best sanitary condition of a dwelling. In selecting a site for a farm-house, therefore, dry land on a slight elevation, which will admit of suitable drainage, should be chosen, if practicable. But there are many sections where the land is wet, and unless some means are employed for under-draining it, the cellars will either contain standing water a large portion of the time, or be dripping with moisture. Such cellars are very unhealthy, and should never be permitted to remain in such a condition while the buildings above them are used as habitations. They are also not fit for the storage of family supplies.

Even dry soils are damp after heavy rain-storms, consequently some means of drainage should be provided in every cellar. But where the land is naturally wet or retentive of moisture, a thorough under-draining is highly essential. This may be accomplished by laying drain-tile a foot or more below the bottom of the cellar, both inside and outside the cellarwalls, which shall go around the cellar, thus securing a drainage on every side. These tiles should communicate with a receiving-drain, which shall take the water quite a distance from the premises. The bottom of the cellar should then be covered with stones or brick (stones 
to be preferred when they can be obtained), and well cemented. Boards should never be used for flooring a cellar, as they soon become damp and moldy, making such houses very unhealthy to live in. Mr. Garduer, in his treatise on Farm Architecture, expresses the following opinion on this subject:-

"By careful draining, it is possible to make a soil naturally wet fit to live upon.

Where any doubt exists, the entire site should be thoroughly under-drained. The foundation-walls should be solid; that is, laid in cement and mortar.

An enterprising rat with a large family on his hands will destroy more in a single winter than the whole extra cost of the mortar.

'Pointing ' the face will not answer; it will stop 'nearly all' the holes, but add nothing whatever to the strength of the masonry.

There are several good reasons why the first or principal floor of a house should not be too high up in the world. From the picturesque stand-point the lowly estate is decidedly preferable, especially as the underpinning is usually treated. But other reasous for keeping the living-rooms well above the surface of the ground are too important to be disregarded.

A free circulation of air and plenty of light underneath the first floor are indispensable to the best sanitary condition. These can be most easily secured when at least half the cellar or basement story is above the ground.

The porch and the main entrance-hall may perhaps be upon a lower level.

For warmth and dryness, the cellar-wall above the ground, commonly called the underpinning, should be hollow-two thin walls of stone or brick, or one of each."

With respect to improving cellars of old buildings that are dark, damp, and moldy, the same writers says:-

"Dig a trench around outside nearly to the bottom of the wall, or at least until the stratum of earth is reached that holds the water, and girdle the foundation with a drain of horseshoe-tiles, having one or more free outlets. Refill the trench with sand, gravel, or cinders, and cover the top with several inches in depth of clay and loam pitching sharply away from the house, and lay a shallow, open gutter of concrete or cobble-stones to catch the water from the roof, if there are no eave-spouts.

If the cellar is not deep enough for the modern furnace or steam-heater, and the walls do not extend below the cellar-bottom, build a new wall of bricks or stones two or three feet inside of the old, and below the cellar-bottom, leaving a sort of platform for bins, barrels, and boxes around the edges, and dig the rest two feet deeper. By this means the old foundations are not disturbed, and the whole can be done in cold or wet weather.

When the old house rests so closely upon the earth that no sunlight enters the cellar through the narrow windows, and the cellar cannot be raised without great expense, nor the earth be removed around it, then build semicircular areas of bricks about the windows, and make the windows themselves large enough to admit plenty of fresh air and sunlight under the house. Darkness is the first station on the road that leads to dampness, decay, disease, and death. This is true of the new house, as well as of the old.'

The present custom of constructing cellars smaller than the area covered. by the house, and also of placing the building higher up from the ground, is a great improvement upon the old-time method of extending large, deep cellars underneath the entire building, and setting the house so low that it was nearly on a level with the soil. Very large cellars, which are unnecessary, are thus prevented from becoming the storage of waste materials, which, by a slow process of decay, would render the buildings above them exceedingly unhealthy ; but by being elevated higher from the ground, more light and better ventilation are secured. The cellar should, however, be of the same dimensions as the house in one direction for the purpose of securing a good ventilation.

It is a good plan to remove the turf from that portion of the earth which is to be covered 
by the building where the cellar does not extend, fll in to the depth of several inches with gravel, afterwards covering with cement. This prevents, in a great measure, the gases that arise from the earth from making their way into the building. Arrangements should also be made in the underpinning, in such places, for permitting a free circulation of air, by leaving spaces or openings, or the unhealthy gases that are carried underneath the building will surely find an entrance. The timbers will also soon be affected with dry rot, if such places are not properly ventilated.

The cellar-walls should be well cemented, and the ceiling lathed and plastered. This latter will render the rooms above warmer in cold weather, and the floor less liable to admit the gases from the cellar, while a well-built and carefully-cemented wall will exclude rats, mice, and other vermin.

There should be several windows in every cellar for the purpose of furnishing light, and a free circulation of air. Additional ventilation can also be secured by means of an aperture comnected with the chimney. A wire netting should be placed over the windows to exclude rats, mice, and insects when the windows are opened. There should be broad, well-lighted stairs leading from the kitchen, or near it, to the cellar, also stairs leading from the cellar out of the building.

Darkness and dampness should be excluded from cellars, and they should also be kept free from any decaying substance, such as rotten wood, decaying vegetables, \&c. It is not well to use the home-cellar as a place of storage for very large quantities of root crops, such as for feeding stock. It renders a house unhealthy. A barn, or root-cellar should be used for this purpose.

Water-Closets and Vaults.-When properly constructed, water-closets are the most coinplete arrangements for their intended purpose that can be found. But few farm-houses are, however, so arranged as to admit of their use. In such as have the facilities, they are not only a great convenience, but also conduce to sanitary measures as well. They should always be furnished with a good supply of water. When a tank is used for supplying water for this purpose, it should be large enough to hold from serenty-five to one hundred gallons, and should be, at least, from seven to eight feet from the floor.

ln order to be arranged, on good sanitary principles, water-closets should always be supplied with strong lead traps underneath, which shall prevent the escape of gases. These should run into large cast-iron pipes which should communicate with the serer. Unless the utmost care is used in providing a sufficient number of suitable traps, such closets become the source of serious annoyance, and unsanitary conditions in a house, boing the means of conducting the poisonous gases from the sewage directly into the building. In constructing them the most competent workmen, those perfectly familiar with their business, should be employed, and the work thoroughly performed.

It is easy to have it well done at first, but generally a difficult and expensive task to have any mistakes corrected, or repairs made afterward. Care should be used to prevent the water in the pipes from freezing in the winter.

Earth clasets are also used to a considerable extent in many localities. These are most of them provided with an automatic arrangement for throwing down a quantity of sifted earth. But on the farm, and in connection with farm buildings, such conveniences are very rarely seen; the most indifierent arrangements for the family use in this respect being unfortunately commonly provided.

As a general rule, such conveniences in the country consist of an old dilapidated build. ing, located at a distance from the house, and in an exposed locality, so that it cannot be reached in stormy weather, or at any time without exposure to health or observation.

No means are provided for deodorizing, lence such places are a source from which poison to the air and water in their vicinity emanates, thus becoming the means of sowing the seeds of disease and death. 


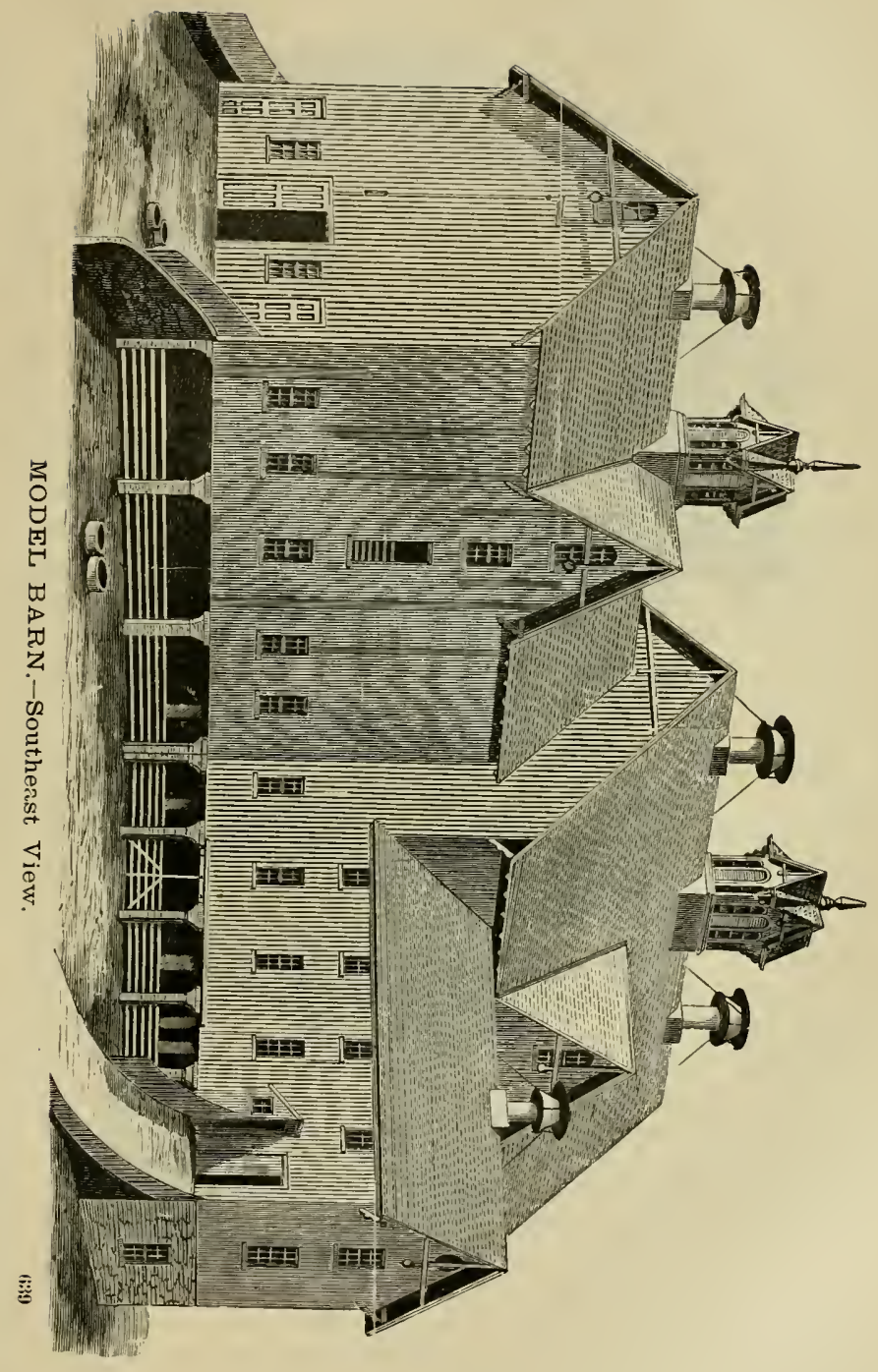




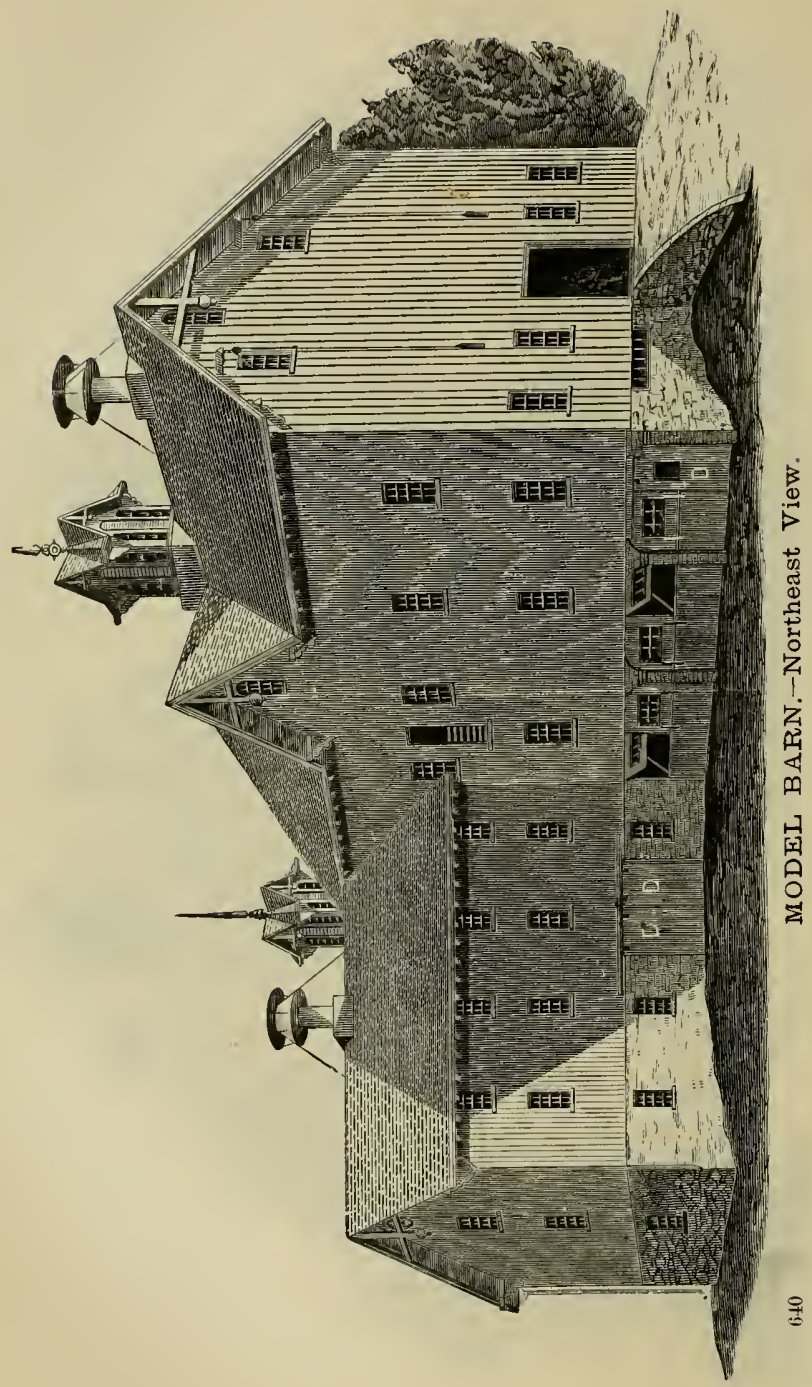


An immense amount of evil is wrought, year after year, simply through indifference and carelessness in this respect on the part of those having charge of the farm management. Typhoid fever, diphtheria, and the many diseases and ills arising from blood-poisoning are often directly traceable to this source. Ignorant of the origin of the evil, no effort is made to remedy it by the family, but that only resort, the doctor, is usually sent for, who may himself be equally ignorant of sanitary laws, or, if not, may perhaps be too ambitious to secure a patient to point ont the real difficulty; consequently pills and powders are administered until the unfortunate victim either dies or eventually recovers, owing to his good fortune in possessing a constitution sufficiently strong to counteract all the unfavorable eonditions. In. telligent and honest physicians, of which there are many, will investigate in order to ascertain the real and true cause of the evil in such cases, and then point them out, and advise the remedy.

Those who drink water that has been contaminated or poisoned in any way cannot fail of being injured by it, although there may be cases, and probably are, where persons inay live to old age, and continue to use such water without any apparent injury. There are exceptions to every rule. There are persons who may be exposed to the small-pox, or other dangerous and contagious diseases, who may chance to escape taking the infection, but such cases are very rare, and are the exception, rather than the rule. Because one person in a hundred might do it, is no reason why the other ninety-nine should run the risk of exposure.

It is the positive testimony of the best medical authorities, that many deaths, and a large percentage of sickness, are caused by unsanitary conditions. When vaults are located near wells, the soil may for a time prevent the evil, by filtering the drainage from such sources; but after the soil has itself become saturated with the poison to a great extent, it cannot purify the water passing through it, hence is the means of conducting it directly into wells that may be located near. By a little care and skill, the evils attending the use of such closets can be avoided.

They should be so located as to be accessible without going out of doors, if practicable, also hidden from the road. If this is not possible, a high tight fence, thick hedge of evergreens, or grape-vine arbor should be made a shield for the walk, while a rustic frame of lattice-work would screen the entrance, over which some kind of climbling vine might be trained. Such unsightly places can thus easily be rendered other than a blemish to the prem. ises at a slight expense, and but little care and labor.

The best vaults are those that are cemented at the bottom and sides. A vault should be closed by a door made of heavy plank, and so hung on hinges that it can be readily opened and hooked up out of the way when it is being cleaned out. Gas-tar, or a similar substance, should be used for coating the inside of the door, while the outside should be painted, by which means it is rendered more impervious to moisture. A little dry road dust, muck, or coal ashes thrown in every two or three times a day, will prove a good deodorizer and absorbent.

A barrel or cask containing this material might stand in the closet, and when farther provided with a long-handled dipper or a small shovel for the purpose, such material could be conveniently thrown into the vanlt, which would certainly be a cheap and easy way of rendering it inoffensive, and of avoiding the evils that now so commonly attend such places. In this way the liquids are absorbed and prevented from filtering into the soil, while the entire contents are deodorized. No soap-suds or other slops should ever be turned into the vaut, but it should be kept as dry as possible, with the absorbents nsed.

The Barn. - As commonly appropriated, farm barns are used for the protection of stock against inclement weather, the storage of their food, the manufacture and preservation of fertilizing materials, and the storage of farm machinery. Aside from these considerations, the convenience in performing the barn work should also be taken into account in construct. 
ing a barn. On very large farms necessitating the extensive use of farm implements, a separate building is sometimes required for their storage.

A good barn is one of the great essentials on a farm. In newly-settled sections, they are sometimes dispensed with for a time, until the land can be put under cultivation, and the owner is able to erect the necessary farm buildings. This is frequently the case in the far West, but even under such circumstances the most thrifty and enterprising farmers will not be long without a barn, and those which are the most enterprising and prosperous will gener. ally erect the best farm buildings and keep them in the best repair. As a general rule, the barns of the W'estern and Southern portions of the country are not equal in architectural structure and convenience to those of New England and the Middle States, although many portions of the older-settled sections of the West are fully equal in this respect to either of the latter mentioned. Next to a good farm house, a good barn is essential, and no farmer can afford to be without one which should be of sufficient size for all the purposes to which it is to be appropriated.

While large barns are more expensive than small ones, and a surplus of room in this respect is therefore a lack of economy, yet it more frequently happens that harns are too small, rather than too large, and the owners are obliged to be subjected to great inconrenience for this reason, or be at the expense of building others, or enlarging the original. A large number of small buildings on a farm are a blemish and an unnecessary expense, and it is better in every respect for the farmer to build one barn of sufficient dimensions for all the practical uses on the farm, than to be obliged to build two or three small ones.

The size of the barn must, of course, be proportionate to the size and productiveness of the farm, and the number of animals to be furnished comfortable quarters. Even in latitudes that do not essentially require the housing of stock during a portion of the year, animals that are kept stabled a part of the time are more valuable, as they have better care, and are more gentle and therefore easily managed, while they can also be fed with less waste of material, and the fertilizers they produce can all be saved with little care, which is no small consideration when we take into account the value of well-composted manure to the farmer. In those latitudes in which the ground is covered with snow a portion of the year, the barn should be large enough to accommodate all the stock on the farm, and their fodder. Animals that are not protected from the cold require more food than those that are, since much of the food which they consume goes torrards the production of animal heat, and unless enough food is given them to satisfy their hunger, this extra demand reduces the supply for repairing the waste of the system; consequently such animals will not only consume larger quantities of food, but will grow thin in flesh and present an emaciated condition in the spring. Young horses and cattle are frequently stunted in their growth by this means.

On the other hand, stock that are provided with warm, comfortable quarters, will consume less food, and be kept in a thriving condition during the winter. Diseases are also more frequently prevented, and more easily cured under such conditions. Cors that are kept in warm stables will give more milk and of better quality than those that are not comfortably housed. This truth is so apparent, that it requires no argument to substantiate it. It is therefore a practice of economy, as well as humanity, for the farmer to furnish food and good shelter for his stock.

There ensilage is used extensively for feeding animals, less room will be required in the barn for the storage of hay. Hay may be stacked in the field, thus rendering less room necessary in the barn; but hay that is thus exposed to the weather, is greatly inferior to that which is stored; besides, stacking involves much waste. A great advantage in this respect will be found in baling hay, as it will then occupy much less room than otherwise. In building a barn the farmer should hare as definite an idea of its use, and the necessities for its convenience, as in building a house, and in many respects what will apply to the one will, with slight modifications, apply to the other. 
In a large portion of the country it will be necessary to build barns in a manner to secure warmth; hence, they must be tightly covered and the floors well laid, that the cold air may not blow in upon the animals. At the same time, good ventilation must be maintained. While warmth is essential in a barn for the comfort and thrift of the animals, it is better that the barn be cold, and good ventilation secured by air blowing through the cracks and about windows, rather than that the animals be made to constantly breathe the offensive and tainted atmosphere of an ill-ventilated building. Many of the diseases to which cattle and horses, as well as other domestic animals, are subject, are due to overcrowding and the breatling of the poisoned atmosphere of badly-ventilated stables. Cattle kept in such enclosures cannot be healthy, and are totally unfit to become food for mankind.

Location of Barus. - The barn should be located at a convenient distance from the farm-house, but sufficiently removed to prevent all contamination of air and water. It should never be placed upon ground higher than the house, in such a manner that the drainage from it, either on the surface or in the soil, will be able to reach the cellar, well, or the surroundings. Unless on a level, with the house, the barn should be placed on a lower level, if practicable. The location should also be so chosen that the drainage from the barn-yard shall flow upon the farm lands, that they may receive and absorb all the fertilizing elements that may be washed from the yards in heavy storms, etc. We have frequently seen barns built upon a road side, with a slope towards the highway into which all the wash from the yard is carried, year after year. By this means much of the fertility that might be derived from this source is lost to the farm. This may seem a small matter to those farmers who till the prairie soil, possessing such a wealth of fertility that it requires no fertilizer in addition, for the production of vast crops; but to the farmer whose soil is of such quality that it necessitates such a large supply of plant-food applied every year to render it productive, that it is difficult to preserve, or secure the requisite amount, it means considerable.

Other important considerations are involved in the location of a barn, such as the health and comfort of the animals to be stabled in it; consequently dry land should be chosen, and damp localities avoided; also cold, bleak sites, or those that are inconvenient of access in hauling loads to and from it. The old-fashioned custom of building a house on one side of the highway and the barn on the other, nearly opposite, should also be regarded as obsolete, and a better one substituted in its place. Frequently a locality will be found admitting of a basement partly under ground, which will furnish the best facilities for a root-cellar, as well as for other purposes.

Plans for Barns. - We shall not attempt to furnish plans that will meet in all respects the wants of the builder, as this would be an impossibility, since the ideas, wants, and tastes of each individual farmer differs so materially in this respect; but rather to give a few general plans that may sorve as suggestions and hints to the farmer in better determining his own wants, and in carrying out such ideas in practice as shall subserve to fully meet those wants in the most convenient and economic manner. While some of the plans of model barns that have been inserted may prove quite too expensive for the ordinary farmer to follow in detail, they may serve to suggest in certain respects what might be done on a smaller scale, and but little or no more expense involved, than in the construction of many of the inconvenient, uncomfortable, and uncouth structures called barns, that we so frequently see.

Utility is one of the main considerations in constructing a building, and those are the best that are best adapted to the purposes for which they are to be appropriated, whether they cost much or little. Expense does not necessarily imply utility. We have seen barns costing several thousand dollars that would not afford room enough for more than half a dozen cows and three or four horses. These barns were highly ornamental in finish, with 


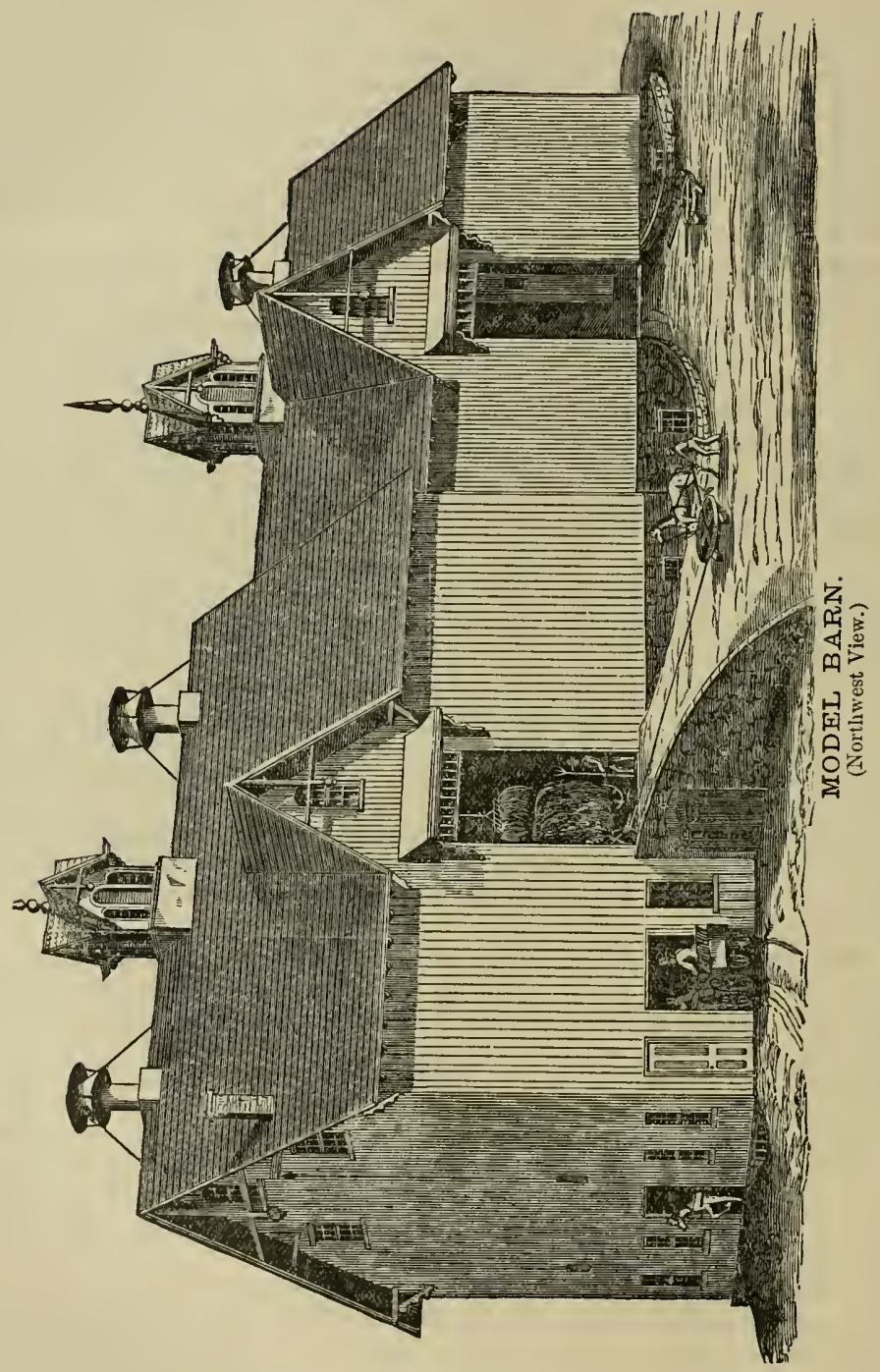


artistic gables, cupolas, etc., and with an abundance of room for the non-essentials, but with little room for the special purposes for which the barn was to be used. Ornamentation is desirable to a certain extent, but it should be of minor consideration when compared with subserving in the best manner the purposes of a building.

On the other hand, we know of barns very convenient in every respect, with ample room for the stabling of a large number of farm animals and the storage of fodder and roots, costing but a small portion of the former structure referrea to, but which, for utility and convenience, was worth more than ten times the former. Good judgment and skill are bighly essential in planning a barn that will subserve the best purpose. The old system of placing small buildings in a hollow square, has been gradually succeeded by that of placing as much as practicable under one roof. This is not only more economic but more convenient. The advantages thus gained are, greater height or depth of bays under the roof for the storage of hay or other fodder, increase of storage, economy in constructing, and economy in labor by having the animals and feed so nearly arranged together. Greater warmth in winter is also secured in this manner. One good, large barn conveniently-arranged, is far more convenient, besides being much more economical in labor and money, than two or three small ones, the combined capacity of which might exceed somewhat that of the former.

The three accompanying plates are representations of different views of a barn located in Connecticut, and which in many respects might very properly be termed a nodel barn, with reference to the purposes for which it was designed, although subject to improvements, perhaps, in some of the minor details. This barn is adapted to all sections-east, west, or south, and shows how space may be economized in planning a building for the various purposes to which a barn may be appropriated; also how the health and comfort of stock should be considered. It is probably a larger and more pretentious structure than many farmers would require; however, it may furnish a model for a smaller and less expensive building, or may suggest improvements upon other plans. The barn stands upon a side-hill which slopes to the east. It has three distinct floors, the structure consisting of a main building and two wings. The main building is 55 by 80 feet; the east wing, 56 feet long and $31 \frac{1}{2}$ feet wicle; the sonth wing, 56 feet long and 35 feet wide,--the total length from north to south being 136 feet.

In constructing any building, it is well to see many models and study various plans, and then select the best they contain for the desired purpose, and combine them into one, unless a better combination can be originated.

The southeast view of this building shows the cattle-yards, both wings, the cellar, etc. Each yard is supplied with iron tanks, which are kept filled with constantly-flowing water for the stock.

The fence and gates which shut off the cellar from the yard are movable, the posts at either end being stepped into sockets. By this means, they may be quickly and easily removed, and the cellar and yard thrown together, thus giving the cattle while in the yard shelter from the eold in winter and heat in summer. Any portion of the cellar may in the same manner be fenced off or opened into the yard.

The northeast view shows the east wing and the cellar or basement wall, with the doors and windows connecting with the pig-pen, etc. The doors (D) are suspended on rollers upon which they slide. The windows are suspended on hinges from the top, and swing open inside.

The northwest side of this building contains the principal embankment, which furnishes facilities for reaching the second floor, with room for power for raising and carrying hay and other feed; also, the horse-stable and entrances to two floors. The drive-ways to the hay or storage floors rise gradually to the required height, and are walled up by substantial masonry. The hay and grain lofts are furnished throughout with facilities for raising and carrying loads, there being no less than six railways for the travelers carrying the forks to run upon. 
The following represents the ground plan of the same building. The heavy black lines indicate the stone wall which supports, in part, the bank of earth on the up.hill side. At the ends, where the cellar-floor is about on a level with the surface, the wall is laid two and a half feet lower. Under the outer edge of the walls the drains are laid with a grouting of stones and cement over them, which prevent any undesirable effects of frost.

The entire floor is grouted three inches deep with stones, covered with gravel, which has a coating of cement.

A root-cellar 18 by 50 feet on this floor affords storage for about 6,400 bushels of roots, secure from frost, yet sufficiently cool to preserve them well. The arrangements are such that the roots may be put in by chutes. This cellar may be sub-divided into three or more bins, as desired.

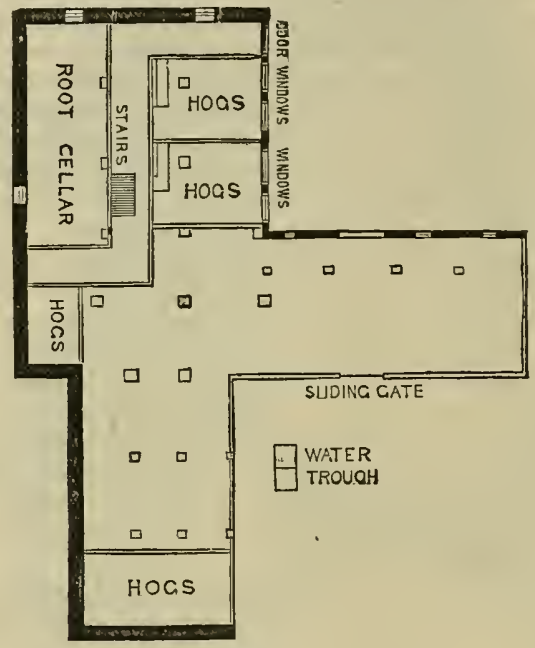

GROUND PLAN.

The following diagram represents the plan of the stock or feeding-floor and yards. On the right of the western entrance of the building is a carriage-room with capacious sliding doors. On the left is a harness-room, in which is a stove for making it comfortable in cold weather. Directly in front are the horse-stalls, and space where horses may be cleaned, and horses harnessed to vehicles may be tied.

There is a clear passage from the north to the south end, through the building, the stairs being so arranged that they may be lifted and fastened up out of the way.

The wings are occupied by cattle-stalls, those in the south wing being wide, and calculated for fattening oxen; those in the east being intended for cows and young stock. There are also three loose boxes on this floor, - one of which is 12 feet square, and two somewhat smaller, - which may be used for horses, or as lying-in stalls for cows.

In the rear of the cattle-stalls, a double line indicates the channel for collecting liquid manure. The points marked $\mathrm{C}$ are openings through which the manure is dropped into cellars. The letter $\mathrm{W}$ shows the location of the water-trough, and $\mathrm{F}$ the trough for mixing the feed. D represents a door through which the cattle have access to the yard. Under the drive-way is an arched rault, which is well lighted, and fire-proof, which forms a very convenient boiler and engine-room, with space for the storage of fuel. 
The steam-pipes for cooking the feed for the stock pass through holes in the wall upon the feeding floor. Steam power is employed for threshing, sarving, fodder-curting, etc. the waste steam being used for steaming hay, roots, etc., the engine-room being contiguous to, and below the threshing floor.

The storage-floor contains the hay, grain, straw, and stalks. Two threshing-floors, 16 feet wide, cross the building: being entered from the west. Here are hay-scales, and hay. cutters. Each grain and meal-bin communicates by a chute with the feeding-floor, where its contents may be drawn off. From this floor there are stairs that ascend to the cupola.

The stables for stock are airy and roomy. The horse-stalls are ten feet from front to rear, a little more than 5 feet wide, and 9 feet and 4 inches high. They are separated by plank partitions $4 \frac{1}{2}$ feet high, surmounted by wire netting, which extends 2 feet higher. The same kind of partition also forms the front of the stalls.

An iron hay-rack is located in one corner, and an iron feed-box in the opposite corner, which is accessible from the passage-way in front of the stalls, by a small door in the wire netting.

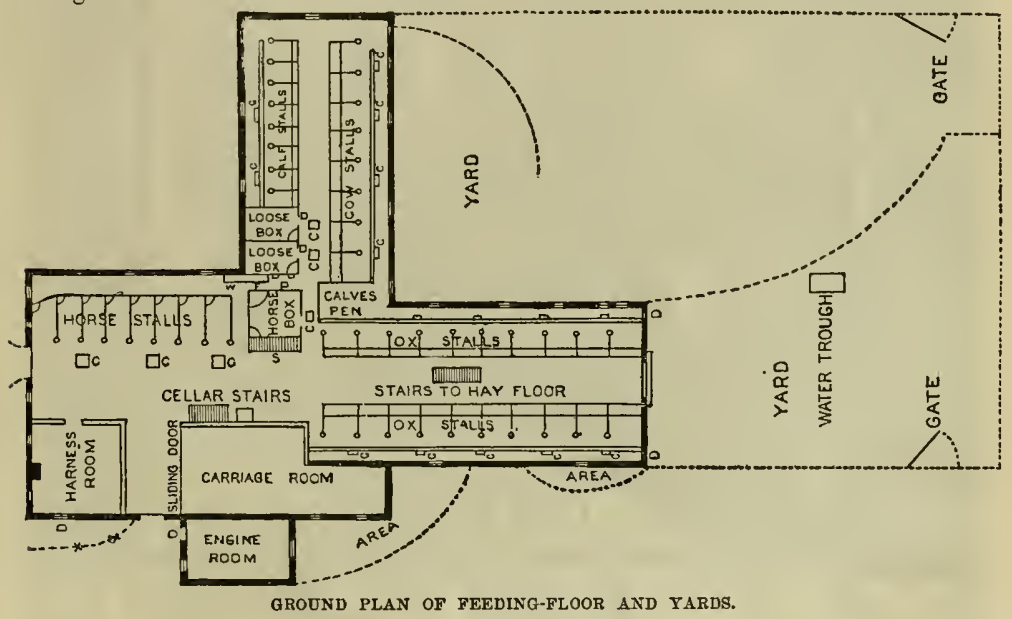

The stalls contain two floors, the lower one being laid of two-inch chestnut plank, with cleats half an inch thick covering the cracks between the planks. Upon this watertight floor is another made of three parts ; two feet of the upper end is made of white oak plank nailed fast. The remainder of the floor is formed of narrow oak plank, fastened to. gether by strong oak cleats in such a manner as to form what is similar to two doors hinged at either side, so as to be lifted and set up as shown in the cut. This arrangement is designed for the more perfect cleansing of the lower floor. A channel at the rear carries off the liquid manure, and the solid manure is thrown into the cellar, through the trap-door seen open in the illustration of the horse-stalls, and indicated by $\mathrm{C}$ in the diagram of the feedingfloors.

Between the cattle-stalls, in the south wing, is a passage-way ten feet wide, through which carts with green food, roots, \&c., may be driven. This arrangement favors a system of soiling in summer when desired. There is also a similar passage-way through the east wing, all the cattle-stalls being made upon the same principle, although of different sizes, being 
designed for fattening cattle, milch cows, and joung stock. The feeding-boxes are $2 \frac{1}{2}$ feet wide, the floors $5 \frac{1}{2}$ feet from the feeding-trough to the gutter (which is 14 inches wide), and the rear passage is 3 fect, making about 12 feet in all for the stalls.

These stalls are $6 \frac{1}{2}$ feet wide, and designed for two animals, which are fastened by a neck-strap or chain, which is attached to a short chain and ring playing up and down upon a rod bolted to the partition between the stalls, thus permitting a free movement of the head.

The rack in front of the manger is perpendicular. A shutter is hinged below it which, when open, is held in an inclined position by a chain. By this means space is given for hay boing passed between the shutter and rack.

This building is well lighted and ventilated. Ventilating trunks, four feet square, exterd from the feeding:floor to the roof, where they each terminate in a large ventilator. The

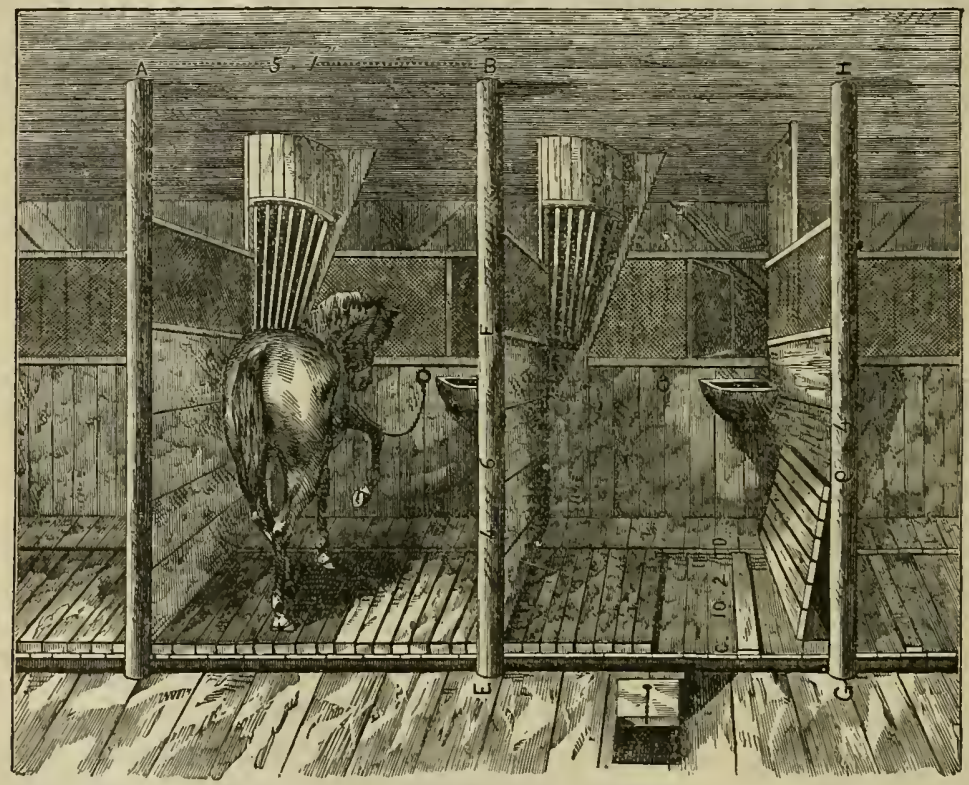

HORSE-STALLS.

windows on the stock floor are numerous, and are each provided with two glazed sashes hung by weights, so that they may be opened and hold in position as desired, making it very cool and airy in hot weather. The yards extend to the south and east.

The water from the eaves is all conducted off, so that none comes into the yard. Thie water for the stock is supplied from an unfailing source, and is brought in pipes to the yards and stock department of the barn.

Whether a barn be large, or small, much may be gained by way of convenience, and the economy of room and expense, by having a good plan for its construction.

A recent writer gives his method of building a bank-barn as follows: "I would say first, that 36 or 40 fcet is ordinarly as wide as it is practicable to build a barn. Additional room can be made up in length, if desired. If there is much of a bank, more dopth 
of basement might be desired, which would be good economy up to 10 or 12 feet, if it is found that the grade will permit. This will allow better ventilation, more security from frost, and room to allow 2 or 3 feet of manure to accumulate under foot, if desired. It will keep in good condition if trodden down as made, but will not freeze to hinder its being drawn out at any time. After digging the cellar, and it is ready for the wall, dig a trench as broad as the wall is to be, and 18 inches deep. Fill this with small, loose, broken stones. Boulders broken up somewhat as they are put in are best, but chips from the stone-yard will do. Fill the trench even full, and on this commence the wall. In case a drain is needed, it should go from the bottom of this trench, but the principal use of this trench is to make the cellar rat-proof. The idea is that rats, after digging down to the bottom of the solid wall,

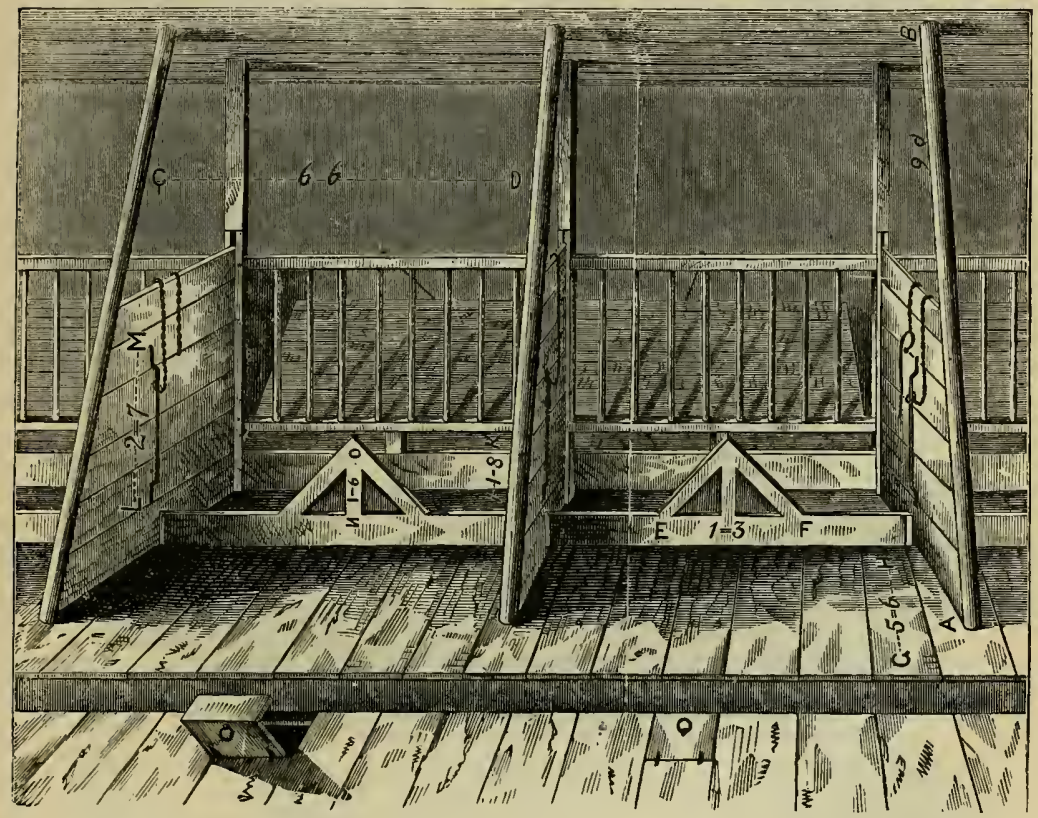

COW STALLS.

find they cannot then get under it through the broken stone, and so abandon it. In practice this has proved effectual. Have the cross sills so heavy and strong as not to require many supports, which would obstruct the free driving of teams around in the basement.

In getting the frame timber, much care should be used to get very stiff, strong beams for the gable ends of the barn, as in barns of such breadth there is great danger of the ends bulging out too much after the bays are filled. The best timbers for the girts are obtained by taking larch or tamarack, from 8 to 10 inches through, and having them sawed through the middle at the saw-mill. Shave off the bark, and use the straight side to nail the siding to. The rounding side next to the mow makes it better and stiffer than common square timber. For sleepers, take elm logs of the proper length, and 12 to 18 inches in size, sawed like the girts, and always peel before using. 
This is the most practical and economical way of furnishing timbers for this purpose. I would advise to have the roof steep; it gives many feet more space without much more cost; is not so apt to leak, and will last better, because it dries off quicker after a rain. Instead of ridge-boards at the top, I would always use tin. Use a strip 10 to 12 or 14 inches wide, made as long as the barn, put in a roll; then, after the barn is shingled and ready, nail the tin as a cap over the ridge. It will answer a much better purpose than boards.

The use of well-seasoned lumber is one of the most important t?ings about building, and too important to neglect, even in making a barn. When building a barn a few years ago, 36 by 100 feet, and 24 -foot posts, I selected old, well-seasoned, sized lumber, 1 by 12 inches, and had it dressed to order. The thickness was so good that I had it dressed a full inch, instead of seren-eighths, as it always is in market, and the width overran nearly enough to make up the waste in matching, so that 37 boards would cover the 36 -foot end of the barn.

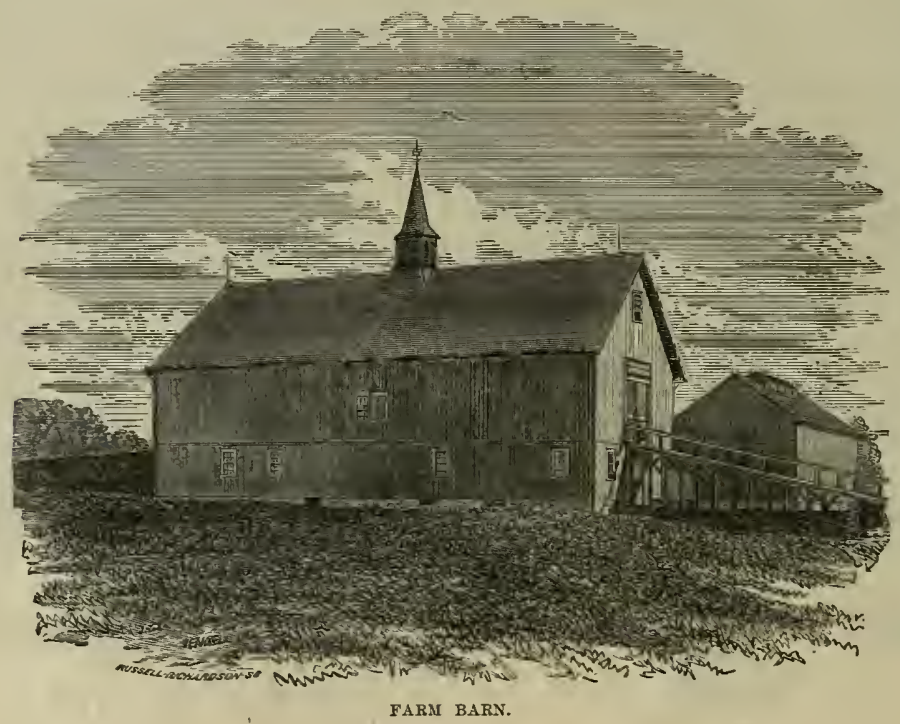

These wide boards have not warped, and their joints have not opened as much, as siding of half its width often does.

The arrangements about such a barn will not be complete without a good cistern, unless it has a better natural water supply than one barn in twenty can hare. To insure a good supply of water, build a large cistern in the bank on the upper side of the barn, and have a pipe go from near the bottom of the cistern through into the basement of the barn, so that water can be drawn by a faucet. Then a pump over the cistern will furnish water for use above. This I have found to give an almost unfailing supply."

It has been estimated by those who have given the subject of economical farm buildings considerable attention, that a basis upon which the lowest possible cost of building a barn mav be calculated is alout $\$ 10$ per head for each horse or cow that it will accommodate with comfortable quarters. and about the same per ton for the safe storage of all crops grown upon the farm. This will, of course, be subject to variation, and admit of considerable 
latitude, but may answer as a basis upon which to calculate the lowest expense of building a cheap and convenient barn suited for common farm purposes.

The expense of building-material varying, as it does, in different localities and at difier-

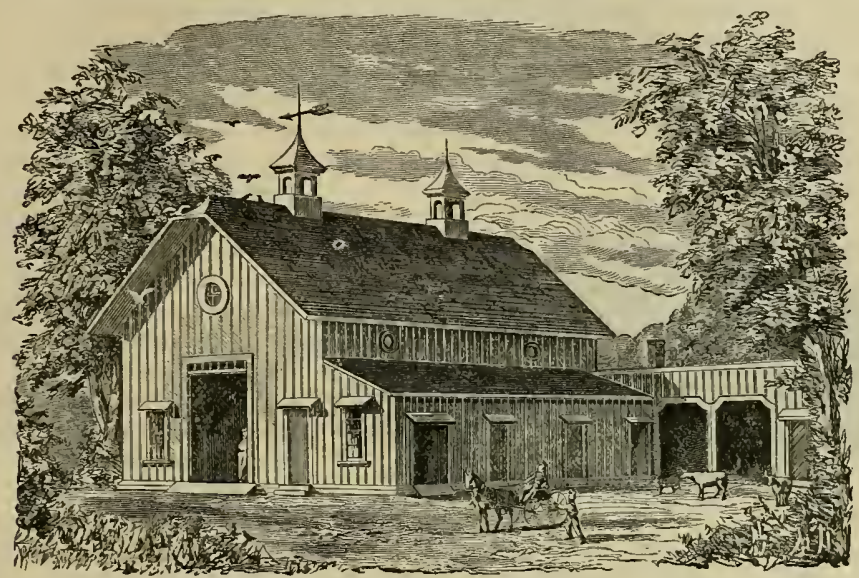

DAIRY BARN.

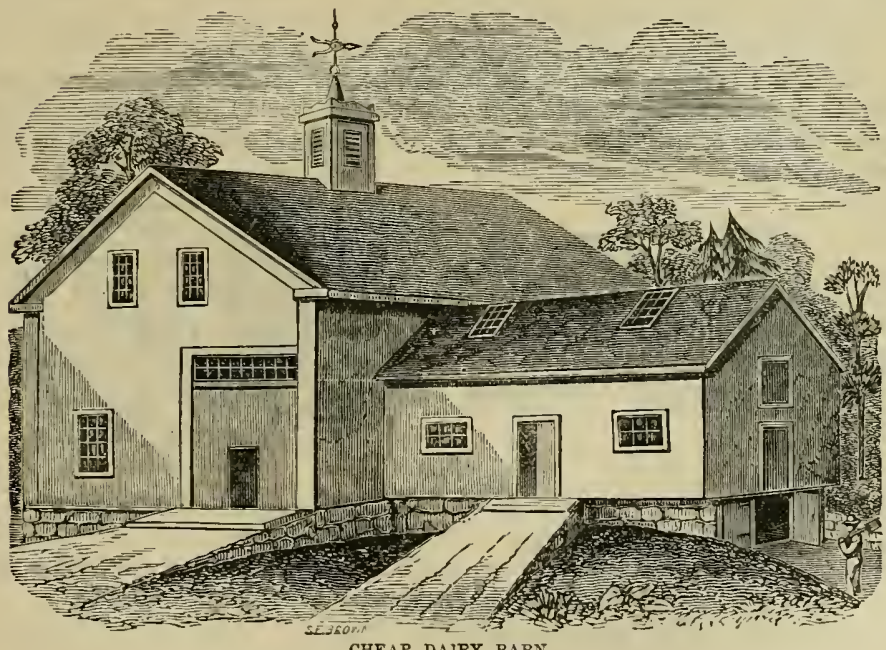

CHEAP DAIRY BARY.

ent periods, as well as that of labor, only an approximate estimate can be reached. It is necessary to have a wide and solid stone foundation in building a barn, and especially so at the lower side when a bank-barn is built, in order that the sills may lie solid and firm.

Barns and sheds should be well raised on substantial underpinnings, in order to prevent 
the rotting of the sills, and admit of the escape of moisture underneath, which would cause the timbers to decay, and also render the stables damp and unhealthy for stock.

While a barn that is externally unattractive may be made internally comfortable for the stock that it shelters, and also to furnish good storage for crops, still there are many reasons why the farmer should endeavor to give his barns and other farm buildings a neat and attractive appearance. A symmetrical barn that is an ornament to the premises will go far towards making the surroundings of a home attractive. It also indicates enterprise and good taste in the owner, and will exert an influence for good on the younger members of the household, causing farm.life and home to be more attractive to them; besides, the same enter.

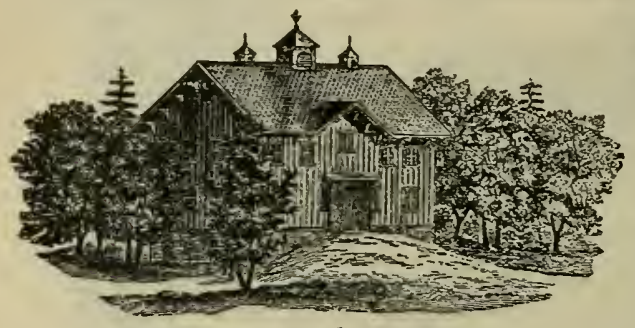

FIG. 1. prising taste will be very likely to be exhibited on all the other portions of the farm, and better fences and improved farming will be the result.

All farm buildings should be constructed in such good taste that they will harmonize with the surrounding landscape, and not prove a blemish to it. A tasty barn need not necessarily be an expensive one. A symmetrical and attractive form, though covered with rough boards, will be far more pleasing and attractive than the most highly-finished building with a disproportioned and awkward outline. Rough, unplaned boards may be made to look very well by applying first a heavy coat of crude petroleum, and afterwards a coat of some of the many kinds of paint that will adhere well. Shade trees around a barn add to the appearance of the surroundings, and will furnish shade to the cattle in the yards during the summer, but they should not be so near the building as to shade it and intercept the sunlight. All buildings should have the sunlight admitted freely for the sake of health, and also for the warmth afforded by this means in colder latitudes, in winter.

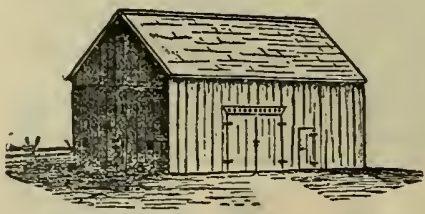

FIG. 2 .

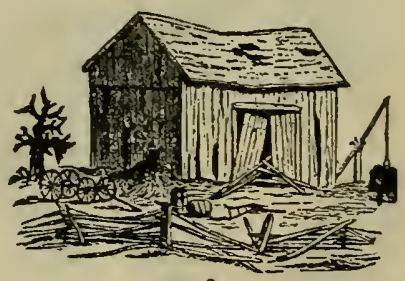

FIG. 3 .

Shade-trees are an ornament to any building, but they must be sufficiently removed to prevent the obstruction of the sun's rays. When trees near a barn or other buildings are interspersed with evergreens, a great protection is thus afforded against the ferce winds of winter. The expense of building a tasty and somewhat ornamental structure will not much exceed that of an excessively plain and unattractive building. Projecting eaves are a benefit as well as an ornament to a building, while ventilators at the top,-which are highly essential where animals are kept, - can be made to subserve an ornamental as well as useful purpose, and will cost but a trifle. The abore cut,-Fig. 1,-representing an attractive, two-story barn, is given in striking contrast with Fig. 2, which illustrates the perfectly plain buildings so commonly seen on the farm. Such a building as this represents does not add to the 
attractive appearance of any farm, and yet, with a slight additional expense in constructing, it might have been made quite the reverse. Fig. 3 is a very good illustration of the same building after the lapse of years, without repairs having been made. Too many such old, dilapidated buildings are to be seen in all sections, and, wherever located, are a standing testimony of the negligence and general shiftlessness of the owner.

If any farmer sees in this latter cut a good representation of any of his own buildings, we hope he will consider carefully the benefits to be derived from a better index of an improved style of farming, and soon proceed to complete the work of demolishing what time has so nearly demolished, and substitute a more commodious, useful, and attractive barn in its place.

Horse Barns.-The illustration of farm buildings on the next page, representing the very extensive horse barns of one of the largest importers of horses in this country, will be found to be quite beyond the requirements of most horsemen; yet the plans they present are the result of long experience and careful investigation. As such, they may prove valuable in suggesting plans and improvements that may be modeled on a smaller scale, and which, with slight modifications, may be adapted to any kind of stock. The description of this barn is by the owner, Mr. Dunham, as follows:-

Barn No. 1 is 160 feet long by 52 feet wide, with an awning adjustable 10 feet wide, to raise and lower. The foundation is of stone, laid below frost; bottom of wall 16 inches, top one foot in thickness. The wall is laid on the east, north, and west sides, and one wall of the same dimensions is laid 16 feet from the north wall and parallel to it. Cross walls, 10 inches thick, support the partitions of the box-stalls. The south outside foundation consiste of piers 20 feet apart, 4 feet square on the bottom and 20 inches square on top, with cap of cut stone, 20 inches square on bottom and 12 inches square on top, to receive the post. Sixteen feet north, and parallel to this line of piers, is another line built in the same manner. The building is constructed, as will be seen (by position of wall), with four rows of poststhe two center rows standing 16 feet from the outside, and 20 feet from each other both ways, extending to the purlin plate, and support the same. The outside posts are 20 feet long, and on the north side are 16 feet apart. All the posts are 8 by 8 inches, and are connected by beams 8 by 10 inches, and 10 feet from the bottom, npon which are laid 2 by 12-inch joists. The two center lines of beams running lengthwise of the building are additionally supported by a cast-iron angle, bolted on the post under the end of each beam, and running down the post and out on the under side of the beam 12 inches. The beams are also trussed on the top, making a solid and safe support for the joists which run crosswise of the building. The roof is one-third pitch, and formed with gables (dormers), and surmounted by a cupola, as shown in the perspective view. The outside is girted with 6 by 6-inch girts, 4 feet apart, and boarded with matched and dressed lumber. The positions of the windows can be seen in the engraving. In the second story there are four doors on the north side, with transoms, and on the south side eight of the same kind. In each end, as high as can be made in the gable, is a door 12 feet high and 10 feet wide, through which the building is filled with hay.

From each door to the center is erected a hay-carrier, as near the ridge as possible. The building is supported by the usual cross-beams and braces. The roof is covered with the very best dry pine shingles boiled in West Virginia oil. To prepare the shingles a vat is used of sheet-iron, 20 inches deep, $2 \frac{1}{2}$ feet wide, and from 2 to 4 feet long, according to the extent of the job. Set the bunches in and have oil enough to come up to the bands; let it boil five minutes, take out, place on an incline with tight bottom and drip back to the vat. In half an hour the other end of the bunch can be dipped, and returned on incline, and in one hour they will be dry. The cost is less than $\$ 1$ per 1000. I believe shingles prepared in this way will, with an occasional coating of oil, last indefinitely, as the water will not penetrate them in the least. 
The squares indicated in the plans are box-stalls 16 by 16 feet square, with one door

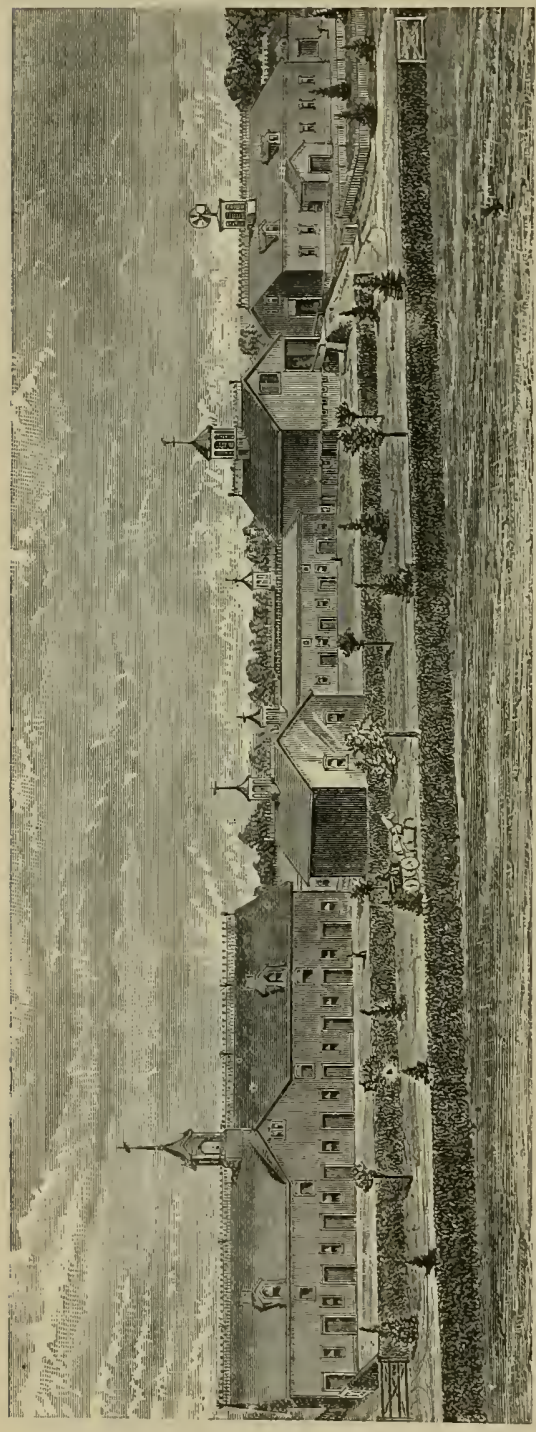
double-thick, 4 feet six inches wide, and 8 fect high. Latch, a straight piece $\frac{3}{t}$ by 1 -inch iron, 1 foot long, mortised into the center edge of the door, end protruding 1 inch, to catch latch hook; an iron plate with slot for latch to play in is screwed on the edge; an inch hole is bored under the latch, to raise with. One window, 12 lights, 12 by 16 on outside, and one 9.light window, from stall to alley, covered with No. 9 wire scrcening. The outside window is grated with inch refuse gas pipe, set three inches apart (cost about $\$ 35$ per ton); win. of dows hang on weights. The north and south sides of stalls are ceiled with 2-inch matched plank, 5 feet high, and from there to top with 1.inch matched stuff.

The hay-shoot is built in the outside corner, with 2 -fect runs, and extends 6 feet above the upper floor, and has a slidedoor on the long side, that can be raised, and leaves an opening in the sloot on a level with the floor when desired. The bottom of the shoot is grated gas pipe 3 feet 6 inches long, set on an incline from the corner to the outside of the bottom of shoot, which is 6 feet from the floor of stall. These pipes are set 6 inches from centers at the top, and one of them moves in a slot, so as to double the distance whon required.

Under the shoot is a manger, made of oak, with a side-run of 3 feet 6 inches. Its height is 3 feet 6 inches, and ceiled in front to the bottom of stall. In the opposite corner is the grain feed-box, opening into an alley by a shide-aoor 1 foot 8 inches by 2 feet 6 inches, hung on weights. The feed-box is made the same as the manger, only smaller.

The partitions between the stalls are made by setting 2 by 4 -inch studding 6 inches apart on sill, and extending 5 feet high. Both sides are then ceiled with common matched and dressed flooring, 
even with the top of studding, and an oak cap 2 feet 6 inches spiked on top. The top of this cap has $1 \frac{1}{4}$-inch holes four inches from center to center and one inch deep, in which inch gas-pipes 3 feet long are inserted, and capped with another oak cap firmly set at both ends. The floors are made of clay and gravel. An alley 6 feet wide runs the entire length of the barn, with manger on the opposite side from stalls.

The large space with posts in center is divided by movable plank partitions 5 feet high, the end bars of which run as high as the beam, and are hung to same with a hinge These partitions can all be raised to the ceiling, and are held there by four wooden hooks, with the lower parts beveled. When the partition (or door) is raised, the hook is thrown back until the door enters the notch (or hook), which falls over it and holds it. In this way the whole south portion of the building can be thrown into one great shed, or divided into small stalls, and when the awning is down everything is perfectly protected. The yard fences are also made movable by sockets being tamped in the ground to receive the posts, which are tapered, and can be taken out with perfect ease, and the holo plugged.

The bins for feed are made in the second story, and are located directly over the hydrant, at which point a box is located for mixing feed.

Barn No. 2 consists en. tirely of box-stalls, made on same plan as those described,

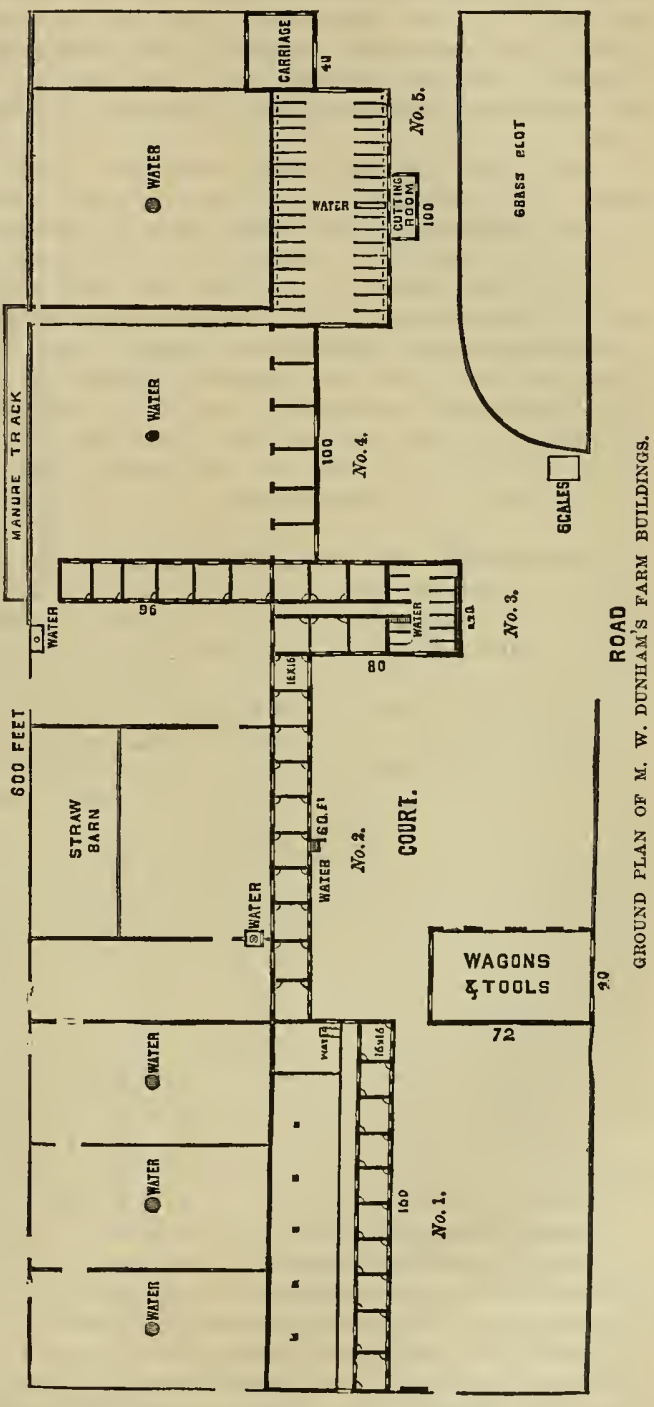
and open into the yards to the south; it is I 6 feet high at eaves, with loft for fodder. Barn 
No. 3 is 40 by 80 feet, 26 -feet posts, with 96 feet extension to the south, all boxes, the same as described. The single stalls are 5 feet wide, and made on the usual plan, with plank floors; hay being fed in shoots from above. The upper part is reached by an embankment and bridge. A hay-carrier is also rigged in it, door opening to the north. Large feed-bins are located over north end of the alley, where water is marked; a mixing-box, filled from spouts from bins, is placed beside the hydrant. No. 4 is an open shed facing south, with yard in front.

No. 5 is 50 by 100 feet; stone basement; the walls 26 inches on the bottom and 16 inches on top. The building rests entirely on the outside walls. The sills are 8 by 10 inches; the posts 20 feet long, and about 14 feet apart. The girts are 6 by 6 inches, and 4 feet apart. The roof is a truss-roof of the strongest kind. (See perspective view for location of windows, cupola, etc.) The boarding is of the best dressed and matched flooring. On the north side and center is a cutting-room, 20 by 24 feet, the cutter standing on a level with the second floor (see view). The basement is divided by three 6 -foot alleys, running north and south, connected by one 4 -foot alley running east and west along the north side. On each side of each alley are four box-stalls, about 12 by 14 feet, with plank partitions 5 feet high, and doors opening from one to the other, to the outside. Hay comes from the third story through shoots opening into the alley, and is fed in mangers.

The second floor is divided entirely into single stalls, as will be seen on the plans; an alley in front of each row for feeding grain and watering. Hay comes from above in shoots, as in the other stalls. The floors are 2.inch matched planks, tarred, and then covered with paper (two thicknesses); on top of this are laid 3-inch planks boiled in oil, knd keyed together every five feet. Between the two floors is an iron gutter, just at the back end of the stalls, with iron outlets running down the basement into the ground for drainage. The stalls are $5 \frac{1}{2}$ feet in the clear, and the partitions are 3-inch planks, doweled together, $4 \frac{1}{2}$ feet high, and the front rises in an oval shape and is barred. The stall-posts are 6 by 6 -inch oak; 3 by 12 . inch joists run from the stall-posts to the outside of the building, and 2-inch matched planks are used for the floor above, so that the space over the horses' heads is perfectly smooth. The ceiling over the floor back of the horses is 12 feet high and 20 feet wide, with a 14-foot slide door at each end. Over each stall is a finished panel, set with pictures of Percheron horses. The stalls and ceiling are painted in nicely-contrasting colors.

The entire water system is supplied from a 2,000-barrel reservoir or cistern, constructed on a hill 60 feet higher than the barns, and 100 rods away, built of stone laid in cement, and completely covered from the frost. The water is forced into this reservoir by wind-power, and is drawn by a $2 \frac{1}{2}$-inch main to the buildings, and distributed through them by $1 \frac{1}{2}$ and and 1 -inch pipes, laid 5 feet under ground.

The wagon-house has a self-supporting roof, and the entire front is composed of sliding doors. Carriage-houses and straw-sheds are ordinary frames. All the yards are graded and graveled in such a manner that they are perfectly free from mud at all times of the year.

The arrangement of the yards can be seen from the diagram. All the manure, except from barm No. 1, goes to the elevated track indicated, and in winter is dumped into wagons and hauled out. The total length of front shown in the diagram is 600 feet.

Cattle Barn.-The following is a description by Prof. Beal of the cattle barn on the grounds of the State Agricultural College, at Lansing, Michigan: It is a side-hill barn, 40 by 60 feet, with the end to the south, at which are double doors, and on each side of these, near the corners, are 4-foot doors for the passage of the cattle. The central alley is 12 feet wide, the foor of which, and that of the stalls on each side, are all tarred and placed upon a grout bottom, so that there can be no rat-holes beneath. The stalls for the cattle extend along on each side of this central alley. The earth being banked against the outer walls of the apartment for roots, prevents freezing; and the room for mixing the feed is partly protected in the same way. 
The planks forming the mangers next the cattle are movable, so that by taking them out and dropping them into grooves for the purpose, the space between the mangers and the manure-gutters may be increased or diminished according to the size of the cattlc. The planks forming the sides of the manger next the alley should be slanting or wider at the top, to make it easier to put in the feed, as well as to prevent the cattle from scattering the food over into the alley. The cattle are fastened with a chain about the neck, with the other end attached to a vertical rod at the side of each stall. As the ring moves freely up and down, ample room is given to the animals. In the rear are low windows. Over the passage at the rear are two ventilators, 3 feet squarc, reaching the roof. The roots are conveyed through side windows into the root-room. Here is a root-pulping machine, driven by a tread-power above.

The stalls vary in width from $3 \frac{1}{2}$ to 4 feet. From the edgo of the manure-trough to the end of the stall next the alley is $7 \frac{1}{2}$ feet for large cows, and 6 feet 7 inches for small ones, with intermediate dimensions as required. The slanting planks dividing the stalls and alley are movable, and drop into grooves at different distances, so that the stalls may be made long or short at pleasure, with similar ones for mangers. The mangers are 2 feet wide inside. The manure-gutters are 20 inches wide, and about 5 inches deep. The manure is wheeled out with a wheelbarrow. The passage at the rear of the stalls is $3 \frac{1}{2}$ feet wide. The basement walls are 2 feet 9 inches thick at bottom, tapering upwards on the inside to 15 inches at the top.

On the floor above is a cutting machine, and stalks, straw, etc., are cut by horse-power, and run down a spout, after cutting, into the feed.room. This cut feed is then placed in thin alternating layers with the pulped turnips. A car or large wheelbarrow is loaded with feed from the feed-room, and run out in front of the stalls.

The granaries are made mouse-tight. The number of bushels held by each is marked by figures on the back side of the bin, at a black perpendicular mark. Over the granary is storage. The barn is vertically boarder, wilh boards a foot wide and 3 -inch battens. The granaries or bins are lined with hard wood. The best way to exclude rats and mice is to pack a space with small fragments of tin-they will not work in it.

Sheep Barn. - The sheep barn at the State Agricultural College of Michigan-which is in many respects a good model for this kind of farm building,-is described as follows: This barn is 40 by 90 feet, and runs north and south. An alley 7 foet wide runs lengthwise through the center. It has a good floor $2 \frac{1}{2}$ feet higher than the pens on each side. At each end of this alley is a sort of step-ladder to go to the hay-loft above. The joists over the alleys are about 7 feet above it. At one corner of the chamber is a wool-room; and at the other a grain-bin. The breadth given to the alley makes it convenient for feeding, and no hay gets on the sheep. In late spring this barn is found a convenient place for young calves.

The floor over the pens and alley is all on the same level. Doors are placed in the sides of the building, opening into the loft, through which to pitch hay. The gates open for the admission of wagon and te $m$ for manure, and other purposes.

Each pen has a low door entering from the alley; and also a door running into the adjoining pen. The sheep-rack forms the boundary of the pens. Water is supplied to each pen from a pipe below ground, and which is pumped up by a wind-mill at some distance from the barn. The water is kept at a uniform level by means of a valve arranged in the reservoir. The back door passing into the yard from each pen is in two parts. The lower door is set in a groove at one edge, and is held to the other with a button. When not in use it is lifted out and set one side. The upper part of the door slides back on rollers above, and is on the outside of the barn. The upper one, when closed, permits a sheep to walk under it; or the lower one may be closed and the upper open when occupied by lambs.

The pens are 12 by 16 feet, and the yards outside and adjoining, are each 12 by 25 feet. 
The pens may be easily varied in width by moving the sheep-rack which divides them. To assist in readily supplying feed from the alley, a slanting board or door inclines towards the alley, and on this the hay or grain is placed on its way to the feeding-trough below. These slanting doors are $2 \frac{1}{2}$ feet high, and are held in place by long hooks at the top. In the summer, these doors are set up vertically against the studs (which form the division between the pens and alley), and are held there by buttons.

Stables. - It is always well to have plenty of stable room, even in a warm climate, where protection from the weather is not as necessary as in the higher latitudes. Animals that are stabled a portion of the time are more tame and gentle, hence more easily managed, and are really more valuable on this account. They can also be kept in better condition by the care they may thus receive, while they will not waste one-lialf the food that they would if fed upon the ground. Animals that are fed on the ground will not only waste a great deal, but are liable to be injured by being hooked by others, while the master spirits of the herd prevent the timid ones from getting their portion. By stable-feeding, these evils are all avoided. Stables should be so arranged as to avoid crowding. Animals that are crowded into narrow quarters will not thrive.

Stables should, as a general rule, be built higher than they commonly are. They should never be less than eight and a half feet, while ten feet would be much better. A better and dryer condition of atmosphere is thus secured. The atmosphere of any stable should be sufficiently dry. to permit the insensible perspiration to pass off, and, at the same time, sufficiently warm to dry a horse readily when its coat is wet with perspiration, and is being well rubbed.

Light in Stables. - Stables should be located in the sunny side of the building, that the animals may lave the benefit of the warmth and sunlight thus afforded. A dark stable should always be avoided. Light is as essential to animals as to men, and no animal will thrive to lee excluded from it for any length of time. At the same time, the arrangement of the stable should be such that the light can be admitted without its being too intense in the face of the animals. We have known horses and cattle to have been made partially or wholly blind by being confined in stables where the eyes were exposed to a strong light most of the time. If practicable, the light should come in from the rear, thus affording a protection to the eyes. Stables located on the south side of a building - which is the most desirable arrangement - will admit of the best means of thus adjusting the light.

Ventilation of Stables. - Good ventilation is also highly essential. So much has been previously stated with reference to this subject, that a repetition here seems unnecessary. No animal can be healthy without pure air. Good ventilators should be arranged at the top of every barn where stock are kept, to permit the foul air to escape, while there should also be openings near the floor for admitting the pure air. Great care should be exercised in order to have the arrangements for ventilation such that no animal shall be exposed to a draught of air. Such exposure will produce the most serious results to man. kind or animals, pneumonia and other diseases being frequently occasioned by it.

We have in mind a farmer who lost, with lung fever, a valuable Alderney cow from his dairy herd for three winters in succession, from having them stand in the stable directly in front of an open window, where they were constantly exposed to a draft of air from the rear. And this farmer was either so indifferent or ignorant, with respect to sanitary laws, that it was only the fact of the cows that died in this manner having each occupied the same place in the stable that aroused his mind to investigate as to the cause.

Stables should be built tight and warm, for the comfort of the stock as well as for economic reasons, while the pure air to be supplied for the animals to breathe should find an entrance in its proper place, and not through the cracks of the barn at the sides, or up 
through the stable floor, or through an open window, in cold, wintry weather, where the air is constantly blowing upon the ani nals. If any farmer doubts the soundness of our position in this respect, let him imagine or experience the discomfort and effects upon the health of being himself confined to a limited space, from which he could not get free, and be obliger to remain constantly in a draft of cold air day and night. If those having charge of animals were themselves obliged to be subjected, for a short time even, to the same treatment they impose upon the dumb, patient, and so often abused creatures under their care, there would soon be a great reformation in this respect, and more kindness and consideration be shown them, than we now o ten see.

Floors, Box-Stalls, etc. - The floors of stables should be strong and well laid. Serious loss sometimes occurs from the giving way of the stable floor. There should be a few box-stalls in every barn where stock are kept, and these should be so arranged that a single animal may be turned in loose when desired. These are very convenient for horses, or sick animals, to occupy. They are also almost a necessity for use as lying-in stalls for cows, while they furnish a convenient place for young calves when separated froin tice mother.

Whatever the method of fastening employerl, it should be such as will admit of the free use of the head and a restful position in lying down. We have seen animals so tied in the stable that they could not lie down without great discomfort. While cattle should be so secured by fastenings that they cannot hook or otherwise injure each other, they should have sufficient freeclom to maintain comfortable positions in standing or lying down. All animals would be more comfortable, when stabled, if they could each have the freedom of a box stall, without being tied at all. This would be impracticable where many cattle are kept, but if all working horses could be favored with such comfortab!e quarters, we believe they would last much longer, and the benefits derived would well repay the expense of this luxury to them.

Freedom of movement is just as essential to animals as to man, for health and comfort, and if horses could each have a large box-stall, where they could run loose and get some exercise in unfavorable weather, and lie down in any position they might choose, it would be much better for them than the common custom of fastening them to one place in a narrow, dingy stable. Mangers should be made perfectly smooth inside, and so well fitted that even dry meal or bran can be fed in them without waste. Where racks are used, a shallow box should be arranged underneath, to prevent the waste of hay.

Manure Gutters in Stables. - In order to prevent the waste of liquid manure, the stable floors should be water-tight, and provided with gutters for conducting it to a place of deposit, or sufficient dry material be supplied in the stable to absorb it. Gutters for both liquid and solid manure are sometimes made in the rear of stables, into which the manure is thrown. These are cleaned out every three or four days, and the manure carted directly upon the soil, or put under a sized, or into a compost pile. These gutters are made in various ways, and of different dimensions. A Western farmer describes, in one of the leading agricultural journals, one which Ire has constructed as being, when finished, about eight inches deep by twenty-four in width, and costing at the rate of two dollars per cow for material and labor. It is made principally of white oak plank, and double throughout, except at the bottom, which has three thicknesses. The gutter is made perfectly water-tight by fitting the boards nicely, and applying a coat of boiling tar between the courses, care being used in putting on the second course to break the joints in the first. It is, of course, necessary in using tar in this manner to nail down the boards while the tar is hot and soft. The same Niter says, respecting this method:-

"After the gutter was done, we laid one counse of tloor, for which we also used one inch 
thick pine boards, and coated it with tar about half way up to the stanchions, and then laid the other course, breaking joints. Always use the tar hot, and use it freely, and you will find it easy to make a tight job. How long it will last I cannot say, but that it will last long enongh to pay many times over, I know from experience. Being built on the ground, separate from the rest of the building, it can easily be replaced when it rots out. It takes about one barrel of tar to 75 feet of stable. Get all the cross-boards and floor-boards sawed at the mill, of the right lengths.

The manure from this gutter is wheeled out once in three or four days. By this plan a man wheels out three days' manure from 50 cows in half a day. If it was wheeled out every day, it would spoil three half-days. The stables are divided so that only 10 or 15 cows are let out at once, and while they are drinking in the yard I wheel out the manure, then put them back and let out another lot. I am keeping 15 three-year old steers on such a floor as I have described."

A farmer in Syracuse, N. Y., describes, in the same journal, a manure-gutter of very different construction, it being covered with a heavy iron grating, which permits the manure to fall through into the gutter beneath. It is as follows:-

"The joists are framed into the sill to hold it in its place. The floor is of 1 or $1 \frac{1}{4}$-inch hemlock lumber, laid lengthwise of the stable in two thicknesses, and so as to break joints, to make it tight. The top of the floor is even with the top of the sill. The iron floor is an invention of E. W. Stewart of Lake View, Erie county, N. Y., and costs $\$ 6$ for each cow. This is hung to the sill, so as to be conveniently raised up to allow the pit to be cleaned. I purchased and put in the floor about the middle of December, having some doubts as to its utility on account of the cows having to stand upon it with their hind feet. I have used it ever since, and I have never tried any experiment which has given me as full satisfaction (except the silo, and that no better) as this. I have not had one-half pound of manure adhering to eight cows in the whole three months, nor has it required one pound of straw or other bedding, and I do not think that I could keep the cows clean with bedding, as it would prevent the droppings from going through into the pit, and the cows would lie down in it.

I use the pit by putting into it about two or three inches deep of swamp muck (not pond mud), and once or twice each day add a little to this by throwing from a pile of muck in the stable a shovelful or two behind each cow on the top of this iron grating, and let it sift down through and mix with the droppings and the urine, and I use also daily a little plaster in the same way. If the cows do not tread all the droppings through, we brush over the top of the grating with a common stable broom (or fork), to break up the droppings and let them go through. Once or twice in a week we drive (or back in) the wagon behind the cows and load into the wagon the contents of the pit (all fine, without straw), carting it directly to the field, and spreading it where it is wanted. I am satisfied, from the little experience which I have had, that besides having something which will last a lifetime, I shall save in labor more than the extra expense every two years, and that the cows will stand or lie down upon it more comfortably than upon a common stable floor, which is not too wide to allow the droppings to fall into the gutter. Another advantage is, that $I$ have no wood coming in contact with the urine to be saturated with it and give off a bad odor. This, and the clean condition of the cows, I consider no small advantage to a cow stable. If I had neither swamp muck nor thoroughly decomposed straw, chaff, weeds, or other vegetable matter for an absorbent, I believe I should use dry road dust. This, however, has little to do with the arrangement of a stable."

The gutter above described is 2 feet deep and 3 feet wide, the sides and bottom being of concrete. The stable floor, from the base of manger to the iron grating, measures 3 feet and 4 inches. By the use of such gutters, as above described, the objections urged against barn 
cellars for the storage of manure are in a great measure obviated, since the manure is taken out before fermentation commences. Gutters for saving the liquid manure separate from the solid, when desired, are shown in previous description and illustrations of horse and cow stables.

Barn Cellars.-Various opinions are entertained with reference to the use of barn cellars for the storage of manure. A few years ago cellars for this purpose were much in favor, but at present many grave objections are being urged against them from some authorities, while others are as strongly as ever in favor of them.

That a barn cellar may be very convenient for many purposes cannot be denied, among which is the storage and manufacture of manure. But in their use for this purpose, unless great care is taken, and the building supplied with a most complete and thorough system of rentilation, they will become the source of disease by the contamination of the atmosphere which the stabled animals must of necessity breathe.

The fumes of decomposing manure in a barn cellar will be sure to find their way to the stable above, where the animals are kept, which will poison their blood and be a source of ill. health, while the quality of the hay and other fodder storel away in such a building will be greatly injured by this means.

Absorbents will prevent decomposition in a measure, but not wholly, even when used in large quantities, while the ordinary facilities for ventilation will prove only partially effectual. But the decomposition of the manure is one of the advantages claimed in the use of the cellar for its storage, and unless this is accomplished, one of the objects sought in their use is not attained.

Wherever so used, great care should be taken to have on hand at all times an abundant supply of dry, absorbing material, which sbould be employed freely, and especial pains taken to have the building thoroughly ventilated. It is also well to make use of deodorizers occasionally to prevent, as far as possible, the evil effects that may result from the storage of such a quantity of fermenting material directly under the building where animals are stabled, and fodder stored. Barn cellars furnish a very convenient place for the storage of roots for feeding stock, where they will also be beyond the reach of the frost. Such places of storage should not be directly under a stable, but under the portion of the building where hay and grain are kept.

Animals should never be stabled in a cellar. Such places are damp and unhealthy, and are injurious to animals that are confined in them. A side-hill or bank-barn may be so constructed as to have a basement on a sunny side of the building, the floor being on a level with the ground, and be less objectionable for stabling cattle than cellar stables, as sometimes arranged; but even then such stables will be more or less damp and unhealthy, and we would not recommend them for that purpose. The practice of confining swine in. barn cellars where manure is stored is open to serious objections. No animal thus kept is fit to become food for mankind. It is very true that swine may thus be able to work over the manure and aid in its thorough mixing, and will eat much of the food wasted by the horses and other stock that may be mixed with it; but no animal can be healthy when forced to live in this manure and breathe such vitiated atmosphere. The "Massachusetts Ploughman" contains the following sensible advice with reference to this subject :-

"It is a fact that is conceded by every intelligent individual, that animals of all kinds, to be healthy, must have plenty of pure air and sunshine. If this be so, then what must be the condition of swine kept in a cellar where no draft of pure air can pass through; where but little sunshine can penetrate, and where the continual decomposition of animal manure is filling the air with carbonic acid and ammonia. To suppose that any animal, except of a low order of existence, can be kept in health in such a position, for any great length of time, is to 
suppose an impossibility. It is true, pigs are able to live and grow in such places until they are large enongh to kill, but it must be remembered that most of them are killed before they are a year old, not giving time for the disease, caused by ill rentilation and darkness, to mature sufficiently to cause death, but it will generally mature enough to make the animal unhealthy, and thus render the pork an undesirable food.

The farmer loses in more than one direction by keeping his animals in an unhealthy place. In the first place, he loses by not, getting as much flesh for a bushel of meal, and in the second place, he loses by feeding his family on the flesh of unhealthy animals. It may not create immediate sickness, but the constant eating of unhealthy food undermines and breaks down the most robust constitution, and renders it a mere wreck, often without the cause being suspected.

The farmer should ever keep in mind the fact, that, if he would keep his family healthy, he must keep the animals, the flesh of which they are to eat, in a healthy, thriving condition, and he should also remember that he can do this only by giving them good food, and keeping them where they can have plenty of pure air and bright sunshine."

It is no wonder that mankind are subject to so many diseases, when the first principles of sanitary laws with respect to the food we eat, the water we drink, and the air we breathe, are so utterly disregarded.

Doors, Seaffolds, etc.-The doors of the main floor of the barn should be wide and high. Narrow or low doors will prove a great inconvenience, and should never be made in any building, especially a barn. Sliding doors set on rollers are generally preferred to hinges. T'here the latter are used, the hinges should be strong, and hooks arranged to fasten them open when desired. Stable-doors should also be of good size.

Means should be provided for safely locking all the barn-doors, in order to keep out intruders. Windows can scarcely be too numerous in a barn. Liglit is essential to the health and comfort of animals, as well as mankind; besides, it is much more convenient to have suffcient light in the barn while performing the necessary work there. Good stairs leading to the scaffolds are much more desirable than the ladders commonly used by farmers. These can frequently be so arranged as to be fastened up out of the way when desired, as in the barn first described in this department. A portable step-ladder will also prove a great convenience for occasional use.

All the bays should have tight floors, and be elevated at least from two to three feet from the ground. The loose floors of scaffolds so commonly seen in barns are very objectionable, as the hay-seed and dust are liable to sift down into the stalles. In order to obviate this difficulty, the floors of the scaffolds that are orer the stables should be lined and made perfectly tight. For the safety of the crops, comfort of the animals, and the prevention of injury to the building, the roof should be kept in good repair. and all leakage preveuted. The walls under the barn should be made tight in the main, but there should be openings left for rentilation on opposite sides. The main floor should be smooth and well laid, and the floors of the stables made of heavy plank, and of the most substantial character.

Eave troughs should be provided for the barn and adjoining sheds, in order to prerent the large quantity of water that falls upon the roof from washing into the yard. By this method a, supply of water could be furnished for the stock, if no better means of watering were provided. Lightning-rods are also a great protection. Some arrangement should be made for protecting the manure from being washed by the rains, and evaporated by the heat of the sun, when not stored in cellars, or carted directly from the stables on to the land. The barn-yards should be so located that the drainage from them will flow upon the farm lands, and also so as to receive water from no other source. It is a good plan to make them a little lower in the center than at the outer limits, in the form of a shallow dish. By this means, the drainage is retained, which may be absorbed by the use of loam or dry muck, and add 
materially to the amount of fertilizers for farm use. If the soil is sandy, and therefore leachy, it should be covered with clay to prevent the escape of the liquid manure by leaching into the subsoil. Some farmers have been at the expense of making a concrete basin of their barn-yards, thus making a complete reservoir for all the fertilizing substances that are con. tained within it, and from which none can escape but by evaporation.

Granary.-Instead of a separate building for the storage of grain, many farmers devote a portion of the barn to this purpose, or the npper pari of some of the other out-buildings, where a series of bins are made to substitute a granary. It is far better, however, to have a separate building for such use, -one built with special reference to the protection of grain from rats, mice, and other vermin. It may not necessarily be a very large or elaborately. built structure. The size and style of its construction will depend npon the size and requirements of the farm, but whether large or small, it should be so arranged as to prevent the entrance of rats and mice, and for the admission of air for keeping the grain dry.

A granary should be set up from three to four feet from the ground, on a perfectly smooth brick wall, or on stone posts. This is to prevent the entrance of rats and mice. In olden times, it was customary to use posts on which the building was elevated, that were capped with large, flat, smooth stones. Although such an arrangement is more useful than ornamental, since it proves an effectual means of securing the object sought, yet if the stone posts or brick wall, as before mentioned, are made as smooth as possible, they will subserve the purpose equally well, and look very much better.

Where brick walls are employed, spaces must be left open sufficient for a free circulation of air under the building. In order to admit of a free circulation of air in the building, two sides of it should be covered with slats from two and a half to four inches wide, placed about half an inch apart. The other sides should bo boarded tight. The eaves should project considerably, and the entire building be kept in good repair, that there may be no leakages from roof or sides. There should be a passage-way through the center of the building, and doors at each end. The doors should be prurided with locks for the purpose of security when desired. There should be a sufficient number of windows to render the building conveniently light. It should also be provided with a good ventilator in the roof. The bins can be arranged according to convenience. It is well, however, to locate the bins for holding ears of corn against the sides of the building, covered with slats, while those for wheat, oats, shelled corn, and meal, should be placed against the sides that are boarded, as a preventive against dampness. Each bin should be provided with a well-fitting cover, and also a lock and key.

Every bin should also have its capacity in bushels plainly marked upon it. It is a good plan to have the number of bushels which each bin contains marked on a slate or blackboard attached to it. An arrangement for this purpose may be painted on each bin at the time of completing the building. By this means the amount it contains can be recorded, as well as the amount taken out from time to time, and by deducting the sum of the latter number of bushels from the former, the amount on hand can at any time be definitely ascertained. The bins should be divided into several compartments by partitions, each holding from ten to forty bushels, or more, of grain.

Seaffolds in the upper part of the building, and which may be reached by stairs, will be found convenient for various purposes. A good step-ladder will also be necessary for use in the granary. The grain should always be perfectly dry and clean when put into the bins, and with the proper provision for ventilation and protection against dampness, as previously indicated, it can be kept in the best condition.

Hog.House.-In the breeding and rearing of swine, buildings will be necessary in which provision is made for warmth in cold weather, and a protection against the hot sun in 
summer; for, although natives of a warm climate, and provided by nature with a protection against the cold of only a thin covering of coarse hair, they are not fond of excessive heat, and will seek shelter from the hot sun when it is accessible.

A mple facilities for ventilation should also be provided, as well as those for keeping the building in as clean a condition as practicable. Orer-crowding should also be aroided where a large number of swine are kept.

The size of the building will of course depend upon the number to be kept. Extensive breeding and rearing of swine necessitates conrenient buildings of large size, consisting of various departments suited to the purpose, such as an apartment for cooking their food, for fattening, and sleeping, and for keeping different portions of the herd that it is desired to keep separate from others. For fattening in the autumn, a large pasture for them to run in during the day, and a dry yard with simply a shelter at night, is what are frequently provided in the Tiestern States; but, even under such circumstances, convenient buildings will be necessary for such as are kept over during the winter. There only a small number are kept, it will be necessary to divide the building into two or more apartments, which are connected with each other by a door, and each connected with the yard. The partitions between the different departments should be sufficiently high to prevent the pigs from jumping over. In front of the pens there should be a wide alley.

A very convenient plan is to hang the lower boards of the partition between the pen and alley, where the feeding-troughs are, on hinges, so that they may be made to swing into the pen and leave the trough in the alley when the food is put in. This renders it more convenient in feeding the pigs, as well as in cleaning out the troughs.

An apartment for sleeping should be separated from the feeding room, and plenty of clean straw provided for their beds. The floors should be of heary planks, well fastened Cown, and laid to slant slightly in the direction of the yard, for the purpose of keeping it well drained and dry. A scaffolding overhead will be found convenient for various purposes. Unless swine are permitted free access to the yard, or have fresh earth, charcoal, \&c., prorided them frequently, they will be uneasy, and will be liable to root up the floor, if possible, to get at the earth.

Special provision shonld be made for readily cleaning out the pens. The pens should be cleaned out every day, the same as stables, and if properly arranged, it can be done with but slight trouble, and in a comparatively rery short time. There is no reason why pigs should not be kept in as clean a condition as other animals, if properly cared for.

Pigs should also, at all times, have access to fresh water, and the pens should be at a convenient distance from a pump, where such supply is not provided in the building. Pigs will thrive better, and be healthier, to have a sufficient amount of room and fresh air.

In raising pigs, more room will be required than simply fattening them. In a cold climate, the sow-pen should be provided with a stove, and other conveniences. Many young pigs are lost in severe weather by getting chilled, and if some means of rendering the pen comfortably warm were provided, it would amply repay for the trouble, where the farmer pays considerable attention to this industry. Harris says with reference to such pens :-

". In pens for breeding sows, we have found it very convenient in cold weather to have a partition between the sleeping and feeding apartments, with a sliding door, that can be easily closed. It is desirable, when pigs are to be made ready for the butcher in eight or nine months, that the sow should farrow early in March; and it often happens that this interesting event occurs during a severe snow.storm. With a warm sleeping apartment, and with a door that can be closed at night, or at any time after the sow has been fed, thousands of pigs that are now lost might be saved. This plan is particularly essential where the feeding apartment is partially or wholly uncovered. But eren where both apartments are covered, it is better to have a partition that can be opened in warm weather, and closed during cold storms. 
The only objection to this plan is, that the sow has not so much room, and there may be increased danger of her crushing the pigs against the sides of the pen. This objection, however, is more apparent than real, from the fact, that no matter how large the pen is, the sow is almost certain to make her bed near one of the sides. She almost invariably, in pigging, places her back against the rail or side of the pen, the object probably being to prevent the little pigs from getting on the wrong side of her, where they would, in cold weather, be likely to perish before they find the teats. Our breeding-pens have a rail on the inside, about six inches from the sides of the pen, and about one foot high, but the sows before pigging take special pains to fill the space with straw, and we are satisfied that if they did not, the little pigs, when born during a cold night, would often get on the backside of the sow, and be chilled to death."

A building for pigs may be made comfortable and convenient, and yet need not necessarily be expensive. Good planning and ingenuity are essential to render such buildings, as well as all others, especially suited to the purpose.

Plans for Piggeries.-The following plans for convenient piggeries will contain valuable suggestions for those farmers who purpose to build a new one, as well as others who are to repair or otherwise improve the old onc now in use.

Mlessrs. H. M. and W. P. Sisson, Galesburg, Illinois, who are extensive breeders of Poland-China hogs, use a breeding-pen, of which the following is a description: The building is $24 \times 40$, with alley, 4 feet wide, through the center; ten pens, each $8 \times 10$ feet-plenty of room for old sows; door to each pen, $2 \times 3 \frac{1}{2}$ feet in size; slide window over each door, excepting two at south end, where window is in the middle of each pen. Door at south end of alley is made in two halves, in order to have upper half open for ventilation. Window over door in south gable. All cross-partitions movable. Two south pens have doors inside, which swing or open into alley, and hook together, forming a passage-way from one side to the other. When partitions are out, both sides can be used for feeding, or one side for feeding, and one for lodging. Partitions can be stowed away on scaffold overhead, and replaced when pens are wanted for sows. The building is set on stone piers, about $1 \frac{1}{2}$ feet high. Sills, $8 \times 8$ inches; joists, $2 \times 8$; shielding, $4 \times 4$; rafters, $2 \times 4,2$ feet apart; roof, $\frac{1}{3}$ pitch, with two good ventilators in peak, right distance apart for appearance. Seven feet from top of sill to top of plate. Stock boards, 14 feet in length, cut in the middle, can be used for the sides; battened all around; sealed up inside with common lumber to bottom of windows; tar paper used on sides and roof; 2-inch plank for floor; shingled roof. This, we beheve, comprises about all needed for the structure.

Mr. Pascal Morris, of Philadelphia, an extensive breeder of Chester Whites, uses a pig. gery which is susceptible of reduction or extension for a larger, or smaller number of pigs, and is intended to show what many of our practical swine-breeders most desire, viz., a cheap and convenient construction of buildings, separate and distinct from each other, thus avoiding the evils that arise from massing large numbers under one roof. It will be seen that Mr. Morris has an eye to cleanliness and pure air in his piggery, as well as the healthful influences of sunlight in his swine quarters. He also looks after the comfort and safety of his breeding-sows, as twenty-five to thirty of these, farrowing at different seasons, can be accommodated under his system of separate pens, by bringing them successively within the enclosure arranged for them. In the sqme way, also, an equal number of hogs can be fattened without crowding or interference with each other.

The following description explains itself: "The entrance is on the north side of the building, which fronts the south, as does also each separate pen. The main building is 32 feet long, by 12 feet wide, with an entrance gate, at each lower corner, to the yard of two first divisions. The entry, or room in the center, is 8 feet wide, allowing space for slopbarrel, feed-chest, charcoal-barrel (almost as indispensable as feed-chest), hatchway, for access 
to root-cellar, underneath the whole building, and also passage-way to second story. This latter is used for storing corn in winter, and curing some varieties of seeds in summer. A wooden spout, with sliding valve, conveys feed to the chest below. The grain is hoisted to the second floor by a pulley and tackle on the outside.

The entrance to the main building is by a door in the center of the north front, the door by which grain is hoisted to the second story being directly above the latter, with a four. paned window on either side for lighting the upper story of the building.

On either side of a central passage-way are six pens, the first two pens to the right and left of the door being $12 \times 12$ feet each, and attached to them are 25 feet in length of yard, by 15 feet wide. All the yards are extended 3 feet wider than the building, which admits of two entrance gates at the corners.

Another division then commences, consisting of a raised platform, 6 to 8 feet wide, and extending the same width as the first pen, with a board roof over it, and also boarded up on the back, which answers the purpose of a division fence, to separate from the pen behind it. Twenty-five feet of yard are also attached to this, and the same arrangement is continued to all the six divisions. We have found, says Mr. Morris, that this board roof and wooden floor, on the north side of each pen, and fronting the south, are ample protection in cold, wet, or stormy weather. The floor is kept perfectly clean, and even the feeding-trough is not on it, on account of more or less of wet and dirt always contiguous to the trough, which freezes in winter and becomes slippery.

Each yard is used for the deposit of refuse vegetables and weeds, litter, etc., thrown in from time to time, to be consumed, or made into manure. This is conveniently loaded into a cart, passing along on the outside of each range of pens. The passage-way between each range of pens gives convenient access to the feeder for all the pens. A door also communicates from one division to the other, to make changes when necessary; and also a door or gate from each pen to the outside, so that one or more can be removed, and others introduced, without any confusion or interference from any of the other pens. The two pens under the main roof of the building, being more sheltered, are reserved for cows that may happen to calve very early in the season, or in extremely cold weather, which is always avoided if practicable. For several reasons, the boiler for cooking food is in a rough shed, adjacent to the piggery and entirely outside of it. There is, indeed, no reason why this should be necessarily a part of the piggery. The above plan is not offered as embracing much that is novel in arrangement, but as one that combines many advantages which may be comprised in the following:-

Complete separation, and easy communication between each pen, as well as to outside from each.

A voiding close and confined air, and admitting of extension or alteration for a large or small number of pigs.

Facilities for keeping clean and receiving refuse vegetables and weeds, etc., for conversion into manure, and also for loading from each pen into a cart, passing along outside.

Cheapness. With the exception of the main building, all the rest can easily be erected by an intelligent farm hand.

Mr. J. W. Morrison of Orange county, N. Y., has for a piggery a two-story building, $26 \times 40$ feet on the ground, the front posts being 16 feet, while the rear posts are 14 feet high. The roof is nearly flat, and covered with gravel and tar.

On the long, forty.feet side of the ground floor are five pens, each $8 \times 14$ feet in size, connected with which are yards $8 \times 16$ feet in size, these being on the south side of the building, which fronts the north. On the north side of these five pens are feeding-troughs, $1 \times 1 \frac{1}{2}$ feet, which connect with the feeding and slaughtering-room by openings suitable for pouring the swill or other feed into the troughs, aside from which the partition between the pens 
and the slaughtering.room is entirely closed, the latter being $40 \times 12$ feet in size. The entrance door to the feeding and slaughtering-room is on the west side, near the northwest corner of the building, This room contains the usual conveniences and implements for feed. ing and slaughtering pigs, such as furnace and boilers, scalding-tub, and dressing-table the boilers being used in cooking food, or heating water for use in dressing the pigs. A door opens from the pens into the slaughter-floor, and the pens are all connected by doors between them.

Feed-tubs are so located as to receive by conducting-pipes the skim and buttermilk from the dairy. The floor of the pens is composed of brick laid in cement, and slopes four inches back to the sills, which are raised that height from the floor, resting on iron pins, thus leaving a space for discharging both solid and liquid manure into the yards. The yards are also provided with a tight bottom, and walls of brick and mortar, thus preserving the manure from waste. The floor of the slaughtering-room slopes one foot in twenty-six in length, and a few inches from the side to the center. The second story contains the sleepingrooms, $8 \times 12$, entrance to which is gained by inclined planes from the pens below. No difficulty was experienced in getting the pigs accustomed to climbing up to their sleeping rooms at night, and they keep them clean and dry; leaves are furnished for bedding. This economical arrangement of pens allows the keeping of about 50 hogs in a comparatively small building. The space over the slaughter-floor extends up to the roof. except that a platform runs around in front of the sleeping-pens, on a level with the second floor:

The arrangement of sleeping-pens in the second story, being similar to that of the feedingpens below, leaves space for two large rooms that may be used for grain-bins and storage. The doors opening into the yards, and from pen to pen, are all managed by ropes and pulleys from in front of the pens. With a view to keeping out vermin, all space around the framework and behind the troughs is filled in with brick and mortar. There seem to be but two defects in the foregoing plans, that might properly be criticised; for while very explicit and minute directions are given on various points, nothing is said respecting the ventilation of this house for 50 hogs. There should be at least three large ventilating cupolas on the top of the building, beside windows and doors that may be opened or closed, as circumstances may seem to direct. There should also be arrangements made for keeping the yards from being flooded by rains, to the great saving of manure.

Poultry-House. - Plans and descriptions of poultry-houses will be given in connection with the poultry department (which see).

Wagon-House, or Shed.-A shelter for the storage of wagons and carts is essential on every farm. On small farms, the wagon-house, tool-house, and repair-shop may be combined in one building; but on large farms considerable room will be required for the wagons and carts alone. A wagon-house should be closely boarded, and kept in good repair. Like all other farm buildings, its size will depend upon the size of the farm, and the purpose to which it is to be appropriated. Care should be taken to have it sufficiently large for all practical purposes. If the upper portion of the building is finished off for other uses, the scaffold or floor above should be close and tight, to prevent the dust from falling through upon the floor below.

There should be a separate department where the best wagons and carriages are kept, while a small room leading into the latter may be finished off for the safe keeping of the harnesses, robes, etc. The door of this department should always

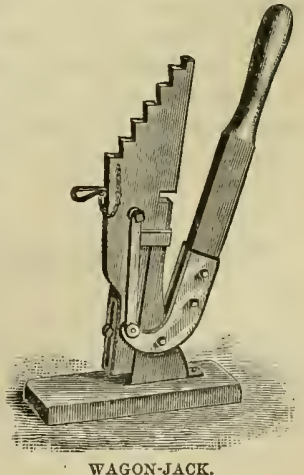

WAGON-JACK. 
be provided with a strong lock. For the sake of convenience in getting wagons in and oul of the building, the latter should be elevated but a little above the surface of the ground Some wagon-sheds are left open in front, but it is much better to have them made tight by doors, thus keeping out the rain, snow, and dust.

The building and doors should be made sufficiently high to permit a high carriage tc pass in and out without letting down the top. A low building that will not admit of this, is a perfect nuisance, as well as a small building that has not sufficient room to store all the wagons, or to get them in and out without great inconvenience. Better, by far, make the building too large than too small, and if there is a surplus of room it can be readily appro priated to other uses. A good wagon-jack is a great convenience in every building where wagons and carriages are stored.

Wood-House. - A convenient place for the storage of fuel is a necessity on every farm. It should be located near the kitchen, and be easy of access from that quarter. If practicable, it should be reached without going out of doors. In any case, it should be near the house. Sometimes a portion of the back part of the dwelling is finished off for this purpose. Its size should be sufficient to hold considerably more wood than would be needed by the family in one year. It should be provided with a good floor, that the wood need not be put upon the ground. There should be a loft over the wood-house, which may be reached by stairs. This will be found rery convenient for the storage of many things. Such places are always arailable for some purpose, on any farm. The building should be tightly boarded and provided with windows sufficient to make it conveniently light. The front of the building should contain large doors, which may be made to slide on rollers, or hung on hinges. The wood should never be piled higher than can be conveniently reached while standing on the floor.

Store-Honse.-A building for the storage of various products is a great convenience on any farm, large or small, while on a large farm it is not only a convenience but a necessity. Such a structure will furnish a place for the storage, ripening, and curing of fruit in the autumn, the drying of nuts and garden seeds, the storage of roots until cold weather, and other purposes too numerous to mention. It should be located near the house, and be closely boarded, well lighted and ventilated. The door should be large, and provided with a strong lock to keep out intruders. It should be supplied with convenient bins, and room for the storage of barrels, which should always be kept clean. Shelves should always be placed against the walls. If designed for ripening pears, a dark place will be essential for this purpose; hence, a dark room, or a large dark closet with shelves against the wall, will be found essential, as pears require a dark place for ripening well.

Such a building should be built sufficiently high above the ground to prevent dampness, and the floors should be made very tight also, for the same purpose. A store-room should always be kept as cool and dry as possible. The underpinning of the building should be so constructed as to admit of a free circulation of air, which will aid greatly in preventing dampness. The size of such a building should be adapted to the size and productiveness of the farm.

Tool-House and Repair-Slıp. - There should be a place on every farm for the storage of tools and farm machines, as well as the repairing of them. On smalı farms the wagon-house may serve for this purpose, in connection with the storage of carts and wagons; but on large farms, the many farm implements that are essential in conducting the business will necessitate a separate building or department for this purpose. The negligent and wasteful practice followed by some farmers of permitting the farm machines to lie in the open field exposed to the storms, year after year. is one not to be commended. Reapers, mowers, hay. tedders, plows, harrows, grain-drills, etc., are injured more by this means than by their use 
on the farm, and the loss thus sustained would very soon defray the expense of providing a convenient place of shelter for all such implements.

Such a building need not be expensive, but should be built tight, to afford protection from the weather. It should be located near to or adjoining the barn, be well covered and lighted, and amply provided with large doors to render it convenient in getting machines in and ont of the building. For the latter reason, it should also be built but a little above the ground. A tight floor should be laid, as it is not well to have machines stand upon the ground, since the dampness arising from the earth would cause the steel and iron portions to - rust badly, as well as the wood-work to swell. A scaffold above will serve as a convenient place of storage for lighter implements, such as rakes, forks, baskets, etc. This scafiold should be reached by a pair of stairs.

One part of the building should be done off for a repair-shop. This can be separated from the former by large folding-doors. Tools are constantly needing to be repaired on even the best regulated farms, and a special place supplied with implements for this purpose is a great convenience, as well as the practice of economy; for if the farmer possesses the means of repairing his farm tools and machines himself, when they require it, he will be more liable to do it in season, before they become more badly broken or injured. The means of repairing them on the premises is also a saving of time and expense in having them taken to a machine-shop for the purpose. With a little practice, any farmer who is handy in the use of tools will soon be able to do many jobs in repairing broken or injured farm implements, that the special mechanic is generally depended upon to perform. The repair-shop should contain a good work-bench, racks and shelves for the purpose of holding tools, a forge, and a lathe.

Two or three convenient horses, upon which planks or farm implements can be laid when desired in repairing, will be necessary. A good set of tools for all the common purposes of working wood and iron will complete the establishment. The repair-shop would require a stove in winter, in order to make it comfortable for working. Such a building, especially where a forge is used, should have a good, substantial chimney, built rather high above the roof. It should also be located sufficiently far from the other farm buildings to obviate all danger from sparks that might escape from the chimney; still, it should be at a convenient distance from them. It would also be well to cover the roof with tin, slate, or some other fire-proof material.

Ice-House. - It was formerly supposed that the construction of ice-houses was an expensive and laborious task, and the keeping of ice through the warm season was attended with many difficulties; therefore, ice was regarded as a luxury that few farmers were financially able to enjoy. But it has been found that very inexpensive structures, or their substitutes, with proper construction and management, are all that is essential for supplying an abundance of ice, which has within a few years ceased to be classed with the list of luxuries, and is now regarded as one of the necessities on every well.conducted farm. There is really no reason why every farmer's family should not be supplied with an abundance, since it can be furnished at so slight an expenditure of money and labor.

Those who have not been favored with the use of ice during the hot weather have no idea of the benefits to be derived from it, both in sickness and health, while those who have been accustomed to an abundant supply would scarcely feel that they could dispense with it for even a single season. Ice is absolutely indispensable for securing the best results in a dairy. In the city, it can always be readily obtained from those who make it their business to furnish it; but in the country no such facilities will be found; therefore, each individual farmer will be obliged to lay up a store during the winter months on his own premises for home consumption, or be deprived of it in the season of its use. 
Essentials in Building Ice-Houses. - Various methods and plans are employed in the construction of ice.houses. Some prefer them built entirely above ground; others recom. mend that they be built partly, or almost entirely, under-ground. When suitably constructed, either method will answer the purpose well. As a general rule, the plan of building way be safely left to the skill of those who know what is required to secure the preservation of the ice when properly packed. When these essentials are well understood, any farmer, with the common tools and materials found on every farm, can easily construct one at but slight expense. The principal rules in building houses that will be successful in preserving ice may be briefly summarized as follows, viz.: a good non-conducting wall; perfect drainage, with. air-tight foundations; ample ventilation at the top of the structure; solid icc, closely packed; sufficient protection between the packed ice and walls; storing the ice in dry, cold weather.

It is always well to construct an ice-house considerably larger than will contain what would be used during the season, as there will always be some waste, while, if the succeeding winter should be too warm to supply a good crop, enough may be left over to supply the deficiency. Besides, it costs but hitle more to build one of considerable size than a small one, and it is better to have an abundance of ice than to be limited in its use. A small ice-house also requires much more care in constructing than a large one, since the larger the quantity of ice stored within it, the better its temperature is preserved, and the less proportionate waste there will be from melting. A small quantity of ice stored requires very careful packing to prevent melting. Some prefer double walls packed tightly between with dry sawdust, or ground bark; others prefer single, tight board walls of one thickness, and fill in a foot of dry sawdust around on all sides between the ice and walls. This may be done to good adrantage, as the ice is deposited in successive layers, and the sawdust may be filled in perhaps more compactly and perfectly, than in the space between two walls or partitions, where it would be liable to settle, or openings form from other causes. Whether double or single walls are constructed, we should recommend that sawdust be always used in packing between the ice and walls.

A hill-side is sometimes chosen for the location of an ice-house, and the gable of one end built above the surface of the ground, in which an opening is made to put in the ice. The other end of the structure in which is the entrance, is exposed, being on a level with the floor. An under-ground ice-house is sometimes made by digging a cellar under a shed or well-ventilated building, and providing means for perfect drainage. An ice-house with a small room partitioned off in such a manner that it shall have ice on two or three sides of it, is a great convenience, as furnishing a nice cool place for keeping milk, meats, butter, or fruit in warm weather. We have seen plans of one that had ice on three sides and the top, and the exposed side opening on the north side of the structure. A milk-room adjoining an ice-house, by which it is kept cool, should have double walls and windows.

Plans for Ice-Houses. - Various plans and methods might be given for constructing ice-houses, but our space will not admit of but two or three, which, together with previous instructions relative to the essentials in such a structure, will serve to furnish suggestions for various plans that may be made according to the requirements of circurnstances.

A dry foundation with perfect drainage is essential. Where the soil is retentive of water, drainage should be provided by the use of drain-tiles or other equally effective means of securing it and conducting the water away. An ice-house 16 feet square and 10 feet high will hold 50 or more tons. The foundations may be of stone or brick; many simply lay down heavy planks or bed the sills in the earth. A stone or brick foundation will, of course, be more durable, although requiring more labor. Inside make a floor of cement, slightly concave, with an opening at the lowest point for conducting the drainage off by means of tiles. The walls may be double or single. If double, there should be a tight packing of sawdust or pulverized bark from eight to ten inches tbickness between them. If single, at least 
twelve inches of sawdust should be closely packed between the ice and wall. The walls, when double, are usually built with separate studding and posts, in which case the studs are joined by cross strips every few feet in order to strengthen the walls. Every precaution should be taken to render the foundation perfectly air-tight.

The drainage from the ice might be utilized in a more elaborate structure, by having it conducted to a milk-room, where it could be used for cooling the milk, or by constructing an apartment for this purpose partioned off from the ice-house. By this means the drainage from the ice could all be utilized, and the consumption of ice economized. The building may be covered with rough unplaned clapboards, and the roof shingled. The covering should he very close and tight, to prevent the entrance of warm air. If the walls are double and filled, the doors should be the same. A small door above the large one, will be convenient for removing the ice until it is taken out down to the lower door, when the latter can be used for the purpose.

Another plan for constructing a cheap ice-house which will hold from 35 to 40 tons is given by a good authority as follows:-The sills to be bedded in the ground, 2 by 12, and the inner studs, 2 by 6 , sheathed on both sides with common boards, the outside to be covered with felt paper, the space formed by sheathing to be filled as compactly as possible with dry sawdust, or tan bark. The outer studding to be 2 by 4 , spiked to outside of sheathing and covered with common siding, leaving a space under frieze, and above base, of three inches. The foundation to be of porous, sandy soil, or if of soil that will not admit of the ready escape of water, to be underdrained with tile. The floor to be constructed by spreading from six to eight inches of sawdust or tan bark, and after leveling it, cover with common boards, leaving about an inch space between each for the water to escape. The plates to be the same as studs, 2 by 12, rafters 2 by 4 . The roof should be shingled. Ventilators in the top of the roof 2 feet 6 inches square, to be surmounted by a small cupola with open slats. Doors double and filled with sawdust.

The full bill of lumber for the above is given as follows:-Eight pieces, 2 by 12 by 14 , for sills and plates; thirty pieces, 2 by 6 by 12, for inner studs; five pieces, 2 by 6 by 12, for hip-rafters and collar-beams; thirty-eight pieces, 2 by 4 by 12, for outer studs; twenty pieces, 2 by 4 by 12, for rafters and the ventilator; 750 feet siding, 14 feet long; 2,000 feet common boards, for sheathing, floor, roof, etc.; twenty-four pieces fencing, surfaced, 12 feet long, for corner-boards, etc.; 80 yards building-paper; 3,000 common shingles.

An elaborate structure is not necessary in order to preserve a good supply of ice; one of rough boards, and so simple in its construction that any farmer can make it who is in the least skilled in the use of tools, will answer the purpose, providing the essential rules previously given for its successful construction be observed.

How to Cut Ice.-In cutting a small supply of ice, such as the quantity required for a farm or dairy, but few tools will be needed. These may be comprised in a cross-cut saw, with one thimble and handle removed, or an ice-saw made especially for the purpose, an ax, and an ice-pole. The latter implement has two sharp points, one projecting at the end to push the blocks of ice, and the other bent down at right angles with the pole to draw with. Where very large quantities of ice are stored, horse-markers and cutters are used. The ice to be stored should always be cut into square, even-sized blocks. These should be marked out from sixteen to twenty inches each way by means of a long line stretched across the ice to guide the saw. Unless the blocks are cut of uniform size, so that they can be packed closely without empty spaces occurring between, ice will not keep well, even in the very best ice-houses. A farmer in Michigan, Mr. N. Atwell, gives his method as follows:-

"A good cross-cut saw is the most convenient tool to use. A good pair of ice tongs is the best instrument with which to haul the cakes out of the water, and also to handle and load them. The cakes should be as large as they can be without inconvenience in handling. 
I make the cakes $21 \frac{1}{2}$ by 27 inches. Twenty cakes of this size will complete one layer nine feet square. The second layer has the cakes placed crosswise of the layer below, and so on to the top. This binds the whole mass together. If the bottom layer is level, and the cakes are of uniform size with square edges, they will fit together nicely, making pounded ice between them unnecessary.

We find that it is less than a day's work for a man to saw out an abundant supply of ice for an ordinary family. During warm weather, ice will unavoidably melt from the outside of the mass, and if neglected, a racancy is soon formed between the rce and the sawdust. It is very important that the sawdust be packed down often, thus preventing the admission of warm air. When we commence marking and sawing ice, we find it an adrantage to make the headings widest where we commence to saw them. They can then be removed without binding or wedging fast."

How to Store Ice.-The floor of the ice-house should be corered from six to eight inches with sawdust, and the ice packed in as closely as possible, the cracks between the blocks carefully filled with broken ice, to make the entire mass perfectly solid, always, how. ever, leaving a space between the body of ice and wall of the building all around, to be filled compactly with sawdust as the packing continues. This space may be from eight to twelre inches, the wider the better protection from the outside heat. Where the walls are constructed double and filled with sawdust or tan bark, this precaution is sometimes not taken, but we should always recommend the practice, as there is less liability of the ice melting, even in such cases. This is especially necessary where the walls are not lined, as there would then be no protection from the heat of the walls, and the ice would soon all melt. In such cases the packing of sawdust between the ice and walls should not be less than a foot in width. Care should be used that no spaces be left open for the air. When the house is well filled, cover the whole mass of ice with two or three feet of sawdust, and keep the building closed as tight as possible, except the ventilators at the top of the structure.

The ice for packing should be solid and clear, and the storing done in dry, cold weather, that the whole mass may becorne thoroughly congealed and perfectly solid. It is well to have a refrigerator, or other means of preserving large pieces as they are taken out of the ice-house, in order to obviate the necessity of admitting the warm outside air to the interior of the building by opening it oftener than is absolutely essential. The best time for doing this is in the evening, or very early in the morning, when the air is coolest. As the ice in the building melts it will become uncovered in places, unless care is taken to keep a good supply of covering added, as it is occasionally removed. Therefore a sufficient amount of sawdust for this purpose should be kept on hand, and care taken to keep the ice well corered. As the ice is taken out from time to time, or has settled away, the sawdust should be packed down at the sides, and kept as compact as possible.

Keeping Ice Without Ice-Houses.-Ice may be kept very successfully without an ice-house, where only a small quantity is needed. A small room may be done off in a wagon. bouse, or other building, or in a clean basement in the barn, where ice may be stored by having a sufficient amount of suitable covering. A large bin with double walls, flled with sawdust or tan bark. and a few holes in the bottom to admit of drainage, will answer the purpose very well. But it must be remembered that in packing the ice under such circum. stances the same rules should be observed as in packing in ice-houses, except more outside covering will be required to exclude the air. For this purpose straw and bay are used very successfully. The American Agriculturist gives the following method of stacking ice, which may prove of benefit to those who have no ice-house:-

"If one has an abundance of ice, but no ice-house, and has straw in plentr, it may be worth while to stack up a lot, though it can hardly be expected to last all summer. The ice- 
stack is especially useful when the ice-house is not large enough to hold a fuil supply, if the ice is freely used. An ice-stack, to be drawn upon during the early part of summer, will allow the store in the house to be a long time undisturbed. If the stack can be made in a shady place, all the better; select a spot where the water will drain off, and lay down a tier of rails a foot or so apart; on these put a layer of brush, and upon the brush, straw to the thickness of a foot. If possible set a strong pole in the center. Now stack up the ice as in an ice-house, taking care that the mass dues not incline to one side. The covering for the sides may be straw, hay, swale hay, or even leaves, but the latter will need to be held in place by boards. A foot in thickness of protecting materials will do, but thicker will be better. Old boards, with braces to press them against the straw, etc., may be used if needed. The stack is to be finished by a roof of straw, put on with pins and ropes, as in finishing off a hay-stack. On grain farms, where straw is abundant, the mass of ice may be covered with a great thickness of straw, by building a stack of it over the ice. In using from such a stack the ice should be taken off on all sides regularly, and care taken to properly replace the covering. The larger such a stack the better. It should not be less than a cube of ice 12 feet on each side."

A cool, shady place should be chosen when the above method of stacking is practiced. Ice is sometimes stored in ice-wells in England, the ice being placed considerably below the surface of the ground. This plan is only practicable where arrangements are made for perpect drainage. The ice-house is a great improvement upon this method. Ice may be kept for a long time by storing it in the corner of some building, such as the wood-house, wagonbouse, etc. A thick bed of straw should first be spread upon the floor, upon which boards are placed loosely. A layer of six or eight inches of sawdust is spread above, and the ice closely packed, to be covered on all sides with sawdust and straw. Boards will be required to hold the straw upon the ice and around it. There should be, at least, two feet of straw on all sides of the ice to preserve it from the warm air. The coldest part of the building should be chosen for this purpose, a northwest corner being the best.

How to Keep Small Quantities of Yce in Sunmer.-A refrigerator is, of course, the best arrangement for keeping food cool by the use of a small amount of ice, and they are a very useful article of household furniture for thus preserving food in warm weather, but where it is desired to keep simply a small quantity of ice for a short time, and there is no ice-house, or if it is not desirable to open the ice-house frequently, we know of no better method than the following:-

Take a clean barrel that is perfectly tight, with the exception of one or two small holes in the bottom for drainage. Have on hand a bushel or more of dry sawdust. Spread about a peck of the saw-dust in the bottom of the barrel, and then put in the large lumps of ice; the larger they are the better they will keep. Be careful not to allow the ice to come in con. tact with the sides of the barrel, but have a thick layer of sawdust between. Cover this with sawdust, and put in more ice as before. Cover the whole with a thick layer of sawdust pressed down tightly, and over all put a folded woolen blanket, pressed down closely to exclude the air. Ice will keep much better in this way than when only folded in a blanket. The barrel should stand in the coolest place that can be found in the cellar.

How to Make Ice.-The securing of a sufficient supply of ice for summer use, although attended with but little labor or expense to the farmer residing near a pond, river, or other body of water, from which to procure it, yet for the farmer living remote from such sources of supply, the task is a more difficult one. For the benefit of such, we quote $\mathrm{Mr}$. Waring's method, which will be found both practicable, simple, and valuable:-

"Select a place on the north side of some building; lay a floor twelve feet square on scantlings, one foot from the ground. Set firmly in the ground, near each corner, two posts, from four to six inches square, and about eight or ten feet long. When the weather becomes 
cold, place on the floor sawdust, tan-bark, or rye-straw, to the depth of eight or ten inches. On the top place another floor of the same size, putting a curb inside the posts to keep the filling between the floors in its place. Next make a curb ten feet square and six inches deep, and fasten the corners with common gate-hooks. On a cold day place the curb on the center of the floor, put in two inches of tan-bark, and dash water over the bottom until it forms a coat of ice that will not leak. Fill the curb with water and let it stand until frozen solid. With boiling water thaw the curb loose, raise it to the top of the frozen mass, fill and freeze as before. Continue so doing until the mass is of the desired height. Place boards on the inside of the posts, and fill the space with tan-bark or rye-straw; nail boards on the outside of tho posts and fill the space with rye-straw; cover the top with tan-bark to the depth of ten inches. Over the whole put a roof, to shield from the sun and rain. Cut and take the ice from the top. Ice can be thus kept the entire season. If a stream of running water can be turned into the curb, the labor of filling will be much lessened."

Another method is to draw water from the well on severe cold days, and pour it into deep square tin pans or wooden boxes that will hold water. When it is frozen solid, apply hot water until the blocks of ice ean be taken out, when they should be stored in an icebouse or other place for the purpose, and the pans or boxes be again filled as before. By this means, ice can be manufactured with but little trouble. This should be done in the very coldest weather.

How to Construct a Cheap Conservatory.-Perbaps some farmers may consider directions with respect to the construction of a conservatory, scarcely admissible among those of farm bnildings, such a structure not being considered an essential adjunct to the farm, and but rarely seen in connection with the farmer's house. But this does not prove that such a desirable addition to any dwelling wouid not be just as bighly appreciated by the farmer's household, as any other, or that the attractions it might give to the farmer's bome, and the happiness and refining influence it would impart, would not many times repay the slight expense of its construction.

If farmers would make farm life attractive to their sons and danghters, and prevent their leaving it for more congenial surroundings and employment in the city, they must do more than is commonly done by the average farmer in this respect. Among the adornments of a home, a simple conservatory would prove a very desirable addition.

A veranda, or a portion of one, on the south side of a dweiling, can be very easily and cheaply made into a conservatory by simply enclosing it with sashes, thus having windows for the walls exposed to the sunlight. This will convert it into a cheap conservatory with an abundance of light which can be regulated by means of shades, if desired. All that remains is to provide shelves and standards for the plants. Heat for such a conservatory can be provided by a warm-air pipe from a furnace, or by a small stove. The sun will add much to the warmth during bright days.

Summer-Houses.-Rustic summer-houses and arbors are very pleasant to have on the premises, and may be very easily constructed. The farmer and his boys may do much in the way of such simple home ornamentation 3 by spending a few days in this manner when the farm work is not pressing. It is astonishing how much can be done towards making a home pleasant and attractive, by devoting an occasional day, or a few hours now and then, to such an object. Such structures need not be elaborate, or expensive. A lattice work of rough, unplaned material, when covered with vines, will answer the purpose just as well as one of nicely finished wood. Various plans for such structures can be devised, according to the skill and taste of the owner. Painting is not essential for the preservation of the wood. A much easier and cheaper method is to thoroughly saturate all the wood-work-as soon as the structure is completed-with crude petroleum, applied with a coarse brush. Light woodwork, when thus treated, will remain perfectly sound for many years, which, if not oiled, would decay in a short time. 



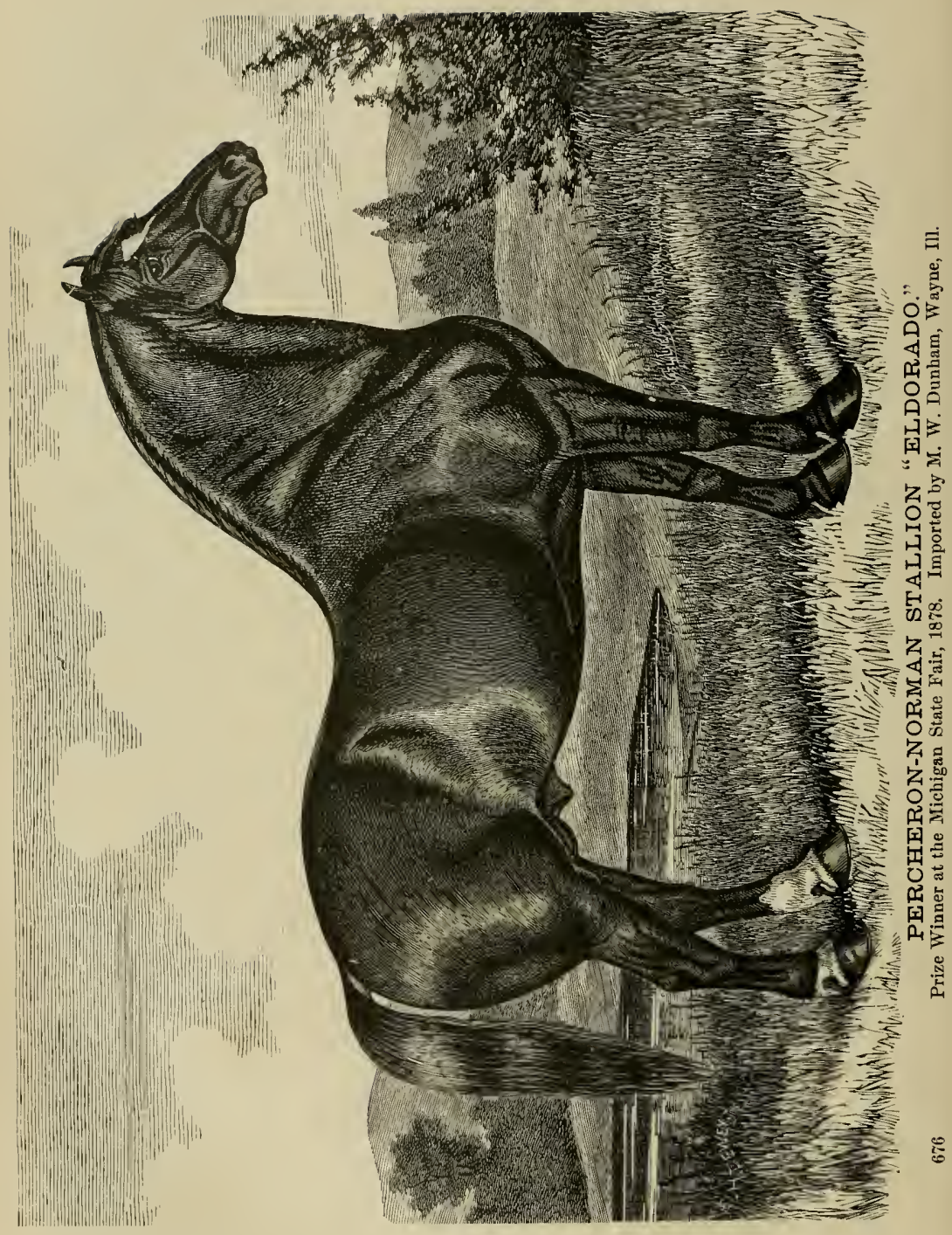




\section{DOMESTIC ANIMALS.}

\section{THE HORSE.}

T

$\mathrm{HE}$ history of the horse is an interesting one, and is closely connected with that of mankind, he having been in all ages of the world's record, man's willing and faithful servant, ready at all times to do his bidding - sharing his toils, hardships, and dangers. Whether on the field of battle, or aiding in the peaceful pursuits of life, he is ever faithful and true to his master.

$\mathrm{He}$ is also one of the most intelligent of the brute creation, second to none in this respect, -unless it be the dog,-while he is unexcelled by any of the other quadrupeds, wild or tame, in the beauty and elegance of form, and gracefulness of motion. Among the domestic ani. mals, the horse has been the one upon which the most time, attention, and affection have been bestowed by not only the most enlightened. but barbarous races also, and it is quite safe to assert that more money and intelligent effort have been expended in attempting to improve and perpetuate the distinguishing traits of the best breeds of this animal, than upon all the other domestic animals combined.

The affection which the wild Arab entertains for his horse, is an interesting feature of that barbarous race, and serves to counteract, in a measure, the undesirable characteristics, or, rather, causes us to regard them with more leniency. A human heart with a love for something, cannot be wholly depraved.

The horse, ass, zebra, quagga, and a few other similar animals, belong to the genus Equus. This term denotes a small group of quadrupeds of the Mammalia class, which have a single hoof, as the horse, ass, etc. The different species of the genus Equus may produce hybrids, but these hybrids are generally sterile, as in case of the mule, which is the product of the male ass and mare, or the hinny, the product of the staliion and female ass.

Horses exist in a wild state in various portions of the globe, but they are easily domesti. cated, even the progeny of those that have run wild for centuries. The period of their first domestication is unknown. Horsemen and chariots are mentioned in Genesis, in connection with the history of Joseph, in transferring his father's remains from Egypt to Canaan. The horse was also in common use among the Egyptians in the time of Moses.

At the time of the exodus of the Israelites, Pharoab had numerous war chariots drawn by horses. It is also stated that Solomon received inany horses from Egypt.

The horse has been domesticated for so many ages, that his original habitat is unknown, or the manner in which he was first trained; in fact, though frequently mentioned in history, but little was known respecting him until about four hundred years before Christ. Xeno. phon was the first writer who left us any statement with respect to the opinion of that age as to what were the best points of a horse, or who described in detail the proper method of training him, which method-strange as it may seem-might, in the main, be regarded as a standard authority at the present time.

From what we are able to learn, the horse was but little used by the ancients, except in war and the chase, and for these purposes the wild horses of the East were well adapted. It 
is, therefore, supposed that up to the commencement of the Christian era, the main characteristics of the horse had been but slightly changed by domestication, the principal changes being such as had been produced by different climates, soil, and food upon which they subsisted. Where the climate is cold and vegetation scanty, the wild horses are dwarfed in size, active and hardy, with compact bodies and long hair. The horses of Iceland, Shetland Isles, and the wild horses of the northern and central portions of Asia and Northern Europe generally, are of this type. These present a striking contrast with the high bred racers of England and the United States, the beautiful Arab, the active Barb, the powerful Clydesdales and Percheron-Normans, or the wild horses of America. The latter are known to be descended from domestic horses brought to this country from Europe by the Spaniards, - the thousands of wild horses on the plains of South America being, according to the best author. ity, descended from only two stallions and four mares which the early Spanish,adventurers left there.

Arabian horses, as a race, have been the most celebrated, while those of Turkey and Barbary (the latter called Barbs) are quite similar. From these, by a thorough and judicious system of breeding, have sprung the English race-horse, which at the present time so far surpasses the original, that scarcely any benefit has been derived from imported stock for more than three-quarters of a century.

The Persian horse has also been quite celebrated. It is more stoutly built than the Arabian, nearly equal to the latter in speed, but possesses less endurance. The Flemish and Dutch horses are generally large, well-formed animals, with fine chests, and an abundance of bone and muscle, and well adapted for purposes of draft. The celebrated draft horses of England were principally descended from these. The French horses are particularly valuable, the Norman breed having long been noted for their excellence. The most celebrated of the Russian breeds is the Orloff.

The Chinese horses are quite inferior. Italian horses were formerly quite highly valued, but do not compare favorably with the present improved breeds of some other countries. England is the source from which the best of our horses have been derived, the thoroughbred blood of our racers being obtained from that source.

The horse is invaluable to man in all climes and conditions, but willing and faithful servant that he is, he too often suffers from ill-treatment and neglect, and is but poorly recompensed for all his fidelity. There is no animal that has proven more useful to man, and none that has been so much abused. There is also no animal that so well deserves or amply repays by his services the best treatment.

The Arabs have the following tradition respecting the origin of the horse: "When God wished to create the horse He said to the south wind, 'I wish to form a creature out of thee, be thou condensed,' and the wind was condensed. And God formed a chestnut horse, saying, 'I have called thee, horse; I have created thee an Arab, and have given thee a chestnut color. I have bound fortune on the mane which falls over thine eyes ; thou shalt be chief among animals; men shall follow thee whithersoever thou goest; good for the pursuit, as for the retreat, thou shalt fly without wings; riches shall repose in thy loins, and wealth shall be made by thy intercession.' Then He marked him with the sign of glory and of happiness, a star shining in the middle of his forehead. After the creation of Adam, God called him by name, and said 'Choose now between the horse and the borak.' Adam replied, 'The more beautiful of the two is the horse.' And God said, 'Excellent, thou hast chosen thy glory, and the glory of thy sons; while they exist my blessing shall be with them, because $\mathrm{I}$ have not created anything that can be more dear to me than man and the horse.' "

Next to man he is one of the noblest and best of God's creations, and under proper training, and kind treatment, can be made to do everything that comes within the limitations of his powers. 
Naturally generous, affectionate, and confiding, he attaches himself to his friend and master, and is ever ready, with kind and yielding disposition, to do within the limits of his capacity all that can reasonably be required of him; while all that is necessary to make him kind, amiable, and gentle, and at the same time increase his knowledge, is to recognize this capacity, and by careful education develop his mental, and (we might almost add) moral qualities, to their fullest extent. Thus domesticated and taught by intelligent owners, the horse will not only increase in intelligence, docility, and consequent value, but will reciprocate the affection bestowed upon him, as is instanced in the well-known Arab custom, where the horse is domesticated to the extent, that he is at the same time servant of the master, and playmate of his children.

Qualities Desirable in a Horse.-While it is essential that the horse should be adapted to the purposes for which he is to be principally used, whether for the common purposes of the farm, for a draft-horse in drawing heavy loads, the carriage, saddle, or racecourse, there are certain characteristics which the animal should possess, aside from the qualities desired for special purposes. These may be included in a good disposition, a desirable temperament, strength, endurance, and activity. Beauty of form and color, gracefulness in motion, are also very desirable, although not absolutely essential for all purposes. There are many good horses that are lacking in the latter qualities, bnt we think it would be better to be at a little more expense in purchasing a horse, and procure one that looks well in all respects, than to be obliged to use an animal that is a constant offence to the eye and taste, even though it might be valuable as far as utility is concerned.

A Good Disposition.-A good disposition is highly essential in a horse, for without this the animal is almost worthless and exceedingly uusafe. Like the human race, horses differ greatly in disposition, no two being alike in this respect. Some are born naturally vicious, and many others are made so by ill-treatment.

A borse with a naturally bad disposition may, with kind treatment, become greatly changed in this respect, and yet such a horse is never really safe, for the evil of his nature may display itself at a time when least expected, and where an animal has so much within his power; it is always best to be on the safe side, which will be by never keeping a bad-tempered horse. A horse may be gentle for years; but if he does not possess a good disposition naturally, he will be liable to display his vicious temper in an unexpected moment, and therefore cannot be safely trusted. The disposition of a horse can be easily determined by even an inexperienced horseman, by the expression of the eye, shape of the head, the manner in which the animal moves his ears, his movements generally, and various other ways, any one of which, or all combined, are a pretty sure index of the temper of the animal.

Intelligence.-Intelligence is also equally essential. An intelligent horse can not only be made more useful, other things being equal, since he can be taught more readily, and to a greater extent, than a stupid animal, but he is generally more docile and kind in disposition, and also more safe, being less liable to be easily frightened and become unmanageable.

Streugth and Endurance.- Strength and endurance are very indispensable qualities. A horse that possesses but little of either is not of much value for any purpose whatever, and whether as a farm, draft, or carriage horse, must necessarily be used to disadvantage. Weak teams for any use are inefficient and unprofitable. Size and strength are not always combined in a proportionate degree in a horse; neither are strength and endurance. While as a general rule a large horse will be much stronger than a small one, yet there are exceptions to this rule, and many large, heavy horses are not as strong as they seem to be. The strength of a horse, while in a measure depending upon his size, depends much more upon his form and muscular development. For this reason, we frequently see medium-sized horses that are stronger than those 
that are much heavier. This is not always so, but is sometimes the case. A large horse with a good form and well-developed muscles, will be stronger, other conditions being equal, than a small or medium-sized one, with an equally well-developed form and muscles, but the size of the animal will not always prove an index of his strength. We specify thus partic. ulariy because we wish to be understood that strength does not always depend upon size. Many horses will also be very strong and able to draw heavy loads for a time, but do not possess the power of endurance that others of less strength may have. A horse may be in disposition, free and willing, and perhaps work beyond his strength, and consequentiy will be liable to be injured by continual severe labor, such as be is obliged to perform at certain busy' seasons on the farm. Irregularity in labor and in feeding, which are frequentily prac. ticed in the busy season on most farms, are very injurions to a horse, and a great strain upon their powers of endurance. A horse, as well as a man, will be able to perform mucb more labor in the aggregate, with less exhaustion to the physical system, by observing regularity in labor, performing about the same amount day after day, than to do an excessive amount of work for two or three weeks, and then lie idie about the same length of time. Regularity is in accordance with Nature's law, and a violation of this law is very liable to bring evil results. Farm labor, being much more severe at some times than at others, will test the powers of endurance of a horse much more than regular labor of some other kind, and therefore the farmer should have for this purpose a horse possessing great powers of endurance in connection with the other good qualities previously mentioned.

Activity.-Activity is also a desirable quality in a horse, and a sluggish drone to be avoided. For a carriage-horse this is very essential, and it is also very desirable in a farm. horse, which, with the majority of farmers, is the "general-purpose horse." A horse with a very nervous temperament, sprightly and active in movement, might prove restive and uneasy under the slow process by which much of the heavy farm work is performed. On the other hand, a sluggish horse, whose activity would not much surpass that of an ox, would prove quite as objectionable. A medium between the two would be an animal possessing a fair amount of activity, yet patient under restraint and admirably suited to all kinds of labor. There is a great difference in animals in this respect, and consequently in the amount of labor they are able to perform in a given time, therefore some horses will prove nearly twice as valuable to the farmer in this respect as others.

The Farm Horse.-As we have previously specified the qualities generally desirable in a horse, which qualities are especially essential in a farm horse, it will only be necessary in this counection to mention some points not referred to in the former, and which have a special application to this subject. On those farms where several horses are required to perform the work, and a special carriage-horse is kept, it is customary in some sections, especial-y in the Western States, to employ the heavy draft-horse for farm purposes, those showing great compactness of body and muscle, low, broad, and heavy-boned, with short neck, wide breast, and withers so formed as to throw the greatest weight into the collar. Such horses are admirably adapted for heavy work. But where the farmer is unable to keep more than one or two horses-and this comprises a large class of farmers-it is generally conceded that, for all the practical purposes of the farm, one of medium size is to be preferred. Under all circumstances, the animal should be adapted to the kind of work for which he is to be principally used. If for general use on the farm, he should neither be too heavy for the road or light work, or too light for heavy work, therefore a medium sized animal, under such circumstances, will best meet the requirements of the farmer. Hon. Geo. B. Loring, Commissioner of Agriculture, sketches in the following a pen-picture of his ideal of a farm horse:-

"When I commenced farming, I made up my mind that my horses should be as good 
as my sheep and cattle; that none of them should be surpassed; and that I would find out a way to breed and rear my own, instead of going into the market to purchase the fruits of other people's industry. I knew very well what I wanted. I did not want a running-horse, nor a saddle-horse, nor a cart-horse. I wanted a horse of all work-a horse weighing a little more than ten hundred pounds, in good road condition; fifteen hands and one inch high (for I had found that this height and weight usually go together); with a head not too fine, wide between the eyes, and high above them; with a good-sized, steady, erect, and lively ear; with every bony process sharp and prominent-even the processes of the first cervical vertebra behind the ears; with a calm and well-set eye, and lips which indicate determination rather than delicacy; a Websterian head, with a neck well muscled, well arched, strong, and elastic; with active motion, and a throttle loose and open; with withers not sharp and thin, but sclid and strong; with a shoulder set loosely on, broad and deep at the base ; with a strong arm, sinewy leg, short cannon-bone, firm and not too long or elastic a pastern, and a firm foot; with a deep chest, without a prominent and bulging breast-bone; with a round barrel, ribbed well back toward the hips, but not so far back as to interfere with the action of the hind-quarters; with a short back, and a slight elevation of the rump just behind the coupling; with a long and strong quarter, well muscled inside and outside; with a hind-leg so set on, that the action shall be free and open, and with the fore-leg so set on, that the toes shall not turn out for fear of brushing the knees at speed, and that they shall not turn in too much for fear of paddling. I wanted a good strong bay color with black points, and a temperament calm, collected, fearless, defiant, and a brain quick to learn, and strong to remember. This was the horse I wanted, and I felt sure I could breed him."

We may add with propriety, that Dr. Loring did breed him successfully, and so may any farmer who understands the true principles of breeding, and conforms to them.

The Carriage Horse.-The English carriage-horses are generally larger than those of this country. The carriages used in England are also larger and heavier than American ones, consequently there is a great demand there for large carriage-horses of elegant style and free, rapid action. In this country we have many trotting horses, but there seems to be a scarcity of the larger carriage-horses of the English type, although there is at present an increasing demand for them. As has been stated by one of our prominent agricultural writers -

"We have as yet no distinctive breeds of driving-horses or roadsters. The horses used for light driving, fast trotting, etc., are largely a conglomeration of all breeds and types. Some approximate the French Canadian pony in form and action, while others possess most of the characteristics of the thoroughbred; but so popular has fast trotting become in this country, and so universal is the fancy for fast driving-horses, that at almost all our fairs the roadster class will be found more largely represented than any other, and usually more largely than all others combined. Indeed, the roadster is more distinctly an American feature than any other in our equine product; and we are fast approaching the time when the American trotting-horse will be classed as a distinct breed. It is the creation of an American fancy - the result of a fashion that has demanded the fastest and stoutest trottinghorses in the world for driving on the road; and to this end we have selected and bred until our horses surpass all others in this particular. Among these horses we have several recog. nized families of especial prominence, all more or less related, but each possessing features that are to some extent peculiarly its own, but none of them entitled to be called a breed."

A good carriage-horse sliould have a moderately small head, free from much flesh, thin lips, open nostril, a kindly, expressive eye, full and lustrous, a broad forehead, wide between the eyes, but not between the ears, ears rathcr small, finely formed, quick and playful in motion; throat broad, neck slender, well set and arching; the skin thin, hair fine and glossy; chest deep, withers high; oblique shoulder; body and limbs well formed and adapted to 
strength and endurance; the hoof round, hard and smooth, wide at the beel, the frog sound and large. As we sluall define more particularly in the pages that follow, the desirable points in a horse, a more minute description in this connection is unnecessary; suffice to say, that intelligence, or what might be called good horse-sense, combined with docility, is one of the most desirable characteristics in a horse for any purpose, and especially a carriage-horse.

The Saddle Horse.-There seems at present to be an increasing demand for good saddle. horses, and we are glad to note the fact, as there is no more healthful or pleasant recreation than is afforded by saddle-riding, when the horse is perfectly adapted to the purpose. The requisites are similar in the main to those of a carriage-horse. For such use a rather small or medium-sized horse is generally preferred. High withers are essential in a saddle-horse, to prevent the weight of the rider from being thrown forward too far.

Saddle-Gaits.-We quote the following on saddle gaits from one of the leading authorities in the country: "The gaits that especially commend a horse for use in the saddle are, the walk, the fox-trot, the single-foot, and the rack. The walk is a gait understood by everybody; but everybody does not understand that a good saddle-horse ought to be able to go a square walk at the rate of five miles an hour. The fox-trot is faster than the square walk, and the horse will usually take a few steps at this gait when changing from a fast walk to a trot. It may be easily taught to most horses by urging them slightly beyond their ordinary walking speed, and, when they strike the fox-trot step, holding them to it. They will soon learn to like it, and it is one of the easiests of gaits for both horse and rider.

The single-foot differs somewhat from the fox-trot, and has been described as exactly intermediate between the true trot, and the true walk. Each foot appears to move independently of the other, with a sort of pit-a-pat, one-at-a-time motion, and it is a much faster gait than the fox-trot.

The rack is very nearly allied to the true pacing-gait, the difference being that in the latter the hind foot keeps exact time with the fore foot of the same side, making it what has been called a lateral or one-side-at-a-time motion, while in the former, the hind foot touches the ground slightly in advance of the fore foot on the same side. The rack is not so fast a gait as the true pace; but it is a very desirable gait in a saddle-horse. In addition, the per. fect saddle-horse should be able to trot, pace, and gallop, and should be quick, nervous, and elastic in all his motions, without a particle of dullness or sluggishness in his nature. His mouth should be sensitive, and he should respond instantly to the slightest motion of the rein in the hands of the rider. A poor and clumsy rider, however, will soon spoil the best-trained saddle-horse in the world, and such a person should never be permitted to mount a horse that is exceptionally valuable for that purpose. A "plug" horse and a "plug" rider may well go togetler; but keep a really good, well-trained saddle-horse for one who knows how to enjoy this most health-giving, exhilarating, and delightful of all out-door exercises."

Points of a Horse, or Marks by Which a Good Horse May be Known.To be familiar with those points, or marks by which a perfect animal may be known, or, in other words, (since absolute perfection in anything is impossible.) to become acquainted with the highest standard of excellence by which to judge of a horse, is of great importance and interest to any one who is about to purchase, breed, or have the care of horses.

As the face of a man, and the conformation of his head, are generally a very sure index of his character, or natural propensities, it is equally true that we may determine the disposition, temperament, and intelligence of the horse by the shape of the face and head, while his strength and power of endurance may be determined by the bones, muscles, and general make-up of the body.

In the same connection, there are other considerations that should not be lost sight of, 
and which are of great importance to the purchaser, whether the animal is designed for the track, carriage, farm, or draft horse, one of which is the pedigree of the animal. It is true that some very fine animals have been produced, as it were by accident, with no pedigree to which to trace their origin; but these are the rare exceptions, the general rule being, that like begets like.

Again, it is of importance to know how a horse has been raised, whether he has received kind treatment or has been ill-treated to the injury of his disposition and physical powers; what has been his principal food, etc. There are localities in the country where young horses are fed very much as swine are, while there are others in which grass, hay, and oats are the principal food of the colt, diet which tends to the production and development of the bone and muscle required for hard service, whether on the road or at the plow. The mature horse, it is true, may be fed moderately on corn without in. jury to the animal; but, in $\mathrm{th}$ is connection, it should be remembered that corn and the production of beef and pork are more intimately related than corn and horse-flesh are, and that what is needed in the horse is hardihood and endurance. If to these we add speed, we have the American trotter; while if to these we add strength, we have the American work-horse

The illustration above given, showing the several parts of the horse, and

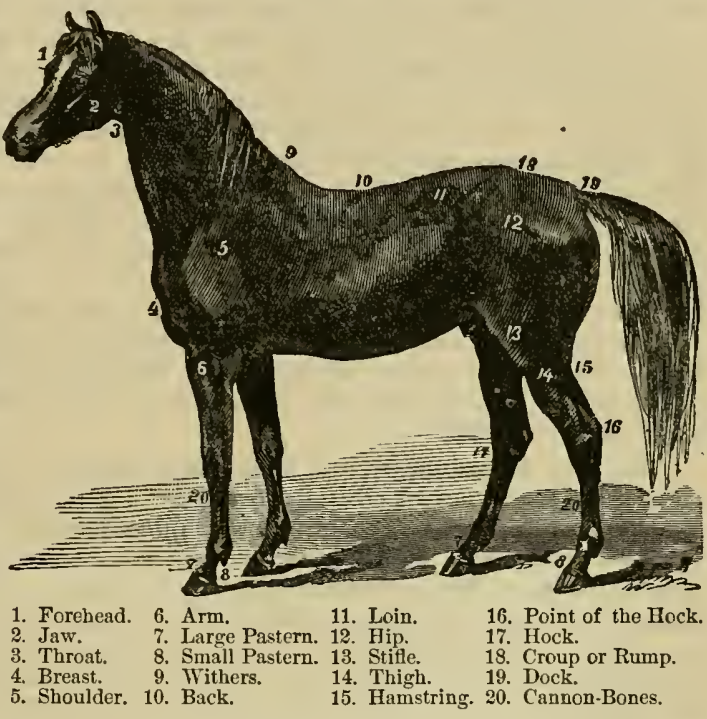
indicated by terms recognized by horsemen generally, will prove of value to those not already familiar with them.

Temperament. - With animals, as with individuals, there are different temperaments. One of the most essential points to be observed concerning horses is the temperament. There should always be a distinguishing difference recognized between temperament and temper, or disposition. This difference is very aptly defined by Murray, as follows:- "The temper is an accident, the result of education, or treatment; in rare instances, of birth; but the temperament is a law or mode of being affecting and modifying the physical structure and the nervous forces. The temper can be modified or changed - the vicious can be made amiable, and the amiable vicious. Not so with the temperament; that is fixed at birth, and remains immutable, dominating over the entire organization. Diet, training, treatment in sickness - these, and much beside, are suggested to the thoughtful mind by the temperament of the horse.

I could show that this matter of temperamental organization of the horse potentially affects the entire animal - even every minute point of the physical structure, and each separate part and function of the body. If the temperament be an active, lively one, then 
will the bones be fine in their texture, ivory-like, and lasting. The muscles, also, will be influenced, and become wiry, compact, and elastic as spiral wire. If the temperament, on the other hand, be sluggish, heavy, lymphatic, the bones will be spongy and porous in their structure, the muscles flaccid and coarse, and the nervous organization low, dull, and inoperant. I am well aware that size, all else being equal, is a true gauge of power; but let it never be forgotten by the breeder and purchaser of the horse, that 'all else' is not equal. Size alone is no measure of power; for all can see, even with the most casual examination of the subject, that the slightest alteration in temperament makes a corresponding alteration in the power and efficiency of every individual part.

A horse does not draw by virtue of his weight, nor in proportion to his size. The public scales and the measuring-tape can never assure us how much a horse can draw, or how many miles he can pull a wagon and its owner in a day. Muscular action and nerve-force must be considered; and these are both closely allied to, and dependent on, the temperament of the animal. The well-bred horse, inch for inch, and pound for pound, is far stronger than the dray-horse; and old 'Justin Morgan,' the founder of the most wonderful family of horses (all things being considered) this or any country ever saw, could draw logs that horses of twelve and thirteen hundred pounds could not even start, albeit he weighed only about nine hundred pounds, and stood barely fourteen and a half hands high. It is the amount of vital force that, at the end of a stick of timber, or on a weary day's journey on a heavy road, tells the story."

There are four distinct temperaments generally recognized, which are, however, but rarely found separate, being usually blended and mingled in a greater or less proportionate degree. They are the nervous, bilious, sanguine, and lymphatic. When not separate, one will be found to so predominate over others as to stamp its characteristic upon the animal, and be easily recognized.

Nervous Temperament.-A horse with a nervous temperament will have an animated expression of face, quick-moving ears, and will be characterized by quickness in all his movements, nervous excitability, and extreme sensitiveness. Combined with these, he will have a large brain. Such a horse will chafe under restraint, like being forced to do work that requires slow movements, as plowing, for instance, and when allowed to test his strength in drawing heavy loads, or doing hard work of any kind, will be liable to over-work himself and be permanently injured. A horse of this kind will suffer extremely from harsh treatment of any kind, even harsh tones of voice, and will be liable to take fright and shy out quickly when on the road, unless the driver is on his guard. The "Vermont Black Hawk" has been instanced as one of the best types of this class of horses.

Bilious Temperament.-The bilious temperament is characterized by a well-developed muscular system, and a horse of this class will have large bones and muscles, and be capable of great strength and powers of endurance. Such a horse, possessing great muscular power, will be able to perform a vast amount of hard labor, without breaking down.

Sargnine Temperament.--Next follows in order the sanguine temperament, which is associated with a large development of the vital organs - the heart, lungs, etc. An animal possessing this temperament will be what is called "long-winded," and whatever he does will be done with ease, and but little appearance of panting or exhaustion after being driven fast. Having large and well-developed vital organs, his digestion will be exceedingly good and the food he eats will be assimilated and appetized, so that food and rest will be to him truly " nature's repair shop," and day by day he will go forth fresh for a trial of speed or strength.

Iymplatic Temperament. - A horse with a lymphatic temperament will be lazy and dull. He will generally be a heavy animal with a surplus of fat, and slow-moving and stumbling in his gait. Such a horse is scarcely worth the whip that drives him, and the driver will well earn the work he gets out of him. 



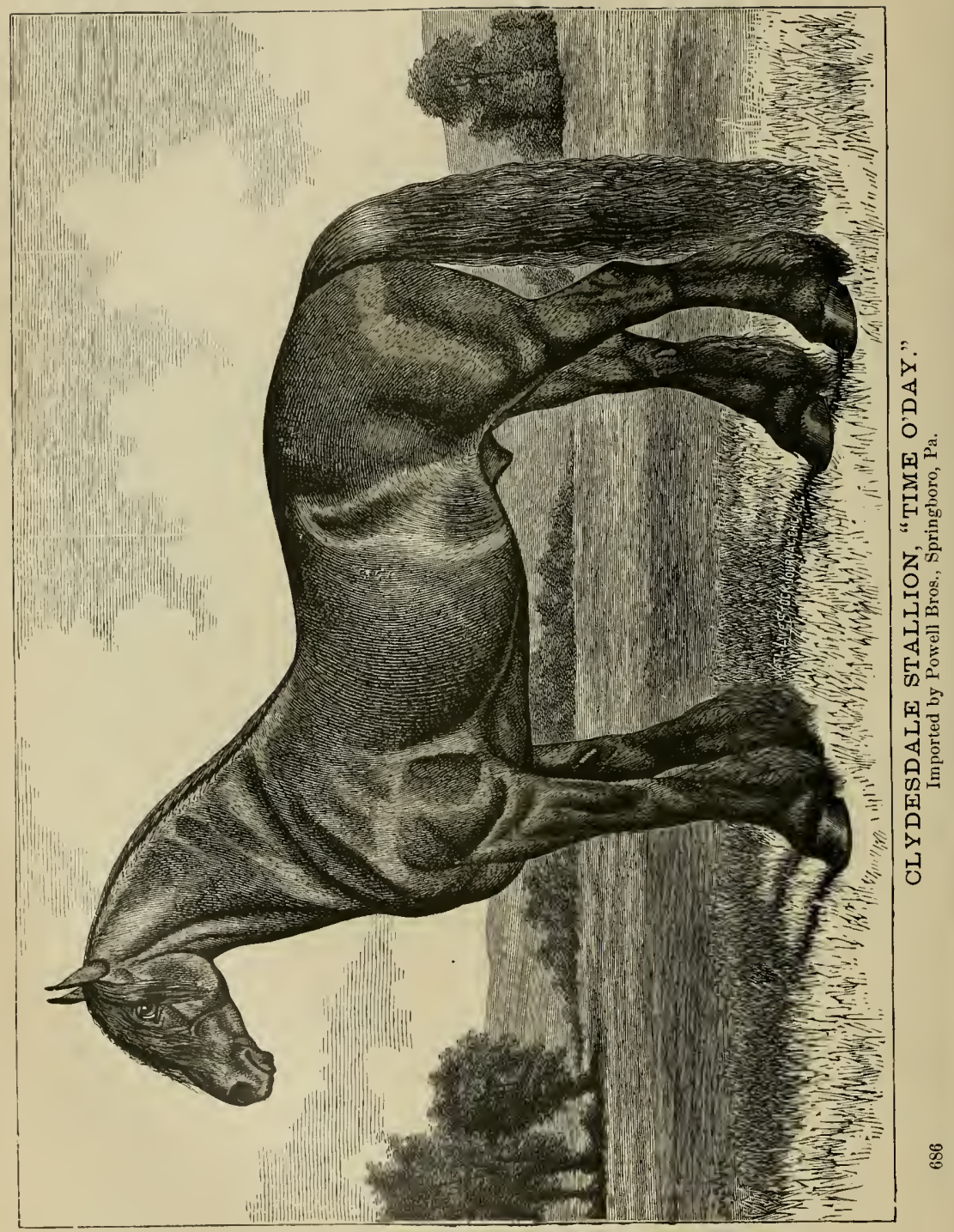


The Eye. - It has been said that the eye is the window through which we may look into the soul of a man. As this is the most expressive feature of the human face, and the one by which we can best determine the disposition of the individual, so with the horse; some of the most important characteristics of the animal may be read in his eye. If you do not like the eye of a horse, have nothing whatever to do with him, for you will not like the animal. The eye of a horse should be intelligent and kindly in expression, yet full of courage. and characterized by mildness and gentleness. It should also be rather prominent and full. The nearer the eye of a horse approaches that of the deer, in expression, the better. A horse that is frequently looking back of him furtively, so as to show the white of the eye, is generally apt to be mischievous, and is not to be trusted.

The Ears. - The ears of a horse, as well as the eye, may express much. They should be fine in texture, that is, thin, in which the veins are readily traced; rather sinall, and not too long proportionately for the head that carries them. They should be curved slightly inward at the tips, and covered with fine, short hair. They should be set rather close together at the base, quick and playful in movement. A horse that is in the habit of laying his ears back, down close to the head, is not trusty, and will be inclined to kick or bite if he gets a chance.

The Head. - The following extract from the description of the head of a perfect horse, by Carson, will be found to contain many excellent suggestions: "The head of every horse should be as small as would be in keeping with the rest of his body. A large, coarse head is a defect in every person's eye; and it has no advantages to counterbalance its deformity. The muzzle should be fine, and of moderate length; the mouth invariably deep for receiving and retaining the bit; and the lip rather thin and firmly compressed. A fine, tight lip is a pretty sure indication of an active temperament, and consequently affords a measure of the energy and durability of the animal. Horses with short, flabby lips, lying wide apart are proverbial for sluggishness. The nostrils should be large, so as to be capable, when open, of allowing the air to have free access to the lungs. In conformity with the uniform condition of the Creator's work, it will be found that there is a direct relation between the development of the nostrils, and the capacity of the lungs for air. Hence arises the necessity of observing the size of the nostrils. Capacious lungs would be of no use if the orifice which connects them with the external atmosphere were so concentrated that they could not get properly filled.

The race-horse must have very wide and dilatable nostrils to admit a large volume of air, with the utmost freedom and greatest speed, into his widely and rapidly distended lungs; but the horse of slow work can take more time in his breathing, and consequently does not require such a very large nostril as the racer, hunter, or steeple-chaser. Care must always be taken, recollect, not to confound a naturally well.developed nostril with one which looks large in consequence of having been kept in a state of permanent distention by disease of the lungs or air-passages.

The muzzle ought to be fine a good way up, and then the parts should enlarge suddenly, in order to give plenty of breadth to the under-jaw, as well as thickness from side to side. This is a point of great beauty, as it gives breadth to the jaw-blade, and breadth from eye to eye, whilst the fineness of the head generally is maintained. A head that is narrow between the eyes, and narrow on the side of the jaw, is painfully disagreeable to the eye of every judge. The space between the two blades of the under-jaw ought to be so broad and so deep as to freely admit the lower edge of the neck when the chin is reined in towards the counter; but it should not be wider than this, as it would then appear coarse. If there is sufficient room in this locality, the horse can be reined up to the proper pitch without stopping up his windpipe. 
The face, on a side-view, should be dipped in the center between the eyes and the nose. This is generally the case in the Arabian and English blood-horse; and it is a much more beautiful formation than either the straight or convex profile. However ornamental it inay be to the human face, a Roman nose certainly does not improve the appearance of the horse. The line of beauty in the one case is very different from the other. A dish-faced horse is admired on all hands; but a pug-nosed man, with a projecting, upturned chin, will have some difficulty in carrying off the prize for beauty. The face must be very broad between the eyes; but it should taper a little as it approaches the ears. If the breadth is carried all the way upwards, the top of the head will be too wide, the ears ill-set, and the horse probably sulky. Now, in respect to the head, it should also be examined in detail, for in it are dis. tiuct organs having distinct uses, and each contributing its share to the proper understanding of the animal to which they belong, and to which they serve. But, of all these organs, perlaps the eye is the most expressive and characteristic of them all.

The portion of the bead lying between the eyes and the ears is worthy of the closest possible attention; for it is the section occupied by the brain itself, - the seat of all intelli. gence, docility, and motive-power. This section of the head can scarcely be too full. I would never breed a mare to a stallion deficient at this point of his structure. I want no colts from a sire with a flat forehead; for such a horse is a savage, sulky, detestable brute. To start with, he will have no memory: he will forget to-morrow what you taught him to-day. Even if he wished to remember it, he could not; for he is incapable. To a bad memory must be added a bad disposition. He is sour, cross, and crabbed, tricky and malignant. His cunning is not playful, but mean; and his tricks are tricks of cruelty. No one ever saw a horse, with such formation of front, tractable and trusty.

But if, on the other hand, you meet a horse with a bold, prominent forehead, a noble fullness at that point where the brain is lodged, you will find him to be of a docile and silky disposition. You can teach him anything; and, when once taught, he will rarely if ever forget. Indeed, his great intelligence suggests to his owner a caution: Never teach him to do anything that you do not desire him to do always, and at all times; for whatever he has once acquired you can only with great difficulty eradicate. I do not wish to be understood as saying that every horse with a fine brain development is gentle; for he may have been trained under a system so essentially vicious, that no natural amiability could withstand its savage friction; but this I do wish to be understood as saying, - that every borse with this full and fine brain development is by nature courageous, docile, and loving; and that, if they become otherwise, it is owing to the vicious management of those who have them in charge."

The Neck. - The neck should be suited to the animal. A neck desirable for the trotter, would be entirely unsuited to the draft horse, and the reverse. The neck should vary according to the service for which the animal is designed. For speed, the nearer the neck approaches the greyhound type, the better, provided it admits of sufficient room for food and air. If, however, the length and lightness of the neck be carried to extreme in breeding, there will be danger of constitutional weakness as a result.

For draft horses, a heavy, thick neck, where it enters the shoulder, is very essential. Mnch of the beauty and elegance of style in a horse depends upon the form of the neck. It is much more easy to the hand in driving a horse with a long neck, than a short one. Many horses have their necks spoiled by the improper use of the check-rein in producing too much of a curvature, or in drawing the head back too far. This practice not only detracts greatly from the beauty of the animal, but is equally cruel, and causes much discomfort and suffering by forcing the neck and head out of its natural position. Many otherwise fine animals are thus spoiled through the ignorance and false notions of beauty in their owners. 
The Chest. - The chest of the horse should be large and roomy, as it contains the vital organs, the heart, lungs, etc., upon the free action of which the perfect health and strength of the whole system so largely depends. If the heart be in a healthy condition, the entire system will be very likely to be healthy; but if the heart be diseased, there will be weakness or disease of the physical system. Neither this organ, or the lungs, can be in a vigorous and healthy state, unless they are well developed, and are allowed sufficient room for action.

The blood is circulated by the action of the heart, but it can only be purified by coming in contact with the air, in passing through the lungs. Now, if the lungs and heart are com. pressed within a narrow chest, it can readily be seen that such an animal will lack one of the great essentials of a perfectly developed, healthy body. The lungs of the horse occupy a much larger space when he is in active exercise than when at rest, consequently the more speed is required of him, the greater the necessity of his being able to increase the size of the chest, to give ample room for these organs.

The following, descriptive of the proper form of the chest, is from Youatt's work, the noted English authority on the horse: "The front of the chest is a very important consideration in the structure of the horse. It should be prominent, broad, and full, and the sides of it well occupied. When the breast is narrow, the chest has generally the same appearance: the animal is flat-sided, the proper cavity of the chest is diminished, and the stamina of the horse is materially diminished, although, perhaps, his speed for short distances may not be affected. When the chest is narrow, and the fore-legs are too close together, in addition to the want of bottom, they will interfere with each other, and there will be wounds on the fetlocks, and bruises below the knee.

A chest too broad is not desirable, but a fleshy and a prominent one: yet even this, perhaps, may require some explanation. When the fore-legs appear to recede, and to shelter themselves under the body, there is a faulty position of the fore-limbs, a bend, or standing over, an unnatural lengthiness about the fore parts of the breast, sadly disadvantageous in progression.

The spaces between the ribs are occupied by muscles firmly attached to their edges, the fibers of which cross each other in the form of the letter $\mathrm{X}$. By the prolongation thus obtained, they have a much greater latitude of action than they would have if they run straight from rib to rib. The ribs, while they protect the important viscera of the thorax from injury, are powerful agents in extending and contracting the chest in the alternate inspiration and expiration of air.

This leads to a very important consideration, the most advantageous form of the chest for the proper discharge of the natural or extraordinary functions of the thoracic viscera. The contents of the chest are the lungs and heart:-the first, to render the blood nutrient and stimulating, and to give or restore it to that vitality which will enable it to support every part of the frame in the discharge of its function, and, devoid of which, the complicated and beautiful machine is inert and dead; and the second, to convey this purified arterialized blood to every part of the frame.

In order to produce, and to convey to the various parts, a sufficient quantity of blood, these organs must be large. If it amounts not to hypertrophy, the larger the heart and the larger the lungs, the more rapid the process of nutrition, and the more perfect the discharge of every animal function.

Then it might be imagined that, as a circle is a figure which contains more than any other of equal girth and admeasurement, a circular form of the chest would be most advan. tageous. Not exactly so; for the contents of the chest are alternately expanding and contracting. The circular chest could not expand, but every change of form would be a diminution of capacity. 
That form of chest which approaches nearest to a circle, while it admits of sufficient expansion and contraction, is the best-certainly for some animals, and for all under peculiar circumstances, and with reference to the discharge of certain functions. This was the grand principle on which Mr. Bakewell proceeded, and on which all our improvements in the breeding of cattle were founded.

In the heavy draft-horse, the circular chest is no disadvantage, and it gives him what we require, weight to oppose the weight of his load. Speed is not demanded of him.

Some of our saddle-horses and cobs have barrels round enough, and we value them on account of it, for they are always in condition, and they rarely tire. But when we look at them more carefully, there is just that departure from the circular form of which mention has been made-that happy medium between the circle, and the ellipse, which retains the capacity of the one, and the expansibility of the other. Such a horse is invaluable for common purposes, but be is seldom a horse of speed. If he is permitted to go his own pace, and that not a slow one, he will work on forever; but if he is too much hurried, he is soon distressed.

Then for the usual purposes of the road, and more particularly for rapid progression, search is made for that form of the chest which shall unite, and to as great a degree as possible, considerable capacity in a quiescent state, and the power of increasing that capacity when the animal requires it. There must be the broad chest for the production of muscles and sinews, and the deep chest to give the capacity or power of furnishing arterial blood equal to the most rapid exhaustion of vitality.

This form of the chest is consistent with all the lightness that can be rationally required. The broad-chested horse, or he that with moderate depth at the girth swells and barrels out immediately behind the elbow, may have as light a forehead and as elevated a wither as the horse with the narrowest chest, but the animal with the barrel approaching too near to rotundity is invariably heavy about the shoulders and low at the withers. It is to the mixture of the Arabian blood that we principally owe this peculiar and advantageous formation of the horse. The arch is light, some would say too much so before, but immediately behind the arms, the barrel almost invariably swells out, and leaves plenty of room where it is most wanted for the play of the lungs, and at the same time where the weight does not press so exclusively on the fore-legs, and expose the feet to concussion and injury.

Many horses with narrow chests, and a great deal of daylight under them, have plenty of spirit and willingness for work. They show themselves off well, and exhibit the address and gratify the ranity of their riders on the parade, or on the park, but they have not the appetite nor the endurance that will carry them through three successive days of hard work.

Five out of six of the animals that die from inflamed lungs are narrow-chested. There are many other importaut points, but that which is most of all connected with the general health of the animal, and with combined fleetness or bottom, is a deep, broad, and swelling chest, with sufficient lengthening of the sternum, or breast-bone, beneath. The impropriety of tightgirthing may readily be seen, especially where it is unnecessary, as in the stable, for instance, or when the rider is off from the saddle.

The sternum, or breast-bone, is a long, flat, spongy bone, forming the floor of the chest. It supports the ribs by the connecting cartilage, and is composed of from seven to nine pieces united together by cartilage. The point of the breast-bone is occasionally injured by blows, and has even been completely broken off. A kind of tumor on it, difficult to heal, has also been produced by some cruelty or violence."

Again the same authority says:- "The question then is, what service is required from the horse? If he has to carry a heavy weight, and has much work to do, he should be ribbed home-the last rib and the hip-bone should not be far from each other. There is more capacity of chest and of belly-there is less distance between the points of support- 
and greater strength and endurance. A hackney (and we would almost say a hunter) can scarcely be too well ribbed home.

If speed, however, is required, there must be room for the full action of the hinder limbs; and this can only exist where there is sufficient space between the last rib and the hip.bone. The owner of the horse must make up his mind as to what he wants from him, and be satis. fied if he obtains that; for let him be assured that he cannot have everything, for this would require those differences of conformation that cannot possibly exist in the same animal."

The Bones.-The bones are the frame-work of the system, and give support and protection to the body. It must be remembered that size is not the true index of strength with respect to the bones of an animal, but rather, size and texture combined. Large bones are often coarse and porous in texture, while small bones will frequently be found hard and firm, and possessing unch more strength than those of larger size, as is shown, for instance, by a comparison made between a section of the cannon-bone of a low-bred cart-horse, and that of a thorough-bred animal. The former was found open and porous, while the latter is firm and solid; in fact, so compact that it may be polished to resemble ivory. The leg of a thor. ough-bred horse, although much smaller than that of a cart-horse, may, as shown, be much stronger than the latter; therefore, although the bones should be of suitable size for the purposes to which the horse is to be used, their texture is of equal importance, and should not be overlooked, by either the purchaser or breeder.

The Shoulder.-The shoulder should vary, according to the type of the horse, and the purposes for which he is to be used. The draught-horse should have a large, powerful neck, an upright, thick, and heavy shoulder, and, withal, there must be an abundance of muscle, flesh, and fiber, so that the weighty collar he is obliged to wear will fit well, and seem, as it were, a part of him, thus distributing the pressure evenly, in drawing heavy loads.

For saddle and carriage use, the shoulder should be oblique, and the withers high, as this gives the animal more freedom in moving, and renders him less liable to stumble when on the road.

The slope of the shoulder also adds to his capacity to stride with ease, and thus renders a horse well adapted to saddle and carriage use. The rule laid down by Youatt and others is, that the oblique shoulder, being less exposed to concussion in rapid action, is indispensable to horses where extensive and rapid action is required, but for horses where use requires them to throw as much weight as possible into the collar, an upright shoulder is of more advantage. Roadsters and what are commonly termed gentlemen's carriage-horses, as well as saddle-horses, should, therefore, have oblique shoulders, while those that are used principally for draught, should have the shoulders upright.

The humerus, or second bone in the shoulder, should be of good length, and supplied with powerful muscles, while a good fore-arm is equally essential.

The Limbs.- However superior a horse may be in all respects relative to his head and body, he becomes ineficient unless these desirable qualities are accompanied with good limbs and feet.

It is, therefore, of the greatest importance that the horse not only bave limbs suited to the body, and the duties he is required to perform, but that they, as well as the feet, receive good care, in order to be kept in a sound and healthy condition.

Youatt says: "Whatever other good points the animal may possess, if the arm is narrow in front and near the shoulders, flat on the side, and altogether deficient in muscular appearance, that horse is radically defective. He can neither raise his knee for rapid action, nor throw his leg sufficiently forward."

The fore-arm, or radius, can scarcely be too long, or well supplied with muscles, as these muscles move the leg and foot. The cannon bone should be small, short, hard, and solid. A short cannon usually accompanies a long fore-arm, especially in horses of great speed. 
The arm should be large where it joins the body, admitting of strength by giving it a frm attachment to the trunk. The sinews of the leg should be large, indicative of large muscles and strength. A broad, flat leg denotes great strength and endurance.

The following cuts will serve to give a better idea of the bones of the lower limbs of a horse, than can be given by a verbal description:-

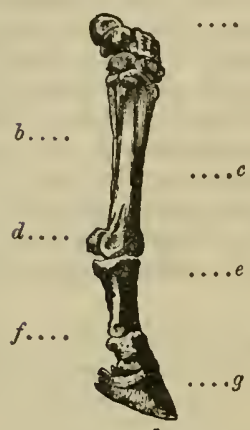

FIG. 1.

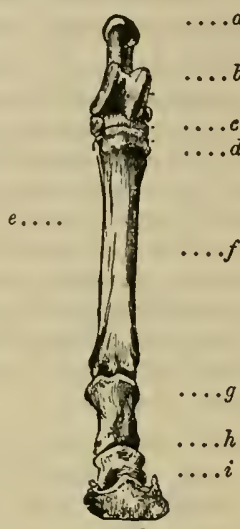

FIG. 2 .

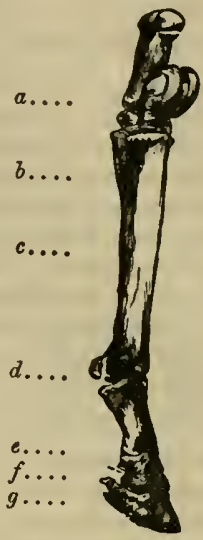

FIO. 3 .

Fig. 1 shows a side view of the bones of the fore leg; $a$ represents the bones of carpus ; $b$, splent, or what is sometimes termed the splint bone; $c$, cannon bone; $d$, sesamoid bone; $e$, pastern bone; $f$, coronet bone; $g$, coffin bone.

Fig. 2 represents the front view of bones of the hind legs; $a, b, c, d, e$, bones of the tarsus ; $f$, cannon bone ; $g$, pastern bone; $h$, coronet bone ; $i$, coffin bone.

Fig. 3 illustrates a side view of the hind leg; $a$, bones of the tarsus ; $b$, splint bone; $c$, cannon bone; $d$, sesamoid bone ; $e$, pastern bone ; $f$, coronet bone ; $g$, coffin bone.

These, together with a cut of the skeleton of a horse hereafter given in connection with diseases of this animal and their treatment, will enable the reader to obtain a correct idea of the location of all the bones.

With regard to the position of the fore leg when standing, Carson says: "The leg should drop perfectly straight from its junction with the shoulder to the ground; and the point of the toe should come as near as possible to a straight line under the point of the shoulder."

The Pastern.--In the draft horse the pastern should be strong, short, and nearly upright, while in the trotter it should be long and well slanted, so as to give the movement of the horse that easy, elastic, springing character that relieves the ends of the upright bones of that fearful concussion which would otherwise invariably occur when trotting upon the racecourse or in runuing and leaping in hurdle races. Besides, when under saddle, horses that have long pasterns are easy and comfortable for the rider. But in this, as in almost everything else, the "happy medium" is to be preferred for general purposes, for if the pasterns are too long, or slope too much, there will be a tendency to spraining or weakness of the hask tendons.

Fig. 4 represents the pasterns of a horse.

a. The shank bone

b. The upper and larger pastern bone. 
c. The sesamoid bone.

d. The lower or smaller pastern bone.

e. The navicular or shuttle bone.

$f$. The coffin bone or bone of the foot.

g. The suspensory ligament inserted into the sesamoid bone.

$h$. A continuation of the suspensory ligament, inserted into the smallcr pastern bone.

$i$. The small inelastic ligament, tying down the sesamoid bone to the larger pastern bone.

k. A long ligament reaching from the pastern bone to the knee.

l. The extensor tendon inserted into both the pasterns and the coffin bone.

$m$. The tendon of the perforating flexor inserted into the coffin bone, after having passed over the navicular bone.

n. The seat of the navicular joint lameness.

o. The inner or sensible frog.

$p$. The cleft of the horny frog.

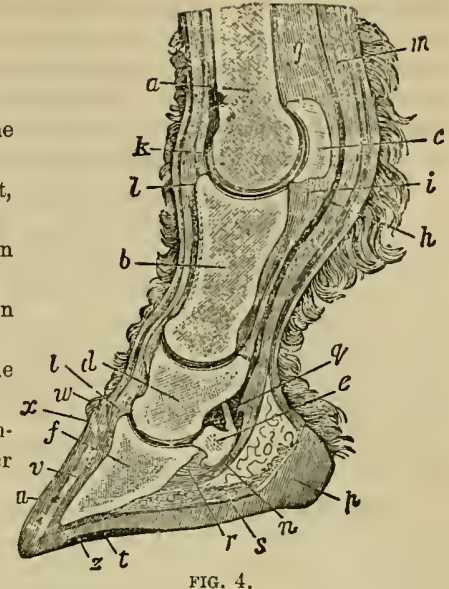

q. A ligament uniting the navicular bone to the smaller pastern.

$r$. A ligament uniting the navicular bone to the coffin bone.

$s$. The sensible sole between the coffin bone and the horny sole.

$t$. The horny sole.

$u$. The crust or wall of the foot.

$v$. The sensible laminæ to which the crust is attached.

w. The coronary ring of the crust.

$x$. The covermg of the coronary ligament from which the crust is secreted.

z. Place of bleeding at the toe.

The Hock.-This is where lameness frequently occurs in a horse, therefore in order to avoid difficulty in this respect, and that it may be strong enough to bear the severe strain that hard labor brings upon this part of the leg, the hock should be large. It is well, then, to look for large, well-proportioned hocks in a horse, whether it be for trotting, draft, or general purposes. The line of distance between the hip and hock should be long, thus bringing the latter low down towards the foot.

The Foot.-The foot of a horse should be of medium size, neither too large nor too small. A large flat foot is very undesirable, and suggestive of a coarse and porous bony structure above it. The animals that are most fleet, such as the antelope and deer, have small hoofs. The wild horse also has a hoof of rather small or medium size. The different parts of the foot are somewhat complicated, but the design in their formation is simple and easily understood. The hoof is made up of a series of thin layers of horn, nearly five hundred in number, nicely fitted the one to the other, and forming a firm and solid lining to the foot itself.

Besides this, there are numerous other layers which are elastic belonging to what is termed the "coffin bone," and which are fitted into this. It is estimated that there are about four thousand elastic layers in the four feet of the horse, upon which the weight of the entire body rests, which enable them not only to bear the great weight of the body, but also the additional burdens that may be placed upon him.

The flat foot is usually weak and troublesome to both the horse and his owner. The 
length of the foot on the ground should be greater than its breadth, and the breadth should be greatest across the center, lessening towards both the toe and heel. The heel should be of medium height, neither too high nor too low. The hoof should also be of fine texture, smooth and tough-looking. A brittle hoof is very objectionable. The frog should be large and healthy, and never be cut, or otherwise tampered with. Reasons for this will be fully given in connection with the subject of shoeing.

The Back.-It is generally conceded by all experienced horsemen the world over that, other conditions being equal, the strength of a horse's back is inversely in proportion to its length, the short-back being the strong back, and the long back the weak one. Consequently the short-backed horse has proportionately greater powers of endurance as well as strength. Flora Temple was a short-backed horse, and usually ended her victorious summer campaign iu a better condition than when she began. Maud S., Governor Sprague, Taggart's Abdallah, Dexter, Thorndale, and other noted animals, are also horses of this type. Such a formation of the spinal column as produces considerable of a dip or hollow immediately back of the withers is objectionable, since it interferes with the space which should be occupied by the heart and lungs, and affords them less room than is essential for their perfect development.

The Hind Quarters.-Here is where both speed and strength lie, while the fore legs are designed, for the most part, for support. They are, in fact, the great moving power of the body. These impel the body forward through the air, and also start the heavy loads. The hind-quarters of the animal should, therefore, be so constructed as to admit of great strength and endurance, which should be with long bones well placed, and long powerful muscles, by means of which these bones are to be moved.

The rump should be of medium width, although narrowness at this point should be avoided, being an indication of a lack of strength.

The thigh bone is heavily overlaid with large, powerful muscles, which constitute the principal moving power of the whole body. This bone should be long, in order to admit of a long stride, and consequently speed in the animal. Shortness of this bone and the one below it indicate that the horse is incapable of taking long strides, and is consequently a short stepper. The muscles that surround them should also be long, heavy, compact, and well developed inside, as well as outside the thigh. Flat or soft muscles here are a sure indication of a want of power.

The Teeth. - Determining the Age of Horses, etc. - It is a truth recognized by horsemen of all nations, and from the earliest records of which we have any knowledge of the horse, that his age can only be determined with any certainty by the appearance of the teeth.

Xenophon, in his writings, alludes to the custom of selecting cavalry horses for the Grecian army by this means, and the rejection of such as had lost the dental mark. Various Roman writers, such as Columella, Virgilius, and Varro, also mention the practice of exam. ining the teeth of horses in order to determine their age.

So many arts and deceptions are practiced by crafty and dishonest dealers at present, in manipulating the teeth, that unless a person is well skilled in judging and handling horses he would be very liable to be deceived in this respect. The adult horse has forty teeth; six incisors or nippers, two canines or tushes, and twelve molars or grinders to each jaw. The canines are generally wanting in the mare. An additional small tooth sometimes makes its appearance in advance of the upper molars. The incisors are placed close together at the terminus of the jaw. They have greater length and curvature than those of ruminants, and differ also by the fold of enamel which penetrates the crown. As the tooth is worn down, this fold becomes a ring of enamel with a cavity filled by cement and particles of food, and is denoted by horsemen as "the mark." 
In aged horses this mark disappears altogether, owing to the wearing down of the tooth below the extent of this enamel fold. This occurs in the lower mid-incisor when the horse is six years of age, in the next pair at seven, and the outer pair at eight years. This "mark" remains considerably longer in the upper teeth.

The grinding teeth are long, and have heavy, square crowns composed of enamel, dentine, and cement intermingled, so that when worn down they present an uneven and ridgy surface, owing to the enamel and dentine being so much harder than the cement. There are two sets, the temporary or milk teeth, which are the first, and the permanent teeth which succeed them. The canines are wanting in the colt, and generally in mares, as previously stated. The following facts relative to this subject are derived mainly from Youatt's noted work on the horse.

The germs of the teeth are visible in the jaw seven or eight months before the birth of the foal. At the time of b:rth, the first and second grinders bave made their appearance, and seem very large in proportion to the size of the jaw. When the colt is seven or eight days old, the two center nippers or incisors appear at the end of the jaw. During the first month, the third grinder appears on the upper and lower jaw, and soon after, and usually by or before six weeks have expired, another incisor comes by the side of the first two, on each jaw. When the colt is two months of age, the center incisors or nippers will have grown their full length, and between the second and third month the second ones attain the same level. Between the sixth and ninth month another pair of incisors appear by the side of the others, making six on each jaw, after which the only apparent difference in these teeth, until between the second and third years, is in the wear. At six months of age, the four incisors are worn to a level.

At the Expiration of the First Year, or shortly after, a fourth grinder is seen, and the colt then has six nippers and four grinders in each jaw, above and below, which will enable one to determine its age at this period. When it has attained the age of a year and a half, the mark in the central incisors will be considerably shorter and fainter; that in the two other pairs will be somewhat changed, and all of them will be worn flat.

At Two Years this change will he still more apparent. When about this age a fifth grinder will be seen, and the new process of shedding the milk or temporary set commences. The necessity of this is readily seen, since the first set are only adapted to the size of the jaw and necessities of the colt, but as these bones expand with the increased growth of the animal the teeth become separated too far apart from each other to be useful, and a larger set will be required to occupy the space, and also to meet the demands of the system.

By the gradual growth and pushing up from below of the permanent teeth, the fangs of the first set are absorbed until the second teeth are near the surface of the gum, when the former drop out. When the second teeth appear by the side of the first, they will be absorbed partially their entire length, will become narrow, and will be pushed out of place. These should always be drawn to give the permanent teeth room, and to prevent injury to the mouth or cheek, which sometimes happens if this is neglected.

At this period the colt should be fed cut or mashed feed, as he will be liable to find diffeculty in eating.

At Three Years of Age the sixth grinder is generally through, or if not, its appearance is indicated by the swelling of the gum at that point.

From three and a half to four years of age, the central nippers will have reached nearly their full growth, the second pair will just be making their appearance above the gum, or a space will be left where the first ones stood. The corner ones will be greatly diminished in breadth, and worn down so that the "mark" will be small and faint. At this time, the second pair of grinders will also be shed. 
It Four Years, the central nippers will be fully developed, the sharp edge somewhat worn off, and the mark shorter, wider, and fainter. The next will be up, but they will be small, with the mark deep and extending across them. The sixth grinders will have attained the level of the others, and the canines or tushes will begin to appear.

At this period, the dishonest dealer will endeavor, more than at any time previous, to make the animal seem older than he really is, as the difference between a four-year old colt and five-year old horse with regard to strength, utility, and value, is great; but the lack of wear in the middle nippers, the small size of the corner ones, and slight growth of the tushes, besides the smallness of the second grinders, lack of depth in the mouth, and disproportionate length of limbs in the animal, will be sufficient to expose the deception.

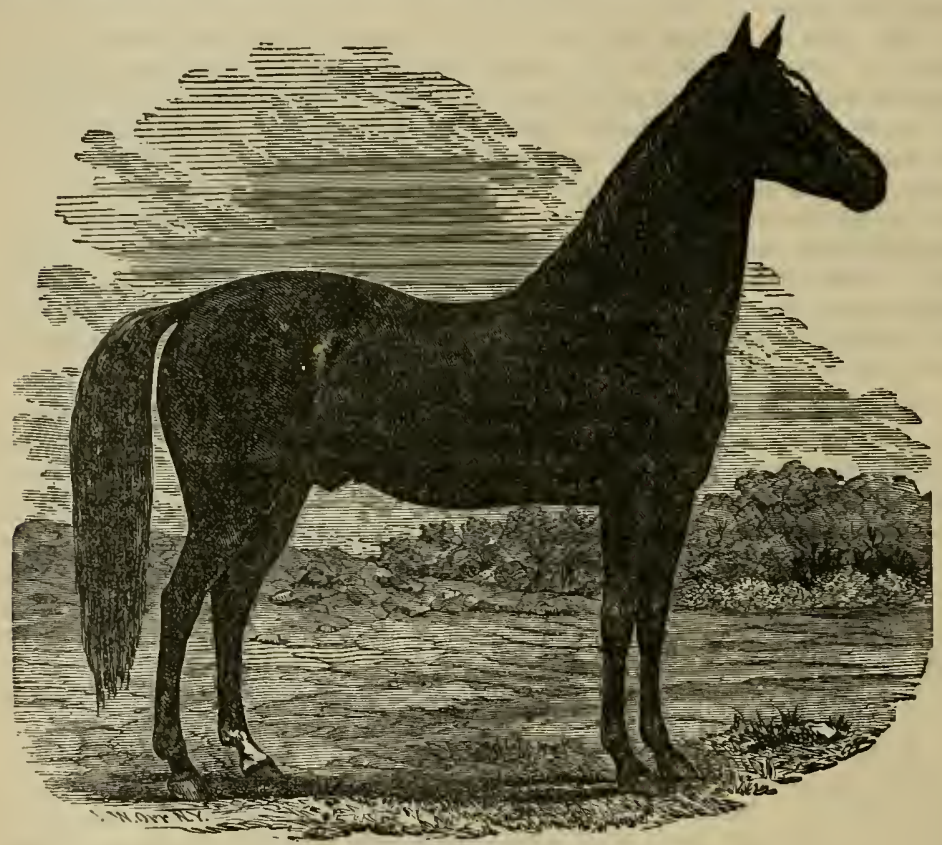

BLACK HAWK, JR.

(Property of S. W. Ficklin, Charlottesville, Virginia.)

The tushes are four in number, two on each jaw, located between the incisors and grinders, and nearer the former than the latter. At four years and a half, or between that and five years of age, the last important change takes place in the mouth of the horse. The corner nippers are then shed, and the permanent ones begin to appear. The central ones are considerably worn, and the next pair begin to show marks of usage somewhat. The tush is now cut through and generally a full half-inch in length. The colt is now considered a horse, and the filly a mare.

At Five Years, the corner nippers are well up, containing the long, irregular, deep mark in the inside, and the other nippers showing evidence of wear. The sixth molar is now up, 
and the third molar wanting. The three last grinders and the tushes are never shed. At six years, the mark on the central nippers is worn out, but there will still be a difference in color in the center of the tooth. In the next pair the mark is shorter, broader, and pointed, and in the corner teeth the edges of the enamel are somewhat worn. The tushes or canine teeth have now attained their full growth, and are nearly or quite an inch long, the grinder is fully grown, and all the grinders on a level. The teeth are now all of them fully grown. At seven years of age, the "mark" is worn out in the four central nippers, and is wearing away in the corner teeth. The tush also at this period will begin to get rounded at the point and edges.

At Eight Years, the mark is gone from all the bottom incisors, and the tush is more rounded in every respect. After this period, nothing remains in the bottom incisors to define definitely the age of the horse.

It is stated by good authority that dishonest dealers resort to the following mean and cruel method of prolonging the mark in the lower nippers of the horse: The horse is thrown down, an excavation dug with an engraver's tool in the plain surface of the corner teeth, in a form resembling the mark of a seven-year old horse, after which the cavity is burned with a hot iron, which leaves a permanent black stain. The next pair of nippers are then slightly touched. By this means a horse nine or ten years old is made to have the appearance of one of seven years of age, to a person inexperienced in judging the age of a horse. But to the experienced eye this deception is easily perceived by the irregular appearance of the cavity, the black stain around the tushes, the sharpened edges and concave inner surface of which can never be given again, the marks on the upper nippers, and the general appearance of the horse.

After eight years of age, sometimes some shght marks may be seen in the nippers of the upper jaw, the marks remaining longer there than in the lower nippers, which is generally believed to be one or two years. At nine years of age, the mark will be obliterated from the middle upper nippers, from the second pair at ten, and from all at eleven. The teeth also undergo a great change, being shorter and more rounded.

After the Horse is Eleven Years 0ld, and until very old, the age may be determined with a considerable degree of accuracy from the shape of the upper surface of the nippers. At eight years they are oval, but as the animal gets older, their surface becomes round instead of oval, the teeth become diminished in size, and by lessening in width, become separated a little apart from each other. At eleven years the second pair of nippers are quite rounded and the corner ones at thirteen. At fourteen, the faces of the central nippers become rather triangular, and at seventeen they all have this appearance. At nineteen, the angles begin to wear off, and the oval form is again assumed, but in a reversed manner, that is, from outward, inward. At twenty-one, all the nippers have this form.

Circumstances will, of course, vary with different conditions, methods of feeding, the care the animal has received, etc., but the above rules for determining the age will be found, in the main, reliable. The diminution of the bars of the mouth denote increasing old age, they becoming less prominent at ten years. Other indications of old age, aside from those of the teeth, are gray hairs over the eyes and about the muzzle; sunken appearance of the eyes, and the deepening of the hollows over them; thinness of lips, as well as their hanging down; sinking of the back, sharpened appearance of the withers, and the disappearance of tumors of every kind.

Horses, when kindly treated, will remain quite vigorous for a long period, and also will attain great age. We have known of their reaching the age of forty-five years, and still be quite active. An account is recorded by Mr. Percival of a horse that died in his sixty-second year. American Eclipse was used successfully in Kentucky for stock purposes at the age of 
thirty-one; but these are very rare instances. From the commonly hard usage that the horse receives, he rarely ever lives beyond the age of twenty-five or thirty; in fact, many horses are subjected to such severe labor, and so much abused, that they are old and decrepit at thirteen or fourteen years of age.

Color.-The color may seem an unimportant point to some persons in regard to a horse, providing he be really a valuable animal; but to individuals of fastidious taste, and who have an eye for beauty, an undesirable color would prove a serious objection. It is generally conceded that bay, chestnut, dark brown, and black are the most desirable colors in a horse, the preference being in the order in which we have given, and, other conditions being equal, horses of these colors will bring a higher price in market than those commonly considered less desirable, such as white, light gray, light sorrel, cream-colored, spotted, etc. A dark bay, with no white about him, and with black tail, mane, and legs from the knees and hocks down, is generally conceded by horse-fanciers to be the most beautiful color, while the chestnut and dark brown might be regarded next in order in this respect.

Hints to Purchasers of Horses. - It would be impossible to lay down any definite rules with respect to judging of the fine points in a horse that would take the place of the experienced and practiced eye of the skilled horseman; but a few hints may be given which will prove of value to the inexperienced in this respect. The first thing to be determined before purchasing a horse is the kind of an animal necessary, and that will be adapted to the labor required of him. This will involve the consideration of size, form, strength, endurance, temperament, disposition, color, and various other things which have already been definitely treated in the preceding pages of this department of the rork. We would advise, first, in all cases to examine carefully the head, note the expression of the eye, and determine the disposition and temperament of the animal, after which the other qualities. The follow. ing directions to inexperienced purchasers of borses, given in a treatise on the horse, by $\mathrm{D}$. C. Linsley, of Vermont, will be found of value to many:-

"When the purchaser is unaccustomed to horses, we would advise him, after determining what kind of a horse he wants, to engage some reliable person to assist him who has had more experience, especially if he is about to purchase of a stranger, for in the latter case he will not only be liable to misjudge as to the justness of the animal's proportions, but also as to the soundness and rices, for a vast number of faults and defects may, by artful jockeys, be concealed from the inexperienced eye, and sometimes even from the closest and most intelligent examination.

If, however, the purchaser avail himself of no such assistance, or chooses to trust to his omn judgment, he cannot be too cautious. When you enter the stable, observe carefully the way in which the owner approaches his horse. If, as soon as he comes within hearing, he speaks to him in a loud, sharp voice that makes the horse spring up in his stall as if frightened, look out for some defect in his limbs. If much lame, it will be seen when he is mored; but if the lameness is very slight, you may not notice it. If the omner comes up to the horse very carefully, and seems a little cautious about going into the stall where he is, you may justly suspect that he is not kind and pleasant-tempered. If he approaches the horse in an easy, careless, but quiet $\pi a y$, you may reasonably expect that the lorse has no such vices to hide, and you will not suspect him of lameness, though it should not prevent your watching carefully every motion of his hims to see if you can detect it.

Mark how the horse stands, how he is hitched, and what kind of a halter he has on. If he stands with one of his fore-feet far. in advance of the other, look out for founder. If he is fastened with more than ordinary care, see if you can observe any signs of his having attempted to untie his halter with his teeth. If the halter is fastened very tight round his throat, he may have a trick of shipping it off, or he may be a cribber. A large strap, buckled close round the throat, will often prevent cribbing. 
Youatt considers crib-biting unsoundness; and it is, at all events, a very bad habit, that materially injures the sale of a horse. If the manger shows signs of being bitten by the horse, do not purchase the animal until you have seen him eat. The teeth of an old "cribber" almost invariably show signs of this habit, being much worn on the outer edge, but in young horses it will not always be noticed.

When the horse is turned out of his stall and faces the light, observe whether he shuns it, and partially closes his eyes; if so, his eyes are weak; if, on the contrary, he looks boldly about, with a bright and lively expression, his eyes are not only good, but you may infer that he is both spirited and tractable. Examine the head carefully. If it is thoroughly good, light, lean, and graceful, with tapering, sharp-pointed ears, ever moving and restless, the eyes animated and prominent, the forehead broad, the muzzle firm, the nostrils large, and the whole well set upon the neck, you may be pretty sure that it belongs to a good horse.

If the eye is not bright and lively, it should be carefully examined. Sometimes the eye will look very natural, and the horse appear bright and well, yet be perfectly blind. If the horse is entirely blind, you may easily detect it, for though some horses move about very easily and handily when perfectly blind, you will soon notice the defect if you watch him and permit him to move about freely. If you are suspicious that, although not entirely blind, his sight is defective, you may determine it by passing some small object like a stalk of hay rapidly before his eyes, and at a short distance from them, taking care that the substance be not large enough to create any perceptible motion of the atmosphere, for a blind horse will wink at the least unnatural current of air.

The appearance of the head is a pretty sure index of the age of the animal, and the inexperienced buyer will do well to rely as much upon this general appearance as upon the appearance of the teeth; for with a saw and a filing-iron old teeth may be made to resemble so closely those of a young horse as to deceive even pretty good judges. In the old horse, the eyes are usually a little sunken, and the hollows over the eyes are deepened, the lips are less firm and close, and the mouth shows the long use of the bit. If the head is satisfactory, next examine the legs, and see that they are not too long, are wide, flat, bony, and free from flesh below the knees and hocks. When the muscles are well developed, and stand well out from the bones, and are free from any fat or meat, they can he distinctly traced by the eye through the skin. See that the horse stands squarely and equally upon them, and that they are neither stretched too far apart nor gathered too much under him. Examine closely whether there be any unnatural enlargements or bunches on either leg. If none can be detected by the eye, then pass the half-closed hand carefully down each one, pressing the leg moderately, to determine whether there are any bunches or scars concealed by the hair.

Look carefully at the hocks for spavins, curbs, or thoroughpin, for, although the latter do not constitute unsoundness, yet they are objectionable. Pass the hand carefully down the back sinews to detect any unevenness in the muscle. If any little bunches are found, or the cord is anywhere enlarged, examine carefully for any scar that may indicate that the injury was an external one. If there are no signs of any such injury, and especially if the muscle at this point feels sore, reject the animal. The back sinews have been strained, if not ruptured, and there is little chance that he will ever be able to endure hard service. Search for bunches on the inside of the fetlocks; if you find them, or scars, you will attribute them to the horse's cutting. If the scars are old, and the horse being in very high flesh yet travels very close, it will be wise to reject him, for in ordinary flesh he will be likely to cut, and this is one of the most unpleasant defects in the horse, and one that is very difficult to remedy. Press the hand closely and carefully around the pastern, for ringbone, or cling-fasts, that are just commencing to form, and will be detected more easily by the hand than the eye. This is the more important, as ringbone is one of the worst kinds of unsoundness, and the horse may not at all times and in all instances be sufficiently lame to enable the buyer to detect it. 
The knees are often too carelessly examined. It is not enough that there is no trembling of the joint, that it shuts back well, and is well shaped. If there are any bunches, hard or soft, or any scars in front, it is a pretty sure sign that the horse is a stumbler, and is unsafe.

Just below the knees, and upon the inside of the leg, look for splints; if small, and removed from the joint, they may in no way affect the usefulness of the animal, but they are unsightly and objectionable. The feet require the closest scrutiny. Reject horses that have split or flat hoofs, as they will be fit only for slow work. Where the feet are high and narrow at the heels, it is a serious objection, as such feet are very liable to founder, and other diseases.

The legs and feet proving satisfactory thus far, take a look at the body. If the ribs are round, the back short, and his wind good, little more need be required. To determine the soundness of the wind is sometimes difficult, especially if the horse is in high condition, and has been dieted and medicated with the view of concealing some defect in it. The best way to determine this is to make him trot briskly about one hundred rods, and as soon as he has done this, let him stand perfectly still and watch closely his breathing. If he breathes easily, and without any apparent effort, and especially if, as soon as he stops, he draws one or two long breaths that fill the lungs to their utmost capacity, without any appearance of distress, his wind may be pronounced good; but if there is the least evidence of painful respiration, or catching for breath, something is wrong, and the animal should be rejected. Heaves, or broken wind, is a common disease at the East, but at the West it is comparatively very rare; hence, the purchaser will be more on his guard to detect the disease in a horse raised at the East, than in one raised at the West.

While the horse is moving, notice carefully his action, and to do this take such a position that he can move by yon on level ground, and at a sufficient distance from you to give a good view of him. Notice particnlarly the action of the forelegs, see that the joint at the shoulder plays freely, that the feet are not raised too high, that he reaches out boldly in his step with his forelegs, and throws his weight freely upon them. If he does this, you may be pretty sure the feet are sound and good, and the shoulders unhurt. On the contrary, if the horse does not carry his feet well forward, but takes a short, mincing step, and puts down his feet cautionsly, they are unsound; and if the legs seem stiff, and tied up at the shoulders, he is chest-foundered. These things should be carefnlly observed when the horse is first moved, for if he is but a little stiff in his shoulders, the stiffness generally disappears entirely after a little exercise.

After having carefully noticed these things, and also his general style and appearance, take such a position that you can watch the movement of his feet as he comes directly towards you, and goes directly from you; see that he carries forward his feet in a straight line, and that he does not travel too close or too wide, for if very close he will be apt to cut, or interfere, and if too wide, his gait will be labored and awkward.

While the horse is moving, see if he hesitates to turn short and quick; if he does, and appears in the least stiff in the back, you may conclude that he has been strained there, and a horse that has once been severely strained in his back rarely, if ever, fully recovers, and is unfit for any kind of business, but if the back is such as we have described, there will be little danger of this complaint.

These defects, at which we have glanced, by no means comprise all the diseases or defects of the horse, nor are the methods we have pointed out in all cases the only ones. We have aimed only to glance at the most serious and ordinary defects, and point ont the simplest means of detecting them. Some quite common defects we have not noticed, as, for instance, string-halt. This is not considered nnsoundness in ordinary circumstances, and where it is so bad as to become seriously objectionable, it cannot fail to be noticed by the most careless observer. 



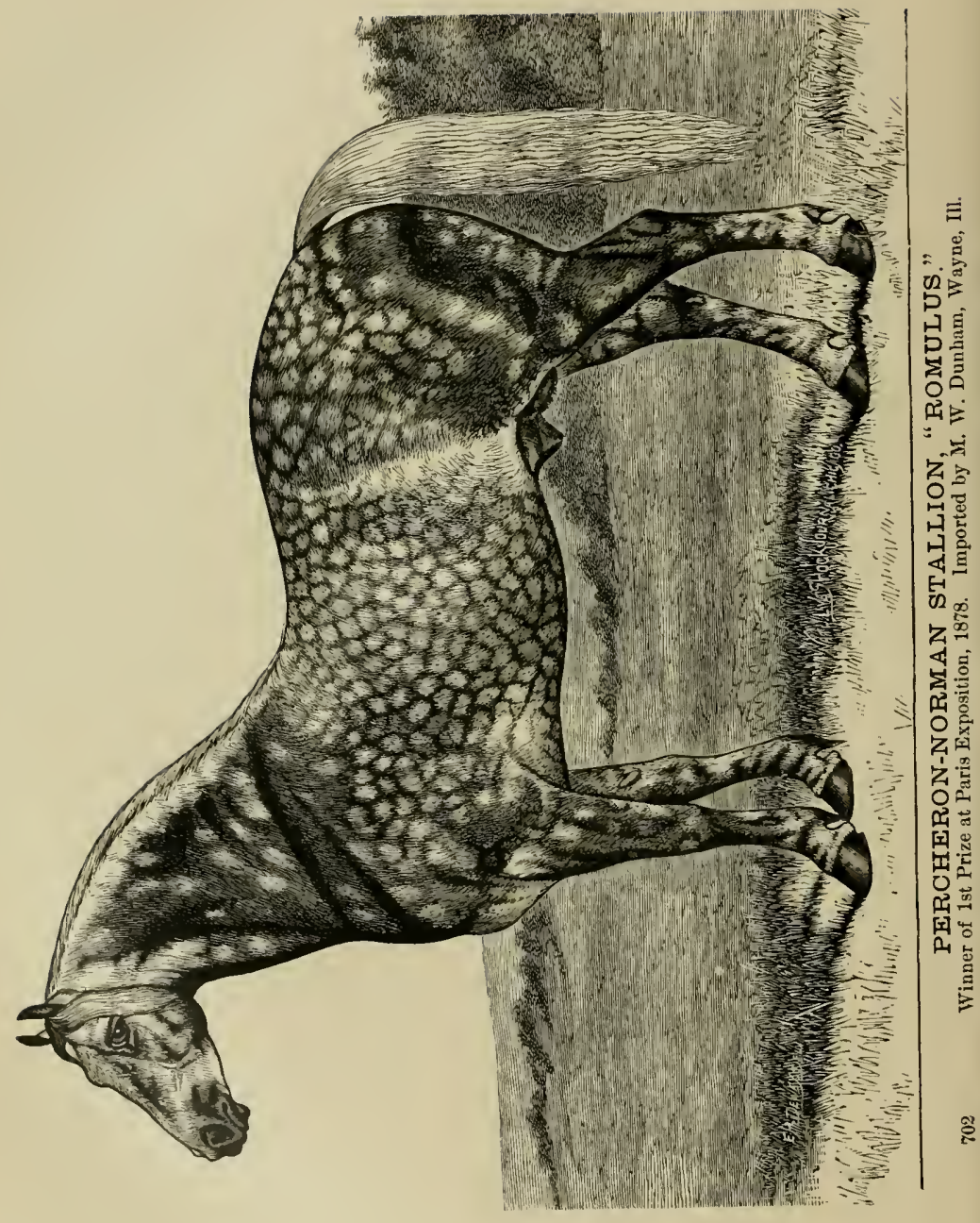


To some, this kind of examination may seem tedious and laborious, and to the inexperienced horseman it may be so; but the horseman of taste, with a practiced eye, will determine the freedom of an animal from the objections we have referred to in a small fraction of the time it has taken us to describe them.

We have said nothing upon those subjects that are merely matters of taste, as to enter into a discussion of them would occupy more space than we could devote to it.

One wishes a horse that shall have a bold, resolute style of action, and a high temper that prompts him to keep a constant and heavy pull upon the reins. A nother wishes a horse of less spirit, with a quiet, pleasant temper, and a mouth that only feels the bit, that is never restless, never pulis, and so gentle that his wife and children can manage him. Yet he does not want a dull, stupid animal; on the contrary, one that is bright and lively, and that when gentily urged will move off at a lively pace.

One wants a horse that is round and smooth, with soft hair, a beautiful color, and a proud, showy style, that will attract the admiration of his neighbors, but cares little about its speed upon the road, or its powers of endurance, while his next-door neighbor cares far less for the showy style and the beautiful color, and values his horse solely on account of his useful qualities. In short, no rules can be laid down that will determine the excellence of the animal in these respects, as so much depends upon the precise service which is demanded of him, and the taste of the purchaser.

Although people differ much in their opinion as to the color, style of movement, etc., of their carriage-horses, yet the anxiety to own a free, nimble driver, is nearly universal, and might almost be styled a national characteristic.

But the passion for horses is co-extensive with man's acquaintance with them, and is confined to no nation, no portion of the human family; indeed, there are comparatively few persons who take no interest in his history, no pleasure in training or using him.

Other animals may be as useful to man, but they do not so lay hold of human affections and sympathies. He is at once his companion and servant in his long and weary journeys. With the same patience and unflinching courage, he carries his rider safely over the scorching sands of the desert, or treads with careful step the icy footpaths of the mountains. He waits with silent patience his master's pleasure, and moves only at his bidding. Heat and cold do not shake his courage, hunger and thirst do not drive him to rebellion. The soothing sound of his master's voice stills his restlessness when he wishes him to be quiet, and the cheering, encouraging tones of the same voice rouse him to the noblest efforts to do his will."

Thorough-Bred. - This is a term that is often improperly used in connection with horses. Technically, it means, when properly applied, that the animal's pedigree can be traced back to the East, or rather, that he is of a breed derived from a Barb or Arabian ancestry.

English juries have frequently decided that a horse that is warranted thorough-bred is returnable, if any defect can be traced in his pedigree, or, in other words, if it can be proven that his pedigree is not directly traceable, on the side of both sire and dam, to imported Barb or Arabian stock. This, then, is the technical meaning of the term, and proves that in this sense there can properly be but one breed of horses designated as thorough-breds: It is the oldest and purest breed in existence, and is noted for speed, endurance, strength, energy, and courage. The genealogy of this race of horses has been carefully recorded in a stud-book for nearly a hundred years, and the records of their achievements upon the race-course, both in this country and Europe, are the most noted in the history of the horse. They were intro. duced into this country at a very early period, and so frequent have been the importations of the choicest animals of this type, that they are identical with those of the old country. A leading authority thus describes the thorough-bred:-

"All running horses are of this breed, and none but thorough-breds can make a credit43 
able showing in long-distance or heat races. A horse with a dash of this blood may make a fast race for a short distance, but it takes the genuine stuff to go a mile and repeat, in good time. They are of all colors, bay, brown, and chestnut predominating; are rather light of bone, and long in the body, with thin neck, small head, wide between the eyes, finely-pointed ears, long quarters, and deep chest, and are usually rather 'leggy.' In height they are from 15 to $16 \frac{1}{2}$ hands, although they may sometimes be found a half a hand above or below these figures. They are nervous, restless, determined, and excitable, and are highly valued for crossing upon all other breeds."

Beyond the technical meaning of the term thorough-bred, some writers give it a practical signification, and employ it to denote certain essential qualities that give superior value to the animal, and determine his rank in the grade to which he belongs. These qualities may comprise beauty of form, compactness and strength of bone and muscles, intelligence, activity, docility, and the various other desirable qualities that give value to the horse. The true thorough-breds, according to the technical and proper signification of the term, are, without doubt, the finest horses in existence, and to that blood are we indebted for some of the finest qualities that our best types of horses possess. Yet, notwithstanding this, a poor horse, as well as a man of inferior ability, will ever remain such, whatever his ancestry may be, and a pure thorough-bred will occasionally, though rarely, be found to be a very inferior animal, since there are exceptions to almost every rule, the world over, and while the law of nature, that "like produces like," will be the general rule, there will occasionally be an exception, and a poor animal will sometimes possess a fine pedigree, and the reverse; some noted animals having sprung from unknown ancestry. Pedigree has, however, great significance, and a good horse with a good pedigree is the one to be desired. The superiority of thoroughbred horses over the common stock, will be found generally to consist in a superior form, physical organization, and intelligence, while from their intelligence, they are more kind and tractable in their disposition. Possessing a superior organization, they are less liable to disease, and live and maintain their usefulness to a much greater age. They have greater endurance, are superior in action, and more elegant in appearance; they are also superior in breathing powers, fleetness, and bravery.

As we have previously stated, the bones of a thorough-bred horse are more solid and fine in texture than those of horses of the common type, while his tendons are much stronger and better defined, and his muscles more firm and elastic. Such a horse will perform much more labor in a given time, with less exhaustion, and be able to repeat the task oftener, than an animal of inferior blood. For all the common and general purposes of farming, or for a carriage horse simply, the thorough-bred horse will be found greatly superior to those of common class, and this should be borne in mind, whether in purchasing, or breeding.

The Percheron-Norman. - These borses are frequently called Normans, or French horses. The breed originated in La Perche, an ancient province of France. Importations from that country to this have been very extensive for more than a quarter of a century past. These horses are noted for great strength and endurance, being much used for draft. They are usually fifteen and a half, or sixteen and a half hands high, although some of the larger types of the breed are occasionally seventeen hands. In temperament they are sanguine, mixed in variable proportions with the musco-lymphatic. In color they are quite uniform, being nearly always gray, although occasionally other colors, such as the black, roan, bay, or chestnut, are seen. It is estimated by good anthority that fully ninety per cent. of them, both in this country and France - from where they are imported - are gray. They are large animals, those imported to this country ranging in weight from 1400 to $1900 \mathrm{lbs}$. They generally have a good head, mild, expressive eyes, rather short neck, denoting strength; high withers, a broad, deep chest, short rump, sometimes slightly drooping, and body well rounded. The mane and tail are abundant. They are very docile, possess great strength and 



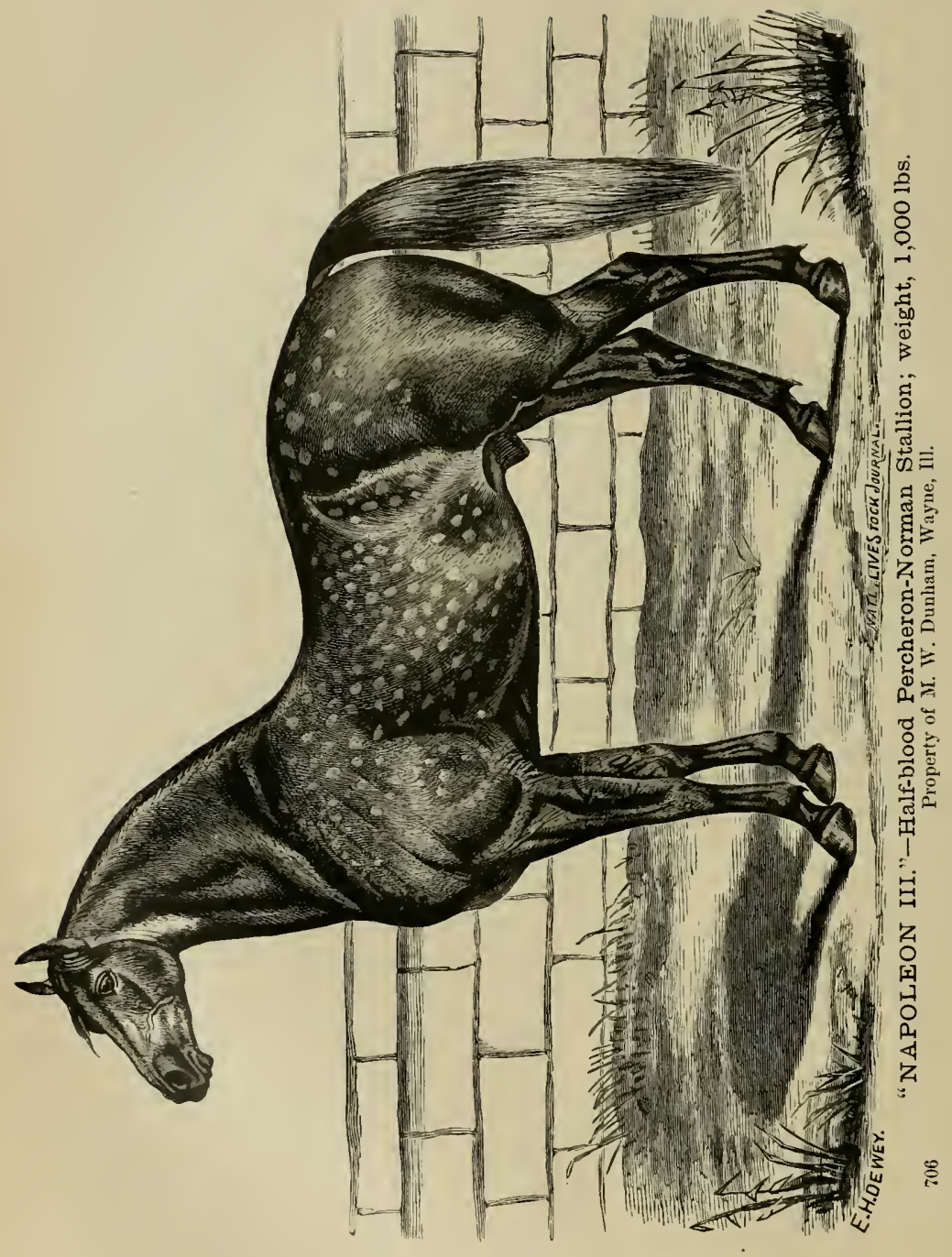


endurance, and, in proportion to their size, are quite active. These horses are much used in the Western States, while an infusion of the blood upon the common stock of the country is very apparent in nearly all sections. It is to this blood that may be attributed largely the excellence that distinguished the Canadian horses about half a century ago, this breed having been introduced into Canada by the French settlers.

While the Percheron-Norman horse is used principally for draft and heavy work, the light or smaller types are frequently employed upon the road. The result of a cross with this breed upon the common horses of the country has been found very satisfactory in producing an improvement in strength, and hardiness.

The Clydesdale. - This breed of horses derives its name from a district on the Clyde, in Scotland, where it was first introduced by one of the dukes of Hamilton, by crossing with the imported Flemish stallions. It is adapted principally for draft, and is much prized for this purpose. Importations of the Clydesdale have been made into this country to the extent that they are now bred here in considerable numbers. In color, the bays and browns are most numerous; but there are occasional blacks, sorrels, and grays among them. Whatever the color of the body, they nearly all have strong, distinct markings of white in the face and on the feet, with, frequently, white spots under the body. An abundance of hair on the legs is considered a good point in a well-bred Clydesdale, while the tail and mane are quite heavy and inclined to be naturally wavy. In height, they range from fifteen to seventeen hands, while sometimes one will be found eighteen hands high. In weight, they range from 1500 to 2100 pounds, one being found occasionally that will reach 2500 pounds.

The pure-bred Clydesdale is somewhat larger than the Percheron-Norman, and is also longer in the body and hind-quarters than the French horses. He is heavier-boned than any other breed, according to his weight, and particularly in his limbs, which are of great width, flat, sinewy, and hard. It is said that at the bone-mills the bones of this breed can be readily distinguished from those of ordinary horses, hy their size and solidity. He has generally a good eye and head, well-shaped neck and shoulders, and a round, straight body. These horses possess great strength and endurance, and are naturally gentle in disposition. The back of the legs, from the knee and hock to the fetlock, are covered with quite long hair, a characteristic greatly prized by the Scotchman, as being an evidence of the purity of the breed. The Clydesdale is frequently crossed with the heavier class of common horses with good results.

English Draft. - The native English breed, variously termed the English Draft, Cart, or Shire horse, is the largest draft horse known. It is supposed to have been first introduced into England through the invasions of war. Their improvement dates from the last century, at which time stallions were imported from Holland. From that time, by judicious crossing and breeding only from the best, and with especial reference to large, powerful draft horses, adding occasionally (as is quite probable) a mixture of the Scotch Clydesdale, a breed has been established of gigantic size, commonly known in Europe and America as the English Draft horse.

The famous brewers' horses of London, which average a full ton in weight, are of this breed. Notwithstanding their immense size and strength, however, they are symmetrical and attractive in form, and may be described as follows: Head short and heavy in appearance; face oval on lower part and broad above; mouth large, with thick lips; eyes small and mild-looking; ears broad, thick, and short; the neck arched; heavy at throat-latch, and thickening towards the shoulder; shoulder with a moderate slant, with withers extending well back; barrel round and "well ribbed up;" rump.bones wide apart, but not prominent, rump drooping to the tail; short quarters, with thick, round buttocks; the upper limbs are not only well-proportioned, but well-shaped; the lower part heavy and bony, with long hair behind, similar to the Clydesdale; feet large. 
They possess great endurance and strength, and make excellent draft horses, although a lighter animal would be desired for common use.

Boulonnaise Draft. - This breed was first brought into notice in the vicinity of Boulogne, but has rapidly spread throughout the region of Paris. They are thus described:-

"They are of about 1,600 or 1,700 pounds weight, and have a handsome form for a large animal. Their action is more clumsy than graceful, and they are principally used for very heavy draft purposes, where great strength is needed instead of speed. The moving of the heavy blocks of stone used in the buildings of Paris is done with these horses. It is not unusual to see six or eight of them hitched in hne, drawing blocks of several tons' weight, which of necessity must move very slowly. They are mostly gray in color, but bays and blacks are not uncommon. For the purposes to which they are by nature adapted, they are entitled to be classed among the best of draft horses."

The Conestoga is descended from early importations from Flanders and Denmark, and is a mixture of several breeds, but owes its principal characteristics to the former. mentioned sources. A heavy roadster, he was more frequently employed as a draft horse, and was formerly extensively reared in Pennsylvania. The best types of this variety possessed fine symmetry and great power, and were much preferred by the German emigrants of that State. They are now rarely met, having been superseded by the Percheron-Norman, Clydesdale, and other breeds for draft purposes.

Clereland Bays. - As the name indicates, the prevailing color of the horses that bear this name is a light bay. They were first known in the district of Cleveland. In the latter part of the eighteenth century, this district became noted for producing a heavy breed of horses suited for cavalry or the coach, and from their history it appears that they are part thoroughbred, being a cross between the race-horse and the large horses found in the country. The old Cleveland horses were very large, and noted for their strength. They are reputed to have carried on their backs a weight of 760 pounds, (or more than 54 stone,) a distance of sixty miles in twenty-four hours. This old race is now nearly extinct, the animals that at present bear that name having but a slight resemblance to them, these having been changed in size and form by careful breeding, to meet the modern ways of travel, since on the improved public highways of England the old-fashioned, cumbersome coach is now for the most part useless. Consequently these animals have been considerably reduced in size by the infusion of thoroughbred blood, so as to conform to the new order of things for which they are in demaud, both at home and in other countries.

They combine the necessary size for ordinary or general-purpose use, with style, action, and endurance, while they have a beautiful color, which is transmitted to their offspring with as much certainty as characterizes the Devon cattle. If exhibited at a fair, they would probably be catalogued as either family, carriage, coach, or saddle-horses, their average weight being about 1,400 pounds.

Youatt, speaking of the improved Cleveland Bay, says: - "Now we have an animal tall, deep-chested, rising in the withers, slanting on shoulder, flat in the legs, with far more strength and treble the speed."

Another writer describes them as follows: - "The modern Cleveland Bay is a large, elegant horse, standing $16 \frac{1}{4}$ to $16 \frac{3}{4}$ hands, and weighing from 1,350 to 1,500 pounds; a fine head; full, bright eye; long, arched neck; oblique shoulders; deep chest; short back; long quarters; strong, clean, cordy legs, and perfect feet. Their color, bay; full, flowing mane and tail, and black legs, usually clear of white.

Cleveland Bays are very strong-blooded, and impress their characteristics on their grade offspring with certainty. They have long been very popular in all parts of Europe for coach purposes, and also for light artillery and cavalry." 



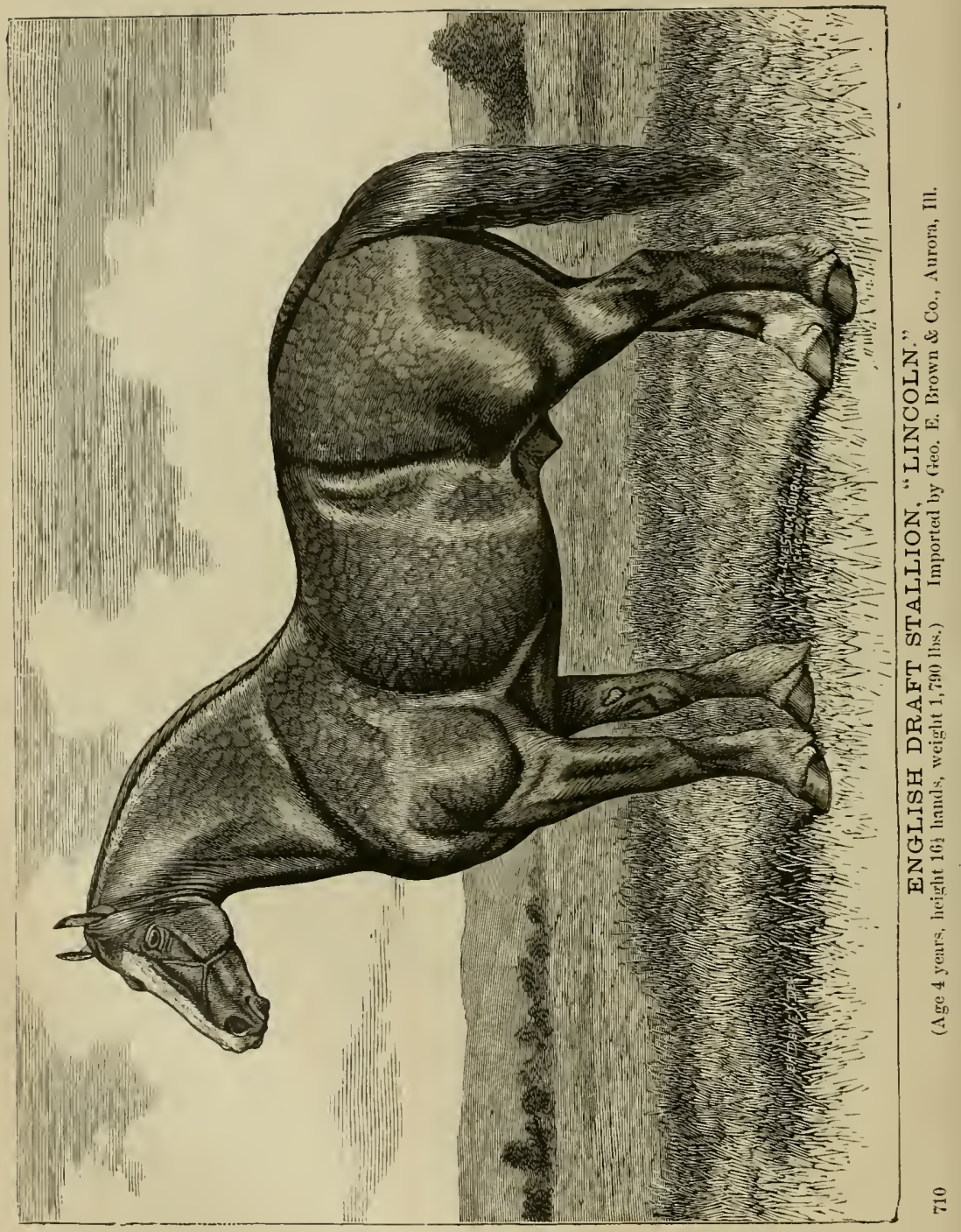


These horses are also valuable in the intelligent breeder's hands for modifying some other cross; but in this matter of producing handsome, stylish carriage and coupé animals, the breeder must have in his mind a standard which he desires to attain, and then govern himself accordingly.

We are certainly not lacking in the elements necessary to produce with certainty the large, stylish carriage-horse in this country, and it only remains necessary for our breeders to direct their attention to supplying this demand, which is growing rapidly both in America and Great Britain.

The Hambletonians. - The Hambletonian family of horses are noted for their speed and power of endurance, and make excellent carriage-horses. Some of the most noted trotters belong to this race. In mentioning a hist of the most noted trotting horses with their record, in connection with this family, a recent authentic writer says:-

"One noticeable feature of this list is that no other family is represented with two or more performers the get of one horse; another is that the fastest record (Maud S., 2.101 ) the second fastest (St. Jukien, 2.11 $\frac{1}{4}$ ) and the third fastest (Goldsmith Maid, 2.14,) are made by two granddaughters and one grandson of the famous horse Hambletonian, showing that he had the power to transmit his excellences to his sons, and thus to perpetuate not only his great fame, but his name and his family as well. Hence, we see not only the importance, but the value of a sire who transmits his qualities in the male line of descent."

This race descended, on the paternal side, from imported Messenger (a thoroughbred), through his son Mambrino (also a thoroughbred), who was the sire of Rysdyk's Hambletonian, the dam' being by Bellfounder, an imported Norfolk trotter, and her dam probably having two direct crosses to Messenger. Through Rysdyk's Hambletonian, on the paternal side, we have a long line of celebrated horses, such as the Volunteers, the Edward Everetts, the Alexander Abdallabs, the Almonts, the Messenger Durocs, the Sentinels, the Happy Mediums, and all the various so-called Hambletonians of the present day.

Hambletonian was a developed trotter, and possessed the power of transmitting his good qualities to his descendants. At three years of age he trotted in $2.48 \frac{1}{2}$, and could trot in 2.40 at any time when matured. In color, he was a bright bay with black extending above the knees and hocks, and white hind feet, also a small star in the forehead. This noted animal was foaled in 1849, bred in Orange County, New York, and sold with his dam when only a few months old to William M. Rysdyk for \$125. He died when twenty-seven years of age, after establishing the most noted family of trotters in existence.

The Messengers.-This class of horses are celebrated as both roadsters and trotters. The founder of this family was Messenger, the son of Mambrino, a thoroughbred, and one of the most celebrated running horses of the English turf. He was imported to this country after having achieved several successful races in England, having won the king's plate in 1785 , being then five years of age. He was in color a beautiful dark gray, fifteen and threequarters hands high, and stoutly built, having a peculiar formation of limbs, large forehead, and deep quarters. He died at the age of twenty-eight years. Some of the most renowned of his immediate descendants are Hambletonian, Lady Suffolk, Abdallah, and Engineer.

The Hambletonians are claimed by some to be a cross between the Morgans and Messengers, uniting the excellences of both. His stock was justly celebrated, and although it may be doubted whether animals of this family may now be found possessing sufficient resemblance to each other or to their progenitor, from whom they derive their name, to be classed as a distinct and permanent variety, yet the effect of this blood is still very perceptible in many sections of the country.

The Morgans.-This is a noted family of horses, of which the famous horse called Justin Morgan was the progenitor. They may perhaps be called our oldest trotting family, 
and although they have not produced animals of the very fleetest type, they doubtless justly merit the very highest rank as fine-tempered, hardy: and desirable roadsters. The popularity of this family of horses seemed at one period unbounded; in fact, no blood, except the thoroughbred, has been inore generally disseminated throughout this country, or more highly esteemed. At the present time, they are not so highly valued by those whose main object is speed, and who place that above all other qualities, but, as a certain writer has said-

"Go where you will annong livery-stable keepers or horse-railroad managers, and ask them what type of horse they have found most profitable to use and wear out on the road, and they will almost invariably answer, 'the old-fashioned Morgan.'"

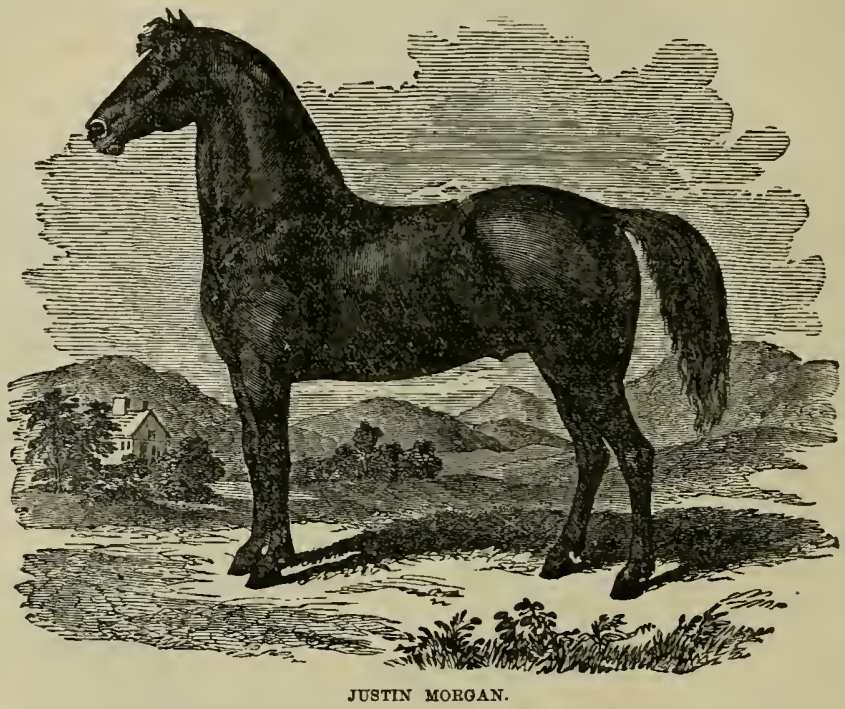

Justin Morgan was bred in Vermont, foaled in 1793, and died in 1821. His ancestry is not positively known, although it is generally beheved that the thoroughbred blood predominated. The following accurate and interesting description and history of Justin Morgan is given in Mr. Linsley's work on "The Morgan Horse ":-

"The original, or Justin Mlorgan, was about fourteen hands high, and weighed about nine hundred and fifty pounds. His color was dark bay with black legs, mane, and tail. He had no white hairs on him. His mane and tail were coarse and heavy, but not so massive as has been sometimes described; the hair of both was straight and not inclined to curl. His head was good, not extremely small, but lean and hony, the face straight, forehead broad, ears small and very fine, but set rather wide apart. His eyes were medium size, very dark and prominent, with a spirited but pleasant expression, and showed no white round the edge of the lid. His nostrils were very large, the muzzle small, and the lips close and firm.

His back and legs were perhaps his most noticeable points. The former was very short; the shoulder-blades and hip-bones being very long and oblique, and the loins exceedingly broad and muscular. His body was rather long, round, and deep, close ribbed up; chest deep and wide, with the breastrbone projecting a good deal in front. His legs were short, close-jointed, thin, but very wide, hard and free from meat, with muscles that were remark- 



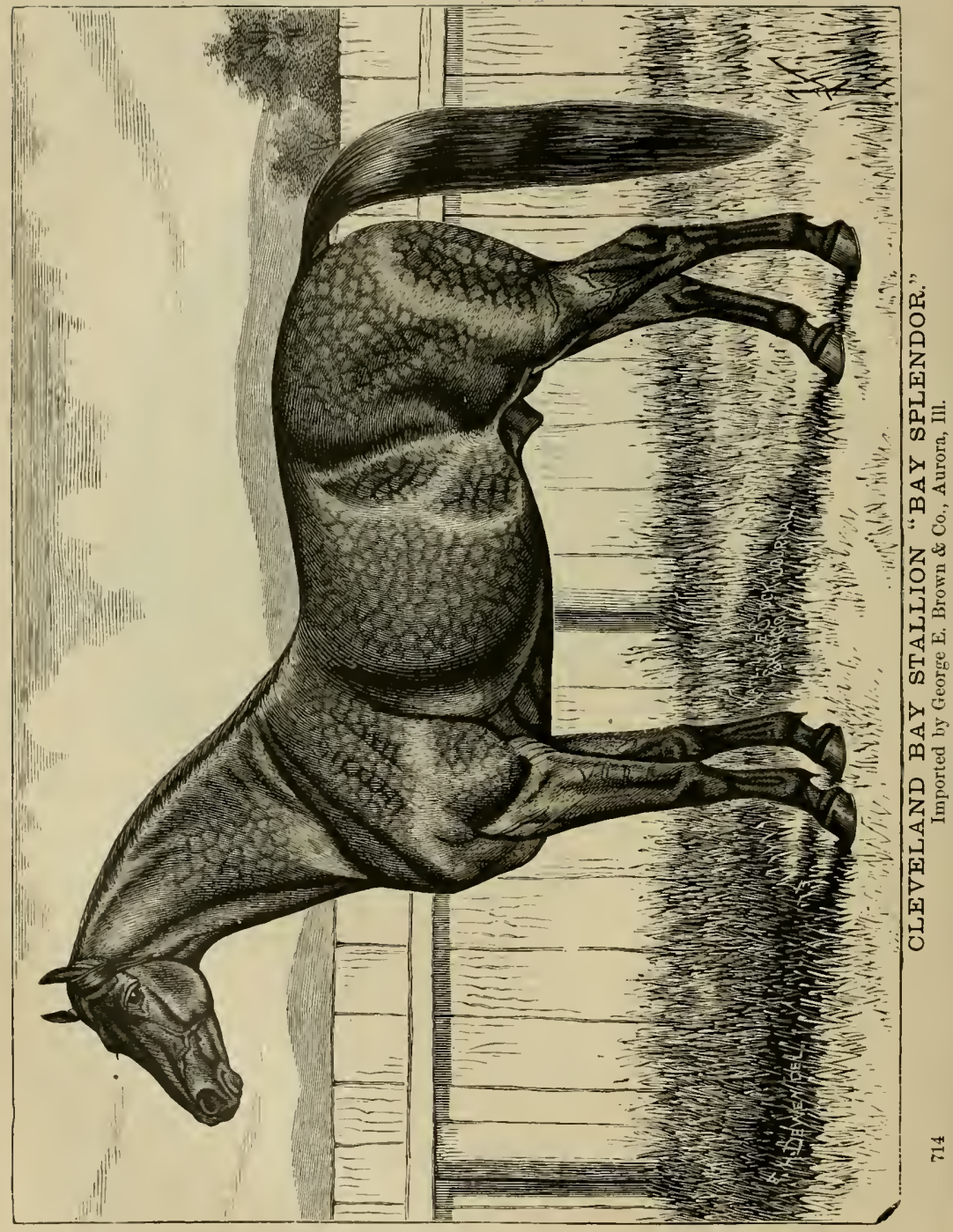


ably large for a horse of his size. This superabundance of muscle exhibited itself at every step. His hair was short, and at almost all seasons soft and glossy. He had a little long hair about the fetlocks, and for two or three inches above the fetlock on the back side of the legs; the rest of the limbs were entirely free from it. His feet were small, but well shaped; and he was in every respect perfectly sound and free from any sort of blemish. He was a very fast walker. In trotting, his gait was low and smooth, and his step short and nervous. He was not what in these days would be called fast; and we think it doubtful whether he could trot a mile much, if any, within four minutes; though it is claimed by many that he could trot it in three.

Although he raised his feet but little, he never stumbled. His proud, bold, and fearless style of movement, and his vigorous, untiring action have perhaps never beeft surpassed. When a rider was on him, he was obedient to the slightest motion of the rein; would walk backwards rapidly under a gentle pressure of the bit; and moved sideways almost as willingly as he moved forward; in short, was perfectly trained to all the paces and evolutions of a parade-horse. When ridden at military reviews (as was frequently the case), his bold, imposing style, and spirited, nervous action attracted universal attention and admiration. $\mathrm{He}$ was perfectly gentle and kind to handle, and loved to be groomed and caressed; but he disliked to have children about him, and had an inveterate hatred for dogs, if loose always chasing them out of sight the instant he saw them. When taken out with halter or bridle, he was in constant motion, and very playful.

He was a fleet runner at short distances. Running horses short distances for small stakes, was very common in Vermont fifty years ago. Eighty rods was very generally the length of the course, which usually commenced at a tavern or grocery, and extended the distance agreed upon, up or down the public road. In these races the horses were started from a 'scratch,' that is, a mark was drawn across the road in the dirt, and the horses, ranged in a row upon it, went off at 'the drop of a hat' or some other signal. It will be observed that the form of Justin Norgan was not such as in our days is thought best calculated to give the greatest speed for a short distance. Those who believe in long-legged racers will think his legs, body, and stride were all too short, and to them it may perhaps seem surprising that he should be successful, as he invariably was, in such contests.

But we think his great muscular development and nervous energy, combined with his small size, gave him a decided advantage in the first start over taller and heavier horses; just as any ordinary horse can distance the finest locomotive in a ten-rod race. At all events, the history of racing in this country and England proves conclusively that small horses may have great speed. In such a race, a horse of great spirit and nervous energy derives a decided advantage from these qualities, especially after being a little accustomed to such struggles. When brought up to the line, his eyes flashed and his ears quivered with intense excitement, he ground the bit with his teeth, his hind legs were drawn under him, every muscle of his frame trembled and swelled almost to bursting, and at the given signal he went off like the springing of a steel trap. His unvarying success in these short races may perhaps be partly accounted for in this way, though he was undoubtedly possessed of more than ordinary speed, and was a sharp runner.

Among the many races of this description that he ran were two in 1796, at Brookfield, Vermont, one with a horse called Sweepstakes, from Long Island, and the other with a horse called Silver J'ail, from St. Lawrence County, New York; both of these he beat with ease. Mr. Morgan (who then owned him) offered to give the owner of Si'ver Tail two more chances to win the stake, which was fifty dollars, by walking or trotting the horses for it, which was declined. There are many accounts of other races which he ran and won; but, these accounts not fully agreeing as to the details, we have not mentioned them.

In harness Justin Morgan was quiet, but full of spirit; an eager and nimble traveler, 
but patient in bad spots; and although for a long time steadily engaged in the beavy work of a new farm, his owner at that time informs us that lie never knew him refuse to draw as often as he was required to; but he pithily adds, 'I didn't very often have to ask him but once; for whatever he was bitched to generally had to come the first time trying.' This uniform kindness at a pull was one of the striking characteristics of the horse, and the same trait may be observed in the greater part of his descendents. 'Pulling matches' and 'pulling bees' were as common in those days as short races, and the 'little horse,' (as he was often called, became quite celebrated for his unvarying willingness to do his best, and for his great power at what is called a 'dead bift.' "

The Morgan horses were spirited, with fine action, but kind and docile in disposition. They were thirteen and a half to fifteen hands high, being of medium size, with finely. formed head and neck, symmetrical bodies, deep chest, short back, and long quarters ; mane and tail heavy. Though horses of fine type, and well adapted to the road, they are regarded by many as too small for a general-purpose horse on the farm, and consequently are not, as previously stated, in as high repute as formerly, although still highly valued by many. Among the noted descendants of Justin Morgan may be mentioned Black Hawk, Gifford, Ethan Allen, Morrill, Taggart's Abdallah, Gen. Knox, Fearnaught, and Lambert. This family of liorses possessed the power of transmitting their excellent qualities to their offspring in a remarkable degree, and dominating over other bloods with which they were crossed, so that wherever found, a Morgan horse would at once be known by the peculiar characteristics which distinguish this family.

The 0rlotf.-This is the most celebrated of the Russian breed of horses. It takes its name from a Russian count who was an enthusiastic horseman, and who, in 1775, imported from Arabia a fine gray stallion of unusual size and strength named Sometaxa. This stallion was the progenitor of the Orloff race, which are noted for large size and fine action, while great pains are taken in training them to trot, and to prevent them from moving in any other gait than a walk, or trot. Many of them are very fine trotters, but do not quite equal in speed the trotting horses of America, as will be seen by the following table giving the best Orloff record.

It is stated that Count Orloff was an intelligent enthusiast in the enterprise he established, and persistently refused to part with any of his breeding stock, preferring to dictate with respect to perpetuating and improving this race of horses. After the Count's death the stud was scattered-a portion of it passing to the crown. Private studs were established about this time, and an Orloff stud-book instituted. As a means of developing the trotting powers of his horses, Count Orloff had been a patron of the race-course, and since his death the Russian Government has furnished more than half of the prize money expended in the established trotting races. Russian trials of speed are regulated by laws, and the result of a violation of any of the rules laid down for the purpose of securing a fair contest, renders the owner or driver liable to a severe penalty, not less than that of a trip to Siberia at the Government's expense. Mons. Jules Goujon, who has resided for many years in Moscow, says:-

"The entries for the races are according to age qualifications, except that for horses past six years old they are free for all. The heats are never less than three versts, and the deciding heat is at the same distance. In races of four and one-half versts, the deciding heat is at three versts. Races of six and twelve versts, are decided in one heat. The first prize is not given to the horse who comes in first in the race, but in the deciding heat. The number of horses entered in each race is unlimited, but they are started three at a time, and the two horses out of the entire number of starters who trot the distance in the least time, according to the watch, take part in the deciding heat. If the horse winning in the first trial comes in second in the second trial, then a third heat settles the question of supremacy. Only two moneys are given. There are three tracks, one for each horse, of oval shape, enclosed one 



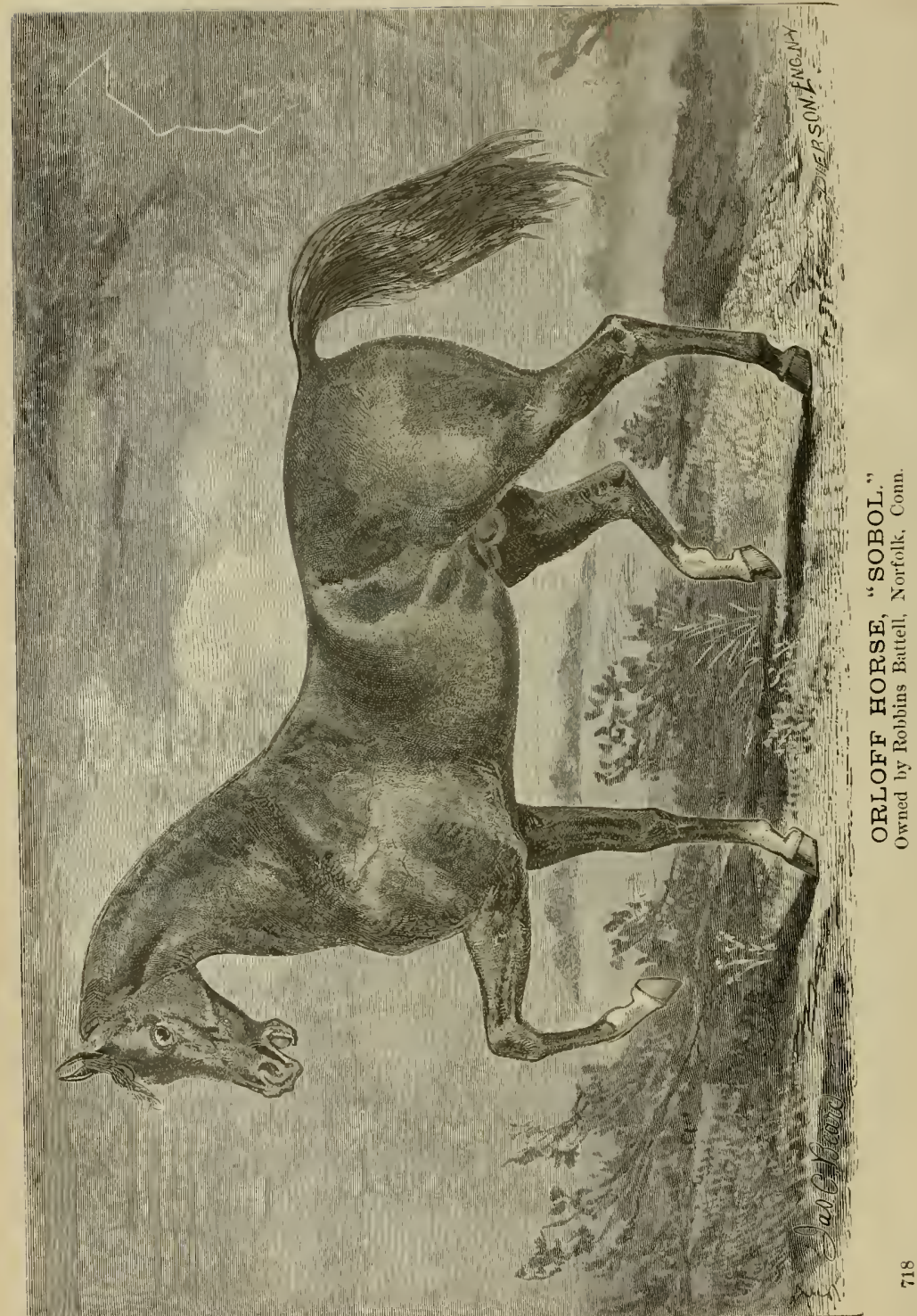


within the other. The outer is $1 \frac{1}{2}$ versts (one mile) long; the two others are shorter, one by 12 sagenes ( 84 feet), and the other by 23 sagenes (161 feet). The first horse is started on the exterior track, in front of the stand, and the two others, in order to equalize the distance, are started in the rear, on their respective tracks, which are selected by lot.

The horses come up at a jog, each one to his place. A judge is placed beside each horse, whose duty it is, by waving a bit of cloth, to notify the starter, who is in the stand, that the horse under his charge has arrived at his position. The horses are started from the stand by the stroke of a bell, which sets in motion at the same time the hands of a great dial, which mark minutes and seconds. At the instant that the first horse passes the winning-score, the judge strikes a blow which stops the first hand of the dial, and in the same way for the second horse. This automatic system gives the time of the race, by means of the clock, without error, and enables the judge, who is placed at the distance-post, to decide which of the horses are distanced, to better know the precise instant when the race is finished. A distance in Russia is 30 sagenes ( 210 feet) for a race of three versts, and 75 sagenes (525 feet) for a race at a greater distance. Only three false starts are allowed; after that number the judges can fine the driver of the horse that is responsible for the false starts, or can refuse to allow him to go the course. In case a driver does not try to win with a horse, the judges may fine him, and on a repetition of the offence he is liable to visit Siberia for a couple of years-a punishment which I imagine, from what I hear, would be salutary for some of your American drivers.

A horse is not allowed to make but three breaks during a heat of three versts, and the same number in the deciding hent. If he makes more, he is out of the race, and so is he if he makes more than thirteen jumps in one gallop. Each horse in the race has a judge especially assigned to watch his movements, whose decision as to whether the horse makes more than three breaks, or more than the permitted number of jumps in one break, is without ap peal; he has only to touch a button of an electric machine designating the offending horse, and a groom on horseback, in front of the stand, at once rushes off to notify his driver to quit the track.

For all the prizes given by the societies, any one can trot as he pleases to a droschka (the national Russian vehicle, four-wheeled, and very clumsy and heavy as compared with the sulky) or to a sulky with two wheels and four reins. The weight of the vehicle and of the sulky is equally ad libitum for all the prizes given by the societies. The Government, for prizes which it gives, specifies for itself the kind of vehicle, its weight, the weight of the driver, and the distance to be trotted.

There are two seasons for racing. The summer races occur in May and June, and are trotted twice a week. They are started at six o'clock in the evening, the days being so long in Russia that it is light until eleven o'clock at night, and thus the heat of the day is avoided, which is an advantage for both the horses and the spectators.

In winter the races are always trotted on Sundays, and on the ice. But two horses are started at a time, and on the opposite sides of the same course, which is but one verst in length."

The droschka referred to, although a clumsy-looking vehicle, is very light, weighing only about seventy pounds. The verst is 3,500 feet in lengtl, being a bittle less than twothirds of a mile.

The best "Orloff" time for thirty versts (twenty miles) is 1 hour 8 minutes 30 seconds. The following table shows favorably for our American trotters :-

Best Russian time.

One mile............. 2:31

Two miles ............ 5:019

Three miles............ $7: 52 \frac{1}{2}$

Five miles ............ 13:56

Twenty miles ...........1:08:80
Best American time. Dif.

One mile ............. 2:101-20:

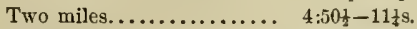

Three miles............ 7:21t-31ts.

Five miles............ 13:00 -56

Twenty miles.......... $58: 25-10 \mathrm{~m}$. $5 \mathrm{~s}$. 
From the foregoing, the superiority of American trotters for speed is clearly seen. Between 700 and 800 of our trotters have beaten the best one-mile time made in Russia, and though two-mile races are somewhat rare in this country, yet the fast Russian time for two miles $\left(j: 01 \frac{3}{4}\right)$, made by their famous trotter, Poitieshnoy, has been beaten by nearly all our great trotters; and to-day there are probably fifty horses in America that can beat that time by several seconds.

The Orloff is a spirited, well-formed animal, of fine action and elegant carriage. But few of this breed have thus far been imported into the United States. The horse "Sobol," of which we give an imperfect illustration, was bred by Count Nicholas Sollogub, of Tambou, Russia, and imported by his present owner, Mr. Robbins Battell, of Norfolk, Conn., in 18i6. He is black in color, and $15 \frac{3}{4}$ hands high. He was foaled in 1870 , sired by Dehrody, 4 th; dam, Pobeida, by Krelika, tracing back directly to Belley Smetanka, who was brought from Arabia by Count Orloff Tehesmenkoi in 1775 .

The Canadian Horse.-This race of horses is of the Norman descent, introduced from France by the early settlers of Canada. For many generations they were bred pure, and possessed the general characteristics of the Norman, except that they gradually became somewhat smaller than their progenitors. This is supposed to be owing to the cold climate, and the more scanty fare on which they have subsisted.

They are annually imported in large numbers into the New Fngland and some of the other northern states, where they have proved valuable for farm use, and as a general-purpose horse.

They are from fourteen and a half to fifteen hands high, have strong, compact muscles, large bones, in proportion to their size, and great power of endurance. They are also active, docile, easy to keep, and will perform a vast amount of hard labor, without any appearance of breaking down. They perpetuate their strong points, and are withal a very valuable animal for the purposes to which they are best adapted. There are many varieties of these, some having been crossed with the English thoroughbred. They are regarded as very valuable for producing crosses with other breeds.

Shetland Ponies.-These are the smallest of the pony breeds, many of them not being more than seven or eight hands high, and, in fact, the average height is not more than nine or ten hands, or from thirty-six to forty inches. It is claimed that no true Shetland ever reached eleven hands in height. They have round bodies, closely ribbed, with heads well shaped, and often with the dish-face of the true Arab. The ears are small and erect, eyes large and intelligent in expression, neck short and strong, shoulders thick and sloping; withers low; back slightly hollowed, loin strong and wide; tail and mane rery heary and long. The legs and hoofs are well-formed and strong, while as a breed, these little creatures possess a wonderful amount of endurance and strength. They are said to perform journeys of forty miles a day, upon the rocky and hilly pathways in their native country. They are generally in color dark sorrel, brown, and black; althongh sometimes spotted with considerable white. When bred in a half wild state, they often suffer for want of food, even subsisting on the coarsest that can be found; such as kelp and sea-weed that are found along the shore. The most perfectly-formed animals of this breed are found in the extreme northern islands of Unst and Tell.

Though they will sometimes resent injuries by the use of the heels, like the mule, yet when kindly treated and petted, they become very docile, being strongly attached to their masters, and make admirable playmates for children, as will be seen by the following respecting them from the London Field:-

"The ponies are not an agricultural, but a domestic necessity. In Shetland, as in parts of Ireland, every family depends for its supply of fuel on peat; and as the peat is seldom 
found near at hand on the shore where the houses stand, but on the hill behind them, there is always a hill in the rear in Shetland, every island consisting mainly of bill, with a patch or two of 'smooth' land in a few snug nooks by the shore, - and as it often is at a distance of several steep and stony miles, each house requires several ponies, the number depending on the distance, and the character of the road. A family living 'convenient' to the peat may require only two peat-carriers, and another family may require half a dozen.

The material, after it has been dug and dried in the usual manner, is carried home on the backs of the ponies in baskets called 'cassies.' It is obvious that the back which has to perform this kind of service should be broad and strong. A pony belonging to a breed which has had to pick its zigzag way down a steep declivity during many generations must be sure-footed. By the same rule, a pony whose grooms and playmates include a dozen juveniles - the children of the neighborhood, who roll about underneath him or upon his back - must be gentle; and the same pony, living on air sometimes, rather than on herbage, must be hardy.

The pony of the Shetiand Isle is, in fact, the offspring of circumstances. He is the pet of the family, gentle as the Arab's steed under similar training. He will follow his friends indoors like a dog, and lick the platters, or the children's faces. He has no more kick in him than a cat, and no more bite than a puppy. There is no precedent for his running away, nor for his becoming frightened or tired, even when he has carried some stout laird from Ler. wick to his house, many Scotch miles across the hills. In crossing boggy spots, where the water is retained and a green carpet of aquatic grass might deceive some steeds and bring them headlong to grief in the spongy trap, he carefully smells the surface, and is thus enabled to circumvent the danger.

In the winter, the Shetland pony wears a coat made of felted hair, and specially suited for the season. His thick winter garment is well adapted for protecting him against the fogs and damps of the climate. It is exceedingly warm and comfortable, fits close to the wearer's dapper form, and is not bad-looking when new. But, when the coat grows old towards spring, at the season when the new one should appear, it becomes the shabbiest garment of the kind that you often see. Its very amplitude, and the abundance of the material, render it the more conspicuous, when it peels and hangs for a while ragged and worn out, and then falls, bit by bit, till the whole of it disappears. No horse looks at his best when losing his old coat; and the more coat there may be to lose, the worse he looks."

Mustang, or Prairie Horses. - These are doubtless of Spanish origin; their promi. nent and general characteristics all bearing unmistakable evidence of this. The Spanish wars with Mexico and also voyages of discovery and exploration are matters of history, and the supposition is that Cortez lost many horses in his conquest of Mexico, also that the death of DeSoto, who discovered the Mississippi River, must have resulted in the abandonment of many horses, since his followers quickly made their escape to Mexico, from the unfriendly savages. It is also quite probable that many of these animals were abandoned by other adventurers in prospecting for gold and silver, all of which suppositions on the origin of this race of animals are well sustained by probable facts. This animal has also been called the avild horse of North America. There were no wild horses found on the American continent when it was discovered, and those that are now found in a wild or undomesticated state are such as have escaped from domesticity, or have been abandoned, and from them have descended the vast herds that may be found on the plains of South America, Central America, Mexico, Texas, California, and some of the territories.

Those found in Mexico and Texas are under-sized, while those of the more northerly section are considerably larger. The former have small limbs, long neck and back, a long, lean head, although well-shaped and well-set, and wide, open nostrils. Their hoofs are somewhat flat, and their tails and manes are generally very fine. These liberty-loving animals 
possess great endurance, are very fleet, and will easily outstrip the fleetest well-bred horse. They are quite useful for saddle purposes when tamed. They are inclined to be vicious, but may easily be managed. They are lightly valued, and may be purchased at a very low rate, owing to the constant and rapid increase of better stock.

Wild horses will always be found divided into squads or herds controlled by the most powerful stallion of the tribe, who holds dominion as long as he is able to do so, or until his place is usurped by a younger and more powerful rival; consequently every herd of wild horses has its leader.

The wild horses of our Western plains have furnished the kernel of many an interest. ing and exciting story of border-life. It is stated that two or three of these leaders, more powerful than all others, lived and led their herds on the plains of Texas for several years. One was white, and a pacer, and such was his speed and endurance, that although he was pursued by parties mounted on the best horses of the frontier for scores of miles together, yet he was never known to fail to keep a good distance ahead of his pursuers.

One of the most famous of these roving kings of the plain was captured many years ago in Matagorda County. He was a dun stallion, with a dark stripe down the back, and faint rings about the legs, as are sometimes seen on the mule. The New York Sportsman gives an account of the capture of this beautiful animal, as follows:-

"Between thirty and forty years ago mustangs were to be found in large numbers on every prairie west of the Brazos, and quite a large herd infested that one bounded by the Colorado on the west, and 'Old Caney' on the east, (about where the boundary between Matagorda and Wharton Counties now runs,) headed by this famous stallion, afterwards called 'Boggy' from the name of a creck by means of which his capture was effected.

These mustangs were very troublesome to the settlers in leading their gentle horses astray, for once with the mustangs they were as wild and unapproachable as the mustangs them. selves, and were scldom or never recaptured. So it was resolved by the settlers that this herd should be driven off, and this was inpossible without the capture of their leader, the dun stallion; consequently that was determined upon. Old Captain John Duncan, who acquired his title, as master of a steamboat on the Alabama River, when that State was but just out of its territorial form, was one of the wealthiest, most energetic, and prominent men of the settlement, and he was selected to plan the capture.

The range of 'Boggy' and his herd was a prairie of about thirty miles circumference, bounded by the bay on the south, Boggy Creek on the north, and Caney and Colorado on the east and west respectively, around which the mustangs always ran when pursued, as they had been 'many a time and oft.' The old captain collected about thirty of the best-mounted light-weights of the settlement, and the day before the chase he distributed them in couples about two miles apart around the prairie, where they camped for the night, and were in the saddle at daybreak the following morning, ready to begin the chase.

About good daylight the captain broke away after the herd, which, under the leadership of Boggy, at once took their usual circuit. As they passed the first couple, the boys whooped them up at their best lick for two miles, when the second couple took it up and drove them to the third, and so on round the prairie. After a while the weaker ones began to fail, and were leit by the herd, but no attention was paid to them, for orders were to capture Boggy. Away and away they went around the thirty-mile track, once, twice, and all were lagging but the magnificent Boggy, whose beautiful wavy mane and tail streamed in the wind as he swept on with reaching stride, running gallantly, at splendid speed, on, on, on. The sun rose and mounted higher and higher, until he stood at zenith, and looked down upon this wonderful struggle, and still the glorious dun kept on his course, nor seemed to fail of speed or wind, until the shadows slanted away to the east, and the shades of evening were warning the captain that he must redouble his efforts or darkness would put out the 



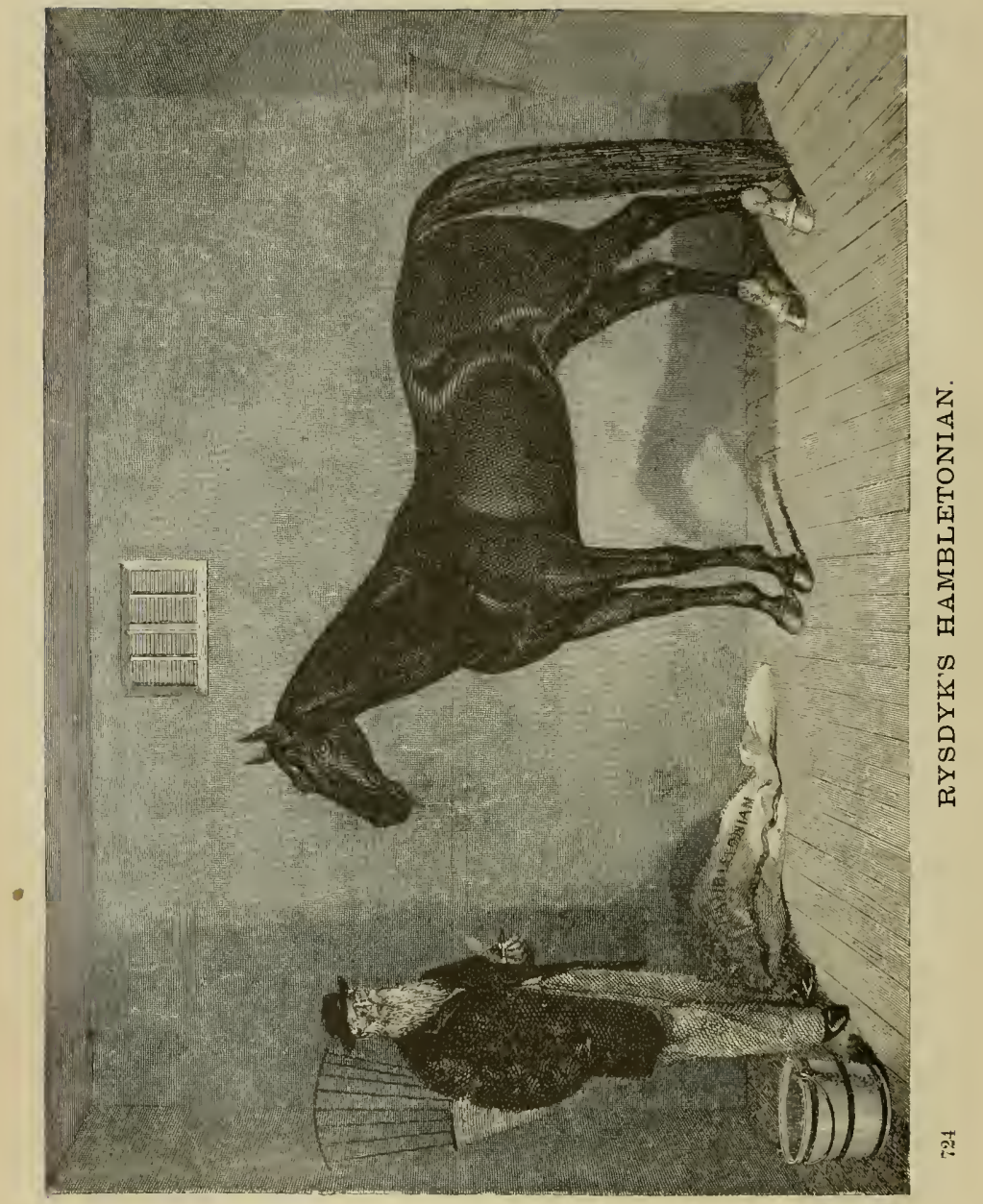


hope of capture, for that day at least, and another cavalcade of horses would have to be gathered to renew the race on the moriow.

So, bringing his ingenuity to bear, he gathered several of the freshest couples together, and held them for a final rush. Girtls were tightened, stirrups shortened, and lariats were coiled for the dash. They were hidden by a clump of trees from the line Boggy was running, and held well in hand until the poor fellow was entering his fourth round, when out they rushed 'with hoop and spur and loud huzzas,' and crowded him from his course. Round and round they twirled their lariats, and spurred and urged to get within throwing distance, when, sudden as a flash of light, Boggy swerved to the right and plunged headlong into the creek, which, as indicated by its name, was to prove a snare to his feet. Floundering, rearing, and plunging along through the treacherous bog which received him up to his very withers, he was gallantly but slowly making his way to the opposite shore, which a few more plunges would have reached, when, alas! alas! a whirr in the air, and over his beautiful head, and encircling his swelling neck, the fatal noose fell, and Boggy, poor, gallant fellow, was a captive.

It is stated that this stallion Boggy, after his captivity, became very quiet and docile. He was extensively used in the stud, but none of his get possessed especial merit."

When attacked by wolves, as the wild horses on the prairies sometimes are, they display much intelligence and courage. The leader of the drove forms the mares in a circle with their heels outward towards the enemy. Within this circle the foals and half-grown colts are collected for protection. Around the outside all the stallions collect to fight the wolves, while the mares use their heels to good advantage whenever the enemy approaches near - enough for the attack.

The Trotting Horse of America.-Horse trotting, as a public amusement, seems to have been inaugurated in this country during the first part of the present century, and since that time the desire for fast horses has been on the increase, so much so that many breeders in making speed the main or principal quality aimed at, have seemed to overlook others equally essential, and there is danger of carrying this point to the extent that speed will be secured at the expense of strength and endurance. The development of speed through systematic breeding and training has become one of the great industries of the United States, where it has attained a degree of perfection truly wonderful, and which is not to be found in any other portion of the world.

This interest dates back to the importation of the celebrated thoroughbred, Messenger, in 1788. At the time of his importation, he was eight years old, and was used in the stud for twenty years, in the vicinity of Philadelphia and New York. This horse is generally conceded to be the foundation or progenitor of the American trotting race, since nearly every trotter of this country, whose pedigree is known, traces to this animal, and the more Messenger strains there are in the pedigree, the greater, as a general rule, is the value.

One of the first horses noticed by the public journals of the country for trotting was the "Rat-tailed Gray," that trotted in 1816 on the Salem turnpike, near Boston. He is said to be the same horse that was afterwards called "Boston Blue," that was matched with Col. Bond, of Maryland, and Major William Jones, of Long Island, to trot one mile in three minutes for $\$ 1,000$. In this race Boston Blue is recorded as winning, and gained a high reputation for his unexampled speed.

New York and Philadelphia were the first to become interested in associations for developing the speed of the trotter, which associations resulted in great success in improving this race of horses.

In contesting for the premiums in trotting, the descendants of Messenger were found to take the lead. The propensity of this family for fast trotting was first discovered in the second generation, and after the death of Nessenger. It is true that there have been many 
fast trotters whose pedigree could not be definitely traced to the thoroughbred stock, but it has been found that, in the large majority of cases, the exceptions being rare, the best trotters can be traced to the thoroughbred stock, and there is no question whatever that this is the original source of the fast-trotting blood, they possessing naturally the rare combination of bone and muscle and other essentials that render them peculiarly adapted to speed.

Some horses trot naturally, while others require to be entirely educated to this gait. While so much depends upon the formation of the bones and muscles, and the adaptation of the limbs and body to the trotting gait, all horses require considerable training to become educated to the degree of developing their best capacity in this direction. The principal families that are at present distinguished as trotters are, the Hambletonians, Mambrinos, Ba. shaws, Clays, Stars, Morgans, Black Hawks (a branch of the Morgan family), Gold Dusts, Blue Bulls, Royal Georges, Canadians, etc., besides various other branches of these leading families.

Progenitors of Trotting Families.-Although England and America are indebted to Arabia for their finest horses-it being the original source of the pure thoroughbredcareful breeding and training, together with judicious crossing, have produced such a change that the American trotter and the English race-horse of to-day are greatly superior in speed and some other qualities to the best types of the thoroughbred that may now be found.

Youatt says, in this connection:- "The Arabian is not equal to his English descendant. This has also been incontestably proved in the United States. Pure-blood Arabians of the highest pretensions have at various times been imported into our country, but they have never compared either in speed or bottom with the English race-horse and his descendants. A few years ago, Recruit, an English horse of moderate reputation, easily beat Pyramus, the best. Arabian on the Bengal side of India."

We see by this the vast field that lies open to the breeder, and the power of intelligent efiort in accomplishing desired results. In breeding trotters, more difficulties have been met than in breeding the thoroughbred, since the latter, being of pure blood of long-known value, could be relied upon with a great degree of certainty, while in the case of trotters, which were produced by crossing with the thoroughbred blood, the breeder would frequently be surprised in obtaining the best results from the most unexpected sources, the union of the thoroughbred with other bloods producing in individual cases very different results.

Messenger, the noted progenitor of the trotting families in this country, foaled in 1780 , had as his first sire Mambrino, second sire Engineer, and third sire Sampson. According to the English stud-book, his first dam was by Turf, second dam by Regulus, third dam by Starling, fourth dam by Fox, fifth dam by Bay Bolton, sixth dam by Duke of Newcastle's Turk, seventh dam by Byerly Turk, eighth dam by Taffolet Barb, and ninth dam by Place's Turk. Beyond Sampson his pedigree may be traced back to Blaze, Flying Childers, and the Darley Arabian. He is described as being gray in color, fifteen and three-fourths bands high, and stoutly built.

Rysdyct's Hambletonian, the founder of the Hambletonian family, was got by Abdallah (who was traced to imported Messenger on the side of both sire and dam) out of a dam by imported Bellfounder, with two crosses to imp. Aessenger on her dam's side; foaled in 1849.

Mambrino Chief, the modern head of the Mambrino family, was foaled in 1844, sired by Mambrino Paymaster, who was a grandson of imp. Messenger.

Young Bushaw, foaled in 1822 by Grand Bashaw, an Arabian horse, heads the Bashaw family; his dam was Pearl, by First Consul.

Henry Clay, a grandson of Young Bashaw through Andrew Jackson, is the founder of the Clays, which are properly a branch of the Bashaw family.

Stockholm's American Star, sired by Duroc, the son of imp. Diomed, is the progenitor of the Star race. 
Justin Morgan, from whom the noted family of Morgans sprang, was foaled in 1793, sired by a horse named True Briton or Beautiful Bay, and without doubt a thoroughbred. Three of Justin Morgan's sons were famous, and each of them became the progenitor of a long line of descendants of marked and superior characteristics; they were known as Bulrush, Sherman, and Woodbury.

Bulrush Morgan was foaled in 1813. He had more muscular development than his brothers, was a dark bay with a few white hairs in the center of the forehead, and no other marks. His legs, mane, and tail were black, his mane and tail being very heavy. His mane is said to have reached nearly to his knees, and his foretop to his nose. His legs were broad, flat, and strong, with a powerful development of muscle. His hips were not as long as Sherman's, and he was not as well quartered as Woodbury, although he was deeper in the chest than either. He had not Woodbury's proud and elegant style of action, although a finelooking animal. His most remarkable characteristic was his great power of endurance. His dam was a dark bay, weighing a thousand pounds. She was bought out of a six-horse team that carried merchandise between Montpelier and Boston, and was a very strong, hardy animal.

Sherman Morgan, foaled in 1808, was a beautiful animal of a bright chestnut color, and possessed most of the fine points of his father. His weight was nine hundred and twenty. five pounds. He had a small white stripe in the face, and his off hind-leg was white from the foot half way to the hock; he also had a fine chest with prominent breast-bone. Although quite spirited, he was very tractable. His descendants were remarkably fine horses of all-work, known as the "general-purpose horse," and had the reputation of being the best stage horses in New England.

Woodbury Morgan was superior to his brothers in style of action, and presented so fine an appearance that he was very much sought after as a parade horse. He was very spirited, bold, and resolute. He was foaled in 1816. His dam was a deep bay, with black mane and tail, a small white spot in the forehead, and no other mark. She was of unknown blood, over fifteen hands high, had a fine heal and shoulders, compact body, and beautiful mane and tail. Her action was bold and spirited, and she had the reputation of being a fast trotter. The following description of Woodbury is given by Linsley:-

"Woodbury was fourteen and three-fourths hands high, and weighed from nine hundred and eighty-eight pounds to ten hundred and forty pounds; he was weighed several times, and these two statements of his weight, at different times, are the extremes. Many persons who have frequently seen him weighed, say they never knew him weigh more than ten hundred and thirty, nor less than ten hundred and fifteen pounds. He was a dark, rich chestnut; his off hind-leg was white from the foot half way to the hock, and he had a white stripe in his face, beginning at the edge of the upper-lip, filling the space between the nostrils, and extend. ing more than half-way to his eyes.

His mane was not very thick or long, and was lighter than either of the others; still it was full. His tail was cut off when a colt, and left about ten inches long; the hair was very full and curly; both mane and tail were about the same color as his body. The hair on the body was fine, short, and soft. He was close and compactly built, with heavy quarters and deep flanks; his chest was good and the shoulders finely shaped; he had a short back, and broad, sinewy loins. His legs had some long hairs on the back side, but were well shaped, somewhat larger than Sherman's and not so large as Bulrush's.

His head was small and lean, with a fine, firm muzzle, the nostrils very large and full, face straight, very wide between the eyes, which were dark hazel, very large and prominent, and showed no white around the edge of the lid. His ears were small and fine, but rather short, and set somewhat wider apart than many would consider consistent with perfect beauty. His style of action was bold and resolute, and his temperament was so nervous 
that when taken out with a bridle it was almost impossible to keep him still. He was a good driver and appeared well in harness, but he appeared to the best advantage under the saddle. Militia colonels and generals were eager to ride him, and no 'musters' or reviews could pasi without his being seen; in his case, to be seen was to be admired. His disposition was pleas. ant and playful.

As has been said, he was taken to Gainesville, Alabama, in the autumn of 1836, being then twenty years old. He was shipped from Boston, on board a small saihing vessel; he suffered much from the long and stormy passage, and never fully recovered from the effects of it. It is altogether likely that the climate and food did not agree with him, for neither were such as he had been accustomed to; however this may be, it is certain that he continued to fail until he died in I833.

Woodbury was the largest of these horses, and possessed in a greater degree the bold, fearless, and showy style of their sire. He was more nervous and less tractabie than Sherman, better under the saddle, not so pleasant in harness, and we are inclined to think hardly

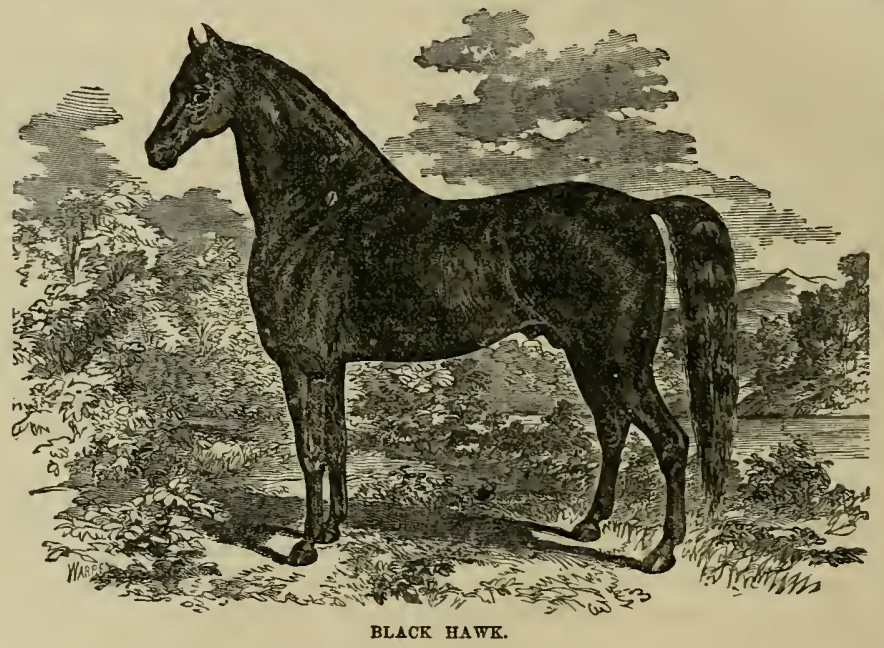

as good a roadster. His form was more symmetrical than either of the others; his breast was not so full and prominent as Sherman's; he was deeper in the flanks and better quartered. No horse ever had less fear. Martial music only roused him; the firing of guns in no way disturbed him; waving flags and gay uniforms seemed hardly able to attract from him a single glance, and he moved about as if he himself were the principal object of attraction, and the cause of all the attending excitement and display."

Black Hawk was sired by Sherman Morgan, the son of old Justin Morgan, the dam being a half-blood English mare of jet-black color, and a fast trotter. From him the race that bears his name are descended, being but another branch of the Morgan family. He was foaled in 1833; was jet-black in color, like bis dam, although in almost every other respect he bore a strong resemblance to the family of which he was a member, such as size, com. pactness, muscular development, temperament, endurance, and style of action. His stock exhibited similar characteristics; though generally larger, they were (when not black) almost without exception a bay, or chestnut. Black Hawk was slightly less than fifteen hands high, and weighed 1,000 pounds. 

In writing of him when he was twenty-three years of age, Mr. Linsley says: - "His compact, symmetrical, and muscular form, and nervous, elastic style of action, give unmistakable evidence of the speed and endurance he has shown upon the turf and road; and although now twenty-three years old, his eye has lost none of its brightness, his health is still excellent, and his movements still graceful and energetic."

He was in all respects a remarkable horse, being beautiful and majestic in appearance, and whether in harness or not, he was ever the same splendid animal, always attracting universal admiration. His blood is very highly prized in New England trotting circles of to-day, as well as other portions of the country. He could trot his mile in 2:40 without training, and was a horse of great intelligence, as well as great endurance. Besides, Black Hawk possessed the power of transmitting his speed and other characteristics to his offspring in a degree unsurpassed by any horse in the country. He died in 1856, being twenty-three years old.

Gold Dust was another branch of the Morgan family of horses, while the Royal Georges sprung from Tippoo, a horse supposed to be from Ogden's Messenger, a son of imported Messenger.

The best representatives of the Canadian race are said to be St. Lawrence and Pacing Pilot, horses of unknown pedigree. Besides these, are the Blue Bulls, which were descended from Doyle's Blue Bull, a pacer that was sired by a pacer of the same name, and numerous other branches of leading families already given. Various opinions are entertained by different breeders, relative to the best methods of perpetuating and improving the trotting horse, but our limits will not admit of farther consideration of the subject in this connection.

Fabulous prices have been paid in this country for trotters, and frequently for colts, simply on the strength of their breeding. It is stated from anthentic sources that in 1876 the sum of $\$ 13,000$ was paid for two two-year-old fillies, and $\$ 11,200$ for thirteen, nearly all of them young. A three-year-old colt, Steinway, was sold in 1879 for $\$ 13,000$ to a party in California. Gov. Sprague was sold for $\$ 27,000$ at the age of five years, and Mand $\mathrm{S}$. for $\$ 21,000$ when four years old, and after she had trotted a mile in $2 \mathrm{~m}, 17 \frac{1}{2} \mathrm{~s}$. As she is now queen of the turf, having reduced her record to $2 \mathrm{~m} .10 \frac{1}{4} \mathrm{~s}$, it would probably require a very much larger sum than that to purchase her, if at all. The sum of $\$ 40,000$ was paid for Smuggler, $\$ 45,000$ for Pocahontas, $\$ 35,000$ for Goldsmith Maid and Dexter, $\$ 36,000$ for Rarus, $\$ 30,000$ for Lady Thorne, while St. Julien was prized at $\$ 50,000$ at the close of 1879 , when he had attained the fastest record known at that time. Messenger, Rysdyk's Hambletonian, Volnnteer, and several other noted horses were valued at $\$ 100,000$ when in their prime.

Noted Trotting Horses. - Among the many noted trotting horses of the country, we have space to give the description of but a few. Trotting time has been gradually reduced until at present it stands at $2 \mathrm{~m} .10 \mathrm{~s}$., that achieved by the queen of the turf, Maud $\mathrm{S}$, whose record has not yet been excelled. Trotting a mile in three minutes was formerly considered very good time. while the opinion entertained by many of the leading horsemen of the country of former days was, that no animal could ever excel the $2 \mathrm{~m}$. $20 \mathrm{~s}$. record, and when little Flora Temple, less than twenty-five years ago, reduced it to $2: 193$, she became the wonder of the world for a brief time. Dexter, after a period of eight years, reduced this record to $2: 17 \frac{1}{2}$. In 1871 Goldsmith Maid reduced this record to $2: 17$, and again in 1873 to $2: 163$. She still continued to reduce her record by degrees, until it reached $2: 14$, remaining queen of the turf until Rarus comes to the front with a record of $2: 13 \frac{1}{4}$. But his reign is short, for from California the following year comes the news that St. Julien has won a victory over time by a record of $2: 12 \frac{3}{4}$. The contest next is between Maud S. and St. Julien, at Rochester, N. Y., in Angust, 18S0, which resulted in a drawn battle for possession of the sceptre, but a joint victory over time, for they each scored on that day a record of $2: 11$ s. This was still further reduced afterward by St. Julien to $2: 11 \frac{1}{4}$, and by Maud S. to 2:101. 
Among the best-informed horsemen there is a diversity of opinion with respect to the limits of trotting speed, but none fix it slower than $2 \mathrm{~m}$. $10 \mathrm{~s}$., while some are confident that a mile will yet be made by a trotter in two minutes. The pacing gait differs from trotting by having the front and hind legs on the same side move in the same direction simultaneously, while in trotting, the near fore-leg and off hind-leg move together. The pacing gait is more rapid than trotting, but the latter is greatly preferred.

Flora Temple was foaled in 1845, and bred by Samuel Welch, Esq., of Oneida county, N. Y. She was sired by Hunter, who was by Kentucky Hunter, her dam being Madam Temple, who was sired by a spotted Arabian horse owned by Mr. Horace Terry of Dutchess county, N. Y. She was purchased of a drover by Mr. Jonathan Vielee for $\$ 175$. At this time she was a little rough-coated animal, not over fourteen hands and two inches high, and was tied behind the drover's wagon by a rope. All that the drover would tell Mr. Vielee about the little bay mare with a docked tail was, that he had purchased her in Utica of a young man who had been trying in vain to dispose of her in connection with another little mare, but that the intractable disposition of this one had invariably prevented a sale, so that finally each animal was sold separately, and Flora, at five years of age, was bought by the drover for $\$ 80$.

It appears, also, that her former owner kept her until she was four years old, and, finding her willful and unserviceable, sold her for the sum of \$13. Mr. Congdon, her new owner, sold her soon after for $\$ 68$, subsequent to which she changed owners two or three times before coming to $\mathrm{Mr}$. Vielee, who, fortunately, was a man who appreciated, to a certain extent, her good points. Being a practical business man, and sufficiently sagacious to see that New York was a place to find a market for such an animal, Mr. Vielee took her there and sold her to Mr. Geo. E. Perrin of that city for $\$ 350$. In his hands she was instructed in trotting and fitted for the track. Her first public appearance upon the turf was at Long Island in 1850 , when, to the astonishment of all, she defeated four horses, winning the purse of $\$ 50$, and the race in $2: 52,2: 55,2: 52,2: 49$.

The next year she was not in training, owing to an accident. The following year she trotted twice, winning both times. In 1853, being then eight years old, she started a wonderful trotting career, which ended only with the advent of the civil war.

In her first race at the old Hunting Park Course, Philadelphia, she was beaten by Black Douglass; a local celebrity, but soon made him lower his colors by defeating him twice without difficulty. She also beat Highland Maid twice, Green Mountain Maid three times, Tacany seven times, Rhode Island three times, and Lady Brooks and Lady Vernon each once. She was beaten twice by Tacony, and once each by Black Douglass and Green Mountain Maid. In her first season upon the turf it will thus be seen that she won nineteen races and lost but four. In her next season she lost in her first race with the gray mare, Sontag, and won in every race besides during the whole season.

In the next two years, she carried off the lion's share of the honors, and reduced her record to $2: 24 \frac{1}{2}$. In 1858 she was sold to $\mathrm{Wm}$. McDonald, Esq., a wealthy gentleman of Baltimore, for $\$ 8,000$, and during the year won thirteen victories, without a single defeat. The next year (1859) was a remarkable year with her, not only for winning in her races with Ethan Allen, beating him twice, and also in other races, but especially for her splendid

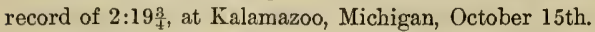

In the many races trotted by little Flora Temple during the rest of her trotting career, her most formidable antagonists were George M. Patchen and Ethan Allen; the former defeating her in one two-mile heat contest, and Flora defeating him twice, and Ethan Allen once. She also went hipporroming with George M. Patchen. Her owner sympathizing with the rebellion, she was confiscated by the governor in 1861, and never trotted again.

In 1864, Flora Temple was purchased by Mr. Welch of Chestnut Hill, Pa., for the sum 
of $\$ 8,000$. Mr. Welch owned her up to the time of her death of old age, which occurred Dec. 21,1877 , aged nearly 33 years.

American Girl.-This noted animal was a bay, about sixteen hands high, was foaled in 1862 , by an unknown mare who was bought for forty dollars from a team in Virginia. She was sired by Amos Clay and bred by Mr. Philip Travis of Westchester County, New York.

Mr. Travis gave the filly to his sons; and they, not seeing her value, sold her for a small sum to a Mr. Odell, who, after testing her value as a roadster, and finding her speedy, disposed of her to Messrs. Travis and Mason, brick-makers, near Peekskill.

The mare now received her name, "American Girl," and was put in training for the trotting-course with a horse known as J. J. Bradley, in the first of which she was to go in wagon, he in harness; in the second, both were to go in harness. The first race occurred on the 15th of November, 1867, and was well contested by American Girl, now five years old, but was won by the horse. The second race, being on even terms, was never trotted, the owner of the gelding paying forfeit.

American Girl was now sold to Mr. William Lovell, and was placed in the hands of John Lovett as trainer, and on the 4th of June, 1868, she beat Goldsmith Maid at Pough. keepsie, after a desperate race of six heats, the first of a series of races in which these renowned mares were rival contestants. In one of the heats of this race, American Girl trotted in 2:28. She showed also that she had remarkable courage and resolution.

Losing in the next race at Narragansett Park, Mr. Lovell selected for her a new driver and trainer, Mr. Hiram Howe. Soon after, there was another trot between American Girl and Goldsmith Nlaid, in which the former defeated the latter after a contest of six heats, the best time being 2:25. American Girl trotted in five other races during the season, winning twice and losing three races. She, however, gained a record of 2:24. After three defeats in the following season, she was placed in the hands of Roden, a man of excellent capacity.

Roden began with her on the 29th of May, 1869, at the Prospect Park course, and had to compete against a large and very strong field, consisting of Lucy, Bashaw, Jr., Goldsmith Maid, Rhode Island, and George Wilkes. In this race she won in three straight heats$2: 23 \frac{1}{2}, 2: 23 \frac{1}{4}, 2: 21$.

This was not only a grand triumph for her, but also shows how much depends upon a driver of the right kind and capacity. This was the first time that this remarkable mare, now in her seventh year, had been properly handled; hence, the result.

In the six ensuing races of the season, she won in every instance; and in one of these races (notably that on the 26th of June) at Narragansett Park, placed herself second on the roll with Dexter, by beating Lady Thorn, Goldsmith Maid, Lucy, and George Palmer, in three straight heats, and also getting a record of $2: 19$.

But at Suffolk Park, Philadelphia, American Girl met Goldsmith Maid for the sixth time and was beaten. Following this defeat, another driver took her in charge, but, as will be seen, he was the wrong man in the wrong place; for in the balance of the season he won but three races, while he lost seven. In his hands also, in 1870 , she won seven races and lost in nine. In 1870 American Girl did not beat Goldsmith Maid once, but was beaten by the latter seven times. During the season of 1871 , she won in six races; none of them very fast, and lost in seven, being beaten six times by Goldsmith Maid.

In 1872, she was placed in the hands of Mr. Mace, and began the season by winning the first three races. In the third race, American Girl met and defeated Goldsmith Maid, Lucy, and Henry, in three heats-the second heat being trotted in 2:191. During this year, she won in all eight races, and in one of them with Lucy, in which she lost, made the time of $2: 17 \frac{1}{4}$. The following year she won five races. In 1874, she won in nine races, the best and crowning one in her career being at Albany, when she made the time of 2:201,2:161, 
$2: 19$, placing the three heats on the average at $2: 18 \frac{1}{2}$ each, the result being all the more remarkable when we remember that she was badly spavined in one leg.

In 1875 she won only one race, when she beat Lula and Nettie, ber remarkable career closing at Elmira, New York, October 2d, where she dropped dead at the quarter-pole in the first heat. She had been ailing with epizootic, and, as the post-mortem showed, ought not to have been put upon the race-course in that condition.

Goldsmitll Maid was foaled in 1857. Her sire was the son of Hambletonian, known in Kentucky as Alexander's Abdallah, Her dam was by the original Abdallah, son of Mambrino (Americus) and sire of Hambletonian. She is said to have been "the smallest of her dam's products, and was by no means exempt from temper and accidents." She was noted for jumping fences, and when put to the harrow, was entirely unmanageable, as she would rear up and fall over; when hitched to a wagon, she would kick herself loose and run away. She was regarded as so very ungovernable as to be practically useless, and her owner, Mr. J. B. Decker, of Sussex County, New York, accordingly sold her for the sum of $\$ 350$. In taking the animal home, the purchaser was offered $\$ 400$ for her, which he accepted. Three months later the last purchaser sold her for $\$ 650$ and a buggy to Mr. Alden Goldsmith, one of the most sagacious, kind, and patient of horsemen. She was at that time very wild, timid, and unruly, although she possessed nothing of what might be termed a malicious disposition. By patient and gentle treatment, Mr. Goldsmith made her more quiet, but she would not go with check-rein or running-martingale, so her owner ordered them taken off, and also took off her blinders. She trotted without check in all ber races for the first three years. She is described as "small of stature, long and low, deep through the heart, of wiry, whalebone texture all over, and with a back of amazing strength for a horse of her size."

In 1865 she trotted her first race, the best time being $2: 26$, and won in three heats. In 1866 she trotted nine or ten times, and won in all but the last. The next year she was beaten by Dexter. In 1868 she won eight times and made a record of $2: 21 \frac{1}{2}$. The next season she

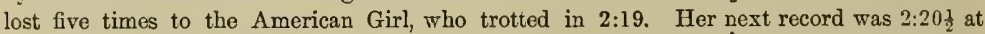
Boston, where she beat Lucy. At Philadelphia she won the victory over American Girl in three heats all better than $2: 20$, which was the first time any horse had beaten $2: 20$ in all the heats of a race.

During this year she won eight races, beating all those that had previously beaten her except Lady Thorne. In 1870 she won eleven times.' In 1871 she beat all her competitors, including American Girl and Lucy. Soon after, she trotted in $2: 17$, beating Dexter's time

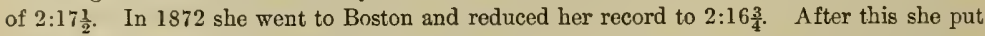
in all the heats for the fourth time in less than $2: 20$. She was then taken to Sacramento and in little more than a month after her previous race trotted in 2:17 $\frac{1}{2}$. In 1874 Goldsmith Maid trotted seventeen times and with increased speed, making a record of $2: 16$. Three times during this year 2:20 was beaten in all the heats. At Rochester a second heat was trotted in 2:143. At Boston in September, 1874, she trotted in 2:14. In 1877 she defeated Rarus in California over a rough track in $2: 19 \frac{1}{2}, 2: 14 \frac{1}{2}, 2: 17$. Shortly after this she was permanently retired from the track, her best record, as previously stated, being 2:14.

The following incident, showing how attached an intelligent horse becomes to his faithful attendant, and also the influence of kindness on dumb animals, will be of interest to many. As the story goes, Charley Cochrane, who was many years the faithful custodian of Goldsmith Maid, went to pay her a visit. It is well known that she was very jealous of her foal, and would not permit any one to come near it, and it was arranged that she should hear Charley's voice before she saw him; and although they had been separated for two years, a loud whinny presently assured the visitors that she had recognized the man's voice. Cochrane next showed bimself, when a touching scene occurred. The old Queen of 
the Turf, who for months would not allow any one to approach her, making use of both heels and teeth if it was attempted, rushed with a bound to her old friend, forgetting even her colt, and rubbed her head upon his shoulders, her nose in his face, played with his whiskers, and showed by her every action that her heart was full of joy to see him. Directly the colt came up to them, and she seemed delighted when Charley placed his hand on the little fellow. When Cochrane left the place she followed him to the gate, whinnying for him even after he had passed out of her sight. This horse is only one of many examples that might be given of what patience and kindness will accomplish with a high-tempered and spirited horse.

Dexter was bred by Jonathan Hawkins, Esq., of Orange county, N. Y. He was foaled in 1858, and purchased when four years old by Mr. George B. Alley for the sum of $\$ 400$. He was sired by Hambletonian, and, although a finely-formed animal, his four white stockings and blaze in the face had caused him to be held in disfavor; so that up to this time it is stated that he was not only practically unbroken, but had never had a feed of oats in his life.

In his early training, while in Mr. Alley's hands, two accidents happened, one while he was attached to a sleigh, and the other to a wagon, resulting in each case in a runaway. Fortunately, in his five-year-old season he was placed in the hands of Hiram Woodruff, and after a short time went in 2:42 to a wagon. Dexter made his first trot on the 4 th of May, 1864 , and was consequently six years old at the time. In this effort his competitors were beaten.

He continued to improve his record until it was reduced to $2: 17 \frac{1}{4}$, being repeatedly matched with the best trotters of the country. After this achievement he was purchased by Mr. Bonner and retired from the turf. Wallace's Monthly says of him:

"During his career of less than four seasons, Dexter won forty-nine races. The great majority of them were mile heats, three in five, in harness. He also won at three-mile heats, and at two-mile heats, in harness, and to wagon he was never defeated. He lost a race to Shark through hitting himself. Lady Thorne defeated him once when he was not seasoned, and was off, as well. He beat her five times in much better races. General Butler beat him once in a poor race, under saddle, when he was off. Ethan Allen, with running mate, beat him twice. Dexter made the best mile under saddle, the best mile in harness, and the best mile to wagon that had been made. His two miles to wagon, second heat, was perhaps his greatest performance."

Rarus.-Of the pedigree of this horse, which was for a brief period king of the turf, there seems to be a mingling of fact and speculation. Mr. Conklin, who bred him, states that his sire was a horse owned by him called Abdallah (the son of old Abdallah). Others trace his pedigree to Rysdyk's Hambletonian, and those who knew the latter horse well see a striking resemblance between him and that famous progenitor of a family of trotters. Mr. Videto, one of his former owners, says he was "marked like the 'old horse,' gaited like him, and formed like him, with the same sway back and white hind-feet."

The investigations which have been made also establish the fact that the dam of Rarus was by Telegraph, and that his grandam was a good mare that was known as a Black Hawk, but farther than this nothing is known of her; from all of which it would seem that the pedigree of Rarus through Conklin's Abdallah is uncertain, and it should be written as unknown.

But a horse that can beat the time of the famous Goldsmith Maid, and win a record of 2:13 $\frac{1}{4}$ as Rarus did, at Bufialo Park, is a kingly horse indeed, and deserving of all the more credit for all his brilliant performances, if he does not inherit speed from his ancestors.

He was a large, strong horse, of an exceedingly unpleasant disposition, and would frequently manifest his temper by both biting and kicking. He was used in the streets of New York for some time, in a butcher's cart, but took a higher position when his real merits became known and appreciated. 
In appearance, Rarus was a fine-looking animal. He was a bay, with white hocks, and a white strip on the nose; was $16 \frac{1}{2}$ hands high, and in many respects resembled Rysdyk's Hambletonian. Rarus's fastest first heat was at Cleveland, Ohio, in 1878, and was $2: 14 \frac{1}{2}$; his fastest second heat was in Hartford, Conn., the same year, and was $2: 13 \frac{1}{2}$. His fastast third

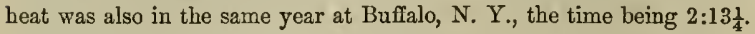

Smuggler.-This horse was bred by Mr. J. M. Morgan, who at that time resided near Columbus, Ohio. He was foaled in 1866, and was taken to Olathe, Kansas, in August, 1872, and at that time is said to have been a confirmed pacer. He was soon after placed in the hands of a very successful trainer and driver, and in about three months made a mile in $2: 30$. From this time his improvement was very rapid. In July, 1873, he made the time of 2:18 He was soon after purchased by Col. Tuffts, of Kansas, and shortly after this taken to Prospect Park, N. Y., and given a public trial of three heats, one mile each, which resulted in the time of $2: 19 \frac{3}{4}, 2: 21 \frac{1}{4}, 2: 21$, making the last half of the third mile in 1:09. Immediately after this performance, he was purchased by Col. H. S. Russell, of Milton, Mass, for the sum of $\$ 40,000$. He continued to gradually lower his record, and at Rochester, N. Y., 1876, he won in three straight heats, in 2:15 $, 2: 18,2: 19 \frac{1}{2}$, making a record four and a quarter seconds lower than had ever been made by any other stallion. At Hartford, Conn., the same year, he trotted in $2: 15 \frac{1}{4}$, the fastest heat ever made by a stallion.

Smuggler is a very dark bay horse, $15 \frac{3}{4}$ hands high, with a blaze lying between the eyes, and widening out until at the end of his nose it extends to either nostril. Like almost all pacers that have been broken to trotting, he wears a heavy shoe in front in order to steady his gait. Of his pedigree, one of the leading stock journals says:

"He was got by Blanco, a son of Iron's Cadmus, and his dam was a bay pacing mare brought from West Virginia. This mare was for a long time reported as by Tuckahoe, but subsequent investigations have exploded that story, and it may safely be said that her blood is hopelessly unknown. The dam of Blanco was by blind Tuckahoe, a son of Herod Tuckahoe. Iron's Cadmus was by Cadmus, son of American Eclipse, out of a mare by Brunswick. This horse, Iron's Cadmus, was the sire of the famous pacing mare Pocahontas, who, in turn, was the dam of Mr Bonner's trotting mare of the same name, by Ethan Allen.

It will be seen from the foregoing that all that is known of the blood of Smuggler is through his sire, Blanco, and that from this source he inherits a good share of pacing blood, mixed with thoroughbred; and that his dam was also a pacer."

St. Julien.-This regal horse, the rival of Maud S., was bred by Mr. Benj. F. Dunning, of Orange county, N. Y. He was foaled in 1869, sired by Volunteer, who was sired by Hambletonian got by Abdallah, while his dam was by Henry Clay. He is a large, powerfully. built animal, and possesses great courage and energy.

He made his entry into the trotting world at Poughkeepsie, N. Y., in August, 1875, and in the short space of three weeks won six races. He was sold in January, 1876, to California parties for the round sum of $\$ 20,000$, and was taken at once to that State, where for three years he was out of condition much of the time. His training during this period, however, was as skillful as it was severe, as his subsequent achieveménts attest.

His fastest record up to the present time is $2: 11 \frac{1}{4}$, being next to Maud S., who stands first, being the fastest trotter known to the turf.

Maul S.-This noted horse, at present queen of the trotting turf, was bred at Mr. Alexander's celebrated "Woodburn Farm." Her sire was Harold, a son of Rysdyk's Hambletonian, whose dam was Enchantress, got by Abdallah. Her dam was Helen Russell, by Pilot, Jr., son of old Pacing Pilot.

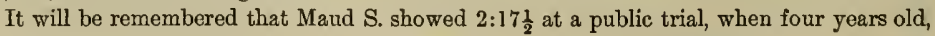
at Lexington, Ky., Oct. 26, 1878; and that thereupon Mr. Wm. H. Vanderbilt, of New York, purchased her for $\$ 21,000$. 



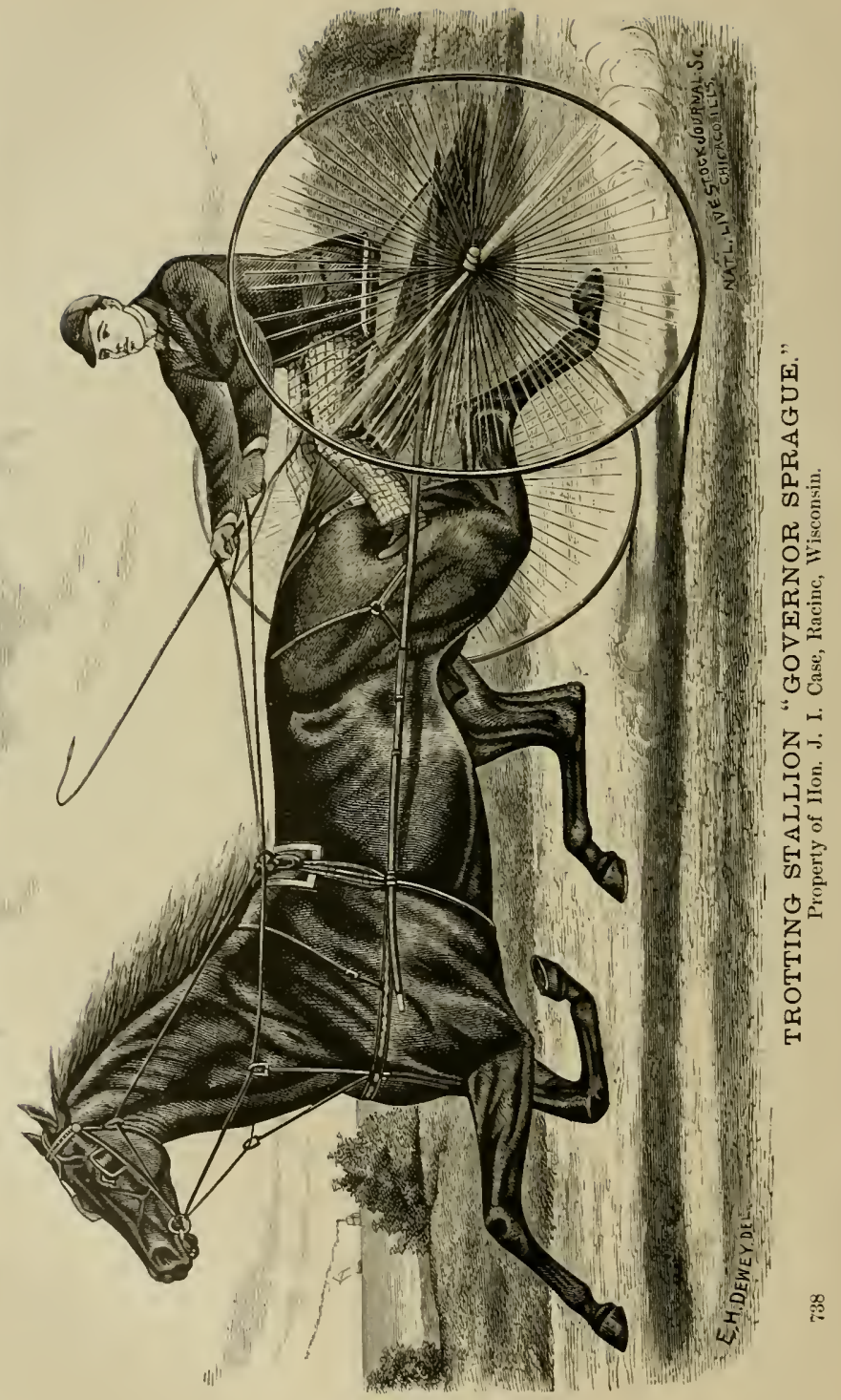


It seems, however, that during the year that Maud S. remained in New York she did not come quite up to Mr. Vanderbilt's expectations, so with his characteristic good sense he sent her back to Capt. Stone, of Cincinnati, the gentleman of whom he bought her, with the request that he would take her in hand and bring her back to her old form.

Upon receiving Maud S. Capt. Stone put her under the charge of her former trainer, in whose hands she rapidly improved, and soon the most remarkable accounts respecting her speed were afloat. She is described as follows:

"Maud S. the champion trotter of the world, is a long-bodied mare, standing 15 hands $2 \frac{1}{2}$ inches high at the withers and fully 16 hands high at the hips. Her weight, in trotting condition, is $960 \mathrm{lbs}$., and her stride, when going at her best, on a straight track, is about 18 feet. In her great feat at Chicago, September 18th, when she trotted a mile in $2: 10 \frac{3}{4}$, her stride, when coming down the home stretch against a high wind, was a little less than 17 feet. She wears 'shin boots' and 'scalpers,' and carries a 15-oz. shoe, with a 4-oz. toe-weight in front, and an 8-oz. shoe behind. She is usually driven in a bridle, without blinds. She is a mare of very strong will, and it is necessary to handle her with great gentleness. A man who would fight her, would soon render her entirely unmanageable. And in this she is the counterpart of her grandam Enchantress, as well as of her sire Harold and his full brother Lakeland Abdallah. The old mare had the courage and resolution of the bull-dog, and this quality descended to all of her produce. Harold and Lakeland Abdallah both possess it to a remarkable degree, and, if they had fallen when young into gentle, careful hands, as Maud S. fortunately did, it is certain that they would have developed into animals of far greater value even than they proved to be."

When brought out for a race, Maud S. is as steady as a clock, and moves with that easy, gliding motion which steals over the ground at a pace that deceives everything but the timer's watch. This gives her a great advantage over horses of nervous and excitable temperaments. The account of her best achievement is thus given by one of the journals of the day:-

"Twenty thousand people gathered at the race-course at Buffalo, N. Y., August 11th, to witness an attempt to lower the record of the noted mare, Maud S. She was accompanied by a running horse who could not keep up with her, the track being in perfect condition for trotting. Maud S. trotted the first quarter in $32 \frac{3}{4}$, the half in 1:051, the three-quarters in

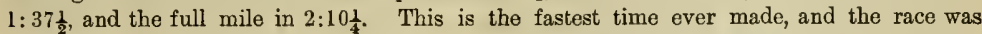
made under the eye of her owner, Wm. H. Vanderbilt, and at his desire. Last year she closed the season with a record of $2: 10 \frac{3}{4}$; at Pittsburgh, Pa., a few days ago, she made a mile in $2: 10 \frac{1}{2}$, and has now still farther reduced her record. Her driver expects to see her make a mile in $2: 08 . "$

Santa Claus. - This famous animal belongs to the Hambletonian family, being the son of Strathmore; a grandson of Rysdyk's Hambletonian, a great grandson of Abdallah. His dam, Lady Thorne, Jr., was by Williams' Mambrino, a grandson of Mambrino Chief. In color, Santa Claus is a dark bay, with black mane, tail, and legs, while his fore-top reaches nearly to his nostrils, and conceals a small star in the forehead, the only white there is on him. He has a well-formed chest and body, and limbs admirably adapted to speed. It is stated that when first foaled he was so ill-formed and ungainly that the owner of his sire endeavored to hire the breeder of the colt to kill him, whose proposition came near*being accepted. This valuable animal is at present owned by Col. P. A. Finigan of California. His best time thus far is $2: 17 \frac{1}{2}$, a record that will probably be reduced in the future, as he is now in his prime, and possesses great endurance and energy.

Gov. Sprague is a coal-black stallion, without a white hair, but his muzzle and flanks show wine-colored tints. He is slightly taller at the withers than at the rump, with a short back, powerfully muscled, and limbs sinewy and strong. He possesses great endurance, as 
is evidenced by his conformation generally, while he is energetic and courageous, yet gentle and kind in disposition. He was foaled in 1871. His sire, Rhode Island, belonged to the Messenger family, and had a record of $2: 23 \frac{1}{2}$, while his dam also possessed thoroughbred blood. During the summer that he was two years old he was broken to the harness, and showed such remarkable promise of speed that he was sold in the autumn to Messrs. Higbie Brothers and Mr. A. C. Babcock of Canton, Ill., for \$1,500. For the next year he was driven but little on the road, and this only with a view to accustom him to the harness; but he was not put to training. In the spring of 1875 he was put to moderate training, but not driven to his full speed. In the spring of 1876 he was again put in training. In the following June he was sold to his present owner, Hon. J. I. Case of Racine, Wis., for $\$ 27,000$. Two weeks afterward he won his second race at Rochester, N. Y., and made a record of $2: 21 \frac{1}{4}$. Shortly afterward he reduced his record to $2: 20 \frac{1}{4}$, being then five years of age.

As a sire of trotters, he has already become noted. Kate Sprague, of his get, a six-yearold mare, made $2: 18$ in the second heat, at Rochester, N. Y., in 1881, trotting the last quarter of the heat in 33 seconds; a 2:15 gait.

Trinket. - This horse was bred by R. S. Veach, Esq., of "Indian Hill Farm," near Louisville. Ky. She was sired by Princess in 1875, who was sired by the famous and fasttrotting stallion Woodford Mambrino, he by Mambrino Chief. The dam of Princess was by Alexander's Abdallah, a son of Rysdyk's Hambletonian. The dam of Trinket was also by Rysdyk's Hambletonian. Thus it will be seen that she possesses a trotting pedigree, built largely upon a thoroughbred foundation. Her owner sold her when a yearling. Then two years old, she showed great speed for her age. Her first appearance upon the turf was in 1879. She is described by one of the leading journals as follows:-

"A handsome, high-bred-looking mare, with a fine, intelligent head, a light, well-shaped neck, and splendid shoulders, with great heart-room, a strong back, well-coupled, wide hips, and sloping quarters and big stifles - a union of great substance along with quality. Her appearance is suggestive of the whalebony, spring-steel style of horse. Her legs are hard, like ivory, and she has not a soft spot about her. A dangerous, resolute-looking mare is Trinket, and when she moves, her action is smooth, frictionless, and stealthy. She covers a vast amount of ground at a stride, but she does it seemingly with a cat-like effort; and when she gets the word 'Gol' she darts as a cat darts from under the bed with a dog to persuade her. She is about $15 \frac{1}{2}$ hands high, and wears an eight-ounce toe-weight when trotting." Her best record, up to this time, is $2: 14 \frac{8}{4}$.

Thorndale is a bay stallion $15 \frac{1}{2}$ hands high, and foaled in 1865 . He was bred by Dr. J. R. Adams of Scott county, Ky., and is owned by Edwin Thorne of "Thorndale Stud Farm," Millbrook, N. Y. He was sired by Alexander's Abdallal, the sire of Goldsmith Maid, for a long time queen of the trotting turf; her record, as previously stated, being 2:14. His first dam was Dolly, by Mambrino Chief, the sire of Lady Thorne, whose record was $2: 18 \frac{1}{4}$. Fis second dam was by a son of Potomac.

Thorndale won the three-year-old trotting stakes at Lexington, Ky., July, 1868, in three straight heats, the record being $2: 49 \frac{1}{2}, 2: 50,2: 55$. Immediately after this race, he was purchased by Mr. Thorne, and placed at the head of his stud. He was awarded the first preminm in the stallion class, three years old and under five, with ten competitors, at the Narragansett Park Fair, 1868. He also won the first premium in the stallion class for getting roadsters, at the New York State Fairs at Albany in 1871, 1873, and 1880.

ln May, 18i6, he was placed in the hands of his trainer, and after eighty days' training he trotted in Buffalo with eleven competitors in the 2:32 class, and won the second, third, and fifth heats, and the race in $2: 22 \frac{1}{4}, 2: 23 \frac{1}{2}$, and $2: 25$. Since that period he has won in several races, in one of which at Fleetwood Park, N. Y., he won not only the race, but a prize of $\$ 2,000$ and a silver cup. 
Trotting Record. - The following is a list of horses that have trotted a mile in $2 \mathrm{~m}$. 20 s., or better, together with their pedigree; also, tables of the fastest trotting to wagon, under saddle, pacing different distances, fleetest running, etc.

$$
\text { 2: } 101 / 4 \text {. }
$$

Yaud S., by Harold, dam Miss Russell, by Pilot, Jr.

$$
\text { 2:113/4. }
$$

St. Julien, by Folunteer, dam by Harry Clsy.

$$
\text { 2: } 13 \frac{1}{4} \text {. }
$$

Rarus, by Conklin'e Abdsllsb, dam by Telegrapb.

$$
\text { 2:14. }
$$

Goldsmith Maid, by Alersnder' 8 Abdallsh, dam by Abdallab. Trinket, by Princeps, dsm by Ryedyk's Hambletonian. Clingstone, by Rysdyck, dam Gretchen by Chosroes.

$$
2: 143 / 4 \text {. }
$$

Hopeful, by Godfrey's Patchen, dam by Bridham Horse. 2:15.

Lnla, by Alexander's Norman, dam by imp. Hooten.

$$
\text { 2: } 151 / 4 \text {. }
$$

Smaggler, by Blancu, son of Iron's Cadmus, dam anknown. (The fastest best by a atalliun.)

$$
\text { 2: } 1516 \text {. }
$$

Hattie Wuudwsrd, by Aberdeen, dam by Henry Clay.

$$
2: 161 / 4 \text {. }
$$

Lncille Golddnst, by Golddust, dam by Bald Hornet.

$$
\text { 2:16\%. }
$$

American Girl, by Amos'i C. M. Clay, dam nnknown.

Darby, by Delmunico, dam by Cox'a Stnmp-tbe-Dealer.

Edwin Thurne, by Thurndale, dam by Lady Lightfoot by Ashland.

$$
\text { 2: } 163 / 4 \text {. }
$$

Charley Ford, by McKisson's Grey Eagle, dam onknuwn.

Oceident, by Doc, son of the paeer St. Clsir, dam unknown. $2: 17$.

Gloster, by Volunteer, dsm by Stockbridge Chief.

$$
\text { 2:171/4. }
$$

Dexter, by Hambletonian, dam by Americsn Star.

Piedmont, by Almont, dam by Mag Ferguaon by Mambrino Chies.

So-So, by George Wilkes, dam by Edwin Furrest.

$$
\text { 2: } 17 \text { L. }
$$

Santa Clana, by Strathmure, dam by Lady Tburne, Jr., by Williams's Mambrino.

$$
\text { 2:173/4. }
$$

Hannis, by Mambrino Pilot, dam s Morgan Mare.

2:18,

Dick Swiveller, by Walkill Chief, dam by Hsrry Clsy.

Edwin Forrest, by Brsnnock's Ned Forrest, dam by Smiling Tom.

Great Esstern, by Walkill Chief, dsm by aon of imp. Consternation.

Josephus, by Green's Bashaw, dam by Copperbottum (?).

Judge Fnllerton, by Edward Everett, dam nnknown.

Kate Sprague, by Gov. Sprague, dam Fan by Lance.

Nettie, by Hsmbletonian, dam by American Star.

Prateine, by Blackwood, dam by Mambrinu Chorister.

Red Clond, by Legal Tender, dam unknown.

Robert McGregor, by Major Edsall, dam by Seely'a American Star.

Jerome Eddy, by Louis Napoleon.

$$
2: 181 / 4 \text {. }
$$

Lady Iand, by Gen. Knox, dam unknown.

Lady Thurne, by Mambrino Chief, dam by Gano.

Lucy, by Geo. M. Patchen, dam by May Day.

Midnigbt, by Pescemaker, dam by son of Hiram Drew.

Monroe Chief, by Jim Monroe, son of Alexander'a Abdallab, dam by Bay Chief.
2:183.

Col. Lewis, by Rifleman, dnm nnknown.

Slow-Go, by Yunng Shautuck, grandsun of Meduc, dam nnlinown.

\section{2: $163 \%$.}

J. B. Thumas, by Sterling, he by Patchen Bay, s son of Gadfrey"a Patehen, dam nuknown.

Nutwoud, by Belmunt, dam by Pilot, Jr.

Patchen, (breeding unknown).

$$
\text { 2:19. }
$$

Albemsrle, by Tom Hunter, dam by Blacher.

Alexander (France's), by Ben Patehen, dam by Cansda Jack. Alley, by Volunteer, dam by New Yurk Black Hawk.

Bonesetter, by Brouks, an of Brown Pilot, dam by Adams'a Stump-the-Dealer.

Cozette, by Blumberg's Blaek Basbaw, dam by Stargazer.

Edward, by Fisk' 8 Hambletouian Star, dam unknown.

Grsves, by Whipple's Hambletunian, dam nnknuwn.

Kitty Bates, by Loder's Clond Mambrino, dam nnknown.

Wedgewrood, by Belmant, dam by Woodford.

$$
2: 191 / 4 \text {. }
$$

Bodine, by Volunteer, dam by Harry Clay.

Comee, by Daniel Lambert, dam by Hiawatha.

Croxle, by Clark Chief, dam by Little Priam.

George Palmer, by Ames's Bogus, dam unknown.

Keene Jim, by Keene's Lookuut, son of Bourbon Chief, dsm by Morgan Rattler.

Parana, by Mambrioo Hambletonian, dam Belle of Cayuga, by Hawbletonian Prince.

\section{2: $194 / 2$.}

Driver, by Volunteer, dam by American Star.

Moose, by Washburn Horse, dam unknown.

Thos. L. Young, by Yeliow Jacket, dam by Dragon.

Tronbadoar, by Revenge, dam Illinois Maid by Black Slssher. Will Cody, by Blue Bull, dam unknown.

Aldine, by Almont.

$$
\text { 2: } 198 / 4 \text {. }
$$

Adelside, by Phil. Sheridan, dam by Sam Houstun, son of Vermunt Black Hawk.

Camore, by Gen. Knox, dam nnknown.

Daisydale, by Thorndale, dam by Burr's Wasbington.

Deck Wright, by Hinsdsle Harse, dam nnknown.

Fanny Witherspoon, by Almont, dam by Gongh'a Wagoner.

Flora Temple, by Loomis's Bugue, dam by a spotted Arabian. John S. Clark, by Thomss Jefferson, dam by Seott'8 Hiatuga.

\section{2: 20 .}

Annie W., by Almont, Jr., dam Belle Forrest.

Belle Brasfield, by Viley'a Cripple, dam by Mambrino Chorister.

Capt. Emmons, by Continental, dam by John Morgan.

Elaine, by Meesenger Duroc, dam by Harry Clay.

Etta Jones, by Parrish's Pilot, dam by Pilot, Jr.

Fleety Golddust, by Golddust, dam Tiger Morgan.

Frank, by Buel's Pathfinder, dam unknown.

Humboldt, by Stocking Chief, dam by Parrish's Crockett.

John H., by Blumberg'a Bixck Bashaw, dam by Morgan Hun ter.

Little Fred, by Eastman'в Morgan, dam by Blackbird.

Ismbrino Gift, by Mambrino Pilot, dam by Pilot, Jr.

May Queen, by Alexander's Norman, dam by Crockett's Arabian.

Nancy Hackett, by Wood's Hsmbletonian, dam unknown.

Orange Gjrl, by Rysdyk's Hambletonian, dam by American Star.

Prospero, by Messenger Duroc, dam by Marry Clay. 


\section{TROTTING TO WAGON.}

One mile, (firat heat)-Hopefal, Chicago, Ill., Oct. 12th, 1878, 2: $16 \%$.

One mile, (aecond heat)-Hopeful, Chicago, 1ll, Oct. 12th, $1878,2: 17$

One mile, (third heat)-Hopefal, Chicago, IIl., Oct. 12th, 1878, 2: 17.

One mile, (drawing 2,000 lba.)-Montain Maid, Long IEland, 1865, $8: 21 \frac{11}{2}$

Two miles, (first heat)-Gen. Bntler, Long Island, 1863, 4:561/4.

Two miles, (second heat)-Dexter, Long Island, Oct. 2ith, $185.5,4: 5614$

Three miles,-Kemble, Jackson, Jone 1st, 1853, 8: 8 .

Four miles,-Longfellow, Cslifornia, Dec. 31st, 1869, 10:3116.

Five miles, -Little Mack, Long Island, Oct. 29th, 1863, 13: 433-

Twenty milea,-Controller, San Francisco, Cal., A pril 20th, $1878,58: 67$.

Fifty miles,-Spangle, 0ct. 13th, 1855, 3 h. 49:4.

\section{TROTTING DOUBLE TEAJS.}

One mile,-Lysander Boy and Willam H., New York, 1881, 2:20.

One mile, (third heat)-Gen, Cobb and Lalu, San Franclaco, Cal., Msy, 18i7, 2: 2616.

One mile, with running-mate, (first heat)-Ethan Allen and mate, Long Island, June 21st, 1867, 2:15.

One hundred miles,-Master Burke and Robin, 10 h. 17:22.

\section{TROTTING UNDER SADDLE.}

One mile,-Great Esstern, Fleetwood Park, N. Y., Sept, 22d, 1877, 2:15\%

Two miles,-Dexter, Long Islsnd, 1865, 5:0\%/.

Three miles,-Dutchman, Beacon Park, N. J., Aag. 18t, 1839, $7: 3246$.

Four wiles,-Dutchmsn, 18 6, 10:31.

\section{PACING.}

One mile in bsrness.-Sleepy Tom, Chicago, Ill, July 28th, $1879,2: 121 / 4$.

One mile nader saddle,-Billy Boyce, Buffslo, N. Y., Aug. Ist, 186s, 1:141/4.

One mile to wagon,-Pocahontas, June 21 st, 1855, 2:1716

Two miles under saddle,-Bowery Boy, Long Is]and, 1839, 5: 116 .

Two miles in hsrness,-Hero, May 17th, 1853, 4:563.

Two miles ander ssddle,-Onedis Chief, New Jersey, 1843, $7: 44$.

Three miles in harness,-Harry White, San Francisco, Cal., Aog. 8th, 1874, 7:571/4.

\section{BEST RECORDS OF FLEETEST RUNNING HORSES.}

Qiving classifled lists of such records, with places, dates, weight carried, age, time in minutes snd seconds. Quarter of a Mrile.

Belle, Gsiveston, Texas, July 3, 1850, 21\%/4 sec. Half a Mile.

Olitipa, 2 jr8., 97 lba., Sarstoga, N. Y., July 25, 1874, 478/4 sec.

Lizzie S , a yr6, 97 lbs., Lexington, Kу., May 8, 1880. $49 \mathrm{sec}$. Harold, 2 jrs., 110 lbs., Saratoga, N. Y., July 23, 18\%8, 491/4 sec.

Idalia, 2 yrs., 10; Jbs., Monmonth Park, July 4, 1876, 491/4 sec.

Vampire, 2 yre., 110 Jba., Sarstoga, N. Y., July 22, 1881, $492 \mathrm{~s}$ sec.

Memento, $2 \mathrm{Jr}_{*}, 107$ lbs,, Sarstoga, N. Y., July 19, 1881, $49 \% \mathrm{sec}$.

\section{Half-Mule Heats.}

Red Oak, aged, $114 \mathrm{lbs}$., Carson Clty, Nev., Sept. 16, 1879,
Half. Mile Heats, 3 in 5.

Mollie M'Cann, 5 yrs, catch weight, Indianapolis, Ind., July $26,18 \div 9,511 / 4,61 \% 4,50 \mathrm{sec}$.

\section{Five Furlongs.}

Olivia, 2 yr8., 97 lbs., Saratoga, N. Y., Aag. 5, 1881, 1:013/4. Lizzie S., 8 yrs., 92 lbs., St. Lonis, Mo., June 10, 1S81, 1:02. Bouncer, 2 jra., 104 lbs., Monmulath Park, N. J., Jaly 4 , 1881, I:02.

Mollie Brown, 2 yrs., 97 lbs., Springfield, MIo., Jane 1\%, 1880 , 1:02.

Brambaletta, 2 yra, 88 lbs., Sheepshead Bay, N. Y., Sept. 4, 1880, 1:021/4.

Gerald, 2 yrs., 110 lbs., Sheepsbend Bay, N. Y., Jone 16, 1881, 1: 0236.

Onondaga, 2 yrs., 115 lbs., Monmouth Park, N. Y., July 7. 1881, 1:0216.

Bend Or, 2 Jr8., 100 lbs., Lexington, Ky., Sept. 18, 18s0, 1:03. Runnymede, 2 yra., 103 1bs., Saratoga, N. I., July 20, 1881, I: $08 \%$.

Pappoose, 4 yra., 131 lbs., Saratoga, N. Y., July 16, IS81, 1: $03 \% / 4$.

Ingomar, 2 yrs., catch weighte, Mount Holly, N. J., Oct. 10, $18 \pi 9,1: 07,1: 03$.

\section{Three-Quarters of a Mile.}

Barrett, 2 yrs.; 110 lbs., Monmonth Park, N. J., Ang. 14 1880, I:14.

Knight Templsr, 3 yrø., 77 Jbs., Sheepshead Bsy, N. Y., Sept. $18,1880,1: 14$.

Gouvernenr, 2 yr8., 82 lbe., Sheepshead Bsy, N. Y., Scpt. 18 $1880,1: 141 / 4$.

Gonverneur, 2 yrs., 98 lbs., Sheepsherd Bay, N. Y., Sept. 25, 1880, 1:141/2; after dead beat witb Ada, 2 gra., 87 lba., 1:148/4. Patti, 8 yrs.,- 92 lbs., Chicsgo, Ill, July 2, 1881, I:1416.

Charley Gorhsm, sged, 103 Ibs., Sheepshead Bay, N. Y. June 29, 1881, 1: 15 .

One dime, 5 yra,, 120 lba,, Saratoga, N. I., July 21, Isst, $1: 15 \% 4$

Bonnie Lizzie, 3 5ra., 101 lbs., Saratoga, N. Y., Ang. 16, $1881,1: 151 / 4,1: 14 \% / 4$

Charley Ross, aged, 118 Ibe., Brighton Besch, N. Y., Joly 1, 1881, 1:16, 1:15. I. B. Sprague, 4 yra, 108 lba., won first heat in $1: 17$.

Knight Templar, 8 yrв., 92 lhs., Lonisville, Ky., May 24, $1880,1: 15,1: 17$.

\section{One Mrile.}

Ten Broeck, 5 yrs., 110 lbs, Louisville, K₹., Msy 24, 1877. "sgainst time," 1:393/4.

Boardmsn, 4 yrs., 91 lba., Sheepshead Bay, N. Y., Sept. 20, $1880,1: 4016$.

Marchioness, $5 \mathrm{yrs}_{*}, 64 \mathrm{lbs}$, Brighton Beach, N. Y., July 6, 188I, I:41.

Victim, 6 yrs., 117 Jbs., Ssratoga, N. Y., Ang. 1, 1881, 1:41\%. Knight Templar, 4 yra,, $106 \mathrm{lbs}_{*}$, Saratoga, N. Y., Ang. 6, 1881, 1:42.

Parole, aged, 102 lbs., Baltimore, Md., Oct. 21, 1880, 1:42.

Hindoo, 3 yrs., 118 lbs., Sheepshead Bay, N. Y., June 15, $1881,1: 431 / 4$.

Mistake, 2 yr8., 100 lba., Lonisville, Ky., Sept. 36, 1879, $1: 42 \% / 4$.

Gabriel, 5 yrs., 115 lb8., St. Louis, Mo., Jane 13, 185I, 1:421/4, $1: 41 \%$.

Dan Sparling, 4 yrs, $106 \mathrm{Jbs}$, Sheepshend Bay, N. T., Sept. 21, 1880, 1:42, 1:44\%. Ada Glenn, 4 yra., $106 \mathrm{lba}$, won first heat in $1: 41 \%$.

Glenmore, 6 yrs., 114 lbs, Sheepshead Bay, N. Y., June 16, 1881, 1:421/4, 1:461/4. Dan Sparling, 6 yrs., $114 \mathrm{Jbs}$, won first beat in 1: $42 \%$. 


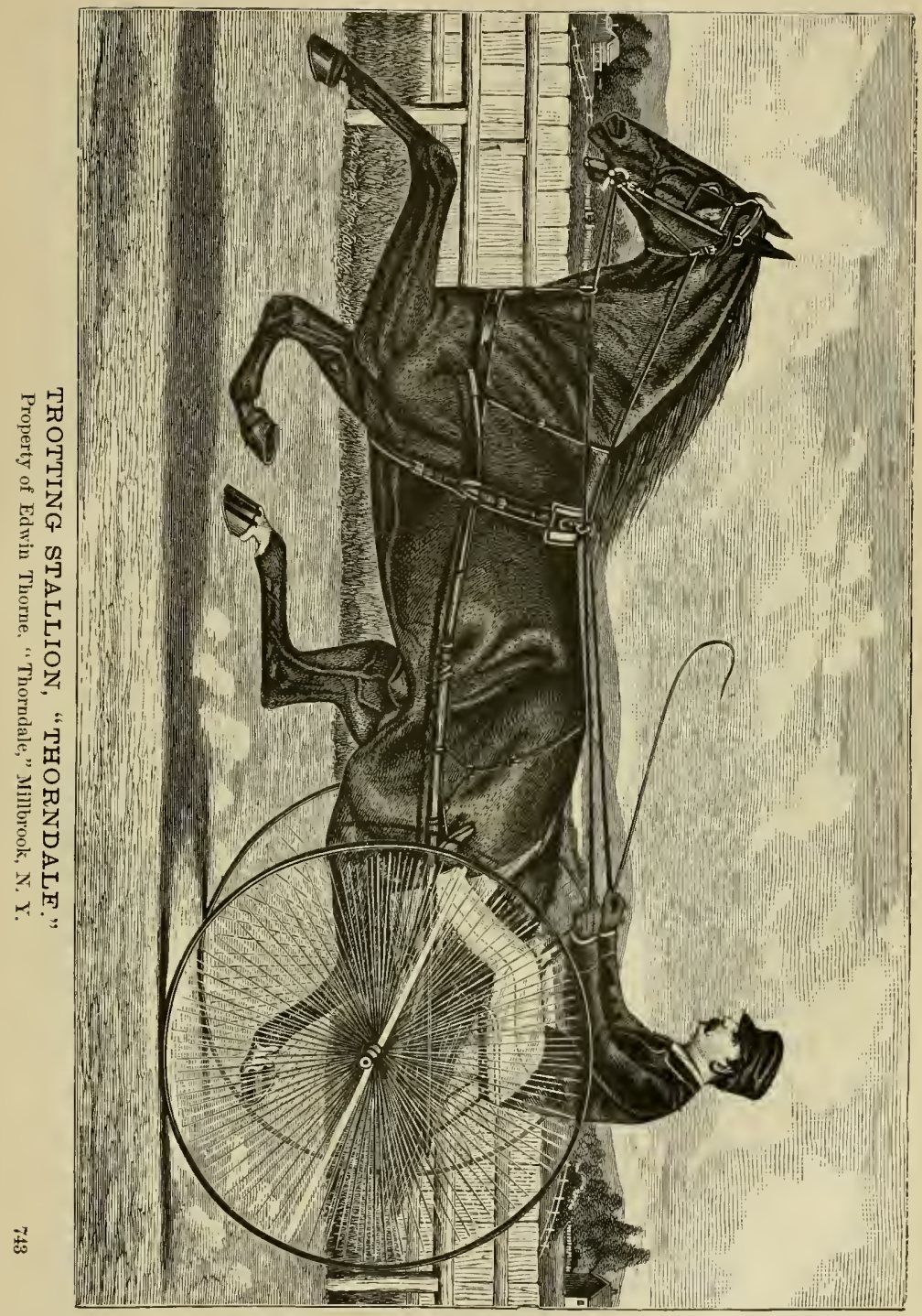



One arile Heats, 3 in 5.

Thad Stevens, sged, $110 \mathrm{lb}$, , Sacramento, Cal., July 8, 18\%3, 1: $431 / 2,1: 461 / 2,1: 45$. Thornhill, 4 yrs., won the firat and second beats in $1: 43,1: 43$.

\section{Mille and a Furlong.}

Bob Woolley, 8 yra., 90 lba., Lexington, Ky., Sept. 6, 1875, 1: 54

Greenland, 9 yrs., 108 1bs., Ssratogs, N. Y., July 23,1881, 1:511/2.

Patti, 3 yr8., 89 Iba., Chicago, Ill., June 28, 1881, 1:519/4.

Kingar, 5 yrs., 115 1bs,, Lowisville, Ky., Sept. 30, 1880, 1:54\%/4.

Baby, 4 yr8., 105 lbs., Brighton Beach, N. Y., July 81, 1880, $1: 55$.

\section{Mile and a Furlong Heats.}

Gabriel, 4 yra., 112 lbs., Sbecpsbead Bay, N. Y., Sept. 23, $1880,1: 56,1: 56$.

\section{Mile and $a$ Quarter.}

Getaway, 3 yra., 100 lbs., Saratoga, N. Y., Ang. 5, 1881, 2:07\%/4.

Blendelasobn, 3 yra., 95 Tba., Lexington, Ky+, Mry 10, 1880, 208.

Sir Hugh, 3 yra., 102 lb8., Saratoga, N. Y., July 19, 1881, 2:081/4.

Checkmate, 6 yrs., 117 lbs., Saratoga, Y. Y., July 16, 18s1, 2:081/2.

\section{Mile and a Quarter Heats.}

Glenmore, 5 yra., 114 1ba., Sheepsbesd Bay, N. Y., Sept. 25, $1850,2: 10,2: 14$.

Mary Anderaon, 3 yra., $83 \mathrm{lbs}$, won first heat in 2:09.

\section{Mile and 500 Yards.}

Valleria, 3 yrs., 94 Jbs., Saratoga, N. Y., Aug. 1, 1881, 2:1216 Ripple, 3 yrs., 109 lbs., Surstoga, N. Y., July 20, 1881, 2:14.

\section{Mile and Three Furlongs.}

Uncse, 4 yrs., $10 \pi$ lbs, Sheepehesd Bay, X. Y., Scpt. 23, $1880,2: 21 \% / 4$.

Luke Blackburn, 3 Fr8., $96 \% 3$ lbs,, Sheepahend Bay, N. Y. June 22, 1880, 2:241/3.

Spendthrift, 3 yra., 123 lbs., Jerome Park, N. Y., June 10, $1879,2: 25 \% / 4$.

\section{Mile and a Holf.}

Luke Blackburn, 8 yrs., 102 lbs., Monmonth Park, Aug. 17, 1880, 2:34.

Tom Bowling, 4 Jrs, 104 lbs., Lexington, Ky., May 12, 1874, 8: $94 \%$.

Hindoo, 8 yr8., 118 lbs, Saratoga, N. Y., Aug, 4, 18s1, 2:36,

Parole, 4 yra., 97 lbe., Sarstoga, N. Y., Aug. 4, 1877, 2:36\%/4.

Liatunale, 5 yrs., 112 lba., Chicago, Il., Jnne 25, 1881, 2;3716

Gabriel, 6 yrs., 116 lbs., St. Lonia, Mo., June 11, 1881, 2: 973 .

\section{Mile and a Half Heats.}

Keno, 6 yrs,, cstch weight, Toledo, O., Sept. 16, 1880 , $2: 4316_{2}, 2: 45$. Belle of Nelaon, 5 yra., won recond best in $2: 45$.

\section{Mile and Five Frurlongs.}

Ten Broeck, 3 yrs, 90 ibs., Lexington, Ky., Sept. 9, 18\%, $2: 491 / 4$.

Eole, 3 yrs., 100 Jbs., Sarstoga, N. Y., Ang. 3, 1881, 2:49\%.

Checkmate, 5 yrs., 111 iba., Sheepshead Bay, N. Y., Sept. $16,1880,2: 50$.

Monitor, 8 yrs., 98 lbs, Prospect Park, N. Y., Sept. 13, 1879, 2: $501 / 2$.

\section{Anle and Three-Quarters.}

Glenmore, 6 yrs., 100 1bs., Sheepshead Bay, N. Y., Jnne 23, 1881, 3:011/4.

Checkmste, 6 yrs., 124 lbe., Sarstoga, N. Y., Ang. 6, 1881, $3: 01 / 3$.

Monitor, 4 yre., 115 1bs., Monmouth Psrk, N. J., Aug. 19, $1880,3: 02 \% / 4$.

Crickmore, 8 yr8., 111 Ibs., Sbeepsbead Bsy, N. Y., Sept. T, 1881, 3:031/4.

Lrke Blackbarn, 3 yrs., 105 lbs., Lonisville, Ky., Sept. 30, 1880, 3:04.

Dartagnan, 3 yrs., 110 lbs., Saratoga, N. Y., July 24, 1875, 3:061/2.

Luke Blackburn, s yr8., 116 lba,, Sarstog8, N. Y., Aug. 7, 1880, 3:07.

Ilindoo, 3 yrs., 118 lbs., Ssratoga, N. Y., July 16, 1881, 3: 0736 .

Two Miles.

Ten Broeck, 5 yrs., 110 lbs, Louisville, Ky., May 29, 1877"aguinst time"

Bushwhacker, aged, 99 lba., Saratoga, N. Y., Ang. 18, 1881 3: 30

McWhirter, 3 yre., 100 lbs., Loaisvllle, Ky., May 28, 1877, 3:301/2.

IIiudoo, 3 yra., 118 lbs., Saratoga, N. Y., Aug. 11, 1881, 3: 32.

\section{Troo-julle Heats.}

Willie D., 4 yrs., 102 Jbs., Prospect Park, N. Y., Sept. 11, $19 \pi 9,8: 341_{3}, 3: 35$

Lottery, 3 yra., 100 lbs., Sacramento, Cal., Sept. 21, 1879, $3: 36,3: 3316$.

Bushwhacker, 4 yr8., 105 lha., Baltimore, MId., Oct. 22, 1878, 3: $36,3: 38 \mathrm{~L}$. Princeton, 4 yrs., $108 \mathrm{lbs}_{,}, \ldots$ won second beat. $3: 36 \%$.

\section{Two Miles and a Fulong.}

Aristldes, 4 yrs., 108 Jbs., Lexington, Ky., May 10, 1876 $3: 451 / 3$.

Mate, 6 yr8., 114 lba., Saratoga, N. Y., July 31, 1875, 3:46\%4 Fuida, 5 yrd, 109 1b8, Sheepahesd Bay, N. Y., June 29, 1891, $3: 43$.

Glenmore, 5 yra., 114 lı.., Sheepshead Bay, N. Y., June 26 , $1880,3: 4816$

Two Nites and a Quarter.

Springbok, 5 yr8., 114 lbs.; Preaknesa, aged, 114 lbs., "dead best," Sarstoga, N. Y., Jnly 29, 1975, 3: 561/4.

Glcnmore, 6 yrs., 114 Jbs., Sheepshead Bay, N. Y., June 18, 1881,$3 ; 58 \%$.

Blue ejea, 5 yrs., 115 Ibs., Chicago, Ill., Jnne 20, 1880, 3: 58\%4 Harry Bassett, 4 yra., 108 lbs., Saratoga, N. Y., July 16, 18\%2, 3: 89

\section{Two Miles and a Half.}

Aristidea, 4 yr8., 104 lbs., Lexington, Ky., May 13, 18\%6, 4:2736.

Katie Peaae, 4 yT8, 105 lbs, Bnffalo, N. Y., Sept. 10, 1874, 4:2816.

\section{Tho Miles and Five Frurlongs.}

Ten Broeck, 4 yrs., 104 lbs., Lexington, Ky., Sept 16, 1876, 4: $58 \%$.

Two Ifiles and Three-Quarters.

Hubbard, 4 yra., $10 \%$ lbs., Sarstoga, N. Y., Ang, 9, 1873 $4: 583 / 4$.

Kentucky, 5 yr8., 124 lbs., Jerome Park, N. Y., Oct. \&, 1866, $6: 04$.

\section{Three Miles.}

Thora, 3 yrs., 99 lbs., Ssrstogs, N. Y., Aug. 27, 1881, 5:251/4. Ten Broeck, 4 yro., 104 lbs., Louisville, Ky., Sept. 23, 1876" againat time" $-5: 261 / 4$.

Elins Lawrence, 3 yr8., 98 lba., Sarstoga, N. Y., Ang. 28, $1890,5: 281 / 4$. 
Three-1rile Heats.

Norfolk, 4 yro., 100 lbs., Sacramento, Cal., Sept. 23, 1895, 5: $2 \pi 3 / 2,5: 291 / 2$.

Brown Dick, 3 yts, 861/2 lbs., New Orleans, La., April 10, $1855,5: 30 \%, 5: 28$.

Note.-Brown Dick would have been a 4year old under present rules.

\section{Four Miles.}

Ten Broeck, 4 yr8, 104 lbs., Louisville, Ky., Sept. 2\%, 18\%6"against time" $-7: 15 \%$

Fellowersft, 4 yrs., 108 lbs., Ssratogs, N. T., Ang. 20, 1874, 7:191/2.

Lexington, 4 yre, 103 lbs., New Orleans, Le., April 2, 1855

- "ggainat time"- $7: 198 / 4$

Janet, 6 yrs., 115 lbs., Louisville, Ky., Sept, 27, 1879, 7:25.

\section{Four-Mule Leats.}

Ferida, 4 Jr8, 105 Ibs., Sheepshcad Bøy, N. Y., Sept. 18, $1880,7: 2315,7: 41$.

Lexington, 4 yr8., 1033/4 lbs., New Orleans, Le., April 14, $1855,7: 23 \%$.

Noтะ,-Lecompte was withdrawn \& fter the first heat.

Lecompte, 3 yrs, 86 lbs., New Orleans, La., April 8, 1854, $7: 26,7: 3814$.

Thad. Stevens, sged, 115 Jbs., San Francisco, Cal., $7: 30$,

7:43. Joe Daniels, 4 yr8., $103 \mathrm{Ibs}$, won first heat in $7: 421 / 4$.

Glemmore, 4 Jrs., 108 Ibs., Baltimore, Md., Oct. 29,1879, $7: 301 / 4,7: 31$.

Wulie D, 4 yrs., $105 \mathrm{lbs}$, won first heat in $7: 2912$.

\section{HURDLE RACES.}

[One Mile, over Four Hurdles.]

Judith, 5 yrs., 145 lbs., Brighton Beach, N. Y., July 17, 1880, 1: 51 .

The Breeding of Horses. - The improvements that have been brought about among the various classes of domestic animals through the efforts of intelligent breeders is truly wonderful, and although many obstacles in this department are to be constantly met, and many intricate questions that present themselves in the pursuance of this art must forever continue to remain mysteries, yet, in the main, breeding has been reduced to a science, and the governing principles and laws in this department of Nature's vast laboratory have come to be so well understood that the skillful breeder can mold, as it were, by a judicious selection and combination, based upon a knowledge of hereditary law, the ideal animal into the real, and can prospectively determine, with considerable certainty, the result of such selection and union. The breeder may, therefore, be said to possess, in a great measure, the creative power, making use of the material that he finds within his reach-as the potter does the clay -to combine and mold the object desired, after the various patterns and designs, for the different uses to which the result of his effort is to be appropriated.

Thus we have to-day among horses, those intended for speed in running and trotting; for style, the different classes of carriage-horses; those adapted especially for the saddle; the heavy draft-horse, and the general-purpose horse, or what might be termed the model farm. horse.

In view of what has thus far been accomplished, the intelligent breeder has great encouragement to continue his efforts towards farther developments and improvements, for the field is indeed a vast one, and it seems almost impossible to reach the limit.

Those animals that combine the greatest number of excellences for the use to which they are to be appropriated are, of course, the best; but as wlat would be desirable in one type or class of horses would be an objection in another class, the purpose for which the animal is to be used should first be considered. For instance, the form of the trotter would 
be incompatible with that of the draft-horse, while the latter would also be objectionable in the carriage-horse, etc.; therefore, many of the desirable qualities in different classes of horses, being antagonistic, cannot be combined in a single animal, and hence the necessity of aivin' ing the animal to its especial use.

The breeding of horses is a very important subject, since it relates to the agricultural interests of the country so extensively. But when we consider the lack of interest that farmers generally take in this department, it is not surprising that we see so many inferior horses. It is rather the occasion of surprise that the common farm-horse is as good an animal as we find him, when so little pains are taken generally towards his improvement.

With most farmers, the opinion is prevalent that when a mare has become useless for any other purpose, having become old, or broken-down by hard work, sprained, spavined, and perhaps diseased, she will do well enough for raising colts.

How in the name of common sense a person can expect a colt worth the raising from such stock is difficult to understand, and yet farmers are constantly doing this all over the country. If good stock is desired, it will be necessary to secure good stock as the progenitors, in order to reach the desired results.

Disqualifications for Breeding.-At the outset, to be successful in breeding requires outlay and careful selection. A person cannot reasonably expect that from a dam that is perhaps not worth twenty dollars, and a sire whose real value may be but little more, that the progeny will be equal to a St. Julien or a Maud $S$. Old age alone is a sufficient disqualification for breeding, and when we add to this, unsoundness and disease, the objections become doubly intensified. The colts from such stock will lack vitality and endurance, since they will inherit these tendencies from their progenitors, and such a method of breeding is not only a direct violation of all physiological law, but of all the teachings of commen sense and experience combined, and cannot be too strongly condemned.

No matter how active and valuable an animal may have been in her prime, if old and broken-down, the foal will inherit the infirmities of old age rather than the excellences of her younger days. Therefore, never breed from a mare that is old and infirm, no matter how pure her "blood" may be.

Never breed from a horse that has the heaves or is spavined. The tendency to transmit disease is as strong in animals as in the human family, and a horse that has any weakness about the organs of respiration will be almost sure to transmit this tendency. The same may be said of any of the other forms of disease. Lameness, although it may have been produced by accident, will also be liable to be transmitted to the foal. Colts from such mares will be apt to become lame in early life, through inherited weakness. In fact, any infirmity, disease, or defect, in either sire or dam, will be very liable to be reproduced in the progeny.

An animal for breeding should be free from a vicious disposition and temper, as well as bad habits of every kind. A cross, ill-tempered mare or stallion will be likely to perpetuate this tendency in the ofispring, while, if both parents possess these traits, an ill-tempered, vicious colt may be expected as an absolute certainty.

Even a tendency to cribbing is known to be inherited. The same law holds true relative to temperament, form, color, size, etc.

Always breed up, and never down; that is, never breed a dam to a sire of inferior blood. It is also bighly essential that the dam possess qualities desirable to be transmitted to the progeny, as her influence in this direction nearly, if not fully, equals that of the sire.

Infinence of the Sire and Dam.-The Arabs have a maxim that "the foal follows the sire," yet at the same time they always select their dams with much care.

Notwithstanding the Arab maxim, we believe the qualities of the dam are of as much importance as those of the sire, and that the progeny will inherit the mingled characteristics 
and qualities of both parents. It is generally thought-although individual cases differ, according to different circumstances-that in the majority of cases, the male parent gives size and form to the bones and muscles generally, while the female parent influences the nervous system, and frequently the form of the head and adjacent portions of the body. W. C. Spooner, a leading English authority, says, respecting this opinion, in the Journal of the Royal Agricultural Society :-

"The influence of male and female parent is not capricious, but jet not always alike; in the majority of instances the male parent gives the size and external shape of the offspring (particularly the back and hind-quarters), while the fomale influences the constitutional, the nervous system, and often the head and fore-quarters. That this combination-which may be more of a mechanical mixture than a chemical union-by no means implies such an equal division of influence, as the mingling of two fluids-in which case the offspring would be unlike either parent, but a juste milieu between the two, and there could be no handing down of type from one generation to another-it is rather such a fusion of two bodies into one, that both defects and high qualifications are passed on from parent to offspring with a sort of regular irregularity resembling the waves of the sea, each parent having the remarkable power of propagating ancestral peculiarities, though latent in itself."

Another English authority, the author of "Breeding, Rearing, Feeding, and General Management of Farm-Horses," says:-

"Instances have come under the notice of the writer where a tribe of horses have been bred in one family for many generations, the mares of which all inlierited from the female ancestors the bad habit of kicking in the yoke; and although crossed with very docile sires, the same propensity and nervous temperament was transmitted from one generation to another; others again preserve the unwelcome and annoying habit of being shy pullers; and others, again, where the mares are hot-tempered, tearing workers, but deficient in stamina or staying power. Owners sometimes breed from a mare that is hot-tempered, or a kicker, to sober her down a bit. They invariably succeed in perpetuating a breed which should be allowed to become extinct. The importance, therefore, of selecting a quiet-dispositioned mare of sound constitution for breeding purposes is apparent. By sober-tempered, a sluggish animal is not meant, activity being very essential in a brood mare, especially in her walk, as this is the most important pace for farm work. Either meeting you or leaving you, a horse should ga square; the fore action should be straightforward, the fore-feet should not be thrown out sideways, as it were. It is also necessary that a farm-horse should be able to acquit itself well in a trot; and the words of an enthusiastic Scotchman when once describing a brood mare can be repeated, when he said, 'Her very step had music in't.' "

The rule previously recognized does not hold true in all cases, since individuals differ so greatly with respect to the power of transmitting their qualities to their progeny. Murray says in this connection:-

"The instances in which the foal does not follow the sire are too numerous for us to allow that the Arabian maxim is worthy of being regarded as a law. Even a casual inspection of my own stables, or the stables of any breeder, would cause a grave suspicion to arise in any thoughtful mind touching the Eastern adage. I have, for instance, in my stables, dams whose foals invariably resemble the sire in size, shape, color, style of going, and even in temperament; and these mares are valued by me as almost beyond price, because of this peculiarity. I know beforehand what $I$ shall get. On the other hand, I have two other mares whose colts invariably resemble themselves, or some one of their parental ancestors. So true is this, that I can calculate before the foal appears what he will not be, although I may not easily tell what he will be. "Such are the facts in my own stables; and they harmonize perfectly with the results of observation in many other breeding establishments. The law plainly suggested by inference from these facts is this, that the animal with the strongest vitality marks the foal. 
If the dam be most highly organized, then the foal will resemble the dam; if the sire, then the foal will resemble the sire. This is the law, as we all know, in the human family: if the mother be of nervous, sanguine temperament, and the father lymphatic and sluggish, the child will take after the mother; if the conditions be reversed, the result will be the reverse. Exceptions there may be and are; but the law stands firm, vindicating its truth with each successive generation. I am bound, nevertheless, to say that this law does not hold good in cases where we should naturally expect it would. To illustrate: According to the law, when a low-blooded mare is bred to a thoroughbred horse, the foal should resemble the sire; but, alas 1 too often he does not. On the other liand, according to the law, a blooded mare bred to a low-blooded horse should bring forth a colt like herself; but neither is this true. What, then, becomes of the law? I confess that I do not know; nor have I been able to find in the works of any author a satisfactory answer to the puzzle. Practically, although I cannot philosophically account for my preference, yet practically, I say, we know that it is far better to have a high, fine organization in the sire, and let the low organization, if it must exist in either parent, be on the side of the dam. The fact is, both parents should be highly organ. ized, and anything short of this introduces uncertainty as to what the result of the experiment will be. The only infallible rule, - the best statement ever given touching the reproduction of any form of life,-was published by God himself in His inspired word, when he said, "Let the earth bring forth the living creature after his kind." This, nevertheless, must be observed, that the power to bring forth after his kind,-if by his kind we mean personal resemblances, rather than generic attributes,--does not belong to the horse as a race, but to the horse as an individual; for, as we have already seen in the case of Justin Morgan, this faculty of reproducing excellences is individual, and not general. And so we come back to the same observation previously made in regard to what constitutes a valuable stock-horse, - -viz, that the best horse is he who, being good in himself, most surely and closely reproduces himself in his offspring; and to this formula should now be added the words, when bred to the mares of the greatest variety of form and temperament."

As a general rule, although, as we have previously seen that individual cases will differ, the purer or less mixed the blood, the more likely are the characteristics of the breed to be transmitted to the progeny, and consequently, the parent that possesses the purest blood, or in whose pedigree the fewest crosses with other breeds are found, will exert the most influence upon the offspring, and be the most largely represented in it.

In the breeding of domestic animals, the male parent is commonly selected with more care than the female, since one animal of this kind impresses itself upon so large a number, consequently it follows for this reason, as the usual result, that the sire exerts more influence than the dam; but when the dam is of superior blood to the sire the result would be the reverse. When both parents are equally well-bred, other conditions being equal, the influence will be divided, the sire perpetuating some characteristics, and the dam others.

What Horses to Breed.-For the reproduction of its kind, whether in vegetable or animal life, the best results can only be reached by employing for this purpose the best and highest types of the species to be perpetuated. For this reason, the most intelligent and successful agriculturists select the seed for future crops from the most thrifty and perfectly developed plants, and by this means not only prevent deterioration of the variety, but frequently improve upon it. In the animal kingdom the same law of selection is equally important. The most perfectly developed, vigorous, and healthy animals of the breed should be selected to reproduce it.

The kind of horse to be bred will, of course, depend upon the use to which it is to be appropriated, whether it be the stylish carriage-horse, the trotter, racer, the saddle-horse, the general-purpose or farm-liorse, or the heavy-draft animal. Whatever breed is to be perpetuated, the best and most perfect types should be selected, and not only this, but those 
possessing the purest blood, whose pedigree may be traced back to the best stock, for it is well known to breeders of experience, that however good an animal may be in himself, if he be of mongrel stock, his progeny will not be likely to represent his accidental good qualities, but will rather be more liable to revert back to their inferior ancestry, it being the natural tendency in animals to revert back to the original type. For this reason those mares should be selected that are the most closely allied to the best blood, and only thoroughbred or highgrade stallions should be employed. Selections, even from thoroughbred stallions, should be made with care, for many will be found objectionable for use; but a low-bred stallion should ever be avoided.

It is a well-known fact that a large majority of the horses that have distinguished themselves by their achievements are the descendants of superior ancestors, the exceptions being very rare. At the same time, circumstances may exert such a controlling influence that some of the most noted horses may produce but ordinary or inferior foals. The expenditure of nervous energies through a long campaign upon the race-course is decidedly opposed to the best physical condition for reproduction, in either sire or dam, and the highest possibilities of either ean never be attained in this respect, while their vital forces are being used up for racing purposes. This truth has been signally exemplified in employing, for breeding purposes, some of the noted trotting mares in their old age, and after their muscles and nerves had been subjected to a severe strain, and in a great measure exhausted in trotting performances.

Neither Flora Temple, Lady Thorne, nor Lucy have ever produced foals that have won recognition in trotting circles, although the sires of their progeny were of most desirable pedigree. Another important point, and yet the most frequently overlooked in breeding, is in the combination; the unskillful mating of the dam and sire being the rule, rather than the exception. There is much truth in the following:-

"Peculiarity of form and constitution will also be inherited. This is a most important but neglected consideration; for, however desirable or even perfect may have been the conformation of the sire, every good point may be neutralized or lost by the defective structure of the mare. The essential points should be good in both parents, or some minor defect in either be met, and got rid of, by excellence in that particular point in the other. The unskillful or careless breeder too often so badly pairs the animals that the good points of each are almost lost, the defects of both increased, and the produce is far inferior to both sire and dam."

Breeders that are careless in this respect can expect nothing but inferior results, for it must be remembered that while the general law, oft repeated, that "like produces like" is true in the main, there are many exceptions, and that where either animal is deficient individ. ually, or their ancestors, it is often the case that the undesirable qualities are more readily perpetuated than the desirable ones, and, therefore, too much care cannot be taken to secure, as far as practicable, a mutual adaptation in form, size, temperament, disposition, etc., between the sire and dam.

Never breed an exceedingly small mare to a large stallion, or the reverse, as the result will be anything but satisfactory. It will sometimes be found that the crossing of certain good strains of horses will invariably, without any known canse, bring poor results. In such instances their crossing should be avoided. The frame of the mare should be roomy, wide at the hips, wide-chested, deep in the girth, quarters strong, and hocks rather wide apart.

In the selection of both dam and sire, those qualities desirable to be possessed by the foal should, as far as practicable, be represented in both parents. As these qualities have already been previously stated, a repetition will be unnecessary in this connection. Suffice it to say, that in breeding, aim to secure all the essential qualities, and as many of the other desirable ones as possible; therefore, breed for beauty, style, speed, docility, strength, endurance, and as many of the other qualities to be preferred as possible, combining with these the size and 
form which are desirable in the horse we wish to produce. Both animals should be strong, vigorous, and in the best condition of health. In this great undertaking, where so many have failed, those will meet with the highest success, who, to zeal and enthusiasm combine knowledge and patient effort, considering well beforehand what they wish to accomplish, and by the use of that knowledge in careful selectiou and combination aim for the best results.

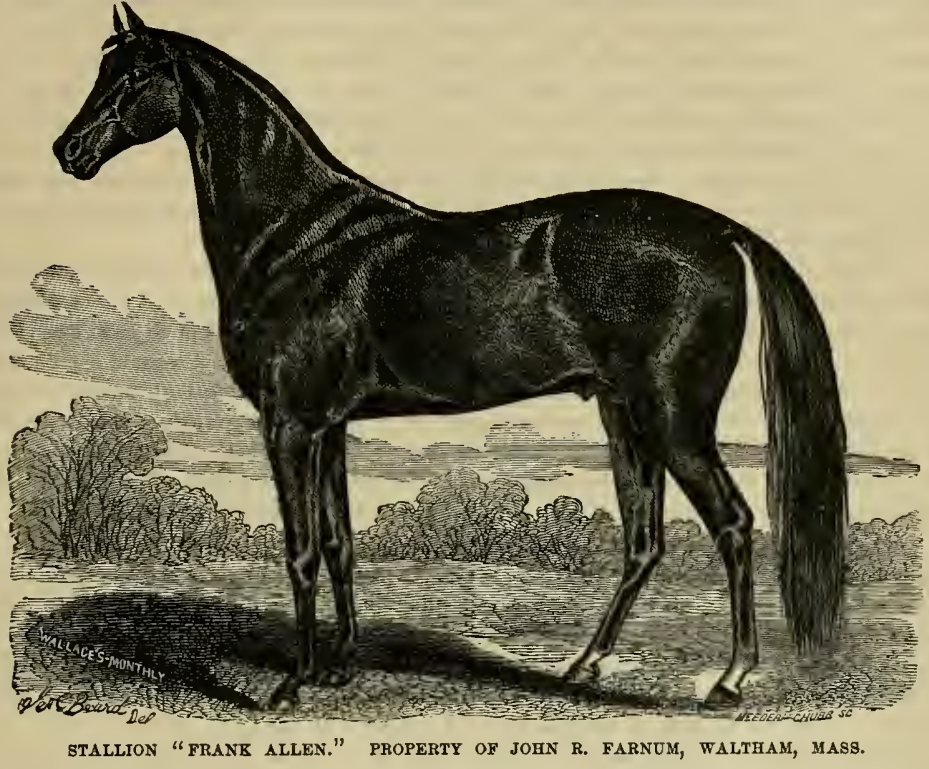

Care of Stallions. - The following sensible method with reference to the care of stallions is recommended by J. E. Russell, Secretary of the Massachusetts Board of Agriculture:-

"When a horse begins a stud career, his owner should absolutely withdraw him from the worry and excitement of training. Horses kept for service, and trained at the same time, will get nervous and excitable stock. But a worse error still is to put a horse into a condition of flesh, like a prize pig, in order to brag of how much he weighs, and to keep him, without exercise, in the close confinement of a box-stall, until he becomes a moody, morose, and often savage brute. Many stallions become partially insane under the common treatment, and are a pest to their owners, dangerous to grooms, and beget vicious stock. A stallion should be kept in good health and moderate flesh. His box should be where he can have the constant company of other horses, or in sight of his mares. He should have a paddock to run in, or have plenty of cut grass during his season. He should be exercised in double harness, or under the saddle accompanied by other horses, as often as convenient. His exercise should be brisk and blood-stirring, with occasional sharp work, so as to get a good sweat. Under such treatment, a stallion, unless he is naturally a vicious brute, will be as cheerful and pleasant to keep as any mare is."

Care of Breeding Mares. - It is very essential that brood mares be kept in good condition. A half-starved dam cannot produce a strong and thrifty foal, since her blood 
must nourish the young life and contribute to its support and growth until it makos its entrance into the world. She should have a liberal supply of food of the best kind, and be provided with warm quarters in winter. Moderate work up to within two or three weeks of foaling will not prove injurious; but excessive labor, such as straining, and drawing heavy loads, should be avoided. She should be kept quiet and apart from other horses, after the first four or five months. The period of gestation varies somewhat with different mares, but is usually from forty-seven to fifty weeks, although sometimes not more than forty-four weeks, and occasionally as long as fifty-six weeks. When the time of foaling is near - say about two or three weeks previous - she should be put into a large box or stall, where she should be allowed her freedom; this should be done especially nights, while during the day she might, if desired, be allowed the freedom of the yard, or near pasture. The box should be of large size, twelve by twenty feet being good dimensions, and thickly strewn with sand, gravel, saw-dust, or tan-bark, over which a bedding of straw or dry leaves should be strewn to the depth of about a foot.

Some mares will eat their bedding. If there is any tendency in this direction, she should have a muzzle of wire put on and wear it, except when eating her rations, as it is not well for a mare to be filled with coarse food at such times. Care should be taken to have everything in the box so arranged that there will be no danger of her getting cast, should she lie down to roll. After foaling, she should still be very liberally fed. Stinting both mother and foal at this period will be found very poor economy, for, if stunted in growth at this early stage of his life, the young colt will never wholly recover from the effect. During all the period of gestation, the mare should be treated with the greatest kindness. She should never receive a blow, or even a harsh word. Either will sometimes cause her to slink the foal.

Care of Young Colts. - If the colt is strong and vigorous at birth, he will not require much special attention; but if he is unable to get on his feet soon after, it is best to assist him to do so. If he is too weak to stand, he should have his body and limbs rubbed, especially the latter, to promote circulation. A woolen cloth dipped in water, blood-warm, should be first used, after which a dry woolen cloth, to dry him off, followed by hand-rubbing. This rubbing will promote circulation throughout the entire system, and give strength to the muscles. It should be kept up until he is able to stand. If left lying unattended in a weak state, to take care of himself, he will be quite as liable to die as to live. He should be held up to the dam, so that he can draw the milk as soon as he is strong enough. He should not be allowed to get chilled, but be kept warm and comfortable. In order to have a colt become a strong, vigorous, and well-developed animal, he should be well fed. Never stint him at all at any period of his life, and especially at the start.

Particular attention must be paid to his growth during his first summer and autumn, for if stunted then, he will never recover from it under any circumstances. There is quite a difference in dams with regard to the quantity of milk they are able to supply. As previously stated, the dam should be very generously fed in order to produce the largest supply of milk possible for the foal, while if lacking at all in quantity, and she fails to keep him in good flesh and steadily improving, a sufficient supply of cows' milk should be given him daily to make up the deficiency. Skimmed milk may be used for this purpose, but in such cases a little flax-seed jelly, oil-meal, or cotton-seed meal (a heaped tablespoonful night and morning, to begin with), should be mixed with it. This can be gradually increased to a pint a day by the time the colt is six months old, or, if he be of the large breed, double this quantity may be given. Oats should also be given as soon as they can be eaten. Many breeders bruise the oats for them at first. When the dam is fed, her rations of oats should be placed where the colt may eat with her, and it can be depended upon that when allowed this opportunity be will not be long in availing himself of it. A half pint of oats at night and in the morning is sufficient to allow him at first, which rations can be increased to four quarts per day, 



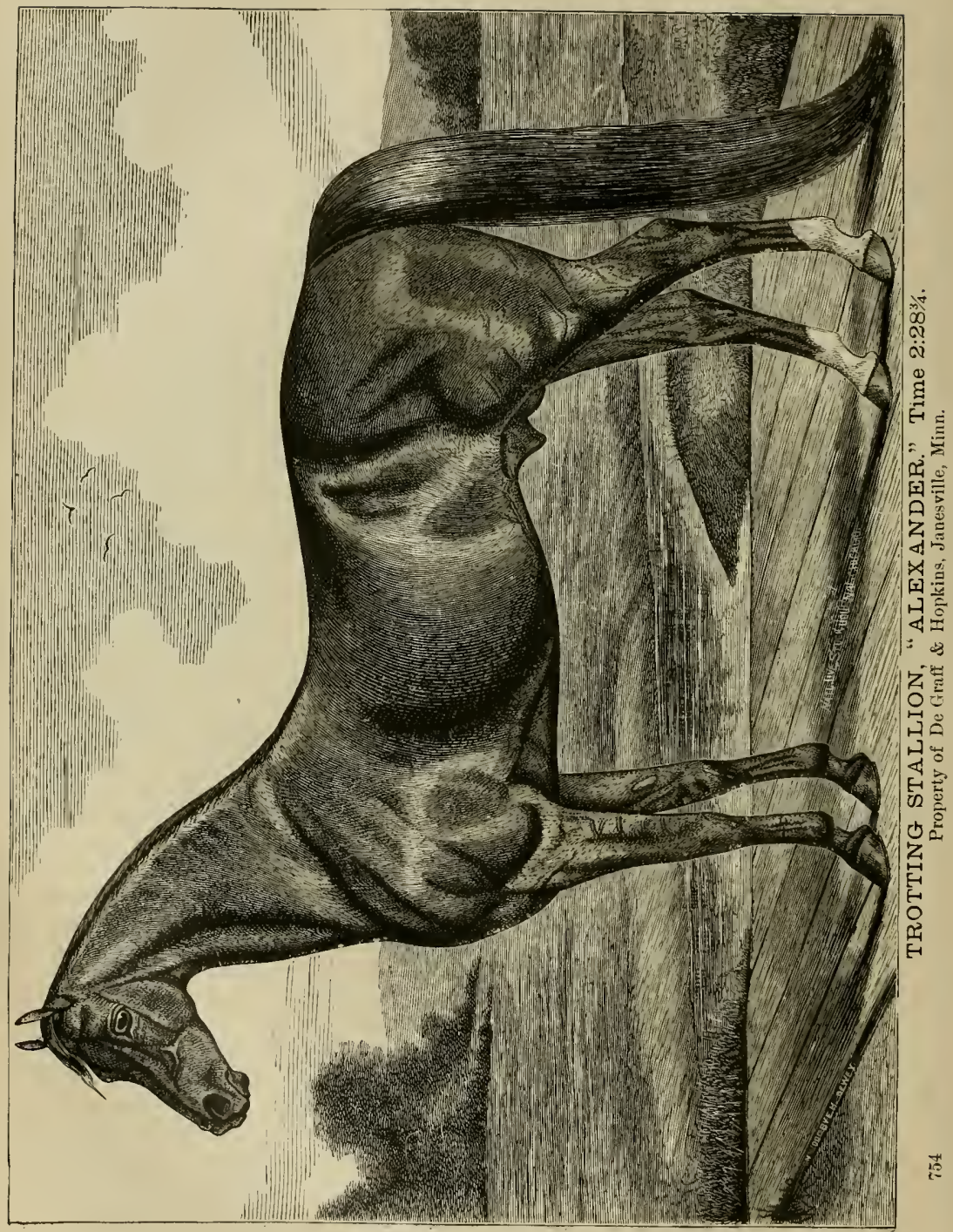


according to his age and size. If to the above a couple of quarts of wheat-bran are added twice a day, great advantage will be derived from it, since the latter is not only nourishing, but helps to keep the bowels in good condition and is an excellent preventative of worms.

Food that will produce bone and muscle should be given at this growing period, rather than such as will divert from this to simply the accumulation of fat. The food for all young animals should be given in small quantities and often, and the rations gradually increased according to the wants of the animal. True economy does not consist in cheap food, but rather such as is adapted to the wants of the animal, of whatever kind it may be.

A floor of plank, cement, or hard material of any kind, will be liable to injure the feet and legs of colts when permitted to stand much on them. Soft, wet ground, or mud, will be very apt to make the hoof tender, no matter how highly-bred or how perfect in texture the hoof of the ancestry may be.

The darn, when fully recovered from foaling, may be put to light work. The foal should at first be shut in the stable during the hours of work; but when he is sufficiently strong to accompany her, it will be better for them both to be together, if the work is slow and does not involve too much travel for him. This will obviate the fretting of the dam for her colt, while he will be able to take more milk from her and thrive better, and will also become familiar with the objects about him, and among which his life is to be spent. When over-heated with work, she should not be allowed to suckle the colt, till she is well cooled off, as her milk in this condition will have a tendency to produce diarrhea in the foal.

When a little older, in case the dam is used on the road, the colt can have his halter on and accompany her, having the halter tied to the shaft or to the harness. By this means he is under control, is not liable to get injured, and will soon become halter-broken.

Weaning. - When five or six months old, according to his vigor and size, the foal may be weaned. It is a good method to halter both dam and colt, giving them a single, large stall, where they can eat and rub noses together at the same manger. The dam and colt will both be contented then, for they wiil not miss each other. The colt should be untied and allowed once a day, for a few days, to relieve the udder of the dam. The colt will also eat at the regular feeding-times, increasing his rations, as he is deprived of his mother's milk. After a while he can be removed to his own stall. By this means the colt is nearly halter-broken without trouble.

Another method frequently practiced is to put the dam and colt in adjoining box-stalls, for a few days, where the partition is so high that they cannot get together, but where thay may see each other, and allow the colt to draw the milk once a day for a few days, after which it may be taken to its winter quarters. When confined in a stall by itself, the colt should have the freedom of it, and the stall should be large, light, and airy. Besides the exercise it may thus get, it should have a run in the yard an hour or two every day, when it is not too stormy. Plenty of clean bedding should also be provided.

A seven-months'-old colt will take from six to seven quarts of wheat-bran per day in connection with other food, while a yearling will take a peck a day, together with oats and hay. It must be remembered that this is the growing season, and material must be furnished for producing bone, muscle, and nerve, or the animal will not have sufficient to build up the structure that should go forward until the complete size and stature of the horse is attained. A few carrots, cut fine, given with oats and wheat-bran or oil-meal, make good rations for colts about the time of weaning and afterwards. They should not, however, be fed to the young colt in too large quantities. Good hay should always be given them in any quantity that they will eat.

- Colts should never be exposed to storms and cold weather, nor the intense heat of the summer sun. When out in the open air in cold weather, a warm shed should be provided, into which they may go in the day-time when they wish, and at night they should be com- 
fortably stabled. In the summer they should be allowed the freedom of the pasture, where there is plenty, of good grass, water, and shade. The shade should consist of not only trees, but a shed where they may find shelter from the hot sun. It is not advisable to put colts into a pasture with cows. When turned together, there is danger of either the colts being hooked by the cows, or the cows kicked by the colts. Neither is it well to put a colt into a pasture alone; two or more colts together will be more contented and do better than when alone, or with cows.

Raising a Colt by Hand. - If the dam should dry her milk, or if for any other reason it becomes necessary to raise a colt by hand, it may be done as easily as raising a calf lamb, or any other young animal. The milk of a cow that has recently calved should be given the foal. This should be mixed at first with about one-third of its quantity of warm water, and sweetened with sugar. After a week or so, the milk will not require watering. He should be fed four times a day until four or five weeks old, after which twice or three times a day may be sufficient, if he has learned to nibble fresh grass a little. It is better to feed small quantities and often, rather than larger quantities at longer intervals. Horses are naturally very particular about their food, and the pail from which the colt is fed should be scalded every time it is used, to keep it sweet and clean. If his bowels become constipated, two or three tablepoonsfuls of olive oil may be necessary to promote the proper laxative condition. Sweetening his milk with molasses will sometimes produce the same result, where the consti. pating tendency is but slight.

He should first be allowed to suck the finger in feeding, but may be taught to drink in a few days. Wheat bran, bruised oats, etc., should also be given him as soon as he will eat them, according to previous directions in feeding colts. Care should be used to give him a proper quantity of food, and at the same time not to over-feed, either being alike injurious. The quantity required will depend upon his size, age, etc.

Castration. - The age at which this operation can best be performed depends upon different circumstances, such as the difference in breeds, the form of the animal, the use to which it is to be appropriated, etc. The longer the colt remains entire, the better developed will the neck and fore parts of the body become; for this reason, we think it best to delay it as long as practicable. It can be safely performed as soon as the testicles have descended into the scrotum, but when performed thus early it will materially affect the development of the forehand. It is frequently done when the colt is a year old, and sometimes before it is weaned, but as a general rule, we think from a year and a half to two years is better. As regards age, the young animal will suffer less than those older, as the parts are not so well developed, consequently smaller, and their removal will not produce such a shock to the con. stitution. Their rapid growth also at this period causes the wound to heal quickly, with less tendency to a fever. The danger increases with colts from two years old and upwards. Castration is generally performed earlier on farm, than carriage horses. The National Live Stock Journal contains the following valuable directions respecting the conditions, prepara tions, etc., for this operation:-

"Castration is often performed by the owner, by a herdsman, or by some other nonprofessional man, rather than the veterinarian, and it is therefore especially important that it should be generally understood what conditions contribute to obviate the attending dangers, and insure a successful result.

Health. - Perfect health of the animal is essential to safety. Any pre-existing disease is pretty certain to be aggravated by the irritation and fever resulting from the operation; any impairment of the nutritive functions will retard the process of healing in the wound, of induce an unhealthy action resulting in permanent injury or death. If disease germs are in the system, their development is hastened, and the system has to bear the attack of two 
different troubles combined; or both concentrate their action on the same point, and the extension of the diseased action to the susceptible structures of the abdomen too often precipitates a fatal result. Thus strangles, so common in young horses, causes a low type of inflammation in the groin, with exudations, adhesions, abscesses, and even gangrene. Glanders, too, is attended by the development of the glanderous material in the wound and elsewhere, and always by a fatal issue. Scarcely less injurious are influenza, catarrhal fever, bilious fevers, etc., etc., the seeds of which find a fertile field for their development in the system fevered by the operation.

The system most favorable to success is one in high condition, with full, hard muscles; clear, bright, prominent eye; smooth, sleek, healthy coat; pulse full, strong, and regular; and spirit lively and ardent. The best condition is, in short, that of the trained animal, in which the wounds heal with that marvelous rapidity which we see in the athlete or.racehorse. It is not the fat animal, soft, flabby, and deficient in endurance, but the one that is all muscle and sinew, and that will not tire. Yet, even with this, it is important to give daily exercise after the operation. If kept up in a stall, the animal accustomed to regular exertion quickly becomes plethoric, and thus his great powers of digestion and assimilation conduce to unhealthy rather than healthy action in the wound. If such an animal must stand in the stable after the operation, his fine condition will be rather prejudicial, and should be reduced somewhat by a dose of physic prior to the operation, and a restricted diet after. A very fat animal may be advantageously treated in the same way. The very poor are liable to have the healing process retarded, and to have a low type of inflammation in the wound, with extensive swelling, gangrene, or inflammation of the lining membrane of the abdomen, or, in less redoubtable cases, local abscess, or tumor of the cord. These should have their condition improved before they are castrated.

No male should be castrated until it has been ascertained whether there is any hernia (rupture) into the scrotum. The sack of the scrotum should contain nothing besides the testicles. Any descent of abdominal organ may be felt at the front and sides of the testicle, and the thickening will be continued upward beside the cord into the abdomen. If pressed, it will return slowly at first, and then suddenly and completely. Such subjects should be left to the veterinarian for a special operation.

The Surroundings. - The season is a most important consideration. In pigs and other animals that tend to heal by adhesion of the lips of the wound, and without the formation of matter, a cool or even a cold season is not prohibitory; but in the horse, in which all wounds tend to form matter, and where the dangers of inflammation extending to the abdomen are so great, a temperate or even warm season is the best. The end of April or May is usually preferable as being mild, but not hot, and at the same time equable. From July onward the intense heats unduly favor putrefaction in the products of the wound, and excessive swelling in its walls. At this season, too, flies prove a source of great annoyance, and are even liable to infect and poison the sore by coming direct from carrion or diseased surfaces. So long as the nights are liable to be frosty or very cold, colts should not be castrated, unless they can be stabled and protected. In all cases the newly-castrated animal should be protected against cold rains or dews, drafts of cold air in buildings, large drinks of ice-cold water, and damp bedding. Wet weather, but above all that which is characterized by a succession of thunderstorms, is to be feared, not alone because of the danger of wetting and chill, but because at such times there is a special tendency to rapid decomposition in all dead organic matter, and therefore to putrefaction in the secretions of the wounds. This tendency is familiar in the souring of milk or dough, and in the penetrating smells that rise from any accumulation of damp vegetable rubbish. In such a season, therefore, the operation should be deferred until the return of steady, clear weather.

For reasons similar to the above, crowded, close, ill-ventilated, and uncleanly buildings 
are most dangerous, and animals from such places are best kept for some time in more healthy quarters prior to castration. The vicinity of slaughter-houses, rendering-works, dissecting-rooms, manure-manufactories, decomposing dung-heaps, etc., are to be avoided as calculated to unduce unhealthy action and gangrene.

The Operator. - The castrator should avoid all dead, decomposing, or unhealthy animal matter. Suppurating wounds, but especially those not doing well, cases of difficult parturition, dead bodies, and all unmanufactured animal products should be carefully avoided. If the operator has run the risk of such contamination, he should wash his hands thoroughly in water containing some carbolic acid or chloride of lime, fumigate his clothes with the smoke of burning sulphur, and allow at least twenty-four hours to elapse before he proceeds to operate. The most scrupulous cleanliness of all instruments is no less essential, as valuable animals are often lost through the retention on these of invisible particles in a state of putrefaction."

The Operation. - This very delicate operation, and the one attended with so much risk to the life of the animal, requires much care and skill on the part of the operator to result successfully, as animals often suffer severely and sometimes die from the effects of an unskill. ful operation, change of weather, or other unfavorable conditions. W. H. Herbert asserts that "it should never be attempted on a living subject except by a person of experience and skill," in which opinion we fully concur. It will certainly be much safer and more economical in the end to employ the services of an intelligent and skillful veterinarian, than for a farmer to attempt to do it himself, unless he has a good knowledge of and experience in such cases. No one should think of attempting it who does not possess these requisites. The use of chloroform will be found highly beneficial in such operations, and should always be employed, as it is more humane, since it obviates all pain, and also prevents the severe struggles of the animal, which are often attended with serious results.

The old method was to make an incision in the scrotum on either side and cut off the testicles, and prevent bleeding by a temporary compression of the cords while they were seared with a hot iron. This was a terribly painful process for the animal to undergo, and is now generally abandoned for the more humane and improved method.

The new method of castrating with the ecraseur is a great improvement upon the old, and also very simple, especially to a person having any experience with the old.

In operating, an incision is made through the subcutaneous tissues, which thus exposes the testicle. The chain of the ecraseur is then placed orer that portion of the cord intended to be severed, and the instrument gradually tightened until the cord is completely severed. This should be done gradually and very carefully. But very little bemorrhage will follow, if it is performed with care. Sometimes tying is resorted to to prevent bleeding.

Another method is by torsion, and is described by Youatt as follows: "An incision is made into the scrotum, and the vas deferens is exposed and divided. The artery is then seized with a pair of forceps contrived for the purpose and twisted six or seven times around. It retracts without untwisting the coils, and the bleeding ceases; the testicle is removed, and there is no sloughing, or danger. The most painful part of the operation, that with the firing iron or the clamp, is avoided, and the wound readily heals."

The old method of castrating with the clamp is an extremely cruel one, and should be forever abolished.

Castrating horses designed for farm, carriage, and general-purpose use, is commonly practiced in this country and England, as they are thus more docile and more easily managed; but in France and in Oriental countries castration is rarely performed, as they are thought to be more hardy and possess greater courage and endurance without it. 
Docking and Nicking.-The custom of docking the tails of horses, so prevalent some years ago, both in England and America, is as absurd as it is cruel, and should become forever obsolete. It is a great disfigurement to a horse, and many an otherwise beautiful animal has been in a great measure shorn of his beauty for life by this means, while there is really no advantage to be gained by it. The argument claimed in favor of docking is that it prevents the animal from switching its tail over the reins, which in many cases, with a high. spirited horse, might cause him to become frightened and the driver to lose control over him. All trouble from this cause may be prevented by having an iron or steel rod fastened from four to six inches above the whole length of the dash-board, for the reins to rest upon. This will keep them so much higher than the rump of the horse that he will not be able to switch his tail over them.

Nicking is a barbarous and inhuman custom, formerly fashionable, but now nearly obsolete. It should be forever prohibited by law. The man who practices it, justly deserves to suffer the severe penalties of the law. A horse with his tail shortened by docking, or weakened by nicking, endures great annoyance from the flies, besides the suffering inflicted at the time of the operation. Horses upon which the latter has been practiced have sometimes died of lock-jaw.

Bleeding.-There are very rare instances in the treatment of horses in which bleeding is beneficial. The practice of frequent bleeding, and for almost every ailment, as followed in the old time heroic treatment, we are glad to record as now obsolete, except it may be still adhered to by a few ignorant quacks, or so called "horse doctors," who, not knowing what else to do for the poor animal under their charge, will generally administer a sufficient amount of powerful drugs to nearly kill him, and then proceed to reduce what little vitality he has left by bleeding, or they will reverse the treatment, and bleed first, and drug afterward, and if the horse manages to live through it all, they will take to themselves great credit for skill in the remarkable eure they have performed!

The blood is that upon which the vital forces depend, containing the very element of life, and the withdrawal of it frequently, or in large quantities, cannot be otherwise than weakening to the vital forces of the animal system. Nature generally supplies the proper amount of blood required by the system, and when this amount is often reduced, there will be an unnaturally vigorous action in the blood-forming process, in the effort to make up for the deficiency of bleeding, which may in time become habitual and result in an apoplectic condition. It is safe to assert as a general rule, with few exceptions, that nature performs her work best without interference from man. In apoplexy, staggers, and a few other diseases or conditions, bleeding may prove very efficacious, but it should be done with the greatest care, and by the direction of an intelligent veterinarian.

In bleeding, a fleam or lancet may be used. The horse should be blindfolded in order to keep him quiet during the operation. The jugular vein is generally selected for the purpose, the point to be chosen being about two inches below the junction of the two portions of this vein, at the angle of the jaw. If a gentle pressure be applied with the finger of the left hand, the vein will enlarge and be distinctly traced at once. The edge of the lance or fleam should be placed directly in line with the course of the vein, and over its center. Great care must be used not to cut so deep that the opposite side of the vein will be opened. A small quantity of blood drawn quickly is much more beneficial than a larger quantity drawn slowly. The quantity to be drawn will depend altogether upon the condition of the animal, and the purpose of the treatment. We are decidedly opposed to large quantities being taken for any purpose.

When sufficient blood has been taken, the edges of the wound should be brought closely and evenly together, and held so by a small sharp pin being passed through them carefully, and a few hairs from the mane, or thread wound around enough to cover the wound. The animal should be so tied, for a few hours, as to prevent rubbing the place against anything. 
The 0ld-Time Method of Managing Colts.-It was the prevalent opinion of our forefathers, that in order to produce a hardy animal, it must be subjected to hardships and privations; hence when a colt was a few months old, he was usually turned out to pick up a living for himself as well as he could in the pastures, generally running with other young horses or cattle. When the cold weather of winter set in, he was in some sections provided with shelter and scantily fed with hay, often having to take up with the refuse and rejected portions of the older and stronger stock; but in many localities colts were turned out to shift for themselves during the entire winter, without shelter except what could be found under trees, fences, or on the leeward side of an occasional hay stack, and would not be seen by the owner for two or three years except by accident. The wild pastures or prairies would in summer afford a comfortable supply of grass, but the scanty feed in the winter would reduce his flesh to such a degree, that were it not for the rough, shaggy coat kind nature gave in her sympathy for his protection in his forlorn condition, he would seem smaller in the spring than the previous autumn, so that in the second year although he would be a little larger, it would be difficult to tell a two-year colt from a yearling by his appearance. When the time came that he was to be "broken," as it was called, the animal would be looked up and driven to his owner, and some rough specimen of humanity known by the professional (?) term of "horsebreaker," - a recognized character in every country town and village,-would be sent for, and the poor animal put under his charge to receive his instructions, which generally consisted of blows, kicks, and the tortures of the bitting bridle; who whipped him to make him go when he did not know what was wanted, or how or which way to go, and then whipped him to make him stop, when he did not know that he must. If in his terror and torture he reared or balked, he received more blows and kicks and jerkings on the cutting bit, until in his fright and sufferings he was incapable of understanding what was wanted of him, and would either be spoiled in temper, if naturally spirited,-or have all the spirit and intelligence taken out of him by such ignorant and cruel treatment. After these experiences, he was taken to the blacksmith's shop where his hoofs were often cut down to the quick, the place where he stood not unfrequently being marked by the blood that oozed out, and then shod in the most awkward manner. Afterward, under the ignorant opinion that nature did not know how to do her work perfectly, and that the horse was not complete without man to rectify her mis. takes, a hot iron was procured, and the roof of his mouth burnt and mangled, to cure him of the "lampers," supposing the ridges found in every young horse's month to be a disease or unnatural, and that they prevented the horse from eating well. We are glad to know that a great improvement has, for the most part, been effected in horse management within a few years past, and that the practices, formerly deemed necessary, have in the main become obsolete, except perhaps here and there in an occasional locality that may be as far behind the present age in other respects as in ideas on this subject, and where the old regime of "breaking colts" is still practiced with all its cruelty, and injurious results.

The Present System of Colt Management.-There is an old adage that "Half the horse goes down his throat." However true this may be, it is apparent to every intelligent supervisor of young stock, that during the growing or building period, when there is such a demand for material for structure, the animal must be fed and cared for in the most judicious manner. Believers in the old time starving and toughening process are at present fortunately hard to find, and it is now a generally accepted fact that the first two years of the animal's life is the time in which to make the horse; in other words, the care and attention bestowed upon the colt during this period have more influence in determining the future value of the animal, than that of subsequent years. It is now generally understood by all intelligent breeders that if the colt is not kept growing he is dwarfed in size, and hence his value lessened, and money lost to the owner. He must be kept growing, and in order to accomplish this, he must be supplied with material for the jurpose. As oats are the natural food of the horse, the little weanling should have from two to four quarts of bruised oats each 
day, according to his age, and occasionally a little wheat bran and oil meal, besides all the good hay or ensilage he will eat. When the colt is a year old or more, he can have a larger quantity of oats, providing he has a large yard for exercise, the aim being to have him fit for light work when three years old; some breeders claim that by this process the colt may be used a little for light work at two years of age; if used at all at this age, however, the work should be very light, or the animal would be liable to be injured seriously. Heavy work at this growing period should always be avoided. It can be readily seen that by this method of caring for colts, horses can be raised with more profit to the owner, than by the former practice of sometimes keeping the animal until he was five or six years old before making him useful; and that keeping a horse that length of time before he earns his living, is a losing course from the time he is three years of age. Mr. J. E. Russell, Secretary of the Massachusetts Board of Agriculture, says:-

"Every breeder should be fond of association with animals: he should be a judge of form, health, and improvement, and he should understand how to handle them. There are no secrets in the horse business that men of average intelligence in regard to animals cannot at once master. Quiet, patient ways, with low voice, and gentle but strong hand, will teach a colt all that he needs to know. There are men in every neighborhood who can break colts safely to harness, and by persistent, careful practice, fit them to stand quietly, to stop at the given word, and to be way-wise on any roads. In my county of Worcester, there are communities where they take great pride in educating oxen; and I have seen, at Worcester and at Franklin County cattle shows, steers trained to an incredible point of intelligence and docility. Boys that can train a steer to walk a chalk-line, and to go on his knees at the word of command, can do wonders with horses; for the horse enjoys the companionship of man, responds at once to kindness, and, next to the dog, has the quickest intelligence of any of our domestic animals. Horses for profit must be sold young, unless they earn their living every day. Leave speculation to jockeys and dealers; sell when you have a customer; sell whenever a reasonable profit is offered to you. The best time to sell is before the colt is foaled, to some one who is in love with the dam: the next best time is when the foal is weaned. To the common eye, all weanlings will make fine horses; and the price of a weanling generally pays more profit than the breeder ever has offered to him again. When your colt is past two, every hour that you delay to sell is 'burning daylight.' Early maturity is one of the great advantages in thorough blood; and no man can afford to breed from any family that is slow in maturing."

The best practice, and the one followed by the most successful managers of horses, is to begin breaking or teaching the colt while he is yet a mere suckling by the side of his dam. A very little pains at this period will teach the baby-horse that it has nothing to fear from the presence of man, and that no harm can come to it from being handled from head to foot. In this way he will soon become perfectly gentle, will easily be led by the halter, and will stand when tied. All the subsequent lessons should be by gradual approaches; the main point being to inspire him with confidence that he will not be harmed. By pursuing this system of gradual approaches with perfect gentleness of manner on the part of the groom or other attendant, there need never be any trouble in breaking the most fiery-tempered colt.

The most intelligent colt will be the most easily managed by this means, for once having learned the lesson of confidence in his master, he can readily be taught to understand and do what is required of him, while a sensitive and naturally timid animal would be spoiled by any other course of treatment. It is not will or obstinacy that are the usual difficulties to be surmounted in breaking colts, but fear, and a failure to understand what is required of them. Rough treatment in the breaking process has spoiled the temper of many a valuable animal, while on the other hand gentle and intelligent instruction improves the disposition and develops the speed and endurance of colts, qualities so excellent in roadsters. The more good qualities developed while young, the greater will be the merits of the adult animal. 
How much the skill and genius of man have added to the value of our improved breed of horses, would be difficult to determine. Many a noted trotter would not have sold for a tenth the price paid for him if his fiery, ungovernable temper or ungainly stride had not been modified and improved by the patience, perseverance, and gentle treatment of his trainer; neither would Maud S. have sold for $\$ 21,000$ if her noted trainer had not taught her at four years old, to trot a mile in $2: 17 \frac{1}{2}$. The same may be said of St. Julien and other noted horses. Untrained horses have seldom been sold for high prices. By patience and kind treatment a horse may be trained to do anything required of him that is reasonable, and not only this, but will become so attached to his master, that he will seem to take a kind of pride and pleasure in trying to please him when he once understands what is desired of him. A recent writer, the owner of several fine horses, expresses the following sensible and humane opinion:-

"We would never have a man on our premises who would abuse a horse. The men who ever worked for us were instructed not only to spare the lash, but to spare the tongue. Rough language is nearly as abusive as actual beating. A horse cannot be screamed at and zursed without becoming less valuable in every particular. To reach the highest degree of value, the animal should be perfectly gentle, and always reliable, but if it expects every moment that it is in the harness to be scolded and struck, it will be in a constant state of nervousness, and in its excitement as liable, through fear, to do something which is not expected as to go along doing what you started it to do. It is possible to train a horse to be governed by the voice almort as completely as it is to train a child, and in such training the horse reaches its highest value. When a horse is soothed by the gentle words of his driver -and we have seen him calmed down from great excitement by no other means-it may be very fairly concluded that he is a valuable animal for practical purposes, and it may be certainly concluded that the man who has such power over him, is a humane man and a sensible one. But all this simply means that the man must secure the animal's confidence. Only in exceptional instances is a horse stubborn or vicious. If he understands his surroundings, and what is required of him he gives no trouble. As almost every reader must know, if the animal when frightened can be brought up to the object, he will become calm. The reason is that he understands that there is nothing to fear. So he must be tanght to have confidence in the man who handles him, and then this powerful animal, which usually a man could not manage, if it were disposed to be vicious, will give no trouble. The very best rule, therefore, which we would lay down for the management of the horse is gentleness and good sense on the part of the driver. Bad drivers almost invariably make bad horses."

If farmers and the owners of horses generally would be more particular in the choice of those whom they employ to have charge of their animals, in all stages of their growth, whether as young colts, draft or carriage horses, they would not only find it more agreeable in having better tempered and more easily managed horses, but also more profitable in a money point of view, since such animals will command a higher price in the market than those made vicious, nervous, and unmanageable from unkind treatment.

The writer has known of many valuable animals being utterly ruined by the bad management and cruel treatment of se-called "professionals" in colt-breaking. In one instance, a valuable colt of four years of age was so wretchedly handled that in three weeks' time she was not worth a dollar for after-use. Another was so cruelly treated in the "bitting process" (a thing which no man who truly ioves his horse will ever allow), that, in horsemen's phrase, "she had no mouth;" and when abused with voice or whip, would drop her jaw back against her neck, and run away and break things, in spite of the efforts of her driver to stop her. In this instance a naturally fine animal was ruined, and practically worthless when "broken," as the expression is. Another animal, from the same stock as the preceding ones, and now in licr llirtieth year, never was broken, in the common mcaning of the term, and will not be when she dies; but willing and true to bit or voice, she has been faithful and fruitful in good 



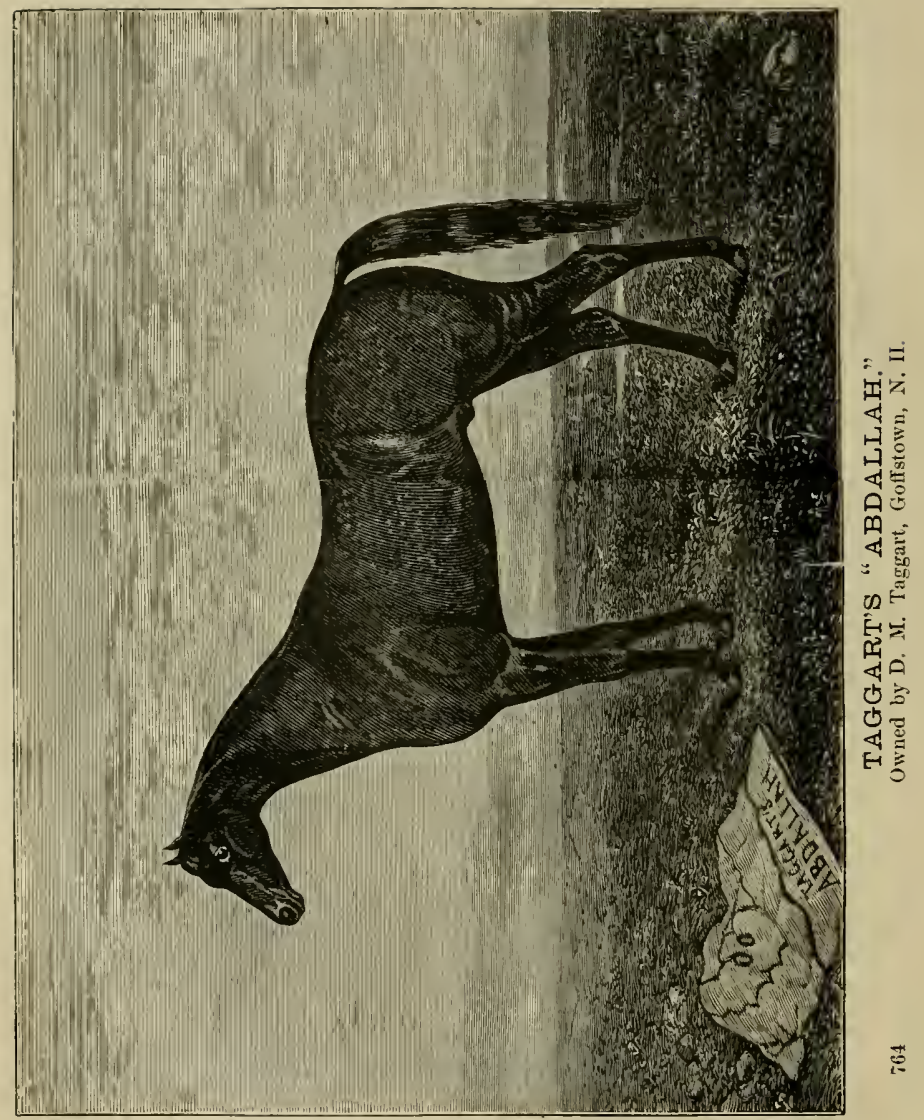


works all her life long, perfectly gentle, although a very spirited animal, but peculiarly sensitive to harsh treatment of any kind, and would have been rendered utterly worthless by it.

- Halter Breaking.-The best method of teaching a colt, is to commence at birth, and by gradual approaches accustom him to be handled. Thus, by kindness and gentleness, his first lesson should be, that he has nothing to fear from man, but that his master is his friend and protector. This lesson must invariably be first taught, in order to attain the highest success in subsequent efforts, in bringing him under complete subjection, and in developing the best qualities of the animal. This method of colt breaking was well understood and practiced by the ancients, as will be seen from the writings of Xenophon, which were penned about twenty-three hundred years ago, as follows:-

"Care must be taken, that when the breaker receives the foal, he be gentle, tractable, and fond of men. For he is generally rendered so at home by the groom, if the foal is made to understand that hunger, thirst, and irritation are procured by solitude; and that meat, drink, and freedom from irritation are procured by men. When these things take place, foals not oniy love, but long for men. It is necessary also to touch those parts which, when touched, give greatest pleasure to the horse; and these are the hairiest, and those parts in which, when he feels any pain, he cannot relieve himself.

The groom should be enjoined to lead him through a crowd, and cause him to approach all kinds of sights and sounds. Whichever of these the foal may dread, it is necessary to teach him, not by harshness, but gentleness, that they are not dangerous."

The young colt should be handled every day, gently and quietly, patting or rubbing him with the hand only in those parts of the body that it is agreeable to him to be touched, such as the back, top of the hips, under the neck and head,- - places that he cannot easily rub him. self, or touch with the mouth or feet. Horses do not usually like to be touched on the

- flanks, or the inside of the stifle, under the breast, or on the ears, and such places should be carefully avoided. Attentions of this kind should be frequent, but never tiresome to him.

These instructions should begin during the first week of his life, for the earlier this is learned, the easier will it be to teach him, and when once taught, it will never be forgotten. Many breeders teach the foal to be led by the halter during the first tiwo or three weeks. If the dam is to be used on the roac', the colt will, when strong enough, soon learn to accompany her by having his halter attached to the harness or shaft.

When being weaned, he may be tied by her in the stall, as previously recommended, which will also further accustom him to the halter. By commencing early, there is no acquired habit to be overcome, and he should be taught only just what you desire him to know. Let him know that you are his friend, and by patient and gentle efforts he will soon be able to understand what is desired of him, and, if he has confidence in you, will willingly yield obedience. When he does well, he should be praised for it, and made to understand, by gentle pats and kind words, that it is appreciated. Animals, even the most stupid, will very quickly translate such acts and words into their true meaning, and the horse, being one of the most intelligent of the brute creation, will soon catch the meaning of what is intended for him to know. Never pull him back on the halter, as so many ignorant, so-called "horse-breakers" do, under the stupid idea that a constant pulling will teach him to walk fast. It will only teach him the reverse, and to hang back, and when this habit is once acquired, it will be almost impossible to break it.

The halter should be of some soft and strong material-leather being best-which will not cliafe or fret him, and it should be made to fit well and not hurt his nose when he pulls. If he should once break the halter and get away, it would be a bad lesson that he might never forget.

Never use a rope for a halter on an unbroken colt, as it would chafe and make him nervous and uncomfortable. With a rope halter, a colt will be very liable to pull, rear, and 
throw himself, since the rope will hurt him, and cause him to try to get his head out of it, and the more he pulls, the more the hard, cutting rope draws upon the sensitive flesh, the slip-noose tightens and pinches his nose, until, with pain and fright, his struggles to get free often result in serious injury to the animal. Besides this, a horse that has once had such an experience of pulling at the halter will always remember it, and cannot be as well broken as one that has never pulled at all.

The best method to teach the colt to lead, if he has not previously been accustomed to it in accompanying the dam, is not to go in front and attempt to pull him after you, but to pull him gently to one side, as he will then not be as liable to brace himself and pull back, and thus acauire the habit of pulling. After he has been a step or two one side, let him rest a moment, while you pat him a little, all the time speaking kindly to him; then pull him gen. tly by the halter to the other side, always speaking the word "come." In a short time he will learn to associate the word with the act of starting, and learn what it means. If he resists, be patient yet firm. If the resistance seems to be through fear, handle and pet him until he loses all fear of you before attempting to force him to yield.

If the resistance appears to be obstinacy, gradually increase the strength upon the halter until he is forced to yield to it. Never jerk or pull suddenly on the halter. After a few moments of rest, this may be repeated, always speaking to him when you want him to go; by this means he will learn to start when told.

Never forget to praise and caress him when he does well, but never punish him at this period.

After a little patience on the part of the trainer, and perhaps resistance on his part, he will soon suffer himself to be led wherever he is desired to go. The trainer may be amused at the exhibition of temper or vexation on the part of the colt during the trial, but he must never allow himself to be angry. He must simply "hold his own," never jerk or twitch on the halter, but steadily and firmly enforce obedience. Obedience, pure and simple, without enmity or fright, must be obtained to reach the best results in halter-breaking, and, in fact, in everything pertaining to the training of the colt at any period. By once learning in this manner that man is stronger than he, he will never forget it, and will the more readily yield obedience when he becomes stronger than man. Don't fail to encourage him by praise and gentle pats when he does well; he will learn to love his master for it, and submission will be pleasure. If he shows much temper, it must be remembered that some of the most intelligent and valuable horses have been naturally high-tempered, but have been subdued and made the valuable animals that they were, through kindness, combined with firmness and persistence.

When visited in the stable, frequently carry him something to eat that he likes; an apple, piece of cake, or lump of sugar will go far towards gaining his affection, and making him docile and submissive. The writer has known older horses with vicious habits entirely reclaimed in this way, and who would by this means learn to regard the master's approach as an omen of good to them, and whinny for him on seeing him in the distance, and when sufficiently near would fumble playfully about his pockets with their lips in endeavors to find the present he had brought. Such a horse, ninety-nine times out of a hundred, will prove an obedient, faithful animal. It is said that the way to an animal's heart, or affections, is down his throat. With how much truth may this not be practically applied to many of the human species. Although it is to be regretted, it will quite too frequently be found with mankind, on a severe analysis of motives for professions of friendship and esteem, that self-interest lies at the foundation. If it does not form the entire basis, it will too often be found to constitute some little "corner-stone" in that foundation.

Whatever may be said of this principle respecting mankind, we believe in a generous toleration of it in the management of animals, and particularly horses. The colt should be 
made familiar with noises and startling objects, gradually at first by beginning with those of a mild character, urtil he fails to be frightened at almost anything in sight or sound. This will prevent him from shying suddenly when used in the harnes:. When well halterbroken, that is, when he will lead readily by the halter, and also stand quietly when tied, he has learned a valuable lesson.

The Bitting Process.-A smooth bit that will not hurt the mouth of the colt should be used; also, one that has a bar at each side to prevent it from pulling through his mouth either way.

This may be attached to the head-stall of the bridle, without reins, and he be allowed to wear it an hour or two each day, until he becomes accustomed to it, and will bear it without trying to get it out of his mouth. As soon as he will bear it, the reins may be attached. Do not tire or worry him, but let everything that is new to him be gradual.

The method of bitting, as followed by many ignorant horse-breakers, cannot be condemned in too strong terms. It is alike inhuman and injurious to the animal, and is practiced under the mistaken idea that by drawing the horse's head out of its natural position, by this torturing process, he will ever afterward carry it in a graceful manner.

The position in which a horse carries his head in harness will depend mainly upon his form and temper. A horse may have ever so much spirit and courage, but, if he have a short neck and perpendicular shoulder, no bitting bridle ever invented will give him a gracefully curved neck, and fine style of carriage. Style is something inherent in the animal, and not in the application of any device that may be invented by man. The great object of the bitting bridle is to cause the horse to yield to the pressure of the bit, and to teach him to obey the guidance of the reins. The mouth of the colt is very tender and sensitive, and the use of any bit that will cut, bruise, and mangle the gums, tongue, or lips, is not only cruel, but in time renders the colt hard-mouthed and unmanageable. Of course, it is desired that the horse's mouth shall become toughened to the use of the bit, but this will come gradually by daily use. It is not, however, desirable that the mouth shall become so injured by it that the bit will make no impression whatever. Mr. Murray's opinion on this subject is so much in conformity with our own, that we quote it for the benefit of our readers:-

"To see the bits that have been invented, and the 'bitting-machines' that have been patented and sold, to serve this purpose, is enough to set the satirist on edge, and arouse the ire of the humane. The truth is, the only use of any 'bitting-machine,' if it is any thing more than a plain bar-bit in a bridle without blinkers or check-line, is to make money for some ignoramus, and torture the horse. The philosophy of bitting horses, upon which these 'bitting-machines' are founded, is a fraud and folly. There is no more need for them in a trainer's yard than there is in a nursery. I make no limitation or modification of this statement at all. Their true name is 'fool's machine,' and not 'bitting-machine:' or, more properly, if you wish to designate their use and result of it, call them 'machines to spoil horses' mouths;' for this appellation precisely describes them. A man using one ought to be indicted before the common law of the country, which should at least be able to prevent such cruelty to animals. If any owner of a colt who reads this owns or uses one of these 'bitting-machines,' I urge him to burn it or bury it, as the most mischievous and hurtful thing that he can have about his stables. If I wished to make my colts ' hogged-mouthed ' and desperate pullers, I would use one of these 'infernal machines,' as I have no doubt the colts themselves call them, and as they deserve to be called by colts and men. I will show you how this plan of using these 'machines' works.

"Now let us begin to bit a colt according to the machine method. The colt, never having been even broke to the halter, perhaps, is led out into a yard, the 'machine' strapped on his back, the bit of iron or steel jammed into his mouth, the check-rein adjusted, and the colt's head drawn suddenly up into the air, and the trainer stands one side. The colt, of course, 
struggles and rears and plunges. He becomes enraged, and 'fights the bit;' foam drops from his lips; pretty soon it is stained with streakings of blood. The iron bit, you see, as he ' fought it,' has grated over the young teeth, cut into the tender tongue, and lacerated the gums and lips. I have seen all this done (it is no fancy sketch), -seen blood come in less than two minutes after the 'bitting-machine' was adjusted. Now, what has been done? Several things, I reply. First, unnecessary pain has been caused an innocent and harmless creature: that alone is enough to condemn any 'machine' ever invented. Secondly, the colt's mouth has been spoiled until the lacerated gums and lips and tongue can heal. Thirdly, the colt's temper has been soured, and no useful knowledge imparted. These truths are self. evident. But this is not all. The 'machine,' instead of being removed, is left on; the trainer goes to his work in the field, or to drive; and the colt is left to 'fight it out.' Now, examine the matter a little. What is the true position of things? This, I respond: The colt is in pain. His head is drawn up to an unnatural height; his neck, pulled into an angle both awkward and painful, aches with exquisite suffering. To appreciate the agony the young thing endures, let some one take hold of your own head, and draw it up and backward as high and as far as the bone-structure and muscles will permit, and compel you to stand with it in that position even for five minutes. In this way you will get some idea of 'bitting-machines,' and the actual torture which colts experience while being 'bitted' by them.

But the evil of this system is not yet fully stated. The colt, with his head drawn up and back, is left in the yard, as I have said, while the trainer goes to his work or to drive. Perhaps he stands an hour; perhaps five hours; very likely all day. For the first few minutes he strives to keep his head up, and the bit loose in his mouth, because it pains him; but pretty soon the muscles of his neck begin to ache. They were never made to hold up the bead in that style, and are actually unable to do it for any considerable length of time. Soon the head sags; the pain in the overtasked muscles of the neck is greater even than that caused by the pressure of the bit. It is, you see, with the colt, a choice between two pairs. Little by little, the head droops; heavier and heavier the weight of it is laid upon the bit; and, in the course of an hour or two, the colt stands weary and stolid, the weight of his head and neck laid solidly down upon the bit. The colt is being taught, you see, to 'take the bit' with a vengeance. He is actually being educated to 'hog on the bit' and be a puller. The true way to bit a colt is not to bit him at all; that is, let him bit himself."

We remember trying the "bitting machine" just once, about twenty-five years ago, in our first attempt at colt-breaking, supposing it to be a necessary part of the operation. It was a pet animal, a fine, spirited bay mare of the Morgan type. We shall never forget how, after the bit was put on, she followed us around with a mute appeal in her eyes to have it taken off, occasionally rubbing her head against our shoulder, the sweat meanwhile starting from every pore, making her bright bay coat nearly black as it became wet.

After about ten minutes of this method of experiment in colt-breaking, we were convinced of the error of using it, and the agony it was inflicting upon our pet horse, and it was immediately removed and never put on again, though we kept her until she died of old age, which was at about thirty years, after having proved one of the most stylish and best roadsters in the country. We have also been equally successful in breaking other horses, but never have tolerated the cruel bitting process. We believe more horses are either injured, or utterly ruined by it, than by almost any other means.

Breaking to Haruess. - This may be done when the colt is about two years old, or even younger, and after he has been well lialter-broken and accustomed to the bit. Many do this when the foal is from twelve to fifteen months old. The more he has been gentled by frequent handling previous to this time, the easier will all subsequent instructions become. He must not only become accustomed to the harness, but must be taught to stand quietly while the harness is being put on and taken off. Portions of the haruess.should be put on 
gradually, permitting him first to smell of it and touch it with his nose. Animals, especially the horse, depend much upon the sense of smell in becoming acquainted with objects about them, and, having once in this way become familiar with things, they will rarely ever be afraid of them afterwards.

The harness should fit him loosely at first, until he becomes a little accustomed to it, when it can be gradually tightened. He should first be allowed to stand an hour or so with the harness on, after which he can be led about, and thus become used to it while walking. Nothing should ever be done to frighten or worry him, but at the same time he should be taught to yield to his trainer by firmness and kindness. When being led about, he should occasionally be stopped at the place of unharnessing, and allowed to stand about the length of time that would be required to remove or put on the harness; by this means he will learn to stand quietly for this purpose. This should be repeated for several days in succession, until this lesson is thoroughly learned. After this be can be taught to obey the motion of the reins. It is a good plan, also, to accustom him to pressure against the breast and shoulders by fastening strong cords or straps into each side of the collar, and by pulling, gently at first, cause him to use his strength to pull against it; by this means he will get an idea of drawing.

The sulky is much the best vehicle to use in breaking a colt. It should be light, but it is important that it have very strong shafts. After becoming accustomed to the harness, and before being attached to the sulky, the colt should first be led up to it, and, by smelling of it and touching it with his nose, learn that it is nothing from which he has any cause to fear. He may then be attached to it, which should be done as quietly and carefully as possible.

Some prefer to have the colt at this point in breaking driven with another horse. If the latter method is practiced, the horse should be one of the most gentle and submissive, also one the colt has been accustomed to be with. We have always been accustomed to drive a colt singly while breaking, and prefer this method. Everything about the harness and sulky should be very strong, that no accident may be liable to occur, for if the colt should chance to break away he would never forget it, and would be liable to be a runaway at any period of his after-life.

The method we have found most successful is to attach the old farm horse, with whom the colt is well acquainted, (or it may be his dam,) to a wagon, and have this team start in front of him slowly, keeping a few yards in advance of the colt team. In this way he will more readily understand what is required of him, and be more willing to follow where he has such company to lead. It will often be found that when a turn in the road may chance to hide the forward team from sight for a moment, the colt will whinny anxiously, and hasten his gait to catch up.

Teach a horse to depend much upon the voice, which should never be harsh and abusive. By this we do not mean to be understood that we favor the custom of such as are constantly shouting and talking to a horse while driving. We have seen some drivers that we would gladly have seen gagged, or their voice stopped by some other effectual means, and whose constant "whoas," "get ups," "take cares," and similar commands and countermands were enough to puzzle and disgust any animal, as well as human beings. A quiet driver is always the best driver, but when he does speak, he should be understood and obeyed.

The horse should be taught the meaning of but few words, yet these he should learn thoroughly, and when they are spoken to him, he should learn to heed them. By this means many a serious result to an accident or fright of the animal may be prevented. A horse who knows his master's voice, and is accustomed to obey it, and who feels that his master is his protector and friend, will be much more likely to stop at his word, when frightened, than one who has learned to fear his master's voice and lash, when anything unusual happened.

If there is any tendency in a colt to balk, let him stand a few moments, and then by 
gentle words or a few pats encourage him forward. Never use the whip under such circum. stances. Never get into a quarrel with him. The best way to avoid this is to be patient, gentle, and firm, giving him time to understand what is wanted, and to make up his mind to do it. A horse is naturally spirited, sensitive, and timid, and although a very intelligent animal, yet he must have time to fully comprehend what is wanted before he can perform what is required of him. Everything is strange and new to him, and a little haste might confuse or frighten him, especially if he be of a nervous temperament, to the extent that he might be wholly unable to understand what was for the time wanted.

Teach him to stand quietly while you are getting into the vehicle, and until you tell him to go. This may be soon accomplished by getting in and sitting a while before starting, always speaking the word when you wish him to start, and never under any circumstances allowing him to start of his own accord. The habit acquired by some horses of starting before the driver is half in the carriage, is a pernicious one, and is more the fault of the trainer in permitting it, than of the colt.

A colt should never have his temper spoiled by being teased or annoyed in any way. More horses are made vicious in this way than in any other. He should never be over-exercised, or discouraged hy loads that are too heavy for him to draw. Let it he remembered that whatever is done should be done quietly and in a gradual way, no hurrying, no bustle or confusion. Always be true to your teachings to your colt; never contradict yourself, teaching him one thing this week, and the opposite the next. He will remember this if you do; it will confuse him, and be as likely to make him do wrong as right. His lesson must be made as plain for him, as for you. Never put blinders on him while being broken, or at any other time.

We know of an owner and trainer of several colts who, when he goes into the pasture among them, will have them all about him, as gentle as pet kittens, rubbing their noses against him, and each seeming to be jealous of the attentions he bestows upon the others; who run to meet him when they see him coming, and who whinny after him when he leaves them. His method is simply to begin gentling the colts from birth by frequently handling them, and by kindness, gentleness, yet firmness, never deceiving them, and always encouraging and rewarding their obedience. Disobedience he rarely punishes with the whip, but generally by abstaining from the little attentions and tit-bits that are given as rewards at other times.

These are the best means of subduing a colt and making him obedient without crushing his spirit. Such training makes much of the difference between a worthless and valuable horse, for it must be remembered that the qualities that make an animal vicious and ungovern. able will, under judicious treatment, maike a spirited yet obedient horse, one possessing great intelligence and appreciative of praise or rebuke. If it is thonght that the directions given require too much time and labor, let it be borne in mind that the horse is a very valuable animal, and that the labor bestowed upon him at this period greatly increases his value by making him more serviceable, readily managed, safe, and reliable, and when once well broken the lesson learned will be for a lifetime, never to be forgotten.

Teaching a Colt to Back.-This may be done by standing in front of him and taking hold of the reins with either hand, give a slight pressure upon the bit, at the same time speaking the word "back."

He will thus be made to step backward a little, and by a few repetitions, always speaking the word, as at first, he will soon learn to associate the movement with it. This is an unpleasant and awkward lesson for him to learn, as it is not natural for any animal to walk backward, and time should be taken, in order not to try his temper and make him obstinate. A few trials each day, for a few days, will generally accomplish it in the most satisfactory manner, and with a slight drawing upon the reins while standing back of him. When he 
has learned to go in a harness, this lesson should be frequently repeated. While being broken to the wagon it is a good plan to have him attached to it, and taken where there is an incline, where the wagon will itself tend to run backward, down hill, thus making it easy for him to back it at first. After having accomplished this to the satisfaction of his trainer, he can be tried on level ground, and thus by degrees, adding after a time a slight weight and increasing it, be may learn perfectly the (to him) difficult lesson.

In the same manner he may learn what the word "whoa" means, and to obey it also, and to stop by a pressure upon the bit.

Breaking to the Sarldle.-This is learned by first putting a saddle upon him, leaving the belts very loose, and omitting the back straps, allowing him to stand in the stable with it on for an hour or two each day, tightening gradually the belly-band. After two or three days put on the back straps, and continue to thaten the belly-band until it is as tight as is customarily worn. He should then be led about until he becomes accustomed to the feeling of the saddle while walking, and when this is thoroughly learned a light weight, like a small bag of grain, may be laid across it. In this way he will learn to carry a weight. He should first be ridden by one he is well acquainted with, and who has charge of him. No colt should, however, carry a heavy weight, such as a rider, for instance, until his back has become sufficiently strong to enable him to do so without injury. The saddle gait can afterward readily be taught. The mounting and dismounting should be done very carefully and quietly at first, in order not to frighten the animal.

The Age for Working Colts.-Although for many reasons it is very desirable to break the colt when young, yet it is not well for him to work hard until he has attained his full growth and strength. The canse of so many horses breaking down early, is doubtless dne to over-work during the growing period, and before the bones have become sufficiently hardened, or the muscles fully developed. By working a colt hard he will become an old horse when he should be only in his prime. Although it may be expensive to kept a colt doing but comparatively little when he is four or five years old, yet it will pay better in the end than to over-work him, for he will last enough longer to more than make up for it. Every horse should work at least two hours a day; they require exercise in order to promote health, and. maty horses suffer for want of such exercise, being kept idle in close, confined stables. Either extreme is injurious. Although a colt may do some light work at four, he should not be put to hard service until he is six or seven years old. By such humane and considerate treatment on the part of his owner, he may be made to do good service until he is from twenty five to thirty years old, or even longer, while many hard-worked colts become horses that are past their usefulness at from twelve to fifteen years.

How to Prevent a Colt from being Easily Frightened.-There is a great difference in horses with respect to their shying and becoming easily frightened while being driven. The temperaments of animals differ as much as those of the human family, and some horses are naturally more nervous and timid than others. But this can be corrected in a great measure by taking pains to permit a horse to examine and smell of everything that looks new and strange to him. Everyone who has had any experience with horses, or has been at all observing, will know that the natural, and, in fact, the only way in which a horse can obtain a (to him) satisfactory knowledge of objects, is to smell of them; also to sometimes feel of them with his nose. He seems to have no confidence in simply looking at an object, and when greatly frightened at anything startling, will generally cease to fear it afterward, if he can apply his nose to it for a moment, and examine it in this way, thus assuring him. self that it will not harm him. And a horse should always be permitted to do this. Some of the most timid and nervous of horses can be greatly improved in this way, and many serious accidents prevented. To whip a horse for being frightened, is the most stupid of 
blunders, for he will be all the more liable to fear the same or similar objects again, and such a course will therefore increase the evil, rather than correct it. When breaking a colt, he should be permitted to examine everything used about him in this way, the harness, saddle, wagon, and when an object on the road startles him, he should be led up towards it carrfully, and by kind words and pats be assured that it is all right by smelling it.

When young horses are being driven to the city or busy town for the first time, and have to encounter so many strange sights and sounds, if carefully and gently treated, they will soon lose all fear without at the same time losing their courage.

Mr. Rarey tells, in his lectures, an incident of a timid horse shying at a buffalo robe. His ignorant and brutal owner tied him fast so that he could not get free, and laid a buffalo robe on him. The poor animal died almost instantly from fright. Had he been permitted to approach the robe gradually and smell of it a moment, ho would probably never have been frightened at a robe afterward.

Balking.-This, in the colt, we beheve to be mainly due to ill treatment or misman. agement on the part of the trainer. In horses this vice may generally be corrected by patience and kindness, but never by blows, kicks, jerking at the bit, and angry tones. Oniy the most ignorant and stupid of drivers will resort to such measures.

Cases have come to our personal knowledge where balky horses have been beaten by brutal men until they died from the effects in a few hours afterward, and yet this excessive cruelty did not result in making the horse go. We recall an incident of this kind, of which we afterward had authentic knowledge, that occurred in a small country town, where a horse persistently refused to draw even an empty buggy. The whip was applied without effect, and as the crowd that collected about the exasperated animal knew of no other way of accomplishing the object, it was continued by different members of the company, taking turns, when one became tired. The poor horse died from the effect of the brutal treatment in the course of twelve hours, but during none of the time that he was receiving it, did it have the effect of making him go. Some of the so-called "leading men" of that country town were among that crowd, and helped administer the blows, one or two members of Christian churches, yet none took measures to have this inhuman treatment stopped.

On the other liand, we have known the most vicious and obstinate balkers cured of this vice by a few kind words or pats, or perhaps a bit of apple or sugar given him to eat. Horses are very susceptible to kindness, and can be persuaded by this means, when the most severe punishment would only result in exasperating and exciting them.

Sometimes very slight causes will tend to make a horse balk; it may be produced by fright or by the collar not fitting well; by its pressing more upon some parts of the shoulder than others; sometimes from the coldness of the collar; or the load. may be too heavy, or the shoulder sore. It is most frequently caused, we believe, by improper treatment while the horse was being broken, and the tendency once acquired, it is not easily cured. Whatever may be the cause, there is one thing that should be remembered and observed in such cases, and that is, never whip a ballku horse. Robert Jennings, veterinary surgeon, says in his work entitled "The Horse and His Diseases:"

"This species of restiveness is one of the most provoking vices of the horse, and it can be successfully combatted only by a man of the most imperturbable temper. The slightest sign of vexation only increases the evil, and makes the animal more and more troublesone each time that he refuses his work. Many a thick-headed, quick-tempered driver flies into a passion and beats or otherwise abuses his horse on the least symptom of balking, until the animal becomes utterly worthless from a confirmation of the habit.

As a rule, it may be stated that horses balk from nervousness or unsteadiness of disposition; if not, indeed, from an over-anxiety to perform their work. Nervous well-bred horses are more susceptible to the influences which induce balking, than are cold.blooded, 
more indolent ones. A high-mettled horse, when carelessly driven, will start suddenly against his collar, fail to start his load, draw back from the pain which the concussion causes, rush at it again, and again draw back, until it becomes impossible for his driver to steady him in his collar for a dead pull. If to all this be added a smart cut with the whip, and it fiercely-spoken word-with, perhaps, a blow over the nose, or a stone in the ear-every fear or vicious feeling of the horse will be summoned into action, and the animal will become entirely unmanageable, requiring to be left for an hour or two in his position, before he gets sufficiently calm to be induced to move. There may occasionally be a horse which cannot be made to draw steadily by the most careful treatment, but the cases are exceedingly rare in which gentle treatment and firmness, a patient persistence in mild, authoritative command, and judicious coaxing, would not either prevent the formation of the habit, or cure it when formed.

To cure the habit of balking is not an easy matter, and it is possible only by the kindest treatment. If the horse shows fear by his excited manner, or, by looking abont him wildly, that he is expecting a blow, you may be sure that he has received hard usage under similar circumstances, and that he must be convinced by caresses and kind words that you will treat him gently. You must recollect that the horse cannot understand your language, and that while he is confused, he will misinterpret every sign which you may make to him. He must feel confidence in your kind intentions, whether it takes an hour or all day to convey it to him, before you can do anything to cure him of his trick.

If you have him harnessed to a light wagon on a smooth road, where it will afford but little resistance, you may, by repeated trials, convince him that it is a simple, easy matter to draw it; and you should continue to exercise him from day to day with the same light load, and afterward increase it gradually, until you have trained him to a quiet manner of starting, or of going up a hill, or elsewhere, where he has been accustomed to balk."

Another writer gives the following sensible advice: "Almost any team, when first balked, will start kindly if you let them stand five or ten minutes, as though there was nothing wrong, and then speak to them in a steady voice, and turn them a little to the right or left, so as to get them both in motion before they feel the pinch of the load. But if you want to start a team that you are not driving yourself, that has been whipped and otherwise mismanaged for some time, go to them and hang the lines on their hames, or fasten them to the wagon, so that they will be perfectly loose; make the driver and spectators (if there are any) stand off some distance to one side, so as not to attract the attention of the horses; loosen the check-lines, so that they can get their heads down, if they choose; let them stand a few minutes in this condition, until you see that they are a little composed. While they are standing, you should be about their heads gentling them; it will make them a bitle more kind. When you are ready to start, stand before them, and, as you seldom have but one balky horse in a team, get as near the front of him as you can, and, if he is too fast for the other horse, let his nose come against your breast: this will keep him steady, for he will go slow rather than run on you; turn them gently to the right, without letting them pull on the traces, as far as the tongue will let them go; stop them with a kind word, gentle them a little, and then turn them back to the left, by the same process. You will have them under your control by this time, and as you turn them again to the right, steady them in the collar. and you can take them where you please.

There is a quicker process that will start a balky horse, but not so sure. Stand him a little ahead, so that his shoulders will be against the collar, and then take up one of his fore feet in your hand, and let the driver start them, and when the weight comes against his shoulders he will try to step; then let him have his foot, and he will go right along. If you want to break a horse from balking, that has long been in that habit, you ought to set apart a half a day for that purpose. Put him by the side of some steady horse; have check-lines 
on them; tie up all the traces and straps, so that there will be nothing to excite them; do not rein them up, but let them have their heads loose. Walk them about together for some time as slowly and lazily as possible; stop often and go to your balky horse and gentle him. Do not have any whip about him, but keep him just as quiet as you can. He will soon learn to s.art off at the word, and stop whenever you tell him.

As soon as he performs right, hitch him in an empty wagon; have it stand in a favorable position for starting. It would be well to shorten the stay-chain behind the steady horse, so that, if it is necessary, he can take the weight of the wagon the first time you start them. Do not drive but a few rods at first; watch your balky horse closely, and if you see that he is getting excited, stop him before he stops with his own accord; caress him a little, and start again. As soon as they go well, drive them over a small hill a few times, and then over a large one, occasionally adding a little load. This process will make any horse true to pull.

When we remember that we are dealing with dumb brutes, and reflect how difficult it must be for them to understand our motions, signs, and language, we should never get out of patience with them, because they don't understand us, or wonder at their doing things wrong. With all our intellect, if we were placed in the horse's situation, it would be difficult for us to understand the driving of some foreigner, of foreign ways and foreign language. We should always recollect that our ways and language are unknown to the horse, and s.hould try to practice what we could understand, were we the horse, endeavoring by some simple means to work on his understanding rather than on the different parts of his body. All balked horses can be started true and steady in a few minutes' time; they are willing to pull as soon as they know how, and I never yet found a balked horse that I could not teach to start his load in fifteen, and often less than three minutes' time.

In the first place, never teach your horse to balk, by giving him a greater load than he can carry, or requiring him to go up too steep a hill without permitting him to stop. If you tell him to stop, in going up a steep hill, it is better than to allow him to do it of his own ascord. If he finds he can stop of his own will, and start when he pleases, he will soon learn t) do it when he ought not to. If at any time he stops without your stopping him, give him a sharp cut, and make him go on, even if you think he ought to stop at that very place; but soon yourself give him an opportunity to stop. This will teach him that he is to stop only at your will, and that you are not unreasonable in your demands."

Use of the Check-Rein. - It is very true, as a certain author has said, that " the horse has to work very hard for his living, and he has a right to be so harnessed as to do his work in the way most easy to himself." If, in drawing a heavy load, a horse has his head held back in an unnatural position by a check-rein, he cannot throw his weight into the collar, and give his body that position that will enable him to use his strength most advantageously. If any one doubts this, let him attempt to draw a heavy load himself, with his head held back by a stout strap attached to a belt about his body in such a manner that he cannot bend forward, but must of necessity maintain an upright position. In England, Ireland, and Scotland it is a very rare thing to see a check-rein used upon a draft horse, while in the United States it is quite too rare to see a horse without one.

The tight check-rein is not only painful to the horse, but no good results to the driver from its nse, except in case of a horse being vicious and unmanageable; he may then be more easily controlled. A horse with his head drawn back in an unnatural position by the check-rein not only has his power to draw lessened, but cannot as well see where to step, and is more liable to stumble, while if he does stumble he cannot as readily recover himself. A tight check-rein has also a tendency to produce disease, injures a horse's mouth, and frets and worries hin. The effects of its use are described as follows by John A. McBride, Professor of Veterinary Medicine and Surgery in the Royal Agricultural College: "I will briefly consider the effects of the check-rein, - (1) upon the circulation of the blood; (2) upon the veins; (3) upon the arteries. 
1. Its effects upon the circulation of the blood.-It will appear very evident that this contrivance must seriously interfere with the return of blood from the brain, and thus lead to an accumulation of venous blood, producing eventually a state of coma, the severity of which will depend upon the duration and intensity of the compression; and, further, it must impede the flow of arterial blood to the brain. This deficiency of arterial blood is characterized by a want of nervous sensation, and a disarrangement of the nervous parts.

2. Its effects upon the veins.-The sudden, intermittent, and violent muscular action of the neck, together with pressure of the lower jaw, would induce more or less permanent distension of the veins, which in all probability would ultimately become varicose.

3. Its effects upon the arteries. - The pressure of the blood within the vessels, and the un. natural muscular contraction of the neck, would lead to laceration of their inner coats, resulting in enlargement of the vessels, and thus form aneurisms.

The consequences of the above conditions would lead to increased pressure upon the nerves of the neck, causing pain to the animal, and, further, it would predispose the unfortunate victim of fashion to such diseases as megrims, apoplexy, coma, inflammation, and softening of the brain."

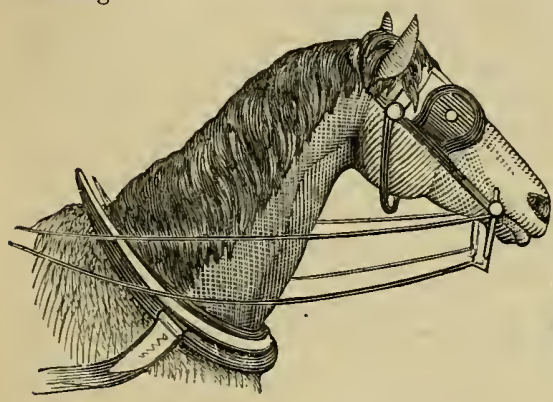

NATURAL POSITION.

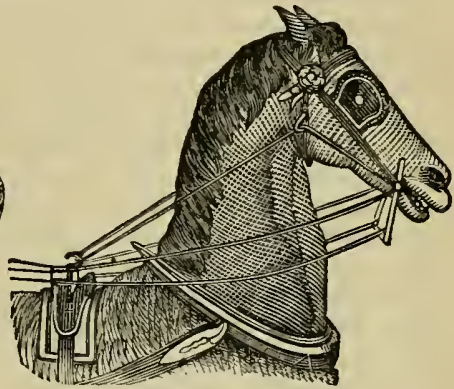

UNNATURAL POSITION.

Youatt says: "Roaring is an unnatural, loud, grunting sound, made by the animal in the act of breathing, when in quick action or on any sudden exertion; it is manifest unsoundness, as impairing the function of breathing. Among other causes of it, is that which the folly, as well as cruelty, of man has introduced - the system of tight reining."

The leading veterinary surgeons of Europe and America denounce its use, both on humane and sanitary principles, and its continued use seems to be simply to conform to the caprice of fashion, regardless of the consequences.

The overhead check is fully as objectionable in all respects as any other, while it produces a very awkward carriage in a horse, since the neck and head are kept drawn back unnaturally high, while to relieve the neck the horse throws his nose out, giving him a gait about as graceful as that of a camel.

Whenever the check-rein is used at all on the horse, it should be made so lon that he can drop his head when going up hill, as far as he would without one. It is to be hoped that farmers and horse owners generally will give this subject more attention, and look upon it in its true light. The logic of the old Scotch stage-driver on this point could not well be controverted, when he said, "WVe dinna use 'em. You winna get half their power with 'em crimped up so."

Frosty Bits. - Never put a frosty bit in a horse's mouth. If iron bits are used in severe weather, always warm them by putting them in water, holding them in the hands a 
moment, or by some other means, rather than cause the animal so much suffering as would be incurred by inserting a frosty bit, which will be liable to result in not only pain at the time, but a sore tongue and mouth which will be constantly irritated, sometimes rendering it difficult to eat the food given them, causing loss of appetite and consequent loss of strerigth.

The use of the India-rubber as well as the leather bit is to be recommended during the cold season, since they obviate the evil effects of iron or steel in frosty weather.

Blinders. - These are not only a nseless but an objectionable appendage to a bridle, and are without doubt in many cases the cause of diseased eyes and blindness. Besides, a horse is more apt to take fright when he can get only a partial view of an object, than if he could obtain a full view of it. Sudden or loud starting sounds, like those from a locomotive, would be much more bikely to frighten a horse when his eyes are so blinded that he cannot tell from whence the sounds proceed. A nervous, timid horse would naturally think it something terrible in pursuit of him, and run to get away from it, and a serious accident might be the result, while if he could see the object, this might be avoided. A recent writer has well said of blinders:-

"We know not who invented this instrument of horse torture, but we know he did not understand the anatomy and physiology of the eye of a horse. Human vision is binocular that is, we see the same object with both our eyes, and so adjust the axis of vision that the object appears single, though seen with two eyes. But the eyes of the horse are placed on the sides of the head, and the axis of each eye is nearly at angles with the longitudinal line of the body, so that it is impossible that the same object can be distinctly seen by both eyes. Now, by blinding the eye in the direction in which it was intended, in its construction, that it should see, it is forced to use an oblique vision, as if we should cover the front of our optics and be compelled to see only by the corners of our eyes. This unnatural and con. strained use of the eye must, to a greater or less extent, impair vision, if not entirely destroy it. The object for which the blind-bridle is used is not accomplished by it. A horse is more readily frightened when he cannot see the object of his dread, than if he can have a fair view of it. Nineteen out of every twenty horses you see in harness have blind-bridles on, and if you ask the owner to explain its benefits, or why he uses it, he will be vtterly unable to give a rational answer."

Clipping Horses. - The advocates of this practice claim it to be a means of promoting the general health of the animal, and a prevention of colds and lung fever, also that it increases, at the same time, his activity and appetite. The main advantage claimed for it is, that when a horse with a heavy coat has been driven in cold weather sufficient to canse his hair to become wet with perspiration, and as soon as he halts is liable to take cold, even when immediately blanketed, since the hair remains wet so long; that clipping and keeping the animal blanketed, except while being driven, will obviate the evil.

The opponents of clipping, on the other hand, assert that it is not only cruel, being the cause of much unnecessary suffering from insufficient protection against the cold, but that it is equally injurious to the health, frequently producing disease and death. There may be instances, and doubtless are, where horses may receive such careful attention that clipping will do injury, and by this means they may be enabled to perform more labor and with greater dispatch than when unclipped; but we are of the opinion that Nature's method is best, and when she gives him a fine, short summer coat that lies smoothly upon his skin, and a heavier and coarser covering for winter, she knows better than man what is best for the animal.

Besides, very few horses when clipped are sufficiently well protected to be furnished with a fair substitute for the coat that has been removed, the hood and blanket only covering the head and upper portion of the body, while the under portions and limbs are entirely exposed. 
But few clipped horses are provided with a protection for the head, the only covering com. monly used being a blanket, so that, even under the most favoring conditions, clipped horses must of necessity suffer more or less from exposure, while, with the common treatment received from careless and indifferent drivers, much injury must result from clipping. It is true that a horse will be more active, and have a more voracious appetite after being clipped than before, but whether this is a healthful indication may be doubted. All animals that are not sufficiently protected from the cold will require a greater amount of food to be kept in good condition, than those that are kept comfortably warm. This is a fact with which every one is familiar.

Now, if by removing the coat we increase the demand for food, thus unduly stimulating the digestive organs, and taxing them to furnish a supply of fat and heat sufficient to compensate for the loss of the covering, the question naturally arises, Will not the extra labor required of the organs of digestion have a tendency to produce disease? And can it be truly a promoter of health?

Again: since clipping has a tendency to increase the activity of a horse, the keen air exciting him and greatly increasing his ambition to go, there is great liability of clipped horses being driven beyond their capacity for endurance, while at the time, there may be no indications of it; and we believe that clipped horses, as a rule, are generally over driven, to say nothing of the results from exposure to colds and other evils from lack of care afterward. That clipped horses really suffer from the cold, need not be questioned by any one who has ever been at all observing in noticing the shivering of the animals on being exposed to the cold atmosphere, after their blankets are removed.

It is the opinion of some of the most intelligent veterinary surgeons, that many diseases are directly traceable to clipping, among which are those resulting from exposure to the cold, the shock occasioned by severe changes in the weather, and over-exertion. The evil effects of clipping may not always be perceived at once, some being gradual in their development. Several cases of string-halt have come to the knowledge of the writer, which were indisputably the result of clipping. Clipped horses are, of course, much more easily taken care of than those unclipped, since they require less grooming to be kept clean; for this reason indolent grooms are very apt to favor the practice, whatever the result to the horse. It is stated by high authority, that inflammation of the lungs has become a prevalent disease among horses since the custom of blanketing them constantly in the stable was introduced. There is no doubt that this practice causes greater sensitiveness to the skin, and increased liability to take cold, than when the blanket is only used as a covering after profuse sweating, or when the animal is standing exposed to the weather.

Where a well-bred horse is put in the stable before cold weather commences, and is well groomed and kept blanketed except when being used, his coat will be about as short as in summer, or at least short enough for appearance, and to admit of drying off readily when sweaty. The coat of a horse that has been clipped does not come out as evenly in the spring as one that is unclipped, and rarely looks quite as well afterward. Never trim the hair at the heels much, if at all. The hair inside the ears should never be cut, nor the long hairs or feelers about the eyes and nostrils; they were designed by nature to be of great utility to the animal.

Stables, and Stable Management. - The stables for horses, and, in fact, for all animals, should be in a healthy location, free from dampness, and with plenty of pure air. They should also be on the sunny side of a building, if practicable, for the sunlight has a healthful and stimulating effect.

The stalls should be roomy, with partitions sufficiently long and high to prevent the horses from kicking, or biting each other. Some recommend having the partition so high that they cannot see each other, but we do not favor this, as horses are social animals, and 
enjoy the company of others. Box-stalls, where the horse can be free from the halter, and also exercise a little, are greatly to be preferred. Horses that are worked hard every day would, we believe, last much longer if they could, when out of harness, have the entire freedom of large box-stalls. The stables should be kept clean, and well ventilated. Horses possess a very acute sense of smell, and greatly dislike offensive odors. Besides, the breathing of air freighted with the odors of excrement, both liquid and solid, and carbonic-acid gas generated from the lungs of the animals in breathing, is very injurious to the system. It poisons the blood, and is the cause of many diseases.

The ammoniacal vapors of ill-kept stables produce inflammation of the eyes, and irritate the throat and lungs, so that blindness, cough, or asthma may be the consequence. Glanders is more frequently caused by breathing the impure air of stables than by contagion. The drainage of stables should receive particular attention, and the arrangement for the manure should be such, whether in pits or otherwise, that the foul odors from it cannot affect the air of the stable. The floor of a stall should be as nearly level as practicable and admit of drainage, for if it slopes much there will be a liability of lameness by straining the ligaments and membranes. The slope should not exceed two inches.

The litter under the horses' feet should be kept dry and clean. Standing on hot, fermenting manure will cause the hoofs to become soft, and will produce lameness. A sufficient amount of light in the stable is indispensable to the health of a horse. No animal can thrive for a long time in a dark stable. Horses that are kept in dark stables will also be more liable to shy when brought out suddenly into the light; but this is of minor importance compared with the injury caused the eye from having this sudden change from darkness to bright sunlight. The sight by this means becomes in time impaired, which frequently results in total blindness.

While dark stables are to be avoided, the opposite extreme of a glare of light is equally objectionable, as far as the effect upon the eye of the horse is concerned. The stables should be so arranged that the light from the windows will not shine into the faces of the animals, or fall upon the eyes from the front, but rather from the rear or side. A strong reflected light should also be avoided. It is well to have the interior of the stables colored with a neutral tint to the distance of seven or eight feet from the floor, to avoid the glare that would be occasioned by a white wall throughout.

We have previously said so much respecting the necessity of pure air for mankind and animals, that a repetition here is unnecessary. All stables should have ventilators so constructed that the foul air can escape, and the pure air find an unobstructed entrance. Great care should be taken that in securing good ventilation the animals are not exposed to a draught of air. When the stables are not occupied, the doors and windows should be left open, if the weather will admit. The stables should be kept as comfortable for the horses as possible, both winter and summer.

As a glossy coat is supposed to be produced by a very warm stable, many grooms sacrifice ventilation to this idea, and not only keep the stable too warm, but poorly ventilated. In a warm, and properly ventilated stable, we doubt whether blanketing is desirable, but in cold stables, where horses will not be sufficiently warm without this protection, it may be found necessary. Where blankets are used as a covering for horses in stables, a moderate temperature and moderate amount of clothing will be better than a low temperature and an undue amount, or a high temperature and slight clotling. About $55^{\circ} \mathrm{Fahr}$. is estimated to be a good mean, or desirable temperature for stables.

The feet and legs of the horse should receive good care, as these are the first to fail, and are subject to the greatest number of diseases. After severe or protracted exertion, a horse should be rubbed down dry. The legs should be well rubbed with the hand, which will tend to prevent swelled legs, stiff joints, contracted tendons, etc. When the legs are of an 
unnatural heat from over-driving, bandaging with wet cloths will reduce the feverish temperature, and prevent wind-galls.

Horses that are not used for work should have some exercise every day. Always use the curry-comb lightly, for the skin of the horse is very sensitive, and rough usage in this respect will cause him much nervous irritation and pain. When tied in the stall, the halter should be sufficiently long to admit of the horse lying down in a comfortable position, yet at the same time care should be used that it be not so long as to permit him to get his foot over it, and thus be thrown down and get cast. Serious results sometimes follow carelessness in this respect.

Feeding.-Improper feeding is the prolific cause of disease in horses, especially to those used upon the farm, and for draught. Horses should always be fed regularly, and in such quantities as will keep them in good condition. Horses that work hard will require nearly twice the amount of food that an idle horse does, other conditions being equal, while, of course, a large horse will need a much larger amount than a small one. The amount of food required will therefore depend upon the size of the animal, and the amount and kind of work performed by him. Enough food should be given under any circumstances to supply the waste of the system.

Due regard should also be had to age and constitution, as well as to size and the amount of labor performed. It is estimated that horses that work require about two per cent. of their weight as a daily allowance of food. From fourteen to sixteen pounds of grain, and the same of good hay, is regarded as a generous allowance for a hard-working horse of large size. The American cavalry horses are allowed about fourteen quarts of oats, and an equal amount of hay, and are fed three times a day. The English cavalry horses have a little less, which is an allowance of ten quarts of oats and twelve pounds of hay, three times a day.

It is very desirable to have a horse a "good feeder," but some horses are gluttons, and in such cases it is not well to give them all the food they will crowd down. A light feeder is apt to be a tender animal, and lack endurance.

Grain of some kind should always be given horses that work hard. Hay or grass alone is not sufficient to sustain a horse that labors hard, because there is not sufficient nutriment in either to supply the waste, while a horse that has his stomach distended with such coarse, bulky material as hay alone, is in no condition to work, providing it met the demand of the system. When worked hard, the food should be chiefly oats, with some hay; when not working, less grain and more hay may be fed, or the feed may be chiefly hay, with a moderate quantity of oats or other grain. Oats seem to be the natural food of horses, and are to be preferred to any other kind of grain. They supply more nourishment and flesh making material than any other kind of food. Barley is also good. Corn is rather heating, and should never be fed alone. It is best when ground, and given in connection with other food. New corn should never be fed to horses in large quantities, as it affects them very injuriously; many valuable animals have been lost by eating soft corn. New oats and new hay should not be fed in connection, and never in large quantities, but should be mixed in part with that which is old.

Roots are excellent for horses, when being fed on dry food. Carrots are the most valuable roots for this purpose, and many horsemen feed regularly with them in considerable quan. tities, they taking the place, in part, of the grain rations.

A ration of one-half carrots and one-half oats is thought to be equivalent to a full ration of oats, while it has been found that horses that perform but little work will keep in good condition on hay and carrots alone. Raw potatoes given occasionally, as well as apples, are also very beneficial. The former are especially valuable for horses troubled with worms. The English feed beans in connection with oats and hay to hunters, and horses that work hard. Beans are heating, and should be given only in small quantities, and never regularly. 
Bran and linseed are used also for special purposes, - sometimes as a substitute for regular feed, and sometimes in addition to it.

Poor hay or damaged corn should never be given to horses. The latter will bring on inflammation of the bowels and skin diseases. Cut hay is much better than that which is not cut. Hay sprinkled with water in which a little salt has been dissolved is much relished by horses. Cut hay moistened with warm water makes a good feed for them. When meal or bran is fed, it is a good plan to mix it with moistened, cut hay. Oats moistened with water are preferred to dry, being more easily masticated and digested. A warm mash is good for horses, especially after a hard day's work, which is made by turning boiling water on bran and stirring it until it is well moistened.

A horse should not be driven hard or put to hard work for an hour or more after eat. ing. The custom of most farmers of taking a horse immediately from his feed to hard work is very injurious. Violent exercise in man or beast should never be taken until digestion has, at least, been partially accomplished. A working horse should also have an hour's rest at noon. A horse designed for rapid work should not be allowed much hay at morning or noon, but grain principally. At night he may be given a larger quantity of hay in connection with grain.

The evening before a long journey, an extra amount of food should be given. Time will thus be allowed for its digestion and extra nourishment will be obtained for the extra labor to be performed.

While on the road, it is always well to feed in small quantities and often, rather than to overload the animal's stomach after a long fast, attended with great exertion and weariness, which would be liable to produce' staggers, or apoplexy. When a horse refuses food and water, he is in no condition to be driven. Never feed a horse much when he is heated and exhausted. Give him a little wet hay, and a few sips of water, and after he is rubbed down and cooled off, he may have his usual drink and rations.

Watering.-Horses that are allowed free access to water will drink little at a time and often, and where stalls are so arranged that a constant supply of pure water can be had at any time, we believe such horses will be kept in much better condition than those that are watered at long intervals, and frequently stinted in the supply, or drink too large a quantity, from the thirst produced by long abstinence. Where horses are stinted in water, a feverish condition of the system will be liable to be the result, together with a loss of condition.

Where horses do not have free access to water, they should be watered four times a day. The old idea of stinting horses in water is not only unreasonable and injurious, but cruel; being fed upon dry hay and grain, they require drink often, and in sufficient quantities to quench thirst. The plan of permitting horses to have access to water at all hours in the stall is meeting with much favor at present, and experience thus far proves that it is highly beneficial, and to be recommended, where practicable.

Spring or well water is not generally as good for horses as that from ponds and streams, as it is generally hard and cold, they much preferring soft water, and that which is slightly warm. It is not a good plan to allow a horse to become accustomed to drinking very warm water, for if obliged to drink cold water at any time it will be liable to cause colic. Never allow a horse to drink freely when heated. Permit him to take a few sips only to cool his mouth, rub him off until he is dry, first with a wisp of straw, and then with a brush, rubbing his legs also well with the hand. When cool, give him his drink first, and then his food. When on the road, always let him drink whenever he likes.

The water given horses should always be of the best quality. Horses are peculiarly sensitjve respecting the water they drink, and are easily made sick by impurities. The pail, tank, or other receptacle for the water should always be kept very clean; in fact, the water given horses should be as clean as that used by the household for drinking purposes. They should never be driven hard immediately after eating or drinking freely. 


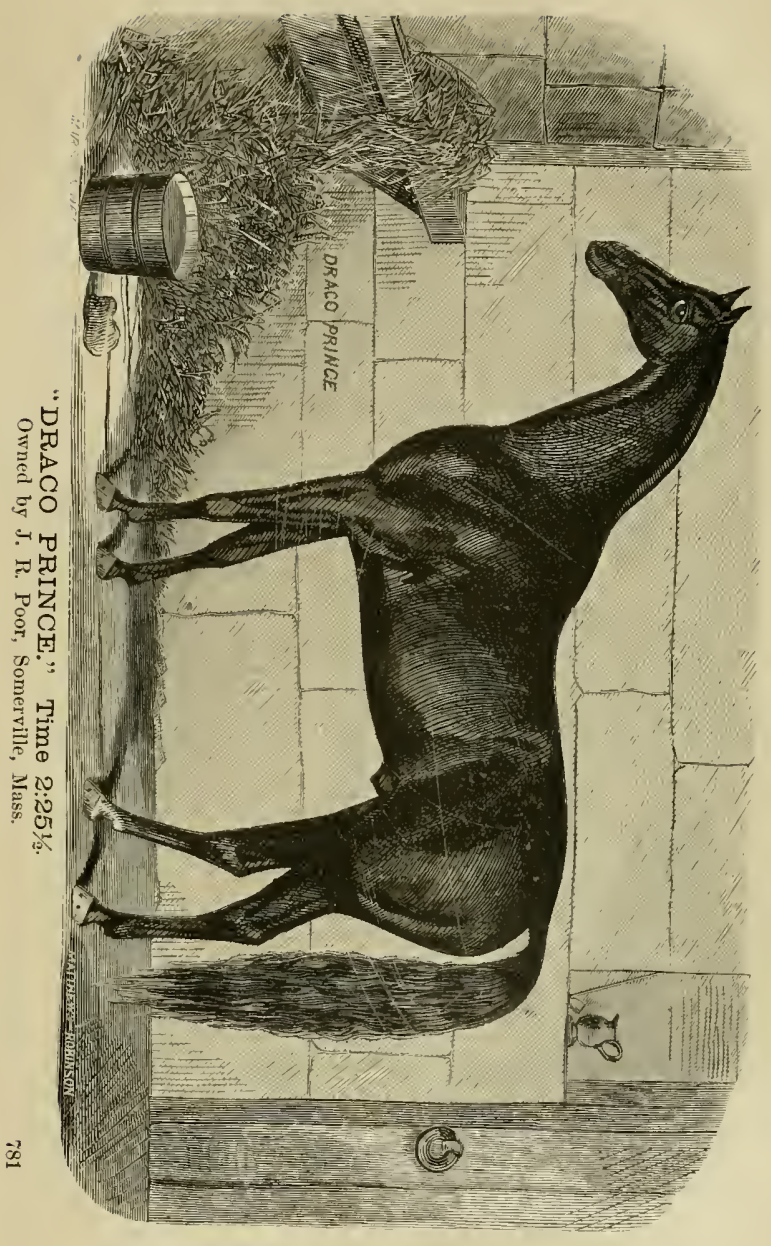



Grooming.- While horses that are constantly used on the farm or in draft and exposed to the weather do not require very much grooming, carriage-horses and those that are stabled a portion of the time require considerable care in this respect. The Agriculturist contains the following sensible hints on this subject, by T. L. Nevill, which so fully coincide with our own views that we give them in his own language:-

"Good grooming is essential for preserving a horse's health and appearance. A fine coat and tender skin should not be touched with the curry-comb, but be brushed very gently. If there are any urine or dung stains, let them be washed, using a sponge deftly, so as not to extend the wet dirty place. By making this the first operation the wet spots have time to dry, while the other parts of the work are being done.

If a curry-comb must be used, have the smoothest one that can be found, and use it but sparingly. In the hands of some men the curry-comb is a barbarous instrument of torture. Applied with a long, sweeping motion, without regard to the shape of the body, or the evenness with which it is held, it will make a poor animal shrink and shiver. The skin is often seriously hurt by the angles of the comb, when carelessly and heavily handled. A short motion, back and forth, does the work more effectively and humanely than the severe application of the comb described. The horse will learn not to dislike it, if he finds he is not hurt.

In cleaning a horse, commence at the head with the brush, having it in one hand, and the curry-comb in the other. Brush every part of the body and limbs thoroughly, laying the hairs in their natural direction, and going through them to the skin. Remove dust from the brush by occasionally scraping it on the curry-comb. The brushing is to be followed by a good rubbing with wisps, and the coat finally laid by the application of a cloth. A thorough cleaning of the head cannot be effected without removing the halter, and be careful not to hurt the eyes, or other sensitive parts.

The mane and tail have now to be combed. If the hairs are knotted, go through them, taking a small lock at a time, straightening and separating the whole. When once properly done, there will be but little trouble afterwards. Sometimes a very thick mane has to be turned over the neck to get at the underside. No two hairs are to be left sticking together. Some short ones may stick up obstinately on the wrong side. If so, damp them with a sponge and try to bring them into place with the brush. If very rebellious, they must be plaited, and a small bit of lead twisted in the ends to bring them right.

Sometimes a horse with very abundant mane will come up from grass with it hanging on both sides of the neck. Do not allow any knowing groom to cut it off from one side. If this is done it will soon present a bristly, unsightly appearance, that will take months of growth to cure. A hittle patience, careful combing, wetting, brushing, and perhaps plaiting and leading of these thick manes, will soon bring them into place, and make a wonderful improvement in the horse's appearance. A portion of the mean on the poll should be cut away to allow the bridle to sit snugly, and to prevent the formation of a bristly tuft."

Hints on Driving, and Draft.--Never use a horse for work of any kind when sick or lame. All horses should have some exercise every day, since exercise promotes a healthy circulation of the blood, and imparts vigor to the whole animal system. In driving, do not keep up a constant talking to the horse; he will soon learn to pay no attention to what you say. Quiet drivers are always the most successful ones. Never trust your horse entirely to himself, but keep your hand steady on the reins, just sufficient to feel his mouth with the bit. This will ouviate accidents from stumbling. By being thus on your guard you can best prevent shying or the accidents that may be caused by it or sudden fright. By this means you keep the animal's attention without irritating him.

There is a vast and important difference between a tight check-rein and a moderately. tightened rein, although not generally understood. The first is injurious in many respects, and may make a horse stumble from not being able to see where to step, and also prevents 
him from recovering himself well when he does stumble. But the latter is a steady support to the head given by the hand of the driver. Avoid a constant hard pull on the bit, for it hardens the horse's mouth and pains and irritates him.

Never pull up suddenly and sharply unless it is necessary, because this is not only painful to the horse, but tries his chest, neck, and fore-legs. Do not acquire the foolish habit of con. tinually jerking on the reins, or using the whip. The first injures and hardens the horse's mouth; besides, he will not know what is really meant when both reins and whip are used in earnest. Be careful to see that the horse's collar fits well and causes him no irritation. Illfitting collars or those that are too tight are the cause of much injury to horses. The following, from the "London Horse Book," contains much truth and may serve to call the attention of those who have been indifferent and careless in this respect:-

"A horse-collar is frequently looked upon as merely a ring for the neck, to which the traces are to be affixed; whereas, there is no part of the harnesis which is so important and which ought to fit so accurately. How often is a little collar only fit for a pony jammed on the neck of a much larger animal, so that every pull he makes must give the feeling of strangulation, and that will in all probability cause some kind of fit if long continued, besides its liability to gall and wring the poor animal's shoulders. When this has taken place, the work cannot be fairly performed; and to do it at all, the anguish of the poor horse must be indescribable."

A badly-fitting collar will also frequently cause horses to balk that are otherwise perfectly docile and easily managed.

In using the saddle, the same care should be exercised to see that it is sufficiently large and bears evenly on the horse's back. A saddle that is too small or does not fit well will chafe and gall the horse most painfully.

The wheels of the vehicle should be kept well greased. This not only saves much of the wear occasioned by constant friction, but renders the draft much easier. It is estimated that well-oiled wheels save one-half the work of draft. In taking a long journey drive slowly at the beginning and near the end, increasing the rate of speed between the two extremes; this permits him to get warm and cool by degrees, which is always the best method. Never stop long in cold, wet, or windy weather, and never in any weather when the horse is warm and sweaty without putting a blanket over him as a protection against taking cold.

When stopping on going up a hill, always block the wheel with a stone, otherwise the whole weight of the wagon and load are drawing on the collar, straining his limbs and back and preventing him from breathing well. Always let a horse have water often when being driven, and in moderate quantities. Never allow him to suffer thirst. Never throw water on his limbs when he is warm. Never overload an animal at any time. This is not only cruel and unjust to the horse, but equally unprofitable to yourself. Always so harness the horse and arrange his load that he may be able to use his strength to the best advantage. Give him the free use of his head, discarding check-reins and blinders, and see that his collar and harness fit well, are properly adjusted, and will neither cramp, chafe, nor irritate him.

There should always be a suitable proportion between the horse and the rehicle. The shafts should be neither too high nor too low, but nearly on a level in draught, though sloping slightly upwards. The horse draws in a level line, and the shafts and traces should therefore be nearly level.

Horses should not be long used without being fed. Nose.bags are very convenient to put the feed in during the intervals of rest when out in the field at work. There should be leather or other durable material at the bottom, in order to retain the food well, while above they should be of open texture or porous, to permit the animal to breathe freely. Something should be given the horse to rest it upon in order to eat at the bottom, otherwise he will be 
obliged to keep tossing it up for nearly every mouthful, which gets the dust, etc., into his eyes and nostrils.

The feet of horses should be examined every night after work, to ascertain whether any stone may have worked its way between the shoe and hoof. If so, it should be removed at once, as lameness will be produced, if it is allowed to remain there over night. In all cases in the care of animals, it is well to remember that they are susceptible to pain, weariness, hunger, and thirst, as well as mankind, and to consider how we should like to be treated were we in their place.

\section{HORSE-SHOEING.}

$\mathrm{T}$ HE foot of the horse is inclosed in a horny case called the hoof, which corresponds to the nail or claw of other mammals. The fore part of the hoof is the thickest, it being about half an inch in thickness, and gradually growing thinner towards the back. Near the heel it curves inward, forming what are called the bars. These are a prolongation of the wall of the hoof, and their object is evidently to protect the frog, and strengthen the foot generally.

In the natural state of the foot, the bars are quite prominent; but in horses that have

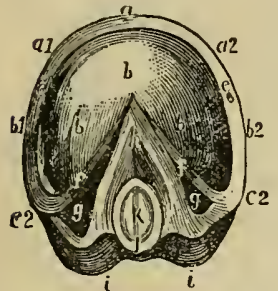

FIG. 1. - HORSE'S FOOT.

The above cut shows the cround surface of the boof prepared for receiving a shoe; and marks very distinctly the difference between the curvature of the outer and inner quarters. $-a$, the toe-rasped away to receive the turned-up shoe; $a 1$, the inner toe; $a 2$, the onster toe: $b 1$, the inner quarter $b 2$, the outer quarter; $c 1$, the
inner heel : $c 2$, the outer heel; $d$ $d d$, the sole; $e e$, the crust or wall of the hoof ; $f f$, the bars; $g g$, the commissures; $\dot{h} k l$, the frog; $h$, the part immediately under the navicular joint; $k$, the oval clee of the frog: $l$, the elevated bound the heels.

The sole is that part of the foot which has a nearly plane or slightly concave surface, and extends from the frog to the wall of the hoof. It is horny and hard, yet, at the same time, somewhat elastic. The outer or non-sensitive portion of the sole protects the inner portion from injury, as it comes in contact with the ground, and also aids the wall in supporting the weight of the horse. The sensitive portion of the sole, lying next to the outer or non-sensitive, aids the horse in traveling, through the sense of touch, so that he can place his foot on the ground in such a way as to favor it. It also furnishes the outer sole with material for its growth or replacement, when worn away. 
The internal structure of the hoof is illustrated on a previous page in this department, in connection with THE PASTERss (which see). At birth, the foot of the colt is in form more like a cloven than a solid foot, the sides being folded or rolled together under the sole. The hoof is soft and fibrous, but soon hardens, and grows rapidly. It, however, wears off quite evenly, as to general appearance, but more at the toe than elsewhere, especially on gravelly soils or stony pastures, giving the foot of a year-old colt a proportionately large frog with prominent bars, as seen in Fig. 2.

Col. M. C. Weld says, in the columns of the American Agriculturist :

"Time makes few changes in the general form of colt's feet up to the age of four, when they begin to be handled by farriers. I suppose, if my pastures had not been so stony, I would have been obliged to rasp off the feet now and then to keep them regular, but as it was, I saw no occasion to do so. One thing I have noticed which has inter. ested me much. It is this: as the weight of the animal increased, the quarters showed a weakness, and notches of greater or less extent were worn at each side of the hoof at the 'quarters,' just in front of the

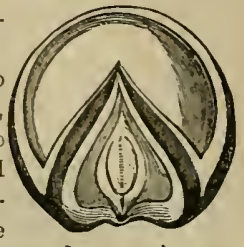

IG. 2. - COLT'S FOOT, AT TWO YEARS. bars. So marked was this in some cases, that the foot lost its round look altogether, and appeared much like Fig. 3 when seen turned up.

It seems that Nature gives great prominence to the bars. It is they which form the inside buttresses to the walls of the foot; and against them the horn grows so tough and strong, that natural calks, as it were, are formed. In our shoeing of horses we disregard some of the plainest and broadest hints given by Nature, for, instead of setting the heel-calks where they are set by Nature, we extend the shoe out behind the heel, and place the calks back of the foot.

The naked foot at the bottom is flattish, somewhat saucer-shaped, rounding up at the edge in front, and much rounded up at the heels, so that when set down in trotting, the heels strike first, and the foot rolls forward upon the flat foot; and when picked up rolls forward upon the Flo. 3, SHowTro QCARtoe. When walking the foot seems to be placed flat upon the ground,

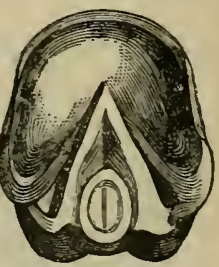

TERS. but the rolling motion is observed when it is picked np. How distressing, then, it must be to the borse to have all this natural roll done away with by the sharp-edged or calked shoes.

It is clear to me that the formation caused by the bars, and the toughness of the horn where the bars are attached, give horses sure footing upon slippery and rocky places. The frog gives surer footing upon the ice, as it is of so soft a nature that it clings to the smooth surface - just as a person slips less upon the ice if he has on india-rubbers."

The hoof is somewhat elastic, and when the weight of the horse is thrown upon it, the softer cushion, or frog, presses firmly upon the earth; consequently, it aids very materially to prevent slipping on the ice, besides giving support to the foot, when it is not cut away in shoeing. Wild horses seem to have hoofs sufficiently strong to support the body without artificial protection; but when used in constant service, on hard roads and stony parements, it has been the custom in all civilized countries to furnish a protection to the foot, and after many experiments, iron was found most serviceable for this purpose. Horse-shoeing was introduced into England by William the Conqueror, and has since that time been generally regarded as a necessity; but within a few years public attention has been directed, to a certain extent, by writers in both Europe and America, to the importance of doing away entirely with shoeing, and permitting the foot to remain as nature formed it, unmutilated and unshod, whatever the service, or the character of the foundation upon which it may rest. A medium between the two extremes of shoeing and bare feet, is the use of "tips," which 
serve as a protection only to the forward part of the hoof, which is most subject to wear, while the other portion remains in its natural state.

There is no doubt that many of the diseases of the feet, and much of the lameness in this locality, is due principally to improper shoeing, and the cutting away of the bars, frog, and sole,-those portions designed by the Creator as a protection and support to the foot of that truly majestic and wonderful animal, - the horse.

If horses are to be shod, it is of the highest importance that the shoeing be performed in such a manner that this process shall prove a benefit, rather than a detriment to them, and the shoe serve as an added protection to that already given by nature, instead of cutting away and mutilating the natural supports, and substituting a poor one in their place. There is no department of horse management that requires so much discretion, and the exercise of such good, sound judgment as shoeing, and yet there is, at the same time, no part of horse management that is, as a general practice, so badly performed, and in which there is so little skill and good sense displayed as in shoeing; ignorance and obstinate stupidity being the rule with those that perform this business, rather than the exception.

We are glad to know that there are exceptions to be met occasionally. Shoeing, when necessary, may justly be regarded as a necessary evil, and too much pains cannot be taken by the blacksmith in preventing the mary ills resulting from faulty work of this kind.

When an unshod hoof that has never been tampered with rests upon an even, hard, unyielding surface, the portions that maintain the weight are the edge of the crust all around, and the frog. Where the surface is uneven, the sole, being slightly concave, sustains a portion or the whole of the weight, according to the degree of unevenness of the surface, caused by stones, frozen clods, etc.

On ordinarily level roads, the hoof wears quite evenly in traveling, but where the sur- face is uneven or gravelly, the toe will wear away faster than the other portions. "Tips," or the "half-moon shoe," made thin and nicely fitted, will protect this portion of the hoof in such cases, and permit the frog to rest upon the ground, as it always should.

A shoe, of whatever style, should always be so fitted that it will permit the frog to rest upon the ground, and the frog and bars should never be cut; simply let them alone, as nature formed them, and never permit a stupid blacksmith to cut and mutilate your horses' feet after the barbarous fashion so commonly seen. The sole should not be cut, and the crust or wall of the hoof should only be cut sufficient to make the shoe fit well, and to reduce it to the form and length to which it would naturally wear away if unshod, as the hoof will continue to grow when protected by the shoc, and will require slight paring on this account.

The wall of the hoof should not be made thin and weak by rasping. The shoe should never extend outside the crust of the hoof; if it does, there will be danger of interfering or cutting. If the calks on the toe of the fore feet are too large, or the hoofs too long, there will be a liability of the horse throwing his hind feet against the heels of the sple of the fore feet in trotting, since, when thus shod, the fore feet will not naturally be placed as far forward, within an inch or two, as they would otherwise be, and over-reaching will generally be found to be caused by such improper shoeing, and remedied by correcting this evil.

It would be a very rare thing indeed to find a horse accustomed to go unshod, to interfere or over-reach. Fleming, one of the best writers on this subject, recommends the follow. ing pattern of shoe:

"If the sole of the hoof has not been mutilated by the knife, it does not require to be covered by the shoe, as Nature has furnished an infinitely better protection. Wide surface shoes can, therefore, be at once dispensed with; and a narrow shoe-made of the very best and toughest iron, adapted for traveling on slippery roads, and for aiding foot and limb, and sufficient to withstand wear for four or five weeks-is all that is required. We will therefore conclude that the upper or foot surface should be the whole width of the shoe, and 
plane,-not beveled, - for we have seen that the sole was destined, particularly at its junction with the wall in front, to sustain weight. We also know that it is advantageous to the whole foot and limb to allow the sole as wide and general a bearing as possible, so that one part may relieve the other; the sole coming to the aid of the wall, and the frog interposing to share the fatigue imposed upon both, as well as to relieve the strain on the hinder parts of the foot, flexor-tendons, and limb, and keep a firm grasp of the ground by its elastic and adhesive properties.

The shoe applied to the foot, then, should have its hoof-surface fiat, in order that it may sustain the wall and as much of this strong portion of the sole as its width permits. This is contrary to the usual practice, which only allows the wall to rest on a narrow surface, and bevels off the remainder of the shoe to prevent contact with the sole. Many years' experi. ence of this plain foot-surfaced shoe in various regions of the globe, and on feet of every kind and quality, has proved the soundness of this view. The foot is brought as near to a state of nature, when the greater part of its plantar surface supports the weight of the body, as man can hope to achieve while submitting the horse to an artificial existence.

A light, thin shoe is always preferable to a heavy, thick one; as the narrowness of the metal insures a good foothold,-in this respect imitating the wall,-while its thinness brings the sole, frog, and bars in closer approximation to the ground."

Miles recommends that the frog, except in very rare instances, should never be cut or pared. He says: "The first stroke of the knife removes this thin, horny covering altogether, and lays bare an under surface, totilly unfitted, from its moist, soft texture, for exposure, either to the hard ground or the action of the air; and in consequence of such unnatural exposure it scon becomes dry and shrinks: then follow cracks, - the edges of which turning outwards form rags; these rags are removed by the smith at the next shoeing, whereby another such surface is exposed, and another foundation laid for other rags; and so on, until at last the protruding, plump, elastic cushion, interposed by nature between the navicular joint and the ground, and so essential to its preservation from injury, is converted by the mischievous interference of art into the dry, shrunk, unyielding apology for a frog, to be seen in the foot of almost every horse that has been regularly shod for a few years. The frog is provided within itself with two very efficient modes of throwing off any superfluous horn it may be troubled with; and it is very unwise in man to interfere with them: the first and most common is the separation from its surface of small bran-like scales, which, becoming dry, fall off in a kind of whitish scurf, not unlike the dust that adheres to Turkey figs. The other, which is upon a larger scale, and of rarer occurrence, is sometimes called "casting the frog." A thick layer of frog separates itself in a body, and shells off as deep as a usual paring with a knife; but it is worthy of remark that there is this very important difference between the two operations: nature never removes the horny covering until she has provided another horny covering beneath, so that, although a large portion of the frog may have been removed, there still remains a perfect frog hehind, smaller, it is true, but covered with horn, and in every way fitted to sustain exposure; while the knife, on the contrary, removes the horny covering, but is unable to substitute any other in its stead. My advice, therefore, is to leave the frog to itself; nature will remove the superfluous horn, and the rags can do no harm, and if unmolested will soon disappear altogether."

Lafosse's System of Shoeing.-Lafosse, the noted French veterinarian and author, recommends the following method of shocing:

"To prevent horses slipping on the dry, glistening pavement-pavés sec et plombé-it is necessary to shoe them with a crescent-shaped shoe,-that is, a shoe which only occupies the circumference of the toe, and whose heels gradually thin away to the middle of the quarters, so that the frog and heels of the hoof bear on the ground, and the weight be sustained behind and before, but particularly in the latter, because the weight of the body falls heaviest 
there. The shorter the shoe is, the less the horse slips; and the frog has the same influence in preventing this that an old hat placed under our own shoes would have in protecting us from slipping on ice.

It is necessary, nevertheless, that hoofs which have weak walls should be a little longer shod, so that the gradually thinning branches reach to the heels, though not resting upon them. For horses which have thin, convex soles,-pieds combles,-these long shoes should be also used; and the toes should be more covered to prevent the sole touching the ground: at the same time the shoe must be so fitted that it does not press upon the sole, and the heels and frog rest upon the ground. This is the only true method of preserving the foot, and restoring it. . . . A horse which has its heels weak and sensitive ought to be shod as short as possible, and with their branches-éponges-so that the frog comes in contact with the ground; because the heels, having nothing beneath them, are benefited and relieved.

Crescent shoes are all the more needful for a horse that has weak, incurvated quarters, as they not only relieve them, but also restore them to their natural condition. Horses which have contusions at the heels-bleimes, corns-should also be shod in this manner; and for cracks-sand-cracks-at the quarter it is also advantageous. The sole, or frog, should never be pared; the wall alone should be cut down, if it is too long. When a horse cuts him. self with the opposite foot, the inner branch of the shoe ought to be shorter and thinner than the outer. In order that the shoe wear a long time, I have used a nail of my invention, the head of which is in the form of a cone, and the aperture in the shoe of the same shape, and exactly filled by the nail. However much the shoe may be worn, it is always retained in its place.

This kind of nail possesses three other advantages: one, that it is less liable to be broken at the neck, because it exactly fits the stamped hole; the other, that it is smaller, and, in con. sequence, not likely to press on the sensitive part of the foot; and, lastly, that it does less damage to the horse."

In another connection, he recommends shoeing horses kept for general service, as follows: - "The shoes must not be too long, or project beyond the heels, but only reach the bars; neither must the hoofs, behind or before, be pared. The wall, or crust, alone should be diminished in proportion as it may be too long. This should be done evenly; and neither the sole nor frog must be cut: the latter should be allowed to project, if possible, above the shoe, so that it may come into contact with the ground. The shoe ought to be about the same strength throughout, or a little thicker and wider in the outer branch of the fore-foot, and thin at the heels of the hind one. Be careful to stamp the nail-boles on the same line, not in a zigzag manner. The holes should not be too coarse, as there is then danger of pricking the horse, or binding the hoof with the stalk of the nail.

The shoe should be stamped coarser outside than inside, because it may be necessary to leave it wider outside. Do not bend the shoes in adjusting them, nor arch them: they ought to be nearly flat; though they might be slightly curved, so as to preserve the wall of the hoof. They should also follow the outline of the hoof, - a little more to the outside than the inside. When fitting, the shoe should not be kept too long a time on the hoof, for fear of heating it. With this shoeing we may travel on slippery ground or grass land, in using for each shoe two nails with long heads, which will prevent the horse from slipping. Also during frost, on paved roads, ice, or snow, use these nails, as they prevent slipping; the roads being hard, three nails are required; two in the outer branch, and one in the inner.

These short shoes, thin at the heels, have caused the horses to walk on their frogs, which are their points of support; and those which were lame at the heels are sound again; those also whose inside quarters were contracted, bent over, and split (sand.crack), have been cured. It has been the same with horses whose quarters and heels have been contracted (encastele): these have been widened, and have assumed a proper shape. The same may be said of those 
whose soles were convex (comble), and which went lame with long shoes. My method has also preserved those horses which had a tendency to thrush (vulgo, fic) and canker of the frog (crapaud)."

The Goodenough Shoe. - This shoe is made upon the principle that the proper way to shoe a horse's foot is to permit the frog to rest upon the ground; the inventor, whose name it bears, having this idea of frog-pressure in mind, succeeded in producing a shoe that has been extensively used and bighly recommended by the best authorities on farriery.

It is stated by those who have become most familiar with its use, that corns, quarter. cracks, thrush, shrunken frogs, contracted heels, and many other of the evil effects of improper shoeing, have been cured by the use of this shoe alone, without any other application.

This shoe is always applied cold, and is fitted by cutting out just enough of the crust to have it fit in well, being imbedded as it were in the crust, and leaving the sole and frog as much exposed as possible, neither of which are ever cut. It is a very light shoe with five calks or bearings, a lower surface similar in form to the natural foot, and is bevelled on both surfaces, the nail-holes being counter-sunk.

Mr. Russell, present Secretary of Massachusetts Board of Agriculture, says of this shoe: "My reason for using the Goodenough shoe was because it kept the principle continually before the man who was shoeing, and enabled him to shoe the horse with frog-pressure. The heel of the Goodenough Shoe is drawn thin. It is a rolled shoe, and it is rolled thin. The shoe is also bevelled on each side. It is bevelled on the foot-surface, the part of the shoe that goes against the foot; so that the bearing of the horse comes upon the outer wall of the hoof entirely. It is bevelled on the inside, which prevents the balling of snow, or suction in mud; which is a very important matter. And then, in rolling up, it is corrugated. There are three depressions in which the nails are counter-sunk, so that the heads of the nails do not strike the ground until the shoe is very well worn down. Any man who will take the pains can shoe his horse in the same way without the use of any special shoe for that purpose."

The Charlier System. - This is an improved French method of shoeing horses, and consists simply of a little rim of iron put about the hoof, set in a groove, so that the whole bottom of the horse's foot, frog, bars, sole, and all, comes upon the ground the same as if the animal were barefoot, the shoe serving as a protection to the rim or crust of the hoof. This mode of shoeing makes a very nice job, requiring the best workman to execute it satisfactorily, and would doubtless prove too expensive to become generally adopted in this country.

Shoe Tips. - These are a narrow, thin, crescent-shaped protection for the front portion of the hoof, which is that part most readily worn away. They leave the sole, bars, frog, quarter, and heel entirely untouched, the hoof having no artificial protection except at the front, as previously indicated. They are highly recommended by those who have used them, and approach the nearest to bare feet of any method of shoeing. Mr. Murray says of them:-

"They are a most excellent form of shoe. I speak from experience, and not from theory alone. I have used horses of eleven hundred pounds weight, in farm-work and ordinary family service, on the road, for months together, with no protection to their feet save these tips, and found that their feet, which, at the beginning of the experiment, were in a most unsatisfactory condition, grew strong and well; and I recommend this form of shoeing to all my readers whose lorses are exercised or worked in the country. Indeed, I am under the impression that the feet of many horses would need no other protection even for city service. It is astonishing how fast the foot will develop and increase when once brought in contact with the ground. Take off those high-beeled shoes from your horse, friend, which you have 
caused to be put on him in order to keep his frog from the ground, and let it come in con. tact with the ground at every step. For a few days, or even for a few weeks, your horse may favor himself somewhat; but Nature will soon accommodate herself to the new liberty granted her; viz., the liberty of helping herself. She will soon build up a frog, such as you never saw in your life, - a large, overlapping pad of gutta-percha-like substance, wide and thick, that feels no more the concussion, when brought in contact with the stone pavement, than the buffer under a railroad car feels the jolts as the train is being whirled along."

Mr. Russell, previously quoted, says:- "A horse condemned to wear heavy shoes to which heel and toe calkins are affixed, begins to fail from that moment. At the age when he should be in the fullest enjoyment of his strength, he is called old. And few of our horses live out half their days, the great cause of their decline being from disease of the feet; all of which are caused by ignorant shoeing. In the management of colts on a farm, they should not be shod until they come to rapid and long-continued labor on hard roads; and then the lightest possible application of iron should be made. The safest way is to let the hind-feet be bare, and to shoe the fore-feet with tips or crescents of iron that only cover the toe. It must be borne in mind that the frog is the natural level of the horse's foot, and the hoof must be trimmed, keeping that ever in view."

Mr. E. F. Bowditch, of Massachusetts, gives his method of shoeing, as follows:- "My way of shoeing is to get a level bearing on the horse's foot, and keep the frog on the ground; never have any heel or toe calk, except when it is absolutely necessary in winter. The last winter, I rode my saddle mare (and of course my neck is worth more to me than anything else I own) on glare ice, with a small bit of iron, about four inches long, curled around her toe, and with a very small toe-calk. I recollect galloping out on the ice, where the men were at work cutting it, and I had no fear of her slipping, although the horse that was marking the ice, that had calks on two inches high, did slip."

Mr. Bowditch further states that he established a little forge on his own farm for his own protection, and because he could not get a blacksmith to do as he wanted him to do. They did not believe in light, three-quarter round shoes, and Mr. B. did; and where horses have had their feet abused for many years he would use nothing but "tips," leaving the heels entirely bare. In reference to preparing the foot for the shoe, he says: -

"One great thing is to take off as little as possible. You merely want to cut a little bit off cf the edge where your shoe is going; so that, when you have got your iron on, the frog will be sure to come down, and take the jar on the foot. No matter if there is a large flake which stands off; leave it there, for it may save the horse from getting hurt when going down bill. There may be a piece of iron in the road (a nut, as happened in one case to myself), and, if the horse steps on that, it may lame him. That flake is dead: it is worn off on the road, and sometimes drops off in the road or in the stable."

He never touches the "frog" in any way, no matter how ragged; and is also very careful not to touch the "bars" unless they strike before the shoe, when he would shave off a little. In reference to a case where the frog had shrunken up into the foot, and the benefits of a frog-bearing in such a case, he says: -

"It will benefit the horse if that method of shoeing is kept up; I know that by experience. I very often have cripples come to my forge; and, when they get to going well, they go back to their own blacksınith; their owners do not think it worth while to bring them to me. When the little mare called the 'corpse,' that I drive, came to me, her heel was about an inch and three-quarters wide, and her frog was the size of my little finger. Now she has a frog that fills up almost the whole of her foot. . . .

I have been interested in shoeing horses for several years, and I never have had any trouble with my own horses' feet from the effects of shoeing. Nine hundred and ninetynine thousandths of all the trouble in horses' feet come from shoeing; in fact, practically all. 
To illustrate: this 'corpse' that I speak of, that I drive fast down bill occasionally, belonged to a friend of mine, and was in the knacker's yard to be killed. She was to be killed because the doctor who had her in charge wanted her legs as specimens of inflammatory rheumatism. I asked my friend, as a favor, if he would let me take her, because I did not think she had inflammatory rheumatism, and I would like to try and cure her; and, if I did not succeed, the legs should go to the veterinary, who wanted them as specimens. I had to bring her sixteen miles; and it took me eight hours, with a man leading her, and a boy behind switching her; and, as they express it in the country, 'every leg was in front of her.' She had a little shrivelled frog. I cut her hoofs very low indeed, until I got a little bit of frog-bearing; but it hurt her to put her foot to the ground. The frog had no life in it, no circulation. About two months after I took her, I thought I would try her, and see if it was inflammatory rheumatism. She took her eighteen miles in an hour and a half, although she was a little lame. I was satisfied she would come out perfectly sound. To-day I am driving that mare. She has never had her frog off of the ground since $I$ had her, winter or summer. Her heel is steadily growing wider, and her frog is growing every day. I have driven her, within the last fortnight, from Boston to my farm, some twenty-three miles. I can do that with this little mare in an hour and forty minutes, and repeat it the same day in an hour and forty-five minutes, and she will not go lame a single step. She will go in the middle of the road, and step on stones without flinching."

The instances already given are sufficient to prove the evil effects of faulty shoeing, and the best method of remedying them.

The Best Method of Shoeing.-That shoeing is undoubtedly best which consists in taking away as small a portion of the natural support of the foot as possible, without cutting the frog bars, or sole, and at the same time giving increased strength and protection, leaving the foot free to maintain those points of support that in a state of nature it always has; in fact, the nearer we follow the natural form and tread of the foot, the better.

Shoes should never be worn longer than four or five weeks without being removed, as contraction of the hoof and corns will be liable to be the result, if resetting is neglected longer than this period.

Barefooted Horses.-Although the shoeing of horses has long been common in most of the civilized portions of the world, still there are many parts where horses are permitted to go year after year without shoes, even on rough roads, and under such conditions are said to remain sound, being less troubled with lameness and diseases of the feet and limbs than where shoeing is practiced.

The attention of some of the leading writers on horse management has for a few years. past been directed towards the benefits to be derived from dispensing with shoes altogether, and permitting horses to go unshod. They claim that more evil than good results from shoeing; that it is entirely unnecessary, and that if horses could be allowed to go unshod, great benefit would be derived. The opponents of the practice claim that horses used on hard pavements, or in constant service on common country roads, will soon become tenderfooted and lame from the wearing away of the hoof, and also that such horses would be liable to serious injury from slipping in freezing weather.

It is a well-known fact that there are hundreds of horses used-unshod-with success in London over the pavements of stone, wood, and asphalt. This method, if practicable, would certainly save the farmer considerable expense for shoeing during the year. There is no doubt that much of the lameness of horses results from improper shoeing, and that when lame from this cause, one of the best remedies in such cases is to remove the shoes, and permit the animal to run in the pasture awhile, or, if in winter, he should be allowed the exercise that a large box-stall affords; also the yard, when the weather will admit. Horses that 
have been accustomed to wear shoes should not have them removed, and be put at once to hard work on rough roads, for this will be liable to make them tender-footed. The hoof will also be apt to split and break. If, in such cases, the animal could be turned into a pasture on damp soil, or where there is wet grass, for a few days, the hoofs will become softened, when they can be trimmed or rasped off evenly. If put to hard work before the nail-holes -or those portions of the wall that have been split or weakened by them-are worn off, there will be a liability of the hoofs being further injured.

It would be well to give horses that have been accustomed to wear shoes, the protection which tips afford for a time, until the hoofs become hardened, if they are to be put to much service.

Probably some horses have had their hoofs injured to such an extent by bad shoeing and nailing, that more time would be required to permit the hoof to become repaired by the natural process, than could be profitably spared by the owner. The quality of the hoof differs with different horses, some having much harder hoofs than others. The only way to tell whether a horse's hoofs are tough or tender is to test them thoroughly after the broken and damaged portions are removed or worn away.

A Western gentleman of large experience with horses says:--" We have long been convinced of the folly of keeping horses shod the year round, and at present we allow all of our horses to go barefoot most of the time, and some of them are constantly on the road, and most of the time on paved streets. We keep our horses shod only during the rongh and slippery roads of mid-winter. If farmers could save a part of their blacksmith bill, and at the same time improve their horses, it would be a decided gain."

Col. M. C. Weld-a high authority on all that pertains to horse-management-gives his practice as follows:- "With me it is a great desideratum to have my horses shod as small a part of the year as possible. They are surer-footed; their feet are in better order; they travel freer on short journeys-I have not tested them on long ones; they do not injure one another by kicking, and it is a saving of expense. In fact, considering the number of ailments brought about by bad shoeing and poor blacksmiths, and the number of horses hopelessly ruined by this means, and the fact that there are parts of the world where the roads are even rockier and rougher than ours, where the art of farriery is unknown, and the horses are all driven and ridden barefoot, that we in the country ought to do the same, and let our horses go barefoot just as much as possible."

We doubt whether horses would be benefited by going entirely without shoes the year round in those latitudes where ice forms during the winter, although many who have tested the plan assert its practicability, but we do believe that the majority of horses might go unshod with benefit during a large portion of the year, and it would be to the advantage of farmers and horse-owners generally to have less iron and steel, and more common sense, employed in the management of their horses' feet.

Results of Improper Shoeing.-Faulty shoeing, as previously stated, is the cause of many of the ills which horses are obliged to endure, good shoeing being the exception rather than the common practice. Among the many evils that result from improper shoeing may be mentioned the cutting away of the bars and frog, thus weakening the support and protection of the foot; the thin paring of the sole, which removes the protection of the inner sole from both the ground and the atmosphere, making the horse tender-footed, and causing the natural moisture of the hoof to become absorbed by the atmosphere, until it becomes dry and cracks. Then there is the breaking off of the walls of the hoof by the nail-holes, the drawing of the nails too tightly, the use of the rasp in making a groove for clinching the nails; the cutting and filing of the edges of the hoof that project beyond the shoe; the opportunity of foreign substances, such as stones, gravel, or earth, becoming wedged between the shoe and sole; the uneven pressure of the shoe on different portions of the sole; the 
searing of the hoof to make the shoe fit better; the uneven growth of the hoof sometimes seen when the shoe is left on too long; the injury to the hoof in removing nails, and from driving them into the sensitive portion of the sole in fastening on a shoe. Sometimes the nail splits in driving, and one thin blade of iron goes into the sensitive portion of the foot, and the other through the wall. Instances of lock-jaw have been known to occur from this very cause. In order to have the foot look round, and give it a finished appearance, some stupid blacksmiths rasp the wall of the hoof, thus weakening it and causing it to become brittle and crack, it not being sufficiently strong, under such treatment, to support the weight of the body, and the concussion produced on hard pavements and rough roads.

Much injury is done the hoof by the violence and carelessness often connected with removing a shoe, thus breaking and tearing portions of the wall. These and many other evils might be mentioned as the direct result of improper shoeing. Blacksmiths cannot take too much pains in informing thenselves respecting the best modes of shoeing, or in the practice of their art when it is thoroughly understood.

Brittle Hoofs.-There is a great difference in animals with respect to the quality of the hoof, - some being of a soft, porous nature, others hard, firm, and capable of enduring much wear, and the extremes of heat and cold, drought and moisture, without being appar. ently affected by them. The quality of the hoof is transmitted with just as much certainty as that of any other characteristics, some breeds or families of horses invariably having large flat feet and hoofs of an open, porous nature, while others-such as the Arabian breed, for instance,-have a small, narrow hoof of the firmest, toughest, and most compact quality. This is not only true of breeds, but of individuals; the quality of the hoof of the parent determining largely that of the offspring. Aside from the difference in quality, there are conditions that affect and modify the character of the hoofs of horses, long-continued warm, dry weather having a tendency to cause the hoof to become brittle, while moisture or dampness has the opposite tendency. Improper shoeing, as previously stated, will cause the worst form of brittleness of hoof. Imperfect nourishmeut will also affect the quality of the hoof, as well as the hair, and cause it to become dry and brittle in character.

The Nutional Live Stock Journal contains the following on this subject: "It is not habitual dryness that injures; it is the alternation of rain and drouth. The evil effects of moisture may be largely warded off by smearing the moistened foot with some impervious, oily agent before exposing it to the drying process. In this way the moisture that has been absorbed by the horn is retained, the sudden drying and shrinking are obviated, and the horn remains elastic and comparatively tough. As it is often needful to soak the foot in warm or cold water, or in poultices in cases of disease, it is all-important that the abovenamed precaution should be constantly borne in mind, and that the softened foot should be smeared throughout with some hoof-ointment before it is allowed to dry and harden. For this purpose nothing is simpler or better than a mixture in equal parts of wood-tar and whale-oil or lard. This may be smeared on the foot every other day.

In addition to the changes of weather, the frequent standing in rotting dung-heaps, or in pools of decomposing liquid manure, may be named as causes of brittle hoofs. In the dungheap there is not only the moisture and steam soaking and softening the hoof, but there is abuudance of ammonia-gas, which is especially calculated to soften, dissolve, and destroy the horn. Rotten manure and putrid liquids, therefore, are much more injurious than pure water, muddy pools, or wet clay. Again, the emanations of this kind are far from conducive to general health, so that they prove hurtful in two ways - first, by directly destroying the substance of the hoof, and, secondly, by reducing the animal vigor, the power of digestion and assimilation, and the power of secreting good horn. Standing in such decomposing organic matter is still more injurious, however, when the animal is confined to a stall or box, for here the injurious effect of inactivity is added to the above-named conditions. 
Not the least among the causes of brittle hoofs is disease of the foot. And this may result from injuries inflicted at a distance, as well as from those acting directly on the foot. Of the first olass are especially to be named founder from an over-feed of grain, or a drink of cold water when heated and fatigued. In both instances the trouble begins with the stomach and digestive organs, which become congested and irritated by the indigestion, or the reaction from the chilling effect of the cold; the irritation extends to the skin; and the foot, being that part of the skin which is most abundantly supplied with blood, and most sensitive, is the most severely affected. Such inflammations of the foot may be so severe as to cause shedding of the hoof, extreme distortion of the hoof, or merely drying, hardening, shrinking, contraction, imperfect nourishment, brittleness, and loss of substance.

The same result sometimes follows on an overdose of purgative medicine, which, primarily, irritates the stomach and bowels, but, secondarily, implicates the skin and feet by sympathy.

The perfect integrity of the hoof is only to be obtained in the progeny of a good stock, in which health, growth, and vigor have been persistently secured, by suitable food, air, exercise, and general care; all inflammation or other diseases of the foot must be carefully guarded against by a most watchful attention to this part alike in shoeing, stabling, and exercise. When a foot has suffered in any way, whether from drying after excessive moisture, from long standing on a dry floor, from freezing in cold weather, from over-driving and concussion, or otherwise, the irritation should be relieved by a soothing application, like a poultice, or a standing place in wet clay, in warm or in cold water, to be followed in every case by a daily, or, at least, a frequent, use of a hoof-ointment. It may be added that the growth of the horn may be hastened by a tar or turpentine ointment, or by the application of a weak solution of cantharides - one part of powdered cantharides to 25 or 30 parts of alcohol - around the hair at the top of the hoof. When a good firm horn is wanted, this must never be applied so as to induce severe inflammation, which would increase the production of horn, but only of an open, unresisting quality of the same, ready to yield or break under tension or concussion.

Gentle daily friction, or the application of the above preparation, as often as may be necessary to keep up a free flow of blood to the part, is all that is required. In very weak, brittle feet there is often an advantage in applying a leather sole beneath the shoe, with a layer of tar and tow between it and the sole. This obviates concussion, and favors growth. Again, broken parts of the wall may sometimes be temporarily repaired by filling the breach with a mixture, in equal parts, of gutta percha and gum ammoniac. This will give some support to the part, and a more equal bearing to the shoe, until the hoof-wall has had time to grow down anew."

Stopping the feet frequently will obviate brittleness of the hoof, which is sometimes caused by the horse being seldom or irregularly worked, and kept most of the time in a dry stable.

Stopping the Feet. - This consists of filling the entire under portion of the hoof with some substance that will serve to impart moisture to the foot. It will always be found beneficial when the hoofs are dry and brittle, or when there is any tendency to feverishness in the feet. For horses that are confined much of the time in dry stables, this process is almost a necessity, but too often neglected in stable management. Some writers on horse management claim that the feet of all horses should be stopped as often as every other night. The best material for this purpose, and what is always convenient to obtain, is fresh cowmanure, mixed with one-fourth part of clay. Either wet clay or cow-manure alone are also highly recommended for the purpose. Wet oakum and linseed-meal are also very good. Youatt recommends, in case of wounds, cow-manure with a fourth part clay beaten into it, and a little tar added, but says that the use of tar in a common stopping is too stimulating 
and drying. The stopning should be always carefully removed before the horse leaves the stable for use.

Lampas. - This is very improperly considered a disease by certain ignorant farriers, and the brutal practice of burning down the bars with a hot iron is frequently resorted to as a remedy. Lampas is a swelling of the bars of the mouth, and is found in all colts to a greater or less extent, and occasionally, though rarely, in old horses, since the teeth of the horse continue to grow during his entire life. Sometimes the swelling extends to a level with the LAMPAS IRON. edge of the teeth, and even beyond them. This condition may be produced from inflammation of the gums when the colt is shedding his temporary teeth, or from some feverish tendency in the system. It frequently occurs when a young horse has beel taken from grass and fed on grain. Over-feeding, with insufficient exercise, will also sometimes cause it. Generally, this difficulty requires no treatment, and, if left to itself, the swelling will soon subside. But when it interferes with the mastication of the food, it will be well to lance the gum or bars of the mouth, the bleeding of which will reduce the inflammation, the lancing to be performed where the most inflammation exists. This may be done with a lance or a common pocket-knife, by making a few slight incisions across. Sometimes the cutting of the tushes and grinders will produce the swelling in the mouth, and when this is the case - which can readily be determined by examining the localities - the gum should be lanced over the tooth, the same as children's gums are sometimes lanced in teething. Warm mashes should be given at such times.

Jennings recommends that, after lancing, the mouth be washed with a solution of tincture of myrrh, two onnces to a pint of water, or a solution of alum and water, this to be repeated twice a day for three or four days, during which time bran mashes or flax-seed gruel, with a small quantity of new grass, should be given. Also, that no hay, oats, or corn be given for a week, after the expiration of which time the teeth will be in condition to masticate such food.

Treatment of old or Disabled Horses. - A horse that has served his master faithfully many years is justly entitled to kind and considerate treatment in old age. It is too frequently the case that after a horse becomes advanced in years, and his usefulness somewhat impaired, he is sold to some unscrupulous person who will either over-work and otherwise ill-treat the faithful creature, or who will sell him to some one of a numerous class of men who are too poor to own a good horse, but who for a few dollars will purchase an old or otherwise partially-disabled animal, and get all the work he can out of him, at the same time keeping him with the least possible expense. There are already too many of these abused, half-starved creatures to be seen in every city and town, the sight of which, more than almost anything else, strikingly impresses one with a sense of man's inhumanity.

The selling of the faithful, patient old farm-horse to a sharp-eyed jockey is frequently done in a thoughtless manner by many a kind-hearted farmer, who, if he wonld stop to reflect a moment upon the suffering this act of his would bring upon the noble animal, would refuse to do it. There is another class of farmers to whom the few dollars that such a sale would bring in return would far outweigh the feeling of gratitude or conscience in the consideration, and to such we suppose this appeal in behalf of the faithful old horse would be futile; but we hope and believe this class to be in the minority, and the nobler elements to largely predominate. He who will cause by his act, or permit cruelty to be perpetrated upon any of the creatures God has given us, is himself guilty, nearly, if not fully, to the extent of him who perpetrates directly the cruel act, since he either causes or permits what he 
might easily prevent, and is therefore responsible. No horse should be made to labor beyond his strength, at any age, and all horses are entitled to kind treatment and good care. When a horse becomes so old or otherwise unfitted for service that it does not pay to keep him, and the owner feels that he cannot afford to keep him simply for the good he has done, or has not sufficient affection for him to still care for him after he ceases to be paying property, let him be humanely killed by a rifle-ball shot through the brain, rather than sell him to be abused and wear out the remainder of his life in suffering and neglect. Old horses should be fed much with warm mashes, oats, and cut feed.

Carrots are especially good for old horses, to the extent that they have been very appro. priately called the regenerator of old, worn-out horses, as well as those that have been over. worked, poorly fed, and otherwise improperly treated. Such horses when allowed a generous diet of carrots, in connection with other food, will gain in condition very rapidly. They will soon put on flesh, and the coat become materially improved. Carrots seem to supply horses with new blood, and thus restore their youth for a time. Horses are very fond of them and will eat them greedily. If fed upon them in large quantities for a long time, however, the effect is lessened, as the body becomes accustomed to them, and the result is less marked. Care should be used while thus feeding to give the animal sufficient exercise, as there will be a tendency to apoplexy where this is neglected, and such stimulating food long given in large quantities.

Care of Harnesses.-Harnesses that are constantly surrounded with the fumes of ammonia will become stiff and hard, as the ammonia will have a tendency to absorb or eat up the oil contained in the leather. For this reason nice harnesses should never be hung where the ammonia from the air of the stable will reach them. A harness-room or closet should be done off in the barn or wagon-house for the purpose, which will also keep them free from dust. The walls should not be whitewashed, as the lime, coming in contact with the leather, would injure it. Long pegs or hooks should be fastened in the wall about six feet from the floor for hanging the harnesses on. Two hooks will be sufficient for one harness, one for the bridle and collar, the latter hung with the small end up and outside the bridle, and the other for the harness, which should be hung by the saddle. By this arrangement they are hung in proper order for putting on the horse.

On taking the harness from the horse, the dust and rain or perspiration should all be wiped off with a piece of chamois skin, or woolen cloth. When nearly dry, the portions that were damp should be again rubbed with another cloth or chamois skin kept for the purpose, until they are soft and piiable. The plated mountings and bits should be cleaned by rubbing with a cloth slightly oiled. Harnesses require occasional special cleaning and oiling in order to prevent the leather from becoming stiff and hard. They are more injured by use and require more care in summer than in winter, as they are more frequently soaked by the rains, wet with perspiration from the horses, and subjected to heated and dry atmosphere. If kept well oiled, the injurious effects of these conditions may be in a great measure obviated.

How to Clean and 0il a Harness. - There are various methods and applications employed for cleaning and softening harnesses, the most common of which is to first wash with soap and warm water, followed by rinsing in clear, soft water, and afterwards apply oil; neats-foot oil being generally used. Another method is to oil the harness well, by which means the dirt on it is softened, after which wash it thoroughly with soap cund water, and rinse it as before, afterwards applying some one of the many preparations for the purpose, which will both oil the leather and improve its color. By oiling before washing, the oil penetrates the leather and prevents the water from saturating it and making it hard. Castor oil is highly recommended by some for this purpose. A preparation of equal parts of oil and tallow, colored with lamp-black and a small portion of Prussian blue, makes a very good application 
for harnesses after they have been washed and dried. Castor oil will endure the effects of the atmosphere and moisture much longer than neats-foot oil, and when used will not require applying as often. A mixture of equal parts of castor and neats-foot oil is frequently used.

Le Bourellier et le Sellier, a French work, gives the following recipe for restoring old and stiff leather:-Melt over the fire, in a metallic vessel, eight pounds of very pure beeswax, stirring it until it is all melted; then introduce one pound of litharge, which has been pulverized in water, dried, and passed through a fine sieve. Leave it on the fire, and stir it until all of the soluble part of the litharge is incorporated with the wax; remove the vessel from the fire, and when the mixture shall have lost a portion of its heat, incorporate with it, little by little, one pound and a half of very fine ivory black of the best quality; replace it on the fre, and stir it incessantly until the wax commences to boil again; then remove it and allow it to get nearly cool. Then add to it spirits of turpentine, until it is of the consistency of a paste. More turpentine may from time to time be added, as may become necessary.

Cleaning the Plate. - When tarnished, the plate of a harness may be cleaned by rubbing with common whiting for polishing silver. If badly tarnished, rub first with whiting wet with soapsuds, afterwards with dry whiting. No acids should be used; it will be liable to destroy the plate. Steel mountings should be kept bright by rubbing with a cloth containing a very little sweet oil, never enough to be perceptible on the surface of the steel.

Treatment of Sick Horses.-As soon as a horse is discovered to be sick he should be placed apart from others, both for his own welfare and that of other horses that may be stabled with him, as contagious diseases may be communicated by the confined air of the stalls. The sick animal should have warm, airy, and well-ventilated quarters, which should be kept scrupulously clean. He should also be provided with a good, soft bed of clean straw or leaves. He should be handled very gently, and always approached in a quiet manner, avoiding all unnecessary noise, or anything that would have a tendency to disturb or irritate him, for sick animals, like sick people, like to be quiet and undisturbed, and are equally affected by irritating causes. Good nursing and simple remedies are much to be preferred for sick animals, as well as for sick persons. Never use a horse that is not well. If he refuses his food, or seems ailing, he is in no condition for work.

Diet for Sick Horses. - This will depend much upon the nature of the disease, the degree of development, its intensity, etc. Rush recommends that " in acute diseases no food whatever be given until improvement has taken place, and even then only in a sparing man. ner; the articles of diet most suitable are bran, oats, hay, carrots, Swede turnips, and green food, either grass or clover. The bran may be given either dry or wet, whichever way the animal prefers it. Oats may be given mixed with bran, either raw and crushed or whole and boiled. It may be necessary to keep the animal without food and water for a half hour before and after administering medicine." A warm mash of bran is excellent, also boiled flax-seed, potatoes, either raw or boiled, and a few sweet apples.

Pulse of the Horse.-It may not be known to all having the care of horses that the pulse of the horse can most conveniently be felt close to the junction of the head with the neck, in the lower jaw, a little back of where the sub-maxillary artery and vein, and the parotid duct come from under the jaw. The ordinary number of pulsations in a common farm horse in temperate climates is thirty-six per minute, while that of the thoroughbred will be from forty to forty-two. It is somewhat increased by hot weather, and greatly so by a hot climate.

It is stated by reliable authority that the pulse of a thoroughbred horse will be about ten degrees higher in New Orleans than in New York. The ordinary practice of feeling the pulsations of the heart through the sides determines only the number of beats in the minute, while by that of the jaw, as described, determines not only this, but the manner in which the blood passes, and the quantity. 


\section{DISEASES OF THE HORSE.}

$\Gamma{ }^{\mathrm{HE}}$ diseases of horses are very numerous, some of which can scarcely be distinguished from others, in their early stages, even by the most skilled veterinarian; it is therefore not advisable for an inexperienced person to attempt to prescribe in such cases, since a successful treatment of sick animals necessitates, not only a thorough knowledge of the whole animal system, but also the symptoms and nature of the disease, as well as the nature and action of the remedies. When a horse seems slightly ailing, it will frequently be found that good nursing, rest, and judicious feeding for a few days are all that will be necessary to restore him.

Nature effects cures more frequently in spite of powerful drugs, than with any assistance they may afford. There are, however, instances of acute disease where prompt attention will be necessary; and under such circumstances a good veterinarian, if procurable, should be sent for, but beware of ignorant "quacks" who administer powerful drugs of which they know little, into animals of which they know less, and which would either render recovery impossible, or cause a needless amount of suffering to the poor animal.

There may be cases of sickness or accidents where immediate action is necessary, which, if delayed a sufficiently long time to send for aid, might endanger the life of the animal.

A gain, there are ailments of a trifling nature, requiring the most simple remedies, where it would be wholly unnecessary to secure the advice of a veterinarian, and which any person of good judgment could treat who was at all familiar with horses and their management. In any case, it is well for those having the care of horses to acquaint themselves with the nature of the more common diseases and their remedies.

We do not, however, approve the practice so frequently seen among horsemen of constantly dosing horses with all sorts of nostrums, torturing them with applications of every nature, both external and internal, which would be sufficient to make even a well animal sick.

Blistering should never be resorted to, except in cases of real necessity. Firing is a barbarous, cruel practice, and should become forever obsolete. Deep burning often results in inflammation and ulceration that may with difficulty be healed, and in the majority of cases more injury than benefit results from this mode of treatment. Blisters are only beneficial as a counter irritant, and are seldom necessary.

Good care and kind treatment, which comprise the observance of sanitary laws and humane considerations in all respects, will have a tendency to keep animals in a vigorous and healthy condition to the extent that but little if any medicine will ever be required. The majority of diseases to which horses are subject are due, either directly or indirectly, to a lack of ventilation in stables, as well as a lack of cleanliness in stable management, in other respects. Herbert very justly remarks:-

"It is not too much to say that more than one-half the ailments of horses arise, in the first instance, from bad management, - or, to speak more correctly, from absence of all management,-from an improper system of feeding, from ill-constructed, unventilated, filthy stabling, from injudicious driving, and neglect of cleaning. When disease has arisen, it is immediately aggravated and, perhaps, rendered ultimately fatal, either by want of medical aid, or, what is far more frequent as well as far more prejudicial, ignorant, improper, and often violent treatment, either on a wrong diagnosis of the affection, or on a still more wrong system of relieving it. Over-medicining and quacking slightly ailing horses is the bane of half the private stables in cities, and of nearly all the farm stables in the country; and one or the other, or both combined, cause the ruin of half the horses which "go to the bad" every year.

There is no quack on earth equal to an ignorant, opinionated groom; and every one, 
now-a-days, holds himself a groom, who is trusted with the care of a horse, even if he do not know how to clean him properly, or to feed him so as not to interfere with his working hours. Every one of these wretched fellows, who has no more idea of a horse's structure or of his constitution than he has of the model of a ship or the economy of an empire, is sure to have a thousand infallible remedies for every possible disease, the names of which he does not know, nor their causes, origin, or operation; and which, if he did know their names, he is entirely incapable of distinguishing, one from the other. These remedies he applies at haphazard, wholly in the dark as to their effect on the system in general, or on the particular disease, and, of course, nine times out of ten he applies them wrongfully, and aggravates fifty-fold the injury lie affects to be able to relieve.

These are the fellows who are constantly administering purgative balls, diuretic balls, cordial balls, on their own hook, without advice, orders, or possible reason-and such balls, too! some of them scarcely less fatal than a cannon ball-who are continually drugging their horses with nitre in their food, under an idea that it is cooling to the system and that it makes the coat sleek and silky; never suspecting that it is a violent diuretic; that its operation on the kidneys is irritating and exhausting in the extreme, and that the only way in which it cools the animal's system is that it reduces his strength, and acts as a serious drain on his constitution.

These, lastly, are the fellows who are constantly applying hot oils, fiery irritants, and stimulants, to wounds, strains, bruises, or contusions, which in themselves produce violent inflammation, and to which, requiring as they do the use of mild and soothing remedies, cold lotions, or warm fomentations, the application of these stimulating, volatile essences is much what it would be to administering brandy and cayenne to a man with a brain fever. It should therefore be a positive rule in every stable, whether for pleasure or farm purposes, that not a drachm of medicine is ever to be administered without the express orders of the master.

The more ordinary diseases and affections of the horse are very similar to those with which we are affected ourselves; their treatment is always analogous, often almost exactly identical; the processes by which relief is to be obtained are the same, and the medicines do not materially differ from those suitable to the human race. It is not too much to say that any intelligent man, gifted with good reasoning powers and not deficient in observation, who knows how to keep his own bodily health in a good state, and to deal with his own ordinary ailments, can, within twelve months, qualify himself to treat a horse in all the cases that are likely to befall him, under ordinary circumstances, as well as anybody else, and fifty times better than the stable-keepers, who will sneer at his efforts until they perceive that they are successful, and then will suddenly discover that the means he took are precisely those which themselves recommended.

The things of great importance which he has to learn, in order to guard against danger, are, how much depletion the system of a horse can endure without danger, and what extent of purgation his bowels can resist undamaged. And to these questions it may be answered, generally, that the horse can bear much more depletion and less purgation than is generally imagined, especially of the drastic drugs usually exhibited. We are very decided opponents of purgatives in general, and have been gratified by observing that the recent cause of veterinary practice, both in France and England, is tending to the entire abandonment of the old system; according to which, every horse, whether anything ailed him or not, was put through two annual courses of purgation, each of three doses, in the Spring and Fall, beside having to bolt a diuretic ball fortnightly, or oftener, according to the whim of the groom, when his kidneys no more required stimulation than his hocks did blistering."

Inasmuch as it is generally easier, by proper management and good care, to prevent dis. eases than to cure them after they have been contracted, the former will always prove the 
better method in all respects. The following cut, copied from Kendall's Treatise on The Horse, shows the location of many of the common diseases of the bones and joints of this animal.

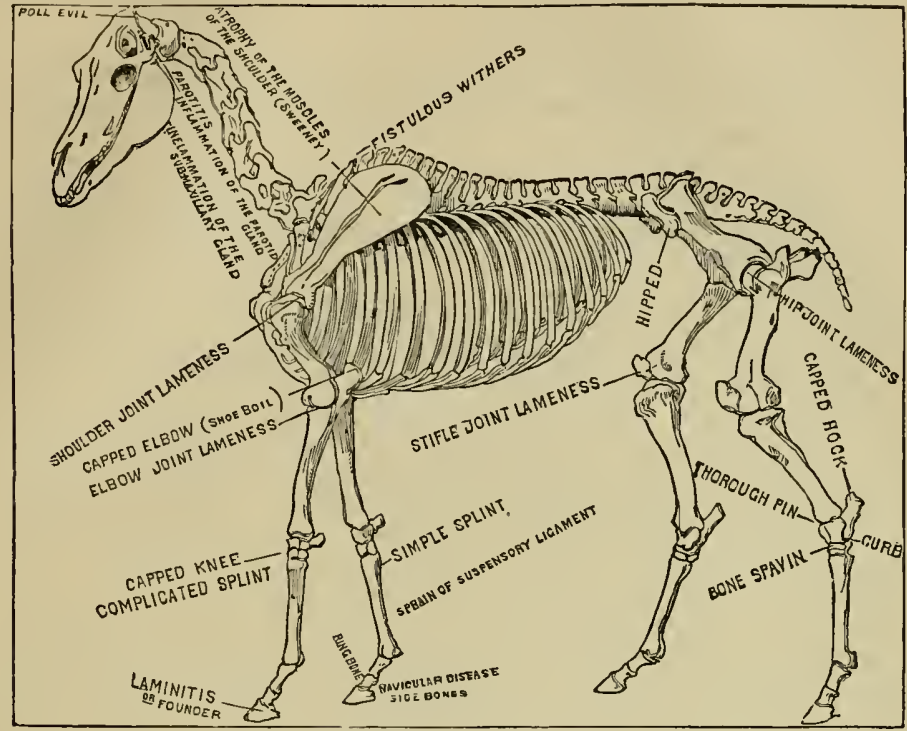

SKELETON OF THE HORSE.

Anchylosis. - This is a stiffening of the joints caused by the bone becoming united by bony material instead of the natural ligament. It is generally produced by extreme hard service, and especially in horses that are too young for hard work and before the bores and joints are sufficiently strong to endure the strain. The bones of the back and loins, as well as the limbs, are liable to become affected in this manner. When this difficulty exists to a considerable extent in the back, the horse will be stiff-backed, and consequentiy turns around, lies down and gets up with some difficulty. There is no cure for it.

Blood Sparin. - This is a somewhat soft swelling caused by an enlargement of the sack which contains the lubricating fluid of the hock joint, giving it a rounded appearance. Young horses and colts, especially if overdriven or worked hard, are more liable to have this form of spavin than older horses. It does not always cause lameness, unless the horse is worked sufficiently hard to aggravate the evil. It may, however, be justly regarded as unsound. $\mathrm{n} 3 \mathrm{ss}$, and lessens very materially the value of the animal. Blood spavin is caused by bog spavin, and results when the distended sack of lubricating fluid, which produces the latter, becomes so enlarged that the vein which passes over it is compressed between this enlargement and the skin, to the extent that the blood is impeded. The accumulation of blood in the vein, in consequence, distends it, this distension generally reaching as far as the next valve, and we have what is called blood spavin. When once reduced, it is liable to occur again.

Entire rest for a time, together with frequent bathing of the affected parts with cold water, rubbing carefully with the hand, and bandaging, are generally the most successful 
methods of treatment. In the use of the bandage, the compression should be upon the swelling. Compresses or spring trusses are very useful for this purpose. Blistering is irequently recommended, and more frequently practiced for this difficulty, but rarely results with any permanent benefit.

\section{Bloody Urine. (See Hematuria.)}

Bog Spavin. - An enlargement generally in front of the hock joint, while in blood spavin the swelling is not only in front but extends to the inside and outside. Treatment the same as for blood spavin (which see).

Bone Spavin. - Bone spavin, or spavin proper, is an enlargement of the hock joints caused by a growth of bony matter which generally involves the heads of the splint and com. mon bones, and the small bones with which they connect. Strains by hard labor will produce it, or anything that causes the weight and concussion to be thrown principally upon the inner splint bone and produce inflammation of the cartilaginous substance that unites it to the shank bone, thus inducing a bony deposit at this point. Raising the onter heel higher than the inner one in shoeing in order to prevent interfering, will sometimes cause spavin.

It generally makes its appearance upon the inside of the hock, and in front of the middle of the joint. There is always a lameness and considerable pain while spavin is forming, even in its earliest stages, and is most noticeable when a horse is first taken out of the stable. After being driven a short distance, the lameness will seem to subside, but will make its appearance again after standing a short time, and starting again. Sometimes horses are so lame from spavin that they are rendered entirely useless, while it is not infrequent that horses with large spavins are only shightly lame, or a little stiff in their gait. The pain and lameness is usually less severe after the bony process is completed.

Bone-spavin is the most serious kind of spavin with which a horse can be affected. If properly treated in the first stages, it is frequently cured, but no cure can be effected after a considerable amount of bony formation has taken place. When taken early, rest, with a little exercise,-such as that taken by a horse in being turned into a pasture,-together with the use of counter irritants on the part affected,- - such as blistering,-will usually prove beneficial. Never resort to firing; it is a barbarous practice, and invariably results, not only with no benefit, but in a positive injury.

After a considerable growth of bony material has taken place, no treatment whatever will be able to cause an amount of absorption sufficient to produce a cure. The wisest course to pursue in the early stages of the disease, and the one recommended by some of the best veterinarians, is, to turn the animal out to grass, supplying him with cooling yet nutritious food, and that which is moderately loosening to the bowels. Constipation or other feverish tendencies should be avoided. During this time the joint affected may be bathed twice a day, for a week or more, with sait and vinegar, after which the blistering salve, or a substitute, may be applied to the spavin, care being used to judiciously regulate the application according to the effect it produces, avoiding over-irritation. Sometimes one application will be sufficient; in other cases it will need to be applied again in three or four days. After producing considerable irritation, the spavin may be permitted to heal until all the heat and inflammation have subsided, when the treatmeut may be again renewed. Avoid blistering too severely, as the difficulty may by this means be aggravated; an inflamed sore may be produced in this way that will be difficult to heal.

Another method of treatment in the first stages, is the application of cold water until the inflammation is reduced; this should be thoroughly applied the same as for any other inflammation, after reducing which an application to cause absorption of the bony material should be made as follows:-Mercurial ointment, four ounces; powdered cantharides, a half ounce; oil of rosemary, two drachms. Mix these well together, and apply to the spavin daily. The 
cartilaginous substance that has not already changed to bone, can generally be absorbed by thoroughly rubbing the part with oleate of mercury daily for a few days.

Another preparation frequently employed by reterinarians in such cases is aqua ammo. nia, one ounce; olive oil, two ounces; mix these and add oil organum, two ounces; half an ounce of oil of wormwood; one ounce spirits of turpentine; camphor gum, one ounce; one pint of alcohol; mix thoroughly, and apply three times a day.

A blister for sparin-aside from that already given-may be made of one ounce of pulverized cantharides, mixed with three or four ounces of lard. In applying it, clip off the hair over the bunch, and rub in this mixture well for ten or fifteen minutes. One such application will generally be sufficient to produce the counter irritant required. After two days, apply sweet oil to soften and heal the skin.

As has previously been stated, a bone-spavin is a serious matter, and when once the bony material is formed to a considerable extent, or when anchylosis has taken place, no cure can be effected.

Although spavin is caused by strains and overwork, yet it is hereditary to a peculiar degree, the progeny of a spavined sire or dam being very liable to be affected in this manner at an early age. For this reason, such horses should never be used for breeding purposes.

Bots.-These are the larra of the bot-fly, which infests the stomach of the horse, and, sometimes, though rarely, produces injurious results. The horse bot-1ly (Gastrophilus equi), a species of the gad-fly, lays its eggs on the hair of the horse, generally on the knees and sides of the animal. In licking himself, the eggs, or small worms that are hatched from them, adhere to the tongue, and are carried with the food into the stomach. The larvæ are provided with a hook, on either side of the mouth, by which means they attach themselves to the inner portion of the stomach, and there remain feeding upon the mucus for about a year. After attaining a considerable size, and undergoing certain changes, they release their hold, and, passing into the oowels, are eventually removed with the natural evacuations. This usually occurs late in the spring. The maggot buries itself in the ground, where it changes to a chrysalis, and, after a few weeks, escapes from its confinement a perfect fly. The female soon lays its eggs upon the parts of the horse which he is accustomed to lick, and in this manner the insect continues to be propagated. It is supposed that the bots, by their attachment to the coats of the stomach, do not produce pain, and that they cannot be removed by any medicine which will not injure the horse to have administered.

Their presence may be indicated by loss of flesh, and an unthrifty coat. The best treat. ment is to improve the condition of the animal by a generous supply of nutritious food, so that the system may not become debilitated, and the general health suffer. Tonics are sometimes administered, but, as a general rule in such cases, good care and generous feeding will obviate the evil without the use of drugs.

Sometimes the bot-worm is found in the rectum, and may be seen about the anus and under the tail, proving very troublesome to the animal. Injections of linseed oil, or tobacco smoke, will generally remove them. There is a disagreement among breeders and veterinarians as to the extent of the injury done by the bot-fly.

\section{Broken Wind. (See Heaves.)}

Bruises or Contusions.-Bathe in lukewarm water as soon as practicable, accom. panied with rubbing by the hand, after which apply freely tincture of arnica two or three times a day. The sooner this treatment is receired after the injury, the better.

Bnrus.-Linseed oil, or sweet oil, should be applied with bandages when the skin has been removed, and the parts be kept as much from the air as possible.

When the skin is unbroken and no blister formed, bathing in pure cider vinegar will be found an excellent remedy. 
Capped Ankles.-This difficulty is generally produced by the same cause as capped hocks, and the treatment should be the same.

Capped Elbow.-(Shoe boil.) This is a tumor, formed just behind the shoulder, caused by the pressure against the calk or heel of the shoe while the horse is lying down. The best treatment is first to remove the cause by the changing or shortening the calks of the shoe, or by the use of pads in the stable that will protect this part of the body. If the tumor contains pus, it will be best to open it, and allow it to discharge, afterwards washing out the wound with castile soap and warm water, and allow it to heal.

If the tumor is hard, seems separate from the skin, and shows no signs of pus formation, it may be carefully removed with a sharp knife, and the location treated as a common wound. If callous simply, the washes or ointments recommended for spavin will prove beneficial, but it will require some time and repeated applications to remove the blemish.

Capped Hock.-The point of the hock is sometimes injured by the horse kicking in the stable, hard blows received in this locality, etc. It sometimes occurs from lying upon an unevenly paved stable with but little bedding. In such cases, there generally appears a soft, watery tumor, which is the enlargement of the mucus sack.

When the injary is not very severe, making cooling applications will sometimes prove beneficial, to be followed by the use of iodine ointment. Blistering will sometimes prove necessary, and quite beneficial, to be repeated after the skin is healed, if the first is not effectual. In extreme cases, where the tumor is large and does not yield to other treatment, a seaton is sometimes passed through it, and kept open until the discharge becomes somewhat of the nature of pus, after which wash with castile soap and warm water, and permit it to heal.

Colic.-This is a very common disease among horses, and frequently proves fatal. There are two forms of it, spasmodic and flatulent colic. The former, as its name indicates, is of a spasmodic nature, and sometimes terminates fatally in inflammation of the bowels.

The causes of spasmodic colic are the drinking of cold water when the animal is in a heated condition; sometimes exposure to cold winds or draft when heated; costiveness; unwholesome food; green food given in too large quantities when the animal is not accustomed to it or when he is heated will sometimes cause it; feeding with new corn will not unfrequently produce it; also an overcrowding of the stomach with food of any kind. Hard water will cause the colic in some horses. There also seems to be a predisposition to it in certain cases, and a horse that has once had it is quite liable to have it again. When recovering from the colic, a horse should be fed on warm bran mashes, and be allowed to drink only water that is blood-warm, for two or three days, as cold water might bring it on again.

Symptoms of spasmodic colic.-Youatt thus describes the symptoms of spasmodic colic, also the method of distinguishing it from inflammation of the bowels, as follows:-

"The attack of colic is usually very sudden. There is often not the slightest warning. The horse begins to shift his posture, look round at his flanks, paw violently, strike his belly with his feet, lie down, roll, and that frequently on his back. In a few minutes the pain seems to cease, the horse shakes himself and begins to feed; but on a sudden the spasm returns more violently, every indication of pain is increased, he heaves at the flanks, breaks out in a profuse perspiration, and throws himself more violently about. In the space of an hour or two, either the spasms begin to relax and the remissions are longer in duration, or the torture is augmented at every paroxysm, the intervals of ease are fewer and less marked, and inflammation and death supervene. 


\section{SYMPTOMS OF COLIC.}

Sudden in its attack, and without any warning. Pulse rarely much quickened in the early period of the disease, and during the intervals of ease, but evidently fuller.

Legs and ears of natural temperature.

Relief obtained from rubbing the belly.

Relief obtained from motion.

Intervals of rest and ease.

Strength scarcely affected.
SYMPTOMS OF INFLAMMATION OF THE BOWELS.

Gradual in its approach, with previous indica. tions of fever.

Pulse very much quickened, but small, and often scarcely to be felt.

Legs and ears cold.

Belly exceedingly painful, and tender to the touch.

Pain evidently increased by motion.

Constant pain.

Great and evident weakness."

As many of the symptoms of spasmodic colic are similar to those of inflammation of the bowels, such as pawing, kicking at the belly, looking round at the flanks, rolling violently, etc., it will be highly essential to distinguish between the two by the difference in symptoms as previously given, since the treatment for the former might prove very injurious, if not fatal, in the latter.

Treatment for spasmodic colic.-Having determined the symptoms to be those of spasmodic colic, and not inflammation of the bowels, the remedy should be resorted to as soon as possible, as delay is hazardous. Various remedies have been found beneficial in such cases, when promptly administered. One tablespoonful of chloroform mixed with a gill of whisky and a pint of warm water will often stop the pain alnost immediately. This should be accompanied with an injection of warm soapsuds, using castile soap, or flax-seed water, made by turning boiling water on flax-seed, letting it stand until blood-warm, then strain out the seeds. A pint of linseed oil and a quart of warm water makes an excellent injection. If chloroform is not at hand, a pint of warm water in which as much salt as possible has been dissolved may be used as a substitute. This can be administered by a drenching horn or long-necked bottle.

Another remedy is one ounce of sulphuric ether, one ounce of laudanum, and a pint of raw linseed oil. At the same time an injection as above given will prove very beneficial in getting the bowels to act promptly. If this does not give relief in an hour the dose may be repeated. A very simple as well as excellent remedy, and one always at hand, is a tablespoonful of saleratus mixed with a pint of sweet milk and given at a single dose.

Some veterinarians use spirits of turpentine in cases of colic, but we consider it very objectionable, as having a tendency to irritate and inflame the throat and bowels, especially if there is any tendency to inflammation of the bowels, as is usually the case. As previously stated, clysters will greatly aid in exciting the bowels to action. Rubbing the bowels with the hand or a warm flannel cloth is also good. Occasionally walking the horse about (never trot or gallop him) will also serve the same purpose. Bleeding is sometimes resorted to when other remedies fail, but we believe that where good may be done once in a thousand cases, injury would result in the nine hundred and ninety.nine.

Flatulent colic, sometimes called "windy colic," is a very different form of disease from spasmodic colic, and is caused by an inflation of the bowels with gas produced by the fermentation of undigested food in the stomach or large intestines. This distention is sometimes so great as to cause strangulation or a rupture of the colon or caecum, producing instant death. The cause is usually overloading the stomach, or giving improper food in large quantities. The symptoms at first are similar to those of spasmodic colic, but after a time (generally from twelve to forty-eight hours), if not previously checked, the belly becomes considerally increased, being most prominent on the right flank.

Treatment for flatulent colic.-Relief will be effected by the getting rid of the gas that is inflating the bowels. For this purpose try first an injection, and if it results in the escape of 
gas. good will have been accomplished. If the animal seems constipated, a dose of oil or aloes may bring rehief. Walk the animal about, but do not permit him to exercise violently, such as rolling or trotting, as in severe cases of distention there would be danger of rupture. In severe cases, where delay might prove hazardous, and all other remedies fail, the truchar is sometimes used for the escape of the gas, the puncture being made in the middle of the right flank. No one should however resort to this remedy, unless skilled in its use.

Corns. - These are found in the angle of the hoof between the bars and quarters, near the heel, and are generally caused by improper shoeing, or the shoe being worn too long, which results in the hoof growing over the shoe, bringing the pressure of the weight upon the sole. Placing the shoe on in such a manner that the pressure comes upon the sole will also cause corns. An established corn is very troublesome, and will sometimes produce permanent lameness. Horses with low, weak heels and thin, flat soles are apt to have bruises of this kind. Old corns are difficult to cure, but those recently formed yield more readily to treatment, frequently disappearing with proper shoeing, that will remove the pressure from the part affected. A bar shoe that relieves the bar from pressure, and throws it upon the frog will often prove highly benefieial. This should not, however, be worn for more than two successive shoeings. The corn should be pared out very thin, and dressed every day with the following mixture: 1 drachm of chloride of zinc, $2 \mathrm{oz}$. of glycerine, and $6 \mathrm{oz}$. of water. Upon cutting away the horn over the corn, a red or dark purple spot will be found, the latter showing the corn to be a deep-seated one. After paring the part, it can be determined whether there is any matter or blood underneath; if there be any, a small opening should be made through the horn for its discharge.

When suppuration has taken place, a poultice should be applied until the corn is softened, after which the horny portion above it can be removed. The eorn should then be kept dressed with a solution of one grain chloride of zinc to one ounce of water.

In all cases the most important part of the treatment is to remove the cause of the diffculty, by proper shoeing;

Cough.-A chronic cough in a horse is difficult to cure, and frequently leads to other troubles.

Strep half a pound of flax-seed in boiling water, and when nearly cold mix both seeds and tea with his food every day, for a week or so. Give him also a tablespoonful of the following once or twice each day: extract of belladonna $1 \mathrm{oz}$; powdered muriate ammonia, $3 \mathrm{oz}$; powdered liquorice, 5 oz.; powdered sal nitre, 2 oz.; honey, 1 lb.; Barbadoes tar, 1 lb. ; powdered ginger, $\frac{1}{2} \mathrm{oz}$. Mix thoroughly before using.

The best way to administer it, is to cover an old bit with a piece of cloth well fastened on, and smear it with a tablespoonful of the paste, before putting it in the mouth. The horse will suck it off in half an hour or so, after which remove the bit.

Another remedy, often very effectual: $1 \mathrm{oz}$. balsam of fir, dissolved in two ozs. sweet spirits of nitre, to which add 4 ozs. of the syrup of garlic; dose, a tablespoonful night and morning.

A simp'e, and sometimes very quick, remedy is found by steeping 2 parts liquorice root, 2 parts slippery elm bark, 4 parts flax-seed together, and giving two or three times a day.

Crib-Biting.-This is a vice that is sometimes acquired by a colt when teething, or by horses that are too highly fed, and but little used. It not unfrequently happens that horses that are not sufficiently fed will form the habit, from not having their hunger appeased. Sometimes it seems to be from mere idleness, and not having anything else to do the horse bites his manger.

When once the habit is well established, it is a difficult one to cure, and an inveterate cribber may be looked upon as a hard case. 
Horses will also form the habit by imitation, from seeing others. A young horse may sometimes be cured by turning him out in a pasture for several weeks, and not permit him to occupy a stall during that time. By this means the habit will be frequently forgotten. A strap buckled rather tightly round the neck, and thus compressing the wind-pipe, is also pretty sure to check the evil, and frequently to cure it; but it is an objectionable one, as it must be constantly worn to prove effectual, and its pressure is apt to bring on a greater evil, which is an irritation of the wind-pipe, and which leads to roaring.

Muzzles and certain patent halters have also been used to some extent; also covering the wood work with sheet-iron, etc.

The best'remedy that we have ever known, is to mix cayenne pepper and brown soap together, forming a paste, and apply it in numerous places within reach of the horse, and especially to those parts of the manger or wood.work wherever the marks of his teeth are discerned. It should be lightly applied, and the place disguised by having a little dust sifted over it, so that he will not be able to distinguish its locality. He will soon evidently entertain the opinion that the whole wood-work tastes the same, and let it entirely alone.

A five-ring halter with a piece of sheet-iron or zinc cut in the shape of a heart, and so attached to the halter that when the neck is arched in the act of cribbing, the point of the piece will prick the skin under the lower jaw, will frequently cure the habit.

Always keep a little rock salt within their reach, where they can get it when they wish.

Curb.-This is one of the numerous diseases of the hock, and consists of an enlargement of the back of it, three or four inches below the point, and results from a strain to either the ligament which binds the tendons or of the sheath of the tendons. Any sudden action of the animal that brings a strain upon this part, such as leaping, severe galloping over uneven ground, or a violent check while galloping, will he liable to produce it. Pulling a horse up suddenly on his haunches while going rapidly at any gait will also not unfrequently cause it.

It will usually produce lameness and may justly be considered unsoundness, although some horses will have curbs for years without being lame. It may be best detected by a side view of the leg.

The inflammation should first be reduced by frequently bathing the part in cold water, or in vinegar, in which a little salt has been dissolved. A wet bandage is good if it can be made to fit the leg, but it is difficult to keep a bandage on without obstructing the movement of the joint.

It is very essential that an animal thus strained should be allowed perfect rest for a time. Arnica is also valuable for relieving the soreness. Cold water in which saltpeter has been dissolved, applied sufficiently to keep the leg constantly wet for a few days, is used very successfully by some veterinarians.

In cases of long-standing blistering may be necessary, and the same treatment given as for ring-bone.

Diabetes.-This disease is rare in the horse, and when once fully established is regarded as incurable. It may, however, be cured in its early stages, by removing the cause, which is generally the use of strong diuretics or unwholesome food, that has a tendency to induce an increased action of the kidneys. The hay and oats should be of the very best quality. Mow burnt hay has a tendency to produce this disease. The administering of powerful nostrums, which act upon the kidneys, as is frequently done by many ignorant persons having the care of horses, (and usually without the knowledge of the owner,) will sometimes prove the secret of this difficulty. In the first place, in this disease, the most careful attention should be given respecting the quality of the food. 
Carrots and other green food, such as potatoes, are very good, given with oats and bran. Flax-seed steeped in water for several hours, should be liberally given. In connection with this, give one drachm of iodine of iron, and from ten to twelve grains of opium once a day. A moderate dose of opium administered twice a day is also an excellent remedy in some cases.

Bleeding for this disease, according to the old time method of practice, is one of the worst things that could be done, and should never be permitted under any circumstances.

Diarlhea.-Flax-seed gruel mixed with the food is very soothing to the bowels in cases of diarrhea or dysentery. In connection with this give 1 oz. prepared chalk, 12 grains pulverized opium; tincture of aconite root, from fifteen to twenty drops. Mix in a little water and give in one dose. If relief does not follow in three or four hours after administering the above, repeat the dose. Feed with good hay and oats, giving a warm bran mash (wheat) every other day; also every day a half pound of flax-seed that has been steeped in hot water several hours.

Another very good remedy, where the disease assumes a chronic form, is to give one of the following balls every day, until three are used; afterwards two the next week, and one the week following: Barbadoes aloes $16 \mathrm{dr}$.; nitrate of potash $18 \mathrm{dr}$.; sulphate of iron $2 \mathrm{oz}$; powdered gentian $2 \mathrm{oz}$; powdered capsicum $3 \mathrm{dr}$., and Venice turpentine sufficient to make into six balls.

Distemper.-This is a kind of contagious disease among horses, characterized by a swelling between the bones of the lower jaw, and which generally terminates in an abscess. Horses that have it should be kept apart from all others. Feed warm bran mash and green food principally; keeping the animal warm and comfortable, while at the same time, the stable should be well ventilated, also kept clean and free from offensive odors.

In some cases it may be necessary to poultice the abscess until it breaks, or is ready to be opened. After the abscess has discharged, the appetite will return. The animal will then require a generous amount of nutritious food to recover its former condition and strength. Rest and good nursing are better than medicine in such cases.

Epizootic. - This disease has been known also under the names of contagious influenza, epidemic catarrh, contagious catarrhal fever, etc. It is estimated that from the time of its first appearance, which was in Canada in Septomber, 1872, and during the winter of 1873, the epizootic influenza destroyed 1,500 horses and mules in New York city, or about 4 per cent. of the entire number it contained. The immediate cause of this disease is, with. out doubt, blood poisoning from germs floating in the air, and it generally proves most malignant and contagious in large, overcrowded and ill-ventilated stables.

The early symptoms of this disease are a general weakness, hanging of the head; shivering of the body and trembling of the limbs; watery discharge from the nose, and sometimes a watery appearance of the eyes; loss of appetite; staring hair; weak, quick pulse and rapid breathing. A horse thus affected seems reluctant to lie down, and stands in an unnatural, strained manner, his legs seeming to prop up his body. The bowels are generally somewhat constipated, and the urine much less in quantity than in health. The discharge from the nose becomes of a white, yellow, or greenish hue, and is quite copious. In some cases the throat becomes very sore and ulcerated, the swelling showing externally, the animal refusing to eat.

It is always best in the first place, since prevention is much easier than cure, to avoid, as far as possible, all diseases of this nature by allowing the animals an abundant supply of pure air, and the observance of other sanitary conditions, which can only be secured through proper ventilation and cleanliness of the stables. The following methods of treatment are condensed from that recommended by Dr. James Law, of Cornell University. 
In the treatment of this disease everything that has a debilitating or depressing tendency should be carefully avoided, such as bleeding, purging, unduly stimulating the kidneys, vio. lent blistering, depressing sedatives, etc. In the regular and uncomplicated form of this disease, nearly all suffering with it will recover without the use of medicine under good nursing, and with an abundant supply of pure, fresh air. The animal should be placed in a cool, dry box that is well ventilated, and provided with a good, clean litter, clothing him comfortably in order to avoid all tendency to a chill, and bandaging his legs loosely. The skin should be carefully brushed or curried, the clothes changed two or three times a day, and the animal kept quiet and still, except perhaps giving him a little exercise in the shelter and sunshine. Feed with bran mashes, boiled oats or barley, turnips, carrots, or other roots in small quantities and often, so as not to cloy the appetite. For drink, a quart or two of water nearly cold, or cold oatmeal or flax-seed gruel, should be frequently given. The action of the kidneys and bowels should be moderate, but never excessive. It will be better to obviate a tendency to costiveness by frequent injections of water that is blood-warm, three or four quarts at a time, - or by a half pint of molasses, or three ounces of sulphate of soda added to the same quantity of water.

If necessary to give a laxative, it should be done with care, the dose rarely exceeding one-third the usual quantity, as violent purging would be very hazardous. Mild febrifuge diuretics may be used to advantage, such as spirits of nitrous ether in half-ounce doses given twice a day, or liquor of acetate of ammonia in ounce doses four times a day, in the watergruel drank. When the cough proves especially violent and painful, a drachm each of anodynes, such as belladonna and camphor, may be added to the above diuretics with advantage.

The cough may be further relieved by causing the animal to inhale warm water vapor several times a day for an hour, which is most conveniently done by saturating chaff bran, or other simple agent, with boiling water and placing it in a nose-bag, which is hung on the animal's nose by means of a strap crossing behind the ears. Counter-irritants often prove beneficial. If, however, inflamed and sore throat seem extreme, a poultice may be applied with advantage a day previous to blistering, or the throat may be well fomented with warm water for an hour, and then wrapped in a sheep-skin with the wool turned inward. Oil of turpentine, which has been largely used as a counter-irritant in many cases, is objectionable on the ground of causing so much local irritation without blistering, as to drive some excitable horses almost to distraction.

The throat and chest where the disease has been located may often be greatly benefited by producing an irritation by rubbing in a thin pulp of ground mustard and water, and then covering up. This may be replaced by a soap liniment, composed of six ounces of soap, three of camphor, and a pint each of proof spirit, liquor ammonia, and linseed oil, to be applied frequently at short intervals, and be well rubbed in. If a more active blister is wanted, an ointment may be used composed of a drachm and a half of powdered cantharides, a scruple of camphor, ten drops of spirits of wine, and an ounce of lard. The hair should be cut off and the blister rubbed in, in a direction contrary to that of the hair. 'After it has acted, the skin should be kept soft and pliant by rubbing it with fresh lard.

Any of these irritants should be applied to a limited space only, and not exceeding the bounds of the inflammatory action, as the best results are thus secured. For this reason a careful examination of the chest especially, should always be made before making such an application. As the mouth becomes cooler and more moist, and the pulse softer and less frequent, a more stimulating treatment is desired. At first, two drachms each of gentian, powdered cinchona, niter and sal ammoniac may be given night and morning, or if the debility is very great, the last-named agent may be replaced by four drachms of carbonate ammonia made into a bolus with linseed-meal, or dissolved in a half pint of water and repeated three or four times a day. 
In cases marked by a daily remission, a dose of thirty grains of sulphate of quinia may prove effectual in preventing the paroxysm, if given an hour or two before the period when it was in the habit of appearing. During convalescence, gentian, cinchona, and other tonics are desirable, with alcoholic, ammoniacal, or other stimulants, if there is much debility or prostration. The diet should be tempting and nutritious, supplied often, fresh and frequently varied, care being taken at all times to counteract any sudden suppression of the bowels and kidneys, or even the nasal passages.

Farcy.-Farcy and glanders are regarded as but different types or stages of the same disease; there is, however, a great difference in their symptoms and progress. They both are caused by blood poisoning, and are generally due to bad stable management, the neglect of proper sanitary conditions, etc. They are liable to be found in poorly-ventilated stables, where many horses are crowded together. Absorption of the virus from glandered or farcied horses is also another cause of farcy, as it is very contagious. This disease is generally characterized by a rough, unhealthy-looking coat, the swelling of one limb, sometimes the swelling of the head, especially the muzzle, loss of flesh and strength accompanied with eruptions on different parts of the body, etc., which break and discharge; in fact, it would be almost impossible to describe, in a limited space, the many forms that this disease assumes in different stages, and in different cases.

Farcy differs from glanders in being much more slow in its progress than the latter, and in involving the lymphatic system, while glanders attacks the nasal mucus membrane. Both are regarded incurable, although in a mild form of farey, nutritious and generous diet, green food, and remedies that may be employed. will prove beneficial to a certain extent. Carrots are excellent to form a part of the diet in such cases.

Farcy is very contagious to man as well as horses, and those having charge of animals thus affected cannot be too cautious with regard to becoming infected with it. For this reason, many of the best veterinarians recommend, when it is ascertained for a certainty that a horse has this disease, that he be killed at once, and the body deeply buried, that no animal may be contaminated by it. From five to ten grains of hydriodate of potash given daily in connection with three drachms of sulphate of iron and two drachms of gentian, have been found beneficial, at the same time using an external application to the swollen parts, of equal portions of mercurial ointment and lard, well rubbed in.

Another method of treatment is as follows: - A tablespoonful of sulphite of soda once a day for a week, to be followed by a tonic to improve the general condition of the animal, consisting of pulv. copperas $\frac{1}{4} \mathrm{lb}$., pulv. gentian $\frac{1}{4} \mathrm{lb}$., pulv. fenugreek $\frac{1}{2} \mathrm{lb}$., pulv. elecampane $\frac{1}{4} \mathrm{lb}$. Mix thoroughly and give a tablespoonful once a day. Everything used about the diseased animal, whether in clothing, grooming, or feeding, should be kept from all healthy animals, while it is of course equally essential that the animal himself should be kept apart from others. This, disease is very deceptive, and it frequently happens that when the symptoms are most favorable, the ulcers healed, and the swelling disappeared, that it will break out again and prove fatal in a short time. (SEE Glaxders.)

Fistula. - In horses, a fistula is most liable to occur upon the withers or top of the head; in the latter case it is called by farriers poll-evil. It is a deep, chronic abscess which discharges pus through fistulous pipes to the surface. They are generally caused by blows, galls, or strains, and are usually formed from wounds of long standing, neglected sores, etc., and are most frequently found in old, overworked, and poorly-kept horses.

In old cases, where the bone is involved, cure will be impossible, and the animal had better be humanely killed, and put out of misery. If the bone has not been reached by the abscess, the pipe leading from it should be cut open carefully with a sharp knife, the direction of the pipe being determined by a probe, and the wound washed thoroughly with 
warm water. Wash two or three times a day with the following mixture: chloride of zinc, $1 \mathrm{dr}$; ; carbolic acid, $3 \mathrm{dr}$.; water, one pint. Sometimes a thorough application of hot caustic solutions is essential before using the wash, in order to make the flesh heal.

Founder (Laminitis).-This is an inflammation of the laminn of the horse's foot, and may be caused by overdriving, exposure to cold when perspiring, thus suddenly checking perspiration, overieeding, or giving food and cold water in large quantities too soon after excessive work; long continued driving on hard pavements or frozen ground, improper slioeing, driving through a deep stream or river when warm, etc.

This disease has two forms-the acute and chronic; the former may be cured, if properly treated, and taken in season, but the latter is considered incurable, although it may be con. siderably relieved. This disease resembles rheumatism in many respects, and, like that, the acute form is attended with fever and pain. The fore-feet are generally affected, and sometimes the muscles of the chest.

A foundered horse will be known by his peculiarly mincing gait, as though it hurt hirn to put his fore-feet upon the ground; by a hot, contracted hoof; by resting his fore-foot upon the toe, and also by keeping his fore-feet stretched out as far as possible, so that the weight of the body will rest on the heel, instead of the toe.

Treatment of Acute Founder.-Perfect rest is essential. Give the horse a large stall, and a good deep bedding of clean straw. He may thus be induced to lie down, which will relieve the pain of the feet by removing the weight from them, and aid in producing a cure. Where the inflammation is very great, it may be necessary to sling the horse up so that his hoofs will not touch the floor.

The shoes should also be removed. Some veterinarians apply warm poultices to the hoofs, but we prefer cloths wet in ice water, applied frequently, to be continued for several hours, and occasionally for two or three days, if necessary. This reduces the inflammation and pain. From fifteen to twenty drops of tincture of aconite should be given in about half a pint of cold water every four hours, until six doses have been given. Give mashes, grass, carrots, or potatoes, with oats, for a few days; also all the water, and as often as the animal will drink. Then recovering, and well enough to exercise some, turn the horse out to grass to remain until the cure is completed.

It is also a good plan to let the horse stand in about six inches of sawdust, wet a little once or twice a day, for two or three weeks, when recovering from acute founder, or in chronic founder. This gives a soft floor to stand upon, and keeps the hoofs moist and soft.

Treatment for Chronic Founder.-Nothing can be done for this form of the disease, except to relieve it somewhat. The entire hoof should be frequently wet; also kept well oiled with castor or linseed oil. The oiling should extend up to the skin, and cover the sole. A soft floor, such as earth, sawdust, or tan-bark, occasionally wet, should be provided in the stable. A protection of sole-leather, or similar substance, sometimes proves highly beneficial by being put on over the sole before setting the shoe, allowing it to come to the edge of the wall of the hoof, so that the nails may be driven through it in fastening on the shoe. A flling of cotton, saturated with tar and oil, should be put in between the leather and the sole.

Fracture.-In surgery, fractures are distinguished, according to their nature, by the terms simple, compound, complicated, comminuted, etc. A simple fracture is when the bone only is divided, and there is no wound to admit air to the seat of the fracture. By compound fracture is meant that there is such a wound. A complicated fracture is one in which some other serious injury is inflicted near it, such as the rupture of the osseous tissues, important nerve trunk, or blood vessel, by the broken bone, or when the fracture extends into the cavity of the joint. 
A comminuted fracture is where the bone is broken or crushed into several small pieces. In cases of simple fracture a horse may (with proper treatment) so far recover from the injury as to be made useful for many purposes, and when such an accident happens to a raluable animal, it will pay to take the trouble to accomplish it. In the other forms of fracture mentioned, it will generally be useless to attempt a cure, and the animal bad better be killed at once, and relieved of his suffering.

The treatment of a fracture should be such that the portions of the bones broken shall be restored to their original position, and held there by some means that will keep them secure in place until they have united, and at the same time will not cause discomfort or injury to the patient.

A friend of the writer had a valuable pet horse that had its leg broken abore the knee, by accidentally falling off a steep declivity while running in the pasture. Although many advised killing the animal, the owner, being greatly attached to him, did not follow the advice, but had the limb properly placed and bandaged, and the horse taken to a large spreading apple tree in the orchard,--it being summer,-and slung up to one of the strong branches, just enough to relieve the limbs of the weight of the body.

A hollow was then made in the ground directly under the broken leg, to prerent the possibility of its hitting anything. An awning tent was constructed about him, so as to keep off the storm, and at the same time to be open on all sides in pleasant weather. This anmal recovered to the extent that he has been used for a carriage-horse for several years, there being no indication of the former injury except that the broken limb is not quite as strong as the others, but even this is only detected when he has become rery tired from an unusually long journey.

In setting a fractured limb, the bones should be placed in their former position so that when grown together the limb will be of the same length as the corresponding one, and also to secure the most strength possible. The broken surfaces should be brought closely together. It is a good plan to apply a coating of tar to the leg a few inches above and below the fracture; then wrap the whole leg in cotton-batting, just enough to keep the splints from hurting the skin, and place over this, splints cut from wood, that will rest erenly upon the leg. There should be four or five splints placed about the leg in such a manner as to keep the bones in place. All uneren spaces under them should be flled with cotton. Orer these, carefully wrap strong bandages several times around, and secure them by pins or soft cord, so that they shall not become loosened. The bandages will require being sufficiently tight to hold all securely in place, and at the same time should not be so tight as to prevent a proper circulation of blood in the limb. The splints should be carefully examined every day to sec that they have not become displaced.

A little tincture of arnica poured orer the fracture will relieve the soreness, and it may be well to give the animal from twenty to twenty-five drops of tincture of aconite root every fire or six hours during the first twenty-four, to lessen the ferer and pain somewhat. The horse should have plenty of nutritious food, and all the water he will drink, if giren frequently. Green food, such as fresh grass, carrots, turnips, or potatoes, should be giren in connection with oats. A bran mash should also be given occasionally.

When a horse thus injured is kept in a stable, it will be well to make a hole in the floor underneath the foot of the broken leg, to avoid its touching anything, and admit of its hanging in a natural position. It is considered unnecessary by some to sling the horse up to relieve the limb in case of fracture, but we regard it as a sure precaution against anj displacement of the bones, which would be liable to occur if this were not done. In slinging the animal, he should not be entirely elerated from the ground, but to the extent that he may be able to relieve himself. and rest from standing. The broken limb should alwars hare the earth hollowed out under it, or some other means emplosed to permit it to hang free from all obstructions and obriate hitting against anything. 
Galls, which in farriery means sores produced by the friction of the saddle, or any part of the harness, are best remedied by removing the cause and see that every part of the harness and saddle fit properly, and there is no uneven pressure, since it is better to prevent such evils than to cure them. Apply twice a day some of the following mixture: Lauda. num, two ounces; tannin, two drachms. When partially healed, pulverized charred leather and lard, well mixed and applied, will aid in the healing process, and also prevent the hair from growing out white.

Washing the galled places with a solution of sugar of lead, or dressing with equal parts of mutton-tallow and beeswax, melted together, are also good remedies. In all cases, there should be allowed no pressure upon the part until it is perfectly healed.

Where there is a bability of an abscess forming, such as where lumps are formed or the skin callous, take out the stuffing from the collar directly over where the injury lies; this will relieve the pressure at that point. In all cases of galls there should be no friction on the part, during the healing process, by the wearing of the collar or harness. Give nature a chance to repair the injury and she will generally do it unaided.

An old stage-driver of long experience that we used to know, and who was noted for keeping his teams sound, always washed the shoulders and breasts of his horses as soon as the harness was taken off, using cold water in the summer and lukewarm water in the win. ter. After rubbing nearly dry, he washed them daily with a decoction of smartweed (Polygonum hydropiper) in the summer, when there was most danger of galled shoulders.

In the winter he used the smartweed only about once a week, and his horses never had sore necks or shoulders, though in constant and hard usage upon the road.

Glanders.-This is the worst disease with which a horse may be afflicted, and a glandered horse is a dangerous animal to have about, since the contagion is very easily communicated both to man and animals. There are instances of those having died of it who have had charge of horses with this disease. It is, therefore, the safer and better way to kill a glandered horse at once. The cause of this disease is lack of cleanliness, poor ventilation in stables, catarrhal diseases, reduced and weakened condition of the system, lung fever, and any disease that may generate pus, and which by being absorbed by the system poisons the blood.

The first stage of this disease is characterized by a dark hue of the nostrils, accompanied by a discharge of thin, transparent fluid. This may possibly continue for months. The discharge eventually becomes thicker, of a gluey consistency, and the lining membrane of the nostrils has sores upon it. This ulceration of the nose will generally extend into the throat. The coat will be rough and unhealthy-looking, and frequently the animal becomes hide-bound. The legs also swell, and the body wastes away in flesh. If the discharge from the nostril of a glandered horse should come in contact with a wound or a mucous suriace, such, for instance, as the lining of the nostril, it will produce a similar disease. The danger attending the persons having the care of such horses is so great, and the disease so very contagious to other animals that, as previously stated, no cure should be attempted, and a well-directed bullet through the brain will prove the best remedy in such cases.

Grease (Scratches). - This is an inflammation of the skin of the heels of the horse. It sometimes is found in the fore-feet, but more frequently in the hind ones. Swelled legs will sometimes degenerate into this disease. Grease is caused mainly by bad stable management; permitting the mud and dirt to dry and remain in the heels, cutting away the hair that gives a natural protection to the heels; wet and filthy stables; standing in the cold when the heels are wet; or washing them and permitting them to dry off by evaporation. Untrimmed horses are less liable to have them than those from whose legs and heels the hair has been closely cut. Some horses seem to have a constitutional tendency to this disease. There is 
generally a white, greasy discharge from the heels of the horse, accompanied with a hot and tender swollen skin, which cracks open and sometimes sloughs off, leaving a sore that is diffcult to heal.

Treatment.- Wash the parts with Castile soap and warm water, then rinse with clear warm water and wipe dry. Apply at once a solution of $30 \mathrm{grs}$. of chloride of zinc and one pint of water. After about ten minutes apply a liberal quantity of glycerine to the parts. If the case is very obstinate and does not yield to this treatment, increase the quantity of zinc to 40 or 45 grs., and apply as before. Feed the animal liberally, giving part green food.

A nother remedy that has proved very effectual in some cases is to wash the parts as before, and wet them three times a day with the following mixture: Sulphate of copper, 2 oz.; hot water, 1 pint; carbolic acid, $\frac{1}{2}$ oz. After a few days of the above treatment, apply the following twice or three times a day: Tincture of aloes comp., $4 \mathrm{oz}$; glycerine, $3 \mathrm{oz}$; tannic acid, $2 \mathrm{dr}$.; tincture of opium, $2 \mathrm{oz}$.

Grease takes many different types, and requires treatment accordingly. In cases that do not yield to local treatment, it may be well to give some simple alterative, such as $2 \mathrm{dr}$. of pure cream of tartar, $2 \mathrm{dr}$. of powdered saltpetre, and $4 \mathrm{dr}$. of flowers of sulphur. Such a dose may be mixed with the food every evening during the week, and continue every other week as long as may be necessary. At the same time some one of the above-mentioned remedies for treatment of the heels should be adopted, or the following: Wash the heels as previously recommended, and apply twice or three times a day this mixture: $2 \mathrm{oz}$. of crude carbolic acid, $2 \mathrm{oz}$. of glycerine, and $15 \mathrm{oz}$. of raw linseed oil.

\section{Gripes. (See Colic.)}

Heares.-This disease greatly lessens the value and usefulness of a horse, and is due to the rupture or enlargement of the air-cells of the lungs. It is either preceded or accom. panied by a cough, which is quite characteristic, being very short and followed by wheezing. When driven quickly for a short time, a horse with this disease breathes with difficulty, the inspirations being very short and rapid. This disease is hereditary, and no animal having it should ever be used for breeding purposes. Narrow-chested horses are more liable to it than those with broad, deep chests, since the latter admit of more room for the expansion of the lungs in breathing. Horses that devour large quantities of coarse food, are worked hard with their stomachs distended with it, and are greedy feeders, are very liable to have the heaves.

This disease cannot be cured, since it is impossible for any medical skill to repair the ruptured or enlarged cells of the lungs, but considerable may be done to palliate the evil. Attention to the quality of the food is of great importance. Instead of large quantities of food being given that require much room, such as coarse hay, etc., that which is nutritious and requires but small compass should be fed, such as a liberal supply of oats and a small amount of hay. Chaff is considered objectionable, as it is rapidly eaten, and thus distends the stomach too much. Carrots, potatoes, and other green food are useful in such cases. It is a good plan to wet the oats and also the little hay that is given. A half pound of flax-seed on which a gallon of boiling water has been turned and permitted to stand over night is also good to give frequently, mixing both the tea and seeds with the food. Bran mashes are excellent. The following, given in tablespoonful-doses daily, is good: Powdered licorice, 3 oz.; powdered nitrate of potash, 4 oz.; Barbadoes tar, $1 \mathrm{lb}$; mix thoroughly.

Hematuria (Bloody Criue).- The cause of this cannot always be determined, but it generally proceeds from some great strain or a severe blow. Sometimes pure blood is discharged, at other times it is more or less mixed with the urine. Perfect rest and cooling food are the very best remedies. 
Hide-Bound.-This usually arises from a deficiency in the quality or quantity of food, and also frequently occurs in lingering diseases that emaciate and weaken the body. In this disease the skin adheres to the body. Nutritious food in liberal quantities and variety should be given. Wheat-bran and corm meal made into a warm mash is excellent. Cut hay, oats, carrots, potatoes, and other green food should also be fed.

\section{Influenza. (See Epizootic.)}

Inflammation of the Bladder.-Inflammation is sometimes located in the body of the bladder, and, again, only in the neck of it. The symptoms are quite similar to those of inflammation of the kidneys. The causes are generally the presence of some irritating matter in the urine, or stone in the bladder. If there is considerable fever, give one scruple of aconite every two hours. Where there is much pain, the same quantity of belladonna may be given. If the evacuations of the bladder are obstructed, the careful use of the catheter should be employed. Difficulty in urinating is sometimes due to a foul sheath, and can frequently be relieved by cleansing the same properly with warm water and a sponge, and giving small doses of nitre.

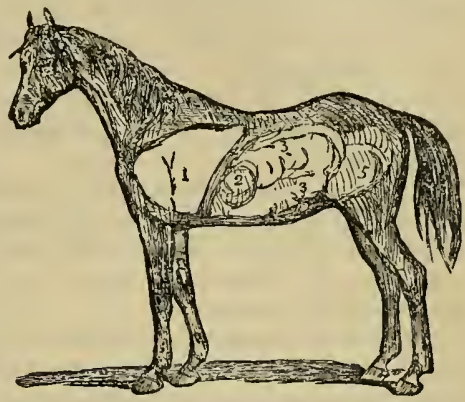

The above cut shows the location of some of the internal organs of the horse. Fig. 1, the lungs; 2 , the stomach 3 , the colon; 4 , the diaphragm; 5 , the bladder.

Inflammiation of the Bowels.-This disease is one which frequently terminates fatally. The causes are various, and may be exposure to the cold, drinking cold water in large quantities when warm, overdriving, diarrhea, constipation, and sometimes it follows violent attacks of colic. The symptoms are very severe, and continuous pain in the bowels. It varies from colic in this respect, the latter being intermittent with intervals of rest. The general difference in the symptoms between this disease and the latter have already been given under CoLIc (which see).

When constipation seems to be the cause, a quart of raw linseed oil should be given. It would also be well to give injections of warm flax-seed tea, or thin gruel, from which the seeds have been carefully strained. These can scarcely be too copious, for the sooner the bowels can be made to act without irritating them, the better. If the disease proceeds from diarrhea, or over-purging, give an ounce of the tincture of opium, and twenty-five drops of the tincture of aconite in a half pint of water. Give for drink, flax-seed gruel. If the cause is other than those mentioned, give from twenty-five to thirty drops every three hours, also injections of thin flax-seed gruel, or warm water, (the first-mentioned is best,) until the bowels move. The belly should have blankets wrung out in hot water applied, and renewed as often as every fifteen or twenty minutes, rubbing on first over the belly with the hands carefully ground mustard made into a thin paste with vinegar. This will act very quickly as a counter-irritant, and will have a tendency to relieve the internal inflammation.

Some veterinarians recommend bleeding, and there is quite a diversity of opinions respecting it in this disease; we are inclined to the belief that in the large majority of cases bleeding would be objectionable. Clysters of gruel should be continued for two or three days in some cases, in order to keep the bowels open and free.

The limbs should be rubbed with the hands to promote circulation, also bandaged to be kept warm. The body should be kept warm, though not uncomfortally so, but the air of the stable should be cool and pure. Avoid drafts of air in securing good ventilation. Bran mashes and flax-seed steeped in hot water, together with green food should be given. 
Oats and hay should not be allowed during the attack, but may be given gradually, a little at a time, as the horse gets better. Anything that would have a tendency to irritate the bowels should be carefully avoided.

Inflammation of the lidneys. - This is by no means a rare disease in horses, and it is also one which is usually treated more unskillfully than almost any other. Some of its causes are the improper use of nitre or other diuretics, poor food, over-work, exposure to cold after being heated, or under the saddle a long time, being out in a cold storm with the rain dripping upon the loins; also a strain in the loins.

The symptoms are short, quick breathing, accompanied with fever, quick pulse, disinclination in the animal to walk or move the hind legs, but when he does so, keeps them unnaturally wide apart. The urine will also be scanty and dark-colored, frequently bloody. A pressure with the hand upon the loins over the kidneys will prove tenderness and soreness in this locality, by the mauner of the animal.

Clothe the horse warmly, and avoid drafts of cold air. Apply a paste of ground inustard wet with vinegar to the loins, by rubbing it into the hair with the fingers; afterwards keep clothes wet in hot water upon the loins; or a sheep-skin with the skin side inward may be kept there, to induce perspiration in that locality, a fresh one being applied after a few hours. as the first becomes wet.

Give all the flax-seed tea the animal will drink, and keep the bowels open by injections of the same, or warm water. About twenty grains of powdered opium may be given two or three times at intervals of two or three hours during the first stages. If there is much fever, twenty drops of tincture of aconite may also be given with benefit, to be repeated a few times at intervals of four hours, until the fever abates. Rest for four or five weeks, and careful usage for some time will be necessary, as this discase is slow in recovery, and will be liable to return unless some such precaution is taken. Always allow plenty of cool drink to the animal with this disease.

Inflammation of the Lungs (Lung Fever-Pneumonia).-This disease is usually caused by taking a sudden cold, such as may be contracted by the horse on being taken from an ill-ventilated, warn stable, and driven against a cold, sharp wind, or in a snow or cold rain storm; a change from a warm stable to a colder one; standing exposed to a draft of air in the stable; exposure to cold after being heated by hard work or driving; turning the horse out to pasture early in the spring before the weather has become sufficiently warm, working the animal when he is not in proper condition, or putting too much hard work upon one that has not been accustomed to it.

The first symptom and the surest in this disease is a chill, the animal almost invariably being taken with an attack of shivering, and seems to be cold all over. This soon passes off and is succeeded by a general warmth of the body, or more commonly by a fever heat, and cold legs and ears. The pulse will be quick and wiry, and the breathing difficult. A peculiar crepitating sound will be heard from the lungs, if the ear is applied to the chest or neck. The horse has a generally dejected air, drops his head, and does not seem inclined to he down, or change his position.

The animal should be kept warmly clothed, and the legs bandaged, but the stable should be supplied with pure fresh air by the most perfect system of ventilation, which will be an abundance of pure air without exposing the animal to a draft.

Keep the bowels open by giving part green food, such as carrots, turnips, and potatoes, if the animal will eat,-feeding little at a time and often; if not, and there is any costiveness, a moderate dose of raw linseed oil may be given (not castor oil). Give plenty of water, or, what is better, flax-seed tea. Rub a mustard paste, made of ground mustard and vinegar, on the sides and chest, in the locality directly over the lungs (which can be determined by the 
previous small cut, showing position of the internal organs), -and give from twenty-five to thirty drops of tincture of aconite root in a half pint of cold water until five or six doses have been given, at intervals of four or five hours.

Hay tea, oat meal gruel, warm bran mashes, and raw eggs may be given with benefit in severe cases. Coarse food, such as hay, should not be allowed until the animal is somewhat relieved.

Keep the animal loose in a large box stall, with plenty of clean bedding. He should be kept quiet and undisturbed. When he lies down (unless it be from very weakness), it may be regarded as a favorable symptom. Do not make him get up after he has lain down; rest will do him more good than medicine.

Great care will need to be used in feeding as he gets better, in order not to overload the stomach, but to give a sufficient amount of nutritious food to strengthen and build up the animal. It should be in small quantities and often, for some time. This is a slow disease, and its effects are very exhausting and lingering, and there is a liability of its recurrence unless great caution is taken against taking cold. Chest protectors for horses that are driven in cold, windy, or stormy weather would be of much utility in preventing this disease.

Laminitis. (See Founder.)

Lice.-The skin of the horse is extremely sensitive, and if for any reason it becomes infested with lice, the irritation and annoyance will be enough to almost distract a nervous animal.

Horses that have been sick with a lingering disease, and are in a run-down condition, or that have a skin disease, will sometimes be troubled in this way. Or if the stall be near where fowls are kept, this may be the cause of the difficulty. Anoint the skin with hinseed oil, sweet oil, or melted fresh lard, and after an hour or two wash off with castile soap and water, taking a portion of the body at a time, afterward rinsing with clear water, and wiping dry.

\section{Lockjaw. (See Tetanus.)}

\section{Lung Fever. (See Inflammation of the Lungs.)}

Mange (Itch). - This disease usually makes its first appearance on the neck at the root of the mane, and unless treated in season will be liable to extend upward to the liead, or down to the withers and back, sometimes extending over the whole body. It is an eruption which causes intense itching, and after the breaking of the watery vesicles the hair falls off, leaving a bare spot covered with scurf. It is a very contagious disease, and is originally caused by neglect of cleanliness, and lack of nutritious food. Cattle and horses with this disease will communicate it to each other by the least contact. The disease is produced by a very small insect which burrows in the skin, causing the irritation and destroying the hair follicles. The most simple, and one of the best methods of treatment, is to wash off the scurf from the skin with castile soap and lukewarm water, and if there are any scabs formed, soak and break them up so that the ointment applied may reach the insect readily. Wipe dry and rub thoroughly into the skin an ointment of equal quantities of sulphur and lard, well mixed.

It may sometimes be necessary to give in the same connection two or three ounces of sulphur mixed with the food. The ointment should be repeated once a day until a complete cure is effected, being washed off with castile soap and lukewarm water after it has been on five or six hours. It is a good plan to let the animal be in the warm sun for a time after it has been applied.

Carbolic acid washes, a decoction of tobacco, and other remedies are frequently used with good effect, but the sulphur ointment is to be preferred. 
Megrims. - This disease is a mild form of apoplexy, and is a pressure of blood upon the brain, caused by an unusual flow of blood to that organ. Various causes contribute to this disease, such as excessive exercise in a hot day, violent blows upon the head, tight collar or check-rein, the two latter of which would prevent the blood from circulating freely, and from returning from the head, which would unduly distend the blood-ressels of the brain. Youatt describes the symptoms as follows:-

"When the horse is driven rather quickly, he will, without any premonitory symptoms, suddenly stop, shake his head, and exhibit evident giddiness, and half-unconsciousness. This will soon pass over, and he will go on as if nothing had happened.

When the attack is more serious, he will fall without the slightest warning, or suddenly run round once or twice, and then fall. He will lie insensible, or struggle with the utroost violence. In five or ten minutes he will begin gradually to come to himself; he will get up and proceed on his journey, yet somewhat dull, and evidently affected and exhausted by what had happened, although not seriously or permanently ill.

A predisposition to a second attack almost always remains, and it is a long time before the blood-vessels recover their former tone. Experience has shown that a horse that has had a second attack of the megrims is nerer to be trusted."

The treatment should be modified according to the cause of the difficulty. Bleeding at the moment of attack should be resorted to, in severe cases. If the animal is constipated, a mod. erate dose of physic should be administered. Rest and careful nursing for a while, together with judicious feeding, will be better for the animal than drugs, in the majority of cases. A covering worn over the head, but not resting upon it, will prove rery beneficial in protecting the brain from the hot sun. These are supported by a wire and fastened to the head-stall, and are much used on draft-horses in nıany localities.

Navicular Disease. - This is sometimes called "foot disease," and is an inflammation of a small bone in the interior of the foot, called the navicular bone. Improper shoeing, and orerwork on rough roads and hard parements, are the principal causes. It may be best determined by an unnatural degree of heat in the foot, although the symptoms are somewhat obscure. Remove the shoe and soak the hoof in water that is quite warm. from half to three-quarters of an hour every day. In some cases it may be necessary to poultice the foot to further relieve the pain and inflammation. The horse should bave a long period of rest, extending from two to six months. It is a disease that is slow of recorery, while if there be a relapse, a cure may be regarded as very doubtful. It is highly important that it receive attention in the first stages. A blister applied around the coronet will frequently prove highly beneficial, while in severe cases a frog-seaton should also be inserted, and a discharge encouraged by a daily dressing of it with the tincture of cantharides. In about three or four weeks the seaton may be remored, and another blister applied. The horse should then be turned out to pasture and permitted to run for three or four montlis. Particular care should be taken to have the horse shod properly after recovery.

Hygienic management in the stable is also very important in sucb cases. Some prefer a cold bath for the foot instead of warm, but we have always found the latter most beneficial.

Ophthalmia. - This is an inflammation of the eye, which is liable to recur at regular intervals, and frequently terminates in total blindness to one, or both eyes. It is sometimes called "moon blindness." The attacks are usually rather sudden; the lid becomes swollen, and water drips from the corner of the eye. The eye is very sensitive, and there is an arersion to the light. This disease frequently terminates in a cataract. Pure air and cleanliness in the stables are of the first importance, and any carelessness in this respect will increase the difficulty.

Treatment should commence during the first stages. Sometimes a seaton put in about 
an inch below the corner of the eye, will result in a cure. Thread a sharp darning-needle with bair from the mane, then pinch up a little skin a full inch directly below the corner of the eye, and push the needle through, drawiug the hair in, and tying a knot in each end to prevent the hair getting out. Leave it there eight or ten days, turning it a little each day. It may be necessary to grind the point of the needle to make it sufficiently sharp, and the hair should be left long enough at each end to take bold of readily. The food should be laxative, and the stable moderately dark, the head to be so placed that the light, either direct or reflected, shall not fall upon the face. Keep wet sponges on the eyes, and frequently bathe the hids with the following lotion:- Tincture of opium, $\frac{1}{2}$ oz.; tincture of belladonna, $3 \mathrm{dr}$.; powdered acetate of lead, I dr.; rose water, $2 \mathrm{oz}$; soft water, $12 \mathrm{oz}$.

Occult Spavin.-This is quite similar to bone spavin, except there is in it no perceptible enlargement, the location of the disease being within the joint. Because there is no enlargement of the joint exteriorly, this difficulty is often mistaken for hip lameness. It should be treated the same as Bone Spavin (which see).

Overreaching. - This frequently results from improper shoeing, and may be remedied by removing the cause. It consists in striking the heel of the fore-feet with the toc of the hind shoe, which sometimes makes a bad wound. It may be prevented by rounding off the inside edge or rim of the hind shoes. When a bruise has been inflicted by overreaching, apply tincture of arnica hiberally as soon as practicable.

Pink Eye.-This is a common term used to denote a form of influenza in which the eyes become bloodshot and swollen, accompanied by a watery discharge more or less abundant. It takes its name from the color of the mucous membrane of the eyelid, which is pink, bright red, or a very dark red, according to the degree of congestion of its blood vessels. Sometimes this inflamed condition of the eye is followed by the formation of pus, and the swelling of the limbs. It is also frequently accompanied by symptoms of lung fever, bronchitis, or an inflamed condition of the bowels, according to the type and severity of the disease, as well as the symptoms of influenza.

Rest and good nursing will do more for the animal than medicine. Care should be taken to observe the best sanitary conditions in stable management, and prevent the animal from taking cold. Judicious feeding is essential, while plenty of drink should be given. Small doses of saltpetre are very beneficial. Avoid a strong light in the stable. Bandaging the limbs to keep them warm, and blanketing according to the temperature of the external air, are of great advantage. The treatment should vary according to the type of the disease, as recommended in epizootic or influenza.

Poll Evil.-This is a fistulous ulcer that sometimes makes its appearance at the top of the head or just behind the ears of the horse. It may be caused by a violent blow, hitting or rubbing this part against anything with sufficient force to produce inflammation, or pulling against a tight halter. Straining the ligaments of the muscles by high checking or sudden tight reining will sometimes cause it.

The skin becomes bot, tender, and swollen, and a tumor frequently forms. The horse should not have a halter pressing upon the locality, but go loose in a shed or large box-stall. In the early stages, the inflammation should be reduced by keeping a sponge or cloth wet in cold water constantly on the parts, or a mixture of equal parts of strong vinegar and water with tincture of arnica added in the proportion of a quart each of the former, to two ounces of the latter. When the inflammation has been reduced, and there is hardness, indicating a tendency to tumor formation, apply daily an ointment of one drachm of iodine mixed thor. oughly with one ounce of lard. If the tumor suppurates, it will be best to open it, which should be done when the pus becomes near enough the skin to be felt by the finger slightly soft beneath. The opening should be lengthwise with the neck. 
When the pus has been discharged cleanse the wound and its surroundings with a sponge and warm water, and afterwards wash two or three times a day with a solution of sixteen grains of chloride of zinc to a pint of soft water. If it does not heal readily under this treatment it may sometimes be necessary to insert a seaton, as in cases of fistulas of long standing.

\section{Pneumonia. (See Inflammation of the Lungs.)}

Quarter-Crack. (See Sand-Crack.)

Ringhone.-This disease is so called because it constitutes a bony growth around the pastern bone. It frequently spreads quite rapidly, and not only involves the pastern bones, but the cartilages of the foot. It is generally found among heavy draft horses and such as are overworked on hard roads and pavements, although, like bone spavin, a tendency to this disease may be hereditary, and occasionally a young colt will be found with this bony enlargement. The treatment should be similar to that for bone spavin (which see).

Roaring.-This is an unnatural sound made by some horses when drawing the air into their lungs, especially while traveling fast, and results from some obstruction of the trachea or windpipe. It is due to various causes, the principal of which is the continued use of a tight check-rein, which keeps the head of the animal in an unnatural position, compressing the larynx until it becomes permanently distorted, and does not permit a free passage of air into the lungs. A tight throat-latch will also produce the same result. Chronic cough and catarrhal colds sometimes terminate in roaring. When this disease is once established it is difficult to cure. The best treatment that we can recommend is to remove the cause as far as possible by discontinuing the use of the check, and permit the throat-latch to be worn as loose as practicable. This disease may be regarded as hereditary.

Sand-Crack.-This is a separation of the laminæ of the hoof, forming a crack up and down its wall. When it occurs in the front part of the hoof it is called a "toe-crack," and when on one side, "quarter-crack." It usually begins at the coronet, the thin edge parting and extends until it often divides the entire hoof. It more frequently occurs in the inner quarters than elsewhere, and is often caused by improper shocing. It also indicates brittleness of the hoof, which is due to a certain extent to its quality, also to diseases in this locality, the frequent wetting and drying of the feet, dry, hot weather, impaired nutrition, overwork, low condition of the animal, and impure state of the blood.

This difficulty should receive attention in its early stages, and the animal given entire freedom from work. Nutritious food should also be given in liberal quantities, and the best sanitary conditions observed in stable management. We believe, in interfering as little as possible with a sand-crack, except to keep it free from sand and dirt. There should be no cutting, firing, or rasping, as sometimes recommended. An application of the solution of three grains of chloride of zine to an ounce of water twice a day, together with daily washing with Castile soap and warm water, will prove very beneficial. Castor or linseed oil applied to the external surface of the hoof will also counteract the brittle tendency of the hoof in a great measure. (See Britrie Hoof, on a previous page.)

If the animal is to be used, the crack should be filled with lint saturated with the solu. tion previously recommended, which should be kept in place with a piece of cloth covered with tar, which will keep out the gravel and other foreign substances. This should be removed and the crack washed out at night. If the crack extends through the crust so as to canse lameness, it will require considerable time to complete a cure, and such are serious cases. The more a horse is used that has a cracked hoof, the more danger is there of increasing the difficulty. It is a good plan to turn a horse ont to pasture,-if it occurs in summer,-and permit him to remain there until completely cured. In severe cases it may be necessary to apply a blister to the coronet, just above the crack. 
M. Defay, a French veterinarian, has discovered a preparation which he recommends for sand-cracks or fractured hoofs, and which he claims forms a hard and durable cement. The precaution necessary for its successful application, is to carefully remove all traces of grease in the crack and about the edges, with spirits of sal ammoniac, sulphide of carbon, or ether. As we have never tested its merits, we are not able to express an opinion as to its utility for this purpose. The recipe for the cement is as follows:-Take one part of coarsely-powdered gum ammoniacum, and two parts of gutta-pcrcha, in pieces the size of a hazelnut. Put them in a tin lined vessel, over a slow fire, and stir constantly until thoroughly mixed. Before the thick, resinous mass gets thoroughly cold, mould it into sticks like sealing-wax. The cement will keep for years, and, when required for use, it is only necessary to cut off a sufficient quantity, and remelt it immediately before application.

Seratches. (See Grease.)

Sore Shoulder. (See Galds.)

Sparill. (See Bone Spavin.)

Splent or Splint. - This is a bony growth, generally located upon the inside of the fore-leg of the horse, a little below the knee-joint. It is usually caused by overworking a young horse. The treatment recommended is the same as that for Bove Spavis (which see).

\section{Stuggers. (Sce MEgrius.)}

\section{Strangles. (See Distemper.)}

Strains or Spraius. - The wrenching or torsion of the muscles or tendons will generally be succeeded by pain, lameness, and swelling. Stonehenge thus defines strains of this nature:- "Muscular strains consist of an absolute tearing of the fibrous tissue composing the muscles; or else of such an approach to a disruption as to have an equally prejudicial effect in producing lameness. In some cases the whole of a small bundle of fibers is torn across; but this is not the usual degree in which strains occur, and the most common amount of mischief is only a slight separation of a few of the very small fibers of which the bundle is composed; and this state is then generally spread over a cousiderable surface, producing considerable soreness from inflammation. Tendinous and ligamentous strains are very similar in their nature, and consist either in an absolute tearing apart of these fibers, or such an approach to this as to cause great inflammation, and consequent incapacity for using them. Sometimes what is supposed to be a strain of the tendon is really an inflammation in its sheath, which causes great swelling and pain, and the limb is thereby rendered quite useless for the time being."

Bathing the parts in tincture of arnica is one of the best remedies for injuries of this nature. Where the skin is not broken, hot fomentations of vinegar and water applied with flannel bandages, and renewed every ten or fifteen minutes until the inflammation is reduced, is an excellent remedy, and considered by many the best possible treatment. Where there is much acute inflammation and swelling, linseed or carrot poultices will prove beneficial. The bandages should also be kept tight in such cases.

Stringhalt. - This is an involuntary action or contraction of the muscles which causes one or both of the hind-legs to be raised higher in walking than is natural. It may be regarded as unsoundness, and greatly injures the appearance of the animal, but does not much interfere with his service. It is incurable.

Tetanus (Lockjaw), - This is one of the most painful and fatal diseases to which a horse is subject. It generally proves fatal, and when recovery does take place it is usually very slow, and extends over several months. The disease derives its name from the fact 
that the muscles of the jaw are so affected that the mouth become immorably closed. It usually results from some injury to the foot, such as the stepping on a nail and driving it into the sensitive portion, or from a prick in shoeing. It not unfrequently follows castration. the docking and nicking of the tail, or other injuries. A sudden exposure to cold after being heated has also been known to produce it.

- Tetanus is a disease of the nerves, caused by an injury to one of them, the effect of which extends to the entire nerve organization. When it terminates fatally, it is generally from the sixth to the eighth day after receiving the injury. The animal should be kept as quiet as possible, and nothing of an exciting nature permitted.

The difficulty with the treatment of this disease is, that it usually becomes well established before it is discovered, when remedies will have less effect than at an earher period. It would always be well to take suitable precautions against it whenever the hoof is injured by being punctured by a nail or other substance. We once lost a beautiful and valuable animal with this disease, from stepping on a nail while on the road. As soon as the accident occurred, the horse stopped, held up her injured foot, and looked back to us.

We immediately got ont of the carriage, and, on examining the foot, found the nail, which, on being withdrawn, did not indicate by any blood following, or the length of the nail, that the puncture was a deep one, but she died of lockjaw from the effect fire days afterward, after the most intense suffering.

When an injury of this kind occurs it would be well to take precautionary measures by removing the shoe and soaking the foot and limb in warm water for an hour or more, as soon as possible afterward, at the same time rubbing it gently with the hand. Then apply tincture of arnica from the knee down, rubbing it in, and getting as much as possible into the hole made by the nail.

A hot poultice for the foot and limb would also prove beneficial. Such treatment followed immediately after receiving the injury, would have a tendency to ward off the disease. The bowels should be kept moderately laxative, and a plenty of water and flaxseed gruel given for drink.

When it is discovered that the animal has the disease, the shoe should be remored, and the foot carefully examined to see if there is any nail or foreign substance in it; if such are found, pull them out, and treat as above recommended. Give the following dose: An ounce of powdered aloes, half a drachm of podophyllin, and two drachms each of nitre and ginger. Make into two balls with mucilage, and give both at the same time. Injections of flaxseed tea or warm water will aid also in keeping the bowels open. If there should be any diffsulty in evacuating the bladder, the catheter should be used for this purpose. Opium is also a valuable agent in this disease, and may be given in doses of from one to two drachms.

The animal should be tempted to eat nutritious food by putting it occasionally to his month, or between his grinders. Carrots or apples are excellent; also warm bran mashes. Rub the body gently, especially along the neck and spine. Keep him warm by blanketing, and induce perspiration.

In the middle of the day, if the weather is warm and pleasant, and not uncomfortably hot, let him be out in the sunshine a while. Keep up the natural functions of the animal system, and, above all, keep him soothed and quiet. Have no strangers about him, or loud talking, or noise of any kind; neither permit him to be harnessed, but keep him as quiet as possible.

Thoroughpin.-This is a disease similar to bog spavin, and projects on both sides of the hock, from one side of the joint to the other, forming a round swelling. The treatment should be similar to that of Bog Spatix (which see). 
Thrush.-This disease consists of inflammation of the lower surface of the inner or sensible frog, attended by a discharge of pus. It is caused by foul stables and neglect of the feet.

The remedy is to remove the cause, by giving proper attention to cleanliness. The feet should be washed with castile soap and warm water at morning and night, carefully removing the loose and decayed portions of the frog; dry the parts thoroughly, after which apply the following mixture between the crevices of the frog: Barbadoes tar, eight ounces; melted lard, one ounce; sulphuric acid, half an ounce. Cover with tow and a leather sole and keep from stepping in the wet.

Another remedy equally good is to cleanse the foot as above indicated, then dust in a litkle of the following: Calomel, two drachms; powdered sulphate of copper, three drachms; carbolic acid ten drops. Cover with tow, etc., as in previously given remedy. When the liquid discharge has ceased, fill the cleft with tar, and continue to apply this if necessary, for a week or two.

\section{Toe-Crack. (See SAND-Crack.)}

\section{Tread. (See Over-Reaching.)}

Warts.- Where the warts are very small, they may be cut off close to the skin with a pair of scissors, and the place touched with lunar caustic. If the stem is large, tie a thread of waxed silk around quite tight, gradually tightening it each day. This method deprives it of its nutriment from the skin, and it will drop off in a few days. It is said that an application of indigo dissolved to the consistency of thick paint, and applied daily to warts will remove them without soreness. Having never tried the latter we cannot vouch for its correctness.

Windgalls. - These are soft swellings or enlargements near the fetlocks of horses, produced by strains or overdriving. Tight bandages, and astringent lotions may be applied with benefit. A piece of cork placed over the swelling, and covered with bandages sufficient to keep it down, is sometimes used. They are similar to blood spavin, and should be treated accordingly. (See Bloon Spavix.)

Worms.- Salt seems to be obnoxious to worms; therefore have a good piece of rock salt in the manger at all times, or a sufficient amount of common salt, within reach of the animal. Common purgatives will often bring away large numbers. The following is a good remedy: Tartar emetic, $2 \mathrm{dr}$.; ginger, 1 scruple; 1 pint linseed oil; half pint molasses, Give half an hour, every other morning, before feeding-time. Also, flaxseed tea every day. An. other good remedy is spirits of turpentine and quassia tea; first beating the turpentine with the yolks of eggs, to cause it to mix readily. To a pound of quassia chips, add three quarts of boiling water. When cold, strain off the tea, (which is a sufficient dose for an adult horse) and add the turpentine; giving in proportionate quantities as follows: For a colt six months old, a half ounce of spirits of turpentine; a year old, one ounce; two years. $1 \frac{1}{2}$ ounces; three years, 2 ounces; four years, three ounces. Give every other day, in the morning before feeding. Feed with nutritious food - a mixture of green food being very beneficial. Raw potatoes are excellent for horses troubled with worms. An injection of a quart of linseed oil will prove the best remedy for removing the smaller worms (ascaris), which often cause such serious irritation and annoyance about the anus. 


\section{THE ASS.}

$\mathrm{T}$ $\mathrm{HE}$ ass is a native of Central Asia and Africa and belongs to the genus Asinus and family Equidæ. It is of smaller size than the horse, and is characterized by long ears, the absence of warts on the hind legs, and a tuft of long hair at the extremity of the tail. Its color is generally gray, marked with a dorsal streak of a darker hue, and a similar one across the shoulders. There are also white and black varieties, though less common than the former. This animal was probably domesticated by man before the domestication of the horse, and has proved a most valuable servant, possessing. as it does, great strength, endurance, patience, and docility. The first mention of the ass in the Scriptures is in the account of the going of Abraham into Egypt, at the time of the great famine in Palestine.

A wild variety of the ass is now found in Abyssinia, and some other localities, and although differing somewhat from the domestic variety, it resembles it in many respects. It has cross-bands on its legs, a mark occasionally seen in the domestic breeds. There seems to be a natural aversion in this animal to cross the smallest stream of water, a trait which is also seen in the camel, while it delights to roll itself in the dust and sand. which fact would argue in faror of the desert regions as its original home. The wild ass is a spirited animal of great speed, and is one of the principal objects of chase in Persia, where its flesh is highly esteemed as food. In Oriental countries they are employed as beasts of burden, and the practice of riding them in trareling is rery common. Careful selection and more humane treatment than this animal usually receives, shows that it is capable of great improvement, and possesses qualities that have never been fully appreciated. In Southern Europe, especially in Spain, Italy, and Nalta, it has been carefully bred and greatly improred.

Varieties, etc.-The different breeds of the ass are supposed to be quite as numerous as those of the horse. The Maltese and Spanish breeds are considered by American breeders the best rariety from which to propagate. The small size of the ass in cold countries is due fully as much to neglect, as to the sererity of the climate. It is said that in the north of India, where it is used among the lowest castes, it does not attain a height greater than that of the Newfoundland dog. In Persia there are two breeds, one large, heary, and slow, used principally for burden, and another, considerably smaller and more actire, used for the saddle. In Spain there is still a greater difference seen. The Arabs and Persians breed them as carefully as we do our best horses, and are perfectly familiar with their pedigree. Darwin states that in Syria there are four distinct breeds:- "A light and graceful animal with agreeable gait, nsed by ladies, an Arab breed, reserved exclusively for the saddle, a stouter animal. used for plowing and various purposes, and the large Damascus breed, with peculiarly long body and ears."

The ass is but little used in the United States except for breeding purposes in producing mules. They are bred to a certain extent throughout the Western and Southern States, but principally in Kentucky, Tennessee, Texas. Ohio, Indiana, Illinois, and Missouri In Kentucky, where mules are in great demand, and where they are raised with much care from imported animals of an arerage height of fourteen hands, they have been raised to fifteen and even sixteen hands in height. Jennets, or female asses, are used principally in this country for breeding jacks, and are not numerous. A superior Maltese jack was presented to General Trashington, in $178 \%$, by La Fayette, and is believed by some to have been the first of this breed ever sent to this country. The description given of him by Mr. Custis is that of a moderate-sized animal, clean limbed, rery active and spirited, and possessing the ferocity of a tiger; color dark brown and nearly black, white belly and muzzle, and manageable with safety unly by one grown. This animal lived to a great age, and his progeny was highly prized. 


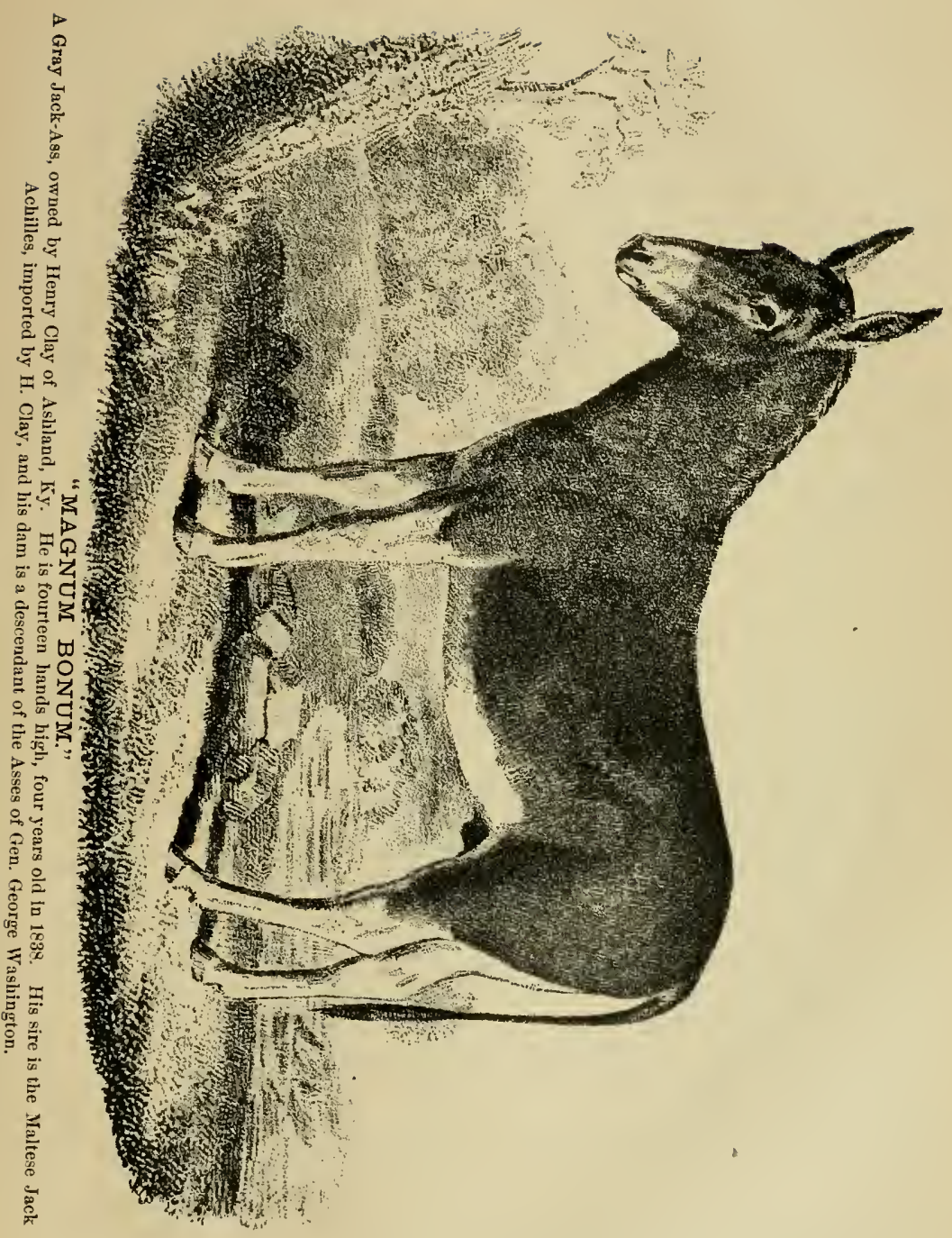



It is believed that Gen. George Washington was the pioneer in the business of mule. breeding in this country. It seems that previous to 1783 there were very few mules in the country; and these were such inferior animals that farmers were very much prejudiced against them; consequently there were few jacks, and no disposition to increase the stock. Washington, however, became convinced that the introduction of mules generally among Southern planters would prove a great blessing to them, because these animals are longer lived, less liable to disease or injury by careless help, are well suited to a hot climate, and will work on shorter feed than horses. A recent writer says, respecting this subject:-

"It becoming known that the illustrious Washington desired to stock his Mt. Vernon estate with mules, the king of Spain, in 1787 , sent him a jack and two jennets from the royal stables, and La Fayette sent another jack and jennet from the Island of Malta. The first was of a gray color, sixteen hands high, and of a sluggish nature. He was named the Royal Gift. The other was called the Knight of Malta; he was about as high as the former, and lithe and fiery, even to ferocity. The two sets of animals gave him the most favorable opportunity of making improvements by cross-breeding, the result of which was the favorite jack Compound, because he partook of the best points in both originals. The General bred his brood mares to these jacks, even taking those from his family coach for that purpose, and produced such superb mules that the country was all agog to breed some of the same sort, and they soon became quite common. This was the origin of improved mules in the United States."

The teeth of the ass are similar to those of the horse, and the age of the animal is indicated, as in the horse, by the changes that occur at different periods during their growth.

\section{THE MULE.}

A $\mathrm{S}$ will be seen from the foregoing, the improved mules of our country owe their origin to the intelligent, and therefore successful management of Gen. Washington, a man who could be "first in war, first in peace, first in the hearts of his country. men," and, when he saw the necessity, also the first in brooding mules for his countrymen to use. As is well known, a mule is a hybrid, the produce of a jackass and a mare, while a hinny is the produce of a female ass and a horse, and is therefore half-horse and half-ass, the same as a mule; but there is a wide difference between them. The hinny has the voice, the mane, and the tail, and very much of the form of the horse; while in all of these particulars the mule resembles the ass more strongly than the dam.

The mule is an exceedingly hardy animal, and admirably adapted to hard work in hot weather, such as would be too severe for either the ox or horse; for this reason it is peculiarly valuable in the Southern States, for farm use, and all kinds of heavy work. Mules are also used quite extensively in the Western States, but only to a limited extent in New England. North of $40^{\circ}$ they are used nuch less than south of this latitude, while beyond $43^{\circ}$ they are rarely employed. There has recently been an increasing demand for mules for exportation to Europe, which has given a new impetus to the trade. St. Lonis is the principal mule market in the United States, although they are raised in various sections, Kentucky, Tennessee, Ohio, Indiana, Illinois, Missouri, and Texas, as proviously stated, being the States in which they are bred in the greatest numbers.

Economy of Mnle-Labor. - Being longer-lived and more hardy than the horse, the working period of the mule is considerably longer, while it will thrive with less care. A well-bred mule, with proper care, will out-wear two horses. Mules do not become frightened 
as easily as horses, and when frightened are not as liable to run away. A good mule-team costs much less than a proportionately good horse-team, while a mule is raised to a working age much more cheaply than a horse.

Mules may be kept at less expense than horses, and are not subject to as many diseases, while those they may have are more easily cured. On the other view of the subject, a horse may be used for pleasure driving, or for all purposes, while a mule is suited only for heavy work, and purposes of draft. Judge Hinckley, of Northampton, Mass., formerly a breeder of mules, always kept a team of them for performing the drudgery of farm work, much prefer. ing them, after an experience of fifty years, to horses for this purpose, although he kept his stables full of horses besides. One pair thirty years old were particularly serviceable, having outlived several generations of horses, and though the latter were often out of condition and sick, the mules never were. One of his stock, forty-five years old, was perfectly able to perform his share of labor.

Another gentleman who used mules for over thirty years, says: - "From repeated experiments I have found that three mules from fourteen and a half to sixteen hands high, capable of performing any work a horse is usually put to, and which were kept constantly at work, consumed about the same quantity of hay, and only one-half the provender which was given to two middling sized coach horses, only moderately worked. I an satisfied that a large-sized mule will not consume more than three-fifths to two-thirds the food to keep him in good order, that will be necessary for a horse performing the same labor. The expense of shoeing a mule the year round, does not exceed one-third that of the horse, his hoofs being harder, more horny, and so slow in their growth, that shoes require no removal, and hold on till worn out; and the wear, from the lightness of the animal, is much less.

Iules have been lost by feeding on cut straw, and corn meal; in no other instance have I known disease in them, except by inflammation of the intestines, caused by the grossest exposure to cold and wet, and excessive drinking cold water, after severe labor, and while in a high state of perspiration. It is not improbable that a farmer may work the same team of mules for twenty years without having a farrier's bill presented to him. In my experience of thirty years, I have never found but one mule inclined to be vicious, and he might have been easily subdued while young. I have always found them truer pullers, and quicker travelers, with a load, than horses. Their vision and hearing are much more accurate. I have used them in my family carriage, in a gig, and under the saddle, and have never knomn one to start or run from any object or noise, a fault in the horse that continually causes the maiming and death of numerous human beings.

The mule is more steady in his draught and less likely to waste his strength than the horse, hence more suitable to work with oxen, and as he walks faster, will habituate them to a faster gait. In plowing among crops, his feet being small and following each other su much more in a line, he seldom treads down the ridges or crops. The facility of instructing him to obey implicitly the voice of the driver is astonishing. The best plowing of tillage land that we ever saw, we have had performed by two mules tandem, without lines or driver."

Mules have been driven eighty miles in a day without injury, although this is not to be recommended, and it is too much to expect of any animal, yet it shows how much they are capable of enduring. Mr. Ellicott, of Patuxent Furnaces, states that out of one hundred mules in the works, they have not lost, on an average, one in two years, while he does not recollect that they have ever had one that was wind-broken. They are rarely defective in the hoof, and though kept shod, shooing is not as necessary as with horses. Their skin is tougher than that of a horse, and they are consequently not as much annoyed by the flies, and do not suffer so much with the heat in summer. Mr. Morris, of Pennsylvania, says: -

"Being a dealer in this class of live-stock, and coming in almost daily contact with their muleships, I have learned their dispositions and habits pretty thoroughly, and having learned 
their full worth on the farm, have entirely discarded horses for farm-work, knowing from actual experience, that mules make a far more economical farm team than do horses."

Althongh mules will thrive on coarser and considerably less fare than horses, yet it is always the most economical and pays best to give either horses or mules, or in fact any kind of stock, a plenty of good food without overfeeding them. We believe the mule should be fed with as gooa food as horses, and that they require as much in proportion to their size.

Breeding Mules.-The breeding of mules in America began with mnch spirit in the New England States, soon after the Revolutionary war. They were then bred as an article of commerce, being at first shipped exclusively to the West Indies, but afterwards to the South and West, for use in the sugar-mills, and other plantation work. At first the breeding animals were very inferior, both sires and dams, the stock produced being held in derision where they were bred, but in those days anything blessed with long ears, and called a mule, commanded a remunerative price and ready sale. As a natural consequence, a prejudice was established against the whole mule family, which has to this period never been entirely removed in certain localities.

The improved mule, an American production and superior animal, has been largely introduced in the West, and to some extent all over the country, besides being imported to Europe quite extensively. The size of the mule most profitable to raise for use in the West and South is from fourteen and a half to fifteen and a half or sixteen hands in height, since, other conditions being equal, size is the measure of strength. It is found in mule-breeding that the jack should be a spirited animal, and not less than fifteen hands high, and that the best mules result from crossing such with improved blooded mares. The noted jacks, Mammouth and Warrior, which have so improved the stock of this country, were imported to Kentucky from Spain about the year 1837 , at a cost of $\$ 5,000$ each. These animals were sixteen hands ligh, of fine quality, and greatly improved the size of the jack stock by their crossing with the common jennets of the State. They were the result of crossing the jacks of Malta with jennets of Spain.

In breeding mules, it is highly important that the jack be of the height previously mentioned, in order that the progeny be of good size; also that they be intelligent, active, and spirited animals, that these other good qualities be also perpetuated. It is likewise of equal importance that the best mares be used,-blooded mares, $\rightarrow$ and, as a general rule, the finer the mare the better the mule product, unless too small.

Mr. E. F. Spencer of Kentucky, a gentleman of large and successful experience in mulebreeding, gives the result of his observation and experience in this department as follows:-

"During the breeding season, beginning here in March, the jack should have a lot sufficiently large to supply him with grass at night; but in the day he should be kept in a tight stable. The lot should be located, if possible, where no horse stock can come to the fence; for if they do, a vigorous jack will fret through the night and may become vicious, and some. times will bite horses through the fence.

They frequently fret from this cause till they get poor in flesh and unserviceable. If permitted to run in the lot during the day, you may feed him, three times a day, four to six ears of good sound corn, and two bundles of sheaf oats, cut up to the band. I have found by experience this to be the better plan. If the breeder has no grass lot, he should feed corn and oats, the latter cut fine enough to make chop feed. This is a feed composed of oats cut up and corn-meal ground fine-fine enough for family purposes. Put a little salt, if not every time it is fed, at least frequently; and don't feed too much at first, to avoid founderor the new meal may cause colic.

A jack should be permitted to serve one or two mares at two years of age; but be careful not to breed the jack intended for mares to jennets before you have bred him to at least half a dozen mares; and, in breeding, do not let him serve more than two a day, - one in the 
morning and the other in the evening, -and not over forty the first year, nor more than sixty any year. If more are bred, permanent injury to the jack is likely to result. In litigated cases which have come under my notice, the testimony has generally shown that more jacks have beeu injured by breeding to too many mares at three years old, than at any other age.

Caution should be used during the season to have the jack securely haltered through tlee day, for some few will wateh for an opportunity, when the groom is off his guard, and bite seriously. I once had a jack, seemingly docile, seize his groom by the back, and bite him severely. Jacks, generally, are docile; but until you find out the disposition of your jack, you had better, during the breeding season, give him no chance to do mischief. The application of a good hickory will soon cure his viciousness.

A jennet will carry her foal 12 months, and a jack foal often 13 months; and the owner should be present, if possible, when she foals, for the young of this kind of stock are more liable to be smothered than mule or horse colts.

It shculd be borne in mind that a young, healthy jack will get his best colts the first season; and if you are using your jack yourself, never permit him to be over-bred.

If your jack is 15 to $15 \frac{1}{2}$ hands, and has that height by inheritance, through his ancestry, you call, by breeding him to mares of good size, confidently rely upon having good. sized mules.

The well-bred mule, if properly treated after being weaned, will grow the first year six, and the second year three inches; and if well kept in this way for two years, will have attained its height. Of course, if poorly fed and cared for during the first and second years, they will not mature until three years old; but the stunting business costs the owner one year's additional interest, feed, attention, and care, which never pays."

Mules bred in Arkansas, Missouri, Tennessee, and Kentucky, are generally known to be superior to those of most of the other sections of the country, owing, probably to the fact of the improved jacks being used there more extensively with blooded mares.

Breaking and General Management of Mules.- The general rules to be observed in breaking colts, and horse management, are equally applicable to mules; hence, do not require repetition in this connection.

Iules are too often neglected and abused, and from such treatment are frequently made vicious and stubborn from mere self-defense. They are frequently termed stupid and obsti. nate, but in nine cases ont of ten where they are found such, it is due more to the ignorance, stupidity, and brutality of those that reared, broke, or have driven them, than that of the animal. II ules are naturally affeetionate and patient, and should be kindly treated. When thus treated, they are very docile, and yield readily.

Their ears, like those of the horse, are peeuliarly sensitive, and tender; hence when handling them, it should be done very earefully, and in such a manner that they will know that they are not to be annoyed, or harmed in any way. This will help wuch in overcoming their naturally tinid dispositions, and when they find that they are not to be abused, they will be kind and submissive.

The mule's ears are so extremely sensitive, that a scratch or the slightest injury to them will make them afraid, and have a tendency to arouse stubbornness; and it will be with the greatest diffleulty in such cases, that they will for a long time afterward submit quietly to be haltered. Mr. MIorris, of Pennsylvania, previously referred to, says respecting mule-breaking: "There is a great prejudice existing against mules in some parts of the United States, but this is most generally the case where they are least used, and, consequently, but little is known of their good qualities, and they are accused of being vicious, stubborn, and ready to kick at anything coming within reaching distance. How common the remark, 'As stubborn as a mule.' 
And yet some of the very traits that are so much decried in the mule, are the very best traits that either mule or horse could possess. What is wanted, is to 'train a mule up properly in the way it should go,' and my word for it, ' when it is old it will not depart from it.' We should nse kindness instead of abuse, aud there would seldom be any kicking or biting, and the mule will learn far quicker than most horses what is wanted. Mnles are naturally very timid animals, and have a great deal of curiosity. The latter trait I have often observed in driving a number of loose mules along the public highway. They will stop and notice every strange object along the road, and will, occasionally, turn off on by-roads to gaze at something, and yet, to show how quickly they learn, and how easily managed, after a day's driving, a few calls from the driver will generally bring them back to the main road. Some, occasionally, are very roguish, and seem to take a delight in running far enough away from the drove to cause the driver some uneasiness, but, with a little patience and firmness, they are soon broken of the habit. Mules liave also a very aflectionate disposition, but one peculiarity of theirs (and this same trait is peculiar to the elephant) is that of remembering and resenting an injury; therefore, the more quietly we go about handling and breaking them, the less trouble we will have with them, and the less disposition they will ever possess to kick at any one. A showman once announced, whilst performing his bear, that 'he broke him with kindness,' adding 'and a club.' My advice in breaking mules is, be sure to adopt the kindness part of it, bnt, under no circnmstances, use the club. Many a mule is spoiled by ignorant and thoughtless persons seizing it by the ear and holding on to that sensitive organ until the mule becomes so much afraid that it is almost impossible to bridle it. Some can never be entirely cnred of the timidity thus produced."

Hon. J. B. Smith, of Pennsylvania, says: "I had the pleasure, once, of owning a pair of mules. I bought them when they were two years old, and made up my mind that they conld be broken the same as a horse, if treated in the same way. I got a friend to assist, and we went to work and broke them the same as colts, using them kindly; and a better pair of mules never were used. Any one could drive them. I could hitch them into a buggy together, and they wonld go well. All you wonld have to say was 'go.' A mule lives on less than a horse, does more work, and is less liable to die.

"In our minds they are very valuable. We drive one into a gang-way half a mile, taking a light for loading. After the car is loaded and the mule started, he will find his way out over the proper track without any light at all. Then all you have to do is to start him again, and he will go back to where they are mining. I believe the mule should have the same treatment as the horse, and then he will have as kind a disposition. If you whip and scold him every time be does anything wrong, and make a 'scape-goat' of him generally, in course of time he will not mind anybody, not even yourself. But if you treat him right, he will do right in return."

We lay it down as a general principle, that any animal is far more valuable for service of any kind, and hence worth more money, for simply the kind and hnmane treatment it has received from birth to maturity, and that much-abused animal, the mule, is no exception to this principle.

Shoeing. - All that has been said with reference to the shoeing of horses applies with equal force to the shoeing of mules,-the half-brother of the horse. Do not permit the frog or sole of the foot of the mule to be cut, any more than that of the horse, for it will be sure to bring injury to the feet sooner or later. If a mule is troublesome about being shod, blind. fold him by putting a bandage before his eyes, and he will generally yield. Timidity and fear of being harmed will generally be the cause of resistance in such cases, rather than obstinacy. When they cease to fear man, and have confidence in his kind intentions, they will use their heels and teeth less in self-defence, and prove less shy and willful.

Diseases of the Mule.-For treatment of diseases of mules, see Diseases of tue Horse, since all remedial agencies apply equally well to both animals. 

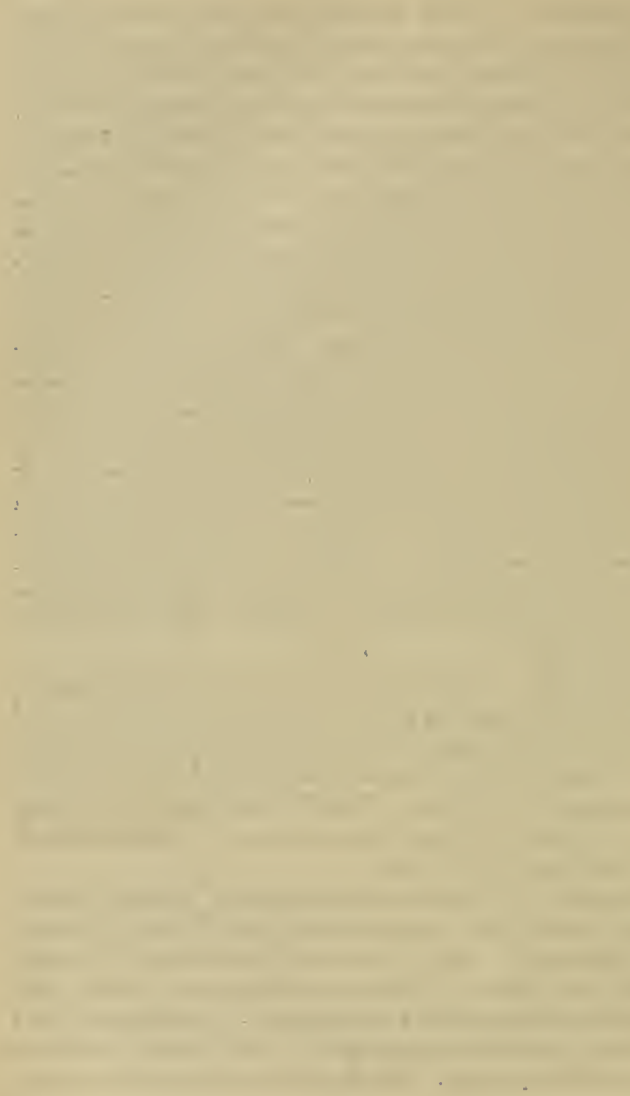

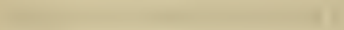

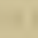

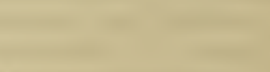

1 -20

in thente

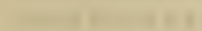
(2)

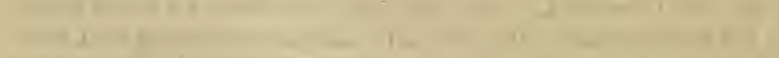

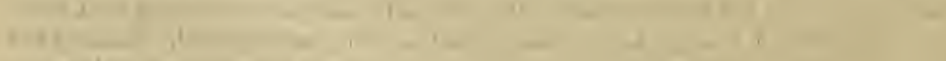

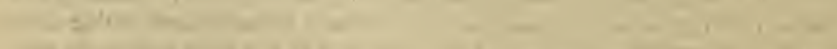

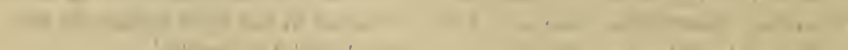

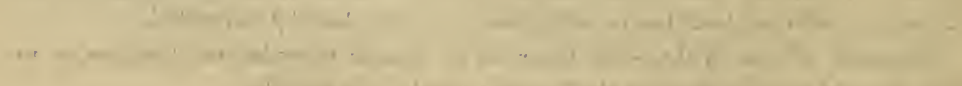

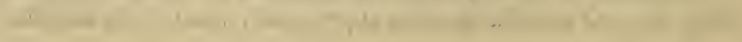




\section{N D E X.}

Activity in the Horse,

Adulteration of Tea,

Advantages of Large Farms, $\quad .583$

" Small Farms, . . . . 584

“ the Ensilage System, . . 509

After-Culture of Wheat, _ . . . 277

Aftermath, or Rowen, . . . . . . 248

Age and Size of Trees, . . . . . 520

$A$ Good Disposition in the Ilorse, . . . 679

Agrieulture, General,

$$
\text { “. Improvements in, . . }
$$

A Judicious Choice of Crops Essential, .

Alfalfa, value of as a forage plant,

$$
\text { cultivation of, }
$$

Alluvial soils,

Alpha potato, .

Alsike clover, .

American cotton, rank of, . . . . 401

Auerican Girl,

$$
\text { guano, }
$$

Ammonia, escape of from fcrmenting manure, 49

". " how preveuted, . . 42

Analysis of Artificial Grasses,

226,227

Ash of " $\quad$. $\quad . \quad 229$

" Natural Grasses, . . . 229

Natural Grasses, . . 229

Corn, . . . 37, 39, 304

" when cut in a green state, 508

Grain and Straw, . . . 37

Green Crops, . . . . 37

IIay, - • • . • • 37

Roots and Tops, . . . . 37

Soils, . . . . . 25

"Table of, . . . 27, 28

Unproductive Soils, Table of, . 28

Sugar-Cane,

Weeds,

Anchylosis,

Animal Frerements, Analysis of,

“ Dust, .

Analysis of, . $\quad . \quad . \quad 57$

mental Cottage

Aplides, or Cotton Lice,

A Picturesque Country Villa,

Application of Commercial Fertilizers, . $\quad 83$

Artesian Wélls,
Artichokes,

A Sail in a Paris Sewer, . . . . 50

Ashes, Coal, . . . . 61

" Leached, . . . . . 61

" of Sea-Weed, . . . . . . 62

" Peat, . . . . . 62

“ Wood, . . . . . . . 60

Asparagus, Colossal, . . . . . 360

Ass, The, . . . . . . . 826

“ " Varieties, etc., . . . . 826

Assorting Tobaceo, . . . . . 448

Balking, . . . . . . . . 772

Baling Hay, . . . . . . . 247

“ Press, Lever, . . . . . 247

“ " . . . . . 24team,

Barb Wire Fence, . . . . . . 570

Barefoot Horses, . . . . . . . 792

Barley, . . . . . . . 297

" Cultivation of, . . . . . 298

"Harvestiug, . . . . . . 299

Barn, The, . . . . . . 641, 650

“ Cellars, . . . . . . 42, 661

“ Loeation of, plans for, etc., . . . . 643

"Ground Plan for, . . . . . 646

Barns, Cattle, . . . . . . . 656

" Dairy, . . . . . . . 651

“ Farm, . . . . . 650

"Horse, . . . . . . . 653

" Model, . . . . . 639, 640, 644

" old and new, . . . . . . 652

Barn-yard Manure, . . . . . . . 40

Bars in Road-Making, . . . . . 552

Bat Guano, . . . . . . . 60

Beans, Varieties, etc., . . . . . 475

“ Cultivation of, . . . . . . 476

“ Harresting, . . . . . 476

“. Field, Formula of Fertilizers for, - 81

“ Castor,. . . . . 47\%

" "Cultivation of, . . . . 477

"Horse, Cultivation, etc., . . . . . 476

‘. Improved Lima, . . . . . . . 366

Beetle, Colorado, . . . . . . . 349 
Beets,

PAGE.

364 Bulrush, Morgan, . . . . . . 727

365 Burr Cucumber, . . . . . . 370

365 Butter-Worker, . . . . . . 594

"Cultivation of,

“ Harresting, .

“ Storage of, .

“ Blood Turnip,

“. Early Red,

“ Imperial Sugar,

" Long Blood,

" Mangel Wurzel,

$$
\text { " " Cultivation of, }
$$$$
\text { “ Swiss Chard, }
$$

367 Calcarcous Soils, . . . . . . 15

366 Canadian Horse, The, . . . . . 720

366 Care of Breeding-Mlares, . . . . 751

370 Care of Cattle at Mountain-Side Farm, . 601

366 “ Cows at Deerfoot Farm, . . . 601

Belgian Carrot,

Best American Trotting-time,

" Method of Shocing Horses, .

"Russian Trotting-time, .

“ Reeords of Flectest Running Horses,

Bitting Process, The,

Black lHawk,

\begin{tabular}{lllll}
\multicolumn{1}{c}{ Junior, } & . & . & . & . \\
Black Tea District, . & . & . & . & . \\
Bleeding llorses, . & . & . & . & . \\
Blinders for Hlorses, & . & . & . & . \\
Blinds for Buildings, & . & . & . & . \\
Blood as a Fertilizer, & . & . & . & . \\
Bones as a
\end{tabular}

" How to Reduce and Pulverize,.

Bokhara Clover,

Boll-Worm, The,

Boulounaise Draft Horses, . . .

Box-Stalls,

Breaking and General Management of Mules,

$$
\text { " Colts to the Harness, }
$$

Breeding of Horses, The,

$$
\begin{aligned}
& \text { " Disqualifieations for, } \\
& \text { Mares, Care of, . }
\end{aligned}
$$

Brick Silos,

Brittle Hoofs, Rensedy for, etc.,

Brooks,

Broom Corn,

"Soil and Preparation for,
" Planting, .
“
" Cultivation of, .
" Curing, .

Buckwheat,

“ Varieties.

Buffalo Clover,

Buildings, Farm, .

$$
\begin{aligned}
& \text { “ “Height of, . } \\
& \text { “ Painting, } \\
& \text { "Plans of, } \\
& \text { “ Repairing, } \\
& \text { “ Necessary on the Farm, }
\end{aligned}
$$

367,370 $36 \tau$

\section{1}

$40 \%$ Cator .

708 Castration of Colts, * *

659 Catch-waters in Road-making, . . . 559

832 Cattle Barn, . . . . . . 656

768 (Yellars, Barn, . . . . . . . 42, 661

771 ". for Dwellings, . . . . . 636

746 Centrifugal Machines of Deerfoot Farm, . 597

747 Cereals, . . . . . . 263

751 Cereal Products in the United States, . . 263

831 Champlain Wheat, . . . . . 266

491 Chareoal Dust, . . . . . . . 62

794 Cheap Conservatory, How to Construet, . 674

529 Clieck-rein, Use cî, . . . . . 774

478 Chest of the Horse, . . . . . . 686

479 Chimneys of Dwellings, . . . . 633

479 Chinch Bug, . . . . . . 283

480 Chinese Yam, Cultivation of, . . . 357

480 Chloride of Sodium, . . . . . 68

481 Chufas, Cultivation of, . . . . 373

299 Choiee of Farms; . . . . . 582

300 Choice of Plants for Produeing Seed, . . 513

300 Cisterus, . . . . . . . 531

80 " How Construeted, . . . . 532

210 Cireular Cisterns, How to find Capacity of, 533

613, 654 Cisterns, Filters for, . . . . . 533

655 Classifieation of Soils, . . . . . 14

616 Clay Loams, $\quad . \quad . \quad . \quad . \quad . \quad . \quad 14$

621 Clay Soils and their Management, . . . 15

616 Cleaning Harness Plate, . . . . 800

615 Cleveland Bay, . . . . . . . 708 
Cleveland Bay Stallion, "Bay Splendor,"

Clipping Horses, .

Closets of Dwellings,

Clover, Alsike,

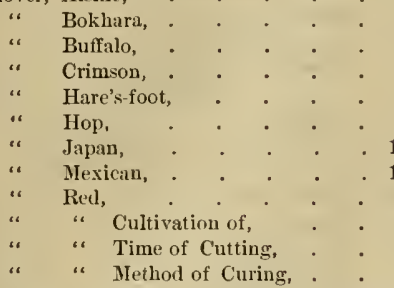

Clover Hay, Conditions Favorable to Fermentition in,

Clover Ilay, How Fermentation can be Prerented in,

Clover Seed, Cultivation of, .

$$
\text { " " " Harvesting of, . }
$$

Clover as a Fertilizer,

“Sickness,

"White,

" Yellow,

“ Zigzag,

Clydesdale, The,

Coal Ashes,

Stallion, "Time o' Day,"

Color in the llorse,

Color of Soils,

Colorado Beetle, The,

Colossal Asparagus,

Colts, Age for Working,

" Breaking to Harness,

“ Breaking to Saldle,

“ Foot at $t$ wo years,

“ Halter Breaking.

“ Old-time Method of Managing,

“ Present System of HIanagement,

“ Raising by Iland,

“ Teaehing to Back,

"' Weaning,

Commercial Fertilizers, .

Common Millet

$$
\text { " } \quad \text { “ } \quad \text { Analysis of, }
$$

Composition of Jafures,

Composition of German Potash Salts,

Composts,

Compost Pile, How to Construet, .

Compost Poudrette,

Compton's Surprise Potatoes,

Concrete Silos,

Conestoga. The,
PAGE.
Conservatory, How to Construct a Cheap, . $6 \pi_{4}$

Corn, Analysis of, . . . . 37, 39, 304 ". ". when cut green, . 508

“ Diseases of, ete., . . . . . 323

" Cultivation of, . $\quad . \quad 314$

" Cultivators, . . . 314, 315, 316

“ Grinding, . . . . . . 320

“ Harvesting, . . . . . 317

“ Hilling and Level Culture, . . . 315

“ Ilill and Drill Planting, . . . 311

“ Hilling Cultivator, . . . . 315

"History of, ete., . . . . . 302

"Insect enemies of, . . . . 324

“ Mills, Farm, * . . . 322, 323

" Planter, . . . . . . 312

“ Planting, . . . . . . 311

“ Preparation of Sced for Planting, 308

“ Selection of Seed, . . . 306

“ Shellers, . . . . . 321, 322

“ Shelling and Grinding, . . . 320

“ Soil for, and its Preparation, . . 309

“ Stalk Cutters, . . . . 317,318

“ Summary of Rules for Securing Large

Crops. . . . . . . 313

"Varieties of, . . . . . 305

Cotton, . . . . . 382

Aphides, or Cotton-lice, . . . 403

Baling of, . . . . . 400

Bolls, Rotting of, . . . . 409

Boll-worm, . . . . . 40\%

Caterpillar, or Cotton-worm, . . 403

“ “. “ "

" " " " Larva of, . 403

". " " " Chrysalis of, 405

Cultivation of, . . . . . 394

Cultivator, . . . . . 395

Fertilizers for, . . . . . 393

Gins, . : . $398,399,400$

Ginning, . . . . . . 399

Growing Bett, The, . . . . 385

Lands, Exhaustion of, . . . 391

“ " IIow Prevented, 391

Harvesting of, . . . . . 396

History of, . . . . . 389

Lice, . . . . . . . 403

Plant, Blight of, . . . . 409

Plant, Diseases of, . . . . 409

Plant, Rust of, . . . . 409

Plant, Encmies of, . . . . 403

Planter, . . . . . . 383

Plants, Thinning of, . . . 396

Press, . . . . . 383

Production in the United States, and

average product per acre, Table of, 386

Rotation for, . . . . . 392

Seed IIuller, · • • • • 402 
Cotton Seel, Selection of for Planting,

PAGE. |

390 -Uses of,

Soil, and its Preparation for, .

Stalk Cutter,

Underdraining of Soil for,

Varieties of,

Torm,

Rank of American,

Covering of Wheat, Deptl of,

Cow, or Field Pea, Cultivation of, ete.,

Cow Stable for Mlodel Burn,

Crops, a Judicious Choice of, Essential,

Crops, Choice of, Mtodified by Demand,

$$
\text { " " " " the character of }
$$

the Soil, etc.,

Crops, Relative Cost in the Production of,

"IRotation of, .

“Various for Green Manure, .

“What to Raise,

Cultivation of Grass Seed,

Cutting Potatoes for Planting,

Cntting Potatoes to a Single Eye, .

Dairy Barns, .

Dariymple Farm, The,

Dcerfoot Farm, The,

Defiance Wheat,

Deficieney of Potash in Common Fertilizers and in Soils,

Deptls of Covering Wheat,

Depth of Drains,

Depth of Plowing,

Design for a Country House, .

Determining the Outlet of Drains, .

Determining the Age of Horses,

Dexter,

Dew Point, The,

Diet for Sick IIorses,

Diseases of the Horse,

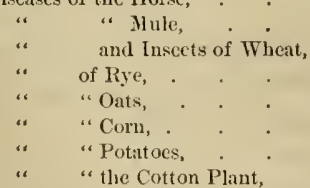

Disqualifications for Preeding,

Docking,

Dog Power,

Doing Work Over Nicely,

Doors,

Doors for Barns,

Domestic Animals, .

Double Row Stalk-Cutter,

Dr. J. B. Lawes' Experiments with Manures on Mealow Lands,

Dr. Bailey's Silos, .

Draco Prince, .

.

- 401

- 391

397

392

38

403

401

275

217

$6 \pm 8$

10.4 105$$
\text { - } 8
$$

\section{- 28}

Drainage,

$\cdot \quad \cdot \quad 12$

for Roads, . . . . . . 552

" Furrow, . . . . . . 138

" Old Time Method of. . . . 127

“ Tools for, . . . . . 134

"What Lands Require, . . " " 128

“ Determing the Depth of, . . 133

" " " Outlet of, . . 130

“ Plans for, . . . . . 137

“ Locating the Main Drains and Tributaries of, . . . . 131

Drainage, Size and Kind of Tile for, . . 134 “. Stone, . . . . 136

“ Tile, . . . . . 130

"Turf, . . . . . . 137

" Obstructions in, . . . . . 132

“ Open Ditclies, . . . . 138

“ Rule to find How Many Acres a

Given Main will Drain, . . . . 134

Dried Blood, . . . . . . . . 56

Drill-Planting of Corn, . . . . . . 311

Driven Wells, . . . . . . . 535

Dwellings, Warmth in, . . . . . 628

". Influence of on Character, . . 620

Early Amber Sugar-Cane, . . . . . 421

Eave Troughs, . . . . . . 633

Peho Farm, . . . . . . . . 587

“ " Buildings, . . . . . 586

Economy of Mule Lahor, . . . . . . 899

Elements of Plant Food, . . . . 31

. " " " Organic, . . 31

" Inorganie, . . 31

Embankments, . . . . . . 567

Enemies and Diseases of the Cotton Plant, . 403 " of the Tobacco Plant, . . . . 444

English Cottage, . . . . . . . 625

English Draft, The, . . . . . 707

" " Stallion, "Lincoln," . . 710

Ensilage, . . . . . . . . 489

." Adapted to the Southern States, . 508

“ Analysis of, . . . . . 509

“ Corn-Planter, . . . . . 501

“ Cutters, . . . . . 503, 504

“ How to Feed. . . . . . 507

" Metlod of Cultivating Maize for, . 500

“. Mr. Mills' System of, . . . 499

“ Mr. Morris" " ". . . . 497

“ Most Profitable Crops for, . . 500

“ Southern Seed Corn for, . . . 500

“ System, Advantages of, . . . 509

“ Experiments Respecting, . . 510

“ Opinions Respecting. : . . 510

" Time of Ilarvesting IIaze for, • 501

". Silos, Brick, . . . . 491

" " . Concrete, . . . . 493

" " Dr. Bailey's, . . . . 494

“ " . . . . 497 
Ensilage, Silos, How to Build,

$$
\text { : }
$$

491

Farm Buildings-Continued.

PAGE.

" How to Fill,

504

" MI. Goffart's,

" Lnited. Plau of,

Silo, Yertical Slicing in,

" Removing Fodder from,

Erecting Barb Wire Fence,

Esssentials in Building an Ice-House,

Evaporating the Juice of Sugar Cane,

Exlraustion of Soils by Crops,

$$
\text { " " " " "Table of, . }
$$

Experiments in Rotation of Crops,

" with Manures on Permanent

" with Manures on Permanent
Meadow-Lands, by Dr. Lawes, .

Extraction of the Juice of Sugar-Cane, . . 417

Fall Fallowing.

Fall Grazing of llowing Lands, . . $\quad 249$

Fallow System, The,

Farm Buildings, · · · • . 582, 613

Design for a Conntry Housc, . . 614 " an Ornamental Cottage, . 629

“ English Cottage, . . 625

" Ground Plan of, . . 625

“ Chamber-Floor Plan, . 625

Designs for a Pieturesque Country Villa, 629 $\begin{array}{ccc}\text { “ a Rural Cottage, . } & 627 \\ \text { " } & \text { " } & \text { " } \\ \text { " Chand Plan, } & 627\end{array}$ Plan,

That are necessary, 615

Should be Made Tasty and Attraetive, . $616,620,621,622,652$

Height of

- 621

Painting,

616

Plans of,

Repairing,

Vesey Homestead Cottage, The, . . 623

The Farm-House, .

" " " $\quad$ Location of, . . .

Influence of Dwellings on Character, .

The Farm-House, Plans for,

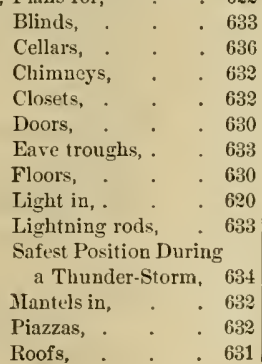

The Farm-House, Stairs, . . . 631

" " Ventilation in, . 634

“ " . Vaults, . . 638

" " " warmth in, . . 629

". water-closets and vaults, . . 638

" " Windows, . . 629

The Barn, . . . . . 641, 650

" “ Box-Stalls, . . . . 659

“ "Cellars, . . . 142, 661

" " Doors of, . . . . 662

" " Location of, . . . . 643

“ "

" " old and New, . . . 652

“ " Scaffolds for, . . . . 662

“ " Stables, . . . 658, 777

" " " Floors of, . . . 659

" " " for Ilorses and Stable

Management, . $i r$

" " " Light in, . . . 658

“ " " "NIannre-gutters in, . 659

“ " " Stalls, box, . . . 659

" " " Ventilation of, . . 658

Cattle Barns, . . . . . 656

Dairy, " . . . . . . 651

Horse " . $\quad . \quad+\quad . \quad+\quad .653$

" "Stables, and Stable Man.

agement in. . . .

Model Barns, . . . $639,640,644$

" . Cow-Stall for, . . . 649

" " Feeding-floor and Yard,

Plan for, . . . 64 i

" " Ground plan for, . . 646

" " Horse-Stall for, . . 648

Sheep Barns, . . . . 657

Granary; . . . . . . 663

Hog-House, . . . . . . 663

“ Plans for Piggeries, . . 665

Ice House, . ․ . 669

." " Essentials in building, . . $6 \boldsymbol{6}_{0}$

" " Plans for, . . . . 670

" "How to cut Ice, . . . 6i1

" " " keep small quantities of ice in summer, . 673

“ " How to make Ice, . . . 673

" "How to store Ice, . . . 67̃

" " Keeping Ice withont IceHouses, . . . . 6r2

Summer-Houses, . . . . . 6it

How to Construet a Cheap Conservatory, 674

Shed, or Wagon-Honse, . . . . 667

Store-House, . . . . . 668

Tool-House and Repair Shop, . . 668

Tagon-House, or Shed, . . . $66 \pi$

Wood-House, . . . . . 668

Piggeries, or Hog-IIouse, ․ . 663

Plans for, . . . . 665 
Farm Buildings-Continued.

Poultry-Houses,

Repair-Shop,

Farming as an Occupation,

“ Permanence and Independence of, .

"Profitable,

Farm Fences,

$$
\begin{aligned}
& \text { " " To what Extent Essential,. } \\
& \text { " “ Removing of, . . . . } \\
& \text { " " " " Stone-heaps, . . 569 } \\
& \text { “ “ Barb Wire, . . . . 570 } \\
& \text { “. ". " Erecting,. . . } 568 \\
& \text { " " } \text { " " Use of Stretcher, . } 570 \\
& \text {. . . . . . . 5ire,. } 5 \text {. . } \\
& \text { ، “ Embankments, . . . . } 50 \% \\
& \text { “ ". Flood, . . . } 569 \\
& \text { “. . Hedges, . . . . } 566 \\
& \text { ". } \text { ". } \\
& \text { " " " } " \text {. . } 566 \\
& \text { " " . } \\
& \text { « “ Portable, . . . . } 56 \% \\
& \text { “ “ Post and Board,. . . . } 564 \\
& \text { " ". . . Rail, . . . } 563 \\
& \text { “ " Preserving Fence-Posts, . . } 564 \\
& \text { “ " Rail, . . . . } 563 \\
& \text { “ ". Stone Tralls, . . . } 505 \\
& \text { " ". Wire,. . . . . } 569
\end{aligned}
$$

Farm Life, Pleasures of, . . . . 9

" Mills, . . . . . 322, 323

“ Roads, . . . . . . 558

Farms aud Farm Buildings, . . . . 582

Farm Teans-Horse, Mule, Ox, etc., . . 54:

Farm, Wastes and Wants of the, . . . 562

Farms, Choice of, . . . . . . 582

“ Large, Adrantages of, . . . . 583

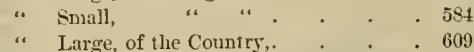

“ ." " " Dalrymple Farm, 610

" " " " "Glenn Farm, . 612

" " " " "Quinn Farm, . 612

- " " " Schaeffer" ete.,

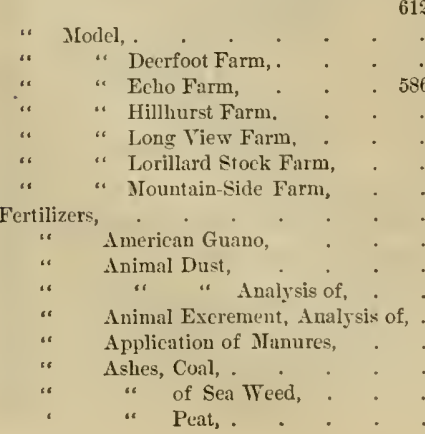

Fertilizers, Ashes, Wood, . . . . 60

Leached, 61

Barn-yard Manure, . . . 40

Bat Guano, . . . . 60

Bones, . . . . . 65

“. Pulrerizing and Reducing of, 65

Chareoal Dust, . . . . 62

Cliloride of Sodium, Salt, . . 68

Clover for Vegetable llanure, . $\quad 75$

Commercial, . . . 36,77

Application of, . 83

Analysis of, . . 38

Composition of German Potash

Salts, . . . 70

Composition of Janures, . . 36

Compost Poudrette, . . . 45

Composts, . . . . 51

“ How to Construct a Pile, 51

Caster Pomace, . . . . $5 r$

Cotton-seed Pomace, . . . 57

Deficiency of Potash in Common Fertilizers aud Soils, . . 39

Dried Blood. . . . . 56

Dr. Lawes' Experiments with IIanures on Meadow Lands, . . 256

Elements Necessary to Supply Soils, 32

Elements of Plant Food, . . 31

Escape of Ammonia from Ferment-

ing Manures, How Prevented, . 42

Exluaustion of Soils by Crops, . $\quad 36$

Formula of, for Buckwheat, . 80

" " Field Beans, . 81

“ “. Fodder Corn, . 80

" " Hay, . . 81

“ "Indian Corn, . 80

“. " Oats, . . 80

“ " Onions, " . 81

" " Potatoes, . . 81

" “ Rye, . . . $\$ 0$

“ " Swede Turnip, or

Rutabagas, . 81

. "Wheat, . 80

Fall Fallowing, . . . . 84

Fallow System, The, . . . 85

Fish, Fish Guano, Fish Pomace, . 57

Flesh, Blood, Hair, etc., as a Fertilizer,

Gas Lime, . . . . . 6.5

General Principles Concerning Plant Food,

Ground Limestone and Shells, . $\quad 65$

Guano. American, . . . 60 “ Peruvian, . . . 58

“ “. Rectified, . . 59

Gypsum, . . . . 6\%, 200

Dr. Franklin's Experi-
ment in the Use of, 68,200
How to Construct a Compost Pile, 51 
Fertilizers, How to use Potash Salts as a Fertilizer,

Leaves of Trees as Fertilizers, .

Lime,

" Gas,

Linseed Pomace,

Magnesia,

Manuring Mowing Lands, . " with Green Crops,

Marl,

Miscellaneous, Analysis of, .

Night Soil,

Nitrogen,

Peat,

"Ashes, .

Peruvian Guano,

" " Rectificd,

Phosphoric Acid,

Plaster,

Potash, . ". Sources of Supply of, “ Strassfurt Potash Mines, .

Poudrette,

". Blood and Meat, .
“ Compost, .
“.

“ Simple,

Poultry Manure,

Salt,

Sewage of Paris,

Sea Weed, . . . . . 62

Soot, . . . . . 61

Sulphate of Lime, • • . 67

Special Fertilizers, . . . 79

Superphosphates, . . . . 38

Table for Calculating the Exhaustion of Soils by Crops, . .

Table Showing the Materials Removed from the Soil by Various Crops,

Tobacco Stems,

“ Various Crops for Green Manure, $\quad 76$

Fertilization of Soil for Wheat, . . . 268

Fertilizers for Tobacco, . . . . . 441

Field Roller, The, . . . . . . . 103

Flax, .

"Cultivation of for Fiber, . . . 466

" " " Seed, . . . 468

" Harvesting of, . . . . . 468

"Preparing the Fiber of, . . . . 469

“ " "Seed for Market, . . 469

Filters for Cisterns, . . . . . 533

Flora Temple, . . . . . . 732

Forage Plants, Grasses and, . . . . 138

Forcing Potatoes, . . . . . . 343

Forests, Influence Exerted by, . . . 517

" " " " on Climate, . 518
Forests, Influence Frerted by, on Painall, PAGB

by, on Rainfall, . 518

Form of a Road-bed, . . . . . 550

Furrow Draining, . . . . . . 138

Gas Lime, . . . . . . 65

Glossary of Terms Used in Describing Grasses, 221

Gold Dust, . . . . . . . 731

Goldsmith Maid, . . . . . . . 734

Good Seed, . . . . . . 512

" " Cloice of Plants in Producing, . 513

“ " Special Cultivation Essential, . 512

“ “ Harvesting Sced, . . . 512

“ " "Storing, " 515

“ " Qualities Desirable in, . . . 516

Gov. Sprague, · • • • . . 738

Grasses and Forage Plants, . . . . 138

Aftermath or Rowen, . . . . 248

Agrostis-like Panic-Grass, . . . 189

Alfalfa, Lucerne, . . . . . 210

“ Value of as a Forage Plant, . 211

“ Manner of Cultivating, . . 212

" Soil to wbich it is Adapted, . 212

Analysis of Green Alfalfa, . . . 215

" " Alfalfa Hay, . . . . 213

Alsike Clover, . . . . . 207

“ " Cultivation of, etc., . 208

" " Analysis of, . . . 209

Analysis of Natural Grasses, 226, 227

“ Ash of Natural Grasses, . 229

“ Artificial Grasses, . 226, 227

“ Ash of Artificial Grasses, . 229

“Wecds, . . . . 229

Alpine Brown Bent, . . 154

Annual Spear-Grass, . . . . 149

Awnless Mublenbergia, . . , 156

Barnyard-Grass, Cock's-foot Grass, 186, 188

Beach-Grass, Sea Sand Reed, Mat-Grass, 157

Bengal-Grass, . . . . . 158

Bermuda-Grass, . . . . 160,163

Blue-Grass, Wire-Grass, . . . 149

Blue-Joint Grass, . . . . . 157

Bonnet-Grass, . . . . . 153

Bottle-Grass, : : . 158

Bristly Fox-Tail, . . . . . 158

Broad-Leaved Panic-Grass, . . . 189

Broom Grass, ～～～～～～180, 182

". Sedge, . . . . 170, 182

Brown Bent, . . . . . 156

Bokhara Clover, . . . . . 210

Buffilo Clover, . . . . . . 210

Clover as a Fertilizer, . . . . 205

Clover, Red, Cultivation of, etc., . 199

" Seed, " " . . 204

“ Sickness, . . . . . 207

Common Canary-Grass, _ . . 172

“ Millet. . . . . 190

Couch-Grass, Quitch-Grass, etc., . . 162

Crab-Grass, . . . . . 186, 187

Creeping Meadow-Grass, . . 150, 151 
Grasses and Forage Plants-Continued.

Crow-Foot, Yard-Grass,

English-Bent, 153

Downy Oat-Grass,

Drop-Seed Grass, Nimble Will, 155, 156

Feather-Grass, Blaek-Oat Grass, 167, 172, 173

Foul Meadow-Grass, . . . 140, 150

Fresh Water, Cord-Grass, . . . 167

Fringed Brome-Grass, . . . . 181

Gama-Grass, . . . . . 164, 166

Giant Sainfoin, . . . . . 215

Glaucous Small Reed, . . . . 157

Glossary of Terns used in Deseribing, 221

Golden Millet, . . . . . 191

Green Foxtail, Bottle-Grass, . 158

Green Meadow-Grass, June-Grass, ete., 147

Guinea-Grass, . . . . . 189

Grouping of, . . . . . 223

Hair-Stalked Meadow-Grass, . . 189

Hair-Grass, Flyaway-Grass, . . . 154

Hard Feseue-Grass, . . . . 152

Hare's-Foot Clover, . . . . 210

Herd's-Grass, Timothy, . . . 144

“ “ Red-Top, etc., . . 152

Hop Clover, Yellow Clover, . . 210

Hungarian-Grass, Hungasian-Millet, . 190

Indian Corn as a Forage Plant, . . 193

Indian-Grass, Wood-Grass, . . 184, 185

" Millet, . . . . . 186

Italian Rye-Grass, . . . . 161

Japan Clover, . . . . 194, 195

Kentueky Blue-Grass, . . . . 139

Kidney Vetch, . . . . . 216

Long-Panicled Manna-Grass, . 167

Lucerne, Value of, ete., . . 210, 211

"Cultivation of, ete., . . 212

Lupine, . . . . . . 218

Lisi of Grasses and Forage Plants, . 220

Maize as a Forage Plant, . . . 193

Meadow Brome-Grass, . . . . 181

Meadow Fescue, . . . . . 151

Meadow Foxtail, . . . . . 146 “ Oat-Grass, . . . . 163

Meadow Soft-Grass, Velvet-Gruss, . 168

Meadow Spear-Grass, Nerved MannaGrass,

Uteaus, • • 148

Mexiean Clover, .. . : . 196, 197

Mexican Muhlenbergia, . . . 156

Millet-Grass, . . . . . 172

Mixture of Grass Seed, . $\quad$. $\quad$. $\quad$. 231

Mixture of Grass Seed for Fine Lawns Frequently Mown,.

Mixture of Grass Seed for Hay and Pasture in Orchards and Shaded Places,

Mixture of Grass Seed for Hay and Pasture Combined,
Grasses and Forage Plants-Continued.

PAGE.

Mlixture of Grass Seed for MIarsby Grounds,

234

Mixture of Grass seed for Meadow Lands, . . . . . . 232

Mixture of Grass Seed for Mowing Lands, . . . . . . 231

Mixture of Grass Seed for Mowing on Light Lands,

Mixture of Grass Seed for Permanent Lawns,

Mixture of Grass Seed for Permanent Pastures, . . . 231, 232, 233

Mixture of Grass Seed for Reelained Peaty Lands, . . . . . 234

Mixture of Grass Seed for Rocky Ilills, 234 " " " "Rotation and Improving the Soil,

Mixture of Grass Seed for Summer Pastnres,

232

Mixture of Grass Seed for Winter Pastures,

$-232$

. . . . 149

Mountain or Northern Red-Top, . . 156

Mountain Rice, . . . . . 167

Nutritive Value of, . . . . . 224

Obtuse Flowered Panic-Grass, . . 189

Obtuse Spear-Grass, . . . . 197

Orchard-Gi'ass, Rough Cock's-Foot, . 147

Pearl Millet, . . . . . . 191

Perennial Rye-Grass, Darnel, . . 161

Pigeon-Grass, Bristle-Grass, . 159, 161

Poverty-Grass, Three-Awned Grass, . 167

Priekly Comfrey, . . . . . 219

Prolific Panic-Grass, . . . . 189

Quaking-Grass; . . . . . 161

Rattlesnake-Grass, . . : . 167

Red-Top, Fine-Top; ete., . . . 152

Rice-Grass, Cut Grass, etc., . . . 152

Reed Canary-Grass, . . . . 171

Rough-Stalked Mleadow-Grass, . . 149

Rush Salt-Grass, . . . . . 168

Sainfoin, . . . . . 215

" Cultivation of, . . . 215

"Giant, . . . . 215

Salt Marsh Grass, . . . . 168

Salt Reed-Grass, . . . . . . 167

Sand-Grass, . $\quad . \quad . \quad . \quad .168$

Sehrader's Grass, . . . . 178, 181

Sheep's Feseue, . . . . . 151

Slender Crab-Grass, . . . . 189

"Spiked-Fescue, . . . . 152

Smut-Grass, . . . 175,176

Southern Bent, . . . . . 154

Spurry, . . . . . . 216

Sweet-Seented Vernal Grass, . . 168

Sylvan Muhlenbergia, . . . . 150

Texas Millet, . . . . . 141 
Grasses and Forage Plants-Continued.

Timothy, or Herds-Grass,

Tall Fescue Grass,

Tall Meadow-Grass, Tall Oat-Grass,

Tall Pauic-Grass, . . . . 189, 192

Tall Red-Top, . . . . 168, 169

Texas-Millet, . . . . . 186

The Florin, Broad-Leared Creeping Bent,

Vanilla-Grass, Seneca-Grass, $\quad 179,181$

Vetch, Tare, . . . . 216

Water-Grass, . $\quad . \quad \ddots \quad .172,174$

Water Spear-Grass, Reed MeadowGrass,

White-Clorer, . . . . 209

Wild Chess, . . . . . . 175

Willdenom's MIuhlenbergia, . . . 157

Wild Fescue, . . . . 175, 177

Wood Meadow-Grass, . . . . 149

Yard-Grass, . . . . . 165

Yellow Oat-Grass, . . . 163

Zigzag Clover, . . . . . 210

Grouping of, . . . . . 223

Ground Limestone and Shells, . . . 65

Guano, American, . . . . . 60

Bat, . . . . . . 60

Peruvian, . . . . 58

Rectified, . . . . . 59

Gypsum, . . . . . . . . 88

Hay Caps, . . . . . . 242

Elevator and Carrier, . . . 244, 247

Forks, . . . . . . 245

Method of Curing, . . . . . 237

Press, . . . . . 247, 248

Pitching Apparatus, . . . . 246

Rakes, . . . . . . 243

Scales, . . . . . . . 415

Stacking, . . . . . . . 245

Storage of, . . . . . . 242

Tedders, . . . . . 240, 241

Time of Cutting Grass for, . . . 237

Mowers, . . . . . . 236, 238

Renovating Grass Lands, . . . 252

Rolling Mowing Lands and Pastures, . 250

Selection of Grass-Seed, . . . . 230

Time to Sow Grass-Seed, . . . . 229

Harrowing, . . . . . . . 100

Height of Buildings, . . . . . 621

Hemp, . . . . . $4 \pi 0$

Cultivation of, . . . . . 470

Harvesting of, . . . . . 471

After-Mlanagement of, . . . . 471

Hessian Fly, . . . . . . . 285

Hill and Drill Planting, . . . . . 311, 315

"Hillhurst," . . . . . . 604
Hilling Cultivator, PAGE.

$\cdot 315$

Hog-House, . . . . . 663

Hops, . . . . . . . 482

Cultivation of, . . . . . . 483

Varieties of . . . . . . 482

Harvesting of, . . . . . . 485

Drying of, . . . . . . 485

Horse, The, . . . . . . . 677

Qualities Desirable in, . . . . 677

Activity, . . . . . 680

A Good Disposition, . . . . 679

Endurance, . . . . . . 679

Intelligence, . . . . . . 679

Strength, . . . . . . 679

The Back, . . . . . 694

Balking of, . . . . . . 772

Black Hawk, . . . . . 728

“ " Junior, • . . . 696

Blinders, Use of, . . . . . 776

Bits, Frosty, . . . . . . 775

The Bones, . . . . . . 691

of the Leg, . . . . 692

Boulonnaise Draft, . . . . 708

Breeding of, . . . . . . 746

Breeding, Disqualifieations for, . . $\quad \mathbf{\$ 4 7}$

Influence of Sire and Dam, . 747

What Horses to Breed, . . 749

Care of Breeding-Mares,. . 751

Care of Young Colts, . . $\quad 752$

Care of Stallions, . . . 751

Chest, The . . . . . . 689

Canadian, The, . . . . $\quad . \quad 720$

Carriage-Horse, . . . . . 681

Cleveland Bay, The, . . . 708, 714

Clipping, . . . . . . 776

Cljdesdale, . . . 686,707

Color in, . . . . . . 698

Colts, Age for Working, . . . 771

Balking of, . . . . 772

Breaking of to Halter, . . . 765

“ Harness, . . 768

Bitting Process, . . 767

“Saddle,. . . . 771

Check Rein, Use of, . . . 774

Natural and Unnatural Position of a Horse's Head, . . . 775

Teaching a Colt to Back, . . 770

Care of,. . . . . . . 752

Castration of, . . . . . 756

How to Prevent a Colt from being Easily Frightened,

Old-Time Method of Managing, . . 760

Present System of Colt Mlanagement, . $\quad 760$

Raising by Hand, . . . . 756

Weaning, . . . . . 755

Colt's Foot at Two Years, . . . 786

Conestoga, The, . . . . 708 
Horse, The-Continued.

Diet for Sick Horses, : • • . 800

Diseases of, and Treatment for, . . 801

Anchylosis, . . . . 803

Blood Spavin, . . . 803

Bloody Urine, . . . . 804

Bog Spavin, . . . . 804

Bone Spavin, . . . . 804

Bots, . . . . . 805

Broken Wind, . . . . 805

Bruises, . . . . 805

Burns, . . . . 805

Capped Ankles, . . . . . 806

“ Elbow, . . . . 806

“ Hock, . . . . 806

Colic, . . . . . 806

“Symptoms of, . . . 807

Contusions, . . . . 805

Corns,. . . . . 808

Cough, . . . . 808

Crib-Biting, . . . 808

Curb, . . . . 809

Diabetes, . . . . 809

Diarrhea, . . . . 810

Distemper, . . . . . 810

Epizootic, . . . 810

Farcy, . . . 812

Fistula, . . . . , 812

Founder, . . . . 813

Fracture, . . . . 813

Galls, . . . . 815

Glanders, . . . . 815

Grease, . . . . 815

Scratehes, . . . . . 815

Gripes, . . . . . 816

Heaves, . . . . 816

-Hematuria,. . . . . 816

Hide Bound, . . . . 817

Inflammation of the Bladder, $\quad 817$

$$
\text { " " " } " \text { "Symp- }
$$

817

Inflammation of the Kidneys,

$\begin{array}{ccc}\text { Inflammation of the Kidneys, " } & 818 \\ \text { " " Lungs, . } 818\end{array}$

Influenza, . . . . . 817

Iteh, . . . . . . 819

Laminitis, . . . . . 819

Lice, . . . . . 819

Lock-Jaw, . . . . 819

Lung Fever, . . . 818

Mange, . . . . . 819

Megrims, . . . . . 820

Navicular Disease, . . . 820

Occult Spavin, . . . . 821

Ophtlualmia, . . . . 820

Overreaehing, . . . . 821

Pink Eye, . . . . . 821

Pneumonia, . . . 818
Horse, The, Diseases and Treatment-Continued.

PAGE.

Horse, The, Diseases and Treatment-Continued.
Poll Evil.

Quarter-Crack, . . . . 822

Ring-Bone, . . . . . 822

Roaring, . . . . . 822

Sand-Crack, . . . 822

Seratches, . . . . 823

Sore Shoulder, . . . . 823

Spavin, . . . . 823

Splent or Splint, . . . . 823

Staggers, . . . . . 823

Străngles, . . . . . 823

Strains or Sprains, . . . 823

String Halt, . . . . 823

Tetanus, . . . . 823

- Thoroughpin, . . . . 824

Toe-Crack, . . . . . 825

Thrush, . . . . . 825

Tread, . . . . . 825

Warts, . . . . . 825

Wind Galls, . . . . 825

Docking,

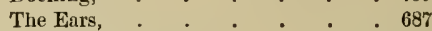

The English Draft, . . . 707, 710

The Eye, . . . . . . 687

The Farm Horse, . . . . . 680

The Foot, . . . . . 693, 785

Gold Dust, . . . . . . 731

The Hambletonians, . . . 711,730

The Head, . . . . . . 687

Henry Clay, . . . . . . 726

The Hind-Quarters, . . . . 694

Hints to Purchasers of, . . . 698

Hints on Driving and Draft, . . 783

The Hock, . . . . . . 693

Lampas, . . . . . . 796

Lampas-Iron, . $\quad . \quad$. $\quad$. 796

The Limbs, . . . . . . 691

The Messengers, . . . . 711, 726

The Morgans, • • • . 711, 727

Mustang Horses, . . . . . 721

The Neck, . . . . . $\quad$ • 686

Nicking, . . . . . . 759

Noted Trotting Horses, . . . 731

American Girl, . . . . 733

Dexter, . . . . 735

Flora Temple, . . . . 732

Goldsmith Maid, . . . 734

Governor Sprague, . . 738, 739

Maud S., . . . . . 736

Rarus, . . . . 735

Santa Claus, . . . . 739

Smuggler, . . . . . 736

St. Julien, . . . . . 736

Thorndale, . . . 740,744

Trinket, ... . . . 740

Orloff, . . . . . . . 716 
Horse, The-Continued.

Orloff Stallion "Sobol," . . . 718

The Pasterns, . . . . . 692

Percheron-Norman, . . 676, 702, 704

" " Grade, . . . 706

Points of, - . . - . 682

Prairie Horses, . . . . . 721

$\begin{array}{cc}\text { Progenitors of Trotting Families, } & 726 \\ \text { Black Hawk, } & .\end{array}$ Gold Dust, . . . . . 731

Henry Clay, . . . . 726

Mambrino Chief, . . . 726

Messenger, . . . . 711, 726

Morgan, Bulrush, . . . 727

“ Justin, . . . . 727

“ Sherman, . . . 727

“ Woodbury, . : . 727

Rysdyk's Hambletonian, . 724, 726

Stockholm's American Star, . 726

Young Bashaw, . . . . 726

Pulse of,

Saddle Gait, . . . . . . 682

Saddle Horse, . . . . . 682

Shetland Ponies, . . . . . 720

Shoeing, . . . . . 785

"Best Method of, . . . 792

Barefooted Horses, . . . . 792

Brittle Hoofs, . . . . . 794

Colt's Foot at Two Years, . . . 786

Horse's Foot,

" " Showing Quarters,

The Charlier System of Shoeing,

The Goodenough Shoe,

The La Fosse System of Shoeing,

Results of Improper Shoeing,

The Shoulder,

Sick Horses, Diet for, .

" " Treatment of,

Skeleton of, .

Stable and Stable Management, .

Care of Harnesses,

How to Clean and Oil a Harness,

How to Clean Harness Plate,

Feeding,

Grooming $\quad * \quad 7$

Stopping the $\cdot{ }^{\circ} \cdot 783$

Watering, .

The Teeth,

Determining the Age of Horses by, .

At the Expiration of the First Year,

At Two Years,

"Three

"Four

" Five

"Six
Horse, The, Teeth of-Continued.

PAGE.

At Seven Years, . . . 697

"Eight " . . . . 697

"Nine " . . . . 697

“Ten and Eleven Years, . 697

After Eleven Years, . . . 697

“ Fourteen ". . . 697

“ Nineteen “. . . 697

“Twenty-one“. . . 697

Temperament in, . . . . . 683

Billions, . . . . 684

Lympathic, . . . 684

Nervous, . . . . 684

Sanguine, . . . . . 684

Thoroughbred, . . . . . 703

Treatment of Old or Disabled Horses, 796

Treatment of Sick Horses, . . . 800

The Trotting Horse of America, . . 725

Best American Trotting Time, . . 719

“ Russian " " . 719

Trotting Record, . . . . . 741

Best Records of Fleetest Running

Horses, . . . . . . 742

Trotting with Double Team, . . 742

Hurdle Races, : . . . . 746

Pacing, . . . . . . . 742

Trotting Under Saddle, . . . 742

Trotting to Wagon, . . . . 742

Horse Hoe, . . . . . . 316, 361

IIorse Power, . . . . . 542, 543

Hybridizing Potatoes, . . . . . 345

Hydraulic Ram, . . . . . 526, 537

Ice House, . . . . . . 669

“ Essentials in Building Ice Houses, 670

“ Plans for, . . . . . $6 \% 0$

“ How to Cut Ice for, . . . 671

“ How to Store Ice in, . . . 6\%2

Ice,-Keeping without an Ice House, . . 6\%:

"How to Keep Small Quantities of in

Summer,

“ How to Make, . . . . . 673

Imphee Sugar Cane, . . . . . . 421

Improved Hoosier Grain Drill, . . . . 274

“ Peach Blow, . . . . . . 333

“ Lima Bean, . . . . . 366

“ Farm Implements Essential, . . 578

Indian Corn, . . . . . . . 37

694 " " Stalks and Leaves of, . . 37

India, Tillage in, . . . . . . 89

694 Indigo, . . . . . . . 464

" Cultivation of, . . . . . 464

695 “ Preparing for Market, . . . 465

695 Incourenience of Farm Buildings, . . 577

695 Inferior Stock, . . . . . 577

696 Insufficient Kelp, . . . . . . 5\%6

696 Interior of Cow Stable, . . . . . 591

697 Influence Exerted by Forests, . . . 517 
Influence Exerted by Forests on Climate,

PAGE. |

518

42,210

Influence of Dwelling on Character,

518 " Value of as a Forage Plant, . . 211

". " the Sire and Dam respectively,

620

Inflammation of the Bladder,

747 M. Goffart's Silos, . . . . . . 496

817 Minguesir, . . . . . . . 73

817 Magnum Bonum, Jack, . . . . . 828

818 McCormick Harvester, . . . . . 262

818 " Dropper, . . . . . 276

Intelligence of the Horse,

" Bowels, .

"Lungs, .

Irrigation,

"Wethods of,

Iron MLwer,

Iron Turbine Wind Mill,

Itch,

Jack, "Magnum Bonum,"

Japan Clover, .

Jersey Bull, Litchfield,

“ Bulls, Group of, .

"Heifers, “

Justin Morgan,

Jute,

"Cultivation of,

"Harvesting of,

Kemp's Manure Spreader,

Keeping Ice Without Ice Houses,

Lack of Economy in Labor,

Lack of Care of Farm Implements,

La Dow's Pulverizing Harrow,

Lampas,

Large Farms of the Country,

Late Beauty of Hebron,

Leached Ashes,

Leaf of Tea, .

Leaves of Trees,

679 Maize, Method of Cultivating for Ensilage, .

117 " Time of Harvesting " " . 501

120 " How to Cut for Silos, , . . . 502

126 Mambrino Chief, . . . . . . 726

236 Mangel Wurzel Beet, . . . 366, 367, 370

525 " " " Cultivation of, . . 367

819 " " " Harresting of, . . 367

“ " Storage of, . . . 367

592 " " and Syrup from Sor-

589 . ghum, . . . 425

588 " " from Beets, . . 428

712,727 Manure Spreader, . . . . . . 30

472 Manuring with Green Crops, . . . . 74

472 " Mowing Lands, . . . . 250

473 Janure Gutters in Stables, . . . . . 659

30

Maple Sugar Making, . . . . . . 432

“ Obtaining Sap for, . 429

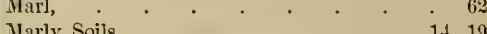

573 Maud S., . . . . . . . . 736

575 Messenger, The, . . . . . 711, 726

100 Metallic Pipes, Injurious Effects of, . . 529

796 Methods of Irrigation, . . . . . 120

796 Methods of Curing Hay, . . . . 237

609 Mills' System of Ensilage, . . . . 499

334 Miscellaneous Fertilizers, . . . . 38

61 Ilixture of Grass Seed, . . . . . . 231

453 for Mowing Lands, . . . . 231

73 for Permanent Pastures, . . . 231

247 for Hay and Pasture Combined, . . 232

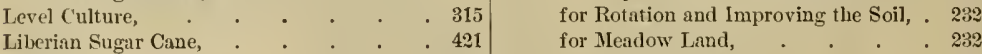

Light in Stables, . . . . . . . 658 for Summer Pasture, . . . $\quad 232$

Lime, 63

for Winter Pasture, . . . . 232

65 for Permanent Pasture, . . 232, 233

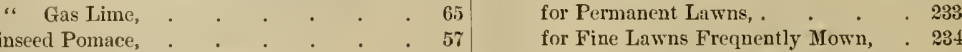

List of Grasses and Forage Plants, . . - $220 \quad$ for Hay and Pasture in Orchards and

Location of Maple Sugar House, . . . 431

" " Farm Roads, . . . . 549

“ " Barns, . . . . . . 643

Locating Main Drains and Tributaries, . . 131

Loamy Soil, . . . . . . 14, 19

Long Blood Beet,

Long Orange Carrot,

Long Yiew Farm, .

"System of Management at,

Lorillard Stock Farm,

\section{4} for Mowing on Light Soils, . . . . 234 for Mowing on Reelnimed Peaty Lands, 234 for Marshy Grounds, . . . . 234 for Rocky Hills, . . . . . 234

Model Barns, . . . . . . 689, 640, 644

Model Farms, . . . . . . . 587 "Deerfoot Farm," . . . . . 595

" "Centrifugal Machines at, 597

" Care of Cows at, . . 597 
Model Farms-Continued.

"Echo Farm,"

Buildings, etc,

Group of Heifers, . . . 588

Interior of Cow Stable, . . 591

Jersey Bull, "Litchfield," . . 592

Stable Mlanagement of, . . 593

The Dairy, . . . 593

" Hillhurst," Management of, etc.,

"Long View Farm,"

System of Management at,

Lorillard's Stock Farm,

"Mountain Side Farm,"

Birns of

“Interior Vier of, Care of Cattle at,

More Capital in Farming,

Morgans, The,

Morris' System of Ensilage,

Most Profitable Crops for,

Moline Stalk-Cutter,

Motive Power for Farms,

"Mountain Side Farm,"

MLuek,

Mule, The,

" Breaking of, .

" Breeding of,

“ Diseases of, .

" Economy of Miule Lahor,

" General Mauagement of,

Mustard,

$$
\text { Shoeing of, }
$$

". Cultivation and Harresting of,

Nature Follows a Course of Rotation,

Natural Positiou of the IIorse's Head,

New Buckeye Table-Rake,

Nicking, .

Night Soil,

Night Soil and Poudrette,

Nishwitz IIarrow,

Nitrogen,

Noted Trotting Horses, .

Nutritive Value of Roots,

$$
\text { " " of Grasses, }
$$

Oats,

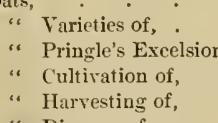

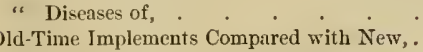

“ " "Farming,

" " Mrethod of Managing Colts,

Oliver Clilled Plow,

Oomseeana Sugar-Cane, . 598

109
Onions, 374

375

376

378

$3 \% 9$

379

379

379

379

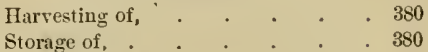

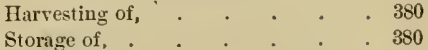

605 Origin and Age of Muck Deposits, . . 20

603 Orloff Trotting Stallipn "Sobol,". . . 718

599, 600 Parsnips, . . . . . . . 371

. 602 " . . . . . . 371

601 " Cultivation of, . . . . 371

580 Peanuts, . . . . . . 460

711 . Cultivation of, . . . . 461

497 " Harvesting of, . . 462

500 . Preparing for Market, . . . . 463

318 Pens, . . . . . . . 478

543 " Cultivation of, . . . . 474

598,600 " Harvesting of, . . . :474

20 Peat, . . . . 62

829 Peat Ashes, . . . . . . . 62

832 Peaty Soils and their Cultivation, . . . 22

831 Plantation Cane-Mill, . . . . . 416

833 Prairie Horses, . . . . . . 721

829 Permanence and Independence of the Farm-

832 er's Tocation, . . . . . . 8

833 Peruvian Guano, . . . . . . . 58

486 Percheron-Norman Stallion, "Romulus," . 702

486 " " " "Eldorado," . 676

man Pleasures of Farm Itife,

Pleasures of Farm Life, . .

279 Piggeries, Plans for, . . . 665

759 Pringle's Excelsior Oat, . . . . . 294

44 Phosphoric Acid, . . . . . . 34

44 Plowing, 91

102 Plow, Subsoil _. . . . . 92

36 . Iron Beam, . . . . . . 93

731 “ Sulky, . . . . . . . 93

859 “ Adamant, . . . . . 94

224 “ Cabbage, . . 94

" Advance Chilled, . . . . . 97

292 “ Oliver, . . . . . . . 97

293 “ Stubble, . . . . . 95

294 " Gilpin Sulky, . . . . 88

295 " Timber Land, . . . . . 88

297 " Cassaday Sulky, . . . . . 88

297 "When to, . . . . . 94

6 " How to, . . . . 95

7 Plowing, Deptl of, . . . . . . 96

760 Profitable Farming, . . . . . . 9

97 Progenitors of Trotting Families of Horses, . 726

421 Poor Teams, . . . . . . . 
Potash,

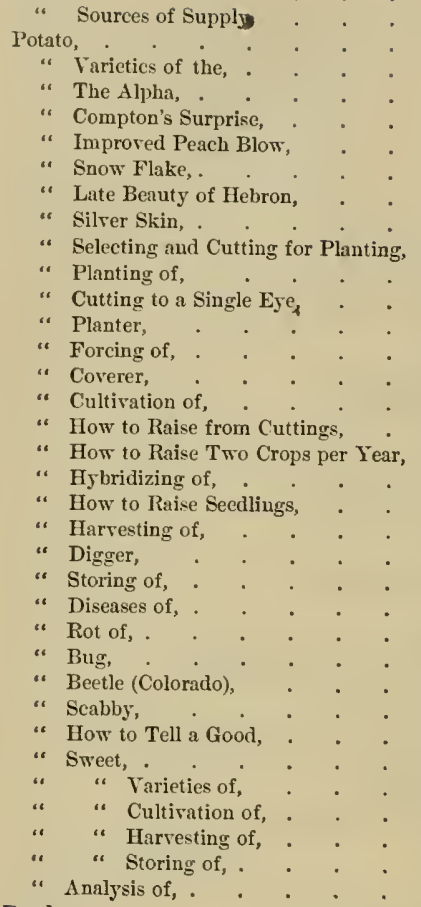

Poudrette

Poultry Houses

Poultry Manure,

Power, Mlotive for Farners,

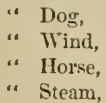

Pulse of the Horse,

Pulverizing and Reducing Bones,

Quinn Farm, The,

Red Clorer,

Relative Cost in Production of Crops,

Repairing Tools,

Rice,

"Cultivation of,

" Harvesting of,

"T Threshing and Milling of,

Roads, Removing Obstructions from,
PAGE.

Roads, Drainage for,

Scene on Grandin Farm,
"Ashes of, . • . 62

PAGE.

Cat. . . . . . 525

Catch-Water for, . . . . . 552

“ Bars for, . . . . . . . . 552

"Repairing of, . . . . . . . 553

"Care of, . . . . . . . . 554

" Objections to Present System of, . . 554

"Grades, . . . . . . . 557

"Farm, . . . . . . 558

Roller, Use of, . . . . . . 103

“ The Field, . . . . . . 103

"How to Construct for Farm Use,. . 104

"Romulus"-Percheron-Norman Stallion, . 702

Roots and Esculent Tubers, . . . . 330

"Cutters, . . . . . . . 363

"Crops, Ridge-Culture for, . . . 373

Rotation of Crops, . . . . . . 107

" " . . . 108

" Nature follows a Course of, . . . 109

"Experiments in, . . . . . 110

" Schemes of, . . . . . 112, 115

Rural Cottage, . . . . . . 627

“ “ Ground plan for, . . . . 627

" "Chamber-floor planfor,. . . 627

Ruta Bagas, Cultivation of, . . . . 361

" " Harresting of, . . . . 362

Rye, . . . . . . . . 326

"Tarieties of, . . . . . . . 288

"Soil for, . . . . . . . 288

"Sowing of, . . . . . . . 288

"Harvesting of, . . . . . . . 288

“ Diseases of, . . . . . . 291

"Thresher, . . . . . . 290

Rysdyk's Hambletonian, . . . 724, 726

Salt, . . . . . . . . 68

Shallot, . . . . . . . . $\quad$. 379

Share's Coulter-Harrow, . . . . 101

Stables, . . . . . . . 659

" Light in, . . . . . . 658

"Ventilation of, . . . . 658

Stacking Hay, . . . . . . 245

Stassfurt Potash Mines, . $\quad$ • $\quad$ • $\quad$. 69

Sea-Weed, . . . . . . 62

Selecting and Cutting Potatoes for Planting,
Sequoia Gigantea,

Sewage as a Fertilizer, . . . . . . 46

Sheep Barn, . . . . . . . 657

"Sherman Morgan," . . . . . . 727

Shetland Ponies, . . . . . . 720

Steam Power,. . . . . . . 544 
Siphon, Use of in Water Supply, .

Spring,

Soils,

" Origin of, .

"Classification of,

" Pure Clay,

" Strongest Clay Soil,

" Clay Loam,

"Loamy,

"Sandy Loam,

" Marly,

"Calcareous,

“Vegetable Molds,

" Clay Soils and their MIanagement,

"Sandy

" Gravelly, .

"Loamy,

“Marly and Calcareous,

" Silt and Alluvial,

" Muck,

" " Origin and Age of Mnek Deposits,.

"Peaty, and their Cultivation,

"Subsoils, .

"Friableness of, .

" Color of,

"Analysis of,

" " Unproductive

"Table of Analysis,

"Soot,

Sorgho, Sugar-Cane,

Special Fertilizers,

Stowe Drains, .

"Heaps,

“ Walls,

Southern Seed Corn,

"Smuggler," .

Stubble Plow,

Subsoil

Sulky

Sugar,

Sugar-Cane, Varieties of,

" Analysis of,

" Cultivation of, .

“ Harvesting of, .

“ Plantation Mills,

“ Extraeting the juice of,

"Defecation or Classifieation of the

$$
\text { “. juice of, }
$$

" Evaporation of the juice of,

" Evaporators,

$$
\text { "Crystallization of Sugar, " }
$$$$
\text { “ Machines for, . . . . } 420
$$$$
\text { “ Seales }
$$

“ Sorgho and Impliee,

Varieties of,

Soil and its Prepa. ration for,
PAGE. PAGE.

540 Sugar-Cane, Sorgho aud Imphee, Planting of, 423

529 " " " Cultivation of, . 424

10 " " " Harvesting, " " 424

10 " " “ Manufacturing Syrup

14 and Sugar from, . . . . . 425

14 . Sugar-Beet, . . . . . . . 426

14 " Cultivation of, . . . . 427

1 " Harvesting of, . . . . 427

14 " . Stotage of, . . . . 427

14 " Manufacturing Sugar from, . . 428

15 Sugar, Maple, . . . . . . 429

15 " Obtaining Sap, . . . . . 429

15 " Loeatiou of Sugar-house, . . . 431

15 " Making, . . . . . . 432

17 " Yield of Maple Trees, . . . 433

19 Summer Fallow, . . . . . . 84

19 Sulphate of Lime, . . . . . . 67

19 Summer Houses, . . . . . . 674

20 Table for Caleulating the Exhaustion of Soil by Crops, . . . . 37

22 Table Showing Materials Removed from the

22. Soil by Various Crops, . . . . 39

24 Table Showing the Nutritive Value of Roots, 359

24 Tare, . . . . . . . 216

$25,27,28$ Tea, . . . . . 452

. 28 " Leaf of, . . . . . . . 453

27, 28 " Leaves, . . . . . . . 454

61 " Varieties of, . . . . . 455

421 " Cultivation of, . . . . 455

79 " Picking of, . . . . . . 456

136 " Curing of, . . . . . . 456

562 " Plant and Root, . . . . 457

565 " Gathering of, . . . . . . 458

500 “ Adulteration of, . . . . . 460

736 Teeth of Horses, Age Determined by, . . 695

98 Teaching a Colt to Back, . . . . 770

92. Treatment of Old and Disabled Horses, . 796

93 " " Sick Itorses, . . . . 800

411 The Trotting Horse of America, . . . 725

411 The Tire Worm, . . . . . . 324

412 "Thorndale,". . . . . . 703

412 Thorouglibred Horse, The, . . . . 703

413 Thresbing, . . . . . . . 281

414, 416 Thresher and Cleaner, . . . . . 282

417 Tile Draining, . . . . . . 130

Tillage, . . . . . . 86

417 " Benefits of, . . . 86, 91

417,418 " Theories Concerning, . 86

419 "Should be Adapted to Nature of Soil, 89

420 " in India, . . . . . . 89

“ for Clay Soils, . . . . . 89

420 “ Benefits to the Soil from Freezing, . 89

415 "

421 " " . . . . . 91 91

421 “ Harrowing, . . . . 100

". Use of the Roller, . . . . 103

423 Timber Culture, . . . . . 516 
Timber Culture, Forests, Influence Exerted by, 517

" $\quad$ " $\quad$ " $\quad$ " " of on Rainfall, 518

“ “ Trees, Varieties of for Planting, .

" ‘. Age and Size of, . . . . 520

“ “ Sequoiı Gigantea, Giant Redwood,

$\begin{array}{lll}\text { " } \quad \text { " Where and When to Plant,. } & 522 \\ \text { " Planting from Sceds, . } & 522\end{array}$

“ . Transplanting, . . . 527

Time of Sowing Spring Wheat, . . . 271

“" " Winter, " . . . 271

Tobacco, . . . . . . 431

“ Tarieties of, . . . . . 436

" Soil for, . . . . . . 436

" Preparation of Plant-beds, . . 43\%

“ Sowing and Weeding, . . . . 438

“ Preparation of Soil for, . . . 441

" Fertilizers for,. . . . . 441

" Ridger, . . . . . . 442

“Transplantiug, . . . . 442

“ Cultiration of, . . . . 443

“ Enemies of. . . . . . 444

" Worming of, . . . . . 445

"Topping, . . . . . 445

“ Suckering, $\quad . \quad+\quad . \quad+446$

“ Cutting, . . . . . . 446

" Curing, . . . . . . 447

" Stripping, . . . . . 448

“ Assorting, . . . . . 448

" Packing, . . . . . . 449

“ Cultivating for Seed, . . . 449

Tobacco Stems for Fertilizers, . . . . 73

Tool House and Repair Shop, . . . 668

"Trinket,"

Trotting Records,

Turf Drains,

Turnips, ('ultivation of, etc.,

Two Crops of Potatoes a Year,

Unnatural Position of Horse's Head.

Underdrainage,

Upland Cotton,

Use of Birds in Protecting Corn,

Use of Poor Tools,

Uses of Cotton Seed,

Various Crops for Green Manures,

Veutilation of Dwelliugs,

$$
\text { " "Stables, }
$$

Vetch, The,

Victor Cane Mill,

Wagon House, The,

$$
\text { “ Jack, The, . }
$$

Warmth in Dwellings,
Wastes and Wants of the Farm,

PAGE.

Better Knowledge of Farming and Less

Drudgery, . . . . . 578

Waste of MIanure, . . . . . . 572

“ Lack of Economy in Labor, . . . 573

“ Slovenly Management, . . . . 5it

“ Doing Work Orer-nicely, . . . . 574

"Use of Poor Tools, . . . . . 5it

" Borrowing Tools, . . . . 575

“ Lack of Care of Farm Implements, . 575

“ Repairing Tools, . . . . . 576

“ Insuffieient Help, . . . . . 576

“ Poor Teams, . . . . . . 5r

“ Inferior Stock, . . . . . $5 \%$

“ Inconvenience of Farm Buildings, . 577

“ Improved Farm Implements Essential, 578

“ Systematic Management Essential, . 580

" More Capital in Farming, . . . 580

Water Closets, . . . . . . 638

Water Supply for the Farm, . . . . 52\%

“ Metallic Pipes, Injurious Effects of, . 529

“ Springs, . . . . . . 529

“ Brooks, . . . . . . 529

"Cisterns, . . . . . . 531

“ Wells, . . . . . . . 534

" Hydraulic Rams, . . . . . 537

“ Wind Mills, . . . . . . 537

“ Wind Engines, . . . . 538, 539

" Siphon, Use of, etc., . . . . 540

Watering IIorses, . . . . . . 780

Weaning Colts, . . . . . . 755

Wells, . . . . . . . . 534

" Artesian, . . . . . . 535

“ Driven, . . . . . . 535

“. Tube, . . . . . . $58 \pi$

What Horses to Breed, . . . . . . 749

770 What Lands Require Irrigating, . . . . 128

137 Wheat, . . . . . . . . 261

358 " Varieties of, . . . . . 261

345 " Champlain, . . . . . 266

“ Defiance, . . . . . . 266

775 " Preparation of Soil for, . . . 267

127 " Fertilizers of Soil for, . . . 268

384 " Selection of Seed for, . . . $2 \pi 0$

$325,326 \quad$ " “ Time of Sowing Spring Wheat, . 271

574 “ " " " " Winter " . 271

401 " Quantity Sown to the Acre, . . 271

" Sowing, . . . . . . 273

“ Grain Drills, . . . . . . 274

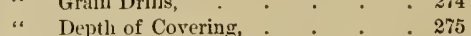

“ Dropper, . . . . . . 276

" After-Culture of Wheat, . . . 277

.. Ilarvesting, . . . . . 2 277

.. Self-Binding Harvester, . . . 278

“ Table Rake, . . . . . 279

". Thresher and Cleaner, . 280, 281, 282

“. Threshing, . . . . . 281 
Wheat, Diseases and Inseet Enemies of,

PAGE.

" “ Smut, . .

" " Rust,

" Inseet Enemies of,

282 Wire Fence,

I'A 7 E.

$$
\text { ". }
$$

" Chinch-Bug",

" Rocky Mountain Locust,

" " Hessian Fly,

When to Irrigate

$\cdot \cdot \cdot \cdot 324$

Wood House, The, . $\quad 60$

284 "Woodbury, . . . 668

When to Plow,

Worming Tobaceo,

Wlite Clover,

Wind Engine,

94 Yam, Cultivation of, etc., . . . . 357

" “ at Rest,

Windows,

" in Motion,

Wind Mills, Use of, ete.

209 " Chinese, Cultivation of, etc., . . . 357

538 Yellow Clover, . . . . . 210

540 Yicld of Maple Trees, . . . . . . 433

629 Zigzag Clover, . . . . . . 210 




\section{)}

(3) 355

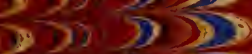

3) 3. 20

$32003,23$.

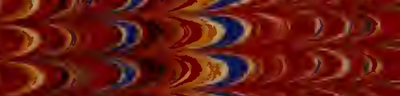

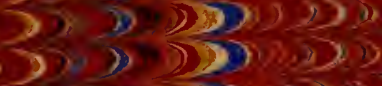

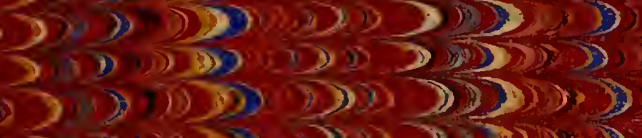

$>3.3$

3) $x, 23 y 3$

D) 39

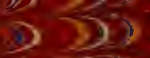

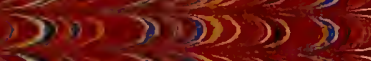

(3) 291

D) 32:

2932

D) 213

2)

3

2) 32012

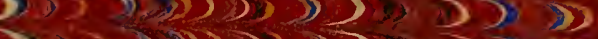

29302

2) $(20) 20$

2932393

D) 20129

(2) $12>10$

()) 20.07

203, is

30 (D) 12

i)) 5939,1013

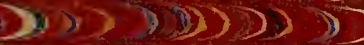

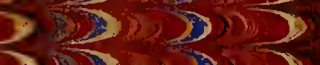

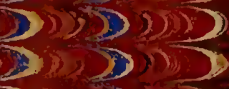

$>20$

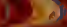

)

(3)

(3) 11

2130.9

3) 303 
If

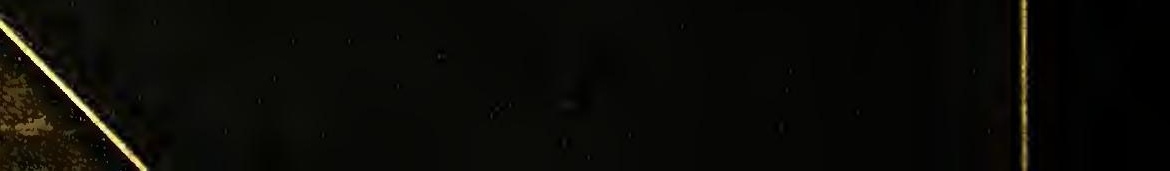

